This electronic thesis or dissertation has been downloaded from the King's Research Portal at https://kclpure.kcl.ac.uk/portal/

\title{
A Study of the Deposition and Distribution of Copper Alloy Vessels in Roman Britain
}

\section{Lundock, Jason Richard}

\section{Awarding institution:}

King's College London

The copyright of this thesis rests with the author and no quotation from it or information derived from it may be published without proper acknowledgement.

\section{END USER LICENCE AGREEMENT}

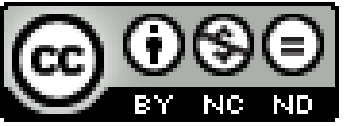

Unless another licence is stated on the immediately following page this work is licensed

under a Creative Commons Attribution-NonCommercial-NoDerivatives 4.0 International

licence. https://creativecommons.org/licenses/by-nc-nd/4.0/

You are free to copy, distribute and transmit the work

Under the following conditions:

- $\quad$ Attribution: You must attribute the work in the manner specified by the author (but not in any way that suggests that they endorse you or your use of the work).

- $\quad$ Non Commercial: You may not use this work for commercial purposes.

- $\quad$ No Derivative Works - You may not alter, transform, or build upon this work.

Any of these conditions can be waived if you receive permission from the author. Your fair dealings and other rights are in no way affected by the above.

Take down policy

If you believe that this document breaches copyright please contact librarypure@kcl.ac.uk providing details, and we will remove access to the work immediately and investigate your claim. 


\title{
A Study of the Deposition and Distribution of Copper Alloy Vessels in Roman Britain
}

\author{
Thesis by Jason Lundock for King's College London in completion of degree Doctor of \\ Philosophy in Classics Research
}

July 2014 


\begin{abstract}
The following thesis surveys the deposition and distribution of copper alloy vessels in Britain during the Roman period and then applies this data to the study of culture change and the construction of identity in the province during this time. The principal research strategy was to categorize the objects from published sources within four groups based on Depositional Context and to then examine these data-sets for patterns in geographic and temporal distribution, object form and decoration as well as patterns among the findspots where these objects were discovered. The copper alloy vessels themselves are classified using forms and typologies familiar from previous scholarship, though a new system for classifying handled pans was found necessary and is introduced in this thesis. Multiple patterns emerged within Depositional Contexts, Site Types and regional distribution relating to vessel selection and decoration which indicate a variety of practice by diverse peoples. This analysis argues that the principal function of copper alloy vessels in Roman Britain was for ablutions, whereas it has been previously proposed that most vessels were used for dining or drinking services. Additionally, the spread of copper alloy vessels was found to be so wide across the province that it was determined that this commodity was utilized and adapted by much of the population of Britain. The conclusions were then applied to the principal paradigms currently ascendant in characterizing culture change in the province. It was found that the predominant theories, which largely rely upon a dualistic view of cultural aggression and resistance, are insufficient to characterize the complex interaction between cultures in Britain and the development of an integrated and fluid material culture as expressed through the repertoire, deposition and distribution of copper alloy vessels evident during the Roman period in Britain.
\end{abstract}




\section{Acknowledgements}

Anyone who has undertaken doctoral research will know that it is not so much a singular effort as the development of one person through the influence and help of many others. Though it is unlikely for me to do justice to the many people who have helped me along the way, I would like to attempt by making the following acknowledgements.

First I must thank my parents, who have stood by me through the process and have proven a great source of emotional and psychological support throughout my educational career. Their reassurance has equipped me with the strength and perseverance to undertake such a work and the current thesis would have been impossible without their belief in me.

I would like to thank my primary supervisor, John Pearce, who made great effort to mould and focus my often rather eclectic and divergent thoughts into something that approaches coherence. Through the process of research and writing up, he gave me irreplaceable guidance and was understanding to the pressures one faces when undertaking such work; he proved to be both a mentor and a friend throughout my doctoral work. I would also like to thank my secondary supervisor, Will Wootton, for his advice and input during the writing process.

There are a number of individuals who aided me through conversation about Roman small finds, offered research advice or helped me with access to the material at their institutions. Of these I would like to extend particular thanks to Ralph Jackson and Richard Hobbs of the British Museum, Francis Grew of the Museum of London, Andrew Birley of the Vindolanda Trust, Felicity Cobbing of the Palestine Exploration Fund, Aaron Irving of Murray State University, Fraser Hunter of the National Museums of Scotland, Sally Worrell of the Portable Antiquities Scheme, Robert Webley (then Portable Antiquities Scheme), Juan Chacon-Fossey (then Archaeological Services and Consultancy) and James Gerrard (then Pre-Construct Archaeology). I would particularly like to thank Sam Moorhead and Philippa Walton, who supervised work that I undertook at the British Museum and took a genuine interest in my doctoral research and my professional development, offering advice and input. Their guidance has been of the greatest help to me and their friendship has proven invaluable. I also owe a great debt of gratitude to Katherine Robbins, for her help in constructing the maps that feature in this thesis. 
I was also greatly helped through conversation with my fellow doctoral researchers and would like to take a moment to acknowledge the particular help of Caroline Barron, Alessandra Esposito, Gabrielle Florence, Victoria Gyori, Yukiko Kawomoto, Alexandra Markou, Vladimir Mihajlovic, Shaun Mudd, Darrell Rohl, Kaja Stemberger, Federico Ugolini, Jeff Veich, David Walsh, Lily Withycombe, Ian Worthington, Bobby Xinyue, and Andrea Zerbini,. Most of all among these, I would like to thank Justin Yoo. His friendship proved invaluable during my doctoral research and he never hesitated to offer his help either in the academic or personal fields of life. His friendship and tenacity to make sure I did the best work I could achieve proved instrumental in bringing this project to fruition.

I would also like to thank the following people for their advice and friendship throughout my doctoral process: James Bedillion, Dewayne Bowlers, Heather Bright, Philip Eldred, Alexander Koefoed, Michael Skeide, Hugh Somerville, Noel Villanueva, and Brandon Ziegler. They helped me cope with a great deal of the stress that accompanies such an undertaking and even would listen with feigned interest when I discussed details of my personal research, a true sign of devotion.

Finally, I would like to thank Rachel Greenberg, without whose aid and companionship I would have been unable to have completed this work. She not only put up with the insanity one inevitably goes through whilst undertaking a doctorate, but she offered understanding and aided me with research, imaging, proof-reading and any number of other tasks throughout the project. She acted as a sounding board whenever I chose to subject her to my developing ideas concerning my research and bore that burden bravely. I could not have asked for a better friend and companion to see me through the doctoral process and her support made possible the following thesis. 


\section{Chapter List}

Chapter 1- Introduction and Review of Previous Research Concerning Roman Britain and Copper alloy Vessels

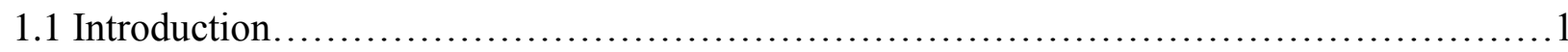

1.2 Review of Historiographic and Theoretical Debates....................................

1.3 Review of previous archaeological investigation relating to copper alloy vessels...........10

1.4 Current understanding of Copper Alloy vessels in Roman Britain.........................16

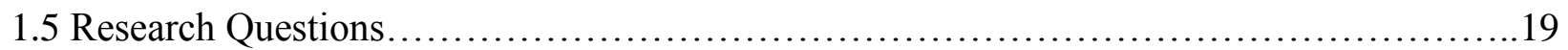

Chapter 2: Materials, Methods and Approaches

2.1 Definition and Parameters of Material under Investigation............................28

2.2 Recording Frameworks Explanatory Notes.....................................40

2.3 Data Collection and Analysis................................................... 46

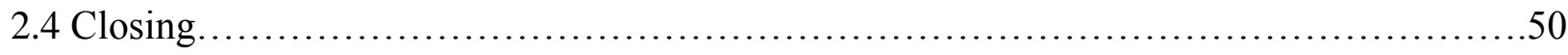

Chapter 3: Structured Deposits

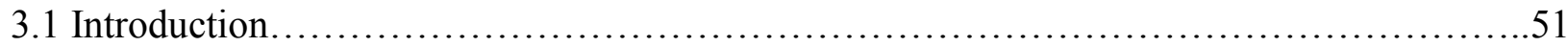

3.2 Geographic Survey of Structured Deposits.....................................51

3.3 Patterns in the depositional circumstances of Structured Deposits......................88

3.4 Form and Typological Patterns in Structured Deposits............................... 100

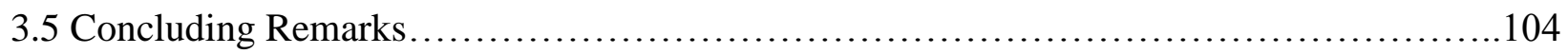

Chapter 4: Grave Deposits

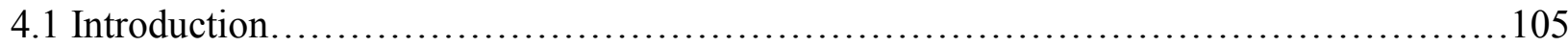

4.2 Geographic Survey of Grave Deposits........................................ 108

4.3 Chronology and its relation to ritual practice and object selection.......................158

4.4 Discussion of Decoration in Grave Deposits..................................... 168

4.5 Associated Finds, Functionality and Burial Status................................. 175

4.6 Concluding Remarks..................................................... 180

Chapter 5: Site Finds

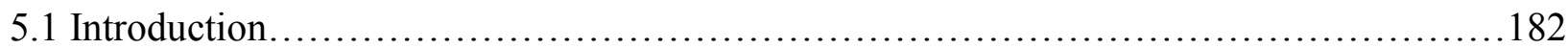

5.2 Geographic Survey of Site Finds............................................ 182

5.3 Site Type and Chronological Comparison of Site Finds Distribution.....................202

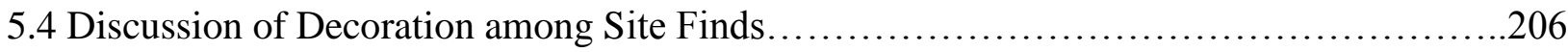




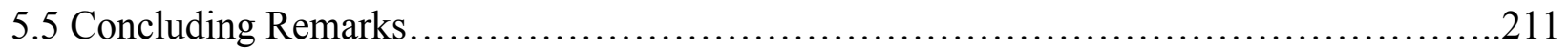

Chapter 6: Single Finds Reported through the Portable Antiquities Scheme

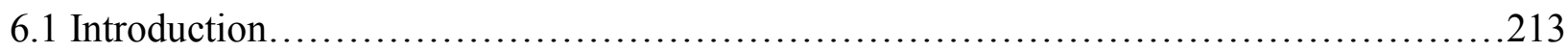

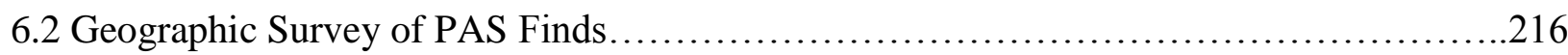

6.3 Associated Finds and Contextualizing PAS Finds..................................227

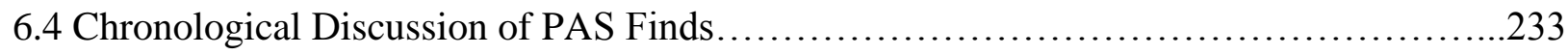

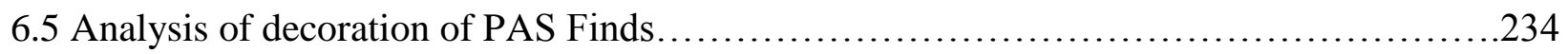

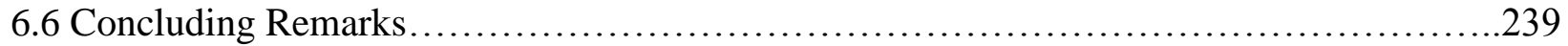

Chapter 7: Synthetic Discussion Addressing Thesis Questions 1-3

7.1 How can individual depositional contexts be characterised and interpreted?....................241

7.2 What is the distribution of copper alloy vessels across time and space?...........................256

7.3 How were copper alloy vessels used and consumed?.................................................264

Chapter 8: Copper Alloy Vessels and Identity in Roman Britain

8.1 Reflection on thesis and future understanding of copper alloy vessels..................280

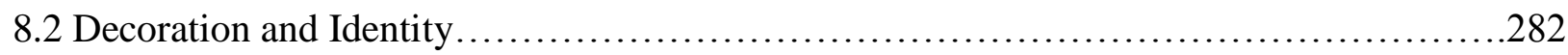

8.3 Copper alloy vessels and the present understanding of identity in Roman Britain...........294

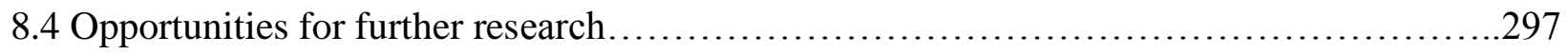

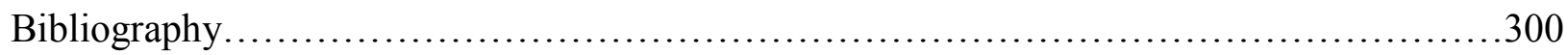


Appendix I: Copper Alloy Vessels from Roman Britain................................

Appendix II: Structured Deposits of Copper Alloy Vessels in Roman Britain...............XXXII

Appendix III: Grave Deposits of Copper Alloy Vessels in Roman Britain...................XL

Appendix IV: Site Finds of Copper Alloy Vessels in Roman Britain.......................XLVI

Appendix V: Single Finds of Copper Alloy Vessels from Roman Britain reported through the Portable Antiquities Scheme.............................................

Appendix VI: Graves from Brougham, Cumbria with Copper Alloy Vessels...............LXXII

Appendix VII: Romano-British Graves in Essex with Copper Alloy Vessels...............XXXI

Appendix VIII: Romano-British Grave Deposits from the South Eastern Counties of England containing Copper Alloy Vessels........................................XXVIII

Appendix IX: Romano-British Site Finds of Copper Alloy Vessels from the South Eastern Counties of England ...........................................XXX

Appendix X: Single Finds of Copper Alloy Vessels of Roman date reported through the Portable Antiquities Scheme in East Anglia and the South East of England.... LXXXII Appendix XI: Finds associated with Copper Alloy Vessels of Roman date reported through the Portable Antiquities Scheme..........................................

Appendix XII: Inscriptions on Copper Alloy Vessels from Roman Britain.............. LXXXVII

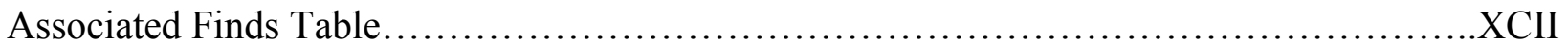




\section{List of Figures}

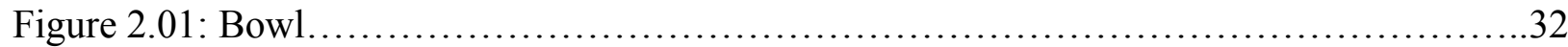

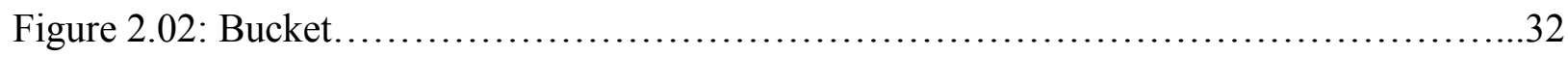

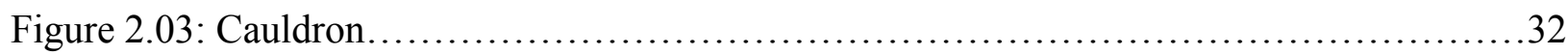

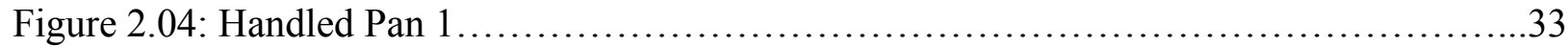

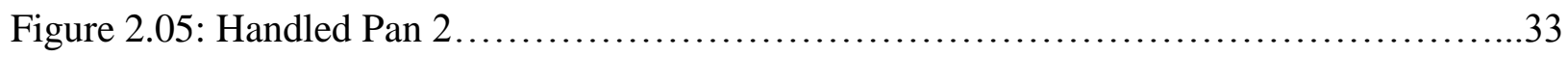

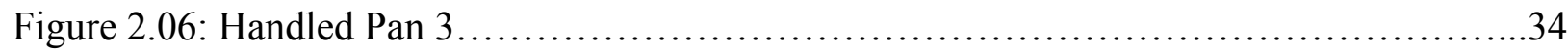

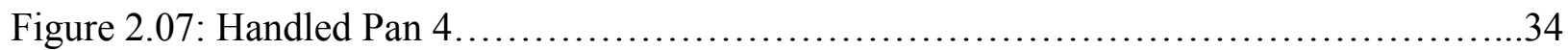

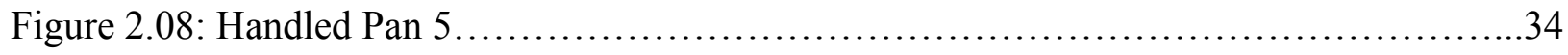

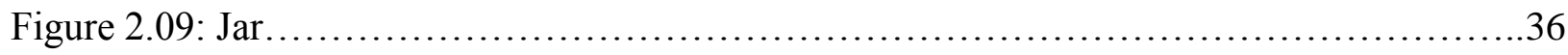

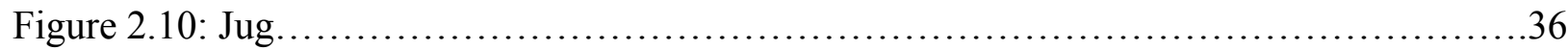

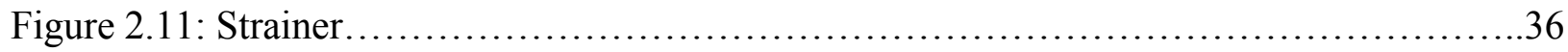

Figure 2.12: Bassin á bord godronne.......................................................

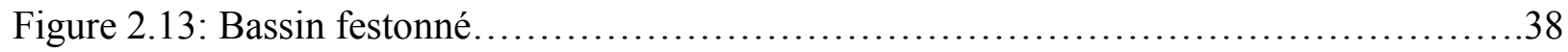

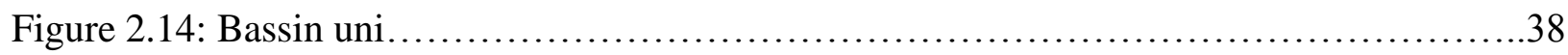

Figure 2.15: Hemmoor Bucket........................................................... 38

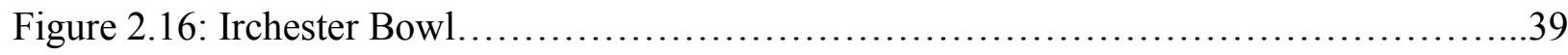

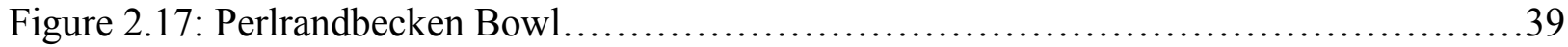

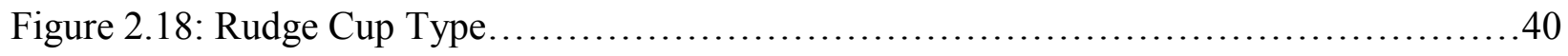

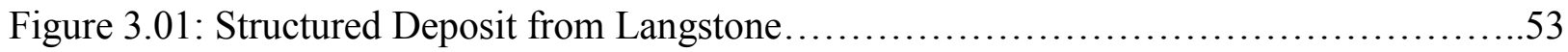

Figure 3.02: Structured Deposit from Coygan Cave .........................................54

Figure 3.03: Structured Deposit from Corbridge on display in the British Museum..............59

Figure 3.04: Jar from Structured Deposit at Whittington.......................................61

Figure 3.05: Fluted Bowl from Knaresborough..........................................64

Figure 3.06: Fluted Bowl from Sutton Hoo Anglo-Saxon Burial...............................64

Figure 3.07: Handled Pan 2 from Structured Deposit at Linton...............................66

Figure 3.08: Votive Bowl from South Shields.............................................67

Figure 3.09: The Ribchester Hoard...................................................... 71

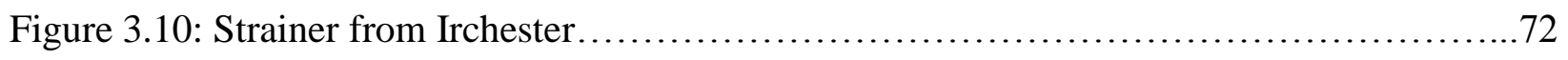

Figure 3.11: Great Dish from Mildenhall.................................................. 73

Figure 3.12: Handled Pan 2 from Oulton.................................................. 
Figure 3.13: Structured Deposit from Drapers' Gardens, London.........................80

Figure 3.14: Zoomorphic mount from Drapers' Gardens, London........................81

Figure 3.15: Bowl from Wotton................................................ 84

Figure 3.16: Structured Deposit from Kingston Deverill................................86

Figure 3.17: Personification of River Danube on Trajan's Column...........................92

Figure 3.18: Regional distribution of aquatic deposits of copper alloy vessels in

Roman Britain............................................................ 93

Figure 3.19: Distribution of large Late Roman Structured Deposits of copper alloy vessels......97

Figure 3.20: Frequency of forms across Structured Deposits of copper alloy vessels in

Britain.

Figure 3.21: Prevalence of Handled Pan 2s and large Late Roman basins in the Structured

Deposits of copper alloy vessels in Roman Britain.............................. 102

Figure 4.01: Welwyn Garden City Late Iron Age Burial............................... 106

Figure 4.02: Regional distribution of sites with AE vessels as Grave Deposits...............109

Figure 4.03: Anthropomorphic Handled Pan 1 from Welshpool............................111

Figure 4.04: Detail of anthropomorphic handle medallion from Handled Pan 1 from

Welshpool.............................................................. 111

Figure 4.05: Zoomorphic Handled Pan 1 from Welshpool...............................112

Figure 4.06: Anthropomorphic jug handle from Welshpool............................... 112

Figure 4.07: Zoomorphic hanging vessel mount from Welshpool........................113

Figure 4.08: Jug from Llantilio Pertholey ....................................... 114

Figure 4.09: Vessel forms in cremation burials at Brougham..........................117

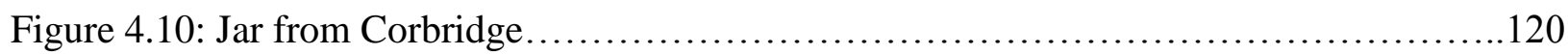

Figure 4.11: Objects from graves at Turners Hall Farm..............................123

Figure 4.12: Jug with Triton from Grave 1 at Turners Hall Farm...........................124

Figure 4.13: Jug with Minerva from Grave 1 at Turners Hall Farm......................... 124

Figure 4.14: Strainer bowl from Grave 1 at Turners Hall Farm.............................125

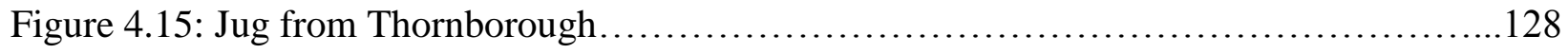

Figure 4.16: Jug from Thornborough............................................ 128

Figure 4.17: Cremation 25 from Stansted.......................................... 130

Figure 4.18: Jug from Cremation 25 at Stansted.....................................131 
Figure 4.19: Handled Pan 1 from Cremation 25 at Stansted............................132

Figure 4.20: Handled Pan 2 from Cremation 25 at Stansted................................133

Figure 4.21: Copper alloy vessel refitted as lock-plate from Cremation 24 at Stansted........134

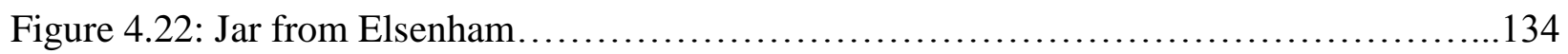

Figure 4.23: Handled Pan 1 from Warrior's Burial at Stanway.......................... 136

Figure 4.24: Jug from Warrior's Burial at Stanway..................................136

Figure 4.25: Detail from strainer bowl in Doctor's Burial at Stanway.......................137

Figure 4.26: Handled Pan 2 from Doctor's Burial at Stanway........................... 138

Figure 4.27: Zoomorphic jug from Rivenhall....................................... 142

Figure 4.28: Child's Burial from Colchester........................................ 143

Figure 4.29: Handled Pan 1 from Grave 6260 at Tollgate.................................146

Figure 4.30: Jug handle from Grave 6260 at Tollgate................................. 146

Figure 4.31: Cauldron from grave 6020 at Tollgate................................ 147

Figure 4.32: Floral decoration on Handled Pan 1 from grave 6635 at Tollgate.................148

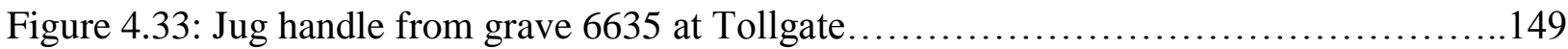

Figure 4.34: Jar with negroid faces from Sittingbourne, Bayford......................... 150

Figure 4.35: Detail of anthropomorphic handle medallion from Sittingbourne, Bayford........151

Figure 4.36: Jug handle depicting madness of Ajax from Sittingbourne, Bayford.............152

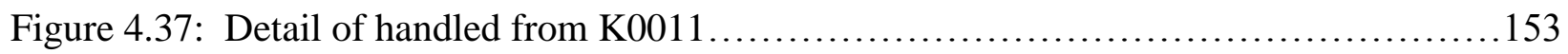

Figure 4.38: Jug from Neupotz.............................................. 153

Figure 4.39: Layout of grave from Lullingstone Roman villa...........................154

Figure 4.40: Numbers of copper alloy vessels across Site Types..........................157

Figure 4.41: Chronology of Grave Deposits....................................... 158

Figure 4.42: Funerary altars from the Appian Way now on display at the

Baths of Diocletian, Rome.................................................. 160

Figure 4.43: Funerary altar on display in the Museo de Arte Romano, Merida.................160

Figure 4.44: Funerary altar on display in the British Museum............................ 161

Figure 4.45: Funerary relief on display in the Kunst Historisches Museum, Vienna...........162

Figure 4.46: Ivory relief of Pilate washing his hands after the judgement of Jesus............162

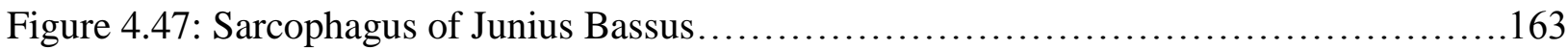


Figure 4.48: Illustration from 4th century manuscript of the Aeneid, currently in the

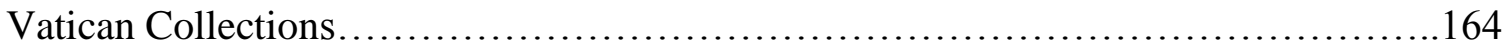

Figure 4.49: Graves with both HP1 \& jugs in Roman Britain over time..................... 164

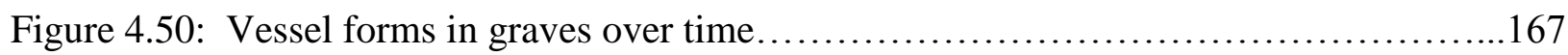

Figure 4.51: Vessel forms deposited in Romano-British graves........................ 168

Figure 4.52: Bust on handle terminals for Handled Pan 1s in Romano-British graves..........169

Figure 4.53: Enamelled rooster from Cople in Bedford.................................170

Figure 4.54: Graves including Associated Finds...................................... 176

Figure 5.01: The Rudge Cup............................................... 183

Figure 5.02: Jug from Carlisle depicting scene of sacrifice............................ 188

Figure 5.03: Site Finds by form in Wales, Hadrian's Wall and the Northern Counties of

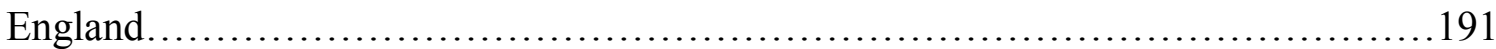

Figure 5.04: Handled Pan 2 from Prickwillow ..................................... 194

Figure 5.05: Vessel forms from the Midlands..................................... 194

Figure 5.06: Site Finds by form from St.Albans/Verulamium.........................195

Figure 5.07: Site Finds by form from London..................................... 197

Figure 5.08: Site Find forms from Colchester.................................... 198

Figure 5.09: Site Finds from all regions...........................................201

Figure 5.10: Distribution of Site Finds by Settlement type...........................202

Figure 5.11: Site finds forms from Urban sites....................................203

Figure 5.12: Site Finds forms from Military sites ..................................203

Figure 5.13: Site Find forms from Rural Settlements..................................204

Figure 5.14: Site Find forms from Rural Unknown sites.............................204

Figure 5.15: Objects with decoration in SF data-set................................206

Figure 5.16: Iconography of Handled Pan 1s in Site Finds data-set........................207

Figure 5.17: Decorated object in the Structured Deposits, Grave Deposits and

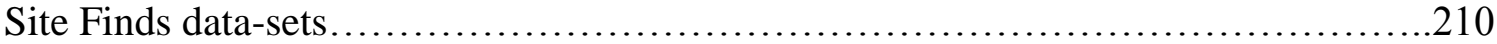

Figure 6.01: Numbers of copper alloy vessels reported as Single Finds through the PAS......216

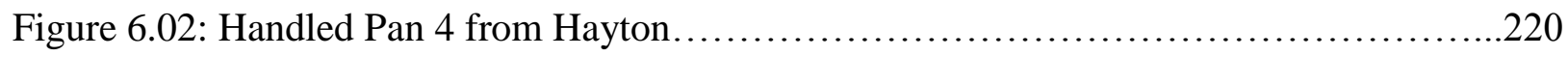

Figure 6.03: Bovine vessel spout from Bilsby in Lincolnshire..........................221

Figure 6.04: Anthropomorphic mount from Caistor.................................. 222 


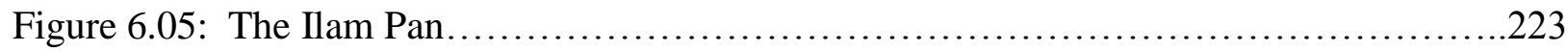

Figure 6.06: Vessel forms of Single Finds PAS in the Midlands............................224

Figure 6.07: Vessel forms for East Anglia and the South East reported as Single Finds

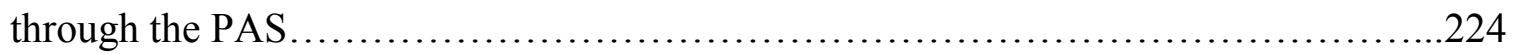

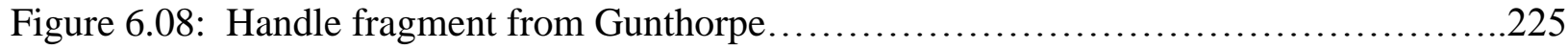

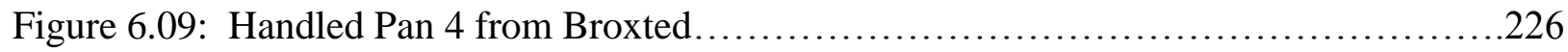

Figure 6.10: Sites with numbers of associated finds reported through the PAS................229

Figure 6.11: Associated finds from assemblages over 100 objects..........................231

Figure 6.12: Anthropomorphic mount from Clothall.....................................235

Figure 6.13: Bacchic mount from Letcombe Regis........................................235

Figure 6.14: Anthropomorphic mount from Fisherwick...................................235

Figure 6.15: Reclining banqueter mount from Bembridge, Isle of Wight...................236

Figure 6.16: Bovine hanging vessel mount from Wymondley, Hertfordshire..................237

Figure 6.17: Detail of zoomorphic spout from Turners Hall Farm..........................237

Figure 6.18: Pelta shaped vessel foot from Gaddesby in Leicestershire.......................239

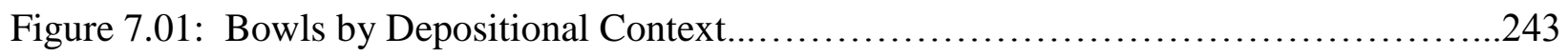

Figure 7.02: Buckets by Depositional Context.................................................

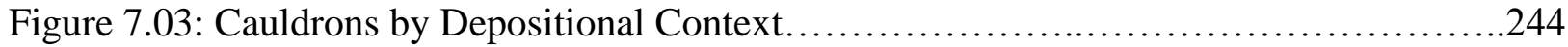

Figure 7.04: Handled Pan 1 by Depositional Context......................................245

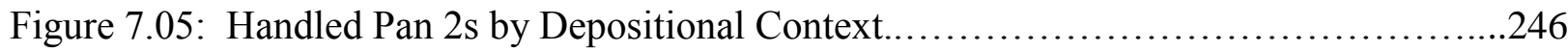

Figure 7.06: Handled Pans 3-5 by Depositional Context....................................247

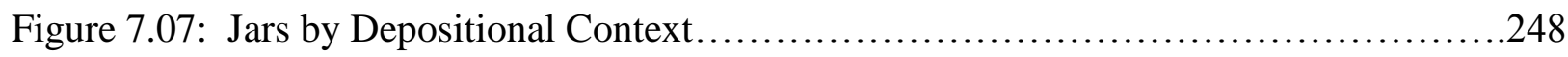

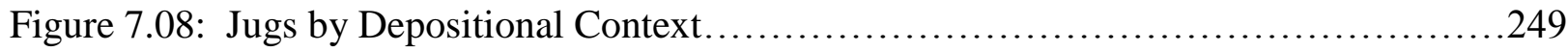

Figure 7.09: Strainers by Depositional Context............................................... 251

Figure 7.10: Undiagnostic vessel fragments by Depositional Context.......................251

Figure 7.11: Broad distribution of copper alloy vessels by geographic region................257

Figure 7.12: Numbers of copper alloy vessels by Site Type.......................................

Figure 7.13: Chronological distribution of copper alloy vessel forms in Roman Britain........263

Figure 7.14: Numerical distributions of copper alloy vessels by form in Urban Site Types.....266

Figure 7.15: Numerical distributions of copper alloy vessels by form in Military Site Types...267 
Figure 7.16: Numerical distributions of copper alloy vessels by form in

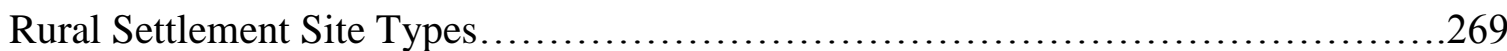

Figure 7.17: Numerical distributions of copper alloy vessels by form in

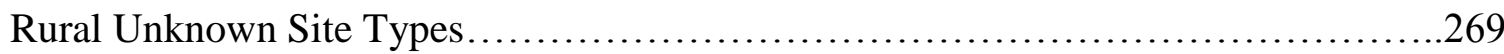

Figure 7.18: Numbers of objects by Depositional Context.................................276

Figure 7.19: Comparative numbers of decorated and undecorated vessels by region............277

Figure 7.20: Comparative numbers of decorated and undecorated vessels from

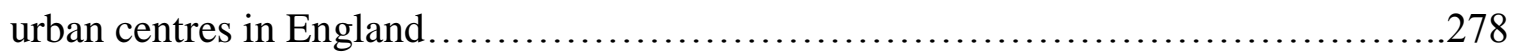

Figure 8.01: Bucranium architectural relief on fragment outside the

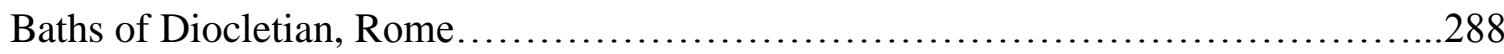

Figure 8.02: Bucranium altar relief on display in the Museo de Arte Romano, Merida........288

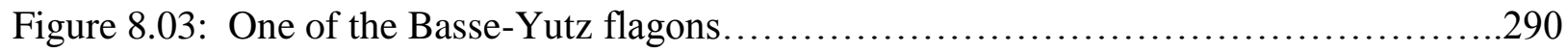

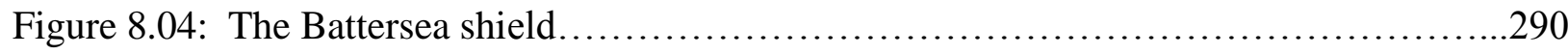

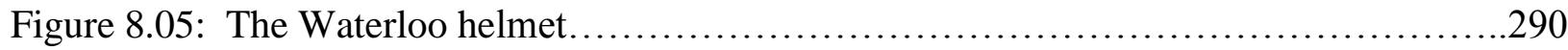

Figure 8.06: Comparative frequency of copper alloy vessels with inscriptions from

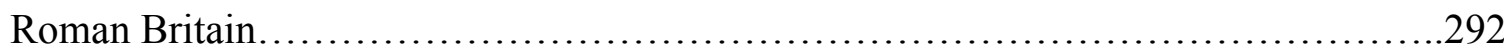




\section{List of Tables}

Table 1a- Examples of usage for Latin terms patera and trulla and their equivalences in this thesis

Table 3a-Structured Deposits from Wales............................................51

Table 3b-Structured Deposits of the hinterland of Hadrian's Wall...........................58

Table 3c- Structured Deposits of the northern counties of England........................62

Table 3d- Structured Deposits from the Midlands......................................71

Table 3e- Structured Deposits from East Anglia........................................ 76

Table 3f- Structured Deposits from the south west of England.......................... 84

Table 4a- Grave Deposits from Wales........................................... 110

Table 4b- Grave Deposits from the Midlands........................................ 121

Table 5a- Site Finds from the South West counties of England............................ 186

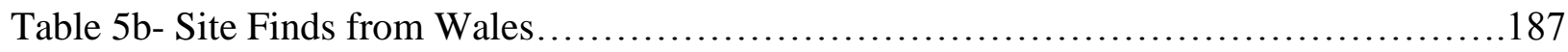

Table 5c- Site Finds from Cumbria and Northumberland..................................189

Table 5d- Site Finds from the Northern Counties of England..............................190

Table 5e- Site Finds from Midlands.............................................. 193

Table 5f- Site Finds from Colchester and London..................................... 196

Table 6a- PAS finds from Wales and the southwest of England ..........................218

Table 6b- PAS finds from the northern counties of England.............................218

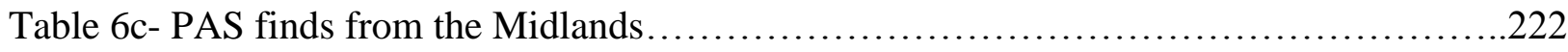

Table 6d- Vessel findspots with more than one hundred associated finds of Roman date

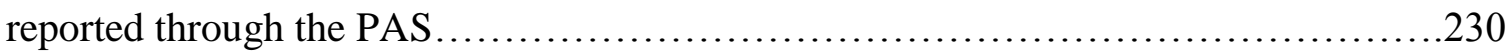




\section{List of Maps}

Map 1: Structured Deposits of copper alloy vessels in Roman Britain........................52

Map 2: Grave Deposits of copper ally vessels from Roman Britain....................... 108

Map 3: Site Finds of copper alloy vessels from Roman Britain......................... 185

Map 4: Single Finds of copper alloy vessels reported through the PAS ....................217

Map 5: Urban sites with AE vessels...............................................260

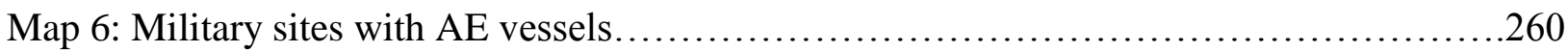

Map 7: Rural Settlements sites with AE vessels....................................260

Map 8: Rural Unknown sites with AE vessels......................................260 


\section{Chapter 1- Introduction and Review of Previous Research Concerning Roman Britain and Copper alloy Vessels}

\section{$\underline{1.1 \text { Introduction }}$}

The following thesis collects together data concerning copper alloy vessels from Roman Britain (with its main focus on present day England and Wales) and relates the evidence they present to prevailing theories of consumption, identity and culture change in Britain during this time. The aims of this thesis are to collect a catalogue of copper alloy vessels from England and Wales, categorise them by form, typology, context, chronology and geographic distribution, offer interpretations concerning their cultural associations, manners of consumption, functionality and development over time before commenting upon their value as small finds material reflective of culture change more broadly within Britain during the Roman period. Copper alloy vessels from the Roman period in Britain have not been the subject of focused scholarly study for over 50 years and have never had a focused examination in English. This thesis not only rectifies this gap in the literature, but proceeds to directly apply this data analysis to the greater theoretical discourse of the development of material culture in Britain during the Roman period, thereby demonstrating the validity and importance of small-finds studies to the larger historiographic and theoretical discourse. This is also the first study of copper alloy vessels in Britain to investigate depositional patterning across contexts and between contexts, a research methodology which proved to be instrumental in understanding the use and consumption of this commodity in Britain as well as demonstrating the importance of understanding contextual circumstances in artefact studies.

The study area of this thesis is limited to England and Wales, excluding Scotland. This choice was made for two principal reasons. First, England and Wales were both part of the Roman provinces of Britain, while only portions of southern Scotland were temporarily incorporated and are therefore more applicable to frontier studies as opposed to provincial studies (this thesis being in the latter category). Secondly, England and Wales both participate within the Portable Antiquities Scheme (PAS) while Scotland does not. ${ }^{1}$ The sheer mass of data that the PAS provided for this thesis ${ }^{2}$ makes it an integral part of the research analysis,

\footnotetext{
${ }^{1}$ PAS is discussed in more detail in Section 1.5 and 6.1.

${ }^{2}$ Over a quarter of the objects in this thesis were reported through the Portable Antiquities Scheme.
} 
problematizing any seamless integration of material from regions that do not have a comparable system of data-collection and reporting.

This first chapter provides an overview of the development of academic theory concerning Roman Britain, so as to establish the intellectual foundations upon which this thesis was built and sets the stage for the reader to understand the relevant scholarship and understanding of material at the commencement of this thesis as well as introduces the problems addressed. Section 1.2 reviews the development of modern historical debate on Roman Britain, in the process discussing the development of theoretical perspectives in which the archaeology and history of Roman Britain have been understood. Section 1.3 provides an overview of previous archaeological scholarship of particular relevance to the understanding of copper alloy vessels in Roman Britain while Section 1.4 outlines the consensus view among current archaeologists of how copper alloy vessel material is interpreted and understood within Romano British contexts. Section 1.5 posits difficulties in current understanding of copper alloy vessels in Roman Britain and how this thesis contributes to the understanding of this material by elaborating on the research aims and thesis questions of this thesis, and then briefly outlining the structure of the remaining chapters.

\subsection{Review of Historiographic and Theoretical Debates}

The synthesis of the material in this thesis is intended to be applicable to the greater historigraphic study of Roman Britain. As such, it is of value at this stage to briefly outline the development of the historical narratives and debates concerning this timeframe. What follows is a brief synopsis of the development of the historical and theoretical debate concerning Roman Britain, which is by no means intended to comprehensively address the plethora of concerns of historiographic study over the $19^{\text {th }}$ to $21^{\text {st }}$ centuries, but rather reviews the most pertinent debates and theoretical approaches for understanding the material in this thesis.

While interest in the history and effects of the Roman period in Britain has existed since the Medieval period, ${ }^{3}$ what may be considered the inception of the 'modern' discussion of Romano-British history really begins with the writings of Haverfield, who outlines Classical civilization's cultural triumph over the indigenous cultures which they encountered. ${ }^{4}$ Haverfield

\footnotetext{
${ }^{3}$ See Hingley 2008 for a detailed discussion of pre-modern Romano-British historiography.

${ }^{4}$ Mattingly 2011, 38.
} 
views the Romans as having a civilizing effect upon the native populations which they conquered, though he admits varying levels of success in this endeavour depending on the social status and location of those involved, with the higher classes in the urban lowlands being more fully 'Romanized' than the peasant herdsmen of the highlands. ${ }^{5}$ Haverfield's overall concept of the civilizing effect of Rome on the people of Britain was also influenced by the contemporary theories regarding race and the civilizing effect of empire that were prevalent in his day, ${ }^{6}$ reminding us of how reflective historical research is of the time in which it is conducted ${ }^{7}$ as even much of the basic terminology used would be highly unacceptable today. ${ }^{8}$ The willing adoption of a higher form of civilization to replace a lower one is instrumental in Haverfield's theoretical construct, reflecting the colonial mind-set of $19^{\text {th }}$ and early $20^{\text {th }}$ century Europe and would likely have been agreeable to most of Haverfield's readers. ${ }^{9}$ This sentiment of consensual assimilation is shared by Collingwood, another prominent archaeologist of that time. Considering ideas of colonial separation between coloniser and subjugated race that were evident in parts of the British Empire during this period, Collingwood was inspired to make the following statement concerning the Roman imperial experience in Britain:

'There was no sharp distinction of race; the distinction of language did not matter; and the difference in civilization was not of such a kind that the Romans could be called civilized and the Britons savages...the Britons became Romans. They did not remain a subject race, held down by the Roman army. They became Romans in speech, in habits, and in sentiment. ${ }^{10}$

Important to both of these historians is the difference between the 'Celtic' culture of the indigenous Britons and the culture which the Romans brought and that theoretically flourished under their rule. Both draw a clear distinction between the culture of the 'Romanized' Britons and the culture of those who lived outside of the sphere of Roman influence. ${ }^{11}$ Both their perspectives on the development of culture in Britain are highly teleological and reflect a belief in the inevitability of triumph of a 'high' culture over a more 'primitive' one, ${ }^{12}$ though

\footnotetext{
${ }^{5}$ Haverfield 1923, 79.

${ }^{6}$ Haverfield 1924, 175.

${ }^{7}$ Gerrard 2013, 2-5.

${ }^{8}$ Hingley 2005, 117.

${ }^{9}$ Lyons \& Papadopoulos 2002, 5.

${ }^{10}$ Collingwood 1932, 6-7.

${ }^{11}$ Haverfield 1923, 79; Collingwood 1932, 48.

${ }^{12}$ Hingley 2004, 39.
} 
Collingwood does state that the culture of Britain as a whole during this period 'was neither merely provincial nor merely cosmopolitan, neither Celtic nor Roman simply, but a fusion of (the) two.' ${ }^{13}$ The concepts of Haverfield and Collingwood are clear reflections of the times in which they worked and have their roots in Hegel's framework of 'theodicy in history', or the pre-determined course of history based upon design and the eventual rise of freedom and reason as fundamental aspects in human civilization, ${ }^{14}$ a view reminiscent of historic views of the role of the Roman empire dating all the way back to imperial Rome itself. ${ }^{15}$ This view is summed up well by Freeman in his critique of $19^{\text {th }}$ century interpretations of imperial Rome when stating, '...the objective of imperialism, and the Romanization which followed it, has been variously seen as a combination of benevolent civilizing, economic advantage, and the cause of good government." 16

The intellectual tide began to shift away from such colonial models following World War II, developing throughout the latter half of the $20^{\text {th }}$ century. The rise of post-colonial thought and changing perspectives on relations between the rulers and the ruled within a colonial context led to the development of arguments such as Legg's 'Perpetual British War' between indigenous Britons and Roman invaders existing throughout the Roman period, referencing the surplus of soldiers regularly garrisoned in Britain and 'frequent historical references' to conflicts in the province to construct and justify his theory. ${ }^{17}$ This relies specifically on a concept of divided identities between 'us' and 'them', ${ }^{18}$ a very different model of identity in Britain from that proposed by Collingwood. Conversely, Frere saw the culture of Britain as being materially very much influenced by Rome, yet maintaining many of its pre-Roman features in its immaterial practice: 'Outwardly it was Roman, inwardly it remained Celtic; yet it would be wrong to suppose an inner conflict between the two aspects. ${ }^{19}$ This is not to say, however, that he felt that the adoption of some aspects of Roman material culture was at all superficial on the part of the inhabitants of Britain. Particularly pertinent to this thesis are his comments on the adoption of Roman dining equipment:

\footnotetext{
${ }^{13}$ Collingwood 1932, 94.

${ }^{14}$ Perhaps most succinctly summarised in Hegel 1956, 103-104.

${ }^{15}$ Whittaker 1997, 143-144; Hingley 2004, 64-67.

${ }^{16}$ Freeman 1997, 27.

${ }^{17}$ Legg 1983, 168-175.

${ }^{18}$ Laurence 1998, 95; Grahame 1998, 159; Malkin 2002, 151-159.

${ }^{19}$ Frere 1967, 342.
} 
'The great variety of plates, dishes, bowls and cooking vessels which were now available, far in excess of anything known in the Iron Age, and many of them of local British manufacture, bears witness to a complete revolution in manners. The widespread use of mortaria for preparing food similarly points to changes in diet, and the vast increase in amphorae shows that wine-drinking was now a luxury not confined to the houses of the aristocracy. ${ }^{20}$

Millett introduced a more complicated and nuanced view concerning the development of power structures and the acceptance of Roman material culture in The Romanization of Britain. ${ }^{21}$ Neither adopting a model based upon complete assimilation nor a model of overt separation, Millett's argument was based upon the willingness of the local aristocracy to take part in the new Roman system as a means of securing their own traditional hold on power as well as to facilitate the development of a greater level of authority. Millett sees the advent of Roman hegemony not so much as a dramatic shift in power structures so much as a re-organization of these same power structures in accordance to Roman systems and structures that precipitated an integration into the wider Roman political and economic world by these tribal elites. Millett specifically emphasises continuity between pre and post-conquest systems of governance and power structures in Britain, as well as the active participation by and benefits to the local elites, when he states:

'The application of the system to Britain means that the incorporated tribal elites transformed themselves into the decuriones of the civitates. In this way they were rewarded by retaining power, control of their tribe and wealth, thus continuing a de facto hereditary system. 22

The physical residue of this acceptance by the native aristocracy is evident in the architecture as well as the material culture, such as ceramic forms adopted following the conquest. $^{23}$ In Millett's framework, the fact that the local elites benefited in some ways from Roman-overlordship and desired association and incorporation within the Roman system would seem to be evident in the acceptance of many of the trappings of Roman material culture. Roymans offers similar perspective to Millett, using examples of the incorporation of tribal elites

\footnotetext{
${ }^{20}$ Frere 1967, 344.

${ }^{21}$ As controversy over the term developed after the book's publication, as discussed later in this section.

22 Millett 1990, 66.

${ }^{23}$ Millett 1979, 35-48; Millett 1990, 157-174; Mattiungly 2011, 234.
} 
in Gaul within the system of the Roman auxiliaries as a means of maintaining pre-Roman customs of aristocratic martial achievement within an integrated Roman system of power. The adoption of sedentary agricultural values evidently played an important role in the construction of elite identity in Gallia Belgica during this time, as indicated by the representations by provincial elites of the Roman values of farming and agriculture on their funerary monuments. ${ }^{24}$ Woolf emphasises the allure that integration into Roman society likely had for many Gallic provincial elites, emphasizing the economic benefits of peace and the role that classical education and the notions of paideia and humanitas had on the development of Roman Gaul. ${ }^{25}$ The work of Millett and others at the close of the twentieth century implies that there tended to be incorporation and compromise between the indigenous British and imported Roman cultures and power structures, with particular emphasis on the role of local elites in the process of the dissemination of Roman political and cultural influence. ${ }^{26}$ While not as dramatic as Collingwood's comment that 'the Britons became Roman', ${ }^{27}$ this model of indigenous acceptance and collaboration favoured by Millett et al. does assert that the Britons, or at least their elites, were receptive to adopting the Roman system as it was often to their own benefit. This sentiment is perhaps best summed up by Miles when he writes, 'Romanization does not represent a complete takeover of local cultures and languages which were used to articulate them. Rather, it was a process that involved appropriations by both rulers and ruled in the creation of new imperial narratives. ${ }^{28}$

This system of inter-cultural developmental exchange is also put forward by the creolization theory of Webster, in which she makes comparisons between Caribbean Creole cultures and the colonial environment of the Western Roman Empire in an attempt to understand how culture may have developed there. Her argument takes into account not only the presence of Romans and Britons, but also the diverse cultures from across the Empire which would have been present in Britain for military or commercial endeavours and how these cultures and peoples would have mixed. She characterises her views on inter-cultural syncretism thusly:

\footnotetext{
${ }^{24}$ Roymans 1996, 61-72.

${ }^{25}$ Woolf $1998,48-76$.

${ }^{26}$ Mattingly 2011, 38-39.

${ }^{27}$ Collingwood 1932, 7.

${ }^{28}$ Miles 2000, 60.
} 
'First, no discourse is purely dominant or oppositional but is to some degree both, and that ought to be the starting point for any analysis of social action within the Roman hegemony. Second, where we do encounter acceptance of colonial concepts, beliefs and material culture, that acceptance requires neither consent nor belief but is often a tactical obedience. $^{29}$

There is a distinct difference between the syncretism of Millett and the creolization of Webster: Millet's requires the acceptance of the Britons of 'Roman' culture while Webster's view emphasises the existence of diverse cultural values competing with each other in a shared landscape, the coloniser and colonised in a sort of cultural negotiation as they both attempt to define and assert their own identities within the greater society.

It is the development of such theories of cultural multiplicity which has led to the terms 'Romanization' and 'Romanized' to fall out of favour with scholars during the final years of the $20^{\text {th }}$ century and the beginning of the $21^{\text {st }}$ century as this phrase was viewed as too simplistic to define the nuances of cultural development which occurred as a result of the interaction between Roman and indigenous cultures of the west, not to mention the cultural pluralism experienced across the entire Roman world, as well as being a term that may have inherent modern political prejudices. $^{30}$ In many ways, the 'Romanization' argument encapsulates the development of the theoretical debate within Roman studies in Western Europe over the past 20 or so years. ${ }^{31}$ Much of this debate centres on how active a role the indigenous population played in 'joining in' on the Roman system, both culturally and politically, and how disenfranchised and subjugated the indigenous populations under Roman authority may have been. There also developed theories concerning the meanings and associations which may have developed concerning 'Roman' objects culminating in Barrett's assertion that there is a distinct problem with trying to understand relations between 'Roman' and 'native', as there is no clear definition of what either of these terms actually represents in terms of individuals or groups. ${ }^{32}$

David Mattingly's concept of Roman Britain is one of pure colonial exploitation and is developed principally from the current state of world affairs with 'the end of the Cold War and

\footnotetext{
${ }^{29}$ Webster 1997, 181-182.

${ }^{30}$ Webster 2001, 216-217; Lyons \& Papadopoulos 2002, 7; Mattingly 2011, 39.

${ }^{31}$ As may be witnessed developing in the annual installments of the Proceedings of the Theoretical Roman Arcaheology Conference, recently summarised in Heeren 2014, 159-161.

${ }^{32}$ Barrett 1997, 51.
} 
the emergence of the United States as a solitary superpower'. ${ }^{33}$ More to the point, his definition of 'empire' is the non-consensual rule of territories and peoples over a large landscape. ${ }^{34}$ His emphasis on imperial power being characterised as inherently non-consensual underscores his view of power relations and what the application of power structures means. He does agree with previous scholars in classifying Roman Britain as being a 'colonial' environment, but states 'Colonialism is essentially about the operation of power in situations that necessarily created or reinforced large inequalities within territories subject to exterior rule'. ${ }^{35}$ This emphasis purposefully downplays any positive effects of empire, asserting that economic and social exploitation lay behind the motivations and practices of empire in all its incarnations throughout time. ${ }^{36} \mathrm{He}$ downplays the importance of native elites in the imperial process, stressing instead the importance of 'discrepant experiences' between different social classes in different areas of the empire. ${ }^{37}$ Mattingly also emphasises the locals' subordinate role in the imperial administration and infrastructure to that of colonisers, ${ }^{38}$ as well as the omnipresent and intimidating threat of force against those not willing to be compliant with the new system of authority which is inherent in imperial systems. ${ }^{39}$ Most explicitly, he states: 'The Roman Empire was not run on altruistic lines: it developed mechanisms for the exploitation of land and people'. ${ }^{4}$

Mattingly also attacks the theory of cultural or social integration by stating that the way land use and settlement has been traditionally approached by scholars directly impacts the conclusions that researchers are able to reach. Arguing that the emphasis placed on towns puts a bias into the understanding of how settlement actually occurred in Britain during this time, he states that what is represented is 'Roman Britain' as opposed to 'Britain in the Roman Empire', a distinction which characterises his view of how the territory and people of Britain were viewed and treated under Roman rule. ${ }^{41}$ Mattingly's approach has struck a powerful chord with the contemporary scholarly community and epitomises a widely held view.

\footnotetext{
${ }^{33}$ Mattingly 2011, xvii.

${ }^{34}$ Mattingly 2011, 75.

${ }^{35}$ Mattingly 2006, 13.

${ }^{36}$ A similar opinion of the imperial process in Roman Britain may be found in Hingley 1982, 17-52.

${ }^{37}$ Mattingly 2011, 206-236.

${ }^{38}$ Mattingly 2006, 355.

${ }^{39}$ Mattingly 2006, 90.

${ }^{40}$ Mattingly 1997, 134.

${ }^{41}$ Mattingly 2006, 357.
} 
Within the greater historiographic and theoretical debate on Roman Britain, little space is given to the study of small finds, which is all too often regarded as a subsidiary study within the field. $^{42}$ Of the reports mentioned above, only in Millett and to a lesser extent Mattingly does small finds data play a significant role in the discussion and in both these cases it is used primarily as supporting evidence for landscape, architectural or other data. A recent publication by Gerrard also incorporates a great deal of small finds data into its argument, but its emphasis is focused principally upon Late Antiquity and does not cover the entire Roman period in as much detail. $^{43}$ This is not to say that small finds have not been applied to the arguments of cultural development and identity in Britain during the Roman period. The work of Swift across objects of dining, grooming and personal adornment has also proved very influential in drawing the study of small finds into the general nexus of debate concerning culture change and adaptation during the Roman period in Britain. ${ }^{44}$ Another notable work that applies small finds data to identity is Eckardt and Crummy's recent monograph on toilet instruments in Late Iron Age and Roman Britain, which illustrates specifically British trends in personal grooming such as the prevalence of nail cleaners in the province throughout the Roman period. ${ }^{45}$ Walton's monograph on coin loss in Roman Britain brings this group of objects into the study of regional and temporal change in culture practice in the province, indicating varying acceptance and use of coinage as a means of monetary exchange from the Iron Age through to the Anglo-Saxon period. ${ }^{46}$ Brooches have also received significant scholarly attention and their typologies are often used to track cultural movement and change in the Romano-British landscape. ${ }^{47}$ The current thesis sits within this tradition of such focused artefact studies.

Over the past century of scholarship, the development of how Britain during the Roman period is understood and perceived has been a dynamic process reflecting the ideas and the prejudices of the times in which the theories have been constructed. As it is true that researchers into antiquity are forced to overlay their own views and prejudices upon it, ${ }^{48}$ this process will continue in the future and will be informed by new developments, discoveries and writings. While rarely integrated into the same investigation, the relationship between historical theory

\footnotetext{
${ }^{42}$ Cooper 2007, 35-53; Johns 2007, 29-34; Swift 2007a, 18-27.

${ }^{43}$ Gerrard 2013.

${ }^{44}$ Swift 2007a; Swift 2007b; Swift 2009.

${ }^{45}$ Eckardt \& Crummy 2008, 69-72.

${ }^{46}$ Walton 2012.

${ }^{47}$ Collins 2010, 64-74; Mackreth 2011; McIntosh 2011, 155-182; Gerrard 2013, 198-203 \& 221.

${ }^{48}$ Mattingly 2006, 3-4; Gerrard 2013, 2.
} 
and artefact studies is reciprocal: one provides perspective within which to characterise finds while the other provides hard data with which to test intellectual paradigms. While informed by the developments in historiographic study outlined above, this thesis contributes to the development of the theoretical debate by offering a focused finds study which is then critically applied to the larger paradigm of the cultural development of Britain during the Roman period. Copper alloy vessels prove especially fruitful for this application, as they are both objects of pragmatic use as well as objects for the transmission of art through decoration and display. Their use both as functional objects and concentrations of wealth adds further layers of how these objects may be understood and interpreted. Finally, their use in the construction and display of cultural identity through table-practice and decoration provides fresh insight into the development of culture change across time and space in Roman Britain. All of these perspectives for viewing copper alloy vessels as cultural objects in Roman Britain will feature throughout this thesis, particularly in Section 1.5 and Chapters $7 \& 8$.

\section{$\underline{1.3 \text { Review of previous archaeological investigation relating to copper alloy vessels }}$}

While the previous section was concerned with the theoretical frameworks constructed within the academic community on how to view Britain during the Roman period, the present section will review the key archaeological investigations that form the foundation upon which the current thesis is constructed. It is important to have knowledge of the previous scholarship in order that the contribution that the present work makes is understood.

The principal starting point for the study of copper alloy vessels in Britain is Eggers' 1968 article. While principally serving as a catalogue, Eggers offers some discussion of the contexts of these objects as well as highlighting some trends in their deposition, such as grave goods and aquatic deposits. ${ }^{49}$ Though ambitiously expansive in its scope, much of the data in Eggers' article comes from military contexts, particularly from Hadrian's Wall and the frontier forts of Wales. This may have been a result of a depositional bias in the material, as it could be expected that the army was more inclined to use Roman material than the indigenous population or that their material practices might be representative of the importation of other customs from elsewhere in the Empire. ${ }^{50}$ Also, there are notable omissions in Eggers' catalogue as well as

\footnotetext{
${ }^{49}$ Eggers 1968.

${ }^{50}$ Cool 2006, 179-180.
} 
discrepancies between his report and other reports of certain objects, which put extra importance on the cross referencing of objects in the catalogue during the process of data collection. Nonetheless, the data collected by Eggers in his study is extensive and proves invaluable to the foundational work of this thesis.

In 1971, Kennett published a complimentary article to Eggers' which focused specifically on Late Roman copper alloy vessel hoards in Britain. His study is comprised of six hoards across Britain, which he inventories and offers brief descriptions of before outlining his theories in their manufacture and possible deposition. Importantly, he identifies several forms using accepted classifications for continental examples: the basin à bord godronné (impressed continuous oval design), Perlandbecken bowl (individual spherical designs), basin festonné (scalloped etched lines along rim), and basin uni (out turned rim). ${ }^{51}$ Kennett's article is principally a catalogue with some chronological explanation; it does not attempt to make associations between vessels and how they may have been used together or functioned. His work nevertheless is invaluable in developing the understanding of the repertoire of British vessels as well as indicating how these vessels may be related to trade and the economy of the province.

Though it does not deal directly with Britain, another important study by Eggers to consider when developing an understanding of Romano-British copper alloy vessels is his study on Roman vessels found beyond the northern boundaries of the Empire in Free Germany. ${ }^{52}$ His classification of vessel types is extensive and proved to be highly useful for this study, as it is widely used and accepted within the scholarly community. Eggers' typology is the system most commonly referenced in academic literature to this day. Radnoti's work on Roman copper alloy vessels in Pannonia is also a useful interpretative catalogue of material with much comparison of vessel forms across the empire and proposals on production centres for specific vessel types. ${ }^{53}$ The two catalogues of copper alloy vessels in the museum collection of Nijmegen are useful resources for the understanding of the repertoire of copper alloy vessels from the Roman period and they are often referenced by scholars for comparisons when describing copper alloy Roman vessels from excavations or museum collections. ${ }^{54}$ Though they prove to be a large sample

\footnotetext{
${ }^{51}$ Kennet 1971, 138.

${ }^{52}$ Eggers 1951.

${ }^{53}$ Radnoti 1938.

54 den Boesterd 1956; Koster 1997.
} 
group, the uncertainty of the context for many of the pieces in the first volume severely limits their usefulness to understanding their function and their relation to other dining objects. Den Boesterd provides a very useful synthesis of the previous, predominantly German, scholarship of copper alloy vessels in the introduction to her volume, ${ }^{55}$ which remains the principal source for identifying dates, function and place of manufacture in English scholarship. Also, as a collection of continental material it proves useful for comparison with the data-set developed in this thesis and helps to orient it within the wider western Roman world.

Tassinari's catalogue of material from Pompeii is also a key text in understanding copper alloy vessel material, as she also provides a discussion of their possible functions as well as methods of manufacture. ${ }^{56}$ She also includes information on findspots, when such information is known. Of course, the objects in her catalogue would have all been manufactured prior to the eruption of Vesuvius in $79 \mathrm{CE}$ and her catalogue is therefore only directly coeval to the first few decades of the Roman period in Britain. This is, however, some of the most comprehensive evidence available to us for the variety of vessels used at an urban site during the Roman period and must be utilised as far as possible without falling into the assumption that Roman-Britons invariably viewed material culture in the same way that Pompeiians did. Tassinari also has a further catalogue of copper alloy vessels from Gaul which proved highly useful for comparative purposes for this thesis, especially as the material within it covers a chronological span more closely comparable to the Roman period in Britain than the material from Pompeii. ${ }^{57}$ Cool's recent work is the most comprehensive archaeological study of the subject of dining in Roman Britain to date and is the foundational text for any current study of the subject. ${ }^{58}$ The scope of evidence is wide, including osteological, archaeo-botanical, and literary sources in order to develop an understanding of the various developments in British dining practice during the Roman period. When she discusses dining-ware as a source of evidence, she utilises grave good assemblages as her principal (though not only) source for discussing them, which could have biased her interpretation as grave contexts are ritual and not necessarily representative of the normal use-life of an object. Cool makes the notable observation that from the Late Iron Age to the Roman period there is a decline in the use of jars and large communal dishes and the

\footnotetext{
55 den Boesterd 1956, XIX-XXXI.

56 Tassinari 1993.

${ }^{57}$ Tassinari 1975.

${ }^{58}$ Cool 2006.
} 
adoption of individual sized bowls and plates in the ceramic record, this she attributes to a greater acceptance of Roman dining in much the same way as she does with the differentiation between cooking and dining vessels. ${ }^{59}$ This is a very useful observation and was valuable for reference when comparing copper alloy vessels over time through the Roman period. As the majority of her study was concerned principally with ceramics and glass, there is not a great deal on copper alloy vessels specifically to be gleaned from the pages as there would have been both cultural as well as practical differentiations between the utilization of earthen ware as opposed to copper alloy vessels, ${ }^{60}$ though some critical discussion of vessel use and context is offered and is cited in the following discussion. Additionally, Cool's over all observations are useful for comparison with this thesis's findings concerning copper alloy vessels.

Lee recently published a detailed investigation on the production, use-life and deposition of pewter vessels in Roman Britain. One of his most intriguing observations is that prior to the $3^{\text {rd }}$ century CE, London seems to have possessed a near monopoly on the distribution of pewter tableware whereas after $200 \mathrm{CE}$ it begins to appear in respectable quantities outside of this city, particularly in civitas capitals. ${ }^{61}$ This he ascribes to the increase in supply of this alloy through increased production and recycling as well as a shifting trend in elite fashion as pewter replaced ceramic and silver in some forms of vessels. This is plausible and further research in the distribution patterns of these materials comparatively could help to further clarify this theory. It is also worth noting that there is an uneven distribution of forms among pewter ware vessels, particularly that cups seem under represented as a whole among the finds. ${ }^{62}$ It would appear that these smaller tableware forms were more often constructed of glass or ceramic. This is a healthy reminder that different materials could have different uses and significance attached to them and that materials are not directly comparable across forms and functions. A vessel may be chosen to be constructed out of ceramic, glass or metal based upon the design and function of the vessel as opposed to the social status of the buyer and the cultural capital invested in different materials. ${ }^{63}$ This proves important when considering objects composed of a specific material, such as copper alloy.

\footnotetext{
${ }^{59} \mathrm{Cool} 2006,54$.

${ }^{60}$ Cool 2006, 47-50; Hurcombe 2007, 109-118 \& 190-208.

${ }^{61}$ Lee 2009, 75.

${ }^{62}$ Lee $2009,63$.

${ }^{63}$ Hurcombe 2007, 109-118.
} 
Allison's work on the household assemblages in Pompeii is of particular use in this study as her approach incorporates material culture studies and uses domestic small finds as a lens with which to understand the larger culture concerned. ${ }^{64}$ In these works, Allison develops groupings and associations between objects from Pompeiian houses, a context which gives us a rare collection of assemblages of household items in their 'original' location, ready for 'normal' everyday use. While these reviews and collections are of great use in developing our understanding of how individual vessels may have related to each other and what one might expect of a vessel assemblage from an elite household, the use of her material also has some important limitations in its applicability to our study. The geographic difference between Italy and Britain may have had a much larger effect on what dining equipment might be present than simply the willingness of one group or another to absorb or adapt the practices of another culture group. To briefly summarise how the geographic and cultural landscape could have led to differing patterns in the use of copper alloy vessels in these two areas, Pompeii itself was a colony of Rome which was established over a previous Greek colony in an Oscan speaking part of Italy, and therefore was itself something of a palimpsest of cultures. ${ }^{65}$ Also, as Pompeii was a maritime Mediterranean city, it might be expected that a comparatively large amount of fish and other seafood was consumed at this city as opposed to more land-locked centres. This would be more a reflection of the functionality of available food sources and less subject to the influence of outside cultures. As it so happens, it does not appear that fish was consumed on any great scale in Britain during the Roman period. ${ }^{66}$ Climate itself also likely played a part in how social customs, such as dining and bathing, may have been performed. As Allison herself suggests, there could have been great variability in how different items would have been used in different parts of the Empire. ${ }^{67}$

One of the most important aspects to Allison's work is her research perspective. Allison views the objects as evidence of domestic consumption. ${ }^{68}$ She seeks to compare these objects to where they are found in relation to their architectural surroundings as well as with other objects

\footnotetext{
${ }^{64}$ Allison 1992; Allison 1999; Allison 2004.

${ }^{65}$ Malkin 2002, 151-181; Beard 2008, 26-52.

${ }^{66}$ Cool 2006, 104-105.

${ }^{67}$ Allison 2004, 61.

${ }^{68}$ Allison 2004, 4.
} 
in order to develop a better understanding of how these objects may have been used or related to one another. ${ }^{69}$ As Allison states:

'Few studies use provenance artefact assemblages to better understand the consumption of Roman material remains. A lack of concern for specific artefact contexts in the published finds catalogues from quite recent excavations makes such studies extremely difficult to pursue., 70

The most recent publication directly concerned with copper alloy vessels in Roman Britain is the volume edited by Breeze which deals with a specifically Romano-British tradition of enamelled copper alloy vessels and other objects. ${ }^{71}$ The various papers in this volume deal with objects included in the data-set of this thesis as well as offering insight into the distribution and cultural significance of these objects. Understandably, this volume is regularly referred to in this thesis when dealing with enamelled vessels in Britain and also proved useful in characterising how vessels may more generally be used as objects of cultural consumption and corporate identity as these vessels reflect a merging of cultural traditions into a new art form. ${ }^{72}$ The recent Breeze volume is also the only study of copper alloy vessels in Britain thus far produced to devote considerable attention to decoration and how it may reflect function and identity. While previous work has used decoration on copper alloy vessels as supporting points to wider arguments of art and decoration in the province during the Roman period, ${ }^{73}$ decoration has not featured largely in the study of Romano-British copper alloy vessels themselves. ${ }^{74}$ This is one of the gaps in the research that this thesis seeks to rectify.

These above sources form the basis of current understanding of copper alloy vessel use in the western empire and in Roman Britain. Though a comprehensive synthesis of this information to offer a unified understanding of copper alloy vessels has not been established prior to this thesis, a negotiation of theories harvested from these previous authors does characterise the basic consensus of scholarly opinion at the inception of this thesis project. A

\footnotetext{
${ }^{69}$ Allison notes that it appears that most dining may have taken place in or near the garden areas of the house and that this may also reflect seasonal dining practice here as some believe that the eruption of Vesuvius took place during summer $(2004,132)$.

${ }^{70}$ Allison 2004, 6.

${ }^{71}$ Breeze (ed) 2012.

72 Künzl 2012, 9-22; Hunter 2012, 98-105; Breeze 2012, 107-111.

${ }^{73}$ Henig 1995.

74 Toynbee 1964, 317-327 being a notable exception.
} 
brief summary of this broad understanding is offered below in order to 'set the scene' for the reader and put into perspective the contribution of the current thesis to the scholarship.

\subsection{Current understanding of Copper Alloy vessels in Roman Britain}

With the previous literature of immediate relevance to this thesis having been reviewed, it is now possible to comment on the current state regarding the understanding of copper alloy vessels in Britain during the Roman period. Since Eggers' 1968 article, there has been no effort to provide a synthesis of copper alloy vessels in Roman Britain; part of the purpose of this thesis is to rectify this gap in the scholarship. Current understanding of copper alloy vessels in Roman Britain is dependent largely on continental literature, the principal authors being Eggers, Radnoti and den Boesterd. These three authors remain the principal sources for finds researchers, providing much of the basis for statements in finds reports on manufacture, origin and dating for this material. There remains no consistent terminology beyond Eggers' typology for labelling copper alloy vessels, which is not universally applied.

Den Boesterd's synopsis of vessel function and origins remains the basis for most English scholarship on the matter, especially as she provides a synthesis of much of the German scholarship on the subject. Additions and supporting material have been incrementally offered piecemeal in the meantime, but these three authors form the principal basis of what is currently understood and believed about copper alloy vessels across the Western Empire, including Roman Britain.

Copper alloy vessels would appear to have been unusual commodities in pre-conquest Britain, ${ }^{75}$ and their advent in the archaeological record may be seen to a greater or lesser extent as a sign of Roman influence much in the same way as mortaria often are. ${ }^{76}$ Throughout the Roman period, vessels are seen largely as import commodities, with Italy and Gaul being the principal areas of manufacture, ${ }^{77}$ though some objects seem to have come from as far away as Alexandria. ${ }^{78}$ The principal exceptions to this rule are Late Roman hanging basins, such as the Irchester bowl, believed to have a British origin. ${ }^{79}$ Exact provenance of manufacture centres is not an immediate concern of this thesis, especially as the theories of provenance are often based

\footnotetext{
${ }^{75}$ Eggers 1968, 67-69; Carver 2001, 2-3, 27 \& 37.

${ }^{76}$ Carver 2001 32-34; Cool 2006, 42-43.

${ }^{77}$ den Boesterd 1956, XIX-XXX.

${ }_{78}^{78}$ Radnoti 1938, 14-25; den Boesterd 1956, XIX-XX; Kennett 1971, 137-138.

${ }^{79}$ Kendrick 1932, 161-184; Kennett 1971, 147-148.
} 
solely on stylistic grounds that are difficult to substantiate empirically. This may best be illustrated by vessels from the river deposit at Neupotz that are believed to be from a workshop in Gaul, ${ }^{80}$ but match very close in form and decorative style copper alloy vessels from Pompeii and elsewhere that are believed to be of Italian manufacture. ${ }^{81}$ Stylistic similarity with objects elsewhere in the Roman world does prove to be useful for analysing culture change and adaptation; this decorative or art historical approach therefore features much more prominently in the discussion of this thesis and in its conclusions than theories of manufacturing centres. Theories on vessel function applied to this material are almost exclusively based on the work of den Boesterd and it is indeed difficult to offer a discussion of this without simply paraphrasing her treatment. ${ }^{82}$ Den Boesterd's influence is perhaps best demonstrated in regards to a vessel type she refers to as a 'bath saucer', ${ }^{83}$ which has come to characterise the understanding of this vessel type's function despite there being little supporting evidence outside of an example found in the Forum Bath at Pompeii. ${ }^{84}$ She describes other handled pans as 'sauce pans' and asserts that they were used mostly for the serving of wine. ${ }^{85}$ An accepted amendment to this is a specific variety of handled pan, ${ }^{86}$ which Nuber has convincingly argued was used for ritual ablutions associated with sacrifice and burial. ${ }^{87}$ The terminology for these various vessels which resemble modern day frying-pans is confused in the scholarly literature, with terms like 'sauce pan', 'skillet', trulla and patera being used interchangeably in the literature without any consistency between authors. ${ }^{88}$ Discrepancy in how some terms are used in the literature has led to a loss of clarity in the reporting of copper alloy vessels. Some have argued that a patera has a handle, ${ }^{89}$ while others contend that a trulla has a handle and that a patera inherently lacks one. ${ }^{90}$ These debates in the literature are fuelled by ancient documents making passing references to these objects, such as RIB 2415 and Vindolanda Tablet 596, which are not conclusive. This has led to a muddled and confused terminology in scholarly usage. This thesis proposes a new

\footnotetext{
${ }^{80}$ Bakker et al. 2006, 62-183.

${ }^{81}$ den Boesterd 1956, ix-xx; Tassinari 1993, 17-24.

82 den Boesterd 1958, XIX-XXX.

${ }^{83}$ Tassinari 1993 I1110-I2400; den Boesterd 1958, XXI; Künzl 1993, 381-382.

${ }^{84}$ Kohlert-Németh 1990, 81-82.

${ }^{85}$ den Boesterd 1958, XIX-XXI; Carver 2001, 15-22 \& 32-33.

${ }^{86}$ Eggers 154-155; refered to in this thesis as Handled Pan 1, see Chapter 2.

${ }^{87}$ Nuber 1973, 1-232.

${ }^{88}$ Compare Toynbee (1964, 317-327), Henig (1984, 131 \&193) and Philpott $(1991,25)$ on their usage. Boon (1988, $525)$ attempted to argue for a consistent definition for these terms, but the use in present literature remains confused. ${ }^{89}$ Henig 1984, 131, 193.

${ }^{90}$ Boon 1988, 525.
} 
terminology for these vessels and a new framework within which to consider them, as discussed in Section 2.1. At present, to help clarify the confused state of the terminology, Table 1a illustrates some the more common ways that handled and un-handled pans have been described.

\begin{tabular}{|l|l|l|}
\hline Latin Term & English Substitutes & $\begin{array}{l}\text { Neutral term used in this } \\
\text { thesis } \\
\text { (approximated, as consensus } \\
\text { does not exist in Latin or } \\
\text { English terms) }\end{array}$ \\
\hline Patera & $\begin{array}{l}\text { Skillet, sauce pan, bowl, cup, } \\
\text { libation bowl, basin, pan }\end{array}$ & Handled Pan 1, Bowl \\
\hline Trulla & $\begin{array}{l}\text { Skillet, sauce pan, bath saucer, } \\
\text { pan }\end{array}$ & Handled Pan 2, Handled Pan 3 \\
\hline
\end{tabular}

Table 1a: Examples of usage for Latin terms patera and trulla and their equivalences in this thesis.

There has been no collective discussion of vessel function in English since den Boesterd and comments about possible vessel function are dispersed in the literature, often as side-notes or supporting statements to other studies. Cool makes a convincing argument that Eggers type 128 \& 128a jugs may be considered as having been used predominantly for water due to lime-scale residues and artistic representations. ${ }^{91}$ While Pompeiian contexts inform us little more than copper alloy vessels are generally associated with kitchen and dining gear, Allison has shown a plausible relation between Eggers type 98-104 vessels, often referred to in the Italian scholarship as pasticcerie, with water and probably personal ablution. ${ }^{92}$ While early interpretations of hanging basins, such as Irchester type bowls, suggested their use as ritual water-clocks, ${ }^{93}$ they are much more convincingly argued presently to have been used as part of a dinner service, perhaps also for hand-washing and ablutions. ${ }^{94}$

Copper alloy vessels remain largely understood through their appearance in Structured Deposits and Grave Deposits, ${ }^{95}$ as these are the objects most likely to appear in publication or on display in museums. This has a large impact on how these objects are perceived by researchers and what objects, and by association activities, are associated with them. As such, they are viewed as something of an elite luxury in Britain and almost exclusively associated with wine-

\footnotetext{
${ }^{91}$ Cool 2006, 137.

92 Allison 2004, 55; Cool 2006, 138 concurs.

${ }^{93}$ Smith 1916, 77-95.

${ }^{94}$ Hawkes 1951, 172-199; Kennett 1971, 147-148; Harris \& Henig 2010, 25-38.

${ }^{95}$ Defined in Section 1.5.
} 
drinking and high dining display. ${ }^{96}$ Apart from Eggers, no work has been done to systematically compare copper alloy vessels across depositional circumstances nor widely across geographic or chronological distribution in Britain. This forms one of the principal aims of this thesis, as discussed in further detail in Section 1.5.

To date, there has been no attempt to develop a comprehensive understanding of the place of copper alloy vessels within Romano-British society as functional objects nor how they were utilised as objects of cultural definition and display. There has also been no discussion of how copper alloy vessels themselves may provide insight into developmental changes in the cultural life and the construction of identity in Britain during the Roman period. This in turn forms the basis and principal aim of the current thesis, as addressed by the research aims in the following section.

\section{$\underline{1.5 \text { Research Questions }}$}

With the aim of making the most comprehensive and innovative use out of the copper alloy vessel material assembled in this thesis in its relation to understanding material culture in Britain during the Roman period, the following research questions form the basic interpretative framework within which the data will be analysed and understood. After expository review and discussion of the material in Chapters 3-6, these questions will be individually addressed through a synthesis of the data in Chapters 7 and 8.

\section{How can individual depositional contexts be characterised and interpreted?}

As has been stated earlier in this chapter, there has been no effort in previous scholarship to examine the depositional circumstances of copper alloy vessels comparatively across Roman Britain to determine patterns in the archaeological record. To clarify what this means and how fundamental it is to the new contributions to the scholarship offered by the current thesis, it is worth explaining what 'depositional context' means and how this relates to our understanding of the material in this thesis.

In the following thesis, the term 'deposition' will be applied to the circumstances by which an object has entered the archaeological record. This is a key component of the data related to an object in this thesis as the method by which a metal vessel enters the archaeological

${ }^{96}$ den Boesterd 1958, XIX-XXX; Carver 2001, 15-22; Cool 2006, 136-140. 
record differs significantly from the way in which a stone or ceramic object may. First, a discussion of object biography and the depositional processes which are relevant to copper alloy vessels will be offered followed by an explanation of the depositional categories used in this thesis: Structured Deposits (SD), Grave Deposits (GD), Site Finds (SF) and Single Finds reported through the Portable Antiquities Scheme (PAS).

To understand how representative the material which occurs in the archaeological record is and what it may be able to tell us about those who first used and deposited the material, we have to examine the processes by which an object enters the archaeological record, making it available for our study, and how its contextual circumstances can inform us of its use-life and deposition. ${ }^{97}$ All artefacts that become part of the archaeological record have undergone a process from manufacturing to reporting and recording which we may define as the 'use life'. This covers the broad phases of an object's existence, namely that it had to have been manufactured, utilised, deposited, recovered and subsequently recorded if it is to become part of the archaeological record and available for academic study. ${ }^{98}$ The life-cycles of objects can be quite complex, especially if the objects bear intrinsic or artistic value. ${ }^{99}$ Schiffer divided the life of objects between 'Systemic Context' and 'Archaeological Context' to differentiate between the influences they would be subject to between their pre and post-depositional existences, dividing the essential stages an object would undergo in the 'Systemic Context' to pass to the 'Archaeological Context' as procurement, manufacture, use, maintenance, and discard. ${ }^{100}$ While both of these concepts prove to be useful tools in conceptualizing the processes by which an object enters the archaeological record by broad-strokes, they do not go very far in informing us of the particular variables which a specific artefact group may have been subject to which led to its deposition into an archaeological context. The material an object is composed of has key importance in how the object will be treated and how it might enter the archaeological record. ${ }^{101}$ This is based upon the material's cultural significance, economic importance and the recyclability and/or reusability of the material in question. Glass often disappears from the archaeological record since it is not easily repairable, yet is easily recyclable. ${ }^{102}$ An object made

\footnotetext{
${ }^{97}$ Hurcombe 2007, 14-53; Swift 2012, 167-168 \& 202-204.

${ }^{98}$ Hurcombe 2007, 38-41.

${ }^{99}$ Swift 2012, 167-215.

${ }^{100}$ Schiffer 1972, 158.

${ }^{101}$ Rissman 1988, 216; Hurcombe 2007, 111-112.

102 Prior 2014, 105-121.
} 
of stone is difficult to repair if it is damaged beyond viable use for the purpose which it was made. Unless the object is recycled into blocks of masonry for later constructions, as happened with many Roman funerary monuments during Late Antiquity, ${ }^{103}$ it is often reduced to lime and therefore the original object does not pass into the archaeological record.

Ceramics are similarly difficult to repair or recycle and are often discarded after they have been broken, allowing them to be preserved for archaeological study. The lifecycle of Roman pottery has been extensively covered previously ${ }^{104}$ and need not be further detailed here. Suffice to say, Peña divides the lifecycle of Roman pottery into 'Primary Use-life' and 'Reuse use-life' to differentiate between periods of primary use of the objects for their manufactured purposes, reclamation after discard and residual use. ${ }^{105}$ This complicates the circumstances by which an object may enter the archaeological record, especially as its reuse may remove it from the cultural and economic circumstances that previously governed the use-life of the object. The life cycle of copper alloy vessels preceding their emergence in the archaeological record could be exceedingly complex involving primary use as dining or bathing vessels, repair, refitting/reuse, discard and recycling into raw metal for manufacture or trade just to name the most evident uses of these objects. These many factors contribute to copper alloy vessels being rare archaeological finds in comparison to pottery. ${ }^{106}$ For example, metal vessels being easily repaired with soldering and patches, they are more likely to have a longer use life and less likely to be discarded upon breaking. ${ }^{107}$ Most important for our consideration of the objects entering the archaeological record is the ease with which metal is recycled, thereby removing the object from the material record of the past and distorting our image of what objects may have been comprised of this material and our perception of the prevalence of these objects in daily life. Additionally, the potential prestige value of metal objects and the economic value of the metal itself, as well as its practical utility, increased the likelihood for the objects to be sold for scrap or to otherwise circulate within the active economy as opposed to being discarded, and being therefore available for current archaeological study. Conversely, the prestige of metal will at times directly lead to its deposition, as may be seen in the hoarding behaviour which appears to have led to many of the Structured Deposits in this study as well as in the inclusion of copper

\footnotetext{
${ }^{103}$ Espérandieu 1913; Renard 1959, 22-25; Terrisse 1982, 10.

104 Tite 1999, 181-233; Arthur 2002, 331-355; Peña 2007; Hurcombe 2007 166-182.

105 Peña 2007, 9-11.

${ }^{106}$ Cool 2006, 47.

107 den Boesterd 1956, XIX; Hurcombe 2007, 190-208.
} 
alloy vessels in Grave Deposits. ${ }^{108}$ This in turn provides its own bias for our understanding of the place of these vessels within society, as material intentionally deposited for a ritual function is likely to have been influenced by this ritual purpose and may not be reflective of the normal use-life of the object in question. ${ }^{109}$ Though it is impossible to fully counteract these factors which influence object deposition and therefore cause the formation of a bias affecting our perspective of the objects under study, it is important to understand them in order to make best use of the material. The objects comprising this data-set are categorised within one of the following four categories: Structured Deposits, Grave Deposits, Site Finds and Single Finds reported through the PAS. A brief description of these categories is offered below.

Structured Deposits: The term 'Structured Deposit' was introduced into archaeological literature during the latter half of the twentieth century in reference to pre-historic assemblages in Britain of what appeared to be intentionally grouped, or 'selectively deposited', ${ }^{110}$ objects and has since gained some level of acceptance as a comparatively value-neutral term for groups of objects that may otherwise have the value-laden descriptions of 'hoards' or 'ritual deposits'. 111 'Structured Deposit' is used in this thesis to describe an object or assemblage of objects that was collected and interred through direct human agency and thus found its way into the archaeological record. Sometimes the term 'special deposit' is preferred in the literature, ${ }^{112}$ but 'structured' is a preferable term for the material treated in this thesis as it reflects the process by which deposition occurred. This need not only be large hoard assemblages, but may also consist of small groups or even singular objects left at probable votive sites, such as aquatic settings or in temples. Detailed discussion of these practices and how they relate to the material in this thesis may be found in Chapter 3. For the purposes of this thesis, Structured Deposits reported through the PAS are qualified as Structured Deposits and considered in this data-set.

Grave Deposits: $\quad$ 'Grave Deposits' are defined in this thesis as an object or group of objects interred with the deceased that would appear to have formed part of the burial ritual. Grave Deposits have their own set of issues that differentiate them from the broader category of other

\footnotetext{
${ }^{108}$ See Chapters 3 and 4 for further discussion.

${ }^{109}$ Needham 1988, 229-248; Hatzaki 2009, 19-30.

${ }^{110}$ Needham 1988, 229-248.

${ }^{111}$ Richards \& Thomas 1984, 189-218; Hill 1993, 57-75; Hill 1995a, 53; Hill 1995b; Hingley 2006, 213-257; Hatzaki 2009, 19-30.

112 Hill 1996, 17-32; Hamerow 2006, 1-30.
} 
deliberate deposits and justify their consideration individually. ${ }^{113}$ As objects that are interred with the dead, there is specific ritual intent involved in their deposition that may have influenced which vessels were selected for interment and which vessels were excluded. ${ }^{114}$ Grave material was collected together for a specific purpose and interred as such and therefore holds clues to uses and associations between vessel forms. The practice of funerary ritual is also important for the construction of social identity and defining cultural allegiance and hierarchy, making Grave Deposits very useful in examining changing concepts of culture and identity. That the ritual context may be somewhat better defined in graves as opposed to the processes leading to the deposition of Structured Deposits, as at least mortuary significance is understood for the rites involved in grave deposition. ${ }^{115}$ This allows more specific scrutiny of their greater significance within that practice, as well as more focused discussion on matters such as ritual use of vessels and the role of iconography in objects included in grave assemblages. For the purposes of this thesis, objects identified as burial assemblages reported through the PAS are qualified as Grave Deposits.

Site Finds: $\quad$ This category comprises all of the objects which do not easily fit into the previous two categories (and not reported through the PAS), most specifically settlement contexts and occupation deposits, and in large part represents objects which likely entered the archaeological record through accidental or indifferent loss and includes objects both from excavations as well as chance finds which cannot be given a specific immediate context or findspot. As such, this category covers a diverse range of objects that likely experienced diverse processes of deposition. It is important to specify that a sole metal object could have been deposited by itself as a votive offering or buried for safe-keeping as a Structured Deposit. ${ }^{116}$ As Paul Rissman explains, 'It may be expected that valuable objects would be guarded against loss and discarded relatively infrequently'. ${ }^{117}$ This sentiment would lead one to believe that most objects of a valued nature, such as complete and intact copper alloy vessels, are very unlikely to have entered the archaeological record through discard or accidental loss. Even damaged objects may be suspected of having undergone a ritually structured process of deposition, ${ }^{118}$ further

\footnotetext{
${ }^{113}$ Pearce 2000, 1-8.

${ }^{114}$ Pollard 2001, 315-333.

115 Pearce 2000, 2-5.

116 Such as DUR0001; Burnham 2007, 264-266.

${ }^{117}$ Rissman 1988, 216.

${ }^{118}$ Bendann 1930, 115-120; Hobbs 2006, 7; Swift 2012, 182-185.
} 
complicating how to interpret objects of such a functionally valuable material as copper alloy occurring in circumstances suggesting discard and abandonment at occupation sites. However, it is often simply impossible to prove incontrovertibly if an object entered the archaeological record through a direct process of intended or ritualised deposition or if it is simply the result of lost and forgotten property or even simple refuse. For this reason, any object found without other objects directly associated with it that cannot be clearly shown to belong to a Structured Deposit or Grave Deposit will be classified as a Site Find and approached accordingly in this thesis.

Single Finds Recorded through the Portable Antiquities Scheme (PAS): $\quad$ Objects reported to the Portable Antiquities Scheme that cannot be clearly identified as Structured Deposits or Grave Deposits are considered in their own depositional category for the purposes of this thesis. This decision was taken because the process of discovery, recording and reporting these objects differs from that used in more traditional scholarly publications and validates the consideration of this data-set separately. ${ }^{119}$ The PAS has opened up a vast amount of new data that would otherwise likely have been lost to scholarship, most specifically material from rural areas of the province that traditionally have been less extensively investigated. This thesis is the first focused work on copper alloy vessels to incorporate this data in a systematic fashion and this results in significantly different interpretations in the use of copper alloy vessels and a much wider perspective on their distribution and availability across the province during the Roman period than would have been available without this wealth of material.

The division and interpretation of data into these contextual categories allows for the focused analysis of trends and the comparison of patterns between them. This has been the most fundamental aspect of the methodology of this thesis, guiding the discussion in Chapters 3-6 as well as defining how many of the conclusions offered in Chapter 7 and 8 were arrived at. The choice to adopt a context driven methodology builds upon previous work on small finds which have also put an emphasis on context ${ }^{120}$ and proves to be an instrumental part in developing an understanding of finds data. While Depositional Context plays a key role in the research approach and discussion throughout the thesis, this thesis question is directly addressed in Section 7.1.

\footnotetext{
${ }^{119}$ This is further explained and elaborated in Chapter 6.
}

120 Allison 2004; Cool 2006; Eckardt \& Crummy 2008. 


\section{What is the distribution of copper alloy vessels across time and space?}

For the purposes of this thesis, distribution will refer to both the geographic area and site type ${ }^{121}$ where an object was found. The geographic location simply means where in Britain this material was found. This includes the greater geographical region, county, parish, site or even specific location within a site depending upon how detailed of context information is available for the object or assemblage in question. The more specific social distribution relies upon recorded find-spot data available in the report. Specific location within an excavation site, or within the natural environment, can be of great importance also in patterning social distribution. For example, the deposition of objects in aquatic environments such as wells, rivers and springs proves of immediate relevance to the interpreting of some of the Structured Deposits in this thesis. ${ }^{122}$ All of this distribution data reflects upon who may have used the object, how it may have been used, how the object was perceived by those who used it and how it was incorporated into the cultural construct of those to whom it was a part of everyday life. Understanding the distribution patterns is instrumental to understanding the use of different forms of material culture by different socio-economic groups in Britain during the Roman period, as it is differences between these patterns which indicate difference in use and availability of material. Distribution forms a key part in the discussion of copper alloy vessels by Depositional Context in Chapters 3-6 as well as features in each of the synthetic discussions. This thesis question is addressed specifically in Section 7.2.

\section{How do copper alloy vessels reflect use and consumption?}

This question aims to investigate how copper alloy vessels were perceived as a consumable commodity and how these objects were utilised to varying degrees across the province during the Roman period. While not an economic investigation in earnest, this problem does have an economic aspect as to how the material was distributed and consumed by those who used it, if not specifically how it was quantified and precisely valued in a modern economic sense. One aim is to understand how patterns across Depositional Contexts, site types ${ }^{123}$ and broad regional geographic distribution may indicate perceived cultural value in these objects as

\footnotetext{
${ }^{121}$ Definition of Site Types used in this thesis may be found in Section 2.2.

122 As detailed in Section 3.3.

${ }^{123}$ Depositional Contexts are explained earlier in this section, Site Types are explained in Section 2.2.
} 
commodities and social capital as opposed to their place within gross economic trade networks as a specific medium of exchange. The functionality of these vessels in their pre-deposition uselife also features in this question, as the normal day-to-day use of these vessels had a direct bearing on their cultural significance and offers insight into the spread of customs in the province over time. The analysis of context plays a key role in understanding the perception of these vessels and how they may have functioned within the greater material practice of British society during the Roman period. How these objects were consumed and used played a key role in the manner in which they were deposited in the archaeological record and influences the interpretations offered in the focused depositional discussions of Chapters 3-6. This thesis question is specifically addressed in Section 7.3.

\section{How can copper alloy vessel material from Roman Britain be applied to the study of cultural identity and culture change in Britain during the Roman period?}

This question is the principal research aim of the study of copper alloy vessels in this thesis and is used to characterise the patterns in use and consumption discernible between Depositional Contexts and how this related to the cultural activity associated with the construction and display of identity surrounding their deposition. As such, the synthetic discussion of the material in this thesis in regard to this question comprises the closing chapter of this thesis. It is apparent that varying culture practices and methods of creating and asserting identity would in turn have led to the differing depositional practices across time and space in the province, reflecting different uses of the objects by specific social and economic segments of society that varied and changed depending on time and space. Practice is inherently and problematically linked with identity, ${ }^{124}$ allowing for the focused study of a functional commodity like vessels or brooches to be of particular use in analysing developing concepts of identity. ${ }^{125}$ Copper alloy vessels are particularly useful for such a focused study as they were not widely used prior to the Roman period, ${ }^{126}$ and their varied forms and functions can indicate progressive change through depositional practice and associated objects. Decoration can also prove to be highly informative in developing theories on identity and cultural construction, offering suggestions to the specific tastes and cultural associations the owners of the objects wished to

\footnotetext{
${ }^{124}$ Pitts 2007, 693-713.

${ }^{125}$ Mackreth 2011, 234-242; McIntosh 2011, 155-182.

${ }^{126}$ Eggers 1968, 67-69 ; Cool 2006, 49.
} 
construct for themselves. This could include figural representation of characters or creatures from Classical mythology as well as more subtle floral and geometric designs which could descend from British or Mediterranean artistic traditions. An examination of the use of these different decorative motifs, with particular care as to how their depositional patterns vary across Britain, helped to illuminate how the construction and expression of identity varied over time and across groups within British society. Inscriptions are also considered in this question, as the epigraphic habit is reflective of linguistic and cultural ties between Britain and the rest of the empire. The comparison of objects between Depositional Contexts is particularly useful in developing arguments of identity, as it allows patterning between divergent cultural practices leading to object deposition and reflecting differing acts in culture practice as used in the process of constructing identity. This thesis seeks to prove how valuable this understanding of contextual variability is in the study of small finds, allowing insight into cultural associations and the development of identity across time and space in Roman Britain. This question proved particularly relevant to understanding the content of each Depositional Context within its own right throughout the thesis, as well as interpreting the comparative evidence from across Depositional Contexts.

These questions aided in structuring the analysis and discussion of material in this thesis. The expository discussion of separate Depositional Contexts in Chapters 3-6 offer specific interpretation of controlled data-sets of material in order to better characterise and understand how each of these Depositional Contexts aids in our understanding of the use of these objects during the Roman period. Chapter 7 will integrate all of the material from the four Depositional Contexts of this thesis to extrapolate information in regards to the first three thesis questions. Chapter 8 will be a concluding assessment of how the material between Depositional Contexts can inform our understanding of material culture in Britain in response to the fourth thesis question, culminating not only in a greater understanding of copper alloy vessels and their place within the material culture of Roman Britain, but also in the application of context specific analysis to the study of finds and the use of this material to the fuller discussion of the cultural landscape of Britain throughout the Roman period. 


\section{Chapter 2: Materials, Methods and Approaches}

\subsection{Definition and Parameters of Material under Investigation}

The following chapter defines and explains the key terms used in identifying and classifying the data-set as well as explaining the methodology behind the research. Section 2.1 is concerned with the material that is under study and how it is defined while Section 2.2 details how this material was quantified. Section 2.3 discusses how this material was researched, offering a brief overview of the principal sources to gather material and the obstacles encountered in the gathering of data. The intent of this chapter is to offer transparency in the research undertaken during this thesis and to offer the reader the opportunity to retrace the steps taken in the course of data collection and analysis, as well as to familiarise the reader with how the data is presented and interpreted throughout the remainder of the thesis.

The research methodology of this study is principally concentrated on context, as this provides the best means for answering the Research Questions outlined in Section 1.5. The contextual emphasis framed the ways in which material was conceptualised and recorded, first through the division of material by the Depositional Contexts outlined in the previous chapter and then in regards to the recording of geographic distribution, chronology and site type data which plays a fundamental role in the interpretation of these objects and their relation to culture change in Britain over the three and a half centuries of Roman rule.

It proves immediately useful to define 'vessel' as it will be understood in this thesis. A 'vessel' is an object made for the containment and serving/dispensing of a commodity. Most often, this commodity is a solid food or liquid. Objects used to contain water or oils for bathing and ablutions are also considered vessels. Boxes, caskets or any other objects that do not appear to have been used in these ways are not considered 'vessels', either generally in the scholarship or in this thesis. The above definition offered may seem straightforward and possibly unnecessary, but such distinctions must be clearly understood as definition inherently leads to exclusion. Several mounts and fittings were excluded from the data of this thesis because they appeared to more probably represent casket fittings and furniture mounts than parts of vessels. ${ }^{127}$

\footnotetext{
${ }^{127}$ Discussed later in this section.
} 
Boxes, such as a cylindrical example from Crundale in Kent ${ }^{128}$ or the so-called Modius Claytonensis with a deleted inscription of the emperor Domitian from Carvoran in Northumberland, ${ }^{129}$ were similarly excluded from the data of this thesis as they seemed more representative of storage containers or devices of measurement than functional vessels and would have their own cultural relevance that is best understood separately from the current survey.

For the purposes of this thesis, the term 'repertoire' is used to define the various forms and types of vessels which compose the data-set, along with their decoration. The need for understanding the differences between vessels and being able to assign a usable term by which to reference them is a fundamental part of the research strategy of this thesis and its goal in providing a useful source of reference for future researchers. For this purpose, a list of important vessel forms and types associated with this study along with a short definition and description is provided in this section.

First, it is important to differentiate what the terms 'form' and 'type' mean as they are used in this thesis, as this distinction is instrumental to the treatment of the data therein. 'Form' refers to the basic shape of a vessel (bowl, cauldron etc.) and is used as a general guide to indicate possible functionality and associations it may have with other objects. This is based on similar distinctions established for distinguishing broad functional categories for the quantification and study of Romano-British ceramics, ${ }^{130}$ as well as being used for understanding broad functional groups of copper alloy vessels in continental studies, ${ }^{131}$ and it was found applicable for the purposes of this thesis. 'Type' is used in the normal typological sense of the word and refers to the stylistic attributes of an object, including decorative motifs and minor shape variations, which are more indicative of particular tastes than to the integral construction of the vessel. 'Form' is therefore concerned with the basic functional shape of a vessel and 'type' reflects the stylistic tastes and choices of the manufacturer and/or consumer. It will be important to understand this difference throughout this thesis, as changes in function and changes in style do not inherently synchronise.

\footnotetext{
${ }^{128}$ Hassall \& Tomlin 1985, 328 (25).

${ }^{129}$ Haverfield 1916, 85-102; RIB II 2415.56.

${ }^{130}$ Millett 1979, 35-48; Tyers 1996, 43.

${ }^{131}$ Particularly den Boesterd 1956.
} 
Form is the base-line categorization used in this thesis, from which further classifications based on decoration and design follow. This allows for broad patterning in the repertoire to be analysed and variances across space and time to be recognised. While most previous studies on copper alloy vessels have used typology as their base-line distinction between varieties of vessels, the use of more general form classifications to recognise patterns is not without precedent. Cool used a classification of broad vessel forms in analysing metal vessel distribution across different kinds of sites in Roman Britain, ${ }^{132}$ and den Boesterd's terms for describing general vessel forms and typological groups remains the standard terminology used in much English language scholarship today. ${ }^{133}$ What has not been previously done is using a systematic classification of forms and types to analyse broad distribution patterns in Britain, as is done in this thesis.

While it has been argued that divisions between functional and stylistic traits in the classification of objects is not useful as it is 'at best an educated guess based on the gut feelings and experience of the archaeologist... and at worst an unnecessary impediment that systematically prevents an accurate evolutionary understanding of the archaeological record, ${ }^{134}$ this distinction is seen as valuable in analysing the data-set of this thesis as it classifies the data into comprehensible and manageable groups that can be logically associated and compared. These data-groups are then available for separate comparison and analysis. This is not so easy to do with typologies alone which combine functional and stylistic characteristics, such as those of Eggers and Tassinari. This division in the data-set has been lacking in previous studies. This facilitates tracing patterns in deposition and distribution, helping to recognise trends in consumption patterns.

The terms used to define objects within these two distinctions are offered and briefly defined below. ${ }^{135}$ These definitions were developed by the author to suit the needs of the study

\footnotetext{
132 Cool 2006, 138-140.

133 den Boesterd 1956.

${ }^{134}$ VanPool 2001, 120.

${ }^{135}$ Handled Pans are differentiated in this report by form, as opposed to previous literature which differentiated them by type, implying that the differences between these vessels were stylistic as opposed to functional. This thesis disagrees with this assumption, as will be discussed later in this chapter.
} 
material, though originated from the terminology already in use for vessels of this period ${ }^{136}$ and adapted to fit the functional attributes of the specific material under current study.

Forms:

Bowl: $\quad$ Open rimmed (neckless/untapered) basin with a height less than the radius of the vessel at its rim (Figure 2.01).

Bucket: $\quad$ Open rimmed (neckless/untapered) basin whose height is greater than the radius of the vessel at its rim. Often has handles, but not essential for classification (Figure 2.02).

Cauldron: Basin greater than $30 \mathrm{~cm}$ in diameter with vertical body coming up from base which is greater than one third the diameter of the rim (Figure 2.03).

Handled Pan 1: $\quad$ A basin with a rounded cylindrical horizontal handle (often fluted). This vessel is often decorated with the head of an animal at the end of its handle, most often as the head of a ram. cf. Eggers 154-155 (Figure 2.04).

Handled Pan 2: $\quad$ A basin with a flat horizontal handle. While the handle terminals can vary significantly in this form, from flat terminals (Eggers 147) to elaborate zoomorphic suspension loop terminals (Eggers 131-133), the principal shape of the vessel's basin and handle indicates that these vessels shared a functional continuity. The most common type of Handled Pan 2 from Roman Britain has a concentric circle suspension loop on its handle (Eggers 139-144). Rudge Cup type handled pans are considered as this form for the purposes of this thesis, though their function may indeed have been somewhat different than other handled pans. ${ }^{137}$ cf. Eggers 131153 (Figure 2.05).

\footnotetext{
${ }^{136}$ Most notably from den Boesterd 1956, Millet 1979 \& Cool 2010.

${ }^{137}$ Breeze 2012, 109-111.
} 


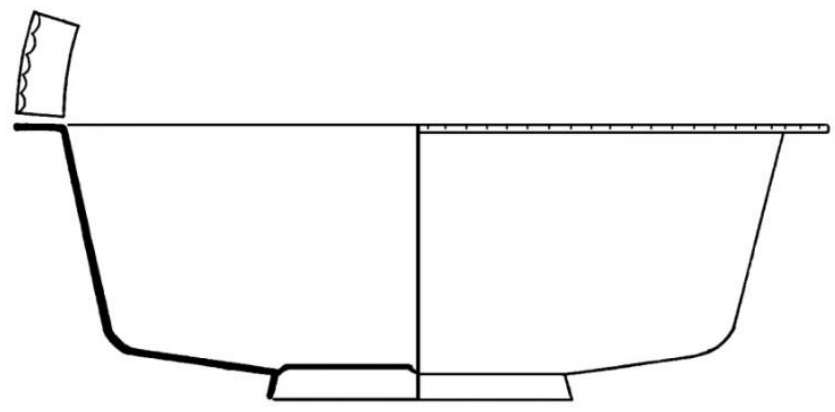

Figure 2.01: Bowl (Kennett 1971, 126 Fig.1, Scale 1:5)

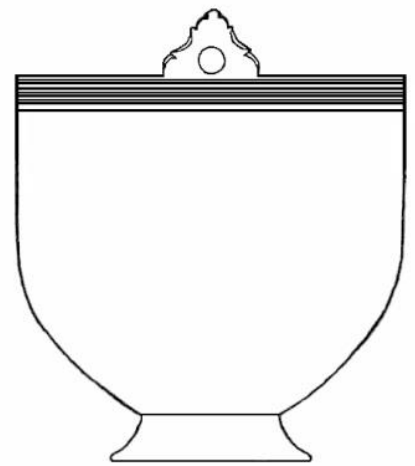

Figure 2.02: Bucket (Eggers 1951, Tafel 7 Fig.58, Scale 1:5)

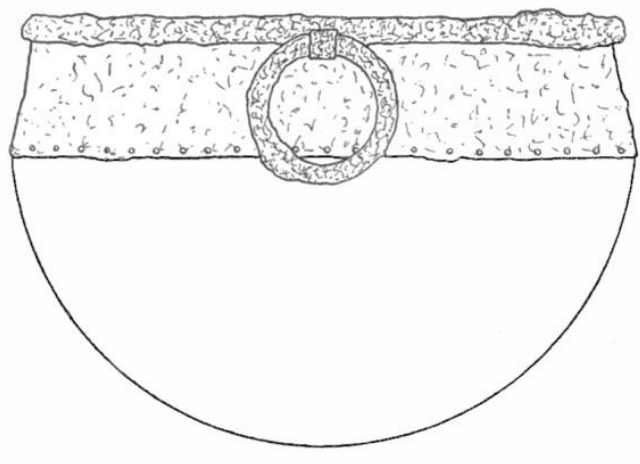

Figure 2.03: Cauldron (Eggers 1951, Tafel 2 Fig.5, Scale 1:12) 
Handled Pan 3: $\quad$ A basin with a flat hilted horizontal handle. cf. Eggers 159-161 (Figure 2.06).

Handled Pan 4: $\quad$ A large, square or oblong basin with a hinged and folding horizontal handle. It is also sometimes referred to as a Coptic type, as several examples are known from Egypt and it is believed to be of Late Roman date (Figure 2.07). ${ }^{138}$

Handled Pan 5: A basin with a long horizontal handle with hooked terminal, often in the shape of a swan or duck's head. cf. Eggers 130 (Figure 2.08).
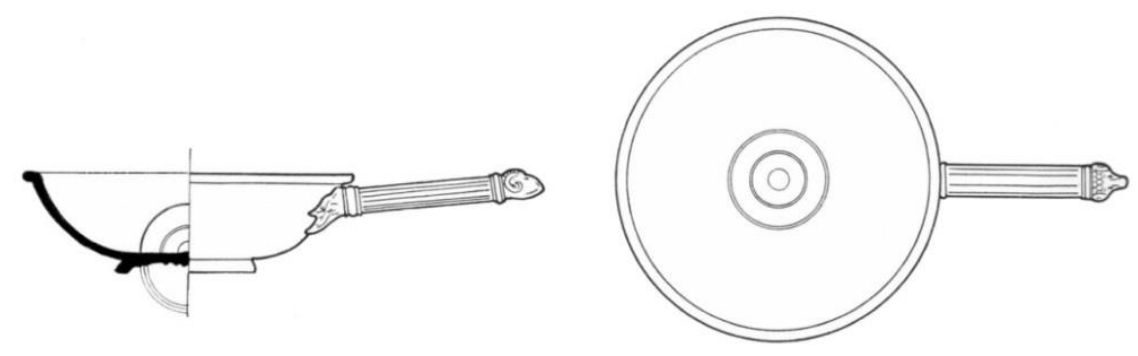

Figure 2.04: Handled Pan 1 (Radnoti 1938, Tafel VI Fig.29, Scale 1:7)

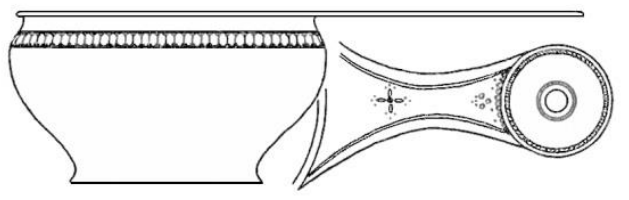

Figure 2.05: Handled Pan 2 (Eggers 1951, Tafel 12 Fig.144, Scale 1:10)

\footnotetext{
${ }^{138}$ Harcum 1921, 44-46; Kennet 1971, 137-138.
} 

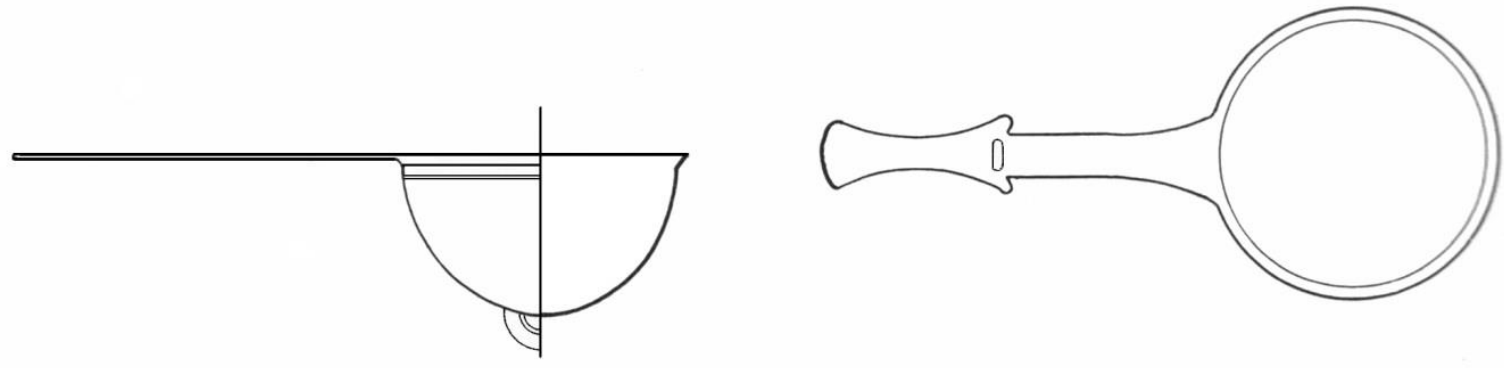

Figure 2.06: Handled Pan 3 (Radnoti 1938, Tafel V Fig.24, Scale 1:10)
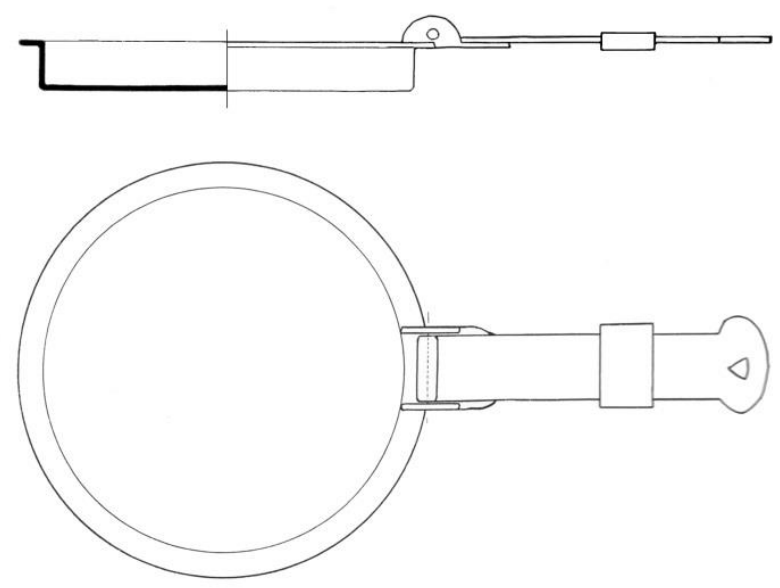

Figure 2.07: Handled Pan 4 (Radnoti 1938, Tafel V Fig.21, Scale 1:11)

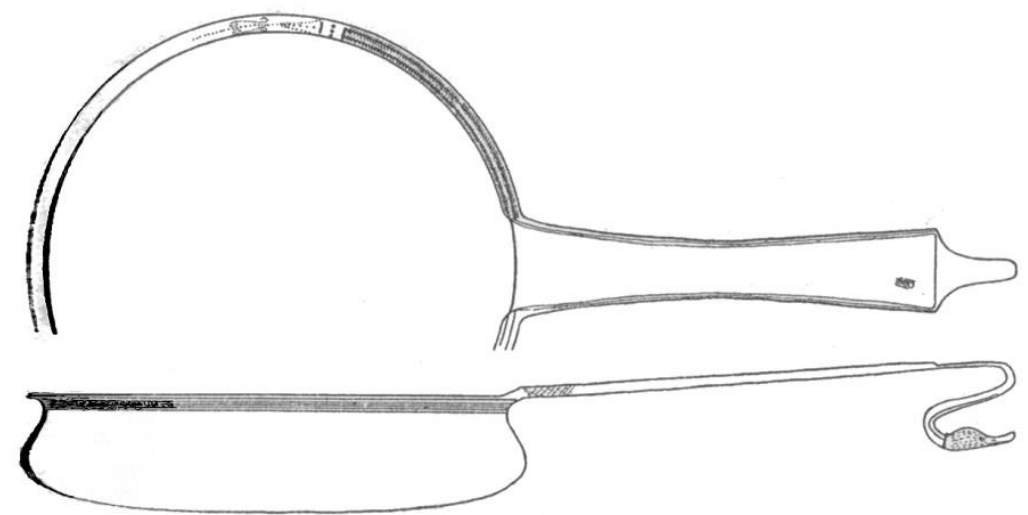

Figure 2.08: Handled Pan 5 (Eggers 1951, Tafel 12 Fig.130, Scale 1:7) 
Jar: A cylindrical vessel form which tapers at the rim. If the vessel has a neck, its height is less than one third the height of the whole (Figure 2.09).

Jug: $\quad$ Necked vessel with a neck height greater than one third the height of the whole. These vessels are almost ubiquitously handled (Figure 2.10).

Strainer: A basin with perforations designed to allow liquid to pass through, commonly believed to be used for the straining and flavouring of wine or other beverages during the Roman period. ${ }^{139}$ This theory is supported by the remains of artemisia found in a strainer from Stanway, ${ }^{140}$ though it must be remembered that the vessel could have had multiple purposes. The design of this vessel could vary significantly from resembling a bowl to resembling a handled pan, though the functional utility of this form of vessel may be presumed to have remained the same (Figure 2.11).

Den Boesterd and Cool provide the general consensus view on the use of different forms of vessels. ${ }^{141}$ Almost all forms of copper alloy vessels are believed to be associated with winedrinking, ${ }^{142}$ bowls and the various handled pans being the principal exceptions, as these are sometimes associated with hand washing and ablutions. Nuber argues on the basis of exhaustive iconographic analysis and contextual association that jugs were used with handled pans in hand washing, ${ }^{143}$ while Allison indicates the use of bowls for the same purpose. ${ }^{144}$ Of course, vessel use is likely to have varied over the some four hundred years under investigation in this thesis and it is not wise to seek a synthetic unified theory of form functionality to fit all vessels in all circumstances. However, the current consensus offers a baseline understanding for contextualizing the vessels and suggesting interpretation.

\footnotetext{
${ }^{139}$ Cool 2006, 143-146.

${ }^{140}$ Crummy et al. 2007, 207.

141 den Boesterd 1956; Cool 2006.

142 Carver 2001, 38.

${ }^{143}$ Nuber 1973, 7-28.

${ }^{144}$ Allison 2004, 55.
} 


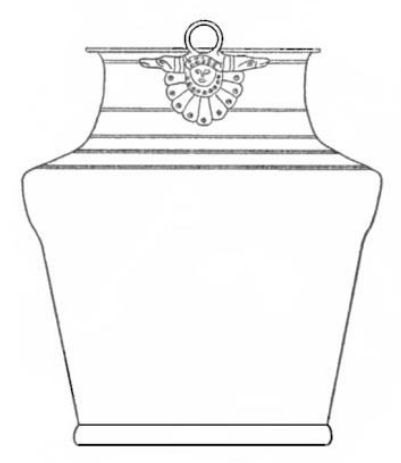

Figure 2.09: Jar (Eggers 1951, Tafel 4 Fig.25, Scale 1:7)

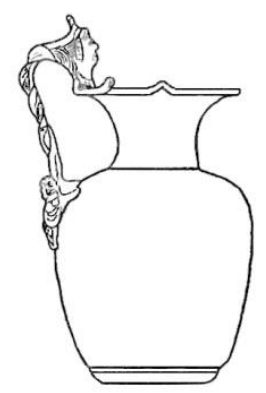

Figure 2.10: Jug (Eggers 1951, Tafel 11 Fig.124, Scale 1:10)

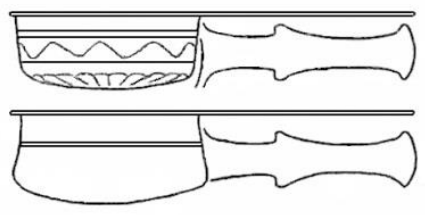

Figure 2.11: Strainer (Eggers 1951, Tafel 13 Fig.161, Scale 1:10) 
Types: In the course of this thesis, typology plays a much lesser role in the identification and charting patterns in deposition and distribution than form. Nonetheless, it is important to understand the typologies in use and the terminology as it is applied in this thesis and the greater scholarship as reference is made to specific types and they do prove to be useful in characterising use and distribution patterns in the British provinces during the Roman period.

Bassin á bord godronné: A bowl with a flat, out-turned rim with a series of oval impressed decorations, which Kennet refers to as godrons or repoussé bosses, along its perimeter (Figure 2.12). ${ }^{145}$

Bassin festonné: This is a bowl with a flat, outturned rim with a series of engraved half-circle grooves as decoration. ${ }^{146}$ This decoration is engraved and does not affect the shape of the rim itself (Figure 2.13).

Bassin uni: This type resembles the bassin á bord godronné and basin festonné, though with plain, outturned rim with no necessary decorative motifs (Figure 2.14). ${ }^{147}$

Hemmoor Bucket: This bucket has a globular body and is footed. It will either have a handle loop as part of its body or will have a separately cast looped mount for the attachment of a handle (Figure 2.15). ${ }^{148}$

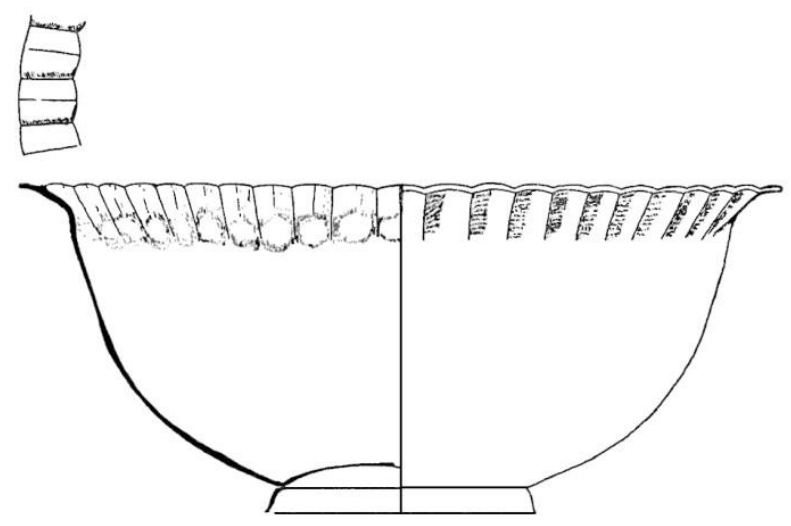

Figure 2.12: Bassin á bord godronne (Kennett 1971, 130 Fig.7, Scale 1:5)

\footnotetext{
${ }^{145}$ Kennett 1971, 138.

${ }^{146}$ Kennett 1971, 142.

${ }^{147}$ Kennett 1971, 138.

${ }^{148}$ Eggers 1951, 55-63.
} 


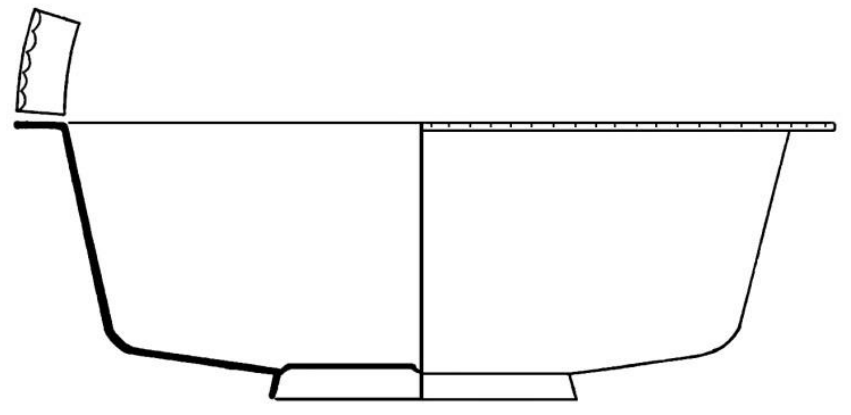

Figure 2.13: Bassin festonné (Kennett 1971, 126 Fig.2.1, Scale 1:5)
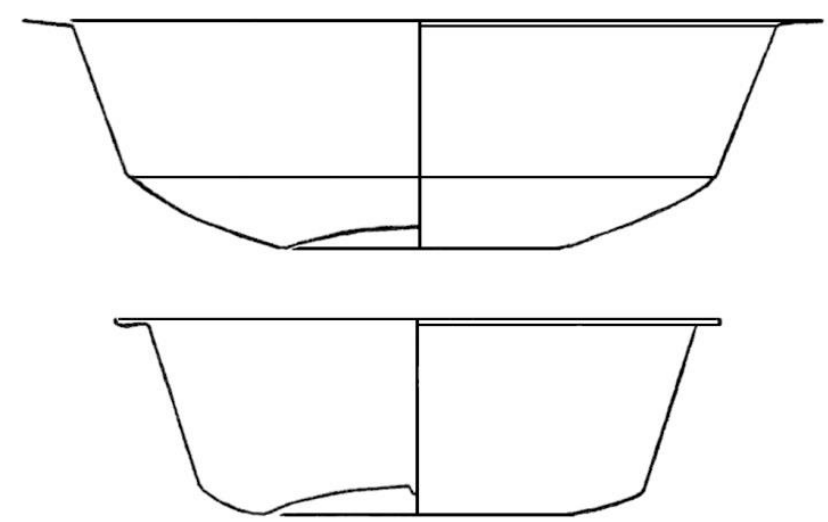

Figure 2.14: Bassin uní (Kennett 1971, 128 Fig4.6-7, Scale 1:5)

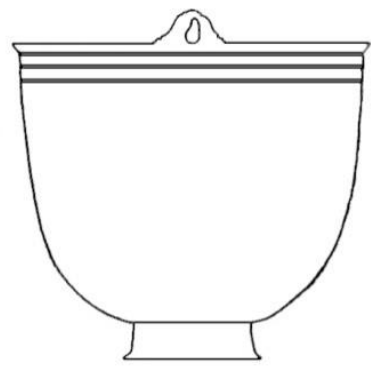

Figure 2.15: Hemmoor Bucket (Eggers 1951, Tafel 7 Fig.56, Scale 1:7) 
Irchester Bowl: This is a large bowl or basin with an in-turned rim and a rounded body. This vessel often has three escutcheons by which it may have been suspended and is believed from the depositional contexts in which it occurs to be of $4^{\text {th }}-5^{\text {th }}$ century manufacture. ${ }^{149}$ It has also been suggested that the uniformity of design of the vessel type implies that these are the products of one or more centralised or associated workshops (Figure 2.16). ${ }^{150}$

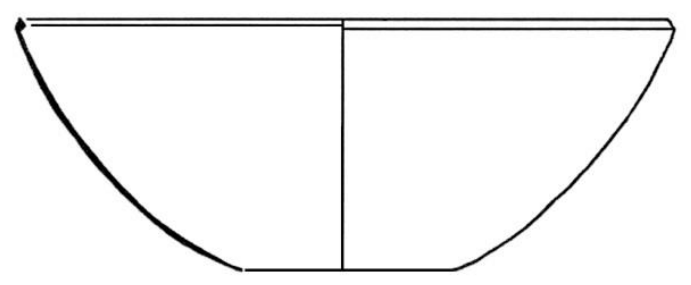

Figure 2.16: Irchester Bowl (Kennett 1971, 127 Fig.3.1, Scale 1:12)

Perlrandbecken Bowl: This bowl is characterised by an out-turned rim with a pearl or beaded decoration along its perimeter (Figure 2.17). ${ }^{151}$

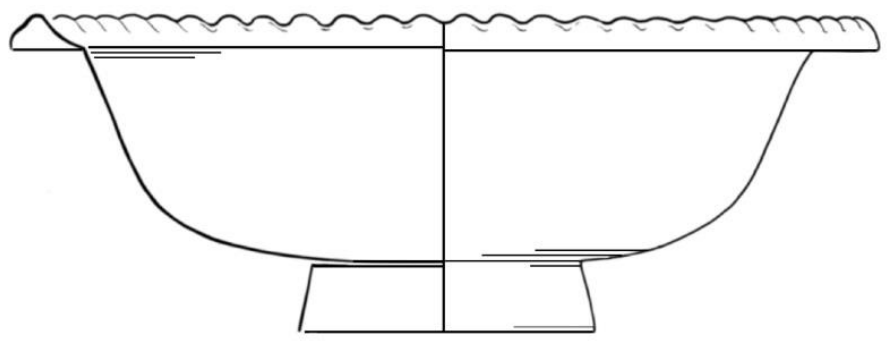

Figure 2.17: Perlrandbecken Bowl (Gerrard 2014, 110 Fig.3.17, Scale 1:6)

Rudge Cup Type: This is a small type of Handled Pan 2 with enameled decoration, generally of floral, geometric or architectural nature. As several examples carry depictions of Hadrian's Wall ${ }^{152}$ along with labelled references to military forts along it (including the Rudge Cup, Ilam Pan, and Amiens Patera) this type is believed to be associated with the military (Figure 2.18). ${ }^{153}$

\footnotetext{
${ }^{149}$ Kendrick 1932, 162.

${ }^{150}$ Farley et al. $1988,363$.

${ }^{151}$ Kennett 1971, 140.

152 Cowen \& Richmond 1935, 317-318.

${ }^{153}$ Breeze 2012, 109-110.
} 


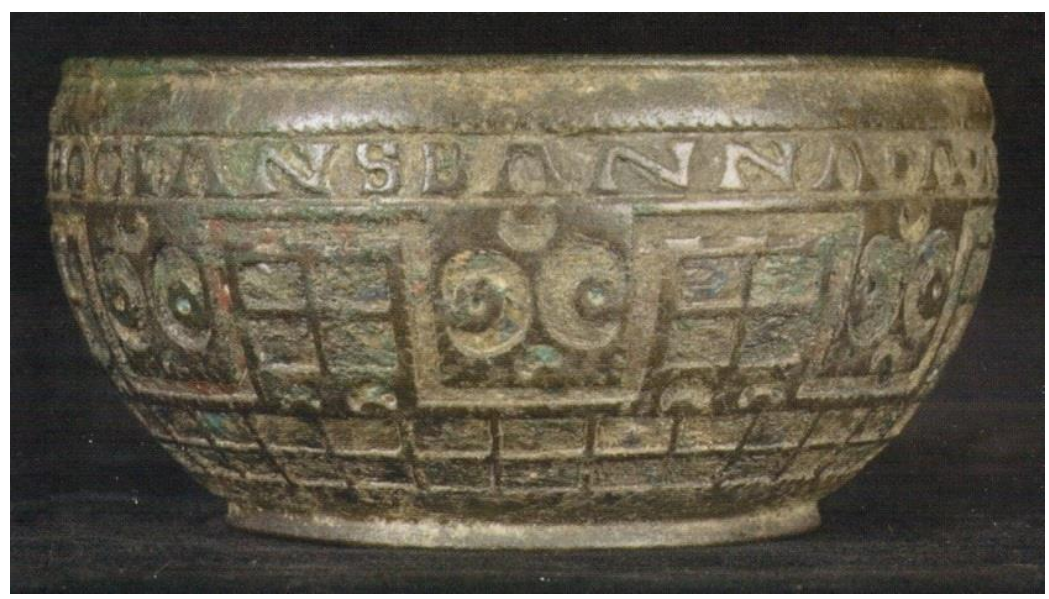

Figure 2.18: Rudge Cup Type (Breeze 2012, 1 Fig. 1.1)

In addition to the type categories just listed, typology numbers from other catalogues will be provided where appropriate to aid in the classification and understanding of the material under discussion. This will most frequently be Eggers' numbers, both because it is the common convention and because his typological catalogue remains the most comprehensive and functional for the purposes of identification and classification.

\section{$\underline{2.2 \text { Recording Frameworks Explanatory Notes }}$}

What follows is an overview and explanation of the research methods used for selecting and recording the copper alloy vessel material for this thesis and a justification for the decisions made during the course of the research. This is offered as a guide to the reader to allow for a better understanding of the methodology of the research as well as enable readers to determine for themselves the relevance of the biases which may or may not have affected the research.

Only material which is deemed diagnostic enough to be identified unequivocally as having consisted as part of a vesse ${ }^{154}$ dating to the Roman period during its pre-deposition use life will be included in the data-set. To be diagnostic, a fragment has to have enough of the body, rim, handle or an identifiable vessel mount surviving to indicate that it originated from a vessel of Roman date as compared with known examples from datable contexts or the object must originate from a stratigraphically datable context itself. Sheet bronze will not be considered diagnostic enough to be considered in this report. This practice differs from some

\footnotetext{
${ }^{154}$ As defined in Section 2.1.
} 
site reports, such as that for the Roman cemetery at Brougham, ${ }^{155}$ which choose to include this undiagnostic material in their quantification of vessels. The inclusion of such material leads to an over-representation of copper alloy vessels in these reports at the expense of other objects which were also constituted of copper alloy such as furniture fittings, decorative leaf, sheeting and jewellery. Additionally, to justify the decision to define this material as undiagnostic for attribution to a vessel, it is worth noting that undiagnostic sheet bronze is a not uncommon find. It would seem likely that some of this material may have at one time constituted parts of vessels, though unfortunately it is not possible to discern what sort of object sheet bronze constituted upon deposition. It is possible that sheet bronze may have been deposited simply as sheet bronze or scrap metal without being further worked or incorporated into any other form of object. Therefore, the inclusion of sheet bronze in this data-set would prove unrepresentative of the deposition of copper alloy vessels during the Roman period in Britain.

Also as a result of the necessity to exclude undiagnostic material, some other groups of data have been omitted. This is most evident in the case of drop-loop handles. Though it is true that several vessel forms possessed drop handles as a normal part of their construction, especially Italic designs as represented in the repertoire of Pompeiian material, ${ }^{156}$ a drop-loop handle alone is not diagnostic enough to necessitate that it originated from a vessel as it may in fact have been a furniture or casket fitting. An example of such a case may be found in the Roman burial discovered near Radnage, Buckinghamshire where a pair of drop-loop handles and several other copper alloy mounts which could have been interpreted as vessel trappings were in fact part of the fittings of a still recognizable wooden box. ${ }^{157}$ More recently, excavations along the A2 in Kent produced a similar casket with drop-loop handles as part of a burial. ${ }^{158}$ Another such box with elaborate copper alloy fittings, including medallion busts and drop-loop handles, is attested from Eigeltingen. ${ }^{159}$ Several other examples are also known and well documented from Augst and Mainz. ${ }^{160}$ Other objects may also have used drop-loop handles, as their presence on a gaming board from the 'Warrior's Burial' in Stanway, Essex demonstrates. ${ }^{161}$ This evidence being considered, only drop-loop handles which are directly associated with other fragments of a

\footnotetext{
155 Cool 2004.

156 Tassinari S2122-4400, for example.

157 Skilbeck 1923, 335.

${ }^{158}$ Allen et al. 2012, 381.

${ }^{159}$ Kohlert-Németh 1990, 9.

${ }^{160}$ Kohlert-Németh 1990, 40-44; Riha 2001, 23-33.

${ }^{161}$ Crummy et al. 2007, 186-193.
} 
vessel will be included in this data set as vessel material. This may lower the quantitative representation of this variety of handled vessel, but its inclusion would prejudice against the other applications of this type of handle and therefore skew the data as to make it unreliable and unrepresentative. While Riha's discussion of drop-loop handles is very detailed in their relation to varying types of boxes, chests and caskets, ${ }^{162}$ there has yet to be an analysis of how drop-loop handles designed for use as casket fittings and those designed for use with vessels typologically relate to each other and if there is significant variation between them or a reliable means to differentiate between handles used for these different purposes. Without such foundational research being available, it proved impossible to reliably and systematically assimilate drop-loop handles into the data-set of this thesis, which led to their omission.

Careful examination of the data was crucial before it was included within the data-set. To be included in this thesis as vessel material of Romano-British origin, the following criteria must have been met by each object:

\section{Its provenance and find circumstances must be known.}

2. It must have analogous examples which are datable and identifiable as Roman vessels, or be stratified in association with material of Roman date.

Though seemingly straight forward, these criteria have led to the exclusion of some material from the data-set and therefore they require explanation and justification.

The first criterion regards location and is absolutely necessary in a study which has a finite geographic boundary. ${ }^{163}$ It is important for the integrity of the study that the object in question exited normal use-life and its deposition into the archaeological record occurred in Britain during antiquity. This, of course, does not exclude material manufactured elsewhere. What is of importance is that the object was used and fell out of regular use in Britain, and for that reason the findspot must be recorded in order for the material to be provably of British archaeological provenance and therefore included in this data-set. Having accurate findspot information also proves instrumental to tracing patterns in depositional practice and for addressing the research aims and thesis questions outlined in Chapter 1.

The second criterion is a temporal criterion and is therefore of prime importance in a study with finite chronological perimeters. Material in this survey is limited to objects that fell

\footnotetext{
162 Riha 2001.

${ }^{163}$ In the case of this thesis, England and Wales.
} 
out of normal use-life and were deposited into their archaeological context within the Roman period of Britain, which for the purposes of this thesis is defined as between 43 and $410 \mathrm{CE}$. This excludes material interred during the Late Iron Age as well as material reused and deposited during the Anglo-Saxon period. This distinction can be difficult to make in some instances, most particularly with Conquest Period burials that have a date range that straddles the 30s to 50s CE. For this reason, material datable to the Conquest Period will be reviewed also in this thesis. By excluding material which would reflect different use-life and depositional patterns, it allows this thesis to more clearly focus on the patterns which develop during the Roman period without distraction and therefore to be able to have a more detailed analysis of this particular material. Of course, unstratified finds are impossible to date on anything besides stylistic grounds and it may be very problematic to determine when an object may have entered the archaeological record, regardless of its date of manufacture. ${ }^{164}$ For this reason, unstratified finds not associated with features of later dates which are stylistically datable to the date range of the Roman Period are included in this report. ${ }^{165}$ It was important also to make sure that objects post-dating the Roman period were not included, this being most problematic for fragmentary and undiagnostic objects from unstratified or poorly dated contexts. Metal working techniques varied relatively little over time prior to the Industrial Revolution and often the most diagnostic assessment for a fragment of undecorated copper alloy may be 'pre-Industrial'. This means that style and form are the key indicators of date, with manufacture method used for specification and authentication of the object. This was particularly applicable to fragmentary material recorded through the PAS, some of which could only be datable to the 'pre-industrial' period. While some of this fragmentary material could well have been of Roman date, lack of stylistic or stratigraphic evidence to indicate this led to exclusion of such objects from the data-set.

The location from which an object has been found is recorded in five different categories (County, Site, Site type, Feature, Context). While these categories may be somewhat artificial, they are valuable in organizing the data into manageable geographic and depositional groups to allow comparisons to be carried out in a variety of different formats in order to trace patterns and study variations in depositional practice. The modern boundaries and place names help to place the objects in an identifiable location and provide a consistent and comparable means by which

\footnotetext{
${ }^{164}$ Gerrard 2013, 9.

${ }^{165}$ Such as material reported through the PAS, further detailed in Chapter 6.
} 
to record the find-spots of the objects under study in this thesis. Of course, the site location and context upon deposition (i.e. the ancient context) is of chief importance in this thesis and this location data is included in the recording and presentation framework. For the sake of clarity, a brief definition of each of the categories of location data follows.

- County- Current county boundaries (as of the principal research phase) ${ }^{166}$ within which the object was found, as defined by the boundaries used by the Portable Antiquities Scheme.

- Site- The site name or modern parish within which the object was found. Parishes are defined by the boundaries used by the Portable Antiquities Scheme.

- Site type- $\quad$ The site as it related to the ancient landscape from the evidence currently available to archaeological study. The choice was made to avoid some of the traditional categorizations used to characterise sites, such as 'villa', 'small town' or 'rural shrine', as they are unlikely to accurately or consistently reflect the perception of these sites to ancient eyes. Also, a divide between ritual and secular space in characterising sites is often used but again does not necessarily reflect how space was qualified in antiquity and is therefore a problematic means by which to characterise sites. For this reason, value neutral distinctions based upon the architectural remains of a site were used as the means to qualify site types. There are four site types used in this thesis. Urban refers to sites that have evidence for three or more features of monumental architecture extant including triumphal arches, amphitheatres, forums, temples, monumental inscriptions, etc. Military refers to a site that can be identified as of principally military function, such as a fort or a fortress, and its surrounding features such as a vicus. Rural Settlement refers to any site which has evidence of settlement that falls outside of the previous two categories, notably including villas and rural shrines, but also including small towns and farmsteads. Rural Unknown refers to sites where there is presently no direct evidence for structures or habitation during the Roman period, though it is understood that such evidence from timber or mud-and-thatch structures may have disappeared through the forces of time and the plough.

- Feature- Specific contextual data of an object within a site upon deposition (i.e. well, cremation pit, house cellar, etc.).

\footnotetext{
${ }^{166}$ End of 2012.
} 
- ContextThe context classification, which in this report is divided into the four categories of 'Structured Deposit', 'Grave Deposit', 'Site Find' and 'Single Find recorded through the PAS'.

Chapters 3-6 are divided based upon depositional context. Within these chapters, the treatment of material is presented initially in geographic terms using approximately the regional distinctions utilised by the journal Britannia in its annual 'Roman Britain in...' column. This regional division provides a well-established framework within which to present and consider the material and allows for larger regional patterns to be recognised and compared. During the writing of this thesis, it was found that depositional trends would often cross borders between these regions. For this reason, the boundaries of regions and counties are not strictly adhered to in the analysis and discussion of the data-groups; they are instead used as loose guide-lines in which to present the data. The larger regional variations that were identified during the course of this thesis prove key in addressing the thesis questions offered in Section 1.5.

As many of the objects were highly fragmentary, decoration proved highly important for identification of objects belonging to this data-set. All decoration is divided into four categories: anthropomorphic, zoomorphic, floral, and geometric. 'Anthropomorphic' refers to humanoid or figural representation, 'zoomorphic' refers to any representation of animals, 'floral' refers to any depiction of vegetation, and 'geometric' is used for abstract shapes, designs and patterns. Decoration has also been recorded separately for the vessel body and the vessel handle. This is because in the course of the research and data collection of this thesis, it was found that often the decorative motifs on these two parts of the vessel would differ significantly. Dividing the data between body and handle during the recording process also allows for more detailed reporting and comparison of decoration between objects. Inscriptions were not common, being present on some 62 objects in the data-set. This information was also recorded as occurring either on the body or the handle of the vessel. Inscriptions will be discussed individually as they occur throughout the thesis and a synthetic analysis of them will be offered in Section 8.3.

Chronological data for objects is divided into two categories: date of manufacture and date of deposition. As copper alloy vessels are durable objects and may have an extensive uselife, objects may well be deposited long after their manufacture and objects of divergent manufacture dates may be deposited together. ${ }^{167}$ For this purpose, date of manufacture and date

\footnotetext{
${ }^{167}$ As is the case with Draper's Gardens (Gerrard 2009; L0001-L0015; Section 3.2).
} 
of deposition were recorded in the cases where approximate date of deposition (beyond that of its known manufacture period) is recorded, i.e. when there is other evidence (such as stratigraphically associated numismatic or ceramic evidence) for the date of deposition; this is the case with a number of Structured Deposits and Grave Deposits, though not common among Site Finds and inapplicable with the disturbed contexts of PAS material.

Objects were assigned catalogue numbers consisting of an abbreviation for the modern county they were found in ( $\mathrm{K}$ for Kent, MON for Monmouthshire, etc.) followed by a numerical designation. These are the numbers that are used to identify and reference the objects in this thesis. The full catalogue of objects is collected in Appendix I, with subsidiary groupings by Depositional Context (Appendices II-V) also offered. These appendices offer full data entries for the objects that were not always prudent or possible to include in the body of the thesis text.

\subsection{Data Collection and Analysis}

What follows is a brief discussion of the resources reviewed in the course of data collection of this thesis. While the data collection phase of this thesis ${ }^{168}$ attempted to be as inclusive as possible within the time and monetary restraints of a doctoral thesis, discrepancies in reporting and the availability of data will inevitably have affected the data-set of this thesis. This problem faces any scholar attempting to undertake a materially based study as the quality and accuracy of reporting will inevitably vary widely from publication to publication. A good example of how accuracy of reporting may be an issue with even a well-publicised group of objects may be found in the Ribchester Hoard. Found in 1796, it consists of various copper alloy objects, most famously a near complete Roman cavalry helmet. ${ }^{169}$ Among the objects possibly associated with this hoard are three Handled Pan 2s. ${ }^{170}$ While all three would appear to have been purchased by Charles Townley along with the rest of the Ribchester Hoard, ${ }^{171}$ Eggers records two of the Handled Pan $2 \mathrm{~s}$ as being a separate aquatic deposit ${ }^{172}$ and it may well be that these two or all three Handled Pan 2s actually do not originate from the rest of the hoard

\footnotetext{
168 The principal data collection phase of this thesis project was undertaken from August 2011 to September 2012 , with minor corrections and amendments added until the submission of the thesis.

${ }^{169}$ Smith \& Shortt 1890, 32; Jackson \& Craddock 1995, 75; Edwards 2000, 95.

${ }^{170}$ LAN0001, LAN0002, LAN0004.

${ }^{171}$ Smith \& Shortt 1890, 32; Jackson \& Craddock 1995, 75.

${ }^{172}$ Eggers 1968, 107 (57a \& b).
} 
assemblage. ${ }^{173}$ This specific instance is highlighted as only one example of how data could be confused and misreported, even in a group of objects that has received considerable popular and scholarly attention. This made cross-referencing information particularly important in cases where multiple sources were available. Under-reporting is also a significant issue with any study of archaeological material, particularly with single finds from excavations or as chance finds by the public that may not be published widely or published at all. For this reason, the data-set comprises of data available through print or online publication including journal articles, published site reports, print and online museum catalogues as well as scholarly publications. The choice to rely on these source materials for this thesis was made principally to help ensure the veracity and quality of the data collected while also providing the widest and most inclusive method for collecting data in order to minimise mistakes and omissions. What follows is a brief description of the source material used to gain data on the various depositional contexts of copper alloy vessel material for this thesis.

\section{Structured Deposits}

The starting point for the gathering of material on Structured Deposits for this report was a combination of two reports, the first by Eggers and the second by Kennett, in 1968 and 1971 respectively. Between these two articles, the principal hoards of copper alloy Roman vessels known in Britain up to 1971 are listed, though some of the data proved to be inaccurate or incomplete and was corrected in the current thesis. These articles also provide sources for cross referencing, which sometimes have greater amounts of detail. Consultation of journal reports also showed some significant omissions from the catalogues of Eggers and Kennett. Individual site reports and the county archaeological journals were referenced for any new finds, as were the pertinent scholarly journals (i.e. Britannia, Antiquaries Journal, etc.). The Portable Antiquities Scheme also proved useful in illuminating this category, from groups of objects either brought in by a finder or collected through excavation when a site has been reported to the local Finds Liaison Officer. Each object in a Structured Deposit was individually recorded and identified as comprising a group with any other vessels associated with it. Other associated finds were also recorded, though with less detail given than the copper alloy vessels. Associations

\footnotetext{
${ }^{173}$ Edwards 2000, 69. As discussed in Section 3.2, it is the opinion of the author that this group of objects is composed of more than one act of deposition.
} 
between vessels, as well as other objects in these Structured Deposits, aided in determining possible depositional processes and patterns between groups of Structured Deposits as detailed in Chapter 3.

\section{Grave Deposits}

Philpott's comprehensive survey of Roman period graves in Britain provided a suitable point of reference to begin this segment of the research. This report detailed the known Romano-British graves as of 1991, along with accounts of their burial method and grave goods. Egger's 1968 article proved useful in amending the Philpott report. For more recent discoveries, review of Britannia and county archaeological journals was necessary. Site reports and monographs were also of invaluable use for the collection of data, as several large cemetery sites of Roman date have been excavated across Britain in the intervening decades and been published as independent monographs. It was imperative to accurately record the finds associated with Grave Deposits as they often proved to be highly variable and complex assemblages. Associated finds also provided a further method to gauge patterns in depositional practice, indicating likely perceived associations between the objects held by those performing the interment.

\section{Site Finds}

The primary source for Site Finds was within the specific excavation reports themselves, when these included finds reports. Particularly unusual or noteworthy finds may also be published in their own rights in applicable journals. Associated finds did not figure heavily with Site Finds, as associated finds almost always indicate some level of intentionality in the depositional process and would therefore be more suggestive of a Structured Deposit. As discussed in Chapter 5, patterns did emerge in the synthesis of this material and proved that Site Finds can be very instructive in our understanding of the use and spread of material culture across Britain.

\section{Single Finds reported through the Portable Antiquities Scheme (PAS)}

The PAS database contributed over 200 objects to the data-set for this report, more than any other single source. Nevertheless, the data from this source had to be sifted and re-examined 
before being included in this study and the steps taken in the utilization of this data-group are explained here.

The PAS database is a means for recording the objects which are processed through the PAS for identification in accordance with the Treasure Act 1996. While projects have been in place to record finds in various parts of England and Wales since 1997, it was only in 2003 that all of England and Wales were incorporated within the Scheme. ${ }^{174}$ Objects are found and collected by the public at large, mostly by metal-detectorists and hobbyists, from across England and Wales and taken to their local Finds Liaison Officer (FLO) for recording. They are identified and recorded on an online database available to the public. The objects are then usually returned to the finder, though some are donated or purchased and find their way into museum collections. The open nature of the PAS allows for much more data to be acquired for scholarly research than would be available through more traditional means, but also leads to a great deal of variation in the quality and quantity of recorded material. This will inevitably have an effect on any study which utilises this resource.

A number of biases influence the material which is entered onto the Portable Antiquities Scheme database. First and foremost, it relies on public interest and vigilance to bring objects in to be identified and recorded. Primarily, it is likely that more decorative and ornate objects will be far more often recorded than plain, unadorned or simple objects. This would indeed appear to be the case, as in the PAS data-set used here some $80 \%$ of the material has iconographic elements whereas non-PAS data has iconography or decoration on approximately $10 \%$. There is also a geographic bias in this collection method, as objects will be found and reported in greater volume in areas where people expect to find objects. This is predominantly in the east Midlands, East Anglia and the south eastern counties of England, as this area is more intensively frequented by metal detector hobbyists searching for finds in the plough-soil. ${ }^{175}$ This geographic bias was anticipated in the collection of data, though in practice it does not appear to have provided any greater geographic bias to the south east than other sources of data and in fact the PAS data

\footnotetext{
${ }^{174}$ Worrell 2004, 317.

${ }^{175}$ Brindle 2011, 21-24 \& 69-71.
} 
helped to illuminate several parts of Britain which had practically no copper alloy vessels recorded in traditional archaeological reports. ${ }^{176}$

The second principal variable is the ability of the Finds Liaison Officer to identify and subsequently record an object once it has been reported by a member of the public. As FLO's have to record objects that span a history of some 500,000+ years, it is not surprising that not all of them are Roman Finds specialists. ${ }^{177}$ With this in mind, careful scrutiny was used in qualifying the PAS data to be included in the data-set. To the credit of the PAS, misidentification did not seem to play any major part in the objects included in this thesis. ${ }^{178}$

These two variables require that each report is individually reviewed before its inclusion in the data-set for this study. The criteria used for qualifying material for this report was outlined previously in this chapter. No source can indeed be taken for granted, however, and it proved necessary to scrutinise and cross-reference scholarly publications with the same level of vigilance as was necessary for the PAS data.

\section{$\underline{2.4 \text { Closing }}$}

Now that the framework for the data collection has been explained, it is possible to turn to the material itself. The following chapters will deal in depth with the data collected and are grouped by their depositional categories (i.e. Chapter 3-Structured Deposits, Chapter 4-Grave Deposits, Chapter 5- Site Finds, Chapter 6- PAS). Data synthesis and overall thesis results will be offered in Chapters 7 and 8.

\footnotetext{
${ }^{176}$ As discussed in Chapters $6 \& 7$.

${ }^{177}$ Brindle 2011, 70-71.

${ }^{178}$ Discussed in Section 6.1.
} 


\section{Chapter 3: Structured Deposits}

\section{$\underline{3.1 \text { Introduction }}$}

In this chapter, Structured Deposits of copper alloy vessels from Roman-Britain will be reviewed. The purpose of the present chapter is to critically analyse this group of data in isolation to determine patterns in object selection and depositional process across time and space in Britain. The expository discussion of the material review will be organised by region, beginning in Wales and then turning north to Hadrian's Wall before progressing south. Discussion of the assemblages within each given region will then be offered. Patterns in deposition for Structured Deposits across Britain will be outlined in Section 3.3, while discussion of patterns observable in the forms and types deposited will be presented in Section 3.4. It is worth noting at the outset of this chapter that while Structured Deposits of materials are well known in Western Europe and Britain dating back as far as the Neolithic period, ${ }^{179}$ there does not appear to have been a widespread tradition for Structured Deposits of copper alloy vessels in Britain prior to the Roman period. ${ }^{180}$ Its ready visibility during much of the Roman period, then, must be seen as a change and adaptation of culture practice. This will be further considered throughout this chapter and in Chapters 7 and 8.

\section{$\underline{3.2 \text { Geographic Survey of Structured Deposits }}$}

\begin{tabular}{|l|l|}
\hline Find Location & Catalogue Numbers \\
\hline Coygan Cave, Kyngadl (Carmarthenshire) & CAR0001-CAR0002 \\
\hline Glyn Dyfrdwy (Denbighshire) & DEN0001-DEN0004 \\
\hline Plas Uchaf, Abergele (Denbighshire) & DEN0008-DEN0016 \\
\hline Halkyn Mountain, Halkyn (Flintshire) & F0001-F0008 \\
\hline Ynys Gwrtheyrn (Gwynedd) & GWY0001-GWY0003 \\
\hline Harlech (Gwynedd) & GWY0004-GWY0008 \\
\hline Llanberis (Gwynedd) & GWY0009-GWY0012 \\
\hline Langstone (Newport) & NE0007-NE0008 \\
\hline
\end{tabular}

Table 3a- Structured Deposits from Wales.

\footnotetext{
${ }^{179}$ Bradley 1990; Hill 1993, 57-75; Hill 1995a, 47-98; Hill 1995b; Hill 1996, 17-32.
}

${ }^{180}$ Carver 2001, 37. 


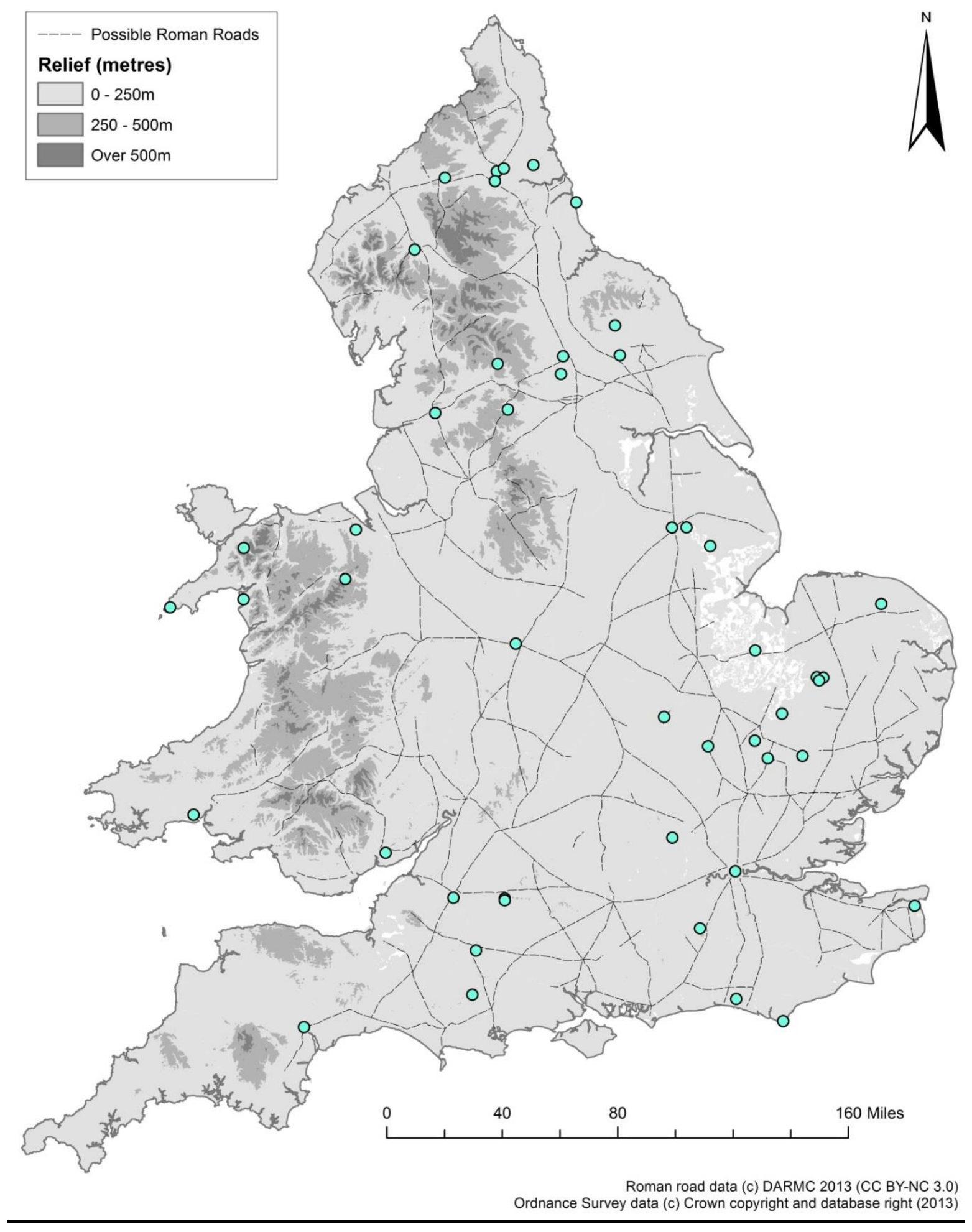

Map 1: Structured Deposits of copper alloy vessels in Roman Britain (K. Robbins) 


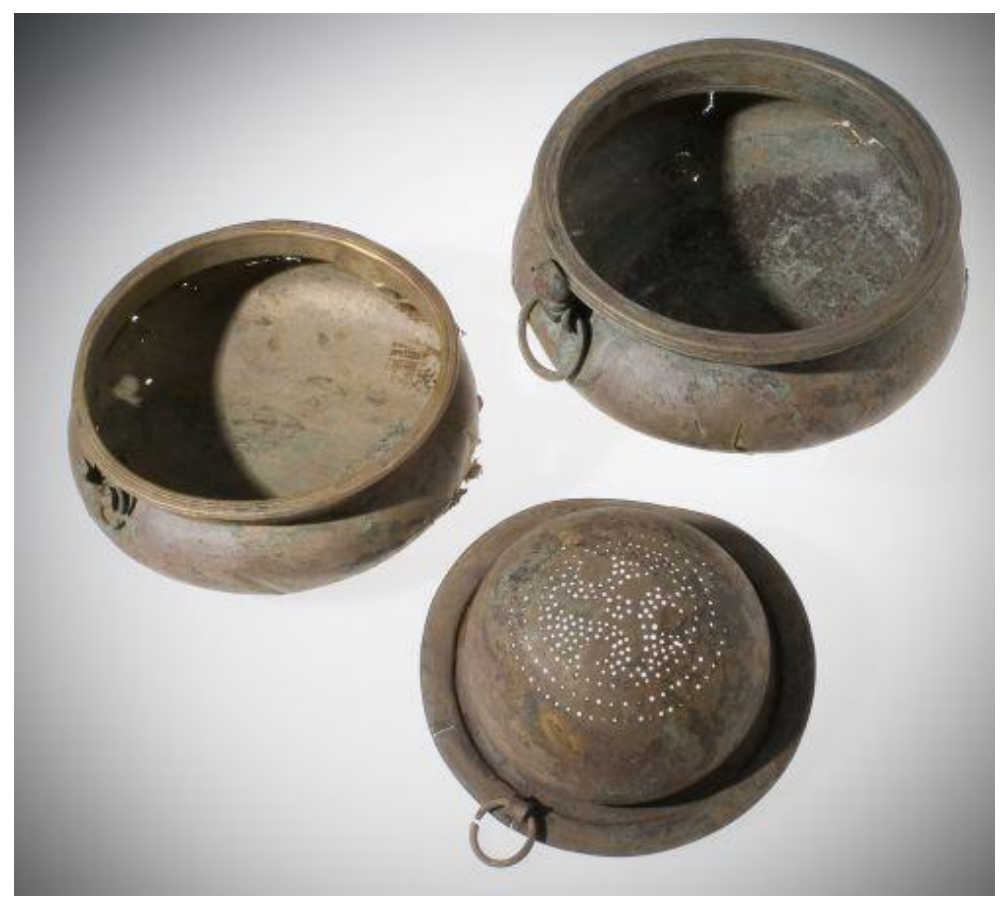

Figure 3.01: Structured Deposit from Langstone (PAS NMGW-9C0216)

Map 1 illustrates the findspots of Structured Deposits of copper alloy vessels in Roman Britain. Though found across Britain, several geographic trends are immediately apparent; a preference to the east and north of England and a marked absence from the centre of the province being two of the most important. The following discussion will detail the individual assemblages from each region and then discuss regional trends in deposition.

There are 8 Structured Deposits from Wales (Table 3a). The Structured Deposit from Langstone, Newport was discovered in 2008 and processed as Treasure through the PAS (Figure 3.01). The group comprises three vessels: two 'Rose Ash' type bowls and a single flange rimmed strainer with a 'triskele' geometric strainer dot pattern of similar design to that found on some Late Iron Age vessels. ${ }^{181}$ The bowls could be of either Late Iron Age or Roman period manufacture, as the namesake vessel of this type discovered at Rose Ash in Devon is thought to have been interred prior to the Claudian invasion. ${ }^{182}$ A wooden tankard of indigenous manufacture with copper alloy fittings was found 12.8 meters away from this Structured Deposit and is likely to have been intentionally deposited in much the same manner, the date of manufacture is believed to be Romano-British due to a parallel example found from a Roman

\footnotetext{
${ }^{181}$ Wainwright 1967, 88; Laing 2000, 10-14; Worrell 2009, 286-287.

${ }^{182}$ Fox 1961, 186-198.
} 
fort in Swansea; Worrell therefore suggests a late $1^{\text {st }}$ century CE date of deposition for all these objects. ${ }^{183}$ The strainers are likely of continental import, indicating their owners had crosschannel contacts. All three vessels have hanging vessel mounts and suspension loops. The hanging vessel mounts of the bowls are decorated by being divided into several separate lobes with insets of red glass. ${ }^{184}$ The objects were deposited in a bog, reflecting a wider trend of vessels deposited in aquatic environments that will be further discussed in Section 3.3.

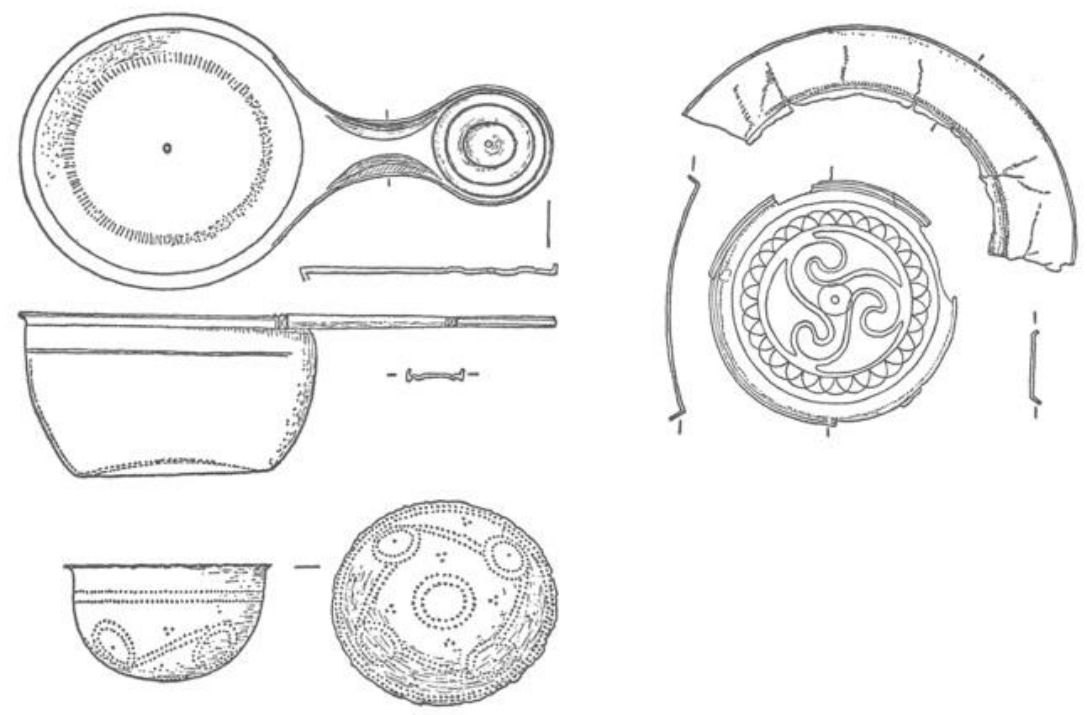

Figure 3.02: Structured Deposit from Coygan Cave (Eggers 1968, 147 Abb. 47)

The Structured Deposit from Coygan Cave in the parish of Kyndgadl in western Wales provides a useful parallel to this group from Langstone (Figure 3.02). It consists of a Handled Pan 2 and a flange rimmed strainer similar to that from the Langstone group. Found in the early $19^{\text {th }}$ century, the Structured Deposit was also stated to have contained 60 coins of the emperor Carausius. Unfortunately, these coins are non-extant and the association cannot therefore be substantiated. A hoard of counterfeit coins dating to roughly the same period found nearby ${ }^{185}$ could help support the claim of these vessels originally containing a coin hoard. The possible association with Carausius indicates that the late $3^{\text {rd }}$ century would be the earliest that this group

\footnotetext{
${ }^{183}$ Worrell 2009, 285-287.

${ }^{184}$ PAS NMGW-9C0216.

${ }^{185}$ Wainwright $1967,85$.
} 
could have been interred. While of likely $1^{\text {st }}$ century CE manufacture, the Handled Pan 2 shows extensive signs of use as a result of repair and/or customization. Its original base plate was removed and replaced with a base decorated with a 'triskele' design, ${ }^{186}$ similar to the decoration of the Langstone group mentioned above.

A further Structured Deposit from Roman Wales comprising a strainer and several basins was found in 1862 at Abergele in Denbighshire. ${ }^{187}$ This group is significantly larger than the Coygan Cave or Langstone groups, comprising some nine extant vessels and believed to have originally contained more, but its composition of a strainer coupled with a collection of Handled Pan $2 \mathrm{~s}$ and bowls is notably similar to other Structured Deposits from the $1^{\text {st }}-2^{\text {nd }}$ centuries in Wales and elsewhere in Britain. ${ }^{188}$ Two of the bowls in the Abergele assemblage bear etched inscriptions on the interior of their basins. ${ }^{189}$ They both have 'INDVS' etched legibly followed by an uncertain combination of letters or symbols that have a confused interpretation, perhaps labelling objects in a numbered set. ${ }^{190}$ The name 'Indus' most likely represents an ownership label, as it would be unusual for this to be a votive inscription as it lacks an apparent reference to a deity. Such etched inscriptions are rare on copper alloy vessels, and the purpose of this graffito must remain somewhat obscure given the current level understanding. The punch-dot pattern of the handled strainers is paralleled by Pompeiian examples, ${ }^{191}$ and the manufacture of all of the objects could easily fall within the 1st century CE, suggesting a date of deposition analogous to the Langstone group.

These three Structured Deposits exhibit very similar features. All three contain basins and strainers. Both the Langstone and Coygan Cave groups exhibit geometric and triskele decoration which could indicate cultural interchange and hybridization between Roman and indigenous artistic traditions while the presence of what are likely Iron Age vessels in the assemblages of Abergele and Langstone show the persistent use of indigenous forms and the incorporation of Roman forms with them following the conquest. While the manufacture date of all the vessels from all three groups would appear to date to the $1^{\text {st }}$ century CE or earlier, the degree of wear on the Handled Pan 2 from Coygan Cave, as well as the possible association of

\footnotetext{
${ }^{186}$ Wainwright $1967,88$.

${ }^{187}$ RCAHM 1914, 7-8; Wright \& Hassall 1972, 363.

188 See later in this section.

${ }^{189}$ DEN0008 \& DEN0009.

${ }^{190}$ RIB II 2415.61-62.

${ }^{191}$ Tassinari 1993 (1347).
} 
Carausian coins, indicate a possible $3^{\text {rd }} 4^{\text {th }}$ century CE or later date of deposition. Langstone and Abergele lack further contextual data to date their deposition beyond their date of manufacture, though they may or may not have been deposited significantly later than this. According to the general consensus regarding vessel use, the strainers suggest that these sets were intended for the drinking of wine or beer. ${ }^{192}$ However, the evidence for Handled Pan 2s having any specific association with wine-drinking is not very strong across the evidence offered in this thesis and it is likely that strainers had uses outside of wine and beer drinking. This will be discussed in further detail in Chapters 7 and 8 after evidence from across other Depositional Contexts is considered.

The Structured Deposits from Llanberis and Glyn Dyfrdwy are unusual as they comprise only Handled Pan 2s, and in the case of Llanberis both specifically of Eggers type 142. One of the Handled Pan 2s of Eggers type 131 from Glyn Dyfrdwy is the most richly decorated item to come from any Structured Deposit in Wales. ${ }^{193}$ The suspension loop at the terminal of the handle is moulded from a design of dual swan's heads, which den Boesterd suggests indicates northern Italian manufacture. ${ }^{194}$ The handle is also stamped with two legends: 'S MERCV' at a 45 degree angle across the handle just beneath the suspension loop and 'CIPINICOMA' in line with the handle. ${ }^{195}$ 'CIPI' is a well attested stamp across the western Roman world and refers to a family of copper alloy manufacturers who were operating in the $1^{\text {st }}-2^{\text {nd }}$ centuries $\mathrm{CE}$, most likely in Capua or southern Italy. ${ }^{196}$ The 'MERCV' legend could be read as '[sor]s Mercur[i]', or 'Mercury's lot', which was used to label goods of high quality. ${ }^{197}$ Additionally, one of the Handled Pan 2s from the Structured Deposit at Llanberis also bears a maker's mark:

'ABVCCV $[. .$.$] '. { }^{198}$ Makers' marks are not common on copper alloy vessels, though are far more common on Handled Pan 2s than other vessel forms, these two particularly suggesting that the objects were manufactured in southern Italy. ${ }^{199}$ A useful parallel may be drawn between these two Structured Deposits and two other Welsh assemblages found not far distant, those from

\footnotetext{
${ }^{192}$ Cool 2006, 143-146.

${ }^{193}$ DEN0001.

${ }^{194}$ den Boesterd 1956, xx.

${ }^{195}$ Eggers 1968, 104 (19a); McPeake \& Moore 1978, 333 (9).

${ }^{196}$ Bennett \& Young 1981, 39-43.

${ }^{197}$ RIB II 2415.16.

${ }^{198}$ GWY0009; Eggers 1968, 104 (21); McPeake \& Moore 1978, 333 (1); RIB II 2415.1.

${ }^{199}$ McPeake \& Moore 1978, 331-334; Bennet \& Young 1981, 37-44.
} 
Harlech $^{200}$ and Yns Gwrtheryw, ${ }^{201}$ which both contain a number of Handled Pan 2s combined with bowls.

The Structured Deposits from Roman Wales thus far discussed have consisted of relatively few numbers of comparatively small vessels such as Handled Pan 2s, bowls or strainers. In contrast to these assemblages, the Halkyn Mountain group is large and diverse. This group consists of eight vessels: three Irchester type bowls, one Bassin Uní type bowl, one shallow bowl, two buckets and one cauldron. This larger assemblage is unusual in Wales and is far more characteristic of large vessel hoards in eastern England, as will be discussed later in this chapter. Unfortunately, this Structured Deposit was lost soon after its discovery and recording, ${ }^{202}$ so it is not possible to comment further on wear patterns or decoration. Also, its specific findspot was not recorded. Mining settlements (mostly lead) are well known in the areas of northern Wales around Flint and Halkyn. ${ }^{203}$ This could indicate that the owner of these vessels was affiliated with the mining operations, or perhaps that the objects were a dedication to the gods of the area in exchange for the mineral resources extracted. The vessel forms would appear to be of multiple manufacture dates with the buckets having the earliest date of manufacture $\left(2^{\text {nd }}\right.$ century CE) and the Irchester bowls and Bassin Uní representing vessels of certain Late Roman manufacture. Such large deposits from the Late Roman period occur throughout Britain, Halkyn Mountain being the western-most example in this data-set. Their composition will often reflect vessels of various manufacture dates and include large basins, such as the Irchester type bowls found here. Further discussion of such large Late Roman Structured Deposits will be offered in Sections 3.3 and 3.4 of this chapter, as well as in Chapter 7.

Taken as a group, the Structured Deposits from Wales show a general patterning in the vessel forms selected for deposition. With the exceptions of Langstone and the Halkyn Mountain assemblages, the regularity of Handled Pan 2s across the Welsh Structured Deposits is a noteworthy pattern. The Structured Deposits from Llanberis, Harlech, Yns Gwrtheryw, Glyn Dyfrdwy and Kyngadl all contain Handled Pan 2s and none of the larger vessels often found in Structured Deposits elsewhere, such as cauldrons or Irchester bowls. The similarity between these deposits would indicate that they were likely related to each other by having been

\footnotetext{
${ }^{200}$ GWY0004-GWY0008.

${ }^{201}$ GWY0001-GWY0003.

${ }^{202}$ Kennett 1971, 128.

${ }^{203}$ Webster 1953, 3-15; O’Leary et al. 1989, 2-62.
} 
assembled by individuals with similar culture practices, or at the very least all sharing ready access to Handled Pan 2s. The military had a high presence in Wales, as is attested by the number of forts, marching camps and other military sites. ${ }^{204}$ The concentration of the Roman military could be the cause of these vessels' presence in the region, especially as comparison between Map 1 and the OS Historical Map for Roman Britain indicates that most of the Structured Deposits are within 3-5 miles of a known military installation, with none further than 10 miles from a military site. However, whether the deposits were made by soldiers themselves or not is more difficult to assert. Further discussion of the probable influence of the Roman military on the material culture of Wales will be discussed in Chapters 5 and 7. The Langstone and Halkyn Mountain groups appear to be related to two depositional trends prevalent elsewhere in Britain, namely aquatic deposition and large Late Roman assemblages, and will be further discussed in comparison with them later in this chapter.

\begin{tabular}{|l|l|}
\hline Find Location & Catalogue Numbers \\
\hline Clifton (Cumbria) & C0029-C0030 \\
\hline $\begin{array}{l}\text { Aesica Roman Fort, Great Chesters } \\
\text { (Northumberland) }\end{array}$ & NU0003-NU0005 \\
\hline Prestwick Carr, Ponteland (Northumberland) & NU0019-NU0025 \\
\hline Whittington (Northumberland) & NU0029-NU0030 \\
\hline Corbridge (Northumberland) & NU0035 \\
\hline Ingoe township, Matfen (Northumberland) & NU0050-NU0051 \\
\hline
\end{tabular}

Table 3b- Structured Deposits from Hadrian's Wall and environs.

Moving to the counties of Northumberland and Cumbria, there are six Structured Deposits that feature in this thesis (Table 3b).A copper alloy jug of Eggers type 128a was found buried immediately north of the Stanegate Roman Road in Corbridge on September $4^{\text {th }}$ of 1911 (Figure 3.03). This vessel contained 160 gold aurei with a coin of Marcus Aurelius as the latest issue and two bronze coins of Trajan and Hadrian respectively acting as a stopper in the neck of the jug. ${ }^{205}$ This is slightly unusual as coin hoards are not often interred in copper alloy vessels and most often occur in organic sacks, ceramic vessels, or wooden chests. In this thesis, there are only six coin hoards that are associated with copper alloy vessels. ${ }^{206}$

\footnotetext{
${ }^{204}$ Haverfield 1910, 20-123; Manning 2001, 44-53; Arnold \& Davies 2000, 58-59; Davies \& Jones 2006.

${ }^{205}$ NU0035; Forester et al. 1912, 210; Abdy 2002, 35.

${ }^{206}$ Roundway Hill (WIL0023) \& Bishop's Cannings (WIL0013-0022), Wall (STA0008), Corbridge (NU0035), Bullock Down Farm in Beachy Head (ESUS0004) and possibly Coygan Cave (CAR0001-CAR0002).
} 


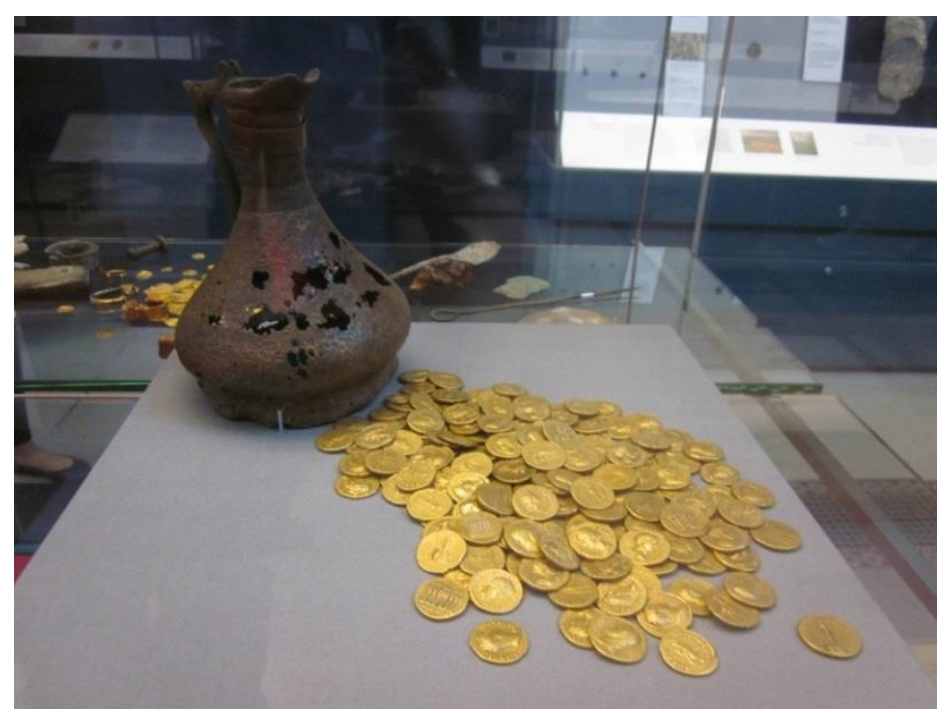

Figure 3.03: Structured Deposit from Corbridge on display in the British Museum (photo by author)

The depositional circumstance of this group proves problematic to discern. It has been suggested that the numismatic evidence indicates that the group was interred around the same time as a possible attack on the site around $160 \mathrm{CE} .^{207}$ Though there are certainly much larger coin hoards than this known from Roman Britain, ${ }^{208}$ the wealth represented by this number of gold coins would not have been discarded lightly and it would be fitting if this was an evacuation hoard, plunder or an assemblage lost in the chaos of an attack. The numismatic evidence conveniently coincides with such a theory. However, the surrounding features associated with the hoard may contradict a $2^{\text {nd }}$ century date of deposition. The jug was found less than two feet below the modern surface and associated with a layer of gravel and a drain of probable later Roman date. $^{209}$ It is possible the group was rediscovered after initial deposition and then redeposited sometime in the late $4^{\text {th }}$ century $\mathrm{CE} .{ }^{210}$ Be this as it may, this does not change the circumstances of its original collection and interring, which numismatically dates to the late $2^{\text {nd }}$ century $\mathrm{CE}$.

\footnotetext{
${ }^{207}$ Forester et al. $1912,156$.

${ }^{208}$ Abdy 2002, 25-66.

${ }^{209}$ McDonald 1912, 1-20; Abdy 2002, 35.

${ }^{210}$ Forester et al. 1912, 157.
} 
The Structured Deposit from Great Chesters was found during excavations of a Roman fort and comprises two handled strainers and one Handled Pan 3, all of Eggers type 161. ${ }^{211}$ Though somewhat fragmentary, there is no reason to assume that the objects were damaged prior to internment. This is particularly true of the strainers, as their fragility makes them prone to breakage and erosion. This assemblage is similar to several found in Wales, though substituting a Handled Pan 3 for the Handled Pan 2s.

The Structured Deposit from the parish of Ponteland consists of seven vessels of varying type interred in a swamp at Prestwick Carr. ${ }^{212}$ It is unclear as to whether this assemblage is the product of a single votive offering, or accumulated through multiple acts of deposition. This group is the second largest Structured Deposit found in aquatic circumstances currently known after the Structured Deposit of 15 vessels from Drapers' Gardens in London. ${ }^{213}$ The findspot was in a boggy and waterlogged area that drained into the River Pont before the entire area was drained over a century ago. ${ }^{214}$ The vessels which comprise this group are also note-worthy: three Handled Pan 2 s of likely $1^{\text {st }}$ century manufacture and three large bowls and a cauldron which would appear to be of Late Roman date. One of the Handled Pan $2 s^{215}$ has a maker's mark of 'DRACCIVS F' ${ }^{216}$ stamped onto the handle. ${ }^{217}$ Tassinari notes several examples of this stamp on the continent and based on form and distribution believes him to be a manufacturer in Gaul of roughly contemporary or slightly later date to C. Cipius Polybius, ${ }^{218}$ whose material is well known from the Pompeian destruction layers, ${ }^{219}$ as well as appearing in Britain. This would indicate that these objects were likely at least 200 years old at the time of their deposition. A series of punch dot inscriptions on one of the bowls proves of great use for understanding this group, indicating that it was owned by at least three different men in succession (Crescens, Senecio and Vannus) from two different troops of soldiers (those of Tiro and Kandianus) during its use-life. ${ }^{220}$ This contextualises this group within a military setting, which is not surprising considering the concentration of Roman forces in northern England. Other objects are currently

\footnotetext{
${ }^{211}$ NU0003-NU0005; Eggers 1966, 108 (59 c-e).

${ }^{212}$ NU0019-NU0025; Eggers 1968, 108 (65).

${ }^{213}$ L0001-L0015, discussed later in this section.

${ }^{214}$ Harbottle 1995, 1.

${ }^{215}$ NU0019.

216 'Draccius f(ecit)', or 'Draccius made it'.

${ }^{217}$ Bennet and Young 1981, 43.

218 Tassinari 1975, 29-30 (13).

219 Tassinari 1993.

${ }^{220}$ NU0023; RIB II 2415.63.
} 
unknown from the site and there is no reason to believe that this particular bog had a continuous tradition of structured deposition. While Handled Pan 2s do not commonly feature in Late Roman Structured Deposits with large basins and cauldrons, it is common for vessels of wide manufacture dates to be interred together in the Late Roman period. ${ }^{221}$ Their presence is particularly intriguing when considered with the military inscriptions, as Handled Pan 2s are commonly found on military sites in Britain. ${ }^{222}$

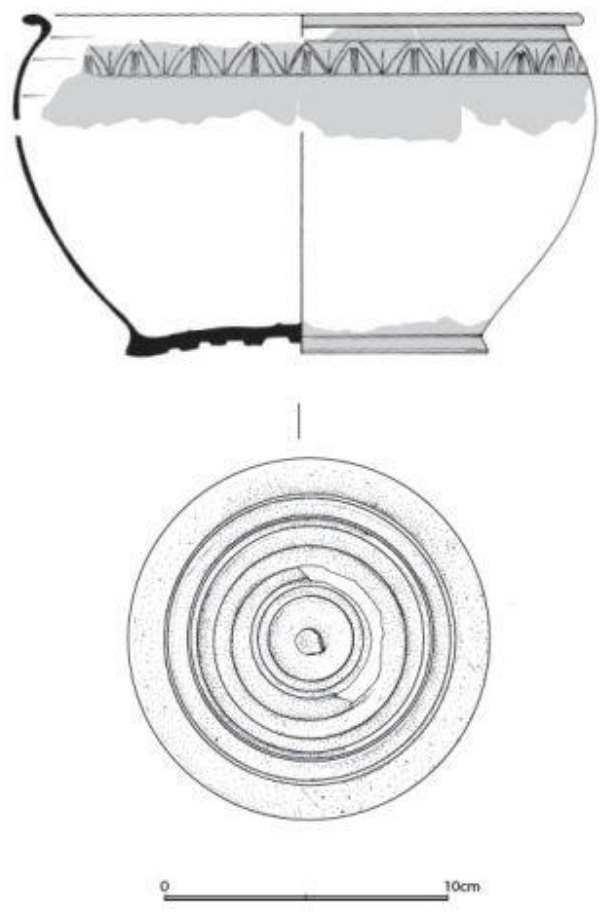

Figure 3.04: Jar from Structured Deposit at Whittington (PAS NCL-33CC76)

The three remaining Structured Deposits from this region also contain Handled Pan 2s. The Whittington assemblage reported through the PAS contains a Handled Pan 2 and a jar, which is not a common pairing in the Structured Deposits of this data-set (Figure 3.04). The objects are highly fragmentary and incomplete, it is indeed difficult to discern if they were interred in a damaged state or if they were damaged after deposition.

Two Handled Pan 2s of Eggers type 139-144 were found in Ingoe township in Matfen and are particularly noteworthy as they both not only bear maker's marks, ${ }^{223}$ but also have etched

${ }^{221}$ Such as Drapers' Gardens (Gerrard 2009; L0001-L0015).

${ }^{222}$ As further discussed in Chapter 7.

223 'SABIANVS F' \& 'MAXIMINVS F' respectively. 
inscriptions on the underside of their handles which could be numerals. ${ }^{224}$ This could perhaps be interpreted as identifying these handled pans as part of a numbered set in much the same way seen on the assemblage from Abergele in Wales, ${ }^{225}$ inviting probable military association. The assemblage from Clifton in Cumbria also has a Handled Pan 2 with a maker's mark and was found paired with a strainer, ${ }^{226}$ a combination that is repeated elsewhere in Britain. ${ }^{227}$

The presence of the army would have undoubtedly influenced the vessels which were to be found in the area of Hadrian's Wall and also most likely influenced the circumstances of their deposition. As cumulative evidence from this thesis shows, this could explain the presence of Handled Pan 2s in four out of six of the Structured Deposits of the region. The sample group from this area is rather small, however, and may not be adequate to indicate such patterning.

\begin{tabular}{|l|l|}
\hline Find Location & Catalogue Numbers \\
\hline Sedgefield (Durham) & DUR0001 \\
\hline South Shields Roman Fort (Tyne and Wear) & DUR0005 \\
\hline Upper Weardale (Durham) & DUR0006-DUR0008 \\
\hline Ribchester (Lancashire) & LAN0001-LAN0002, LAN0004 ${ }^{228}$ \\
\hline Lincoln (Lincolnshire) & LIN0028 \\
\hline River Witham, Fiskerton (Lincolnshire) & LIN0029-LIN0030 \\
\hline River Witham, Kirkstead (Lincolnshire) & LIN0031 \\
\hline River Witham, Fiskerton (Lincolnshire) & LIN0032 \\
\hline Linton (North Yorkshire) & NYR0003-NYR0004 \\
\hline Knaresborough (North Yorkshire) & NYR0008-NYR0028 \\
\hline Beadlam Roman Villa (North Yorkshire) & NYR0037 \\
\hline Stittenham (North Yorkshire) & NYR0040-NYR0044 \\
\hline
\end{tabular}

Table 3c- Structured Deposits of the northern counties of England.

The remaining counties of northern England contain 12 Structured Deposits (Table 3c). A preferential distribution across the north east, 11 out of 12 assemblages, is visible in these Structured Deposits. Although this could reflect a somewhat the higher level of population settlement and greater ease of movement and communication networks as opposed to the west, ${ }^{229}$ the north west of England was well populated, particularly with military garrisons. The possible

\footnotetext{
${ }^{224}$ Wright 1969, 1-5; McPeake \& Moore 1978, 334 (30); RIB II 2415.40 \& RIB II 2415.44.

${ }^{225}$ DEN0008-DEN0016; see earlier in this section.

226 'Talio F'; RIB II 2415.47.

${ }^{227}$ Discussed further in Section 3.4.

${ }^{228}$ This group could be either one or two different Structured Deposits, see discussion.

${ }^{229}$ Margary 1967, 400-401.
} 
cultural and economic mechanisms which could have influenced this are discussed in Chapter 7 after material from other Depositional Contexts is reviewed.

The Stittenham assemblage is noteworthy for comprising only Handled Pan 2s of Eggers type 142, two of which bear the maker's mark of P. Cipius Polybius, ${ }^{230}$ who is believed to have been active in the second half of the first century $\mathrm{CE}^{231}$ The uniformity of this group is striking and begs explanation. The Roman military again provides a plausible candidate for an organization whose purchasing power and uniformity of material could account for such an assemblage, though other scenarios, such as the vessels being lost in shipment, should not be discounted.

The Knaresborough group of vessels, ${ }^{232}$ numbering some 21 vessels, is the largest single Structured Deposit of copper alloy vessels currently known from Britain. It is likely that what is extant is only a fraction of the original size of the hoard. Discovered in 1864, when originally found it was said to be enough to 'have filled a cart' and it would seem that the majority of the hoard was melted down for reuse shortly after discovery. ${ }^{233}$ Nevertheless, the scale and variety of vessels still extant is unprecedented in Britain, consisting of one bucket of Eggers type 37-40, six strainers, 12 bowls of varying size and type, a handle fragment from a Handled Pan 5 and an undiagnostic rim fragment. ${ }^{234}$ The strainer-dot patterns on the strainers are particularly ornate, including flower patterning, ${ }^{235}$ vine leaf designs framing swastika patterns, ${ }^{236}$ and elaborate geometric swirl patterning. ${ }^{237}$ Handles survive on three of these strainers, making them recognisable as Eggers type 161. It is likely that the three other strainers also had handles of this type.

\footnotetext{
${ }^{230}$ Eggers 1968, 107 (54); RIB II 2415.19 \& 2415.26.

${ }^{231}$ Bennet \& Young 1981, 37-44.

${ }^{232}$ NYR0008-NYR0028.

${ }^{233}$ Kennett 1971, 132.

${ }^{234}$ Eggers 1966, 107 (55).

${ }^{235}$ NYR0013.

${ }^{236}$ NYR0014.

${ }^{237}$ NYR0009-NYR0011.
} 


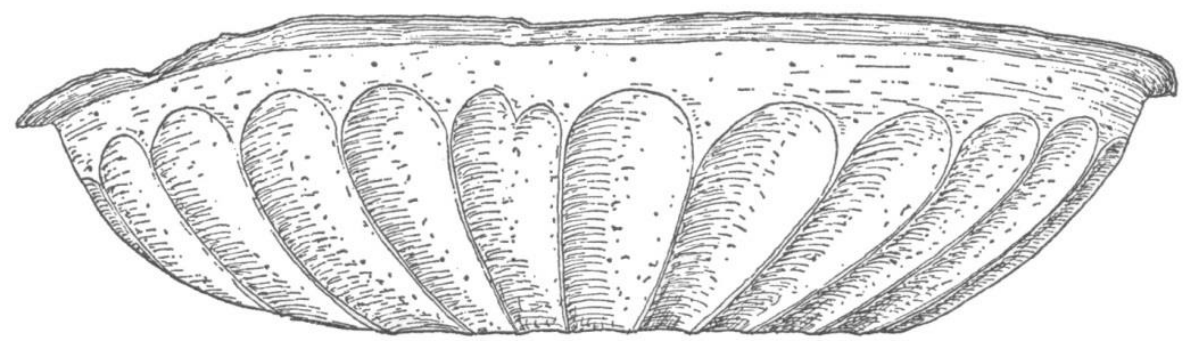

Figure 3.05: Fluted Bowl from Knaresborough (Eggers 1968, 142 Abb. 42.8, Scale 1:7)

Most of the bowls are fairly common types: there are two Bassins à bord godronné types and six Irchester types. However, there is also a bowl with a fluted body which is of unusual type (Figure 3.05). ${ }^{238}$ Fluting is not known elsewhere among copper alloy vessels in Roman Britain and is indeed better paralleled with objects of other materials, such as several silver fluted bowls from the Roman hoard at Traprain Law, Scotland ${ }^{239}$ or in the Sutton Hoo burial from the early Medieval period (Figure 3.06). ${ }^{240}$

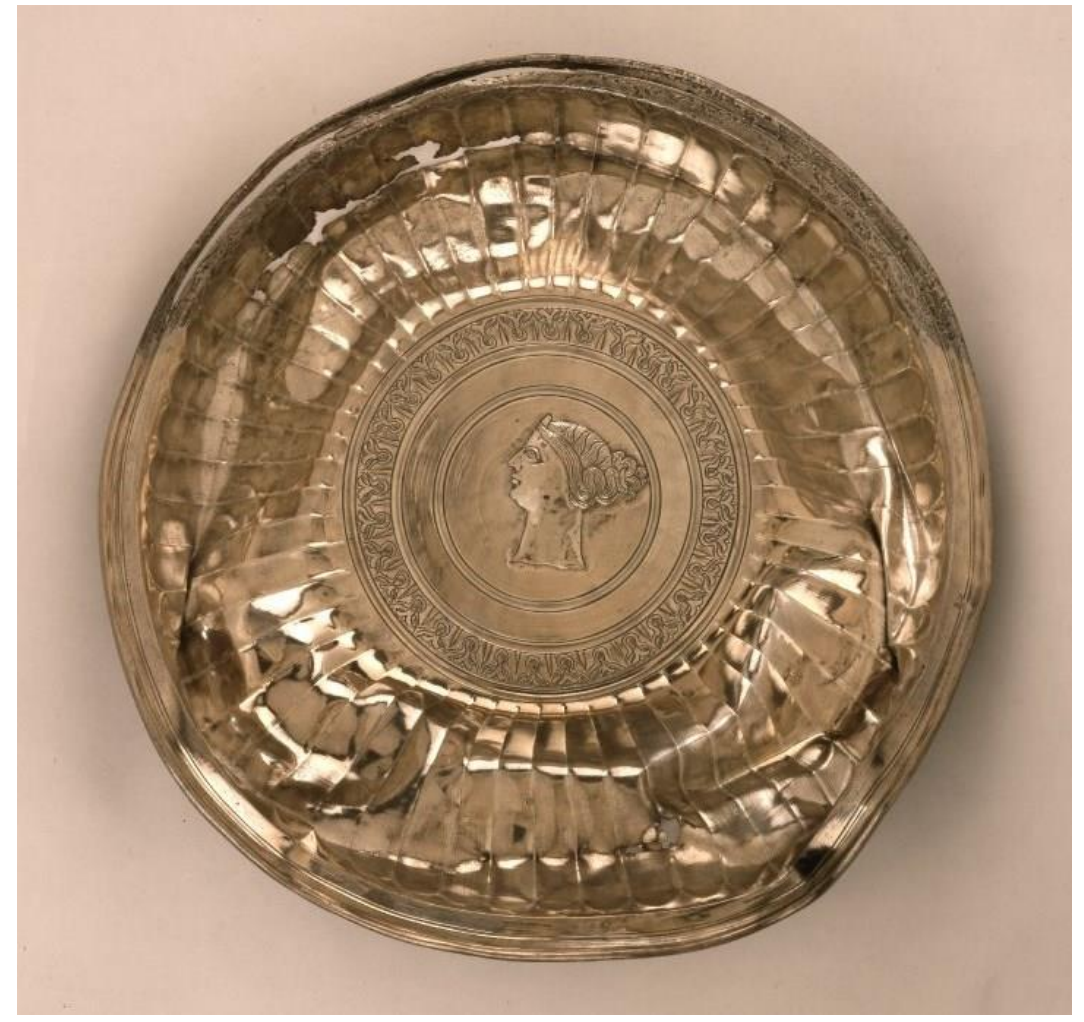

Figure 3.06: Fluted Bowl from Sutton Hoo Anglo-Saxon Burial (@ the British Museum)

\footnotetext{
238 NYR0015.

${ }^{239}$ Curle 1923, 36-41.

${ }^{240}$ Bruce-Mitford \& Raven 2005, 16; BM 1939,1010.77.
} 
Irchester bowls are of late Roman manufacture, ${ }^{241}$ implying a probable $4^{\text {th }}-5^{\text {th }}$ century CE date for the deposition of this group. The size of this assemblage, as well as its varied composition, makes it comparable to the Neupotz group and other large assemblages from southern Germany, ${ }^{242}$ though the Knaresborough hoard would post-date these $2^{\text {nd }}-3^{\text {rd }}$ century groups. The $4^{\text {th }}-5^{\text {th }}$ century date of its deposition does suggest this group could be associated with some kind of Late Roman crisis response. However, if this assemblage does represent stored wealth or plunder, it would seem unusual that there are only copper alloy vessels interred and not any other forms of portable wealth that one might expect to find in such a group, such as jewellery or coinage. This implies a highly selective process involved with the deposition of this assemblage, arguing against hasty assemblage and abandonment that might associate it with a response to immediate danger or indiscriminate looting. This is a conundrum encountered in several Late Roman Structured Deposits and will be discussed further in Section 3.3. The size of this assemblage, as well as its proximity to a trunk route, is intriguing and it remains unfortunate that we are unlikely to have the full assemblage available for current study. A hoard of 283 bronze radiate coins of the late $3^{\text {rd }}$ century $\mathrm{CE}$ found nearby is an intriguing discovery, ${ }^{243}$ though there is no evidence that these two deposits are related.

A further Structured Deposit of Late Roman vessels from North Yorkshire was discovered in the parish of Linton and processed through the PAS. The assemblage consists of two vessels: one is a Handled Pan 2 of unusual type (Figure 3.07) and the other a bowl of late Roman manufacture. ${ }^{244}$ The Handled Pan 2 appears to have been sheet hammered, whereas solid body casting with lathe finishing was the conventional method of manufacture of similar vessels during the Roman period. ${ }^{245}$ The functional relation of these two objects is uncertain, though perhaps it may be related to ablutions.

\footnotetext{
${ }^{241}$ Kendrick 1932, 162-163.

${ }^{242}$ Bakker et al. 2006.

243 Barclay 1997, 279-283.

${ }^{244}$ NYR0003, PAS SWYOR-E51F57; NYR0004, PAS SWYOR-E4D7D0.

245 Radnoti 1938, 9-67.
} 


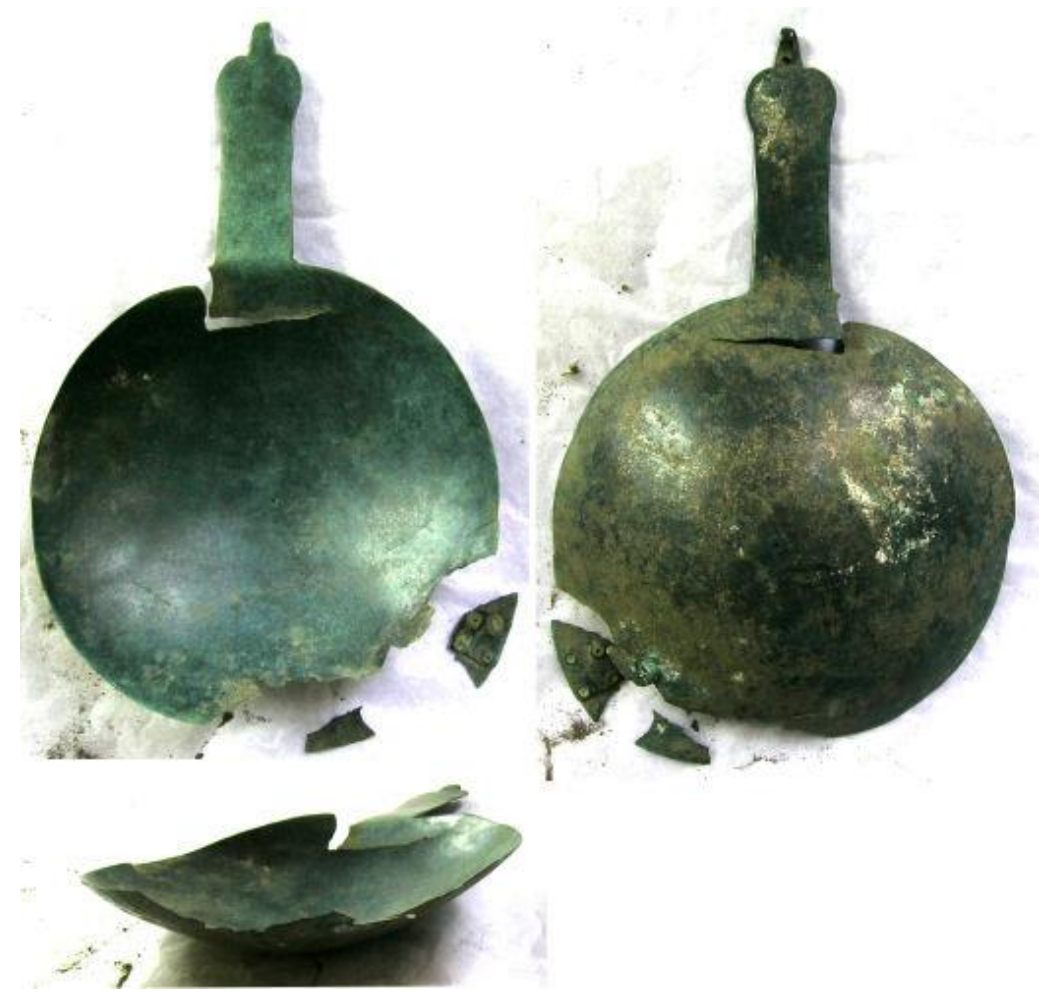

Figure 3.07: Handled Pan 2 from Structured Deposit at Linton (PAS SWYOR-E51F57)

A Structured Deposit of metal scrap comprising some 59 objects, including two vessels, was found on a villa site near Beadlam, North Yorkshire. ${ }^{246}$ The scrap included jewellery and toilet items in various states of fragmentation. Associated copper alloy vessel material associated with this assemblage comprised a strainer ${ }^{247}$ and a Handled Pan 2 of Rudge Cup type ${ }^{248}$ both in highly fragmented states. The Rudge Cup type vessel fragment is enamelled with geometric zig-zag and lines and waves with a broken inscription reading '[...] ICITR' for feliciter, meaning 'good luck' ${ }^{249}$ This deposit reflects the transition of a villa site into an industrial site, a process common in the fourth and fifth centuries in England, ${ }^{250}$ and almost certainly represents scrap from a metal-smith's workshop. This makes the Beadlam group particularly interesting, as it is the assemblage in this thesis that most clearly appears to be a scrap or metal worker's hoard. ${ }^{251}$

\footnotetext{
${ }^{246}$ Wright \& Hassall 1973, 334 (38); Neal 1996, 49.

${ }^{247}$ NYR0036.

${ }^{248}$ NYR0037.

${ }^{249}$ Neal 1996, 49.

${ }^{250}$ Gerrard 2013, 255.

${ }^{251}$ Though the Santon Downham group (SUF0003-SUF0004 \& SUF0051) could also represent such an assemblage.
} 
There are three Structured Deposits from county Durham, two of which are composed of single vessels. The large bowl from the Roman fort at South Shields deserves special recognition, as it is specifically labelled as a votive offering with its inscription $M$. A. SAB. APOLLINI ANEXTIOMAROM, which should be read as 'to Apollo the Great Protector (Anextiomarus) from Marcus Antonius Sabinus' (Figure 3.08). ${ }^{252}$ Although there was an active tradition for the votive deposition of vessels carried out through the Iron Age and Roman Periods. ${ }^{253}$ Votive dedicatory inscriptions are not common on copper alloy vessels in Roman Britain. This example from South Shields is the principal exception, a further example from Bath being the only other known example from Roman Britain. ${ }^{254}$ The form of this vessel is similar to vessels found in Pompeii, ${ }^{255}$ indicating a likely $1^{\text {st }}$ or $2^{\text {nd }}$ century date of manufacture.

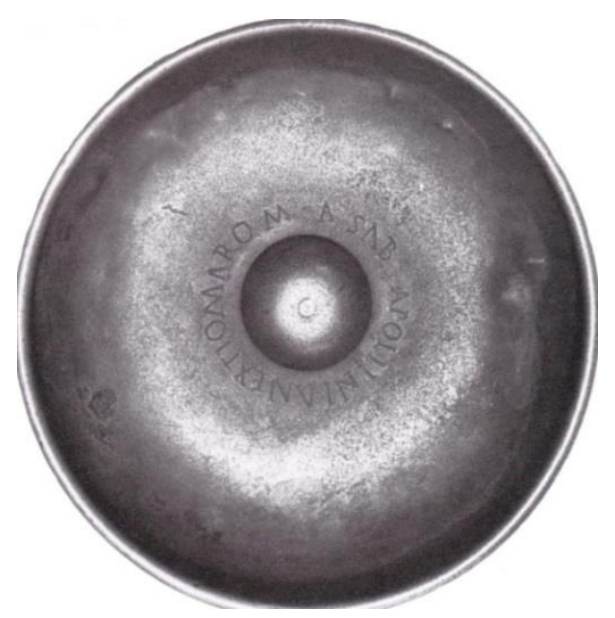

Figure 3.08: Votive Bowl from South Shields (Henig 1984, 133 Fig.56)

The jug from Sedgefield was found complete in the same refuse fill as a complete ceramic jar, implying that these vessels are best interpreted as Structured Deposits. ${ }^{256}$ The jug itself is adorned with a gorgon head handle medallion and zoomorphic aquatic bird's heads framing the rim of the vessel where the handle connects. The type and decoration for this vessel places its date of manufacture in the $1^{\text {st }}-2^{\text {nd }}$ centuries CE and is comparable to examples believed to have been manufactured in Italy and Gaul. ${ }^{257}$ This is an unusual addition to the Structured Deposit material for this thesis, as jugs are not common in this depositional context.

\footnotetext{
${ }^{252}$ DUR0005; Henig 1984, 132; RIB II 2415.55.

${ }^{253}$ Henig 1984, 145-152; Bradley 1990; Bradley 2000.

${ }^{254}$ AV0002; Cunliffe 1988, 14-16 (23); Hassall \& Tomlin 1981, 381 (20); RIB II 2415.60.

255 Tassinari 1993 (S1300).

${ }^{256}$ DUR0001; Burnham 2007, 264 (5).

257 Tassinari B1261 \& E3000; Tassinari 1975, 66-67 (171).
} 
Additionally, highly decorated vessels as a whole are not commonly interred as Structured Deposits. Finally, Structured Deposits of single copper alloy vessels are a rarity, though this could be influenced by the stringent classification criteria used in this thesis. ${ }^{258}$ While the jug from Sedgefield was found with a complete ceramic jar nearby, it would not appear that the two objects are part of the same depositional act. However, some association may be drawn between them in that they were both selected to be deposited in this location in complete form, perhaps indicating that they served similar ritual functions that could, by extension, be associated with their practical functions.

The third Structured Deposit from county Durham was found in a peat bog in Upper Weardale and is composed of three Handled Pan 2 s of Eggers type $142 .^{259}$ In addition to its aquatic deposition and its narrow composition, this assemblage is also noteworthy as two of the vessels bear inscriptions. ${ }^{260}$ While both bear maker's marks for the workshop of the family of Polybius, ${ }^{261}$ the personalised punch-dot inscription of 'LICINIANI' on the handle of DUR0007 is indicative of ownership. ${ }^{262}$ The placement of this group of imported vessel forms in an aquatic environment reflective of indigenous votive practice ${ }^{263}$ is particularly interesting and implies a level of cultural interchange and amalgamation, or 'syncretisation' between imported and indigenous ritual practice, ${ }^{264}$ taking place.

The three remaining Structured Deposits from northern England all appear to be aquatic votive deposits. In 2001, work on Depot Street in Lincoln led to the discovery of a late Roman Irchester type bowl ${ }^{265}$ and a glass vessel in the same area along what would have been the riverside during the Roman period. ${ }^{266}$ The presence of the glass vessel makes this deposit particular interesting, as glass is not commonly found interred with copper alloy vessels in Britain outside of burial contexts. ${ }^{267}$ However, there is no need to assume that the two were deposited together in the same ritual act, but could indeed represent two independent votive acts.

\footnotetext{
${ }^{258}$ See Section 2.2 and 2.3.

${ }^{259}$ DUR0006-DUR0008; Egglestone 1915, 9-11.

${ }^{260}$ DUR0006 \& DUR0007.

261 'P. CIPEPOLI' \& 'POLIBI.M' respectively.

${ }^{262}$ Egglestone 1915, 9-11; Bennet \& Young 1981, 43 (40 \& 42); RIB II 2415.20 \& 2415.27.

263 Bradley 1990, 1-42.

${ }^{264}$ Webster 1997, 165-182.

${ }^{265}$ LIN0028.

${ }^{266}$ Burnham et al. 2002, 305.

${ }^{267}$ See Chapter 4.
} 
In either case, the presence of the glass vessel strengthens the claim that this is a votive offering made in an aquatic environment.

The site of Fiskerton, Lincolnshire provides us with much more contextual data than is often present for aquatic votives. At the site of a Late Iron Age timber causeway which extended into the River Witham, many objects (including jewellery fragments, tools, and coins) ranging from Late Iron Age to Early Medieval date have been found. ${ }^{268}$ Among the various finds are two Irchester bowls ${ }^{269}$ and a Handled Pan 2 with the maker's mark 'FLORVS F', ${ }^{270}$ The existence of a causeway here, seemingly specifically designed to facilitate the votive deposition of material in the river, reveals the importance felt for the location and the care taken to prepare and maintain it. A similar causeway with an even greater variety of votive offerings was recently discovered near modern day Piercebridge in Durham ${ }^{271}$ and it is likely that these two locations served much the same purpose. The River Witham would appear to have served a ritual purpose of some significance as is evidenced by an additional deposit made in this same river at Kirkstead which is composed of a single Handled Pan 2 of Eggers type 139-144 with a maker's mark 'C.ARAT' stamped onto the handle. ${ }^{272}$

A Structured Deposit of two Handled Pan 2s of Eggers type 150, commonly referred to as 'bath saucers' in the literature, ${ }^{273}$ was discovered in the wetlands of the parish of Ribchester, Lancashire. $^{274}$ A third Handled Pan 2 of Eggers type 140-144 in a fragmentary state of preservation with a partially legible stamped inscription on its handle reading '[...]CONP[...]' was also found in the vicinity, ${ }^{275}$ though it may be instead associated with the more famous Ribchester Hoard (Figure 3.09). The Ribchester Hoard consists of several objects of Roman date, both of ceramic and metalwork, and is most famous for its near complete Roman cavalry helmet. $^{276}$ The hoard is also noteworthy for its military horse trappings including an anthropomorphic copper alloy mount of Minerva. ${ }^{277}$ It is worth noting that the objects are said to have been discovered in the late 1700s near a house 'on the western side of the main street

\footnotetext{
${ }^{268}$ Field \& Pearson 2003.

${ }^{269}$ LIN0029 \& LIN0030; Field \& Pearson 2003, 118 (1 \& 2).

${ }^{270}$ LIN0032; McPeake \& Moore 1978, 334 (26); RIB II 2415.35.

${ }^{271}$ Walton 2012, 152-166; Walton forthcoming.

${ }^{272}$ LIN0031; McPeake \& Moore 1978, 333 (6); RIB II 2415.12.

273 den Boesterd 1956; Cool 2006.

${ }^{274}$ LAN0001 \& LAN0002; Eggers 1968, 107 (57a\&b).

${ }^{275}$ LAN0004; Jackson \& Craddock 1995, 87 (24-25).

${ }^{276}$ Smith \& Shortt 1890, 32-35; Jackson \& Craddock 1995, 75-102; Edwards 2000, 65-72.

${ }^{277}$ Smith \& Shortt 1890, 32-35; Jackson \& Craddock 1995, 81-82.
} 
leading down to the river'. ${ }^{278}$ While it would seem that LAN0001, LAN0002 and LAN0004 were all purchased by Charles Townley synchronously along with the Ribchester Hoard, ${ }^{279}$ it remains unclear which, if any, of these Handled Pan $2 \mathrm{~s}$ is directly associated with the Ribchester Hoard and which may have been independently found. ${ }^{280}$ The various military objects would prove likely to have been deposited together, and this could perhaps include LAN0004 as this type of Handled Pan 2 appears to have military associations in Britain. ${ }^{281}$ Presence of a crest which was not likely attached to the helmet in this assemblage has led to speculation that the majority of the objects could come from a scrap hoard, ${ }^{282}$ though the circumstances and recording of the find makes any such assertion difficult to prove. However these objects are divided or grouped into individual assemblages, all of the objects were deposited near the River Ribble and likely reflect similar depositional processes behind their interment. All three Handled Pan $2 \mathrm{~s}$ from Ribchester are of $1^{\text {st }}$ century manufacture, the proposed early $2^{\text {nd }}$ century CE date of deposition for all of the material conveniently coincides with a rise in coin hoards in the immediate area, perhaps suggesting possible social disruptions or increased military activity in the Ribble Valley during this time. ${ }^{283}$

The Structured Deposits from the northern counties of Britain are diverse, but largely follow some patterns evident elsewhere across the province. This is most prominent in the presence of large Late Roman Structured Deposits and aquatic votive deposits, both of which will be considered in greater detail in Section 3.3. There is no clear patterning in the Site Types where Structured Deposits are found in this region. Unlike Wales and the Hadrian's Wall region, the Structured Deposits for this area do not appear to be necessarily associated with the Roman military specifically (with the notable exception of the Ribchester objects). This could help explain the lesser representation of Handled Pan $2 \mathrm{~s}$ in these assemblages compared with Wales and the hinterland of Hadrian's Wall, though this may also be a reflection of chronological variance as most of the assemblages from this area would appear to be of $3^{\text {rd }}-5^{\text {th }}$ century date.

\footnotetext{
${ }^{278}$ Jackson \& Craddock 1995, 75; Edwards 2000, 65.

${ }^{279}$ Smith \& Shortt 1890, 32; Jackson \& Craddock 1995, 75.

${ }^{280}$ Edwards 2000, 69; Eggers records LAN0001 and LAN0002 as an isolated Structured Deposit and makes no record of LAN0004.

${ }^{281}$ As discussed later in this Chapter and in Chapter 7.

${ }^{282}$ Breeze \& Edwards 2012, 65-69.

${ }^{283}$ Jackson \& Craddock 1995, 99.
} 


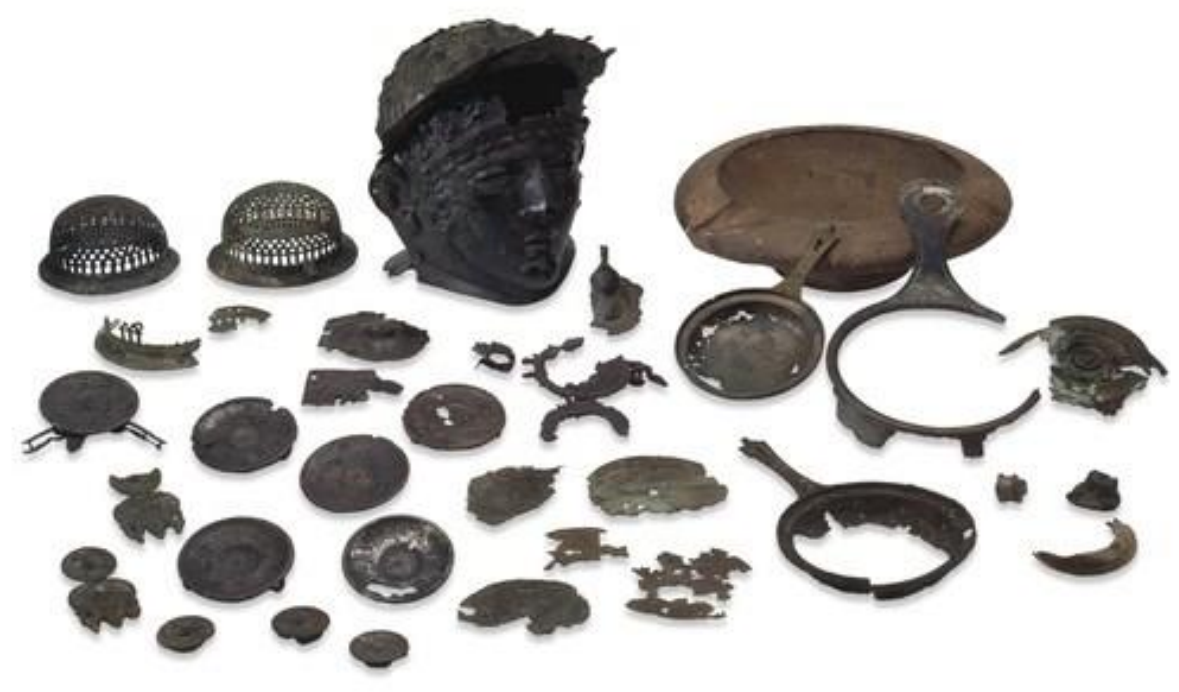

Figure 3.09: The Ribchester Hoard (ㄷ the British Museum)

\begin{tabular}{|l|l|}
\hline Find Location & Catalogue Number \\
\hline Sandy (Bedforshire) & BE0001-BE0003 \\
\hline Amersham (Buckinghamshire) & BUC0001-BUC0011 \\
\hline Coldham, Elm (Cambridgeshire) & CAM0001-CAM0002 \\
\hline Burwell (Cambridgeshire) & CAM0010-CAM0024 \\
\hline Irchester (Northamptonshire) & NH0001-NH0009 \\
\hline Wall (Staffordshire) & STA0008 \\
\hline
\end{tabular}

Table 3d- Structured Deposits from the Midlands.

There are six Structured Deposits of copper alloy vessels of Roman date from the Midlands that feature in this thesis (Table 3d). Irchester type bowls derive their name from the Structured Deposit found in the town of Irchester. Discovered in 1874, it contains nine vessels, ${ }^{284}$ four of which are Irchester type bowls. ${ }^{285}$ The bowls are all of similar size, ranging between 23 and 28.8 centimetres rim diameter. Also in the group is a rim fragment of what would seem to be a very large bucket or cauldron, with an approximate rim diameter of 42.3

\footnotetext{
${ }^{284}$ NH0001-NH0009.

${ }^{285}$ Kennett 1971, 128.
} 
$\mathrm{cm} .{ }^{286}$ As there are several examples of Irchester type bowls being deposited with cauldrons, ${ }^{287}$ the presence of such a vessel would be expected. Also in the group are a single Handled Pan 2, a bassin á bord godronné, and two strainers. The strainers have elaborate geometric strainer-dot patterns which incorporate wave patterns as well as cruciform motifs (Figure 3.10). ${ }^{288}$
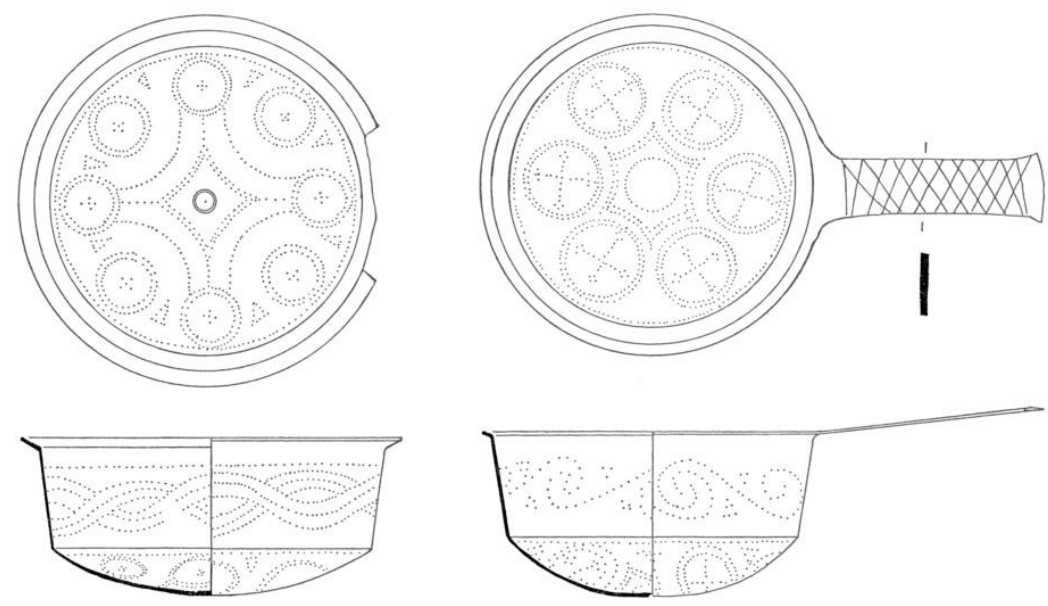

Figure 3.10: Strainer from Irchester (Kennett 1971, 133 Fig.10)

The $4^{\text {th }}-5^{\text {th }}$ century date of this deposit makes it probable that the owners and manufacturers of these vessels were at least aware of Christianity, if not necessarily adherents; though the vessels are a bit early in date to assume that the crosses have inherent Christian significance. The wave patterns on the strainers also suggest the possible spiritual metaphor of the passing from one life to another, as a journey across waters is often used metaphorically for this journey. This association is played out perhaps most elaborately in the famous silver Great Dish from the Mildenhall Treasure (Figure 3.11) ${ }^{289}$ In this platter, a central bust of the god Oceanus is ringed by registers depicting the sea before concluding in a scene of Bacchic revelry. As this same combination of aquatic motifs and Bacchic ecstasy occur together often on

\footnotetext{
${ }^{286}$ Ibid.; NH0001.

${ }^{287}$ Such as those from Burwell (CAM0011-CAM0024) and Halkyn Mountain (F0001-F0008).

${ }^{288}$ NH0008-NH0009.

${ }^{289}$ Hobbs 2012, 19-28; BM 1946.10-7.1.
} 
sarcophagi, it is likely to represent 'the journey of souls across the ocean to the after-life'. ${ }^{290}$ The presence of spoons in the Mildenhall Treasure decorated with the chi-rho and inscribed with Christian blessings suggests the owners of the group were likely Christian themselves, or at least comfortable appropriating Christian ideas. ${ }^{291}$ It would not be difficult for a Christian to use such a pagan scene as a metaphor for the Christian journey through death and rebirth, ${ }^{292}$ and the connection between Bacchus and Jesus both being gods from the east who died and were resurrected is not a difficult one to make. This connection could be useful in interpreting the strainer from Irchester, especially as aquatic motifs appear to have particular importance in burial ritual. $^{293}$

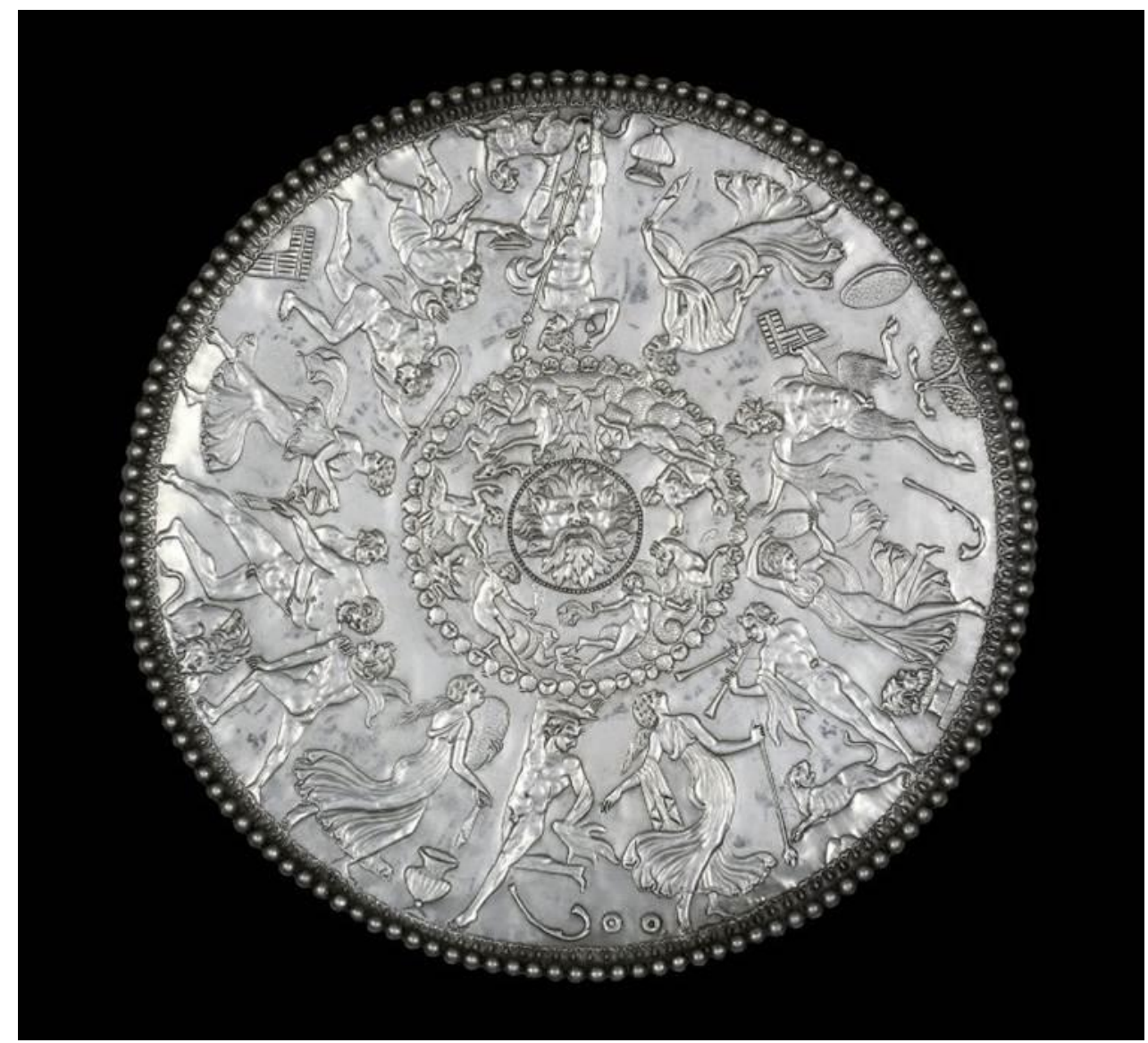

Figure 3.11: Great Dish from Mildenhall (C the British Museum)

\footnotetext{
290 Toynbee 1964, 309.

${ }^{291}$ Hobbs 2012, 38-39.

${ }^{292}$ Painter 1977, 18.

${ }^{293}$ As discussed in Section 4.4.
} 
A further Late Roman Structured Deposit with possible Christian significance was discovered in Wall in Staffordshire during an unrecorded excavation and may have originated from a cemetery. ${ }^{294}$ This assemblage is unlikely to have been a Grave Deposit, though, as the bowl contained some 31 coins dating mostly to the $4^{\text {th }}$ century CE. This number of coins would be an unusual inclusion as a grave offering and depositing copper alloy vessels in graves is also highly uncommon by the $4^{\text {th }}$ century CE in Britain. ${ }^{295}$ The bowl is decorated with a chi-rho and it has been suggested that this could represent church treasure. ${ }^{296}$ This group could represent a secular coin hoard just as easily, and it would be unhealthy to jump to conclusions on way or another.

The Structured Deposit from Sandy, Bedfordshire consists of three footed bowls with out-turned rims of bassin festonné, bassin á bord godronné, and bassin uní type respectively. ${ }^{297}$ These types of bowls are well represented in the archaeological record from the $4^{\text {th }}$ century on into the Merovingian and Anglo-Saxon periods, though more commonly associated with graves in the medieval period. ${ }^{298}$ As these are not believed to be part of a grave assemblage and their date of manufacture and principal use is generally believed to be Late Roman, it is likely that this was deposited in the late $4^{\text {th }}$ century or early $5^{\text {th }}$ century. Unfortunately, as the find occurred in $1856,{ }^{299}$ detailed archaeological context data is wanting.

A Structured Deposit of six copper alloy bowls ${ }^{300}$ nesting inside of each other was found near a known Romano-British site in the Misbourne Valley within the parish of Amersham, Buckinghamshire in $1982 .^{301}$ In the vicinity were found several unstratified coins, the latest identifiable coin being a FEL TEMP REPARATIO type of the House of Constantine dating to between 348-360 CE. ${ }^{302}$ The manufacture date of the bowls themselves also indicates a Late Roman date of deposition, as four of the six are Irchester bowls, all of which also show significant signs of wear and repair. One of the Irchester bowls has the letters ' $\mathrm{X}$ ' and a sideways ' $A$ ' stamped on the side. ${ }^{303}$ Also nearby were found two anthropomorphic terminal

\footnotetext{
${ }^{294}$ Mawer 1995, 19.

${ }^{295}$ Philpott 1991, 222-226; See Chapter 4.

${ }^{296}$ Mawer 1995, 19.

${ }^{297}$ BE0001-BE0003.

${ }^{298}$ Kennett 1971, 138-141.

${ }^{299}$ Kennett 1971, 124.

${ }^{300}$ BUC0006-BUC0011.

${ }^{301}$ Farley et al. 1988, 357.

${ }^{302}$ Farley et al. 1988, 363-364.

${ }^{303}$ BUC0006.
} 
busts, which may have originally come from sceptres or furniture. The busts likely depict gods, and seem to be indigenous syncretisation with Classical deities. ${ }^{304}$ While located in a valley which leads into the Thames, the finds themselves do not appear to be closely related to any known water feature. It is believed that the find spot is on the periphery of what was once a villa complex, perhaps with industrial features, and it is suggested that the finds in question could have been associated with a shrine or cult location associated with the complex, ${ }^{305}$ though direct evidence for this is lacking. A hoard of some 1,500 bronze coins was also found in the vicinity, apparently deposited around $380 \mathrm{CE} .^{306}$ The presence of these several finds lends credence to the assertion that the area had ritual significance, which suggests a ritual function for the vessels found on the site. This assemblage helps to further clarify the use and ritual significance of such basins in the late Roman period, offering insight into the possible depositional circumstances of other such Structured Deposits.

A large Structured Deposit of some 15 vessels stacked inside each other was discovered in Burwell, Cambridgeshire in 1967 during the levelling of a natural lime knoll. ${ }^{307}$ The Burwell assemblage consists of one heavily worn and patched sheet bronze cauldron, a Handled Pan 2, and some 13 bowls of varying size. ${ }^{308}$ Of these bowls, seven are of Irchester type. ${ }^{309}$ This is a particularly large Structured Deposit, and there is the likelihood that it represents the possessions of more than one individual, likely being the assemblage of a temple, guild or extended household. It was deposited in association with a natural landmark and it is possible that such natural topographic features may have held ritual significance. ${ }^{310}$ However, further excavation of the area around the findspot could find no archaeological evidence to imply spiritual significance to the location or imply habitation of the site during the Roman period, ${ }^{311}$ it is just as likely that the landmark was used as an easy point to identify for the retrieval of objects stored for safekeeping.

\footnotetext{
${ }^{304}$ Farley et al. 1988, 366.

305 Farley et al. 1988, 358.

${ }^{306}$ Bland 1997, 410.

${ }^{307}$ Wilson 1971, 270; Gregory 1976, 63.

${ }^{308}$ Gregory 1976, 63-77.

${ }^{309}$ MAA 1994-11-11; CAM0012-CAM0018.

${ }^{310}$ Yeates 2006.

${ }^{311}$ Browne 1965, 81-92.
} 
Two Handled Pan 2s of Eggers type $140^{312}$ and $144^{313}$ were found at Coldham in the parish of Elm. ${ }^{314}$ They are of $1^{\text {st }}-2^{\text {nd }}$ century date, making this Structured Deposit the only assemblage not of Late Roman date to come from the Midlands. In fact, this group was discovered in the far eastern part of Cambridgeshire, and therefore may be more closely related to finds from western East Anglia discussed below.

The Structured Deposits of the Midlands are almost ubiquitously large Late Roman assemblages of large basins. The high concentration of Irchester type bowls, 15 in total, from these Structured Deposits signifies a specific social imperative to inter these sorts of vessels in this region. This pattern is particularly significant as this preference for Late Roman material in the Midlands is not reflected in the other depositional contexts of this thesis, ${ }^{315}$ indicating a shift in depositional practice here in the Late Roman period that is not necessarily reflective of the wider consumption patterns of this time. This indicates that these Irchester bowls may well have had a ritual significance that led to their preferential selection for interring in these Structured Deposits. This helps to indicate the depositional circumstances of these large Late Roman assemblages, as discussed in Section 3.3.

\begin{tabular}{|l|l|}
\hline Find Location & Catalogue Numbers \\
\hline Sturmere (Essex) & EX0008-EX0014 \\
\hline Bors Field, Chesterford (Essex) & EX0022-EX0023 \\
\hline Weeting (Norfolk) & NOR0033-NOR0040 \\
\hline Outlon (Suffolk) & SUF0001-SUF0002 \\
\hline Santon Down (Suffolk) & SUF0003-SUF0004, SUF0051 \\
\hline Brandon (Suffolk) & SUF0043-SUF0046 \\
\hline
\end{tabular}

Table 3e- Structured Deposits from East Anglia.

As Table 3e illustrates, there are six Structured Deposits from East Anglia. The group from Weeting, comprising 8 vessels, is the largest Structured Deposit yet found in East Anglia and its composition matches closely with the large assemblages known from the Midlands and elsewhere in Britain, indicating a $4^{\text {th }}-5^{\text {th }}$ century date of deposition. Within one large cauldron were found two smaller cauldrons, a bassin á bord godronné, a bassin uní, a Helmsdale type bowl, and two Irchester type bowls. ${ }^{316}$ Two Iron Age brooches and a group of pewter plates

\footnotetext{
312 CAM0001.

313 CAM0002.

${ }^{314}$ Eggers 1968, 106 (40).

${ }^{315}$ See Chapters 4, 5 and 6 for further discussion.

${ }^{316}$ Gregory 1977, 265-272; NOR0033-NOR0040.
} 
have also been found at the site, ${ }^{317}$ indicating a possible tradition for the votive deposition of metal material at this site. One unusual addition to this group is the presence of iron suspension equipment, ${ }^{318}$ which is not recorded with other similar finds. Ferrous material being highly corrodible, it is likely that such material may have simply deteriorated beyond recognition in other Structured Deposits before retrieval. Additionally, as many of the large assemblages of comparable material were discovered in the $18^{\text {th }}$ and $19^{\text {th }}$ centuries, it is possible that corroded ironwork may have been simply discarded without recognition or recording. ${ }^{319}$ It is worth noting that the recently discovered Structured Deposit of Late Roman copper alloy vessels from Drapers'Gardens in London was accompanied by ferrous material, ${ }^{320}$ perhaps increasing the precedence for this association with copper alloy vessel assemblages more generally.

The Structured Deposit of a Handled Pan 2 of Eggers type 150 and a strainer of Eggers type 161 from Chesterford included a coin hoard of 195 coins dating from Caligula to Commodus. ${ }^{321}$ The numismatic evidence would indicate a late $2^{\text {nd }}$ century CE date of deposition, which is appropriate for a handled pan manufacture in the $1^{\text {st }}$ or $2^{\text {nd }}$ century CE. The composition of this group is paralleled by the Coygan Cave assemblage from Wales with its purported coin hoard, ${ }^{322}$ though of earlier date.

A Structured Deposit of nine copper alloy vessels was discovered near Sturmere in Essex, of which seven are currently extant. ${ }^{323}$ The group includes three Irchester type bowls and two bassin uní. An unusual addition to this group is the Handled Pan 4, or Coptic Pan. ${ }^{324}$ A Handled Pan 4 is also associated with the Structured Deposit from Wotton, ${ }^{325}$ which is similar to the Sturmere group also in containing mostly large Late Roman basins. ${ }^{326}$ Handled Pan 4s are otherwise rather uncommon for Britain, with only five included in the material of this thesis. The vessels comprising this group are all of Late Roman date and indicate a $4^{\text {th }}-5^{\text {th }} \mathrm{CE}$ deposition.

\footnotetext{
${ }^{317}$ Grew 1980, 375.

${ }^{318}$ Gregory 1977, 265.

${ }^{319}$ Gregory $1977,270$.

${ }^{320}$ Gerrard 2009; L0001-L0015.

${ }^{321}$ EX0032-EX0033; Eggers 1968, 106 (38).

${ }^{322}$ CAR0001-CAR0002.

${ }^{323}$ Kennett 1971, 124; EX0008-EX0014.

${ }^{324}$ EX0011.

${ }^{325}$ SUR0010.

${ }^{326}$ Section 3.8.
} 
A cauldron containing a strainer, Handled Pan 2 and bucket were found in Brandon, Suffolk in $1979 .{ }^{327}$ The manufacture of all of the vessels is likely to be $1^{\text {st }}$ or early $2^{\text {nd }}$ century CE. The wear on the vessels is limited and there is no evidence of repair, suggesting that they were not antiquated at the time of their deposition. This implies that this group was probably deposited in the $2^{\text {nd }}$ century CE; this is a time period with few Structured Deposits outside of coin hoards recorded in Britain, making this assemblage somewhat unusual. The Structured Deposit from Santon Downham was also contained in a $1^{\text {st }}$ century cauldron, ${ }^{328}$ though its assemblage was far more eclectic in composition and would appear to represent a metalworker's hoard, including some of his tools. ${ }^{329}$ While most all of the material in the Santon Downham assemblage is in some degree of disrepair, a complete trefoil mouth copper alloy jug of Eggers type 125 was included in the group. ${ }^{330}$ The jug has canine iconography both on its thumb-rest and handled medallion, which may not seem immediately significant save that the handle fragment of a Handled Pan 1 that is included in the group is also decorated with a canine handle terminal. $^{331}$ The use of canine iconography could be of significance for understanding this assemblage, as dogs were believed to have had curative or purifying properties. ${ }^{332}$ The combination of this handled pan and jug would be more characteristic of burial practice than of hoarding behaviour, ${ }^{333}$ as will be seen in Chapter 4. This group may have constituted a grave assemblage, though no body or cremation was recorded upon its discovery in the late $19^{\text {th }}$ century. ${ }^{334}$ The assorted scrap material found in the cauldron also suggests that this is unlikely to be a grave, though if this is a simple metalworking hoard or if it was deposited with ritual significance is impossible at this point to determine.

\footnotetext{
${ }^{327}$ Grew 1980, 376; SUF0043-SUF0046.

${ }^{328}$ SUF0051.

${ }^{329}$ Smith 1909, 146-163.

${ }^{330}$ SUF0004; Eggers 1968, 106 (44b).

${ }^{331}$ SUF0003; Eggers 1968, 106 (44a).

${ }^{332}$ Plutarch Roman Questions, 68; Toynbee 1973, 122-124.

333 Nuber 1973.

${ }^{334}$ Smith 1909, 146-147.
} 


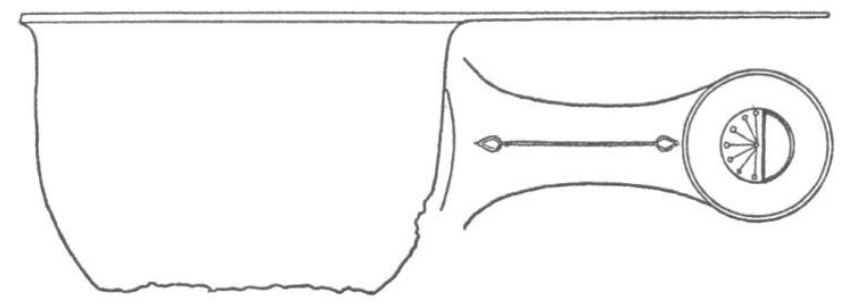

Figure 3.12: Handled Pan 2 from Oulton (Eggers 1968, 130 Abb. 26a, Scale 1:8)

A Structured Deposit of two Handled Pan 2s was found in a bog near Oulton in Suffolk. ${ }^{335}$ One of the pans, an Eggers type 137-138, has a handle decorated with a caduceus running down it terminating in a half sun-burst design beneath a half-circle handle loop (Figure 3.12). ${ }^{336}$ The other Handled Pan 2, Eggers type 144, is decorated with a floral leaf band around the exterior of its basin. ${ }^{337}$ It is likely that this is an intentional votive deposit of the aquatic type which shall be discussed in further detail in Section 3.3.

It is worth noting that while East Anglia would appear to have been a particularly active place for the hoarding of precious metal during the Iron Age and Roman period, ${ }^{338}$ it would not appear that this is particularly reflected in the depositional patterns of copper alloy vessels. On the contrary, while the Sturmere and Weeting assemblages are of Late Roman date, the remaining three assemblages would appear to have been deposited prior to the $3^{\text {rd }}$ century. This clearly reflects a difference in treatment of copper alloy as opposed to precious metal in this depositional context and suggests that copper alloy may have been seen as a more utilitarian metal as opposed to a high end means of wealth concentration in East Anglia. The depositional practice for Structured Deposits of copper alloy vessels in the southern Midlands and southeast is characterised almost exclusively by large Late Roman Structured Deposits, making the presence of these earlier assemblages in East Anglia even more significant.

\footnotetext{
${ }^{335}$ Eggers 1968, 106 (45).

${ }^{336}$ SUF0001.

${ }^{337}$ SUF0002.

${ }^{338}$ Hobbs 2006, 133.
} 


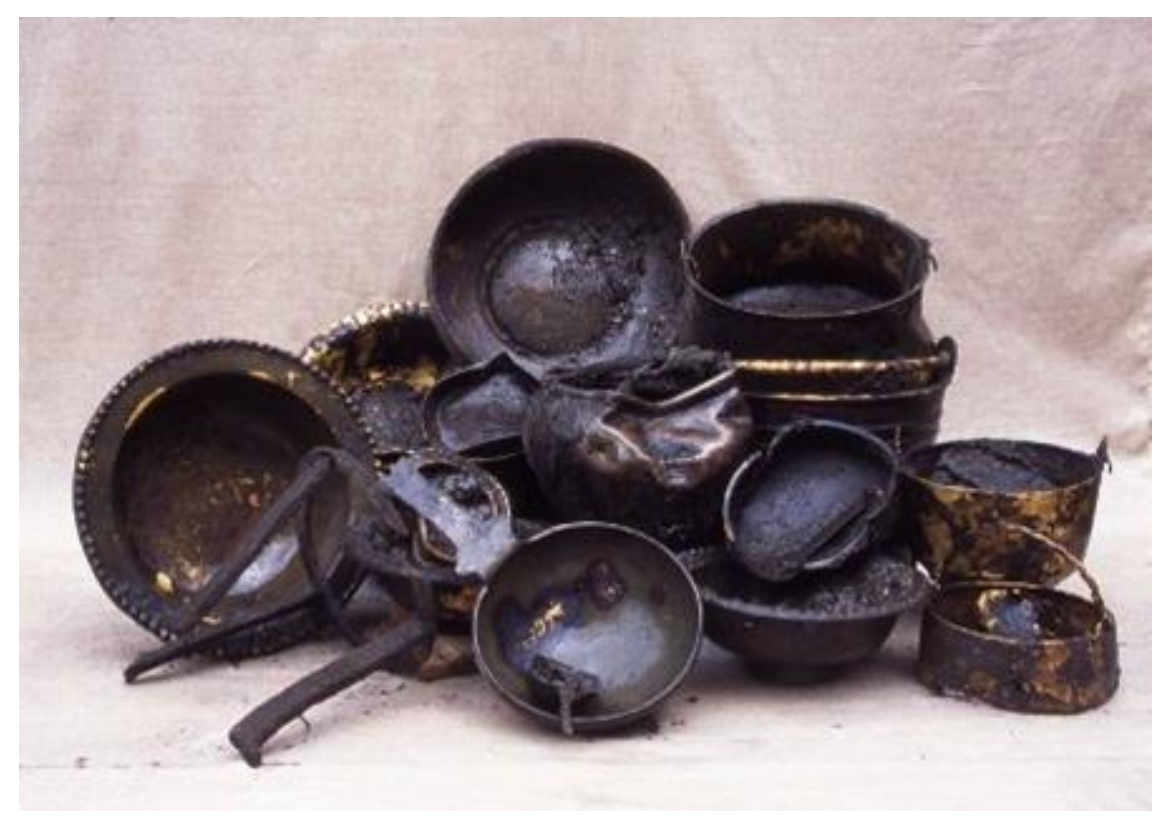

Figure 3.13: Structured Deposit from Drapers' Gardens, London

( Pre-Construct Archaeology)

There is only one Structured Deposit of copper alloy vessels so far known from Greater London, and that would be the group found during excavations undertaken by Pre-Construct Archaeology at the site of Drapers' Gardens in the City of London in 2007 (Figure 3.13). ${ }^{339}$ This is the highest profile Structured Deposit of copper alloy vessels from Roman Britain found in recent years and proves to be one of the most informative, largely due to the excellent work done in its excavation, reporting and documentation of contextual material. The Walbrook valley was an area of mixed residential and industrial occupation during the Roman period, ${ }^{340}$ though significant ritual activity is attested as well by the presence of a Mithraeum as well as assemblages of ceramics and animal bone that are identified as Structured Deposits. ${ }^{341}$

The Structured Deposit was found stacked at the bottom of a stone lined well and consists of 15 copper alloy vessels, ${ }^{342}$ three lead alloy vessels, an iron tripod and an iron ladle. ${ }^{343}$ The copper alloy vessels in the Structured Deposit include an Irchester type bowl, ${ }^{344}$ three

\footnotetext{
${ }^{339}$ L0001-L0015; Gerrard 2009, 163.

${ }^{340}$ Leary \& Butler 2012, 10-20 \& 86.

341 Toynbee 1986; Leary \& Butler 2012, 84-86.

${ }^{342}$ L0001-L0015.

${ }^{343}$ Gerrard 2009, 167-176.

${ }^{344}$ L0001; Gerrard describes it as 'similar' to an Irchester type bowl.
} 
Perlrandbecken bowls, ${ }^{345}$ five buckets of various type,${ }^{346}$ and two Handled Pan $2 \mathrm{~s} .{ }^{347}$ Found below these objects were two well preserved and apparently unworn coins struck by Gratian between 367-375 and 375-378 CE respectively, indicating a date of deposition after $375 \mathrm{CE}{ }^{348}$ The Irchester type bowl from Drapers'Gardens ${ }^{349}$ has only one extant suspension ring and hanging vessel mount extant, but would have originally had three. ${ }^{350}$ The mount is of zoomorphic design, which Gerrard believes to be a lion, ${ }^{351}$ though the mount is well worn and identification of the creature difficult beyond it being a beast with a maw (Figure 3.14). If the animal was accepted to be canine, it could indicate a possible cleansing association with this vessel as part of ritual ablutions. ${ }^{352}$

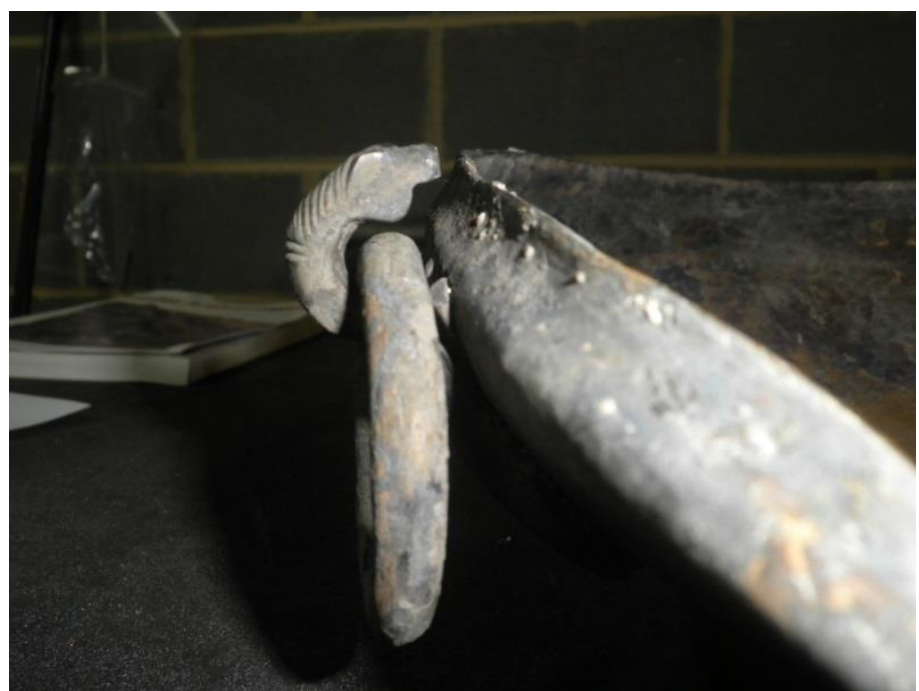

Figure 3.14: Zoomorphic mount from Drapers' Gardens, London (photo by author)

The composition of this assemblage is chronologically diverse, a characteristic shared by other large Late Roman Structured Deposits. The two Hemmoor buckets ${ }^{353}$ were probably antiques when they were deposited, being manufactured some two centuries before the numismatic evidence and Irchester bowl indicate the group was deposited. ${ }^{354}$ The incomplete

\footnotetext{
${ }^{345}$ L0007-L0009.

${ }^{346}$ L0002-L0006.

${ }^{347}$ L0014-L0015.

${ }^{348}$ Gerrard 2009,166.

${ }^{349}$ L0001.

${ }^{350}$ Gerrard 2009, 168.

${ }^{351}$ Gerrard 2009, 169.

${ }^{352}$ As may be seen from discussions in Chapters 4 and 7 .

${ }^{353}$ L0002-L0003.

${ }^{354}$ Gerrard 2009, 178.
} 
skeleton of a juvenile red deer was interred just above the Drapers'Gardens assemblage and indicates that the entire group is part of a ritual deposit. ${ }^{355}$ This is especially true as red deer are known from other Structured Deposits in Britain, for example in wells at Baldock ${ }^{356}$ and Rudstone Roman Villa. ${ }^{357}$

While the Drapers'Gardens material would appear to be a ritual deposit, the date for its deposition would suggest that it was likely influenced by the late $4^{\text {th }}-5^{\text {th }}$ century political and social events in Britain that mark the end of the Roman period. The find is notable, not only being the only definite Structured Deposit of copper alloy vessels of Roman date yet found in London, but also by the ritual evidence provided by the red deer and the chronological frame offered by the numismatic material. The contextual information provided by the Drapers'Gardens assemblage proves of great use in understanding the probable depositional circumstances of other assemblages of similar date and composition. ${ }^{358}$

There are three Structured Deposits from the south eastern counties of England that feature in this thesis: one a large Late Roman Structured Deposit from Wotton, the second a group of $3^{\text {rd }}$ century coins interred in a bucket found at Bullock Down Farm in East Sussex and finally a curious collection of objects found at Richborough Roman fort in Kent. Of these three, only Wotton fits into the wider patterns of Structured Deposition of copper alloy vessels in Britain, indicating that this region did not have the same cultural of political impetus that led to Structured Deposition of copper alloy vessels in other parts of the province.

The coin hoard from Bullock Down farm is composed of some 5,495 debased silver radiate coins of the $3^{\text {rd }}$ century. ${ }^{359}$ The inclusion of this hoard in a copper alloy vessel is unusual, though not wholly without precedent in Britain. This group reflects more upon the processes of coin hoarding that were active in the province during the $3^{\text {rd }}$ century and is best understood and discussed within that set of data as opposed to informing our understanding of the Structured Deposition of copper alloy vessels more broadly.

A very unusual collection of objects comes from the bottom of a pit at the Roman fort at Richborough where remains of a Handled Pan 1 were found with ten glass game pieces, two copper alloy spoons, one copper alloy rod, four blue paste beads, one bone spoon, $161^{\text {st }}$ century

\footnotetext{
${ }^{355}$ Gerrard 2009, 179-180.

${ }^{356}$ Chaplin \& McCormick 1986, 410-411.

${ }^{357}$ Chaplin \& Barneston 1980, 155.

358 Section 3.3.

${ }^{359}$ Bland \& Johns 1979, 61-107; BM 1973.0404.1.
} 
CE sestertii, one amber bead, one set of iron shears, one bone comb, and various hobnails and iron nails and unknown iron fragments associated with the remains of a wood and iron box that may have originally contained them. ${ }^{360}$ As will be seen in Chapter 4, this would not be an unusual collection for a grave assemblage, but its placement at the bottom of a purpose dug pit without any human remains recorded is something of an enigma. All of the coins in this group were minted under the emperor Claudius, ${ }^{361}$ suggesting a Conquest Period deposition to the group. The Handled Pan 1, decorated with a ram's head handle medallion, is well attested from Pompeii $^{362}$ and other first century contexts, especially funerary contexts. ${ }^{363}$ Though this group was professionally excavated and well documented, its depositional process is difficult to reconstruct, though some process of ritual deposition may be inferred. It does not coincide with other known patterns in the Structured Deposition of copper alloy vessels in Roman Britain that are currently known and serves as a reminder of how complex and varied depositional processes may be, inevitably resulting in anomalous groups that defy simple explanation. The discovery during this same series of excavations (though not bearing a close spatial relationship) of a much larger coin hoard of circa 1,200 coins of largely $4^{\text {th }}$ century date, with a minimum of issues dating as far back as the Republic, is worth mention as perhaps showing a long term continuity of the deposition of coinage at the site. ${ }^{364}$

The large Late Roman assemblage of ten vessels from Wotton in Surrey is the only such deposit from the south eastern counties of England currently known. ${ }^{365}$ It contains three cauldrons and several bowls, including two Irchester type bowls, ${ }^{366}$ as well as a Handled Pan 4 or Coptic Pan. ${ }^{367}$ A hand-written note by an anonymous hand stored with the Wotton assemblage in the archives of the British Museum mentions that the objects were stacked and residue of straw packing was evident when the objects were first examined, ${ }^{368}$ providing evidence of a careful deposition. One of the bowls is of quite unusual type, ${ }^{369}$ with 11 pairs of shallow channels decorating the walls of the basin (Figure 3.15). Its unusual form and the presence of a

\footnotetext{
${ }^{360}$ Bushe-Fox 1928, 31.

${ }^{361}$ Bushe-Fox 1928, 32.

362 Tassinari 1993 (H2311).

${ }^{363}$ Nuber 1973, 38-50 \& 144-188; See Chapter 4.

${ }^{364}$ Bush-Fox 1932, 192-235.

${ }^{365}$ Kennett 1971, 130-132 (5); SUR0001-SUR0010.

${ }^{366}$ SUR0006-SUR0007.

${ }^{367}$ SUR0010.

${ }^{368}$ BM 1915.0406.5.

${ }^{369}$ SUR0001.
} 
lathe-stability perforation in its centre led Smith to count this as one of his proposed groups of waterclocks. ${ }^{370}$ A comparable example from Pannonia is noted by Radnoti, ${ }^{371}$ but little is known of its probable place of manufacture or date. As the other vessels in this group are datable to the Late Roman period, it might be implied that this bowl is also of Late Roman manufacture.

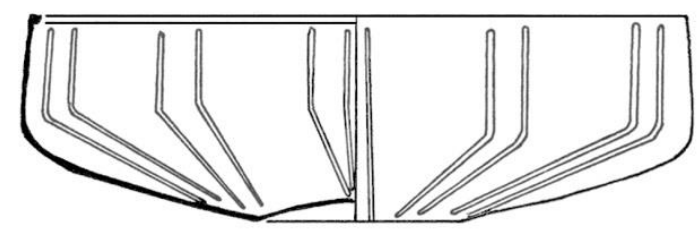

Figure 3.15: Bowl from Wotton (Kennett 1971, 135 Fig.12.1, Scale 1:7)

\begin{tabular}{|l|l|}
\hline Find Location & Catalogue Number \\
\hline Bath (Avon) & AV0002 \\
\hline Exeter (Devon) & DEV0003-DEV0004 \\
\hline Hod Hill, Cranbone (Dorset) & DOR0001-DOR0005 \\
\hline Kingston Deverill (Wiltshire) & WIL0004-WIL0008 \\
\hline Bishop's Cannings (Wiltshire) & WIL0013-WIL0022 \\
\hline Roundway Hill (Wiltshire) & WIL0023 \\
\hline
\end{tabular}

Table 3f- Structured Deposits from the south west of England.

In the south west of England, six Structured Deposits of copper alloy vessels from the Roman period are currently known (Table 3f). The Handled Pan 2 from Bath in Avon is of particular interest because it was found at a well-known temple site that has received a great deal of scholarly attention, the baths of Sulis Minerva. ${ }^{372}$ This object was found in the reservoir spring which feeds into the main bath complex within a mixed and highly complicated context as wide as from the $2^{\text {nd }}-5^{\text {th }}$ century CE along ${ }^{373}$ with many other votive objects including other metal vessels (mostly of pewter, but also silver), a tin face mask, gemstones, jewellery, coins and hair combs. ${ }^{374}$ Of the 23 metal vessels recorded from the excavations undertaken during the nineteenth as well as during latter half of the twentieth century by Nigel Sunter and David

\footnotetext{
${ }^{370}$ Smith 1916, 81-82.

${ }^{371}$ Radnoti 1938, 97 \& Plate XXX (3).

${ }^{372}$ Cunliffe 1988, 14 (23).

${ }^{373}$ Cousins 2014, 53-62.

${ }^{374}$ Cunliffe 1988, 6-35; Gerrard 2005, 371-373.
} 
Brown, only one is of copper alloy. ${ }^{375}$ This highlights the fact that copper alloy vessels are not common votive deposits at temple sites, a fact which is clearly made evident from the data-set of this thesis. This vessel also stands out as it is a Rudge Cup type. This particular vessel, though now missing its enamel, has clear depictions of crenulations around its body very much like those found on the Rudge Cup $^{376}$ and the Amiens Patera. ${ }^{377}$ The argument put forward by Cowen and Richmond that these are an artistic representation of Hadrian's Wall itself, ${ }^{378}$ supported by inscriptions on other Rudge Cup type vessels of the names of forts along Hadrian's Wall, ${ }^{379}$ seems to be well founded and is the generally accepted view among the academic community. Even though the Handled Pan 2 from the sacred spring of Sulis Minerva lacks the place-names along Hadrian's Wall that the Rudge Cup and Amiens Patera have, ${ }^{380}$ it is almost without doubt a depiction of the same structure and should be considered to have the same cultural associations of these other vessels. On the underside of the handle, which is now broken roughly in half, is a punch dot inscription in two lines that reads DIISVM[...]/CODON[...]. This has been reconstructed as possibly reading $\mathrm{De}[\mathrm{ae}] \mathrm{Su}(\mathrm{li}) \mathrm{M}$ [inervae] followed by a probable Celtic name of the donor, ${ }^{381}$ indicating this is clearly a votive offering made to the goddess at her spring.

Excavations carried out in 1981 at Friernhay Street in Exeter uncovered two Handled Pan 2 s within a $2^{\text {nd }}$ century CE stone-lined pit next to a timber building. ${ }^{382}$ There is no evidence suggesting that there was any particular ritual significance to the pit or the building and the objects could simply be part of the goods of a private household, though as the full excavation report has not been published it is difficult to make many judgements regarding the immediate context of the vessels. The group of objects from Hod Hill proves equally enigmatic as it was found in a highly fragmented state and the inclusion of a Handled Pan 1 along with three Handled Pan 2s and a bucket fragment is rather unusual for the composition of Structured Deposits in Britain. ${ }^{383}$ The proximity of the finds to the Iron Age Hill fort and Roman military installations of Hod Hill indicates a likely military association, as does the presence of the

\footnotetext{
${ }^{375}$ Cunliffe 1988, 9-21.

${ }^{376}$ Cowen \& Richmond 1935, 310-342; Allason-Jones 2012, 25-26.

${ }^{377}$ Cunliffe 1988, 14-15 (23).

${ }^{378}$ Cowen \& Richmond 1935, 317-318.

${ }^{379}$ Holder 2012, 65- 70 .

${ }^{380}$ Cunlifee 1988, 16 (23).

${ }^{381}$ Hassall \& Tomlin 1981, 381 (20).

${ }^{382}$ Rankov 1982, 382; DEV0003-DEV0004.

${ }^{383}$ DOR0001-DOR0005; Eggers 1968103 (9).
} 
Handled Pan 2 s, though the exact nature of the hoard and if it was originally interred in a damaged state or not is impossible currently to say.

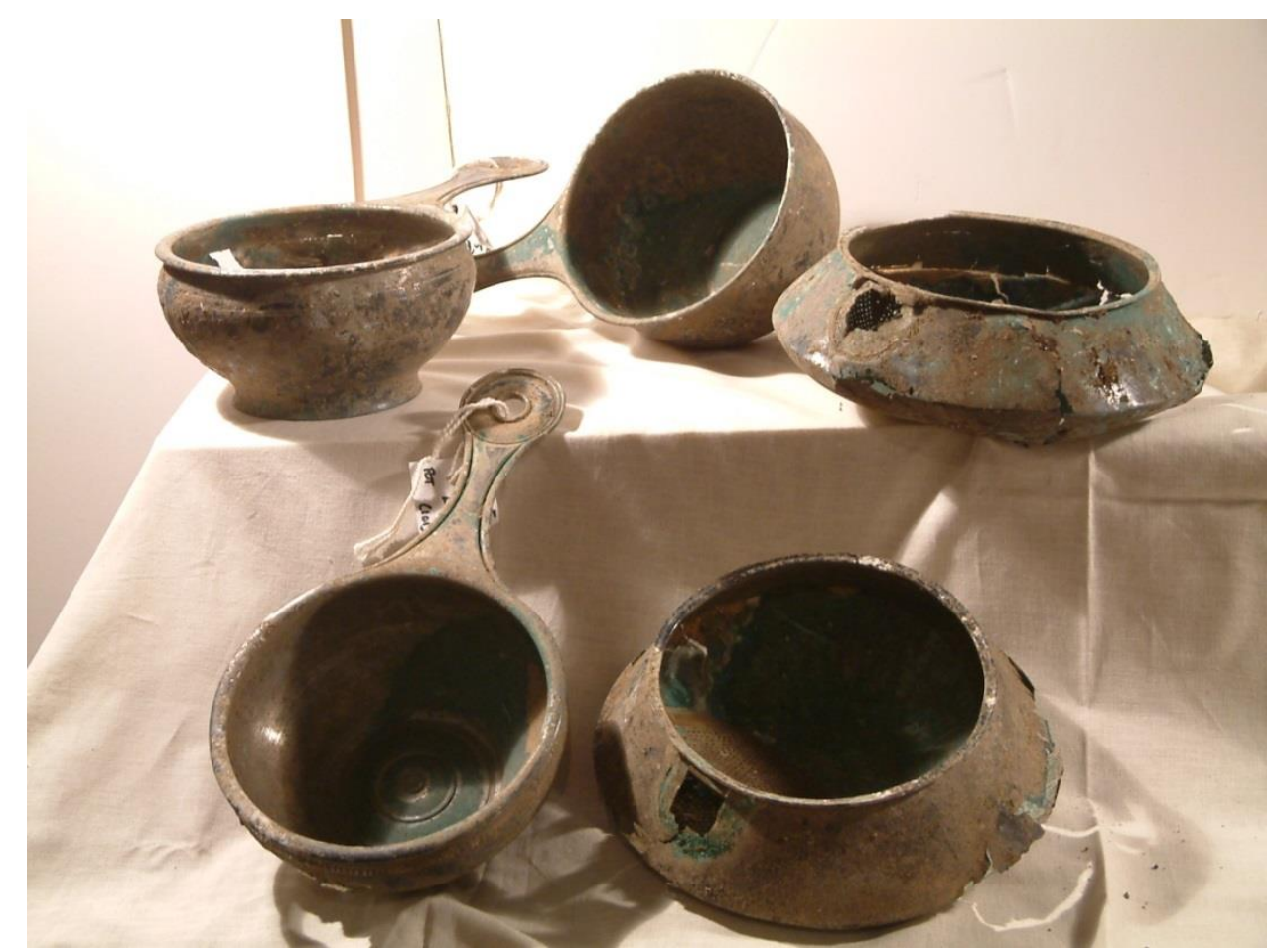

Figure 3.16: Structured Deposit from Kingston Deverill (PAS WILT-92B052)

The Structured Deposit discovered by metal detectorists and reported through the PAS from Kingston Deverill is well documented, but it proves difficult to fully understand the depositional circumstances (Figure 3.16). ${ }^{384}$ Containing three Handled Pan $2 \mathrm{~s}$ and two strainers, this assemblage was discovered about half a meter below the plough soil within the remains of what has been described as a 'large semi-circular feature'. ${ }^{385}$ Like the group from Exeter, it is unknown if any ritual significance was assigned to the structure. One of the Handled Pan 2s from Kingston Deverill bears the inscription P.CIPI.POLIBI, ${ }^{386}$ a maker's mark that appears in Pompeii and would appear to have been in manufacture throughout the second half of the $1^{\text {st }}$ century CE in Italy. ${ }^{387}$ The strainers from Kingston Deverill are of indigenous type and have

\footnotetext{
${ }^{384}$ PAS WILT-92B052; WIL0004-WIL0008.

${ }^{385}$ Worrell 2006, 458.

${ }^{386}$ Worrell 2006, 458 (27); WIL0004.

${ }^{387}$ Bennett \& Young 1981, 39.
} 
zoomorphic spouts that are possibly bovine or dragonesque, but are too badly damaged to be certain. $^{388}$ Comparable vessels are known from the Iron Age, such as the 'fish-headed' strainer bowls from Felmersham in Bedfordshire found with other bronze objects and pottery in what the workmen who discovered the objects described as a 'barrow'. ${ }^{389}$ A close parallel to these strainers is also included in the Turner's Hall burial of early Roman date. ${ }^{390}$ Further afield, a vessel of similar design to these was found in a $1^{\text {st }}$ century CE grave in Poland, hinting at extensive exchange of these vessels in northern Europe. ${ }^{391}$ It is difficult to claim that the objects from Kingston Deverill are votive deposits with there being no direct evidence for ritual significance at the site and they may indeed just as easily be accidental losses, accidentally left behind or left for safe keeping with the intent of retrieval.

An elaborate Structured Deposit which included five copper alloy bowls was found at Blagan Hill within the parish of Bishop's Cannings. ${ }^{392}$ The contents of the assemblage, which were likely originally contained within a wooden chest or box, include beads of both glass and coral, jewellery, 5,535 bronze coins, 1,646 silver coins (mostly siliquae) and 1 gold solidus. ${ }^{393}$ The latest coin issue in the assemblage is dated to $402 \mathrm{CE}^{394}$ In date and composition, this group might easily be compared to the more famous Hoxne treasure which contained jewellery, silver bowls and spoons as well as some 24 bronze coins, 14,630 silver coins and 579 gold solidi whose latest issue is dated to $408 \mathrm{CE} .^{395}$ Both the numismatic dating and the diversity of these assemblages suggest the hasty collection and hiding of portable objects of value from a wealthy household evading a threat at the beginning of the $5^{\text {th }}$ century CE. Though it is unwise to attempt to link a Structured Deposit directly to a response to a particular event or crisis, ${ }^{396}$ it is indeed difficult to construct a depositional circumstance for these two groups that does not relate to the purported incursions and political instability of the late $4^{\text {th }}$ and $5^{\text {th }}$ centuries that led to the end of Roman administration in Britain. The jug from Roundway Hill is also purported to have contained a coin hoard, though unfortunately the number or dates of the coins were not recorded

\footnotetext{
${ }^{388}$ WIL0007-WIL0008.

${ }^{389}$ Watson 1949, 37-61; Kennett 1970, 86-88.

${ }^{390}$ HER0031-HER0040; Burnham et al. 2003, 327.

${ }^{391}$ Megaw 1963, 27-37.

${ }^{392}$ WIL0013-WIL0017; Guest et al. 1997, 426-462.

${ }^{393}$ Cleary 1994, 286.

${ }^{394}$ Cleary 1994, 286.

395 Johns 2010.

${ }^{396}$ Hobbs 2006; Gerrard 2013, 59-63.
} 
and are now lost. ${ }^{397}$ This severely limits the scholarly value of this deposit, though the inclusion of an etched inscription reading 'ASPAE[...]' on the inside of the rim is worth noting. ${ }^{398}$

The Structured Deposits from the south west have parallels with elsewhere in England, but do not seem to follow some of the normal patterns that might be expected. For instance, while there is a Late Roman assemblage from Blagan Hill, it does not share characteristics with other Late Roman hoards of large vessels such as cauldrons and Irchester type bowls and is singular in the data-set for its eclectic composition of material. Also, while the votive offering at the spring of Sulis Minerva at Bath is an aquatic votive deposit, it was made at a temple site while the majority of the examples of copper alloy vessels interred as aquatic deposits in other areas of Britain are made in bogs and seemingly out-of-the-way places.

\subsection{Patterns in the depositional circumstances of Structured Deposits}

Before discussing patterns in depositional practice that are evident in the data-set of Structured Deposits, it is worth briefly mentioning some noteworthy absences that proves just as informative to understanding this material. While in the past it has often been assumed that hoarding reflects a crisis response, there is little evidence in the data-set of this thesis to support this claim in regards to assemblages of copper alloy vessels. There are a small number of Structured Deposits in this data-set that contain coin hoards that may be dated to periods or places of possible turmoil in the province. These include the groups from Corbridge $\mathrm{e}^{399}$ and Chesterford ${ }^{400}$ in the late $2^{\text {nd }}$ century, Bullock Down Farm ${ }^{401}$ in the $3^{\text {rd }}$ century and Blagan Hill in the early $5^{\text {th }}$ century CE. ${ }^{402}$ Of these, Blagan Hill is particularly likely to reflect the collection of household treasure in response to a crisis due to the variety of objects found and the numismatic evidence which dates it to after $402 \mathrm{CE}^{403}$ Even if all these examples are assumed to be evidence of crisis response, they comprise only a small amount of the data-set for Structured Deposits of copper alloy vessels and cannot be used to justify any assertions for regular depositional practice. There could be an argument made for considering many Late Roman Structured Deposits as deposited in response to Germanic invasions of the province or internal

\footnotetext{
${ }^{397}$ WIL0023; Taylor \& Collingwood 1923, 283 (12).

${ }^{398}$ RIB II 2415.8 .

${ }^{399}$ NU0035.

${ }^{400}$ EX0022-EX0023.

${ }^{401}$ ESUS0004.

${ }^{402}$ WIL0013-WIL0022.

${ }^{403}$ Guest et al. 1997, 427.
} 
turmoil, though the depositional circumstances for these assemblages (and other $4^{\text {th }}-5^{\text {th }}$ century assemblages previously thought to be conflict related $)^{404}$ complicate such an interpretation, as will be further discussed later in this section.

From his broad survey of precious metal hoarding behaviour in the Late Roman world, Hobbs concludes that it is impossible to develop anything akin to a 'unified theory of hoarding' simply because the depositional processes behind objects that enter the archaeological record as Structured Deposits are simply too diverse to make this exercise useful. ${ }^{405}$ This proves equally useful in theorizing the depositional processes of Structured Deposits of copper alloy vessels in Britain as well and should discourage the search for any such 'unified theory' or simple explanation for what appear to be diverse reasons and practices resulting in the Structured Deposition of this material. Instead, the individual patterns that have emerged through examination of Structured Deposits of copper alloy vessels in Britain will be discussed and possible explanations for their depositional processes put forth.

The geographic patterning of Structured Deposits of copper alloy vessels reviewed in the previous section reflects a range of practices across the entire chronological span of the Roman occupation of Britain, but it would appear that Structured Deposition was practiced with varying frequency and method across the geographic and chronological span of the province. While there was a general preference for the practice in the east of Britain, areas of high military concentration such as the far north and Wales also had numerous Structured Deposits.

Structured Deposits of copper alloy vessels are not commonly found within urban centres, with Drapers'Gardens being the most notable exception. This is in contrast to other votive deposit assemblages, notably of ceramics and animal remains, which are well attested in urban sites such as Silchester, London and Verulamium. ${ }^{406}$ It is possible that deposits of metals discovered in pre-modern times would have been plundered and recycled, while the chance find of ceramics or animals bones may have been ignored. It is also likely that most deposits of valuable material would be made in a location remote enough to reduce the chances of unintended discovery, leading to metal vessels and coin hoards being found predominantly in more remote locations. To better understand the practice of Structured Deposition of copper alloy vessels in Britain,

\footnotetext{
${ }^{404}$ Gerrard 2013, 15-207 \& 274-276.

${ }^{405}$ Hobbs 2006, 134.

${ }^{406}$ Fulford 2001, 201-216.
} 
analysis of forms and depositional circumstances across the province must now be analysed in order to recognise patterns across geographic and chronological space.

Across the entire study area, two trends dominate the structured depositional behaviour. First, the deposition of copper alloy vessels in bogs, rivers or other aquatic environments is evident across Britain, with enough examples to indicate that this represents a consistent culture practice sustained over a long period of time. The second type is the large Late Roman Structured Deposits that date to the $4^{\text {th }}$ and early $5^{\text {th }}$ centuries CE.

The spiritual importance of water sources and rivers to Iron Age peoples in Western Europe is a well-documented phenomenon. ${ }^{407}$ The deposition of metal objects in water would appear to trace its origins at least back to the beginning of the La Tene period. At Lake Neuchâtel in Switzerland, for example, an Iron Age timber causeway was built out into the lake for the apparent purpose of facilitating the casting of metal objects of various kinds into the waters. ${ }^{408}$ Hundreds of wooden votive statuettes recovered from the source of the Seine and elsewhere in western Gaul attest to the importance given to aquatic environments there, ${ }^{409}$ and the deposition of military equipment in their thousands in wetland areas of Jutland ${ }^{410}$ indicates similar ritual practice involved in these areas during the Iron Age and Roman periods. ${ }^{411}$ In England, ritual deposition in bodies of water may in fact pre-date the advent of metalworking, as flint and bone weapons dating as early as $1500 \mathrm{BCE}$ have been found in sufficient quantities in bodies of water to suggest intentional interment. ${ }^{412}$ Copper alloy vessels were also interred as aquatic deposits during the Iron Age such as the groups containing cauldrons and hanging basins of continental import from Llyn Fawr in Glamorgan and Sompting in Sussex attest. ${ }^{413}$ Vessels were also interred in the River Thames in the Late Iron Age, as extant examples from Hammersmith $^{414}$ and Battersea ${ }^{415}$ attest. The practice of ritual deposition in water is also well attested in Ireland and Scotland. ${ }^{416}$ The 'Golden Bog of Cullen' is likely the most prolific of

\footnotetext{
${ }^{407}$ Bradley 1990, 1-42 \& 155-190; Arnold \& Davies 2000, 126; Bradley 2000, 23-54 \& 132-146; Ingate 2013, 135136.

${ }^{408}$ Ross 1967, 22; Bradley 1990, 157.

${ }^{409}$ Bradley 1990, 167-168.

${ }^{410}$ One of the most famous of which is the silver-gilt Gundestrup Cauldron (Aldhouse-Green 2004, 117-121).

${ }^{411}$ Isserlin 2007, 189.

${ }^{412}$ Hutton 1991, 184.

${ }^{413}$ Cunliffe 1974, 137-138; Green 1998, 68-74.

${ }^{414}$ BM 1862.0912.01.

${ }^{415}$ BM 1857.0720.01.

${ }^{416}$ Green 1998, 68-80.
} 
such sites with cauldrons, weapons and jewellery all having been found in numbers so large as to remain unquantified. ${ }^{417}$ This ritual practice proceeds through the Late Iron Age and is perhaps at this time associated with liminal regions and transitions between one territory and another. ${ }^{418}$ Some of these continental sites of worship and internment of wealth were certainly known to the Romans, as Strabo comments on the extravagant wealth that could be interred in lakes and even cites a case where the Roman authorities auctioned off such a lake for the purpose of dredging the waters to recover the submerged treasures (Strabo 4.1.13). The above examples are by no means an exhaustive account of the practice of ritual deposition in watery environments in Europe, but simply set the stage by establishing how prevalent of a practice aquatic deposition appears to have been in Iron Age Western Europe and the British Isles in order to contextualise and explain the phenomenon as it persisted during the Roman period.

Though the advent of the Roman period appears to have resulted in a decreased use in such places of ritual deposition, ${ }^{419}$ it would seem that many sites continued in use and that the practice was far from forgotten. ${ }^{420}$ Indeed, the Romans even seem to have founded new aquatic votive sanctuaries such as those of Sulis Minerva at Bath ${ }^{421}$ and the well of Coventina in Northumberland. ${ }^{422}$ The site of Piercebridge in Durham was evidently a site of aquatic votive deposition throughout the Roman period, containing objects ranging from coins and jewellery to statuettes and 'curse' tablets. ${ }^{423}$ The reverence that could be offered to bodies of water by the Romans is also demonstrated by the fact that personifications of rivers and other bodies of water was not uncommon in the Roman world, ${ }^{424}$ and is well represented in Roman art, the personification of the River Danube, bridged and defeated underfoot of the Roman army, at the base of Trajan's Column being a prime example (Figure 3.17).

\footnotetext{
${ }^{417}$ Hutton 1991, 186-187.

${ }^{418}$ Garland 2013, 195-193.

${ }^{419}$ Hutton 1991, 186; Arnold \& Davies 2000, 126.

${ }^{420}$ Mattingly 2006, 315 .

${ }^{421}$ Stewart 1981.

${ }^{422}$ Smith 1962, 59-69; Allason-Jones \& McKay 1985, 1-12.

${ }^{423}$ Walton forthcoming.

${ }^{424}$ Yeates 2006, 53-56.
} 


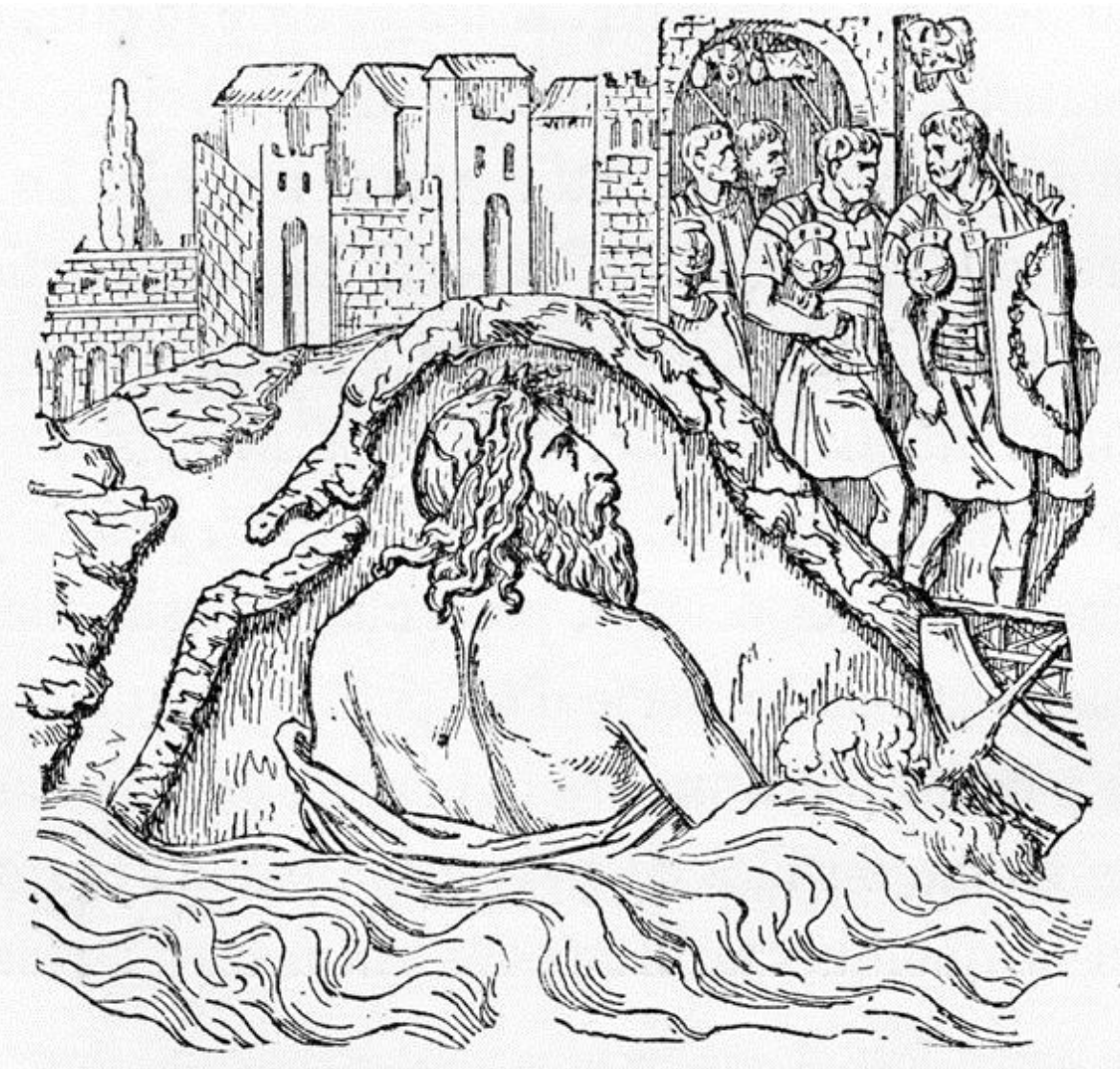

Figure 3.17: Personification of River Danube on Trajan's Column (Froehner 1865, 68)

Bodies of water have a liminal quality, both acting as a separation of territories and as a means of passage between them as well as between realms above the surface and below the surface of the water. ${ }^{425}$ The possible associations between water and the passing between the lands of the living and the lands of the dead have been previously discussed in the iconography of vessels from Mildenhall and Irchester; ${ }^{426}$ the iconographic significance of this idea will be discussed in further detail in Chapters 4 and 7. The indigenous peoples of Western Europe would also appear to have held a belief that water sources were connected to a spirit-world ${ }^{427}$ and such an association proves very useful to keep in mind when trying to understand the importance of such aquatic locations during the Roman period.

The significance of such aquatic sites being recognised and discussed, it is now important to look at the evidence offered specifically by copper alloy vessel deposits. A clear geographic

\footnotetext{
${ }^{425}$ Bradley 1990, 1-42 \& 97-190; Bradley 2000, 23-32.

${ }^{426}$ See Section 3.2.

${ }^{427}$ Stewart 1981, 21-25; Bradley 1990, 1-42 \& 99-109; Bradley 2000.
} 
pattern is evident in the prevalence of aquatic deposits in northern England compared with elsewhere, such zero examples from the Midlands, is clearly illustrated in Figure 3.18.

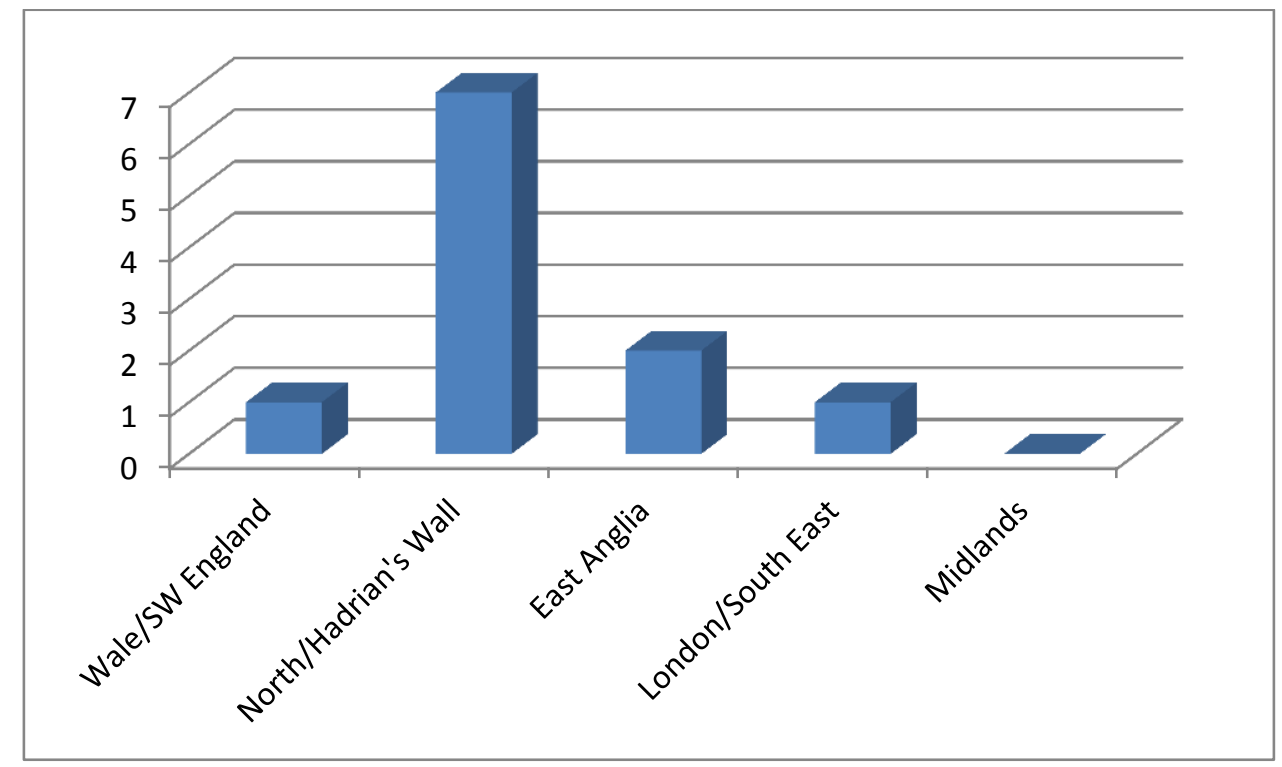

Figure 3.18: Regional distribution of aquatic deposits of copper alloy vessels in Roman Britain.

This could reflect the liminal value that may have been placed on these aquatic environments as transitional zones between two environments (dry and wet, upper world and under world, civilised and barbarian, etc.). Such liminality of space could have been palpable as one moved between securely militarised and urban zones to areas of relative wilderness in northern Britain. ${ }^{428}$ The four remaining deposits that are outside of the northern counties are fairly widely dispersed and would not appear to reflect any specific geographic trends.

It may at this point be appropriate to discuss a few of these aquatic deposits in slightly more detail and note some key features of them before making concluding arguments on the character of aquatic votive deposits in Britain. The site at Fiskerton is worth some specific consideration, as the archaeological evidence indicates that it was in use during the Late Iron Age and continued in use through the Roman period, ${ }^{429}$ thus providing an example of continuity and adaptation over this period, though such continuity of place (as opposed to practice) is more difficult to locate in Britain. The presence of a purpose built causeway associates it with such sites as Neuchâtel and Piercebridge, both mentioned earlier in this section. An examination of the objects offered at Fiskerton suggests that while weapon offerings were popular during the

\footnotetext{
${ }^{428}$ Bradley 2000, 18-32 \& 132-146; Walton 2012, 165-166; Walton forthcoming.

${ }^{429}$ Field \& Pearson 2003; Section 3.4.
} 
Iron Age, the Roman period was characterised by more domestic objects such as ceramic vessels. This could reflect a change in values among the dedicators, reflecting a demilitarised mentality and an emphasis placed more upon objects of civil domestic life as local elites began to define their power less through military achievement and more through civic office, such as would appear to be the case among the Batavians in northern Gaul. ${ }^{430}$ In such a context, the depositing of copper alloy vessels may have become a more symbolically valuable sacrifice than weaponry.

The offering at Bath is also worth special mention in this section of the thesis. It stands out in the data-set not only because it is a Rudge Cup type vessel, but also because it is the only copper alloy vessel that appears to have been offered at a site that is prolific in finds of a probable votive nature. Of the 13 vessels known to have been found at the spring of Sulis Minerva ten are pewter, two are silver and one is copper alloy. ${ }^{431}$ This seems to imply a preference specifically to pewter as a choice of material for votive offerings, at least at this particular site. Pewter vessels appear in rivers, bogs and lakes with enough frequency to imply their deliberate ritual deposition, ${ }^{432}$ much in the same way that copper alloy vessels are found to have been. Their preferential selection at sites such as Bath likely reflects a higher availability of pewter and does not necessarily reflect a conscious preference for interment of this material over copper alloy. ${ }^{433}$ The rich decoration of the Rudge Cup type Handled Pan 2 from Bath is also worth special consideration, as the only other copper alloy vessel with a votive inscription from this data-set is an unadorned bowl ${ }^{434}$ while other aquatic votive deposits of copper alloy vessels are also decidedly plain in their decoration. This seems to be a particular feature at Bath, as the other handled pans of pewter and silver also exhibit elaborate decorative schemes on their handles. ${ }^{435}$ That the temple site at Bath was richly elaborate is well understood ${ }^{436}$ and, while plenty of more mundane offerings such as combs and bracelets are known from this site, ${ }^{437}$ the prestige of the shrine may have encouraged some dedicators to offer richer votives than they may have at more remote locations.

\footnotetext{
${ }^{430}$ Roymans 1996, 13-41.

${ }^{431}$ Cunliffe et al. 1988, 9-20.

${ }^{432}$ Lee 2009, 81-82.

${ }^{433}$ Lee $2009,82$.

${ }^{434}$ DUR0005.

435 Cunliffe et al. 1988, 14-20 (24-25, 28-32).

${ }^{436}$ For a probable representation of the shrine, see the Capheaton Treasure (BM 1824.4-89. 59-65).

${ }^{437}$ Cunliffe et al. 1988, 1-33 \& 279-337; Cousins 2014, 55-61.
} 
Two of the Structured Deposits of copper alloy vessels in aquatic circumstances, those from Prestwick Carr and Drapers'Gardens, share many features with other large Late Roman assemblages not deposited in water. A ritual motivation for these deposits is very likely, especially for Drapers'Gardens. ${ }^{438}$ Ritual deposits of ceramic and stone objects to terminate the use of a well are known from London ${ }^{439}$ and the presence of a partial red deer skeleton above the vessels does suggest some form of ritualised behaviour. ${ }^{440}$ It is possible that some of the objects deposited in the reservoir at Bath, particularly groups of the coins, could also be characterised as a single aquatic deposit of material made during the Late Roman period. ${ }^{441}$ Reaction to crisis and ritual behaviour do not have to be mutually exclusive phenomenon, however, and it is indeed difficult not to try to associate such deposits with the changing social and political landscape around them. How this ritual behaviour was influenced by the rapidly changing world of Late Antiquity must for the time remain one of the frustratingly many unknowns of ancient ritual practice.

Before moving on, it is necessary to mention five objects classified in this thesis as Site Finds that could indeed have been ritually deposited in water: the Handled Pan 2 from Prickwillow near the Isle of Ely, ${ }^{442}$ a jar found in the River Ouse at York, ${ }^{443}$ a cauldron found at the bottom of a well in Blossom's Inn in the City of London, ${ }^{444}$ an Irchester type bowl found in the River Thames in London, ${ }^{445}$ and a two-handled bowl found in the River Thames in Surrey ${ }^{446}$ (all of which are discussed in Chapter 5). All of these objects were found in aquatic environments and are in a complete enough condition for one to make the argument that they were intentionally deposited. However, there is no evidence that their deposition was structured or intentional and they do not appear to be associated with any objects that were structurally deposited or in areas where aquatic structured deposition is otherwise prominent or particularly suspected. Therefore, in adhering to the strict necessity for clear evidence before identifying Structured Deposits, these objects are categorised as Site Finds for the purpose of this thesis,

\footnotetext{
${ }^{438}$ Gerrard 2009, 163-183.

${ }^{439}$ Merrifield 1987, 48-49; Gerrard 2009, 179-180.

${ }^{440}$ Gerrard 2009, 179.

${ }^{441}$ Gerrard 2005, 371-373.

442 CAM0009; Smith 1922, 85.

${ }^{443}$ NYR0029; Eggers 1968, 107 (52a).

${ }^{444}$ L0039; Burnham et al. 2002, 329.

${ }^{445}$ L0055; BM 1891.0320.10.

${ }^{446}$ SUR0012.
} 
though it may be healthy to keep in mind that these examples could be used to strengthen the argument for a tradition of aquatic deposition in Roman Britain.

There are 12 large Late Roman Structured Deposits containing copper alloy vessels in the data-set of this thesis that were interred during the $4^{\text {th }}$ and into the early $5^{\text {th }}$ centuries CE, a time of political instability resulting eventually to the withdrawal of the imperial administration and Roman military presence in Britain. ${ }^{447}$ With the notable exception of the Bishop's Cannings assemblage, the Late Roman Structured Deposits of copper alloy do not contain coin material and are instead assemblages that are composed almost exclusively of copper alloy vessels. Conversely, Hobbs notes that coinage, generally silver, is often associated with precious metal hoards in the Late Roman World. ${ }^{448}$ While this may be attributed to the higher value of silver coinage, the widely attested existence of large hoards of copper alloy coinage, such as the recently discovered Frome hoard, ${ }^{449}$ begs the question of why coinage, of copper alloy or other metal, is not associated with large Late Roman Structured Deposits of copper alloy vessels. The Structured Deposition of copper alloy vessels could reflect their encumbering nature in relation to their comparative value as it may simply have not been worth trying to haul large assemblages of copper alloy vessels during a crisis driven evacuation. This explanation seems unlikely, as several of the Structured Deposits appear to have been carefully arranged and show very deliberate and time consuming depositional behaviour, most explicitly displayed with the careful deposition of the Drapers'Gardens assemblage at the bottom of a well. ${ }^{450}$

It seems most likely that the deposition of copper alloy vessels in some deposits and coinage in others reflects a selection bias in the goods chosen for burial, which in turn implies cultural differences in the method chosen for the concentration of portable wealth within Late Roman societies in Britain. The choice of how one concentrates wealth is determined by the needs one has for that wealth. While coinage is highly partible and easily distributed among many individuals as payment for goods and services, whether military or civilian, the coin itself serves no inherent functional purpose outside of being a means of economic exchange. Apart from any aesthetic appeal a coin may have, it does not serve a functional purpose and must be reworked to be incorporated into an object that can be used. In other words, coins have no

\footnotetext{
${ }^{447}$ Gerrard 2013, 208-273.

${ }^{448}$ Hobbs 2006, 130.

${ }^{449}$ Moorhead 2010a.

${ }^{450}$ L0001-L0015.
} 
functional utility outside of the economic, ritual or sentimental value that their owners place upon them. Vessels, on the other hand, are not easily partible and therefore are not a useful means of wealth concentration if regular division for payment is necessary, unless broken as in the case of Hacksilber or scrap, but this action removes the objects' from the functional use-life as vessels. Vessels do have a functional use and can serve to concentrate the wealth of an individual or of a family, guild, cult, tribe or any such assembly in a tangible manner that can prove of shared use to the entire group affiliated with it. The needs which these different forms of wealth concentration fill inform us about the individuals who made the choice to concentrate wealth in one form or another and what their requirements from that wealth were. This is not to say that large vessel hoards in any way reflect a removal from a moneyed economy, as coin hoards are found fairly ubiquitously throughout Britain. Review of coin hoard distribution does show a preference for the south of England, with hoarding in the Midlands and the North always present but consistently less frequent; ${ }^{451}$ the Late Roman period sees the deposition of both silver and bronze coinage becoming more focused in East Anglia and the southern counties. ${ }^{452}$ Such geographic bias is difficult to discern in the deposition of copper alloy vessels, with a relatively even distribution across the landscape of these Structured Deposits with a slight preference in the North for vessel deposition (Figure 3.19).

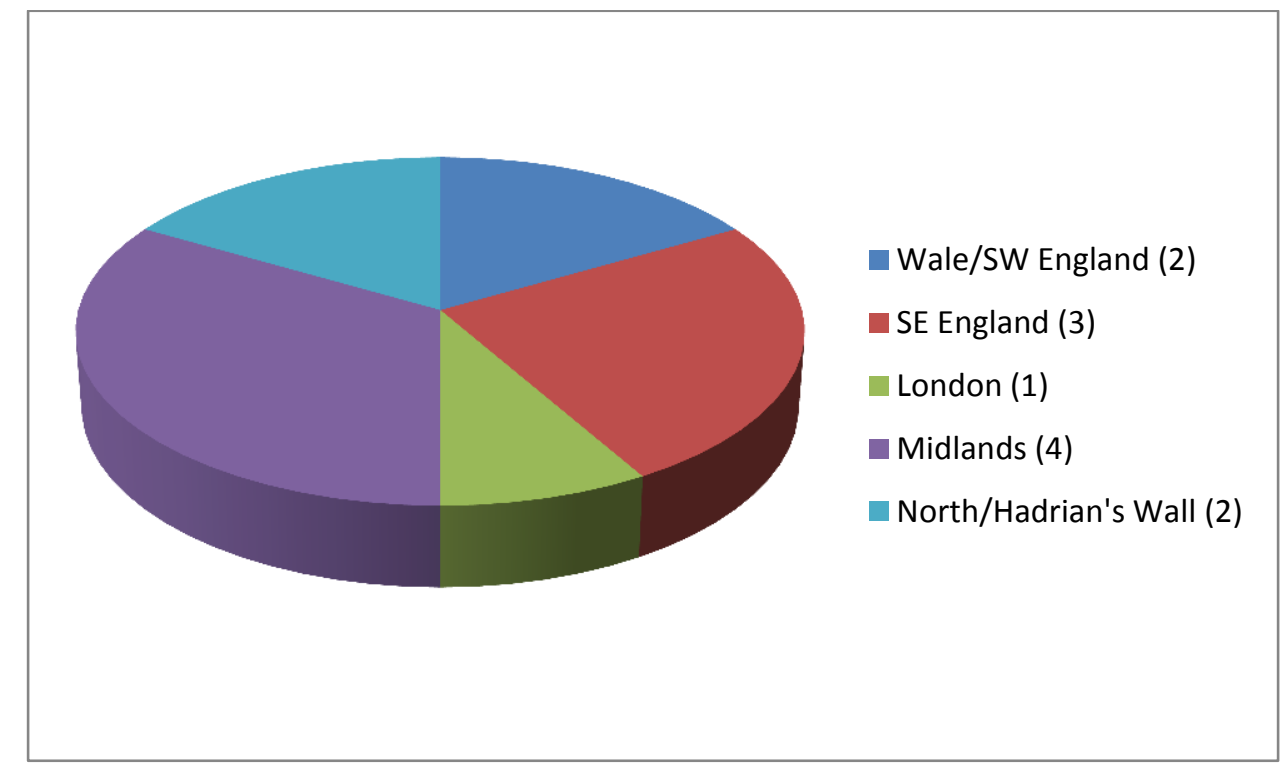

Figure 3.19: Distribution of large Late Roman Structured Deposits of copper alloy vessels.

\footnotetext{
451 Abdy 2002.

${ }^{452}$ Guest $1997,411-423$.
} 
There is no apparent patterning in the immediate topographic features or areas in which the large Late Roman Structured Deposits occur. Though a few, such as the Burwell assemblage, appear to have been interred near geographic features in the landscape, there are not enough instances to suggest this may have been a pattern in depositional practice. There is also a noticeable absence of immediate settlement evidence associated with the majority of these Late Roman deposits, again with the notable exception of Drapers'Gardens. Though it must be kept in mind that the context of many of these assemblages is poorly documented, this absence of predictable context features indicates that the deposition of these vessels was not specifically associated with any shrine or specific type of religious site that we are currently capable of recognizing in the archaeological record. Additionally, this practice is not associated closely within villa precincts as though they were hastily stored on the estates of their wealthy owners. Their apparently removed rural context implies a conscious separation of space between where the vessels were to have had their use-life and where they were interred, a separation of space that could have been both symbolic and functional to ensure the safe interring of the material, though to what end remains somewhat obscure.

The question of whether these large Late Roman Structured Deposits represent hoards stored for safe keeping in the face of a crisis with the intention of retrieval or if they are ritual deposits that reflect a particular cultural trend in Late Antique Britain is central to understanding these assemblages. Michael Grant characterises the older popular view of Late Roman hoarding behaviour in the western provinces when he states:

'When the various people beyond the frontier started dangerously overrunning Roman imperial territory in the fourth century AD, many of these well-provided owners in threatened lands buried their silverware underground, and were never able afterwards to recover what they buried. 453

Hobbs suggests that it is likely that many Late Roman precious metal hoards from the province may reflect the 'demise of the province', but warns against directly associating the peak in Structured Deposition of metalwork in the province during this period as ubiquitously the hiding of wealth in the wake of invaders. ${ }^{454}$ Indeed, recent archaeological examination of sites and human remains from this period in England throws doubt onto the idea of it being the time of

\footnotetext{
${ }^{453}$ Grant 1995, 116.

${ }^{454}$ Hobbs 2006, 128-130.
} 
intense violence and crisis often thought. ${ }^{455}$ The group from Bishops Cannings, ${ }^{456}$ which is a diverse assemblage of valuables representing the collection of household wealth, might more plausibly be read as a Crisis Hoard that was never recovered. On the other hand, it seems unlikely that the group deposited at the bottom of a well in Drapers'Gardens under the sacrificed remains of a red deer was ever intended for recovery and would appear to be most certainly a ritual votive deposit. The majority of large Late Roman Structured Deposits sit in the spectrum between these two extremes, being neither obviously secular hoarding of household wealth for safekeeping nor obviously ritual in deposition in a site of probable religious significance or with associated finds that might indicate ritual activity.

The specific selection of copper alloy vessels for these assemblages, excluding any other form of household wealth, indicates a methodical approach to the process of deposition which would be contrary to the hurried storage of material for evacuation purposes. The best explanation, given the data currently available, is that there was conscious intent involved in the collection and deposition of this copper alloy vessel material, to the exclusion of coinage or other objects, during the Late Roman period in Britain and that this selective deposition of large groups of vessels is indicative of ritual behaviour. It is evident that there was the specific choice for the interring of these large groups of vessels comprising predominantly large basins such as Irchester bowls and cauldrons. These assemblages often represent objects from a wide chronological period of manufacture and would appear to constitute assemblages for large communal ritual. As such, they could represent the depositing of sets belonging to communal groups such as cults or guilds that represent material accumulated and used over a long period of time and then 'put to rest' and interred after the completion of their use-life. In any event, the collection and depositing of these large assemblages of copper alloy vessels in the Late Roman period is indicative of a specific ritual culture practice and likely represents material and religious adaptation to the circumstances of the Late Antique world.

The two predominant practices of Structured Deposition of copper alloy vessels in Roman Britain both seem to be indicative of ritual behaviour and there is little evidence of these objects being regularly hidden for safe keeping. This is in contrast to popular conceptions of hoarding behaviour during the Roman period. It is likely that the value of gold and silver

\footnotetext{
${ }^{455}$ Gerrard 2013, 15-117.

${ }^{456}$ Guest et al. 1997, 426-462; WIL0013-WIL0017.
} 
precluded its use in ritual depositional practices on the scale possible with such material as ceramic or copper alloy.

\subsection{Form and Typological Patterns in Structured Deposits}

Two principal patterns emerge in the composition of forms that make up Structured Deposits of copper alloy vessels in Roman Britain, as Figure 3.20 illustrates. The first trend is collections of Handled Pan 2s, which seem to characterise early Roman Structured Deposits. The second is large basins, principally cauldrons and Late Roman bowl types such as the Irchester, Perlrandbecken, and bassins á bord godronné. These large vessel hoards in turn define the composition of copper alloy deposits of the Late Roman period. Figure 3.21 illustrates the prevalence of these two patterns among Structured Deposits of copper alloy vessels in Roman Britain. Handled Pan 2s occur in 29 out of the 47 Structured Deposits of this thesis, and these assemblages often contain multiple examples. This pattern is almost exclusively composed of Eggers type 139-144 type handled pans, and in the following discussion these are the objects principally referred to. It would appear that there was a conscious choice for interring this form of vessel during the early Roman period, causing its more frequent occurrence in the wider archaeological record of this time. ${ }^{457}$ While these deposits of Handled Pan 2s could be made in water, such as at Prestwick Carr and Oulton, they are also found in remote locations lacking any apparent evidence for ritual significance, such as at Kingston Deverill and Coygan Cave. The geographic distribution of this vessel in Structured Deposits is heavily weighted towards Wales and the North, both areas that had intensive military occupation over the first two centuries CE. Additionally, several of the Structured Deposits from Wales were interred very near to known military sites such as forts and camps. ${ }^{458}$ Handled Pan 2 s may well have had particular associations with the military in Britain, as suggested throughout this chapter and as will be discussed further when considering the evidence from Site finds and PAS data in Chapters 5 and 6 respectively. Handled Pan 2s of Eggers type 139-144 are a distinctly Roman form of vessel that were manufactured principally in Italy and southern Gaul during the $1^{\text {st }}-2^{\text {nd }}$ centuries $\mathrm{CE}$, and their use and adaptation into ritual deposition in Britain is indicative of

\footnotetext{
${ }^{457}$ See Chapter 5 for discussion of Handled Pan 2s as Site Finds.

${ }^{458}$ Such as those at Halkyn Mountain (F0001-F0008), Harlech (G0004-G0008) \& Coygan Cave (CAR0001CAR0002).
} 
cultural collusion and amalgamation in ritual practice; their absorption into the daily practice of Briatin's inhabitants is better illustrated by Site Finds discussed in Chapter 5. Examples of hybrid art on these vessels, such as in Coygan Cave, ${ }^{459}$ is further evidence of this cultural interchange and negotiation.

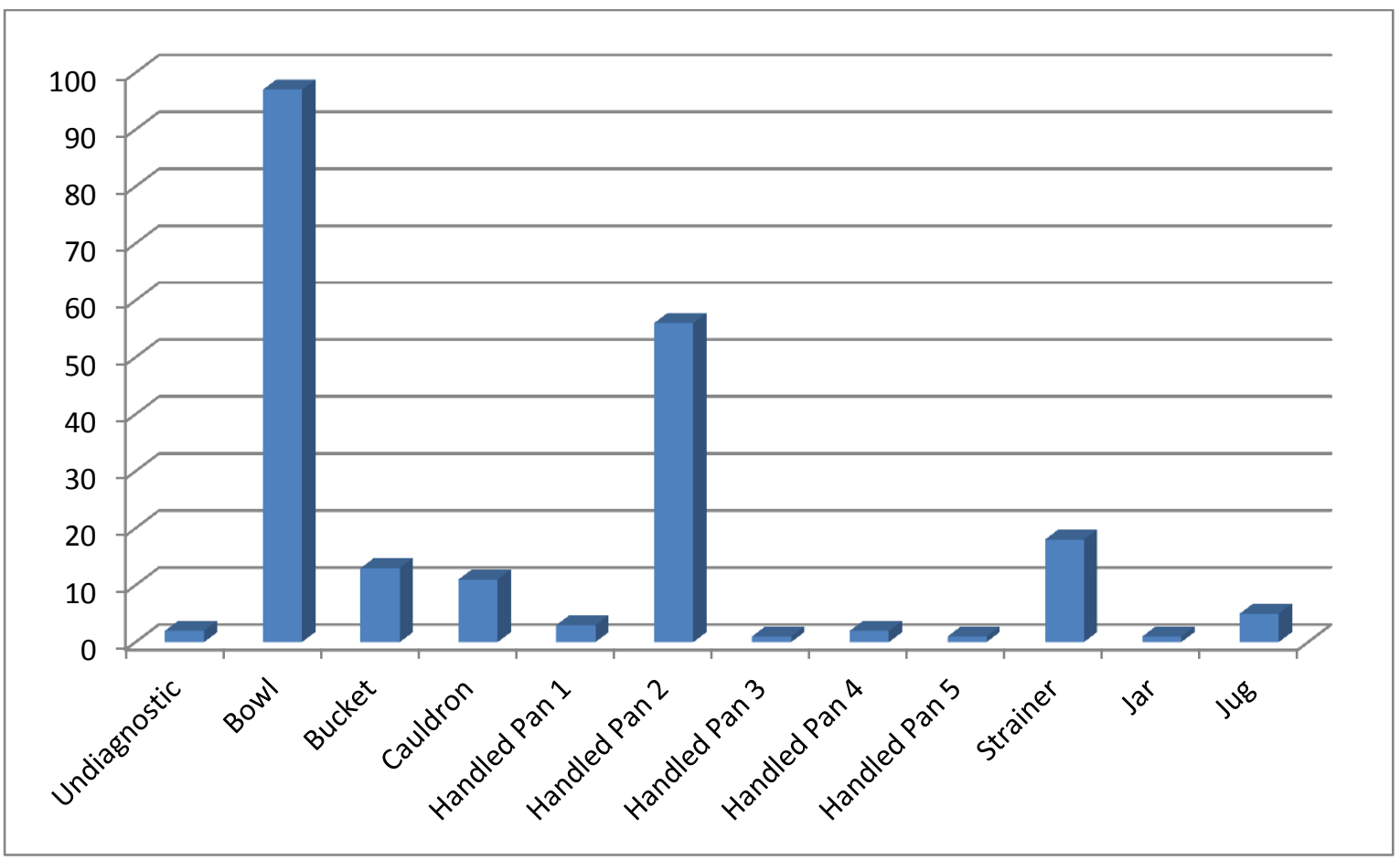

Figure 3.20: Frequency of forms across Structured Deposits of copper alloy vessels in Britain.

${ }^{459}$ CAR0001-CAR0002; Wainwright 1967, 85-88; Eggers 1968, 103 (12). 


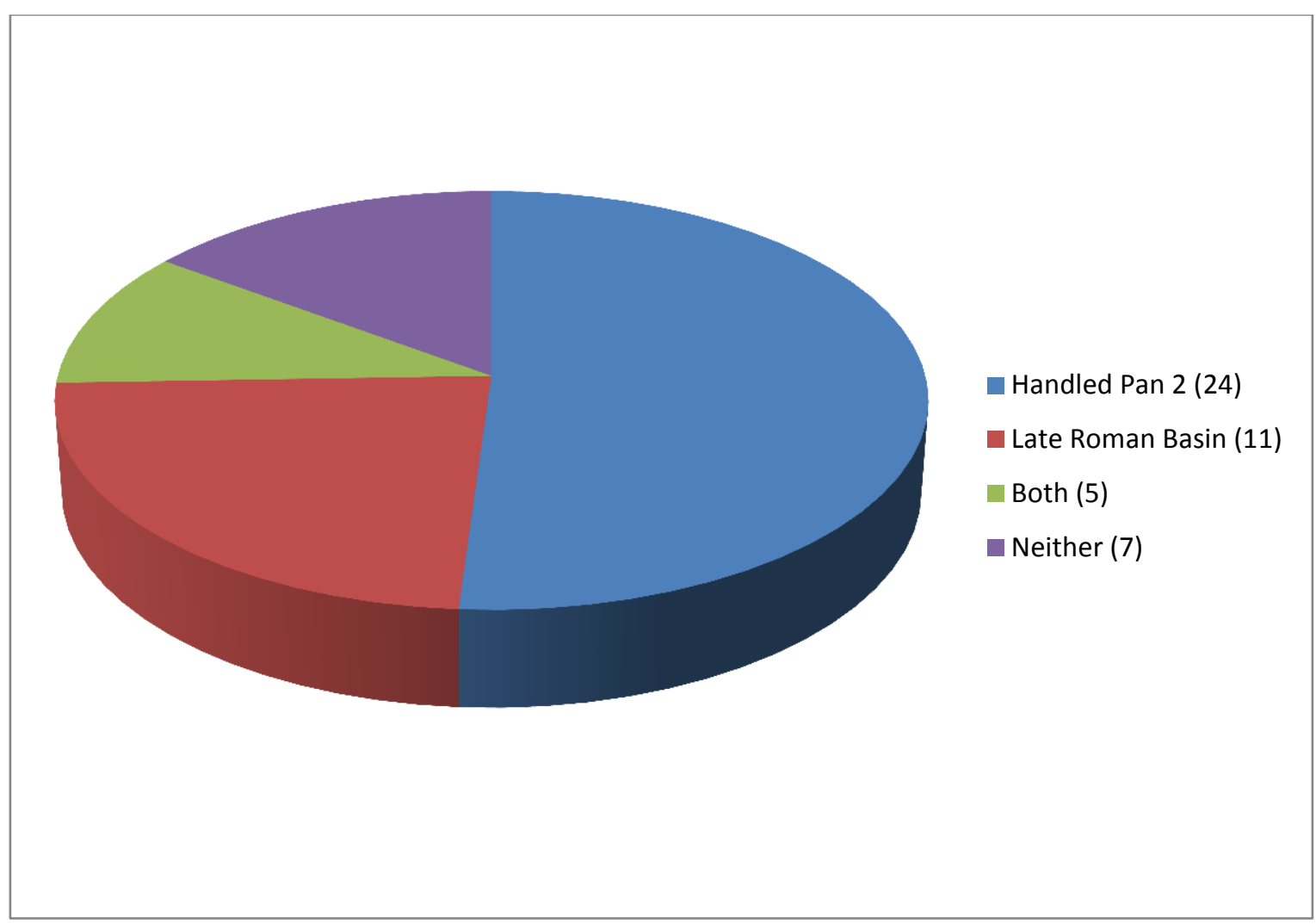

Figure 3.21: Number of Handled Pan 2s and large Late Roman basins in the Structured Deposits of copper alloy vessels in Roman Britain.

Though it is impossible to determine the identity of the dedicators, that they chose to incorporate vessel forms that were unknown to the indigenous repertoire prior to the Roman period within an indigenous ritual tradition symbolises either indigenous Britons adopting these vessels into their practice or Roman and continental colonisers participating in indigenous ritual behaviour. Of course, these two possibilities are not mutually exclusive. Furthermore, certain auxiliary troops from Western Europe may well have been familiar with similar traditions of deposition from their homelands as is clearly evident from northern Gaul. ${ }^{460}$ Some of these deposits, such as Kingston Deverill, ${ }^{461}$ may well have not been ritual, but accidental deposits or storage deposits with the intent of recovery, though the integration of some of these vessels into aquatic deposits at such places as Upper Weardale in Durham and Oulton in Suffolk indicates the incorporation of these Roman objects into an indigenous ritual. It is important to make a brief statement about the probable chronology of the deposition of Handled Pan 2s as Structured

\footnotetext{
${ }^{460}$ Roymans 1996, 9-103.

${ }^{461}$ PAS WILT-92B052; WIL0004-WIL0008.
} 
Deposits in Britain, as copper alloy vessels can have an extensive use-life. Though some examples of Handled Pan 2s contained within Structured Deposits in Britain do show evidence for wear and repair, ${ }^{462}$ the absence of this form of vessel from Late Roman assemblages implies that these vessels were used and interred earlier. From the evidence available, it would seem certain that Handled Pan 2s of Eggers type 139-144 and strainers reflect a Romano-British tradition of depositional practice that spans from the first century to no later than the third at roughly the same time that the preferential deposition of larger basins seen in Late Roman Structured Deposits becomes evident. This reflects not only a change in object selection, but most likely a change in the ritual practice in Late Roman Britain as Handled Pan 2s do not commonly appear in other Late Roman contexts either.

The composition of large Late Roman Structured Deposits was treated in some detail in the previous section. What is important presently for understanding the evidence offered is the patterning of forms within these assemblages and recognizing what these trends may indicate about how the vessels were used and why they were selected for Structured Deposition. Irchester type bowls are particularly prevalent in Late Roman Structured Deposits, accounting for some 42 objects in this Depositional Context. The high presence of this vessel type is not mirrored in other depositional contexts, indicating that this represents deliberate preferential selection which was most likely influenced by the vessel's function or its ritual significance. As these large Late Roman Structured Deposits represent sets of vessels for communal ritual, ${ }^{463}$ it may seem unfortunate that these assemblages lack ceramic or glass vessels that may well have been used with these groups of objects and may have helped to explain the functions of these vessels. However, the absence of these vessels is just as informative as it indicates that any association that these copper alloy bowls and cauldrons may have had with vessels of other material was not strong enough to warrant their sharing a depositional context and therefore implies that these objects were seen as a closed set of inter-related objects complete in and of themselves. The nature of the deposition of these groups implies ritual behaviour and, by extension, ritual significance to the objects interred within these assemblages. The ritual significance of these large copper alloy basins is particular to themselves and led directly to their

\footnotetext{
${ }^{462}$ Such as at Coygan Cave (CAR0001) and Llanberis (GWY0009-GWY0012).

${ }^{463}$ Discussed in detail in Section 7.2.
} 
selective deposition. The importance of these objects as focal points of communal ritual behaviour is evident by the nature and regular patterning of their Structured Deposition.

\section{$\underline{3.5 \text { Concluding Remarks }}$}

Within the Structured Deposits of copper alloy dining vessels from Roman Britain, some noticeable patterns emerge which illuminate ancient perceptions of this material within the wider cultural practice of the province and how these related to the selective deposition of certain forms and types of vessels in particular circumstances. That there are numerous Structured Deposits of copper alloy vessels at all is significant in and of itself, implying that these were viewed as having intrinsic or implied value, making them worth accumulating and methodically depositing. Secondly, the preponderance of aquatic votive deposits and large Late Roman deposits that constitute the majority of the assemblages suggests there was particular ritual intent in the collection and interring of these objects. The regular deposit of copper alloy vessels in exclusion to other objects also indicates a conscious selection process in the collection and deposition of this material, further indicating ritual behaviour and implying specific significance to these vessels outside of their purely functional purposes.

In the following chapters, vessels found in other depositional circumstances will be considered in detail and the patterns evident in them analysed. It will then be clear how unrepresentative Structured Deposits are of the corpus of Romano-British copper alloy vessels and that they reflect specific culture practices in the selection of vessels interred. 


\section{Chapter 4: Grave Deposits}

\section{$\underline{4.1 \text { Introduction }}$}

In this Chapter, copper alloy vessels found in British graves of Roman date will be examined to determine patterns of object selection and distribution that may offer information on culture practice and identity, particularly in relation to the perception and function of copper alloy vessels in the Roman period. This chapter begins with an expository examination of the data modelled on the geographic regional division of Britain as used in Chapter 3. Section 4.3 will then discuss the chronological development of this depositional practice in Britain during the Roman period, with particular focus in the discussion offered to forms and types. Section 4.4 will then discuss the decoration of copper alloy vessels in grave contexts. Associated finds in the graves will then be examined in Section 4.5 in order to discuss probable functional associations before commenting on burial status and the place of copper alloy vessels in the burial tradition of Britain during the Roman period.

The practice of interring goods with the deceased was certainly a long established tradition in Britain by the time of the first recorded Roman contact with Britain during the $1^{\text {st }}$ century BCE. ${ }^{464}$ There are a number of large grave deposits in south-eastern England dating to the Late Iron Age that include copper alloy vessels of continental import, probably the most famous of which being the Welwyn Garden City burial (Figure 4.01). ${ }^{465}$ The presence of wine amphorae and ceramic fine wares has led to the argument that most of the objects in these graves, including the copper alloy vessels, formed part of the wine service. ${ }^{466}$ These grave assemblages form a crucial link between LIA and Roman practice. ${ }^{467}$ As some of the burials of this form are Iron Age as opposed to Roman in date, as defined by a cut-off date of $43 \mathrm{CE},{ }^{468}$ they are not included in the data-set. Of course, exactly dating an assemblage so precisely proves practically impossible. Therefore, assemblages that are 'Conquest Period', i.e. dating to the 40's-50's CE will be considered Romano-British for the purposes of this thesis.

\footnotetext{
${ }^{464}$ Philpott 1991, 6-7; Cunliffe 2005, 543-561.

465 Eggers 1968, 69 \& 105 (31); Pitts 2005, 143-166; BM 1967 2-2 44, 48, 54, 63.

${ }^{466}$ Carver 2001, 31-39.

${ }^{467}$ Philpott 1991, 6-8; Cunliffe 2005, 543-561; Fitzpatrick 2007 134-137; Pearce forthcoming.

${ }^{468}$ Section 2.2.
} 


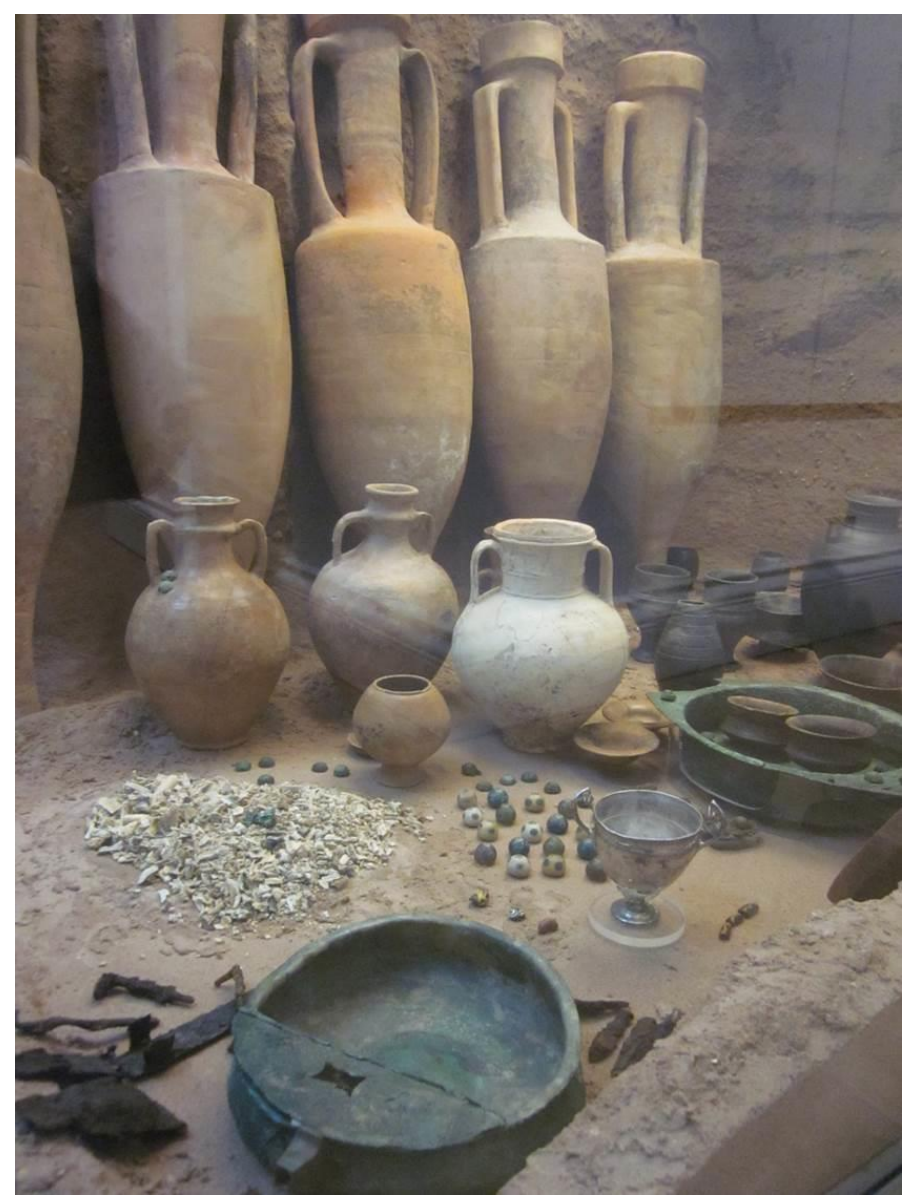

Figure 4.01: Welwyn Garden City Late Iron Age Burial (photo by author)

The depositing of grave goods continued throughout the Roman period and into the early Medieval period. ${ }^{469}$ In fact, objects of Roman date were deposited in Anglo-Saxon graves as items of prestige, ${ }^{470}$ making it very important to properly identify the chronological context of an object before including it within the data-set. While drawing on the broader literature on grave practice in Britain and the western empire, this chapter will focus its discussion on patterns that emerge within the copper alloy vessels deposited in graves and trends in which type of graves containing these sorts of objects as well as their geographic and chronological distribution. ${ }^{471}$

\footnotetext{
${ }^{469}$ Gerrard 2013, 263.

${ }^{470}$ White 1988.

${ }^{471}$ For a useful discussion on Late Iron Age and Roman graves with their regional and chronological variation, see Philpott 1991, 6-102, Fitzpatrick 2007, 123-142 and Pearce forthcoming.
} 
There are two principal paradigms by which Romano-British grave goods and funerary practice have been interpreted: spiritual and social. The spiritual interpretation asserts that the objects and ritual of burial reflects upon the religious belief of those performing the ceremony and that both the practical and symbolic importance of the goods interred reflect upon this belief, indicating a belief in the afterlife in ancient Britain and north-western Europe. The principal proponents of this interpretation are Toynbee and Henig, ${ }^{472}$ supported by work as recent as Crummy's investigation of Late Roman infant burials in Britain. ${ }^{473}$ The social interpretation suggests that the funerary ritual was principally, if not solely, a means by which the relatives or extended network of the deceased could take part in a group display of conspicuous consumption to reflect their communal and individual power and their relation to the deceased. This interpretation in the context of Roman studies was popularised by Morris ${ }^{474}$ and since the early nineties has greatly influenced current interpretive theory in this field. ${ }^{475}$ In this thesis, both interpretations will be considered applicable and by no means mutually exclusive as the funerary ritual was almost certainly interpreted in varying ways at the very time of its performance.

The following sections detail the known grave assemblages with copper alloy vessels included. Copper alloy vessels were rare grave goods in relation to objects made of ceramic or glass. However, they do occur in graves across the province and would appear to have a reasonably broad chronological span.

\footnotetext{
472 Toynbee 1971; Henig 1984, 190-205.

${ }^{473}$ Crummy 2010, 37-93.

${ }^{474}$ Morris 1992, 1-30 \& 200-204.

${ }^{475}$ Pearce 2000, 1-2.
} 


\subsection{Geographic Survey of Grave Deposits}

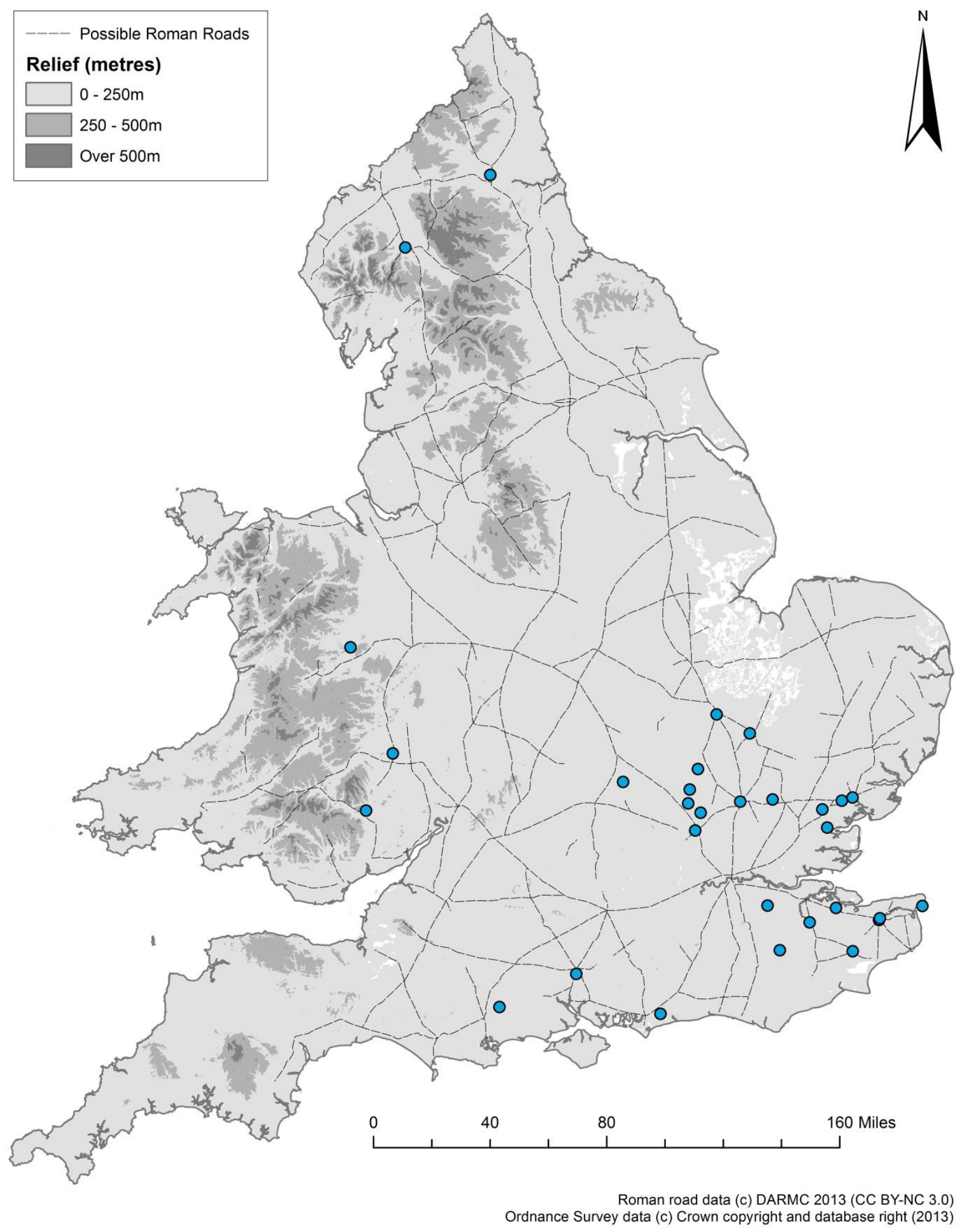

Map 2: Grave Deposits of copper ally vessels from Roman Britain (K. Robbins) 
Map 2 and Figure 4.02 both illustrate a clear geographic discrepancy between different regions of England, most notably the concentration of burials that include copper alloy vessels as grave goods in the southern Midlands that becomes particularly prominent in Essex and Kent. This is broadly in line with a tradition of richer burial assemblages in this region. ${ }^{476}$ These regional divisions will be discussed in greater detail at the end of this section after the assemblages in each of the regions is discussed in turn. However, it is worth bearing in mind these general geographic patterns at the start in order to better interpret the individual groups under discussion.

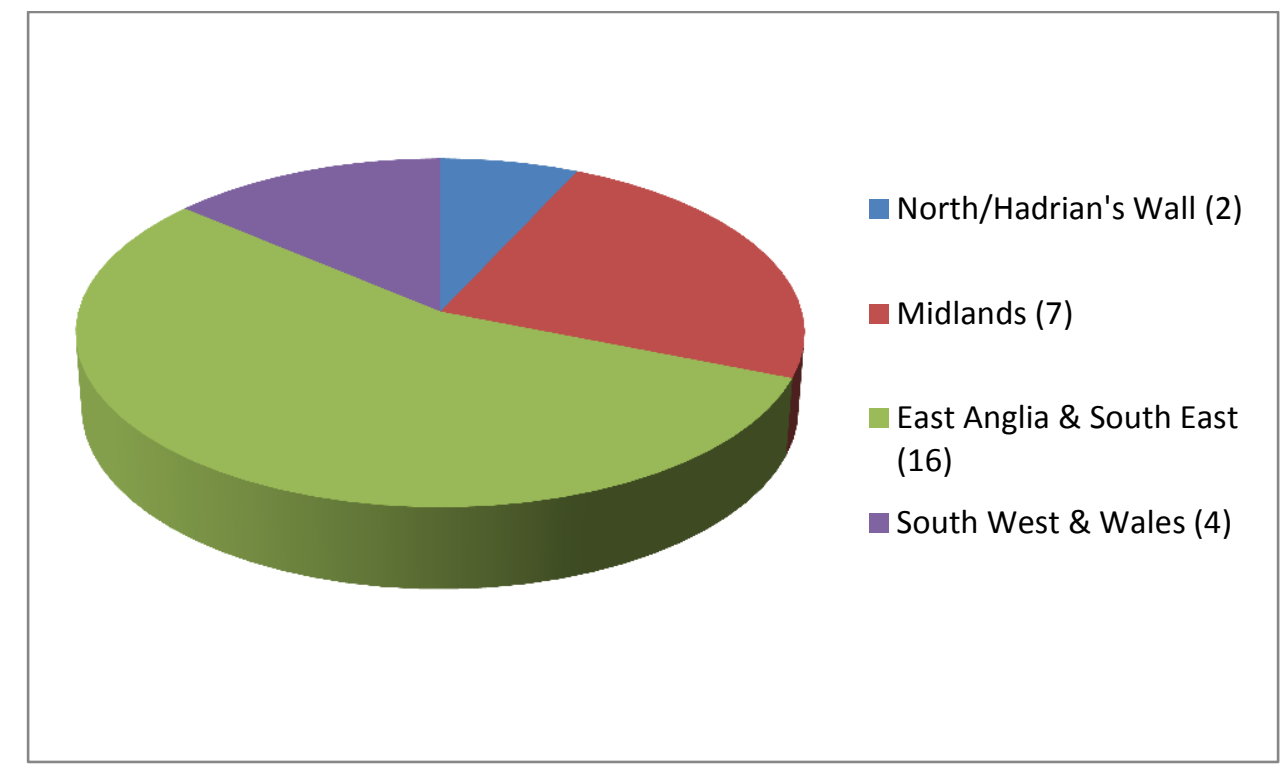

Figure 4.02: Regional distribution of sites with AE vessels as Grave Deposits.

There are only two grave groups known in Wales from the Roman period that include copper alloy vessels: Welshpool in Powys and Llantilio Pertholey in Monmouthshire (Table 4a). While this number is not nearly high enough for us to make qualified statements about regional trends in grave practice save that the interring of copper alloy vessels as grave goods is uncommon in Wales during the Roman period, these two graves are note-worthy and help in characterising the practice of interring copper alloy vessels across Britain more generally.

\footnotetext{
${ }^{476}$ Philpott 1991, 217-218.
} 


\begin{tabular}{|c|c|c|}
\hline Findspot/Date & Copper Alloy Vessels & Associated Finds \\
\hline $\begin{array}{l}\text { Llantilio Pertholey, } \\
\text { Monmouthshire/ } 1^{\text {st }}-2^{\text {nd }}\end{array}$ & MON0007 (Jug) & $\begin{array}{l}\text { unspecified pottery sherds of } \\
\text { Roman date }\end{array}$ \\
\hline Welshpool, Powys/ 1st & $\begin{array}{l}\text { PO0002 (Jug) } \\
\text { PO0003 (Handled Pan 1) } \\
\text { PO0004 (Handled Pan 1) } \\
\text { PO0005 (Handled Pan 1) } \\
\text { PO0006 (Cauldron) } \\
\text { PO0007 (Vessel Mount) }\end{array}$ & $\begin{array}{l}1 \text { iron fire dog and } 2 \text { stands, } \\
1 \text { glass jar, } 1 \text { glass bowl, } \\
\text { unspecified sherds of at least } \\
\text { two ceramic vessels }\end{array}$ \\
\hline
\end{tabular}

Table 4a: Grave Deposits from Wales.

The area around Welshpool in the Severn Valley is known to have been inhabited during the Roman period with military and rural agricultural settlements excavated in the vicinity. ${ }^{477}$ The Welshpool assemblage was discovered during drainage work on a cattle market in 1959 and is a rich burial group of various objects including a large fire-dog characteristic of Late Iron Age design. ${ }^{478}$ Unfortunately, the grave was greatly disturbed by the construction crew that discovered the material ${ }^{479}$ and therefore the objects' exact context and relation to each other in the grave must remain unknown. It is therefore possible that they came from more than one disturbed grave. Boon suggests that as the goods appear to have been found at Roman period 'ground level' that the objects originated from a barrow type tomb, though adds this is not fully provable. $^{480}$ This is an appealing theory, however, as several of the large grave assemblages from eastern England come from excavated barrows, ${ }^{481}$ and could help in our interpretation of the group by analogy. Perhaps this group could even represent an outlier of this grave tradition, drawing association with distant kinsmen through shared burial practice. Even if this were to be assumed that this group is analogous to the assemblages such as those from Bartlow Hills in Essex, ${ }^{482}$ the Welshpool group would be unusual for its far western provenance and must be considered exceptional. The copper alloy vessels included in the grave assemblage are three Handled Pan 1s, a jug, a cauldron and the hanging vessel mount with the fragmentary remains of a vessel that was most probably a bucket. The copper alloy vessels appear to have not been new

\footnotetext{
${ }^{477}$ Collingwood \& Taylor 1927, 186; Longley et al. 1988, 205; Arnold \& Davies 2000, 40-118; Frere 2004, 115 120 .

${ }^{478}$ Boon 1961, 13; Eggers 1968, 103 (16).

${ }^{479}$ Boon 1961, 13-16.

${ }^{480}$ Boon 1961, 16.

${ }^{481}$ Liversidge 1953, 29-32; Eggers 1968, 110 (89a); Philpott 1991, 261\& 256; Cunliffe 2005, 543-578; Eckhardt 2009, 65-98.

${ }^{482}$ EX0058-EX0067; Gage 1832, 1-23; Philpott 1991, 256; Eckhardt 2009, 65-98.
} 
upon interment and some show considerable signs of wear, such as PO0005 that has two repairs. ${ }^{483}$ The five smaller vessels were stacked inside the cauldron and appear to have been packed with straw and covered with a cloth ${ }^{484}$ which is interesting to note as most graves in this data-set were reported without such organic remains being extant. This suggests that such perishable materials were a more common feature in burial practice than is generally visible, due to the common decomposition of this material. The group is iconographically rich. One of the Handled Pan 1s has two anthropomorphic representations: one on the handle terminal and one gripping the rim (Figures 4.03 and 4.04). The form of these faces appears Bacchic and it is possible that the worn drapery of the figure at the rim of the vessel could represent a panther skin. $^{485}$

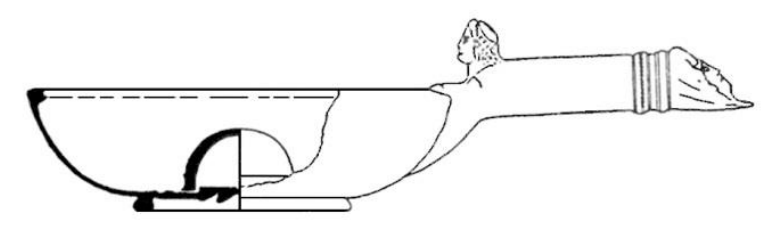

Figure 4.03: Anthropomorphic Handled Pan 1 from Welshpool (Eggers 1968, 133 Abb. 31.2, Scale 1:7)

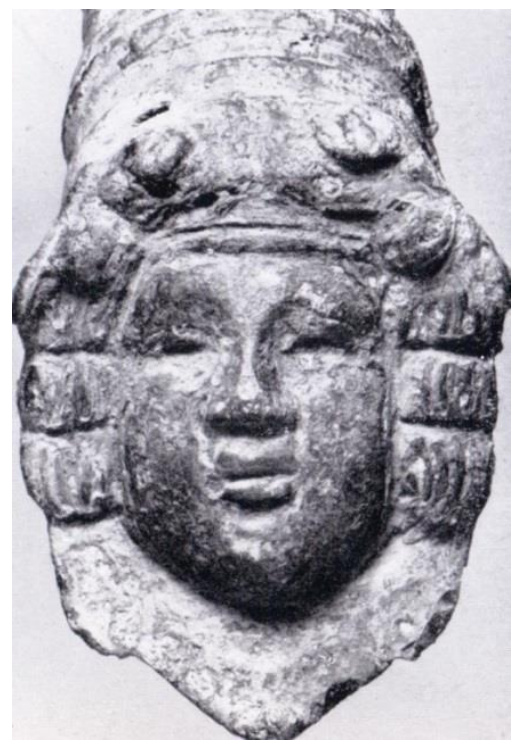

Figure 4.04: Detail of anthropomorphic handle medallion from Handled Pan 1 from Welshpool (Boon 1961, Plate VII)

\footnotetext{
${ }^{483}$ Boon 1961, 24.

${ }^{484}$ Boon 1961, 16-17.

${ }^{485}$ Boon 1961, 22.
} 

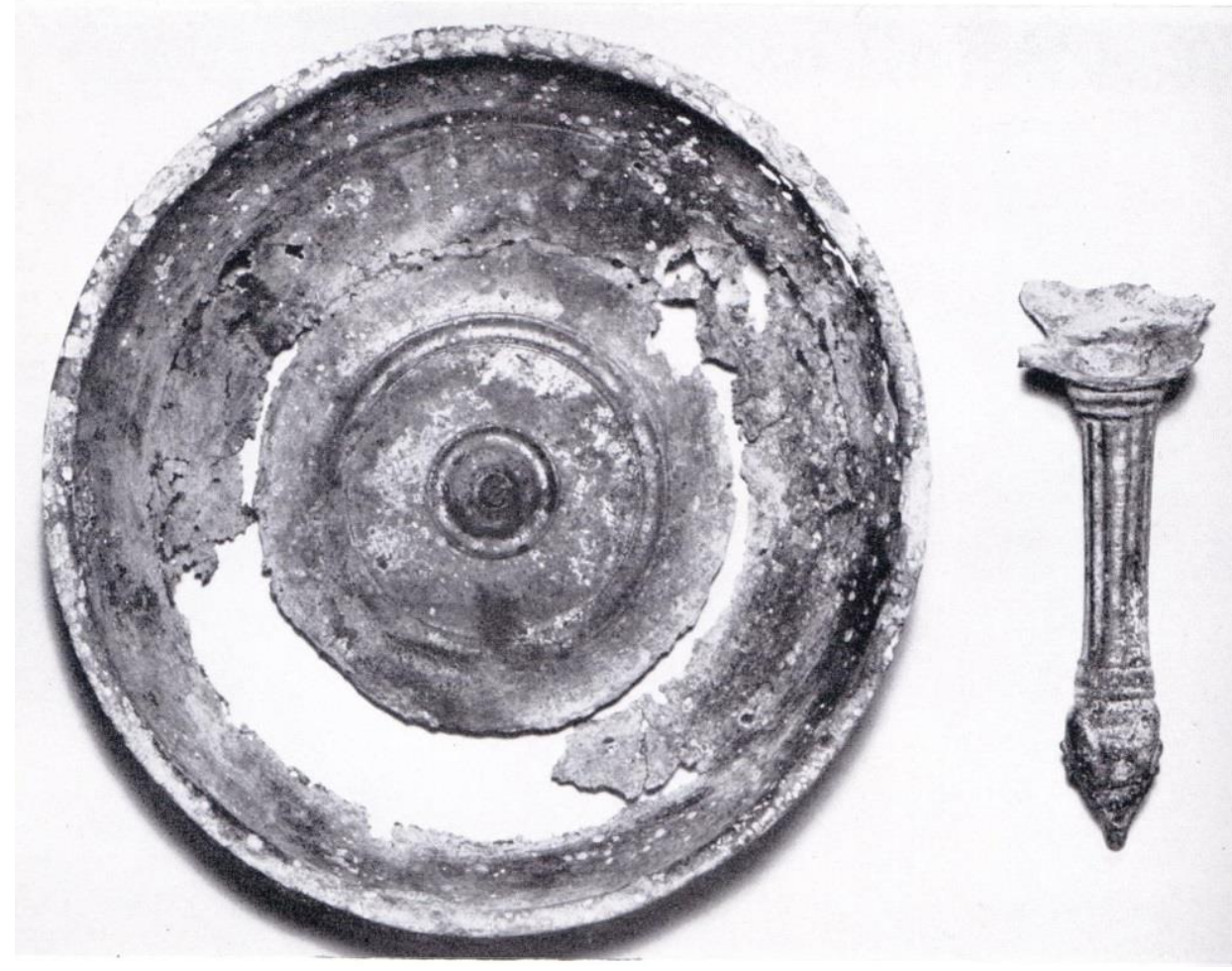

Figure 4.05: Zoomorphic Handled Pan 1 from Welshpool (Boon 1961, Plate VIIIb)

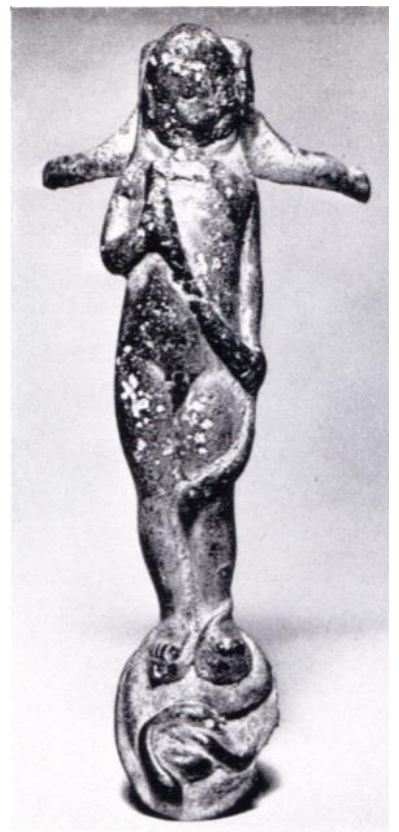

Figure 4.06: Anthropomorphic jug handle from Welshpool (Boon 1961, Plate VIb) 
The other two Handled Pan 1s have zoomorphic ram's head handle terminals, a common design for this form (Figure 4.05). ${ }^{486}$ The jug's highly decorative handle depicts a boy wearing a lion skin holding one snake while another is beneath his feet (Figure 4.06). ${ }^{487}$ Though Eggers suggests this is a satyr, ${ }^{488}$ the lion's skin means it almost certainly is a depiction of Hercules strangling serpents as a child. ${ }^{489}$ The bull-head hanging vessel mount ${ }^{490}$ found in the grave carries on a tradition of vessel decoration from the Late Iron Age into the Roman period (Figure 4.07). ${ }^{491}$

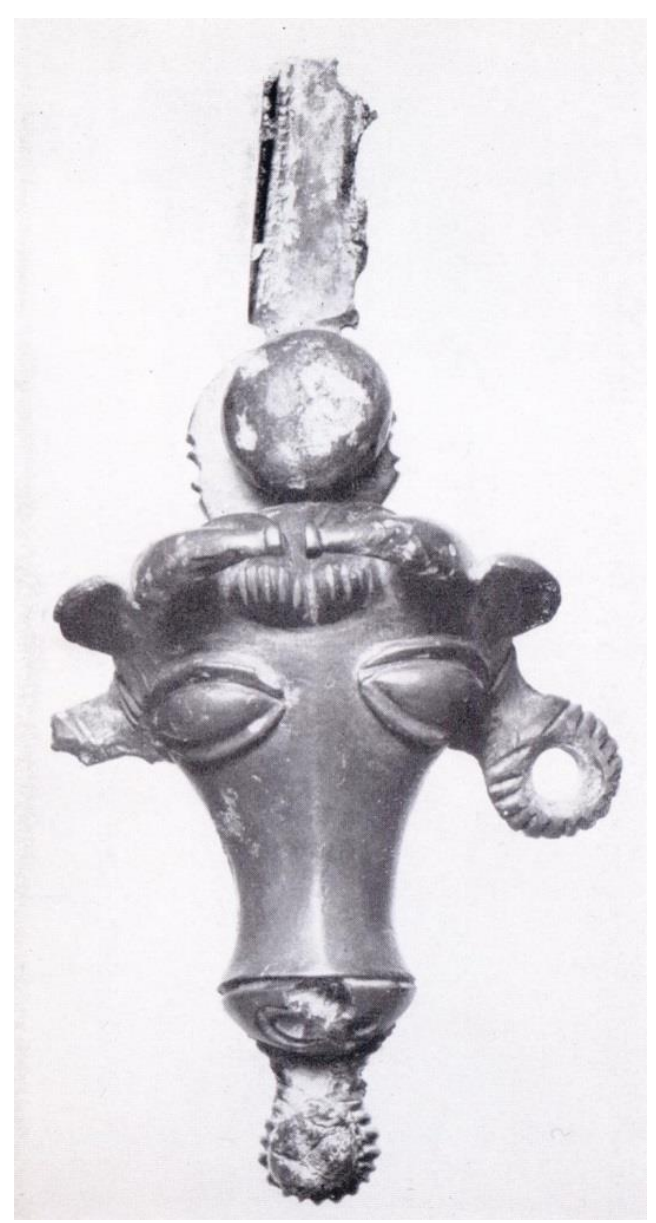

Figure 4.07: Zoomorphic hanging vessel mount from Welshpool (Boon 1961, Plate Xa)

\footnotetext{
${ }^{486}$ Nuber 1973, 33-54; See Sections $4.10 \& 4.11$.

${ }^{487} \mathrm{PO} 0002$.

${ }^{488}$ Eggers 1968, 103 (16.1).

${ }^{489}$ Even though, as Boon (1961) points out, the imagery is not chronologically correct to the myth, as Hercules is already in possession of his lion skin in this depiction. It is most likely that this use of iconographic attributes is to aid in the viewers' understanding and that chronological accuracy was not neccassary to the enjoyment of the object. ${ }^{490}$ PO0007.

${ }^{491}$ Hawkes 1951, 191-198; to be discussed in further detail in Chapter 7.
} 
The combination of Handled Pan 1's and jugs as a pair is characteristic of Roman burial practice in the $1^{\text {st }}-2^{\text {nd }}$ centuries $C E^{492}$ and will be discussed in further detail in Section 4.3 and Chapter 7. Whereas most of the 'Jug and Pan' type burials from the material in this thesis as well as in the examples from Nuber's study only contain one pan, the Welshpool group is unusual in that it contains three. It is also unusual that a cauldron is associated with the deposit, as cauldrons are not common among the repertoire of copper alloy vessels deposited as grave goods during the Roman period in Britain. The size of the deposit, as well as the inclusion of both Roman and indigenous artistic elements, suggests association with burial practice from the south east of Britain during the Late Iron Age. ${ }^{493}$

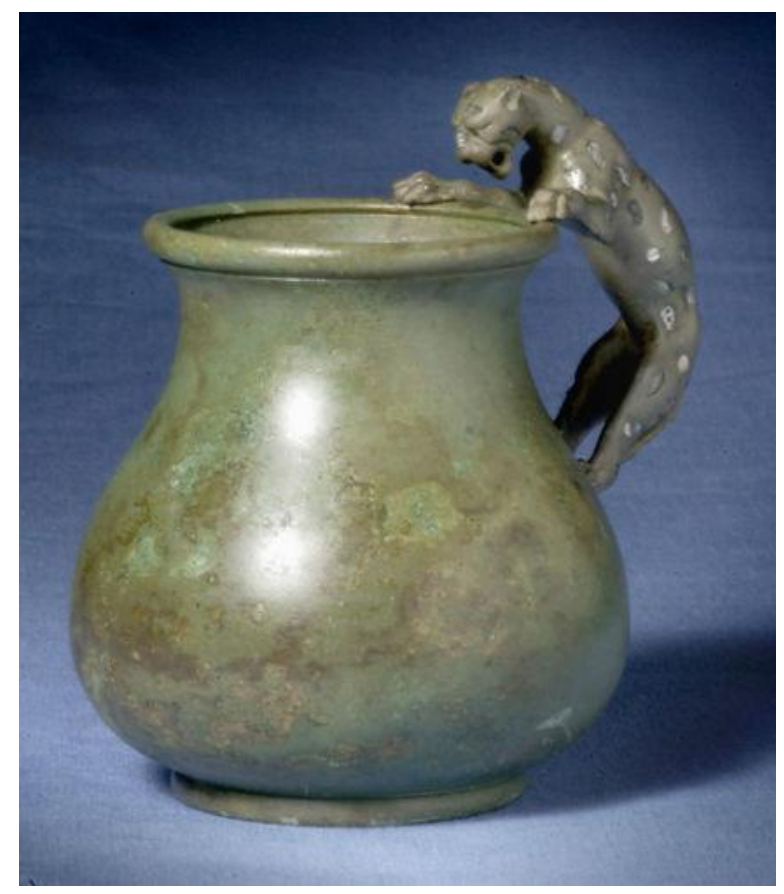

Figure 4.08: Jug from Llantilio Pertholey (PAS NMGW-9A9D16)

The copper alloy vessel recorded through the Portable Antiquities Scheme from Llantilio Pertholey, Monmouthshire is a highly unusual object without parallel in Roman Britain (Figure 4.08). ${ }^{494}$ Excavation undertaken after the find was reported revealed cremated bone and sherds of ceramic associated with it, ${ }^{495}$ clearly identifying it as a Grave Deposit. It is suggested to be of

\footnotetext{
${ }^{492}$ Philpott 1991, 123; Nuber 1973, 144-188.

${ }^{493}$ Boon 1961, 19; Philpott 1991, 123; See later in this section.

${ }^{494}$ MON0007.

${ }^{495}$ PAS NMGW-9A9D16.
} 
$1^{\text {st }}$ century CE Italian manufacture. ${ }^{496}$ This is entirely likely as the body shape is paralleled by examples from Pompeii. ${ }^{497}$ The handle depicts a leopard with its head leaning over the rim of the vessel. The spots of the leopard are ornamented with silver inlay. The handle is closely paralleled by a bronze leopard figurine of uncertain provenance in the collection of the Musée départemental des antiquités de Rouen, ${ }^{498}$ its stance indicating it too was likely a vessel handle. A silver tigress of similar shape found with the Hoxne Hoard provides evidence for the use of this decorative motif stretching to the very end of the Roman period in Britain. ${ }^{499}$ The leopard is associated with Bacchus, which plausibly links this vessel with wine drinking. ${ }^{500}$ The findspot, near the Roman fort at Abergavenny, may also offer some insight into the likely owner of the vessel. $^{501}$

An unusual grave assemblage was discovered at Knob's Crook in Dorset. Beneath a barrow, three cremation pits were found. In Pit I, over 750 torn and heat stressed fragments of copper alloy were discovered. ${ }^{502}$ Unfortunately, the fragments are highly damaged and it is impossible to discern if they came from vessels, furniture fittings, sheet metal or other sources. A single foot ring decorated with concentric circles that coincides with examples from Roman handled pans and basins was discovered, ${ }^{503}$ indicating that at least one copper alloy vessel was interred with the grave. In addition, broken sherds of samian pottery and glass were also discovered in the cremation along with several heat damaged steatite objects. The trepanned disc from a human skull was also included with the cremated bone. It does not appear to itself have been burnt on the pyre ${ }^{504}$ though its presence is otherwise difficult to account for. The samian ware offers a likely early Flavian date to the burial. ${ }^{505}$ This type of large cremation pit would seem to be unusual for south western England and indeed Philpott's survey suggests that inhumation was the preferred form of burial in this region throughout the Roman period, ${ }^{506}$ though cremation is much more common in burials that include copper alloy vessels than

\footnotetext{
496 PAS NMGW-9A9D16.

497 Tassinari 1993 (B1252, B1250, B1261, B1262).

${ }^{498}$ Espérandieu \& Rolland 1959, 63-64 (127).

499 Johns 2010, 62-69 (30).

${ }^{500}$ Carver 2001, 13.

501 Ochota 2013, 104 .

${ }^{502}$ Fowler 1965, 35-36.

${ }^{503}$ DOR0006.

${ }^{504}$ Fowler 1965, 40.

${ }^{505}$ Fowler 1965, 31.

${ }^{506}$ Philpott 1991.
} 
inhumations. ${ }^{507}$ The grave is also unusual for this region in that it contains at least one copper alloy vessel and is further unusual in Britain as the copper alloy material appears to have been burned on the pyre. While damaged fragments of heat stressed copper alloy were found at the Iron Age burials at Folly Lane and King Harry Lane, St. Albans. ${ }^{508}$ From the (admittedly limited) evidence currently available, cremations with copper alloy vessels included as pyre goods are rare in Britain. As such, they may represent intrusive grave practices from elsewhere in the Empire.

The practice of including copper alloy vessels as pyre goods to be ritually destroyed prior to burial was practiced at the Roman cemetery at Brougham in Cumbria, which is the only site in the hinterland of Hadrian's Wall currently known where the deposition of copper alloy vessels in graves appears to have been regularly practiced, though for only roughly a century. ${ }^{509}$ Brougham, Brocavum from the Antonine Itinerary, is located in the western Eden valley at the confluence of the river Eamont and the river Lowther. ${ }^{510}$ Its location appears to have been chosen to guard the main roads that communicate the western portion of Hadrian's Wall with settlements further to the south. ${ }^{511}$ This single cemetery site contributes 20 objects to the data-set from 18 different graves (see Appendix VI). ${ }^{512}$

The site is principally a cremation cemetery and the majority of the vessels buried here appear to have been pyre goods, showing signs of thermal stress and partial melting. ${ }^{513}$ The destruction of copper alloy vessels in this way is highly unusual in Britain. It would appear that the burial customs at Brougham were highly localised and probably associated with a military regiment recruited from a specific geographic location that imported and adapted its own native burial practices. ${ }^{514}$ Before reviewing the diagnostic copper alloy vessels from this cemetery, it is important to note the large quantity of undiagnostic burn copper alloy sheeting that was found in these graves. Undiagnostic copper alloy sheet might originate from caskets, furniture, or vessels. ${ }^{515}$ Several graves from this cemetery which contain undiagnostic copper alloy fragments

\footnotetext{
${ }^{507}$ As discussed further in Sections 4.3 and 4.5.

${ }^{508}$ Niblett 1999, 177-179; Stead \& Rigby 1989, 111.

${ }^{509}$ Cool 2004, 463.

${ }^{510}$ Wilmott 2004, 2-4.

511 Allan 1994, 6.

${ }^{512}$ C0005 was unstratified, but most likely originated from a disturbed grave.

${ }^{513}$ Excluding C0001,C0005 and C00010.

${ }^{514}$ Cool $(2004,464)$ suggests Pannonia, while Philpott $(1991,9)$ suggests Gaul or Germania.

515 Section 2.2.
} 
also contain nails and mounts that may have been fixtures for furniture or chests. This makes it possible that this material did not originate from vessels and that its inclusion would inflate the representation of copper alloy vessels at this site.

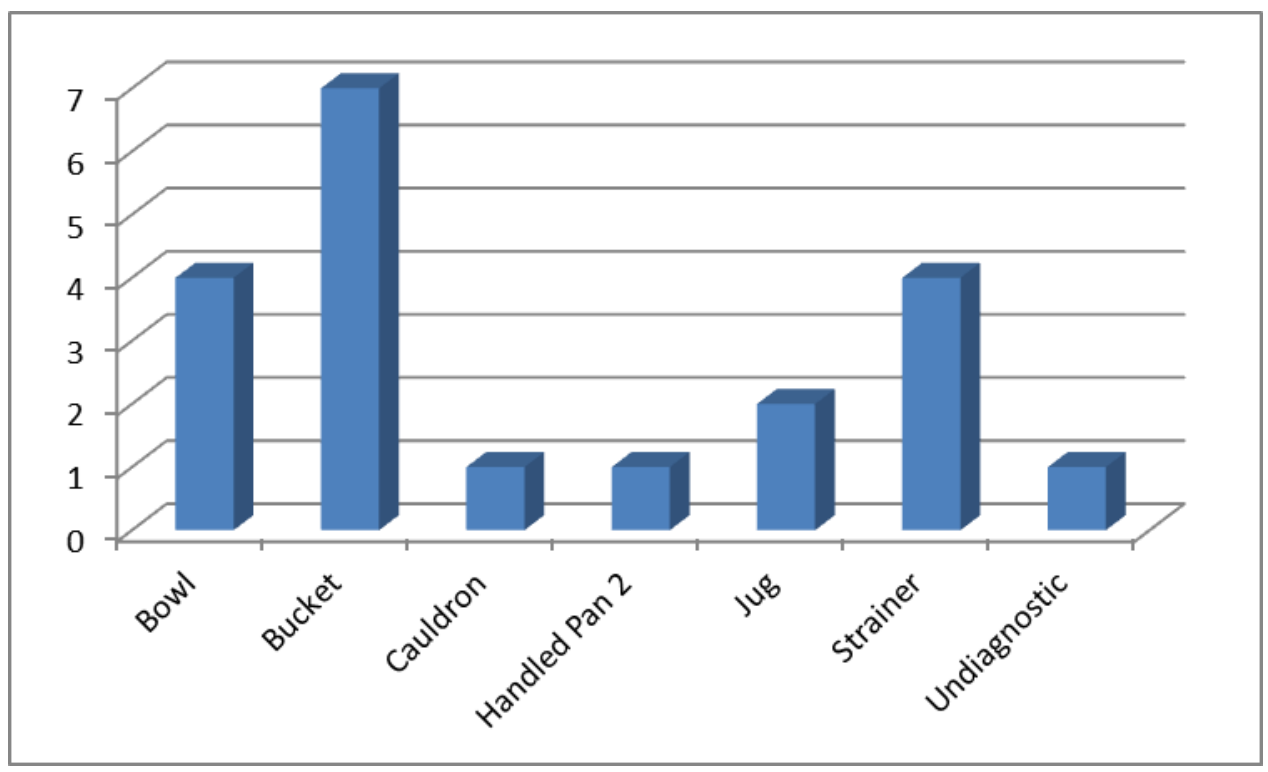

Figure 4.09: Vessel forms in cremation burials at Brougham.

As Figure 4.09 illustrates, buckets are the most common vessel form found in burials at Brougham, followed by bowls and strainers. The vessels were deposited in the cremation burials of both men and women. ${ }^{516}$ The chronology of the cemetery is relatively narrow, with burials taking place between approximately 200-310 CE at the longest estimation of use. ${ }^{517}$ As much of the datable copper alloy vessel material from Structured Deposits and Grave Deposits in Roman Britain is believed to be either chronologically Early or Late Roman, the Brougham assemblage is useful for filling a gap in the data-set and offers clues to what changes in the repertoire of copper alloy vessels may have been occurring during the $3^{\text {rd }}$ century CE. It is worth noting that seven out of twenty of the vessels found at Brougham are buckets, but the only other grave from Roman Britain containing a bucket is from Ramsgate in Kent. ${ }^{518}$ Also noteworthy is the absence of Handled Pans apart from C0001, as these are among the vessels most likely to be interred as grave goods. ${ }^{519}$ This selection of vessel forms would appear to coincide with trends for the $2^{\text {nd }}$

\footnotetext{
${ }^{516}$ Cool 2004, 378-379.

${ }^{517}$ Cool 2004, 463.

${ }^{518}$ K0025; Eggers 1966, 102 (5A); Smith 1922, 93-94.

${ }^{519}$ Nuber 1973, 144-188.
} 
and $3^{\text {rd }}$ centuries evident in the Site Finds and PAS Finds from this thesis. ${ }^{520}$ This indicates that while the burial practice of Brougham may be something of an anomaly for Roman Britain, it may reflect the wider trends in the use of copper alloy vessels during the $3^{\text {rd }}$ century.

The Rudge Cup Type Handled Pan 2 from Grave 107 is an unusual inclusion. This type of Handled Pan 2 is thought to be of British manufacture, ${ }^{521}$ while most of the other copper alloy vessels in this cemetery are probably continental imports. It also is dissimilar to the other vessels chosen for burial in this group of graves, predominantly buckets and bowls. It also does not appear to have been burnt on the pyre, but instead was deposited intact and undamaged by thermal stress. It appears to have been well used at the time of its burial, perhaps as much as 100-150 years old. ${ }^{522}$ This makes it very likely to have been an heirloom piece, perhaps passed down through a military family as this type of vessel would appear to have specific associations with the military and Hadrian's Wall. ${ }^{523}$ In any case, it is clear that it was viewed and treated differently from the other copper alloy vessels used for funerary purposes at Brougham. While it would appear that both a Hemmoor bucket ${ }^{524}$ and a bowl ${ }^{525}$ were also interred in graves without being burned on the pyre, the presence of these forms in and of themselves is therefore not surprising. These objects may represent a variant practice of depositing vessels in graves at Brougham or the objects may simply had been placed too late on the pyre to have become damaged.

The practice of including copper alloy basins as pyre goods seems highly localised and does not appear across Britain more widely, with the isolated exceptions of Ramsgate in Kent and Knobs Crook in Dorset. ${ }^{526}$ Comparison with another cremation cemetery associated with a Roman fort in Cumbria at the site of Beckfoot displays how particular a practice this form of cremation burial was. While the pottery found in the graves at Beckfoot closely matches that of Brougham and dates the use of the site to the $2^{\text {nd }}-3^{\text {rd }}$ century $C E,{ }^{527}$ no identifiable copper alloy vessels have been found at the site either as pyre goods or as post-cremation interments associated with the graves. While some undiagnostic copper alloy fragments were found in a

\footnotetext{
${ }^{520}$ See Chapters 5 and 6.

${ }^{521}$ Moore 1978, 325; Breeze 2012, 108.

522 Cool 2004, 128.

${ }^{523}$ Cool 2004, 128; Breeze 2012, 105-111.

${ }^{524} \mathrm{C} 0005$.

${ }^{525} \mathrm{C} 0010$

${ }^{526} \mathrm{~K} 0025$ \& DOR0006.

${ }^{527}$ Caruana 2004, 143-146.
} 
pyre grave at the site in $1948,{ }^{528}$ it is unclear if this could have belonged to a vessel, furniture fitting or sheet bronze. Unlike Brougham, Beckfoot would appear to be devoid of copper alloy or glass vessels in its cremations. ${ }^{529}$ Low Borrowbridge and Petty Knowes are further examples of cemeteries near Brougham with no evidence for copper alloy vessels in graves, as pyre goods or otherwise. ${ }^{530}$ This comparison makes it clear that the inclusion of copper alloy vessels as pyre goods at Brougham was a practice of an isolated minority group, almost certainly within the military, ${ }^{531}$ in northern England during a limited chronological period.

The only other grave known from the region of Hadrian's Wall to contain a copper alloy vessel comes from Corbridge in Northumberland. It is a small, hexagonal jar with enamelled geometric and floral decoration that was found with a lamp of early $2^{\text {nd }}$ century CE date (Figure 4.10). ${ }^{532}$ Its size, about 8 centimetres tall, makes it an appropriate size for perfume or cosmetic ointments. While other examples of this type of vessel are known from Europe and North Africa, this is the first example found in a datable context and is the first such vessel found in Britain. The cremation which produced this vessel was the richest burial found in a funerary enclosure during work on the Corbridge by-pass in $1974 .{ }^{533}$ Other burials in this group produced ceramics, glass, jewellery and animal bones but no other metal vessels. ${ }^{534}$

It is worth noting that the data-set for the far north of England is limited by the low number of Roman burial sites that have been identified and excavated in the hinterland of Hadrian's Wall in comparison to other areas of Britain. Future exploration and discovery could significantly alter the present data-set and the findings in this thesis. This being said, there are no copper alloy vessels recorded as grave goods among the known and published Roman graves from the rest of northern England. Although absence of evidence is not necessarily evidence of absence, the lack of material may be assumed to be representative. Most of the Roman cemeteries in this region that have been well excavated and documented, such as those in York, ${ }^{535}$ are of Late Roman date and the absence of copper alloy vessels there may be representative more of a chronological shift in burial practice as opposed to regional variation.

\footnotetext{
${ }^{528}$ Caruana 2004, 137.

${ }^{529}$ Caruana 2004, 134-161.

${ }^{530}$ Lambert 1996; Petts 2009, 160-162; Cool 2010, 41-42.

${ }^{531}$ Cool 2004, 463-468; Cool 2010, 27.

${ }^{532}$ Casey \& Hoffmann 1995b, 24 (6); NU0049.

${ }^{533}$ Casey \& Hoffmann 1995b, 17-21.

${ }^{534}$ Casey \& Hoffmann 1995b, 17-44.

535 Ottaway 1993, 120-126 \& 138-140.
} 

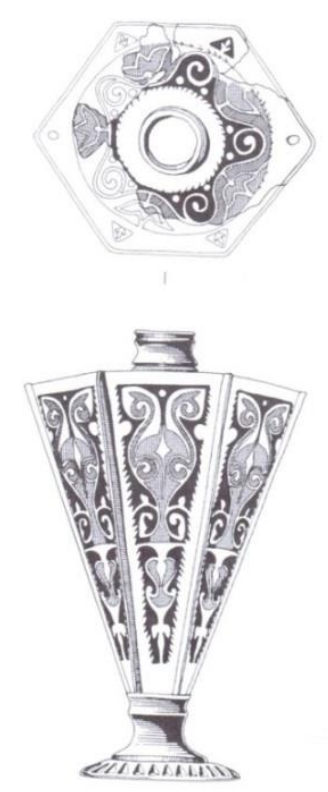

Figure 4.10: Jar from Corbridge (Casey \& Hoffmann 1995a, 24, Fig.3, Scale 1:3)

However, it may be assumed that if the interring of copper alloy vessels was as popular a practice in this region as it was in more southerly regions in the $1^{\text {st }}$ and $2^{\text {nd }}$ centuries $\mathrm{CE},{ }^{536}$ some evidence of this would have come to light. For this reason, it may be currently assumed that copper alloy vessels were not common objects to be included as grave goods in the northern counties of England during the Roman period.

The practice of including copper alloy vessels as grave goods becomes more prevalent in the southern and eastern Midlands, as illustrated in Table 4b. There are no known instances of copper alloy vessels occurring in Romano-British graves from Greater London. This is particularly surprising as London is situated in the southeast, within the broad area of the province that is most prolific in Roman graves with copper alloy vessels. This lack of evidence is certainly not due to lack of excavation, as the Roman cemeteries around London have been subject to considerable attention and it would appear to have a low frequency of grave goods generally throughout the early Roman period. ${ }^{537}$ In any case, London and its immediate surroundings do not appear to have taken part in this particular cultural practice, reflecting a degree of cultural separation between London and the regions surrounding it during the Roman period. London is home to other anomalies in the deposition of copper alloy vessels, being

\footnotetext{
${ }^{536}$ As will be discussed later in this section; Philpott 1991,128-160.

${ }^{537}$ Barber \& Bowsher 2000; Barber \& Hall 2000, 102-120; Swift 2003; Watson 2003.
} 
further indicative of its individual character even during the Roman period and will be discussed further in Chapters 5 and 7.

\begin{tabular}{|c|c|c|}
\hline Findspot/Date & Copper Alloy Vessels & Associated Finds \\
\hline $\begin{array}{l}\text { Stanfordbury Farm, } \\
\text { Bedfordshire (Grave 1) / c. } \\
150 \mathrm{CE}\end{array}$ & $\begin{array}{l}\text { BE0004 (Handled Pan 2) } \\
\text { BE0005 (Jug) }\end{array}$ & \\
\hline $\begin{array}{l}\text { Stanfordbury Farm, } \\
\text { Bedfordshire (Grave 2) / c. } \\
150 \mathrm{CE}\end{array}$ & $\begin{array}{l}\text { BE0006 (Handled Pan 1) } \\
\text { BE0007 (Jug) }\end{array}$ & $\begin{array}{l}2 \text { wine amphorae, } 1 \text { ceramic } \\
\text { bowl }\end{array}$ \\
\hline $\begin{array}{l}\text { Thornborough Barrow, } \\
\text { Buckinghamshire/ } 1^{\text {st }} \mathrm{CE}\end{array}$ & $\begin{array}{l}\text { BUC0001 (Jug) } \\
\text { BUC0002 (Jug) } \\
\text { BUC0003 (Bowl) } \\
\text { BUC0004 (Handled Pan 1) }\end{array}$ & $\begin{array}{l}2 \text { amphorae, } 3 \text { glass bowls, } 1 \\
\text { copper alloy lamp, } 1 \text { wooden } \\
\text { box }\end{array}$ \\
\hline $\begin{array}{l}\text { Huntington Road, Gravel Hill, } \\
\text { Cambridge, Cambridgeshire } \\
\text { (Grave a) } / 1^{\text {st }}-2^{\text {nd }}\end{array}$ & CAM0007 (Vessel) & $\begin{array}{l}4 \text { glass jugs, } 3 \text { stone beads, } 1 \\
\text { ceramic jug }\end{array}$ \\
\hline $\begin{array}{l}\text { Hinchingbrooke, Nun's } \\
\text { Bridge, Godmanchester, } \\
\text { Cambridgeshire } / 1^{\text {st }}-2^{\text {nd }}\end{array}$ & CAM0008 (Jar) & $\begin{array}{l}3 \text { glass bottles, } 1 \text { ceramic } \\
\text { vessel fragment, } 1 \text { silver coin }\end{array}$ \\
\hline $\begin{array}{l}\text { Verulamium, Silchester Gate, } \\
\text { Mayne Avenue, St. Albans, } \\
\text { Hertfordshire (Cremation } \\
\text { Pit)/ } 1^{\text {st }}\end{array}$ & HER0019 (Bowl) & $\begin{array}{l}15 \text { ceramic bowls, } 1 \text { glass } \\
\text { bottle, } 2 \text { glass jugs, } 1 \text { iron } \\
\text { folding chair, } 1 \text { bronze } \\
\text { cosmetic tool, } 4 \text { ceramic } \\
\text { lamps, } 20 \text { glass gaming } \\
\text { counters }\end{array}$ \\
\hline $\begin{array}{l}\text { Ermine Street, Stanton, } \\
\text { Hertfordshire }\left(\text { Site D)/ } 1^{\text {st }}\right.\end{array}$ & HER0030 (Bowl) & 3 ceramic bowls \\
\hline $\begin{array}{l}\text { Turners Hall Farm, } \\
\text { Hertfordshire (Grave 1)/ } 1^{\text {st }}\end{array}$ & $\begin{array}{l}\text { HER0031 (Jug) } \\
\text { HER0032 (Jug) } \\
\text { HER0033 (Jug) } \\
\text { HER0034 (Bowl) } \\
\text { HER0035 (Strainer) } \\
\text { HER0036 (Strainer) } \\
\text { HER0037 (Handled Pan 1) } \\
\text { HER0038 (Handled Pan 2) } \\
\text { HER0039 (Handled Pan 2) } \\
\text { HER0040 (Bowl) }\end{array}$ & $\begin{array}{l}1 \text { copper alloy funnel, } 24 \text { iron } \\
\text { arrowheads, } 6 \text { iron blades, } 3 \\
\text { 'punches', } 2 \text { glass bowl, } 2 \text { glass } \\
\text { hexagonal bottles, } 1 \text { glass jug, } \\
8 \text { ceramic vessels }\end{array}$ \\
\hline $\begin{array}{l}\text { Turners Hall Farm, } \\
\text { Hertfordshire (Grave 2) } / 1^{\text {st }}\end{array}$ & $\begin{array}{l}\text { HER0041 (Jug) } \\
\text { HER0042 (Jug) }\end{array}$ & $\begin{array}{l}1 \text { copper alloy lamp holder, } 5 \\
\text { ceramic bowls, } 2 \text { glass bottles } \\
\text { (one containing cremated } \\
\text { human bone), } 1 \text { glass bowl }\end{array}$ \\
\hline
\end{tabular}

Table 4b: Grave Deposits from the Midlands. 
While over 1,000 graves from the Roman period have been identified in the immediate vicinity of St. Albans, ${ }^{538}$ only one contains a copper alloy vessel. ${ }^{539}$ This gives some indication of how rare the practice of interring copper alloy vessels was, even in the middle of the 'hotspot' area of this depositional practice. The vessel in question is a two handled bowl that comes from an unusually rich cremation burial on Mayne Avenue. ${ }^{540}$ The site is located near the King Harry Lane Cemetery, which is the largest excavated cemetery of ancient St. Albans which has produced some 455 cremation burials and 17 inhumations. ${ }^{541}$ The lamps in this burial bear the maker's mark of Eucarpus and are believed to have been manufactured in Gaul between 75 and $90 \mathrm{CE}$, while the legible stamps from the fineware vessels date from approximately $80-85 \mathrm{CE} .^{542}$ The bowl's handles curve over the side of the basin and connect at the inside of the rim. Both handles have a female bust under a leaf shaped thumb-rest and are decorated with the heads of aquatic birds, possibly swans, along the grip of the rim. The body of the vessel is lathe-finished and has a concentric circle foot-ring. Vessels of similar form have been found at Pompeii, ${ }^{543}$ and the high quality of manufacture for this vessel suggests probable continental import. ${ }^{54}$ The late $1^{\text {st }}$ century CE date offered by the Pompeian examples coincides with the lamp and ceramic evidence from this grave.

A bowl, probably originally tinned and of late $1^{\text {st }}$ century CE date, was discovered in association with three Samian vessels of Neronian-Flavian date during work on Ermine Street in the parish of Stanton, Hertfordshire. ${ }^{545}$ This assemblage most likely originates from a grave, as it was found in association with Iron Age burials. ${ }^{546}$ Also, the evidence presented in this thesis indicates that ceramic vessels are not commonly found grouped with copper alloy vessels in Structured Deposits, ${ }^{547}$ while they often accompany them in burials, also offering credence to the claim that this is a burial deposit. The bowl itself is undecorated and lathe-finished with an outturned rim. A similar bowl is also among the objects found at Turner's Hall Farm, discussed below.

\footnotetext{
${ }^{538}$ Niblett 2000, 97.

${ }^{539}$ HER0019.

${ }^{540}$ Niblett \& Reeves 1990, 441.

${ }^{541}$ Niblett \& Thompson 2005, 36.

${ }^{542}$ Niblett \& Reeves 1990, 444.

543 Tassinari 1993, S3110.

${ }^{544}$ Niblett \& Reeves 1990, 444.

${ }^{545}$ Potter \& Trow 1988, 58-60.

${ }^{546}$ Potter \& Trow 1988, 60.

${ }^{547}$ See Chapter 3.
} 


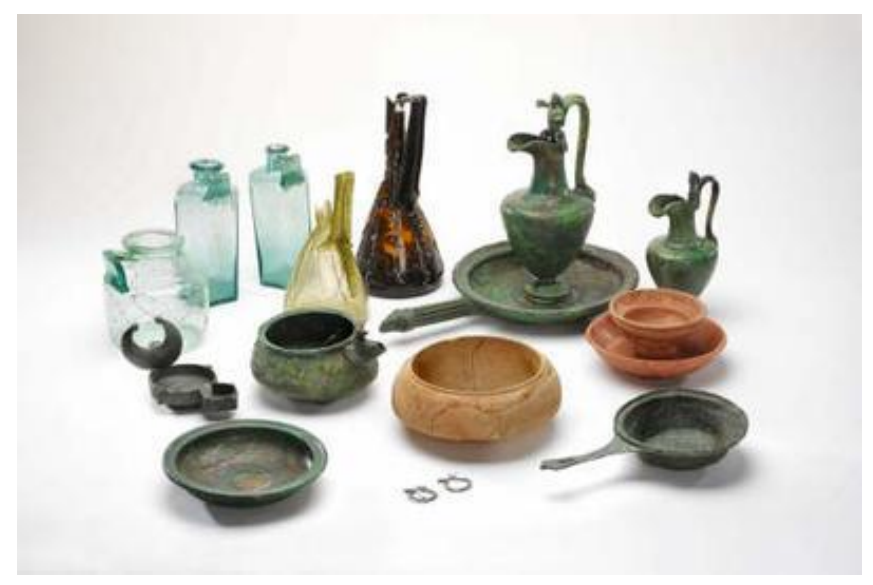

Figure 4.11: Objects from graves at Turners Hall Farm (C Verulamium Museum)

Two lavishly furnished graves of Roman date containing copper alloy vessels were discovered by metal-detectorists at Turner's Hall Farm in Harpenden, Hertfordshire in 2002 (Figure 4.11). ${ }^{548}$ After subsequent excavation, both graves' occupants proved to be female according to the osteological evidence, Grave 1's occupant aged 20-45 and Grave 2's occupant aged $35-50 .{ }^{549}$ Of the two burials, Grave 1 was by far the more lavish in regards both to the number of objects as well as the iconographic complexity of the objects' decoration. A jug of Egger's type 127 from Grave 1 has a particularly elaborate statuette depicting a Triton at the crest of the handle at the thumb rest, overlooking the rim (Figure 4.12). ${ }^{550} \mathrm{He}$ holds two objects that appear to be a jug and a pan, though corrosion makes their exact identification difficult. The handle terminates in a Gorgon head medallion. The Triton has obvious aquatic associations, whose possible symbolic implications were mentioned in Chapter 3 and will be treated in Section 4.4.

\footnotetext{
${ }^{548}$ West 2005, 14.

${ }^{549}$ Ibid.

${ }^{550}$ HER0031.
} 


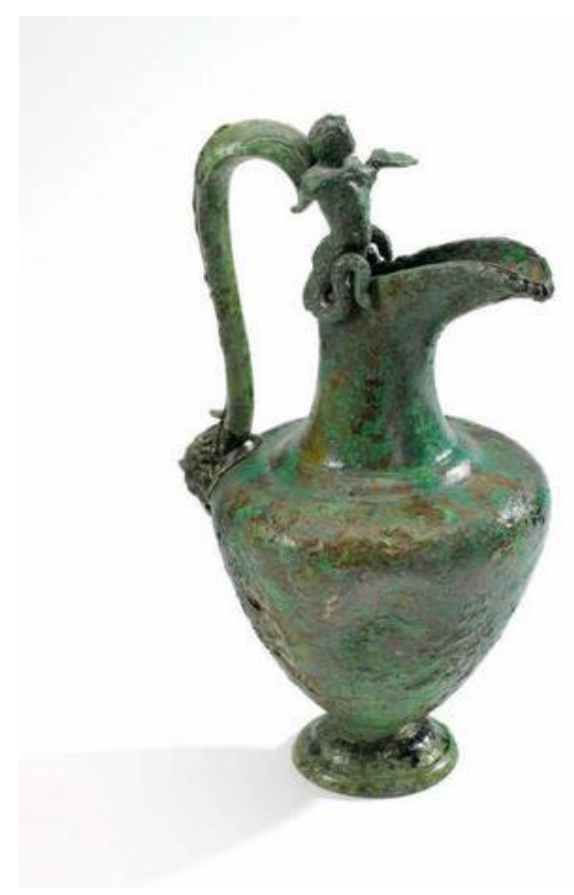

Figure 4.12: Jug with Triton from Grave 1 at Turners Hall Farm (C Verulamium Museum)

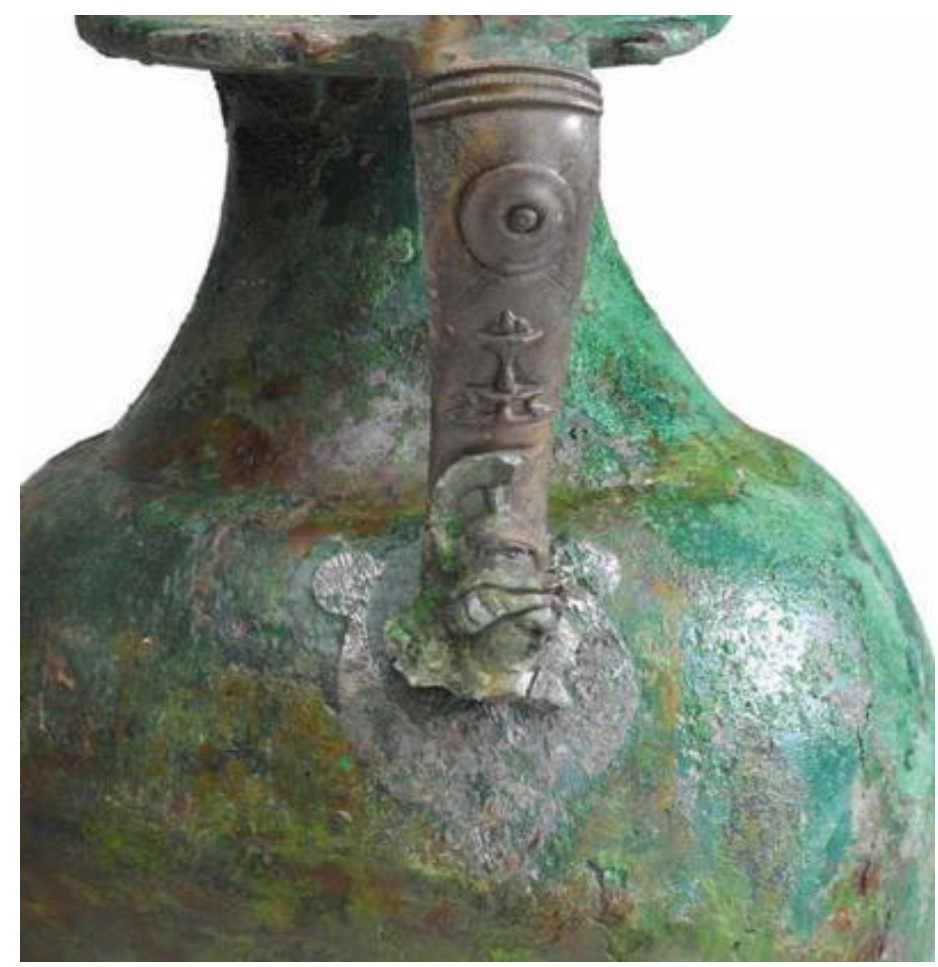

Figure 4.13: Jug with Minerva from Grave 1 at Turners Hall Farm (@ Verulamium Museum) 
The other jug from this grave has a handle medallion depicting the helmeted head of the goddess Minerva (Figure 4.13). ${ }^{551}$ Above the bust of Minerva is a three footed table with a basket of fruit atop it and a round shield in front of two crossed spears in a register above that. Minerva's association with warfare could be connected to the blades and arrow heads also found in the grave.

The Handled Pan 1 from Grave 1 is of the fluted handle and ram's head handle terminal design prevalent in graves from Britain and the continent. ${ }^{552}$ The remains of two Handle Pan 2's of Eggers type 150 (c.f.) are also present in this grave group, as is a wide rimmed bowl. The strainer-bowl from this grave is of a globular design similar to those from the Structured Deposit at Kingston Deverill. ${ }^{553}$ It has three pelta shaped feet at its base and its spout is of uncertain zoomorphic design, depicting an uncertain animal with large ears or horns. It is possible that it depicts a bull, a common decorative motif for metal vessels in Iron Age and Roman Britain (Figure 4.14, detail of zoomorphic mount Figure 6.17). ${ }^{554}$
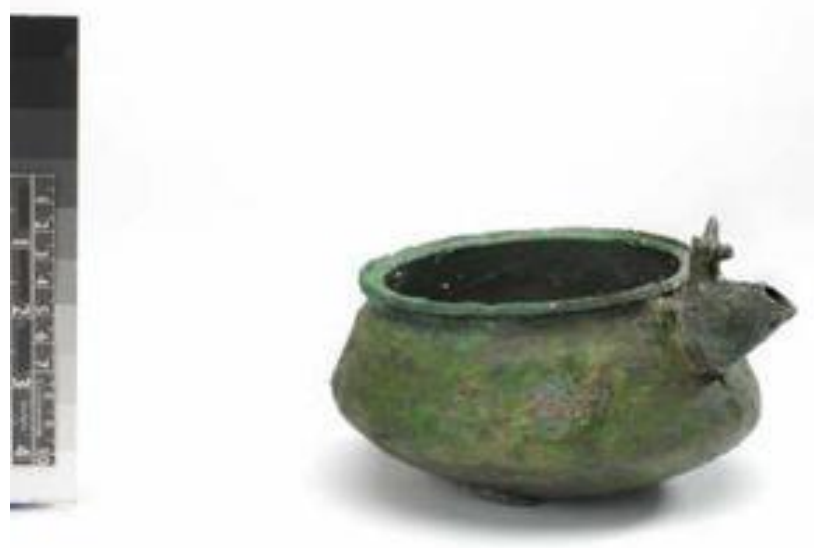

Figure 4.14: Strainer bowl from Grave 1 at Turners Hall Farm (C Verulamium Museum)

\footnotetext{
${ }^{551}$ HER0041.

552 Nuber $1973,38-73$.

${ }^{553}$ PAS WILT-92B052; WIL0007-WIL0008.

${ }^{554}$ Hawkes 1951; See Section 8.3.
} 
Grave 2 from Turner's Hall Farm, though not as lavish as Grave 1, was still a rich burial. One jug from this grave is an Eggers type 127 that has a highly decorative handle,${ }^{555}$ with a bull protome at the top of the handle at the thumb rest looking over the rim of the vessel, its front legs framing the rim. The jug is very similar in form to the example from Grave 1, though slightly smaller. The position and posture of the bull resembles the depiction of a horse on a jug from Martyrs Field Road in Canterbury, also found in a grave. ${ }^{556}$ The other jug is of a globular design similar to Eggers type 122 and has a simpler floral leaf decoration on its handle medallion. The quantity of objects from the Turner's Hall Farm burials and the fact that they include weapons in the burial of women has direct ramifications in how gender roles were perceived and negotiated during the Roman period in Britain. Examples such as these graves and that of the 'Catterick transvestite ${ }^{557}$ indicate that such roles were not as rigid or as predictable as was once believed. Additionally, it is possible that grave goods may not be tied to the gender of the deceased, but may also have played a symbolic function in the grave furniture.

A curious group of jugs comes from graves found near Hauxton in southern Cambridgeshire during the $19^{\text {th }}$ century. Unfortunately, the records are incomplete and it is uncertain if all of the jugs come from a single or from different burials. They are associated with two glass bowls and two glass jugs that are dated to between 150-250 CE and believed to have been manufactured in the Rhineland, perhaps specifically Cologne. ${ }^{558}$ The copper alloy vessel types are of varying date of manufacture, spanning the $1^{\text {st }}$ to $3^{\text {rd }}$ centuries CE. This discrepancy in date does not prove that the vessels come from different graves, as copper alloy vessels had long use-lives ${ }^{559}$ and vessels manufactured in different centuries are commonly found together in the same Structured Deposits. ${ }^{560}$ The absence of associated Handled Pan 1's, commonly found with them in graves is noteworthy. ${ }^{561}$ The two glass bowls associated with the jugs may have served the function of a Handled Pan 1, as glass replaced copper alloy as a material for this combination in Pompeii ${ }^{562}$ as well as in Grave 2 from Harpendon. ${ }^{563}$ The jugs are all in

\footnotetext{
555 HER0032.

${ }^{556}$ Eggers 1966, 102 (4); Philpott 1991, 314; K0012.

${ }^{557}$ Cool 2002 41-42; Pinto \& Pinto 2013, 169-179.

${ }_{558}^{558}$ Liversidge 1977, 29.

${ }^{559}$ See Chapter 2.

${ }^{560}$ See Chapter 3.

${ }^{561}$ Nuber 1973, 144-188.

${ }^{562}$ Nuber 1973, 75-77.

${ }^{563}$ HER0041-HER0042; Burnham et al. 2003, 327.
} 
relatively good condition, and it is suggested that they were interred within a wooden box in a barrow or similar group type tomb structure that has since been destroyed. ${ }^{564}$ Though it is possible that some organic objects such as food stuffs or other grave offerings were unrecorded upon excavation, it is safe to assume that something as substantial as wine amphorae or samianware would have been recorded if they had been found. Unfortunately, as it is impossible to say if the group originates from one or more graves, it is impossible to scrutinise the possible relation to specific grave assemblages much further.

During excavation of Huntington Road at Gravel Hill in Cambridge, a shallow copper alloy bowl was found associated with the inhumation grave of a woman along with four glass flasks, two ceramic vessels and three jet ornaments. ${ }^{565}$ Unfortunately, the copper alloy vessel is no longer extant and its contextual association with the other objects is unclear. The grave was unusual as it was an inhumation, while most of the Romano-British graves in the area were cremations. ${ }^{566}$ Cremation also seems to have been the preferred method of burial practiced by those who chose to include copper alloy vessels in their grave furnishings in Roman Britain. ${ }^{567}$ However, another inhumation burial of Roman date containing a copper alloy handled jar decorated with three anthropomorphic bearded male heads was found during road construction near Nun's Bridge in the parish of Godmanchester in $1967 .{ }^{568}$ The faces on the Godmanchester jar most likely represent satyrs. ${ }^{569}$ A coin dating to circa $180 \mathrm{CE}$ was found in the mouth of the interred individual $^{570}$ giving a terminus post quam.

A large grave assemblage was found in a barrow type grave in Thornborough, Buckinghamshire in the early part of the $19^{\text {th }}$ century. ${ }^{571}$ It included two amphorae, ${ }^{572}$ three glass bottles (one of which contained the cremated remains), a copper alloy lamp with a crescent moon shaped flame guard as well as four copper alloy vessels. ${ }^{573}$ The vessels included two jugs, a shallow bowl and a Handled Pan 1. One of the jugs has an uncertain anthropomorphic figure on

\footnotetext{
${ }^{564}$ Liversidge 1977, 29.

${ }^{565}$ Liversidge 1977, 15-16; Philpott 1991, 325.

${ }^{566}$ Liversidge 1977, 15; Philpott 1991, 217-224.

${ }^{567}$ As will be discussed later in this chapter .

${ }^{568}$ Wilson 1968, 191 (5); Liversidge 1977, 24-25; Philpott 1991, 332.

${ }^{569}$ Liversidge 1977, 25.

${ }^{570}$ Wilson 1968, 191.

${ }^{571}$ Liversidge 1953, 29-32; Eggers 1968, 110 (89a); Philpott 1991, 261.

572 One for wine and one for olive oil.

573 BUC0001-BUC0004.
} 
its handle medallion, perhaps a Cupid (Figure 4.15). ${ }^{574}$ The second jug is a trefoil Eggers type 125 and has a lion's head thumb rest looking over the rim of the vessel with a lion's paw for the handle medallion (Figure 4.16). ${ }^{575}$
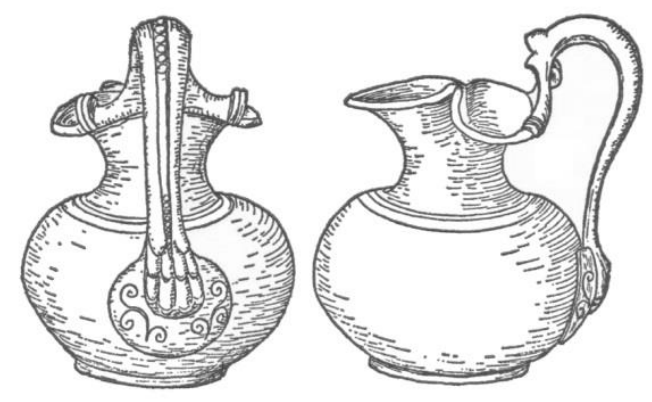

Figure 4.15: Jug from Thornborough (Eggers 1968, 138 Abb.38a, Scale 1:5)
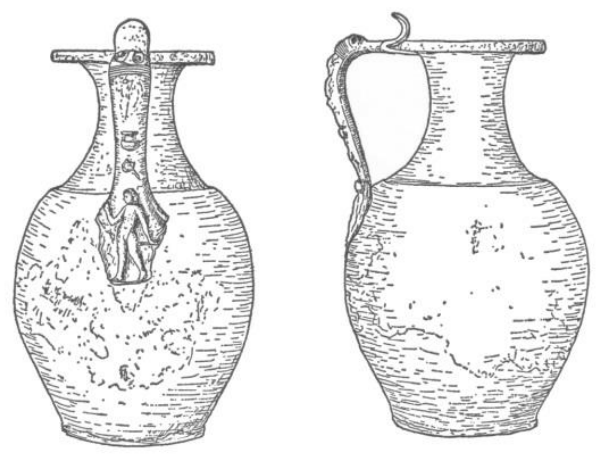

Figure 4.16: Jug from Thornborough (Eggers 1968, 138 Abb.38b, Scale 1:5)

The Handled Pan 1 terminates in the zoomorphic head of a canine or ursine creature. ${ }^{576}$ The bowl was likely used for ablutions ${ }^{577}$ and was almost certainly paired with one of the jugs in this assemblage for precisely that purpose. Several ceramic vessels of Antonine date are present in the grave group. The objects were located on a limestone shelf at the approximate Roman period ground level next to what is believed to be the pyre and were covered with a wooden

\footnotetext{
${ }^{574}$ BUC0002; Liversidge 1953, 30.

575 BUC0001; Liversidge 1953, 31.

${ }^{576}$ BUC0004.

${ }^{577}$ Allison 2004, 54-55.
} 
frame or box, of which some of the planks survived at the time of excavation. The grave itself is of $2^{\text {nd }}$ century date and was likely a site of family reverence for the owners of the property. ${ }^{578}$ The Thornborough group is diverse and iconographically rich, recalling assemblages from Turners Hall Farm and Bartlow Hills in Ashdon, Essex. ${ }^{579}$

Two graves of Roman date containing copper alloy vessels were found in Stanfordbury in Shefford, Bedfordshire. ${ }^{580}$ They both contained a single handled pan and a single jug. While the Handled Pan 1 from Grave 2 decorated with a ram's head terminal is commonplace among copper alloy grave vessels, ${ }^{581}$ the Handled Pan 2 in Grave 1 is more unusual as a grave inclusion, though Handled Pan $2 \mathrm{~s}$ are recorded in a few other graves in Britain. ${ }^{582}$ The main functional difference between these forms is the depth of the basin, Handled Pan 2s being significantly deeper. It would be likely that the Handled Pan 2 was interred with the same functional intention as the Handled Pan 1 in Grave 2, though this does not appear to be a common substitution. The caduceus depicted on the handle of the Handled Pan 2 from Stanfordbury is also worth brief mention here. The caduceus is associated with both Bacchus and Aesculapius. While these two gods had arguably very different functions, both are gods who perished and were resurrected and could therefore offer comforting associations in the context of funerary ritual. The presence of Bacchic iconography in other graves and the apparent absence of Aesculapian iconography would suggest that this caduceus could be considered Bacchic in this instance, though there still remains room for varying interpretation. The caduceus is also an attribute of Mercury, ${ }^{583}$ whose relevance to funerary ritual will be discussed in Section 4.4.

The modern county of Essex is home to a large number of early Roman period graves that contain copper alloy vessels (Appendix VII). Excavations at Stansted Airport revealed a rural settlement that saw near continual occupation from the Iron Age to the post-Medieval period. ${ }^{584}$ This included an enclosed settlement area of several round-houses and a cemetery that was in use during the Late Iron Age and early Roman periods. ${ }^{585}$ Cremation burial 25 from the Stansted Airport excavations proved to be particularly rich (Figure 4.17). The cremated remains

\footnotetext{
${ }_{578}^{579}$ Liversidge 1953, 29-32.

${ }^{579}$ Gage 1832, 1-23; Philpott, 1991, 256; Eckardt 2009, 65-98.

${ }_{581}^{580}$ Eggers 1968 p. 104-105 (29Aa-29Ab).

${ }^{581}$ Section 4.4.

${ }^{582}$ Such as at Stansted (EX0018) and Colchester (EX0034).

${ }^{583}$ Adkins \& Adkins 1996, 152.

${ }^{584}$ Havis and Brooks 2004, xiii.

${ }^{585}$ Havis and Brooks 2004, 79-254.
} 
were laid on a pewter platter surrounded by five copper alloy vessels, five glass vessels, five samian vessels, a small 'carrot' amphora, and various iron objects such as tools and nails. ${ }^{586}$

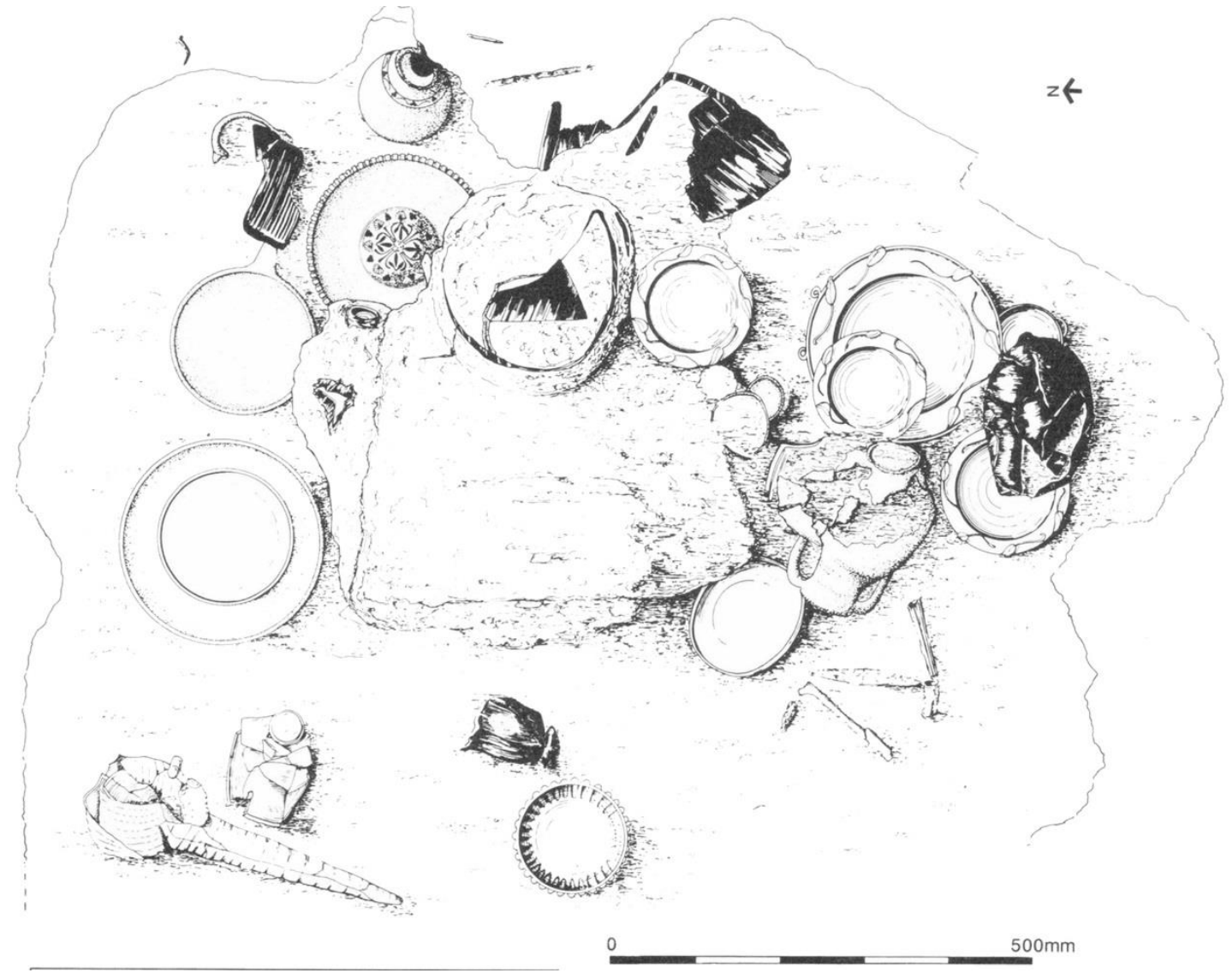

Figure 4.17: Cremation 25 from Stansted (Havis \& Brooks 2004, 217 Fig.145)

The copper alloy vessels consist of two jugs, a Handled Pan 1, a Handled Pan 2, and a bowl. Two of the vessels, one of the jugs and the Handled Pan 1, ${ }^{587}$ are richly decorated. The jug is a trefoil Eggers type 125 and has a handle medallion depicting the face of a young man with pointed ears beneath an eagle atop a globe and a basket of fruit (Figure 4.18). The thumb rest of the handle is in the shape of a thumb and the handle connects to the rim with a frame depicting the legs and hooves of a deer. The youth depicted on the handle medallion may be a satyr and the eagle likely represents Jupiter. ${ }^{588}$ The body has a band of floral leaves around the neck which resemble palms with scalloped embellishments of inlaid silver and the body terminates with a concentric circle foot ring. Jugs with ornamented handles and with thumb rests

\footnotetext{
${ }^{586}$ Havis \& Brooks 2004, 216.

${ }^{587}$ EX0016 \& EX0017.

${ }^{588}$ Havis \& Brooks 2004, 216.
} 
in the shape of thumbs are well represented in the Pompeian destruction layers ${ }^{589}$ and are found in several graves across the continent of Flavian and Antonine date. ${ }^{590}$

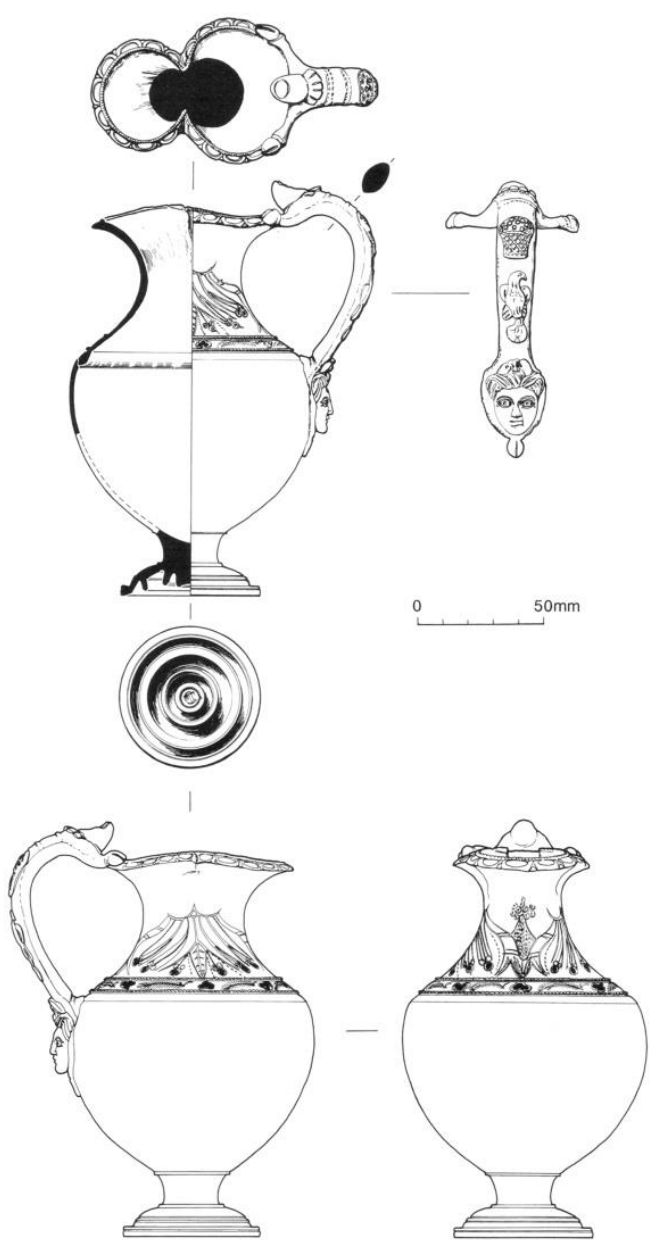

Figure 4.18: Jug from Cremation 25 at Stansted (Havis \& Brooks 2004, 218 Fig.146)

Both of the handled pans were found near the trefoil jug on the north side of the pewter platter and are likely to be associated with it. The Handled Pan 1 has a raised umbo with palm branches inlaid with silver, a scalloped rim and a concentric circle foot ring (Figure 4.19). The handle medallion is the head of either a bear or a dog, with a similar creature peering over the rim of the vessel at the opposite end of the handle. There also appear to be dolphin tails on the damaged undercarriage of the handle connecting with the vessel. The Handled Pan 2 is

${ }^{589}$ Tassinari B1222.
${ }^{590}$ Havis \& Brooks 2004, 216. 
undecorated and has a key-hole shaped handle loop, and resembles the Handled Pan 2s from the Grave 1 at Turners Hall Farm in Harpenden (Figure 4.20). ${ }^{591}$

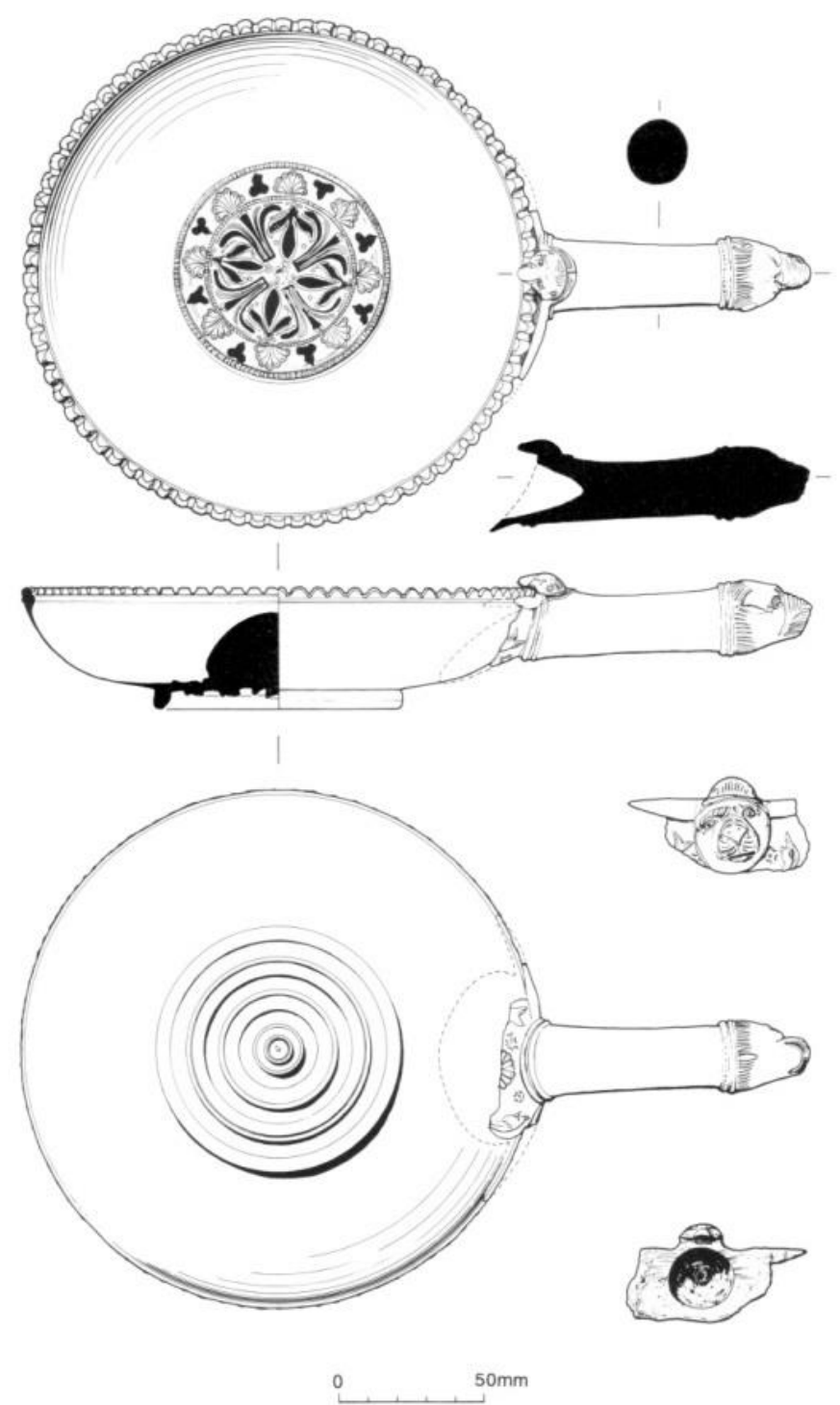

Figure 4.19: Handled Pan 1 from Cremation 25 at Stansted (Havis \& Brooks 2004, 219 Fig.147)

\footnotetext{
${ }^{591}$ Section 4.5; HER0031-HER0040.
} 


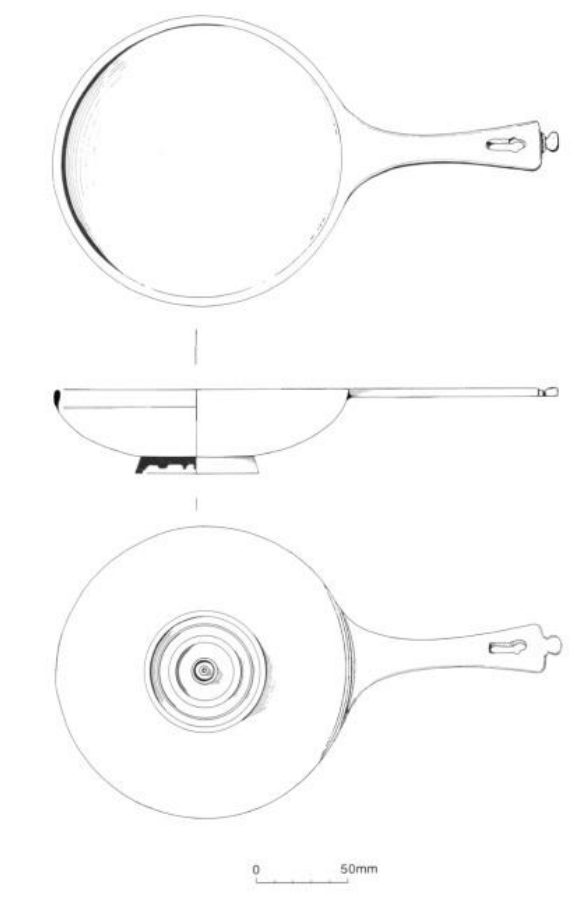

Figure 4.20: Handled Pan 2 from Cremation 25 at Stansted

(Havis \& Brooks 2004, 220 Fig.148)

A two handled jug of Eggers type 129 and the remains of a bowl were found near each other on the south side of the pewter platter. They both were undecorated and perhaps are functionally associated with the samian ware bowls of various sizes around them. It is likely that the copper alloy finds on the north side of the platter represent objects for ablutions and the objects on the south side of the platter are associated with wine drinking, though it would seem that all of these vessels may be associated with the dining table to a greater or lesser extent.

Cremation 24 also had the remains of a copper alloy vessel with a concentric circle foot ring, but refashioned to be used as the lock-plate for a chest (Figure 4.21). It was roughly refitted to the purpose and suggests it was the result of emergency repair. ${ }^{592}$ It could also represent a scarcity of material or a prohibitive expense for professional refashioning of the object, though the grave assemblage suggests that the occupant was not without means. Whatever motivated this alteration, it is a useful example of how this material could be reused and refashioned over its use-life.

${ }^{592}$ Havis \& Brooks 2004, 215-216. 


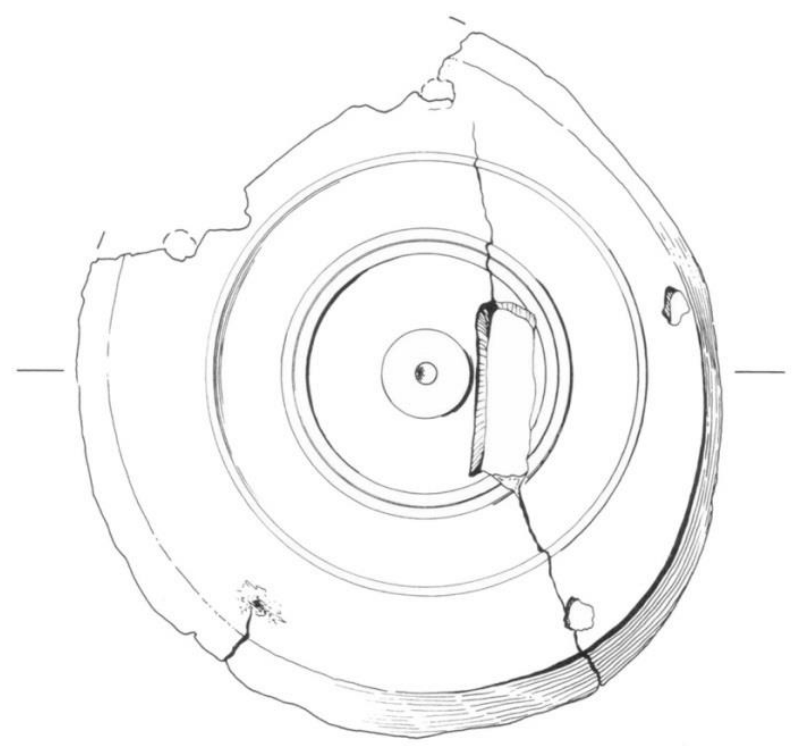

Figure 4.21: Copper alloy vessel refitted as lock-plate from Cremation 24 at Stansted (Havis \& Brooks 2004, 215 Fig.144a, Scale 1:3)

One of the most curious vessels to be included in this thesis is from a disturbed grave context discovered by metal detectorists in Elsenham near Stansted in Essex. It is a small hexagonal jar or pyxis with chequer-boarded enamelling that is unparalleled in Britain and the first to be excavated from a datable context anywhere (Figure 4.22). ${ }^{593}$

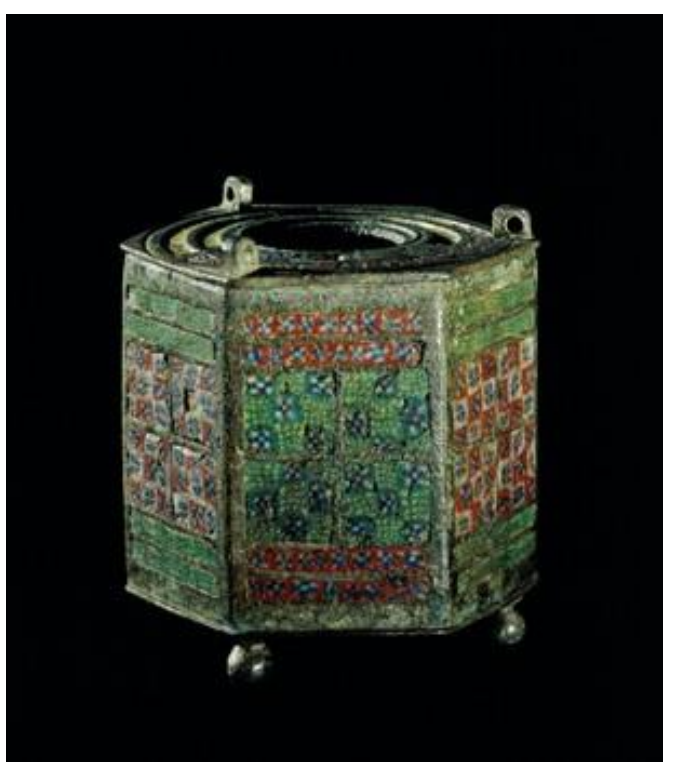

Figure 4.22: Jar from Elsenham (ㄷ the British Museum)

${ }^{593}$ Johns 1993, 161-163; BM 1991,1201.1. 
Its exact use is not precisely known. The other objects found with it in the grave not immediately shedding light on its function. This object belongs to a tradition of enamelled vessel manufacture in Britain during the Roman period that included the Rudge Cup type vessels. ${ }^{594}$ While it has been suggested that such vessels would have served as inkwells, ${ }^{595}$ a theory supported by ceramic inkwells found in burials such as one at Stanway, ${ }^{596}$ it may have served as a cosmetic container and would have had similar use to an enamelled vessel from a Corbridge grave. ${ }^{597}$ These two burials remain unusual for the inclusion of these small vessels. It is possible they served similar functions to glass unguent bottles known from Roman grave contexts for purifying ointment offered to the deceased, ${ }^{598}$ including what would likely have been a higher status object than glass as part of the interment, but otherwise fitting the same ritual function.

The Stanway burial site near Colchester is in a Conquest Period cemetery that is useful in understanding the development and continuity of burial ritual in this part of England in the early Roman period. The use of the site as a cremation cemetery began in the century before the Roman conquest and went out of use before the end of the $1^{\text {st }}$ century $\mathrm{CE} .^{599}$ Of interest to the current thesis are the 'Warrior's Burial' and the 'Doctor's Burial', both of which are rich assemblages containing copper alloy vessels.

The 'Warrior's Burial' is so named for the presence of a spear and shield fragments in the burial. It is a rich assemblage that also contains 15 ceramics of various forms including platters, jugs, and cups, the remains of a Dressel 2-4 amphora, box fittings and a game board with 20 glass gaming pieces, and a Handled Pan 1 with a zoomorphic ram's head handle medallion placed next to a trefoil mouthed jug of Eggers type 125 (Figure 4.23 and 4.24). ${ }^{600}$ The placement of the Handled Pan 1 and jug next to the ceramic plates in the assemblage indicates its association with dining, almost certainly for hand washing. ${ }^{601}$ Both the forms and decorative schemes are well attested at Pompeii ${ }^{602}$ and are likely of Italian manufacture. ${ }^{603}$

\footnotetext{
${ }^{594}$ See Breeze (ed) 2012 for discussion of these objects.

595 Johns 1993, 163.

${ }^{596}$ Crummy et al. 2007, 197-201.

${ }^{597}$ Casey \& Hoffman, 1995b, 24 (6); NU0049.

${ }^{598}$ De Santis 2000, 238-243.

${ }^{599}$ Crummy et al. 2007, 10-13.

${ }^{600}$ Crummt et al. 2007, 170-196.

${ }^{601}$ Crummy et a. $2007,171$.

602 Tassinari 1993 (H2311 \& D2300).

${ }^{603}$ Nuber 1973, 46; Crummy et al. 2007, 185.
} 


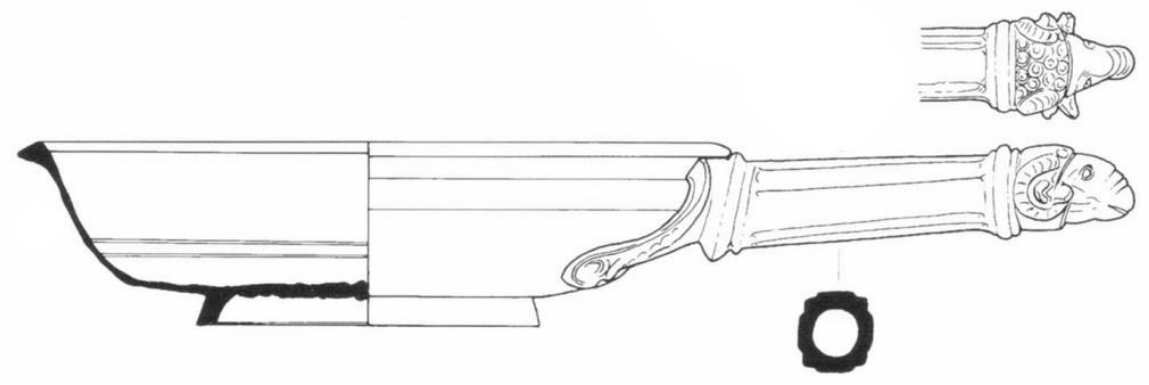

Figure 4.23: Handled Pan 1 from Warrior's Burial at Stanway (Crummy et al. 2007, 185 Fig.87, Scale 1:3)

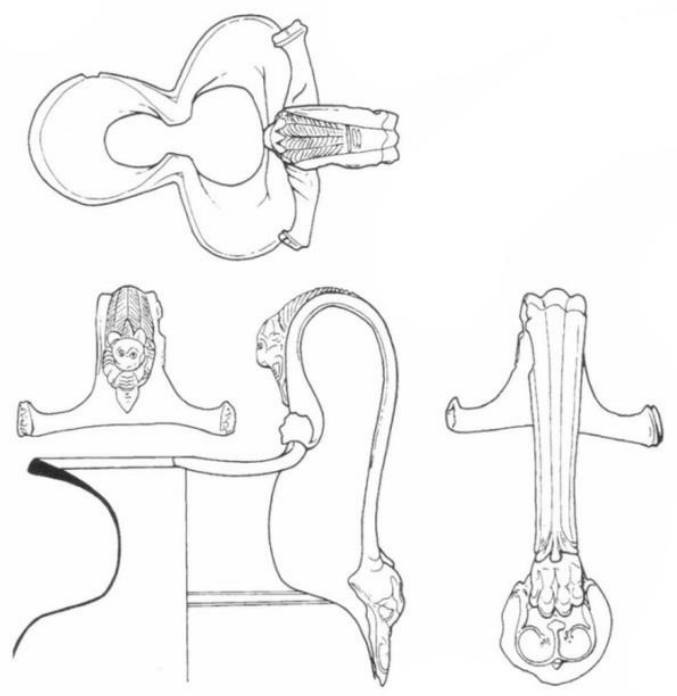

Figure 4.24: Jug from Warrior's Burial at Stanway (Crummy et al. 2007, 185 Fig.87, Scale 1:3)

The sex of this cremation is unknown. ${ }^{604}$ While one may like to assume that martial elements are indicative of a male warrior elite, the female cremation interred with weapons at Turner's Hall Farm is a reminder that it is unwise to make such assumptions. ${ }^{605}$ Whilst the jug and Handled Pan 1 combination found in the 'Warrior's Burial' is well attested in Britain and elsewhere, ${ }^{606}$ the combination of a Handled Pan 2 with a strainer bowl in the 'Doctor's Burial' is more unusual (Figure 4.25). The strainer bowl is similar to those found in one of the Turner's

\footnotetext{
${ }^{604}$ May 2007, 377.

${ }^{605}$ HER0031-HER0042; Burnham et al. 2003, 327; West 2005, 14.

${ }^{606}$ Nuber 1973, 210-220.
} 
Hall Farm burials ${ }^{607}$ as well as from the Structured Deposit from Kingston Deverill, where this vessel type was also paired with Handled Pan 2s. ${ }^{608}$ It would seem that the strainer bowl and most of the ceramics in the grave constitute a drinking set, or perhaps used for mixing medical and/or ritual elixirs; this is supported by the remains of artemisia in the spout of the strainer, indicating it was used as an infuser for a tea-like beverage. ${ }^{609}$ The presence of a Dressel 8 amphora, used for Spanish fish sauce,${ }^{610}$ displays the consumption of imported luxury items as well as a taste for Mediterranean flavouring. The Handled Pan 2 was found next to a ceramic jug, suggesting association between these two vessels and perhaps imitating the more common pairing of Handled Pan 1s with jugs.

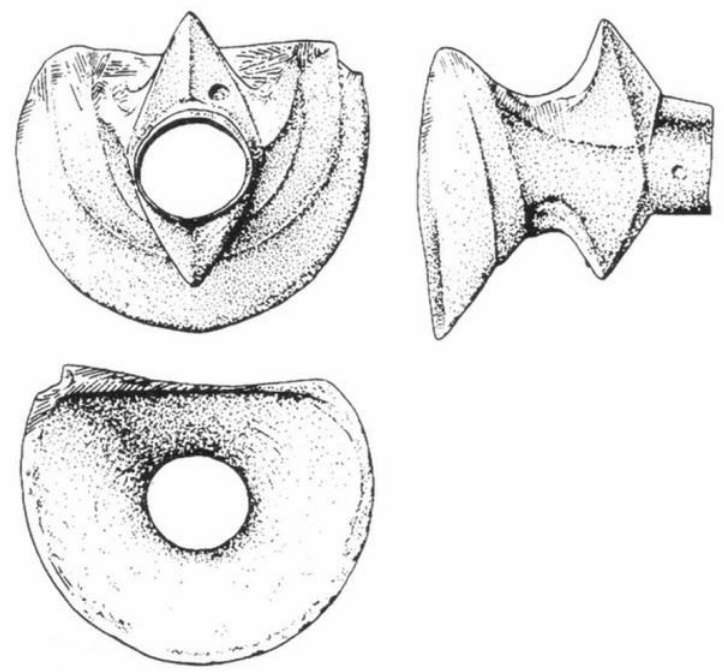

Figure 4.25: Detail from strainer bowl in Doctor's Burial at Stanway (Crummy et al. 2007, 222 Fig.113, app. life size)

\footnotetext{
${ }^{607}$ HER0036.

${ }^{608}$ WIL0007-WIL0008; PAS WILT-92B052.

${ }^{609}$ Carver 2001, 33; Crummy et al. 2007, 207.

${ }^{610}$ Peacock \& Williams 1991, 117-119; Peña 2007, 109.
} 


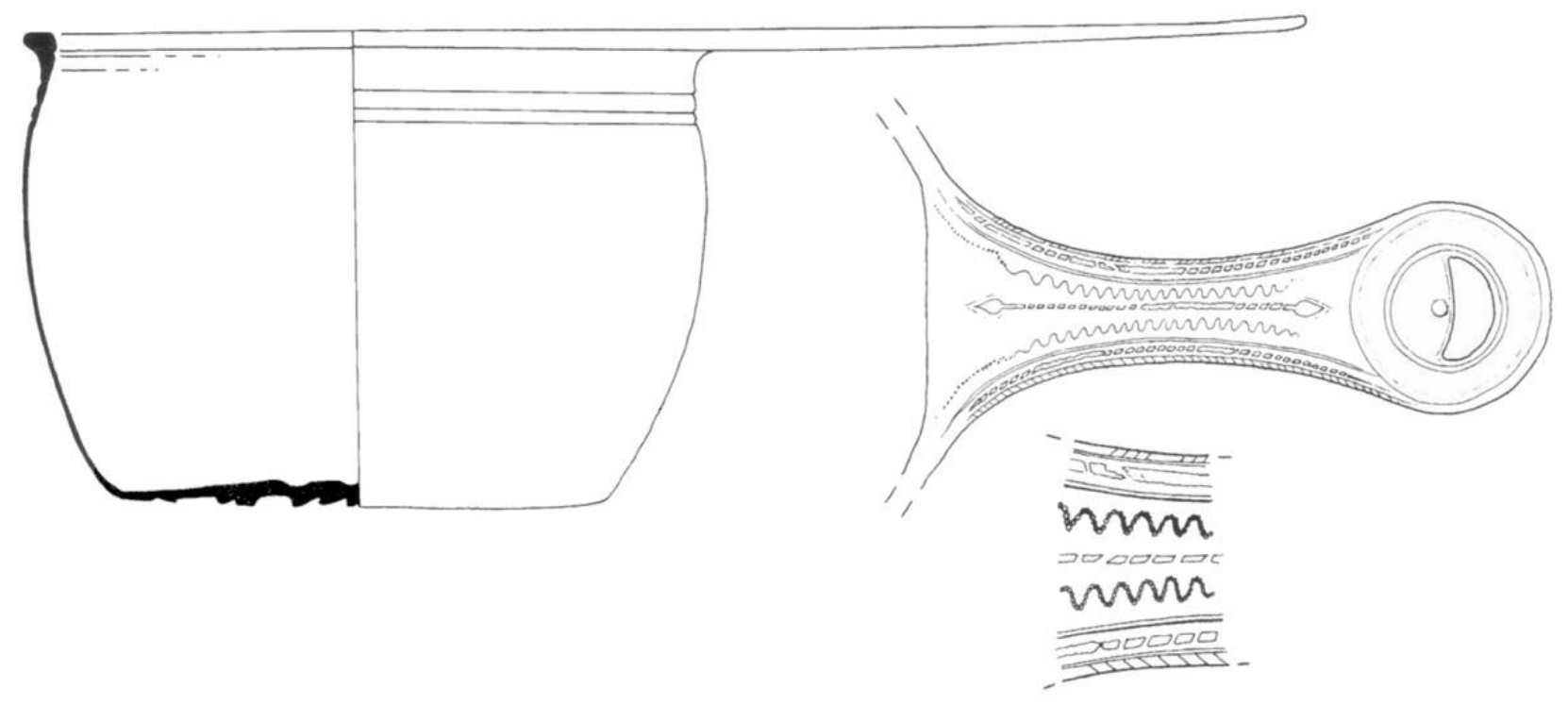

Figure 4.26: Handled Pan 2 from Doctor's Burial at Stanway

(Crummy et al. 2007, 221 Fig.112, Scale 1:2)

The Handled Pan 2 is decorated with a caduceus on its handle, perhaps referencing Mercury (Figure 4.26). ${ }^{611}$ The presence of the gaming board and various medical/ritual implements further complicates interpretation of this grave and makes it of particular importance in understanding the relationship between magic and medicine in early Roman Britain, ${ }^{612}$ though it is unlikely that these play a key role in understanding the presence or significance of the copper alloy vessels. Like the 'Warrior's Burial', the sex of the cremation is unknown. ${ }^{613}$ Strainer bowls may have been used to infuse beverages with either flavour enhancing or intoxicating additives. ${ }^{614}$ That this was a medical practitioner of elite standing would appear evident from the various medical instruments among the grave furniture. ${ }^{615}$ While it is possible that wine had a ritual significance beyond its domestic consumption, ${ }^{616}$ it is likely that the wine accoutrements are more of a reflexion of his station in life and his leisure activity than of his vocation. Though the 'Doctor's Burial' is unusual in many ways, it is still very much a part of a

\footnotetext{
${ }^{611}$ Adkins \& Adkins 1996, 152; See Section 4.4.

${ }^{612}$ Crummy et al. 2007, 224-250.

${ }^{613}$ May 2007, 380-381.

${ }^{614}$ Cool 2006, 144-146.

${ }^{615}$ Crummy et al. 2007, 250.

${ }^{616}$ Carver 2001, 35-38.
} 
wider cultural phenomenon and reflecting that the individual was part of an elite group who represented themselves and communicated their status in much the same way.

The largest group of extant barrows from the Roman period in Britain are found in Bartlow Hills in Essex, ${ }^{617}$ four of its cremation burials contain copper alloy vessels. ${ }^{618}$ Barrow IV contained four copper alloy vessels: two jugs, a Handled Pan 1 and an enamelled jar. Other objects included in the grave included a Dressel 20 olive oil amphora, two copper alloy strigils, five glass bottles of varying size, a copper alloy frame for a folding chair and a copper alloy lamp with a large oak-leaf flame shield. The jug was lying inside the Handled Pan 1 upon discovery. This jug is decorated with a sphinx standing on the necks of two storks at the top of the handle along the rim and has a handle medallion in the shape of a bucranium. While bulls' heads are relatively common decorations on Romano-British copper alloy vessels, ${ }^{619}$ the use of the bucranium is more unusual for copper alloy vessels. The sphinx decoration, rather unusual for Britain, hints to probable Mediterranean manufacture. The handle of the Handled Pan 1 from this grave group terminates in the head of a ram. The other jug in this group ${ }^{620}$ is a simpler Eggers type 128a and is analogous to the jug that contained the Corbridge Hoard. ${ }^{621}$ The jar is of unusual type and its enamelled decoration closely resembles that used on Rudge Cup type vessels, indicating that it is almost certainly an object of British manufacture. ${ }^{622}$ It could be a vessel designed for use in bathing as opposed to dining and the presence of strigils in the grave supports this probability. A vessel of comparable shape with figural relief decoration is known from a probable grave context in Gaul, ${ }^{623}$ indicating that this could have been part of a wider, if occasional, funerary tradition. The presence of a Dressel 20 amphora suggests late $1^{\text {st }}$ to early $2^{\text {nd }}$ century CE deposition, ${ }^{624}$ which the copper alloy vessel forms corroborate. While the use of Spanish olive oil amphorae in graves for containing cremated bones appears to have been a common practice in Britain, ${ }^{625}$ the addition of copper alloy vessels would appear to be something of a novelty, with the exception of a similar grave from a Roman cemetery in Maidstone,

\footnotetext{
${ }^{617}$ Gage 1832, 1-23; Eckhardt et al. 2009, 69.

${ }^{618}$ Philpott 1991, 256; VCH 3 (Essex) 1963, 40-43.

${ }^{619}$ Hawkes 1951, 172-199.

${ }^{620}$ EX0067.

${ }^{621}$ See Chapter 3.

${ }^{622}$ EX0060; Breeze 2012, 108-110.

${ }^{623}$ Espérandieu \& Rolland 1959, 69-70 (139).

${ }^{624}$ Peacock \& Williams 1986, 136-140.

${ }^{625}$ Callender 1965, 26; Philpott 1991, 23.
} 
Kent. ${ }^{626}$ It has also been suggested that the presence of the folding chair may indicate that the occupant was a local magistrate working within the Roman system of government. ${ }^{627}$ The presence of imported olive oil and tableware would indicate that the occupant wished to show their status through the display of Roman material and imports, even while choosing to attempt a link with the past by engaging their burial with a monument dating back to Bronze Age Britain. ${ }^{628}$

Barrows III and V from Bartlow Hills each contain the familiar Handled Pan 1 and jug combination. ${ }^{629}$ In Barrow III, the jug was placed atop the Handled Pan $1 .{ }^{630}$ The jug is a trefoil Eggers type 125 and the Handled Pan 1's handle terminates in a ram's head. Also in this grave was an iron lamp, three glass bottles and fragments of an unidentified ceramic vessel. In Barrow V, the Handled Pan 1 was turned upside down and the jug was placed atop it; both objects are richly decorated. ${ }^{631}$ The jug bears a lion's head with open mouth devouring a bucranium at the top of the handle adjoining the rim, with a handle medallion in the shape of a lion's paw. The handle of the Handled Pan 1 is decorated with four male theatrical masks: one with long hair serving as the handle medallion, two with battle helmets on the underside of the handle flanking a pedestal topped by a basket of fruit, and one on the top of the handle above a basket of fruit. The handle terminates in a ram's head at the basin and is framed by two hooved feet along the rim. Also in this grave are two glass bottles, two glass bowls, an iron lamp and three ceramic vessels. Two of the samian vessels bear stamps, 'IANVARS' and 'MACERATI' respectively, that indicate the likely date of burial to be early Antonine. ${ }^{632}$

Barrow VII from Bartlow Hills contains a trefoil Eggers type 125 jug sitting inside a two handled bowl, ${ }^{633}$ in much the same way often seen done with jugs and Handled Pan 1s. The jug has an anthropomorphic handle medallion, probably female due to the long hair style. The handle of the bowl is topped by the anthropomorphic bust of a veiled female. Also in the grave

\footnotetext{
${ }^{626}$ K0002-K0005; Scott Robinson 1883, 78 (Xb); Jessup 1958, 26 (3); Philpott 1991, 254.

${ }^{627}$ Eckardt 2009, 80.

${ }^{628}$ Eckardt 2009, 85.

${ }^{629}$ Nuber 1973, 210-220; Section 4.11.

${ }^{630} \mathrm{VCH} 3$ (Essex) 1963, 40.

${ }^{631} \mathrm{VCH} 3$ (Essex) 1963, 42.

${ }^{632}$ VCH 3 (Essex) 1963, 42.

${ }^{633}$ VCH 3 (Essex) 1963, 43; only one of the handles of the bowl is currently extant.
} 
were ten ceramic vessels, two glass bottles and one iron lamp. One of the ceramic cups was stamped 'POTTACV $\Sigma$ ', suggesting an early Antonine date for the burial. ${ }^{634}$

The graves of Bartlow Hills are enigmatic for their size, exceptional for Britain. ${ }^{635}$ Barrows were a feature of the British landscape well before the Roman period, with examples reaching as far back as the Bronze Age. ${ }^{636}$ It is likely that these sites would have been recognised in antiquity and that they would have been esteemed for their value as places of heritage and indigenous identity. It would seem likely that the occupants of the Bartlow Hills barrows were keen to associate themselves with this heritage and develop a sense of cultural continuity with a distant past as they constructed their barrows within sight of several prehistoric barrow sites in the area. ${ }^{637}$ The inhabitants were also keen to draw cultural associations with their present societal situation as elites within a Roman province, as their grave goods are reflective of Roman high dining and leisure. That these two cultural associations do not appear to have clashed with each other here is evidence of how multiple sources may influence the construction of a cultural identity and that there need not be mutually exclusive delineations in culture practice, being especially true in the complex ritual which may be associated with burial practice.

A jug and Handled Pan 1 were discovered during excavations of a Roman Villa at Barrows Field in Rivenhall and almost certainly originate from a grave. ${ }^{638}$ Pottery assemblages indicate occupation of the site reaching back to the Mesolithic period and there is evidence of Bronze Age and Iron Age rural settlements and farmsteads in the area around where Rivenhall Roman Villa was later built. ${ }^{639}$ The site appears to have been occupied throughout the Roman and into the Anglo-Saxon period. ${ }^{640}$ The jug is a trefoil Eggers type 125 with a separately moulded handle depicting a lion at the thumb rest and the paw of a lion at the handle medallion (Figure 4.27). This iconographic scheme is well attested from examples in Pompeii. ${ }^{641}$ The Handled Pan 1 is currently missing its handle, but solder marks and variation in patination

\footnotetext{
${ }^{634}$ VCH 3 (Essex) 1963, 43.

${ }^{635}$ Gage 1832, 1-23.

${ }^{636}$ Eckardt 2009, 83-87.

${ }^{637}$ Eckardt 2009, 87.

${ }^{638}$ Rodwell 1978, 15; Rodwell \& Rodwell 1993, 35-36.

${ }^{639}$ Rodwell \& Rodwell 1985, 13-19.

${ }^{640}$ Rodwell \& Rodwell 1985, 13-74.

${ }^{641}$ Tassinari 1993 (10061, 1143, 10662, 5017 and 12712).
} 
indicate where it originally connected to the body of the vessel. ${ }^{642}$ The handle would almost certainly have been fluted and borne a ram's or canine's head at its terminal.

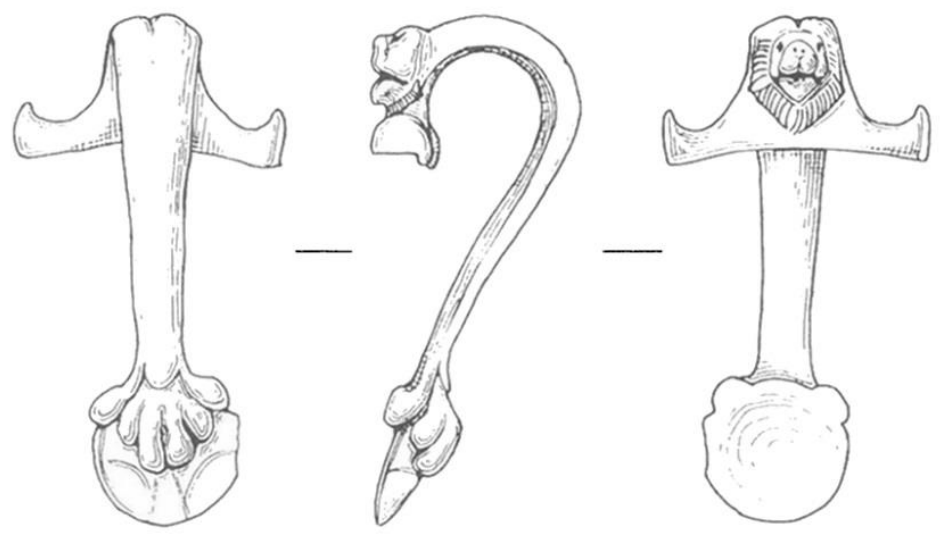

Figure 4.27: Zoomorphic jug from Rivenhall (Rodwell \& Rodwell 1993, 34 Fig.14.3, Scale 1:3)

The jug and Handled Pan 1 combination is again present in the assemblage from Heybridge in Essex which is particularly fragmentary, though the jug handle survives well enough to show a harpy adorning its thumb rest. ${ }^{643}$ Excavations on nearby Elm's Farm have revealed the remains of a rural settlement that saw occupation from the early Iron Age and into the Anglo-Saxon period, including houses, pottery kilns, a probable temple site and several LIA cremations. ${ }^{644}$ That the site would appear to have had spiritual significance that carried over from the Iron Age and into the Roman period is intriguing, ${ }^{645}$ showing a continuity of belief and practice.

\footnotetext{
${ }^{642}$ Rodwell \& Rodwell 1993, 35.

${ }^{643}$ Eggers 1968, 105 (35a); Wickenden 1986, 55 (4A).

${ }^{644}$ Atkinson \& Preston 1998, 85-102.

${ }^{645}$ Atkinson \& Preston 1998, 92-100.
} 


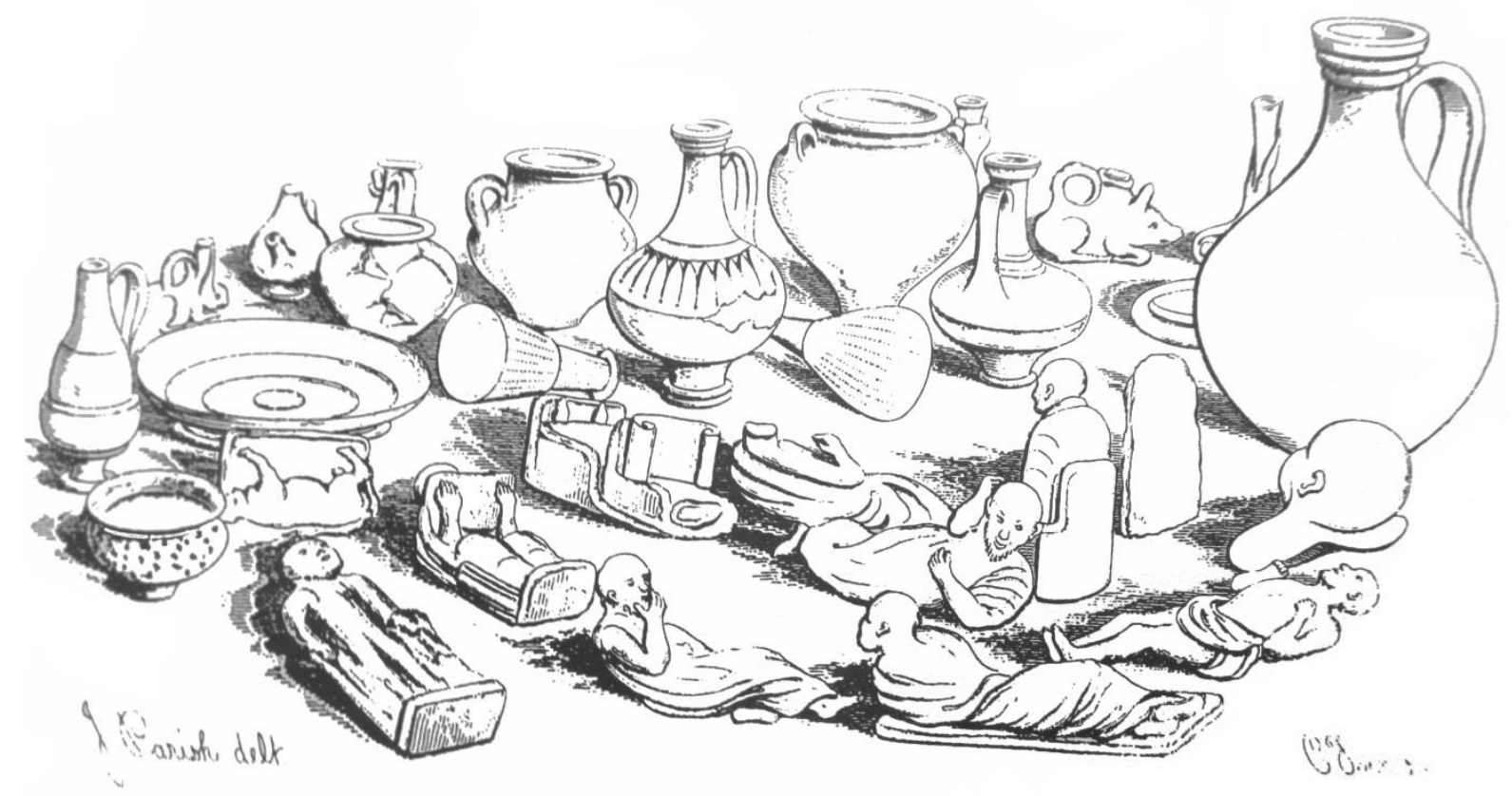

Figure 4.28: Child's Burial from Colchester (Eckardt 1999, 59 Fig.2)

A grave assemblage of unusual composition was found in Colchester. In addition to a Handled Pan 2 with a key-hole suspension loop on its handle of Eggers type $150,{ }^{646}$ it contained 36 Claudian copies along with various bone toilet objects, 21 figurines, ten ceramic vessels and three glass bowls (Figure 4.28). ${ }^{647}$ The figurines are of various types. Several depict animals including hares, monkeys and lions while others depict people, some of which are grotesques. There is also a figurine of Hercules with club and lion skin, which is the only recognizable mythological figure in the group. This grave does not appear to follow the patterns of composition that may be expected for Roman Britain, particularly the large quantity of coins ${ }^{648}$ and the Handled Pan 2. The coins are enigmatic, being highly specific in their issues, though it is difficult to determine what direct relevance or association they may have to this particular burial. The figurines are also puzzling as they could have been simply toys or perhaps have been ritual charms. The grotesques almost certainly served a ritual purpose, as such motifs have been recognised for some time to have served an apotropaic function. ${ }^{649}$ This grave is typically

\footnotetext{
${ }^{646}$ EX0034.

${ }^{647}$ May 1930, 251-253 (1141); Philpott 1991, 289; Eckardt 1999, 57-78.

${ }^{648}$ Philpott 1991, 209.

${ }^{649}$ Wace 1904, 110-114.
} 
believed to be a child's burial, ${ }^{650}$ though it would seem that this is largely based on the assumption that some of the figurines in this grave are toys and is not based on any osteological evidence, as there is none extant. ${ }^{651}$ It seems unlikely that the objects in this grave served as a coherent collection of interdependent objects, simply because of how large and diverse the assemblage is. Therefore, it is unlikely that the presence of the toilet implements or the figurines will offer any clues as to the possible intended function of the Handled Pan 2 in this group. The presence of the findspot near a road which contained several large Roman funerary monuments associated with the Claudian expeditions and the unusual nature of the burial is unparalleled in Roman Britain, making it unlikely to educate us on further burial practice in the province during this period.

The high concentration of graves with copper alloy vessels in Essex is reflective of the great concentration across the south eastern counties of England more generally. The majority of these graves are located in northern Kent and represent a continuation of the wider geographic trend seen stretching from the south-eastern Midlands (see Appendix VIII).

A jug and Handled Pan 1 type grave accompanied by three copper alloy armlets, a glass phial and two toilet instruments was discovered in Martyr's Field in Canterbury. ${ }^{652}$ The Handled Pan 1 has a fluted handle terminating in a canine head. ${ }^{653}$ The jug is slightly more unusual. It resembles an Eggers type 151 and is decorated with the head of a horse looking over the rim of the vessel at the thumb rest and an anthropomorphic bust for the handle medallion. An incomplete example believed to have originally been decorated with a horse was discovered in Pompeii, ${ }^{654}$ suggesting possible $1^{\text {st }}$ century date of manufacture for the example from Martyr's Field. An example from a grave in Pannonia with its horse statuette well preserved is also dated to the $1^{\text {st }}$ century $\mathrm{CE},{ }^{655}$ with an analogous example coming from Grave 2 at Turners Hall Farm. ${ }^{656}$ A further grave in Canterbury (at Palace Street) consisted of a bowl of probable early Roman date turned upside down covering cremated remains. ${ }^{657}$ A single jug was discovered

\footnotetext{
${ }^{650}$ VCH 3 (Essex) 1963, 116 (13).

${ }^{651}$ Eckardt 1999, 57.

652 Philpott 1991, 314; Eggers 1966, 102 (4); Museum of Canterbury 7847-7848.

${ }^{653}$ VCH Kent 3, 78-79.

654 Tasanari 1993, 45 (E3000 Inv. 4291).

${ }^{655}$ Radnoti 1938, 137-139 (Taf. XIII, 71).

${ }^{656}$ HER0041; Burnham et al. 2003, 327; West 2005, 14.

${ }^{657}$ Smith 1922, 86.
} 
interred in a cremation pit in Ashford, Kent. ${ }^{658}$ In addition to the cremated remains, a single terra nigra vessel and the remains of a wooden box and bucket as well as the undiagnostic remains of a second copper alloy vessel were also discovered in the grave. While it is impossible to definitively identify the form of the second vessel, it is most likely from a basin; a Handled Pan 1 would be the expected form based on the presence of the jug. ${ }^{659}$ A grave of Roman date containing a jug and a bowl of Eggers type 99 along with four ceramic vessels (three bowls and a cup), a glass bowl and a glass bottle was discovered in Luton in the late $19^{\text {th }}$ century. ${ }^{660}$ It would appear that this group comprises a drinking and dining set with the glass bottle, copper alloy jug and ceramic cup all being identifiable as accoutrements for drinking. The copper alloy bowl has two handles and is similar to that found in a lavish grave in St. Albans, ${ }^{661}$ though of more modest decoration, with further examples found in Pompeii, Gaul and Germany. ${ }^{62}$ This type of bowl has been identified as being used for ablutions ${ }^{663}$ and it is likely fulfilling a similar function in this grave as a Handled Pan 1 in so many other examples.

During work on the A2, two rich cremation graves that contained copper alloy vessels were excavated at Tollgate near Springhead in Kent near the Roman site of Vagniacae. ${ }^{664}$ Grave 6260 contained a Handled Pan 1 with a ram's head handle terminal (Figure 4.29) paired with a trefoil Eggers type 124-125 jug decorated with an anthropomorphic female handle medallion and thumb rest similar in type to a find from Winchester (Figure 4.30). ${ }^{665}$

\footnotetext{
${ }^{658}$ Burnham et al. 2001, 382.

${ }^{659}$ Nuber 1973; See Section 4.3.

${ }^{660}$ Eggers 1966, 102 3a \& 3b; BM 1894. 8 3. 58; Jessup 1958, 27-28.

${ }^{661}$ HER0019; Section 4.5.

662 Tassinari S3110; den Boesterd 172; Radnoti 60; Eggers 91, 92 \& 94.

${ }^{663}$ Allison 2004, 55; Cool 2006, 138.

${ }^{664}$ Mynott 2008, 15.

${ }^{665}$ K0015-K0016; Allen et al. 2012, 335-342; HAM0003.
} 


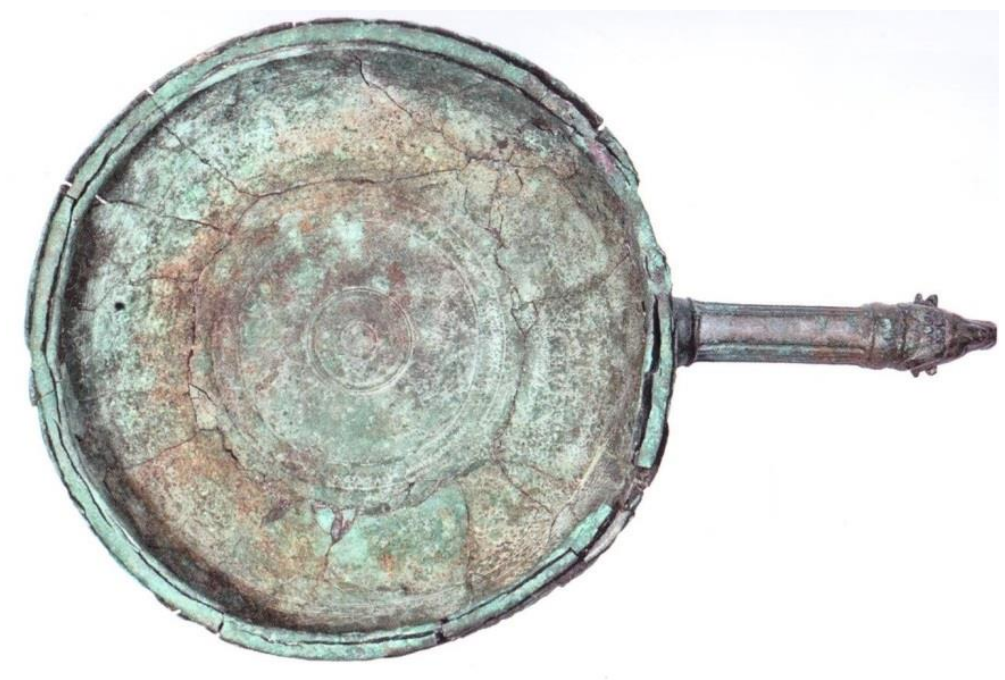

Figure 4.29: Handled Pan 1 from Grave 6260 at Tollgate (Allen et al. 2012, 340 Fig.4.12)

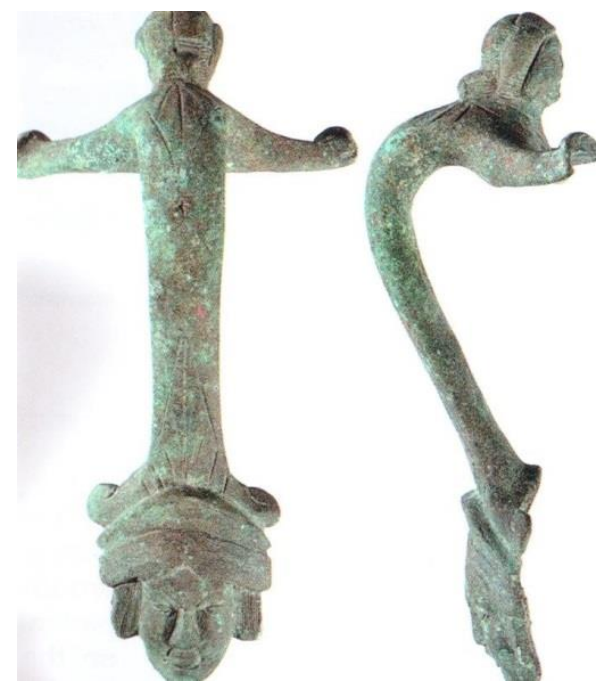

Figure 4.30: Jug handle from Grave 6260 at Tollgate (Allen et al. 2012, 343 Fig.4.15)

A copper alloy cauldron was also interred in this grave that was decorated with ducks' heads on either end of its drop-looped handle and a ring of scalloped ovals in a body band below the rim that may represent eyes (Figure 4.31). ${ }^{666}$ The possibility of these representing eyes, in which case almost certainly performing an apotropaic function, is intriguing as the use of this would be the only currently known instance of such decoration on copper alloy vessels from the Roman period in the West. Until such time as parallels may be found to support the claim of this

${ }^{666}$ K0014; Allen et al. 2012, 334-335. 
decoration acting as an 'evil eye' charm, it would seem more likely that this is a simple geometric scalloped motif without specific iconographic or apotropaic functions. A gaming board, dice, and the remains of a pig were also found in this grave along with 18 ceramic vessels of Flavian date. ${ }^{667}$

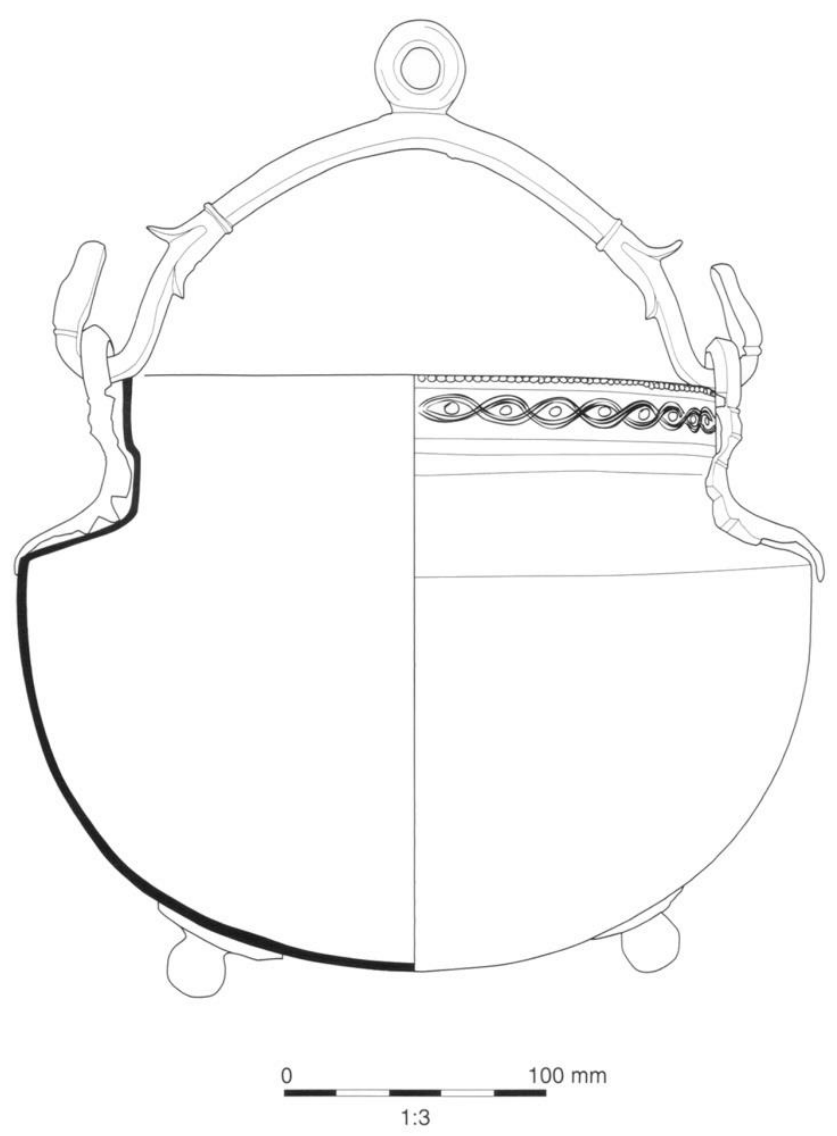

Figure 4.31: Cauldron from grave 6020 at Tollgate (Allen et al. 2012, 334 Fig.4.7)

The second grave in this group that contains copper alloy vessels, Grave 6635, had only the jug and Handled Pan 1 combination constituting its copper alloy vessel assemblage. ${ }^{668}$ The Handled Pan $1^{669}$ again has a ram's head handle terminal, but is also decorated with a floral motif around its umbo instead of the more common concentric circle decoration (Figure 4.32). This same decoration is found on an example from Prague-Bubeneč. ${ }^{670}$

\footnotetext{
${ }^{667}$ Allen et al. 2012, 325-333.

${ }^{668}$ Allen et al. 2012, 363-368.

${ }^{669} \mathrm{~K} 0017$.

${ }^{670}$ Nuber 1973, T. 3, 1a-1b; Allen et al. 2012, 366.
} 


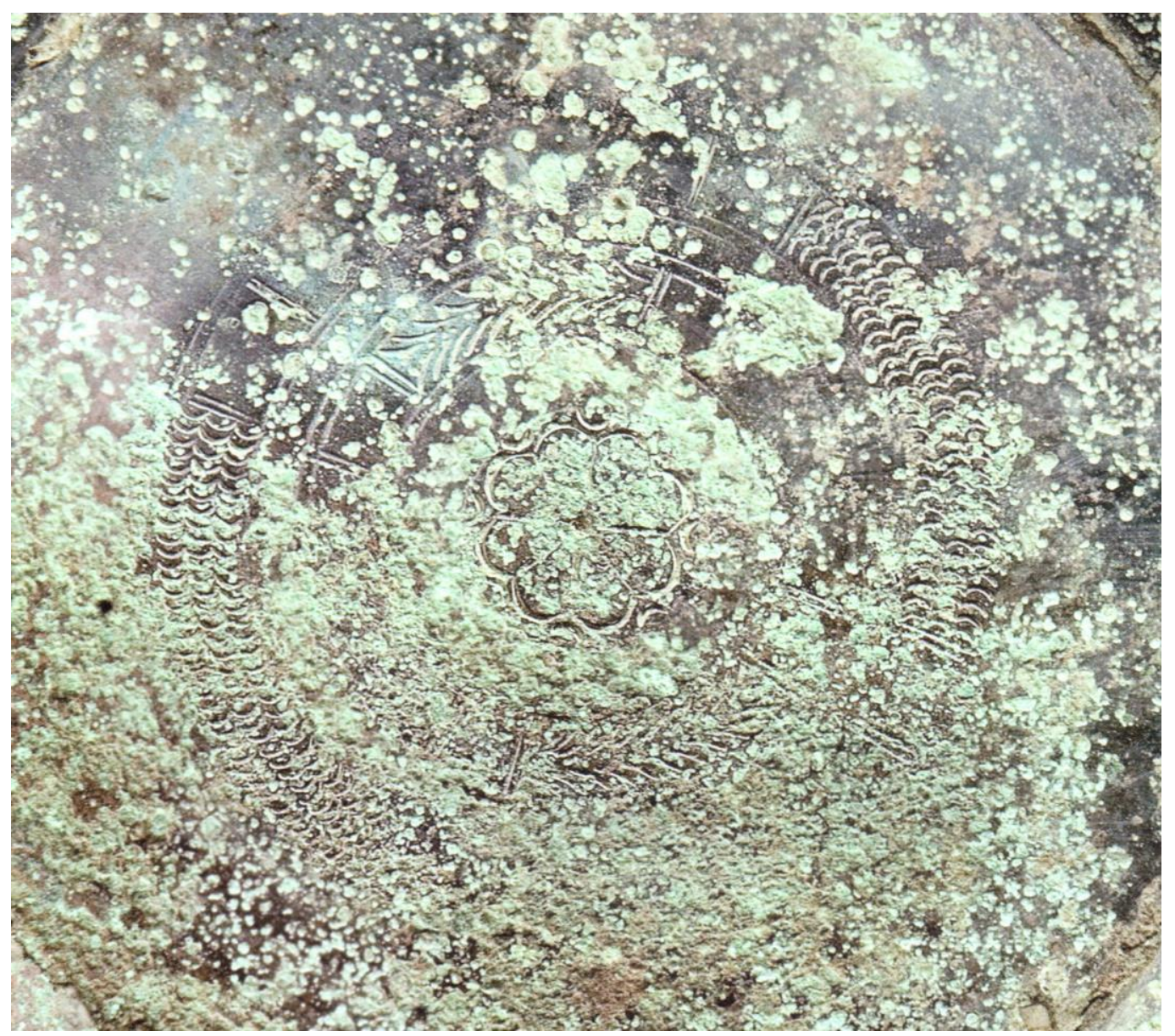

Figure 4.32: Floral decoration on Handled Pan 1 from grave 6635 at Tollgate

(Allen et al. 2012, 365 Fig.4.27)

The jug is an Eggers 124-125 and decorated with a lion's head thumb rest and an anthropomorphic female face for the handle medallion (Figure 4.33). ${ }^{671}$ Grave 6635 also contained what may have been a cosmetics box, with a spatula probe associated with it. ${ }^{672}$ Due to the fragmentary and damaged state of the bones, sex was not identified for the occupants of either of these graves. ${ }^{673}$ A large Roman cemetery of more modest graves is located nearby at Pepper Hill and the Tollgate graves likely represent elite burial partly removed from the principal cemetery area. $^{674}$

\footnotetext{
${ }^{671} \mathrm{~K} 0018$.

${ }^{672}$ Allen et al. 2012, 369-371.

${ }^{673}$ Allen et al. 2012, 454.

${ }^{674}$ Allen et al. 2012.
} 

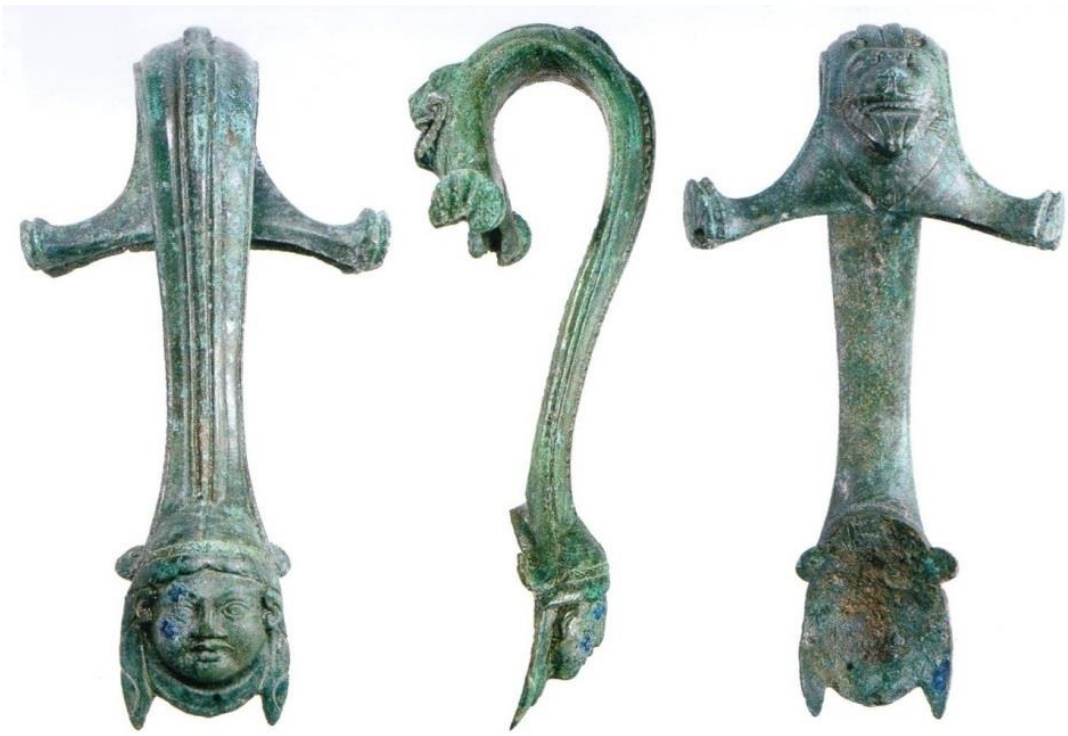

Figure 4.33: Jug handle from grave 6635 at Tollgate (Allen et al. 2012, 367 Fig.4.29)

A grave assemblage found in Cremation Deposit 3 at the Roman cemetery at Joy Wood in Maidstone, Kent included two copper alloy jugs as part of the burial assemblage. ${ }^{675}$ While most of the jugs found in graves in Britain are single handled, one of the jugs interred in the burial is of a two handled Eggers type $129 .{ }^{676}$ The second jug is of a type similar to Tassinari E3000, but with a more angular, octagonal body. A similar example was discovered at Aquincum. ${ }^{677}$ Cremation Deposit 3 in Maidstone also contained a Dressel 20 amphora. A further cremation in this cemetery contained within a glass bottle was also found associated with the undiagnostic remains of a copper alloy vessel. ${ }^{678} \mathrm{~A}$ samian vessel found in one of the graves is stamped 'OFMVRA' and provides a date of around $150 \mathrm{CE}$ for the burial. ${ }^{679}$

\footnotetext{
${ }^{675}$ Philpott 1991, 254; Scott Robinson 1883, 78 (Xb \& Xc); Jessup 1958, 26 (3).

${ }^{676}$ See also Tassinari A3220.

${ }^{677}$ Radnoti 1938, Taf XLV (1).

${ }^{678}$ K0006; Philpott 1991, 259.

${ }^{679}$ Jessup 1958, 27.
} 


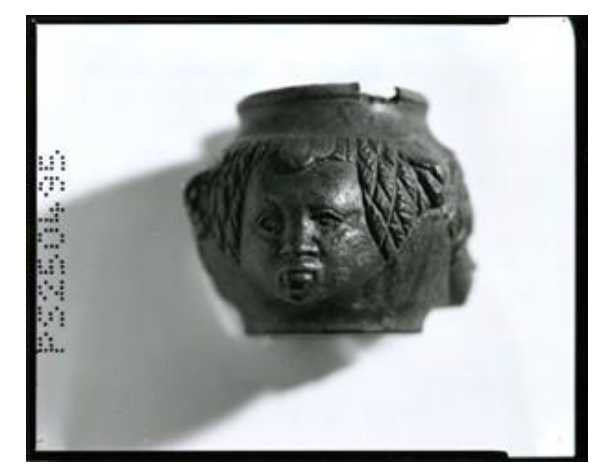

Figure 4.34: Jar with negroid faces from Sittingbourne, Bayford (@ the British Museum)

A cremation burial contained within a glass bottle found at Bayford in Sittingbourne, Kent has four copper alloy vessels as part of its assemblage: a trefoil Eggers type 124-125 jug, a Handled Pan 1, a two handled bowl, and a jar ${ }^{680}$ decorated with negroid faces that almost certainly represent African slaves, or pueri delicati. ${ }^{681}$ This jar has an iron strigil attached to it by a small length of chain (Figure 4.34). ${ }^{62}$ The anthropomorphic decoration of the jar is also found on several other examples in Western Europe. ${ }^{683}$ The jug in this group is decorated with an anthropomorphic winged female figure, most probably depicting either a harpy or siren (Figure 4.35), ${ }^{684}$ while the Handled Pan 1 has a fluted handle that terminates with the anthropomorphic bearded head of a satyr with goat horns and pointed ears. The grave was also furnished with 12 ceramic vessels, a glass jar and a copper alloy lamp with a crescent moon shaped flame guard. While most of the contents might be easily associated with drinking and dining practice, the copper alloy jar and strigil are toilet implements, Payne suggesting they were used for bathing. ${ }^{685}$ The jar is of small size (approximately 2 inches in height) and would have contained something utilised in sparing quantities, such as unguents or perfumes. Copper alloy cosmetic vessels are not common grave inclusions, but are not without precedent in Roman Britain, ${ }^{686}$ and were likely interred in graves as a symbol of leisure and wealth.

\footnotetext{
${ }^{680}$ Referred to as a lecythus by Payne $1877,47-48$.

681 Tanner 2010, 34.

${ }^{682}$ K0007-K0010; Payne 1877, 47-48; Philpott 1991, 259.

${ }^{683}$ Braun 2001, 119-121 (23-27).

${ }^{684}$ Payne 1877, 47-48.

${ }^{685}$ K0009; Payne 1877, 47-48.

${ }^{686}$ The cosmetic jar from Corbridge being a good example of just such a vessel; NU0049; Casey \& Hoffman, 1995b, 24 (6).
} 


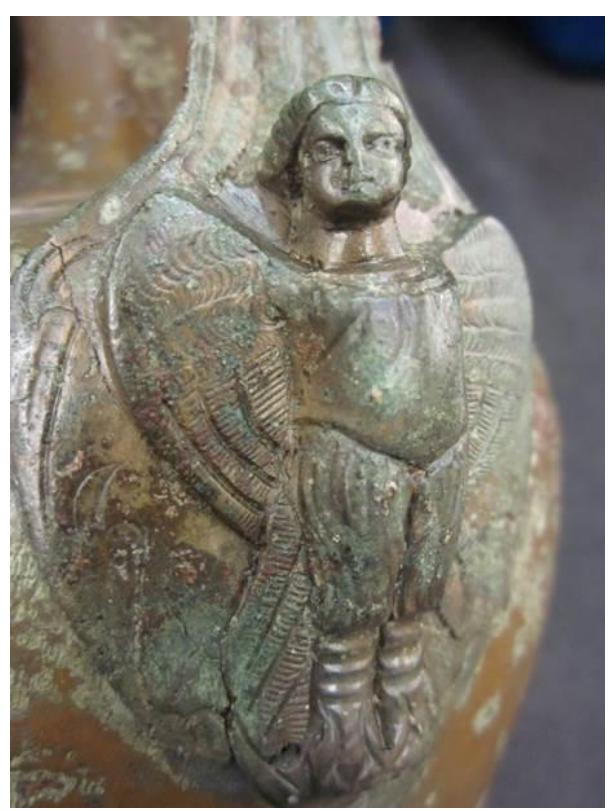

Figure 4.35: Detail of anthropomorphic handle medallion from

Sittingbourne, Bayford in British Museum archives (photo by author)

A jug of Eggers type 122 with an elaborately decorated handle depicting the madness of Ajax was discovered in a cremation burial that was also found among the graves at Bayford. ${ }^{687}$ The handle medallion of the jug depicts Ajax in the process of slaughtering the animals of the Greek armies at Troy after being denied the armour of Achilles (Figure 4.36). ${ }^{688} \mathrm{He}$ is surrounded principally by livestock, including cattle, pigs and goats. There is the depiction of what appears to be a monkey on the upper portion of the handle that would seem out of place with the rest of the decorative scheme (Figure 4.37). It is possible that the monkey was included to indicate place, showing that Troy was located in an exotic location (i.e., not Greece or Italy). Similar representations of the madness of Ajax motif are known from the Roman world, notably on a balsamarium from Nijmegen and in relief sculpture from Besançon. ${ }^{689}$ Vessels of similar form and decorative type are attested from the destruction layers of Pompeii ${ }^{690}$ and support the pre-Hadrianic date ascribed to the cemetery as a whole. ${ }^{691}$ However, such jug handles with

\footnotetext{
${ }^{687}$ K0011; Philpott 1991, 259; VHC (Kent) 3, 97.

${ }^{688}$ As told in Sophocles' Ajax.

${ }^{689}$ Braun 2001, 61-62 \& 108 (3).

${ }^{690}$ Tassanari B1222.

${ }^{691}$ VHC (Kent) 3, 97.
} 
action divided into registers similar to this are also found in $\mathrm{Gaul}^{692}$ and as part of the deposit at Neupotz (Figure 4.38), ${ }^{63}$ indicating that this type of decoration was likely produced, or at least available, in Gaul as late as the $3^{\text {rd }}$ century CE. A further example of such decoration on a jug handle is also extant from Carlisle, which depicts a more traditional scene of sacrifice. ${ }^{694}$

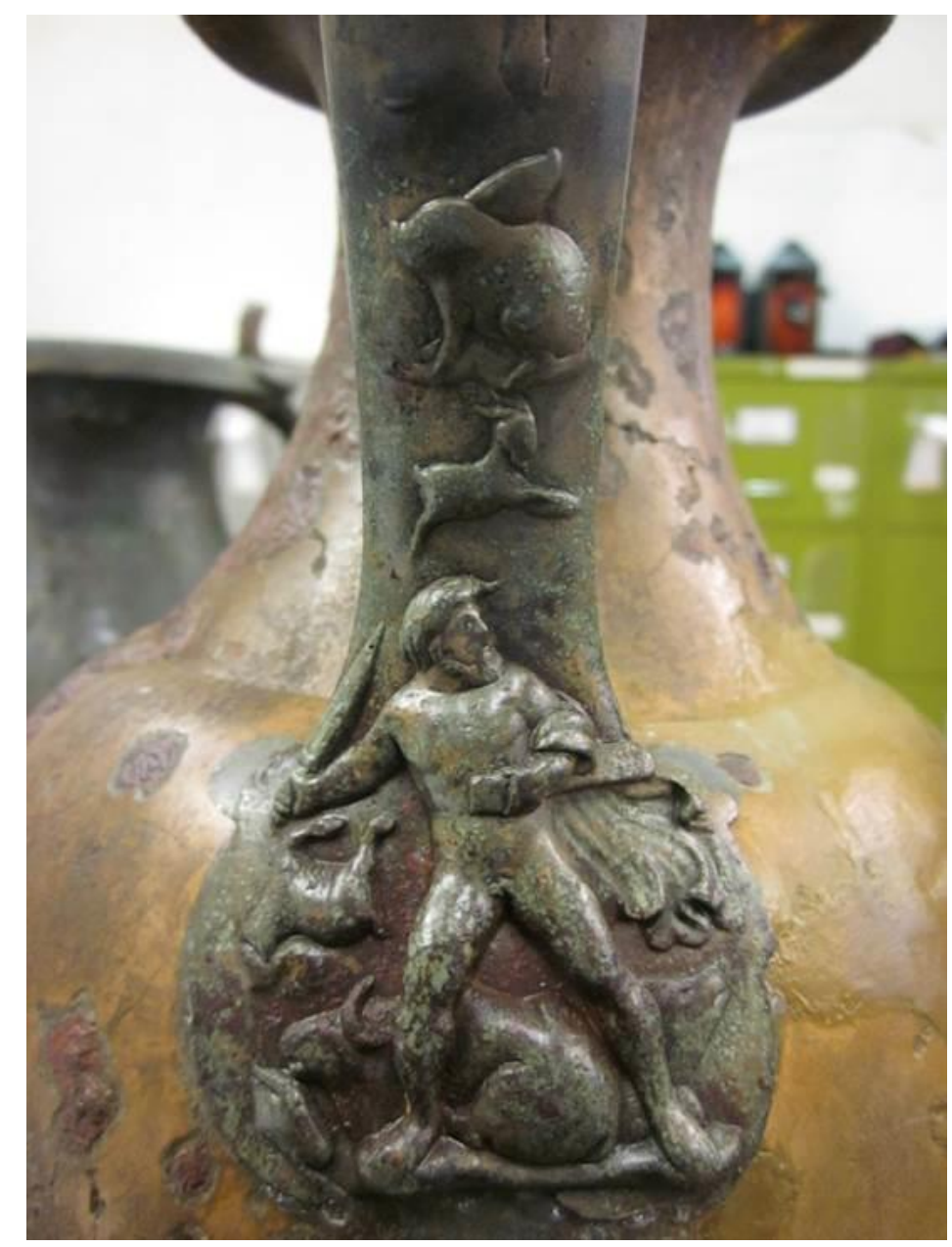

Figure 4.36: Jug handle depicting madness of Ajax from

Sittingbourne, Bayford in the British Museum archives (photo by author)

\footnotetext{
${ }^{692}$ Tassinari 1975, 66-67 (171-172).

${ }^{693}$ Künzl 1993, 122-149 (D4).

${ }^{694}$ C0028; BM 1814,0705.37; Henig 1984, 132-134; See Chapter 5.
} 


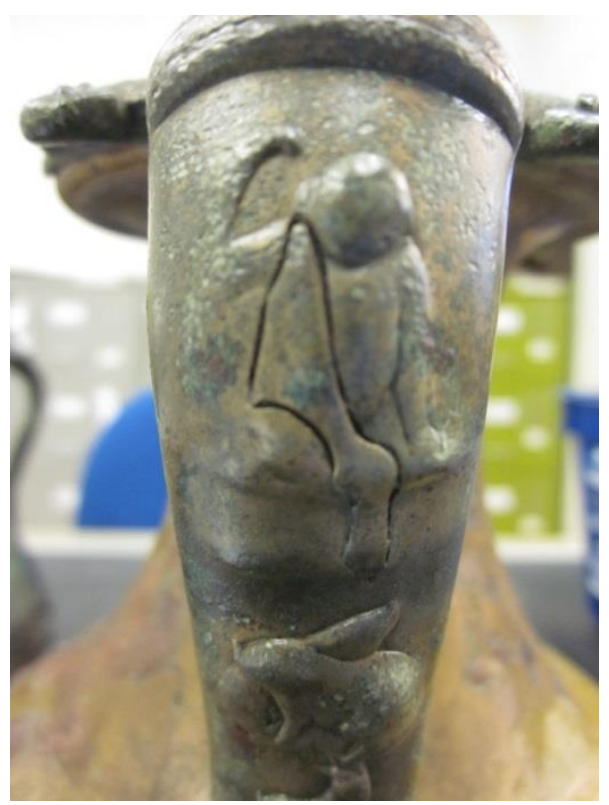

Figure 4.37: Detail of handled from K0011 in the British Museum archives (photo by author)

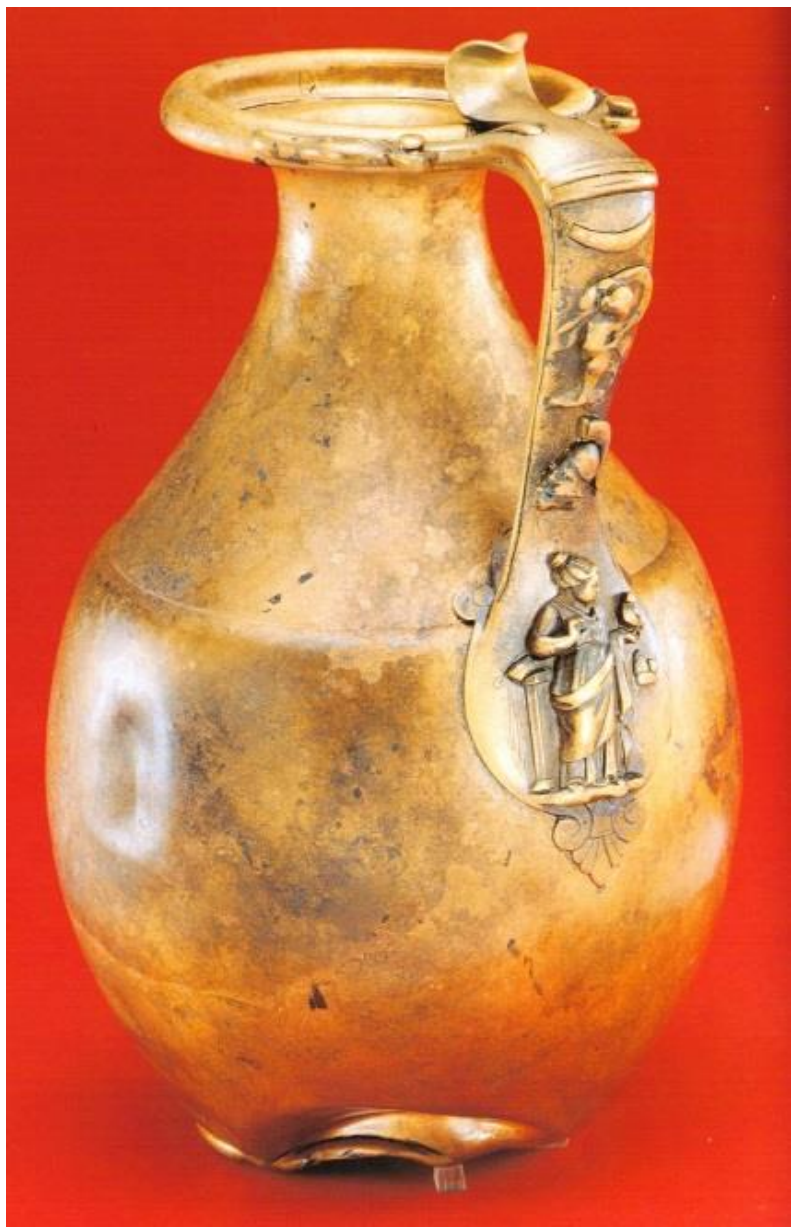

Figure 4.38: Jug from Neupotz (Künzl 1993, Farbtafel 18) 
During excavations at Lullingstone Roman Villa, a farmstead occupied from Claudian times through to the end of the Roman period, ${ }^{695} \mathrm{a}$ tomb was discovered beneath the remains of a Romano-Celtic style temple. It would appear that the structure served as a Temple-Mausoleum for the family of the estate during the late $3^{\text {rd }}$ and $4^{\text {th }}$ centuries CE. ${ }^{696}$ The tomb originally had two lead coffins containing inhumations and a collection of grave goods arranged parallel to them on the west side of the grave.

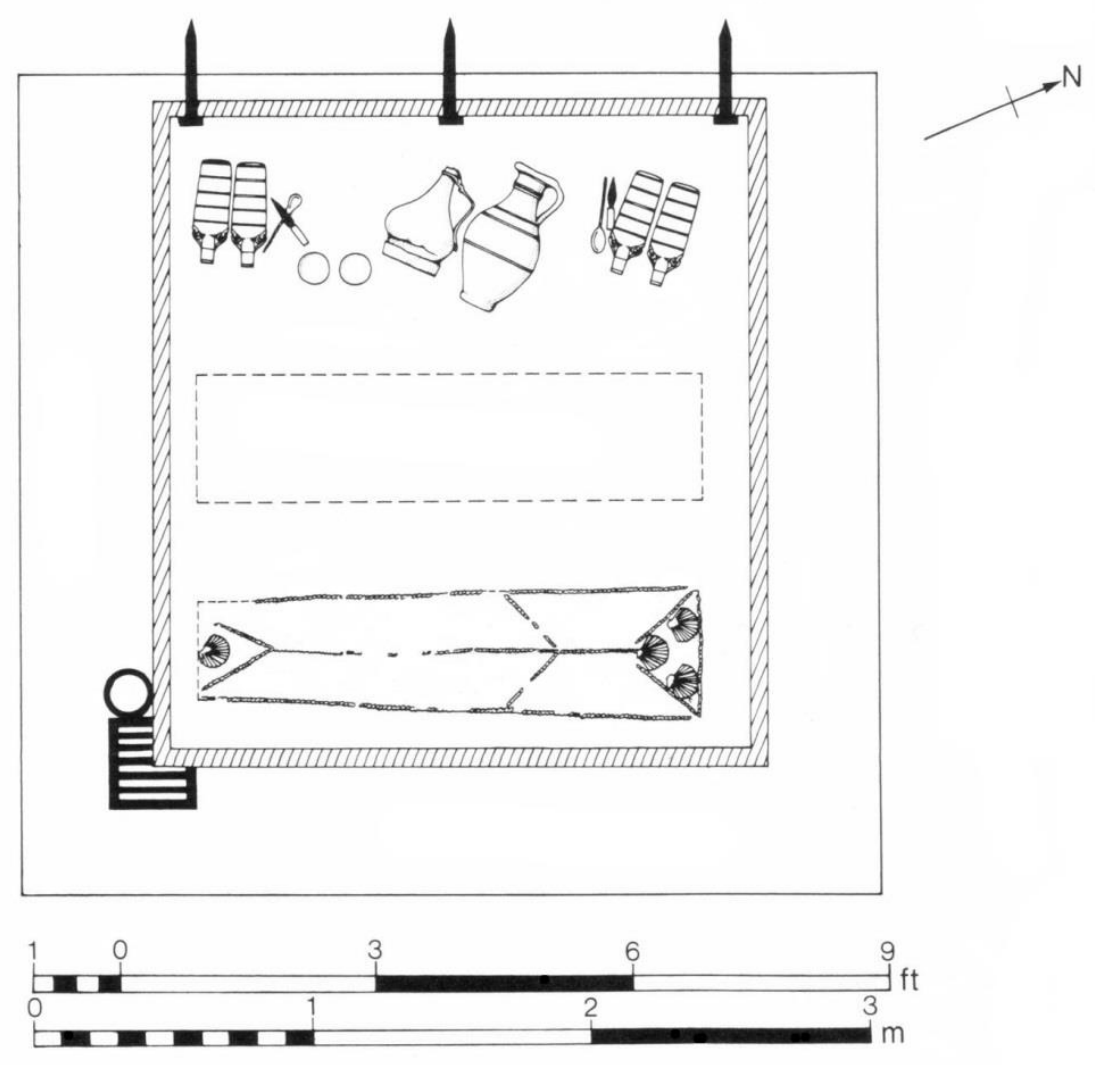

Figure 4.39: Layout of grave from Lullingstone Roman villa (Henig 1983, 197 Fig.97b)

Though the tomb was robbed in antiquity, it would appear that the robbers only took one of the coffins, the second coffin and grave goods escaping notice (Figure 4.39). The grave goods that remained upon excavation consisted of a copper alloy jug and a ceramic jug, four glass bottles, two knives, two spoons, two glass bowls and a bone roundel of a gorgon face. The function of the gorgon roundel is uncertain, though it may well have been votive or

\footnotetext{
${ }^{695}$ Meates 1955.

${ }^{696}$ Meates 1979, 131; Henig 1984, 196-197.
} 
apotropaic. $^{697}$ The glass bottles and cutlery were divided into two groups, suggesting they were set out separately for the two individuals in the tomb and may well represent the extent of the grave material interred. ${ }^{698}$ From the placement of the grave material, it would appear that there is an association between the two jugs and the two glass bowls. In citing a similar pairing in the sculptural relief of the Simpelveld sarcophagus from the Netherlands, Cool suggests that this pairing represents a water and wine jug, ${ }^{699}$ a suggestion that would seem plausibly applicable to the use of these vessels in this circumstance. The copper alloy vessel ${ }^{700}$ is similar to the vessel that contained the hoard of $2^{\text {nd }}$ century coinage from Corbridge discussed in Chapter $3,^{701}$ indicating that the Lullingstone vessel is of likely $1^{\text {st }}$ to early $2^{\text {nd }}$ century manufacture. The pottery and the glass vessels are datable to the late $3^{\text {rd }}$ or early $4^{\text {th }}$ century CE and suggest that the burial took place sometime around $300 \mathrm{CE} .^{702}$ While ancestor worship is well attested in the Roman world, the use of a Romano-Celtic temple as a kind of mausoleum for the cult of the dead is an unusual practice not attested elsewhere in the Roman world, though both Meates and Henig suggest the possible existence of similar subterranean tombs beneath other temple structures during this period. ${ }^{703}$ This burial is also somewhat unusual as it is an inhumation, while most burials containing copper alloy vessels are cremations. This is most likely related to its later date, when inhumation was replacing cremation as the more common form of burial. ${ }^{704}$ Another aspect of this tomb worth brief consideration is the presence of two spoons for the two inhabitants of the grave. While it has been argued that spoons were seen as objects of communal use, ${ }^{705}$ the placement of two spoons here for two individuals would imply that this was not the case. The temple and tomb would appear to have fallen into disrepair during the $4^{\text {th }}$ century CE, perhaps coinciding with the rise of Christianity and the abandonment of pagan rites at the site. ${ }^{706}$

A Hemmoor bucket of Eggers type 56-58 was discovered in several fragments in a cremation burial in Ramsgate, Kent. ${ }^{707}$ This is an unusual inclusion in a burial for this region

\footnotetext{
${ }^{697}$ Meates 1987, 142; Wilk 2000, 31-54.

${ }^{698}$ Meates 1979, 128.

${ }^{699}$ Cool 2006, 197.

${ }^{700} \mathrm{~K} 0001$.

${ }^{701}$ NU0035; Forester et al. 1912, 154; Abdy 2002, 35.

${ }^{702}$ Meates 1979, 128; Henig 1984, 196-197.

${ }^{703}$ Meates 1979, 127-132; Henig 1984, 196-197.

${ }^{704}$ Philpott 1991, 58.

${ }^{705}$ Cool 2006, 53-54.

${ }^{706}$ Meates 1979, 130.

${ }^{707}$ Eggers 1966, 102 5A; Smith 1922, 93-94.
} 
and is more characteristic of the graves found at Brougham in Cumbria. ${ }^{708}$ The fragmentary state of the Ramsgate vessel, if it were to be the result of ritual destruction on the pyre, also suggests analogy with the Brougham group. The Hemmoor bucket is a difficult vessel type to date, as they had a long period of manufacture and could also have a long use-life. ${ }^{709}$

A copper alloy jug was discovered in Winchester interred with one of the two graves of Roman date excavated at Grange Road in late $1964 .^{710}$ The jug was placed next to three samian cups and a glass jug and would appear to have formed part of a wine service. ${ }^{711}$ Also included in the grave were objects seemingly intended for leisure and banqueting, such as gaming counters and samian bowls. The jug is a trefoil Eggers type 125 and has an anthropomorphic female bust as a thumb rest and a face of a similar female as the handle medallion. Both figures wear solid, semi-circular diadems. Jugs very similar to this have been discovered in Pompeii, ${ }^{712}$ and its good state of preservation suggests it was likely not of great age when it was interred. This is corroborated by the dating of the ceramics in this burial to between 70 and $95 \mathrm{CE}$, offering a probable Flavian date for the burial. ${ }^{713}$ It is worth noting that no copper alloy vessels were found from the nearby Lankhills Roman cemetery, ${ }^{714}$ though six pewter vessels have been recorded. ${ }^{715}$ Furthermore, a recent survey of Roman burials from Winchester and its environs also did not note any copper alloy vessels, ${ }^{716}$ further indicating that the use of these objects as grave furniture was not widely practiced in this area. This is likely a reflexion of the $4^{\text {th }}-5^{\text {th }}$ century CE date of this cemetery, ${ }^{717}$ showing the decline in the practice of depositing copper alloy vessels in graves in the Late Roman period.

A single copper alloy jug was found included in a grave assemblage in Chichester, West Sussex. ${ }^{718}$ A ceramic bowl and a pair of brooches found in the grave offer an approximate date of 150 CE. ${ }^{719}$ Fishbourne Roman palace is not far distant, the likely residence of an aristocrat connected with the settlement at Chichester, possibly even the Roman 'client-king'

\footnotetext{
${ }^{708}$ Cool 2004, 144-374; C0002-C0005.

${ }^{709}$ Erdrich 1995, 71-80.

${ }^{710}$ Biddle 1967, 224-250.

${ }^{711}$ Biddle 1967, 231.

712 Tassinari 1993, 65 (11482 \&18763).

${ }^{713}$ Biddle 1967, 245.

${ }^{714}$ Clarke 1979; Booth et al. 2010.

715 Brown 1979, 206-207.

${ }^{716}$ Ottaway et al. 2012, 243-370.

${ }^{717}$ Clarke 1979, 113-122; Booth et al. 2010, 455-462.

${ }^{718}$ WSU0010.

${ }^{719}$ Smith 1922, 98.
} 
Cogidubnus. $^{720}$ The proximity of this grave to an urban centre is unusual, as most of the grave assemblages in this thesis are located near rural settlements such as villas or farmsteads. As Figure 4.40 illustrates, copper alloy vessels are deposited almost exclusively on Rural Settlement site types. This pattern is even more pronounced when it is accounted that 20 out of the 25 vessels from military sites come from Brougham alone.

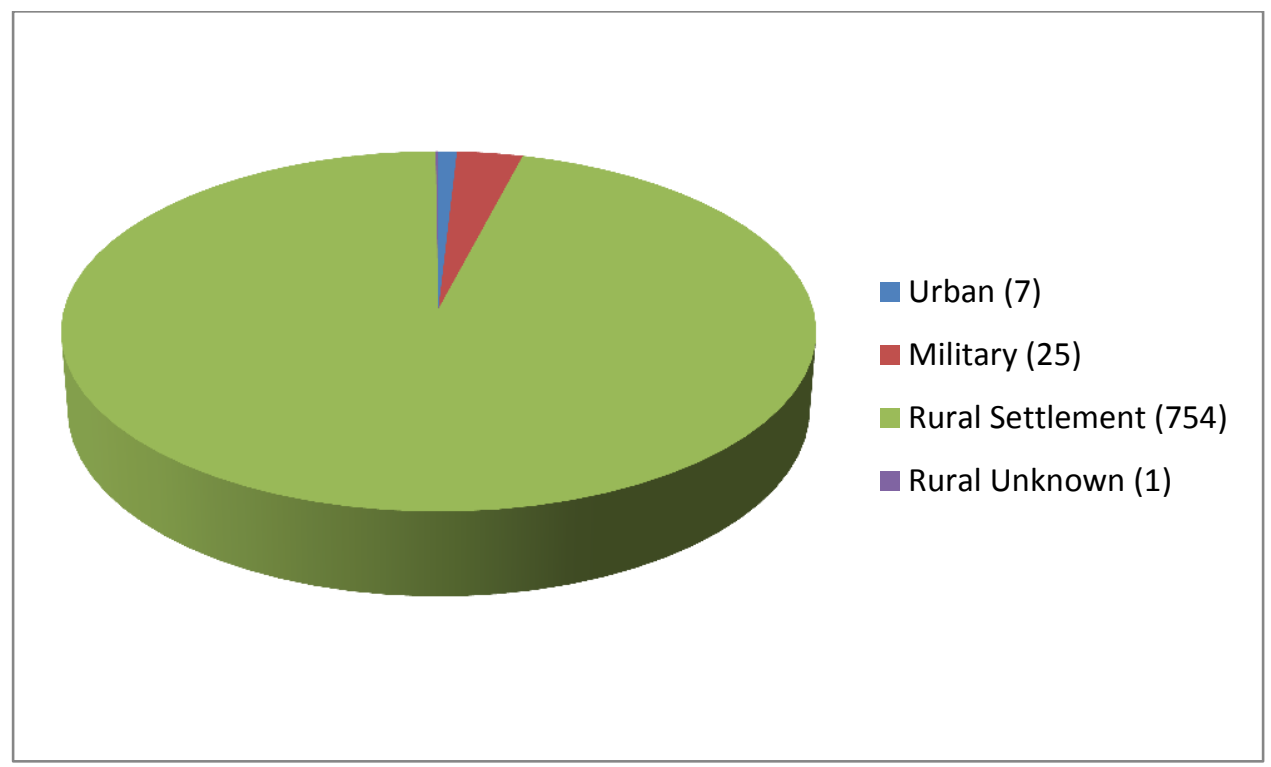

Figure 4.40: Numbers of copper alloy vessels across Site Types.

As the deposition of this material is so ubiquitously at such Rural Sites, geographic patterning of Site Type proved impossible for Grave Deposits. This consistency of Site Type deposition helps to characterise the practice with a rural landed elite, as discussed later in this chapter as well as in Chapters 7 and 8 . The principal geographic patterning which may be deduced from the evidence presented above is a strong preference towards grave deposition in the south east of England as opposed to elsewhere in Britain, as illustrated by Map 2. Where it does appear elsewhere, it appears to be isolated incidents or insular groupings, such as at Brougham in Cumbria. Two principal reasons present themselves for this distributional patterning, the first being cultural and the second being financial. The interring of copper alloy vessels in graves was never a wide-spread practice in Roman Britain, so it is appealing to suggest that the graves in the south east were part of the practice of a local elite class which began to operate in the Late Iron Age and continued into the late $2^{\text {nd }}$ century CE. To include copper alloy

\footnotetext{
${ }^{720}$ Cunliffe 1971, 10-16; Down 1988, 17-27; Manley \& Rudkin 2003, 131-146.
} 
vessels as grave goods takes more than cultural motivation, it requires economic resources sufficient to take part in such acts of conspicuous consumption. The majority of examples of copper alloy vessels come from rural contexts, and may well represent burials of landed elites. This does not explain the isolated anomalies, which most likely represent intrusive burial practices from elsewhere in the empire brought by members of the imperial establishment or by merchants wishing to carry on cultural traditions identifying them with religious or cultural groups from their homeland.

\section{$\underline{4.3 \text { Chronology and its relation to ritual practice and object selection }}$}

The deposition of copper alloy vessels in graves was never a wide spread practice in Britain during the Roman period. This being said, the variations in burial trends that include copper alloy vessels appear to be both geographically and chronologically based and are best framed in these terms. Geographic trends were discussed in the previous section, with the principal concentration of material being from the southern Midlands and south eastern counties of England with few examples elsewhere in the province. Figure 4.41 offers a broad view of the use of copper alloy vessels over time in Roman Britain by dividing the deposits by the broad chronological period of their deposition, as most of the graves can only be dated within a broad period of deposition, often of 50-75 years or more.

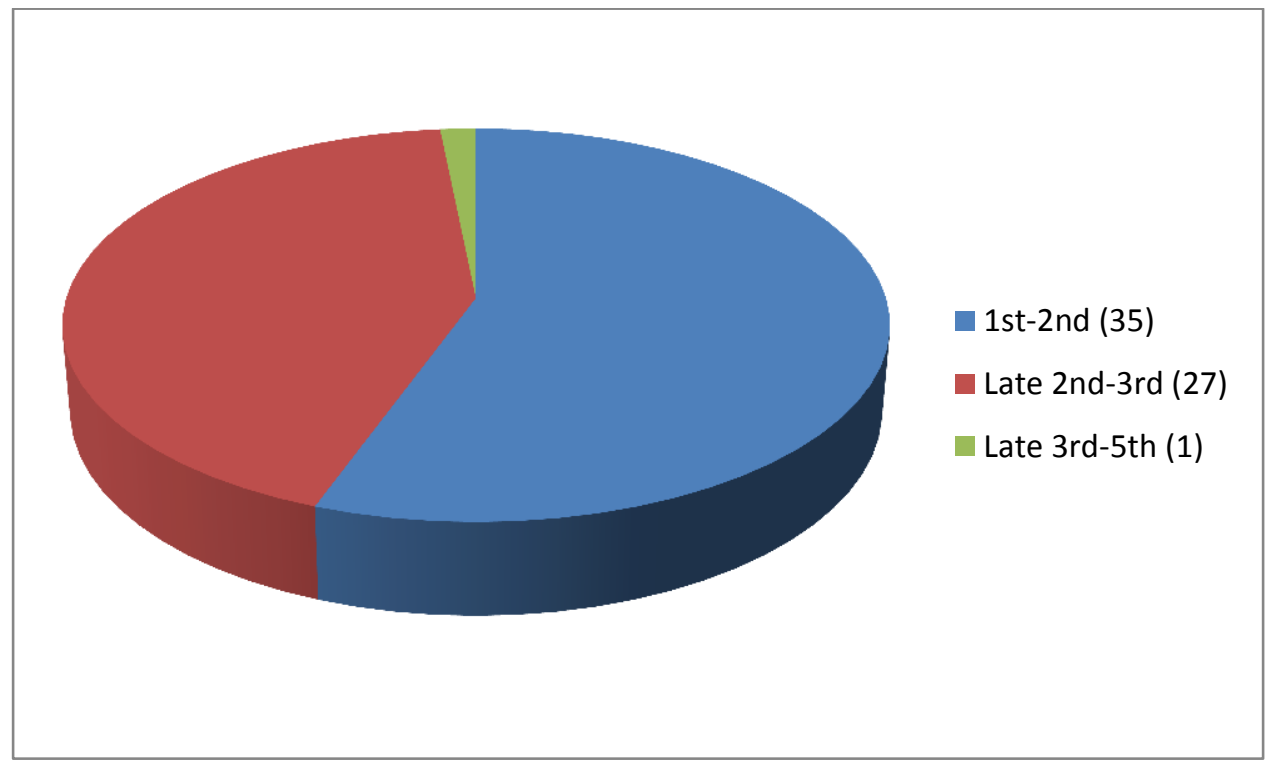

Figure 4.41: Number of Grave Deposits by date. 
The Late Iron Age in Britain saw the rise in large grave assemblages focused on the lower Midlands in the region of Hertfordshire. ${ }^{721}$ This burial practice typically involved the interring of wine amphora and other luxury goods in a cremation burial. ${ }^{722}$ A good example of this type is found in the early to mid- $1^{\text {st }}$ century BCE burial at Baldock, Hertfordshire and included three copper alloy vessels as well as an iron cauldron, firedogs and an Italian wine amphora. $^{723}$ The material interred in such burials would appear to have continued to grow more lavish as time progressed, growing in the numbers of objects as well as a greater amount of imported objects interred. This perhaps reflects a higher level of affluence among the ruling class, or at least an increased desire for the conspicuous consumption of luxury goods by said elites. This eventually culminates in lavish Conquest Period burials such as the King Harry Lane burial from St. Albans ${ }^{724}$ or the burials from Stanway in Colchester. ${ }^{725}$

A separate, though by no means mutually exclusive, custom begins to appear in the neighbouring regions of East Anglia and Kent just as the lavish Late Iron Age and Conquest Period burials recede in practice: the Jug and Pan type burial. This type of burial is characterised by the inclusion of a jug with a Handled Pan 1 and this burial custom has been recognised as a pattern in Hellenistic and Roman early Imperial burial practice. ${ }^{726}$ It is a practice that is by no means specific to Britain and indeed appears to have been well practiced across the empire, particularly along the limes. ${ }^{727}$ The association of jugs and Handled Pan 1s can also be seen in Rome itself. Their depiction in funerary art is particularly prolific, further evidencing the association these objects had with grave ritual. Depictions of jugs and handled pans appear on funerary monuments from Rome itself, such as on funerary altars from the Appian Way now on display at the Baths of Diocletian (Figure 4.42).

\footnotetext{
${ }^{721}$ Cunliffe 2005, 559-570; Fitzpatrick 2000, 15-29; Fitzpatrick 2007 123-142; Pearce forthcoming.

${ }^{722}$ Carver 2001, 31-39.

${ }^{723}$ Niblett 2004, 31 .

${ }^{724}$ HER0019; Niblett \& Reeves 1990, 441-446; Frere 1991, 259; Niblett 1999, 394-418.

${ }^{725}$ Crummy et al. 2007.

${ }^{726}$ Nuber 1973, 33-73 \& 144-188; Philpott 1991, 124.

${ }^{727}$ Nuber 1973, 210-220.
} 


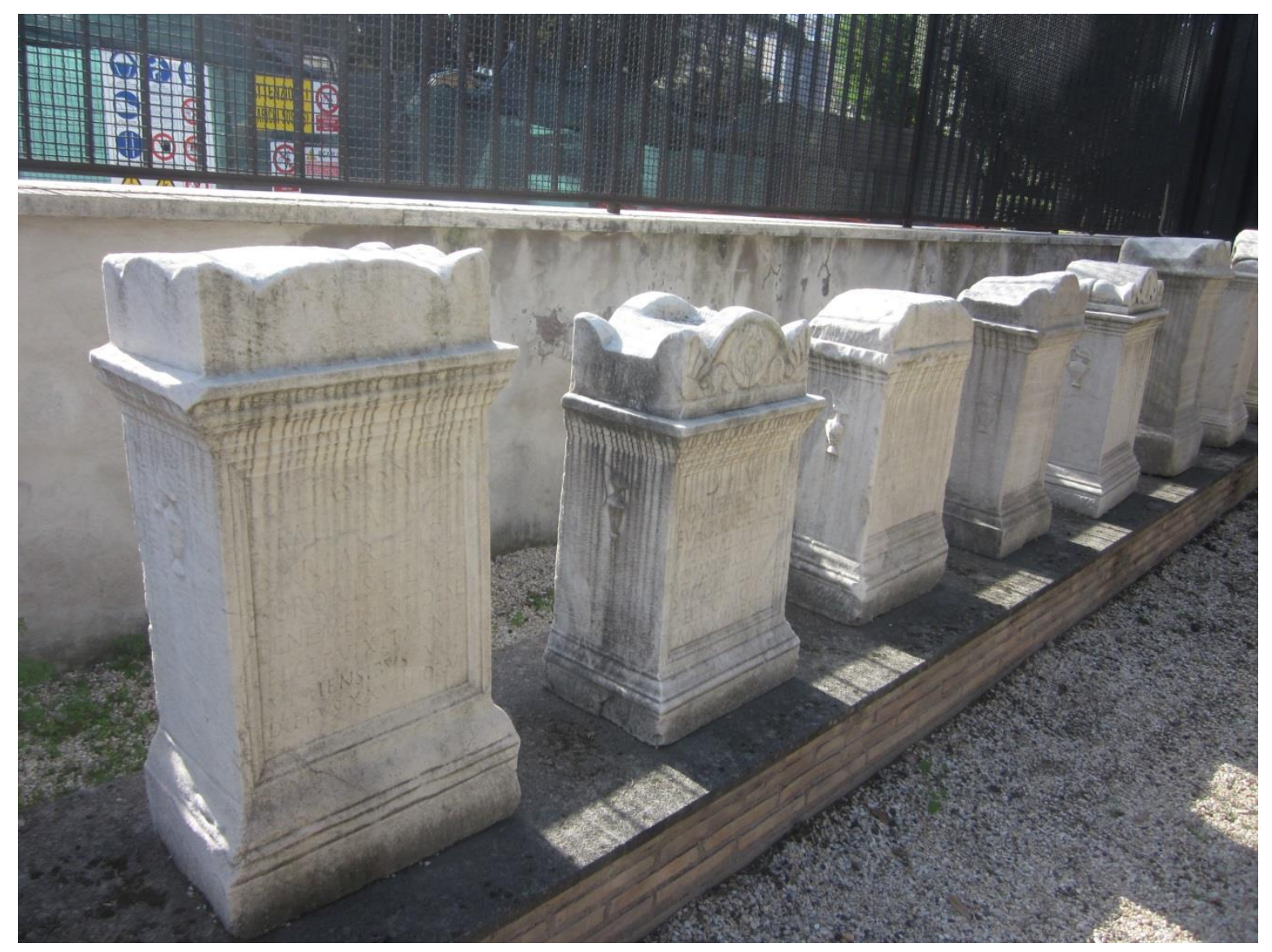

Figure 4.42: Funerary altars from the Appian Way now on display at the Baths of Diocletian, Rome (photo by author)

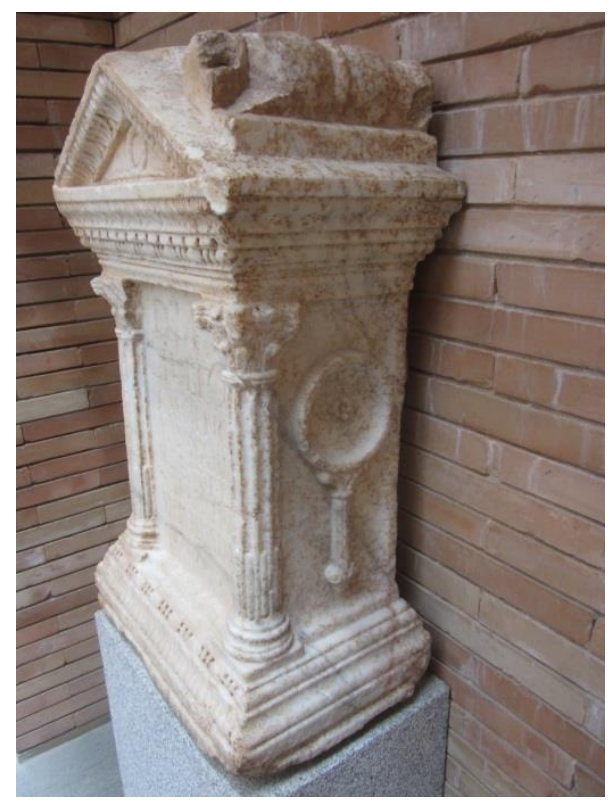

Figure 4.43: Funerary altar on display in the Museo de Arte Romano, Merida (photo by author) 
These depictions are also present in numerous funerary altars of Merida in Spain currently on display in the Museo de Arte Romano (Figure 4.43). The combined use of jugs and pans in the religious ritual of Roman Britain is well attested by the presence of some 46 altars depicting this combination represented in the Corpus Signorum Imperii Romani, a good example of such objects being the sandstone altar from Maryport in Cumbria on display at the British Museum (Figure 4.44). ${ }^{728}$

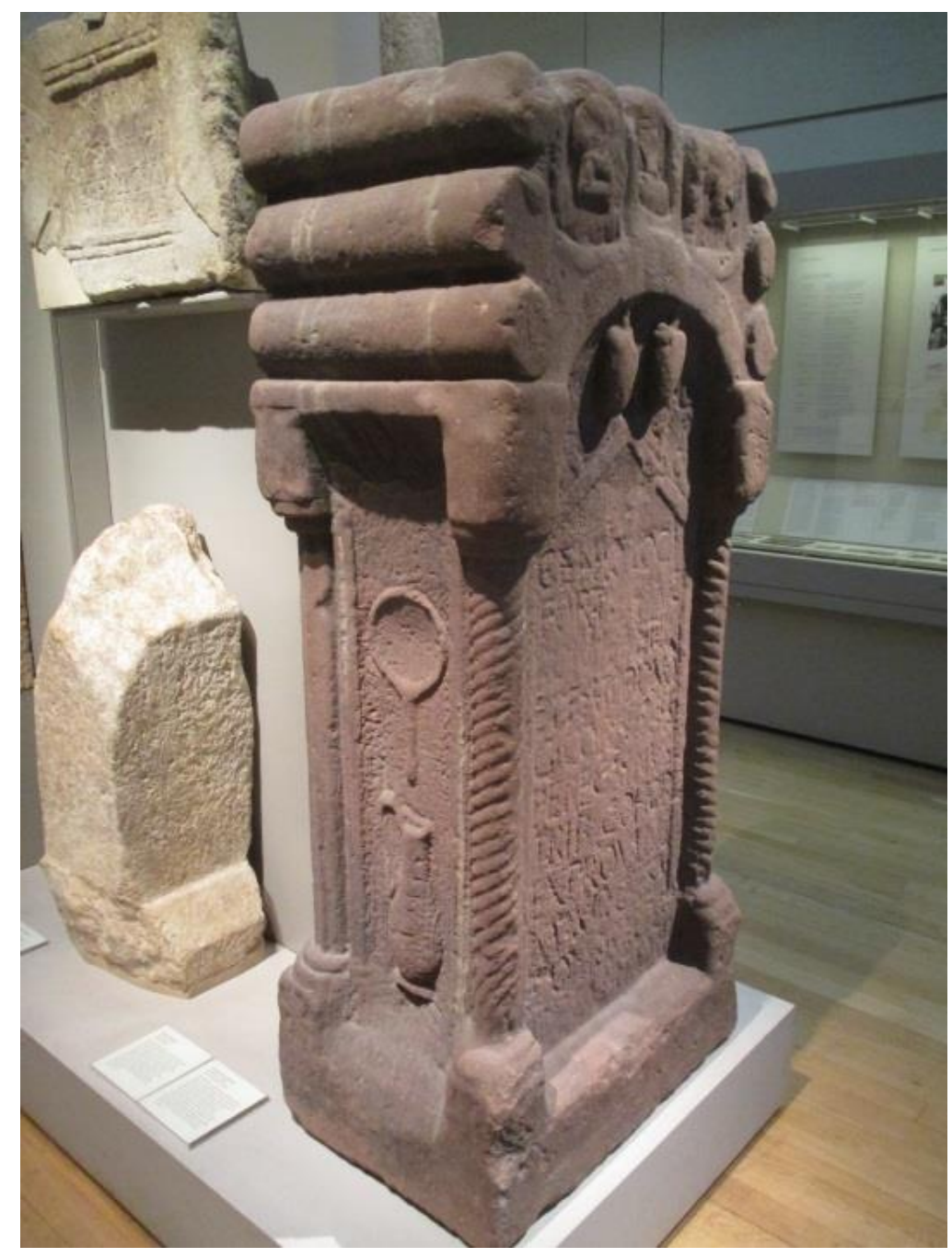

Figure 4.44: Jug and Handled Pan 1 depicted on a funerary altar on display in the British Museum (photo by author)

${ }^{728}$ RIB 812 


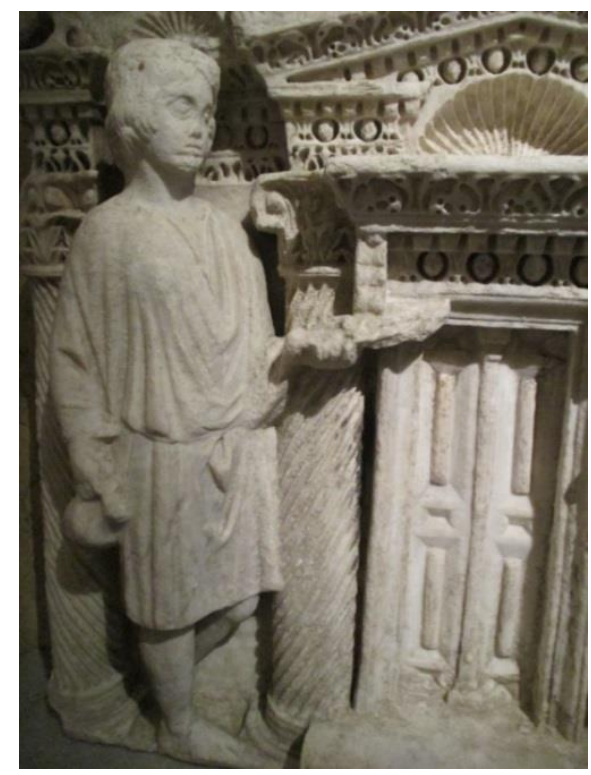

Figure 4.45: Funerary relief on display in the

Kunst Historisches Museum, Vienna (photo by author)

A funerary relief currently in the Kunst Historisches Museum in Vienna shows a servant bearing these two vessels ${ }^{729}$ (Figure 4.45) while direct reference to the vessels being paired for hand-washing are shown in depictions of Pilate washing his hands after the judgement of Jesus both on an ivory relief in the British Museum collection ${ }^{730}$ (Figure 4.46) and on the sarcophagus of Junius Bassus in the Vatican Museums (Figure 4.47). ${ }^{731}$

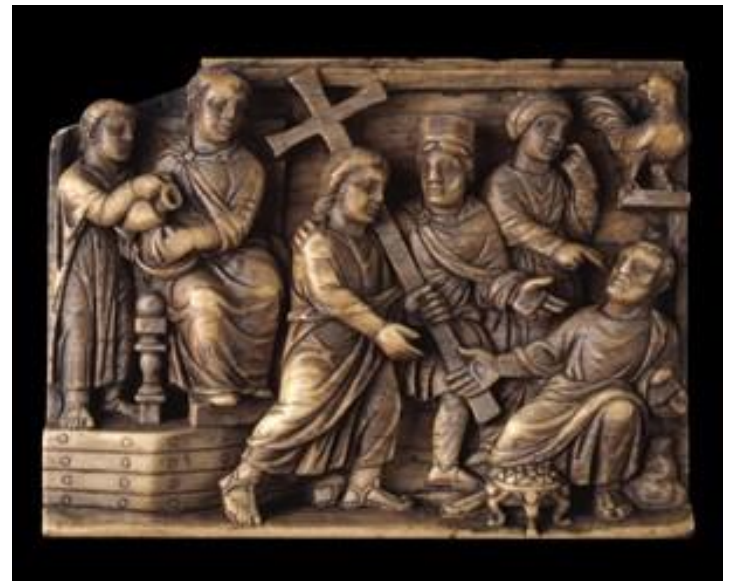

Figure 4.46: Ivory relief of Pilate washing his hands after the judgement of Jesus ( (C) the British Museum)

\footnotetext{
${ }^{729}$ KMN I 1143.

${ }^{730}$ BM 1856,0623.4.

${ }^{731}$ Malbon 1990.
} 


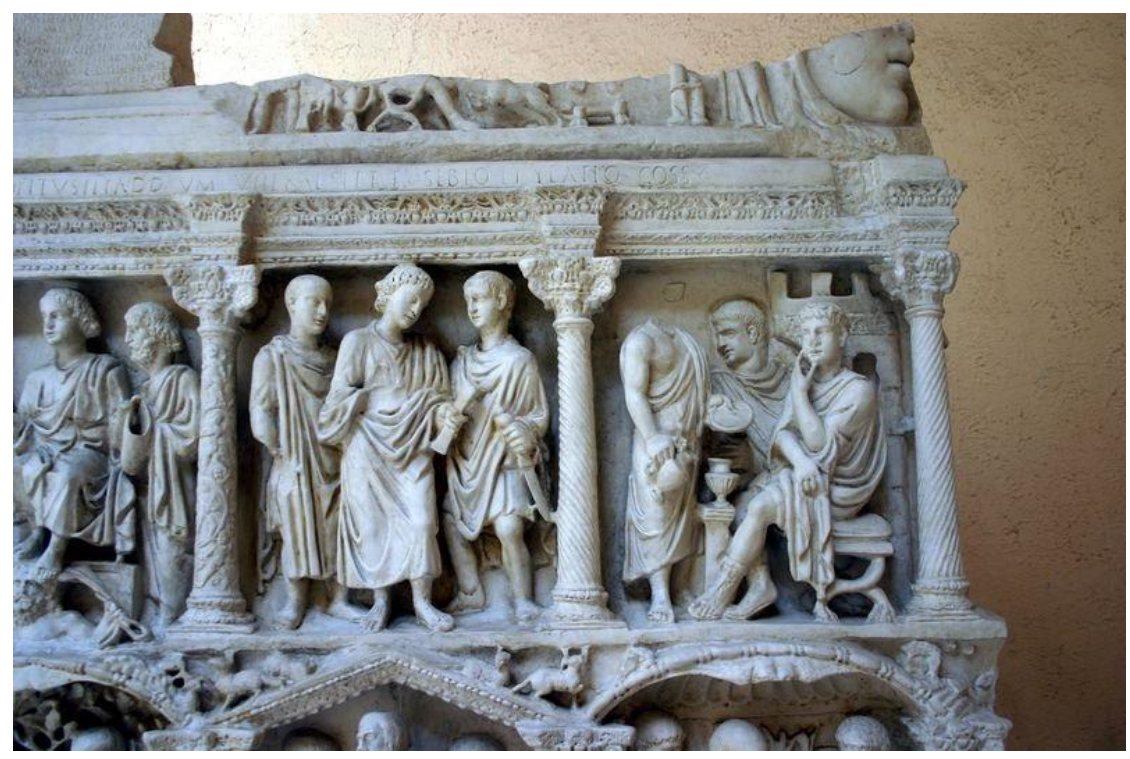

Figure 4.47: Sarcophagus of Junius Bassus (ㅇ the Vatican Museums)

Additionally, a manuscript of the Aeneid, also in the Vatican collections, has an illustration of a banquet scene where a servant is holding a jug and handled pan (Figure 4.48). ${ }^{732}$ These examples are by no means extensive, though they serve to illustrate the association between these vessel forms and their function for hand washing as well as their association with funerary ritual. At the funerary service, they could have played a part in the purification ablutions associated with offering a sacrifice or perhaps served for hand washing at a funerary feast; these would also be the most likely symbolic purposes they would serve in the grave furniture. It is also worth brief mention that while paired vessels of these forms have been found constituted of other materials, ${ }^{733}$ copper alloy is far more common both on the continent and in Britain.

\footnotetext{
732 Hobbs 2012, 49.

${ }^{733}$ Nuber 1973, 73-82; Tuffreau-Libre 2000, 53-54.
} 


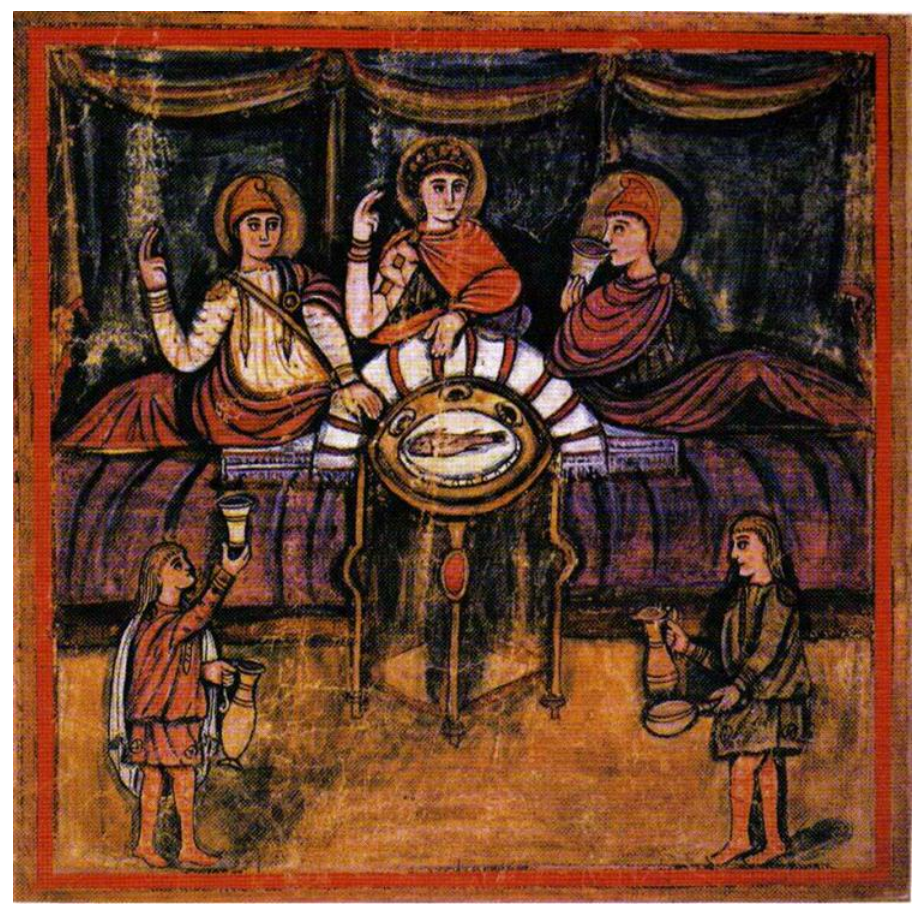

Figure 4.48: Illustration from 4th century manuscript of the Aeneid, currently in the Vatican Collections (Hobbs 2012, 49 Fig.32)

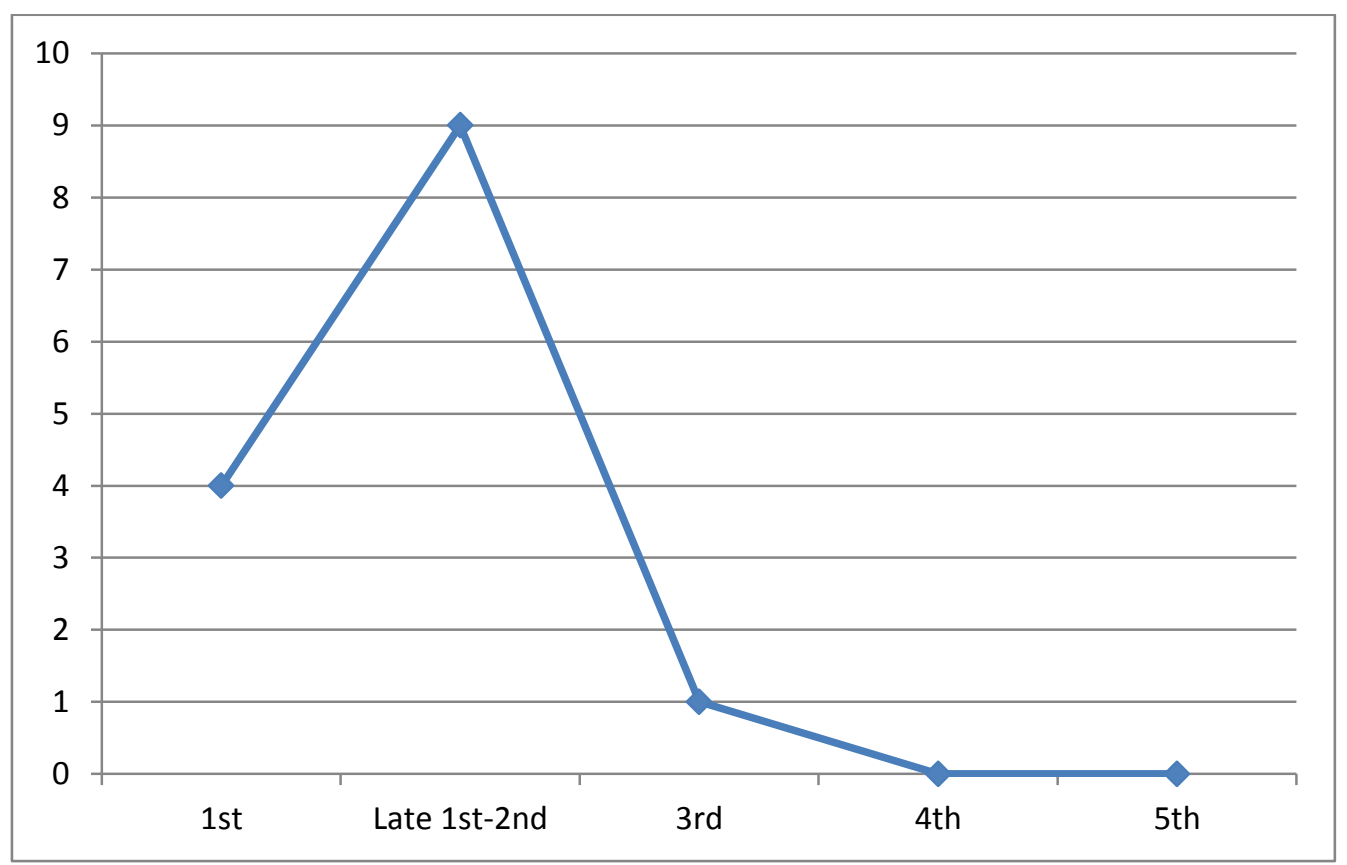

Figure 4.49: Graves with both HP1 \& jugs in Roman Britain over time. 
As Figure 4.49 illustrates, the practice of interring a jug and a Handled Pan 1 with cremation burials in Britain was practiced during the $1^{\text {st }}-2^{\text {nd }}$ century CE and then fell out of general practice during the course of the $3^{\text {rd }}$ century CE, ending the interring of copper alloy vessels with any sort of regularity in Britain until the Anglo-Saxon period. There are occasional exceptions to this, most notably the Roman Cemetery at Brougham where buckets and bowls are the principal vessels interred or the solitary jug buried in a cremation at Llantilio Pertholey. ${ }^{734}$ Such burials would seem to reflect practices of a small imported minority group and reflect isolated anomalies as opposed to any lasting culture practice in Britain, ${ }^{735}$ though they do in turn offer an example of the cultural diversity that existed in Britain during the Roman period.

The period of depositing copper alloy vessels as grave goods in Britain coincides with a period of cultural change and amalgamation between Iron Age tribal and Roman central hegemonies in Britain and the use of these objects as grave furniture expresses cultural identity, status and authority in terms of leisure and ritual activity as opposed to a primarily martial understanding of power and authority. ${ }^{736}$ Similar expressions of elite identity developing through the Iron Age and into the early Roman period have been noted in Belgic Gaul ${ }^{737}$ and the increased value placed on domestic goods as opposed to militaria is noted in the development of aquatic votive deposits in Britain, with weapons dominating the deposits of the Iron Age being replaced with vessels and dress accessories during the Roman period. ${ }^{738}$ It would appear that the burial practice of including large assemblages of luxury objects would reflect the developing identity of a landed elite from a warrior elite, using wealth display in the same way Bronze and Iron Age predecessors did, but substituting objects that drew association with a leisure-loving landed aristocracy operating within the civil sphere of authority in line with the rising acceptance of the Roman administrative model.

With a few possible exceptions, the inclusion of copper alloy vessels as grave goods was not a part of Late Roman burial practice in Britain. A bowl containing coins of $4^{\text {th }}$ century CE date was allegedly found associated with a Late Roman cemetery in Wall, Staffordshire. ${ }^{739}$ However, this is far more likely to be a Structured Deposit of coins than a Grave Deposit as it is

\footnotetext{
${ }^{734}$ Cool 2004; C0001-C0020; PAS NMGW-9A9D16; MON0007.

${ }_{735}$ Cool 2004, 463-467; Cool 2010, 27-43; Pearce 2010, 83 \& 89-93.

${ }^{736}$ Roymans 1996, 9-103.

${ }^{737}$ Roymans 1996, 9-126.

${ }^{738}$ See Section 3.4.

${ }^{739}$ STA0008; see Section 3.5.
} 
not recorded as found in a grave and a coin hoard is not a common grave inclusion. ${ }^{740} \mathrm{~A}$ bowl of undiagnostic date from a cremation in Canterbury could also be Late Roman, ${ }^{741}$ but could be as early as $2^{\text {nd }}$ century CE. Even if such isolated cases are taken into account, it would seem that the inclusion of copper alloy vessels ceased to be practiced on anything near the scale with which it had been during the early Roman period. This relative paucity of material for the Late Antique period should not be surprising, as Philpott notes a general decline in the interment of grave goods of all varieties in the Late Roman period, ${ }^{742}$ though grave goods would again become prominent in the Anglo-Saxon or early medieval period. ${ }^{743}$ This decline in the use of grave goods as a means of constructing and communicating identity may reflect a decrease in the need to express cultural understanding and allegiance through such objects as they became more available to a wider segment of the population. ${ }^{744}$ It would consequently seem that the values and principles of identity being expressed through the use of luxury objects, such as copper alloy vessels, in dining and funerary ritual were so ubiquitously understood by the surrounding society that explicitly expressing such understanding through grave furniture proved repetitive and unnecessary (such an assertion would be supported by the high level of $3^{\text {rd }}$ and $4^{\text {th }}$ century material in the Site Finds and PAS data sets, discussed in Chapters 5 and 6 respectively). This is a particularly intriguing possibility when considering the domestic values implied by the use of copper alloy vessels in graves, indicating that it was not seen as necessary to indicate one's association with a culture of leisurely wining-and-dining as this was taken as a given among the elites of Britain after a century of Roman occupation. This decline in grave furniture could also be related to shifts in religious belief and how this would be expressed in ritual practice, such as the impact the rise of Christianity may have had on ritual practice and object assemblages. ${ }^{745}$ The chronological patterns in vessel size and forms selected for interment seen in Structured Deposits also apply to a limited degree to the finds from graves: namely that smaller vessels such as handled pans were preferred in the early Roman period and that buckets came to be favoured in the middle to later Roman periods (Figure 4.50). As the inclusion of copper alloy vessels dropped out of favour in Britain by the Late Roman period, the absence of large basins such as

\footnotetext{
${ }^{740}$ Philpott 1991, 209.

741 Smith 1922, 86.

${ }^{742}$ Philpott 1991, 225; Cool 2010, 3-4.

${ }^{743}$ Gerrard 2013, 195-205 \& 263.

744 Struck 2000, 87.

745 Philpott 1991, 220-228; Mawer 1995; Harris \& Henig 2010, 25-38.
} 
Irchester bowls is natural and does not necessarily reflect a conscious exclusion of these objects from burial ritual in favour of other vessel forms.

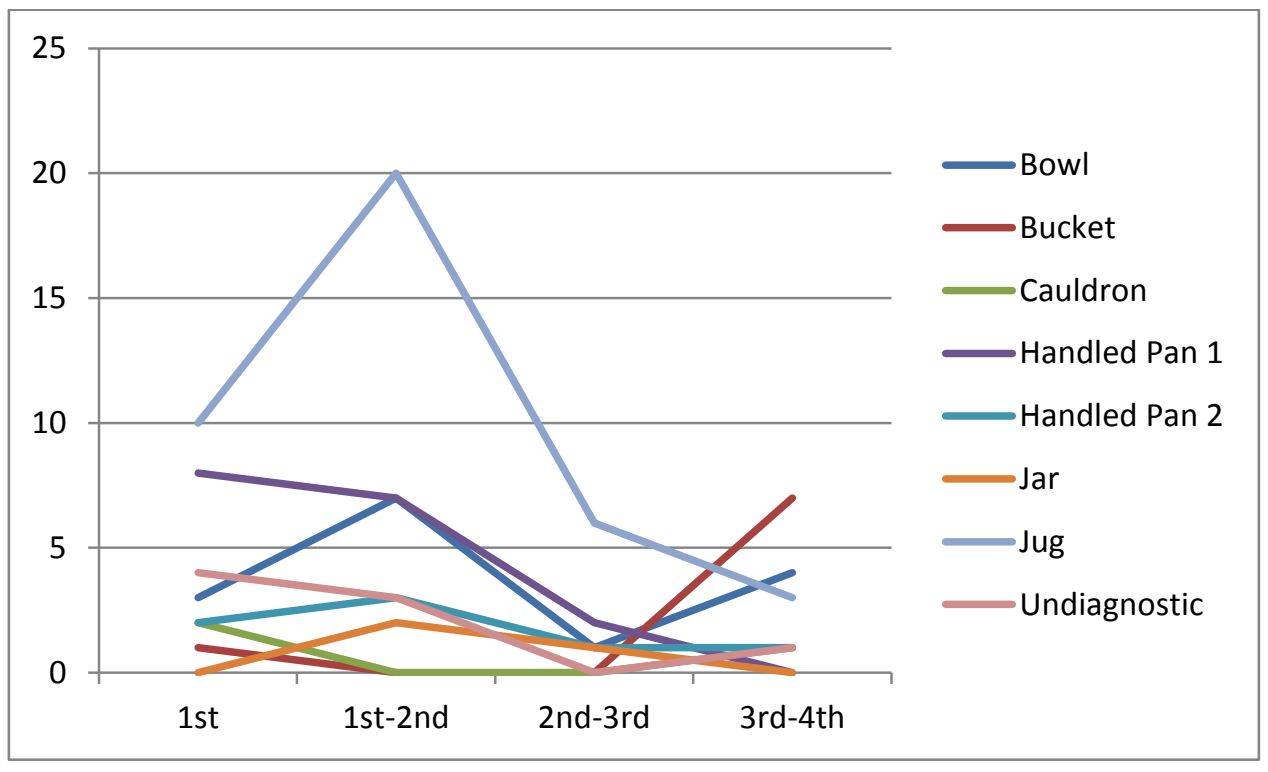

Figure 4.50: Vessel forms in graves over time.

From the data available, Handled Pan 1s are seen as preferentially interred as grave objects compared to any other method and this implies that they were viewed and treated in a different manner than other copper alloy vessels. It would appear that this vessel was of particular significance directly related to funerary and ritual practice. ${ }^{746}$ The significance of Handled Pan 1s and their probable importance within grave ritual is best understood through the decoration which they feature and will be discussed in the following section.

${ }^{746}$ Nuber $1973,90-95 \& 144-188$. 


\section{$\underline{\text { 4.4 Discussion of Decoration in Grave Deposits }}$}

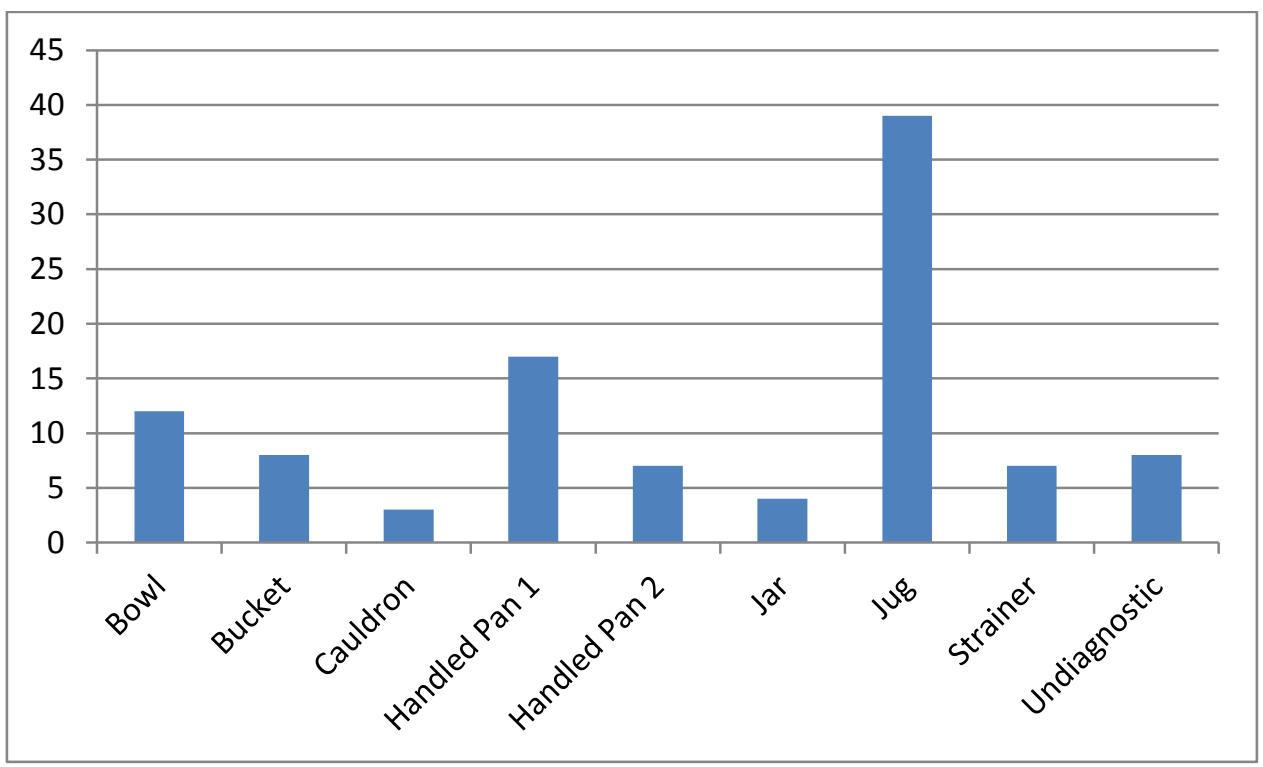

Figure 4.51: Vessel forms deposited in Romano-British graves.

The material that constitutes the Grave Deposits portion of this thesis is more richly embellished than that of the Structured Deposits data-set. This could be principally due to the selection of vessels interred in graves, whose forms are summarised in Figure 4.51. Jugs, the most frequent vessels to be found in Romano-British graves, often have decorated handles while jars, bowls and cauldrons may often be undecorated. This discrepancy in decoration between forms accounts for the higher level of decoration in Grave Deposits in comparison to Structured Deposits. Another interpretation is that the iconography and decorative motifs exhibited on vessels interred as grave goods had directly applicable significance to Roman funerary ritual and practice. Upon reviewing the data available in this thesis, this would appear to be the best explanation for the presence of decoration in grave vessels, as will be explained below. Inscriptions only appear on two of the vessels in the Grave Deposits data-set of this thesis, ${ }^{747}$ neither of which appear to be dedicatory inscriptions and neither of which appear to have direct relation to the funerary ritual besides representing vessels that had a functional use-life prior to their interment in graves.

Concerning decoration on specific forms and types of vessels, the jugs and Handled Pan 1s are by far the most elaborately adorned. The most consistent iconographic feature of these

\footnotetext{
${ }^{747}$ K0020 \& BE0004.
} 
vessels would be the zoomorphic handle terminal on the Handled Pan 1s. It is most commonly in the form of a ram's head, but also takes the form of a canine or a bear and is occasionally anthropomorphic (Figure 4.52).

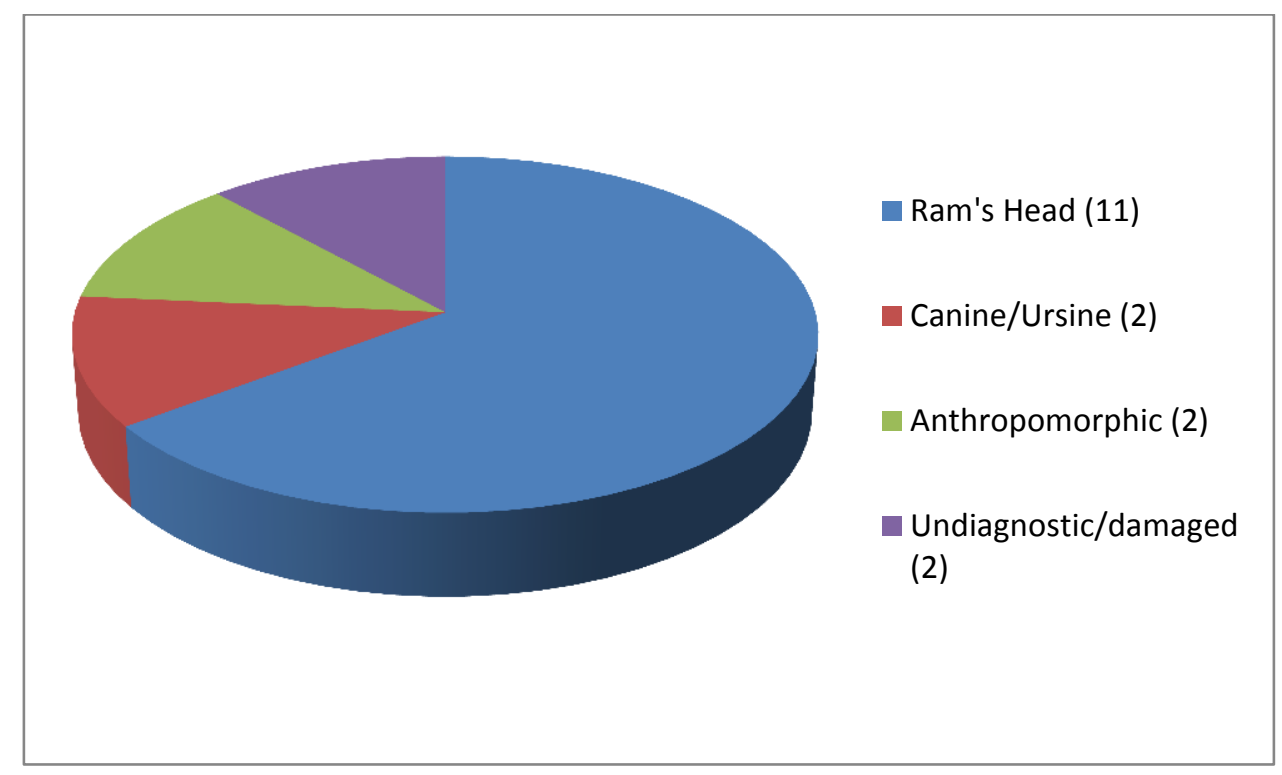

Figure 4.52: Busts on handle terminals for Handled Pan 1s in Romano-British graves.

This consistent feature led for this variety of handled pan to be considered separately from other forms of handled pans as well as its consistent association with funerary contexts as opposed to other Depositional Contexts. Comparison with the depositional patterning of other forms of handled pans appears to justify this distinction as it indicates that it served specific and separate functional and associative roles. As the decorative scheme of these objects is so narrowly uniform, it is tempting to assert that this decoration was somehow referential to the use of the object itself or at least symbolically associated with its functions through metaphor. Nuber makes a comprehensive argument that Handle Pan 1s were used in hand washing, ${ }^{748}$ an argument that is generally accepted in modern scholarship and that the evidence from this thesis does not contradict. The importance of ritual bathing of hands and feet for purification, both in monotheist and polytheist traditions is well attested in antiquity. ${ }^{749}$ This is evidenced by the presence of a Handled Pan 1 in the copper alloy vessel hoard found in the Cave of Letters dating to the Bar Kokhba rebellion during the reign of Hadrian and argued by some to be part of the

\footnotetext{
${ }^{748}$ Nuber 1973.

${ }^{749}$ Harris \& Henig 2010, 25-29.
} 
Temple Treasure from Jerusalem, it is decorated with a ram's head handle medallion as well as a surprisingly pagan scene of Thetis and Achilles. ${ }^{750}$ Rites of purification are often associated with funerary ritual across cultures, ${ }^{751}$ further reinforcing Nuber's argument. This functionality being the likely case, the head of a ram may be an appropriate design for an object intended for ritual hand washing associated with a sacrifice. The iconography was then transferred to the vessel form in its wider usage outside of the specifically sacrificial, such as for hand washing for dining or as part of funerary ritual. In general terms, the decoration of Handled Pan 1s is largely selfreferential.

Rams are also associated with Mercury, ${ }^{752}$ and in such capacity may have had a symbolic significance in a funerary setting as Mercury was a messenger between the lands of the living and the lands of the dead. ${ }^{753}$ This association with Mercury in Romano-British graves is reinforced by the presence of a Handled Pan 2 from a Grave Deposit at Stanfordbury Farm in Shefford that was decorated with a caduceus, ${ }^{754}$ also an attribute of Mercury. ${ }^{755}$

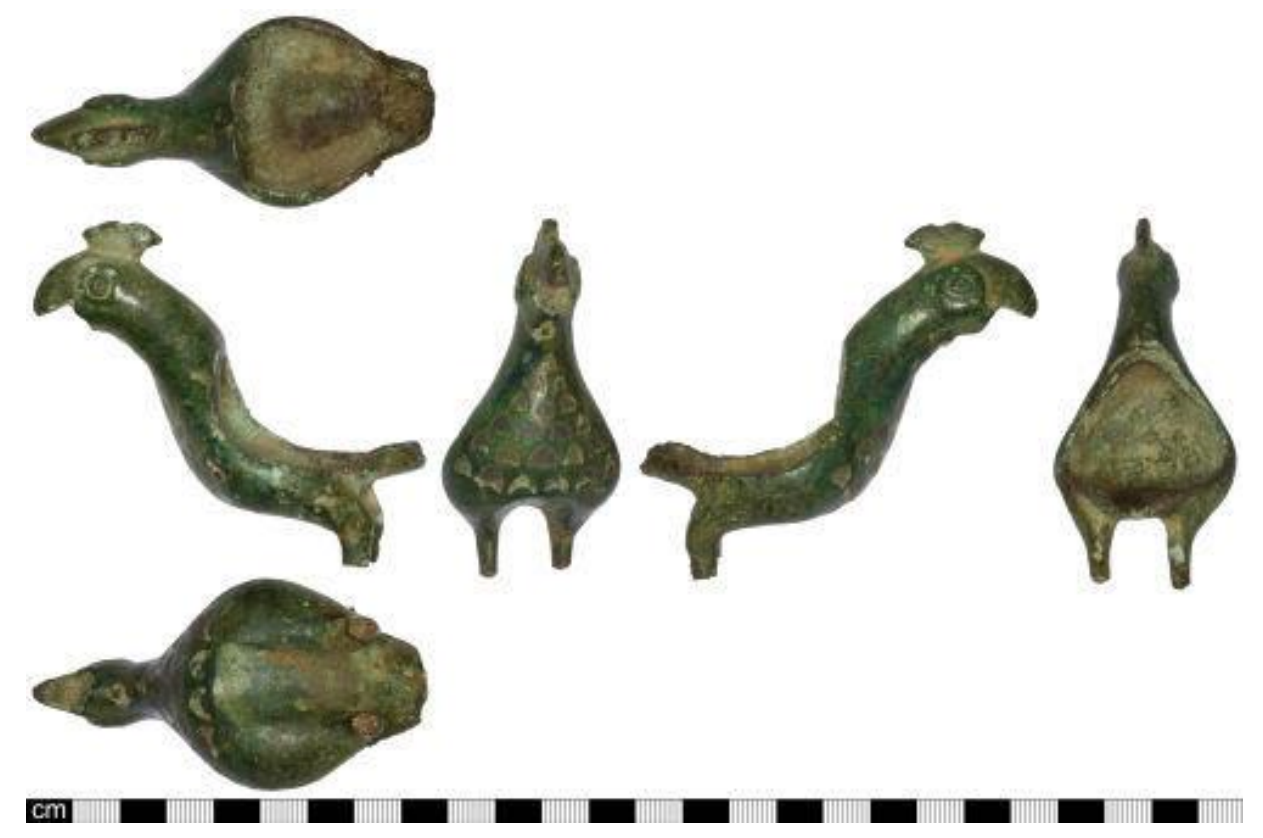

Figure 4.53: Enamelled rooster from Cople in Bedford (PAS SOM-745EA2)

\footnotetext{
${ }^{750}$ Freund 2004, 141-146 \& 219-221.

${ }^{751}$ Bendann 1930, 121-135; Metcalf \& Huntington 1991, 62-132.

${ }^{752}$ Howatson 1989, 273; Adkins \& Adkins 1996, 152.

${ }^{753}$ Howatson 1989, 273.

${ }^{754}$ BE0004; Eggers 1968 104-105 (29Aa); RIB II 2415.51.

${ }^{755}$ Adkins \& Adkins 1996, 152.
} 
A further example of the use of attributes of Mercury in a funerary setting is the curious phenomenon of enamelled rooster statues, discovered sporadically in Britain and the continent (Figure 4.53). ${ }^{756}$ A recently excavated example from Cirencester comes from a grave, ${ }^{757}$ drawing a further direct association between Mercury and burial rites in Britain during the Roman period. It therefore seems probable that the use of ram's head iconography on Handled Pan $1 \mathrm{~s}$ is to be associated directly with sacrifice, purification and funerary ritual, the more so being that this decoration is specifically linked to Mercury, messenger between the living and the deceased.

Two of the Handled Pan 1s from Grave Deposits in this thesis were decorated with canine handle terminals, with further examples found in unstratified contexts through the PAS. ${ }^{758}$ Dogs in ancient literature were identified as being objects that absorb negative and impure energies. Plutarch comments very specifically on this aspect as dogs were sacrificial victims in both Greek and Roman rites of purification (Plutarch, Roman Questions, 68). In this light, dogs as handle medallions for this type of vessel could offer a symbolic association with the object's use in rites of purification and the basin acting as a receptacle for the unclean influences washed away through the ablution process. Additionally, the association of dogs with the underworld, often as guardians of the spirits of the dead, is a common idea found throughout the art and literature of the ancient Mediterranean world as well as being attested as far afield as Kazakhstan, Scandinavia and even pre-Columbian North America. ${ }^{759}$ The goddesses Diana and Hecate are particularly known to have had dogs sacrificed to them ${ }^{760}$ and the ritual torturing and killing of dogs as part of funerary and fertility rituals was a feature of rural Greek folk practice until as recently as the 1980s. ${ }^{761}$ The presence of dogs as ritual deposits at funerary sites in ancient Gaul and Britain is also indicative of this association between dogs and the spirit world. ${ }^{762}$ The importance of dogs to ritual belief in Britain is further supported by the presence of canine statuary at temples sites such as at Lydney in Gloucestershire and Pagans Hill in

\footnotetext{
${ }^{756}$ Worrell 2012, 81-84.

${ }^{757}$ McSloy \& Watts 2013, 1-5.

${ }^{758}$ Chapter 6.

${ }^{759}$ Toynbee 1973, 102-124; Aldhouse-Green 2004, 136; Sergis 2010, 64-65.

${ }^{760}$ Reitler 1949, 30; Sergis 2010, 61-74.

761 Sergis 2010, 61-80.

762 Aldhouse-Green 2004, 138; Score 2011, 103-135.
} 
Somerset. ${ }^{763}$ All this would make the presence of vessels with such iconography particularly appropriate in funerary or ritual settings.

To offer additional complication to the iconographic patterning of these vessels, the presence of anthropomorphic Bacchic busts on Handled Pan 1s suggests a possible use in drinking ceremonies, ${ }^{764}$ though this would also likely have been in the capacity of hand washing. It would seem likely that Handled Pan 1s were manufactured with several circumstances of ablutions in mind, both for secular household and religious use, ${ }^{765}$ and that the iconography chosen to adorn them was associated with this aspect of their use.

Many of the jugs accompanying these Handled Pan 1s have proficiently and elaborately decorated handles, some of which depict scenes featuring gods or mythological heroes and creatures. The iconography on the jugs does not have any immediate funerary significance and it would appear that the vessels were not manufactured with ritual function specifically in mind, as would certainly appear to have been the case with Handled Pan 1s. The iconography on the jugs from the Turners Hall Farm graves provides a good example of the elaborate, though not necessarily referential decoration that features on jugs in funerary settings in Roman Britain. ${ }^{766}$ The ornate statuette of a Triton from one of the jugs is a direct reference to the aquatic realm. The possible associations between aquatic motifs and the underworld or passage between life and the afterlife have been previously discussed in Section 3.4 of this thesis and it could be argued that such association was meant to be drawn with the use of this object in this setting. The Gorgon head on a further jug in this group could also serve a ritual function in funerary ritual, as the Gorgon bust had an apotropaic function. ${ }^{767}$ However, the presence of Minerva does not appear to have any specific funerary attachments in-and-of herself. This could be more symptomatic of the particular tastes and passions of the deceased interred than reflecting iconography that may be generally associated as funerary. Indeed, the presence of weapons in this female grave would suggest association with martial femininity and makes it likely that the jug in question does reflect a predilection of the interred to the worship of Minerva or an indigenous deity syncretised with Minerva, such as is evident with the goddess Senuna from

\footnotetext{
${ }^{763}$ Boon 1989, 201-217.

764 Such as K0010.

765 Nuber 1973, 83-95.

${ }^{766}$ HER0031-HER0042; Section 4.5.

${ }^{767}$ Wilk 2000, 31-54.
} 
Ashwell. ${ }^{768}$ Direct relevance with this sort of decoration with a funerary setting is difficult to draw and it proves unwise to force such association.

The jugs from the Bayford graves, one decorated with a harpy and the other depicting the madness of Ajax, ${ }^{769}$ also do not appear to have immediate relevance to a funerary setting. This suggests that these vessels were not specially made for burial and that they were functional objects used in life prior to being deposited. Their subject matter does instead indicate knowledge of classical motifs, particularly the Ajax jug which portrays a very specific mythological scene, and would likely have been an expression of paideia and identified their owners as belonging to the cultured class of individuals who would understand these images.

Another god, besides Mercury, who appears to be commonly referenced in the decoration of copper alloy vessels in Romano-British graves is Bacchus. This is most obvious in satyr handle medallions, such as the satyr head handle medallion from a Handled Pan 1 from Bayford, Kent. ${ }^{770}$ Bacchic imagery may also be seen in floral decorations that could represent vines that are exhibited on a number of objects in this data-set. Perhaps the clearest example of this is the leopard-handled jug from Llantilio Pertholey in Monmouthshire. ${ }^{771}$ Bacchus, as a god of ecstasy and resurrection, is an appropriate god for a funerary context. In fact, Bacchic iconography is also prevalent in funerary art across the western empire, particularly funerary relief sculpture. ${ }^{772}$ However, his association with copper alloy funerary vessels specifically should not be taken too far, as he is the god of wine and therefore is perfectly suitable to the decoration of drinking or table vessels outside of the funerary context. Bacchus consequently appears to be the most common god associated with copper alloy vessels in Roman Britain across depositional contexts and therefore Bacchic iconography present in grave assemblages of copper alloy vessels could simply be reflecting the wider usage of this iconographic motif on drinking and dining vessels and is not specifically referential to the funerary setting. It is also likely that the objects bearing Bacchic imagery were not designed specifically for funerary purposes and were used as objects in the living world before being interred, such as at dining parties or as part of a symposium, and were seen as objects with fitting and comforting symbolism for the journey to the afterlife. ${ }^{773}$

\footnotetext{
768 Jackson 2007, 37-54.

${ }^{769} \mathrm{~K} 0007, \mathrm{~K} 0011$.

${ }^{770}$ BM 1883,1213.299.

${ }^{771}$ MON0007.

772 Turcan 1966.

${ }^{773}$ Hutchinson 1986, 12-121.
} 
Another decorative element which occurs repeatedly in these grave assemblages is the use of the bull's head or the bull's skull (bucranium). While it is most common as a hanging vessel mount, it also appears on jug handles. The use of the bull's head has a long history in Britain, spanning from the Iron Age well into the Late Antique Period, ${ }^{774}$ so its prevalence in Romano-British graves simply reflects the popularity of this decorative motif in the society generally as opposed to reflecting specific tastes for funerary representation, though its symbolism for virility and sacrifice is obvious and may have influenced the objects' placement in graves. This broad popularity is evident from the multiple examples in the Site Finds and PAS data-sets of this thesis.

A further example of decorative patterning in Grave Deposits is seen in the small enamelled jars found in several graves across Britain, whose multi-coloured geometric patterning is indicative of an indigenous decorative tradition. ${ }^{775}$ Though the decoration on these vessels is ornate, it is difficult to draw any particular significance between the geometric designs and burial ritual specifically. It is far more likely that they represent largely cultural trends in decoration, which are discussed in Chapters 7 and 8.

The decoration of the funerary vessels in the later Roman period is simpler than from $1^{\text {st }}$ and $2^{\text {nd }}$ century contexts, though this may be due more to the choice of forms interred than any conscience choice or economic factor contributing to the decrease in complex iconography. Buckets and bowls predominate the forms in later graves, as indeed these forms also predominate later Roman Structured Deposits. ${ }^{776}$ These forms are less richly decorated in the other data-sets of this thesis, as well as in examples from continental surveys such as those of Eggers, Tassinari, and Radnoti. It would appear that there may have been a general decrease in complex figural decoration even in the less decorated bucket forms than may have been exhibited earlier in the Roman period, with examples from Pompeii and Belgic/Treveran graves providing highly decorated earlier examples of the same forms. ${ }^{777}$ Consequently, the drop in complex iconography in the few graves of post $2^{\text {nd }}$ century date in Roman Britain should not be seen as a conscience rejection of the use of this iconography in funerary settings, but simply

\footnotetext{
${ }^{774}$ Hawkes 1951.

${ }^{775}$ Breeze 2012, 107-112; Künzl 2012, 9-22.

${ }^{776}$ See Section 3.4.

777 Tassanari 1975; Roymans 1996, 35-37; See Chapter 7-8.
} 
reflecting the general decrease in the use of elaborate decorative schemes on copper alloy vessels more generally during this period.

Unlike the decoration of funerary monuments, copper alloy vessels interred in graves appear to have had use-lives before their use as grave furniture and their decoration reflects this, though it appears likely that objects may have been selected on the basis of how appropriate their decoration was to a funerary setting. While the jugs and Handled Pan 1s do seem specifically to have potential ritual and/or funerary associations, there is no reason to believe that they were specifically manufactured for funerary contexts. Instead, much of the decoration may more likely have reflected the tastes of the interred or the individuals performing the ritual of burial. Mercury and Bacchus do have specific funerary associations, but their prevalence on copper alloy vessels broadly across depositional contexts means that their presence on such objects in graves does not inherently represent preferential iconographic selection. The range of decorative schemes and motifs, as well as the wear patterns and repairs visible on many examples, suggests that objects of active use in life were selected for funerary ritual as opposed to specific copper alloy vessels being manufactured for the purpose exclusively. The decoration offers comforting analogies for ease and plenty, perhaps in this world to facilitate the funerary feast of the mourners or perhaps in the next to comfort the departed on their journey to the here-after.

\section{$\underline{4.5 \text { Associated Finds, Functionality and Burial Status }}$}

The Grave Deposits data-set of this thesis comprises Roman burials excavated from antiquarian times to the present date and recorded with varying degrees of accuracy and completeness. For this reason, it is impossible to make a completely accurate survey of how objects were placed in relation to other objects in the graves or what sex the interred may have been. Only 57 graves in total are figured in the current thesis, which increases the impact of recording and reporting discrepancies on the visualizing of any meaningful patterns. However, there is enough knowledge concerning the grave goods as a whole to discuss common trends, especially as some of the graves have been meticulously excavated and reported thoroughly enough to make statements concerning the relation between grave goods in the burial possible.

As illustrated in Figure 4.54, the most common variety of objects to be found in graves containing copper alloy vessels is ceramic vessels. Most, though not all, of these ceramics are 
fine-ware as opposed to coarse. These occur in the largest numbers in the lavish Late Iron Age burials of south-eastern England that continued through to the latter half of the $1^{\text {st }}$ century CE. Though most of the ceramics included in graves with copper alloy vessels are bowls, platters and cups are present in much smaller numbers. It should not come as a surprise that ceramics are found in many of the graves that include copper alloy vessels, as ceramics are very often found in graves from the Roman period more generally. ${ }^{778}$ It may be assumed in some cases that the copper alloy vessels were intended to fulfil the same functions as the ceramic vessels, but in a material of higher expense and prestige. This may be seen in the grave from King Harry Lane in St. Albans, where the copper alloy bowl was placed near ceramic bowls of similar size and dimension and would likely have had a complimentary function to this samian ware set. ${ }^{779}$

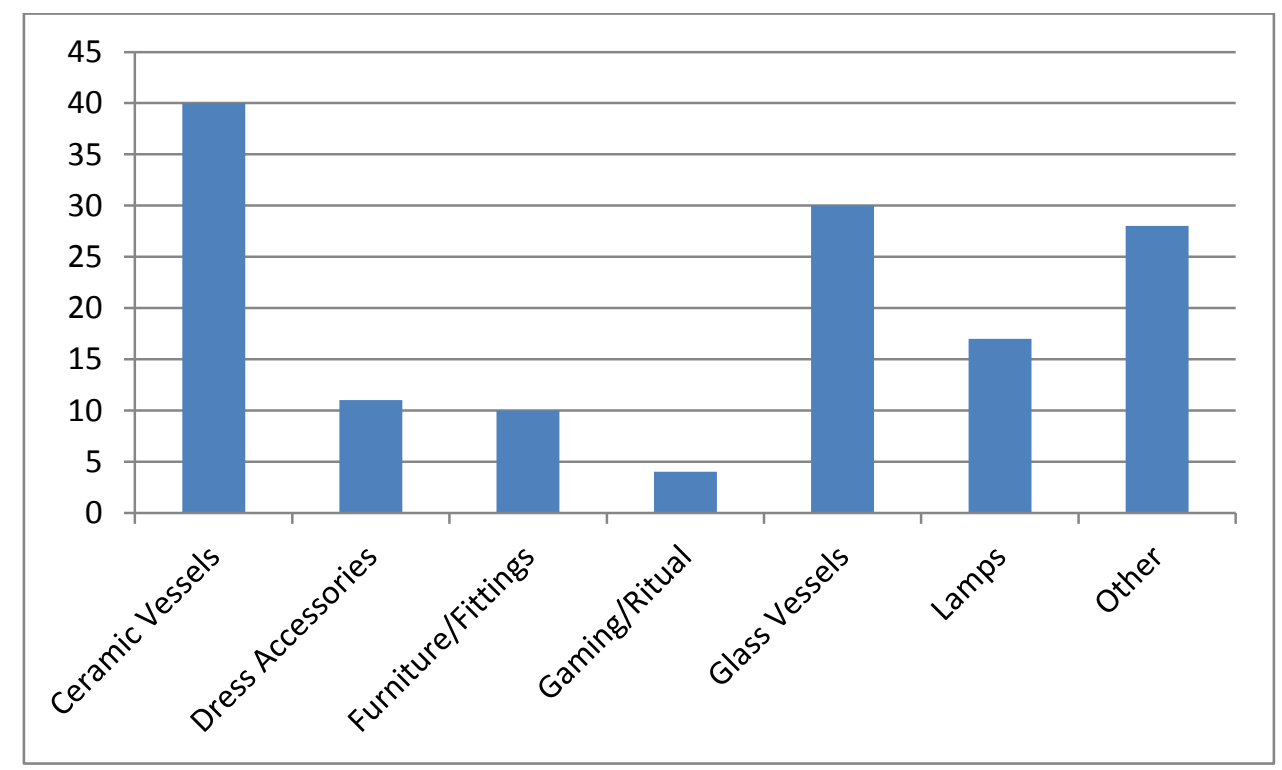

Figure 4.54: Graves including Associated Finds.

In other cases, placement within the grave may have reflected an inter-related function in the forms. This might be best illustrated by the well documented Cremation 25 from excavations at Stansted Airport. While the handled pans and the trefoil mouthed jug were located on one side of the burial, the bronze jug with two handles was placed with a collection of ceramic bowls, ${ }^{780}$ suggesting a differentiation of role and function between these copper alloy vessels in the grave.

\footnotetext{
${ }^{778}$ Philpott 1991 30-44 \& 103-114.

779 Niblett \& Reeves 1990, 443-446 (Fig. 3).

${ }^{780}$ Havis \& Brooks 2004, 216-217 (Fig. 145).
} 
In this case, it is probable that the handled pans and accompanying jug were intended for libation or ablution purposes while the jug associated with the ceramic bowls may have had a role in the dining itself as a serving vessel. Another instance where a copper alloy vessel may have been playing a role inter-related with ceramic vessels is from the Temple-Mausoleum grave at Lullingstone Roman Villa, where a copper alloy jug and a ceramic jug were placed next to each other between glass jugs and ceramic bowls. ${ }^{781}$ Where ceramics are present in graves, they may best be understood as serving a dining or drinking function, either being used by the participants in the funeral or providing the deceased with the necessary equipment for recreation in the next life. $^{782}$ It would not appear that the copper alloy vessels may always be directly associated with the ceramic vessels in a grave assemblage, as direct patterning between forms and types that occur in graves does not appear possible.

Glass also appears with considerable frequency in Romano-British graves that contain copper alloy vessels, in some 30 out of 57 graves reviewed in this thesis. There are instances of glass vessels being used to contain cremated remains, though the patchy recording of osteological data in the reports makes discerning exact figures on the relation to glass container vessels with copper alloy vessels in graves impossible. There are also several examples of glass vessels playing complimentary roles to the copper alloy vessels in the grave, aiding in our understanding of functionality. This is again easiest to discern in the larger grave assemblages of the southeast of England and are coupled with ceramic assemblages that also appear functionally related to dining and imbibing. Glass most often appears in graves with copper alloy vessels as either jugs or bottles, though bowls also appear.

On the whole, the glass and ceramic vessels in Romano-British graves appear to be associated with dining, which should come as no surprise as this is the principal function of most such vessels in any context. The function of the copper alloy vessels in these assemblages was probably complimentary to this, either as accoutrements for wine services or for hand washing associated with dining ritual. There are notable exceptions to this, most notably an anthropomorphic jar with strigils from Bayford ${ }^{783}$ and a small flask from Corbridge ${ }^{784}$ which would both be associated with bathing and hygiene. While the Handled Pan 1 and jug

\footnotetext{
${ }^{781}$ Meates 1979, 128; Henig 1995, 196-197.

${ }^{782}$ Henig 1995, 190-193.

${ }^{783}$ K0009.

${ }^{784}$ NU0049.
} 
combination was used for hand washing and ritual ablutions, ${ }^{785}$ this was in a ritual or dining context as opposed to what might be referred to as recreational or cosmetic bathing.

Lamps are included in several of the graves that contain copper alloy vessels and most often are of simple ceramic form. There is no apparent pattern in the decoration of these lamps as they appear in graves with copper alloy vessels. A number of copper alloy lamps also appear in graves from this data-set including Turners Hall Farm, Thornborough Barrow, Bayford and Bartlow Hills, each of which has a decorative flame guard such as a crescent moon or leaf shape. The crescent moon is the symbol of Diana and it is possible that imagery of the goddess was intentionally invoked in some cases. This is perhaps most intriguing at Turners Hall Farm where the bust of Minerva, also a virgin goddess, appears on a jug handle and a variety of weapons are interred in the grave. ${ }^{786}$ Such a possibility seems ever more likely as the syncretisation of deities is well attested in the Classical world, particularly in Gaul and Britain, ${ }^{787}$ and the syncretism of aspects both of Diana and Minerva to an indigenous goddess would not seem unlikely.

Other objects turn up sporadically in the graves with copper alloy vessels including gaming counters, animal bone, strigils and jewellery. These objects seem to be evoking leisured pursuits and could reflect the hope of a pleasurable afterlife. Conversely, they could reflect the particular tastes and pass-times of the deceased themselves, referencing their identity as a member of an elite class who appreciated wine drinking and convivial dining. The presence of gaming counters and boards in several of the graves is note-worthy; far more could be gleaned if the function of these objects was better understood (i.e. if it was known if these objects were for leisure, magic or a combination of the two). Careful study of the board and counters from the Stanway 'Doctor's Burial' with what is understood about ancient Roman and Celtic games indicates that it is most likely to have been a gaming board as opposed to a counting or ritual device, ${ }^{788}$ though it is worth remembering that these distinctions are not mutually exclusive. When considering all the various grave furniture that can be found in these large burial groups, these grave assemblages on the whole reflect a leisured life lived well and the display of comfort to see the deceased to the next world.

\footnotetext{
${ }^{785}$ Nuber 1973, 83-137; Nenova-Merdjanova 2002, 201-204; See Chapter 7.

${ }^{786}$ HER0032.

${ }^{787}$ Webster 1997, 165-182; Jackson 2007, 37-54.

${ }^{788}$ Crummy et al. 2007, 352-375.
} 
Osteological reporting has only recently been undertaken with a consistent level of accuracy to provide usable data, particularly in cremation burials, and therefore not as much may be said of the individuals from most of these graves. Most were not gendered or assigned age based on osteological evidence. Even in well excavated graves it can be difficult to assign sex on osteological evidence as the lack of complete skeletal remains from cremation burials indicates that careful collection and interment of all of the burnt bone was not seen as an essential part of the ritual during the Roman period. ${ }^{789}$ To further complicate present interpretation, some early grave groups were labelled to a certain sex or age based upon the grave assemblage itself, most notable in this data-set is the grave from Colchester that is commonly referred to as a child's burial. ${ }^{790}$ Assigning identity based on grave goods is a circular argument that can lead to inaccurate identification. The well-known 'transvestite grave' from Catterick is a good example of how grave goods may not provide accurate gender information. ${ }^{791}$ The Turners Hall Farm burials from this data-set, in which weapons were found in the burials of two females, is also a good example of how important it is to study the skeleton itself for identification purposes (though it must be remembered that this burial is not yet fully published). ${ }^{792}$ The information available for the graves in this data-set regarding age and sex of the individuals was too often unrecorded or assigned without adequate osteological proof. For this reason it has not been thoroughly incorporated in the present thesis as the data would prove inconsistent at best and inaccurate at worst. From the graves that do have reliable age and gender identification of the interred, it would appear that no pattern is recognizable across Britain and that it was appropriate for copper alloy vessels to be interred with men, women and possibly children. This in itself is noteworthy, as it indicates that copper alloy vessels were not assigned gender specific roles in Britain during the Roman period, at least not among the groups of people who chose to inter them as grave goods.

Taking all of this information into consideration, there are frustratingly few patterns in the associated finds in graves discernible to inform us about those who chose to include copper alloy vessels in their funerary assemblages. It would seem that copper alloy vessels as a grave good was favoured by a rural elite class, or a class wealthy enough to furnish the dead with

\footnotetext{
${ }^{789}$ McKinley 2000, 42.

${ }^{790}$ Eckardt 1999, 57-90; EX0034.

${ }^{791}$ Cool 2002, 41-42; Pinto \& Pinto 2013, 169-179.

${ }^{792}$ HER0031-HER0042.
} 
substantial burial assemblages, concentrated mostly in southern England from the Late Iron Age into the late $2^{\text {nd }}$ century CE. While judging the status of a burial from the archaeological remains can prove highly problematic, ${ }^{793}$ the interring of material of value, such as copper alloy vessels, requires a degree of disposable wealth that is well beyond subsistence level. It would seem that copper alloy as a grave good did imply a certain level of status, both by its rarity of interment and the elaborate nature of the graves that make up the data-set of this chapter. The associated goods from graves do indicate Classical pagan associations in their decoration and leisure in their function, indicating that the practice of interring copper alloy vessels in graves was a practice exercised by the pagan elites of the province who wished to express their identity through association with luxury dining practice.

\section{$\underline{4.6 \text { Concluding Remarks }}$}

While patterning remained a problem due to the limited number of graves containing copper alloy vessels, patterns in Grave Deposits did reflect different preferences from those found in Structured Deposits. This is particularly evident in the scale of complex iconography preferred in grave assemblages as well as the preference for jugs and Handled Pan 1s. The inclusion of these objects with glass and ceramic vessels that may have likely filled complimentary functions at the dining table shows the likelihood that the majority of copper alloy vessels we have as grave goods were designed for drinking and ablution purposes.

The chronological and geographic distribution of this material was significantly limited in comparison to the distribution of Structured Deposits, being confined almost exclusively to the south and east of England during the first two centuries of Roman rule. Grave deposition of copper alloy vessels appears to have been a minority practice throughout the Roman period and had very distinct areas of practice, reflecting regional cultures (such as in the south east of England) or imported practice by a minority group within the military or merchant classes (such as at Brougham in Cumbria). Conversely, Structured Deposition of copper alloy vessels alone or in combination with coin hoards seems to have been practiced with more consistency across a wider geographic area, even though regionality and variation in practice was evident. Both of these processes of deposition were the product of conscious human agency and are therefore

\footnotetext{
${ }^{793}$ Struck 2000, 85-86.
} 
subject to preferential selection of object inclusion which reflects the tastes of those making the deposits as well as the cultural association that these objects had. In the next two chapters, objects that have entered the archaeological record through more random processes will be examined. The differences between the objects within these depositional contexts reflects greatly on the identity and culture practice of the people who deposited these vessels in the archaeological record as well as indicating the high specificity with which preferential selection shaped the data in Structured Deposits and Grave Deposits, indicating the conscious ritual intent behind these practices of interment. That certain vessels were selected specifically for grave contexts is highly informative, indicating that the objects interred had specific cultural significance for those performing the ritual and that burial goods did not simply reflect a random assortment of goods as they may have been used in life. Association with eating, drinking and leisure activities may be seen from the objects in these graves, as well as iconographic reference to Mercury and Bacchus which would indicate some probable belief in a life after death. Though there was not a consensus attitude regarding the afterlife, belief in some persistence of the soul after death was held by many during the Roman period and this formed the foundations for much of the funerary ritual, ancestor worship and is also attested by many of the popular epitaph formulae in both Greek and Latin. ${ }^{794}$ While some of those participating in the burial may have had no belief in an afterlife and were taking part in the ceremony as a form of conspicuous consumption and display of social status, ${ }^{795}$ the consistent preferential selection of copper alloy vessels associated with drinking, and more particularly with ritual ablutions and purification, indicates a recurring formula dictated by the symbolic significance of these vessels within the burial ritual that would seem to be based on some concept of a life after death. ${ }^{796}$ The examination of copper alloy vessels in Grave Deposits provided in this chapter offers some fresh insight into burial ritual in Roman Britain and offers a fruitful perspective for further examination of religious belief and practice in the province during this period.

\footnotetext{
794 Toynbee 1971, 33-39.

795 Morris 1992, 200-202; Fitzpatrick 2000, 15-27; Struck 2000, 85-94.

${ }^{796}$ Henig 1995, 190-205.
} 


\section{Chapter 5: Site Finds}

\subsection{Introduction}

The following chapter surveys single finds, i.e. not Structured Deposits and burials, from archaeological excavations and independent finds recorded through traditional scholarly means such as site reports and journal articles; this excludes Single Finds recorded through the Portable Antiquity Scheme, which shall be treated independently in Chapter 6. This includes both stratified and unstratified finds found during excavation or accidentally through construction work or other serendipitous circumstances. This makes this group of data rather complex to characterise.

The current data set consists of objects from published site reports (both in journal articles and monographs), finds catalogues from excavations and museums as well as articles on specific objects. This choice was made to ensure quality and veracity of data, consistency in recording as well as adhering to the monetary and time constraints that a doctoral thesis is inherently bound to. The current data set should provide a representative sample of the material, though developments and further publication could affect the results. This is particularly true of further research into un/under-published archives and grey literature, such as Michael Fulford's current project at the University of Reading on rural Britain.

The principal applications of this data-set will be for comparison between Site Types and geographic regions to judge how site assemblages vary, indicating differences in consumption and culture practice. As will be seen, Site Finds contribute vital information that fills in some of the geographic and chronological gaps in the data-sets of Structured Deposits and Grave Deposits and helps to draw a more complete picture of the development of this facet of material culture in Britain during the Roman Period.

\section{$\underline{5.2 \text { Geographic Survey of Site Finds }}$}

As is evident from Map 3, the objects that compose the Site Finds portion of the data-set are broadly distributed across the study area with some 40 objects from Wales, 21 from the south western counties of England, 74 objects from the Northern Counties (including the areas of Hadrian's Wall), 50 objects from the Midlands, and 88 from East Anglia and the south eastern counties (including London). This is a much wider and more varied distribution pattern than was 
evident in either Structured Deposits or Grave Deposits. ${ }^{797}$ As Table 5a illustrates, the data-set for Site Finds from the south western counties of England is more diverse than that offered either by Structured Deposits or Grave Deposits for this region, promising to illuminate our understanding of the use of these objects in this region. The urban assemblages from Exeter and Dorchester offer a variety of vessels, though jugs predominate at Dorchester. The military site of Hod Hill offered two Handled Pan 2 fragments. The presence of Handled Pan $2 \mathrm{~s}$ is a trend seen at other military sites throughout the province across Depositional Contexts. Excavations at a rural settlement in Wanborough, Wiltshire have wielded several copper alloy vessels: two vessel fragments of late $2^{\text {nd }}$ to early $3^{\text {rd }}$ century date of deposition and two Handled Pan 2 fragments from the Late Roman period. The most famous Site Find from Wiltshire is certainly the Rudge Cup (Figure 5.01). This object has received significant scholarly attention over the years and has played an important role in the development of antiquarian and archaeological study of the Roman past in Britain. ${ }^{798}$ It is decorated with what appears to be a crenelated wall and an inscription that reads: .A.MAISABALLAVAVXELODVMCAMBOGLANSBANNA. ${ }^{799}$ This has been interpreted as referring to five forts along Hadrian's Wall, ${ }^{800}$ indicating that the crenulations depicted most probably represent Hadrian's Wall itself. ${ }^{801}$ The Rudge Cup was originally decorated with enamelling, though only traces survive. ${ }^{802}$ It was found in 1725 by a farmer in what later excavations would reveal was a villa complex. ${ }^{803}$

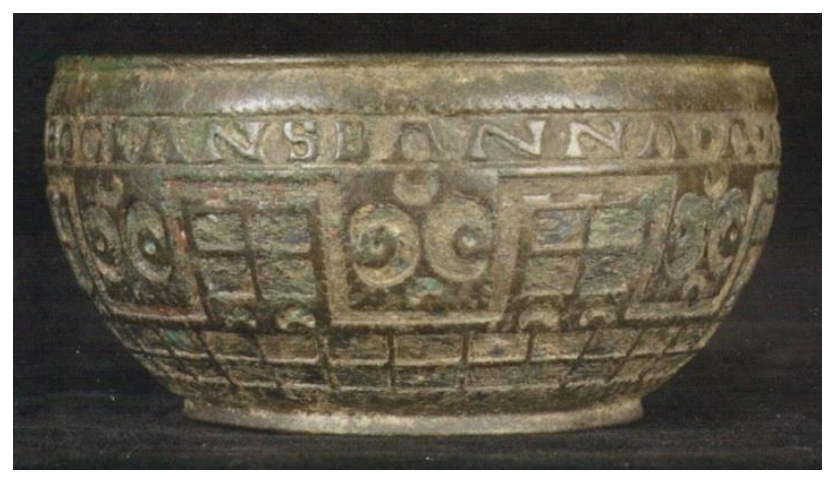

Figure 5.01: The Rudge Cup (Breeze 2012, 1 Fig.1.1)

\footnotetext{
${ }^{797}$ Illustrated in Map $1 \& 2$ respectively

798 Allason-Jones 2012, 33-36.

${ }^{799}$ RIB II 2415.53.

${ }^{800}$ Allason-Jones 2012, 27.

${ }^{801}$ Breeze 2012, 109-110.

${ }^{802}$ Allason-Jones 2012, 23-25.

${ }^{803}$ Allason-Jones 2012, 33-34.
} 


\begin{tabular}{|c|c|}
\hline Findspot (Site Type) & Vessel/Catalogue Number \\
\hline Bath, Avon (RS) & Jug/AV0001 \\
\hline Thatcham, Berkshire (RU) & Jug/BERK0001 \\
\hline Exeter, Devon (U) & $\begin{array}{l}\text { Vessel(fragment)/DEV0001 } \\
\text { Handled Pan 5/DEV0002 }\end{array}$ \\
\hline Dorchester, Dorset (U) & $\begin{array}{c}\text { Jug/DOR0012 } \\
\text { Bowl/DOR0013 } \\
\text { Vessel(fragment)/DOR0014 } \\
\text { Jug/DOR0015 } \\
\text { Jug/DOR0016 } \\
\text { Jug/DOR0017 }\end{array}$ \\
\hline Hod Hill, Dorset (M) & $\begin{array}{l}\text { Handled Pan 2/DOR0018 } \\
\text { Handled Pan 2/DOR0019 }\end{array}$ \\
\hline Lyndey, Gloucestershire (RS) & $\begin{array}{c}\text { Strainer/G0001 } \\
\text { Vessel(fragment)/G0018 }\end{array}$ \\
\hline Cirencester, Gloucestershire (U) & $\begin{array}{c}\text { Handled Pan 2/G0002 } \\
\text { Jug/G0008 } \\
\text { Bucket/G0009 } \\
\text { Jug(lid)/G0010 } \\
\text { Vessel(mount)/G0011 }\end{array}$ \\
\hline Kingholm, Gloucestershire (M) & Handled Pan 2/G0003 \\
\hline Woodchester, Gloucestershire (RS) & Handled Pan 2/G0004 \\
\hline Uley, Gloucestershire (RS) & $\begin{array}{c}\text { Bowl/G0013 } \\
\text { Jug(lid)/G0014 }\end{array}$ \\
\hline Tewkesbury, Gloucestershire (RS) & Jug(handle)/G0015 \\
\hline Wanborough, Wiltshire (RS) & $\begin{array}{c}\text { Handled Pan 2/WIL0009 } \\
\text { Handled Pan 2/WIL0010 } \\
\text { Jug(lid)/WIL0011 } \\
\text { Vessel(fragment)/WIL0012 }\end{array}$ \\
\hline Rudge, Wiltshire (RU) & Handled Pan 2/WIL0024 \\
\hline
\end{tabular}

Table 5a: Site Finds from the South West counties of England. 


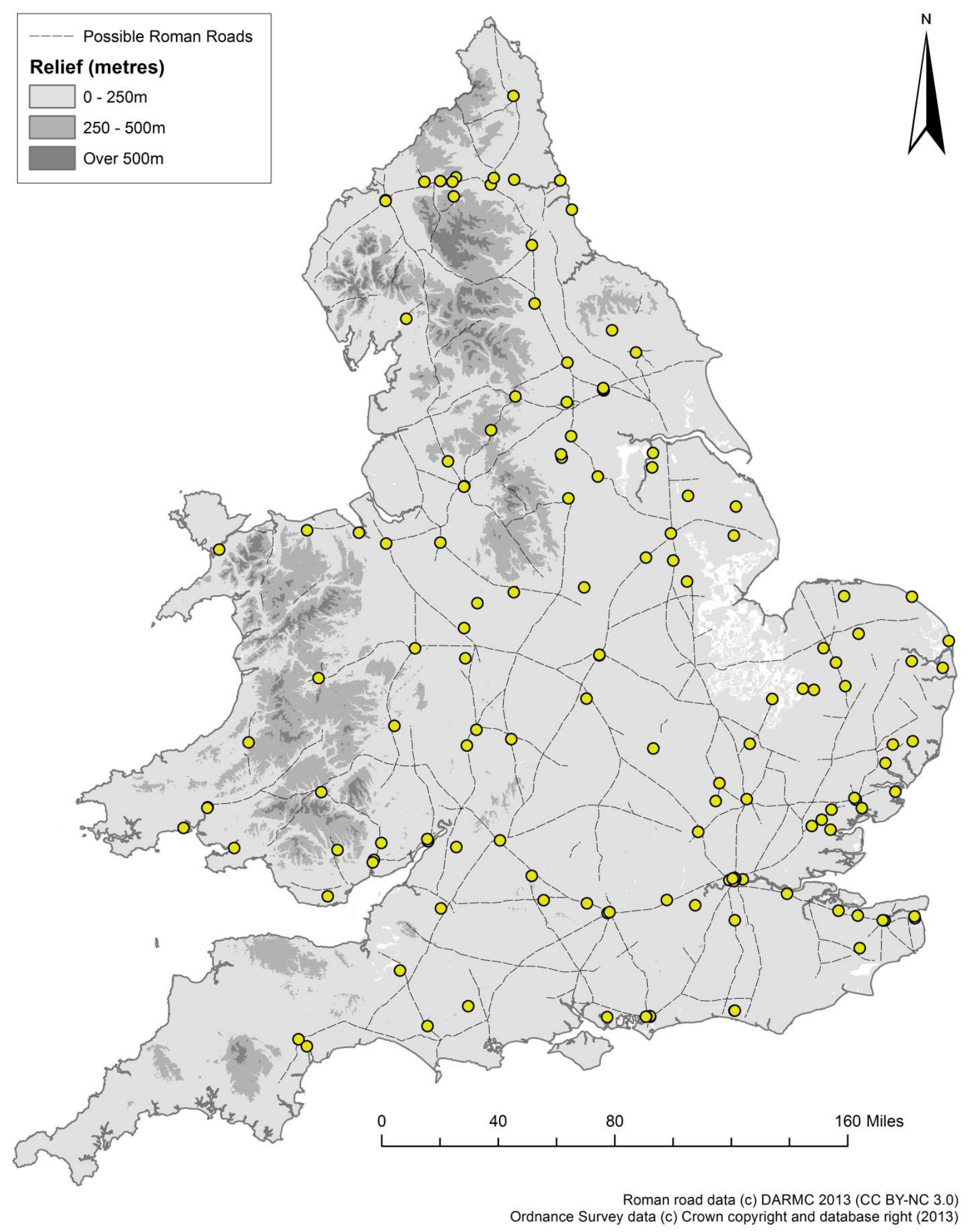

Map 3: Site Finds of copper alloy vessels from Roman Britain (K. Robbins) 
The diversity of site types in the south west of England offers a microcosm of the composition of assemblages across Britain and serves as a good area of comparison for the other areas of Britain, where the predominance of one site-type often dictates the composition of the regional assemblage. Conversely, there are no vessel forms or types that dominate the assemblages of this region as a whole, providing no indication of a localised preference for any vessel form or decorative style. While Site Finds provide us with a fuller picture of what vessels were available to the inhabitants of this region than would be available through either Structured Deposits or Grave Deposits, it does not indicate any regionally specific identity expressed through vessel selection or decorative preferences.

The finds from Wales are predominantly in the coastal regions (Table 5b). Additionally, nearly all were found within settlements that contained significant military components, either as forts themselves or within settlements that had a fort in their immediate vicinity. Of the 14 Welsh sites that contributed material to the Site Finds data-set of this thesis, all except one ${ }^{804}$ fall into these categories. ${ }^{805}$ This should not be surprising, as the documented Roman settlements of Wales had a strong military element to them. ${ }^{806}$ In fact, it is likely that Roman forts and fortresses were placed specifically to coincide with pre-Roman settlement patterns. ${ }^{807}$ The three Welsh sites that have proved most prolific in objects for this data-set (Burrium, Coygan Camp ${ }^{808}$ and Caerleon) are all Roman forts. It should be noted that selection bias may have played a part in this, as the military history of Roman Wales receives particular attention and military sites have been preferentially identified, excavated and subsequently published. The same trend of copper alloy vessel material occurring on military sites appears to be evident in the northern frontier zone along Hadrian's Wall (Table 5c), persisting to a lesser extent into the remaining northern counties (Table 5d). This again may be in part the result of the sort of settlement which occurred in this region more generally, as Hadrian's Wall by its very nature was a heavily militarised zone which also influenced settlement patterns and troop distribution in the rest of northern England. ${ }^{809}$

\footnotetext{
${ }^{804}$ VGL0001, Wenvoe/Whitton in the Vale of Glamorgan, identified as a farmstead or villa complex.

${ }^{805}$ Within 1 kilometer of findspot.

${ }^{806}$ Manning 2001, 8-48; Arnold \& Davies 2000, 40-64; Gerrard 2013, 29.

${ }^{807}$ Nash-Williams 1954, 6-10; Manning 2001, 24-48.

${ }^{808}$ Additionally, a Structured Deposit of copper ally vessels and coins were found at Coygan Cave; CAR0001 CAR0002; See Chapter 3.

${ }^{809}$ Mattingly 2007, 146-149.
} 


\begin{tabular}{|c|c|}
\hline Findspot (Site Type) & Vessel/Catalogue Number \\
\hline Carmathen, Carmarthenshire (M) & $\begin{array}{c}\text { Handled Pan 2/CAR0003 } \\
\text { Jug (lid)/CAR0004 }\end{array}$ \\
\hline Coygan Camp, Carmarthenshire (RS) & $\begin{array}{l}\text { Vessel(fragment)/CAR0005 } \\
\text { Vessel(fragment)/CAR0006 } \\
\text { Vessel(fragment)/CAR0007 } \\
\text { Vessel(fragment)/CAR0008 } \\
\text { Vessel(fragment)/CAR0009 }\end{array}$ \\
\hline Llanio, Ceredigion $(\mathrm{M})$ & Handled Pan 2/CER0001 \\
\hline Dinorben/Rhuddlan, Denbighshire (M) & $\begin{array}{l}\text { Vessel(mount)/DEN0005 } \\
\text { Vessel(mount)/DEN0006 } \\
\text { Handled Pan 2/DEN0007 }\end{array}$ \\
\hline Pentre Farm/Flint, Flintshire (RS) & Jug(lid)/F0010 \\
\hline Caernarvon, Gwynedd (M) & Handled Pan 2/GWY0013 \\
\hline Gelligaer, Monmouthshire (M) & Handled Pan 2/MON0001 \\
\hline Usk, Momouthshire (M) & $\begin{array}{c}\text { Jug(handle)/MON0008 } \\
\text { Jug(lid)/MON0009 } \\
\text { Jug(lid)/MON0010 } \\
\text { Jug(lid)/MON0011 } \\
\text { Jug(lid)/MON0012 } \\
\text { Handled Pan 2/MON0013 } \\
\text { Vessel(fragment)/MON0014 } \\
\text { Vessel(fragment)/MON0015 } \\
\text { Vessel(mount)/MON0016 } \\
\text { Handled Pan 4/MON0017 }\end{array}$ \\
\hline Gwent, Monmouthshire (M) & Handled Pan 2/MON0018 \\
\hline Caerleon, Newport (M) & $\begin{array}{c}\text { Handled Pan 2/NE0007 } \\
\text { Bucket/NE0008 } \\
\text { Handled Pan 2/NE0009 } \\
\text { Vessel(mount)/NE0010 } \\
\text { Vessel(mount)/NE0011 } \\
\text { Bucket/NE0012 } \\
\text { Handled Pan 1/NE0013 } \\
\text { Handled Pan 2/NE0014 }\end{array}$ \\
\hline Caersws, Powys (RU) & Handled Pan 1/PO0001 \\
\hline Brecon, Powys (M) & $\begin{array}{c}\text { Handled Pan 2/PO0008 } \\
\text { Bowl/PO0009 } \\
\text { Vessel(fragment)/PO0010 } \\
\text { Jug(handle)/PO0011 }\end{array}$ \\
\hline Loughor, Swansea (M) & $\begin{array}{c}\text { Vessel(fragment)/SWA0001 } \\
\text { Handled Pan 2/SWA0002 }\end{array}$ \\
\hline Wenvoe, Vale of Glamorgan (RU) & Jug(handle)/VGL0001 \\
\hline
\end{tabular}

Table 5b: Site Finds from Wales.

Another contributing factor is likely to be preferential excavation and publication of forts and urban centres over smaller rural settlements and the comparably small site assemblages of 
rural settlements in reference to forts and urban centres. The prevalence of Handled Pan $2 \mathrm{~s}$ among the data-set of the north is striking and may be considered to define the character of these assemblages. A noteworthy exception to this trend is the ornate jug from Carlisle, C0028, depicting the sacrifice of a pig attended by a priest and magistrates on its handle (Figure 5.02). The near complete state of this vessel, as well as its high degree of detail, suggests that it may not have been accidentally lost or misplaced and that it could have instead come from a disturbed Structured Deposit or Grave Deposit.

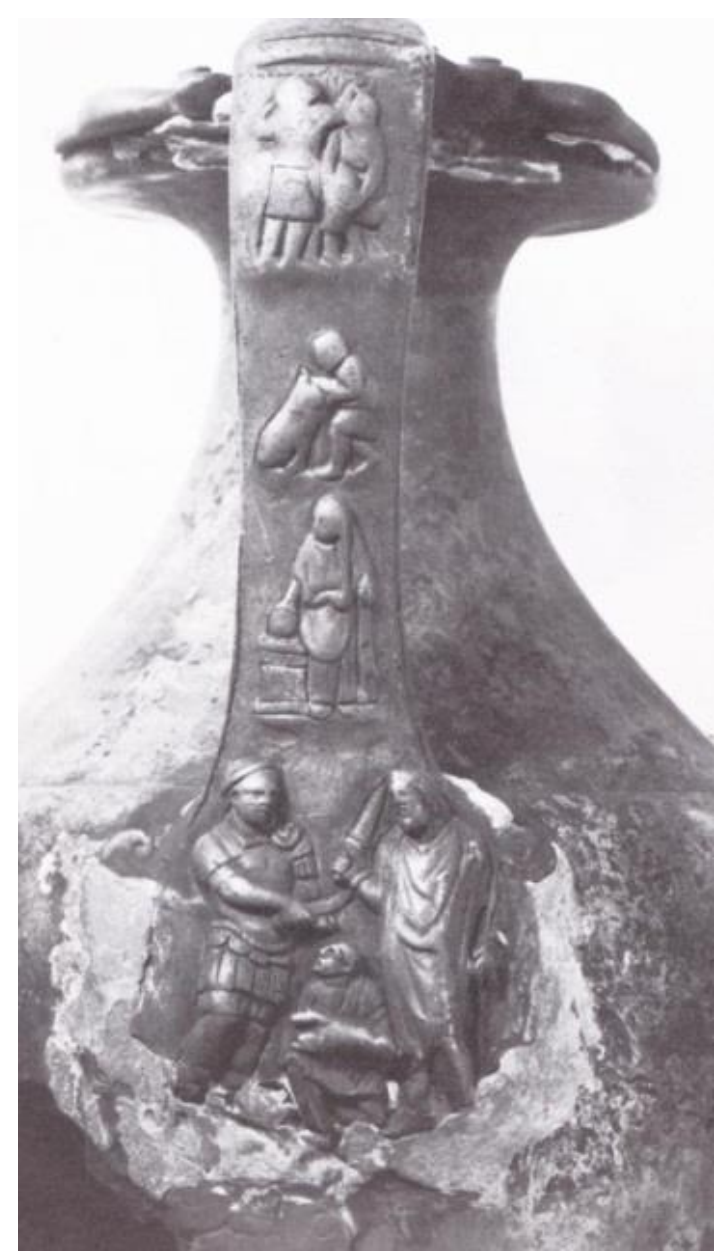

Figure 5.02: Jug from Carlisle depicting scene of sacrifice (Henig 1983, 134 Fig.57) 


\begin{tabular}{|c|c|}
\hline Findspot (Site Type) & Vessel/Catalogue Number \\
\hline Carlisle, Cumbria (M) & $\begin{array}{c}\text { Jug/C0021 } \\
\text { Jug/C0022 } \\
\text { Bowl/C0023 } \\
\text { Handled Pan 2/C0024 } \\
\text { Handled Pan 2/C0025 } \\
\text { Handled Pan 2/C0026 } \\
\text { Jug/C0028 }\end{array}$ \\
\hline Watercrook, Cumbria (M) & Handled Pan 2/C0027 \\
\hline Great Chesters, Northumberland (M) & $\begin{array}{l}\text { Vessel(fragment)/NU0001 } \\
\text { Vessel(fragment)/NU0002 }\end{array}$ \\
\hline Housesteads, Northumberland (M) & $\begin{array}{c}\text { Handled Pan 2/NU0006 } \\
\text { Handled Pan 2/NU0007 } \\
\text { Handled Pan 2/NU0008 } \\
\text { Vessel(fragment)/NU0009 } \\
\text { Handled Pan 2/NU0010 } \\
\text { Jar/NU0033 } \\
\text { Jar/NU0038 } \\
\text { Vessel(fragment)/NU0039 } \\
\text { Vessel(mount)/NU0040 }\end{array}$ \\
\hline Chesters, Northumberland (M) & Vessel(fragment)/NU0011 \\
\hline Corbridge, Northumberland (M) & $\begin{array}{c}\text { Handled Pan 2/NU0012 } \\
\text { Vessel(fragment)/NU0013 } \\
\text { Jug/NU0034 } \\
\text { Jug/NU0036 } \\
\text { Vessel(fragment)/NU0037 }\end{array}$ \\
\hline Rudchester, Northumberland (M) & $\begin{array}{l}\text { Vessel(fragment)/NU0014 } \\
\text { Handled Pan 3/NU0015 }\end{array}$ \\
\hline Southshields, Northumberland (M) & $\begin{array}{c}\text { Handled Pan 2/NU0016 } \\
\text { Handled Pan 3/NU0017 } \\
\text { Vessel(fragment)/NU0018 } \\
\text { Vessel(fragment)/NU0041 } \\
\text { Vessel(fragment)/NU0042 }\end{array}$ \\
\hline Harwood, Northumberland (RU) & Handled Pan 2/NU0026 \\
\hline Hexham, Northumberland (RS) & Strainer/NU0027 \\
\hline Bolton, Northumberland (RU) & Vessel(fragment)/NU0028 \\
\hline Birdoswald, Northumberland (M) & $\begin{array}{c}\text { Handled Pan 2/NU0031 } \\
\text { Jug/NU0032 }\end{array}$ \\
\hline Vindolanda, Northumberland (M) & $\begin{array}{c}\text { Handled Pan 2/NU0043 } \\
\text { Bowl/NU0044 } \\
\text { Handled Pan 1/NU0045 } \\
\text { Handled Pan 2/NU00046 } \\
\text { Handled Pan 2/NU0047 } \\
\text { Handled Pan 2/NU0048 }\end{array}$ \\
\hline
\end{tabular}

Table 5c: Site Finds from Cumbria and Northumberland. 


\begin{tabular}{|c|c|}
\hline Findspot (Site Type) & Vessel/Catalogue Number \\
\hline Middlewich, Cheshire (RS) & $\begin{array}{c}\text { Cauldron/CHE0001 } \\
\text { Vessel(fragment)/CHE0002 }\end{array}$ \\
\hline Chester, Cheshire (M) & Handled Pan 2/CHE0003 \\
\hline Hurworth-on-Tees, Durham (RS) & Bowl/DUR0002 \\
\hline Binchester, Durham (M) & $\begin{array}{l}\text { Handled Pan 2/DUR0003 } \\
\text { Handled Pan 3/DUR0004 }\end{array}$ \\
\hline Leicester, Leicestershire (U) & $\begin{array}{c}\text { Handled Pan 2/LEI0001 } \\
\text { Handled Pan 2/LEI0004 } \\
\text { Vessel(fragment)/LEI0005 } \\
\text { Vessel(fragment)/LEI0006 }\end{array}$ \\
\hline Osgodby, Lincolnshire (RU) & $\begin{array}{l}\text { Vessel(fragment)/LIN0001 } \\
\text { Strainer/LIN0002 }\end{array}$ \\
\hline Ashby, Lincolnshire (RU) & Vessel(fragment)/LIN0003 \\
\hline Sleaford, Lincolnshire (RS) & $\begin{array}{l}\text { Handled Pan 2/LIN0025 } \\
\text { Handled Pan 2/LIN0026 }\end{array}$ \\
\hline Navenby, Lincolnshire (RS) & Handled Pan 2/LIN0027 \\
\hline Humberside, Lincolnshire (RS) & Handled Pan 2/LIN0033 \\
\hline Louth, Lincolnshire (RU) & Handled Pan 2/LIN0034 \\
\hline Lincoln, Lincolnshire (U) & Handled Pan 2/LIN0035 \\
\hline Normanby, Lincolnshire (RU) & Handled Pan 2/LIN0036 \\
\hline Manchester, Greater Manchester (M) & $\begin{array}{c}\text { Jug/M0001 } \\
\text { Jug(lid)/M0002 }\end{array}$ \\
\hline York, North Yorkshire (U) & $\begin{array}{c}\text { Jar/NYR0029 } \\
\text { Vessel(fragment)/NYR0030 } \\
\text { Vessel(fragment)/NYR0039 }\end{array}$ \\
\hline Malton, North Yorkshire (RS) & $\begin{array}{c}\text { Bowl/NYR0031 } \\
\text { Handled Pan 2/NYR0032 }\end{array}$ \\
\hline Aldborough, North Yorkshire (U) & $\begin{array}{c}\text { Vessel(fragment)/NYR0033 } \\
\text { Jug/NYR0034 } \\
\text { Vessel(mount)/NYR0035 }\end{array}$ \\
\hline Beadlam Roman Villa, North Yorkshire (RS) & Strainer/NYR0036 \\
\hline Catterick, North Yorkshire (RS) & Jar/NYR0038 \\
\hline Doncaster, South Yorkshire (M) & Handled Pan 2/SYR0002 \\
\hline $\begin{array}{l}\text { Templeborough/Rotherham, South Yorkshire } \\
\text { (M) }\end{array}$ & $\begin{array}{l}\text { Jug(lid)/SYR0003 } \\
\text { Jug(lid)/SYR0004 }\end{array}$ \\
\hline Castleford, West Yorkshire (M) & $\begin{array}{c}\text { Cauldron/WYR0001 } \\
\text { Handled Pan 1/WYR0006 }\end{array}$ \\
\hline Dalton Parlours, West Yorkshire (RS) & $\begin{array}{l}\text { Vessel(fragment)/WYR0002 } \\
\text { Vessel(fragment)/WYR0003 }\end{array}$ \\
\hline Ilkley, West Yorkshire (M) & $\begin{array}{l}\text { Vessel(fragment)/WYR0004 } \\
\text { Handled Pan 2/WYR0005 }\end{array}$ \\
\hline Slack/Hebden Royd, West Yorkshire (RU) & Handled Pan 2/WYR0007 \\
\hline
\end{tabular}

Table 5d: Site Finds from the Northern Counties of England. 


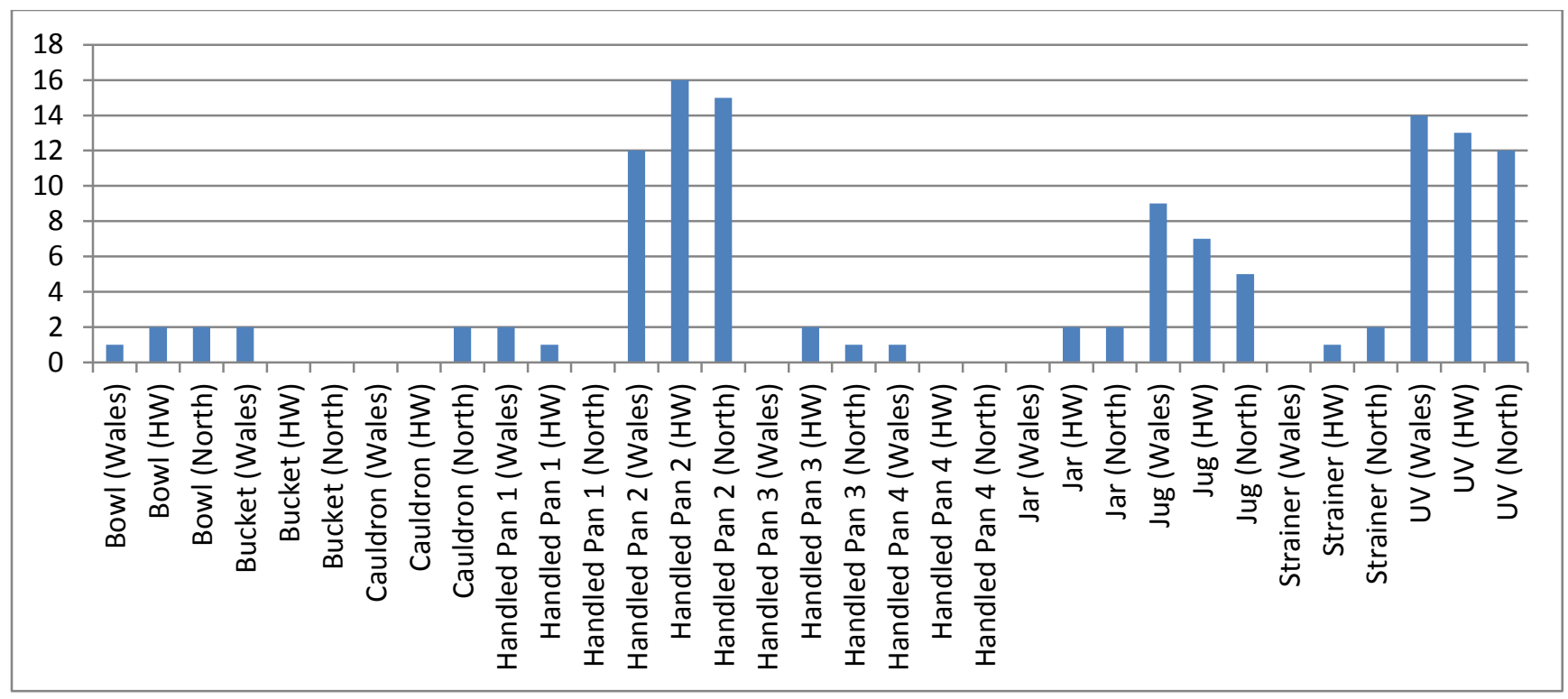

Figure 5.03: Site Finds by form in Wales, Hadrian's Wall and the Northern Counties of England.

As evidenced by Figure 5.03, the predominant diagnostic find both in Wales and in northern England are Handled Pan 2s, which constitute $43 \%$ of the material collected. The second most common vessels found are jugs, being particularly prevalent in Wales. However, it is important to keep in mind that a sizable number of undiagnostic fragments are present in these assemblages that could have affected the data significantly if they had been complete enough to be identified as a specific vessel form or type. A noteworthy anomaly in this region is the two fragments of Rudge Cup type Handled Pan 2s that were found in Sleaford, both of which have enamelled floral decoration. ${ }^{810}$ Another enamelled vessel from this region is the enamelled jar found during excavations of the Catterick Bypass not only as it adds to the corpus of these vessels of apparently British manufacture, but also as the presence of what would seem to be a tar based adhesive product found inside the vessel indicates a possible function for these sorts of vessels, at least as part of a secondary (re-use) existence as opposed to during their primary uselife. $^{811}$

\footnotetext{
${ }^{810}$ LIN0025 \& LIN0026; Elsdon 1997, 190-191.

${ }^{811}$ Wilson 2002, 78-81.
} 


\begin{tabular}{|c|c|}
\hline Findspot (Site Type) & Vessel/Catalogue Number \\
\hline Olney/Milton Keynes, Buckinghamshire (RS) & Handled Pan 1/BUC0005 \\
\hline Barton, Cambridgeshire (RU) & Vessel(mount)/CAM0006 \\
\hline Isle of Ely/Prickwillow, Cambridgeshire (RU) & Handled Pan 2/CAM0009 \\
\hline Braughing, Hertfordshire (RS) & Handled Pan 2/HER0001 \\
\hline St.Albans, Hertfordshire (U) & $\begin{array}{c}\text { Vessel(mount)/HER0002 } \\
\text { Vessel(mount)/HER0003 } \\
\text { Vessel(mount)/HER0004 } \\
\text { Jug(handle)/HER0005 } \\
\text { Jug(handle)/HER0006 } \\
\text { Jug(handle)/HER0007 } \\
\text { Jug(handle)/HER0008 } \\
\text { Jug(handle)/HER0009 } \\
\text { Vessel(fragment)/HER0010 } \\
\text { Jug/HER0011 } \\
\text { Jug/HER0012 } \\
\text { Handled Pan 1/HER0013 } \\
\text { Handled Pan 1/HER0014 } \\
\text { Strainer/HER0015 } \\
\text { Handled Pan 3/HER0016 } \\
\text { Handled Pan 3/HER0017 } \\
\text { Jug(lid)/HER0018 }\end{array}$ \\
\hline Baldock, Hertfordshire (RS) & Handled Pan 2/HER0029 \\
\hline Higham Ferrers, Northamptonshire (RS) & $\begin{array}{l}\text { Vessel(fragment)/NH0010 } \\
\text { Vessel(fragment)/NH0011 } \\
\text { Vessel(fragment)/NH0012 }\end{array}$ \\
\hline Broxtowe, Nottinghamshire (M) & Handled Pan 2/NOT0001 \\
\hline Brough-on-Fosse, Nottinghamshire (M) & Handled Pan 2/NOT0005 \\
\hline North Leigh, Oxfordshire (RS) & $\begin{array}{c}\text { Vessel(mount)/OX0003 } \\
\text { Vessel(fragment)/OX0004 } \\
\text { Vessel(fragment)/OX0005 }\end{array}$ \\
\hline Wroxeter, Shropshire (U) & $\begin{array}{c}\text { Jar/SH0004 } \\
\text { Handled Pan 2/SH0005 } \\
\text { Handled Pan 2/SH0006 } \\
\text { Jug(lid)/SH0006 } \\
\text { Jug(lid)/SH0007 } \\
\text { Jug(lid)/SH0008 } \\
\text { Jug(lid)/SH0009 } \\
\text { Jug/SH0010 } \\
\text { Handled Pan 2/SH0011 } \\
\end{array}$ \\
\hline Otron's Farm/Rocester, Staffordshire (RS) & Handled Pan 1/STA0006 \\
\hline Castle Croft/Wall, Staffordshire (RS) & Vessel(mount)/STA0007 \\
\hline Alcester, Warwickshire (M) & $\begin{array}{c}\text { Bowl/WAR0003 } \\
\text { Vessel(fragment)/WAR0004 } \\
\text { Vessel(mount)/WAR0005 } \\
\text { Vessel(mount)/WAR0006 }\end{array}$ \\
\hline
\end{tabular}




\begin{tabular}{|c|c|}
\hline Cave's Inn/Churchover, Warwickshire (RU) & Handled Pan 2/WAR0007 \\
\hline Droitwich, Worcestershire (RS) & Bowl/WOR0003 \\
\hline Worcester, Worcestershire (RS) & Vessel(fragment)/WOR0004 \\
\hline
\end{tabular}

Table 5e: Site Finds from Midlands.

The variety of site types increases in the Midlands, complicating both the assemblages and their interpretation (Table 5e). Some sites have clear military origins and a probable continued military significance through the Roman period, even after they appear to emerge as civic centres. A good example of this is Wroxeter, ${ }^{812}$ a city contributing 9 objects to the Site Finds data of the current thesis. The objects from this urban centre comprise five jugs, three Handled Pan $2 \mathrm{~s}$ and one jar. It is worth noting that all the copper alloy vessels assigned a stratigraphic date were deposited between the mid- $1^{\text {st }}$ and mid- $2^{\text {nd }}$ century $\mathrm{CE},{ }^{813}$ bridging the period of transition at this site from a military to urban/civilian centre. ${ }^{814}$

However, the majority of the contributing sites in the Midlands appear to be villas or small rural settlements, with little to no evidence of a military presence what-so-ever. For example, the site at Rocester which furnished a Handled Pan 1 handle decorated with a Gorgon headed handle terminal in fact appears to be the site of a rural shrine. ${ }^{815}$ Some objects in the Midlands prove difficult to contextualise due to unclear or unexcavated findspots. This is perhaps most frustratingly apparent with the elaborately decorated Handled Pan 2 found at the Isle of Ely, Prickwillow in Cambridgeshire in 1883 (Figure 5.04). ${ }^{816}$ This apparent diversity in settlement types in turn appears to lead to a more diverse vessel assemblage, with a greater variety of forms present in higher numbers than found in either Wales or the North (Figure 5.05).

Another key factor that differentiates the Midlands from the areas thus far discussed is the presence of a large urban assemblage of objects from Verulamium (modern St. Albans), which contributes 17 objects to this data-set. The most common copper alloy vessels found at St. Albans are jugs, which account for just fewer than $50 \%$ of the finds (Figure 5.06). ${ }^{817}$

\footnotetext{
${ }^{812}$ White \& Barker 1998.

${ }^{813}$ Atkinson 1942, 208-212; Ellis 2000, 133.

${ }^{814}$ White \& Barker 1998, 32-83.

${ }^{815}$ Ferris et al. 2000; STA0006.

${ }^{816}$ CAM0009.

${ }^{817} 8$ out of a total of 17 objects.
} 


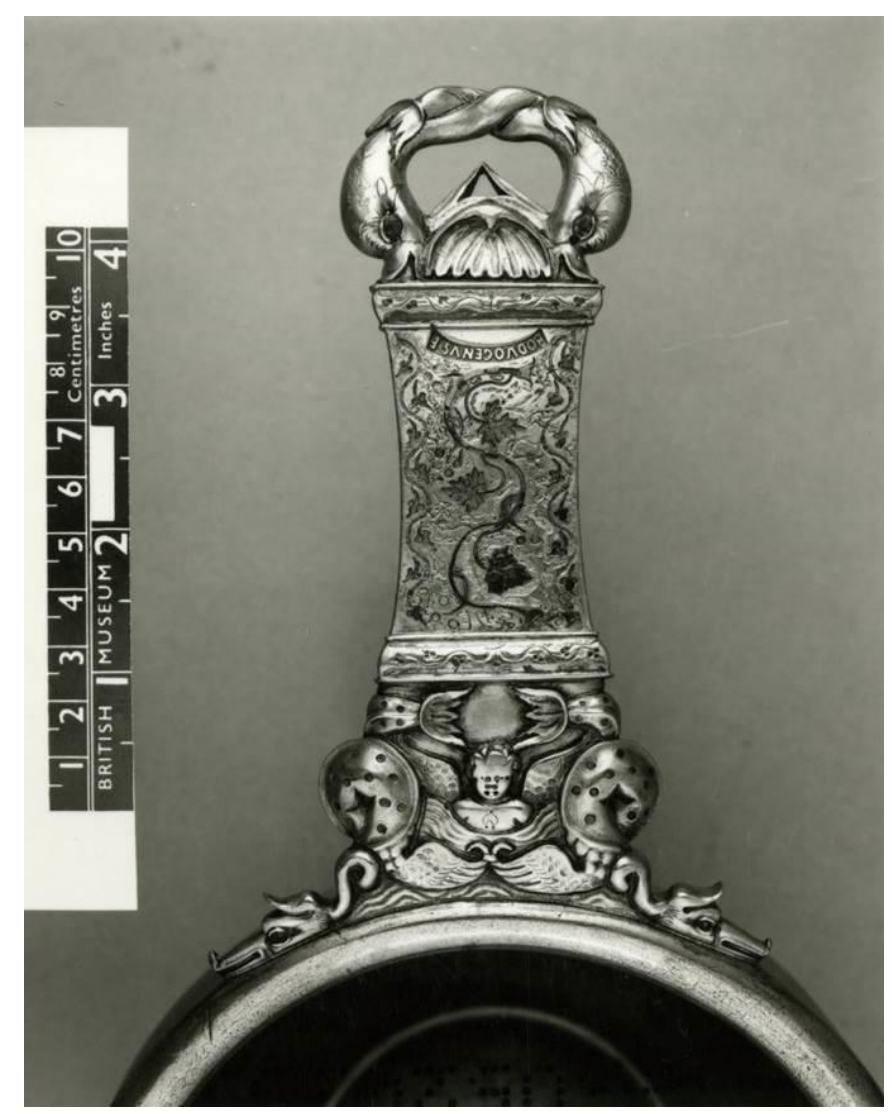

Figure 5.04: Handled Pan 2 from Prickwillow (C the British Museum)

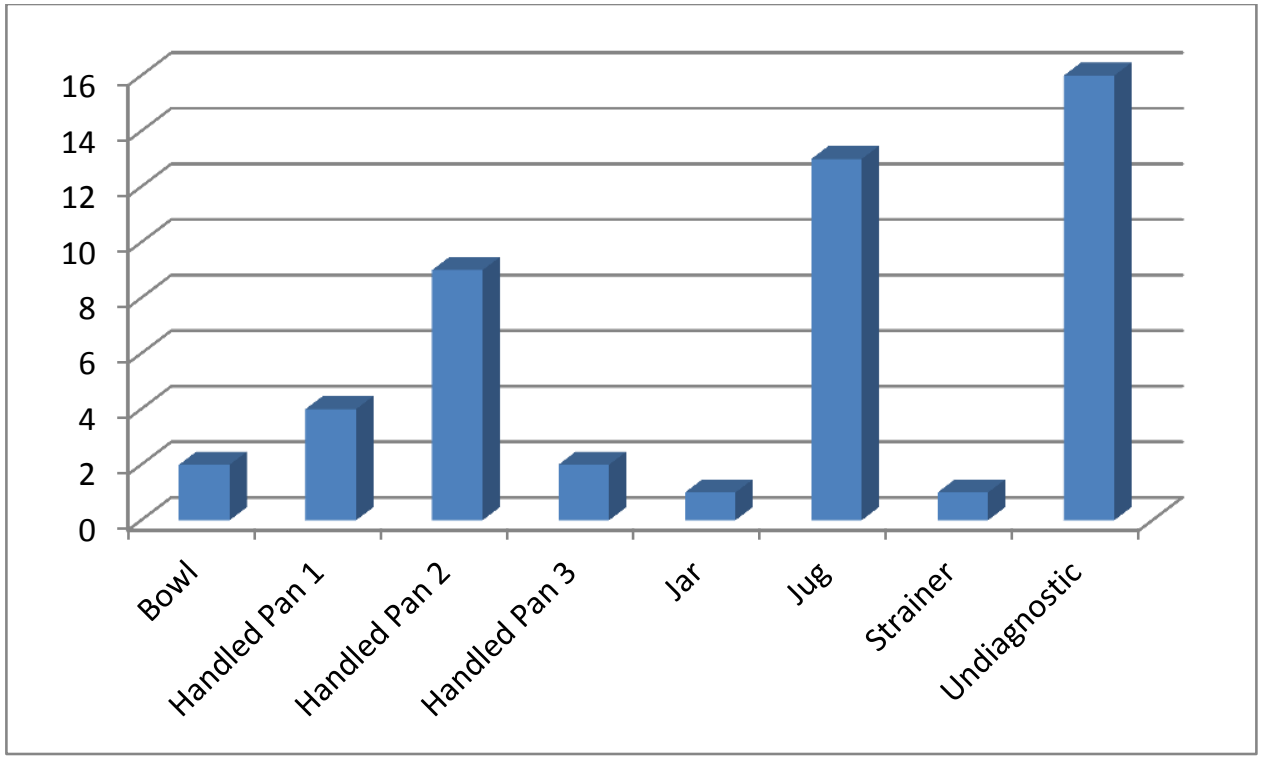

Figure 5.05: Vessel forms from the Midlands. 


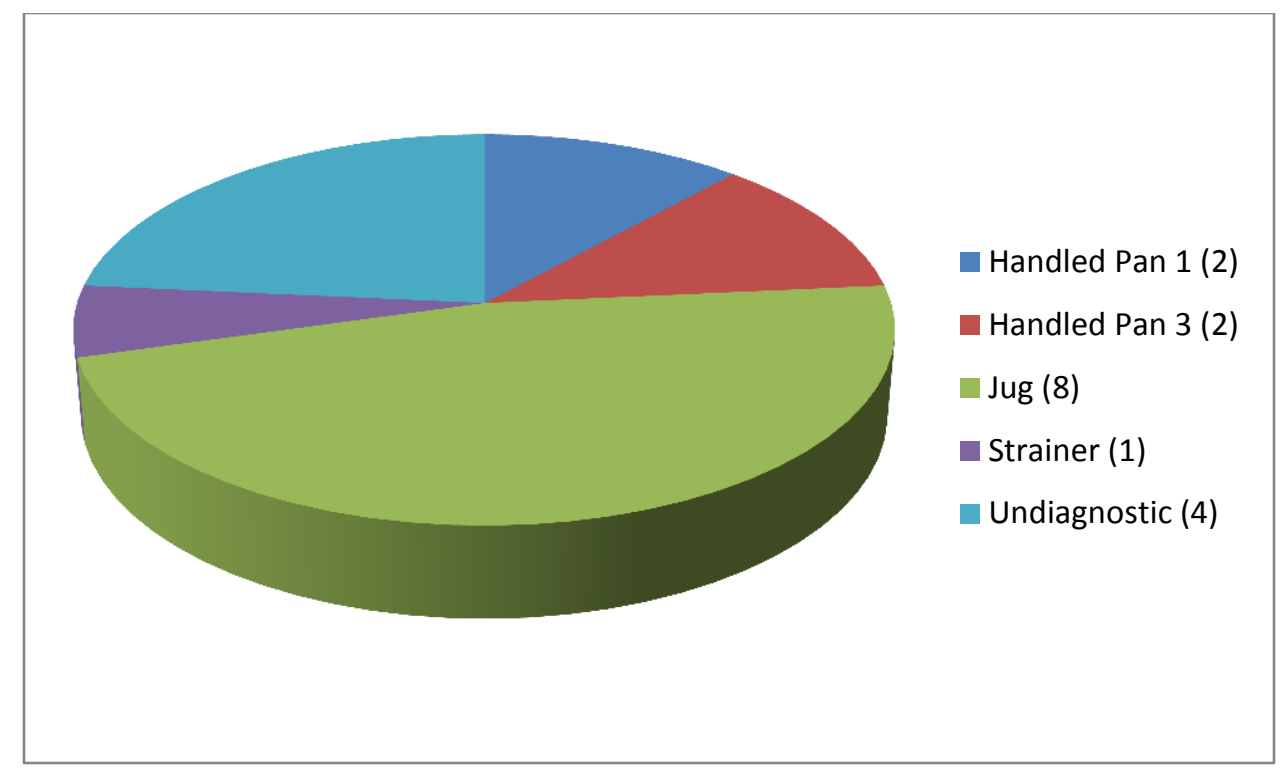

Figure 5.06: Site Finds by form from St.Albans/Verulamium.

While handles from Handled Pan 1s and Handled Pan 3s are present, Handled Pan 2s are conspicuously absent at Verulamium. Fragment HER0010 with a concentric circle umbo could well have originated from such a vessel, though it could also have originated from a bowl or hanging basin. Most of the copper alloy vessels found at St. Albans appear to have been deposited in the Late Roman period, ${ }^{818}$ possibly being more representative of the decline of the town in the $4^{\text {th }}$ century and a breakdown in the mechanisms of production and recycling than any increase in popularity and consumption of the material itself, as there is indeed evidence for a sharp downturn in manufacturing and commerce in the urban centres of Britain generally during the Late Roman period. ${ }^{819}$ This coincides with what is currently understood of the slow decline of the Roman settlement at Verulamium during the $4^{\text {th }}$ century, leading to its eventual abandonment as an urban centre sometime in the $5^{\text {th }}$ century. ${ }^{820}$

Two other important urban centres which contributed significant numbers of objects to this data-set are present in the areas of East Anglia and the greater south east of England, these being Colchester with 17 objects and London with 42 objects (Table 5f). The high numbers from these sites both reflect their importance during the Roman period as well as the archaeological attention and subsequent publication they have received in modern times.

\footnotetext{
${ }^{818}$ Frere 1972, 130-144.

${ }^{819}$ Mattingly 2006, 325-350.

${ }^{820}$ Niblett \& Thompson 2005, 164.
} 


\begin{tabular}{|c|c|}
\hline Site Finds from Colchester & Site Finds from London \\
\hline $\begin{array}{c}\text { Strainer/EX0001 } \\
\text { Bowl/EX0002 } \\
\text { Bowl/EX0003 } \\
\text { Bowl/EX0004 } \\
\text { Vessel(mount)/EX0005 } \\
\text { Handled Pan 3/EX0006 } \\
\text { Jug(handle)/EX0007 } \\
\text { Handled Pan 2/EX0024 } \\
\text { Handled Pan 2/EX0025 } \\
\text { Handled Pan 2/EX0026 } \\
\text { Handled Pan 2/EX0027 } \\
\text { Jug/EX0028 } \\
\text { Jug/EX0029 } \\
\text { Handled Pan 2/EX0037 } \\
\text { Jug(handle)/EX0056 } \\
\text { Handled Pan 2/EX0068 } \\
\text { Jug(handle)/EX0069 }\end{array}$ & $\begin{array}{c}\text { Bowl/L0016 } \\
\text { Jug(lid)/L0017 } \\
\text { Vessel(fragment)/L0018 } \\
\text { Jug(handle)/L0019 } \\
\text { Handled Pan 3/L0020 } \\
\text { Bucket/L0021 } \\
\text { Jug(lid)/L0022 } \\
\text { Handled Pan 1/L0023 } \\
\text { Handled Pan 2/L0024 } \\
\text { Handled Pan 2/L0025 } \\
\text { Jar/L0026 } \\
\text { Jug(fragment)/L0027 } \\
\text { Handled Pan 3/L0028 } \\
\text { Bowl/L0029 } \\
\text { Vessel(fragment)/L0030 } \\
\text { Vessel(fragment)/L0031 } \\
\text { Handled Pan 5/L0032 } \\
\text { Strainer/L0033 } \\
\text { Vessel(fragment)/L0035 } \\
\text { Bowl/L0036 } \\
\text { Handled Pan 2/L0037 } \\
\text { Vessel(fragment)/L0038 } \\
\text { Cauldron/L0039 } \\
\text { Jug(lid)/L0040 } \\
\text { Vessel(fragment)/L0041 } \\
\text { Jug(lid)/L0042 } \\
\text { Jug(lid)/L0043 } \\
\text { Jug(lid)/L0044 } \\
\text { Jug(lid)/L0045 } \\
\text { Jug(lid)/L0046 } \\
\text { Jug/L0047 } \\
\text { Jug/L0048 } \\
\text { Jug(handle)/L0049 } \\
\text { Jug(handle)/L0050 } \\
\text { Jug(handle)/L0051 } \\
\text { Vessel(mount)/L0052 } \\
\text { Bucket/L0053 } \\
\text { Bucket/L0054 } \\
\text { Bowl/L0055 } \\
\text { Jar/L0056 } \\
\text { Bowl/L0058 }\end{array}$ \\
\hline
\end{tabular}

Table 5f: Site Finds from Colchester and London (all urban contexts). 
One find from London is worthy of particular note, and that is the cauldron found in the bottom of a well at Blossom's Inn associated with domestic pottery and refuse suggesting a late $2^{\text {nd }}$ century CE date. ${ }^{821}$ Its complete nature and its presence at the bottom of the well suggests a possible purposeful intent in its deposition, but as there is no other evidence for this being a ritual deposit, it is regarded here as a Site Find. Found at the bottom of the well with the cauldron were the remains of several wooden buckets and lengths of bucket chains. While the material found in the well with the cauldron dates to 150-200 CE, rubbish and coin evidence in the area suggests that the site was in continued use well into the $4^{\text {th }}$ century. ${ }^{822}$ It would seem that the well-house may have burned down and the well itself gone into disuse by the end of the $2^{\text {nd }}$ century, ${ }^{823}$ indicating that the cauldron could have been an accidental loss associated with this event. Large basins of this kind are not common prior to the Late Roman period, so its association here with material of the $2^{\text {nd }}$ century is intriguing and may represent one of our earliest datable examples of this vessel form. ${ }^{824}$

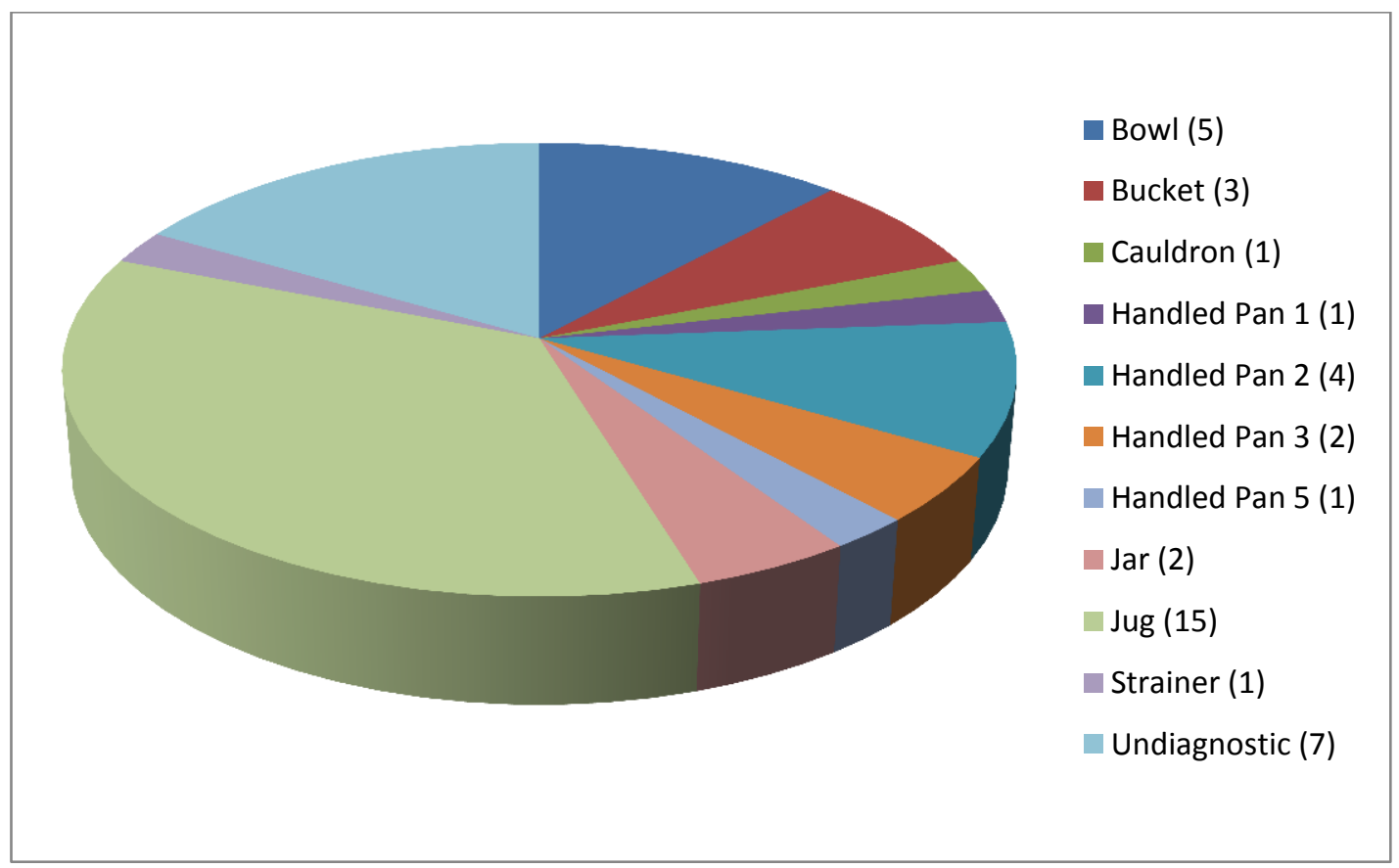

Figure 5.07: Site Finds by form from London.

\footnotetext{
${ }^{821}$ L0039; Burnham et al. 2002, 326-331; Blair et al. 2006, 24.

${ }^{822}$ Burnham et al. 2002, 329-331.

${ }^{823}$ Blaire et al. 2002, 24.

${ }^{824}$ See Chapters 2 and 7 for further discussion.
} 
As illustrated by Figure 5.07, the Site Finds for London more generally are diverse and represent a variety of vessels being used by the population, reflecting both London's complex and multi-faceted character during Roman times as well as the comparatively large assemblage size from this site. Colchester's assemblage is also more diverse than those found on most other sites in Britain (Figure 5.08), though Handled Pan 2s have a noticeable predominance, accounting for about $33 \%$ of the material.

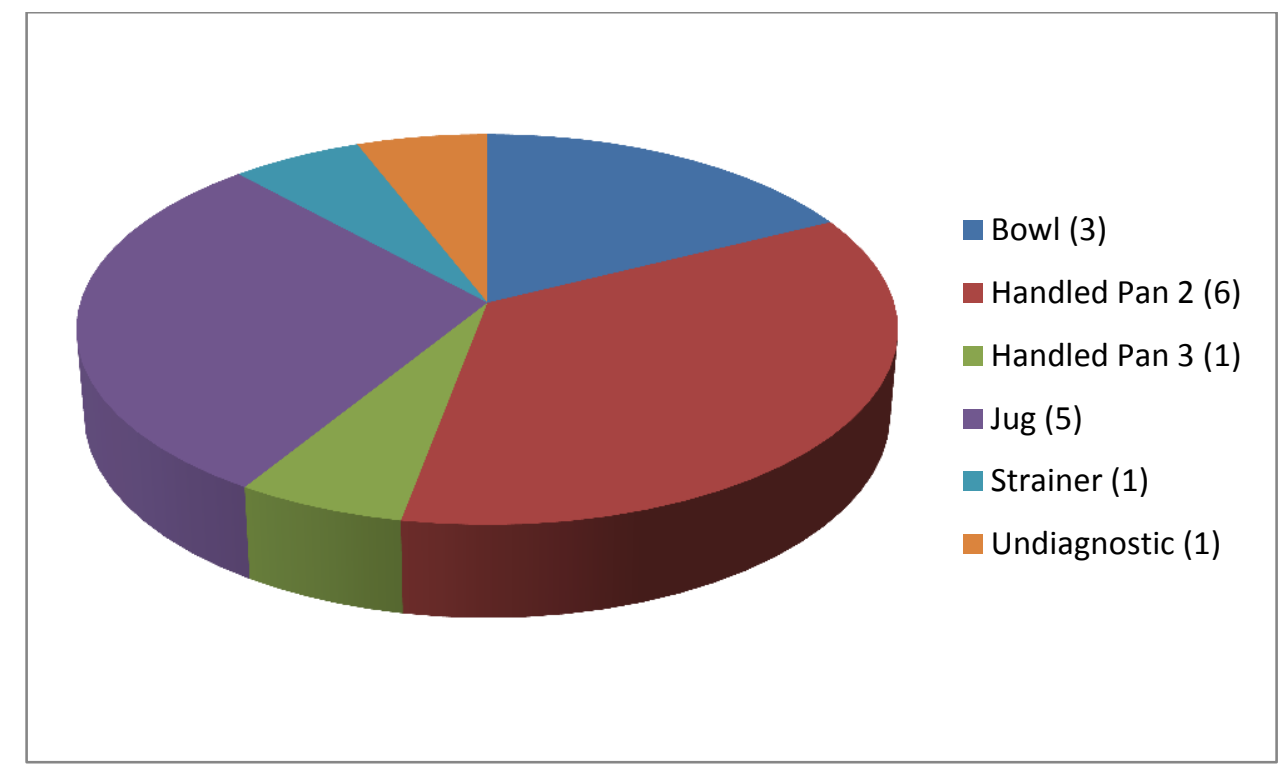

Figure 5.08: Site Find forms from Colchester.

It is also noteworthy that most of the datable finds from the sites of St. Albans, Colchester and London postdate the Boudiccan rebellion. ${ }^{825}$ This would imply that either copper alloy vessels were not common domestic items prior to this period in urban Britain, or more likely that copper alloy vessels were seen as worthy and useful plunder to be extracted from the town by the attackers or carted away by the refugees, thus removing them from the archaeological record of the Boudiccan destruction layers. It should also be noted that domestic assemblages from the Boudiccan destruction layers are not particularly rich in any objects, though some ceramics and glass has been recorded, which has been suggested as indicating either a modest level of material culture available at the early Roman settlements or the thorough ransacking by the Boudiccan forces. ${ }^{826}$

\footnotetext{
${ }^{825}$ EX0002,EX0003 \& EX0069 in Colchester being the only two objects that seem clearly datable to this calamity.
} ${ }^{826}$ Gascoyne \& Radford 2013, 96. 
The distribution of Site Finds in East Anglia and the south east of England is wide and thinly dispersed, with most sites being rural and only having one copper alloy vessel, problematizing the characterization of the use of copper alloy vessels at these sites (See Appendix IX). However, there are a number of sites (generally of an urban or military character) that do contribute multiple objects to this data-set, these are: Hacheston (Suffolk), Richborough and Canterbury in Kent, Chichester and Fishbourne Villa in West Sussex, and Silchester in Hampshire.

The Site Finds from Hacheston are unusual as four out of five of them are leaf shaped hanging vessel mounts. ${ }^{827}$ This type of hanging vessel mount features commonly among Site Finds and Portable Antiquities Scheme data in this thesis and is likely to be predominately Late Roman in origin. ${ }^{828}$ The site of Hacheston sits on the edge of the northern floodplain of the River Deben and would appear to have provided access between that river and the River Ore, making it a suitable site for a village with a role in trade. The site does not appear to have had any strong military presence during the Roman period, and the settlement does not appear to have been inhabited much later than $370 \mathrm{CE}{ }^{829}$

The fort at Richborough in east Kent has received considerable archaeological attention with excavations performed on-and-off since $1922 .{ }^{830}$ This settlement was established early in the Roman occupation as a shore fort. It continued in use through the Roman period and would appear to have had both significant military and civil components, ${ }^{831}$ its shell-fish exports being of enough renown to warrant mention by Juvenal (4.141). Fragments of a Handled Pan 1 and three different jug fragments in the vicinity may indicate a disturbed grave context for these finds, though this is unprovable. ${ }^{832}$ The other finds from this site are highly fragmentary and undiagnostic, offering little aid in evaluating the place and function of copper alloy vessels at this settlement.

Though it was a prominent urban centre that lay along key communication routes by road and river, ${ }^{833}$ there has been comparatively little excavation and publication of the remains of Roman Canterbury. This is reflected in there being only two objects that it contributes to the

\footnotetext{
${ }^{827}$ Blagg et al. 2004, 123-124.

${ }^{828}$ Kendrick 1932, 162-163; Bruce-Mitford 2005 34-37.

${ }^{829}$ Blagg et al. 2004, 197-199.

${ }^{830}$ Bushe-Fox 1926.

${ }^{831}$ Cunliffe 1968, 231-251.

${ }^{832}$ See Chapter 4.

${ }^{833}$ Jessup 1930, 194-199.
} 
current data-set. This paucity of data likely under-represents its probable importance during the Roman period and is a sign of under-publication as opposed to offering a representative view of the Roman material at the site. The Rudge Cup type Handled Pan 2 is worth noting, ${ }^{834}$ both for its aesthetic appeal and as it contributes to an ever growing corpus of these vessels.

The sites of Roman Chichester and Fishbourne Villa are within 5 kilometres of each other, the latter probably representing the lavish residence of an aristocrat associated with the former urban establishment. ${ }^{835}$ Both have received extensive archaeological attention during the twentieth century and have provided several fragmentary remains of copper alloy vessels, though most of them too fragmentary for exact identification. The most impressive fragment to come to light is the jug handle found during construction of the Havant-Chichester bypass, which is decorated with floral vine leaves along the handle with a feline paw directly above a cherubic handle medallion. ${ }^{836}$ A close parallel to this object from the Palace at Boscoreale implies a preVesuvian date to this object's manufacture ${ }^{837}$ and the finding of both of these objects at high status villas enticingly suggests the high value and luxury of the object. It also hints at the probable high status of the owner of the villa in the $1^{\text {st }}$ century $\mathrm{CE}$, as he had access to the same objects that are believed to have been enjoyed by those associated with the imperial family in Italy.

The site of Silchester would appear to have been of some importance as a commercial centre by the Late Iron Age, ${ }^{838}$ though its construction as a Roman urban settlement probably dates to the Flavian period. ${ }^{839}$ The copper alloy vessels found at this site are in a highly fragmented state and it would appear that none of them come from layers later than the $3^{\text {rd }}$ century CE. However, this does not reflect a downturn in the settlement as there is plenty of numismatic evidence from the site to suggest an active economy until the late $4^{\text {th }}$ or early $5^{\text {th }}$ century CE. ${ }^{840}$ It may reflect a decline in the use of copper alloy vessels in this town during the Late Roman period, as there is plenty of ceramic and glass vessel material from this site that

\footnotetext{
834 K0026.

${ }^{835}$ Popular tradition attributing it to Tiberius Claudius Cogidubnus, a client king mentioned by Tacitus (Down 1988, 17-27).

${ }^{836}$ WSU0007; Down \& Henig 1988, 308-309.

${ }^{837}$ Cunliffe et al. 1996, 200 (45).

${ }^{838}$ Boon 1974, 36-42.

${ }^{839}$ Boon 1974, 53.

${ }^{840}$ Fulford \& Clarke 2011, 350-364.
} 
could have fulfilled the same functions as copper alloy vessels for the city's inhabitants. ${ }^{841}$ This could relate to how this metal was perceived and how its intrinsic wealth was chosen to be concentrated; this shall be discussed in greater detail in Chapter 7. Apart from a single jug handle ${ }^{842}$ the fragments from Silchester are undiagnostic as to vessel form or type, though the two pelta shaped vessel feet ${ }^{843}$ may have come from Hemmoor type buckets or similar vessels of a likely $2^{\text {nd }}$ century CE manufacture date. No handled pans or hanging basins are identifiable among the copper alloy vessel remains from Silchester, the latter perhaps reflecting the lack of Late Roman copper alloy vessels more generally.

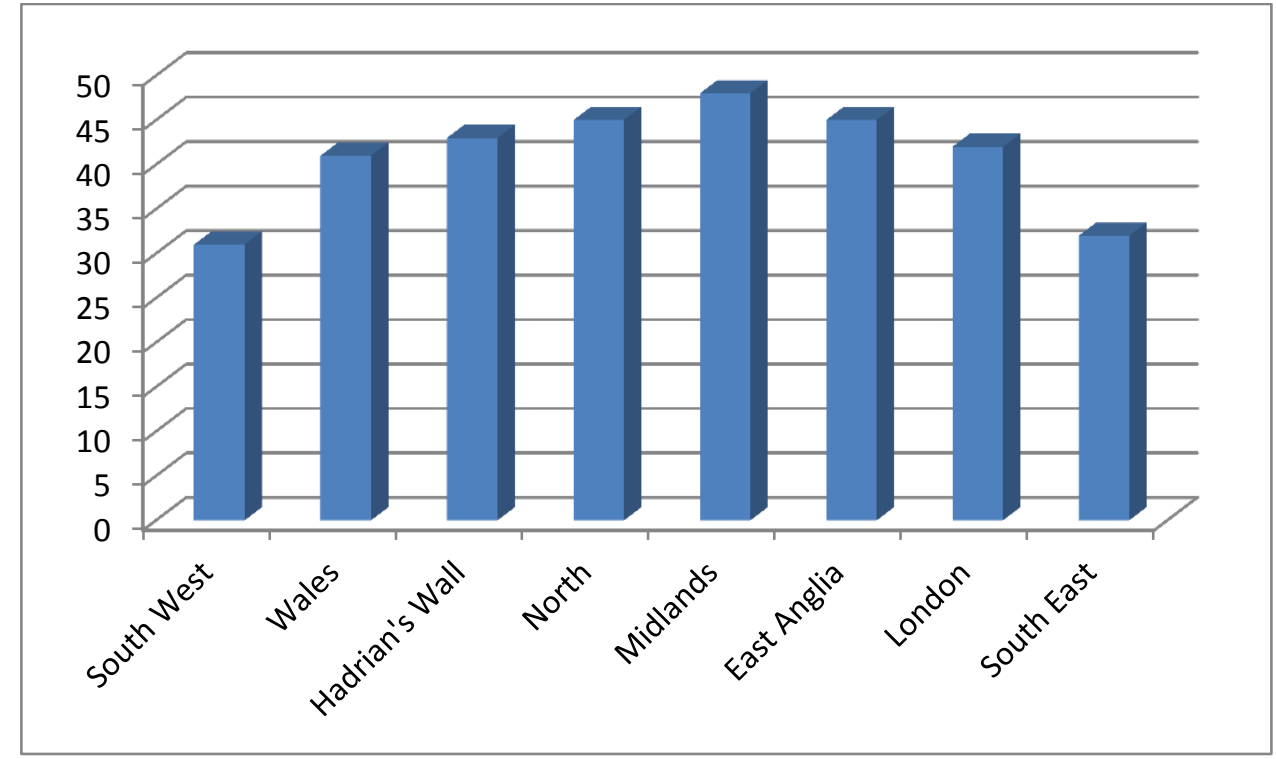

Figure 5.09: Site Finds from all regions.

As both Figure 5.09 and Map 3 illustrate, copper alloy vessels are widely distributed across Britain as Site Finds during the Roman period and have a far more extensive distribution, with a greater consistency in the number of objects across regions, than either Structured Deposits or Grave Deposits. This would indicate that any geographic bias witnessed in these other data-sets has to do with regional practices in the deposition of copper alloy vessels as opposed to representing the wider availability and usage patterns of this commodity.

The presence of material in the northern Midlands fills in a significant geographic area not seen in Structured Deposits and Grave Deposits. While more examples may be available for

\footnotetext{
${ }^{841}$ Fulford et al. 2006, 86-119.

842 HAM0020.

${ }^{843}$ HAM0017-HAM0019.
} 
the southeast, the spread of material indicates that copper alloy vessels would have been available across the span of the province and most likely to a wider segment of society than may be indicated by the data in Structured Deposits and Grave Deposits, which seems to provide data most predominantly for elites and/or sub-elites. ${ }^{844}$ Patterns in decoration amongst the Site Finds data-set of this thesis will be examined in Section 5.4. Presently, Site Types and chronology will be examined and related to patterns of distribution and consumption.

\section{$\underline{5.3 \text { Site Type and Chronological Comparison of Site Finds Distribution }}$}

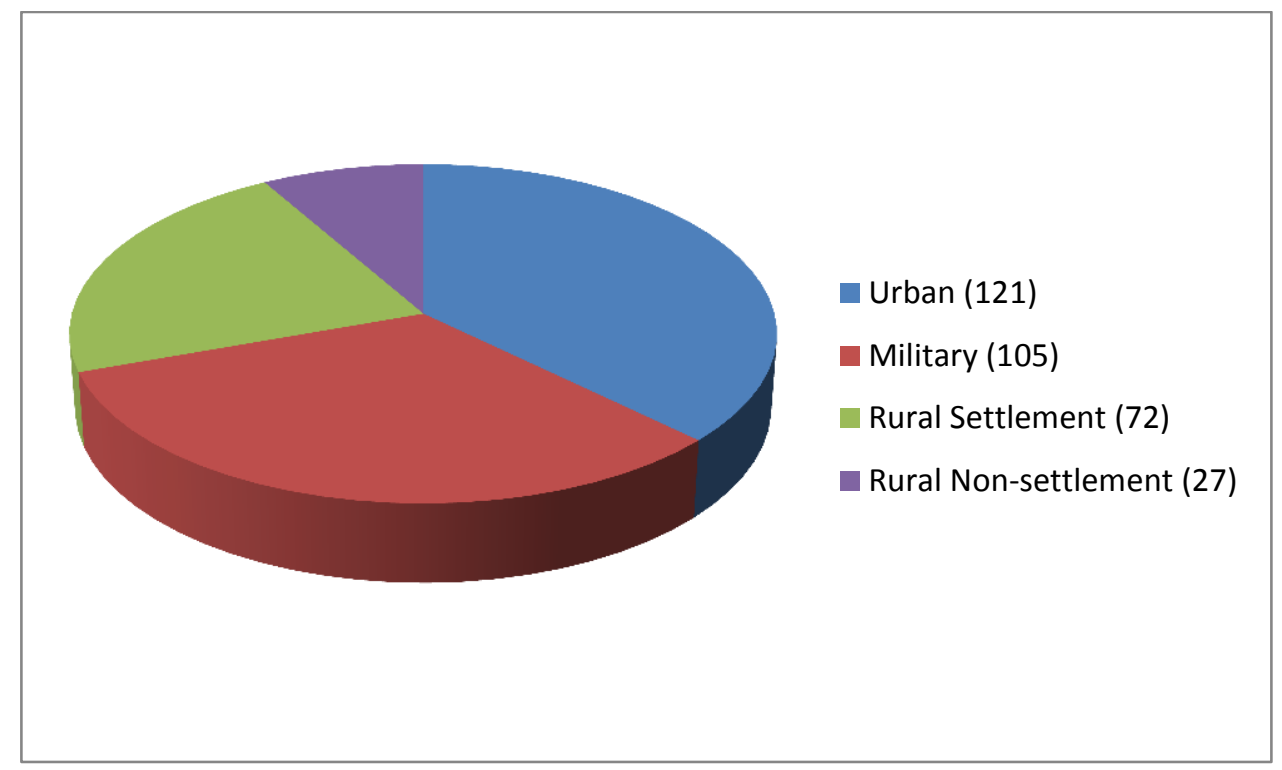

Figure 5.10: Distribution of Site Finds by Settlement type.

As may be seen from Figure 5.10, copper alloy vessels as Site Finds are most commonly found in Urban and Military settings. This may be due in part to how these sites are preferentially targeted for excavation in comparison to rural settlements, though study of the rural environment has gained increasing ground in recent decades. ${ }^{845}$ The urban bias may also be in large part due to the high level of study and publication of the sites of Colchester, London and St. Albans specifically. These three sites alone count for 75 out of the 121 Site Finds from urban contexts in this thesis. Even so, it may be expected that urban and military centres would produce a greater amount of finds simply do to a higher level of population density and a greater number of higher status individuals at these sites with the purchasing power for such

\footnotetext{
${ }^{844}$ See Chapters 3 \& 4.

${ }^{845}$ Hingley 1982; Millett 1990; Hingley 1997; Brindle 2010; Brindle 2012.
} 
commodities than may be expected for the majority of non-villa rural settlements. Though it has been mentioned that excavation bias likely plays a role in the prominent visibility of material from urban and military centres, there are enough finds reported from other settlement types in this thesis to assume that the distribution of finds is approximately representative of how this material is indeed distributed across the landscape and not a result of modern bias. The distribution implies that copper alloy vessels were available and consumed in the countryside and it is very likely that the population density of cities and forts has led to the larger numbers of objects from these sites as opposed to reflecting relative availability of this commodity.

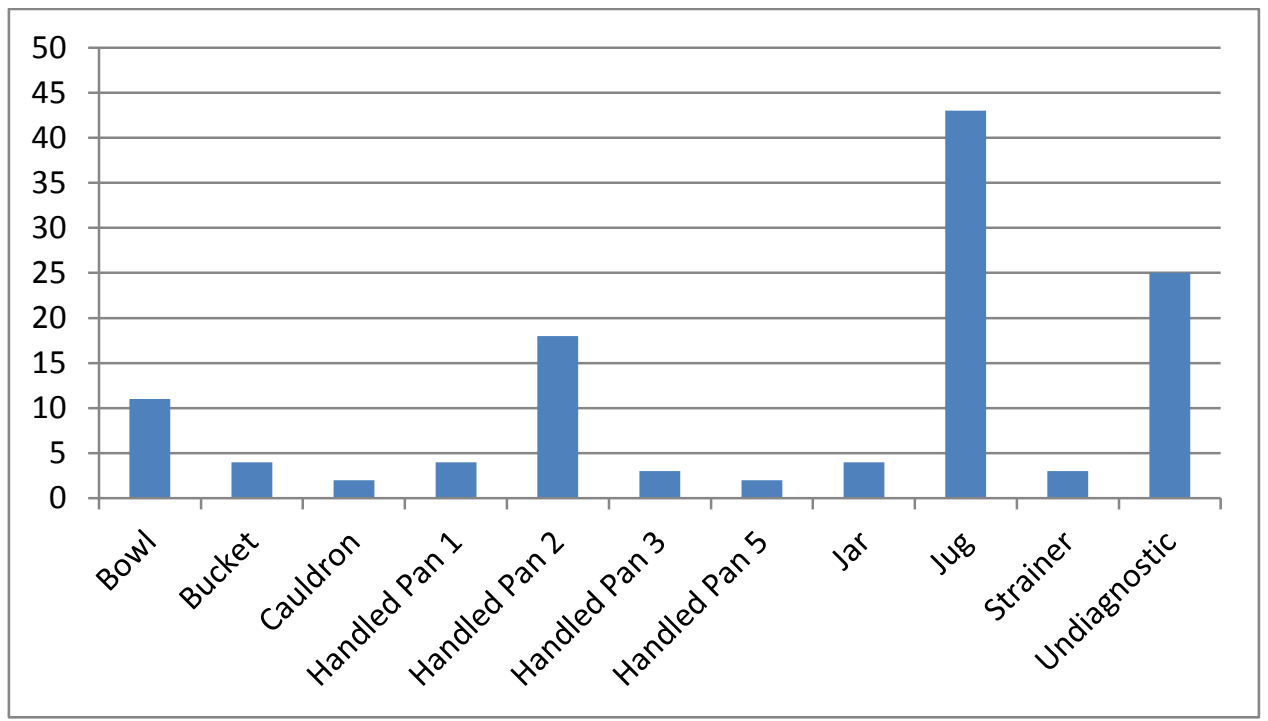

Figure 5.11: Site finds forms from Urban sites.

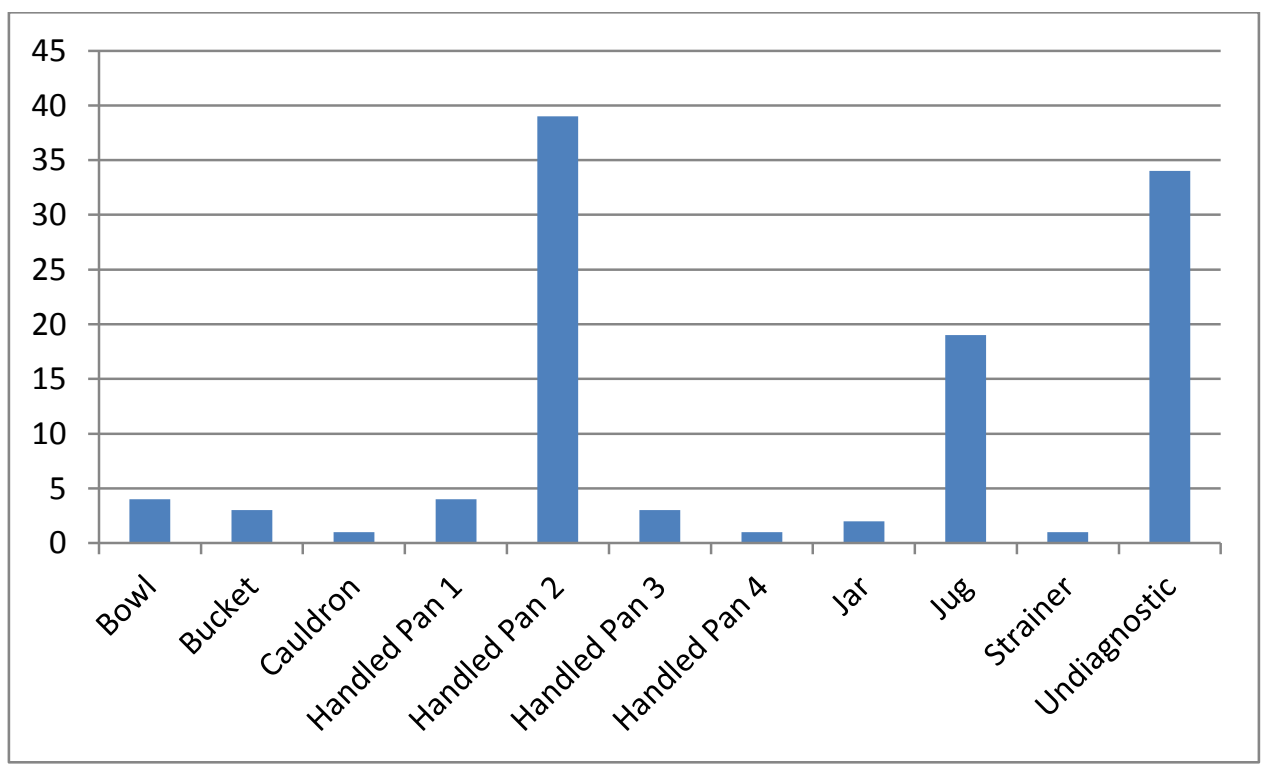

Figure 5.12: Site Finds forms from Military sites. 


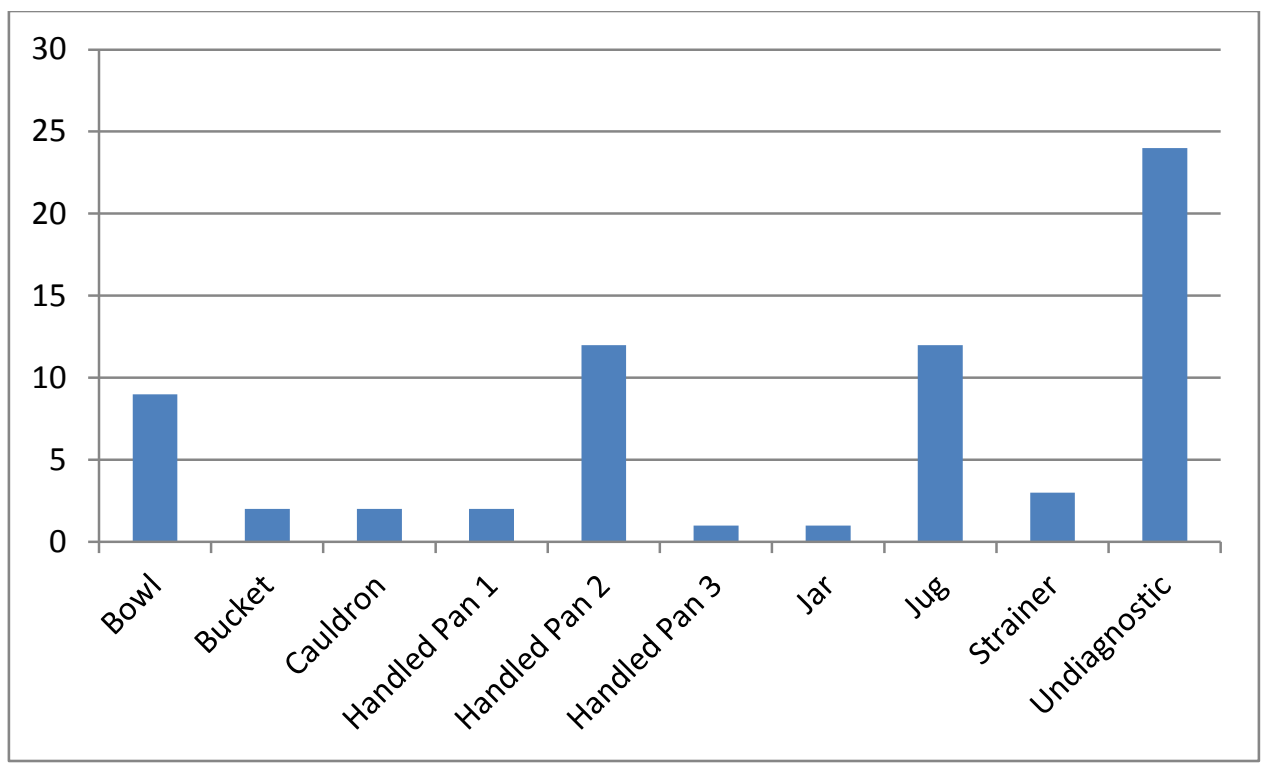

Figure 5.13: Site Find forms from Rural Settlements.

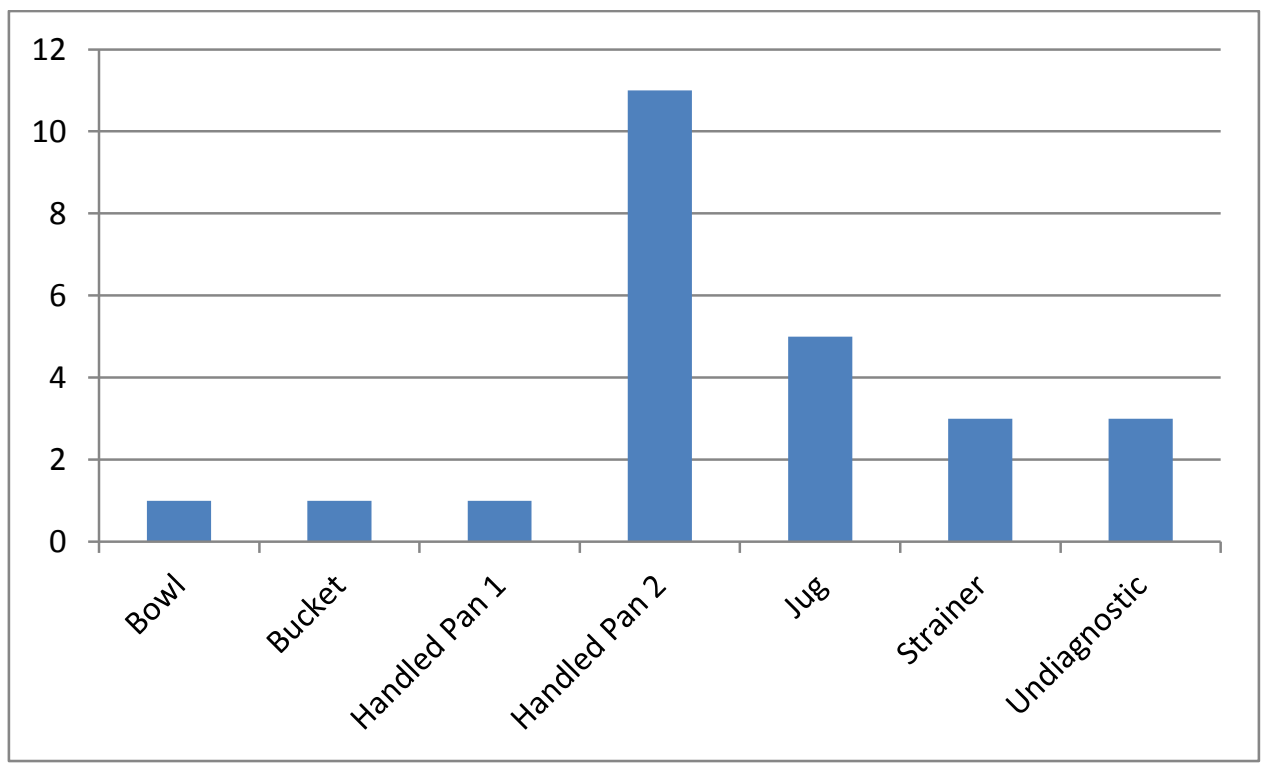

Figure 5.14: Site Find forms from Rural Unknown sites.

More useful information may be gleaned from comparing object forms across Site Types to distinguish patterns in deposition that may reflect preferential consumption. As Figures 5.115.14 make clear, the most obvious trend that appears in the data-set is the preponderance of Handled Pan 2s (specifically of Eggers types 139-144) on military sites as well as urban sites with a strong military presence. This association has been highlighted ${ }^{846}$ will be further examined

${ }^{846}$ Sections 3.2, $3.3 \& 3.4$. 
in Chapter 7, but the evidence from the Site Finds data-set has been instrumental in supporting this conclusion. Jugs occur regularly on all Site Types in fragmentary form, but this may be due at least in part to the fact that jugs have handles and lids that may break off and be misplaced while other forms, such as bowls, are less partible in their composition. Even with this in mind, the frequency of jugs in the data-set is note-worthy and it would seem that a functional and utilitarian vessel form such as this would have been widely utilised.

Chronological shifts in the presence of forms and types seem to generally reflect those seen in Structured Deposits, ${ }^{847}$ representing a shift from smaller vessels at the beginning of the Roman period to larger bowls and hanging basins by the end of the Roman period. ${ }^{848} \mathrm{~A}$ significant gap between the early and late Roman periods is filled in by the Site Finds data-set which is not present in Structured Deposits. This is most evident in the popularity of buckets as Site Finds, most of which were manufactured in the $2^{\text {nd }}-3^{\text {rd }}$ centuries CE. ${ }^{849}$ This preference is also witnessed in the grave assemblages of Brougham, also dating from the late $2^{\text {nd }}$ to mid- $3^{\text {rd }}$ century. ${ }^{850}$ This suggests that while Brougham may have been something of an anomaly in its grave treatments, it likely represented rather characteristic tastes in the choices of copper alloy vessels used during the $3^{\text {rd }}$ century CE in Britain.

\footnotetext{
${ }^{847}$ See Chapter 3.

${ }^{848}$ An exception to this would appear to be CAR0003, a Rudge Cup type handle found in a post hole of Late Roman date. This handle likely dates to significantly earlier than this period and may be considered an accidental or intrusive inclusion.

${ }^{849}$ Erdrich 1995, 71-80.

${ }^{850}$ See Chapter 4.
} 


\section{$\underline{5.4 \text { Discussion of Decoration among Site Finds }}$}

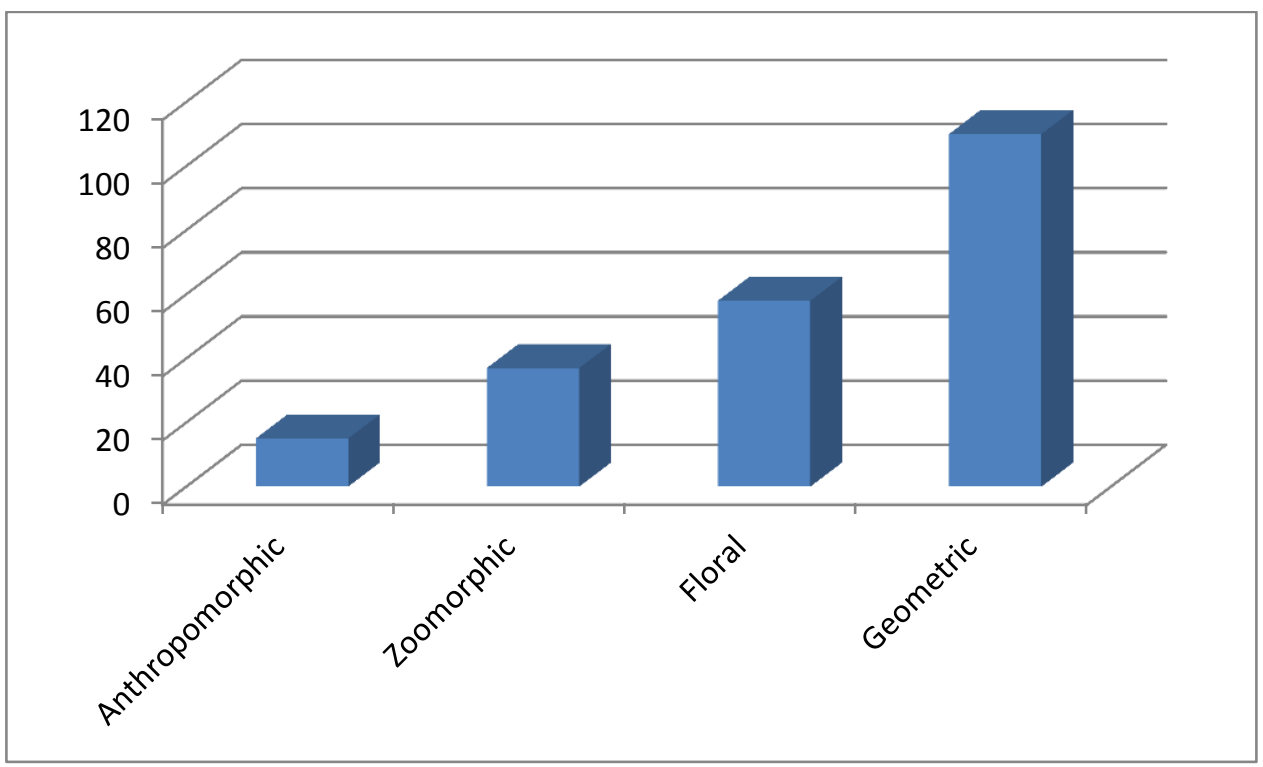

Figure 5.15: Objects with decoration in SF data-set.

As seen in Figure 5.15, there is a high frequency of decoration in the Site Finds data-set. Though the decoration of these vessels is diverse, there are distinct preferences evident. Geometric decoration is far more common than any other types. Other patterns in the use of decoration may also be noted. These include the zoomorphic handle terminals of Handled Pan 1s, bovine head hanging vessel mounts, anthropomorphic/mythological handle medallions, and the recurring use in different manners of birds, dolphins and floral/vine motifs. As there are 11 Rudge Cup type vessel/fragments in this data-set, enamelled decoration also features noticeably among Site Finds of copper alloy vessels. ${ }^{851}$

\footnotetext{
${ }^{851}$ The decoration of Rudge Cup type HP2s among Site Finds is consistent with other examples of this type of vessel, so comment on Rudge Cup type decoration will not be discussed in detail in the present Chapter; See Section 8.3.
} 


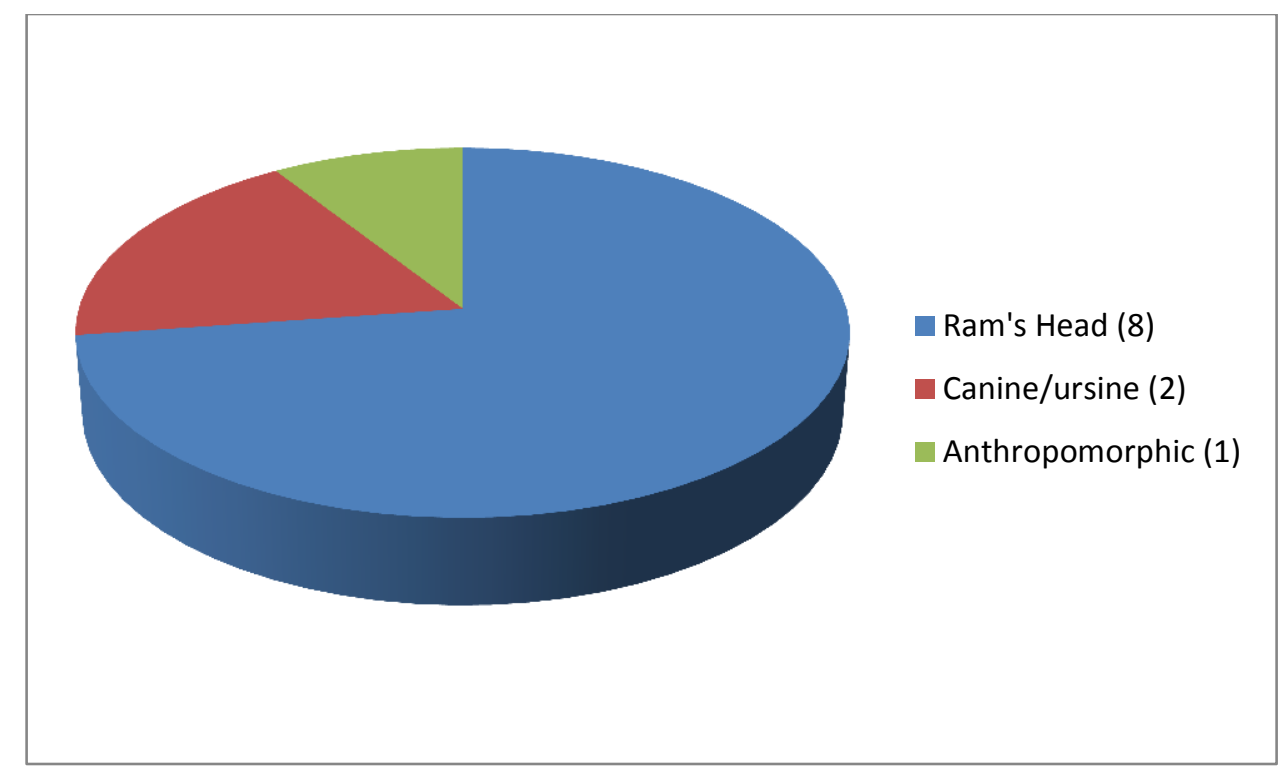

Figure 5.16: Iconography of Handled Pan 1s in Site Finds data-set.

Ram's heads predominate in the zoomorphic handle terminals from Handled Pan 1s as Site Finds (Figure 5.16). This trend is also seen in the selection of material in Grave Deposits and seems to be representative of the preferred iconography of this type of vessel. The majority of these finds come from within settlements, and are therefore unlikely to represent disturbed grave material. ${ }^{852}$

Five hanging vessel mounts of bull/ox heads, most likely from buckets, are included in this data-set. Though this is an iconographic feature, it must be remembered that these hanging vessel mounts served a functional purpose for a vessel. Its presence in the Site Finds data-set therefore has dual significance both as a stylistic choice as well as implications of practical application. While bull-headed hanging vessel mounts occur less frequently in Grave Deposits than as Site Finds, this is likely due mostly to the chronology of this practice as such Roman period mounts are most common on buckets or hanging basin of $2^{\text {nd }}$ century CE or later manufacture, ${ }^{853}$ falling mostly after the principal period of the grave deposition of copper alloy vessels. Where bovine hanging vessel mounts do occur in graves, ${ }^{854}$ these appear to be exclusively from later deposits. The greater frequency of this iconographic element will be

\footnotetext{
${ }^{852}$ As opposed to HP1 handles recorded through the PAS, see Chapter 6.

${ }^{853}$ Hawkes 1951; Kennett 1971; Erdrich 1995; Bruce-Mitford \& Raven 2005.

${ }^{854}$ Such as Brougham in Cumbria.
} 
further seen in the material provided through the Portable Antiquities Scheme database ${ }^{855}$ and its presence plays an important role in characterising changing patterns in vessel use. ${ }^{856}$

Four of the fifteen anthropomorphic representations in this data-set are Gorgon busts. The Gorgon bust is a familiar motif in classical art, and its apotropaic functions are well recognised. ${ }^{857}$ The necessity of such functions on metal vessels is not immediately apparent. Where it appears on Handled Pan 1s or jugs it could well serve as an aid to the process of purification by warding off evil influences. The Gorgon bust also reflects familiarity with Classical learning, and could have been used as a sign of formal education and intellectual class, though it is not possible to determine if the owner of such vessels fully comprehended their iconographic decoration or not. ${ }^{858}$

The frequency in the use of birds for the decoration of copper alloy vessels among Site Finds is not paralleled by either Structured Deposits or Grave Deposits. The same is true, to a lesser extent, of dolphins. This may partly be explained in the vessel forms for which this decoration was most often associated, as many of the avian representations feature as small duck statuettes on trefoil jug lids. This could help explain the absence of the birds in Structured Deposits, particularly Late Roman Structured Deposits, as jugs were simply not commonly selected for inclusion as part of these assemblages. This explanation does not apply when considering Grave Deposits, as jugs were a common feature in Grave Deposits partnered with Handled Pan 1s. ${ }^{859}$ There are no lids associated with the jugs that feature in the Grave Deposits, ${ }^{860}$ which would inherently exclude the 'sitting duck' statuette. This is likely the result both of chronology and geography, as the lidded jugs would appear to be from contexts of $2^{\text {nd }}$ and $3^{\text {rd }}$ centuries CE (as opposed to the $1^{\text {st }}-2^{\text {nd }} \mathrm{CE}$ dates for most graves including jugs) and they are most popular to the west of the principal centres for Jug and Pan type graves, such as Hampshire and the western Midlands. Such a regional preference to this decorative feature suggests local production centres, perhaps simply for the jug lids if not for the entire vessel. Avian iconography is also present on handles and rim accents to hanging basins and handled

\footnotetext{
${ }^{855}$ Chapter 6.

${ }^{856}$ Chapters $7 \& 8$.

${ }^{857}$ Wilk 2000, 31-54.

${ }^{858}$ See Sections 4.4 \& 8.3.

${ }^{859}$ See Chapter 4 throughout, specific discussion in Section 4.4.

${ }^{860}$ As will be further discussed in Chapter 7.
} 
pans. These also appear to be aquatic birds, either ducks or swans, which occur widely without any geographic patterning immediately apparent.

Dolphins appear as decorations on six of the jug handles in the Site Finds data-set of this thesis. ${ }^{861}$ This in itself is not overly surprising, as dolphins were popular motifs in Roman art with examples in every medium from stone carving to mosaics to terra cotta. ${ }^{862}$ Like avian iconography, dolphin motifs appear with a greater frequency as Site Finds than in either Structured Deposits or Grave Deposits. With the exception of the Prickwillow Handled Pan 2, ${ }^{863}$ all the examples of dolphin iconography from Site Finds appear to originate from jugs. This means that like avian iconography, its absence in Structured Deposits may be explained by a variance in the forms this decoration is applied to. However, this explanation does not justify its absence in Grave Deposits. Nor would an exclusion of aquatic motifs from funerary depictions explain it, as the Turner's Hall Farm graves show. ${ }^{864}$ The discrepancy between data-sets is perhaps best explained by geographic variation, as dolphin handles do not appear in the iconography from Site Finds from the areas where the Jug and Pan type of burial was practiced.

Floral decoration is also prevalent in this data-set. These are composed of leaf and vine decorations, predominantly on jugs and on pan handles. There is nothing singularly noteworthy in the floral decoration among Site Finds to differentiate it in any way from what is found more generally across all of the data-sets of this thesis, though floral decoration is more visible among Site Finds than among Structured Deposits.

By far the most iconographically complex object from the Site Finds data-set of this thesis is the Handled Pan 2 found at Prickwillow (Figure 5.04). ${ }^{865}$ Two ketoi frame the rim at the handle and morph into waves that descend down the grip. An anthropomorphic winged male bust in relief wearing a crescent medallion around his neck is framed by waves and the tail fins of the sea-dragons on either side. The middle of the handle is decorated with elaborate grape vines in niello with the handle loop comprising two dolphins. The inscription BODVOGENVS F[ECIT] is stamped just above the handle loop. It is worth noting that Bodvogenus could indeed be a name of British origin, ${ }^{866}$ implying that workshops in Britain were capable of highly

\footnotetext{
${ }^{861}$ They also feature on the Handled Pan 2 from Prickwillow, which is discussed separately later in this section.

${ }^{862}$ Pisano 2008.

${ }^{863}$ CAM0009.

${ }^{864}$ See Chapter 4; West 2005.

${ }^{865}$ CAM0009.

${ }^{866}$ Laing 2000, 65.
} 
detailed work and willing to integrate Classical iconography in their decorative repertoire. The high quality and complexity of its design makes it an anomalous object. Its presence implies an access to artistic luxury and craftsmanship in Britain, even in a rather removed rural setting such as where this object was found.

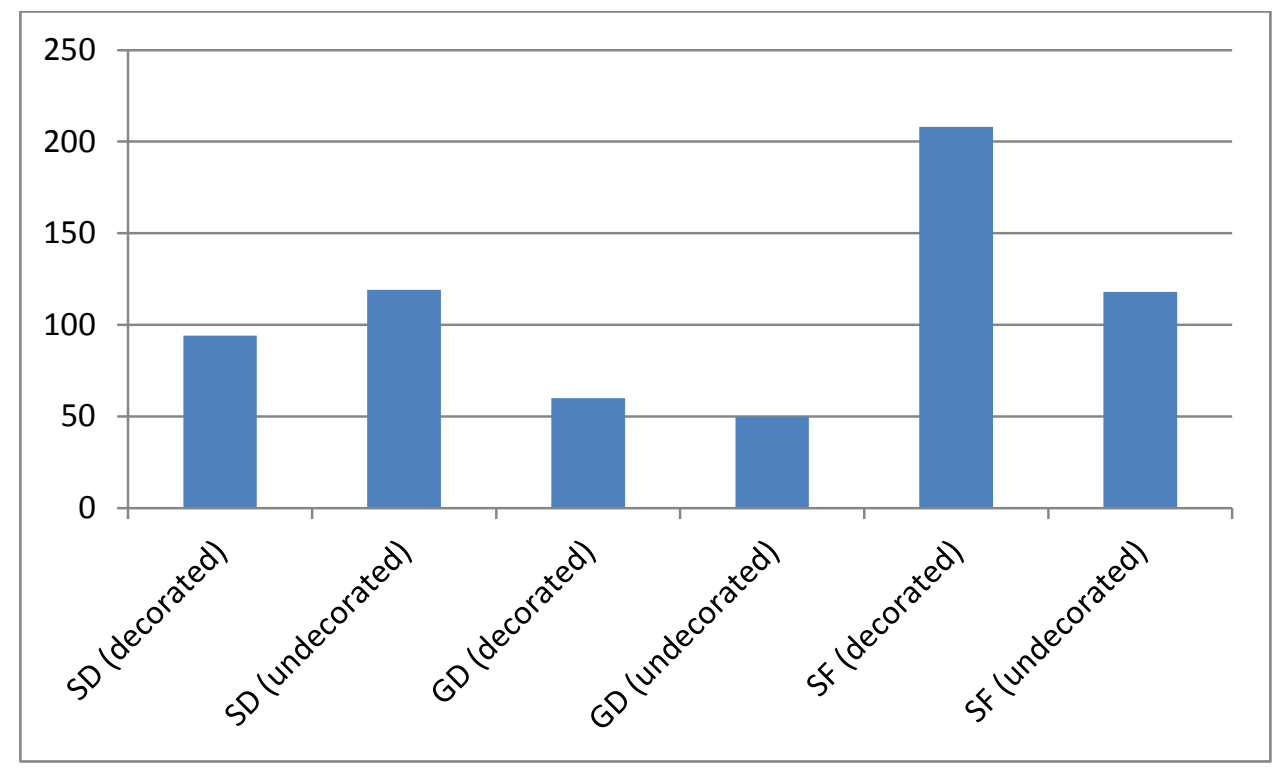

Figure 5.17: Decorated object in the Structured Deposits, Grave Deposits and Site Finds data-sets (does not include inscriptions)

The richness of the iconography of copper alloy vessels found as Site Finds is proportionately far higher than that found among Structured Deposits and roughly equivalent to that represented among Grave Deposits (Figure 5.17). This may, in part, be due to how this material is identified and reported. As complete vessels are very rare Site Finds, often the only distinguishing feature a fragment may have to indicate what type of vessel it originated from is its decorative elements. Some brittle and fragmentary remains of copper alloy may be in such a poor state as to be impossible to identify the object they originate from and could go unmentioned upon publication. This is not the case with all sites, as is evident by the inclusion of a significant number of undiagnostic fragments in this chapter. ${ }^{867}$ The increased likelihood of decorated fragments to be identified and published over undiagnostic fragments is a bias that likely did affect the data-set all-the-same, though probably less so in more recent reports than in older excavations. The wide distribution of decorated objects from across Britain does imply

\footnotetext{
${ }^{867}$ Such as Coygan Camp (Wainwright 1967, 93; CAR0005-CAR0009) or Silchester (Fulford \& Timby 2000, 345 \& 350; HAM0017 \& HAM0018; Fulford \& Clarke 2011, 108; HAM0019).
} 
that ornamented copper alloy vessels were available to a relatively wide section of society during the Roman period.

Site Finds are again perhaps most useful in filling in our understanding of the Midlands during the Roman period. Iconographically rich material comes from across this region and from various types of sites, suggesting that decorated copper alloy vessels were enjoyed both by rural inhabitants and city-dwellers. Anthropomorphic iconography appears to be the reserve of high-end imported luxury goods and is relatively rare. Far more common are zoomorphic and floral decorations. In particular, bovine hanging vessel mounts would appear to be representative of the embellishment of a utilitarian bucket form as opposed to the elaborate decoration seen on such handled pans as the Prickwillow Pan or the HP1 from Rocester. ${ }^{868}$ This differentiation in the level of complexity of the iconographic embellishment further indicates that the material was available to a wider audience, varying in complexity as individual means would allow.

\section{$\underline{5.5 \text { Concluding Remarks }}$}

Site Finds offer a wider and presumably more randomised data-set of copper alloy vessels in Roman Britain than the data-sets of Structured Deposits or Grave Deposits provide. Since this data-set is composed of deposits that have no concrete evidence of purposeful deposition related to ritual or secular motivations, it may be inferred that they are generally accidental losses that rely on little more than human mistake or indifference for entering the archaeological record. This randomness of deposition proves useful in expanding our chronological and geographic understanding of the use of metal vessels, as this data-set is not based upon particular human behaviours of deposition that can be confined within specific chronological and geographic parameters and would vary accordingly. As has been noted above, this is most evident when considering material from the $2^{\text {nd }}-3^{\text {rd }}$ centuries CE or the geographic area of the Midlands, though there is no area of Roman Britain that is not illuminated by copper alloy vessel Site Find material. As seen in Section 5.2, it is evident by comparing Site Finds from the highly militarised frontier zones with those found in the Midlands and the southeast that there was significant variation in the preferences of vessels utilised, which in turn suggests difference in the manner in which these vessels would be put to use and the cultural connotations

${ }^{868}$ CAM0009 and STA0006, respectively. 
attached to them. This could be most apparent in the presence of Handled Pan 2s at sites known to have had a military association. Though it is difficult to determine the status of a given location within a city or a rural settlement, the number and wide dispersal of the material would suggest that these vessels were available to more than just elites and may have been available to most individuals of at least moderate means during the Roman period in Britain.

Before comparing the data-sets to make conclusions about the development and utilization of copper alloy vessels across Britain during the Roman period in the final chapters of this thesis, the data-set of Single Finds recorded through the Portable Antiquities Scheme database will be reviewed in the next chapter. 


\section{Chapter 6: Single Finds Reported through the Portable Antiquities Scheme}

\section{$\underline{6.1 \text { Introduction }}$}

The following chapter will outline the Single Finds data collected through the Portable Antiquities Scheme (PAS) and published on their online database. While the PAS was explained in Chapter 2, it is worth brief consideration presently to clarify the data-set and the biases which it is subject to. The PAS relies on members of the public to come forward to report finds they have made, which are then reviewed by Finds Liaison Officers (FLOs) and recorded when appropriate. The PAS' greatest attribute is its collection and the cataloguing of data from the general public that would easily escape scholarly attention otherwise. Conversely, this reliance on the involvement of the public is the principal source for bias in this data-set. The first important difficulty to understand is the variable level of enthusiasm and interest exhibited by the public to report finds they have made, if indeed they are aware of the PAS at all. This is especially important as the PAS has been established in some parts of Britain for significantly longer than others, which could influence not only the numbers of objects reported for regions but also how well known and understood the PAS is in various parts of England and Wales. ${ }^{869}$ However, the PAS has been on a national basis for over a decade and it is likely that the statistical bias should be evened out. ${ }^{870}$ There is also the fact that the public will often only search for, discover and report objects where they believe it is likely for them to find something of importance. This is particularly true for metal-detector hobbyists, who make up the majority of the public participants in the PAS and will likely only be searching in regions where they believe that finds are likely to occur. These areas are principally the east of England from Yorkshire to Essex, with particular interest paid to areas of plough-land that are easy to search with a metal detector. This will produce an unavoidable geographic skew in the data and must be remembered when reviewing the material. The practice of seeking out finds 'hot-spots', as well as the likelihood of an individual landowner to grant permission for their field to be searched, will also affect which fields receive thorough detecting and which are only treated in a cursory manner, having a significant effect upon which fields and parishes have large numbers of finds assemblages, ${ }^{871}$ but is less significant on a national level.

\footnotetext{
${ }^{869}$ Worrell 2004, 317-318.

${ }^{870}$ Worrell 2007, 306.

${ }^{871}$ Brindle 2011, 71-72; Walton 2012, 27-30 \& 167-168.
} 
Another important bias is the knowledge of the individual members of the public as well as the particular FLO involved in the reporting of any particular object. ${ }^{872}$ While most of the people who report through the PAS are history or archaeology enthusiasts to one degree or another, they are generally not qualified to identify archaeological finds accurately and may not be aware of what objects are old enough or diagnostic enough to take to their local FLO for identification and recording. This could also lead to a bias in what material is brought in for reporting, as decorative or figurative material may be more easily recognisable as significant finds. ${ }^{873}$ Once the objects are brought to the FLO for identification, it is the knowledge and expertise of the FLO in question that affects the material and how it is reported. FLOs become specialised in the particular region under their survey and are trained to identify the sorts of objects that they may reasonably be expected to find in that area. ${ }^{874}$ This in turn may cause an FLO to misidentify an object if it is something they may not be expecting to find in their region, which could in turn skew the data of a Roman based study, such as this thesis, towards the southeast as this is the region expected to produce the most Roman finds. ${ }^{875}$ To combat this potential bias, material dated from the Iron Age to the Medieval period on the PAS database was reviewed in order to counterbalance the possibility of misidentification by members of the public and FLOs. It would appear that chronological misidentification did not affect this data-set to any significant degree. Though some objects of Roman date were recorded with a wider date range than necessary (i.e. 'Iron Age to Early Medieval', for example), this has not compromised the quality of information available.

There are some particular problems when utilizing PAS records for a group of material such as Romano-British copper alloy vessels, most prominent amongst these is the difficulty in accurately identifying this material from fragments. Data recorded through the PAS often has acceptably accurate findspots, but the greater context of the finds are typically unknown; most often times lost through the ravages of development, the plough, or time more generally. Though the finds assemblage of any given location may help to clarify the nature of the site, as discussed in Section 6.3, the context within a site for any of these objects must remain unknown and their relation to the assemblage as a whole will always be obscure (except in the relatively

\footnotetext{
${ }^{872}$ Brindle 2011, 68-76; Walton 2012, 29-30.

${ }^{873}$ Section 6.5 .

${ }^{874}$ Walton 2012, 29-30.

${ }^{875}$ Brindle 2011, 70-71.
} 
rare instances where findspots are subsequently excavated). This leads to difficulty in dating objects that are fragmentary and undiagnostic. Sometimes the best that can be stated with certainty is simply that an object is pre-industrial in its method of manufacture. This problem is particularly important when considering objects such as copper alloy vessels, which are best dated by form and type that may not be recognizable in highly fragmented states. The data included in the thesis does not include undiagnostic material, and it is likely that a certain amount of these undiagnostic fragments would have originated from vessels manufactured during the Roman period. As an example of how this exclusion may have affected the data, fifteen instead of nine objects would be under consideration if the undiagnostic fragments were reinserted into the data-group for Hertfordshire. This would have resulted in a significant quantitative increase; nearly doubling the number of objects recorded for this area, and resulted in the conclusion that this material was far more frequent in this region than is suggested by the current thesis. This conclusion would be inherently fallible, however, because it would disregard the deposition of copper alloy vessels in this region of any date other than the Roman period.

The final bias to consider is the nature of the identifiable material and how this reflects what objects could be included in this thesis. As the objects are unstratified, fragmentary material must consist of diagnostic portions of vessels or identifiable iconography to be included in this data-set. In order to determine if decoration and style was of Roman period origin, iconography and style were compared with dated examples from excavations to determine the likely time-frame of manufacture. As iconography and decorative style play such a key role in the identification of unstratified objects, the PAS data-set is proportionately richer in iconographic representations than the other data-sets of this thesis. This will be discussed in further detail in Section 6.5.

One final point of consideration before reviewing the data-set of this chapter is that it includes only the Single Finds reported through the PAS. Any objects reported through the PAS that could be identified as Structured Deposits or Grave Deposits were discussed in the appropriate chapters of this thesis, although material reported through the PAS that could be thus classified is certainly in the minority. This is not to say that other objects in this current data-set could not have originated from Structured Deposits or Grave Deposits. However, the lack of evidence to imply that they did originate from such contexts forces us to consider them separately. 
The layout of this chapter will closely follow the preceding chapter on Site Finds, with a regional survey highlighting some of the key finds as well as a discussion of the over-all regional trends that are discernible. The other finds reported through the PAS in the vicinity of finds of copper alloy vessels will then be integrated into a discussion of the wider material assemblages of the findspots in Section 6.3. This is then followed by an examination of the iconography utilised in the decoration of these vessels.

\section{$\underline{6.2 \text { Geographic Survey of PAS Finds }}$}

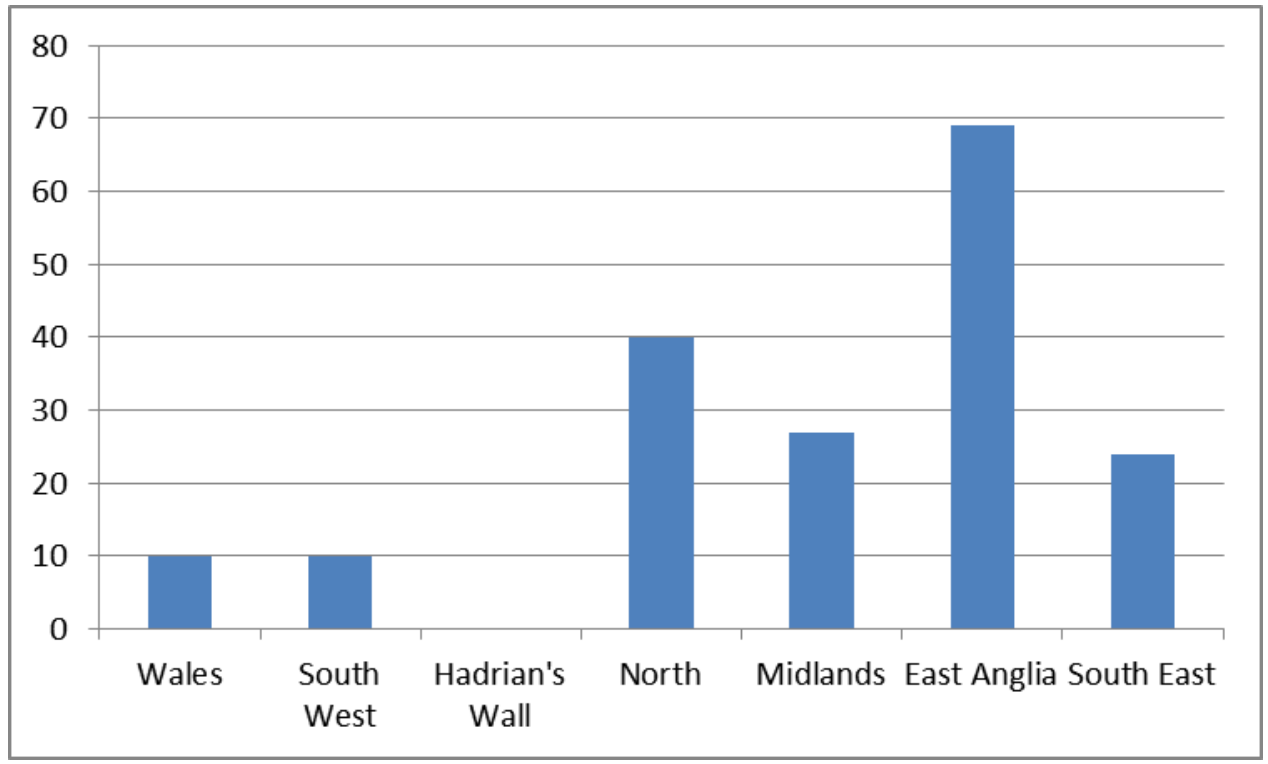

Figure 6.01: Numbers of copper alloy vessels reported as Single Finds through the PAS.

In all, some 195 objects from the PAS database constitute the current data-set, whose regional distribution is illustrated in Figure 6.01 and Map 4 which show a clear distributional bias to the east of England generally and East Anglia most specifically. The data-sets from Wales and the south western counties of England (Table 6a) comprise 10 objects each, all highly fragmentary. While the PAS contributed significant finds to both the Structured Deposits and Grave Deposits data-sets of this thesis for this geographic area, the Single Finds are less spectacular. Their fragmentary nature prevents specific identification of vessel type for the majority of this material, but they are mostly hanging vessel mounts or bucket feet whose date of manufacture is likely from the late $1^{\text {st }}$ to the $3^{\text {rd }}$ centuries CE. 


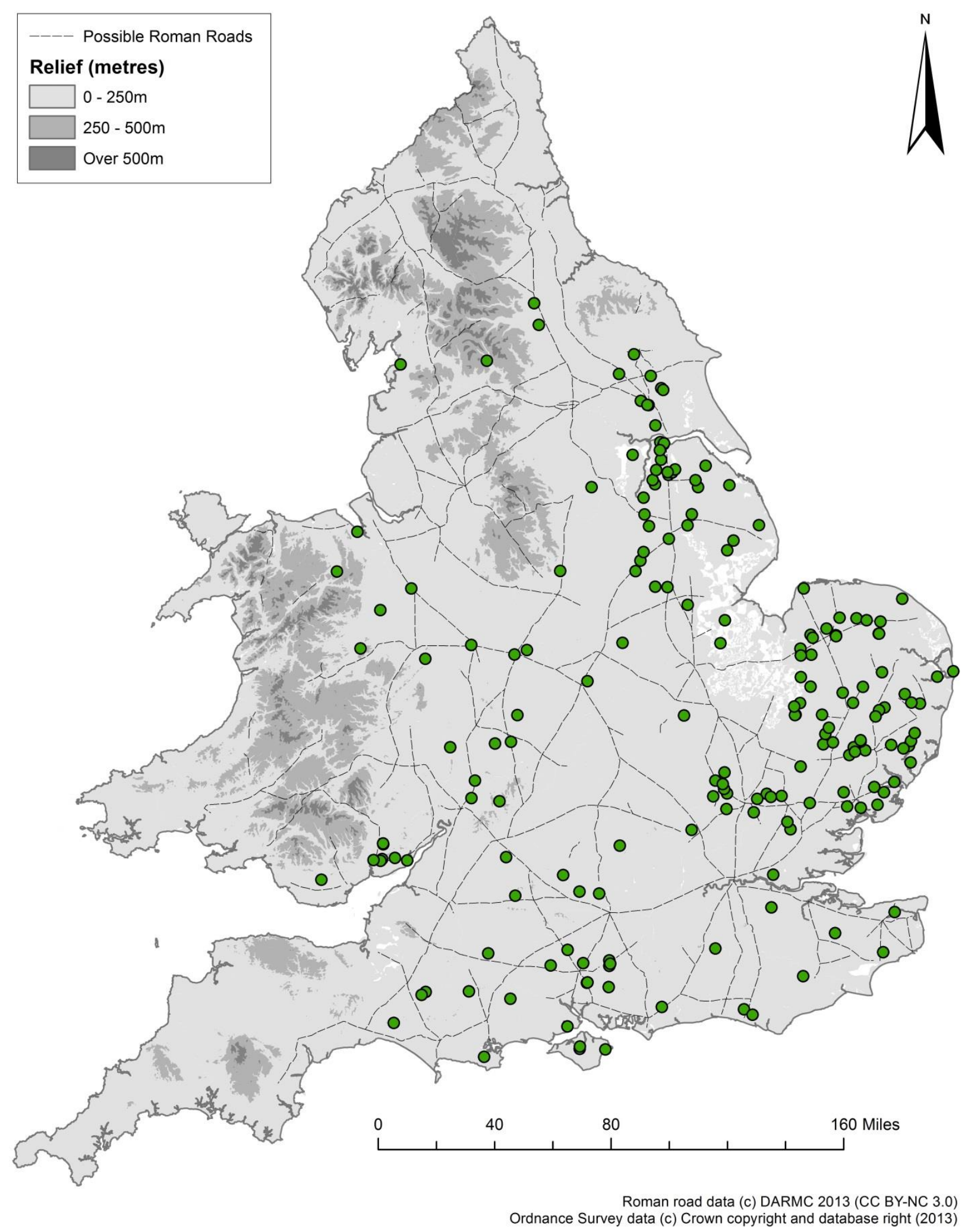

Map 4: Single Finds of copper alloy vessels reported through the PAS (K. Robbins) 


\begin{tabular}{|c|c|}
\hline Findspot (Site Type) & Vessel/Catalogue Number \\
\hline Chieveley, Berkshire (RU) & Vessel(mount)/BERK0002 \\
\hline Castleton, Dorset (RS) & Jug/DOR0007 \\
\hline Nether Compton, Dorset (RS) & Handled Pan 2/DOR0008 \\
\hline Corfe Castle, Dorset (RS) & Jug/DOR0009 \\
\hline Stoke Abbott, Dorset (M) & Bucket/DOR0010 \\
\hline Compton Abbas, Dorset (RS) & Vessel(fragment)/DOR0011 \\
\hline Flint, Flintshire $(\mathrm{RS})$ & Vessel(mount)/F0009 \\
\hline Twyning, Gloucestershire (RU) & Vessel(mount)/G0004 \\
\hline Leigh, Gloucestershire (RS) & Vessel(mount)/G0005 \\
\hline Sudeley, Gloucestershire (RS) & Vessel(mount)/G0006 \\
\hline Mathern, Monmouthshire (RU) & Vessel(mount)/MON0002 \\
\hline Usk, Monmouthshire (M) & $\begin{array}{c}\text { Vessel(fragment)/MON0003 } \\
\text { Jug(lid)/MON0004 }\end{array}$ \\
\hline Llantrisant Fawr, Monmouthshire (RU) & Vessel(fragment)/MON0005 \\
\hline Caerwent, Monmouthshire (U) & Vessel(mount)/MON0006 \\
\hline Langstone, Newport (RS) & $\begin{array}{c}\text { Bucket/NE0001 } \\
\text { Vessel(mount)/NE0002 }\end{array}$ \\
\hline Caerleon, Newport $(\mathrm{M})$ & Vessel(mount)/NE0003 \\
\hline Pont Y Clun, Rhondda Cynon Taf (RU) & $\operatorname{Jug}($ lid)/RC0001 \\
\hline Avebury, Wiltshire (RS) & Vessel(mount)/WIL0001 \\
\hline Leigh, Wiltshire (RU) & Vessel(fragment)/WIL0002 \\
\hline Codford, Wiltshire (RU) & Jug(handle)/WIL0003 \\
\hline
\end{tabular}

Table 6a: PAS finds from Wales and the southwest of England.

No objects from the region of Hadrian's Wall have been reported that feature in this data-

set. This may be in large part due to the fact that much of this area is national parkland, is largely pastoral (as opposed to plough land) and has many Scheduled Monuments and is therefore unavailable for metal detecting. ${ }^{876}$ Two copper alloy vessels discovered in Northumberland that were recorded through the PAS comprise a Structured Deposit and are discussed in Chapter 3. ${ }^{877}$ By contrast, the Northern Counties contribute 40 objects to this dataset (Table 6b), most of these coming from Yorkshire (15) and Lincolnshire (20).

\begin{tabular}{|c|c|}
\hline Findspot (Site Type) & Vessel/Catalogue Number \\
\hline Fridaythorpe, East Riding of Yorkshire (RU) & Vessel(fragment)/EY0001 \\
\hline Humbleton, East Riding of Yorkshire (RU) & Handled Pan 2/EY0002 \\
\hline North Cave, East Riding of Yorkshire (RU) & Vessel(mount)/EY0003 \\
\hline Hayton, East Riding of Yorkshire (RU) & Handled Pan 4/EY0004 \\
\hline Shipton Thorpe, East Riding of Yorkshire (RS) & $\begin{array}{c}\text { Handled Pan 2/EY0005 } \\
\text { Jar/EY0006 }\end{array}$ \\
\hline Thwing, East Riding of Yorkshire (RS) & Bowl/EY0007 \\
\hline
\end{tabular}

${ }_{877}^{876}$ Walton pers comm; Pearce pers comm; Brindle 2011, 32-57. 


\begin{tabular}{|c|c|}
\hline North Dalton, East Riding of Yorkshire (RS) & $\begin{array}{l}\text { Vessel(mount)/EY0008 } \\
\text { Vessel(mount)/EY0009 }\end{array}$ \\
\hline Slyne with Hest, Lancashire (RU) & Handled Pan 3/LAN0003 \\
\hline Gaddesby, Leicestershire (RU) & Vessel(fragment)/LEI0002 \\
\hline Leire, Leicestershire (RU) & Vessel(fragment)/LEI0003 \\
\hline Ancaster, Lincolnshire (RS) & Vessel(fragment)/LIN0004 \\
\hline Scotton, Lincolnshire (RU) & Handled Pan 1/LIN0005 \\
\hline North Thoresby, Lincolnshire (RU) & Vessel(fragment)/LIN0006 \\
\hline Torksey, Lincolnshire (RU) & Bowl/LIN0007 \\
\hline Nettleton, Lincolnshire (RU) & $\begin{array}{l}\text { Vessel(fragment)/LIN0008 } \\
\text { Vessel(fragment)/LIN0009 }\end{array}$ \\
\hline Thonock, Lincolnshire (RU) & Handled Pan 2/LIN0010 \\
\hline Scotter, Lincolnshire (RU) & Bowl/LIN0011 \\
\hline Weston, Lincolnshire (RU) & Vessel(fragment)/LIN0012 \\
\hline Folkingham, Lincolnshire (RU) & Jug(lid)/LIN0013 \\
\hline Bilsby, Lincolnshire (RU) & Vessel(fragment)/LIN0014 \\
\hline Lincoln, Lincolnshire (U) & Jug(handle)/LIN0015 \\
\hline Keelby, Lincolnshire (RU) & Handled Pan 2/LIN0016 \\
\hline Wickenby, Lincolnshire (RS) & Vessel(fragment)/LIN0017 \\
\hline Crowland, Lincolnshire (RU) & Vessel(mount)/LIN0018 \\
\hline Stainton By Langworth, Lincolnshire (RU) & Vessel(fragment)/LIN0019 \\
\hline Gate Burton, Lincolnshire (RU) & Vessel(fragment)/LIN0020 \\
\hline Marston, Lincolnshire (RU) & Vessel(fragment)/LIN0021 \\
\hline Spilsby, Lincolnshire (RS) & Vessel(mount)/LIN0022 \\
\hline Caistor, Lincolnshire (M) & Vessel(mount)/LIN0023 \\
\hline Revesby, Lincolnshire (RU) & Vessel(mount)/LIN0024 \\
\hline Crowle, North Lincolnshire (RS) & Handled Pan 2/NLIN0001 \\
\hline Winteringham, North Lincolnshire (RS) & $\begin{array}{c}\text { Vessel(fragment)/NLIN0002 } \\
\text { Vessel(fragment)/NLIN0003 } \\
\text { Vessel(mount)/NLIN0005 }\end{array}$ \\
\hline Appleby, North Lincolnshire (RS) & Jug(handle)/NLIN0004 \\
\hline Winterton, North Lincolnshire (RS) & Handled Pan 2/NLIN0006 \\
\hline Scawby, North Lincolnshire (RS) & $\begin{array}{c}\text { Vessel(fragment)/NLIN0007 } \\
\text { Handled Pan 2/NLIN0009 } \\
\text { Vessel(mount)/NLIN0011 }\end{array}$ \\
\hline Holme, North Lincolnshire (RU) & Vessel(mount)/NLIN0008 \\
\hline Brigg, North Lincolnshire (RU) & Jug(lid)/NLIN0010 \\
\hline Malton, North Yorkshire (RS) & Handled Pan 2/NYR0001 \\
\hline Brough with St. Giles, North Yorkshire (RU) & Handled Pan 2/NYR0002 \\
\hline Hawkswick, North Yorkshire (RU) & Handled Pan 2/NYR0005 \\
\hline Claxton, North Yorkshire (RU) & Vessel(mount)/NYR0006 \\
\hline Bedale, North Yorkshire (RU) & Vessel(mount)/NYR0007 \\
\hline Edlington, South Yorkshire (RU) & Handled Pan 2/SYR0001 \\
\hline
\end{tabular}

Table 6b: PAS finds from the northern counties of England. 
Handled Pan 2s are the most common diagnostic PAS finds from Yorkshire, accounting for over $30 \%$ of the material from this area. The association between this vessel and the Roman military has been evidenced by the Structured Deposits and Site Finds data-sets of this thesis, ${ }^{878}$ and this could well explain the high presence of this material in Yorkshire, an area which saw regular military movement during the Roman period. ${ }^{879}$ However, this is quite in contrast to the data-set of PAS material from Wales, an area also known to have had a high level of military activity $^{880}$ and which has contributed Handled Pan 2 s to the data-sets of Structured Deposits and Site Finds but which has not contributed any Handled Pan 2 s to the PAS data-set. It is worth noting that Wales has comparatively low numbers of PAS material generally, owing largely to patterns of modern land-use, ${ }^{881}$ which could explain such discrepancies. The finds in Yorkshire are focused mostly in the East Riding, which also has the greatest diversity of objects. Worth specific note is the Handled Pan 4, or Coptic pan, from Hayton, ${ }^{882}$ which deserves attention simply because of the relative rarity of this vessel form and this particular example being the northern-most such pan in Britain (Figure 6.02).

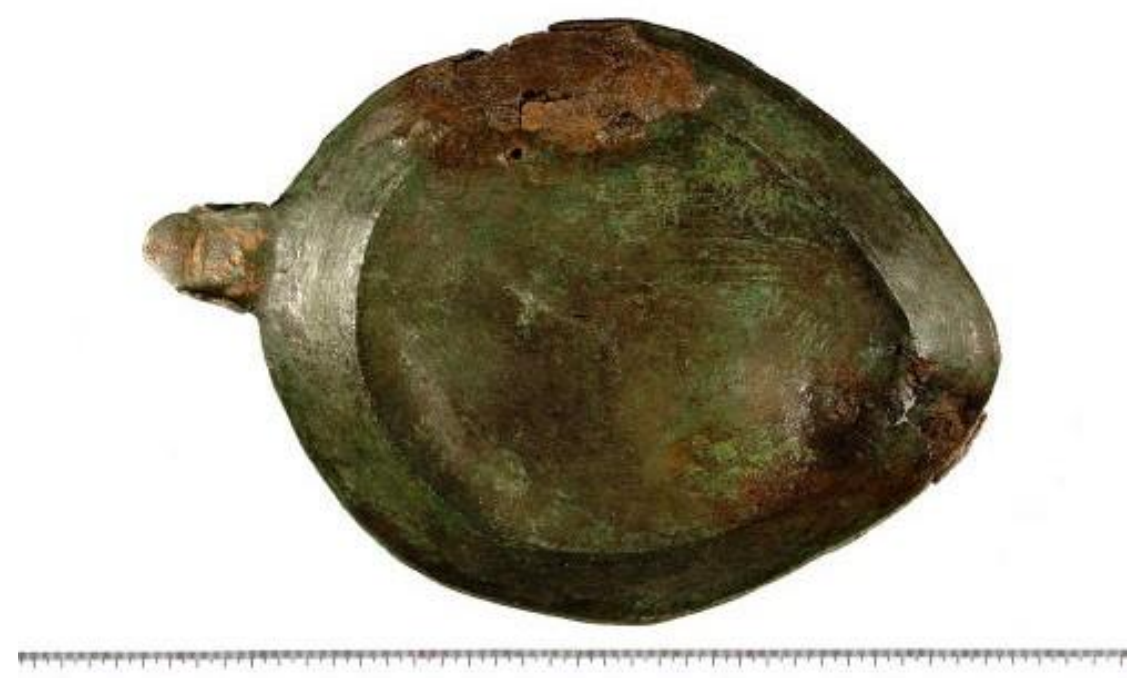

Figure 6.02: Handled Pan 4 from Hayton (PAS RESEARCH-230A51)

\footnotetext{
${ }^{878}$ As discussed in Chapters 3 and 5, respectively; further discussed in Chapter 7.

${ }^{879}$ Hartley 1971, 56-69; Mattingly 2006, 132-136 \& 146-149.

${ }^{880}$ Arnold \& Davies 2000, 58-59.

${ }^{881}$ Pearce pers comm.

${ }^{882}$ EY0004; PAS RESEARCH-230A51.
} 
The material from Lincolnshire is diverse in its composition. The presence of three bovine vessel spouts is particularly interesting, ${ }^{883}$ contributing to a small body of these fittings currently known (Figure 6.03). Such spouts were attached to strainer bowls, such as those found in a Structured Deposit at Kingston Deverill. ${ }^{884}$ Though the find-spots for all three of these vessels are in the northern part of Lincolnshire, ${ }^{885}$ they are not near enough to one another to suggest a production centre. Furthermore, this vessel type and decorative motif is completely absent from this region in the other data-sets. This could be in part explained by the relative rarity of this vessel spout across the Roman world, but makes the presence of three such spouts in the PAS material of this county all the more unusual. The other finds from Lincolnshire are varied enough not to indicate any particular patterns that stand out. One object worth specific mention is an anthropomorphic vessel mount found near Caistor depicting a woman wearing what could be a votive crown; such anthropomorphic representations are relatively rare in the copper alloy vessel material for Britain (Figure 6.04). ${ }^{886}$ Similar mounts (possibly for furniture) have been noted at Hockwald and Cavenham, perhaps relating to a broader artistic tradition. ${ }^{887}$ The other finds from the north are few, isolated and offer little substantive data in themselves aside from contributing to a wider picture of the broad distribution of copper alloy vessel material in Roman Britain.

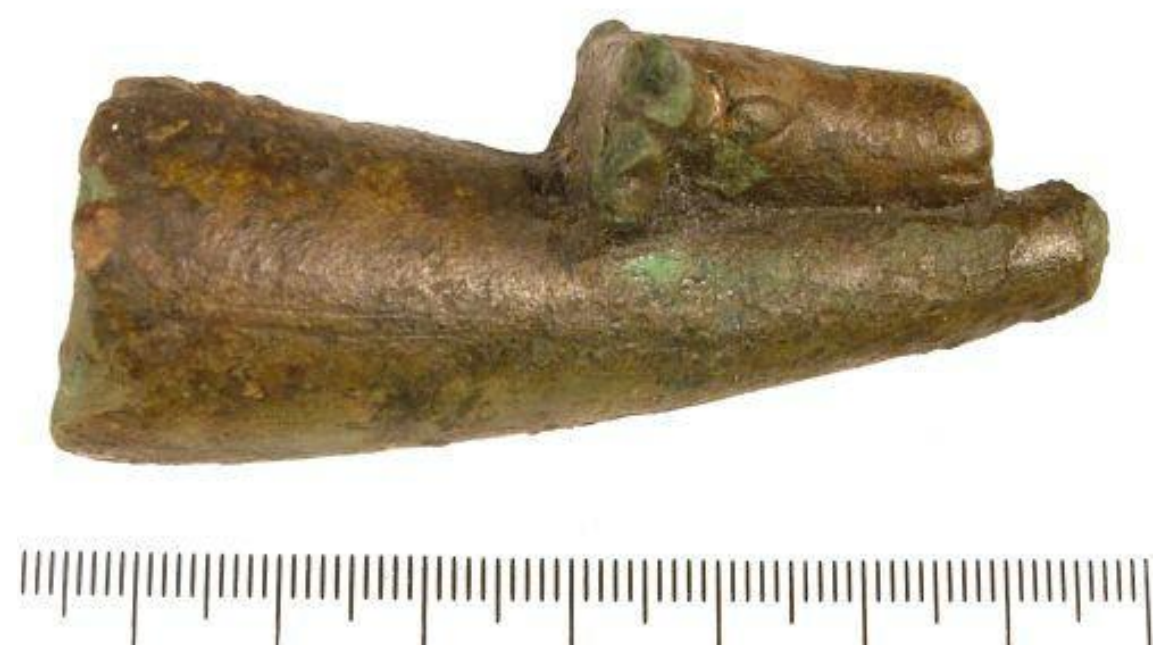

Figure 6.03: Bovine vessel spout from Bilsby in Lincolnshire (PAS LIN-F8BC42)

\footnotetext{
${ }^{883}$ LIN0014, PAS LIN-F8BC42; LIN0017, PAS NLM-5DF5D6; LIN0022, PAS LIN-40CE20.

${ }^{884}$ WIL0007 \& WIL0008; PAS WILT-92B052; Worrell 2006, 458-462.

${ }^{885}$ Bilsby, Wickenby and Spilsby (respectively).

${ }^{886}$ LIN0023; PAS NLM-AEA444.

${ }^{887}$ Toynbee $1964,177-178$ (127 \& 128).
} 


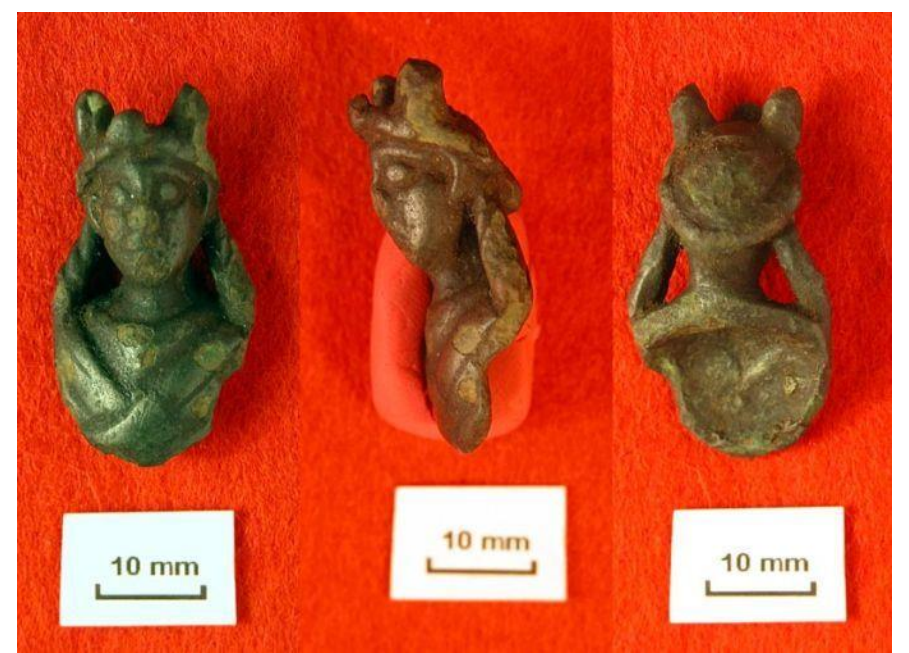

Figure 6.04: Anthropomorphic mount from Caistor (PAS NLM-AEA444)

\begin{tabular}{|c|c|}
\hline Findspot (Site Type) & Vessel/Catalogue Number \\
\hline Ripley, Derbyshire (RU) & Vessel(fragment)/DER0001 \\
\hline St. Michael, Hertfordshire (RU) & Vessel(mount)/HER0020 \\
\hline Clothall, Hertfordshire (RS) & $\begin{array}{l}\text { Vessel(mount)/HER0021 } \\
\text { Vessel(mount)/HER0022 } \\
\text { Vessel(mount)/HER0024 }\end{array}$ \\
\hline Watton-at-Stone, Hertfordshire (RS) & Jug(lid)/HER0023 \\
\hline Ashwell, Hertfordshire (RS) & Vessel(mount)/HER0025 \\
\hline Albury, Hertfordshire (RU) & Bucket/HER0026 \\
\hline Much Hadham, Hertfordshire (RU) & Vessel(mount)/HER0027 \\
\hline Wymondley, Hertfordshire (RS) & Vessel(mount)/HER0028 \\
\hline Winthorpe, Nottinghamshire (RU) & Handled Pan 2/NOT0002 \\
\hline Hawton, Nottinghamshire (RU) & Vessel(fragment)/NOT0003 \\
\hline Collingham, Nottinghamshire (RU) & Vessel(mount)/NOT0004 \\
\hline Adwell, Oxfordshire (RU) & Vessel(mount)/OX0001 \\
\hline Letcombe Regis, Oxfordshire (RU) & Vessel(mount)/OX0002 \\
\hline Hordley, Shropshire (RU) & Vessel(fragment)/SH0001 \\
\hline Sheinton, Shropshire (RS) & Jug(lid)/SH0002 \\
\hline Whitchurch, Shropshire (RS) & Vessel(mount)/SH0003 \\
\hline Shenstone, Staffordshire (RS) & Vessel(mount)/STA0001 \\
\hline Fisherwick, Staffordshire (RU) & Vessel(mount)/STA0002 \\
\hline Brewood, Staffordshire (RS) & Handled Pan 2/STA0003 \\
\hline Ilam, Staffordshire (RU) & Handled Pan 2/STA0004 \\
\hline Thorpe Constantine, Staffordshire (RU) & Vessel(mount)/STA0005 \\
\hline Tanworth In Arden, Warwickshire (RU) & Vessel(fragment)/WAR0001 \\
\hline Alcester, Warwickshire $(\mathrm{M})$ & Vessel(mount)/WAR0002 \\
\hline Inkberrow, Worcestershire (RU) & Vessel(fragment)/WOR0001 \\
\hline Leigh, Worcestershire (RU) & Vessel(fragment)/WOR0002 \\
\hline
\end{tabular}

Table 6c: PAS finds from the Midlands. 


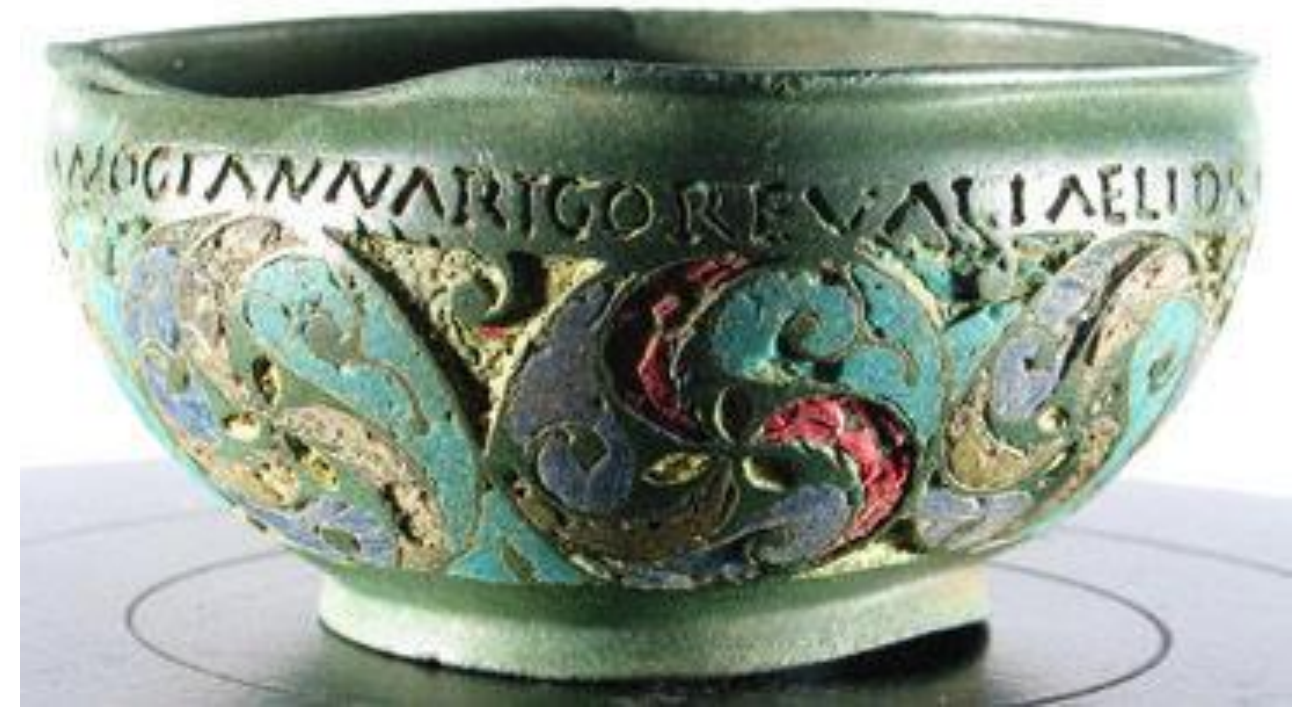

Figure 6.05: The Ilam Pan (PAS WMID-3FE965)

The Midlands contribute 27 objects to the PAS data-set (Table 6c). The best known object from this data-group is the Ilam Pan (Figure 6.05). ${ }^{888}$ This is an elaborately decorated enamelled Handled Pan 2 of the Rudge Cup type. In addition to being decorated with elaborate 'Celtic' type roundels, ${ }^{889}$ a long inscription runs beneath the rim of the vessel: Mais Coggabata Uxelodunum Cammoglanna Rigoreval(l)i Aeli Draconis. The first four words reference known sites from the west end of Hadrian's Wall, being Bowness (MAIS), Drumburgh (COGGABATA), Stanwix (UXELODUNUM) and Castlesteads (CAMMOGLANNA). The reference, RIGOREVALI, is unknown. However, it could be interpreted as meaning 'on the line of the wall', ${ }^{890}$ whether this indicates a specific place or is descriptive of the settlements along Hadrian's Wall more generally is uncertain. The last portion would seem to be the name of the owner or manufacturer of the object, Draco(n). ${ }^{891}$ This object stands out for being one of the best preserved Rudge Cup type Handled Pan 2s known to date and this type of object will be discussed in further detail in Chapter 7.

The other objects in the data-set from the Midlands are highly fragmentary and represent several vessel forms, with a large number of mounts that could have originated from a number of bucket, hanging basin or jar types dating between the $1^{\text {st }}-3^{\text {rd }}$ centuries CE (Figure 6.06). It is not

\footnotetext{
${ }^{888}$ STA0004; PAS WMID-3FE965.

889 Jackson 2012, 46-47.

${ }^{890}$ PAS WMID-3FE965; Tomlin \& Hassall 2004, 344-345; Jackson 2012, 43-45.

${ }^{891}$ Jackson 2012, 45.
} 
surprising that East Anglia and the south eastern counties of England have the highest number of objects in this data-set of the thesis, with East Anglia contributing 69 objects and the south eastern counties, including Greater London, contributing 25 (Appendix X). ${ }^{892}$

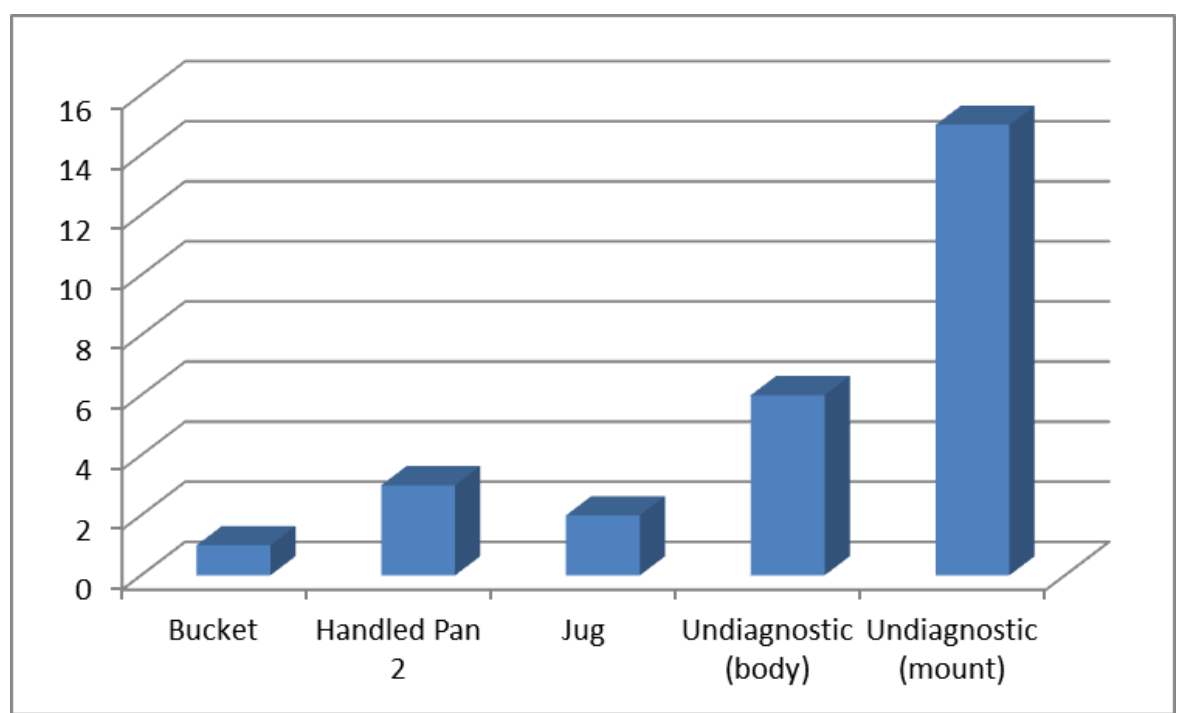

Figure 6.06: Vessel forms of Single Finds PAS in the Midlands.

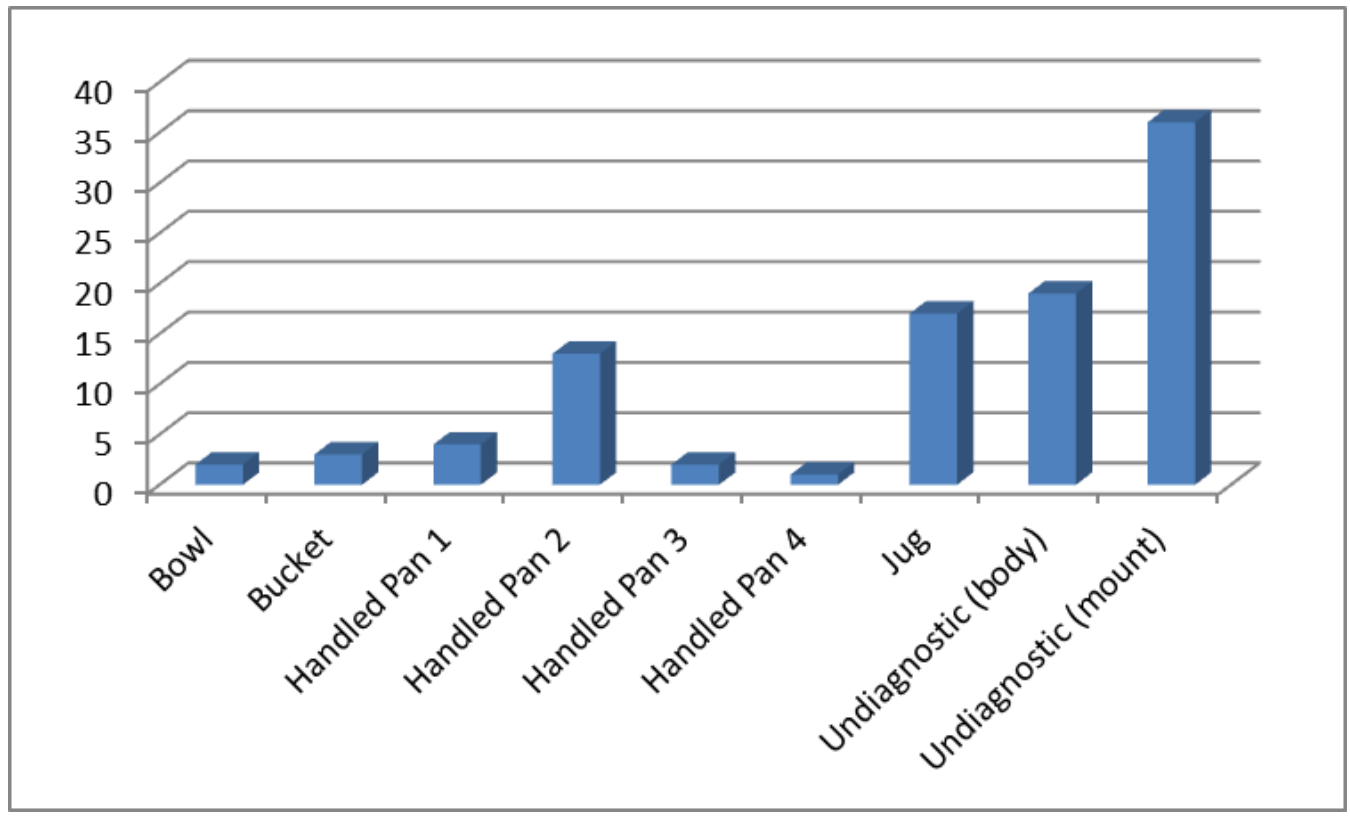

Figure 6.07: Vessel forms for East Anglia and the South East reported as Single Finds through the PAS.

${ }^{892}$ See Map 4. 
The materials from East Anglia and the south eastern counties are for the most part very fragmentary, but nevertheless show a reasonable selection of vessel forms (Figure 6.07). The identifiable objects from this region are principally jugs and Handled Pan 2s. One object that must be mentioned here is a handle fragment from Gunthorpe in Norfolk (Figure 6.08). ${ }^{893}$ While it is recorded in the PAS as a probable fragment from a Rudge Cup type Handled Pan 2, its decoration and manufacturing technique does not easily parallel other known examples of Roman date. Its inscription, 'BEBE SESE', can be plausibly argued to be a misspelled Latin or Latin \& Greek text meaning something akin to 'drink and long life'. 894

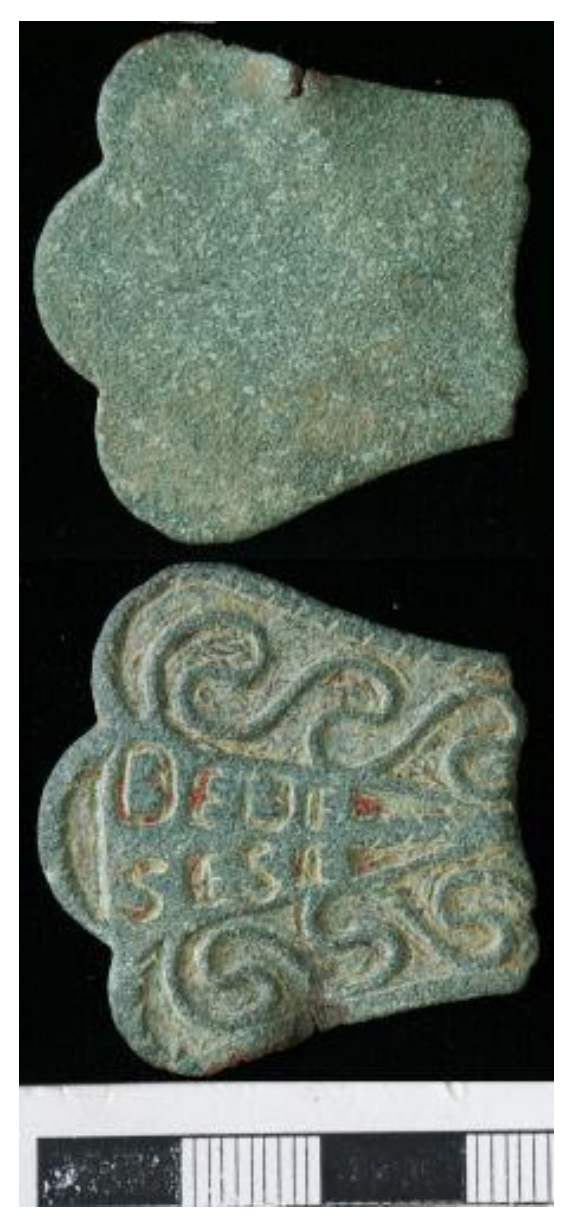

Figure 6.08: Handle fragment from Gunthorpe (PAS NMS-7BC635)

\footnotetext{
${ }^{893}$ PAS NMS-7BC635.

${ }^{894}$ Worrell 2012, 74.
} 
Another object worth brief mention is the small bowl of circa $18 \mathrm{~mm}$ from Manningtree in Essex. ${ }^{895}$ The small size of this vessel makes it unlikely to be a serving vessel, so it is likely a balsamarium or perhaps a cosmetic vessel. Also worth particular note is the fragmentary Handled Pan 4 from Broxted in Essex, ${ }^{896}$ as these Coptic pans are not common finds (Figure 6.09).
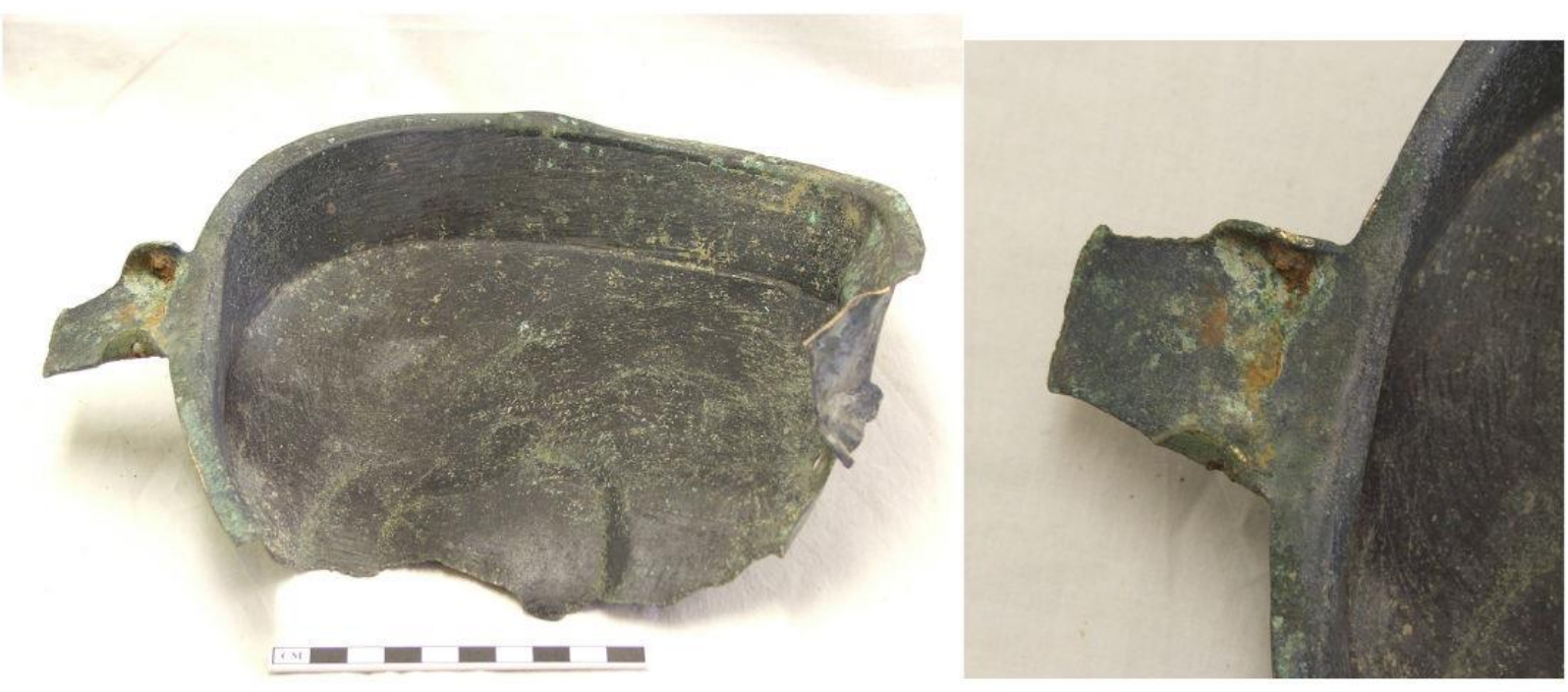

Figure 6.09: Handled Pan 4 from Broxted (PAS ESS-1D3342)

As discussed at the beginning of this section and illustrated in Map 4, the geographic distribution of this material has a strong bias to the south and east, as may be expected for PAS distribution more generally and is likely influenced by the method by which material is found and reported. That being said, it must be remembered that Single Finds from the PAS originate almost exclusively from the country-side, where the majority of the population lived during this time. This means PAS data offers a possible view into rural material culture that is nearly invisible using other archaeological methods and that this data-set is therefore somewhat selfcontained and should not be expected to be immediately reflexive of trends from other data-sets. This would seem to imply that, whilst copper alloy vessel material was readily available in settlements across Britain during the Roman period, their use in rural environments was also widespread, though perhaps more so in the south and east of England than elsewhere. This area

\footnotetext{
${ }^{895}$ PAS ESS-45C445.

${ }^{896}$ PAS ESS-1D3342.
} 
of England was the most fertile and easiest to farm in antiquity much in the same way as it is today. This is attested by the intensification of field division seen in the south east of England compared with elsewhere in the province during the Roman period. ${ }^{897}$ This could perhaps have led to a higher level of affluence which, in turn, permitted the acquisition of objects such as copper alloy vessels. Other regional variations were also noted such as: the high presence of hanging mounts and bucket fragments in Wales and the south west, the relatively high number of Handled Pan 2s in Yorkshire and the bovine vessel spouts from Lincolnshire, all of which help to develop an appreciation for a diverse and highly complex landscape of regional tastes and patterns of consumption. ${ }^{898}$ This discussion has been built upon viewing the copper alloy vessels reported through the PAS in isolation. The next section will expand upon this discussion by examining other finds reported through the PAS in the vicinity of these vessels to contextualise these objects within the wider physical and cultural landscape.

\section{$\underline{6.3 \text { Associated Finds and Contextualizing PAS Finds }}$}

This section will integrate other objects of Roman date reported through the PAS found in the vicinity of the copper alloy vessel material reviewed in the previous section in order to place these vessels within the wider assemblage of the sites. Associated finds were identified on the PAS database by searching for objects of Roman date within the same kilometre grid square of each copper alloy vessel featured in this data-set. ${ }^{899}$ When considering rural finds assemblages, such as those reported through the PAS, it is important to bear in mind that postdepositional processes may have impacted the distribution of these objects. Ploughing, field levelling or destoning, natural rain wash, and the slow impact of fauna or gravitational soil erosion may have a highly significant impact on the distribution of surface objects, scattering objects from a single act of deposition over a wide area or leading to the concentration of objects from multiple points of origin in the same location. ${ }^{900}$ While this would not affect the broad regional distribution patterns, it could affect localised object distribution and findspot assemblages. The possibility for post-depositional processes affecting object distribution should

\footnotetext{
${ }^{897}$ Gerrard 2013, 217.

${ }^{898}$ How this relates to the larger patterns in regional variation will be further explored in Chapters $7 \& 8$.

${ }^{899}$ This was performed by doing a four-figure grid reference search for the findspots of each vessel on the PAS online database.

${ }^{900}$ Brindle 2011, 64-68.
} 
be kept in mind in this section, though, as attempts will be made to characterise how the copper alloy vessel material may fit in with the greater assemblages within which they were discovered.

By far the type of object most commonly found in the vicinity of copper alloy vessels is coins. The majority of these are single coin finds, though sometimes small groups of coins may be recorded as a single find. This should come as no surprise, as Roman coinage is the most common find reported through the PAS, ${ }^{901}$ accounting for $82 \%$ of the total objects recorded in 2012. ${ }^{902}$ Structured Deposits of coins and other objects are also found and reported through the PAS, most notably at Fingringhoe in Essex ${ }^{903}$ and West Stow in Suffolk ${ }^{904}$ from this data-set. Structured Deposits found at sites with copper alloy vessels reported through the PAS also include Hoxne ${ }^{905}$ and Mildenhall. ${ }^{906}$ Interesting as this might be, it is unlikely to be indicative of any direct pattern of association, as Roman coinage is widely distributed across the British landscape and is the most common find of Roman date reported through the PAS. ${ }^{907}$ The same may be said for brooches or small jewellery, which are also found on the same rural sites as copper alloy vessels. A detailed look at the assemblages is required before such theories of association may be tested. The following figure illustrates the numbers of finds of Roman date associated with the findspots of copper alloy vessels:

\footnotetext{
${ }^{901}$ Moorhead 2010b, 143.

${ }^{902}$ Worrell \& Pearce 2013, 345.

${ }^{903}$ PAS Treasure Number 2012 T729.

${ }^{904}$ PAS SF-D4D044.

905 Johns 2010.

${ }^{906}$ Painter 1977; Hobbs 2012.

${ }^{907}$ Moorhead 2013, 3-5.
} 


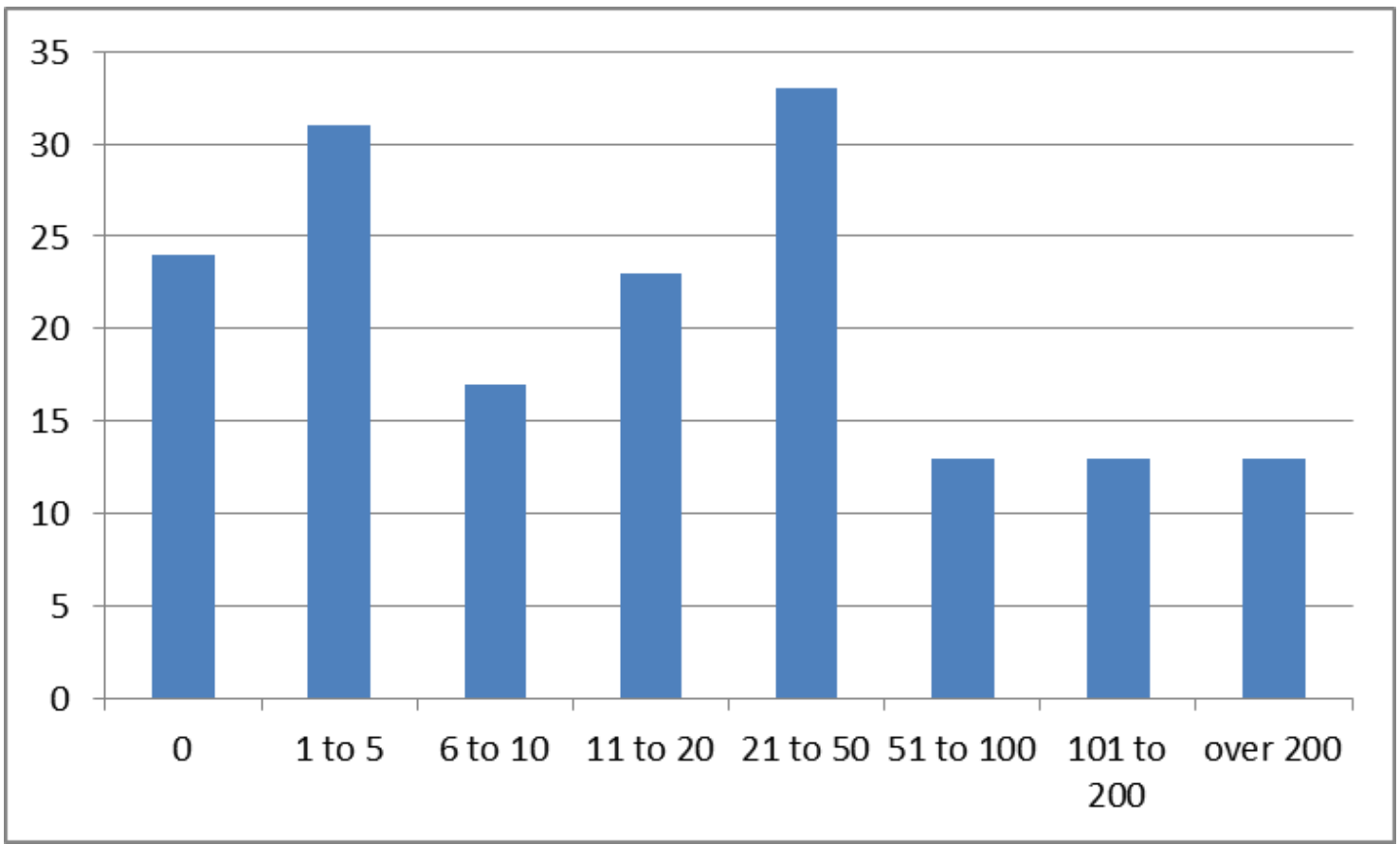

Figure 6.10: Findspots of copper alloy vessels ( $y$ axis) with numbers of associated finds ( $x$ axis) reported through the PAS.

Given the tendency for detectorists to search in locations where they believe clusters of finds to be ${ }^{908}$ it is somewhat surprising to note the high numbers of sites that had fewer than five other finds of Roman date associated with them. Moorhead suggests that any parish with more than 20 coins could be argued to be indicative of a Roman settlement site of one kind or another, ${ }^{909}$ with 100 or more indicating a site likely to be of some significance. ${ }^{910}$ Most findspots that have produced copper alloy vessels fall below this base-line and the vast majority of findspots have produced fewer than 100 total objects of probable Roman date. 26 findspots had over 100 finds associated with them, these listed in Table $6 \mathrm{~d}$ :

\footnotetext{
${ }^{908}$ Brindle 2011, 69-72.

${ }^{909}$ Moorhead 2013, 4.

${ }^{910}$ Moorhead 2010b, 157.
} 


\begin{tabular}{|l|l|}
\hline County, Parish & Number of Associated Finds \\
\hline Essex, Steeple Bumpstead & 255 \\
\hline Essex, Good Easter & 203 \\
\hline Essex, Ardleigh & 127 \\
\hline East Riding of Yorkshire, Fridaythorpe & 210 \\
\hline East Riding of Yorkshire, Shipton Thorpe & 351 \\
\hline Hampshire, Ropley & 126 \\
\hline Hampshire, Wherwell & 138 \\
\hline Hertfordshire, Clothall & 129 \\
\hline Hertfordshire, Watton-at-stone & 148 \\
\hline Leicestershire, Gaddesby & 143 \\
\hline Lincolnshire, Ancaster & 204 \\
\hline Lincolnshire, Thonock & 742 \\
\hline Lincolnshire, Keelby & 174 \\
\hline Lincolnshire, Wickenby & 178 \\
\hline Lincolnshire, Stainton by Langworth & 117 \\
\hline Norfolk, Beebton with Bittering & 241 \\
\hline Norfolk, Shouldham & 822 \\
\hline Oxfordshire, Letcombe Regis & 138 \\
\hline Suffolk, Brockley & 101 \\
\hline Suffolk, Hitcham & 107 \\
\hline Suffolk, West Stow & 924 \\
\hline Suffolk, Barking & 403 \\
\hline Suffolk, Otley & 155 \\
\hline Suffolk, Sutton & 373 \\
\hline Warwickshire, Alcester & 218 \\
\hline West Sussex, Eartham & 283 \\
\hline Table 6d: Vessel findspots with more than 100 associated finds of Roman date reported through \\
& the PAS. \\
\hline
\end{tabular}

It becomes immediately apparent that the majority of rich sites come from East Anglia and the south eastern parts of Britain. This should be no surprise, as these are the areas most frequented by metal-detectorists where reported artefact densities are at their highest. As it is clear that individual PAS finds of copper alloy vessels do not exist in a vacuum, the next step is to attempt to characterise the sites where these objects are found. This is problematic where the associated finds amount to only a few coins or other commonly reported objects, as these finds are widespread and are indicative of little else than there was someone present in the area who could have dropped or discarded the object in question. Therefore, the following discussion is limited to evidence gleaned from the 26 findspots listed in Table $6 \mathrm{~d}$. 


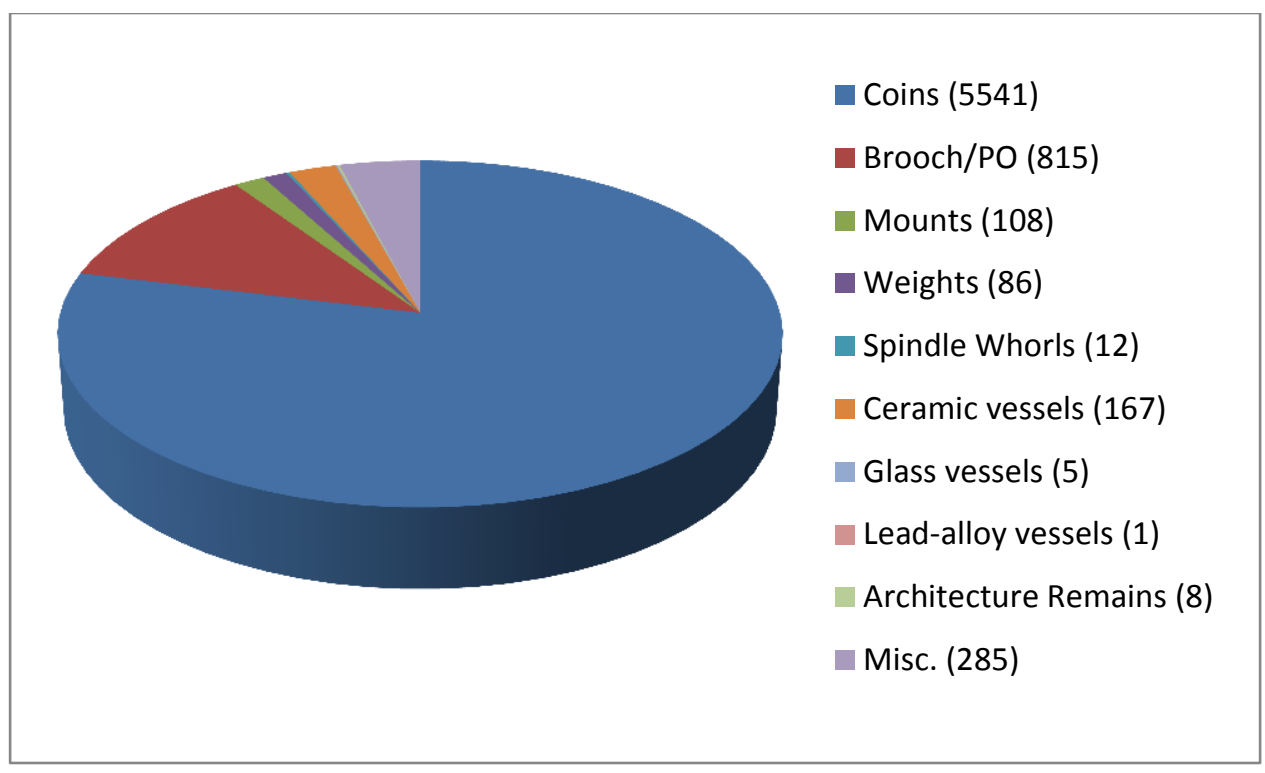

Figure 6.11: Associated finds from assemblages over 100 objects.

As Figure 6.11 shows, the vast majority of finds across these sites are coins $(5,541$ records) with objects of personal adornment (such as brooches, finger rings and bracelets) being the second most frequent class of objects reported (815 records). In occasional cases, such prolific PAS findspots assemblages could be argued to be disturbed Structured Deposits or Grave Deposits. ${ }^{911}$ This is especially relevant at a site such as West Stow, where both an intact cremation burial $^{912}$ and a Structured Deposit of coinage in a greyware ceramic ${ }^{913}$ have been reported through the PAS. However, as seen in Chapter 3, copper alloy vessels are rarely directly associated with Structured Deposits of coinage or even among scrap assemblages. Handled Pan 1s, the vessel most easily associable with graves, is not a common find in the PAS as only five feature in the current data-set, though four of these occur in East Anglia and the south eastern counties, the areas most associated with this form of burial practice. The predominance of coinage among the associated finds also suggests that these are unlikely to be graves, though coinage was included in the furnishing of graves during the Roman period, ${ }^{914}$ it was not interred in the large quantities that would lead to it dominating a site assemblage. ${ }^{915}$ From the presence of other objects in the assemblages such as brooches, finger rings, nail

\footnotetext{
911 Brindle 2011, 61-64.

912 PAS SF-ABCF44.

${ }^{913}$ PAS SF-D4D044.

${ }^{914}$ Philpott 1991, 208-216; Brown 2008, 121-130.

${ }^{915}$ Philpott 1991, 209.
} 
cleaners and ceramics it also becomes apparent that these sites were complex and exhibit the characteristics of long term habitation, which indeed seems the most likely scenario (see Appendix XI).

When looking at the individual sites for patterns and anomalies, it is possible to notice distinct characteristics that may be indicative of the occupation of these places during the Roman period. For instance, the reporting of metalworking debris at six of the sites suggests a possible industrial nature to occupation, ${ }^{916}$ as does possible glass working debris found at Wickenby in Lincolnshire. However, this debris could in fact be post-Roman and, while interesting, cannot be used empirically as a means of characterising the site during the Roman period. More useful are the architectural remains of Roman date such as domestic tiles, tesserae and wall plaster remains found at Steeple Bumpstead (Essex), Wherwell (Hampshire), Wickenby (Lincolnshire), Barking (Suffolk) and Hitcham (Suffolk) that clearly indicate the presence of a structure. The likelihood that these sites represent rural habitation centres is strengthened by the presence of significant numbers of ceramics at several of the sites and perhaps most convincingly by the frequent presence of weights and spindle whorls. The likelihood that these could represent small rural settlements proves the most likely scenario and this is the current theory most prevalently applied to the study of PAS material. ${ }^{917}$ Ritual deposition cannot be ruled out, as such assemblages of coinage and assorted small finds of metal and ceramic are known from other votive deposits of Roman date in Britain. ${ }^{918}$ Particularly worth mentioning is Coventina's Well near Hexham which has a large and diverse assemblage which includes a significant number of coins and brooches, ${ }^{919}$ as is the assemblage from the Temple of Sulis Minerva at Bath that contains metal vessels, Iron Age and Roman coins as well as brooches and other objects of personal adornment. ${ }^{920}$ Another ritual site to produce profuse finds of coins and objects of personal adornment is seen in the Conquest period site at Hallaton in Leicestershire. ${ }^{921}$ Similarly, the assemblage from Piercebridge proves that while predominated by coinage and objects of personal adornment, such votive deposits may be rather eclectic in their composition. ${ }^{922}$

\footnotetext{
916 Steeple Bumpstead (Essex), Thonock (Lincolnshire), Keebly (Lincolnshire), Barking (Suffolk), Otley (Suffolk) and Hitcham (Suffolk).

${ }^{917}$ Brindle 2011, 345-372; Moorhead 2013, 3-7.

${ }^{918}$ Bagnall-Smith 2008, 161-162.

919 Allason-Jones \& McKay 1985, 6-11 \& 20-34.

${ }^{920}$ Cunliffe 1988, 1-54 \& 279-337; Cousins 2014, 52-64.

${ }^{921}$ Score 2011, 1-102.

${ }^{922}$ Walton 2012, 152-166; Walton forthcoming.
} 
These large PAS findspot assemblages that feature copper alloy vessels most likely indicate long vanished rural communities. The most significant thing that these assemblages indicate is that these finds do not occur in isolation within the landscape and are indicative of the thriving material practice of Roman-British rural communities. That copper alloy vessels were used in these communities is important as it indicates that these objects had a wide penetration into the culture practice of the province. This line of thought will be further explored when material from all Depositional Contexts are synthetically discussed to address the thesis questions in Chapters 7 and 8. Presently, chronological patterns in PAS material will be reviewed before the use of decoration in copper alloy vessels reported through the PAS will be examined and its implications in regard to culture change in Britain during the Roman period discussed.

\subsection{Chronological Discussion of PAS Finds}

The chronological information for the current data-set is dependent entirely on stylistic grounds, which was discussed in detail in the previous section. For this reason, the discussion of the dates for material in this data-set will be much abbreviated in comparison to other chapters. As the material lacks datable context, the chronological information gleaned from this data-set can pertain only to the date of manufacture. As has been commented on throughout this thesis, copper alloy vessels could and often did have extensive use-lives that could span over a century. ${ }^{923}$ This makes the construction of chronological arguments regarding deposition with this data-set somewhat problematic. While this remains true, the date of manufacture has much to inform us in regards to the extent of production and consumption, even if its archaeological deposition was significantly later than its date of manufacture. For this reason, the information that the Single Finds of the PAS regarding chronology is still highly significant to our understanding of the availability and use of copper alloy vessels during the Roman occupation of Britain.

The most surprising and most important trend in the chronological information for this data-set is the prevalence of bucket feet and vessel mounts datable to the $2^{\text {nd }}$ and $3^{\text {rd }}$ centuries, with 21 examples, as this is precisely the timeframe which is least represented in Structured Deposits and Grave Deposits. There is comparatively little material that can be securely dated to

\footnotetext{
${ }^{923}$ Specific discussion may be found in Section 2.2.
} 
the early Roman period and even less that can be ascribed to the $4^{\text {th }}$ or $5^{\text {th }}$ centuries in the PAS data-set. It would seem that the $2^{\text {nd }}$ and $3^{\text {rd }}$ centuries saw a distinct rise in the popularity and availability of copper alloy vessels in the countryside while this material's visible impact in urban centres during this period is less noticeable. ${ }^{924}$

\section{$\underline{6.5 \text { Analysis of decoration of PAS Finds }}$}

It was anticipated that this data-set would be richer in decoration than others because, as the material is without specific chronological context, it relies almost exclusively on style and decoration for identification and dating. The data-set for Single Finds from the PAS is indeed iconographically rich, with some $85 \%$ of the data-set being decoratively embellished. ${ }^{925}$

However, the decoration of the individual objects and fragments themselves is not as complex as that found on some of the vessels from other contexts, perhaps best represented in the objects from Turner's Hall Farm burials, ${ }^{926}$ or in the exceptionally elaborate Prickwillow Pan. ${ }^{927}$

Anthropomorphic representations are rare among this data-set. Many anthropomorphic mounts recorded on the PAS database are more likely to be furniture than vessel mounts, and were thus excluded. However, it is worth noting the presence of several anthropomorphic bust vessel mounts from the Midlands, as these are comparatively rare among finds of copper alloy vessels in Britain and the wider Roman world. These are an anthropomorphic vessel mount from in Hertfordshire, perhaps depicting a satyr (Figure 6.12), ${ }^{928}$ a bust of Bacchus from Letcombe Regis in Oxfordshire ${ }^{929}$ (Figure 6.13) and a rather curious male face with almond shaped eyes from Fisherwick in Staffordshire (Figure 6.14). ${ }^{930}$ While anthropomorphic mounts are known to have featured as casket and furniture mounts, ${ }^{931}$ the above mounts are likely to have come from vessels due to their curved profile, implying they were affixed to the rounded body of a vessel. These objects are widely distributed and indicate a wide access to this intricately cast material in the countryside.

\footnotetext{
${ }^{924}$ Discussed further in Chapter 7 in relation with objects from other Depositional Contexts.

925167 out of 195 objects .

926 See Chapter 4; West 2004.

927 CAM0009; see Chapter 5.

${ }^{928}$ HER0022; PAS BH-C89753.

${ }^{929}$ OX0002; PAS BERK-C01546.

930 STA0002; PAS WMID-26ACD7.

931 See Riha 2001 and Kohlert-Németh 1990, 7-10 for examples of such anthropomorphic furniture mounts.
} 

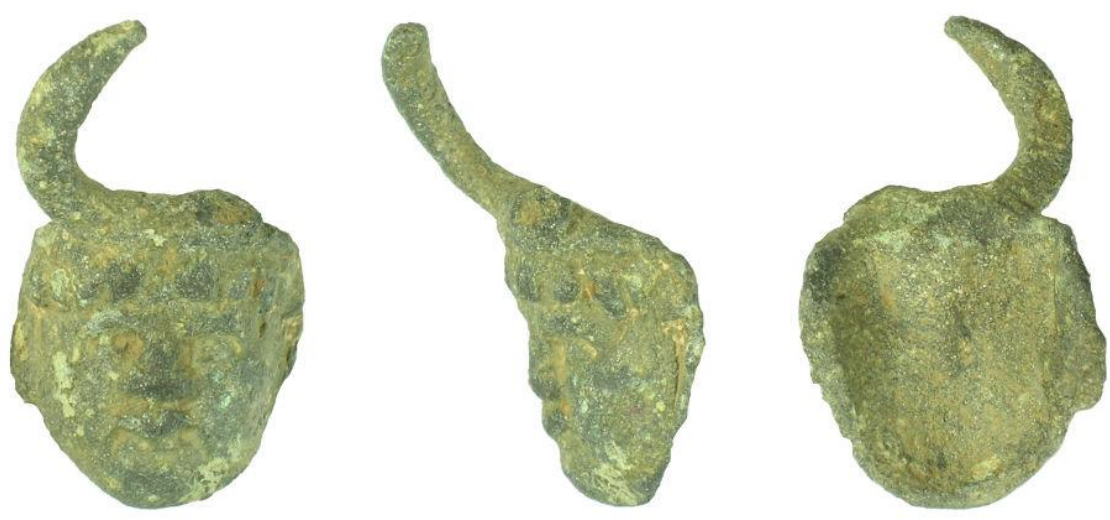

Figure 6.12: Anthropomorphic mount from Clothall (PAS BH-C89753)
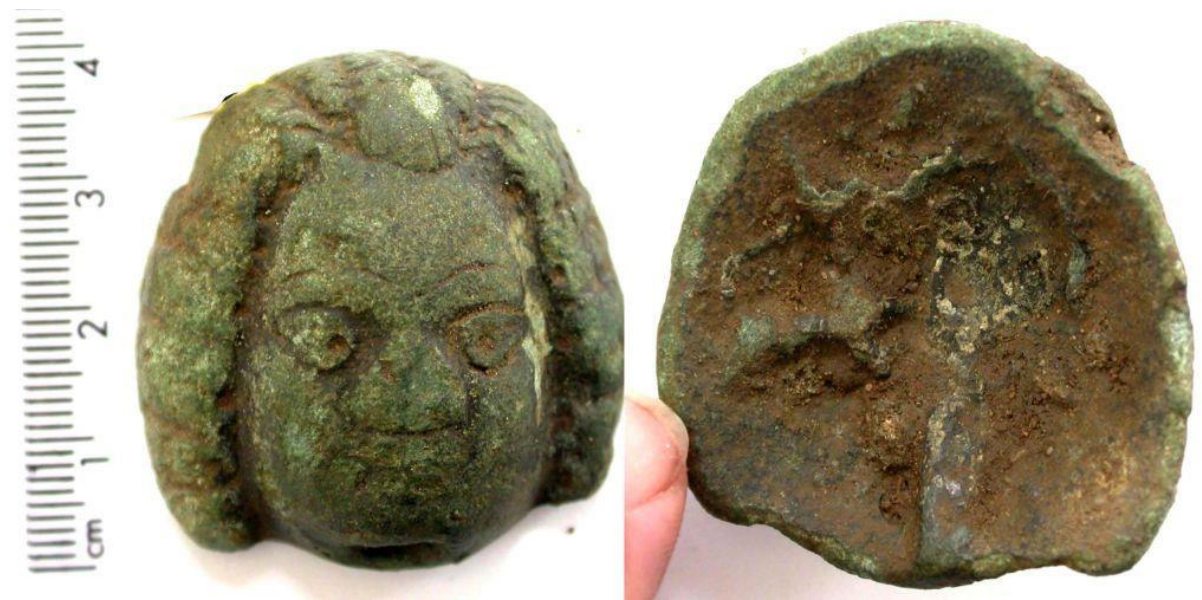

Figure 6.13: Bacchic mount from Letcombe Regis (PAS BERK-C01546)
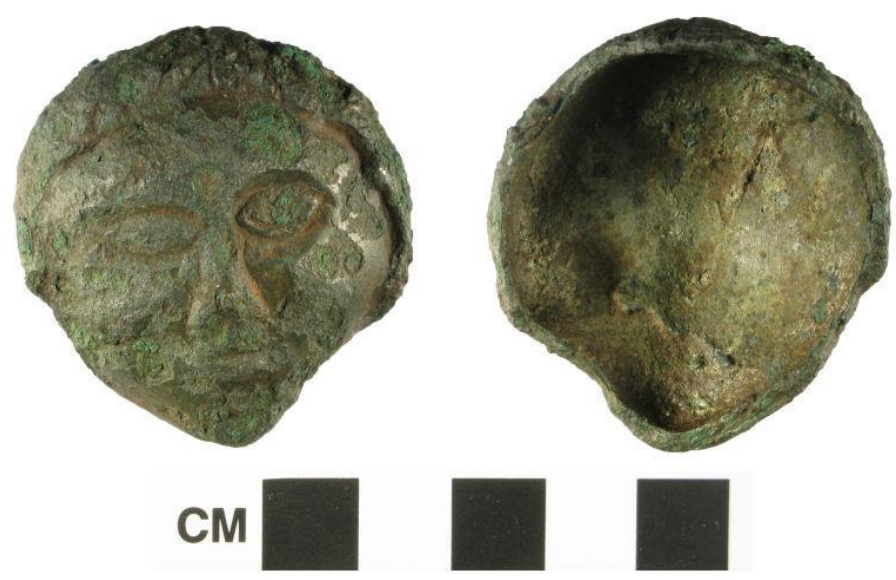

Figure 6.14: Anthropomorphic mount from Fisherwick (PAS WMID-26ACD7) 
One form of anthropomorphic representation extant that is worth particular mention is the 'reclining banqueter' decorative rim statuette, as evidenced by examples from Newport, Staffordshire and the Isle of Wight (Figure 6.15). ${ }^{932}$ Each of these depicts a banqueter in a reclined position, in accordance with Roman traditions of high dining, and is between 30 and 40 mm long with a curved base to fit onto the rim of a vessel. The curvature of the base of these statuettes indicates that it was originally fixed to a rounded rim. A similar statuette of a reclining banqueter was found at Richborough, ${ }^{933}$ though it is unclear if it originated from a vessel rim or perhaps is a box fitting. The presence of this decorative motif communicates an understanding (and probable emulation) of Roman high status leisured dining.

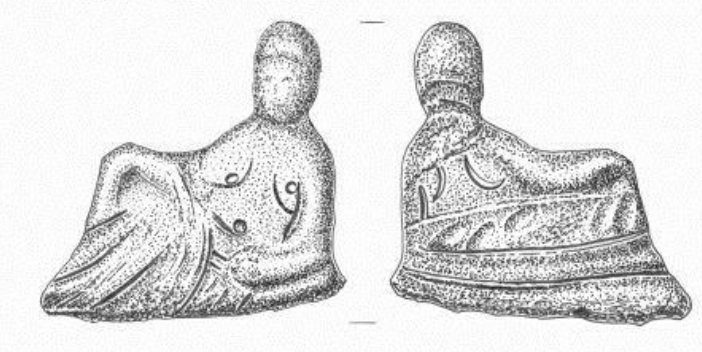

Figure 6.15: Reclining banqueter mount from Bembridge, Isle of Wight (PAS IOW-2F7DD1)

The most common zoomorphic design among the PAS data is the bovine hanging vessel mount, already seen represented among Site Finds (Figure 6.16). ${ }^{934}$ This form of decorative embellishment is used in hanging basins and is present in dated contexts from the Late Iron Age to the $2^{\text {nd }}$ century $\mathrm{CE},{ }^{935}$ representing continuity in decorative art between the Iron Age and Roman periods. The bull's head is also seen in this data-set used as a spout, probably for a strainer vessel similar in form to Eggers type 90. This spout is most attested in Lincolnshire, with three examples. ${ }^{936}$ A comparable spout is also seen on a vessel from the Turner's Hall Farm burial, where there is a zoomorphic representation of a creature with large ears or horns of some

\footnotetext{
${ }^{932}$ NE0002, STA0001, and IOW0003 respectively.

${ }^{933}$ Bush-Fox 1949, 138 (162).

934 Section2 $5.2 \& 5.3$.

${ }^{935}$ Hawkes 1952, 172-199.

${ }^{936}$ LIN0014, LIN0017 \& LIN0022; See Section 6.2 for a fuller discussion of these particular objects.
} 
kind (Figure 6.17). ${ }^{937}$ This indicates a $1^{\text {st }}-2^{\text {nd }}$ century CE date for these particular fragments, which also roughly coincides with the use of this decoration on hanging basins and buckets.
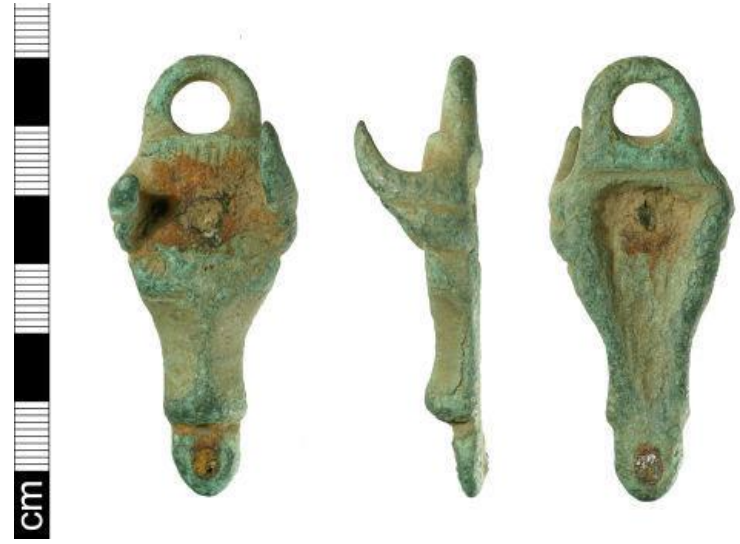

Figure 6.16: Bovine hanging vessel mount from Wymondley, Hertfordshire (PAS BH-1729A7)

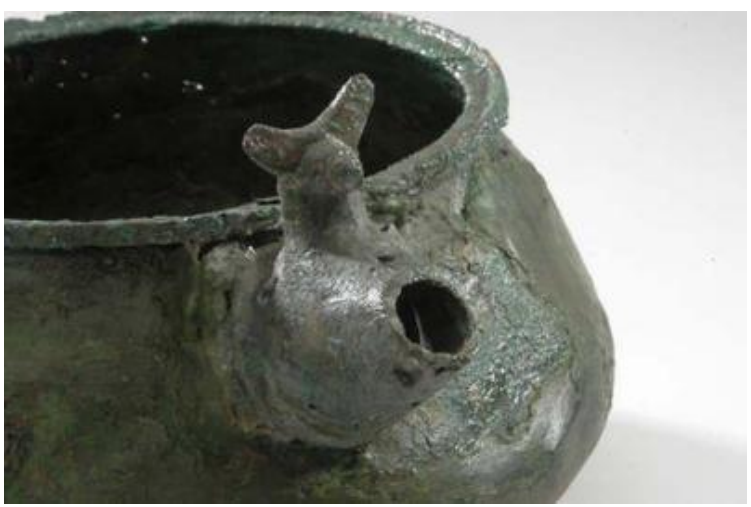

Figure 6.17: Detail of zoomorphic spout from Turners Hall Farm (@ Verulamium Museum)

Avian iconography is also frequent among this data-set, present on 27 objects, again reflecting a trend seen in the Site Finds chapter. Almost all of the birds depicted are aquatic, either ducks or swans. Aquatic birds are well represented in the iconography of this thesis, being present in each data-set. The presence of four aquatic avian decorative fragments from Hampshire alone is noteworthy, ${ }^{938}$ reflecting also the high presence of duck decoration in the Site Finds data-set of this thesis. ${ }^{939}$ A swan headed mount found near the Winchester Hoard could also help support a particular regional preference for such decoration. ${ }^{940}$ Though the fragments are different enough to allow the possibility of being from different workshops, they

\footnotetext{
${ }^{937}$ See Chapter 4; West 2004.

${ }^{938}$ HAM0005 (PAS HAMP-258E52); HAM0008 (PAS SUR-411370); HAM0013 (PAS HAMP3382); HAM0015 (PAS HAMP336).

${ }_{939}$ Section 5.3.

${ }^{940}$ Hill et al. 2004, 14.
} 
none-the-less reflect a regional aesthetic fashion that is not evident elsewhere in Britain and could hint toward localised manufacturing centres.

Pelta shaped bucket feet are well represented among PAS finds, accounting for some $8.6 \%$ of the total material of Single Finds recorded through the PAS (Figure 6.18). ${ }^{941}$ The pelta design is a popular decorative trope in the Roman world which appears in a number of diverse settings and artistic mediums from relief sculpture on distance slabs from Braidfield and Bridgeness along the Antonine Wall ${ }^{942}$ to mosaic floors found in North Africa. ${ }^{943}$ The specific type of mount under question is indicative of vessel feet from buckets of Hemmoor type and similar vessels which appear to have been manufactured predominantly in the $2^{\text {nd }}-3^{\text {rd }}$ centuries $\mathrm{CE},{ }^{944}$ though are also found deposited in Late Roman contexts. ${ }^{945}$ This type of vessel foot is ubiquitous, with findspots from Hampshire in the south to Yorkshire in the north and from East Anglia to Wales. The ubiquity of this material indicates that the use of these forms of buckets and jars was widely accepted in different regions. Unfortunately, as the regions of Hadrian's Wall do not provide any material for the present data-set, it is difficult to compare this area of well-known military concentration with trends from the rest of the province, though pelta shaped vessel feet are conspicuously absent from the Site Finds data-set for this region. ${ }^{946}$ One military setting in which this form of vessel is predominantly present is the Roman Cemetery of Brougham,${ }^{947}$ likely representing the isolated burial practice of an immigrant community in northern Britain. ${ }^{948}$ However, evidence from the Site Finds data-set and the PAS data-set of this thesis would indicate that the choice of Hemmoor and similar vessel forms likely represents equipment that was characteristic of Romano-British table and ritual practice during the $2^{\text {nd }}$ and $3^{\text {rd }}$ centuries CE.

\footnotetext{
${ }^{941} 17$ out of 195 objects.

${ }^{942}$ Breeze 2006, 64-65.

${ }^{943}$ Dunbabin 1999, 103-119.

${ }^{944}$ Erdrich 1995, 71-80.

${ }^{945}$ See the Drapers' Gardens Hoard, L0001-L0015 in Chapter 3; Gerrard 2009.

${ }^{946}$ See Chapter 5.

${ }^{947}$ See Section 4.2; Cool 2004.

${ }^{948}$ Cool 2004, 463-467; Cool 2010, 27-44.
} 


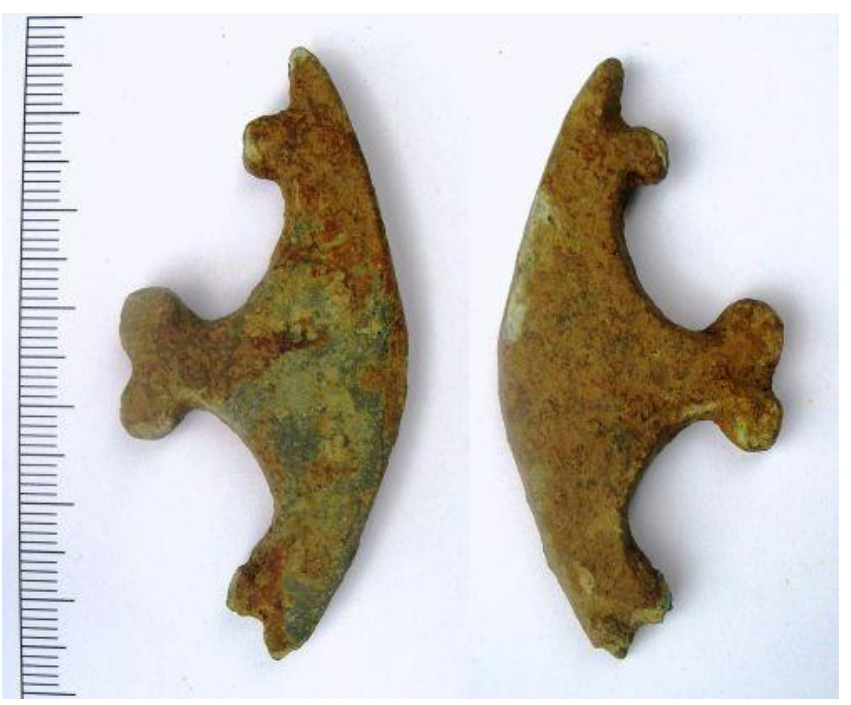

Figure 6.18: Pelta shaped vessel foot from Gaddesby in Leicestershire (PAS LEIC-92A461)

Making wide conclusions concerning the use of decoration among PAS material in this thesis proves slightly problematic as it must remain impossible for us to know how great a percentage of the copper alloy vessels material in the Romano-British countryside was indeed embellished. The variety of decoration was broad, including a surprising number of figural objects. Enamelling, especially on Rudge Cup type vessels, ${ }^{949}$ also contributed to the richness of the decoration in the data-set. Taken all together, the frequency of decorative fragments in the PAS data-set indicates the widespread availability of decorated vessels in the countryside and a ready acceptance of this material associated with ritual and display. ${ }^{950}$

\section{$\underline{6.6 \text { Concluding Remarks }}$}

The Single Finds material recorded through the PAS would seem to indicate that there was a widespread use of copper alloy vessels in the countryside of Britain during the Roman occupation and that the use of this material was most prevalent during the $2^{\text {nd }}$ and $3^{\text {rd }}$ centuries. Much of the material comes from the highly farmed, and therefore easily accessible to metal detectorists, fields of eastern England. Additionally, significant amounts of material came from across the Midlands and there were objects contributed from every part of the province, with the

\footnotetext{
${ }^{949}$ Such as NLIN0001 (PAS FAKL-9900E3) and STA0004 (PAS WMID-3FE965; Jackson 2012, 41-60).

${ }^{950}$ Swift 2009, 105-138; Discussed further in Chapter 8.
} 
exception of the hinterland of Hadrian's Wall. ${ }^{951}$ This widespread dispersal of the material is not as evident in the other data-sets of this thesis, which relied upon excavations that tend to have a military or urban bias as well as the specific ritual and cultural practices that led to deposition in Structured or Grave contexts.

The PAS data illuminates the material lives of the rural population of Roman Britain, a swathe of the population that had been less archaeologically visible prior to the PAS' advent, and seems to indicate that these inhabitants had ready access to moderate luxury items such as copper alloy vessels. The ready availability of moderate luxuries in the Romano-British countryside is corroborated by the wide distribution of lesser commodities such as copper alloy brooches in Britain (particularly the north) during the Roman period, ${ }^{952}$ as well as the blanketing of the British countryside with copper alloy coinage of Roman date. ${ }^{953}$

In the next chapter, the material from Chapters 3-6 will be integrated in order to make comprehensive statements and conclusions about the evidence provided by copper alloy vessel material in regards to the practice of material culture during the Roman occupation of Britain.

\footnotetext{
${ }^{951}$ A gap in the data-set discussed in Section 6.2.

${ }_{952}$ McIntosh 2011, 155-179.

${ }^{953}$ Walton 2012; Moorhead 2013, 1-7.
} 


\section{Chapter 7: Synthetic Discussion Addressing Thesis Questions 1-3}

\subsection{How can individual depositional contexts be characterised and interpreted?}

With copper alloy vessels in Roman Britain being treated through individual analysis of their Depositional Contexts in the previous four chapters, the following chapter offers a synthetic analysis of all of the material in this thesis applied to three of the thesis questions outlined in Section 1.5. The fourth and final question will be addressed in Chapter 8 and forms the concluding statement of this thesis.

During the initial research and data collection phase of this thesis, it became clear that certain vessel forms occurred preferentially in different contexts and that this depositional variation would play a key role in developing an understanding of these vessels. It is principally to track and interpret these trends that the research and recording was carried out by Depositional Context (Chapters 3-6). The present section will compare and contrast the forms of vessels that were found in different Depositional Contexts in order to illustrate the patterns of preferential selection inherent in each. The discussions by form will be short and remain limited to patterning across Depositional Contexts, as opposed to patterns within single Depositional Contexts which were dealt with in each appropriate chapter of this thesis. This section will conclude with a discussion characterising each Depositional Context and how each may be applied to the understanding of the place of copper alloy vessels within Romano-British material culture.

It is worth taking a moment before continuing to address the issues of sample size and statistical validity which affect a study of such a narrowly focused set of material. Copper alloy vessels of Roman date are not common finds in Britain, especially when compared with objects such as coins, brooches or ceramics. The sample size available for analytical study in this these represents this, comprising 840 objects. This number allows for valid discussion in the examination of macro trends across the province to be constructed. Each Depositional Context also had enough objects to be able to make informed arguments, as offered in Chapters 3-6 and will be further addressed throughout the following two chapters. The statistical validity of some of the sub-groups of data, specifically regarding form and type classifications, is tenuous as there simply are not enough examples currently extant to eliminate statistical error. This is perhaps most evident in the case of cauldrons; with only eighteen examples featured in this thesis, it is 
problematic making definitive conclusions on depositional character and how this data may be used to address the thesis questions presented in Section 1.5. However, it is important to make use of all the data and to construct arguments as fully as possible with the information currently available. For this reason, analysis has been conducted on each form and type in the dataset with discussion offered in the following two chapters. In most cases, the number of objects allows for statistical validity to the argument. Discussion of forms such as cauldrons and Handled Pan 4s, based on low numbers of examples, should be understood to have limitations but to be constructed in relation to level of knowledge currently available for these objects and conclusions offered seen as the best available given the current level of data.

As is clearly evident in Figure 7.01, bowls are interred far more often in Structured Deposits than any other Depositional Context. The relatively low numbers represented as Site Finds or PAS Finds may be at least in part due to the difficulty in correctly identifying a fragment of copper alloy scrap as a bowl if it does not consist of a rim fragment or otherwise diagnostic part of the vessel. Even so, the discrepancy is too great for this to be the sole explanation and it is evident that these numbers are the result of preferential selection of bowls during the formation process of Structured Deposition of copper alloy vessels during the Roman period in Britain.

In contrast to bowls, buckets are much more evenly distributed across Depositional Contexts (Figure 7.02). Unfortunately, the numbers cannot be taken completely at face-value as seven out of eight of the buckets found as Grave Deposits come from the single site of Brougham, ${ }^{954}$ making this figure unrepresentative of grave practice in Britain as a whole. Notwithstanding, the distribution between the remaining three Depositional Contexts is fairly even. This reduces the likelihood of these vessels having specific ritual significance comparable to that of Handled Pan 1s, though its presence in Structured Deposits suggests this vessel form was also used in ritual practice.

Cauldrons are not a common find during the Roman period and their relatively low numbers demand caution before extrapolating conclusions from such a small data-set across Depositional Contexts, as even one or two new finds could significantly alter the evidence. They would be almost invisible in this thesis if it were not for their presence in Late Roman Structured

${ }^{954}$ Cool 2004, 373-380. 
Deposits, most of which are composed of large bowls, buckets and cauldrons (Figure 7.03). ${ }^{955}$ This narrow focus on the selection and deposition of this material, which is not prevalent in any other depositional context, illustrates the preference for the deposition of this vessel form in Structured Deposits to a degree that was likely well beyond its relative presence in the repertoire of copper alloy vessels. Its absence from the PAS material could however be in part due to the difficulty in identifying or accurately dating fragmentary remains of this vessel form, while the low numbers of this vessel form among Site Finds suggests that it was never as widely available as bowls, buckets or handled pans. Both folk tradition and archaeological evidence indicates an association between cauldrons and the supernatural dating back as early as the Bronze Age and persisting well into Medieval Europe. ${ }^{956}$ They appear to have had particular connection to water and sacrifice in Celtic folk belief, ${ }^{957}$ a pattern reflected by their presence in Structured Deposits. Their near absence from Grave Deposits is somewhat surprising, as these vessels also seem to have had connections with death and resurrection. ${ }^{958}$ Perhaps either the object was endowed with so much mystical power by the occupants of Britain during the Roman period to dissuade their interring such vessels directly with the deceased or the peoples who practiced the interring of copper alloy vessels as grave goods were not the same peoples who put great significance on the spiritual powers of cauldrons.

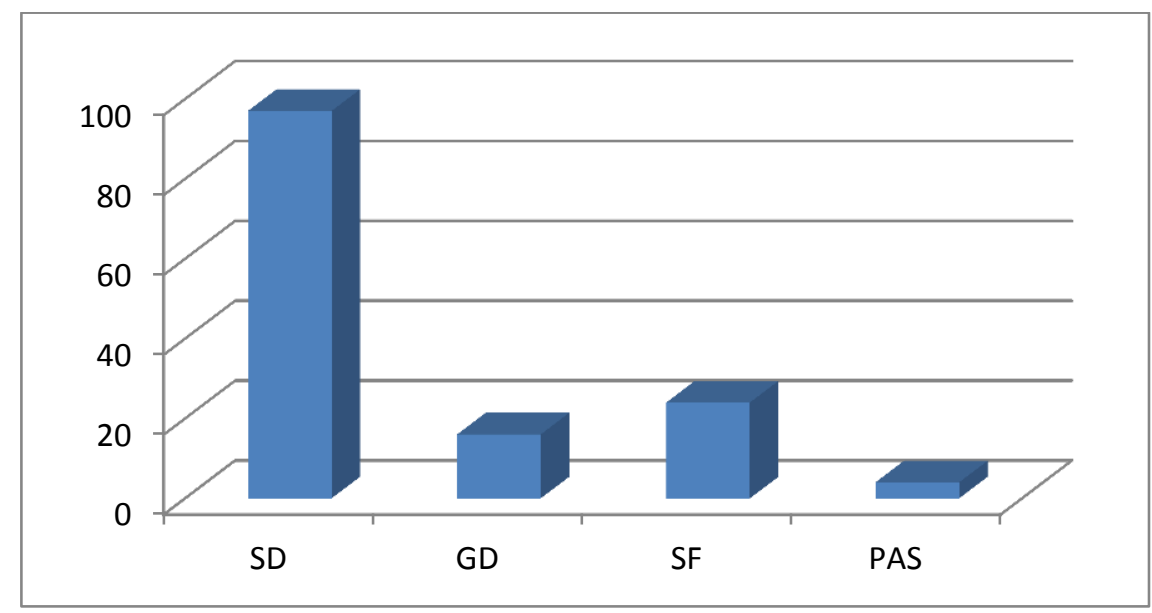

Figure 7.01: Bowls by Depositional Context.

\footnotetext{
955 See Chapter 3 for full discussion.

956 Green 1998, 63-84.

${ }^{957}$ Green 1998, 63-75.

${ }^{958}$ Green 1998, 63-68.
} 


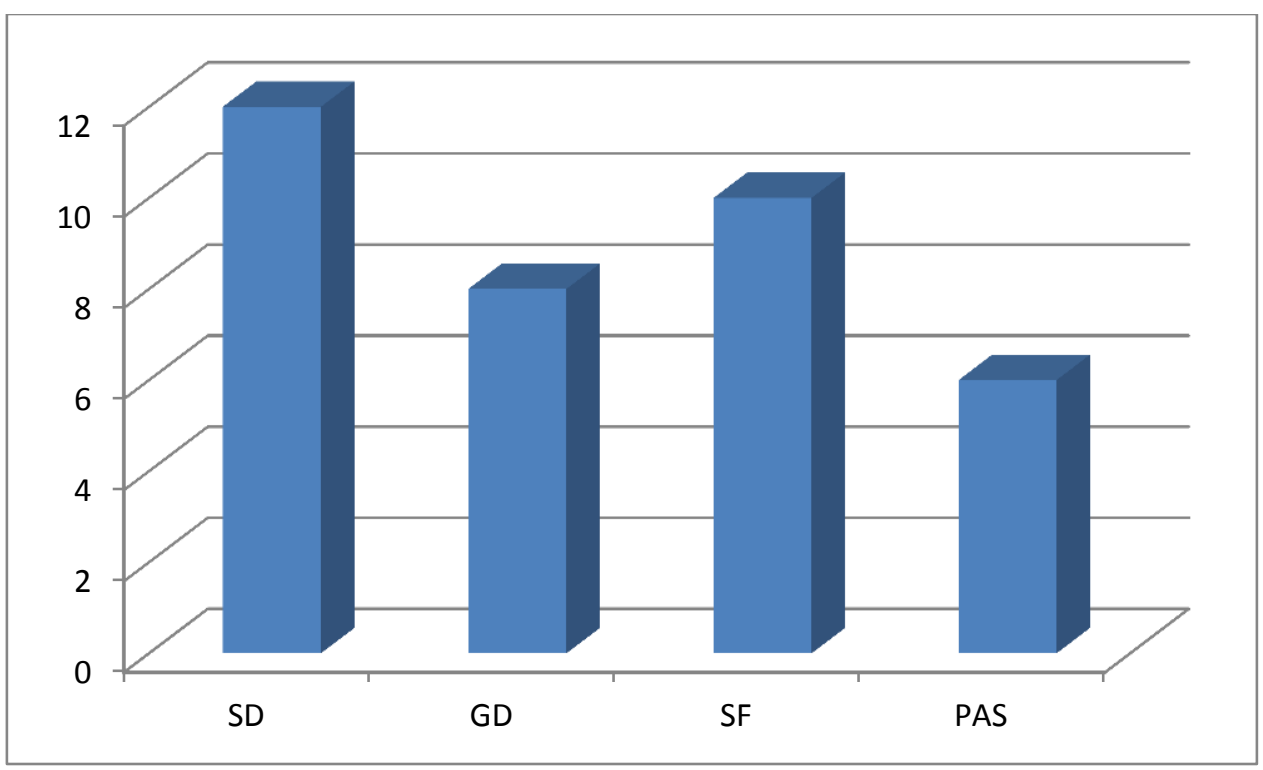

Figure 7.02: Buckets by Depositional Context.

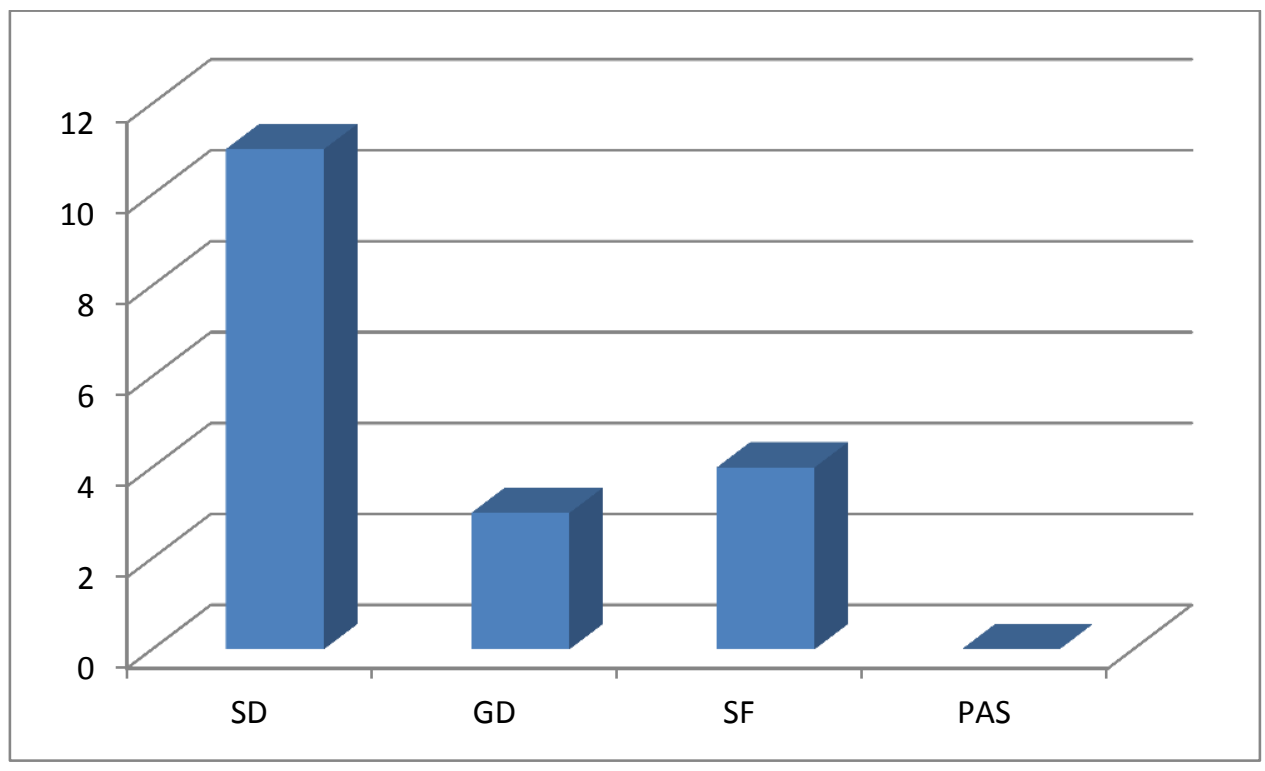

Figure 7.03: Cauldrons by Depositional Context. 


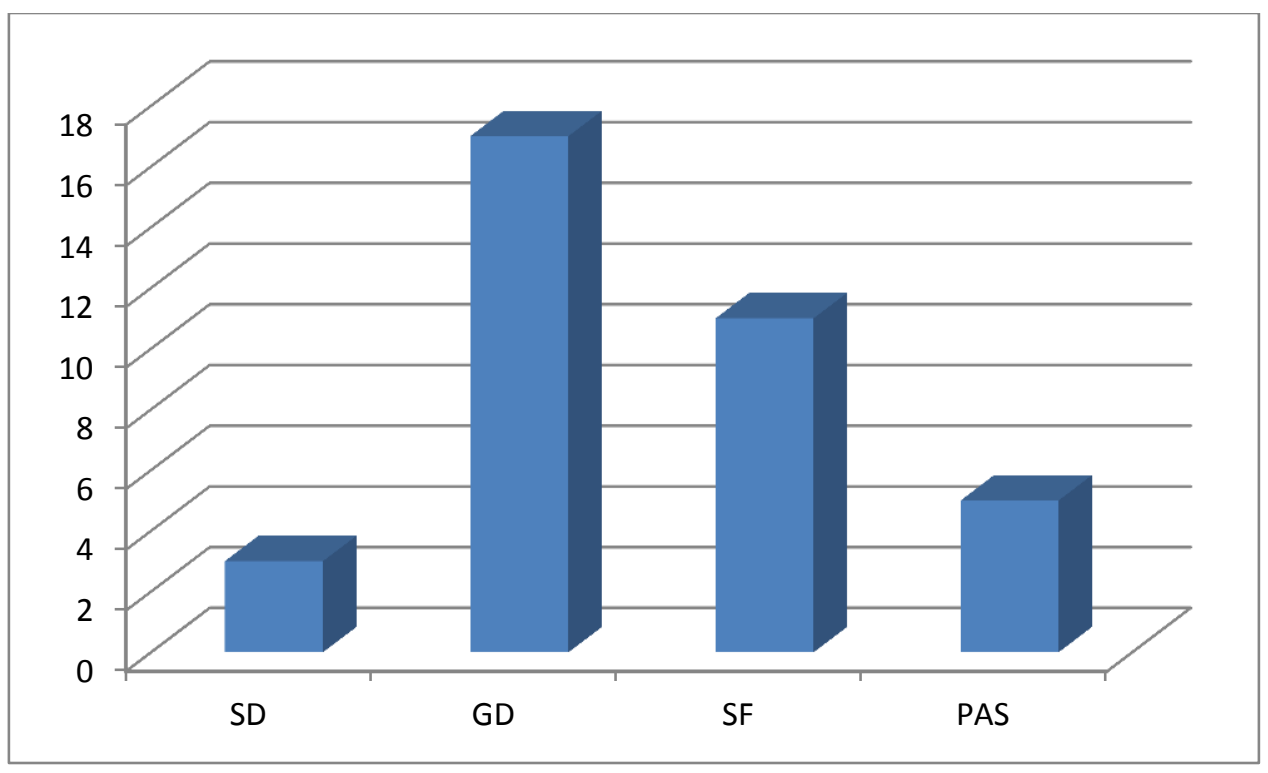

Figure 7.04: Handled Pan 1 by Depositional Context.

As anticipated based on the previous research of Nuber, ${ }^{959}$ Handled Pan 1s are seen more frequently as Grave Deposits than in any other Depositional Context (Figure 7.04). However, with 17 reported as Grave Finds and 11 recorded as Site Finds, the preferential selection of these objects as part of funerary ritual was not as pronounced as expected. This implies that the vessels were unlikely to have been designed with funerary usage specifically in mind to the exclusion of uses in daily life. Nuber comments on the probable use of these forms of vessels for purification rights associated with ritual devotion and perhaps sacrifice. ${ }^{960}$ The Handled Pan 1 from a rural shrine in Rocester ${ }^{961}$ suggests this use outside of a funerary context. That Handled Pan 1s were used outside of burial practice is also shown by their representation on altars to multiple gods. ${ }^{962}$ Their presence within funerary ritual reflects their normal use-life as objects of ritual ablution as opposed to a self-contained ritual entirely removed from more general practice. The presence of Handled Pan 1s in Structured Deposits such as Santon Downham, Hod Hill and Richborough in the UK, ${ }^{963}$ and the Cave of Letters Hoard from the environs of the Dead Sea in Israel $^{964}$ also shows their use in a wider ritual environment than simply funerary and highlights

\footnotetext{
${ }^{959}$ Nuber 1973.

960 Nuber 1973.

${ }^{961}$ Ferris et al. 2000; STA0006.

962 Section 4.3.

963 SUF0003; Eggers 1968, 106 (44a); DOR0005; Eggers 1968103 (9e); K0038; Bushe-Fox 1928, 31 (12).

${ }^{964}$ Freund 2004, 40-45 \& 141-144.
} 
the importance of this object in widely practiced ritual purification across the Roman world. This is further illustrated by artistic representations. ${ }^{965}$ Some finds recorded through the PAS may have originated from disturbed grave contexts, ${ }^{966}$ which would further tilt the balance of the depositional bias in the expected direction of funerary ritual, but these finds could just as well have originated from unrecognised rural settlements and shrines. ${ }^{967}$

With 179 examples, Handled Pan 2s are the most common diagnostic vessel form in this thesis. Their distribution across Depositional Contexts is also interesting, as they dominate Structured Deposits and Site Finds assemblages from the $1^{\text {st }}-3^{\text {rd }}$ centuries CE, though are not terribly common among PAS finds and are barely represented amongst grave assemblages (Figure 7.05). They are also well represented amongst the material documented beyond the Roman frontiers, both in Scotland and in free Germany, ${ }^{968}$ suggesting a high level of availability and acceptance of this particular form. As they are the most common form of identifiable copper alloy vessel in the archaeological record of Roman Britain, their paucity among grave goods is noteworthy and emphasises how specifically selected for their ritual and symbolic value. As Handled Pan 2 s are associated with military sites throughout this thesis, ${ }^{969}$ it is likely that this would have influenced the depositional process of this vessel form.

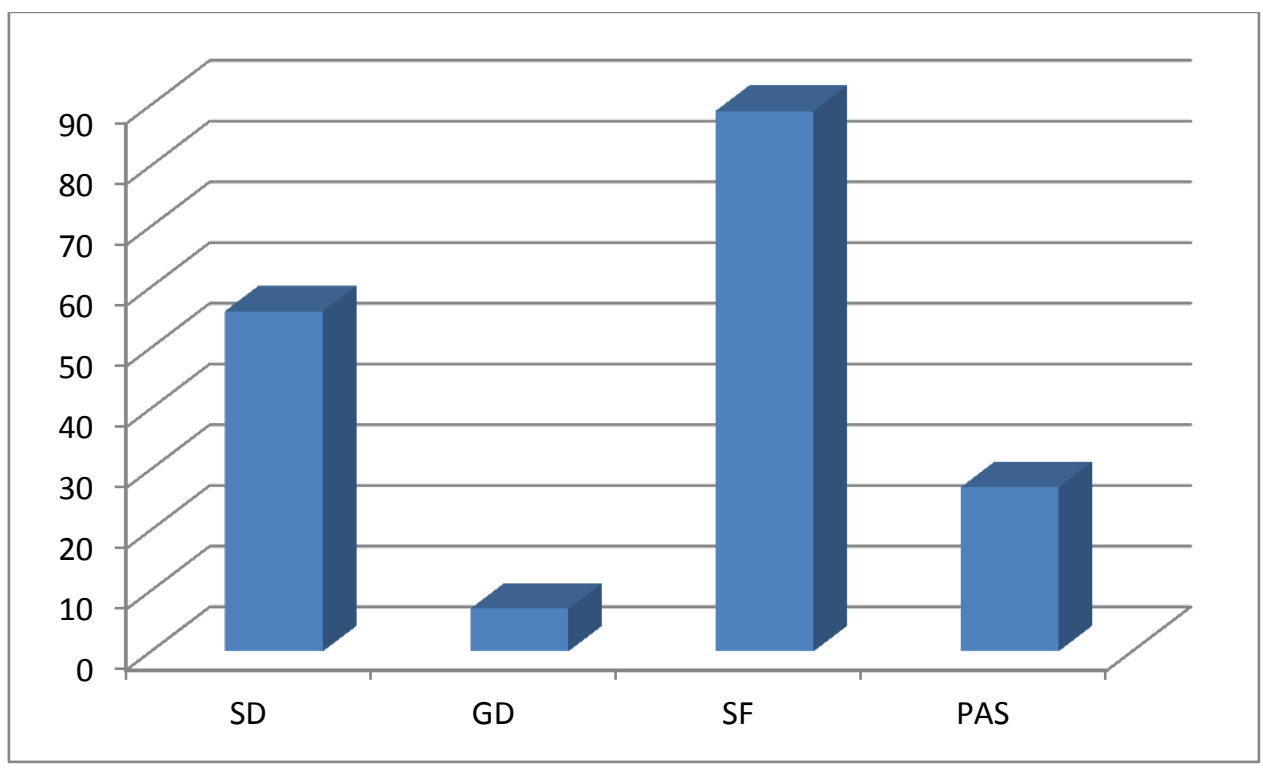

Figure 7.05: Handled Pan 2s by Depositional Context.

\footnotetext{
965 Section 4.4 .

966 Brindle 2011, 61-64.

${ }^{967}$ Brindle 2011, 345-372; Moorhead 2013, 3-7.

${ }^{968}$ Eggers 1951, 11-77; Eggers 1968, 100-110; Hunter 2001, 298-304.

${ }^{969}$ Discussed in Chapters 3 and 5.
} 


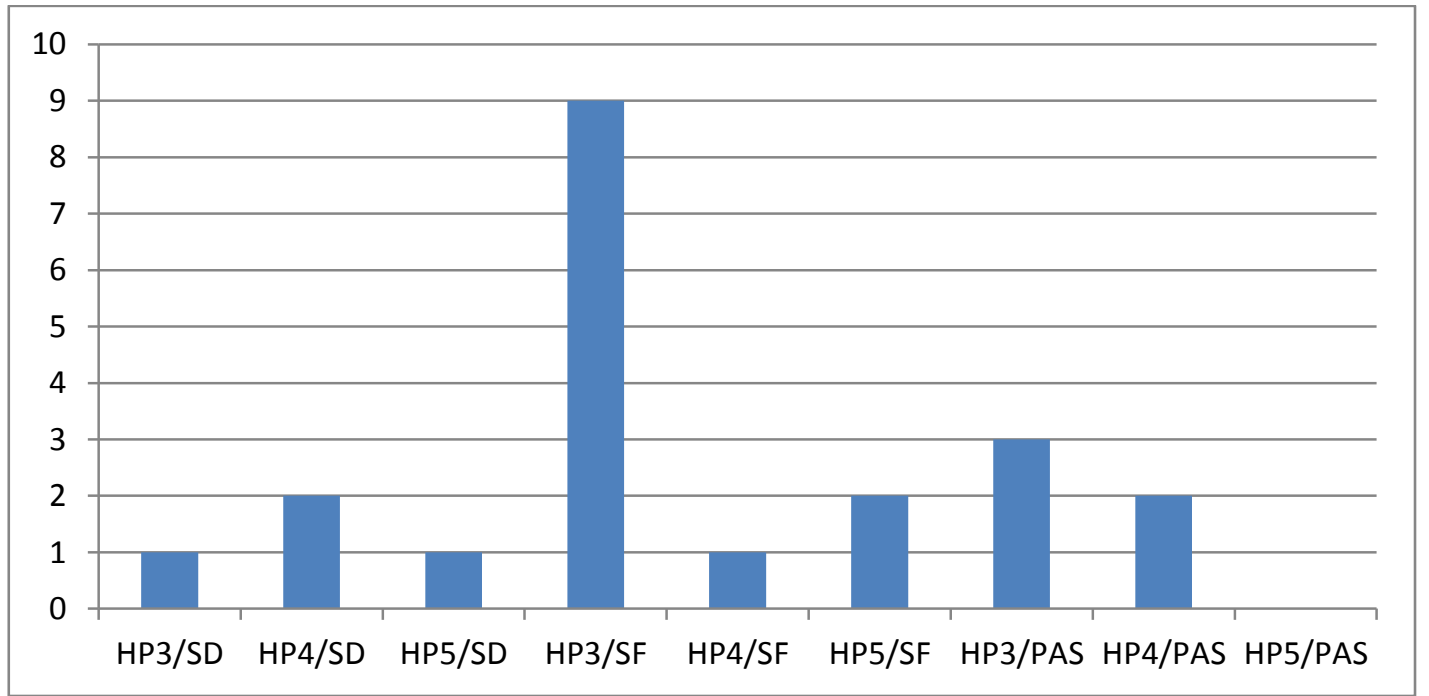

Figure 7.06: Handled Pans 3-5 by Depositional Context.

The remaining three forms of handled pans are not common among any depositional context, making it exceedingly difficult to apply their distribution to the analysis of the depositional processes affecting the formation of these archaeological contexts (Figure 7.06). One of the most striking features is that none are represented among Grave Deposits. This, combined with the low numbers present in Structured Deposits, implies that these vessels did not have ritual significance and were more utilitarian in their function or that they were never common enough to be regularly incorporated into such ritual behaviour. Handled Pan $3 \mathrm{~s}$ are far less common across Britain than Handled Pan 2s, a pattern that is mirrored in assemblages from Pompeii, Pannonia and Western Europe. ${ }^{970}$ The presence of nine Handled Pan 3 s as Site Finds is noteworthy, as this indicates their presence could have been more visible in daily life than the other data-sets would suggest. The general paucity of data for these three vessel forms indicates that none of them were ever integrated into the regular material repertoire of Britain during the Roman period, the functions they served being performed by other vessel forms such as Handled Pans $1 \& 2$ or bowls. It should be noted that these vessel forms are also rare among continental assemblages. ${ }^{971}$ This reflects on how these objects were perceived and utilised in antiquity, as their specific utilization was never imperative to any widespread practice evident through the

\footnotetext{
${ }^{970}$ See Radnoti 1938, Eggers 1951, den Boesterd 1956, Tassinari 1975 \& Tassinari 1993.

${ }^{971}$ Radnoti 1938; Eggers 1951; den Boesterd 1956; Tassinari 1975; Tassinari 1993.
} 
archaeological record. They must have been viewed as varied types of other vessel forms and seem unlikely to have served a function specific or exclusive to themselves.

Jars are another form of vessel that is not particularly common in Roman Britain, with only 13 examples across all Depositional Contexts (Figure 7.07). The great variance in the depositional pattern of jars and buckets is interesting, given that they are both cylindrical vessels which might have had similar functions. This would suggest that they were not visualised this way during the Roman period, each having its own sphere of use and application. The small numbers of these objects makes interpretation problematic, however, and it would be unwise to conjecture too far concerning their role in the repertoire of copper alloy vessels based on patterning among so few examples. While examples of ceramic and glass jars are far more common, it would not be appropriate to suppose that objects of similar shape but of different material had immediately comparable functions. ${ }^{972}$ Like Handled Pan 3s, the fact that jars are found more commonly as Site Finds than in other depositional contexts hints that they were often overlooked in the selection processes that led to Structured Deposition and Grave Deposition. Their relative rarity in PAS data may be explained by there being little diagnostic about these vessels to identify them if they are found in a highly fragmented state, as much of the PAS data inevitably is. A number of small jars, likely used for perfume or unguents, were deposited as grave goods in much the same way that similar vessels of glass were interred. ${ }^{973}$ It is likely in these cases that the prestige of the display of consumption was shared between the expense of the liquids combined with the ornate vessels in which they were contained, enamelled copper alloy jars likely being more expensive and prestigious than their glass cousins.

\footnotetext{
${ }^{972}$ Hurcombe 2007, 109-118 \& 190-208.

${ }^{973}$ De Santis 2000, 238-243.
} 


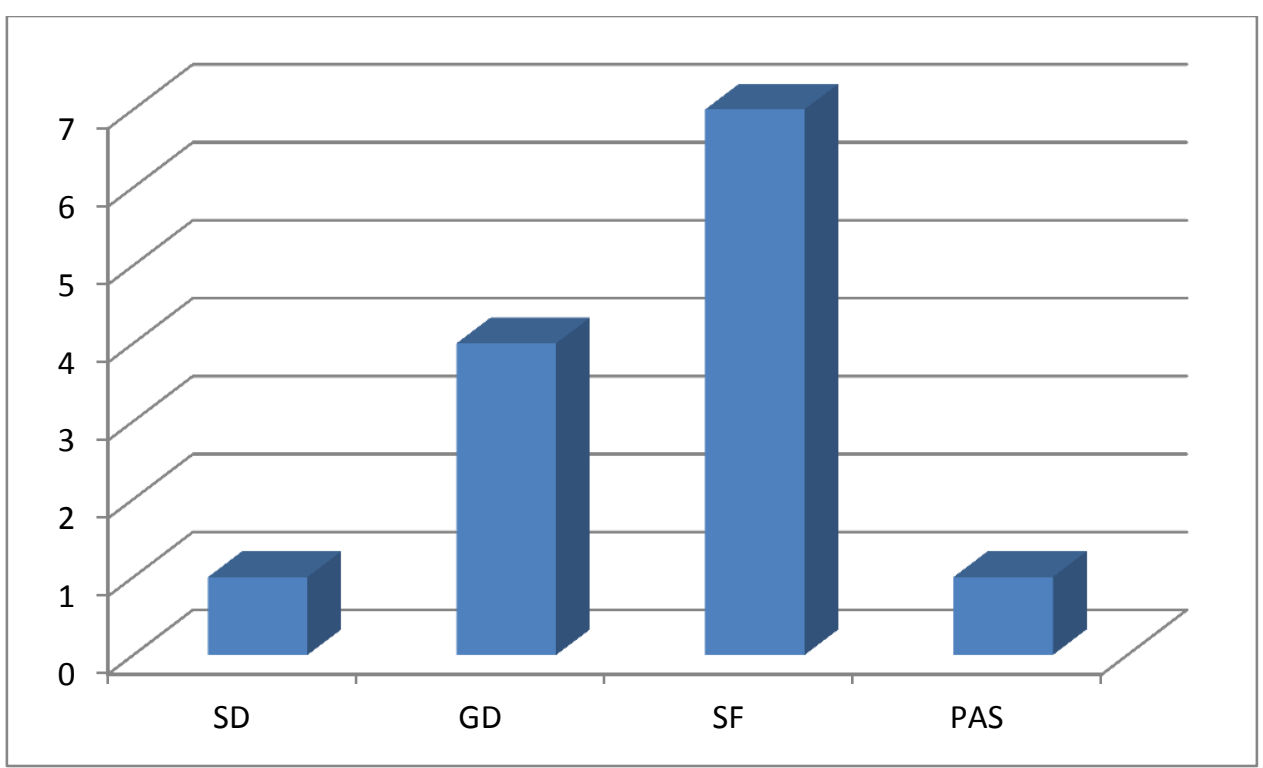

Figure 7.07: Jars by Depositional Context.

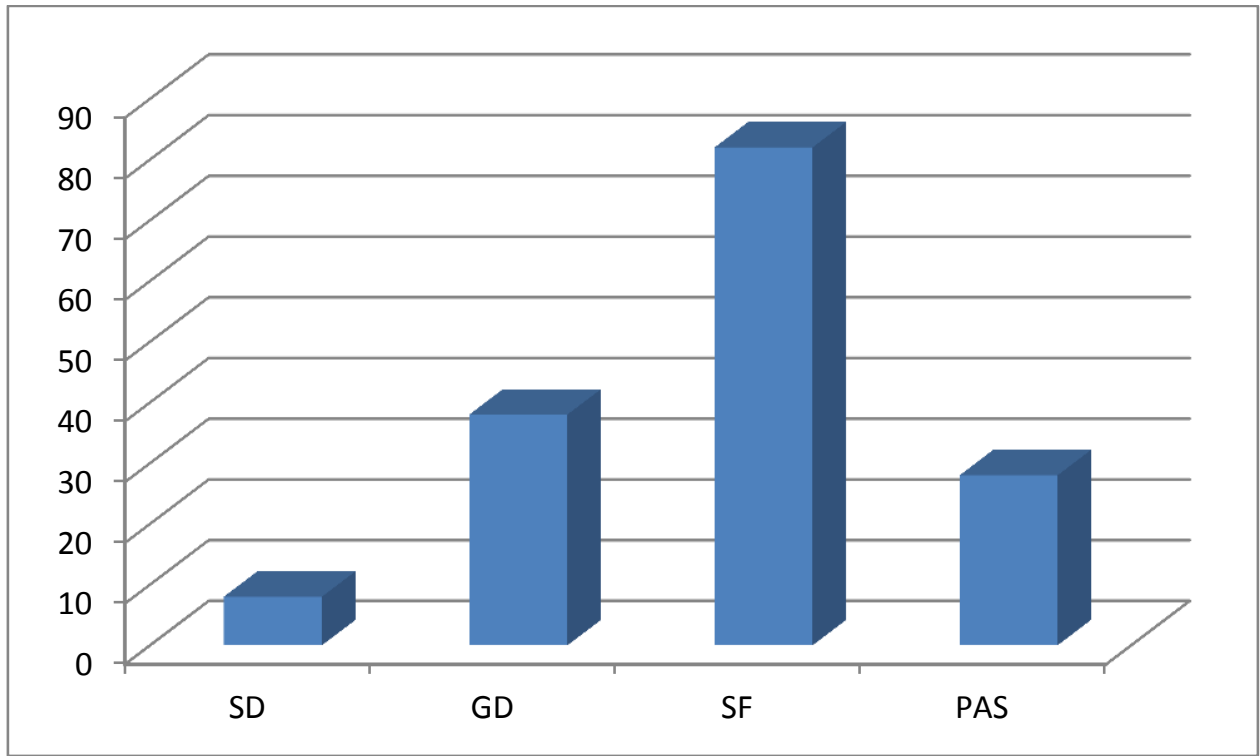

Figure 7.08: Jugs by Depositional Context.

Due to their very function as containers of liquids, jugs are well represented across Depositional Contexts and feature as one of the most common vessel forms (Figure 7.08). Their pairing with Handled Pan 1s in Grave Deposits has been noted ${ }^{974}$ and accounts for their relatively high numbers in funerary contexts. That only eight examples are known from

\footnotetext{
${ }^{974}$ Nuber 1973; Cool 2006, 47-48; see Chapter 4 for fuller discussion.
} 
Structured Deposits, three of these being associated with coin hoards, ${ }^{975}$ indicates a conscious exclusion of this vessel form from the ritual practice leading to such deposition in the archaeological record. The prevalence of jug fragments as Site Finds indicates that these vessels would have formed a common part of the material assemblage in use during the Roman period, supported by its visibility within the PAS data. The visibility of this vessel form in Site Finds and PAS data may be influenced by the diagnostic character of their handles, which were soldered on and can break off from the vessel bodies and are readily diagnostic when found. This is in contrast to bowls and jars, which typically do not have such easily detachable nor diagnostic fragments and which appear far less frequently in either of these depositional contexts. For this reason, the visibility of jugs in the archaeological record may be proportionally inflated when compared with less diagnostic vessel forms. Nonetheless, the visibility of jugs also represents their popularity and their acceptance into the dining and ablution rituals.

Strainers are more ubiquitously associated with imbibing in the scholarly literature of Roman drinking and dining practice than any other vessel form, being seen as used for the infusing of alcoholic beverages with spices or herbs for flavour or narcotic effects, ${ }^{976}$ though they likely served as filtration devices more generally as well. Their rather narrow function, combined with the fragile composition of their construction, accounts for their relative small numbers when compared with jugs and handled pans. Their concentration among Late Roman Structured Deposits is therefore very helpful in constructing the functionality of these assemblages as a whole, indicating that communal imbibing could well have been the principal shared feature in the vessel forms that constitute these assemblages (Figure 7.09). Strainers are also present in earlier Structured Deposits paired with Handled Pan 2s, implying that these too could have been used as part of a drinking service.

\footnotetext{
${ }_{975}^{975}$ Roundway Hill (WIL0023), Corbridge (NU0035) and Blagan Hill/Bishop's Cannings (WIL0020).

${ }^{976}$ Cool 2006, 144-145.
} 


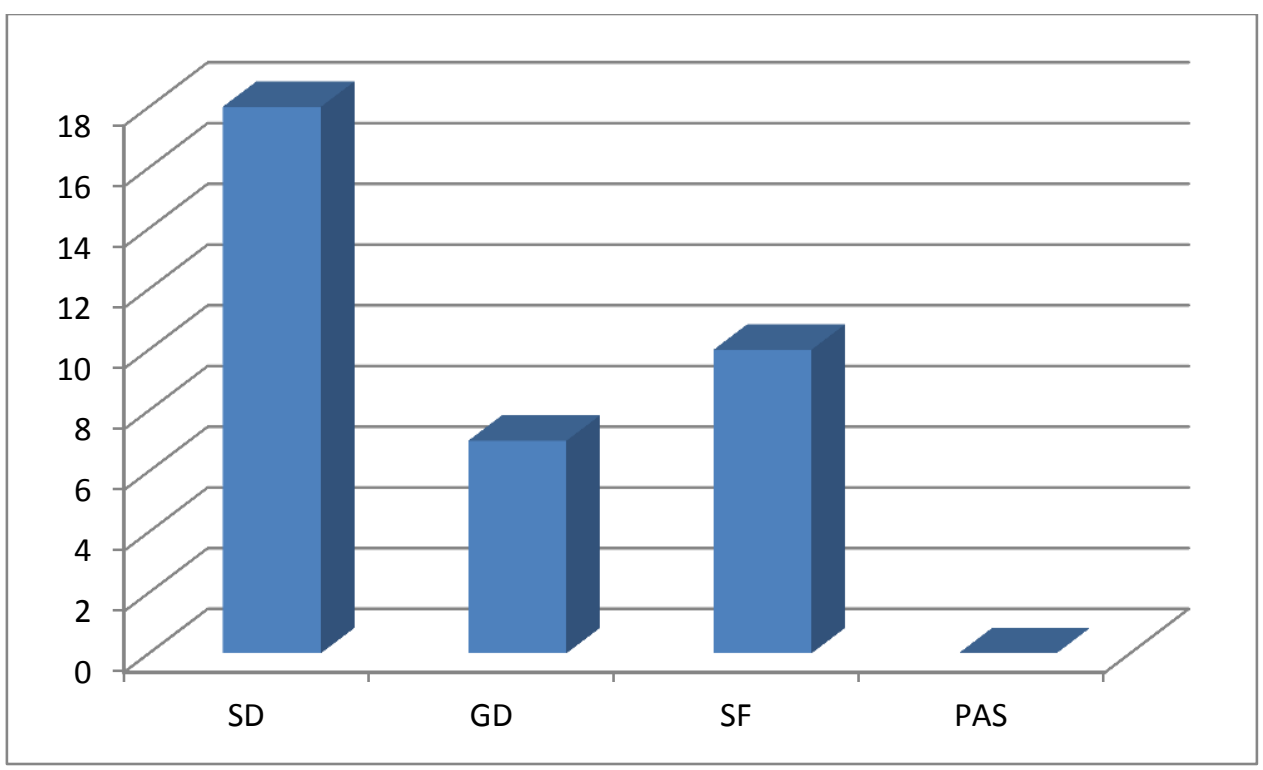

Figure 7.09: Strainers by Depositional Context.

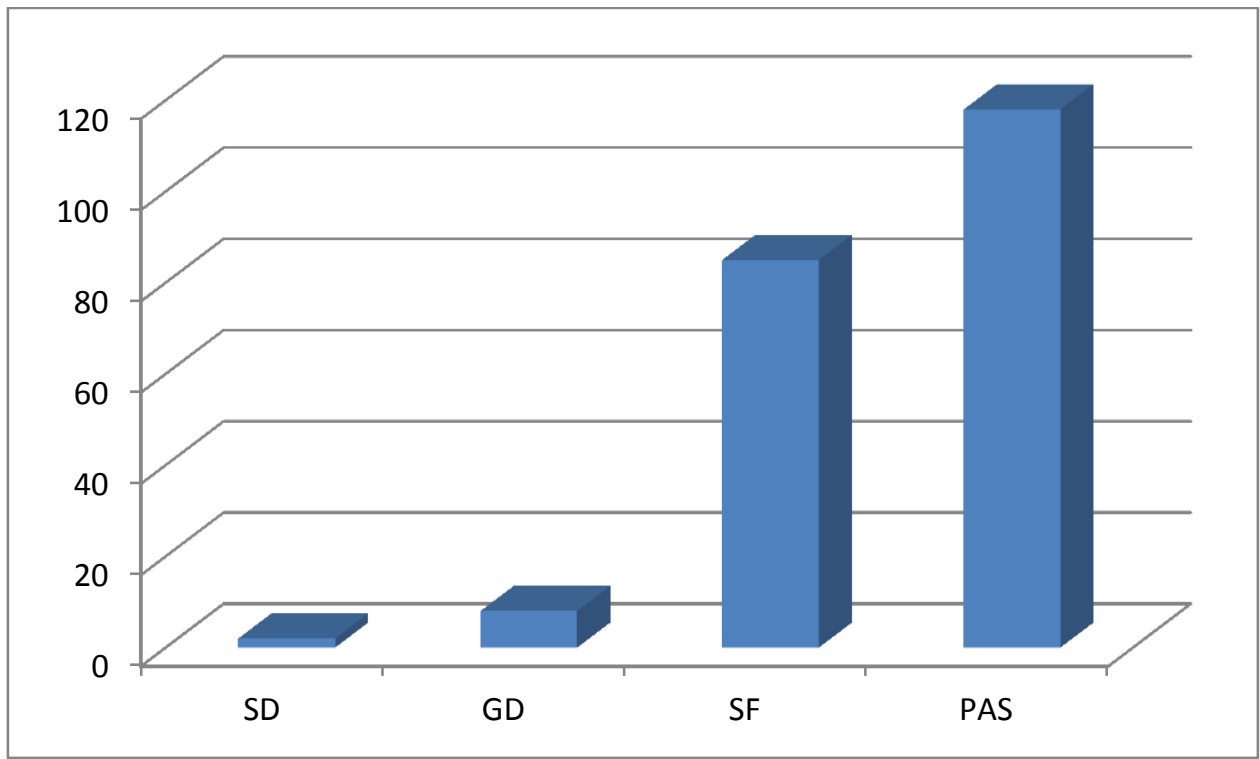

Figure 7.10: Undiagnostic vessel fragments by Depositional Context.

As Figure 7.10 makes clear, undiagnostic vessel fragments provide the starkest contrast among any category of vessels between Depositional Context in this thesis and therefore provide some of the most informative data concerning formation process. As nearly all of the vessels from Structured and Grave Deposits are complete enough to be identified, this indicates they were deposited into their archaeological context in a complete or near complete state of 
preservation. This helps to eliminate scrapping/salvaging as a likely formation process for the majority of Structured Deposits containing copper alloy vessels, as fragmentary material would be thought to be ideally suited and even explicitly selected in the formation process of such an assemblage. By contrast, the high number of undiagnostic fragments that are recorded as Site Finds or Single Finds though the PAS indicate the likelihood that these objects passed out of regular use-life and entered the archaeological record in a fragmentary state, perhaps from having been worn and damaged beyond the point of pragmatic repair or misplaced before they could be recycled into the active economy.

The defining principal behind all of the Structured Deposits in this thesis is the preferential selection of material which fits together to form integrated assemblages and the exclusion of material that the assemblers perceived as incongruous to the integrity of the assemblage as a whole. This is readily evident in the two principal patterns in form selection in Structured Deposits over time, with Handled Pan 2 s predominating earlier assemblages and large bowls and hanging basins dominating those of the Late Roman period. The general exclusion of jugs from such deposits also illustrates how vessels were specifically selected and excluded from these assemblages as jugs are well represented in all other Depositional Contexts. The regularity and predictability of the form and typological composition of these assemblages indicates the application of systematic processes of object selection, being highly indicative of ritual behaviour. This is evident in both of the principal patterns of Structured Deposits discussed in Chapter 3. The votive/ritual nature of aquatic deposition has been extensively addressed above, requiring no further explanation here than already offered in Chapter $3 .^{977}$ The consistent selection of bowls and basins for assemblages of copper alloy vessels in the Late Roman period indicates ritual behaviour in the forming of these deposits, even though there is no recognisable pattern in the landscape or evidence of sites of ritual significance to link them. That these deposits are only comprised of copper alloy vessels, generally in a good state of repair, indicates that these are not household assemblages hastily hidden from abandoned properties in the wake of some barbarian invasion, Bishop's Cannings likely being the exception which proves the rule. ${ }^{978}$ From comparison of the objects forming Structured Deposits of copper alloy vessels in this thesis, it is evident that nearly all of them are the result of conscious selection and ritual

\footnotetext{
${ }^{977}$ See also Merrifield 1987, 23-30; Bradley 1990, 1-42 \& 97-190; Dowden 2000, 39-57.

${ }^{978}$ WIL0013-WIL0022; Guest 1997, 415; Guest et al. 1997, 426-462.
} 
interring. Though some form of votive dedication would seem to be the most convenient explanation currently available to explain such deposits, ${ }^{979}$ there is not any certain way to reconstruct the ritual intent behind such deposits and the lack of regular patterning in the landscape warrants scepticism for these groups being dedicated to any deity in particular. Similarly, the symbolic intent of these assemblages is also difficult to reconstruct, though Roymans' model of domestic wealth replacing militaria in votive traditions of Western Europe with the advent of Roman hegemony offers a likely interpretation of this material, at least during the first centuries of the Roman period in Britain. ${ }^{980}$ Furthermore, the likelihood that many of these vessels were used for ablutions could have added a symbolic importance to their role in a ritual deposit, perhaps as a wider purification rite. ${ }^{981}$ The comparative scale of participation in these rituals also is hinted at by the increase both in the size of vessels and the numbers of vessels included in these deposits over the course of the Roman period, showing that a larger number of people were participating in the ritual activity associated with these vessels in the late Roman period than during the early Roman period. In any event, the important conclusion offered by the Structured Deposit material in this thesis is that there is no grounding in the old 'hoards equate to hordes' paradigm, a theory seriously challenged by recent work, ${ }^{982}$ and that these assemblages are indicative of a more complex social practice resulting from ritual constructs that developed and adapted during the course of the Roman occupation of Britain.

Though accurate reconstruction of past funerary ritual shall always prove problematic, there can be no doubt that the presence of copper alloy vessels as grave goods is the result of a planned ritual practice as opposed to any spontaneous depositional process. The range of vessels commonly found in graves is even narrower than those for Structured Deposits, comprising almost exclusively jugs and Handled Pan 1s. That these vessels feature in the dining ritual of the funeral, or their symbolic importance as objects of purification in such funerary contexts, is the most plausible conclusion and the one generally held. ${ }^{983}$ The evidence from this thesis would support this probability. It is most likely that Grave Deposits were never intended for recovery and are therefore objects that are being removed from active use-life. ${ }^{984}$ By extension, any

\footnotetext{
${ }^{979}$ Merrifield 1987, 30-40; Bradley 1990, 28-29; Dowden 2000, 175-177.

${ }^{980}$ Roymans 1996, 13-103.

${ }^{981}$ Vessel function will be discussed in further detail in Section 7.3.

${ }^{982}$ Hobbs 2006; Gerrard 2013, 59-63.

983 Nuber 1973; Cool 2006, 47.

${ }^{984}$ Johns 1996, 2.
} 
object interred within a grave is an object that the dedicator is capable of relinquishing, i.e. they are wealthy enough to absorb such a loss. Though it is possible that the use of these objects for ritual purification during a funerary ceremony could stigmatise them, making them unusable for the living and necessitating their consignment to the deceased, ${ }^{985}$ those performing the ceremony would still have to absorb this loss of material wealth and must have been willing to do so for the sake of ritual and display. This facet likely plays into the significance of the dedication of such objects to the living participants in the funerary ritual, with the objects acting as a tangible representation of the power and importance of the dedicator and their connection with the revered deceased. That such ritual dedication could only have been carried out by individuals and families of wealth may be assumed simply by the raw material value of copper alloy vessels and the fact that they occur almost exclusively as part of larger grave assemblages including ceramics and other leisure objects such as gaming counters. By extension, copper alloy vessels interred in graves are an indication of elite conspicuous consumption and serve as a means of class definition and the formulation of elite identity. Shared acts of funerary dedication show continuity and solidarity among the elite class, ${ }^{986}$ with the jug and Handled Pan 1 burials proving to be an enduring tradition linking groups of people across the Empire and even to the east coast of the Black Sea. ${ }^{987}$ The appropriation and emulation of rites from earlier periods, such as the use of barrow graves at Bartlow Hills, is further evidence of the use of funerary practice as a means of creating a sense of continuity of authority and prestige by elites during the Roman period. $^{988}$ Even the occurrence of anomalies such as those at Brougham is evidence of acts that forge social cohesion and continuity, this time among what may be considered a warrior elite emigrating to the province from the continent. ${ }^{989}$ Like Structured Deposits, the narrow selection of forms interred as grave goods indicates a symbolic value to the objects included in the ritual. More so than Structured Deposits, Grave Deposits of copper alloy vessels were utilised as a means of constructing and exhibiting cultural continuity among the elites of the province. As the centuries of Roman rule progressed, the importance of such constructs of continuity became devalued and the practice is abandoned by the Late Roman period. Instead of large grave

\footnotetext{
985 Scheid 2008, 5-8; Ochota 2013, 107.

986 Philpott 1991, 228-235.

${ }^{987}$ Nuber 1973, 210-220.

${ }^{988}$ Gage 1832, 1-23; Eckardt et al. 2009, 80-87, 91.

${ }^{989}$ Cool 2004, 463-468.
} 
assemblages associated with dining, other forms of wealth and status display were practiced, such as funerary monuments and lead coffin or plaster burials. ${ }^{990}$

Site Finds prove more problematic to characterise and interpret than either Structured Deposits or Grave Deposits simply because of the greater diversity of the objects encountered, making predictable patterning of such depositional material difficult. The processes of their deposition are certainly more challenging to reconstruct than either Structured Deposits or Grave Deposits, the likelihood being that most of these objects exited active use-life and entered the archaeological record completely by accident or neglect. As the material from this group is more randomised than that of Structured Deposits or Grave Deposits, Site Finds may be best characterised as a broad survey of the material culture available for use (and subsequent loss) by the population. This assertion is supported by the diversity of the objects themselves as well as the greater geographic and chronological range of findspots. Site Type plays an important role in interpreting Site Finds material, as the preference for certain vessel forms on certain types of sites helps to indicate the acceptance and use of these forms varied across the population. ${ }^{991}$ Some of the most noticeable patterns were the prevalence of Handled Pan $2 \mathrm{~s}$ on military sites and the prevalence of buckets and hanging vessel mounts in the rural south and Midlands. This geographic variation and the variation between Site Type indicates that different customs were in place and practiced by different groups of people inhabiting and coexisting in Britain during the Roman period.

Characterising Single Finds recorded through the PAS is perhaps most problematic of all, as the circumstances of their discovery and the highly fragmentary and undiagnostic nature of many of the objects hamper direct interpretation of individual objects. Through contextualization with other finds discovered in their vicinity, it appears that the majority of these objects derive from rural settlements. While it is possible to conjecture that some could actually represent disturbed Structured Deposits from lost and forgotten rural sites of votive significance, ${ }^{992}$ the eclectic nature of the objects comprising the PAS assemblages from these findspots makes it difficult to argue that these are not site assemblages. When viewed as a collective body of data, the PAS material is very helpful in illuminating the wider scope of circulation of this commodity in a rural context than would have been evident in the other three

\footnotetext{
${ }^{990}$ Philpott 1991, 225-332.

${ }^{991}$ This is discussed in Chapter 5 and need not be revisited here in detail.

${ }^{992}$ See Section 6.3 for full discussion.
} 
Depositional Contexts. An interpretative conundrum still exists into how this material would have been viewed and valued by those who possessed it in these rural areas, especially as certain depositional processes must remain a mystery. Nevertheless, the volume of copper alloy vessel material, even though it is certainly not nearly as abundant as other Roman objects reported through the PAS, ${ }^{993}$ indicates an availability which suggests a relatively high degree of accessibility to copper alloy vessels by a wide segment of the rural population of Britain during the Roman period.

The investigation of material by Depositional Context was an integral part of the research process of this thesis. While Chapters 3-6 were able to offer conclusions on the place of copper alloy vessels in cultural practice based upon comparison within a single Depositional Context, this section illustrates the cumulative value of this approach by the synthesis of data across Depositional Contexts in order to better characterise and understand how they affect our understanding of the archaeological data. The variances of vessel forms deposited between Depositional Contexts relate both to the availability of the objects in question and to culture practices undertaken by different groups of people. This has direct bearing on the application of copper alloy vessel material to the broader arguments of theory and approach to the study of the Roman past in Britain as it is through variances in the archaeological record that variances in the construction and application of identity, as well as cultural change and adaptation, may be characterised. The following sections will continue to assimilate data from across Depositional Contexts to further analyse patterns and discrepancies that prove informative to the development of culture practice during the Roman period as well as the developing role of these objects within Romano-British society.

\subsection{What is the distribution of copper alloy vessels across time and space?}

While earlier chapters discussed the geographic and temporal distribution of copper alloy vessel material in Roman Britain divided within each Depositional Context, the current section will synthesise this material into a unified discussion of the overall distribution patterns. The arguments will be based on patterns discernible across periods, regions and site types. This patterning will then be considered in relation to the cultural and economic mechanisms by which copper alloy vessels were distributed in Britain during the Roman period. The first part of this

\footnotetext{
${ }^{993}$ Worrell \& Pearce 2013, 345.
} 
section will focus on broad geographic patterning, examining simply how widely distributed copper alloy vessel material was across the province. This will be followed by an examination of how distribution across space and Site Type varies chronologically.

As Figure 7.11 shows, there is considerable geographic variation in the amount of copper alloy vessel material dating to the Roman period across England and Wales. Not surprisingly, the counties of East Anglia see the highest concentration of material at 170 objects. What is perhaps more surprising is that comparable figures may be found in the Midlands (134 objects) and the North (139). These areas are generally believed to be far less agriculturally cultivated or urbanised (and, by extension, perhaps less influenced by Roman material culture) than the south east due to topographic characteristics which influenced the accessibility of some of these areas for agricultural development and trade as well as possible cultural factors which influenced settlement patterns. ${ }^{994}$ Additionally, such vessels are also well attested beyond the Roman frontier in Scotland, ${ }^{995}$ indicating that urban population density and Roman administration were not inherently essential to the distribution of this material.

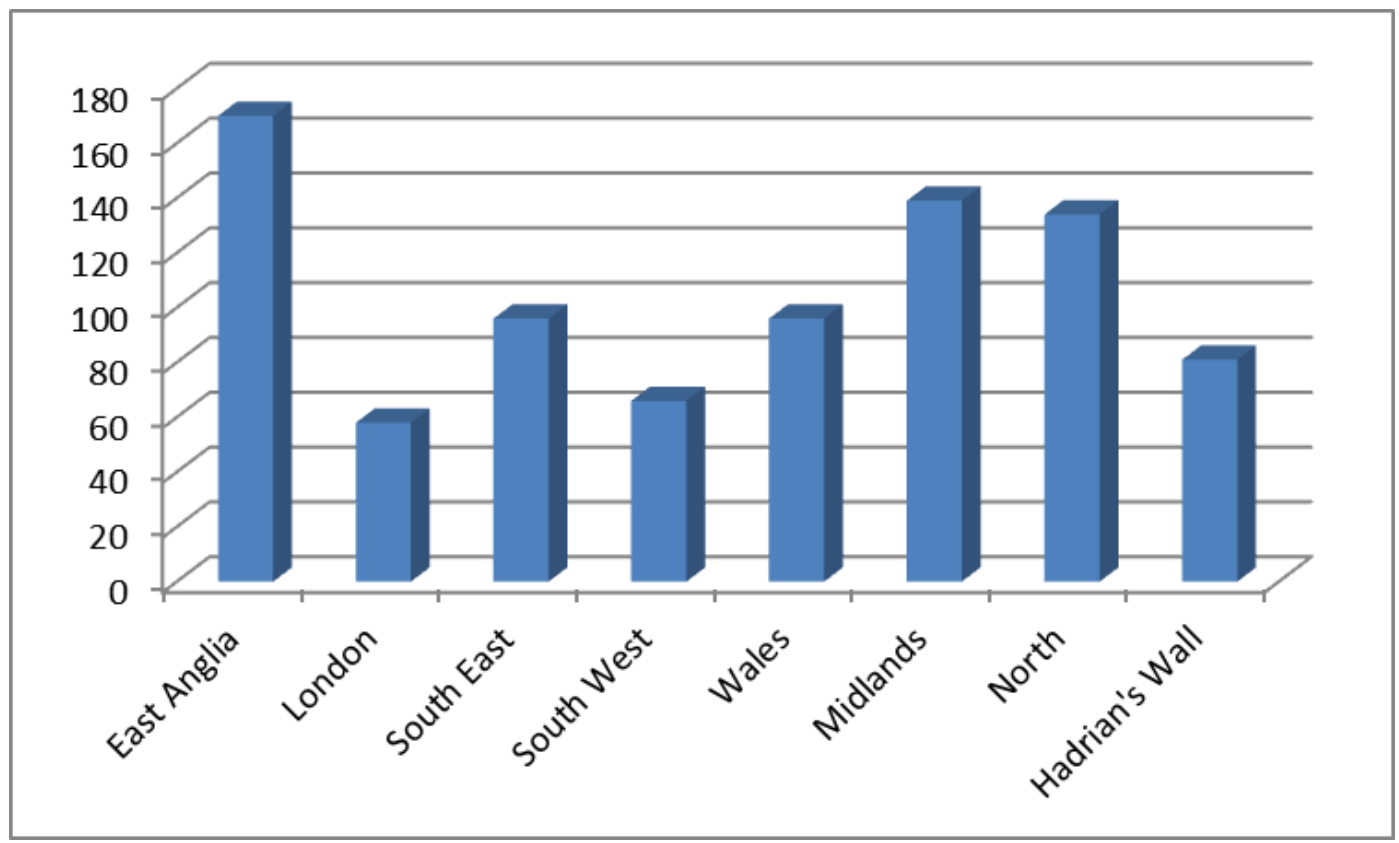

Figure 7.11: Broad distribution of copper alloy vessels by geographic region.

\footnotetext{
${ }^{994}$ Mattingly2006, 379-427; Gerrard 2013, 208-244.

995 Eggers 1968, 100-110; Hunter 2001, 100-110; Hunter 2012, 85-106.
} 
The number of objects from London (58) is significantly higher than the figures for any other urban centre. London's place of primacy in the province, seemingly the largest city for most of the Roman period, ${ }^{996}$ is one factor likely influencing the numbers. Also important is the level of archaeological attention and publication London receives, which may have had an effect on the comparative frequency of this material.

Another useful means of conceptualizing the geographic distribution of this material is by grouping the finds in an east-to-west progression, as is clearly visible by reviewing Maps 1-4. The stark contrast of the distribution of material between east and west demands explanation, which is most immediately offered by settlement patterning and the distribution of the population in Britain during the Roman period. Most of the principal urban centres of the Roman period (including London, Colchester and York) are in eastern England as is the densely populated countryside of East Anglia. The geography of Britain plays some part in this as well, as the Pennines cutting through the centre of Britain has made habitation of these central areas historically sparse in comparison to both the east and west. However, the countryside of western Britain was far from unoccupied ${ }^{997}$ and it is unlikely that variances in population density between east and west in Britain alone would produce such a stark contrast in the distribution of copper alloy vessels. It is likely that avenues of exchange, both economic and cultural, account for the great discrepancy of objects. For example, it is understood that in antiquity transport by road was far more expensive than transport by river, which in turn was far more expensive than transport by sea. ${ }^{998}$ This would have had a very direct impact on the goods available and their quantity in places with differing access to these trade routes, which were inevitably coastal. Though a detailed study of the economic mechanics and distribution networks of copper alloy vessels is beyond the scope of this thesis, it may be assumed that they relate closely to other distribution networks at play during this time between Britain and the continent and may be most relatable to commodities such samianware, whose distribution hubs for the province seem to have been often (though not exclusively) located in the east of England. ${ }^{999}$ The road networks also seem to have played an important role in the distribution and availability of this material (Maps 1-3). It is particularly noteworthy that PAS distribution, illustrated in Map 4, also appears

\footnotetext{
${ }^{996}$ Hassall 1996, 19-26.

${ }^{997}$ Leech 1982, 209-268; Millett 1990, 123-280.

${ }^{998}$ Finley 1999, 126-129.

${ }^{999}$ Willis 2011, 167-242; King 2013, 110-134.
} 
to be heavily influenced by the road network as the method by which these objects are discovered would direct this data away from known settlement sites associated with these road networks. In any event, the mechanics of trade was likely the fundamental cause of the heavier distribution of copper alloy vessels in the east of England in comparison to the west, with population density and cultural preferences in object selection playing subsidiary roles.

The geographic distribution of copper alloy vessels indicates there was variable access and use of these objects during the Roman period, which may be best characterised by the notion of discrepant or divergent experience. ${ }^{1000}$ To understand how these different experiences and cultural choices may be characterised, it is important now to turn to an examination of distribution across Site Type. It is important to note that geographic patterning and Site Type patterning are inter-related, as there is regional variation in the distribution of Site Types across Britain, as Maps 5-8 illustrate. For example, the influence of the Roman military on the material culture of Wales and the hinterland of Hadrian's Wall is well documented. ${ }^{1001}$ This in turn affects the types of settlements and the archaeological work which has been done in these regions, with eight of the fourteen sites from Northumberland being classified as Military Site Types. Alternatively, most of the sites classified as Urban Site Types are in the southern and eastern portions of the province. The distribution of finds across Site Type is nonetheless helpful in visualising how copper alloy vessels would have featured within the material culture of these different types of settlements (Figure 7.12).

The distribution bias towards rural environments is somewhat surprising as urban and military centres have received significantly more scholarly attention and publication, which would theoretically lead to a comparatively greater representation of material from such sites. The numbers from Rural Unknown sites are greatly bolstered by Structured Deposits, which are often found in remote places with no overt evidence of settlement during the Roman period. PAS finds from Rural Unknown sites further complicate how to interpret such distribution patterns as such finds could originate from disturbed Structured or Grave Deposits, though they more likely are indicative of an as-yet unrecognised rural settlement. ${ }^{1002}$ It should be remembered

\footnotetext{
${ }^{1000}$ Mattingly 2011, 205-236.

${ }^{1001}$ Breeze \& Dobson 1987; Arnold \& Davies 2000, 45-64; Manning 2001, 8-48; Bidwell \& Hodson 2009.

${ }^{1002}$ Brindle 2010, 128-132; Moorhead 2010b, 157; Brindle 2011, 61-64 \& 345-372; Walton 2012, 15-16; Moorhead 2013, 3-7.
} 


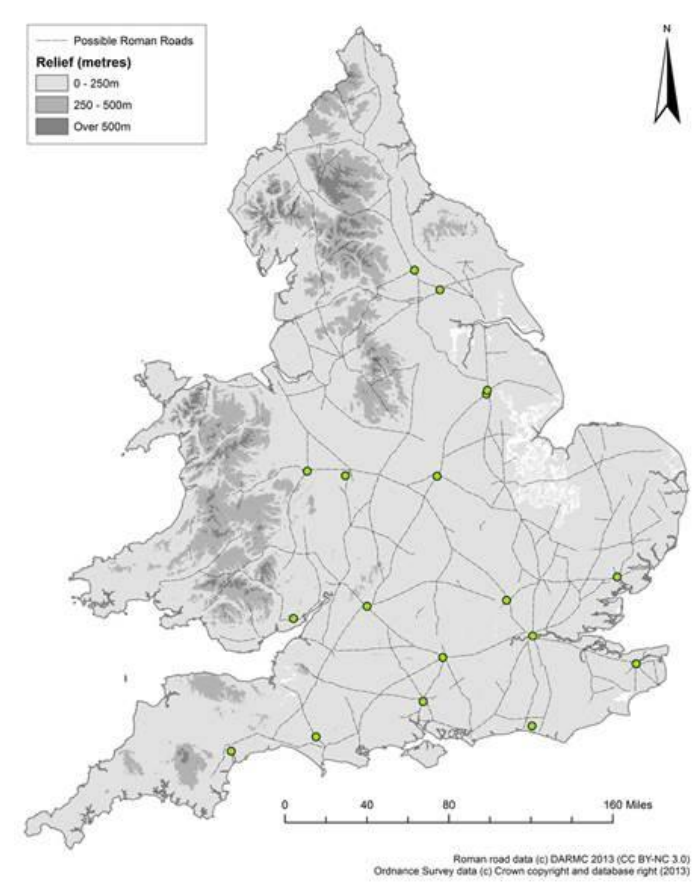

Map 5: Urban sites with $\mathrm{AE}$ vessels.

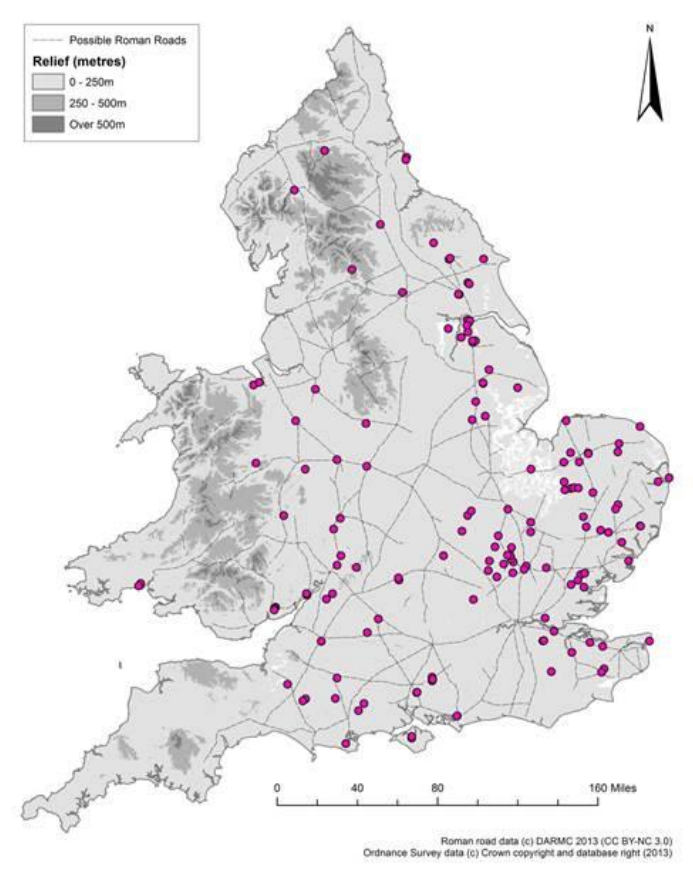

Map 6: Rural Settlement sites with $\mathrm{AE}$ vessels.

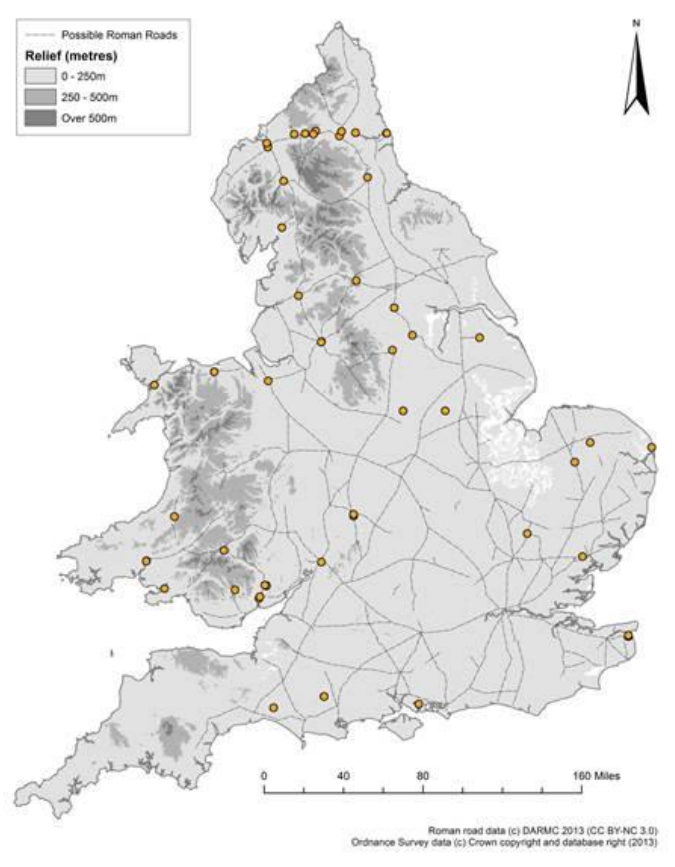

Map 6: Military sites with $A E$ vessels.

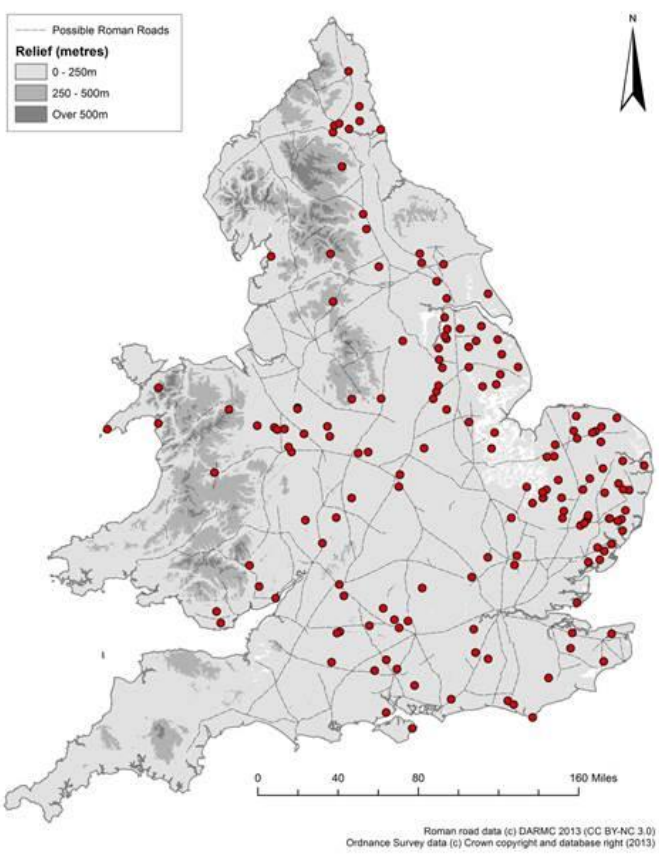

Map 7: Rural Unknown sites with $A E$ vessels. 
that Rural Unknown sites are locations where there is no architectural evidence extant to indicate habitation during the Roman period, but this is certainly not to say that such settlement could not have been present. Many settlements and structures made of wood or other organic materials would very easily have disappeared from the landscape over the past millennia and a half. Richard Hingley has perhaps best expressed the reality of settlement in antiquity, and the difficulty in defining it, when stating, '(during the Roman period) the vast majority of people lived in types of settlements that do not fall easily into the main categories (city, villa, fort, etc.) that have dominated archaeological research. ${ }^{1003}$ As the majority of the population of Britain would have lived and worked in the countryside, ${ }^{1004}$ it could be expected that greater numbers of vessels should be found in rural settings. This statement supposes, however, that the rural inhabitants of Britain would have had access to the same manufactured commodities as urban inhabitants, a statement which is highly problematic as rural and town economies are likely to have had a highly complex and often divergent relationship during the Roman period. ${ }^{1005}$ Nevertheless, the comparatively high numbers of objects from rural sites do show a significant accessibility to this material in the countryside, reflective of its dynamic integration within the wider social fabric of Britain during the Roman period. ${ }^{1006}$

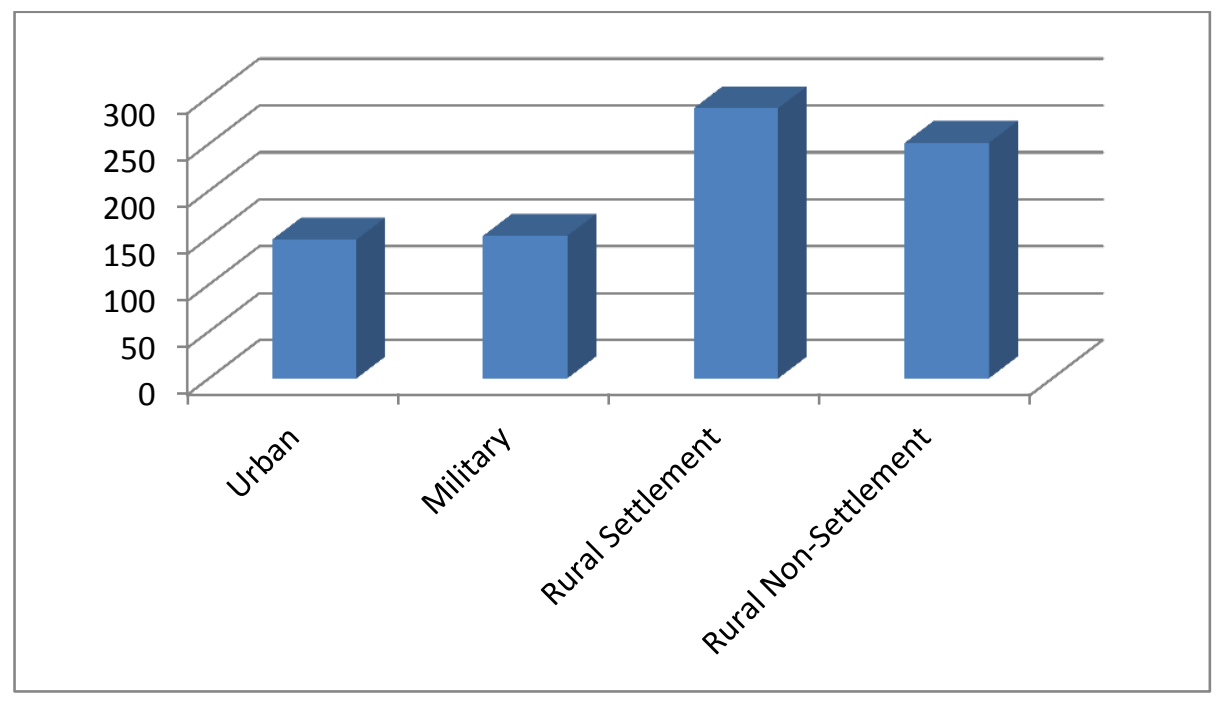

Figure 7.12: Numbers of copper alloy vessels by Site Type.

\footnotetext{
${ }^{1003}$ Hingley 2005, 93.

1004 Laing 2000, 93; Mattingly 2006, 356 \& 453; Gerrard 2013, 55.

1005 Mattingly 2006, 255-490; Gerrard 2013, 86-100.

${ }^{1006}$ Hingley 1982, 17-27.
} 
As this study encompasses some three and a half centuries of Roman rule in Britain, temporal variation in distribution patterns are only to be expected. This is particularly the case as there is clear chronological patterning in different Depositional Contexts resulting from changing social practice over time. Chronology is also important to keep in mind as the cultural perspectives and experiences of the inhabitants of the province would have undoubtedly changed over so many generations. This would have inevitably resulted in differing culture practice and how this may be expressed through the use and archaeological distribution of copper alloy vessels.

Divergent patterns in the chronology of the deposition and distribution of copper alloy vessel material is clearly evident across Depositional Contexts. Structured Deposits sandwich the Roman period, with aquatic votives of small bowls and Handled Pan 2s characterising the early Roman period and large Late Roman vessel forms predominating the last century of the Roman occupation. Grave deposition of copper alloy vessels was practiced by a minority elite in southern Britain from the Late Iron Age through the first century and a half of the Roman period before declining in the following centuries, but was never a wide spread nor ubiquitous practice. Site Finds and PAS Single Finds are most visible in the $2^{\text {nd }}$ and $3^{\text {rd }}$ centuries, precisely the time period which is obscure in the Structured Deposits and Grave Deposits data-sets. This reflects on the availability and consumption patterns of the vessels, which will be discussed in further detail in Section 7.3 and Chapter 8.

Differing patterns in the forms visible is also evident across time and indicates changing culture practice (Figure 7.13). Handled pans are significantly more visible during the first two centuries of Roman rule as opposed to the final century and a half. Buckets and jars are most prevalent during the $2^{\text {nd }}$ and $3^{\text {rd }}$ centuries across Depositional Contexts, though on the whole remain far less visible than basins or handled pans. Large cauldrons and basins such as Irchester type bowls appear in the $3^{\text {rd }}$ century and characterise Late Roman deposits of copper alloy vessels. This chronological variation clearly illustrates the development and adaptation of vessel use over time as fashions and customs emerge and subsequently fall out of practice. The principal shift in the forms of vessels during the Roman period is away from smaller sized vessels in the early Roman period to larger vessels more suitable for convivial events involving relatively large numbers of people during Late Antiquity. It is important to note that this 
movement from smaller to larger forms over the course of the Roman period has been noted in other vessel repertoires during the Roman period, such as glassware ${ }^{1007}$ and ceramics ${ }^{1008}$ in Roman Britain as well as in silver and ceramic vessel assemblages from the Eastern Empire. ${ }^{1009}$ This is particularly noteworthy as a shift from larger to small vessel forms marks the transition from the Late Iron Age to the Roman period in Britain, ${ }^{1010}$ implying that dining and handwashing practice in Roman Britain was particular and removed both from what came before and what came after. A move away from small vessels such as Handled Pan 1s \& 2 s in favour of larger buckets and basins would indicate more importance being placed on the communal aspect of vessel use as opposed to personal use and ownership. That larger vessel forms continue to dominate in the Anglo-Saxon period ${ }^{1011}$ is also symptomatic of there being a gradual, long term shift within British society in preference for group use of this property.

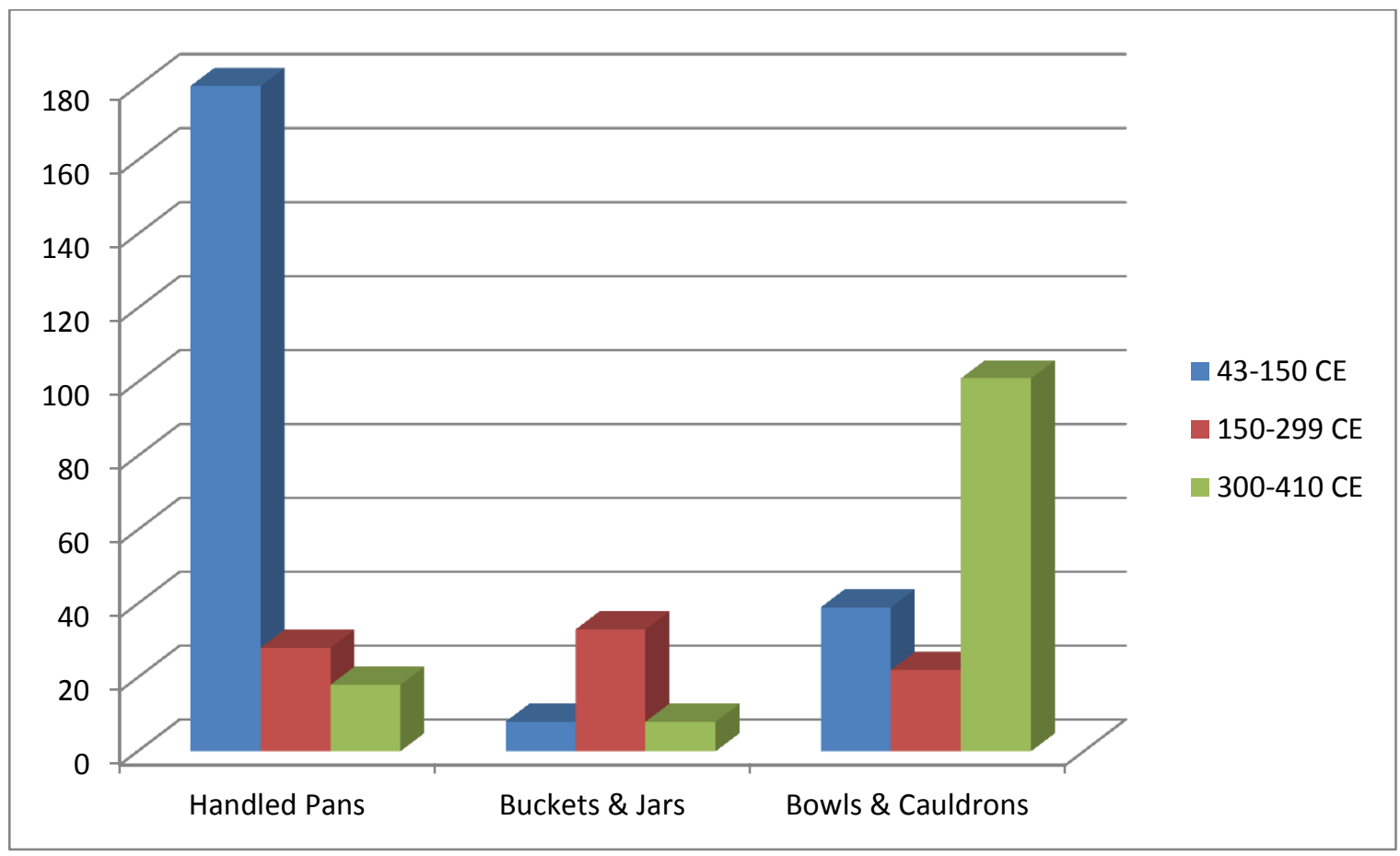

Figure 7.13: Chronological distribution of copper alloy vessel forms in Roman Britain.

${ }^{1007}$ Cool 2006, 223-225; Price 2010, 37-49.

${ }_{1008}$ Cool 2006, 227-235; Gerrard 2013, 87-89.

${ }^{1009}$ Hudson 2010, 672-693.

${ }^{1010}$ Cool 2006, 54.

${ }^{1011}$ Kennett 1971 138-145; Bruce-Mitford \& Raven 2005. 
The fact that copper alloy vessels were adapted to the varying cultural needs of the peoples inhabiting Britain is shown by variations in the vessel forms present across different Depositional Contexts, Site Types as well as geographic and temporal distance. These variations reflect differing tastes and cultural necessities which dictated the functional application of these objects to the culture practice of any particular group. It is now necessary to examine how these vessels were viewed as objects of functionality as well as cultural consumption and display.

\subsection{How were copper alloy vessels used and consumed?}

'It is through the processes of daily life that identities are formed, and it is through the mundane generalities of day-to-day life that individuals are inculcated with the structures and strategies that form their society. ${ }^{, 1012}$

The functional use of objects is an important part in the construction and practice of identity; the objects one uses, how they are used and integrated with other objects and how they are displayed and shared all associate the user with a set of socio-cultural norms and practices which serve to identify who they are and where they stand in regards to their surrounding community. ${ }^{1013}$ There are frustratingly few representations of copper alloy vessels among the decorative arts from the Roman world. A survey of representations of such vessels among the wall paintings of Pompeii and Herculaneum ${ }^{1014}$ proving unhelpful in discerning the function these vessels were intended to perform while the majority of instances where copper alloy vessels are shown in use apply specifically to the use of jugs and Handled Pan 1s for ablutions. ${ }^{1015}$ The following section will discuss copper alloy vessels as commodities of functionality and cultural exchange, relating this to their distribution patterns across Depositional Contexts and Site Types. This will reveal patterns in consumption that relate to the perceived value of these objects as commodities of practical and cultural significance during the Roman period. The diverse economic mechanisms at play during the Roman period (such as free market exchange, gift giving, reciprocity, coerced material redistribution, etc.), ${ }^{1016}$ due in large part to the divergent social experiences of the many different peoples across the provinces of the

\footnotetext{
1012 Petts 1998, 79.

${ }_{1013}$ Lyons \& Papadopoulos 2002, 8.

${ }^{1014}$ Riz 1990.

1015 Nuber 1973; Chapter 4.

${ }^{1016}$ Finley 1999; Harris 2011, 257-313; Hobson 2014, 11-26; Jongman 2014, 27-36.
} 
empire ${ }^{1017}$ prohibits the application of this data-set to anything approaching a unified economic discussion (at least in the time and space allowed for this thesis). Instead, copper alloy vessels will be viewed in their cultural environment as objects of use and consumption.

Before continuing the discussion, it is best to clarify the terminology and theoretical approach which defines the argument. The term 'commodities' is understood here in its social and anthropological sense, which is perhaps best defined as 'a socially desirable 'thing' which has an intrinsic, culturally defined use-value and with an exchange-value that potentially allows its owners to obtain other things'. ${ }^{1018}$ The objects in this thesis are here reviewed as objects of cultural capital, utility and exchange that are awarded value and desirability principally for their application within a wider social matrix. It is justified to view copper alloy vessels in Roman Britain from this perspective due to the patterns in deposition relating to social display as well as the nature of the design and decoration of the objects in this thesis which indicates their use for display in social rituals, similar arguments are used in the characterisation of decorated ceramic fineware in Roman Britain. ${ }^{1019}$ These two aspects relating to how the consumption of copper alloy vessels may be perceived in Britain during the Roman period are discussed below.

It must be remembered that it is problematic to make direct or empirical connections between where an object was found and the identity of who may have used the object or the purpose to which it may have been applied in its pre-deposition use-life. Military Sites are a good case in point, as it is very likely that many people besides military personnel would have passed through or lived around Roman military installations. ${ }^{1020}$ These people could have included merchants, local farmers and herdsman coming to pay taxes, prisoners/slaves, wives and children of the soldiers, ${ }^{1021}$ or officials of the Roman civil administration just to name a few examples of the variety of people who may have lived or worked at a Roman military installation in Britain at one point or another. ${ }^{1022}$ This obviously complicates the process of characterising identity through analysis of Site Type. However, prevalent patterns of deposition discernible within a Site Type are still representative of a common and repeated use of material at this site, indicative of shared culture practice and relatable ideas of cultural identity. Illustration and short

\footnotetext{
${ }^{1017}$ Mattingly 2006, 491-528.

1018 Panagopoulou 2007, 315.

1019 King 2013.

${ }^{1020}$ Collins 2008, 49.

${ }^{1021}$ Birley 2002, 121-156.

1022 Mattingly 2006, 170-176.
} 
discussion of the distribution of forms within each of the Site Types used in this thesis will be offered presently, followed by a synthetic discussion of the material comparatively across Site Types.

As Figure 7.14 illustrates, jugs are the most commonly found diagnostic vessel found at urban sites. As mentioned earlier in this chapter, ${ }^{1023}$ the visibility of jugs in the data-set may be increased due to the diagnostic character of jug handle. Even so, the prevalence of this form across urban sites reflects that these functional vessels were well integrated into the cultural practice of urban dwellers during the Roman period, even though cheaper alternatives would certainly have been available in ceramic and wood. The use of copper alloy as opposed to ceramic shows a self-conscious desire for wealth display that goes beyond pragmatic functionality of the vessel itself, indicating that urban dwellers would have taken part in such display ritual and appreciated such objects both as signs of wealth and as objects of aesthetic and artistic quality. Bowls and Handled Pan 2s are also well represented in this data-group, with 22 and 23 examples respectively, evidencing their relatively wide availability and acceptance as well. One of the most interesting aspects of this group of data is what is absent, namely significant numbers of other vessel forms, which will be discussed in more detail after reviewing the distribution of forms across the other three Site Types.

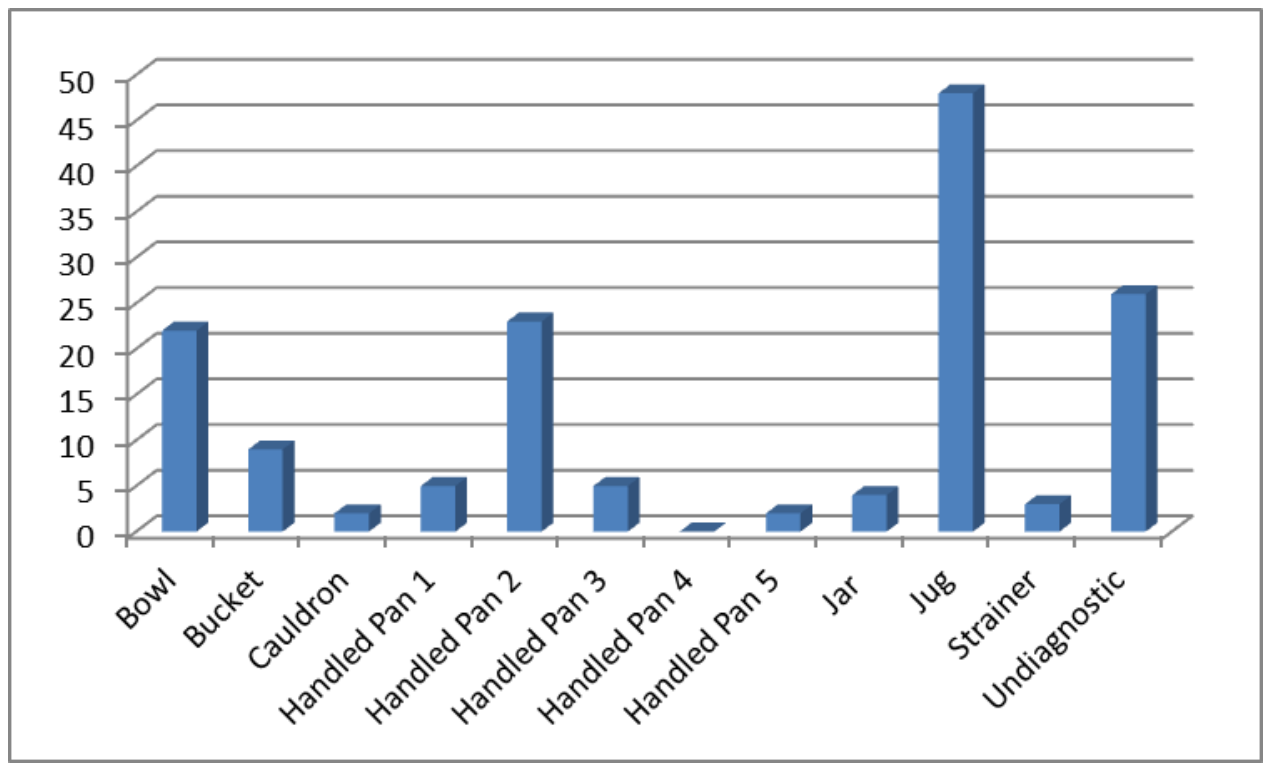

Figure 7.14: Numerical distributions of copper alloy vessels by form in Urban Site Types.

${ }^{1023}$ Section 7.1 . 


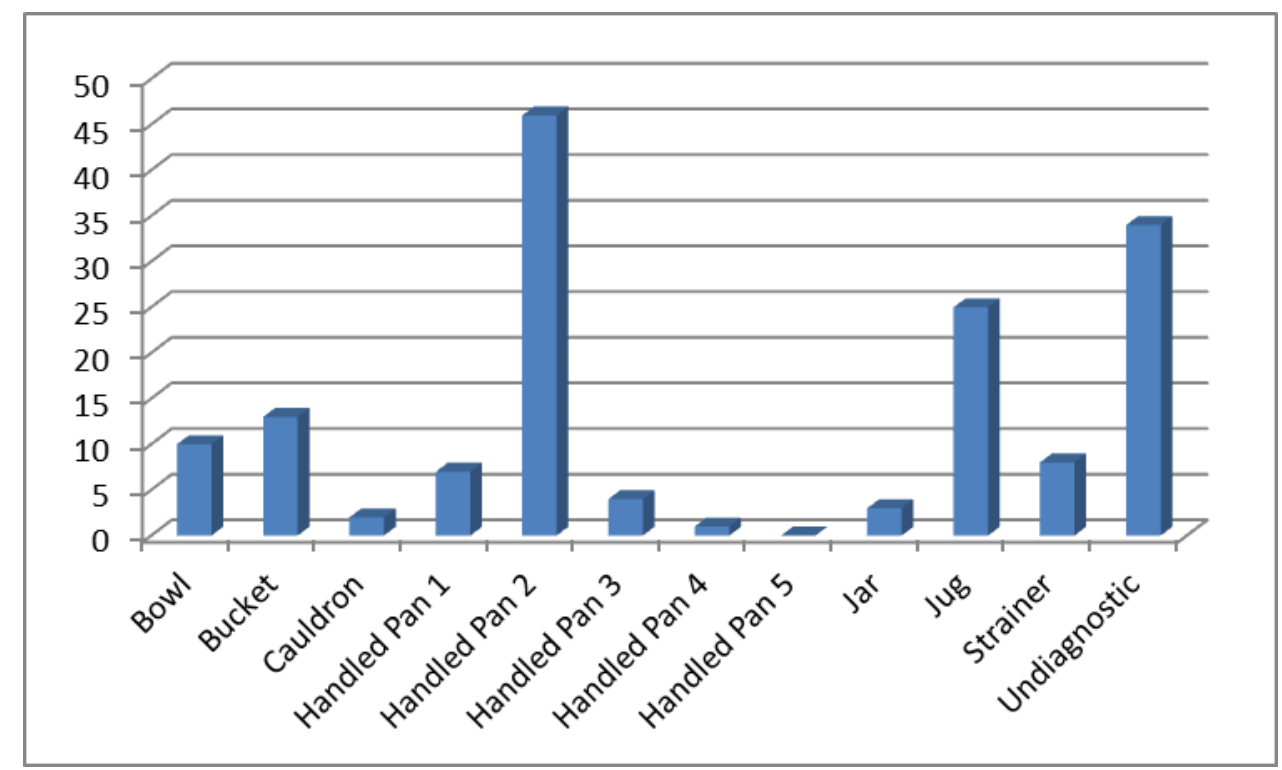

Figure 7.15: Numerical distributions of copper alloy vessels by form in Military Site Types.

Handled Pan 2s characterise the assemblage at Military Site Types with 46 examples (Figure 7.15). Jugs come in a far second at 25 examples recorded. It is not surprising that military sites may contain a narrow range of objects as the uniformity of kit would suggest that most of the soldiery would have utilised the same forms and objects in the daily practice of their lives. ${ }^{1024}$ It is worth noting also that several Handled Pan 2 s have been found beyond the northern frontier of Britain in Scotland, ${ }^{1025}$ likely symptomatic either of Roman incursions or trading between military personnel and local tribesmen. The low numbers of buckets in this data-set is surprising when it is considered that Hemmoor buckets were particularly prevalent in the military cremation cemetery at Brougham. ${ }^{1026}$

There is a greater level of diversity among vessel forms in Rural Settlement Site Types than is visible in either of the previous categories (Figure 7.16). It is somewhat surprising that this Site Type would have a significantly more diverse assemblage than urban settlements, as it might be suspected that urban centres would have had a greater fluidity of peoples and, by extension, cultural practices. In turn, this reflects the diverse character of the rural occupation during the Roman period caused not only by different varieties of settlement such as farmsteads,

\footnotetext{
${ }^{1024}$ Bishop \& Coulston 1993, 2-40 \& 233-265.

${ }^{1025}$ Eggers 1968, 109-110; Hunter 2001, 298-304.

${ }^{1026}$ Cool 2004, 374-379.
} 
shrines etc. but also reflects a diversity of peoples of different cultural backgrounds and economic standing. ${ }^{1027}$ This being said, the three most prevalent forms are the same as those from urban settings (namely jugs, bowls and Handled Pan 2s), though the numerical discrepancy between them is far less significant. The presence of other vessel forms that are rare in urban or military settings is noteworthy. The higher numbers of Handled Pan 1s are explained by their presence among grave furniture in rural settings as opposed to urban grave yards such as those around London. ${ }^{1028}$ This discrepancy in practice shows a distinct appreciation for the social and geographic setting of culture practice and that ritual behaviour was very much tied to the space and area within which it was performed. The high numbers of bowls is also symptomatic of this, as they were common inclusions in rural Structured Deposits throughout the Roman period.

As can be seen in Figure 7.17, the profile of vessel forms in Rural Unknown contexts is surprisingly uniform as this category may have been anticipated to include a diverse group of settlements. The evidence from the distribution of forms refutes such an assumption. The dominance of Handled Pan 2s in the data-set is particularly surprising, as this vessel form has been shown to have a close association with military sites. The high visibility of this vessel form in unknown rural contexts reflects the integration of these 'Roman' forms into the cultural materiality of the countryside, perhaps through the agency of settled veterans or through trade and exchange mechanisms. Alternatively, some of this material may have been deposited by troops on the move who lost their kit in transit, though it seems unlikely that this variable would have produced such a prevalence of this vessel form in remote rural locations. The relatively low numbers of jugs compared to other Site Types is also worth comment, as it suggests that the display purpose that these vessels would have served did not have as much influence on the culture practice of the removed countryside as it did for urban centres, military centres and rural settlements such as villas and rural shrines. ${ }^{1029}$ Nonetheless, significant numbers of vessels from Rural Unknown Site Types is indicative of the integration of this commodity into the lives of the rural inhabitants of Britain. This increase in the consumption of material culture by the rural

\footnotetext{
${ }^{1027}$ Mattingly 2006, 379-427.

${ }^{1028}$ Chapter 4.

${ }^{1029}$ As mentioned in Section 7.2.
} 
inhabitants of Britain is reflected in other commodities as well, such as toilet instruments, ${ }^{1030}$ ceramics and coins. ${ }^{1031}$

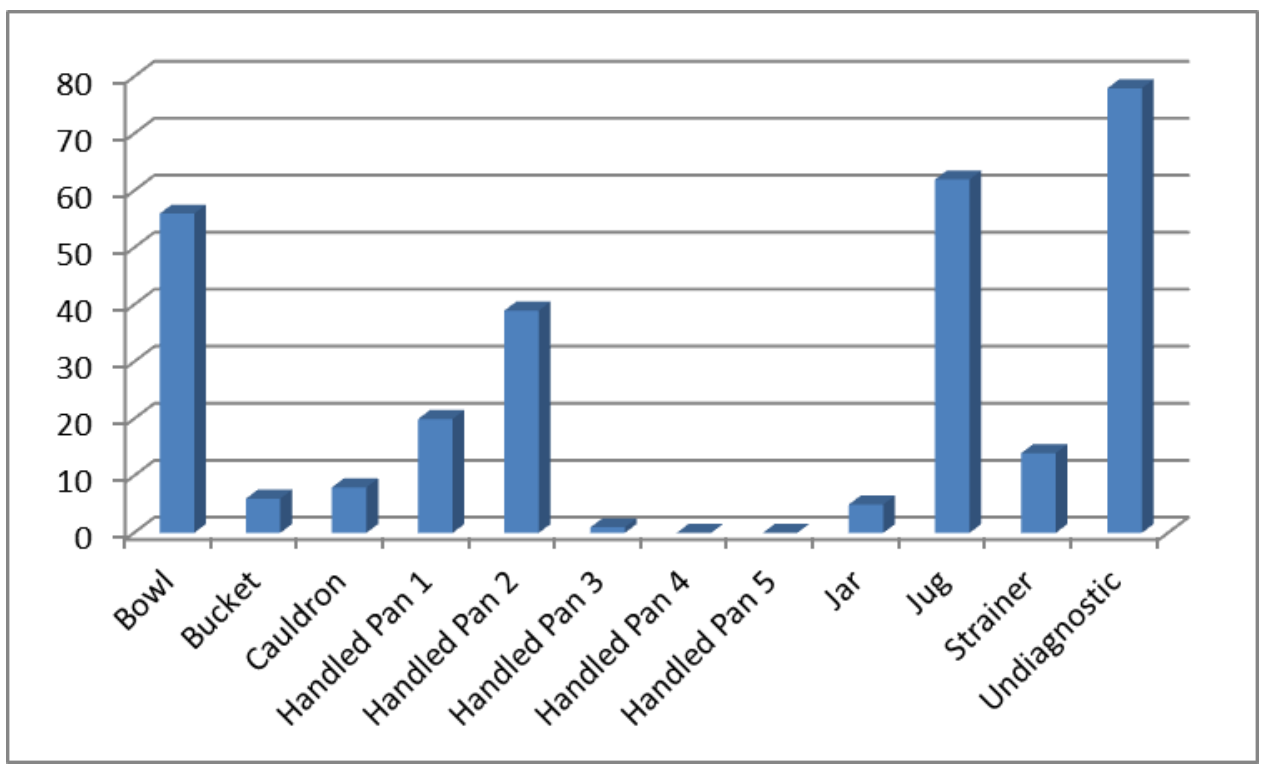

Figure 7.16: Numerical distributions of copper alloy vessels by form in Rural Settlement Site Types.

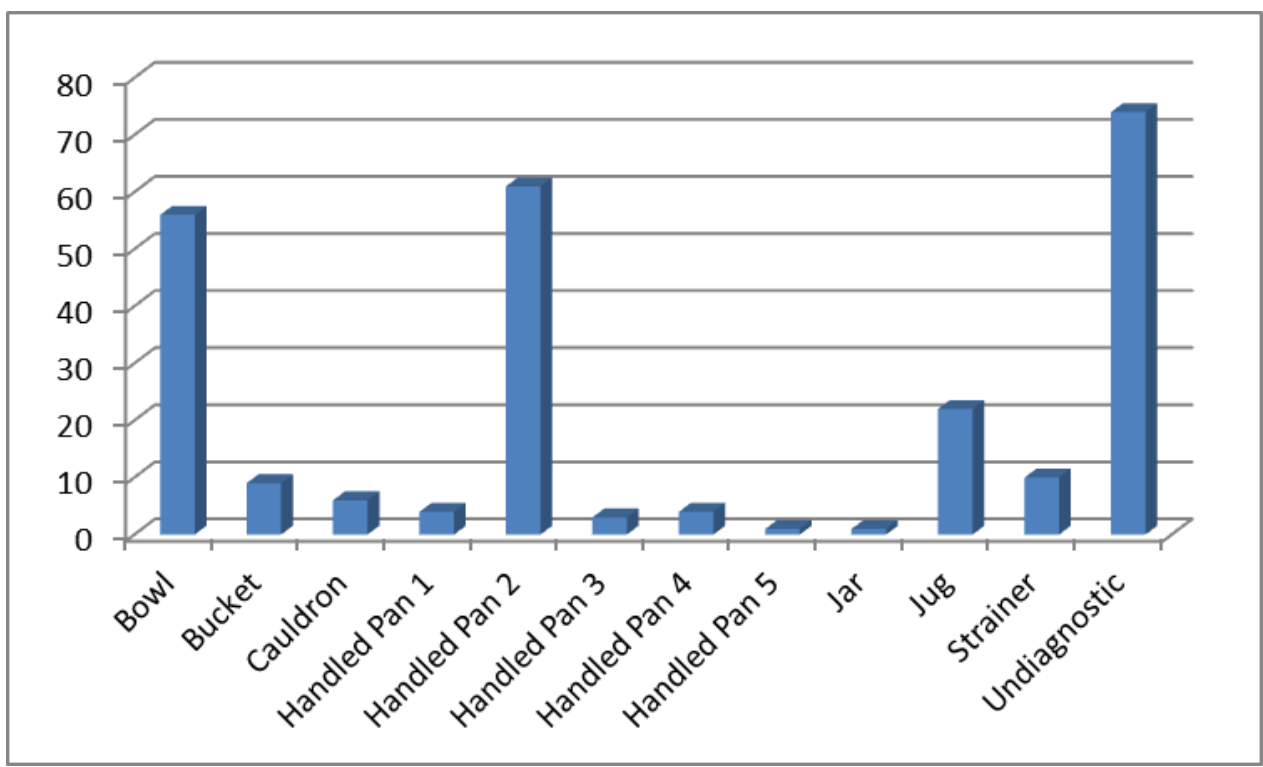

Figure 7.17: Numerical distributions of copper alloy vessels by form in Rural Unknown Site Types.

${ }^{1030}$ Eckardt \& Crummy 2008, 12 \& 117.

${ }^{1031}$ King 2013; Gerrard 2013, 86-94. 
One of the principal facts that can be seen from the distribution of vessel forms across Site Types is the common availability of bowls, Handled Pan $2 \mathrm{~s}$ and jugs. This implies both the relative availability of these forms compared to others as well as reflecting a fairly uniform application of these vessels to the functional process of display culture. There was not, however, uniformity of practice across the province. Whilst this was shown by the distribution of material across Depositional Contexts ${ }^{1032}$ as well as broad geographic distribution, ${ }^{1033}$ the variance in forms across Site Type is useful for conceptualizing the material expression of identity in Britain during the Roman period. It is perhaps most surprising that other vessels forms never rose to a high prominence in any of the Site Types, though fluctuations they show across Depositional Contexts and geography do indicate differing material practice and consumption patterns at play between different peoples in Britain during this time.

While it is obvious that there were differing practices at play in Britain during the Roman period in regards to the use and consumption of copper alloy vessels, the question remains as to how individual vessel forms fit into and were adapted by people within the British provinces. The function of Handled Pan 1s in ritual ablutions was discussed in some detail earlier in this thesis, as it was necessary to do so to characterise grave ritual. ${ }^{1034}$ The use of this vessel for ablutions is well attested by its pairing with jugs in the archaeological record as well as by artistic representation of this vessel in use. ${ }^{1035}$ That these vessels fall out of use during the $2^{\text {nd }}$ century CE is likely to be unreflective of a decline in the practice of ablutions, but instead indicates the rise of other vessel forms in the repertoire to fill the gap. Though buckets may well have served a role in the mixing of alcoholic beverages, ${ }^{1036}$ it is probable that such functionally useful vessels could serve multiple functions. If buckets could also serve for ablutions, and by extension ritual purification, this could help to explain their significance in the grave assemblages at Brougham in the $2^{\text {nd }}$ century when Handled Pan $1 \mathrm{~s}$ are seen falling out of use. It is most likely that the larger basins that characterise late antique assemblages such as Irchester type bowls and Perlrandbecken bowls also filled this necessity. ${ }^{1037}$ Particularly informative for the function of such basins in the Anglo-Saxon period is the hanging bowl found with the Sutton

\footnotetext{
1032 Section 7.1 .

1033 Section 7.2.

1034 Sections 4.3-4.5; Section 7.1.

1035 Nuber 1973; Cool 2006, 47-48; Haris \& Henig 2010; See Sections 4.3 and 4.4.

1036 Cool 2006, 136-140.

${ }^{1037}$ Harris \& Henig 2010.
} 
Hoo Anglo-Saxon burial where a statuette of a fish is placed on a pedestal in the centre of the bowl with seals or otters acting as the hanging mounts, ${ }^{1038}$ indicating that in all likelihood that this vessel was intended to contain water. Indeed, it is likely from contextualised examples in Pompeii that large copper alloy basins shared ablution functions with the Handled Pan 1s at least as early as the $1^{\text {st }}$ century $\mathrm{CE}^{1039}$ and that the Handled Pan 1 falling out of use at the same time that other basins became more prominent indicates a changing of fashion as opposed to a disappearance of ablutions from ritual practice.

While it seems certain that the principal use of copper alloy vessels was for ablutions at dining and other ritual events, there are some noteworthy examples where vessels are likely to have been used for the preparation or serving of food themselves. The principal example of this is the cauldron, which seems to have been used for the preparation of meats and stews. ${ }^{1040}$ There is a long artistic tradition of these vessels being depicted as part of the cooking process of meat dating back to the Bronze and Iron Age Near East ${ }^{1041}$ and their place within folk tradition in Western Europe makes the association between cauldrons and cooking very clear. ${ }^{1042}$ The presence of lipids in the Late Iron Age cauldrons from Chiseldon cauldrons offers an example of this same practice in Britain. ${ }^{1043}$ These vessels need not have been single purpose, however, and it is likely that they also served as containers for large amounts of water for use in multiple functions. Indeed, these large vessels could be heated relatively easily, purifying water for purposes of cooking, cleaning or bathing.

Jugs seem to have fulfilled functions both as vessels for ablutions and as service vessels at the table, particularly for wine. Cool suggests that the plainer jug types were used for dispensing water (sometimes heated), whilst more elaborate vessels, such as the trefoil Eggers type 125 jugs, were used for wine. ${ }^{1044}$ While her theory is sound for the use of plainer jugs for water at the dining table based on calcified deposits found in some examples, the presence of elaborate jugs of trefoil shape or with elaborate handles in graves paired with Handled Pan 1s as ablution sets indicates that these vessels were not intrinsically earmarked for use in wine service. Instead, the elaboration of the vessel used had more to do with the importance of the ceremony

\footnotetext{
${ }^{1038}$ Henig 1995, 105; Bruce-Mitford \& Raven 2005, 258-266 (88).

1039 Allison 2004, 55.

${ }^{1040}$ Green 1998, 63-84.

${ }^{1041}$ Shafer-Elliot 2013, 133-134.

1042 Green 1998, 63-83; Cool 2006, 49.

1043 Baldwin, A. pers. comm.

${ }^{1044}$ Cool 2006, 136-140.
} 
as opposed to what was actually contained within it. Elaborate decoration would be used on vessels in wine services for the elites, but would also service within the ritual context of purification at dining, religious and funerary ceremonies.

Small enamelled jars, often termed balsamaria in the text, would appear by their size and shape to be unguent bottles, a probability supported by their placement in grave contexts similarly to examples in glass, ${ }^{1045}$ though residue of a tar based adhesive in an example from Catterick suggests that they could have served other purposes throughout their use-life. ${ }^{1046}$ These are comparatively rare finds and likely have more in common with the more prevalent corpus of glass unguent bottles and inform us comparatively little on copper alloy vessels as a group of objects. Nonetheless, their enamelled decoration draws them into the wider British tradition of enamelled metalwork, offering a further example of the application of a provincial technique applied to a more widely accepted repertoire of objects. ${ }^{1047}$

The relative rarity of Handled Pan $4 \mathrm{~s}$ in the data makes identifying the function of this vessel form particularly problematic. It is found in Late Roman Structured Deposits, ${ }^{1048}$ indicating its association with rituals of dining and/or ablutions. Like other basins, its appearance in the record in the Late Roman period does suggest it may have also replaced Handled Pan 1s in their function as vessels of purification. While the relatively simple examples of this vessel may seem less than suggestive of ritual function, elaborate examples from the Eastern Empire in the collection of the Royal Ontario Museum do show that prestige could be attached to these objects. ${ }^{1049}$ Their rarity indicates that they were never widely adapted into the material practice of the province and it is unlikely that their function was such that it could not have been easily fulfilled by other vessels forms.

Strainers have been believed to have been principally used for the straining of wine or herbal elixirs. ${ }^{1050}$ This is plausible, especially with strainer bowls of types such as Eggers 90. Indeed, it is just such vessels that are found in the grave assemblages of Stanway and Turner's Hall Farm associated with drinking and dining equipment. ${ }^{1051}$ It should be kept in mind that strainers in antiquity could have had multiple functions and purposes. The presence of strainers

\footnotetext{
${ }^{1045}$ De Santis 2000, 238-243.

1046 Wilson 2002, 81; NYR0038.

1047 Breeze 2012, 107-112.

${ }^{1048}$ EX0011; Kennet 1971, 126 (2.4); SUR0010; Kennett 1971, 132 (5.10); BM 1915.04 06.10.

${ }^{1049}$ Harcum 1921, 37-54.

1050 den Boesterd 1956, XXII; Cool 2006, 143-146.

${ }^{1051}$ EX0076, HER0035 \& HER0036.
} 
in large Late Roman Structured Deposits is also curious, as these assemblages also lack the ceramics and glass to make them functional wine services and are instead more likely to represent the assemblages for ablution rituals. These vessels would have served as functioning filters of impurities from water as well as wine and it would seem likely that this could explain their presence in the Late Roman Structured Deposits. As water purifiers, they would have been an integral part of such assemblages of ablution vessels. This function of purification could have offered these vessels symbolic significance, as alluded to by the cross and wave decoration of the strainers in the assemblage from Irchester. ${ }^{1052}$ Buckets are another form of vessel that some identify with wine service, ${ }^{1053}$ but again it is likely that these vessels were used for containing liquids in drinking as well as washing rituals. This is not to say that buckets were never used to mix wine or any other combination of ingredients, but simply that their use likely encompassed several functions and that they were not indispensable components of either wine or washing services.

Lastly, it is important to discuss the function of the most common form among the repertoire of copper alloy vessels in Roman Britain, Handled Pan 2s. A good case can be made for the Rudge Cup type of Handled Pan 2s to have been used for drinking, either as cups or dippers. This is based not only on their size, which is analogous to cups, but from the inscription seeming to reference imbibing found on a handle from Gunthorpe. ${ }^{1054}$ Nevertheless, it is problematic to draw this association to this vessel's larger cousins. Though they are found in every Depositional Context and Site Type distinction used in this thesis, it is rare that they are found with assemblages that can be firmly identified as wine services. Their pairing in some early Structured Deposits with strainers, such as at Coygan Cave, ${ }^{1055}$ could be evidence of such a use, though it was just mentioned that strainers themselves would have had multiple purposes and can therefore not be taken as direct evidence for a wine service specifically. The absence of this vessel from the wine services in Late Iron Age and early Roman graves in south eastern England also gives pause to assigning a wine drinking function to these vessels, or at least that they were not viewed as an indispensable part of the service. Far more likely, these vessels were multi-functional and could be used for eating, drinking or perhaps cleaning in much the same

\footnotetext{
1052 NH0008 \& NH0009.

${ }^{1053}$ Cool 2006, 136-140.

${ }^{1054}$ Worrell 2012, 73-74.

1055 CAR0001 \& CAR0002.
} 
way that modern bowls fulfil a variety of functions. This would help to explain their proliferation as compared with other vessel forms, as the objects were adapted to serve the use their owners saw fit.

The principal conclusion of this thesis regarding the function of copper alloy vessels is that they are most readily associated with ablutions, as opposed to the common perception previously that these objects were predominantly for wine service and dining. This requires that the place of these vessels within social practice must be reconsidered. Though these vessels may have indeed formed a part in the elite display of leisured dining practice, their application was not directly related to the dining table, as such, but formed a very specific function in washing and hygiene that would communicate familiarity with the customs of 'civilised' people across the empire. In this way, these vessels played an important role in the construction and display of identity in the province.

As has been discussed throughout this thesis, different Depositional Contexts represent differing culture practice and, by extension, differing manners of use and consumption. This inherently reflects on how the objects were perceived and the social value attached to their use and consumption. While the broad formation process of Structured Deposits is able to be understood as the accumulation of interrelated goods into a unified assemblage that is then deposited, ${ }^{1056}$ the principal issue determining how this Structured Deposit material was viewed in relation to its consumption and use hinges on if these groups were intended to be removed from active use-life or if they were intended for recovery at some point. This would have varied with each assemblage, as each assemblage had its own particular formation process with its different motivations and expectations. Nevertheless, it is certain that much of the Structured Deposit material in this thesis relates to ritual practice. ${ }^{1057}$ This would be highly suggestive of the removal of these commodities from active use-life and their delegation to functions outside of normal material practice. ${ }^{1058}$ This indicates that Structured Deposits may not have much to offer to our understanding of the active consumption of these commodities, as the very act of their deposition removed them from this cycle. Much the same could be said for Grave Deposits, as these objects are again being removed from active use-life. ${ }^{1059}$ The process of their deposition

\footnotetext{
1056 Section 1.5; Chapter 3.

${ }_{1057}$ See Sections 3.3-3.5 for discussion justifying this conclusion; Fulford 2001, 199-201 \& 215-216.

1058 Though, there is the possibility such objects could be reclaimed; see Dowden 2000, 176.

${ }^{1059}$ Johns 1996, 2.
} 
would most likely have been a final act of conspicuous consumption, in which the relinquishing of wealth by an individual or group is displayed in an act of piety and/or dedication which also serves to highlight their affluence and power. This does not inform us greatly as to how this material would have been viewed and consumed in its more common incarnation in daily uselife, though it does indicate that copper alloy vessels were perceived as goods of sufficient intrinsic or symbolic significance to lend validity to such acts of dedication. This helps place copper alloy vessels within a greater hierarchy of goods of value, as a sacrifice or act of conspicuous consumption only proves valid if what is relinquished is of value. ${ }^{1060}$ Where exactly copper alloy vessels sat within this hierarchy of materials is difficult to discern in definitive terms. The relation between copper alloy and pewter is likely to have been the closest, as Structured Deposits of these materials share several characteristics and they were both utilitarian alloys of functional purpose well below the status or expense of silver or gold. ${ }^{1061}$ The exact nature of this relation is problematic to reconstruct and it is likely that their relation varied significantly over time and space in Britain, reflecting differing cultural perspectives to these materials.

In contrast to Structured or Grave Deposits, Site Finds and PAS data represent what may be seen as broader patterns of consumption. This is expressed not only in the diversity of objects in these contexts, but also the diversity of locations and Site Types which indicates a greater variety in usage and consumption from those represented by the high status rituals of Structured Deposition or the depositing of copper alloy vessels in graves. While it proves difficult to estimate the amount that non-elites may have been able to act as selective consumers, ${ }^{1062}$ the finding of objects in small rural settlements with no apparent evidence for particular affluence indicates that there was a level of availability across a wide segment of Romano-British society and that this material was not solely used by the urban or villa dwelling elites. Furthermore, it indicates that there was a desire to take part in such consumption and that luxuries such as copper alloy vessels were valued and appreciated by people who were not of particularly high economic or class status.

That significant numbers of these vessels occur in each Depositional Context is evidence to the wide use of these objects (Figure 7.18). The comparatively low numbers for Structured

\footnotetext{
${ }^{1060}$ Merrifield 1987, 22.

${ }^{1061}$ See Lee 2009 for full discussion of pewter tableware, its perceived value and ritual deposition.

1062 Ratliff 2011, 43; King 2013, 128-131.
} 
Deposits and Grave Deposits in relation with Site Finds relates to what has already been communicated at various points throughout this thesis: that Structured and Grave Deposits represent the great minority of practice in the use and disposal of copper alloy vessels in Britain during the Roman period and that the general process of consumption and use of this commodity is better visualised through the distribution of Site Finds and PAS data. This being understood, it is helpful to turn attention to how these patterns relate to Site Type and the greater geographic distribution patterns introduced earlier in this chapter. ${ }^{1063}$

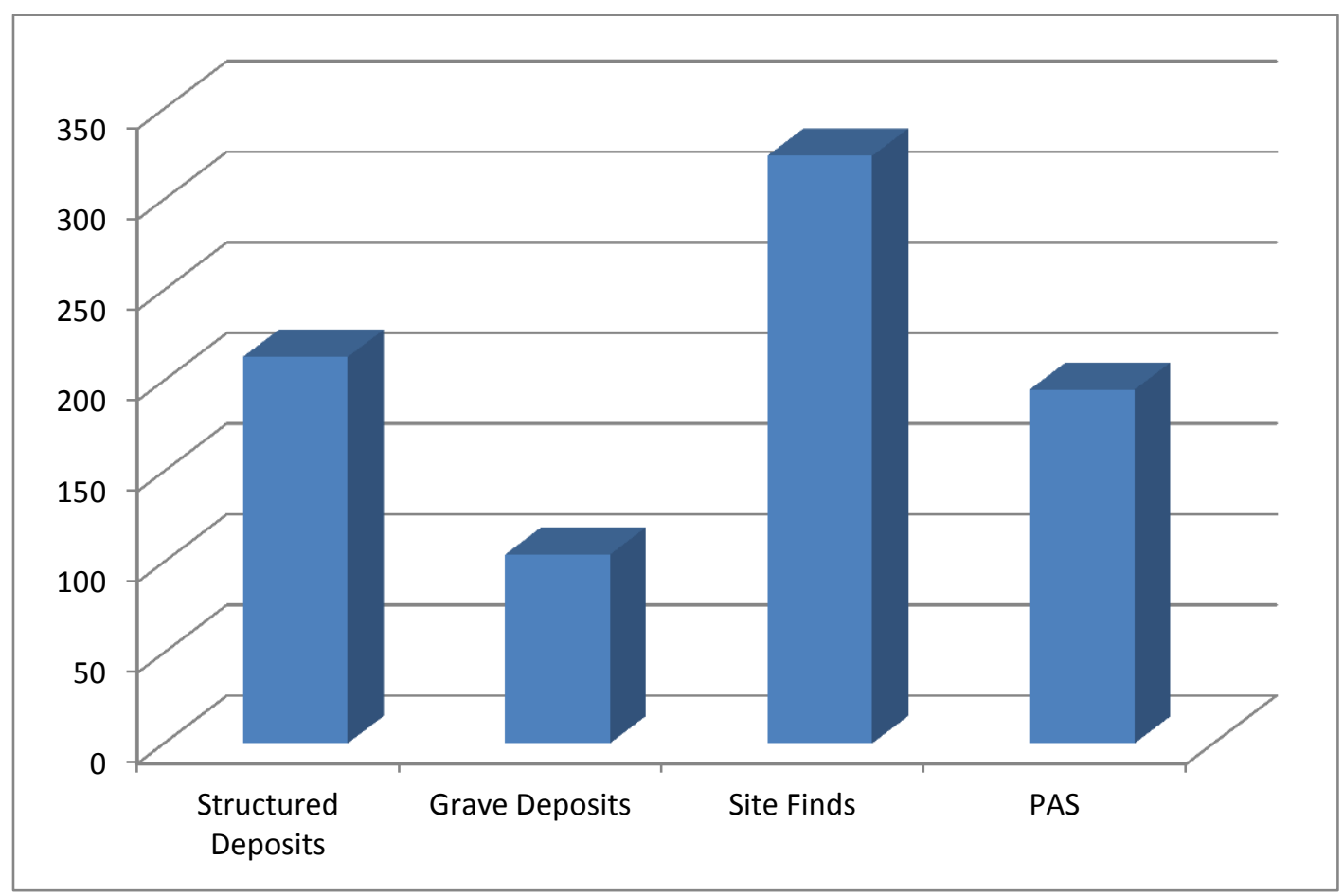

Figure 7.18: Numbers of objects by Depositional Context.

${ }^{1063}$ Section 7.2. 


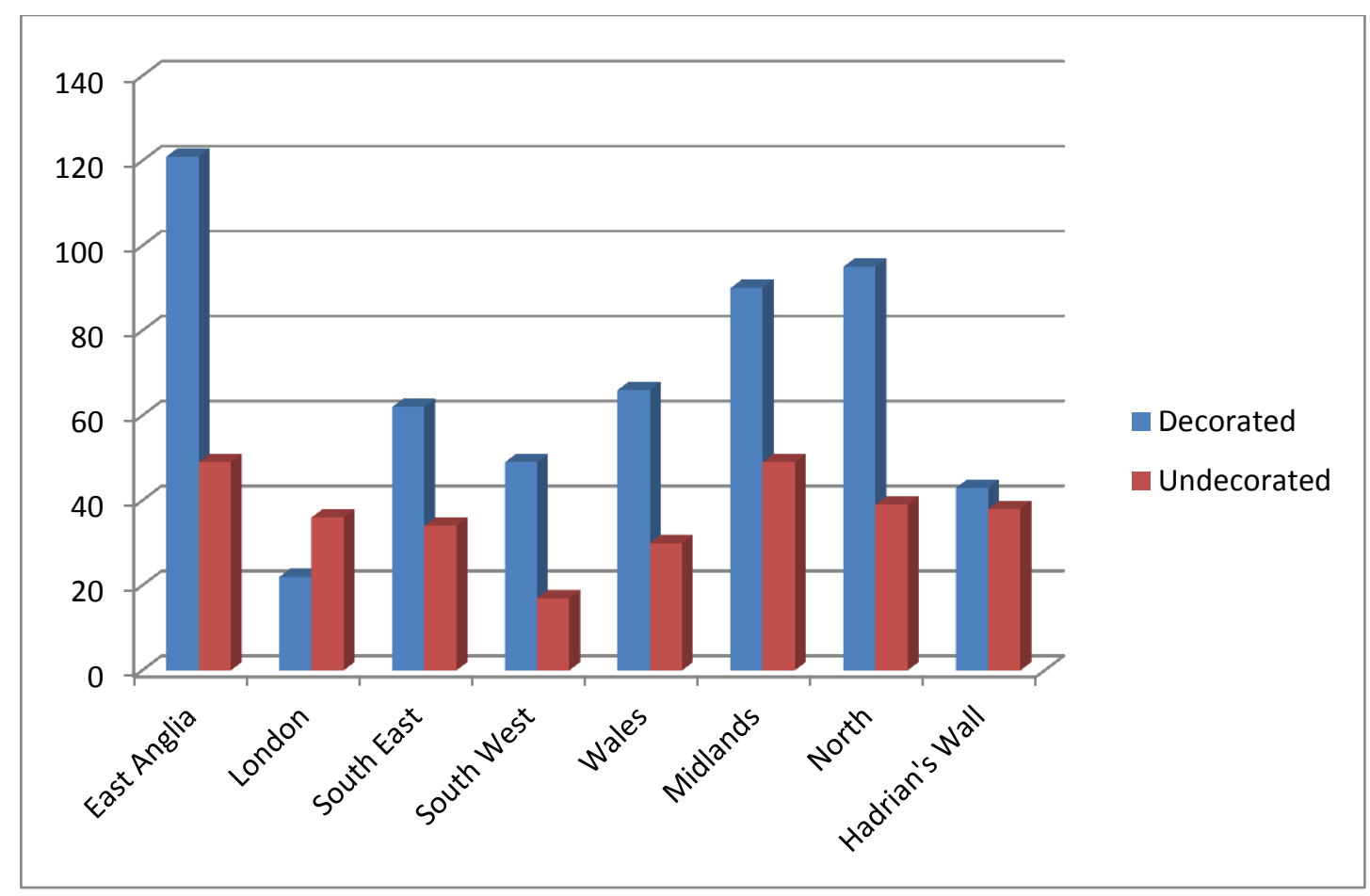

Figure 7.19: Comparative numbers of decorated and undecorated vessels by region.

When viewing these objects as cultural commodities, it is also important to consider the level of decoration exhibited by the objects across the landscape, as illustrated in Figure 7.19. Decoration, serving no inherent use in practical function, ${ }^{1064}$ relates directly to display and identifies these as objects of aesthetic, as well as material and functional, value. ${ }^{1065}$ Tracing the distribution of decoration across the landscape identifies how culturally relevant this artistic value may have proved to people across the province during the Roman period. The following discussion is about decoration broadly, as opposed to decoration which may be directly associated specifically with indigenous traditions or classical paideia. ${ }^{1066}$

As may be expected, East Anglia has the greatest number of decorated objects and also a high level of decorated compared with undecorated vessels. This reinforces the assertion that decoration may be broadly applied to the use of these vessels as cultural commodities of prestige and display. Though smaller in number, the ratio between decorated and undecorated vessels in the North, the Midlands and in Wales is surprisingly comparable to that of East Anglia (about

\footnotetext{
${ }^{1064}$ Though decoration could certainly prove important as allegory in ritual use or be self-referential (Swift 2009); discussed further in Chapters 4 \& 8 .

1065 King 2013, 128-134.

${ }^{1066}$ Discussed in Section 8.2.
} 
$30 \%+/$ - undecorated in each region), indicating a similar level of cultural value put upon the aesthetic appeal of these objects as opposed to their simple functionality.

That London has a greater proportion of undecorated vessels to decorated vessels at first appears surprising, especially as it is nestled between the regions of East Anglia and the South East which both have significantly more decorated than undecorated vessels. London proves to be something of an anomaly in this regard, even compared with other urban centres as Figure 7.20 illustrates.

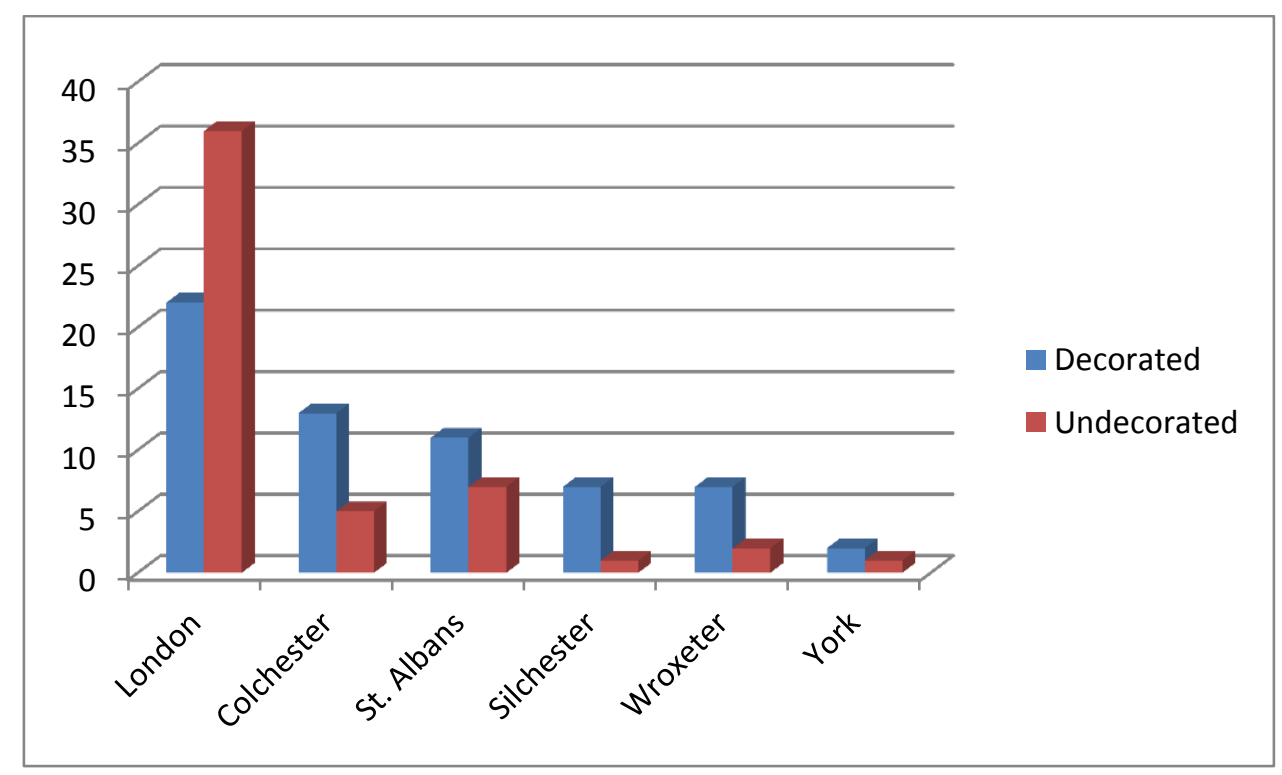

Figure 7.20: Comparative numbers of decorated and undecorated vessels from urban centres in England.

This may be in large part due to the focused archaeological attention London has received and the high level of on-line and print publication available for the city's archaeological sites. If this were to be the case, it would have to be supposed that other urban centres would exhibit similar figures if they received similar attention and publication. It is unlikely that underpublication or oversight would have greatly affected the cities of Colchester and St. Albans, which have both received great attention and publication over the course of the last century. This would suggest that these ratios are fairly representative of the respective assemblages of these sites and that the discrepancy between the different cities represents a difference in how vessels were consumed and utilised functionally and artistically. Another factor could be the absence of grave evidence from London, as objects in burials were disproportionately decorated 
when compared to Structured Deposits or Site Finds. ${ }^{1067}$ That London is the anomaly feeds into the greater difficulty of characterising Roman London in its relation to the rest of the province. ${ }^{1068}$ That London was a great importer and mercantile city is well understood, ${ }^{1069}$ its famously large forum attesting to its economic importance. ${ }^{1070}$ This would suggest affluence among the population and a willingness of them to take part in display culture. A further possible explanation for the relative lack of decorative copper alloy vessels is the intense habitation that London has experience in the fifteen hundred years following the Roman period, which could in turn have led to the finding and removal of ornate vessels and fragments during construction prior to the advent of archaeological recording. This combination of factors led to a prevalence of undecorated vessels in London in comparison to other urban centres. As they are a mix of historical and modern biases, it proves difficult to determine if this accurately reflects comparative consumption patterns in London during the Roman period.

The data available from this thesis indicates that copper alloy vessels were a widely circulated commodity throughout the Roman period in Britain, consumed and enjoyed by a great cross-section of the population of the province. Though never present in numbers comparable to ceramics or coins, ${ }^{1071}$ the broad distribution of copper alloy vessel material across time and in contexts that are unlikely to represent elite consumption indicates that these commodities were consumed and utilised by a larger segment of Romano-British society than would previous have been assumed. Though the use of copper alloy vessels as cultural commodities figured principally in this discussion, the role they played in the formation of identity and culture change as a whole was not addressed. While concepts of identity certainly played a role in the use of these objects, the concept of identity is far too broad of an issue to address in the present section. For this reason, identity will be the focus of the next and final chapter of this thesis, offering a cumulative discussion of how the deposition and distribution of copper alloy vessels during the Roman period relates to the study of culture change and the construction of identity in Britain during this time.

\footnotetext{
1067 Section 4.4.

1068 Morris 1982, 1-3; Perring 1991, ix-x; Hassall 1996, 19-26; Wilkes 1996, 27-32; Millett 1996, $33-38$.

${ }^{1069}$ Milne 1985; Mattingly 2006, 500 \& 511; Cowan et al. 2009, 91-105.

${ }_{1070}$ Merrifield 1983, 68-72.

${ }^{1071}$ Moorhead 2010b; Willis 2011, 167-242; Walton 2012; King 2013.
} 


\section{Chapter 8: Copper Alloy Vessels and Identity in Roman Britain}

\section{$\underline{8.1 \text { Reflection on thesis and future understanding of copper alloy vessels }}$}

This chapter will apply the arguments presented throughout this thesis to some of the wider historiographic issues regarding the interpretation of the Roman period in Britain. In so doing, it will show the relevance of focused artefact studies to understanding the theoretical and practical aspects concerning issues of identity and culture change. First, it will prove useful to summarise and comment on some of the findings made in this thesis thus far and offer a consideration of how this could impact the perception of copper alloy vessels in Roman Britain as well as how this data may apply to the study of identity and historical theory in the interpretation of the Roman period.

The first item worth mention is the impact of the methodology centred on Depositional Contexts in determining the use and cultural significance of these objects. This allowed for patterning to be analysed and for the characterising of each context. In turn, this allowed the development of hypothesises with which to characterise the people who deposited the items and how this related to the objects' use and cultural significance. For instance, it has in the past been common to characterise both Structured Deposits and Grave Deposits as examples of conspicuous consumption on the part of the elite, ${ }^{1072}$ while the data in this thesis has shown that the communal nature of the assemblages of copper alloy vessels in some Structured Deposits indicates that it is far more likely that they represent group ritual as opposed to the conspicuous consumption of one individual or family. Likewise, examining Site Finds material separately indicated that there was sub-or-non elite consumption of these objects, which is a facet of their role in society that has been largely overlooked in the past. Considering these objects within geographic groups was also instrumental in the development of their analysis, it became clear that these vessels were used by a multiplicity of people across space and time, reflecting an availability and acceptance of this commodity which would hitherto not have been expected.

The principal examination of the objects based on forms, as opposed to more specific typologies, allowed for the broader examination of distribution patterns and their relation to the general use and perception of these vessels by the peoples of Roman Britain, as was the recognising that handled pans comprise several distinct forms as opposed to simple typological

\footnotetext{
1072 Hobbs 2006, 132-134.
} 
variances within a single form. These two factors contributed to the realization of the broad shift from smaller vessel forms to larger ones and that this reflected a shift from individual to group use of these objects. In turn, this indicated that the vessels likely served the same principal functions with one form replacing another as the requirements demanded of these objects shifted over time with the rise of convivial ritual during Late Antiquity. ${ }^{1073}$

This methodological approach allowed for the development of comparative patterning between Depositional Contexts, object forms, geographic and temporal distribution, Site Types and decoration which were used in the development of the theories regarding the function and cultural significance of these objects offered in this thesis. ${ }^{1074}$ Principal among these was that copper alloy vessels were utilised by a wide segment of the population that seemed to span both military and civilian, affluent and modest. This indicates that copper alloy vessels were readily received by the inhabitants of Britain and the forms in manufacture applied to the material needs of their lives.

The likelihood that copper alloy vessels were chiefly for ablutions, as opposed to the general assumption in previous literature that they were part of the food or wine service, is also an important conclusion from this thesis based on the distribution across contexts and chronology as well as the close interrelation of these objects to each other in their depositional patterning seemingly to the exclusion of any other objects that would facilitate their use in dining or drinking. ${ }^{1075}$ Handled Pan 2 s are the principal exception to the broad pattern of copper alloy vessels as forming ablution sets, but this form's likely multi-functional nature also suggests that they could have purposes outside of the wine service. While the application of some forms of copper alloy vessels to ritual purification or bathing has been generally recognised, ${ }^{1076}$ the scholarly consensus has previously been that most of these vessels were used in the wine or food service. ${ }^{1077}$ A departure from this model of vessel use and the adoption of these vessels more closely with ritual and bathing equipment, as this thesis advocates based on the evidence above, would lead to a shift in how these objects should be interpreted within site assemblages and within the greater material practice of Britain and, perhaps, the empire more widely.

\footnotetext{
${ }^{1073}$ Hudson 2010, 663-695; Section 7.2.

1074 Principally in Chapter 7.

1075 Section 7.3.

1076 Nuber 1973; den Boesterd 1956, XXI; Harris \& Henig 2010, 25-38.

1077 den Boesterd 1956, XIX-XXXI; Carver 2001, 15-22 \& 32-33; Cool 2006, 136-140.
} 
The above observations are the key contributions of this thesis to the study of copper alloy vessels as a group of objects and form the basis for the observations on the larger theoretical and historiographical debates in the study of Roman Britain addressed later in this chapter. Before progressing further in the application of the evidence offered by copper alloy vessels to the development and creation of identity in Britain during the Roman period, it is worth considering a cautionary statement offered by Cool:

'...people's use of material culture tends not to be straightforward. They use it to manipulate their personas. Sometimes this persona may be straightforward and reflect their actual nationality or ethnicity, but that is not always the case., ${ }^{, 1078}$

The sentiment of this statement offers a suitable segue between the conclusions made thus far in this thesis and those to be presented in the following chapter.

\section{$\underline{\text { 8.2 Decoration and Identity }}$}

While the functional use and display of vessel forms described in the last chapter may inform us about broad patterns of the function of these objects in Roman Britain, it is the finer variances in decoration and adornment that may help clarify nuances in taste that are indicative of the construction and display of identity. It is to the examination of this aspect of the material cultural of these objects that we turn in the following section. The use of decorative art as a means for creating identity and conveying cultural connections is ubiquitous across human societies, it may even be said that the principal purpose of decorative art is to convey cultural allegiance and build social identity. ${ }^{1079}$ There has been much recent work in the fields of anthropology and social science in regard to the application of art and material culture towards identity, ${ }^{1080}$ which proves to be one of the most fruitful fields of research into the construction and expression of cultural identity in past societies. ${ }^{1081}$ The place of decorative art in the culture of Roman Britain has been extensively investigated ${ }^{1082}$ and it is not the aim of the current section to offer a broad history of the subject. Instead, the use of decoration on copper alloy vessels as

\footnotetext{
1078 Cool 2010, 27.

${ }^{1079}$ Lyons \& Papadopoulos 2002, 8; Swift 2009, 187.

${ }^{1080}$ Lyons \& Papadopoulos 2002, 8-11; Aldhouse-Green 2004; Hurcombe 2007, 3-13 \& 209-211; Swift 2009.

${ }^{1081}$ Lyons \& Papadopoulos 2002, 8; Hurcombe 2007.

1082 Millett 1990, 112-117; Henig 1995.
} 
objects of use and display will be examined as a means for investigating the development and negotiation of identity across Britain during this period.

Before continuing, a brief summary of the decoration on copper alloy vessels will prove helpful in understanding how it relates to ideas of identity discussed in this section. Much of the surface area of copper alloy vessels is left undecorated, with areas around the rim and handles being the most likely place for decoration to occur. Figural representation is not common, occurring on 180 out of 840 objects in the data-set of this thesis. Human representations are mostly of mythological subject, with a notable absence of hunting and gladiator scenes. ${ }^{1083}$ Zoomorphic themes include birds, bulls and mythological creatures. Ram's head and dog's head handle terminals to Handled Pan 1s are representative of these objects and were discussed in detail in Chapter 4. Floral design, in the form of leaf and vines, is found on handles and around the rims of vessels. On the whole, more decorated objects are found in the south and east than in the north, though highly decorated objects are known from both the area of Hadrian's Wall and Wales.

In the first line of her book on art in Roman Britain, Laing states 'Classical and barbarian art were fundamentally opposed in their aims'. ${ }^{1084}$ Perceptions such as this of the dichotomy in the artistic aims and expressions at play in the Roman world has led many scholars to attempt to find 'Roman' and 'native' voices and peoples in ancient art from the provinces. A further application of this dichotomy to the study of Roman art has seen the presence of 'Romanized' art as symptomatic of Rome's colonial success in assimilating native peoples and 'provincial' art as a poor imitation of a higher culture that the native craftsmen were unable to attain. ${ }^{1085}$ This is a view that Mattingly has pointed out has led to a centralist approach to the study of Roman art that is unrepresentative of the artistic landscape of the time. ${ }^{1086}$ Such approaches to art in the Roman world necessitate a rigidly defined perception of the concept of culture and identity, of being part of one group and inherently opposed (at least culturally) to another group. ${ }^{1087}$ This perspective on culture is in many ways contrary to the reality of how identity is individually negotiated and expressed. The process of forming identity is not a linear process, but is built upon the multiple roles that one has in relation to family, real or imagined ancestry, previous and

\footnotetext{
${ }^{1083}$ L0043, a jug lid in the form of a gladiator's helmet, being the sole exception.

${ }^{1084}$ Laing 2000, 8.

1085 Millett 1990, 113-117; Scott 2003, 1-5.

${ }^{1086}$ Mattingly 2011, 246.

${ }^{1087}$ Laurence 1998, 95; Grahame 1998, 159; Sen 2006, 20.
} 
current professions, religious affiliation and any number of social or civil allegiances. ${ }^{1088}$ This could make for a complex and multi-layered identity in a society as complex as that of the Roman world. ${ }^{1089}$

The malleability and complicated nature of identity in the Roman world is readily expressed through its visual arts. Huskinson points out the complex relationship between Roman/Hellenistic models of representation and local identities in the personifications of Rome and the provinces in a famous mosaic from El Djem in Tunisia, acknowledging an acceptance (if not all-out approval) of the cultural diversity and complexly layered identities active in the empire. ${ }^{1090}$ A good example of such complexity in the communication of identity through visual culture is the tomb of Philopappos in Athens, dating to 114-116 CE. The sculptural representations which adorn it are varied and complex in the messages they convey about Philopappos and his place in society, as are the both Greek and Latin inscriptions which list his lineage and honours. Miles explains the monument rather succinctly when writing: 'The fact that Philopappos can represent himself as a Roman Consul, an Athenian archon, a Commagenian king and even as a god on the same monument shows that identity is a constructed rather than a fixed reality. ${ }^{1091}$ A further example of the presence of plural identities in Britain comes from the tombstone of Regina at South Shields, depicting her as a Roman matron though the epitaph identifies her as a member of the British Catuvellaunian tribe and she was married to a man from Palmyra who subsequently chose to have her funerary inscription written in both Latin and Palmyrene. ${ }^{1092}$ It is just such complexity in the process of building and communicating identity that has led to the term 'Romanization' falling out of use; ${ }^{1093}$ though it has, interestingly enough, not led to a departure from the conflict between 'Roman' and 'native' peoples and cultures being the predominant paradigm in conceptualizing cultural interaction during the Roman period.

One means of expressing identity is through showing familiarity with the traditions and stories associated with a certain culture, by showing the objects and owner as they should be in relation to their world, which may not be wholly congruent with how this relation indeed is. ${ }^{1094}$ In regards to Classical civilization, this is often termed paideia and is meant to express

\footnotetext{
${ }^{1088}$ Huskinson 2000a, 10-25; Lomas et al. 2013, 1-8.

${ }^{1089}$ Collins 2008, 47; Gerrard 2013, 246-263.

${ }^{1090}$ Huskinson 2000, 3-10.

${ }^{1091}$ Miles 2000, 34.

1092 RIB I 1065; Mattingly 2011, 217-218; Carroll 2012, 281-311.

1093 Barrett 1997, 51-64; Mattingly 2011, 38-42.

1094 Swift 2009, 190.
} 
familiarity with Classical education and socialization to the customs, language and status it entails, which in turn defines the more elite honestiores from the crude humiliores. ${ }^{1095}$ Though in the Greek use of the word it denotes specifically aristocratic upbringing within a polis structure, it is by extension used to characterise familiarity and association with Classical culture and mythology as a component in the construction of personal or group identity (which is the sense in which it is commonly used in scholarship). While there is evidence that some decoration on vessels was self-referential to their use, ${ }^{1096}$ it would seem this was not always the case and that decoration was generally more for cultural display. ${ }^{1097}$ Even without considering decoration, the use of Mediterranean copper alloy vessel forms unknown in Britain until contact with Rome (such as Handled Pan 1s and Handled Pan 2s) should be interpreted as expressing familiarity with the Classical world and its material traditions. ${ }^{1098}$

It is through the decoration of these vessels that more direct evidence of the influence and acceptance of Classical ideas and customs may be discerned. An argument can be made that the presence of Classical iconography in the decoration of portable objects cannot be taken as a sign of cultural allegiance or even cultural awareness, but could simply represent acquisition and display of exotic imported luxuries by a purchaser who may well be ignorant to the objects' uses or artistic significance. This argument is made most frequently to explain the presence of objects with Classical iconography in Free Germany and the far North. ${ }^{1099}$ This same argument does not graft seamlessly onto Britain as it was subject to Roman rule for centuries and inhabited by peoples from across the empire who were familiar with Greek and Latin language and customs, producing sculpture, mosaics and portable art referencing classical themes in profusion. ${ }^{1100}$ The relation of decoration to the identity and convictions of the users of these vessels becomes further complicated when considering the presence of pagan imagery on the Cave of Letters Hoard in Israel, sometimes associated with the Temple Treasure of Jerusalem, as well as the depiction of Thetis on the Temple Menorah in the triumphal procession on the Arch of Titus in Rome and scenes from Classical mythology depicted on the door and ceiling tiles of the

\footnotetext{
1095 Gerrard 2013, 124.

1096 Swift 2007b, 388-399; Swift 2009, 122-136.

1097 Swift 2007b, 399-407; Swift 2009, 114 \& 138; King 2013, 128-134.

${ }_{1098}$ Cool 2006, 46-50.

${ }^{1099}$ Eggers 1951; Aldhouse-Green 2004, 117-121.

${ }^{1100}$ Henig 1995, 42-173.
} 
synagogue of Dura Europos, ${ }^{1101}$ complicating our understanding of how accepted such depictions of paideia and romanitas may have been. In any case, ignorance of the subtleties of myth need not prevent someone from appreciating an object, decorative motif or idea. The very act of incorporating the object into culture practice makes the object and decoration a part of the material practice and cultural understanding of those owning, viewing and utilizing the object.

It must be said that copper alloy vessels do not show the same coverage of their surface that vessels of other materials such as ceramic, glass and silver often exhibit, ${ }^{1102}$ the principal exception to this being enamelled vessels. ${ }^{1103}$ This is another manner in which the enamelled vessel tradition in Britain is distinct, a point that will be further touched upon later in this chapter.

Though anthropomorphic representations represent a minority among the data-set of this thesis, occurring on only 60 objects, they represent the most direct examples of decoration that appear to represent the expression of paideia. This is especially true as anthropomorphic art is uncommon in British metalwork prior to the Roman Conquest. ${ }^{1104}$ Interpreting anthropomorphic decoration after the advent of the Roman period is problematized by the patchy nature of the evidence as well as the poor preservation of many of the examples that hinder the recognising of attributes that might aid in understanding. This being said, several representations of Classical Roman gods and heroes are identifiable while it is difficult to identify any native deity represented on copper alloy vessels. This may in large part be symptomatic of the syncretisation of indigenous deities to the Roman pantheon and the hybridization or creolization of religious practice in Britain, ${ }^{1105}$ merging identities of local and pan-Roman deities as to make them indistinguishable from each other. There are a number of examples of just this sort of syncretism in Britain, with the hybrid deity of Sulis-Minerva at Bath being the most famous. ${ }^{1106}$ A further example of a localised deity taking on the appearance and trappings of classical identity may be seen in the goddess Senuna from Ashwell. ${ }^{1107}$ It becomes apparent that it is not a useful exercise to look for 'native' identities in the anthropomorphic decoration of copper alloy vessels simply because the conception of such decoration was synthesised and combined with the Roman

\footnotetext{
${ }^{1101}$ Freund 2004, 141-146 \& 219-221.

${ }^{1102}$ Swift 2009, 105-138.

${ }^{1103}$ Breeze (ed) 2012.

${ }^{1104}$ Henig 1995, 13-23.

${ }^{1105}$ Henig 1984, 36-67; Webster 1997, 165-182; Webster 2001, 209-225.

${ }^{1106}$ Stewart 1981; Cunliffe 1988; Cousins 2014 52-64.

1107 Jackson 2007, 37-54; Ochota 2013, 50-53.
} 
representation as to make any distinction between the two unrecognisable. This helps characterise the form as either wholly colonial and never accepted by the indigenous inhabitants of Britain or as an art form that was fully integrated and adapted to the needs of the multiple groups that inhabited Britain during the Roman period. As further discussion of the use of decoration will help indicate, the latter proves to be by far the more likely scenario.

While the anthropomorphic representations on copper alloy vessels may be viewed as somewhat classicising in their style and syncretising in their subject matter, the zoomorphic imagery used is more difficult to classify and comprehend in relation to identity. This is in part due to the ubiquitous nature of animal iconography in the societies of ancient Europe, ${ }^{1108}$ a good example of this being the use of bulls. Bovine vessel mounts feature in the art of Iron Age and Roman Britain as well as play an important part in the art and folk belief of many indigenous societies of northern Europe. ${ }^{1109}$ The use of such decoration would therefore at first indicate continuity in decorative practice and social affiliation. However, bulls, bulls' heads and bucrania (specifically referencing sacrifice) are commonly found depicted elsewhere in the Roman world and are highly visible in the sculptural relief of Rome itself (Figure 8.01) as well as in the provinces (Figure 8.02). This indicates that this decorative scheme would not have been opposed to or disapproved of by the 'Roman' constituent of Romano-British society. Indeed, the exact opposite would likely have been the case. The use of bovine iconography could well have been a point of similarity and understanding between different peoples, a readily used and understood symbol across ancient European societies which was widely relatable. This reflects how easily cultural customs may have been syncretised between different European groups during this time and reflects how symbols may be used and adapted fairly seamlessly between peoples.

\footnotetext{
${ }^{1108}$ Laing 2000, 160.

1109 Aldhouse-Green 2004, 113-148.
} 


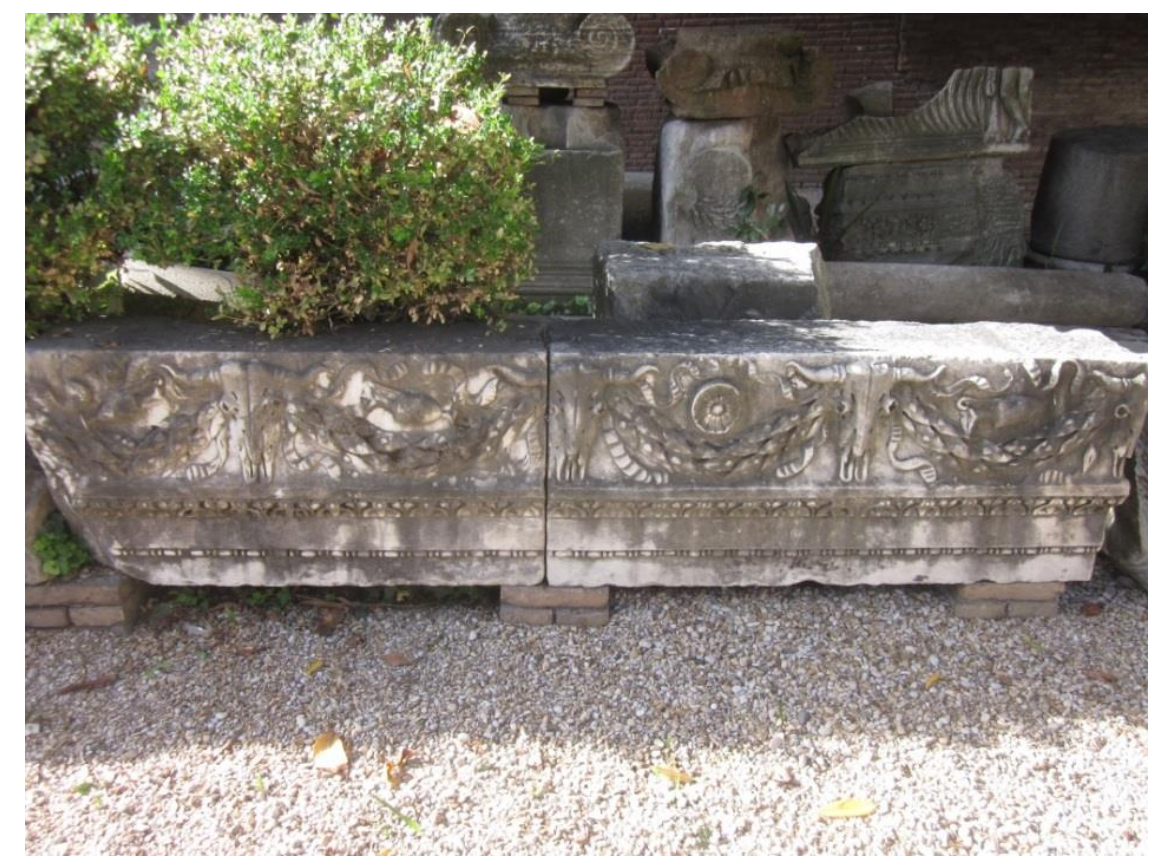

Figure 8.01: Bucranium architectural relief on fragment outside the Baths of Diocletian, Rome (photo by author)

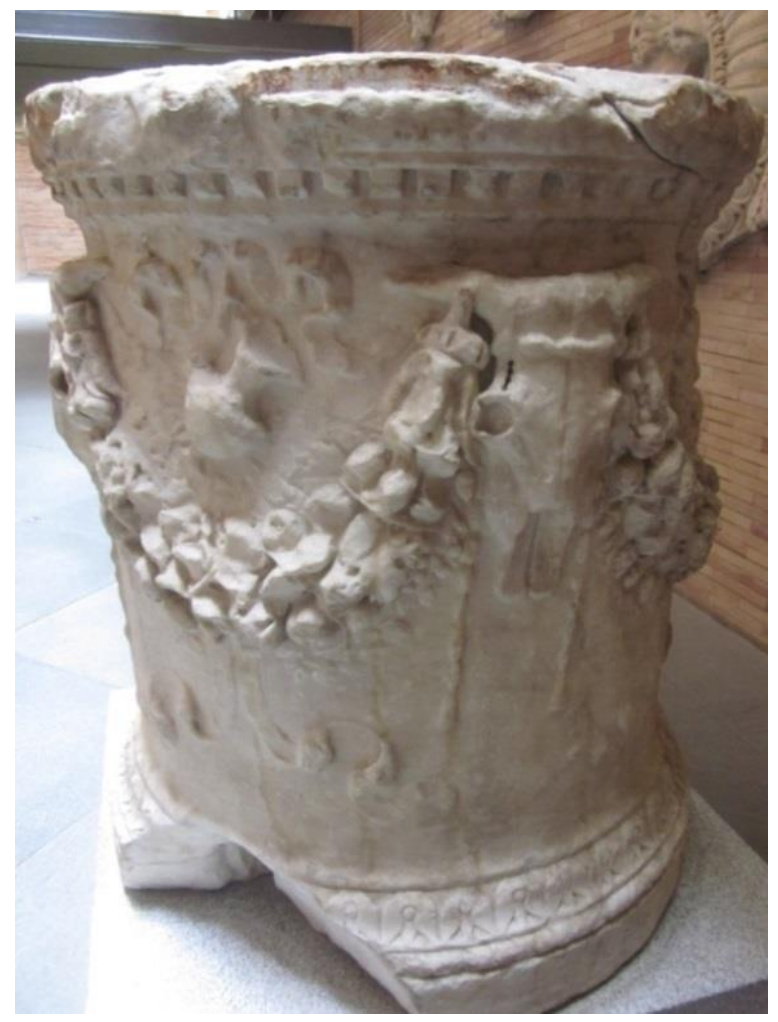

Figure 8.02: Bucranium altar relief on display in the

Museo de Arte Romano, Merida (photo by author) 
Another common use of zoomorphic iconography on copper alloy vessels is on the handle terminals of Handled Pan 1s. These comprise principally ram's heads and a discussion of these vessels may be found in Section 4.4. What concerns the present discussion is how these objects were used in the construction and maintenance of identity. Their use in the expression of social identity is clear in their common usage in funerary ritual. ${ }^{1110}$ By the consistency of the zoomorphic decoration, a ritual symbol could be identified and readily recognised among members of the elite sharing this funerary practice. The practice of showing shared knowledge and cultural understanding is an important component for the construction of identity and the maintenance of social order and cohesion, creating social bonds and reinforcing concepts of status and identity.

While the use of floral and geometric decoration is commonly used on copper alloy vessels, how to interpret its use in relation to identity is problematic. Like bovine busts, floral and geometric decoration is widely used in ancient Europe and is not by necessity indicative of any particular social or religious group. A good example of the use of floral decoration in preRoman western Europe is the use of acanthus scrolls on the Basse-Yutz flagons which have been interpreted as Greek influence on these Gaulish Iron Age vessels (Figure 8.03), ${ }^{111}$ whilst the Battersea shield $^{1112}$ (Figure 8.04) and Waterloo helmet ${ }^{113}$ (Figure 8.05) both attest to the use and skill in geometric enamel work design in pre-Roman ornamented metalwork in Britain.

In like manner, both floral and geometric design figure heavily in Roman art across the empire, exhibited in all forms of decorative art all the way up to expensive and prestigious mosaic floors. ${ }^{114}$ Additionally, that classical imagery was utilised and adapted onto the Late Iron Age coinage of Britain, perhaps adapted from intaglios or other non-numismatic models, ${ }^{1115}$ shows the malleability and versatility of such images and their associations. These could all be signs of the transmutability of art across cultural landscapes and may well have offered familiar points of reference between peoples.

\footnotetext{
${ }^{1110}$ Nuber 1973; Sections $4.3 \& 7.3$.

1111 McGreagor 2010, 177-182.

1112 BM 1857.7-15.1.

${ }^{1113}$ BM 1988.10-4.1.

1114 Dunbabin 1999, 291-298.

${ }^{1115}$ Henig 1972, 209-223.
} 


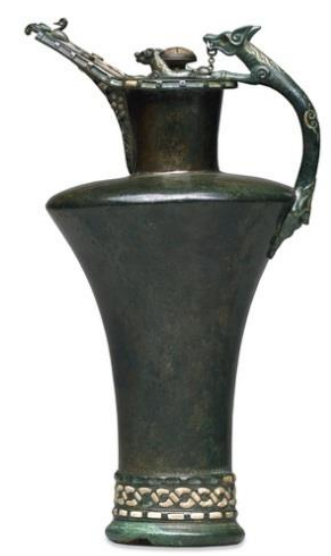

Figure 8.03: One of the Basse-Yutz flagons (ㅇ the British Museum)

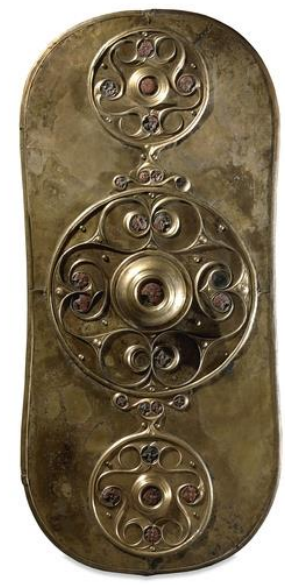

Figure 8.04: The Battersea shield (C the British Museum)

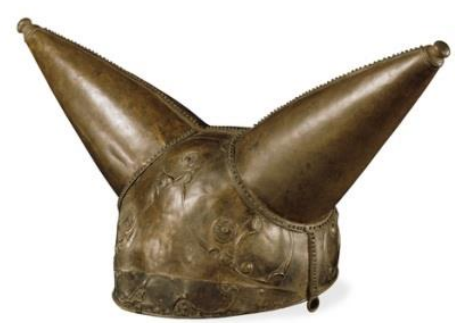

Figure 8.05: The Waterloo helmet (C the British Museum) 
A method prevalent in decorative metalwork, including copper alloy vessels, in Britain during the Roman period is the use of enamelling. The practice of enamelling copper alloy objects seems to be a particularly British craft during the Roman period, associated with a 'home-grown' tradition of manufacture. ${ }^{1116}$ The discovery of moulds for the production of such enamelled vessels found at Castleford supports this hypothesis. ${ }^{117}$ This being said, this practice incorporates components that are more distinctly Roman in their origin. Nowhere is this more evident than in the enamelled Handled Pan 2s classified as Rudge Cup type pans in this thesis, which incorporate the use of a Roman vessel form, Latin inscriptions and probable representations of military architecture decorated in enamelling that in some cases is reflective of British artistic tradition. ${ }^{1118}$ These objects show that native decoration could be incorporated into the imported vessel forms and that the syncretism of these styles created a new form of art. By extension, this new art would have with it new possibilities for defining and displaying one's culture and identity within this blended form of expression. This may have been particularly appealing to colonists and soldiers who came from elsewhere, bringing other traditions and identities, but wishing to express their connection to their new homeland as well. It is evident that there was a great deal of migration to Britain during the Roman period, ${ }^{1119}$ providing fertile ground for the development and appreciation for hybrid forms of art and expression.

Though only a small number of the objects in this thesis bear inscriptions, only some 62 vessels, these inscriptions are worth consideration as to how they relate to the construction and display of identity. Most fundamentally, they are in Latin, implying and displaying a familiarity with the language of administration and, by extension, association with the ruling cultural hegemony. Interpreting the use of inscriptions as aspects of decoration beyond this is problematic, as they often fulfil a different purpose than other forms of adornment. The inscriptions of individual vessels were discussed as they appeared throughout this thesis. Additionally, they are listed in Appendix XII of this thesis. At present, it proves useful to analyse the forms and typology of the vessels that bear inscriptions.

\footnotetext{
1116 Breeze 2012, 107-112.

1117 Bayley \& Budd 1998, 203-222; Künzl 2012, 12-13.

1118 Laing 2006, 172-177; Künzl 2012, 9-22; Breeze 2012, 107-112.

${ }^{1119}$ Eckardt et al. 2010, 99-130; Fulford 2010, 67-78.
} 


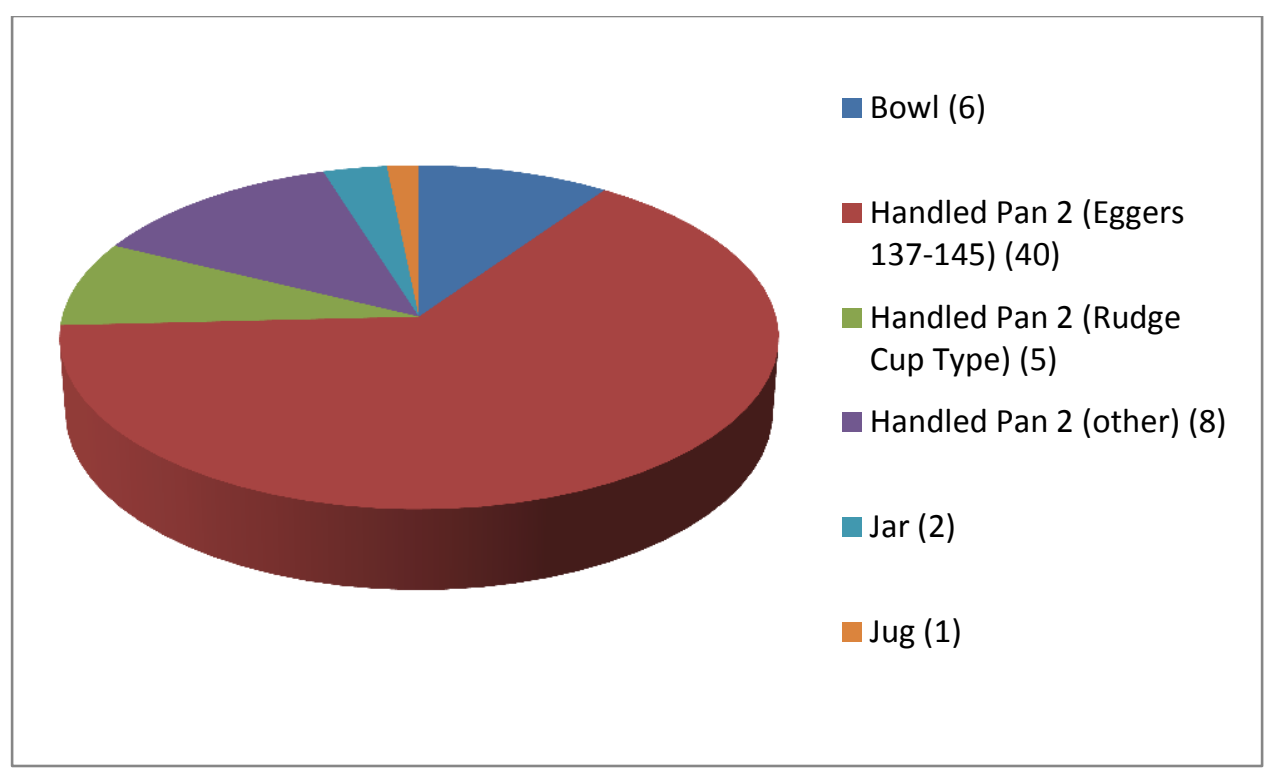

Figure 8.06: Comparative frequency of copper alloy vessels with inscriptions from Roman Britain.

The limited range of vessels that bear inscriptions show it was never a widely adopted practice in Britain and would have served a very narrow function limited to a narrow range of objects. It is evident that this relative lack of epigraphic evidence on copper alloy vessels is reflected outside of Britain as well, as assemblages from the Bay of Naples, Gaul and Free Germany make clear. ${ }^{1120}$ As Figure 8.06 makes perfectly clear, the vast majority of inscriptions (40 out of 62) are found on Handled Pan 2s of Eggers types 137-145. This type occurs throughout the Roman empire and has been found to be associated foremost with the military in Roman Britain. The majority of these inscriptions are makers' marks and designate the objects as goods of some quality or reputation. Punch dot inscriptions on these vessels principally refer to the owners of the vessels or the military unit with which he was associated. Rudge Cup type Handled Pan 2s that bear inscriptions often refer to installations along Hadrian's Wall and its hinterland, offering a rare and invaluable window into how these vessels helped to represent the owners' identities through (probably) commemorating their time spent living and working there. ${ }^{1121}$ Votive inscriptions are exceptionally rare among copper alloy vessels in Roman

\footnotetext{
${ }^{1120}$ Eggers 1951; den Boesterd 1956; Tassinari 1975; Tassinari 1993.

${ }^{1121}$ Künzl 2012, 18-22; Breeze 2012, 107-112.
} 
Britain, with the bowl offered to Apollo at South Shields ${ }^{1122}$ and the Rudge Cup Type Handled Pan 2 dedicated to Sulis Minerva at Bath ${ }^{1123}$ being the only examples in this thesis.

Though comprising only a small number of objects, these inscriptions prove very useful in developing how the concept of identity was constructed through the use of copper alloy vessels during the Roman period in Britain. It is most likely that the labelling of these objects came from the close quarters with which the military lived and the uniformity of their material possessions, while private citizens would have likely had greater room and greater privacy to keep their possessions safeguarded. This being the case, the labelled objects would have associated their owners with the military whether they were on or off duty. The Rudge Cup type vessels are also likely to be associated with the military ${ }^{1124}$ and perhaps the epigraphic habit they exhibit is reminiscent of the labelling of the soldiers' dining gear, thus serving as an even greater memento of their military career. The two dedicatory inscriptions in the data-set of this thesis may both also very likely be associated with the military stationed in Britain, one found at a military site and the other being a Rudge Cup type vessel. It is likely that this recognition and display of literacy was an important part of constructing and communicating identity, especially as literacy rates would likely not have been high in Britain during this period. ${ }^{1125}$

What is not present is often as important, if not more important, than what is present. This is just as true when analysing the decoration of copper alloy vessels as it is when examining any other aspect of material culture. One thing noticeably absent from the decoration of copper alloy vessels in Roman Britain is reference to martial or hunting scenes. Though decoration depicting hunting is attested in silver both within and outside the Roman Empire, ${ }^{1126}$ such references are nearly non-existent among copper alloy vessels in Britain. The two principal exceptions to this would be the depiction of Minerva on a jug from Turner's Hall Farm ${ }^{1127}$ and a jug depicting the madness of Ajax from Bayford, Kent, ${ }^{1128}$ though both of these examples could also be more representative of paideia more generally than of specifically martial significance. The absence of this imagery is particularly interesting as many of the vessels in this thesis come from military sites and some, like Rudge Cup type vessels, were almost certainly manufactured

\footnotetext{
${ }_{1122}^{1123}$ DUR0005; Henig 1984, 132.

${ }^{1123}$ AV0002; Hassall \& Tomlin 1981, 381 (20); Cunliffe 1988, 14 (23).

${ }^{1124}$ Künzl 2012, 18-22; Breeze 2012, 107-112.

${ }^{1125}$ Mattingly 2006, 39-41.

${ }^{1126}$ Such as the Gundestrup cauldron (Aldhouse-Green 2004, 154-156) and the Milden Hall Hoard (Hobbs 2012).

${ }^{1127}$ HER0032.

${ }^{1128}$ K0011.
} 
specifically for a martial clientele. ${ }^{129}$ This could partly be explained by the types of vessels that are present in Britain, as the jugs, bowls and handled pans that comprise the repertoire of copper alloy vessels in Britain do not have the surface areas typically used for such relief depictions on silver platters or ceramic cups. Where figural relief is more typically found on copper alloy vessels is on small jars, or balsamaria, and continental examples will feature hunting scenes and mythological scenes of combat. ${ }^{1130}$ These vessels are practically unknown from Britain, ${ }^{1131}$ though they are not common anywhere in the empire. ${ }^{1132}$

What is evident from the use of decoration in the copper alloy vessels of Roman Britain is that it is distinctly difficult to recognise particular and disconnected traditions, let alone specifically 'Romanist' and 'nativist' opposing traditions. The Prickwillow Pan, signed by a probable British artisan, ${ }^{1133}$ is a good example of the integration of all the decorative themes associable with 'Celtic' or 'Roman' art into an object of singular artistic achievement. Objects such as the enamelled Rudge Cup type pans also show how art was integrated and developed into new forms during the Roman period and refute the concept of self-contained traditions of artistic expression existing, at least among artisans working in copper alloy metalwork. ${ }^{1134}$ This indicates that these objects were malleable forms for the construction and communication of identity and that their symbols had appeal to the various peoples who inhabited Britain during the Roman period. It is likely that a multitude of interpretations for an object were open for the owners, users and viewers of these pieces and that these interpretations may well have varied considerably over the long use-life that these objects had.

\section{$\underline{8.3 \text { Copper alloy vessels and the present understanding of identity in Roman Britain }}$}

The present section will discuss how the evidence offered by copper alloy vessels relates to the greater discussion of identity in Roman Britain and the application of present theoretical paradigms to the study of the Roman past and offer concluding remarks and synthesis of the copper alloy vessels in this thesis and how such an artefact study is relevant to the more general conception and understanding of the Roman world.

\footnotetext{
${ }^{1129}$ Künzl 2012, 18-22; Breeze 2012, 107-112.

${ }^{1130}$ Braun 2001, 107-144.

${ }^{1131}$ K0009 from Bayford in Kent being a notable exception (Philpott 1991, 259; Braun 2001, 120).

1132 Braun 2001.

${ }^{1133}$ Laing 2000, 65.

${ }^{1134}$ Künzl 2012, 9-22; Breeze 2012, 107-112.
} 
At present, the consensus in the theoretical framing of identity in Roman Britain relies on an imperial paradigm of dominance and resistance. ${ }^{1135}$ However, the application of the material in this thesis to support a paradigm of dominance and resistance would be a forced argument and incongruous to what the deposition and distribution of copper alloy vessels indicates of the material practices of the peoples inhabiting Britain during the Roman period. This, in turn, calls into question the application of other varieties of small finds research for this purpose. Whilst plurality of culture practice is evident in differing traditions of Structured and Grave deposition, there is no reason to imply any interaction between these culture traditions as consistently either dominant or resistive. What may be seen instead is a complex layering of material culture which reflected plurality and fluidity of practice, expressing multiple identities intermingling with no necessity of overt conflict in the minds or conscience of those adopting and adapting these identities to their material realities. This indicates the existence of regional variability in culture practice, ${ }^{1136}$ expressed also in other forms of material culture such as ceramics, coins, cosmetic implements and brooch use. ${ }^{1137}$ This is most clearly expressed by the fact that no single group or cultural identity is identifiable through their use of copper alloy vessels. Though the military may appear to have close ties with Handled Pan 2s and rural elites of the south east with Handled Pan 1s and jugs (commonly Eggers type 125), these vessels occur in a variety of settings and Depositional Contexts that indicate these objects were viewed and utilised in diverse ways. ${ }^{1138}$ While contemporary scholars such as Mattingly are very happy to say there were multiple identities existent in the Roman world, ${ }^{1139}$ they prove unwilling or unable to divorce this from a post-colonial view of imperial dominance and indigenous resistance; a perspective that perpetually drives and directs the current interpretation of data. While changes in the deposition were noted over time and differing practices were exhibited by elites in their forms of conspicuous consumption in Structured Deposits and Grave Deposits, ${ }^{1140}$ the profiles of the distribution of copper alloy vessels across the landscape and across Site Type does not indicate that there was a clear cut difference in practice that may be traceable or associable with particular groups who sought to define their identity through their application of these objects to their daily

\footnotetext{
1135 Hingley 2005, 6-48; Jiménez 2008, 15-17; Mattingly 2011, 30-124.

${ }^{1136}$ Mattingly 2006, 522-524.

${ }^{1137}$ Eckardt \& Crummy 2008, 65-72;McIntosh 2011, 155-182; Willis 2011, 219-228; Walton 2012 ; King 2013.

${ }^{1138}$ See Chapter 7.

1139 Mattingly 2011, 214.

${ }^{1140}$ See Chapters 3, 4 and 7.
} 
culture practice that was in any meaningful sense in opposition or divergence with another identifiable group inhabiting the province. This is in direct opposition with what the present accepted paradigm ascribes to. ${ }^{1141}$

It must be questioned as how representative this evidence may be to the broad material cultural experience of inhabitants of the province. The visibility of copper alloy vessels in the archaeological record does not approach the numbers exhibited by ceramics or coins, ${ }^{1142}$ for example, and it may be argued that copper alloy vessels would not be a viable object group to apply to questions of identity and interpretive historic paradigms. This does have a negative effect on the statistical value of some of this material when considering specific questions regarding particular vessel forms and their uses. Nevertheless, these vessel do occur in sizable enough of numbers in the archaeological record and occur widely enough distributed across the geographic landscape and covering sites of apparently diverse social and cultural standing to make their use in analysis of culture change and identity useful. As the broad distribution patterns nonetheless show, it would appear that every area of the province had access to copper alloy vessels and sites that otherwise have no evidence for affluent inhabitants have produced copper alloy vessel fragments, ${ }^{1143}$ indicating an incorporation of this material into the broad material practice of the province over the course of the Roman period.

While broader theoretical works, such as those of Millett and Mattingly, ${ }^{1144}$ have the luxury of sifting through a great variety of data in the construction of their arguments, artefact studies are limited in the evidence they can draw upon which, in turn, limits the malleability of this evidence to preconceived paradigms. At the outset of this thesis work, I had anticipated the work to indicate colonial identities present and in opposition to each other. On the contrary, this focused artefact study has proven such an oppositional paradigm not only insufficient to conceptualise the cultural application of these objects, but that such an approach oversimplifies and dehumanises the users of these vessels, making them statistical points in a greater theoretical narrative as opposed to the independently acting agents participating in the construction and definition of themselves and their identity within a wider and ever changing world that they indeed were. The complexity of the development of the use and decoration of these vessels

\footnotetext{
${ }^{1141}$ Mattingly 2011, 215.

1142 Willis 2011; Walton 2012; King 2013.

1143 Section 7.2; Section 8.1.

${ }^{1144}$ Millett 1990; Mattingly 2006; Mattingly 2011.
} 
attests to this and proves that the study of the Roman period in Britain cannot be simply the study of the 'Romano-British', nor the 'Roman' and the 'British', but is instead the study of a polyethnic and diverse social landscape that was never static enough to develop into longer term oppositional identities. What must be studied is the plurality of cultural materiality that helped individuals define themselves in relation to the world they found themselves in and that they used objects such as copper alloy vessels as a means of negotiating and displaying this identity. Far from having a narrow scope, this focused study of the distribution and deposition of copper alloy vessels illustrates the use of their application to the understanding of the greater cultural climate within which these objects were utilized.

\subsection{Opportunities for further research}

The preceding thesis has attempted to develop understanding of copper alloy vessels and their place in the material culture of Roman Britain through investigating their deposition and distribution patterns across the province. It has also opened up the possibility for further research to be done, utilizing the material developed herein and applying it further to the study of these objects and material culture in Britain. In closing, I would like to suggest ways in which this research may develop and how the present thesis may contribute to future work.

One of the areas for further research that becomes immediately apparent is to widen the study area and to incorporate neighbouring provinces and liminal regions into the data-set. This would allow for wider understanding of the availability and prestige of these objects during the Roman period and could help to indicate if patterns of deposition and distribution in the British provinces were exceptional or comparatively standard. In Chapter 1, it was explained that the lack of a comparable data gathering entity to the Portable Antiquities Scheme made it impossible to incorporate Scottish material seamlessly into the data-set of this thesis as it would have resulted in an unfair comparison of data that would have wielded fallible conclusions. This limitation remains with incorporating data from neighbouring areas such as France or the Netherlands and this biasing of the data would have to be taken into account when drawing conclusions between comparative distribution patterns in these regions. Much work has previously been done on the copper alloy vessels of Scotland, ${ }^{1145}$ and it would prove unnecessary to re-tread this ground, though integrating Scottish research into a broader study of depositional

\footnotetext{
${ }^{1145}$ Hunter 2001, 289-309; Hunter 2012, 85-106.
} 
patterns in north western Europe during the Roman period would prove to be a fruitful avenue of further research.

Another extension of the research from this thesis would come in the chronological expansion of the material under consideration, particularly into the examination of copper alloy vessel deposition and distribution during the Anglo-Saxon period. Fruitful work has already been undertaken in studying copper alloy vessels from Anglo-Saxon England. ${ }^{1146}$ Much could potentially be gleaned from applying the analysis of the deposition of these vessels by the Depositional Contexts used in this thesis and patterning the vessels' distribution across the landscape. As most archaeological evidence from the Anglo-Saxon world comes from burials, ${ }^{1147}$ the depositional patterning for this period would be significantly different and would likely affect the repertoire of vessels present in such a data-set. The previous belief that Britain remained somewhat sequestered from Europe and the developing Byzantine world until the $7^{\text {th }}$ century has been challenged by recent work, ${ }^{1148}$ which raises questions as to how much of a break in continuity did occur with the withdrawal of Roman administration and how much the developing Anglo-Saxon world exhibited a continuation of cultural practices such as wealth display and ritual ablution, which could be reflected in the use and deposition of copper alloy vessels. Such a study would also aid in clarifying some of the data in the current thesis as it would place it within a wider chronological frame and could illuminate culture change and continuity between these two periods, a subject which has gained in popularity in recent scholarship. ${ }^{1149}$

The current thesis is in much the same vein as Eckardt and Crummy's 2008 monograph on toilet instruments in Britain, bringing together a corpus of small finds data from published sources in order to develop theories of cultural development and adaptation in the province. ${ }^{1150}$ As such artefact driven research projects develop, it will prove ever more intriguing to chart similarities and variances between distribution patterns of different object types. Of particular interest will be how objects of different type, but made from the same material, differ in their depositional and distribution patterns. In this thesis, mention was made to comparable patterns in the distribution and deposition of copper alloy vessels, coinage and brooches during the

\footnotetext{
${ }^{1146}$ White 1988; Bruce-Mitford \& Raven 2005.

${ }^{1147}$ Leeds 1936, 1-3; White 1988, 1-5; Harris 2001, 163.

${ }_{1148}$ Harris 2001, 139-188; Gerrard 2013.

1149 Harris 2001; Gerrard 2013.

${ }^{1150}$ Eckardt \& Crummy 2008.
} 
Roman period. The patterning of these objects should be more intensively studied in the future and could prove a fruitful line of inquiry into the conceptualization of materiality and prestige in the province as well as to illustrate the availability and desirability of such objects across the British landscape during the Roman period. Related to this, though likely proving of enough scope to warrant its own project, is the analysis of all Roman copper alloy small finds reported through the Portable Antiquities Scheme to analyse patterns of deposition and distribution across the landscape during the Roman period.

As the data of this thesis was applied to the debate within archaeological theory and historiography, it is worth considering how further work on this subject may impact the development of the theoretical paradigms of the discipline. The development of interpretive frameworks with which to approach the material under study is largely subjective upon the individual undertaking the investigation and is wholly dependent upon the social and cultural climate of the times within which the scholar lives. ${ }^{1151}$ This being said, one of the purposes of an artefact study is to be used by current researchers to test their paradigms and develop new theories which may better fit their own interpretation of the data within such reports. In this way, artefact led studies such as this thesis are crucial to the future development of archaeological theory and interpretive models, ${ }^{1152}$ though it remains largely impossible to conjecture what exactly will be the impact that any particular study may have on the development of scholarship. It is most appropriate for artefact based studies to remain as practical in their data analysis as possible in order to minimise the influence of unnecessary or unhelpful paradigms during the presentation of the data and making the material as useful as possible to scholars on every side of the interpretative discourse, making way for new interpretive theories to develop as the discipline progresses into the unknown future.

\footnotetext{
${ }^{1151}$ Mattingly 2006, 3-14; Gerrard 2013, 1-7.

1152 Swift 2007a, 24-25.
} 


\section{Bibliography}

Abdy, R. 2002. Romano-British Coin Hoards. Shire Archaeology: Princes Risborough.

Adams, G. \& Tobler, R. 2007. Romano-British Tombstones between the $1^{\text {st }}$ and $3^{\text {rd }}$ centuries $A D$. BAR British Series 437.

Adkins, L. \& Adkins, R. 1996. Dictionary of Roman Religion. Facts on File, Inc.: New York.

Aldhouse-Green, M. 2004. An Archaeology of Images: Iconography and cosmology in Iron Age and Roman Europe. Routledge: London.

Allan, M. 1994. The Roman Route across the Northern Lake District: Brougham to Moresby. University of Lancaster Press: Lancaster.

Allason-Jones. L. 2012. "The Rudge Cup". In Breeze, D. (ed.) The First Souvenirs: Enamelled Vessels from Hadrian's Wall. Cumberland and Westmorland Antiquarian and Archaeological Society: Kendal. 23-36

Allason-Jones, L. \& McKay, B. 1985. Coventina's Well: A shrine on Hadrian's Wall. Oxbow Books: Oxford.

Allen, T. et al. 2012. A Road Through the Past: Archaeological Discoveries on the A2 Pepperhill to Cobham road-scheme in Kent. Oxford Archaeology Monograph 16.

Allison, P. 1992. The Distribution of Pompeian House Contents and its Significance. Unpublushed Doctoral thesis: University of Sydney.

Allison, P. 1999. "Labels for Ladles: Interpreting the Material Culture of Roman Households". In Allison, P. (ed) The Archaeology of Household Activities. Routledge: London. 57-77.

Allison, P. 2004. Pompeian Households: An Analysis of the Material Culture. Cotsen Institute of Archaeology UCLA Monograph 42.

Anderson, A. et al. 2001. The Romano-British 'Small Town' at Wanborough, Wiltshire. Britannia Monograph Series 19.

Arnold, C. \& Davies, J. 2000. Roman and Early Medieval Wales. Sutton Publishing: Stroud.

Arthur, J. 2002. "Pottery Use-Alteration as an Indicator of Socioeconomic Status: An Ethnoarchaeological Study of the Gamo of Ethiopia”. Journal of Archaeological Method and Theory 9.4. 331-355

Atkinson, D. 1942. Report on Excavations at Wroxeter (the Roman City of Viroconium) in the County of Salop 1923-1927. Oxford University Press: Oxford. 
Atkinson, M. \& Preston, S. 1998. "The Late Iron Age and Roman Settlement at Elms Farm, Heybridge, Essex, Excavations 1993-1995: A Interim Report”. Britannia 29. 85-110

Bagnall-Smith, J. 2008. “Aspects of votive offerings in south-east Britain”. In Rudling, D. (ed.) Ritual Landscapes of Roman South East England. Oxbow Books: Oxford. 161-162

Bakker, L. et al. 2006. Geraubt und im Rhein versunken: Der Barbarenschatz. Historischen Museum der Pfalz Speyer: Stuttgart, Germany.

Barber, B. \& Bowsher, D. 2000. The Eastern Cemetery of Roman London: Excavations 19831990. Museum of London Archaeological Service Monograph 4.

Barber, B. \& Hall, J. 2000. "Digging up the people of Roman London: Interpreting evidence from Roman London's Cemeteries." In Haynes, I. et al. (eds) London Under Ground: The Archaeology of a City. Oxbow Books: Oxford. 102-120

Barclay, C. "Knaresborough, North Yorkshire". 1997. Coin Hoards from Roman Britain: Volume X. British Musuem Press: London.

Barford, P. 2002. Excavations at Little Oakley, Essex, 1951-1978: Roman Villa and Saxon Settlement. East Anglian Archaeology Report 98.

Barrett, J. 1997. "Romanization: a critical comment". In Mattingly, D. (ed) Dialogues in Roman Imperialism: Power, discourse, and discrepant experience in the Roman Empire. JRA Supplemental Series 23. 51-65

Bateman, N. et al. 2008. London's Roman Amphitheatre: Guildhall Yard, City of London. MOLAS Monograph 35.

Bayley, J. \& Budd, P. 1998. "The Clay Moulds". In Cool, H. \& Philo, C. (eds) Roman Castleford: Excavations 1974-1985 Volume I; Small Finds. West Yorkshire Archaeology Service: Wakefield. 195-222.

Beard, M. 2008. Pompeii: The Life of a Roman Town. Profile Books: London.

Bendann, E. 1930. Death Customs: An Analytical Study of Burial Rites. Alfred A. Knopf Publishing: New York.

Bennett, J. \& Young, R. 1981. "Some New and Some Forgotten Stamped Skillets, and the Date of P. Cipius Polybius”. Britannia 12. 37-44

Biddle, M. 1967. "Two Flavian Burials from Grange Road, Winchester”. Antiquaries Journal 47. 224-250 
Biddulph, E. et al. 2011. Settling the Ebbsfleet Valley: High Speed I Excavations at Springhead and Northfleet, Kent. The Late Iron Age, Roman, Saxon, and Medieval Landscape Volume II: Late Iron Age to Roman Finds. Oxford Wessex Archaeology: Oxford.

Bidwell, P. \& Hodson, N. 2009. The Roman Army in Northern England. The Arbeia Society on behalf of the Organising Committee of the XXIst International Limes (Roman Frontiers Studies) Congress: Newcastle upon Tyne.

Bidwell, P. \& Speak, S. 1994. Excavations at South Shields Roman Fort: Volume I. The Society of Antiquaries of Newcastle upon Tyne Monograph Series 4.

Bishop, M. \& Coulston, J. 1993. Roman Military Equipment from the Punic Wars to the Fall of Rome. B.T. Batsford: London.

Birbeck, V. and Schuster, J. 2009. Living and Working in Roman and Later London:

Excavations at 60-63 Fenchurch Street. Wessex Archaeological Report 25.

Birley, A. 2002. Garrison Life at Vindolanda: A Band of Brothers. Tempus: Stroud.

Birley, A. \& Blake, J. 2005. Vindolanda Excavations: 2003-2004. Vindolanda Trust: Bardon Mill.

Birley, A. \& Blake, J. 2007. Vindolanda Excavations: 2005-2006. Vindolanda Trust: Bardon Mill.

Birley, R. 2009. Vindolanda: A Roman Frontier Fort on Hadrian's Wall. Amberley Publishing: Stroud.

Bishop, M. \& Dore, J. 1988. Corbridge: Excavations of the Roman fort and town 1947-1980. English Heritage Archaeological Report 8.

Blagg T. et al. 2004. Excavations at the large Romano-British settlement at Hacheston, Suffolk in 1973-4. East Anglian Archaeology Report 106.

Blaire, I. et al. 2006. "Wells and Bucket-chains: Unforeseen Elements of Water Supply in Early Roman London.”. Britannia 37. 1-52.

Bland, R. 1997. “Amersham, Buckinghamshire.” In Bland, R. \& Orna-Ornstein, J. (eds) Coin Hoards from Roman Britain: Volume X. British Museum Press: London. 410

Bland, R. \& Johns, C. 1979. “The 1973 Beachy Head Treasure Trove”. Numismatic Chronicle 139. $61-107$

Blockey, K. et al. 1995. Excavations in the Marlowe Car Park and Surrounding Areas. Canterbury Archaeological Trust: Canterbury. 
Booth, P. et al. 2010. The Late Roman Cemetery at Lankhills, Winchester: Excavations 20002005. Oxford Archaeology Monograph 10.

Boon, G. 1961. "Roman Antiquities at Welshpool”. Antiquaries Journal 41. 13-31

Boon, G. 1974. Silchester: The Roman Town of Calleva. David \& Charles Publishing: London.

Boon, G. 1988. "Review of Coventina's Well: a Shrine on Hadrian's Wall, by L. Allason-Jones and B. McKay". Britannia 19. 524-5

Boon, G. 1989. “A Roman Sculpture Rehabilitated: The Pagans Hill Dog”. Britannia 20. 201217

Bosanquet, R. \& Richmond, I. 1936. “A Roman Skillet from South Shields”. Archaeologia Aeliana 13. 139-151

Bowman, A. 1998. Life and Letters on the Roman Frontier. British Museum Press: London.

Bradley, R. 1990. The Passage of Arms: An archaeological analysis of prehistoric and votive deposits. Cambridge University Press: Cambridge.

Bradley, R. 2000. An Archaeology of Natural Places. Routledge: London.

Braun, C. 2001. Römische Bronzebalsamarien mit Reliefdekor. BAR International Series 917.

Breeze, D. 2006. The Antonine Wall. John Donald Publishing: Edinburgh.

Breeze, D. 2012. “Conclusions”. In Breeze, D. (ed.) 2012. The First Souvenirs: Enamelled Vessels from Hadrian's Wall. Cumberland and Westmorland Antiquarian and Archaeological Society: Kendal. 107-112

Breeze, D. (ed). 2012. The First Souvenirs: Enamelled Vessels from Hadrian's Wall. Cumberland and Westmorland Antiquarian and Archaeological Society: Kendal.

Breeze, D. \& Dobson, B. 1987. Hadrian's Wall $3^{\text {rd }}$ edition. Penguin Books: London.

Breeze, D. \& Edwards, B. 2012. "The Sphinx Crest in the Ribchester Hoard”. Antiquaries Journal 92. 65-69

Brindle, T. 2010. "The Portable Antiquities Scheme and Roman Rural Settlement: some preliminary work on Wiltshire". In Worrell, S. et al. (eds) A Decade of Discovery: Proceedings of the Portable Antiquities Scheme Conference 2007. BAR British Series 250. 121-133

Brindle, T. 2011. The Portable Antiquities Scheme and Roman Britain: An Evaluation of the Potential for using Amateur Metal Detector Data as an Archaeological Resource. Unpublished doctoral thesis: King's College London. 
Brodribb, A. et al. 2005. The Roman Villa at Shakenoak Farm, Oxfordshire Excavations 19601976. BAR British Series Monograph 395.

Brown, D. 1979. "Pewter Vessels". In Clarke, G. The Roman Cemetery at Lankhills. Winchester Studies 3.2.

Brown, L. 2008. "Charon's Obols? A Case Study in the Role of Coins in Roman Burial Ritual”. In Driessen, M et al. (eds) TRAC 2007: Proceedings of the seventeenth Theoretical Roman Archaeology Conference. Oxbow Books: Oxford. 121-130

Browne, D. 1976. "Excavations at Burwell, Cambridgeshire". Proceedings of the Cambridge Antiquarian Society 66. 81-92

Bruce-Mitford, R. \& Raven, S. 2005. A Corpus of Late Celtic Hanging-Bowls. Oxford University Press: Oxford.

Bruton, F. 1909. The Roman Fort at Manchester. Sherratt \& Hughes, Publishers to the Victoria University of Manchester: Manchester.

Buckland, P. \& Magilton, J. 1986. The Archaeology of Doncaster: Part 1- The Roman Civil Settlement. BAR British Series Monograph 148.

Burn, A. 1969. The Romans in Britain: An Anthology of Inscriptions with Translations and a Running Commentary. Basil Blackwell: Oxford.

Burnham, B. et al. 1994. "Roman Britain in 1993: Sites Explored”. Britannia 25. 245-292

Burnham, B. et al. 2001. "Roman Britain in 2000: Site Explored”. Britannia 32. 311-386

Burnham, B. et al. 2002. "Roman Britain in 2001: Sites Explored". Britannia 33. 275-354

Burnham, B. et al. 2003. "Roman Britain in 2002: Sites Explored”. Britannia 34. 293-359

Burnham, B. et al. 2004. "Roman Britain in 2003: Sites Explored”. Britannia 35. 253-316

Burnham, B. et al. 2005. "Roman Britain in 2004: Sites Explored". Britannia 36. 383-446

Burnham, B. et al. 2007. "Roman Britain in 2006: Sites Explored”. Britannia 38. 241-302

Burnham, B. et al. 2008. "Roman Britain in 2007: Sites Explored”. Britannia 39. 263-336

Bushe-Fox, J. 1926. First Report on the Excavation of the Roman Fort at Richborough, Kent. Reports of the Research Committee of the Society of Antiquaries of London 6. 
Bushe-Fox, J. 1928. Second Report on the Excavation of the Roman Fort at Richborough, Kent. Reports of the Research Committee of the Society of Antiquaries of London 7.

Bushe-Fox, J. 1932. Third Report on the Excavation of the Roman Fort at Richborough, Kent. Reports of the Research Committee of the Society of Antiquaries of London 10.

Bushe-Fox, J. 1949. Fourth Report on the Excavation of the Roman Fort at Richborough, Kent. Reports of the Research Committee of the Society of Antiquaries of London 16.

Callender, M. 1965. Roman Amphorae with Index of Stamps. Oxford University Press: London.

Carroll, M. 2012. “The Insignia of Women': Dress, Gender and Identity on the Roman Funerary Monument of Regina from Arbeia". The Archaeological Journal 169. 281-311

Caruana, I. 2004. “The Cemetery at Beckfoot Roman Fort”. In Wilson, R. \& Caruana, I. (eds)

Romans on the Solway: Essays in Honour of Richard Bellhouse. Cumberland and Westmorland Antiquarian \& Archaeological Society on behalf of the Trustees of the Senhouse Roman Museum, Maryport: Kendal.

Carver, E. 2001. The Visibility of Imported Wine and its Associated Accoutrements in Later Iron Age Britain. BAR British Series 325.

Casey, P. \& Hoffmann, B.1995a. "Excavations at Alstone Cottage, Caerleon". Britannia. Volume 26. 63-106

Casey, P. \& Hoffmann, B. 1995b. “Excavations on the Cordbridge Bypass, 1974” Archaeologia Aeliana 23. 17-46

Chaplin, R. \& Barnetson, L. 1980. “Animal Bones”. In Stead, I. Rudston Roman Villa. The Yorkshire Archaeological Society: Leeds. 149-161

Chaplin, R. \& McCormick, F. 1986. “The Animal Bones”. In Stead, I. \& Rigby, V. Baldock: The Excavations of a Roman and pre-Roman Settlement, 1968-1972. Britannia Monograph Series 7. $396-415$

Clarke, G. 1979. The Roman Cemetery at Lankhills. Winchester Studies 3.2.

Clay, P. \& Pollard, R. 1994. Iron Age and Roman Occupation in the West Bridge Area, Leicester: Excavations 1962-1971. Leicestershire Museums Arts \& Records Service: Leicester.

Collingwood, R. 1932. Roman Britain. Oxford University Press: London.

Collingwood, R. \& Taylor, M. 1927. "Roman Britain in 1926”. Journal of Roman Studies. 184219 
Collingwood, R. \& Wright, R. 1995. The Roman Inscriptions of Britain: Volume I. Alan Sutton Publishing: Stroud.

Collins, R. 2008. "Identity in the Frontier: Theory and Multiple Community Interfacing". In Driessen, M. et al. (eds) TRAC 2007: Proceedings of the Seventeenth Annual Theoretical Roman Archaeology Conference. Oxbow Books: Oxford. 45-52

Collins, R. 2010. "Brooch use in the $4^{\text {th }}-$ to $5^{\text {th }}$-century frontier". In Collins, R. and AllasonJones, L. (eds) Finds from the Frontier. CBA Research Report 162. 64-77

Cool, H. 2002. "An overview of the small finds from Catterick". In Wilson, P. et al. Cataractonium: Roman Catterick and its hinterland. Excavations and research, 1958-1997Part 2. CBA Research Report 129. 24-44

Cool, H. 2004. The Roman Cemetery at Brougham, Cumbria. Britannia Monograph Series 21.

Cool, H. 2006. Eating and Drinking in Roman Britain. Cambridge University Press: Cambridge.

Cool, H. 2007. "Telling Stories about Brougham, or the Importance of the Specialist Report". In Hingley, R. \& Willis, S. (ed) Roman Finds: Context and Theory. Oxbow Books: Oxford. 54-58

Cool, H. 2010a. "Finding the Foreigners". In Eckardt, H. (ed) Roman Diasporas:

Archaeological Approaches to Mobility and Diversity in the Roman Empire. JRA Supplemental Series 78. 27-44

Cool, H. 2010b. “A different life". In Collins, R. \& Allason-Jones, L. (eds) Finds from the Frontier. CBA Research Report 162. 1-9

Cool, H. \& Philo, C. 1998. Roman Castleford: Excavations 1974-1985 Volume I: The Small Finds. West Yorkshire Archaeological Service: Wakefield.

Cooper, N. 2007. "Promoting the Study of Finds in Roman Britain: Democracy, Integration and Dissemination". In Hingley, R. \& Willis, S. (eds) Roman Finds: Context and Theory. Oxbow Books: Oxford. 35-53

Cosh, S. \& Neal, D. 2002. Roman Mosaics of Britain: Volume I. Society of Antiquaries London: London.

Cosh, S. \& Neal, D. 2009. Roman Mosaics of Britain: Volume III. Part 2 Society of Antiquaries London: London.

Cosh, S. \& Neal, D. 2010. Roman Mosaics of Britain: Volume IV. Society of Antiquaries London: London. 
Cousins, E. 2014. "Votive Objects and Ritual Practice at the King's Spring at Bath". In Platts, H. et al. (eds) TRAC 2013: Proceedings of the Twenty-Third Theoretical Roman Archaeology Conference King's College, London 2013. Oxbow Books: Oxford. 52-64

Cowan, C. et al. 2009. Roman Southwark settlement and economy: Excavation in Southwark 1973-91. Museum of London Archaeology Monograph 42.

Cowen, J. \& Richmond, I. 1935. “The Rudge Cup”. Archaeologia Aeliana 12. 310-342

Cracknell, S. \& Mahany, C. 1994. Roman Alcester: Southern Extramural Area 1964-1966 Excavations Part 2: Finds and Discussion. CBA Research Report 97.

Cra’ster, M. 1970. “A hoard of Romano-British Bowls from Burwell, Cambridgeshire”. Antiquaries Journal 50. 344

Crummy, N. 1983. The Roman Small finds from Excavations in Colchester: 1971-1979. Colchester Archaeological Trust: Colchester.

Crummy, N. 2010. "Bears and Coins: The Iconography of Protection in Late Roman Infant Burials". Britannia 41. 37-93

Crummy, P. 1992. Colchester Archaeological Report 6: Excavations at Culver Street, the Gilberd School and other sites 1971-85. Colchester Archaeological Trust: Colchester.

Crummy, P. 1997. City of Victory: The Story of Colchester-Britain's first Roman town. Colchester Archaeological Trust: Colchester.

Crummy, P. et al. 2007. Stanway: An Elite Burial Site at Camulodunum. Britannia Monograph Series 24.

Cuff, D. 2011. "The King of the Batavians: Remarks on Tab. Vindol. III, 628”. Britannia 42. 145-156

Cunliffe, B. 1971. Excavations at Fishbourne: 1961-1969 Volume I: The Site. Reports of the Research Committee of the Society of Antiquaries of London 26.

Cunliffe, B. 1975. Excavations at Portchester Castle-Volume I: Roman. Reports of the Research Committee of the Society of Antiquaries of London 32.

Cunliffe, B. et al. 1988. The Temple of Sulis Minerva at Bath-Volume 2: The Finds from the Sacred Spring. Oxford University Committee for Archaeology Monograph 16.

Cunliffe, B. et al. 1996. Chichester Excavations IX: Excavations at Fishbourne 1969-1988. Chichester District Council: Chichester. 
Cunliffe, B. 2005. Iron Age Communities in Britain (Fourth Edition). Routledge \& Kegan Paul: London.

Curle, A. 1923. The Treasure of Traprain: A Scottish Hoard of Roman Silver Plate. Maclehose, Jackson and Company: Glasgow.

Curle, J. 1932. "Roman Drift in Caledonia”. Journal of Roman Studies 22. 73-77

Dalwood, H. \& Edwards, R. 2004. Excavations at Deansway, Worcester 1988-89: RomanoBritish small town to late medieval city. CBA Research Report 139.

Darling, M. \& Gurney, D. 1993. Caistor-on-Sea: Excavations by Charles Green 1951-1955. East Anglian Archaeology Report 60.

Darvill, T. 2010. Pre-Historic Britain. $2^{\text {nd }}$ edition. Routledge World Archaeology: London.

Davies, J. \& Jones, R. 2006. Roman Camps in Wales and the Marches. University of Wales Press: Cardiff.

den Boesterd, M. 1956. The Bronze Vessels in the Rijmuseum G. M. KAM at Nijmegen. Volume I. Provinciaal Museum G.M. Kam: Nijmegen.

De Santis, P. 2000. "Glass Vessels as grave goods and grave ornaments in the catacombs of Rome: some examples". In Pearce, J. et al. (eds) Burial, Society and Context in the Roman World. Oxbow Books: Oxford. 238-243

Dodd, P. \& Woodward, A. no date. Excavations at Slack 1913-1915. Yorkshire Archaeological Journal Supplement: York.

Dore, J. 2010. Haltonchesters: Excavation directed by J.P. Gillam at the Roman Fort, 1960-61. Oxbow Books: Oxford.

Douglas, A. et al. 2011. A Roman Settlement and Bath House at Shadwell: Excavations at Tobacco Dock and Babe Ruth Restaurant, The Highway, London. Pre-Construction Archaeology: London.

Dowden, K. 2000. European Paganism: The realities of cult from antiquity to the Middle Ages. Routledge: London.

Down, A. 1978. Chichester Excavations III. Chichester Civic Society Excavations Committee: Chichester.

Down, A. 1988. Roman Chichester. Phillimore \& Company Ltd.: Chichester.

Down, A. 1989. Chichester Excavations VI. Chichester Civic Society Excavations Committee: Chichester. 
Down, A. 1993. Chichester Excavations VIII. Chichester Civic Society Excavations Committee: Chichester.

Down, A. \& Henig, M. 1988. “A Roman askos handle from Fishbourne”. Antiquaries Journal 68. $308-310$

Drinkwater, J.F. 1978. "Rise and Fall of the Gallic Iulii”. Latomus 37. 817-850

Drummond-Murray, J. et al. 2002. Settlement in Roman Southwark: Archaeological Excavations (1991-1998) for the London Underground Limited Jubliee Line Extension Project. Museum of London Archaeological Service Monograph 12.

Dunbabin, K. 1999. Mosaics of the Greek and Roman World. Cambridge University Press: Cambridge.

Eckardt, H. 1999. "The Colchester 'Child's Grave”". Britannia 30. 57-90

Eckardt, H. \& Crummy, N. 2008. Styling the body in Late Iron Age and Roman Britain: a conceptual approach to toilet instruments. Monographies Instrumentum 36.

Eckardt, H. et al. 2009. "Roman Barrows and their Landscape Context: a GIS Case Study at Bartlow, Cambridgeshire”. Britannia 40. 65-98

Eckardt, H. et al. 2010. “A long way from home: diaspora communities in Roman Britain”. In Eckardt, H. (ed) Roman Diasporas: Archaeological Approaches to Mobility and Diversity in the Roman Empire. JRA Supplemental Series 78. 99-130

Edwards, B. 2000. The Romans at Ribchester: Discovery and Excavation. University of Lancaster Press: Lancaster.

Eggers, H. 1951. Der Römische Import im Freien Germanien. Hamburgisches Museum für Völkerkunde und Vorgeschichte: Hamburg.

Eggers, H. 1968. "Römische Bronzegefäße in Britannian”. Jarhbuch des Römische Germanischen ZentralMuseums Mainz 13 Jahrgang 1966. 67-164

Egglestone, W. 1915. "Two bronze inscribed skillets and a ladle, of Roman date, found in a peat bog in Upper Weardale”. Proceedings of Society of Antiquaries of Newcastle-Upon-Tyne 7. 911

Ellis, P. 2000. The Roman Baths and Macellum at Wroxeter. English Heritage Archaeological Report 9. 
Elsdon, S. 1997. Old Sleaford Revealed: A Lincolnshire settlement in Iron Age, Roman, Saxon and Medieval times: excavations 1882-1995. Oxbow Monograph 78. Nottingham Studies in Archaeology 2.

Erdrich, M. 1995. “Zu den Messingeimern vom Hemmoorer Typ: Verbreitung, Datierung und Herstellung”. Rom an der Niederelbe Katalog zur Austellung: Neumünster. 71-80

Espérandieu, E. 1913. Recueil Général des Bas-Relies, Statues et Bustes de la Gaulle Romaine.V. 5. Imprimerie Nationale: Paris.

Espérandieu, E. \& Rolland, H. 1959. Bronzes Antiques de la Seine-Maritime. Gallia Supplemental Series 8.

Evans, E. 2000. The Caerleon Canabae: Excavations in the Civil Settlement 1984-90. Britannia Monograph Series 16.

Farley, M. 1977. "Archaeological Notes from the Buckinghamshire County Museum”. Records of Buckinghamshire 20.3. 478-491

Farley, M. et al. 1988. "A Hoard of Late Roman Bronze Bowls and Mounts from the Misbourne Valley, near Amersham, Buckinghamshire". Britannia 19. 357-366

Ferris, I. et al. 2000 The Excavation of a Romano-British Shrine at Orton's Pasture, Rocester, Staffordshire. BAR British Series 314.

Ferris, I. 2010. The Beautiful Rooms are Empty: Excavations at Binchester Roman Fort, County Durham 1976-1981 and 1986-1991. Durham County Council: Durham.

Field, N. \& Pearson, M. 2003. Fisherton: An Iron Age Timber Causeway with Iron Age and Roman Votive Offerings: the 1981 Excavations. Oxbow Books: Oxford.

Finley, M. 1999. Ancient Economy. University of California Press: London.

Fitzpatrick, A. 2000. "Ritual, sequence, and structure in Late Iron Age Mortuary practices in North-West Europe". In Pearce, J. et al. (eds) Burial, Society and Context in the Roman World. Oxbow Books: Oxford. 15-29

Fitzpatrick, A. 2007. "The fire, the feast and the funeral: Late Iron Age mortuary practices in south-eastern England”. Revue du Nord. Hors Série Collection Art et Archéologie 11. 123-142

Forester R. et al. 1912. “Corstopitum: Report on the Excavations in 1911”. Archaeologia Aeliana 8. 137-263

Forester R. et al. 1913. "Corstopitum: Report on the Excavations in 1913”. Archaeologia Aeliana 9. 230-280 
Fowler, J. 1965. “A Roman Barrow at Knob’s Crook.” Antiquaries Journal 45. 22-52

Fox, A. 1961. “An Iron Age Bowl from Rose Ash, North Devon”. Antiquaries Journal 41. 18698

Froehner, W. 1865. La Colonne Trajane. Museé Impérial de Louvre: Paris.

Fulford, M. 2001. “Links with the Past: Pervasive 'Ritual' Behaviour in Roman Britain”.

Britannia 32. 199-218

Fulford, M. 2010. "Roman Britain: immigration and material culture”. In Eckardt, H. (ed)

Roman Diasporas: Archaeological Approaches to Mobility and Diversity in the Roman Empire. JRA Supplemental Series 78. 67-78

Fulford, M. \& Clarke, A. 2011. Silchester: City in Transition- The Mid-Roman Occupation of Insula IX c. A.D. 125-250/300. A Report on Excavations undertaken since 1997. Britannia Monograph Series 25.

Fulford, M. \& Timby, J. 2000. Late Iron Age and Roman Silchester: Excavations on the site of the Forum-Basilica 1977, 1980-86. Britannia Monograph Series 15.

Fulford, M. et al. 2006. Life and Labour in Late Roman Silchester: Excavations in Insula IX Since 1997. Britannia Monograph Series 22.

Freeman, P. 1997. "Mommsen to Haverfield: the origins of studies of Romanization in the late $19^{\text {th }}$-c. Britain". In Mattingly, D. (ed.) Dialogues in Roman Imperialism: Power, discourse, and discrepant experience in the Roman Empire. JRA Supplementary Series 23. 27-50

Frere, S. 1972. Verulamium Excavations Volume I. Reports of the Research Committee of the Society of Antiquaries of London 28.

Frere, S. 1983. Verulamium Excavations Volume II. Reports of the Research Committee of the Socitey of Antiquaries of London 41.

Frere, S. 1984. Verulamium Excavations Volume III. Oxford University Committee for Archaeology Monograph 1.

Frere, S. 1985. "Roman Britain in 1984: Sites Explored”. Britannia 16. 251-316

Frere, S. 1991. "Roman Britain in 1990: Sites Explored”. Britannia 22. 221-292

Frere, S. 2004. "The Roman Fort at Colwyn Castle, Powys (Radnorshire)". Britannia 35. 115120

Freund, R. 2004. Secrets of the Cave of Letters. Humanity Books: Amherst. 
Gage, J. 1832. "A plan of barrows called Bartlow Hills, in the parish of Ashdon, in Essex, with an account of Roman sepulchral relics recently discovered in lesser barrows". Archaeologia 25. $1-23$

Gardner, A. 2001. "Identities in the Late Roman Army: Material and Textual Perspectives." In Davies, G. et al. (eds) Proceedings of the Tenth Annual Theoretical Roman Archaeology Conference London 2000. Oxbow Books: Oxford.

Gardner, W. 1927. "Four Roman Bronze Vessels found at Glyn Dyfrdwy”. Archaeologia Cambrensis 7. 129-140

Gardner, W. \& Savoy, H. 1964. Dinorben: A Hill-Fort Occupied in Early Iron Age and Roman Times. The National Museum of Wales: Cardiff.

Garland, N. 2013. "Ritual Landscapes of Pre-Roman Britain: The Margins of Practice on the Margins of the Empire". In Bokern, A. et al. (eds) TRAC 2012: Proceedings of the TwentySecond Theoretical Roman Archaeology Conference, Frankfurt 2012. Oxbow Books: Oxford. 183-198

Gascoyne, A. \& Radford, D. 2013. Colchester: Fortress of the War God-An Archaeological Assessment. Oxbow Books: Oxford.

Germany, M. et al. 2003. Excavations at Great Holts Farm, Boreham, Essex, 1992-1994. East Anglian Archaeology Report 105.

Gerrard, J. 2005. “A Possible Late Roman Silver 'Hoard' from Bath”. Britannia 36. 371-373

Gerrard, J. 2009. “The Drapers' Gardens Hoard: A Preliminary Account”. Britannia 40. 163183

Gerrard, J. 2013. The Ruin of Roman Britain: An Archaeological Perspective. Cambridge University Press: Cambridge.

Gillian, D. 2008. “Other Copper Alloy Objects and Iron chain”. In Williams, M. \& Reid, M. (eds) Salt: Life and Industry- Excavations at King Street Middlewich, Cheshire, 2001-2002. BAR British Series 456. 43-46

Goodburn, R. 1979. "Roman Britain in 1978: Sites Explored”. Britannia 10. 267-338

Gould, J. 1964. Excavations at Wall (Staffordshire), 1961-3. On the site of the early Roman Forts and of the Late Roman Defenses. Lichfield and South Staffordshire Archaeological and Historical Society Transactions 1963-64 7 supplemental publication.

Grahame, M. 1998. "Material Culture and Roman Identity: The spatial layout of Pompeian houses and the problem of ethnicity". In Laurence, R. \& Berry, J. (eds) Cultural Identity in the Roman Empire. Routledge: London. 156-178 
Grant, Michael. 1995. Art in the Roman Empire. Routledge: London.

Gregory, A. 1976. "A Hoard of Roman Bronze Bowls from Burwell, Cambridgeshire”.

Proceedings of the Cambridge Antiquarian Society 66. 63-80

Gregory, R. 2007. Roman Manchester. Oxbow Books: Oxford.

Gregory, T. 1977. “A Hoard of Late Roman Metalwork from Weeting, Norfolk”. Norfolk Archaeology 36. 265-272

Green, M. 1998. "Vessels of Death: Sacred Cauldrons in Archaeology and Myth". Antiquaries Journal 78. 63-84.

Grew, F. 1980. "Roman Britain in 1979: Site Explored”. Britannia 11. 346-402

Grisell, L. 1961. "The Breaking of Objects as a Funerary Rite”. Folklore 72.3. 475-491

Guest, P. 1997. "Hoards from the End of Roman Britain". In Bland, R. \& Orna-Ornstein, J. (eds) Coins Hoards from Roman Britain: Volume X. British Museum Press: London. 411-423

Guest, P. et al. 1997. "Bishop's Cannings (Blagan Hill), Wiltshire”. In Bland, R. \& OrnaOrnstein, J. (eds) Coins Hoards from Roman Britain: Volume X. British Museum Press: London. 426-462

Hamerow, H. 2006. “ 'Special Deposits' in Anglo-Saxon Settlements”. Medieval Archaeology 50.1. $1-30$

Hands, A. 2004. The Romano-British Roadside Settlement at Wilcote, Oxfordshire: III. Excavations 1997-2000. BAR British Series 270.

Harbottle, B. 1995. "Prestwick Carr: Its Draining and Enclosure”. Archaelogia Aeliana 23. 115

Harcum, C. 1921. "Roman Cooking Utensils in the Royal Ontario Museum of Archaeology". The American Journal of Archaeology 25. 37-54

Harris, A. 2003. Byzantium, Britain \& the West: The Archaeology of Cultural Identity AD 400650. Tempus Publishing: Stroud.

Harris, A. \& Henig, M. 2010. "Hand-washing and Foot-washing, Sacred and Secular, in Late Antiquity and the Early Medieval Period”. In Henig, M. \& Ramsay, N. (eds) Intersections: The Archaeology and History of Christianity in England, 400-1200. BAR British Series 505. 25-38

Harris, W. 2011. Rome's Imperial Economy: Twelve Essays. Oxford University Press: Oxford. 
Hartley, B. 1971. "Roman York and the northern military command to the third century A.D.". in Butler, M. (ed) Soldier and Civilian in Roman Yorkshire. Leicester University Press:

Leicester. 55-70

Hassall, M. 1996. "London as a provincial capital”. In Bird, J., Hassal, M. \& Sheldon, H. (eds) Interpretting Roman London: Papers in memory of Hugh Chapman. Oxbow Monograph 58. 1926

Hassall, M. \& Tomlin, R. 1981. "Roman Britain in 1980: Inscrpitions”. Britannia 12. 369-396

Hassall, M. \& Tomlin, R. 1984. "Roman Britain in 1983: Inscrpitions". Britannia 15. 333-356

Hassall, M. \& Tomlin, R. 1985. "Roman Britain in 1984: Inscrpitions”. Britannia 16. 1985. 317-332

Hatzaki, E. 2009. "Structured Deposition as Ritual Action at Knossos". In Wiseman, J. \& Zachos, K. (eds) Landscape Archaeology in Southern Epirus, Greece I. Hesperia Supplements 42. $19-30$

Haverfield, F. 1910. Military Aspects of Roman Wales. Honourable Society of Cymmrodorion: London.

Haverfield, F. 1916. "Modius Claytonensis: The Roman Bronze Measure from Carvoran”. Archaeologia Aeliana 13. 85-102

Haverfield, F. 1923. The Romanization of Roman Britain (Fourth Edition). Oxford University Press: London.

Haverfield, F. 1924. The Roman Occupation of Britain. Oxford University Press: London.

Havis, R. \& Brooks, H. 2004. Excavations at Stansted Airport, 1986-91 Volume I: Prehistoric and Romano-British. East Anglian Archaeology Report 107.

Hawkes, C. 1951. "Bronze-workers, Cauldrons, and Bucket-Animals in Iron Age and Roman Britain". In Grimes, W. (ed.) Aspects of Archaeology in Britain and Beyond. H.W. Edwards: London. 172- 199

Haynes, I. 2000. "Religion in Roman London". In Haynes, I. et al. (eds) London Underground: The Archaeology of a City. Oxbow Books: Oxford. 85-101

Hegel, G. 1956. The Philosophy of History. J. Sibree (tr.). Dover Publications: London.

Henig, M. 1972. “The Origin of Some Ancient British Coin Types”. Britannia 3. 209-223

Henig, M. 1984. Religion in Roman Britain. B.T. Batsford: London. 
Henig, M. 1995. The Art of Roman Britain. B.T. Batsford: London.

Heurgon, J. 1951. "The Amiens Patera". Journal of Roman Studies 41. 22-24

Hill, J. 1993. "Can we recognize a different European past? A contrastive archaeology of late Prehistoric settlements in Southern England". Journal of European Archaeology 1. 57-75

Hill, J. 1995a. "The Pre-Roman Iron Age in Britain and Ireland (ca. 800 B.C. to A.D. 100): An Overview". Journal of World Prehistory 9. 47-98

Hill, J. 1995b. Ritual and Rubbish in the Iron Age of Wessex: A Study on the Formation of a Specific Archaeological Record. BAR British Series 242.

Hill, J. 1996. "The identification of ritual deposits of animals. A general perspective from a specific study of 'special animal deposits' from the Southern English Iron Age". Ritual Treatment of Human and Animal Bones: Proceedings of the first meeting of the Osteoarchaeological Research Group held in Cambridge on $8^{\text {th }}$ October 1994. Oxbow Books: Oxford. 17-32

Hill, J. et al. 2004. "The Winchester Hoard: A Find of Unique Iron Age Gold Jewellery from Southern England". The Antiquaries Journal 84. 1-22

Hill, J. \& Woodger, A. 1999. Excavations at 72-75 Cheapside/83-93 Queen Street City of London. Museum of London Archaeological Service Archaeological Studies Series 2.

Hiller, J \&Wilkinson, D. 2005. Archaeology of the Jubilee Line Extension: Prehistoric and Roman Activity at Stratford Market Depot West Ham, London 1991-1993. Museum of London Archaeology Service: London.

Hingley, R. 1982. "Roman Britain: The Structure or Roman Imperialism and the Consequences of Imperialism on the Development of a Peripheral Province". In Miles, D. (ed.) The RomanoBritish Countryside: Studies in Rural Settlement and Economy. BAR British Series 103(i). 1752

Hingley, R. 1990. "Iron Age 'currency bars': The archaeological and social context". Archaeological Journal 147. 91-117

Hingley, R. 1997. "Resistance and domination in Roman Britain". In Mattingly, D. (ed.) Dialogues in Roman Imperialism: Power, discourse, and discrepant experience in the Roman Empire. JRA Supplementary Series 23. 81-102

Hingley, R. 2005. Globalizing Roman Culture: Unity, diversity and empire. Routledge: London.

Hingley, R. 2006. "The Deposition of Iron Objects in Britain during the Later Prehistoric and Roman Periods: Contextual Analysis and the Significance of Iron”. Britannia 37. 213-257 
Hingley, R. 2008. The Recovery of Roman Britain: 1586-1906. Oxford University Press: Oxford.

Hobbs, R. 2006. Late Roman Precious Metal Deposits c. AD 200-700:Changes over time and space. BAR International Series 1504.

Hobbs, R. 2012. The Mildenhall Treasure. British Museum Press: London.

Holbrook, N. \& Bidwell, P. 1991. Roman Finds from Exeter. Exeter Archaeological Reports 4.

Holbrook, N. et al. 1998. Cirencester Excavations V: Cirencester: The Roman Town Defense, Public Buildings and Shops. Cotswold Archaeological Trust: Cirencester.

Holder, P. 2012. "The Inscriptions on the Vessels.” In Breeze, D. (ed.) 2012. The First Souvenirs: Enamelled Vessels from Hadrian's Wall. Cumberland and Westmorland Antiquarian and Archaeological Society: Kendal. 65-70

Hopewell, D. 2009. G1632 Roman Military Sites 2008: The Roman Road between Segontium and Aber. Gwynedd Archaeological Trust Report 779.

Howatson, M. 1989. The Oxford Companion to Classical Literature. Oxford University Press: Oxford.

Howe, E. \& Lakin, D. 2004. Roman and medieval Cripplegate, City of London, Archaeological Excavations 1992-1998. Museum of London Archaeological Services Monograph 21.

Hudson, N. 2010. "Changing Places: The Archaeology of the Roman Convivium". American Journal of Archaeology 114. 663-695.

Hunter, F. 2001. "Roman and Native in Scotland: New Approaches". Journal of Roman Studies 14. 289-309.

Hunter, F. 2012. "Frontier Finds, Frontier Art- Views of Enamelled Vessels". In Breeze, D. (ed) The First Souvenirs: Enamelled Vessels from Hadrian's Wall. Cumberland and Westmorland Antiquarian and Archaeological Society. Kendal. 85-106.

Hurcombe, L. 2007. Archaeological Artefacts as Material Culture. Routledge: New York.

Hurst, D. et al. 2006. Roman Droitwich: Dodderhill fort, Bays Meadow villa and roadside settlement. CBA Research Report 146.

Huskinson, J. 2000a. "Looking for culture, identity and power". In Huskinson, J. (ed) Experiencing Rome: Culture, Identity and Power in the Roman Empire. Routledge in association with The Open University: London. 3-28 
Huskinson, J. 2000b. "Élite culture and the identity of empire". In Huskinson, J. (ed) Experiencing Rome: Culture, Identity and Power in the Roman Empire. Routledge in association with The Open University: London. 95-124

Hutchinson, V. 1986. Bacchus in Roman Britain: The Evidence for his cult. BAR British Series 151 (i).

Hutton, R. 1991. The Pagan Religions of the Ancient British Isles: Their Nature and Legacy. Blackwell Press: Oxford.

Hutton, R. 2011. "Romano-British Reuse of Prehistoric Ritual Sites". Britannia 42. 1-22

Ingate, J. 2013. "Hybrid Bridges: An Explorations into how Traditionally 'Romanised' Elements of the Town Interacted with Meaning-Laden Pre-Historic Waterscapes". In Bokern, A. et al. (eds) TRAC 2012: Proceedings of the Twenty-Second Theoretical Roman Archaeology Conference, Frankfurt 2012. Oxbow Books, Oxford. 133-150

Isserlin, R. 2007. “Deposit of Withdrawal?”. In Hingley, R. \& Willis, S. (eds) Roman Finds: Context and Theory. Oxbow Books: Oxford. 186-198

Jackson, R. 2007. "The Senuna Treasure and Shrine at Ashwell". Continuity and Innovation in Religion in the Roman West: Volume I. JRS Supplemental Series 67. 37-54

Jackson, R. \& Craddock, P. 1995. "The Ribchester Hoard: A Descriptive and Technical Study". In Rafterty, B. et al. (eds) Site and Sights of the Iron Age. Oxbow Monograph 56. 75-102

James, H. 2003. Roman Carmarthen: Excavations 1978-1993. Britannia Monograph Series 20.

Jarrett, M. \& Wrathmell, S. 1981. Whitton: An Iron Age and Roman Farmstead in South Glamorgan. University of Wales Press: Cardiff.

Jessup, R. 1930. The Archaeology of Kent. Metheun \& Company Limited: London.

Jessup, R. 1958. "Barrows and Walled Cemeteries in Roman Britain". Journal of the Archaeological Association 22. 3

Jiménez, A. 2008. "A Critical Approach to the Concept of Resistance: New 'Traditional' Rituals and Objects in Funerary Contexts of Roman Baetica". In Driessen, M. et al. (eds) TRAC 2007: Proceedings for the seventeenth Theoretical Roman Archaeology Conference. Oxbow Books: Oxford. 15-30

Johns, C. 1993. "An enamelled bronze 'pyxis' from a Roman grave at Elsenham, Essex". The Antiqauries Journal 73. 161-165

Johns, C. 1996. "The Classification and Interpretation of Romano-British Treasures". Britannia 27. $1-15$ 
Johns, C. 2003. “Art, Romanization, and Competence”. In Scott, S. \& Webster, J. (eds) Roman Imperialism and Provincial Art. Cambridge University Press: Cambridge. 9-23

Johns, C. 2007. "The Last Chance”. In Hingley, R. \& Willis, S. (eds) Roman Finds: Context and Theory. Oxbow Books: Oxford. 29-34

Johns, C. 2010. The Hoxne Late Roman Treasure: Gold Jewellery and Silver Plate. British Museum Press: London.

Jones, A. et al. 2003. Settlement, Burial and Industry in Roman Godmanchester. BAR British Series 346.

Jones, C. \& Sherlock, D. 1996. "Early decorated spoons from London”. In Bird, J. et al. (eds) Interpreting Roman London. Oxbow Books: Oxford. 165-176

Kendrick, T. 1932. "British Hanging-Bowls”. Antiquity. 6. 161-184

Kennett, D. 1970. “The Felmersham Fish-Head Spout: A Suggested Reconstruction”. Antiquaries Journal 50. 86-88

Kennett, D. 1971. "Late Roman Bronze Vessel Hoards in Britain” Jahrbuch des RömischGermanischen Zentralmuseums Mainz 16 Jarhgang, 1969. 123-148

Kenyon, K. M. 1948. Excavations at the Jewry Wall Site, Leicester. Oxford University Press: Oxford.

King, A. 2013. Coins and Smaian Ware: A study of the dating of coin-loss and the deposition of samian ware (terra sigillata), with a discussion of the decline of samian ware manufacture in the NW provinces of the Roman Empire, late $2^{\text {nd }}$ to mid $3^{\text {rd }}$ centuries $A D$. BAR International Series 2573.

Kohlert-Németh, M. 1990. Archäologische Reihe Römische Bronzen II aus Nida-Hedderheim Fundsachen aus dem Hausrat. Museum fur Vor-und Frühgeschichte Archäologisches MuseumFrankfurt am Main: Frankfurt.

Koster, A. 1997. Description of the Collections in the Provincial Museum G.M. Kam at Nijmegen: The Bronze Vessels 2. Provinciaal Museum G.M. Kam: Nijmegen.

Künzl, E. et al. 1993. Die Alamannenbeute aus dem Rhein bei Neupotz Teil 1: Untersuchungen. Römisch-Germanischen Zentralmuseums: Mainz.

Künzl, E. 2012. "Enamelled vessels of Roman Britain”. In Breeze, D. (ed) The First Souvenirs: Enamelled Vessels from Hadrian's Wall. D. Cumberland and Westmorland Antiquarian and Archaeological Society: Kendall. 9-22 
Laing, J. 2000. Art \& Society in Roman Britain. Wrens Park Publishing: Stroud.

Laing, L. 2006. The Archaeology of Celtic Britain and Ireland c. AD 400-1200. Cambridge University Press: Cambridge.

Lambert, J. 1996. Transect through time. The archaeological landscape of the Shell northwestern ethylene pipeline. Lancaster Imprints 1.

Laurence, R. 1998. "Territory, Ethnonyms and Geography: The Construction of identity in Roman Italy". In Laurence, R. \& Berry, J. (eds) Cultural Identity in the Roman Empire. Routledge: London. 95-110

Lawerence, S. \& Smith, A. 2009. Between Villa \& Town: Excavations of a Roman roadside settlement and shrine at Higham Ferrers, Northamptonshire. Oxford Archaeology Monograph 7.

Leary, J. \& Butler, J. 2012. Roman Archaeology in the Upper Reaches of the Walbrook Valley: Excavations at 6-8 Tokenhouse Yard, London EC2. Pre-Construction Archaeology Limited Monograph 14.

Lee, R. 2009. The Production, Use and Disposal of Romano-British Pewter Tableware. BAR British Series 478.

Leech, R. 1981. "The Excavation of a Romano-British Farmstead and Cemetery on Bradley Hill, Somerton, Somerset”. Britannia 12. 177-252

Leech, R. 1982. "The Roman Interlude in the South-West: The Dynamics of Economic and Social Change in Romano-British South Somerset and North Dorset". In Miles, D. (ed) The Romano-British Countryside: Studies in Rural Settlement and Economy. BAR British Series 103(i). 209-268.

Leeds, E. 1936. Early Anglo-Saxon Art and Archaeology. Oxford University Press: London.

Liversidge, J. 1953. "The Thornborough Barrow”. Records of Buckinghamshire 16.1. 29-32

Liversidge, J. 1977. "Roman burials in the Cambridge Area". Proceedings of the Cambridge Antiquarian Society 67. 11-38

Lomas, K. et al. 2013. "Creating Ethnicities and Identities in the Roman World". Creating Ethnicities and Identities in the Roman World. Institute of Classical Studies, School of Advanced Study, University of London: London. 1-8

Longley, D. et al. 1988. "Excavations on Two Farms of the Romano-British Period at Bryn Eryr and Bush Farm, Gwynedd". Britannia 29. 185-246 
Lyons, C. \& Papadopoulos, J. 2002. “Archaeology and Colonialism”. In Lyons, C. \& Papadopoulos, J. (eds) The Archaeology of Colonialism. The Getty Research Institute: Los Angeles. 1-26

MacDonald, G. \& Park, A. 1906. The Roman Forts on The Bar Hill Dumdartonshire. James Maclehose \& Sons: Glasgow.

Mackreth, D. 2011. Brooches in Late Iron Age and Roman Britain: Volume I. Oxbow Books: Oxford.

Malbon, E. 1990. The Iconography of the Sarcophagus of Junius Bassus. Princeton University Press: Princeton.

Malkin, I. 2002. "A Colonial Middle Ground: Greek, Etruscan, and Local Elites in the Bay of Naples". In Lyons, C. \& Papadopoulos, J. (eds) The Archaeology of Colonialism. The Getty Research Institute: Los Angeles. 151-181

Mango, M. et al. 1989. "A $6^{\text {th }}$-century Mediterranean bucket from Bromeswell Parish, Suffolk". Antiquity 63. 295-311.

Manley, J. \& Rudkin, D. 2003. Facing the Palace: Excavations in front of the Roman Palace at Fishbourne. Sussex Archaeological Collections 141.

Manning, W. 1995. Report on the Excavations at Usk 1965-1976: The Roman Small Finds. University of Wales Press: Cardiff.

Manning, W. 2001. A Pocket Guide: Roman Wales. University of Wales: Cardiff.

Margary, I. 1967. Roman Roads in Britain. John Baker Publishers: London.

Marvell, A. \& Owen-John H. 1997. Leucarum: Excavations at the Roman Auxiliary Fort at Loughor, West Glamorgan 1982-84 and 1987-88. Britannia Monographs Series 12.

Mattingly, D. 1997. “Africa: A Landscape of Opportunity?”. In Mattingly, D. (ed) Dialogues in Roman Imperialism: Power, discourse, and discrepant experience in the Roman Empire. JRA Supplemental Series 23. 117-135

Mattingly, D. 2006. An Imperial Possession: Britain in the Roman Empire 54 BC-AD 409. Penguin Publishing: London.

Mattingly, D. 2011. Imperialism, Power, and Identity: Experiencing the Roman Empire. Princeton University Press: Princeton.

Mawer, C. 1995. Evidence for Christianity in Roman Britain: The small-finds. BAR British Series 243. 
May, S. 2007. “The Cremated Human Remains". In Crummy, P. et al. (eds) Stanway: An Elite Burial Site at Camulodunum. Britannia Monograph Series 24. 377-381

May, T. 1922. The Roman Forts of Templeborough near Rotherham. The County Borough of Rotherham: Rotherham.

May, T. 1930. Catalogue of the Roman Pottery in the Colchester and Essex Museum. Cambridge University Press: Cambridge.

McCarthy, M. 1990. A Roman, Anglican and Medieval Site at Blackfriars Street, Carlisle:

Excavations 1977-1979. Cumberland and Westmorland Antiquarian and Archaeological Society Research Series 4.

McCarthy, M. 2000. Roman and Medieval Carlisle: The Southern Lanes. Carlisle Archaeology Limited. Research Report 1.

McDonald, G. 1912. “The Corbridge Gold Find of 1911”. Journal of Roman Studies 2. 1-20

McIntosh, F. 2011. "Regional brooch-types in Roman Britain: Evidence from Northern England”. Archaeologia Aeliana Fifth Series 40. 155-182

McKinley, J. 2000. "Phoenix Rising: Aspects of Cremation in Roman Britain". In Pearce, J. et al. (eds) Burial, Society and Context in the Roman World. Oxbow Books: Oxford. 38-44

McPeake, J. \& Moore, C. 1978. "A Bronze Skillet-Handle from Chester and other vessels from the British Isles". Britannia 9. 331-334

McSloy, E. \& Watts, M. 2013. "Excavation at Bridges Garage, Tetbury Road, Cirencester: Interim Report”. Glevensis 46. 1-5

McWhirr, A. 1986. Cirencester Excavations III: Houses in Roman Cirencester. Cirencester Excavation Committee: Cirencester.

Meates,G. 1955. Lullingstone Roman Villa. William Heinemann Limited: London.

Meates, G. 1979. The Roman Villa at Lullingstone, Kent Volume I: The Site. Kent Archaeological Society Monograph Series 1.

Meates, G. 1987. The Roman Villa at Lullingstone, Kent Volume II: The Site: The Wall Paintings and Finds. Kent Archaeological Society Monograph Series 3.

Megaw, J. 1963. "A British Bronze Bowl of the Belgic Iron Age from Poland." Antiquaries Jounral 43. 27-37

Menzel, H. 1986. Die Römischen Bronzen aus Deutchland III: Bonn. Philipp von Zabern: Mainz am Rhein. 
Merrifield, R. 1983. London: City of the Romans. B T Batsford Limited: London.

Merrifield, R. 1987. The Archaeology of Ritual and Magic. B.T. Batsford Limited: London.

Metcalf, P. \& Huntington, R. 1991. Celebrations of Death: The Anthropology of Mortuary Ritual. Cambridge University Press: Cambridge.

Miket, R. 1983. The Roman Fort at South Shields: Excavations of the Defenses 1977-1981. Tyne and Wear County Council Museums: Gateshead.

Miles, R. 2000. “Communicating culture, identity and power”. In Huskinson, J. (ed.) Experiencing Rome: Culture, Identity and Power in the Roman Empire. Routledge in association with The Open University: London. 29-62

Millett, M. 1979. "An Approach to the Functional Interpretation of Pottery" in Pottery and the Archaeologist ed. Martin Millett. Institute of Archaeology Occasional Publication 4. 35-48

Millett, M. 1990. The Romanization of Britain: An essay on Archaeological Interpretation. Cambridge University Press: Cambridge.

Millett, M. 1995. Roman Britain. B.T. Batsford/ English Heritage: London.

Millett, M. 1996. “Characterizing Roman London”. In Bird, J., Hassal, M. \& Sheldon, H. (ed.) Interpretting Roman London: Papers in memory of Hugh Chapman Oxbow Monograph 58. 3338

Milne, G. 1985. The Port of Roman London. B.T. Batsford Limited: London.

Moore, C. 1978. "An Enamelled Skillet-Handle from Brough-on-Fosse and the distribution of similar vessels". Britannia 9. 319-327

Moorhead, S. 2010a. The Frome Hoard. British Museum Press: London.

Moorhead, S. 2010b. "Expanding the Frontiers: how the Portable Antiquities Scheme database increases knowledge of Roman coin use in England". In Worrell, S. et al. (eds) A Decade of Discovery: Proceedings of the Portable Antiquities Scheme Conference 2007. BAR British Series 250. 148-160

Moorhead, S. 2013. A History of Roman Coinage in Britain. Greenlight Publishing: Witham.

Morris, I. 1992. Death-ritual and social structure in classical antiquity. Cambridge University Press: Cambridge.

Morris, J. 1982. LONDINIVM: London in the Roman Empire. Book Club Associates: London. 
Mynott,E. 2008. "Early Roman Graves Discovered at Tollgate". Kent Archaeological Review 171.

Nash-Williams, V. 1954. The Roman Frontier in Wales. University of Wales Press: Cardiff.

Neal, D. 1996. Excavations on the Roman Villa at Beadlam, Yorkshire. Yorkshire Archaeological Report 2.

Needham, S. 1988. "Selective Deposition in the British Early Bronze Age". World Archaeology 20.2. $229-248$

Nenova-Merdjanova, R.2002. "Bronze vessels and the toilette in Roman times". Mattusch C. et al. (eds) From the Parts to the Whole Volume 2: Acta of the $13^{\text {th }}$ International Bronze Conference held at Cambridge, Massachusetts, May 28-June 1, 1996. Journal of Roman Archaeology Supplementary Series 39. 200-204

Newman, J. 1963. "Ennius the Mystic I: The Terms of Debate" Greece and Rome 10. 132-139

Niblett, R. 1985. Sheepen: an Early Roman industrial site at Camulodunum. CBA Research Report 57.

Niblett, R. 1999. Excavation of a Ceremonial Site at Folly Lane, Verulamium. Britannia Monograph Series 14.

Niblett, R. 2000. "Funerary Rites in Verulamium during the Early Roman Period". In Pearce, J. et al. (eds) Burial, Society and Context in the Roman World. Oxbow Books: Oxford. 97-104

Niblett, R. \& Reeves, P. 1990. “A Wealthy Early Roman Cremation from Verulamium”. The Antiquaries Journal Volume 70. 441-446

Niblett, R. \& Thompson, I. 2005. Alban's Buried Towns: An Assessment of St. Albans' Archaeology up to AD 1600. Oxbow Books: Oxford.

Nuber, H. 1973. "Kanne und Griffschale. Ihr Gebrauch im täglichen Leben und die Beigabe in Gräbern der römischen Kaiserzeit”. Bericht der Römisch-Germanischen Kommission 1972. 1232

O’Leary, T. et al. 1989. Pentre Farm, Flint 1976-81: An official building in the Roman lead mining district. BAR British Series 207.

Ochota, M. 2013. Britain's Secret Treasures. Headline Publishing Group: London.

Osbounre, R. 2004. "Hoards, Votives, Offerings: The Archaeology of the Dedicated Object". World Archaeology 36.1. 1-10 
Oswald, A. 1939. "A Roman Skillet from Broxtowe, Nottinghamshire". Antiquaries Journal 19. 441

Ottaway, P. 1992. Archaeology in British Towns: From the Emperor Claudius to the Black Death. Routledge: London.

Ottaway, P. 1993. Roman York. Tempus Publishing: Stroud.

Ottaway, P. et al. 2012. The Roman Cemeteries and Suburbs of Winchester: Excavations 1971-86. Winchester Museums: Winchester.

Painter, K. 1977. The Mildenhall Treasure: Roman Silver from East Anglia. British Museum Publications: London.

Panagopoulou, K. 2007. "Between Necessity and Extravagance: Silver as a Commodity in the Hellenistic Period". Annual of the British School at Athens, 102. 315-343

Partridge, C. 1981. Skeleton Green: A Late Iron Age and Romano-British Site. Britannia Monograph Series 2.

Payne, G. 1877. "Roman Interment Discovered at Sittingbourne”. Archaelogia Cantiana 11. 47-48

Peacock, D. \& Williams, D. 1986. Amphorae and the Roman Economy: An Introductory Guide. Longmann Publishing: London.

Pearce, J. 2000. "Burial, Society and context in the Provincial Roman World". In Pearce, J. et al. (eds) Burial, Society amd Context in the Roman World. Oxbow Books: Oxford. 1-8

Pearce, J. 2008. "Burial evidence from Roman Britain: the un-numbered dead." In Scheid, J. (ed) Pour une archéologie du rite. Nouvelles perspectives de l'archéologie funéraire. Collection de L'École française de Rome 47. 29-42.

Pearce, J. 2010. "Burial, Identity and migration in the Roman World". In Eckardt, H. (ed) Roman Diasporas: Archaeological Approaches to Mobility and Diversity in the Roman Empire. JRA Supplemental Series 78. 79-98

Pearce, J. (forthcoming). "A 'civilised' death? The interpretation of provincial Roman grave good assemblages". in Brandt, J. et al. (eds) Death and Changing Rituals. Function and Meaning in Ancient Funerary Practices. Oxbow Books: Oxford.

Pearce, J. et al. (eds). 2000 Burial, Society amd Context in the Roman World. Oxbow Books: Oxford.

Peña, J. 2007. Roman Pottery in the Archaeological Record. Cambridge University Press: Cambridge. 
Pernice, E. 1900. "Bronzen aus Boscoreale”. Jahrbuch des Kaiserlich Deutchen Archäologischen Institutes, Archäologischer Anzeiger Beiblatt 15. 177-198

Perring, D. 1991. Roman London. Seaby: London.

Petts, D. 1998. "Landscape and Cultural Identity in Roman Britain”. In Laurence, R. \& Berry, J. (eds) Cultural Identity in the Roman Empire. Routledge: London. 79-94

Petts, D. 2009. “Burial on Hadrian's Wall”. In Symonds, M. \& Mason, D. (eds) Frontiers of Knowledge: A Research Framework for Hadrian's Wall. Durham County Council: Durham. 160-162

Philpott, R. 1991. Burial Practices in Roman Britain: A Survey of the Grave Treatment and Furnishing A.D. 43-410. BAR British Series Monograph 219.

Pinto, R. \& Pinto, L. 2013. "Transgendered Archaeology: The Galli and the Catterick Transvestite". In Bokern, A. et al. (eds) TRAC 2012: Proceedings of the Twenty-Second Theoretical Roman Archaeology Conference Frankfurt 2012. Oxbow Books: Oxford. 169-183

Pisano, F. 2008. Hic Sunt Delphini: La Singolare Propagazione del Simbolo del Delfino nei Flegrei in Etá Antica. Antonio Pisano: Pozzuoli.

Pitts, M. 2005. "Pots and Pits: Drinking and Deposition in Late Iron Age South-East Britain". Oxford Journal of Archaeology 24.2. 143-166

Pitts, M. 2007. “The Emperor's New Clothes? The Utility of Identity in Roman Archaeology”. American Journal of Archaeology 111. 693-713

Pollard, J. 2001. "The Aesthetics of Depositional Practice”. World Archaeology 33.2. 315-333

Potter, T. 1979. Romans in North-West England. Excavations at the Roman Forts of Ravenglass, Watercrook and Bowness on Solway. Cumberland and Westmorland Antiquities and Archaeological Society Research Series 1.

Potter, T. \& Johns, C.1992. Roman Britain. British Museum Press: London.

Potter, T. \& Trow, S. 1988. Puckeridge-Braughing, Hertfordshire: The Ermine Street Excavations 1971-72. Hertfordshire Archaeology Monograph 10.

Price, J. 2010“Late Roman glass vessels in the Hadrian's Wall frontier region”. In Collins, R. \& Allason-Jones, L. (eds) Finds from the Frontier. CBA Research Report 162. 37-49

Prior, J. 2014. "Methods and Difficulties in Quantifying Archaeological Vessel Glass Assemblages". In Platts, H. et al. (eds) TRAC 2013: Proceedings of the Twenty-Third 
Theoretical Roman Archaeology Conference King's College, London 2013. Oxbow Books: Oxford. 105-121

Radnoti, A. 1938. Die Römischen Bronzegefässe von Pannonien. Institut für Münzkunde und Archäologie der P. Pázmány-Universität: Budapest.

Rankov, N. 1982. "Roman Britain in 1981". Britannia 13. 328-395

Ratliff, M. 2011. "Gloabisation, Consumerism and the Ancient Roman Economy". In

Mladenovíc, D. \& Russel, B (eds) TRAC 2010: Proceeding of the Twentieth Annual Theoretical Roman Archaeology Conference. Oxbow Books: Oxford. 32-46

Reitler, R. 1949. "A Theriomorphic Representation of Hekate-Artemis". American Jounral of Archaeology 53. 29-31

Renard, M. 1959. “Technique et agriculture en pays trévire et rémois”. Latomus 38. 307-333

Richards, C. \& Thomas, J. 1984. "Ritual Activity and Structured Deposition in Later Neolithic Wessex". In Bradley, R. \& Gardiner, J. (eds) Neolithic Studies: A Review of Some Current Research. BAR British Series 133. 189-218

Richmond, I. 1968. Hod Hill Volume II: Excavations carried out between 1915 and 1958 for the trustees of The British Museum. British Museum Press: London.

Ridgeway, V. et al. 2009. Secrets of the gardens : archaeologists unearth the lives of Roman Londoners at Drapers' Gardens. Pre-Construction Archaeology: Brockley

Riha, E. 2001. Forschugen in Augst Band 31: Kästchen, Truhen, Tische-Möbelteile aus Augusta Raurica. Römermuseum: Augst.

Rissman, P. 1988. "Public Displays and Private Values: A Guide to Buried Wealth in Harappan Archaeology". World Archaeology 20.2. 209-228

Riz, A. 1990. Bronzegefässe in der Römish-Pompejanischen Wandmalerei. Deutsches Archäologisches Institut Rom Sonderschriften Band 7.

Robertson, A. 2000. An Inventory of Romano-British Coin Hoards. Royal Numismatic Society Special Publication 20.

Rodwell, K. 1988. The prehistoric and Roman settlement at Kelvedon, Essex. CBA Report 63.

Rodwell, W. 1972. “The Roman Fort at Great Chesterford, Essex”. Britannia 3. 290-293

Rodwell, W. 1978. "Rivenhall and the emergence of first-century villas in northern Essex". In Todd, M. (ed) Studies in the Romano-British Villa. Leicester University Press: Leicester. 11-32 
Rodwell W. \& Rodwell, K. 1985. Rivenhall: Investigations of a Roman villa, church and village, 1950-77. CBA Research Report 55.

Rodwell, W. \& Rodwell, K. 1993. Rivenhall: Investigations of a Roman villa, church and village, 1950-77 Volume II. CBA Research Report 80.

Ross, A. 1967. Pagan Celtic Britain: Studies in Iconography and Tradition. Routledge and Kegan Paul Press: London.

Roymans, N. 1996. "The sword or the plough: Regional dynamics in the romanisation of Belgic Gaul and the Rhineland area" in Roymans, N. (ed) From the Sword to the Plough: Three studies on the Earliest Romanisation of Northern Gaul. Amsterdam University Press: Amsterdam. 9-103

Rushworth, A. 2009. Housesteads Roman Fort- The Grandest Station: Excavation and Survey at Housesteads, 1954-95, by Charlse Daniels, John Gillam, James Crow and others. English Heritage Archaeological Reports.

Sandars, N. 1971. "Orient and Orientalizing in Early Celtic Art”. Antiquity 45. 103-112.

Scheid, J. 2008. "En guise de prologue : de l'utilisation correcte des sources écrites dans l'étude des rites funéraires". In J. Scheid (ed) Pour une archéologie du rite. Nouvelles perspectives de l'archéologie funéraire. Collection de L'École française de Rome 47. 5-8

Schiffer, M. 1972. "Archaeological Context and Systemic Context". American Antiquity 37. 156-165.

Scholz, H. 1937. Der Hund in der Griechisch-Römischen Magie und Religion. Unpublished doctoral thesis. University of Berlin: Berlin.

Score, V. 2011. Hoards, Hounds and Helmets: A conquest-period ritual site at Hallaton, Leicestershire. Leicester Archaeology Monograph 21.

Scott, S. 2003. "Provincial Art and Roman Imperialism: An Overview". In Scott, S. \& Webster, J. (eds) Roman Imperialism and Provincial Art. Cambridge University Press: Cambridge. 1-8

Scott Robinson, W.1883. "Traces of Roman Occupation in and near Maidstone". Archaeologia Cantiana 15. 68-80

Sen, A. 2006. Identity and violence: The illusion of destiny. W.W. Norton: New York.

Sergis, M. 2010. "Dog Sacrifice in Ancient and Modern Greece: from the sacrifice ritual to dog torture (kynomartyrion)". Folklore: Electronic Journal of Folklore 45. 61-88

Shafer-Elliot, C. 2013. Food in Ancient Judah: Domestic Cooking in the Time of the Hebrew Bible. Equinox Publishing: Sheffield. 
Skilbeck, C. 1923. "Notes on the Discovery of a Roman Burial at Radnage, Bucks." Antiquaries Journal 3. 334-337.

Smith, D. 1962. "The Shrine of the Nymphs and the Genius Loci at Carrawburgh". Archaeologia Aeliana 11. 59-81

Smith, J. 1999. "Votive Objects and Objects of Votive Significance from Great Walsingham". Britannia. 30. 21-56

Smith, R. 1909. "A Hoard of Metal Found at Santon Downham, Suffolk". Proceedings of the Cambridge Antiquarian Society 13. 146-163

Smith, R. 1916. "Paper delivered 1915, Jan. 28". Proceedings of the Society of Antiquaries 27. 77-95

Smith, R. 1922. A Guide to the Antiquities of Roman Britain in the Department of British and Mediaeval Antiquities. Oxford University Press: Oxford.

Smith, T. \& Shortt, J. 1890. The History of the Parish of Ribchester in the County of Lancashire. Bemrose \& Sons Publishing: London.

Speidel, M. 1992. “Roman Army Pay Scales”. The Journal of Roman Studies 82. 87-106

Stead, I. \& Rigby, V. 1986. Baldock: The Excavation of a Roman and Pre-Roman Settlement, 1968-1972. Brittania Monograph Series 7.

Stead, I. \& Rigby, V. 1989. Verulamium: the King Harry Lane site. English Heritage Archaeology Report 12.

Stewart, B. 1981. The Waters of the Gap: The Mythology of Aquae Sulis. Bath City Council: Bath.

Struck, M. 2000. "High Status burials in Roman Britain (first-third century AD)- potential interpretation". In Pearce, J. et al. (eds) Burial, Society amd Context in the Roman World. Oxbow Books: Oxford. 85-96

Swan, V. 1992. "Legio VI and its men: African legionaries in Britain". The Journal of Roman Pottery Studies 5. 1-33

Swift, D. 2003. Roman Burials, medieval tenements ad suburban growth. Museum of London Archaeology Studies Series 10.

Swift, E. 2007a. "Small objects, Small Questions? Perceptions of Finds Research in the Academic Community". In Hingley, R. \& Willis, S. (eds) Roman Finds: Context and Theory. Oxbow Books: Oxford. 18-28 
Swift, E. 2007b. "Decorated Vessels:The Function of Decoration in Late Antiquity". In Lavan, L. et al. (eds) Objects in Context, Objects in Use: Marterial Spatiality in Late Antiquity. Brill Publishing: Boston. 385-412

Swift, E. 2009. Style and Function in Roman Decoration: Living with Objects and Interiors. Ashgate Publishing: Farnham.

Swift, E. 2010. "Identifying Migrant Communities: A Contextual Analysis of Grave Assemblages from Continental Late Roman Cemeteries”. Britannia 41. 237-282

Swift, E. 2012. "Object Biography, Re-use and Recycling in the Late to Post-Roman Transition Period and Beyond: Rings made from Romano-British Bracelets”. Britannia 43. 167-215

Tanner, J. 2010. "Race and Representation in Ancient Art: Black Athena and after". In Bindman, D. and Gates, H. (eds) The Images of the Black in Western Art Volume I: From the Pharohs to the Fall of the Roman Empire. Harvard University Press: Cambridge, MA. 1-39

Tassinari, S. 1975. La Vaisselle de Bronze, Romaine et Provinciale, au Musée des Antiquités Nationale. Gallia Supplément 29.

Tassinari, S. 1993. Il Vasellame Bronzeo di Pompei. «L'ERMA» di Bretschneider: Rome.

Taylor, M. \& Collingwood, R. 1922. "Roman Britain in 1923”. Journal of Roman Studies 12. 240-313

Terrissee, Jacques. 1982. La moissonneuse gauloise, des Rèmes aux Trévires. Groupe d'Etudes Archélogiques Champagne Ardenne: Reims.

Thomas, C. 2004. Life and Death in London's East End: 2000 years at Spitalfields. Museum of London Archaeological Service: London.

Tite, M. 1999. "Pottery Production, Distribution, and Consumption: The Contribution of the Physical Sciences”. Journal of Archaeological Method and Theory 6.3. 118-233

Tomlin, R. \& Hassall, M. 2004. "Roman Britain in 2003 III: Inscriptions”. Britannia 35. 335350

Toynbee, J. 1964. Art in Britain under the Romans. Oxford University Press: London.

Toynbee, J. 1971. Death and Burial in the Roman World. Thames and Hudson: London.

Toynbee, J. 1973. Animals in Roman Life and Art. Thames and Hudson: London.

Toynbee, J. 1986. The Roman Art Treasures from the Temples of Mithras. London and Middlesex Archaeological Society Special Paper 7. 
Tuffreau-Libre, M. 2000. "Pottery Assemblages in Gallo-Roman Cemeteries". In Pearce, J. et al. (eds) Burial, Society amd Context in the Roman World. Oxbow Books: Oxford. 52-60

Turcan, R. 1966. Les Sarcophages Romains á Représentations Dionysiaques: Essai de chronologie et d'histoire religieuse. Éditions E. De. Boccard: Paris.

Turner, R. 1999. Excavations of an Iron Age Settlement and Roman Religious Complex at Ivy Chimneys, Witham, Essex 1978-83. East Anglian Archaeology Report 88.

Tyers, P. 1996. Roman Pottery in Britain. B.T. Batsford: London.

VanPool, T. 2001. "Style, Function and Variation: Identifying the Evolutionary Importance of Traits in the Archaeological Record". In Hunt, T. \& Rakita, G. (eds) Style and Function:

Conceptual Issues in Evolutionary Archaeology. Bergin \& Garvey: Westport. 119-140

Wace, A. 1904. "Grotesques and the Evil Eye". Annual of the British School at Athens 10. 103114

Wacher, J. \& McWhirr, A. 1982. Cirencester Excavations I: Early Roman Occupation at Cirencester. Cirenchester Excavation Committee: Cirenchester.

Wainwright, G. 1967. Coygan Camp: A Prehistoric, Romano-British and Dark Age Settlement in Carmarthenshire. Cambrian Archaeological Association: Cardiff.

Walton, P. 2012. Rethinking Roman Britain: Coinage and Archaeology. Moneta: Wetteren.

Walton, P. (forthcoming) "Is the Piercebridge Assemblage a Roman Military Votive Deposit?" Journal of Roman Military Equipment Studies.

Watson, S. \& Heard, K. 2006. Development on Roman London's Western Hill. Museum of London Archaeological Service Monograph 32.

Watson, S. 2003. An Excavation in the Western Cemetery of Roman London: Atlantic House, City of London. Museum of London Archaeology Service Archaeology Study Series 7.

Watson, W. 1949. "Belgic Bronzes and Pottery found at Felmersham-on-Ouse, Bedfordshire". Antiquaries Journal 29. 37-61

Webster, G. 1953. "The Lead-Mining Industry in North Wales in Roman Times". Proceedings of the Flintshire Historical Society 13. 3-31

Webster, J. 1997. "Negotiating syncretism: readings on the development of Romano-Celtic religion". In Mattingly, D. (ed) Dialogues in Roman Imperialism: Power, discourse, and discrepant experience in the Roman Empire. JRA Supplemental Series 23. 165-182 
Webster, J. 2001. "Creolizing the Roman Provinces". Archaeological Jounral of America 105. 209-225

West, S. 2005. "Rich Graves in the Verulamium area". Lucerna: The Roman Finds Group Newsletter 29. 14

Wheeler, R. 1926. The Roman Fort near Brecon. Cymmrodorion Society Publications: London.

Wheeler, R. \& Wheeler, T. 1932. Report on the Excavations of the Pre-historic, Roman, and post-Roman Site in Lydney Park, Gloucestershire. Reports of the Research Committee of the Society of Antiquaries of London 9.

Whimster, R. 1981. Burial Practices in Iron Age Britain: A Discussion and Gazeteer of the Evidence c. 700 B.C.- A.D. 43. BAR British Series 90.

White, R. 1988. Roman and Celtic Objects from Anglo-Saxon Graves: A catalogue and an interpretation of their use. BAR British Series 191.

White, R. \& Barker, P. 1998. Wroxeter: Life \& Death of a Roman City. Tempus Publishing: Stroud.

Whittaker, C. 1997. "Imperialism and culture: the Roman initiative”. In Mattingly, D. (ed) Dialogues in Roman Imperialism: Power, discourse, and discrepant experience in the Roman Empire. JRA Supplementary Series 23. 143-164

Wickenden, N. 1986. "Prehistoric Settlement and Romano-British 'small town' at Heybridge, Essex". Essex Archaeology and History 17. 7-68

Wilk, S. 2000. Medusa: Solving the Mystery of the Gorgon. Oxford University Press: Oxford.

Wilkes, J. 1996. “The status of Londinium”. In Bird, J., Hassal, M. \& Sheldon, H (eds)

Interpretting Roman London: Papers in memory of Hugh Chapman. Oxbow Monograph 58. 2732

Williams, T. 1993. Public Buildings in the Sotuh-West Quarter of Roman London. CBA Research Report 88.

Willey, G. 1953. “Archaeological Theories and Interpretation: New World”. Anthropology Today. 361-385

Willey, G. \& Phillips, P. 1958. Method and Theory in American Archaeology. University of Alabama Press: London.

Willis, S. 2011. "Samian Ware and Society in Roman Britain and Beyond”. Britannia 42. 167242. 
Wilmott, T. 1997. Birdoswald: Excavations of a Roman for on Hadrian's Wall and its successor settlements 1987-1992. English Heritage Archaeological Report 14.

Wilmott, T. 2004. "Roman Brougham in its Regional Context". In Cool, H. The Roman Cemetery at Brougham, Cumbria. Britannia Monograph Series 21. 2-8

Wilson, D. 1968. "Roman Britain in 1967 I: Sites Explored”. Journal of Roman Studies 58. 176214

Wilson, D. 1971. "Roman Britain in 1970”. Britannia 2. 242-304

Wilson, P. 2002. Cataractonium: Roman Catterick and its hinterland. Excavations and research, 1958-1997 Part II. CBA Report 129.

Witts, P. 1994. “Interpreting the Brading ‘Abraxas’ Mosaic. Britannia 25. 111-117

Woods, P. and Hastings, S.1984. Rushden: The Early Fine Wares. Northamptonshire County Council: Rushden.

Woodward, A. 1925. The Roman Fort at Ilkley. Yorkshire Archaeological Society: York.

Woodward, A. \& Leach, P. 1993. The Uley Shrines: Excavations of a ritual complex on West Hill, Uley, Gloucestershire 1977-1979. English Heritage Archaeological Report 17.

Woodward, P. et al. 1993. Excavations at the Old Methodist Chapel and Greyhound Yard, Dorchester, 1981-1984. Dorset Natural History and Archaeological Society Monongraph Series 12.

Woolf, G. 1998. Becoming Roman. Cambridge University Press: Cambridge.

Worrell, S. 2004. "Finds Reported under the Portable Antiquities Scheme". Britannia 35. 317334

Worrell, S. 2006. "Finds Reported under the Portable Antiquities Scheme". Britannia 37. 429466

S. Worrell 2007. "Finds reported under the Portale Antiquities Scheme". Britannia 38. 303-344

Worrell, S. 2009. "Finds Reported under the Portable Antiquities Scheme”. Britannia 40. 281312

Worrell, S. 2012. "Enamelled Vessels and Related Objects Reported to the Portable Antiquities Scheme 1997-2010". In Breeze, D. (ed.) The First Souvenirs: Enamelled Vessels from Hadrian's Wall. Cumberland and Westmorland Antiquarian and Archaeological Society: Kendal. 71-84 
Worrell, S. \& Pearce, J. 2013. "Finds Reported under the Portable Antiquities Scheme". Britannia 44. 345-380

Wrathmell, S. \& Nicholson, A. 1990. Dalton Parlours: Iron Age Settlement and Roman Villa. Yorkshire Archaeology 3.

Wright, R. 1944. "Roman Britain in 1943: Inscriptions". Journal of Roman Studies 34. 85-91

Wright, R. 1958. "Roman Britain in 1957: Inscriptions". Journal of Roman Studies 48. 150-155

Wright, R. 1961. "Roman Britain in 1960: Inscriptions". Journal of Roman Studies 51. 191-198

Wright, R. 1964. "Roman Britain in 1963: Inscriptions". Journal of Roman Studies 53. 177-185

Wright, R. 1969a. "Two bronze vessels from Northumberland and new readings of the names of other makers". Archaeologia Aeliana 47. 1-5

Wright, R. 1969b. "Roman Britain in 1968: Inscriptions". Journal of Roman Studies 59. 235264

Wright, R. \& Hassall, C.1971. "Roman Britain in 1970: Inscriptions". Britannia 2. 289-318

Wright, R. \& Hassall, C. 1972. "Roman Britain in 1971: Inscriptions". Britannia 3. 352-370

Wright, R. \& Hassall, C. 1973. "Roman Britain in 1972: Inscriptions". Britannia 4. 324--345

Yeates, S. 2006. Religion, Community and Territory: Defining Religion in the Severn Valley and Adjacent Hills from the Iron Age to the Early Medieval Period. BAR British Series 411.

Zienkiewicz, J. 1986. The Legionary Fortress Baths at Caerleon II: The Finds. National Museum of Wales Press: Cardiff. 
Appendix I: Copper Alloy Vessels from Roman Britain

\begin{tabular}{|c|c|c|c|c|c|c|c|c|c|c|c|}
\hline Catalog Number & object & Classification & County & Town/Site & Site Type & Feature & Context & Associated Finds & Decoration (body) & Decoration (handle) & Reference \\
\hline AVoo01 & Jug (fragment) & & Avon & Bath & RS & Swallow Street, Context 390 & $\mathrm{SF}$ & & Zoomorphic duck thumb-rest & & Davenport 1991, 81 (1 \\
\hline Av0002 & Handled Pan 2 & Rudge Cup Type & Avon & Bath & RS & $\begin{array}{l}\text { Reservoir of the Roman Baths, } \\
\text { (CS layer } 3 \text { sf 20,008) }\end{array}$ & sD & & $\begin{array}{l}\text { Geometric squares and triangles; Geometric } \\
\text { depiction of wall crenalations; Concentric circle foot } \\
\text { ring }\end{array}$ & \begin{tabular}{|l} 
Floral (possible) leaf and/or vine motif; \\
Geomentric wave shapes; punch dot inscription \\
"DIISVM[.../CODON[...]"
\end{tabular} & $\begin{array}{l}\text { Cunlifie 1988, 14-16 } \\
\text { (22); Hassall \& Tomlin } \\
\text { 1981, } 381 \text { (20); RIB II } \\
2415.60\end{array}$ \\
\hline BE0001 & Bowl & Bassin festonné & Bedfordshire & Sandy & RS & & SD & BE0002, BE0003 & & & \\
\hline BE0002 & Bowl & Bassin á bord godronné & Bedfordshire & Sandy & RS & & SD & BE0001, BE0003 & Geometric repoussé bosses (godrons) 59 in number & & Kennet $1971 \quad 124,1.2$ \\
\hline $\mathrm{BE} 0003$ & Bowl & Bassin uni & Bedfordshire & Sandy & RS & & SD & BE0001, BE0002 & Geometric rim bosses & & Kennet $1971 \quad 124,1.3$ \\
\hline BE0004 & Handled Pan 2 & Eggers $137-138$ & Bedfordshire & Shefford & RS & Stanfordbury Farm, Grave 1 & GD & BE0004-BE0005 & & $\begin{array}{l}\text { Geometric half subburst on handle medallion, } \\
\text { caducaus on handle; inscription below sunburst } \\
\text { "d....ESSPY" }\end{array}$ & $\begin{array}{l}\text { Eggers } 1968104-105 \\
\text { (29Aa); RIB II 2415.51 }\end{array}$ \\
\hline BE0005 & Jug & Eggers $124-125$ & Bedfordshire & Shefford & RS & Stanfordbury Farm, Grave 1 & GD & BE0004-BE0005 & & $\begin{array}{l}\begin{array}{l}\text { Anthropomorphic human bust on thumbrest and } \\
\text { handle medallion }\end{array} \\
\end{array}$ & $\begin{array}{l}\text { Eggers } 1968 \quad 104-105 \\
(29 \mathrm{Ab})\end{array}$ \\
\hline BE0006 & Handled Pan 1 & Eggers 155 & Bedfordshire & Shefford & RS & Stanfordbury Farm, Grave 2 & GD & $2 \mathrm{Am}[\mathrm{c}], 1 \mathrm{~B}[\mathrm{c}]$ & Concentric ring umbo & $\begin{array}{l}\text { Zoomorphic rams head handle terminal, fluted } \\
\text { handle, floral second handle }\end{array}$ & $\begin{array}{l}\text { Eggers } 1968 \quad 104-105 \\
(29 \mathrm{Ab})\end{array}$ \\
\hline BE0007 & Jug & Eggers 125 & Bedfordshire & Shefford & RS & Stanfordbury Farm, Grave 2 & GD & $2 \mathrm{Am}[\mathrm{cc}, 1 \mathrm{~B}[\mathrm{c}]$ & & & 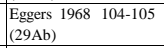 \\
\hline$\frac{\text { BERK }}{\text { BER0001 }}$ & \begin{tabular}{|l|} 
Jug \\
Vessel (mount)
\end{tabular} & Eggers 128 & \begin{tabular}{|l|} 
Berkshire \\
Berkshire
\end{tabular} & \begin{tabular}{|l|} 
Thatcham \\
Chieveley \\
\end{tabular} & \begin{tabular}{|l|l}
$\mathrm{RU}$ \\
$\mathrm{RU}$
\end{tabular} & Near spring & $\begin{array}{l}\text { SF } \\
\text { PAS }\end{array}$ & & \begin{tabular}{|l} 
Concentric rings on neck \\
Zomorothic non-auatic bird mount
\end{tabular} & & \begin{tabular}{|l} 
Eggers $1966,103(7)$ \\
PAS BERK-291567
\end{tabular} \\
\hline BUC0001 & Jug & Eggers 125 & Buckinghamshire & Thornborough & RS & $\begin{array}{l}\text { Thornborough Barrow, } \\
\text { possible family tomb }\end{array}$ & GD & $\begin{array}{l}2 \mathrm{Am}[\mathrm{cl}], 3 \mathrm{Bo}[\mathrm{gg}], 1 \mathrm{~L}[\mathrm{ca}], 1 \\
\mathrm{Bc}, \mathrm{BUCO001-0004}\end{array}$ & Trefoil mouth & $\begin{array}{l}\text { Zoomorphic lion thumb rest with paw as handle } \\
\text { medallion }\end{array}$ & $\begin{array}{l}\text { Eggers 1968, } 110 \\
\text { (89a); Philpott 1991, } \\
\text { 261; Liversidge 1953, } \\
\text { 29-32 }\end{array}$ \\
\hline BUC0002 & Jug & Eggers $128 \mathrm{a}$ & Buckinghamshire & Thornborough & RS & $\begin{array}{l}\text { Thornborough Barrow, } \\
\text { possible family tomb }\end{array}$ & GD & $\begin{array}{l}2 \mathrm{Am} \text { [c], 3 Bo [g]], } 1 \mathrm{~L} \text { [ca], } 1 \\
\mathrm{Bc}, \mathrm{BUC0001-0004}\end{array}$ & & $\begin{array}{l}\text { Anthropomorophic standing figure on handle } \\
\text { medallion, floral thumb rest }\end{array}$ & $\begin{array}{l}\text { Eggers 1968, } 110 \\
\text { (8gob; Philpott 1991, } \\
\text { 261: Liversidge 1953, } \\
\text { 29-32 }\end{array}$ \\
\hline BUC0003 & Bowl & Eggers 94 & Buckinghamshire & Thornborough & RS & $\begin{array}{l}\text { Thornborough Barrow, } \\
\text { possible family toom }\end{array}$ & GD & $\begin{array}{l}2 \mathrm{Am}[\mathrm{cc}, 3 \mathrm{Bo}[\mathrm{ggl}], 1 \mathrm{~L} \text { [ca], } 1 \\
\text { Bc, BUC0001-0004 }\end{array}$ & & & $\begin{array}{l}\text { Philpott 1991, 261; } \\
\text { Liversidge 1953, 29-32 }\end{array}$ \\
\hline 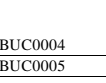 & \begin{tabular}{|l|} 
Handled Pan 1 \\
Handled Pan 1 \\
\end{tabular} & \begin{tabular}{|l} 
Eggers $154-155$ \\
Egers $154-155$
\end{tabular} & \begin{tabular}{|l} 
Buckinghamshire \\
Buckinghamshire
\end{tabular} & \begin{tabular}{|l} 
Thornborough \\
Milton Keynes
\end{tabular} & $\frac{\mathrm{RS}}{\mathrm{RS}}$ & $\begin{array}{l}\text { Thornborough Barrow, } \\
\text { possible family tomb } \\
\text { Olney }\end{array}$ & \begin{tabular}{|l} 
GD \\
$\mathrm{SF}$
\end{tabular} & $\begin{array}{l}2 \mathrm{Am} \text { [c], } 3 \mathrm{Bo}[\mathrm{gg}], 1 \mathrm{~L} \text { [ca], } 1 \\
\mathrm{Bc}, \mathrm{BUC0001-0004}\end{array}$ & Concentric cirlce umbo and foot ring & $\begin{array}{l}\text { Zoomorphic bear/wolf handle terminal } \\
\text { Zoomorphic bear/wolf handle terminal }\end{array}$ & \begin{tabular}{|l} 
Philpott 1991, 261; \\
Liversidge 1953, 29-32 \\
Farley 1977, 485
\end{tabular} \\
\hline BUC0006 & Bowl & Irchester & Buckinghamshire & Amersham & RS & Misbourne Valley & SD & & $\begin{array}{l}\text { Concentric circle body bands; Inscription "X" and } \\
\text { sideways "A" on exterior body of vessel; Coins } \\
\text { found in area date between Trajan and Constantine I } \\
\text { with two "unidentifiable 4th century" coins }\end{array}$ & & \begin{tabular}{|l} 
Farley et al. $988,359-$ \\
366 (1)
\end{tabular} \\
\hline BUC0007 & Bowl & Irchester & Buckinghamshire & Amersham & RS & Misbourne Valley & SD & & $\begin{array}{l}\text { Concentric circle body bands; omphalos dot; Coins } \\
\text { found in area date between Trajan and Constantine I } \\
\text { with two "unidentifiable 4th century" coins }\end{array}$ & & $\begin{array}{l}\begin{array}{l}\text { Farley et al. } 1988,359- \\
366(2)\end{array} \\
360\end{array}$ \\
\hline BUC0008 & Bowl & Irchester & Buckinghamshire & Amersham & RS & Misbourne Valley & SD & & $\begin{array}{l}\text { Raised umbo and omphalos dot; Coins found in } \\
\text { area date between Trajan and Constantine I with } \\
\text { two "unidentifiable eth century" coins }\end{array}$ & & $\begin{array}{l}\text { Earley et al. } 1988,359- \\
366(3)\end{array}$ \\
\hline BUC0009 & Bowl & Irchester & Buckinghamshire & Amersham & RS & Misbourne Valley & SD & & $\begin{array}{l}\text { Raised umbo and omphalos dot; Coins found in } \\
\text { area date between Trajan and Constantine I with } \\
\text { two "unidentifiable th century" coins }\end{array}$ & & $\begin{array}{l}\text { Farley et alia } 1988,359 \\
366(4)\end{array}$ \\
\hline BUC0010 & Bow1 & & Buckinghamshire & Amersham & RS & Misbourne Valley & SD & & $\begin{array}{l}\text { Coins found in area date between Trajan and } \\
\text { Constantine I with two "unidentifiable 4th century" } \\
\text { coins }\end{array}$ & & $\begin{array}{l}\text { Farley et alia } 1988,359 \\
366(5)\end{array}$ \\
\hline BUC0011 & Bowl & & Buckinghamshire & Amersham & RS & Misbourne Valley & SD & & $\begin{array}{l}\text { Coins found in area date betwenn Trajan and } \\
\text { Constantine I with two unidentifiable th century" } \\
\text { coins }\end{array}$ & & $\begin{array}{l}\text { Farley et alia } 1988,359 \\
366(6)\end{array}$ \\
\hline CAR0001 & Handled Pan 2 & Eggers $139-144$ & Carmarthenshire & Kyngadl & $\mathrm{RS}$ & Coygan Cave & SD & CAE0002 & Geometric trefoil swastika umbo & Concentric circle handle medallion & $\begin{array}{l}\text { Eggers 1968, } 103 \\
\text { (12a); Wainwright } \\
\text { 1967, 85-88 }\end{array}$ \\
\hline
\end{tabular}




\begin{tabular}{|c|c|c|c|c|c|c|c|c|c|c|c|}
\hline CAR0002 & Strainer & & Carmarthenshire & Kyngadl & RS & Coygan Cave & SD & CAE0001 & Geometric circle/wave strainer dot pattern & & \begin{tabular}{|l}
$\begin{array}{l}\text { Eggers 1968, } 103 \\
\text { (12b); Wainwight } \\
\text { 1967, 85-88 }\end{array}$ \\
\end{tabular} \\
\hline CAR0003 & $\begin{array}{l}\text { Handled Pan } 2 \\
\text { (handle) }\end{array}$ & Rudge Cup Type & Carmarthenshire & Carmarthen & M & Priory Street & SF & & & Floral vine and leaf enameled in blue & James 2003, 317 (57) \\
\hline CAR0004 & Jug (lid) & Eggers $123-126$ & Carmarthenshire & Carmarthen & M & Church Street & SF & & Floral (possibly) leaf shaped jug lid & & James 2003, $319(60)$ \\
\hline CAR0005 & Vessel (fragment) & & Carmarthenshire & Coygan Camp & RS & & SF & & Concentric circle umbo & & $\begin{array}{l}\text { Wainwright 1967, } 93 \\
\text { (24.7) }\end{array}$ \\
\hline CAR0006 & Vessel (fragment) & & Carmarthenshire & Coygan Camp & RS & & SF & & & & $\begin{array}{l}\text { Wainwright 1967, } 93 \\
(24.9)\end{array}$ \\
\hline CAR0007 & Vessel (fragment) & & Carmarthenshire & Coygan Camp & RS & & SF & & & & $\begin{array}{l}\text { Wainwright 1967, } 93 \\
(24.10)\end{array}$ \\
\hline CAR0008 & Vessel (fragment) & & Carmarthenshire & Coygan Camp & RS & & SF & & & & $\begin{array}{l}\text { Wainwight 1967, } 93 \\
(24.11)\end{array}$ \\
\hline CAR0009 & Vessel (fragment) & & Carmarthenshire & Coygan Camp & RS & & SF & & & & $\begin{array}{l}\text { Wainigright 1967, } 93 \\
\text { (24.12) }\end{array}$ \\
\hline Сам0001 & Handled Pan 2 & Eggers 140 & Cambridgeshire & Elm & RS & Coldham & SD & САM 0002 & Concentric circle umbo & Circle handle loop & Eggers 1968, 106 (40a) \\
\hline САм0002 & Handled Pan 2 & Eggers 144 & Cambridgeshire & Elm & RS & Coldham & SD & CAM0001 & Concentric circle umbo & Circle handle loop & Eggers 1968, $106(40 \mathrm{~b})$ \\
\hline САм0003 & Jug & Eggers 125 & Cambridgeshire & Hauxton & RS & & SD & САM0003-СAM0005 & Trefoil mouth & $\begin{array}{l}\text { Anthropomorphic human face, perhaps cherub, on } \\
\text { handle medallion }\end{array}$ & \begin{tabular}{|l|l|} 
Eggers 1968,106 \\
$(39 a) ;$ Liversidge, 1977 \\
29
\end{tabular} \\
\hline Сам0004 & Jug & & Cambridgeshire & Hauxton & RS & & SD & САM0003-САM0005 & & \begin{tabular}{|l}
$\begin{array}{l}\text { Anthropomorphic human face with wings above } \\
\text { head, perhaps somen form of Mercury, on handle } \\
\text { medallion; high handle with floral thumb rest }\end{array}$ \\
\end{tabular} & \begin{tabular}{|l} 
Eggers 1968,106 \\
39b); Liversidge 1977, \\
29
\end{tabular} \\
\hline Cам0005 & Jug & & Cambridgeshire & Hauxton & RS & & SD & САМ 0003 -САM 0005 & & \begin{tabular}{|l}
$\begin{array}{l}\text { Anthropomorphic human feet handle medallion, } \\
\text { flat thumb rest, semi-fluted handle }\end{array}$ \\
\end{tabular} & $\begin{array}{l}\text { Eggers 1968, } 106 \\
\text { (39c); Liversidge } 1977, \\
29\end{array}$ \\
\hline CAM0006 & Vessel (mount) & Eggers 79 & Cambridgeshire & Barton & $\mathrm{RU}$ & & SF & & Zoomorphic bird (dove) & & Eggers 1968, $106(41)$ \\
\hline САм0007 & Vessel & & Cambridgeshire & Cambridge & RS & $\begin{array}{l}\begin{array}{l}\text { Huntington Road, Gravel Hill, } \\
\text { Grave a }\end{array} \\
\end{array}$ & GD & $4 \mathrm{~F}[\mathrm{gl}], \mathrm{Po} 3[\mathrm{st}], 1 \mathrm{~F} \mathrm{[c]}$ & & & $\begin{array}{l}\begin{array}{l}\text { Philpott 1991, } 325 ; \\
\text { Liversidge 1977, } 15-16\end{array} \\
\end{array}$ \\
\hline Сам0008 & Jar & & Cambridgeshire & Godmanchester & RS & Hinchingbrooke, Nun's Bridge & GD & 3 Bo $[\mathrm{g}], 1 \mathrm{~V}[\mathrm{cc}, 1 \mathrm{Cn}[\mathrm{s}]$ & 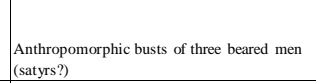 & Floral drop handle & $\begin{array}{l}\text { Philpott 1991, 332; } \\
\text { Whilison, } 1918 \text { 191 (5); } \\
\text { Liversidge 1977, 24-25 }\end{array}$ \\
\hline CAм0009 & Handled Pan 2 & Eggers $131-133$ & Cambridgeshire & Ely & $\mathrm{RU}$ & Isle of Ely, Prickwillow & SF & & & $\begin{array}{l}\text { Anthropomorphic elements on top, dolphins } \\
\text { below, floral vines; inscription "BODVOGENVS } \\
\text { F" }\end{array}$ & $\begin{array}{l}\text { Smith, } 1922 \text { 85; RIB II } \\
2415.11\end{array}$ \\
\hline Cам0010 & Cauldron & & Cambridgeshire & Burwell & $\mathrm{RU}$ & natural chalk knoll & SD & CAM0011-САM0024 & & & \begin{tabular}{|l} 
Cráster 1970, 344; \\
Wilson 1971, 270; \\
MAA 1996.44; MAA \\
1994-11-11 \\
\end{tabular} \\
\hline Сам0011 & Bowl & Basin á bord godronne & Cambridgeshire & Burwell & $\mathrm{RU}$ & natural chalk knoll & SD & САM0011-САM0024 & Geometric repoussé bosses (godrons) & & $\begin{array}{l}\text { Cráster 1970, 344; } \\
\text { Wilion 1971 277; } \\
\text { MAA 1969.44; MAA } \\
\text { 1994-11-11 } \\
\end{array}$ \\
\hline САM0012 & Bowl & Irchester & Cambridgeshire & Burwell & $\mathrm{RU}$ & natural chalk knoll & SD & САM0011-САM0024 & (2) & & $\begin{array}{l}\text { Cráster 1970, 344; } \\
\text { Wilion 1971, 277; } \\
\text { MAA 1969.44; MAA } \\
\text { 1994-11-11 } \\
\end{array}$ \\
\hline САм0013 & Bowl & Irchester & Cambridgeshire & Burwell & $\mathrm{RU}$ & natural chalk knoll & SD & САM0011-САM0024 & & & $\begin{array}{l}\text { Cráster 1970, 344; } \\
\text { Wilion 1971, 277; } \\
\text { MAA 1969.44; MAA } \\
\text { 1994-11-11 } \\
\end{array}$ \\
\hline САм0014 & Bowl & Irchester & Cambridgeshire & Burwell & $\mathrm{RU}$ & natural chalk knoll & SD & САM0011-САМ0024 & & & $\begin{array}{l}\text { Cráster 1970, 344; } \\
\text { Wilison 1971, 277; } \\
\text { MAA 1969.44; MAA } \\
\text { 1994-11-11 } \\
\end{array}$ \\
\hline САм0015 & Bowl & Irchester & Cambridgeshire & Burwell & $\mathrm{RU}$ & natural chalk knoll & SD & САM001-САM0024 & & & $\begin{array}{l}\text { Cráster 1970,344; } \\
\text { Wilson 1971, 277; } \\
\text { MAA 1969.44; MAA } \\
\text { 1994-11-11 } \\
\end{array}$ \\
\hline
\end{tabular}




\begin{tabular}{|c|c|c|c|c|c|c|c|c|c|c|c|}
\hline CAM0016 & Bowl & Irchester & Cambridgeshire & Burwell & $\mathrm{RU}$ & natural chalk knoll & SD & CAM0011-САM0024 & & & $\begin{array}{l}\text { Cráster 1970, 344; } \\
\text { Wilson 1971, 270; } \\
\text { MAA 1969.44; MAA } \\
\text { 1994-11-11 MA } \\
\end{array}$ \\
\hline CAM0017 & Bowl & Irchester & Cambridgeshire & Burwell & $\mathrm{RU}$ & natural chalk knoll & SD & CAM0011-САM0024 & & & $\begin{array}{l}\text { Cra'ster 1970, 344; } \\
\text { Wilson 1971, 270; } \\
\text { MAA 1969.44; MAA } \\
\text { 1994-11-11 } \\
\end{array}$ \\
\hline CAM0018 & Bowl & Irchester & Cambridgeshire & Burwell & $\mathrm{RU}$ & natural chalk knoll & SD & САM0011-САM0024 & & & $\begin{array}{l}\text { Cratster 1970, 344; } \\
\text { Wilion 1971, 270; } \\
\text { MAA 1969.44; MAA } \\
\text { 1994-11-11 }\end{array}$ \\
\hline CAM0019 & Bowl & & Cambridgeshire & Burwell & $\mathrm{RU}$ & natural chalk knoll & SD & CAM0011-САM0024 & & & $\begin{array}{l}\text { Cra'ster 1970, 344; } \\
\text { Wilson 1971, 270; } \\
\text { MAA 1969.44; MAA } \\
\text { 1994-11-11 } \\
\end{array}$ \\
\hline Сам0020 & Bowl & & Cambridgeshire & Burwell & $\mathrm{RU}$ & natural chalk knoll & SD & САМ0011-САМ0024 & Floral vine circling umbo & & $\begin{array}{l}\text { Cráster 1970, 344; } \\
\text { Wilson 1971, 270; } \\
\text { MAA 1969.44; MAA } \\
\text { 1994-11-11 } \\
\end{array}$ \\
\hline Сам0021 & Bowl & & Cambridgeshire & Burwell & $\mathrm{RU}$ & natural chalk knoll & SD & САM0011-САM0024 & Floral vine circling umbo & & $\begin{array}{l}\text { Cráster 1970, 344; } \\
\text { Wilion 1971, 270; } \\
\text { MAA 1969.44; MAA } \\
\text { 1994-11-11 }\end{array}$ \\
\hline САM0022 & Bowl & & Cambridgeshire & Burwell & $\mathrm{RU}$ & natural chalk knoll & SD & CAM0011-CAM0024 & & & $\begin{array}{l}\text { Cráster 1970, 344; } \\
\text { Wilion 1971, 270; } \\
\text { MAA 1969.44; MAA } \\
\text { 1994-11-11 }\end{array}$ \\
\hline САM 0023 & Handled Pan 2 & & Cambridgeshire & Burwell & $\mathrm{RU}$ & natural chalk knoll & SD & CAM0011-СAM0024 & & & \begin{tabular}{|l} 
Cra'ster 1970, 344; \\
Wilson 1971, 270; \\
MAA 1969.44; MAA \\
1994-11-11 \\
\end{tabular} \\
\hline Сам0024 & Bowl & & Cambridgeshire & Burwell & $\mathrm{RU}$ & natural chalk knoll & SD & САM0011-САM0024 & & & $\begin{array}{l}\text { Cráster 1970, 344; } \\
\text { Wilson 1971, 270; } \\
\text { MAA 1969.44; MAA } \\
\text { 1994-111-11 } \\
\end{array}$ \\
\hline CER0001 & Handled Pan 2 & Eggers 139-144 & Ceredigion & Llanio & M & & SF & & Concentric circle umbo and omphalos dot & $\begin{array}{l}\text { Concenticic circle handle medallion and } \\
\text { suspension loop; } 3 \text { concentric licle punch dots } \\
\text { forming a triangle where handle medallion meets } \\
\text { the handle }\end{array}$ & $\begin{array}{l}\text { Burnham et alia 2005, } \\
386\end{array}$ \\
\hline CHE0001 & Cauldron & & Cheshire & Middlewich & RS & $\begin{array}{l}\text { King Street; Shallow Hearth } \\
\text { Pit withing Building B }\end{array}$ & SF & & & & Dunn 2008, 43-44 (1) \\
\hline CHE0002 & Vessel (fragment) & & Cheshire & Middlewich & RS & King Street; post hole fill & SF & & $\begin{array}{l}\begin{array}{l}\text { Floral (possibly) vines circling omphalos dot; } \\
\text { geometric linear etched umbo ring; concentric circle } \\
\text { foot ring }\end{array} \\
\text { for }\end{array}$ & & Dunn 2008, $45(6)$ \\
\hline СНЕ0003 & $\begin{array}{l}\begin{array}{l}\text { Handled Pan } 2 \\
\text { (handle) }\end{array} \\
\end{array}$ & Eggers 139-144 & Cheshire & Chester & M & Goss Street & SF & & & $\begin{array}{l}\begin{array}{l}\text { Concenrtic cirlce handle medallion and } \\
\text { suspension loop; stamped inscription reads CIPI } \\
\text { POLLII...] }\end{array} \\
\end{array}$ & $\begin{array}{l}\text { McPeake \& Moore } \\
\text { 1978, 331 (11); RIB II } \\
2415.25\end{array}$ \\
\hline Co001 & Handled Pan 2 & Rudge Cup Type & Cumbria & Brougham & M & Roman Cemetary, Grave 107 & GD & 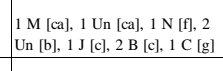 & Floral and Geometric, enameled & & Cool 2004, 124-128 \\
\hline $\mathrm{C} 0002$ & Bucket (fragment) & Hemmoor, Eggers 58 & Cumbria & Brougham & M & Roman Cemetary, Grave 237 & GD & 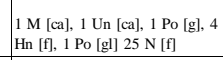 & Floral and Geometric & & Cool 2004, 374 \\
\hline $\mathrm{C} 0003$ & Bucket & Hemmoor, Eggers 58 & Cumbria & Brougham & M & Roman Cemetary, Grave 235 & GD & $\begin{array}{l}1 \mathrm{M} \text { [ca], } 1 \text { Un [ca], } 11 \mathrm{~N}[\mathrm{ff}, 4 \\
\mathrm{V}(\mathrm{f})[\mathrm{cc}\end{array}$ & & & Cool 2004, 195 \\
\hline $\mathrm{C} 0004$ & Bucket & Hemmoor, Eggers 58 & Cumbria & Brougham & M & Roman Cemetary, Grave 141 & GD & $20 \mathrm{Hn}[\mathrm{ff}, 5 \mathrm{Po}[\mathrm{gl}], 2 \mathrm{~V}(\mathrm{~F})[\mathrm{c}]$ & & & Cool 2004, 144 \\
\hline C0005 & Bucket & Hemmoor, Eggers 58 & Cumbria & Brougham & $\mathrm{M}$ & Roman Cemetary & $\mathrm{GD}$ & & Geometric Beaded rim & & Cool 2004, 374 \\
\hline c0006 & Bucket & & Cumbria & Brougham & M & Roman Cemetary, Grave 326 & GD & $\begin{array}{l}8 \mathrm{Un}(\mathrm{F})[\mathrm{cal}], 6 \mathrm{~N}[\mathrm{ff}, 1 \mathrm{~B}[\mathrm{Bc}, 1 \\
\mathrm{Jccl}, 26 \mathrm{~V}(\mathrm{~F})[\mathrm{cc}], 1,1 \mathrm{Po}[\mathrm{gg}]\end{array}$ & & & Cool 2004, 254 \\
\hline C0007 & Bucket & & Cumbria & Brougham & M & Roman Cemetary, Grave 114 & GD & 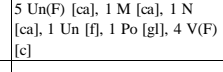 & & & Cool 2004, 129 \\
\hline Co008 & Bucket & & Cumbria & Brougham & M & Roman Cemetary, Grave 289 & GD & $1 \mathrm{M}[\mathrm{b}], 1 \mathrm{~V}[\mathrm{c}]$ & & & Cool 2004, 230 \\
\hline Co009 & Cauldron (fragment) & & Cumbria & Brougham & M & Roman Cemetary, Grave 198 & GD & $\begin{array}{l}1 \mathrm{Un}[\mathrm{b}], 4 \mathrm{Un}[\mathrm{cal}, 1 \mathrm{~N}[\mathrm{ca}], \\
1 \mathrm{Kn}[\mathrm{ff}], 130 \mathrm{~N}[\mathrm{fl}], 36 \mathrm{HN}[\mathrm{ff}] \\
1 \mathrm{Wf}[\mathrm{bb}, 13 \mathrm{Ar}[\mathrm{b}], 3 \mathrm{~V}[\mathrm{gl}]\end{array}$ & & & Cool 2004, 173 \\
\hline
\end{tabular}




\begin{tabular}{|c|c|c|c|c|c|c|c|c|c|c|c|}
\hline $\mathrm{C} 0010$ & Bowl & & Cumbria & Brougham & M & Roman Cemetary, Grave 127 & GD & & & & Cool 2004, 136 \\
\hline coo11 & Bowl & & Cumbria & Brougham & M & Roman Cemetary, Grave 245 & GD & & & & Cool 2004, 203 \\
\hline Co012 & Bowl & & Cumbria & Brougham & M & Roman Cemetary, Grave 239 & GD & $\begin{array}{l}28 \mathrm{Un}[\mathrm{ca}], 2 \mathrm{Hn}[\mathrm{ff}, 10 \mathrm{~N}[\mathrm{ff}] \\
1 \mathrm{~V}[\mathrm{gl}]\end{array}$ & & & Cool 2004, 198 \\
\hline $\mathrm{C} 0013$ & Bowl & & Cumbria & Brougham & M & Roman Cemetary, Grave 270 & GD & $\begin{array}{l}1 \mathrm{Un}[\mathrm{s}], 1 \mathrm{M}[\mathrm{ca}], 2 \mathrm{Un}[\mathrm{ca}], 1 \\
\mathrm{Hn}[\mathrm{ff}, 2 \mathrm{~N}[\mathrm{f}, \mathrm{A}[\mathrm{b}]\end{array}$ & & & Cool 2004, 217 \\
\hline Co014 & Strainer & & Cumbria & Brougham & M & Roman Cemetary, Grave 133 & GD & $\begin{array}{l}23 \mathrm{Un}[\mathrm{cal}], 1 \mathrm{Po} \text { off], } 17 \mathrm{~N}[\mathrm{ff}, 1 \\
\mathrm{V}[\mathrm{gl}], 1 \mathrm{I}[\mathrm{cc}\end{array}$ & & & Cool 2004, 138 \\
\hline Co015 & Strainer & & Cumbria & Brougham & M & Roman Cemetary, Grave 81 & GD & $\begin{array}{l}\begin{array}{l}2 \mathrm{~N}[\mathrm{f}], 1 \mathrm{~A}[\mathrm{~b}], 1 \mathrm{~J}[\mathrm{cc}], 1 \mathrm{Mo} \\
{[\mathrm{cc}}\end{array} \\
\end{array}$ & & & Cool 2004, 106 \\
\hline $\mathrm{C} 0016$ & Strainer & & Cumbria & Brougham & M & Roman Cemetary, Grave 217 & GD & 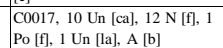 & & & Cool 2004, 185 \\
\hline $\mathrm{C} 0017$ & Vessel (mount) & & Cumbria & Brougham & M & Roman Cemetary, Grave 217 & GD & 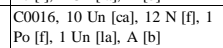 & & & Cool 2004, 185 \\
\hline Coo18 & Jug & & Cumbria & Brougham & M & Roman Cemetary, Grave 221 & GD & 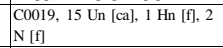 & & & Cool 2004, 188 \\
\hline $\mathrm{C} 0019$ & Strainer & & Cumbria & Brougham & M & Roman Cemetary, Grave 221 & GD & $\begin{array}{l}\text { Coolis, } 15 \text { Un [ca], } 1 \text { Hn [f], } 2 \\
\mathrm{~N} \text { [f] }\end{array}$ & & & Cool 2004, 188 \\
\hline $\mathrm{C} 0020$ & Jug & & Cumbria & Brougham & M & Roman Cemetary, Grave 77 & GD & $\begin{array}{l}21 \mathrm{Un}[\mathrm{cal}], 2 \mathrm{Hn}[\mathrm{fl}], 15 \mathrm{~N}[\mathrm{fl}] \\
1 \mathrm{Po}[\mathrm{gl}], 1 \mathrm{~V}[\mathrm{gl}], \mathrm{A}[\mathrm{b}], 1 \mathrm{~J} \\
{[\mathrm{cc}}\end{array}$ & & & Cool 204, 104 \\
\hline $\mathrm{C} 0021$ & Jug (fragment) & Eggers $123-126$ & Cumbria & Carlisle & M & South Lanes & $\mathrm{SF}$ & & & & $\begin{array}{l}\text { McCarthy } 2000,105 \\
\text { (C30) }\end{array}$ \\
\hline $\mathrm{C} 0022$ & Jug (fragment) & Eggers $123-126$ & Cumbria & Carlisle & M & South Lanes & SF & & & & $\begin{array}{l}\text { McCarthy 2000, } 105 \\
\text { (C31) }\end{array}$ \\
\hline $\mathrm{C} 0023$ & Bowl (fragment) & & Cumbria & Carlisle & M & Blackfriars Street, BLA D 101 & SF & & & & $\begin{array}{l}\text { Mocarthy 1990, } 132 \\
\text { (95) }\end{array}$ \\
\hline $\mathrm{C} 0024$ & Handled Pan 2 & Eggers $145-147$ & Cumbria & Carlisle & M & Blackfriars Street, BLA G + & SF & & & Trefoil suspension loop on handle medallion & \begin{tabular}{|l} 
Mccarthy 1990, 132, \\
$135(96,99)$
\end{tabular} \\
\hline $\mathrm{C} 0025$ & $\begin{array}{l}\begin{array}{l}\text { Handled Pan } 2 \\
\text { (fragment) }\end{array} \\
\end{array}$ & Eggers 139-144 & Cumbria & Carlisle & M & Blackfriars Street, BLA D 501 & SF & & & $\begin{array}{l}\text { Concentric circle handle medallion with circular } \\
\text { suspension lopis inscripion in rectangular field } \\
\text { reads "ANSL. DIODORII...]" }\end{array}$ & 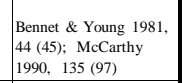 \\
\hline C0026 & $\begin{array}{l}\text { Handled Pan } 2 \\
\text { (fragment) }\end{array}$ & Eggers 139-144 & Cumbria & Carlisle & M & Blackfriars Street, BLA B 600 & SF & & & $\begin{array}{l}\text { Concentric circle handle medallion with circular } \\
\text { suspension loop; inscription in rectangular field } \\
\text { reads ".C.I.A[...]" }\end{array}$ & $\begin{array}{l}\text { Bennet \& Young 1981, } \\
\text { 44 (46); Mc arthy } \\
\text { 1990, } 135 \text { (98) }\end{array}$ \\
\hline $\mathrm{C} 0027$ & $\begin{array}{l}\begin{array}{l}\text { Handled Pan } 2 \\
\text { (fragment) }\end{array} \\
\end{array}$ & Eggers $139-144$ & Cumbria & Watercrook & M & & SF & & & $\begin{array}{l}\text { Concentric circle hande medallion with circular } \\
\text { suspension lopp; inscription }[[\ldots] \text { ANDID }[\ldots . .]\end{array}$ & $\begin{array}{l}\text { Potter 1979, } 215 \text { (48); } \\
\text { Bennet \& Young 1981, } \\
\text { 44 (44); RIB II } \\
\text { 2415.13 } \\
\end{array}$ \\
\hline C0028 & Jug & & Cumbria & Carlisle & M & & $\mathrm{SF}$ & & & $\begin{array}{l}\text { Anthropomorphic and zoomorphic scene of } \\
\text { magistrates sacrificing a pig in four registers }\end{array}$ & $\begin{array}{l}\text { BM 1814,0705.37; } \\
\text { Henig 1984, 132-134 }\end{array}$ \\
\hline $\mathrm{C} 0029$ & Handled Pan 2 & Eggers 140 & Cumbria & Clifton & RS & & SD & & & inscription "TALLO F" & RIB II 2415.47 \\
\hline $\mathrm{C} 0030$ & Strainer & & Cumbria & Clifton & RS & & SD & & & & RIB II 2415.47 \\
\hline DEN0001 & Handled Pan 2 & Eggers 131 & Denbighshire & Gilyn Dyfrdwy & $\mathrm{RU}$ & & SD & DEN0001-0004 & Concentric circle umbo & $\begin{array}{l}\text { Zoomorphic dual swan head handle loop, } \\
\text { geometric scalloping boarder, inscription "s } \\
\text { MERCV" in horizontal rectangualr field, } \\
\text { inscription "CPIIIICOMA" in veritcal } \\
\text { rectangular field }\end{array}$ & \begin{tabular}{|l|} 
Gardner 1927, 129-140 \\
Eggers 1968 104 (19a) \\
McPeake \& Moore \\
1978, 333 (9); RIB II \\
2415.16
\end{tabular} \\
\hline DEN0002 & Handled Pan 2 & Eggers $137-138$ & Denbighshire & Giln Dyfrdwy & $\mathrm{RU}$ & & SD & DEN0001-0004 & $\begin{array}{l}\text { Concentric circle umbo, concentric circle on exterior } \\
\text { body }\end{array}$ & $\begin{array}{l}\text { Geometric half circle handle loop, caducaus on } \\
\text { handle, "A[.... IVIT" }\end{array}$ & $\begin{array}{l}\text { Gardner 1927, } 129-140 \\
\text { Eggers 1968 104(19b) } \\
\text { RIB II 2415.10 }\end{array}$ \\
\hline
\end{tabular}




\begin{tabular}{|c|c|c|c|c|c|c|c|c|c|c|c|}
\hline DEN0003 & Handled Pan 2 & Eggers 146 & Denbighshire & Glyn Dyfrdwy & $\mathrm{RU}$ & & SD & DEN0001-0004 & Concentric circle umbo & $\begin{array}{l}\text { Geometric trefoil dots on handle medallion, } \\
\text { "[...III...II SAT[...]" }\end{array}$ & $\begin{array}{l}\text { Gardner 1927, 129-140, } \\
\text { Eggers 1968 104 (19c) } \\
\text { RIB II 2415.28 }\end{array}$ \\
\hline DEN0004 & $\begin{array}{l}\begin{array}{l}\text { Handled Pan } 2 \\
\text { (fragment) }\end{array} \\
\end{array}$ & Eggers 144 & Denbighshire & Glyn Dyfrdwy & $\mathrm{RU}$ & & SD & DEN0001-0004 & Geometric rectagular sectioned ring on exterior body & & \begin{tabular}{|l} 
Gardner 1927, 129-140; \\
Eggers 1968 104 (19d)
\end{tabular} \\
\hline DEN0005 & Vessel (mount) & & Denbighshire & Rhuddlan & M & Dinorben & $\mathrm{SF}$ & & Zoomorphic ox head hanging vessel mount & & $\begin{array}{l}\begin{array}{l}\text { Gardner \& Savoy 1964, } \\
144-148\end{array} \\
\end{array}$ \\
\hline DEN0006 & Vessel (mount) & & Denbighshire & Rhuddlan & M & Dinorben & SF & & Zoomorphic ox head hanging vessel mount & & $\begin{array}{l}\begin{array}{l}\text { Gardner \& Savoy 1964, } \\
144-148\end{array} \\
\end{array}$ \\
\hline DEN0007 & \begin{tabular}{|l}
$\begin{array}{l}\text { Handled Pan } 2 \\
\text { (handle) }\end{array}$ \\
\end{tabular} & Rudge Cup Type & Denbighshire & Rhuddlan & M & Dinorben & $\mathrm{SF}$ & & & $\begin{array}{l}\begin{array}{l}\text { Geometric linear etchings and swirls (possibly } \\
\text { floral vines) }\end{array} \\
\end{array}$ & \begin{tabular}{|l} 
Gardner \& Savoy 1964, \\
$148-149$
\end{tabular} \\
\hline DEN0008 & Bowl & & Denbighshire & Abergele & RS & Plas Uchaf & SD & DEN0008-DEN0016 & $\begin{array}{l}\text { Concentric circle umbo; etched inscription "INDVS } \\
\text { LX" }\end{array}$ & & $\begin{array}{l}\text { Wright \& Hassall 1972, } \\
\text { 363; NMGW 71.27H; } \\
\text { RCAHM 1914, 7-8; } \\
\text { RIB II 2415.61 } \\
\end{array}$ \\
\hline DEN0009 & Bowl & & Denbighshire & Abergele & RS & Plas Uchaf & SD & DENO008-DEN0016 & $\begin{array}{l}\text { Concentric circle umbo; etched inscription "INDVS } \\
\text { SH(?)" }\end{array}$ & & $\begin{array}{l}\text { Wright \& Hassall 1972, } \\
\text { 363; NMGW 71.27H; } \\
\text { RCAHM 1914, 7-8; } \\
\text { RIB II 2415.62 } \\
\end{array}$ \\
\hline DEN0010 & Bowl & & Denbighshire & Abergele & RS & Plas Uchaf & SD & DEN0008-DEN0016 & & & $\begin{array}{l}\text { Wright \& Hassall 1972, } \\
\text { 363; NMGW 71.27H; } \\
\text { RCAHM 1914, 7-8 }\end{array}$ \\
\hline DEN0011 & Bowl & & Denbighshire & Abergele & RS & Plas Uchaf & SD & DEN0008-DEN0016 & & & $\begin{array}{l}\text { Wright \& Hassall 1972, } \\
\text { 363; NMGW 71.27H; } \\
\text { RCAHM 1914, 7-8 }\end{array}$ \\
\hline DEN0012 & Strainer & & Denbighshire & Abergele & RS & Plas Uchaf & SD & DEN0008-DEN0016 & $\begin{array}{l}\begin{array}{l}\text { Geometric meander, concentric circle and floral } \\
\text { strainer dot pattern }\end{array} \\
\end{array}$ & & $\begin{array}{l}\text { Wright \& Hassall 1972, } \\
\text { 363; NMGW 71.27H; } \\
\text { RCAHM 1914, 7-8 }\end{array}$ \\
\hline DEN0013 & Handled Pan 2 & & Denbighshire & Abergele & RS & Plas Uchaf & SD & DEN0008-DEN0016 & & Concentric circle handle loop & $\begin{array}{l}\text { Wright \& Hassall 1972, } \\
\text { 363; NMGW 71.27H; } \\
\text { RCAHM 1914, 7-8 }\end{array}$ \\
\hline DEN0014 & Handled Pan 2 & & Denbighshire & Abergele & RS & Plas Uchaf & SD & DEN0008-DEN0016 & & Trefoil handle loop & $\begin{array}{l}\text { Wright \& Hassall 1972, } \\
\text { 363; NMGW 71.127H; } \\
\text { RCAHM 1914, 7-8 }\end{array}$ \\
\hline DEN0015 & Bowl & & Denbighshire & Abergele & RS & Plas Uchaf & SD & DENO008-DEN0016 & & & \begin{tabular}{|l} 
Wright \& Hassall 1972, \\
363; NMGW 71.27H; \\
RCAHM 1914, 7-8
\end{tabular} \\
\hline DEN0016 & Bowl & & Denbighshire & Abergele & RS & Plas Uchaf & SD & DEN0008-DEN0016 & & & $\begin{array}{l}\text { Wright \& Hassall 1972, } \\
\text { 363; NMGW 71.27H; } \\
\text { RCAHM 1914, 7-8 }\end{array}$ \\
\hline DER0001 & Vessel (fragment) & & Derbyshire & Ripley & $\mathrm{RU}$ & & PAS & & Slightly bulged rim & & PAS DENO-D72802 \\
\hline DEV0001 & Vessel (fragment) & & Devon & Exeter & u & Topsham & $\mathrm{SF}$ & & Concentric circle rim & & $\begin{array}{l}\text { Hollrook \& Bidwell } \\
1991,257(106)\end{array}$ \\
\hline DEV0002 & Handled Pan 5 & Eggers 130 & Devon & Exeter & $\mathrm{U}$ & wS 22 & SF & & & & $\begin{array}{l}\text { Hollbrook \& Bidwell } \\
1991,257 \text { (107) }\end{array}$ \\
\hline DEV0003 & Handled Pan 2 & Eggers $139-144$ & Devon & Exeter & $\mathrm{u}$ & $\begin{array}{l}\text { Stone lined pit next to timber } \\
\text { house }\end{array}$ & SD & & & & Rankov 1982,383 \\
\hline DEV0004 & Handled Pan 2 & Eggers 139-144 & Devon & Exeter & u & $\begin{array}{l}\text { Stone lined pit next to timber } \\
\text { house }\end{array}$ & SD & & & & Rankov 1982, 383 \\
\hline DOR0001 & Bucket (fragment) & Eggers 24 & Dorset & Cranborne & M & Hod Hill & SD & $\begin{array}{l}\text { DOR0002, DOR0003, } \\
\text { DORO004, DOR0005 }\end{array}$ & $\begin{array}{l}\text { Anthropomorphic human bust and animal (canine?) } \\
\text { heads on rim }\end{array}$ & & Eggers $1968103(9 \mathrm{a})$ \\
\hline DOR0002 & \begin{tabular}{|l|}
$\begin{array}{l}\text { Handled Pan } 22 \\
\text { (handle) }\end{array}$ \\
\end{tabular} & Eggers 133 & Dorset & Cranborne & M & Hod Hill & SD & \begin{tabular}{|l} 
DOR0001, DOR0003, \\
DORO004, DOR0005
\end{tabular} & & Geometric punch dots & Eggers $1968103(9 \mathrm{~b})$ \\
\hline DOR0003 & $\begin{array}{l}\begin{array}{l}\text { Handled Pan } 2 \\
\text { (handle) }\end{array} \\
\end{array}$ & Eggers 138 & Dorset & Cranborne & M & Hod Hill & SD & $\begin{array}{l}\text { DOR0001, DOR0002, } \\
\text { DORO004, DOR00055 }\end{array}$ & & $\begin{array}{l}\text { Geometric half sunburst on handle medallion, } \\
\text { caducaeus on handle }\end{array}$ & Eggers $1968103(9 \mathrm{c})$ \\
\hline DOR0004 & $\begin{array}{l}\text { Handled Pan } 2 \\
\text { (handle) }\end{array}$ & Eggers $140-141$ & Dorset & Cranborne & M & Hod Hill & $\mathrm{SD}$ & $\begin{array}{l}\text { DORO001, DOR0002, } \\
\text { DOR0003, DOR0005 }\end{array}$ & & Concentric ring handle medallion & Eggers 1968103 (9d) \\
\hline DOR0005 & $\begin{array}{l}\begin{array}{l}\text { Handled Pan } 1 \\
\text { (handle) }\end{array} \\
\end{array}$ & Eggers $154-155$ & Dorset & Cranborne & M & Hod Hill & SD & $\begin{array}{l}\text { DOR0001, DOR0002, } \\
\text { DOR0003, DOR0004 }\end{array}$ & & $\begin{array}{l}\text { Zoomorphic ramshead handle terminal, fluted } \\
\text { handle }\end{array}$ & Eggers $1968103(9 \mathrm{e})$ \\
\hline
\end{tabular}




\begin{tabular}{|c|c|c|c|c|c|c|c|c|c|c|c|}
\hline OOR0006 & Vessel (fragment) & & Dorset & Woodlands & RS & Knob's Creek, Pit I, Cemetary & GD & $\begin{array}{l}28 \mathrm{~N}[\mathrm{ff}],+\mathrm{V}[\mathrm{c}],+\mathrm{Un}[\mathrm{cal}, 7 \\
\mathrm{Un}[\mathrm{ff},+\mathrm{V}[\mathrm{g}], 5 \mathrm{Un}[\mathrm{st}]\end{array}$ & & & Fowler, $196536(1)$ \\
\hline DOR0007 & Jug (fragment) & & Dorset & Castleton & RS & & PAS & & & Anthropomorphic feet handle terminal medallion & PAS DOR-2DCB21 \\
\hline OOR0008 & $\begin{array}{l}\text { Handled Pan } 2 \\
\text { (handle) }\end{array}$ & Eggers $139-144$ & Dorset & Nether Compton & RS & & PAS & & & Concentric ring handle medallion & $\begin{array}{l}\text { PAS SOMDOR- } \\
\text { OFE673 }\end{array}$ \\
\hline OR00099 & Jug (fragment) & Eggers 128 & Dorset & Corfe Castle & RS & & PAS & & Geometric circular lid & & PAS WAW-4B7FA1 \\
\hline OOR0010 & Bucket (fragment) & & Dorset & Stoke Abbott & M & & PAS & & Geometric guilloche running along top of foot & & $\begin{array}{l}\text { PAS SOMDOR- } \\
\text { 53DF91 }\end{array}$ \\
\hline OOR0011 & Vessel (fragment) & & Dorset & Compton Abbas & RS & & PAS & & Zoomorphic bull's head mount & & $\begin{array}{l}\text { PAS SOMDOR- } \\
\text { B23561 }\end{array}$ \\
\hline OOR0012 & Jug & & Dorset & Dorchester & $\mathrm{u}$ & Greyhound Yard & SF & & & Anthropomorphic face handle medallion & $\begin{array}{l}\text { Woodward et al. } 1993, \\
127(80) \text {, }\end{array}$ \\
\hline OOR0013 & Bowl & & Dorset & Dorchester & $\mathrm{u}$ & Greyhound Yard & SF & & Concentric circle umbo & & \begin{tabular}{|l|}
$\begin{array}{l}\text { Woodward et al. 1993, } \\
127(81)\end{array}$ \\
\end{tabular} \\
\hline DOR0014 & Vessel (fragment) & & Dorset & Dorchester & $\mathrm{u}$ & Greyhound Yard & SF & & & & 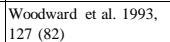 \\
\hline OOR0015 & Jug (fragment) & Eggers $123-126$ & Dorset & Dorchester & $\mathrm{u}$ & Greyhound Yard & $\mathrm{SF}$ & & \begin{tabular}{|l}
$\begin{array}{l}\text { Zoomorphic dolphin thumb-rest on lid; Floral } \\
\text { (possible) heart shaped lid }\end{array}$ \\
\end{tabular} & & $\begin{array}{l}\text { Woodward et al. } 1993, \\
127(83)\end{array}$ \\
\hline OOR0016 & Jug (fragment) & & Dorset & Dorchester & $\mathrm{u}$ & Greyhound Yard & $\mathrm{SF}$ & & & Zoomorphic lion's paws and tail handle terminal & $\begin{array}{l}\text { Woodward et al. } 1993, \\
127(84)\end{array}$ \\
\hline DOR0017 & Jug (fragment) & & Dorset & Dorchester & $\mathrm{u}$ & Greyhound Yard & SF & & & Floral (possible) leaf thumb rest & \begin{tabular}{|l|}
$\begin{array}{l}\text { Woodward et al. } 1993, \\
127(85)\end{array}$ \\
\end{tabular} \\
\hline DOR0018 & \begin{tabular}{|l|}
$\begin{array}{l}\text { Handled Pan } 2 \\
\text { (handle) }\end{array}$ \\
\end{tabular} & & Dorset & Blandford Forum & M & Hod Hill & $\mathrm{SF}$ & & & Geometric scalloping on terminal of handle & $\begin{array}{l}\text { Richmond } 1968,114 \\
(26)\end{array}$ \\
\hline OOR0019 & $\begin{array}{l}\begin{array}{l}\text { Handled Pan } 2 \\
\text { (handle) }\end{array} \\
\end{array}$ & & Dorset & Blandford Forum & M & Hod Hill & $\mathrm{SF}$ & & & Geometric incised line border & $\begin{array}{l}\text { Richmond } 1968,114 \\
(27)\end{array}$ \\
\hline OUR0001 & Jug & & Durham & Sedgefield & RS & & SD & & & $\begin{array}{l}\text { Anthropomorphic medusa handle medallion; } \\
\text { Zoomorphic birds' heads where handle frames } \\
\text { rim; fluted handle }\end{array}$ & $\begin{array}{l}\text { Burnham 2007, } 264(5, \\
\text { Fig. 13) }\end{array}$ \\
\hline OUR0002 & Bowl & & Durham & Hurworth-on-Tees & RS & & $\mathrm{SF}$ & & Concentric circle umbo & & $\begin{array}{l}\text { Burnham 2008, } 2286- \\
287 \text { (1, Fig. 13) }\end{array}$ \\
\hline DUR0003 & \begin{tabular}{|l}
$\begin{array}{l}\text { Handled Pan } 2 \\
\text { (handle) }\end{array}$ \\
\end{tabular} & Eggers $139-144$ & Durham & Bishop Auckland & M & Binchester Roman Fort & SF & & & Concentric circle handle medallion and loop & Ferris 2010, $352(90)$ \\
\hline OUR0004 & \begin{tabular}{|l}
$\begin{array}{l}\text { Handled Pan } 3 \\
\text { (handle) }\end{array}$ \\
\end{tabular} & Eggers 160-161 & Durham & Bishop Auckland & M & Binchester Roman Fort & $\mathrm{SF}$ & & & & Ferris 2010, 352 (91) \\
\hline OUR0005 & Bowl & & Durham & South Shields & M & South Shields Roman Fort & SD & & $\begin{array}{l}\text { Inscription "M A SAB APOLLINI } \\
\text { ANEXTIOMAROM" circling raised umbo }\end{array}$ & & $\begin{array}{l}\text { Henig 1984, 132-133; } \\
\text { RII II 2415.55 }\end{array}$ \\
\hline DUR0006 & Handled Pan 2 & Eggers 142 & Durham & Upper Weardale & $\mathrm{RU}$ & Peat bog & SD & DUR0006-DUR0008 & Concentric circle umbo & $\begin{array}{l}\text { Concentric circle handle loop; inscription "P. } \\
\text { CIPEPOLI" }\end{array}$ & $\begin{array}{l}\text { Egglestone 1915, 9-11; } \\
\text { Bennett \& Young 1981, } \\
\text { 43 (41); RII II 2415.20 }\end{array}$ \\
\hline OUR0007 & Handled Pan 2 & Egger 142 & Durham & Upper Weardale & $\mathrm{RU}$ & Peat bog & SD & DUR0006-DUR0008 & $\begin{array}{l}\text { Concentric circle umbo; punch dot inscription } \\
\text { around umbo read "LCINIANI" }\end{array}$ & \begin{tabular}{|l} 
Concentric circle handle loop; inscription \\
"PoLLBI.M"
\end{tabular} & $\begin{array}{l}\text { Egglestone 1915, 9-11; } \\
\text { Bennet \& Y Young 1981, } \\
\text { 43 (42); RII II 2415.27 }\end{array}$ \\
\hline OUR0008 & Handled Pan 2 & Eggers 142 & Durham & Upper Weardale & $\mathrm{RU}$ & Peat bog & SD & DUR0006-DUR0008 & & & Egglestone 1915, 9-11 \\
\hline Esusoool & Jug (handle) & & East Sussex & Etchingham & $\mathrm{RU}$ & & PAS & & & $\begin{array}{l}\text { Anthropomorphic cherub with prominent curls } \\
\text { and possible phrygian cap }\end{array}$ & PAS SUSS-C411A6 \\
\hline SSUS0002 & Bucket (fragment) & & East Sussex & St. Ann Without & $\mathrm{RU}$ & & PAS & & Geometric fan design, probably foot & & PAS SUSS-85ASE2 \\
\hline esuso003 & \begin{tabular}{|l|}
$\begin{array}{l}\text { Handled Pan } 2 \\
\text { (handle) }\end{array}$ \\
\end{tabular} & Rudge Cup Type & East Sussex & Southease & $\mathrm{RU}$ & & PAS & & & $\begin{array}{l}\text { Geometric etched lines form diamond and } \\
\text { triagular shapes; possible enameling }\end{array}$ & PAS SUR-4DEOE1 \\
\hline ESUSO004 & Bucket & & East Sussex & Beachy Head & $\mathrm{RU}$ & Bullock Down Farm & SD & $5495 \mathrm{Cn}[\mathrm{s}]$ & & & $\begin{array}{l}\text { Bland \& Johns 1979, } 61 \\
\text { 107; BM 1973.0404.1 }\end{array}$ \\
\hline
\end{tabular}




\begin{tabular}{|c|c|c|c|c|c|c|c|c|c|c|c|}
\hline Ex0001 & Strainer & & Essex & Colchester & u & Lion Walk (Domestic Fill) & $\mathrm{SF}$ & & Geometric swastica meander band & & \begin{tabular}{|l|}
$\begin{array}{l}\text { Crummy 1983, } 71 \\
2029\end{array}$ \\
\end{tabular} \\
\hline Ex0002 & Bowl (fragment) & & Essex & Colchester & $\mathrm{u}$ & Lion Walk (Destruction layer) & $\mathrm{SF}$ & & & & $\begin{array}{l}\text { Crummy 1983, } 71 \\
2030\end{array}$ \\
\hline EX0003 & Bowl (fragment) & & Essex & Colchester & u & \begin{tabular}{|l} 
Lion Walk (Destruction \\
Layer)
\end{tabular} & SF & & Geomertic and floral beaded rim & & \begin{tabular}{|l|} 
Crummy 1983,71 \\
2031
\end{tabular} \\
\hline EX0004 & Bowl & Bassin Uní (possibly) & Essex & Colchester & $\mathrm{U}$ & Balkerne Lane (Domestic fill) & $\mathrm{SF}$ & & & & \begin{tabular}{|l} 
Crummy 1983,71 \\
2034
\end{tabular} \\
\hline Ex0005 & Vessel (mount) & & Essex & Colchester & $\mathrm{u}$ & Balkerne Lane & $\mathrm{SF}$ & & & & $\begin{array}{l}\text { Crummy 1983, } 71 \\
2040\end{array}$ \\
\hline EX0006 & \begin{tabular}{|l|}
$\begin{array}{l}\text { Handled Pan } 3 \\
\text { (handle) }\end{array}$ \\
\end{tabular} & Eggers $160-161$ & Essex & Colchester & $\mathrm{u}$ & Balkerne Lane & SF & & & & \begin{tabular}{|l|}
$\begin{array}{l}\text { Crummy } 1983,73 \\
2043\end{array}$ \\
\end{tabular} \\
\hline EX0007 & Jug (handle) & & Essex & Colchester & u & Cups Hotel & $\mathrm{SF}$ & & Geometric & & $\begin{array}{l}\text { Crummy 1983, } 73 \\
2044\end{array}$ \\
\hline Exo008 & Bowl & Irchester & Essex & Sturmer & $\mathrm{RU}$ & & SD & EX0008-EX0014 & & & Kennet 1971, 1242.1 \\
\hline EX0009 & Bowl & Irchester & Essex & Sturmer & $\mathrm{RU}$ & & SD & EX0008-EX0014 & & & Kennet 1971, 1242.2 \\
\hline EX0010 & Bowl & Irchester & Essex & Sturmer & $\mathrm{RU}$ & & SD & EX0008-EX0014 & & & Kennet 1971, 1262.3 \\
\hline Ex0011 & Handled Pan 4 & Coptic & Essex & Sturmer & $\mathrm{RU}$ & & SD & EX0008-EX0014 & & & Kennet 1971, 1262.4 \\
\hline EX0012 & Bowl & & Essex & Sturmer & $\mathrm{RU}$ & & SD & EX0008-EX0014 & & & Kennet 1971, 1262.5 \\
\hline \begin{tabular}{|l} 
EX0013 \\
EX014
\end{tabular} & $\begin{array}{ll}\text { Bowl } \\
\text { Bown }\end{array}$ & \begin{tabular}{|l|l|} 
Bassin Uní \\
Basin Uní
\end{tabular} & \begin{tabular}{|l} 
Essex \\
FEsex
\end{tabular} & $\begin{array}{l}\text { Sturmer } \\
\text { Sturmer }\end{array}$ & $\frac{\mathrm{RU}}{\mathrm{RU}}$ & & $\begin{array}{llll}S D \\
S D\end{array}$ & \begin{tabular}{|l|} 
EX0008-EX0014 \\
FX008-E0014
\end{tabular} & $\begin{array}{l}\text { Concentric circle umbo } \\
\text { Comenentic cirle umbo }\end{array}$ & & Kennet 1971, 1272.6 \\
\hline EX0014 & Bowl & Bassin Uní & Essex & Sturmer & $\mathrm{RU}$ & Standsted Airport, Cremation & SD & EX0008-EX0014 & Concentric circle umbo & & \begin{tabular}{|l|l|} 
Kennet 1971, 1282.7 \\
Havis and Brooks 2004.
\end{tabular} \\
\hline EX0015 & Bowl & & Essex & Stansted Mountiftchet & RS & $\begin{array}{l}\text { Standsted Arrport, Cremation } \\
24 \text { (DCS 251) }\end{array}$ & GD & Bc (F)[ca], $13 \mathrm{~N}$ [f], $\mathrm{V}[\mathrm{cl}, \mathrm{J}[\mathrm{c}]$ & Concentric circle umbo & & $\begin{array}{l}\text { Havys and Brooks 2004, } \\
215\end{array}$ \\
\hline EX0016 & Jug & Eggers 123-126 & Essex & Stansted Mountiftchet & RS & $\begin{array}{l}\text { Stansted Airport, Cremation } \\
25 \text { (DCS 247) } \\
\end{array}$ & GD & 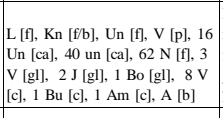 & \begin{tabular}{|l} 
Floral neck with palm and trefoil leaves, trefoil \\
mouth with godronné border, concentric circles on \\
foot ring (body); bust of youth/satye, aagle atop a \\
globe, basket of fruit, thumb shaped thumb rest, \\
hoof footed rim frame (handle)
\end{tabular} & & $\begin{array}{l}\text { Havis and Brooks } 2004 . \\
216\end{array}$ \\
\hline EX0017 & Handled Pan 1 & Eggers 154-155 & Essex & Stansted Mountiftchet & RS & \begin{tabular}{|l} 
Standsted Airport, Cremation \\
25 (DCS 247)
\end{tabular} & GD & 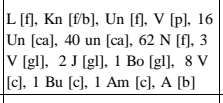 & Floral umbo with palm and trefoil leaves & $\begin{array}{l}\text { Zomororphic handle with bearcaninellion terminal } \\
\text { and in ornamentation as well as dolphins on the } \\
\text { underside of the body }\end{array}$ & $\begin{array}{l}\text { Havis and Brooks } 2004 . \\
216\end{array}$ \\
\hline Ex0018 & Handled Pan 2 & Eggers 150 & Essex & Stansted Mountiftchet & RS & $\begin{array}{l}\text { Standsted Airpot, Cremation } \\
25 \text { (DCS 247) }\end{array}$ & GD & 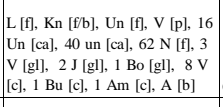 & Concentric circle foot ring & Geometric keyhole shaped handle loop & $\begin{array}{l}\text { Havis and Brooks } 2004 . \\
224\end{array}$ \\
\hline EX0019 & Jug & Eggers 129 & Essex & Stansted Mountiftchet & RS & \begin{tabular}{|l} 
Standsted Airport, Cremation \\
25 (DCS 247) \\
\end{tabular} & GD & 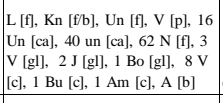 & Concentric circle foot ring & & $\begin{array}{l}\text { Havis and Brooks } 2004 . \\
226\end{array}$ \\
\hline EX0020 & Bowl & & Essex & Stansted Mountiftchet & RS & \begin{tabular}{|l} 
Standsted Airport, Cremation \\
25 (DCS 247)
\end{tabular} & GD & 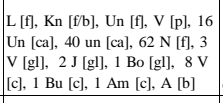 & Concentric circle umbo & & $\begin{array}{l}\text { Havis and Brooks } 2004 . \\
226\end{array}$ \\
\hline Ex0021 & Vessel (fragment) & & Essex & Stansted Mountiftchet & RS & $\begin{array}{l}\text { Standsted Airpot, Cremation } \\
25 \text { (DCS 247) }\end{array}$ & GD & 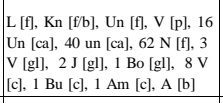 & & & $\begin{array}{l}\text { Havis and Brooks } 2004, \\
226\end{array}$ \\
\hline EX0022 & Handled Pan 2 & Eggers 150 & Essex & Chesterford & M & Bors Field & SD & EX0023 & Geometric linear rim embellishment & Geometric sub rectangular handle loop & Eggers 1968, 106 (382) \\
\hline EX0023 & Strainer & Eggers $160-161$ & Essex & Chesterford & M & Bors Field & SD & EX0022 & Geometric strainer dots & Hilted handle & Eggers 1968, $106(38 \mathrm{~b})$ \\
\hline Ex0024 & Handled Pan 2 & Eggers 196 (c.f.) & Essex & Colchester & u & & SF & & Concentric cirlce foot ring & $\begin{array}{l}\text { Geometric trefoil handle loop, inscription in } \\
\text { rectangular field "PCIPI.POLYBI". }\end{array}$ & \begin{tabular}{|l} 
Eggers 1968. 106 \\
(37); ; Mceake \& \\
Moore 1977, 333 (13); \\
RII II 2415.23
\end{tabular} \\
\hline EX0025 & Handled Pan 2 & Eggers 150 & Essex & Colchester & $\mathrm{u}$ & & SF & & Concentric circle foot ring & Floral (possibly) tear drop/leaf shaped handle loop & Eggers 1968, $106(37 \mathrm{f})$ \\
\hline
\end{tabular}




\begin{tabular}{|c|c|c|c|c|c|c|c|c|c|c|c|}
\hline ex0026 & \begin{tabular}{|l|}
$\begin{array}{l}\text { Handled Pan } 2 \\
\text { (handle) }\end{array}$ \\
\end{tabular} & Eggers 139 (c.f.) & Essex & Colchester & $\mathrm{u}$ & & SF & & & Geometric circle handle loop & Eggers 1968, $106(37 \mathrm{~g})$ \\
\hline ex0027 & $\begin{array}{l}\text { Handled Pan } 2 \\
\text { (handle) }\end{array}$ & Eggers 140 & Essex & Colchester & u & Trinity Street & $\mathrm{SF}$ & & & \begin{tabular}{|l} 
Geometric circle handle loop; inscription \\
"TVGIM"
\end{tabular} & $\begin{array}{l}\text { Eggers 1968, } 106 \\
\text { (37h); Wright } 1944,89 \\
\text { (12): RII II 2415.50 }\end{array}$ \\
\hline ex0028 & Jug (fragment) & Eggers 128 & Essex & Colchester & $\mathrm{u}$ & & $\mathrm{sF}$ & & Concentric circle neck, single spout, stopper & Floral thumb rest & Eggers 1968, 106 (37i) \\
\hline Ex0029 & Jug & Eggers 128 & Essex & Colchester & $\mathrm{u}$ & & $\mathrm{SF}$ & & Concentric circle neck, single spout, stopper & & Eggers 1968, $106(37 \mathrm{k})$ \\
\hline Ex0030 & Strainer & Eggers 161 & Essex & Great Wackering & $\mathrm{RU}$ & & $\mathrm{SF}$ & & Geometric sunburst and ring strainer dot pattern & & Eggers 1968, $105(34)$ \\
\hline ex0031 & \begin{tabular}{|l} 
Handled Pan 1 \\
(handle)
\end{tabular} & Eggers $154-155$ & Essex & Heybridge & RS & The Towers & GD & $1 \mathrm{~A}[\mathrm{c}], \mathrm{EX} 0038$ & Floral Umbo & Fluted handle & \begin{tabular}{|l|}
$\begin{array}{l}\text { Eggers 1968, } 105 \\
\text { (35a); Wickenden 1986, } \\
\text { 55 (4A) }\end{array}$ \\
\end{tabular} \\
\hline Ex0032 & Vessel (fragment) & Eggers 25 & Essex & Heybridge & RS & The Towers & SF & & & & Eggers 1968, 105 (35b) \\
\hline ex0033 & Bucket & Ostland, Eggers $40-41$ & Essex & Fingringhoe & $\mathrm{RU}$ & Wick Fame Gravel Pits & $\mathrm{SF}$ & & & & Eggers 1968, 105 (36) \\
\hline ex0034 & Handled Pan 2 & Eggers 150 & Essex & Colchester & $\mathrm{u}$ & & GD & $\begin{array}{l}10 \mathrm{~V}[\mathrm{cl}, 3 \mathrm{Bo}[\mathrm{gg}], 1 \mathrm{Bc}, 36 \\
\mathrm{Cn}, 21 \mathrm{Ar}[\mathrm{cl},+\mathrm{Co}[\mathrm{b}]\end{array}$ & & Keyhole shaped handle loop & 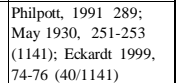 \\
\hline Ex0035 & Jug & Eggers $123-126$ & Essex & Rivenhall & RS & Barrow Field, Possible Grave & GD & EX0045 & & $\begin{array}{l}\text { Zoomorphic lior's head thumb rest, zoomorphic } \\
\text { lion's paw handle medallion }\end{array}$ & \begin{tabular}{|l|} 
Rodwell, 1978 15; \\
Rodwell \& Rodwell \\
$1993,35-36$
\end{tabular} \\
\hline Ex0036 & Handled Pan 1 & Eggers $154-155$ & Essex & Rivenhall & RS & Barrow Field, Possible Grave & GD & EX0044 & Concentric circle foot ring & & $\begin{array}{l}\text { Rodwell, 1978 15; } \\
\text { Rodwell \& Rodwell } \\
1993,35-36\end{array}$ \\
\hline Ex0037 & $\begin{array}{l}\text { Handled Pan } 2 \\
\text { (handle) }\end{array}$ & Eggers 136 & Essex & Colchester & $\mathrm{u}$ & & $\mathrm{SF}$ & & & $\begin{array}{l}\text { Zoomorphic birds framed by floral vines, two } \\
\text { handle loops; inscription in fectangular field } \\
\text { "T.POMP.NIC" }\end{array}$ & $\begin{array}{l}\text { Smith, } 1922 \text { 85; RIB II } \\
2415.48\end{array}$ \\
\hline Ex0038 & Jug (handle) & & Essex & Heybridge & RS & The Towers & $\mathrm{GD}$ & 1 A [c], EX0031 & & $\begin{array}{l}\begin{array}{l}\text { Anthropomorphic harpy thumb rest and handle } \\
\text { medallion }\end{array} \\
\end{array}$ & \begin{tabular}{|l|}
$\begin{array}{l}\text { Wickenden 1986, } 55 \\
(4 \mathrm{~A})\end{array}$ \\
\end{tabular} \\
\hline ex0039 & Bucket (fragment) & & Essex & $\begin{array}{l}\text { Greenstead Green And } \\
\text { Halstead Rural }\end{array}$ & RU & & PAS & & Geometric 'pelta' design & & PAS ESS-8C8A14 \\
\hline $\begin{array}{c}\mathrm{E} \times 0040 \\
\end{array}$ & Vessel (mount) & & Essex & \begin{tabular}{|lll} 
Ugley \\
\end{tabular} & $\mathrm{RU}$ & & PAS & & Geometric sub-triangular, top shaped mount & & PAS BH-118825 \\
\hline EX0041 & \begin{tabular}{|l|l|l|l|l|l|l} 
Vesel (mount) \\
Vessel (mount)
\end{tabular} & & \begin{tabular}{|l} 
Essex \\
Essex
\end{tabular} & \begin{tabular}{|l|l} 
Birch \\
Wix
\end{tabular} & $\frac{\mathrm{RU}}{\mathrm{RU}}$ & & $\begin{array}{l}\text { PAS } \\
\text { PAS }\end{array}$ & & Geometric sub-triangular, top shaped mount & & \begin{tabular}{|l} 
PAS ESS-7F3EB2 \\
PAE ESS-332F1
\end{tabular} \\
\hline Ex0043 & $\begin{array}{l}\text { Handled Pan } 3 \\
\text { (handle) }\end{array}$ & Eggers 160 & Essex & Great Bentley & $\mathrm{RU}$ & & PAS & & & Hilted Handle & PAS ESS-6BE383 \\
\hline ex0044 & $\begin{array}{l}\text { Handled Pan } 1 \\
\text { (handle) }\end{array}$ & Eggers 154-155 & Essex & Steeple Bumpstead & RU & & PAS & & & Zoomorphic handle terminal, either lion or a bear & PAS SF-9C7FA4 \\
\hline Ex0045 & Handled Pan 4 & Coptic & Essex & Broxted & $\mathrm{RU}$ & & PAS & & & & PAS ESS-1D3342 \\
\hline ex0046 & Vessel (fragment) & & Essex & Roxwell & RU & & PAS & & $\begin{array}{l}\text { Zoomorphic birds head suspension hook, probably a } \\
\text { swan or duck }\end{array}$ & & PAS ESS-E58103 \\
\hline Ex0047 & Bowl & & Essex & Manningtree & $\mathrm{RU}$ & & PAS & & & & PAS ESS-45C445 \\
\hline EX048 & \begin{tabular}{|l} 
Vessel (mount) \\
Vsel (nout
\end{tabular} & & Essex & Elsenham & $\mathrm{RU}$ & & PAS & & Floral (possibly) leaf shaped mount & & 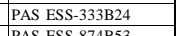 \\
\hline E0049 & Vessel (mount) & & Essex & Fordham & $\mathrm{RU}$ & & PAS & & Zoomorphic birds head suspension hook, probably a & & PAS ESS-874B53 \\
\hline ex0050 & Vessel (fragment) & & Essex & Fingringhoe & $\mathrm{RU}$ & & PAS & & $\begin{array}{l}\text { Zoomorpritic birds head suspension hook, rrobably a } \\
\text { swan or duck }\end{array}$ & & PAS ESS-2B77F7 \\
\hline Ex0051 & Vessel (mount) & & Essex & Good Easter & $\mathrm{RU}$ & & PAS & & Floral leaf shaped hanging vessel mount, five points & & PAS ESS-DD8738 \\
\hline E0052 & Vessel (mount) & & Essex & Ardleigh & $\mathrm{RU}$ & & PAS & & Geometric pelta vessel foot & & PAS ESS-A61324 \\
\hline ex0053 & Vessel (fragment) & & Essex & Boreham & RS & & $\mathrm{SF}$ & & Geometric 'pear-shaped' suspension loop & & $\begin{array}{l}\text { Germany et al. } 2003, \\
75 \text { (18) }\end{array}$ \\
\hline ex0054 & $\begin{array}{l}\begin{array}{l}\text { Handled Pan } 2 \\
\text { (handle) }\end{array} \\
\end{array}$ & Rudge Cup type & Essex & Kelvedon & RS & & $\mathrm{SF}$ & & & \begin{tabular}{|l|} 
Geometric checker board enamel pattern in black, \\
yellow, blue and red enamel
\end{tabular} & \begin{tabular}{|l}
$\begin{array}{l}\text { Rodwell, } 198857 \text { (Fig. } \\
\text { 48.43) }\end{array}$ \\
\end{tabular} \\
\hline ex0055 & Jug (handle) & & Essex & Little Oakley & RS & Site II & $\mathrm{SF}$ & & & Floral leaf shaped handle medallion & \begin{tabular}{|l|}
$\begin{array}{l}\text { Barford 2002, } 88 \\
\text { (CU11) }\end{array}$ \\
\end{tabular} \\
\hline ex0056 & Jug (handle) & & Essex & Colchester & U & Sheepen Hill & $\mathrm{SF}$ & & & & Niblett 1985, 119 (21) \\
\hline ex0057 & Vessel (mount) & & Essex & Witham & RS & Ily Chimneys & $\mathrm{SF}$ & & Geometric triangualr vessel mount & & Turner 1999.83(47) \\
\hline ex0058 & Jug & & Essex & Ashdon & RS & \begin{tabular}{|l}
$\begin{array}{l}\text { Bartlow Hills, Barrow IV, } \\
\text { Cemetary }\end{array}$ \\
\end{tabular} & GD & 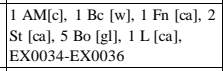 & Floral neck ring & \begin{tabular}{|l} 
Zoomorphic bull skull handle medallion, \\
zoomorphic sphinx standing on storks thumb rest
\end{tabular} & \begin{tabular}{|l} 
Philpott, 1991 256; \\
VCH 3 (Essex) 1963 \\
41
\end{tabular} \\
\hline ex0059 & Handled Pan 1 & Eggers 154-155 & Essex & Ashdon & RS & $\begin{array}{l}\text { Bartlow Hills, Barrow IV, } \\
\text { Cemetary }\end{array}$ & GD & 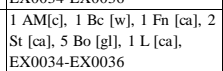 & theng & $\begin{array}{l}\text { Zoomorphic rams head handle terminal, fluted } \\
\text { handle }\end{array}$ & $\begin{array}{l}\text { Philpott, 1991 256; } \\
\text { Phy } 3 \text { (Essex) } 1963 \\
41\end{array}$ \\
\hline
\end{tabular}




\begin{tabular}{|c|c|c|c|c|c|c|c|c|c|c|c|}
\hline EX0060 & Jar & & Essex & Ashdon & RS & $\begin{array}{l}\begin{array}{l}\text { Bartlow Hills, Barrow IV, } \\
\text { Cemetary }\end{array} \\
\text {. }\end{array}$ & GD & 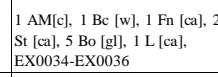 & $\begin{array}{l}\text { Floral leaves and vines, geometric triangle incised } \\
\text { bands, linear etchings on rim, enameleded }\end{array}$ & $\begin{array}{l}\text { Geometric linear etchings, geometric tapered } \\
\text { terminals to drop loop handle }\end{array}$ & $\begin{array}{l}\text { Philpot, 1991 256; } \\
\text { VCH 3 (Essex) 1963 } \\
\text { 41; BM 1868.0801.1 }\end{array}$ \\
\hline EX0061 & Jug & Eggers $123-126$ & Essex & Ashdon & RS & $\begin{array}{l}\begin{array}{l}\text { Bartlow Hills, Barrow III, } \\
\text { Cemetary }\end{array} \\
\end{array}$ & $\mathrm{GD}$ & $\begin{array}{l}3 \mathrm{Bo}[\mathrm{gl}], 1 \mathrm{~L}[\mathrm{ff}, 1 \mathrm{~V} \text { [c], } \\
\text { EX0038 }\end{array}$ & & & \begin{tabular}{|l} 
VCH 3 (Essex) 1963 \\
40
\end{tabular} \\
\hline EX0062 & Handled Pan 1 & Eggers $154-155$ & Essex & Ashdon & RS & $\begin{array}{l}\text { Bartlow Hills, Barrow III, } \\
\text { Cemetary }\end{array}$ & GD & $\begin{array}{l}3 \text { Bo [g]], } 1 \mathrm{~L} \text { [f], } 1 \mathrm{~V} \text { [c], } \\
\text { EX0037 }\end{array}$ & & $\begin{array}{l}\text { Zoomorphic ram's head handle terminal, fluted } \\
\text { handle }\end{array}$ & $\begin{array}{l}\text { VCH } 3 \text { (Essex) } 1963 \\
40\end{array}$ \\
\hline EX0063 & Jug & & Essex & Ashdon & RS & $\begin{array}{l}\text { Bartlow Hills, Barrow V, } \\
\text { Cemetary }\end{array}$ & $\mathrm{GD}$ & 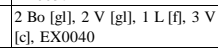 & & $\begin{array}{l}\text { Zomomorphic lion's head above cow skull thumb } \\
\text { rest, zoomorphic lion's paw handle medallion }\end{array}$ & $\begin{array}{l}\text { VCH } 3 \text { (Essex) } 1963 \\
42\end{array}$ \\
\hline EX0064 & Handled Pan 1 & Eggers $154-155$ & Essex & Ashdon & RS & $\begin{array}{l}\begin{array}{l}\text { Bartlow Hills, Barrow V, } \\
\text { Cemetary }\end{array} \\
\end{array}$ & $\mathrm{GD}$ & 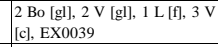 & & $\begin{array}{l}\text { Zoomorphic ram's head handle terminal, fluted } \\
\text { handle }\end{array}$ & $\begin{array}{l}\text { VCH } 3 \text { (Essex) } 1963 \\
42\end{array}$ \\
\hline EX0065 & Jug & Eggers $123-126$ & Essex & Ashdon & RS & $\begin{array}{l}\text { Bartlow Hills, Barow VII, } \\
\text { Cemetary }\end{array}$ & GD & $\begin{array}{l}2 \mathrm{~F}[\mathrm{cc}, 5 \mathrm{C}[\mathrm{cc}, 2 \mathrm{Bo}[\mathrm{gl}], 3 \mathrm{~V} \\
{[\mathrm{c}], 1 \mathrm{~L}[\mathrm{f}], \mathrm{EX} 0042}\end{array}$ & & Anthropomorphic human bust handle medallion & \begin{tabular}{|l} 
VCH 3 (Essex) 1963 \\
43
\end{tabular} \\
\hline EX0066 & Bowl & & Essex & Ashdon & RS & \begin{tabular}{|l} 
Bartlow Hills, Barrow VII, \\
Cemetary
\end{tabular} & $\mathrm{GD}$ & $\begin{array}{l}2 \mathrm{~F}[\mathrm{cc}], 5 \mathrm{C}[\mathrm{cc}, 2 \mathrm{Bo}[\mathrm{gl}], 3 \mathrm{~V} \\
{[\mathrm{cc}, 1 \mathrm{~L}[\mathrm{ff}, \mathrm{EX} 0041}\end{array}$ & & $\begin{array}{l}\text { Anthropomorphic human bust veiled thumb rest } \\
\text { standing on two birds }\end{array}$ & $\begin{array}{l}\text { VCH } 3 \text { (Essex) } 1963 \\
43\end{array}$ \\
\hline EX0067 & & Eggers $128 \mathrm{a}$ & Essex & Ashdon & RS & $\begin{array}{l}\text { Bartlow Hills, Barrow IV, } \\
\text { Cemetary }\end{array}$ & GD & 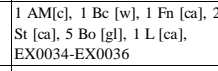 & & & $\begin{array}{l}\text { Philpott, } 1991 \text { 256; } \\
\text { VCH 3(Essex) 1963 } \\
41\end{array}$ \\
\hline EX0068 & $\begin{array}{l}\text { Handled Pan } 2 \\
\text { (handle) }\end{array}$ & Eggers 139-144 & Essex & Colchester & u & & $\mathrm{SF}$ & & & Concentric Circle handle loop & $\begin{array}{l}\text { Crummy 1992, } 156 \\
\text { (543) }\end{array}$ \\
\hline EX0069 & Jug (handle) & Eggers 123-126 & Essex & Colchester & u & & SF & & & Floral (?) thumbrest; jug lid attached & $\begin{array}{l}\text { Crummy } 1992,156 \\
(544)\end{array}$ \\
\hline Ex0070 & Jar & & Essex & Elsenham & RS & & GD & 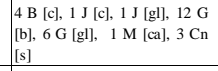 & Geometric enameled chequer-board & & $\begin{array}{l}\text { Johns 1993, 161-165 } \\
\text { Breeze 2012,16; BM } \\
\text { 1991.1201.1; }\end{array}$ \\
\hline EX0071 & Bowl & & Essex & Elsenham & RS & & GD & 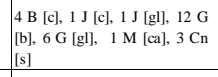 & & & $\begin{array}{l}\text { Johns } 1993,161-165 \\
\text { BM **1991.1202.1- } \\
43(?)\end{array}$ \\
\hline EX0072 & Strainer & Eggers 160 & Essex & Great Wakering & $\mathrm{RU}$ & & SF & & Geometric waves and trefoil strainer dot patterns & Hilted Handle & BM 1892.1104.14 \\
\hline EX0073 & Jug & Eggers 125 & Essex & Stanway & M & $\begin{array}{l}\begin{array}{l}\text { Grave BF64, "The Warrior's } \\
\text { Burial" }\end{array} \\
\end{array}$ & GD & 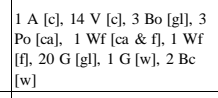 & & $\begin{array}{l}\begin{array}{l}\text { Zoomorphic lions thumbrest and paw handle } \\
\text { medallion }\end{array} \\
\text { a }\end{array}$ & $\begin{array}{l}\text { Crummy et al. 2007, } \\
170-196 \text { (BBF64.25) }\end{array}$ \\
\hline EX0074 & Handled Pan 1 & Eggers 154 & Essex & Stanway & M & $\begin{array}{l}\begin{array}{l}\text { Grave BF64, "The Warrior's } \\
\text { Burial" }\end{array} \\
\text { (a) }\end{array}$ & GD & 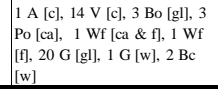 & & Zoomorphic ram's head handle terminal & \begin{tabular}{|l}
$\begin{array}{l}\text { Crummy et al. 2007, } \\
170-196 \text { (BF64.26) }\end{array}$ \\
\end{tabular} \\
\hline
\end{tabular}




\begin{tabular}{|c|c|c|c|c|c|c|c|c|c|c|c|}
\hline EX0075 & Handled Pan 2 & Eggers 137 & Essex & Stanway & M & $\begin{array}{l}\begin{array}{l}\text { Grave CF47, "The Doctor's } \\
\text { Burial" }\end{array} \\
\end{array}$ & GD & 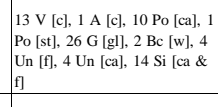 & & Crescent handle loop; Caducaeus & $\begin{array}{l}\text { Crummy et al. 2007, } \\
201-253 \text { (CF47.21) }\end{array}$ \\
\hline EX0076 & Strainer & & Essex & Stanway & M & $\begin{array}{l}\begin{array}{l}\text { Grave CF47, "The Doctor's } \\
\text { Burial" }\end{array} \\
\end{array}$ & GD & 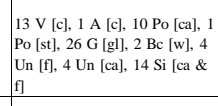 & Zoomorphic dragonesque spout & & $\begin{array}{l}\text { Crummy et al. 2007, } \\
\text { 201-253 (CF47.22) }\end{array}$ \\
\hline EX0077 & Vessel (fragment) & & Essex & Stanway & M & Chamber BF6 & GD & $\begin{array}{l}21 \mathrm{~V}[\mathrm{cc}], 2 \mathrm{~A}[\mathrm{cc}, 1 \mathrm{Fn}[\mathrm{ca}], 1 \\
\mathrm{G}[\mathrm{w}], 1 \mathrm{Po}[\mathrm{f}]\end{array}$ & & & $\begin{array}{l}\text { Crummy et al. 2007, } \\
\text { 104-127 (BF6.25) }\end{array}$ \\
\hline EY0001 & Vessel (handle) & & East Riding of Yorkshir. & Fridaythorpe & $\mathrm{RU}$ & & PAS & & & Zoomorphic swan's head & PAS NLM-596735 \\
\hline EY0002 & \begin{tabular}{|l}
$\begin{array}{l}\text { Handled Pan } 2 \\
\text { (handle) }\end{array}$ \\
\end{tabular} & & East Riding of Yorkshir & Humbleton & $\mathrm{RU}$ & Burton Constable & PAS & & & & PAS FAKL-58F455 \\
\hline EY0003 & Vessel (mount) & & East Riding of Yorkshir. & North Cave & $\mathrm{RU}$ & & PAS & & Geometric fan-shaped mount & & PAS SWYOR-53D721 \\
\hline EY0004 & Handled Pan 4 & Coptic & East Riding of Yorkshir & Hayton & $\mathrm{RU}$ & & PAS & & & & $\begin{array}{l}\text { PAS RESEARCH- } \\
\text { 230A51 }\end{array}$ \\
\hline EY0005 & Handled Pan 2 & Eggers $139-144$ & East Riding of Yorkshir & Shipton Thorpe & RS & & PAS & & & Geometric framing lines & PAS SWYOR-8F20AS \\
\hline EY0006 & Jar & & East Riding of Yorkshir & Shipton Thorpe & RS & & PAS & & Geometric punch dots along base of the neck & & PAS YORYM-103E35 \\
\hline EY0007 & Bowl & & East Riding of Yorkshir. & Thwing & RS & Bridlington Area & PAS & & & & PAS NCL-55ECD6 \\
\hline EY0008 & Vessel (mount) & & East Riding of Yorkshir. & North Dalton & RS & Bone Field & PAS & & Geometric pelta shaped vessel foot & & PAS NCL-B86E85 \\
\hline EY0009 & Vessel (mount) & & East Riding of Yorkshir & North Dalton & RS & & PAS & & Geometric pelta shaped vessel foot & & PAS YORYM-6A0083 \\
\hline Fo001 & Bowl & & Flintshire & Halkyn & RS & Halkyn Mountain & SD & F0001-F0008 & & & Kennet 1971, 1283.1 \\
\hline F0002 & Bucket & & Flintshire & Halkyn & RS & Halkyn Mountain & SD & F0001-F0008 & $\begin{array}{l}\begin{array}{l}\text { Geometric triangular mount fittings with trefoil } \\
\text { hoops }\end{array} \\
\end{array}$ & & Kennet $1971,1283.2$ \\
\hline F0003 & Bucket & & Flintshire & Halkyn & RS & Halkyn Mountain & SD & F0001-F0008 & $\begin{array}{l}\text { Goometric triangular mount fittings with trefoil } \\
\text { hoops }\end{array}$ & & Kennet $1971,1283.3$ \\
\hline $\begin{array}{l}\text { F0004 } \\
\text { F0005 } \\
\end{array}$ & \begin{tabular}{|l} 
Cauldron \\
Bowl \\
\end{tabular} & Bassin Uní & \begin{tabular}{|l|} 
Flintshire \\
Flintshire \\
\end{tabular} & $\begin{array}{l}\text { Halkyn } \\
\text { Halkyn }\end{array}$ & \begin{tabular}{|l} 
RS \\
RS \\
\end{tabular} & \begin{tabular}{|l|} 
Halkyn Mountain \\
Halkyn Mountain \\
\end{tabular} & \begin{tabular}{|l}
$\mathrm{SD}$ \\
$\mathrm{SD}$ \\
\end{tabular} & \begin{tabular}{|l} 
Fo001-F0008 \\
F0001-F0008
\end{tabular} & $\begin{array}{l}\begin{array}{l}\text { Geometric triangular mount fittings with trefoil } \\
\text { hoops }\end{array} \\
\end{array}$ & & $\begin{array}{l}\text { Kennet 1971, } 1283.4 \\
\text { Kennet 1971, } 1283.5 \\
\end{array}$ \\
\hline Fo006 & Bowl & Irchester & Flintshire & Halkyn & RS & Halkyn Mountain & SD & F0001-F0008 & & & Kennet 1971, 1283.6 \\
\hline F0007 & Bowl & Irchester & Flintshire & Halkyn & RS & Halkyn Mountain & SD & F0001-F0008 & & & Kennet 1971, 1283.7 \\
\hline Fo008 & Bowl & Irchester & Flintshire & Halkyn & RS & Halkyn Mountain & SD & F0001-F0008 & & & Kennet 1971, 1283.8 \\
\hline F0009 & Vessel (mount) & & Flintshire & Flint & RS & & PAS & & & & PAS HESH-A0AE36 \\
\hline Fo010 & Jug (lid) & & Flintshire & Flint & RS & Pentre Farm & $\mathrm{SF}$ & & & & $\begin{array}{l}\text { O'Leary et alia 1989, } 64 \\
(4)\end{array}$ \\
\hline G0001 & Strainer (fragment) & Eggers $160-162$ & Gloucestershire & Lydney & RS & Lydney Park & SF & & Geometric linear strainer dot pattern & & Eggers $1968,104(27)$ \\
\hline G0002 & $\begin{array}{l}\text { Handled Pan } 2 \\
\text { (handle) }\end{array}$ & Eggers 140 & Gloucestershire & Circentre & $\mathrm{RU}$ & & SF & & & Concentric circle handle loop & Eggers 1968, $104(28)$ \\
\hline G0003 & Handled Pan 2 & Eggers 146 & Gloucestershire & Kingholm & M & & SF & & Concentric circle foot ring, body band & $\begin{array}{l}\text { Concentric circle handle loop, linear etched } \\
\text { boarder }\end{array}$ & Eggers 1968, 104 (26) \\
\hline G0004 & \begin{tabular}{|l|l} 
Vessel (mount) \\
Vosel (ment)
\end{tabular} & & 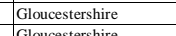 & Twyning & $\mathrm{RU}$ & & $\begin{array}{l}\text { PAS } \\
\text { PAS }\end{array}$ & & 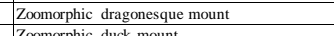 & & $\begin{array}{l}\text { PAS WAW-CEOACS } \\
\text { PAS WWW-CCFOPI }\end{array}$ \\
\hline G0005 & \begin{tabular}{|l} 
Vessel (mount) \\
Vosel (moutt
\end{tabular} & & 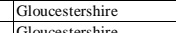 & $\begin{array}{ll}\text { Leigh } \\
\text { Sddery }\end{array}$ & $\frac{R S}{\text { PS }}$ & & PAS & & 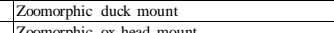 & & PAS WAW-C7FOF1 \\
\hline G0006 & Vessel (mount) & & Gloucestershire & Sudeley & RS & & PAS & & $\begin{array}{l}\text { Zoomorphic ox head mount } \\
\text { Geometric rectangle and triangle body band; }\end{array}$ & Floral wheat stalks on perimeter; Concentric & PAS GLO-63AEF2 \\
\hline G0007 & Handled Pan 2 & Eggers 139-144 & Gloucestershire & Woodchester & RS & DM I 137 & \begin{tabular}{|l}
$\mathrm{SF}$ \\
$\mathrm{SF}$ \\
\end{tabular} & & Concentric circle umbo and foot ring & circle handle medallion & \begin{tabular}{|l|} 
BM 1811.0607 .9 \\
$\begin{array}{l}\text { Wacher \& McWhirr } \\
\text { 1982, 93 (30) }\end{array}$
\end{tabular} \\
\hline G0009 & Bucket (fragment) & & Gloucestershire & Cirencester & u & CY V 8 (house) & SF & & Floral leaf-shaped hanging vessel mount & & $\begin{array}{l}\text { McWhirr 1986, } 111 \\
(71)\end{array}$ \\
\hline
\end{tabular}




\begin{tabular}{|c|c|c|c|c|c|c|c|c|c|c|c|}
\hline G0010 & Jug (lid) & Eggers 123-126 & Gloucestershire & Cirencester & u & ARI 46 & $\mathrm{SF}$ & & Floral (possible) leaf-shaped jug lid & & $\begin{array}{l}\begin{array}{l}\text { Holbrook } 1998,318 \\
(40)\end{array} \\
\end{array}$ \\
\hline G0011 & Vessel (mount) & & Gloucestershire & Cirencester & $\mathrm{u}$ & AHVIII 26 & $\mathrm{SF}$ & & Anthropomorphic reclicing banqueter vessel mount & & $\begin{array}{l}\text { Holbrook 1998, } 318 \\
(43)\end{array}$ \\
\hline G0012 & Vessel (fragment) & & Gloucestershire & Lydney & RS & Bath Building & $\mathrm{SF}$ & & Floral vine pattern on body; pearl scalloping on base & & Wheeler 1932, 87 (112) \\
\hline G0013 & Bowl (fragment) & & Gloucestershire & Uley & RS & Structure IX & $\mathrm{SF}$ & & & & \begin{tabular}{|l|}
$\begin{array}{l}\text { Woodward \& Leach } \\
1993,209(5)\end{array}$ \\
\end{tabular} \\
\hline G0014 & Jug (lid) & Eggers 128 & Gloucestershire & Uley & RS & Structure IV & $\mathrm{SF}$ & & Geometric circular lid & & \begin{tabular}{|l} 
Woodward \& Leach \\
$1993,209(9)$
\end{tabular} \\
\hline G0015 & Jug (handle) & & Gloucestershire & Tewkesbury & RS & & $\mathrm{SF}$ & & & Zoomorphic clawed foot handle medallion & BM 1903.014.1 \\
\hline GWY0001 & Handled Pan 2 & Eggers 140 & Gwynedd & Ynys Gwrtheyrn & $\mathrm{RU}$ & & SD & GWY0001-GWY0003 & Concentric circle umbo & Concentric circle handle medallion & \begin{tabular}{|l|}
$\begin{array}{l}\text { Eggers } 1968,103-104 \\
\text { (17a) }\end{array}$ \\
\end{tabular} \\
\hline GWY0002 & Handled Pan 2 & Eggers 142 & Giwnnedd & Ynys Gwrtheyrn & $\mathrm{RU}$ & & SD & GWY0001-GWY0003 & Concentric circle umbo & $\begin{array}{l}\text { Concentric circle handle medallion, geometric } \\
\text { waves on handle }\end{array}$ & \begin{tabular}{|l|}
$\begin{array}{l}\text { Eggers 1968, } 103-104 \\
\text { (17a) }\end{array}$ \\
\end{tabular} \\
\hline GWY0003 & Bowl & Eggers 110 & Gwynedd & Ynys Gwrtheyrn & $\mathrm{RU}$ & & SD & GWY0001-GWY0003 & & & \begin{tabular}{|l|}
$\begin{array}{l}\text { Eggers } 1968,103-104 \\
\text { (17a) }\end{array}$ \\
\end{tabular} \\
\hline GWY0004 & Handled Pan 2 & Eggers 140 & Gwynedd & Harlech & $\mathrm{RU}$ & & SD & GWY0004-GWY0008 & & Geometric circle handle loop & Eggers 1968, $104(18 \mathrm{az})$ \\
\hline GWY0005 & Handled Pan 2 & Eggers 139-144 & Giwnnedd & Harlech & $\mathrm{RU}$ & & SD & GWY0004-GWY0008 & & Geometric circle handle loop & Eggers 1968, 104 (18b) \\
\hline GWY0006 & Vessel (fragment) & & Gwynedd & Harlech & $\mathrm{RU}$ & & SD & GWY0004-GWY0008 & & & Eggers 1968, $104(18 \mathrm{c})$ \\
\hline GWY0007 & Bowl & Eggers 110 & Gwynedd & Harlech & $\mathrm{RU}$ & & SD & GWY0004-GWY0008 & & & Eggers 1968, $104(18 \mathrm{~d})$ \\
\hline GWY0008 & Bowl & & Gwynedd & Harlech & $\mathrm{RU}$ & & SD & GWY0004-GWY0008 & & & Eggers 1968, $104(18 \mathrm{e})$ \\
\hline GWY0009 & Handled Pan 2 & Eggers 142 & Giwynedd & Llanberis & $\mathrm{RU}$ & & SD & GWY0009-GWY0012 & Concentric cirlce umbo and foot ring & \begin{tabular}{|} 
Concentric circle handle medallion; stamped \\
inscription reads "ABVCCVI..." "
\end{tabular} & \begin{tabular}{|l} 
Eggers 1968, 104 (21); \\
Mcreake \& Moore \\
1978, 333 (1); RIB II \\
2415.1
\end{tabular} \\
\hline GWY0010 & Handled Pan 2 & Eggers 142 & Giwnnedd & Llanberis & $\mathrm{RU}$ & & SD & GWY0009-GWY0012 & Concentric cirlce umbo and foot ring & Concentric circle handle medallion & Eggers 1968, 104 (21) \\
\hline GWY0011 & Handled Pan 2 & Eggers 142 & Gwynedd & Llanberis & $\mathrm{RU}$ & & SD & GWY0009-GWY0012 & Concentric cirlce umbo and foot ring & Concentric circle handle medallion & Eggers 1968, 104 (21) \\
\hline GWY0012 & Handled Pan 2 & Eggers 142 & Gwynedd & Llanberis & $\mathrm{RU}$ & & SD & GWY0009-GWY0012 & Concentric cirlce umbo and foot ring & Concentric circle handle medallion & Eggers 1968, 104 (21) \\
\hline GWY0013 & $\begin{array}{l}\text { Handled Pan } 2 \\
\text { (handle) }\end{array}$ & Eggers 142 & Gwynedd & Caernarvon & M & & SF & & & $\begin{array}{l}\text { Concentric circle handle medallion, inscription } \\
\text { "SAGAVGVF" in rectangular field }\end{array}$ & \begin{tabular}{|l|} 
Eggers 1968, 104 \\
(22a); Wright 1969, \\
238 (20); RIB II \\
2415.45
\end{tabular} \\
\hline НАМо0о1 & Handled Pan 2 & Eggers 140 (c.f.) & Hampshire & Silchester & u & Pit 10, Insula XXIII & $\mathrm{SF}$ & HAM0002 & Concentric circle umbo & $\begin{array}{l}\text { Concentric circle handle terminal, "PIAV" } \\
\text { inscription on handle }\end{array}$ & $\begin{array}{l}\text { Eggers 1966, } 102 \text { (6a); } \\
\text { RgI II 2415.37 }\end{array}$ \\
\hline НАм0002 & Handled Pan 1 & Eggers $154-155$ & Hampshire & Silchester & $\mathrm{u}$ & Pit 10, Insula XXIII & SF & НАМ 0001 & Concentric circle umbo & Zoomorphic ram's head handle terminal & \begin{tabular}{|l|} 
Eggers 1966, 102(6b); \\
Boon 1974 2228-230 (1)
\end{tabular} \\
\hline$\frac{\text { HАMO0003 }}{\text { HAMOOOO4 }}$ & \begin{tabular}{|l} 
Jug \\
Bucket (fragment)
\end{tabular} & Eggers 123-126 & $\begin{array}{l}\text { Hampshire } \\
\text { Hampshire }\end{array}$ & \begin{tabular}{|l} 
Winchester \\
Beaulieu
\end{tabular} & \begin{tabular}{|l}
$\mathrm{U}$ \\
$\mathrm{RU}$
\end{tabular} & Grange Road, Grave 2 & $\frac{\mathrm{GD}}{\mathrm{PAS}}$ & 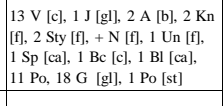 & $\begin{array}{l}\text { Trefoil mouth } \\
\text { Geometric tan/'pelta' shaped foot }\end{array}$ & $\begin{array}{l}\text { Anthropomorphic female bust thumb rest and } \\
\text { handle medallion }\end{array}$ & $\begin{array}{l}\text { Philpott, 1991 270; } \\
\text { Biddle, } 1967 \text { 230-231, } \\
\text { 240-242 } \\
\text { PAS HAMP-EFA6E4 }\end{array}$ \\
\hline НАмо005 & Bucket (fragment) & Fagers $123-126$ & Hampshire & Medstend CP & $\mathrm{RU}$ & & PAS & & \begin{tabular}{|l} 
Goomentric fan'/pelta' 'shaped foot \\
Zoorophic duck statuette, feather design
\end{tabular} & & PAS HAMP-EFA6E4 \\
\hline HАM0006 & $\begin{array}{l}\text { Handled Pan } 1 \\
\text { (handle) }\end{array}$ & Eggers $154-155$ & Hampshire & Ropley & Rs & & PAS & & Zoomorphic ram's head handle terminal & & PAS HAMP-D46597 \\
\hline HAM0007 & Vessel (fragment) & & Hampshire & King's Worthy & $\mathrm{RU}$ & & PAS & & & Floral leaf thumb rest, possibly palm & PAS HAMP-D685D2 \\
\hline НАмо0ов & Vessel (fragment) & & Hampshire & Broughton & $\mathrm{RU}$ & & PAS & & \begin{tabular}{|l}
$\begin{array}{l}\text { Zoomorphic bird statuette on lid, perhaps a duck or } \\
\text { chicken }\end{array}$ \\
\end{tabular} & & PAS SUR-411370 \\
\hline HAM0009 & \begin{tabular}{|l|l} 
Vessel (fragment) \\
Vocsl frament
\end{tabular} & & $\begin{array}{l}\text { Hampshire } \\
\text { Hompchire }\end{array}$ & Ropley & $\begin{array}{ll}\mathrm{RS} \\
\mathrm{RS}\end{array}$ & & $\begin{array}{l}\text { PAS } \\
\text { PAS }\end{array}$ & & 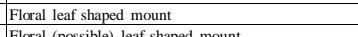 & & \begin{tabular}{|l|l|} 
PAS SUR-934DA8 \\
PAS SIP 20151
\end{tabular} \\
\hline HAM0010 & Vessel (fraggent) & & Hampshire & & & & PAS & & & & \\
\hline
\end{tabular}




\begin{tabular}{|c|c|c|c|c|c|c|c|c|c|c|c|}
\hline HАM0011 & Vessel (fragment) & & Hampshire & Damerham & RS & & PAS & & & Geometric etched lines on handle sides & PAS WILT-149137 \\
\hline $\begin{array}{ll}\text { HAM0012 } \\
\text { HAM013 }\end{array}$ & \begin{tabular}{|l} 
Vessel (fragment) \\
Vessel (frament)
\end{tabular} & & $\begin{array}{l}\text { Hampshire } \\
\text { Hampstire } \\
\end{array}$ & 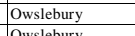 & $\frac{\mathrm{RS}}{\mathrm{PS}}$ & & $\begin{array}{l}\text { PAS } \\
\text { PAS }\end{array}$ & & & 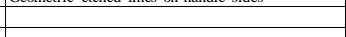 & PAS FASW-4CB045 \\
\hline HAM0013 & Vessel (fragment) & & Hampshire & \begin{tabular}{|l|} 
Owslebury \\
Corhampton And
\end{tabular} & $\mathrm{RS}$ & & PAS & & Zoomorphic duck statuette & & PAS HAMP3382 \\
\hline HAM0014 & Vessel (fragment) & & Hampshire & $\begin{array}{l}\text { Corhampton And } \\
\text { Meonstoke }\end{array}$ & $\mathrm{RU}$ & & PAS & & Geometric fan'pelta' shaped foot & & PAS HAMP2278 \\
\hline HAM0015 & Vessel (fragment) & & Hampshire & Wherwell & $\mathrm{RU}$ & & PAS & & & Zoomorphic birds head terminal, perhaps a swan & PAS HAMP336 \\
\hline HАM0016 & Bucket (fragment) & & Hampshire & Fareham & M & Portchester Castle & $\mathrm{SF}$ & & Zoomorhpic ox head hanging vessel mount & & Cunliffe $1975,212(61)$ \\
\hline HАM0017 & Vessel (fragment) & & Hampshire & Silchester & $\mathrm{u}$ & Forum-Basilica & SF & & Geometric fan'pelta' shaped foot & & $\begin{array}{l}\text { Fufflfor \& Timby 2000, } \\
345 \text { (46) }\end{array}$ \\
\hline HАм0018 & Vessel (fragment) & & Hampshire & Silchester & u & Forum-Basilica & SF & & Concentric circle umbo & & $\begin{array}{l}\text { Fulford \& Timby 2000, } \\
350(84)\end{array}$ \\
\hline HАм0019 & Vessel (fragment) & & Hampshire & Silchester & $\mathrm{u}$ & Insula IX & SF & & Geometric fan $/$ pelta' shaped foot & & \begin{tabular}{|l} 
Fulford \& Clarke 2011. \\
$108(46)$
\end{tabular} \\
\hline НАм0020 & Jug (handle) & & Hampshire & Silchester & u & Insula IX & $\mathrm{SF}$ & & \begin{tabular}{|l} 
Anthropomorphic human foot handle terminal; \\
zoomorphic leaf thumb rest
\end{tabular} & & \begin{tabular}{|l|}
$\begin{array}{l}\text { Fulford \& Clarke } 2011, \\
114(65)\end{array}$ \\
\end{tabular} \\
\hline HАм0021 & Jug & Egger 129 & Hampshire & Silchester & $\mathrm{u}$ & Insula XXIII, Well & SF & & & $\begin{array}{l}\text { Anthropomorphic handle medallion of bearded } \\
\text { man, perhaps Silenus }\end{array}$ & \begin{tabular}{|l} 
Boon $1974,85-86$ \& \\
$228-229(3)$
\end{tabular} \\
\hline НАм0022 & Cauldron & & Hampshire & Silchester & u & & SF & & & & Boon 1974, $228-229$ (4 \\
\hline HER0001 & Handled Pan 2 & Rudge Cup Type & Hertfordshire & Braughing & RS & & $\mathrm{SF}$ & & Floral vine and leaf, enameled; foot ring & & $\begin{array}{l}\begin{array}{l}\text { Eggers } 1968,105 \\
(31 \mathrm{~A})\end{array} \\
\end{array}$ \\
\hline $\begin{array}{ll}\text { HER00002 } \\
\text { HERPO03 }\end{array}$ & \begin{tabular}{|l|l|l} 
Vessel (mount) \\
Veses (mont)
\end{tabular} & & Hertfordshire & \begin{tabular}{|l|l} 
St. Albans \\
sitas
\end{tabular} & $\mathrm{U}$ & $\begin{array}{ll}\text { Verulamium } \\
\text { Groluminam }\end{array}$ & $\mathrm{SF}$ & & & & \begin{tabular}{|l|l|} 
Frere 1972, 130 \\
Fin? 130
\end{tabular} \\
\hline $\begin{array}{l}\text { HERR0003 } \\
\text { HER0004 }\end{array}$ & \begin{tabular}{|l} 
Vesse (mount) \\
VVsese (mount)
\end{tabular} & & $\begin{array}{l}\text { Hertrfordshire } \\
\text { Herffordshire }\end{array}$ & $\frac{\text { St. Abbans }}{\text { St. Albans }}$ & $\frac{U}{U}$ & \begin{tabular}{|l} 
Verulamium \\
Verulamium
\end{tabular} & $\frac{S F}{S F}$ & & & & \begin{tabular}{|l|l|} 
Frere $1972,2,130$ \\
Frere 1972, 130
\end{tabular} \\
\hline HERO0005 & Jug (handle) & & Hertfordshire & St. Albans & u & $\begin{array}{l}\text { Verulamium } \\
\end{array}$ & SF & & Zoomorphic Dolphin & & Frere 1972, 130 \\
\hline HER0006 & Jug (handle) & & Hertfordshire & \begin{tabular}{|l|l|} 
St. Albans \\
S. lan
\end{tabular} & $\mathrm{U}$ & Verulamium & $\mathrm{SF}$ & & Zoomorphic Dolphin & & Frere 1972, 130 \\
\hline 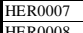 & Jug (handle) & & Hertfordshire & \begin{tabular}{|l|l|} 
St. Albans \\
s.
\end{tabular} & $\mathrm{U}$ & Verulamium & $\frac{S F}{S F}$ & & Zoomorphic Dolphin & & \begin{tabular}{|l|l|l|l|l} 
Freer $1972,130-132$ \\
\end{tabular} \\
\hline $\begin{array}{l}\text { HERRO008 } \\
\text { HEROOO9 }\end{array}$ & Jug (handle) & & $\begin{array}{l}\text { Hertffordshire } \\
\text { Hertofdshire }\end{array}$ & \begin{tabular}{|l|l} 
St. Albans \\
S. Albans
\end{tabular} & $\mathrm{U}$ & $\begin{array}{l}\text { Verulamium } \\
\text { Verralminiming }\end{array}$ & $\frac{S F}{S F}$ & & Floral leat moulding Face female & & $\begin{array}{ll}\text { Freere 1972, } 132 \\
\end{array}$ \\
\hline $\begin{array}{l}\text { HERRO099 } \\
\text { HERO010 }\end{array}$ & $\begin{array}{l}\text { Jug (handle) } \\
\text { Vessel (fragment) }\end{array}$ & & $\begin{array}{l}\text { Hetrtorddhhire } \\
\text { Hertfordshire }\end{array}$ & \begin{tabular}{|l} 
St. Albans \\
St. Albans \\
\end{tabular} & $\frac{u}{u}$ & $\begin{array}{l}\text { levulamium } \\
\text { Verulamium }\end{array}$ & $\begin{array}{l}\mathrm{SF} \\
\mathrm{SF}\end{array}$ & & \begin{tabular}{|l} 
Foral leaf moulding, Face (female) \\
Concentric circle umbo
\end{tabular} & & \begin{tabular}{|l|l|} 
Frere 1972,132 \\
Frere 1972,132 \\
\end{tabular} \\
\hline HERO011 & Jug & & Hertfordshire & St. Albans & U & Verulamium & $\mathrm{SF}$ & & & & Frere 1972,132 \\
\hline HERO012 & Jug & & Hertfordshire & St. Albans & $\mathrm{u}$ & Verulamium & $\mathrm{SF}$ & & & $\begin{array}{l}\text { Floral handle with trefoils and heart shapes } \\
\text { terminating in bulls head at vessel rim }\end{array}$ & Frere 1972, 132 \\
\hline HER0013 & $\begin{array}{l}\begin{array}{l}\text { Handled Pan } 1 \\
\text { (handle) }\end{array} \\
\end{array}$ & Eggers 154-155 & Hertfordshire & St. Albans & $\mathrm{u}$ & Verulamium, unstratified & SF & & & Zoomorphic ram's head terminal, fluted handle & Frere 1972, $138(148)$ \\
\hline HER0014 & $\begin{array}{l}\begin{array}{l}\text { Handled Pan } 1 \\
\text { (handle) }\end{array} \\
\end{array}$ & Eggers $154-155$ & Hertfordshire & St. Albans & $\mathrm{u}$ & Verulamium, B I 55 & $\mathrm{SF}$ & & & Zoomorphic ram's head terminal, fluted handle & Frere 1972, $138(149)$ \\
\hline HER0015 & \begin{tabular}{|l|l|l|l} 
Strainer \\
\end{tabular} & & Hertfordshire & St. Albans & u & Verulamium & $\mathrm{SF}$ & & Geometric punch-dot circle, 'S', and 'Z' & & Frere 1972, 144 \\
\hline HER0016 & \begin{tabular}{|l} 
Handded Pan 3 \\
(handle)
\end{tabular} & Eggers $160-161$ & Hertfordshire & St. Albans & $\mathrm{u}$ & Verulamium & SF & & & & Frere 1984, 51173 \\
\hline HER0017 & $\begin{array}{l}\begin{array}{l}\text { Handled Pan } 3 \\
\text { (handle) }\end{array} \\
\end{array}$ & Eggers $160-161$ & Hertfordshire & St. Albans & $\mathrm{u}$ & Verulamium & SF & & & & Frere 1984, 51174 \\
\hline HER 0018 & Jug (lid) & Eggers $123-126$ & Hertfordshire & St. Albans & $\mathrm{u}$ & Verulamium & $\mathrm{SF}$ & & Zoomorphic Duck sitting or swimming & & Frere 1984, 51175 \\
\hline HER0019 & Bowl & Eggers 98-99 & Hertfordshire & St. Albans & $\mathrm{u}$ & $\begin{array}{l}\text { Verulamium, Silchester Gate, } \\
\text { Mayne Avenue (Cremation } \\
\text { Pit) }\end{array}$ & GD & $\begin{array}{l}15 \mathrm{~V}[\mathrm{cl}, 1 \mathrm{Bo}[\mathrm{gl}], 2 \mathrm{~J}[\mathrm{gl}], 1 \\
\mathrm{Fn}[\mathrm{ff}, \mathrm{Co}[\mathrm{cal}, 4 \mathrm{~L}[\mathrm{cl}, 20 \mathrm{G} \\
{[\mathrm{glg}]}\end{array}$ & $\begin{array}{l}\text { Concentric circle interior and exterior of body; } \\
\text { Concentric cirlce foot ring; omphalos evident }\end{array}$ & $\begin{array}{l}\text { Anthropomorphic female bust thumb rest; } \\
\text { zoomorphic birds heads; Floral leaf handle and } \\
\text { lower handle medallion }\end{array}$ & $\begin{array}{l}\text { Niblett \& Reeves 1990. } \\
\text { 441-446 (Fig. 1) }\end{array}$ \\
\hline HERO020 & $\begin{array}{l}\text { Bowl (mount) } \\
\text { Vessel (mout) }\end{array}$ & tegers $8>-999$ & $\begin{array}{l}\text { Hertrofordshire } \\
\text { Herthire } \\
\end{array}$ & \begin{tabular}{|l} 
St. Mibans \\
St. Michal \\
\end{tabular} & $\mathrm{RU}$ & & PAS & & 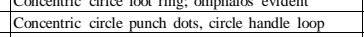 & & PAS BH-5ECIF6 \\
\hline HER0021 & Vessel (mount) & & Hertfordshire & Clothall & RS & & PAS & & Geometric triangular shaped mount & & PAS BH-FB17E1 \\
\hline HER0022 & Vessel (mount) & & Hertfordshire & Clothall & RS & & PAS & & $\begin{array}{l}\text { Anthropomorphic bust, round headed male with } \\
\text { wide nose, large eyes, and wavy hair, possibly satyr } \\
\text { or Pan }\end{array}$ & & PAS BH-C89753 \\
\hline HER 0023 & Jug (lid) & Eggers 123-126 & Hertfordshire & \begin{tabular}{|l} 
Watton-at-Stone \\
atton
\end{tabular} & RS & & PAS & & $\begin{array}{l}\text { Floral leaf (possibly) shaped lid } \\
\text { lat }\end{array}$ & & PAS BH-57C9D5 \\
\hline HER0024 & Vessel (mount) & & Hertfordshire & Clothall & RS & & PAS & & Zoomorphic bird mount & & PAS BH-231086 \\
\hline
\end{tabular}




\begin{tabular}{|c|c|c|c|c|c|c|c|c|c|c|c|}
\hline HER0025 & Vessel (mount) & & Hertfordshire & Ashwell & RS & & PAS & & Zoomorphic bird mount, appears to be a duck & & PAS BH-5D2737 \\
\hline HER0026 & Bucket (fragment) & & Hertfordshire & Albury & $\mathrm{RU}$ & & PAS & & Geometric crescent bucket foot & & PAS BH-4FF557 \\
\hline HER0027 & Vessel (mount) & & Hertfordshire & Much Haaham & RU & & PAS & & $\begin{array}{l}\text { Anthropomorphic bust, female with two buns in } \\
\text { hair, possibly Diana }\end{array}$ & & PAS ESS-C55282 \\
\hline HER0028 & Vessel (mount) & & Hertfordshire & Wymondley & RS & & PAS & & Zoomorphic bull's head hanging vessel mount & & PAS BH-1729A7 \\
\hline HER0029 & Handled Pan 2 & Eggers 153 & Hertfordshire & Baldock & RS & $\begin{array}{l}\text { Upper Wall's Common, Site A } \\
268\end{array}$ & SF & & & Flared handle terminal & \begin{tabular}{|l|}
$\begin{array}{l}\text { Stead \& Rigby 1986, } \\
139(394)\end{array}$ \\
\end{tabular} \\
\hline HER0030 & Bowl & & Hertfordshire & Stanton & RS & Ermine Street; Site D & GD & $3 \mathrm{~V}[\mathrm{cc}]$ & Concentric circle umbo and omphalos dot & & $\begin{array}{l}\text { Potter \& Trow 1988, 58- } \\
59(7)\end{array}$ \\
\hline HER0031 & Jug & Eggers 127 & Hertfordshire & Harpenden & RS & Turners Hall Farm, Grave 1 & GD & 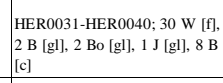 & & $\begin{array}{l}\text { Anthropomorphic Triton statuetete at crest of } \\
\text { handle by rim holding ajug and pan: } \\
\text { Anthropomorphic Medusa head handle medallion }\end{array}$ & $\begin{array}{l}\text { Burnham et al. } 2003, \\
327\end{array}$ \\
\hline HER0032 & Jug & & Hertfordshire & Harpenden & RS & Turners Hall Farm, Grave 1 & GD & $\begin{array}{l}\text { HER0031-HER0040; } 30 \mathrm{~W} \text { [f], } \\
\text { 2 B [gl], } 2 \text { Bo [gl], 1 ] [gl], 8 B } \\
\text { [c] }\end{array}$ & & 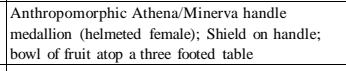 & $\begin{array}{l}\text { Burnham et al. 2003, } \\
327\end{array}$ \\
\hline HER 0033 & Jug & & Hertfordshire & Harpenden & RS & Turners Hall Farm, Grave 1 & GD & 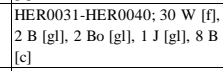 & & & $\begin{array}{l}\text { Burnham et al. } 2003, \\
327\end{array}$ \\
\hline HER 0034 & Bowl & & Hertfordshire & Harpenden & RS & Turners Hall Farm, Grave 1 & GD & 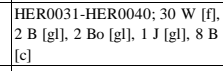 & & & $\begin{array}{l}\text { Burnham et al. } 2003 \text {, } \\
327\end{array}$ \\
\hline HER 0035 & Strainer & & Hertfordshire & Harpenden & RS & Turners Hall Farm, Grave 1 & GD & $\begin{array}{l}\text { HER0031-HER0040; 30 W [f], } \\
\text { 2 B [gl], } 2 \text { Bo [gl], I J [gl], 8 B } \\
\text { [c] }\end{array}$ & & & $\begin{array}{l}\text { Burnham et al. } 2003, \\
327\end{array}$ \\
\hline HER0036 & Strainer & & Hertfordshire & Harpenden & RS & Turners Hall Farm, Grave 1 & GD & 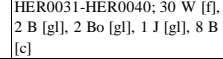 & & & $\begin{array}{l}\text { Burhham et al. 2003, } \\
327\end{array}$ \\
\hline HER 0037 & Handled Pan 1 & Eggers $154-155$ & Hertfordshire & Harpenden & RS & Turners Hall Farm, Grave 1 & GD & 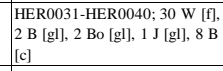 & & Zoomorphic ram's head terminal, fluted handle & $\begin{array}{l}\text { Burhham et al. } 2003, \\
327\end{array}$ \\
\hline HER 0038 & Handled Pan 2 & Eggers 150 & Hertfordshire & Harpenden & RS & Turners Hall Farm, Grave 1 & GD & 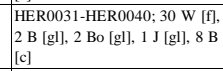 & & (1) & $\begin{array}{l}\text { Burnham et al. } 2003, \\
327\end{array}$ \\
\hline HER0039 & Handled Pan 2 & Eggers 150 & Hertfordshire & Harpenden & RS & Turners Hall Farm, Grave 1 & GD & 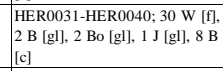 & & & $\begin{array}{l}\text { Burnham et al. } 2003, \\
327\end{array}$ \\
\hline HER0040 & Bowl & & Hertfordshire & Harpenden & RS & Turners Hall Farm, Grave 1 & GD & 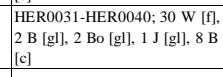 & & & $\begin{array}{l}\text { Burhham et al. } 2003, \\
327\end{array}$ \\
\hline HER0041 & Jug & Eggers 127 & Hertfordshire & Harpenden & RS & Turners Hall Farm, Grave 2 & GD & 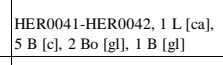 & & $\begin{array}{l}\text { Zoomorphic bull thumb rest; Anthropomorphic } \\
\text { gorgon head handle medallion }\end{array}$ & $\begin{array}{l}\text { Burnham et al. 2003, } \\
327\end{array}$ \\
\hline HER0042 & & & Hertfordshire & Harpenden & RS & Turners Hall Farm, Grave 2 & GD & $\begin{array}{l}\text { HER0041-HER0042, } 1 \mathrm{~L}[\mathrm{ca}], \\
5 \text { B [c] } 2 \text { Bo [gl]], } 1 \text { B [gl] }\end{array}$ & & & $\begin{array}{l}\text { Burnham et al. 2003, } \\
327\end{array}$ \\
\hline IOW0001 & Vessel (fragment) & & Isle of Wight & Newport & RS & ( & PAS & & Geometric crescent shaped bucket foot & & PAS IOW-9CE336 \\
\hline IOW0002 & Vessel (mount) & & Isle of Wight & Newport & RS & & PAS & & Zoomorphic bird mount, perhaps a swan or duck & & PAS IOW-BDD755 \\
\hline Iow0003 & Vessel (mount) & & Isle of Wight & Bembridge & $\mathrm{RU}$ & & PAS & & $\begin{array}{l}\text { Anthropomorphic mount of a reclining male } \\
\text { banquetor }\end{array}$ & & PAS IOW-2F7DD1 \\
\hline K0001 & Jug & & Kent & Lullingston & RS & & GD & 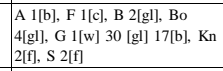 & & & Meates, 1979 122-132 \\
\hline K0002 & Jug & & Kent & Maidstone & RS & $\begin{array}{l}\text { Cremated Deposit 3, Amphora } \\
\text { X }\end{array}$ & GD & $\begin{array}{l}\text { Bo 1[g]], L1[f], T1[uo], Am } \\
\text { I[c] K0003 }\end{array}$ & & & $\begin{array}{l}\text { Philpott, 1991 254; } \\
\text { Scott Robinson, } 1883 \\
78 \text { ( (b); Jessup, } 1958 \\
26 \text { (3) }\end{array}$ \\
\hline K0003 & Jug & Eggers 129 & Kent & Maidstone & RS & $\begin{array}{l}\text { Cremated Deposit 3, Amphora } \\
\mathrm{X}\end{array}$ & GD & \begin{tabular}{|l}
$\begin{array}{l}\text { Bo 1[g], L1[f], T1[uo], Am } \\
\text { I[c] K0002 }\end{array}$ \\
\end{tabular} & & & 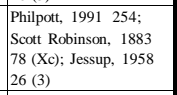 \\
\hline K0004 & Jug & & Kent & Maidstone & RS & $\begin{array}{l}\text { Cremated Deposit 3, Amphora } \\
\mathrm{X}\end{array}$ & $\mathrm{GD}$ & Bo 1[g], T 1[uo], L1[f] K0005 & & & $\begin{array}{l}\text { Philpott, } 1991 \text { 259; } \\
\text { Jessup } 1958 \text { 27(5) }\end{array}$ \\
\hline Ko005 & Jug & & Kent & Maidstone & RS & $\begin{array}{l}\text { Cremated Deposit 3, Amphora } \\
\text { X }\end{array}$ & $\mathrm{GD}$ & Bo 1[g]], T I[uo], L 1[f] K0004 & & & Philpott, 1991259 \\
\hline K0006 & Vessel & & Kent & Maidstone & RS & & $\mathrm{GD}$ & & & & Philpott 1991259 \\
\hline
\end{tabular}




\begin{tabular}{|c|c|c|c|c|c|c|c|c|c|c|c|}
\hline K0007 & Jug & & Kent & Sittingbourne, Bayford & RS & & GD & 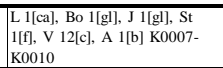 & & \begin{tabular}{|l|}
$\begin{array}{l}\text { Anthropomorphic winged female (?) figure, possiblya } \\
\text { harpy or siren }\end{array}$ \\
\end{tabular} & Philpott 1991259 \\
\hline K0008 & Bowl & & Kent & Sittingbourne, Bayford & RS & & GD & 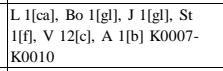 & & & \\
\hline K0009 & Jug & & Kent & Sittingbourne, Bayford & RS & & GD & 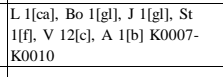 & Anthropomorphic negroid faces & & Philpott 1991259 \\
\hline K0010 & Handled Pan 1 & Eggers 155 & Kent & Sittingbourne, Bayford & RS & & GD & $\begin{array}{l}\text { L I 1[ca], Bo 1[g]], J1[g], St } \\
\text { I[f], V 12[c], A 1[b] K0007- } \\
\text { Ko010 }\end{array}$ & & $\begin{array}{l}\text { Anthropomorphic satyr head with beard, horns and } \\
\text { pointed ears; eyes have space where semi-precious } \\
\text { stones or gems may have once been inserted }\end{array}$ & $\begin{array}{l}\text { Philpott 1991 259; BM } \\
1883.1213 .299\end{array}$ \\
\hline K0011 & Jug & & Kent & Sittingbourne, Bayford & RS & & GD & 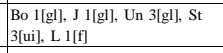 & & \begin{tabular}{|l||}
$\begin{array}{l}\text { Anthropomorphic Ajax in frenzy attacking } \\
\text { zoomorphic beasts }\end{array}$ \\
\end{tabular} & $\begin{array}{l}\text { Philpott 199. 259; BM } \\
\text { 1883.1213.320 }\end{array}$ \\
\hline K0012 & Jug & & Kent & Canterbury & u & Martyrs Field Road & GD & 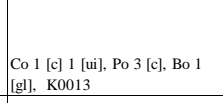 & & & $\begin{array}{l}\text { Philpott } 1991 \text { 314; } \\
\text { Eggers } 19661024 ; \\
\text { Museum of Canterbury } \\
\text { 7847-7848 } \\
\end{array}$ \\
\hline K0013 & Handled Pan 1 & Eggers 155 & Kent & Canterbury & $\mathrm{u}$ & Martyrs Field Road & GD & $\begin{array}{l}\text { Co } 1 \text { [c] } 1 \text { [u]], Po } 3 \text { [c], Bo } 1 \\
\text { [gl], Ko012 }\end{array}$ & Zoomorphic ram's head terminal, fluted handle & & $\begin{array}{l}\text { Philpott } 1991 \text { 314; } \\
\text { Eggers } 1966 \text { 102 4; } \\
\text { Museum of Canterbury } \\
\text { 7847-7848 }\end{array}$ \\
\hline K0014 & Cauldron & & Kent & Springhead & RS & $\begin{array}{l}\text { Vagniacae, Toll Gate, } \\
\text { cremation Pit }\end{array}$ & GD & V 18 [c], G [v], K0015, K0016 & Geometric scalloped rim, perhaps depicting the evil eye & $\begin{array}{l}\text { Zoomorphic duck's head drop-loop handle; Floral leaf } \\
\text { shaped hanging vessel mount }\end{array}$ & \begin{tabular}{|l|l|} 
Mynott 2008, 15 \\
\end{tabular} \\
\hline K0015 & Handled Pan 1 & Eggers 155 & Kent & Springhead & RS & $\begin{array}{l}\text { Vagniacae, Toll Gate, } \\
\text { Cremation Pit }\end{array}$ & GD & V 18 [c], G [v], K0014, K0016 & Concentric circle umbo & Zoomorphic ram's head terminal, fluted handle & Mynott 2008, 15 \\
\hline K0016 & 4 & & Kent & Springhead & RS & $\begin{array}{l}\text { Vagniacae, Toll Gate, } \\
\text { Cremation Pit }\end{array}$ & GD & V 18 [c], G Gl], K0014, K0015 & & $\begin{array}{l}\text { Anthropomorphic female bust thumb rest and handle } \\
\text { medallion }\end{array}$ & Mynott 2008, 15 \\
\hline K0017 & Handled Pan 1 & Eggers 155 & Kent & Springhead & RS & $\begin{array}{l}\text { Vagniacae, Toll Gate, } \\
\text { cremation Pit }\end{array}$ & GD & V 15 [c], K0018 & $\begin{array}{l}\text { Floral flower in centre of basin circled by two bands of } \\
\text { flloral wreathes }\end{array}$ & Zoomorphic ram's head terminal, fluted handle & Mynott 2008, 15 \\
\hline K0018 & Jug & & Kent & Springhead & RS & $\begin{array}{l}\text { Vagniacae, Toll Gate, } \\
\text { Cremation Pit }\end{array}$ & GD & V $15[\mathrm{cc}, \mathrm{K} 0017$ & & $\begin{array}{l}\text { Anthropomorphic female handle medallion; } \\
\text { Zoomorphic lion'd head thumb rest }\end{array}$ & Mynott 2008,15 \\
\hline K0020 & Bowl & Eggers 99 (c.f.) & Kent & Luton & RS & & GD & $\begin{array}{l}1 \mathrm{Bc}[\mathrm{cal}], 1 \mathrm{Bo}[\mathrm{gll}], 1 \mathrm{~B}[\mathrm{gl}], 3 \\
\mathrm{v}[\mathrm{c}, 2 \mathrm{C}[\mathrm{cl}, \mathrm{K} 0027\end{array}$ & Footed bowl, "AFRICANVS" inscription & & $\begin{array}{l}\text { Eggers 1966, 102 3a; } \\
\text { BM 1894. 3 3.58; } \\
\text { Jessup, 1958 27-28; } \\
\text { MMPeake \& Moore } \\
\text { 1978, 333 (2); RIB II } \\
\text { 21415.2 }\end{array}$ \\
\hline K0021 & Jug & & Kent & Faversham & RS & & $\mathrm{SF}$ & & Concentric circle body bands & $\begin{array}{l}\text { Anthropomorphic Cupid with sword and sling } \\
\text { handle medallion }\end{array}$ & $\begin{array}{l}\text { BM 1882.0405.2; BM } \\
1882.0405 .3\end{array}$ \\
\hline K0022 & \begin{tabular}{|l|}
$\begin{array}{l}\text { Handled Pan } 1 \\
\text { (handle) }\end{array}$ \\
\end{tabular} & Eggers 154-155 & Kent & Richborough & M & & $\mathrm{sF}$ & & Zoomorphic ram's head terminal, fluted handle & & Eggers 1966, $1025 \mathrm{a}$ \\
\hline K0023 & \begin{tabular}{|l} 
Jug (fragment) \\
\end{tabular} & \begin{tabular}{|l|l|} 
Eggers $123-126$ \\
\end{tabular} & Kent & Richborough & M & & $\mathrm{SF}$ & & Floral & & $\begin{array}{l}\text { Eggers 1966, } 1025 \mathrm{c} \\
\text { Feors } 1066 \text { 10 5 }\end{array}$ \\
\hline K0024 & Vessel (mount) & & Kent & Richborough & $\mathrm{M}$ & & $\mathrm{SF}$ & & & & Eggers 1966, $1025 \mathrm{~d}$ \\
\hline K0025 & Bucket & $\begin{array}{l}\text { Hemmoor, Eggers 56- } \\
58\end{array}$ & Kent & Ramsgate & RS & Cremation Pit & GD & & $\begin{array}{l}\text { Concentric cirlce rim, drop handle, trefoil handle } \\
\text { fittings }\end{array}$ & & $\begin{array}{l}\text { Eggers 1966, 102 5A; } \\
\text { Smith 1922, 93-94; } \\
\text { BM 1901.0716.1 }\end{array}$ \\
\hline K0026 & $\left\{\begin{array}{l}\text { Hadaded Pan } 2 \\
\text { (fragment) }\end{array}\right.$ & Rudge Cup type & Kent & Canterbury & $\mathrm{u}$ & & SF & & Geometric circles and swirls inside pentagons & & Eggers 1968, 148 (4) \\
\hline K0027 & Jug & & Kent & Luton & RS & & GD & 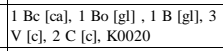 & & & $\begin{array}{l}\text { Jessup 1958, 27-28; } \\
\text { BM 1894.0803.59 }\end{array}$ \\
\hline K0028 & Bowl & & Kent & Canterbury & U & Palace Street & GD & 2 Un $[f]$ & & & \begin{tabular}{|l|l|} 
Smith, 192286 \\
mo 20270017
\end{tabular} \\
\hline K0029 & Vessel (mount) & & Kent & Lyminge & $\mathrm{RU}$ & & PAS & & Geometric sub-triangular mount & & PAS KENT-9604E7 \\
\hline K0030 & $\begin{array}{l}\text { Handled Pan } 1 \\
\text { (handle) }\end{array}$ & Eggers 154-155 & Kent & Eynsford & RS & & PAS & & & \begin{tabular}{|l|}
$\begin{array}{l}\text { Zoomorphic ram's head handle terminal, fluted } \\
\text { handle }\end{array}$ \\
\end{tabular} & PAS LON-B47821 \\
\hline K0031 & $\begin{array}{l}\text { Handled Pan } 3 \\
\text { (handle) }\end{array}$ & Eggers 160-161 & Kent & Lenham & $\mathrm{RU}$ & & PAS & & & Hilted Handle & PAS KENT-8DAE18 \\
\hline K0032 & Bowl & & Kent & Chislet, Upstreet & $\mathrm{RU}$ & & PAS & & & & PAS KENT-6E89B4 \\
\hline K0033 & Jug & & Kent & Chislet, Upstreet & $\mathrm{RU}$ & & PAS & & Concentric circle foot ring & $\begin{array}{l}\begin{array}{l}\text { Anthropomorophic bust handled terminal, appears } \\
\text { to be youthul male with hat (perhaps Phrygian); } \\
\text { Floral leaf thumb rest }\end{array} \\
\end{array}$ & PAS KENT-6E5FE6 \\
\hline K0034 & Jug (fragment) & Eggers 123-126 & Kent & Faversham & RS & & $\mathrm{SF}$ & & Floral palm pattern on neck (resembles fleur-de-lys) & & BM 1882.0405.2 \\
\hline
\end{tabular}




\begin{tabular}{|c|c|c|c|c|c|c|c|c|c|c|c|}
\hline K0035 & Vessel (mount) & & Kent & Ashford & RS & Westhawk Farm, Context 7004 & $\mathrm{SF}$ & & \begin{tabular}{|l}
$\begin{array}{l}\text { Floral leaf shaped (five pointed) hanging vessel } \\
\text { mount }\end{array}$ \\
\end{tabular} & & 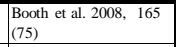 \\
\hline K0036 & Jug (fragment) & Eggers $123-126$ & Kent & Canterbury & $\mathrm{u}$ & Marlowe Car Park & $\mathrm{SF}$ & & & & \begin{tabular}{|l|}
$\begin{array}{l}\text { Blockley et alia 1995, } \\
1018(336)\end{array}$ \\
\end{tabular} \\
\hline K0037 & Bowl (fragment) & & Kent & Springhead & RS & Roman Building 300522 & $\mathrm{SF}$ & & $\begin{array}{l}\text { Zoomorphic spout, highly styized and difficult to } \\
\text { ascribe; possibly a bear }\end{array}$ & & 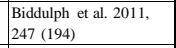 \\
\hline K0038 & Handled Pan 1 & Eggers 154-155 & Kent & Richborough & M & Pit 20 & SD & 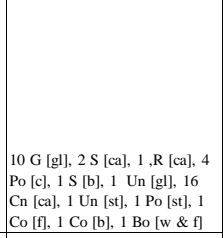 & & 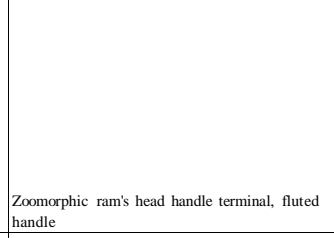 & $\begin{array}{l}\text { Bushe-Fox } 1928,31 \\
\text { (12) }\end{array}$ \\
\hline K0039 & Jug (fragment) & & Kent & Richborough & M & west of Site I & $\mathrm{sF}$ & & & Floral (possible) leaf thumb rest & $\begin{array}{l}\mid \begin{array}{l}\text { Bushe-Fox 1932, } 83 \\
(49)\end{array} \\
\end{array}$ \\
\hline K0040 & Vessel (mount) & & Kent & Richborough & M & & $\mathrm{SF}$ & & & & \begin{tabular}{|l|l|}
$\begin{array}{l}\text { Bushhe-Fox 1932, } 83 \\
\text { (52) }\end{array}$ \\
\end{tabular} \\
\hline K0041 & Vessel (mount) & & Kent & Richborough & M & & $\mathrm{SF}$ & & & & $\begin{array}{l}\text { Bushh-Fox 1932, } 83 \\
(54)\end{array}$ \\
\hline K0042 & Jug (fragment) & & Kent & Richborough & M & South west area of Stone Fort & SF & & & $\begin{array}{l}\text { Anthropomorphic female bust handle medallion; } \\
\text { Floral leaf thumb rest }\end{array}$ & $\begin{array}{l}\text { Cunlifie } 1968,102-103 \\
\text { (189) }\end{array}$ \\
\hline K0043 & Jug & & Kent & Ashford & RS & Cremation Pit & GD & $\begin{array}{l}\begin{array}{l}\text { K0043-K0044, 1 B [c], 1 Bo } \\
{[\mathrm{ww}], 1 \mathrm{Bu}[\mathrm{w}]}\end{array} \\
\end{array}$ & & & $\begin{array}{l}\text { Burnham et alia } 2001, \\
382\end{array}$ \\
\hline K0044 & Vessel (fragment) & & Kent & Ashford & RS & Cremation Pit & GD & $\begin{array}{l}\mathrm{K} 0043-\mathrm{K} 0044,1 \text { B [c], } 1 \text { Bo } \\
{[\mathrm{ww}], 1 \mathrm{Bu}[\mathrm{w}]}\end{array}$ & & & \begin{tabular}{|l} 
Burnham et alia 2001, \\
382
\end{tabular} \\
\hline K0045 & Jug (handle) & & Kent & Chalkwell & $\mathrm{RU}$ & & $\mathrm{SF}$ & & & $\begin{array}{l}\text { Anthropomorphic (perhaps Gorgon?) handle } \\
\text { medallion }\end{array}$ & BM 1883.1213.369 \\
\hline LANO001 & Handled Pan 2 & Eggers 150 & Lancashire & Ribchester & M & & SD & LAN0001-LAN0002 & $\begin{array}{l}\text { Concentric circle umbo, geometric linear } \\
\text { embellished rim }\end{array}$ & Geometric keyshaped suspension loop & \begin{tabular}{|l|}
$\begin{array}{l}\text { Eggers 1968, } 107 \\
\text { (g7): Jackson \& } \\
\text { Craddock 1995, 86 (22) }\end{array}$ \\
\end{tabular} \\
\hline LANO002 & Handled Pan 2 & Eggers 150 & Lancashire & Ribchester & M & & SD & LANO001-LAN0002 & Geometric linear embellished rim & Geometric keyshaped suspension loop & \begin{tabular}{|l}
$\begin{array}{l}\text { Eggers 1968, } 107 \\
\text { (57b); Jackson \& } \\
\text { Craddock 1995, 86 (23) }\end{array}$ \\
\end{tabular} \\
\hline LAN0003 & $\begin{array}{l}\text { Handled Pan } 3 \\
\text { (handle) }\end{array}$ & Eggers 160-161 & Lancashire & Slyne with Hest & $\mathrm{RU}$ & & PAS & & & Hilted handle & $\begin{array}{l}\text { PAS LANCUM- } \\
101193\end{array}$ \\
\hline LAN0004 & Handled Pan 2 & Eggers 139-144 & Lancashire & Ribchester & M & & SD & LAN0001-2 (?) & Concentric circle foot ring & $\begin{array}{l}\text { Inscription "CONP[...]" on handle; Concentric } \\
\text { circle handle loop }\end{array}$ & 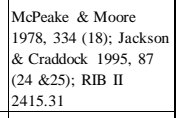 \\
\hline LEI0001 & $\begin{array}{l}\text { Handled Pan } 2 \\
\text { (handle) }\end{array}$ & Eggers 140 & Leicestershire & Leicester & $\mathrm{u}$ & Jewry Wall & SF & & & Concentric circle hanlde loop with dot in centre & \begin{tabular}{|l|} 
Eggers 1968, 107 (49); \\
Kenyon 1948, 260 (6)
\end{tabular} \\
\hline LE10002 & Vessel (fragment) & & Leicestershire & Gaddesby & $\mathrm{RU}$ & & PAS & & $\begin{array}{l}\text { Geometric pelta shaped bucket foot, duel-globular } \\
\text { "heart-shaped" foot in centre of cresent }\end{array}$ & & PAS LEIC-92A461 \\
\hline LE10003 & Vessel (fragment) & & Leicestershire & Leire & $\mathrm{RU}$ & & PAS & & Gemommtric pelta shaped bucket foot & & PAS LEIC-055A12 \\
\hline LEI0004 & \begin{tabular}{|l|}
$\begin{array}{l}\text { Handled Pan } 2 \\
\text { (handle) }\end{array}$ \\
\end{tabular} & Rudge Cup Type & Leicestershire & Leicester & $\mathrm{U}$ & West Bridge, Site I & $\mathrm{sF}$ & & & Floral (possible) vine pattern enameled in blue & \begin{tabular}{|l|}
$\begin{array}{l}\text { Clay \& Pollard 1994, } \\
147(41)\end{array}$ \\
\end{tabular} \\
\hline LEI0005 & Vessel (fragment) & & Leicestershire & Leicester & $\mathrm{u}$ & West Bridge, Site I & SF & & Concentric ring umbo and omphalos dot & & \begin{tabular}{|l} 
Clay \& Pollard 1994, \\
$147(42)$
\end{tabular} \\
\hline LEI0006 & Vessel (fragment) & Perrandbecken & Leicestershire & Leicester & $\mathrm{u}$ & West Bridge, Site I & SF & & Geometric 'pearl' beaded out-urned rim & & \begin{tabular}{|l|l|}
$\begin{array}{l}\text { Clay \& Pollard 1994, } \\
147 \text { (43) }\end{array}$ \\
\end{tabular} \\
\hline LIN0001 & Vessel (fragment) & & Lincolnshire & Osgodby & RU & & SF & & & & Eggers 1968, 107 (50a) \\
\hline LIN0002 & Strainer (fragment) & & Lincolnshire & Osgodby & $\mathrm{RU}$ & & $\mathrm{SF}$ & & Geometric circle and square dot strainer dots & & Eggers 1968, 107 (50b) \\
\hline LIN0003 & \begin{tabular}{|l} 
Vessel (fragment) \\
Vor
\end{tabular} & Eggers 79 & Lincolnshire & Ashby & $\mathrm{RU}$ & & SF & & $\begin{array}{l}\text { Zoomorphic bird escutcheom, perhaps a dove or } \\
\text { duck }\end{array}$ & & 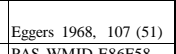 \\
\hline LIN0004 & Vessel (fragment) & & Lincolnshire & Ancaster & RS & & PAS & & & \begin{tabular}{|l} 
Zomorphic birds's head \\
\end{tabular} & PAS WMID-E86F58 \\
\hline LIN0005 & Handled Pan 1 & Eggers 155 & Lincolnshire & Scotton & $\mathrm{RU}$ & & PAS & & Concentric circle foot ring & & PAS SWYOR-FB6262 \\
\hline
\end{tabular}




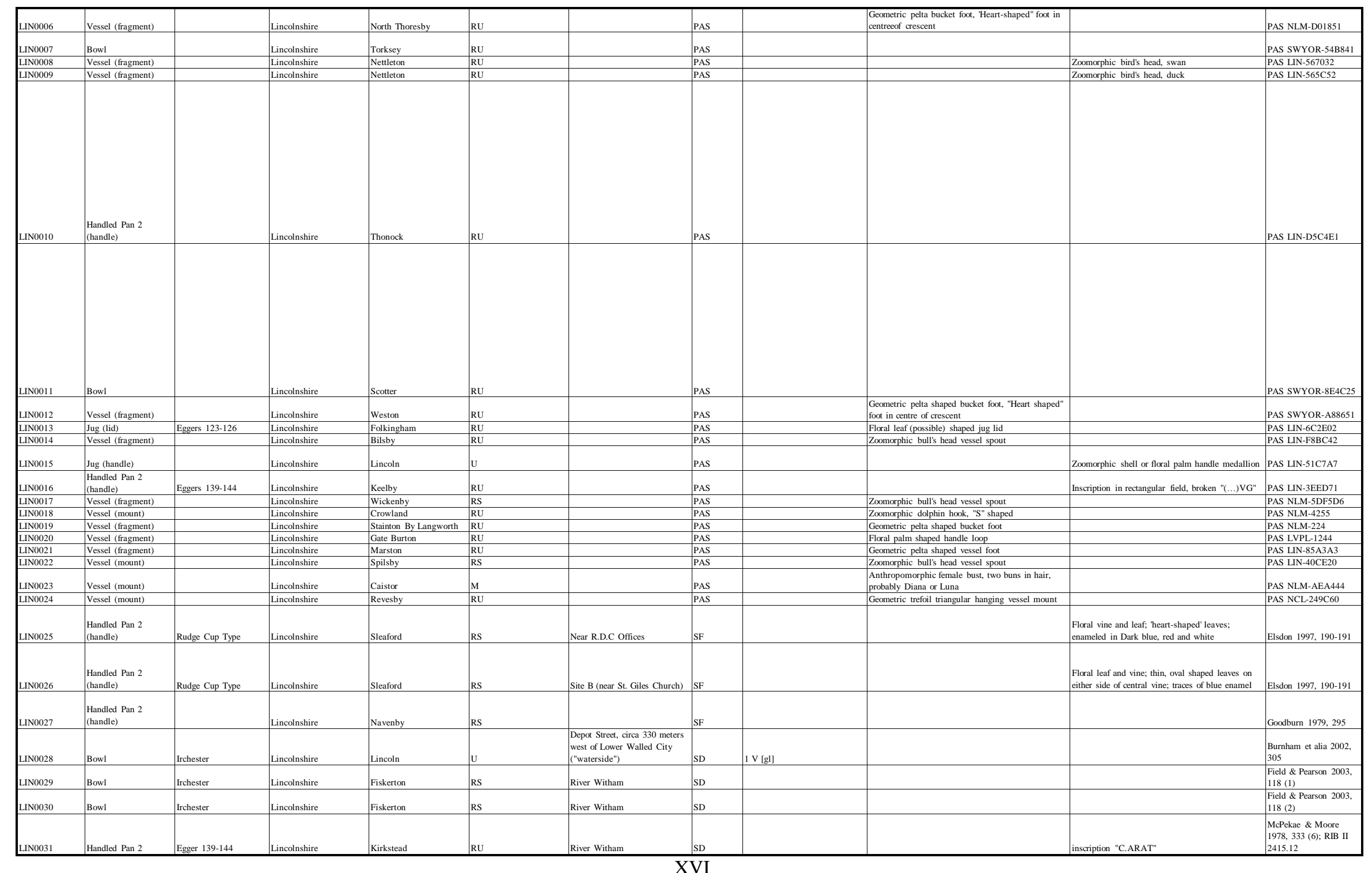




\begin{tabular}{|c|c|c|c|c|c|c|c|c|c|c|c|}
\hline LIN0032 & $\begin{array}{l}\begin{array}{l}\text { Handled Pan } 2 \\
\text { (handle) }\end{array} \\
\end{array}$ & & Lincolnshire & Fiskerton & RS & River Witham & SD & & & $\begin{array}{l}\text { Floral vine leaves and caducaeus; inscription } \\
\text { "FLORVS F" }\end{array}$ & $\begin{array}{l}\text { McPeake \& Moore } \\
\text { 1978,334 (20); RIB II } \\
2415.35\end{array}$ \\
\hline LIN0033 & $\begin{array}{l}\begin{array}{l}\text { Handled Pan } 2 \\
\text { (handle) }\end{array} \\
\end{array}$ & Eggers 139-144 & Lincolnshire & Humberside & RS & & $\mathrm{SF}$ & & & |inscription "[....]IAPPIA" & $\begin{array}{l}\text { Hassall \& Tomlin } 1984 \\
\text { 345 (49); RIB II } \\
2415.36\end{array}$ \\
\hline LIN0034 & $\begin{array}{l}\begin{array}{l}\text { Handled Pan } 2 \\
\text { (handle) }\end{array} \\
\end{array}$ & Eggers $139-144$ & Lincolnshire & Louth & $\mathrm{RU}$ & & $\mathrm{SF}$ & & & \begin{tabular}{|l} 
stamped inscription "MLNFEC"; punch dot \\
inscription "C CLSENIORIS ANNI" \\
\end{tabular} & $\begin{array}{l}\text { Wright 1964, } 180 \text { (16); } \\
\text { RIB II 2415.41 }\end{array}$ \\
\hline LIN0035 & $\begin{array}{l}\begin{array}{l}\text { Handled Pan } 2 \\
\text { (handle) }\end{array} \\
\end{array}$ & Eggers $146-147$ & Lincolnshire & Lincoln & $\mathrm{u}$ & & $\mathrm{SF}$ & & & $\begin{array}{l}\begin{array}{l}\text { inscription "MLN [....]" on top of handle; } \\
\text { inscription "[...] C. A. S. DC" on underside of } \\
\text { handle }\end{array} \\
\end{array}$ & $\begin{array}{l}\text { Bennett \& Young } 1981 \\
4 \begin{array}{l}4(43) ; \\
2415.42\end{array}\end{array}$ \\
\hline LIN0036 & $\begin{array}{l}\begin{array}{l}\text { Handled Pan } 2 \\
\text { (handle) }\end{array} \\
\end{array}$ & Eggers 139-144 & Lincolnshire & Normanby & $\mathrm{RU}$ & near Scunthorpe & $\mathrm{SF}$ & & & \begin{tabular}{|} 
Concentric cicle handlele lopp; punch dot \\
inscription reads "ALPRI"
\end{tabular} & $\begin{array}{l}\text { Wright \& Hassall } 1971 \\
\text { 299 (60); RIB II } \\
2415.57\end{array}$ \\
\hline Lo001 & Bowl & Irchester & London & City of London & u & Drapers' Gardens, Well & SD & L0001-L0015 & Zoomorphic hanging vessel mount (bear or lion) & & Gerrard 2009168854 \\
\hline L0002 & Bucket & Henmoor, Eggers 58 & London & City of London & $\mathrm{u}$ & Drapers' Gardens, Well & SD & L0001-L0015 & & & Gerrard 2009171821 \\
\hline Lo003 & Bucket & Hemmoor, Eggers 58 & London & City of London & $\mathrm{U}$ & Drapers' Gardens, Well & SD & L0001-L0015 & & & Gerard 2009171824 \\
\hline Lo004 & Bucket & & London & City of London & $\mathrm{u}$ & Drapers' Gardens, Well & SD & L0001-L0015 & & & Gerrard 2009171820 \\
\hline Lo005 & Bucket & & London & City of London & $\mathrm{U}$ & Drapers' Gardens, Well & SD & L0001-L0015 & & & Gerrard 2009171816 \\
\hline Lo006 & Bucket & Westland & London & City of London & $\mathrm{u}$ & Drapers' Gardens, Well & SD & Lo001-L0015 & & & Gerrard 2009171813 \\
\hline Lo007 & Bowl & Perlandbecken & London & City of London & u & Drapers' Gardens, Well & SD & L0001-L0015 & & & Gerrard 2009173818 \\
\hline Lo008 & Bowl & Perlandbecken & London & City of London & $\mathrm{U}$ & Drapers' Gardens, Well & SD & Lo001-L0015 & & & Gerrard 2009173829 \\
\hline Lo009 & Bowl & Perlandbecken & London & City of London & $\mathrm{U}$ & Drapers' Gardens, Well & SD & Lo001-L0015 & & & Gerrard 2009172822 \\
\hline Loo10 & Bowl & Bassin á bord godronné & London & City of London & $\mathrm{u}$ & Drapers' Gardens, Well & SD & L0001-L0015 & & & Gerrard 2009173826 \\
\hline Lo011 & Bowl & Bassin á bord godronné & London & City of London & $\mathrm{U}$ & Drapers' Gardens, Well & SD & L0001-L0015 & & & Gerrard 2009173853 \\
\hline Lo012 & Bowl & & London & City of London & $\mathrm{U}$ & Drapers' Gardens, Well & SD & L0001-L0015 & & & Gerrard 2009173817 \\
\hline Lo013 & Bowl & & London & City of London & $\mathrm{u}$ & Drapers' Gardens, Well & SD & L0001-L0015 & & & Gerrard 2009173819 \\
\hline Lo014 & Handled Pan 2 & Eggers 161 (c.f.) & London & City of London & $\mathrm{U}$ & Drapers' Gardens, Well & SD & L0001-L0015 & & & Gerrard 2009176823 \\
\hline Lo015 & Handled Pan 2 & Eggers 160 (c.f.) & London & City of London & u & Drapers' Gardens, Well & SD & L0001-L0015 & & Geometric diamond-shaped expansion & Gerrard 2009176814 \\
\hline Lo016 & Bowl & & London & City of London & $\mathrm{u}$ & Shadwell, Well (A) & $\mathrm{SF}$ & & & & $\begin{array}{l}\text { Burnham et alia } 2003, \\
\text { 347; Douglas et. alia } \\
\text { 2011 }\end{array}$ \\
\hline Lo017 & Jug (lid) & & London & City of London & $\mathrm{u}$ & Paternoster Square & SF & & & & Watson 2006, $97 \mathrm{S37}$ \\
\hline Lo018 & Vessel (fragment) & & London & City of London & $\mathrm{u}$ & Paternoster Square & SF & & & & Watson 2006, $97 \mathrm{~S} 38$ \\
\hline Lo019 & Jug (handle) & & London & City of London & U & Paternoster Square & SF & & Zoomorphic, possibly lion & & Watson 2006, $97 \mathrm{~S} 39$ \\
\hline Lo020 & \begin{tabular}{|l} 
Handded Pan 3 \\
(handle)
\end{tabular} & Eggers $160-161$ & London & Southwark & u & & SF & & & & $\begin{array}{l}\text { Drummond-Murray } \\
\text { 2002, } 218 \text { R11 }\end{array}$ \\
\hline Lo021 & Bucket (fragment) & & London & City of London & $\mathrm{u}$ & Fenchurch & SF & & & & Birbeck 2009, 823611 \\
\hline L0022 & Jug (lid) & Eggers $123-126$ & London & City of London & u & Fenchurch & SF & & Zoomorphic Dolphin & & Birbeck 2009, 82468 \\
\hline L0023 & $\begin{array}{l}\text { Handede Pan } 1 \\
\text { (handle) }\end{array}$ & Eggers 154-155 & London & & u & & $\mathrm{SF}$ & & Zoomorphic ram's head terminal, fluted handle & & Eggers 1966, $100 \mathrm{la}$ \\
\hline Lo024 & $\begin{array}{l}\begin{array}{l}\text { Handled Pan } 2 \\
\text { (handle) }\end{array} \\
\end{array}$ & Eggers 142-144 & London & Cit of London & u & Christ's Hospital & SF & & & \begin{tabular}{|l} 
Geometric circle terminal, linear border; \\
inscription "L....SII"
\end{tabular} & $\begin{array}{l}\text { Eggers } 1966,100 \text { lb; } \\
\text { McPeake \& Moore } \\
1978,334 \text { (25); RIB II } \\
2415.8\end{array}$ \\
\hline Lo025 & Handled Pan 2 & Eggers 141 & London & Bermondsey & $\mathrm{u}$ & Potter's Fields (south London) & SF & & $\begin{array}{l}\text { Concentric circle umbo and foot ring, "TRVFC" } \\
\text { insscription in rectangular field }\end{array}$ & & \begin{tabular}{|l|} 
Eggers 1966, 101 1c; \\
Mcreake \& Moore \\
1978, 334 (33); RIB II \\
2415.49 \\
\end{tabular} \\
\hline
\end{tabular}




\begin{tabular}{|c|c|c|c|c|c|c|c|c|c|c|c|}
\hline L0026 & Jar & Eggers 39 & London & & u & & $\mathrm{SF}$ & & & & Eggers 1966, 101 ld \\
\hline L0027 & Jug (fragment) & Eggers 125 & London & & u & & $\mathrm{SF}$ & & Concentric circles on neck & & Eggers 1966, $101 \mathrm{lg}$ \\
\hline L0028 & Handled Pan 3 & Eggers 161 & London & City of London & $\mathrm{u}$ & & $\mathrm{SF}$ & & & & Eggers 1966, $101 \mathrm{lh}$ \\
\hline L0029 & Bowl & Eggers 71 & London & City of London & u & Bucklersbury House & $\mathrm{sF}$ & & & & Eggers 1966, 101 (i) \\
\hline L0030 & Vessel (fragment) & & London & & u & & $\mathrm{SF}$ & & Anthropomorphic medusa head & & Eggers 1966, $101(\mathrm{lr})$ \\
\hline Lo031 & Vessel (fragment) & Eggers 160-161 & London & City of London & $\mathrm{u}$ & Bucklersbury House & SF & & & Inscription "NDINVSF" in rectangular field & 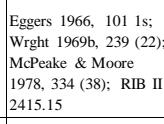 \\
\hline L0032 & Handled Pan 5 & Eggers 130 & London & City of London & $\mathrm{u}$ & Creed Lane & SF & & & & $\begin{array}{l}\text { Eggers 1966, } 101 \text { lu; } \\
\text { BM 1855.0804.23 }\end{array}$ \\
\hline$L 0033$ & Strainer & Eggers $160-161$ & London & City of London & u & & $\mathrm{SF}$ & & & & Eggers 1966, $102 \mathrm{lw}$ \\
\hline L0034 & Vessel (mount) & & London & Rainham & $\mathrm{RS}$ & & PAS & & Geometric sub-triangular mount & & PAS ESS-1DAE38 \\
\hline L0035 & Vessel (fragment) & & London & City of London & $\mathrm{u}$ & Angel Court & $\mathrm{SF}$ & & Possible engraved lines & & $\mid \begin{array}{ll}\mathrm{MoL} \\
\mathrm{ACW} 74[\mathrm{~B} 12]<19>\end{array}$ \\
\hline L0036 & Bowl & & London & City of London & u & Peninsular House & SF & & & & $\mid \begin{array}{l}\text { MoL } \\
\text { PEN79[1142]<53> }\end{array}$ \\
\hline L0037 & Handled Pan 2 & & London & City of London & $\mathrm{u}$ & $\begin{array}{l}\text { 145-146 Leadenhall Street, } \\
\text { EC3 }\end{array}$ & SF & & & & MoL LEN89[604]<55> \\
\hline L0038 & Vessel (fragment) & & London & City of London & u & \begin{tabular}{|l|l|}
$160-162$ Fenchurch Street, 22- \\
3 Lime Street, EC3
\end{tabular} & SF & & Concentric circle umbo & & MoL FSE76[0]|<23> \\
\hline L0039 & Cauldron & & London & City of London & u & Blossom's Inn (well) & SF & $20 \mathrm{Bu}[\mathrm{w}]$ & & & $\begin{array}{l}\text { Burrhham et alia } 2002 \text {, } \\
329\end{array}$ \\
\hline L0040 & Jug (lid) & Eggers $123-126$ & London & City of London & $\mathrm{U}$ & \begin{tabular}{|l|}
$19-25$ Birchin Lane, Bengal \\
Court, 1-3 Castle Court, EC3
\end{tabular} & SF & & Trefoil lid & & \begin{tabular}{|l} 
MOLA \\
BRL87[595] 667$\rangle$
\end{tabular} \\
\hline L0041 & Vessel (fragment) & & London & City of London & u & $\begin{array}{l}\begin{array}{l}\text { Southwark, Tabbard Square } \\
\text { (drain) }\end{array} \\
\end{array}$ & SF & & & & $\begin{array}{l}\text { Burnham et alia } 2004, \\
301\end{array}$ \\
\hline L0042 & Jug (lid) & & London & City of London & $\mathrm{u}$ & Amphitheater & SF & & & & \begin{tabular}{|l|}
$\begin{array}{l}\text { Bateman } 2008,197 \\
\text { (S82) }\end{array}$ \\
\end{tabular} \\
\hline L0043 & Jug (lid) & & London & City of London & $\mathrm{U}$ & Hibernia Wharf, Southwark & SF & & $\begin{array}{l}\text { Anthropomorphic(?) jug lid in the shape of a } \\
\text { conical Gladiator's helmet }\end{array}$ & & \begin{tabular}{|l|l|} 
MOLA \\
HIB79[53]<28>
\end{tabular} \\
\hline L0044 & Jug (lid) & Eggers $123-126$ & London & City of London & U & Hibernia Wharf, Southwark & SF & & 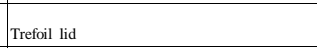 & & \begin{tabular}{|l|} 
MOLA \\
HIB79[260]<80>
\end{tabular} \\
\hline L0045 & Jug (lid) & Eggers 123-126 & London & City of London & u & 23-25 Austin Friars, EC2 & SF & & Trefoil lid & & \begin{tabular}{|l|} 
MOLA \\
AST87[233]<69>
\end{tabular} \\
\hline L0046 & Jug (lid) & Eggers $123-126$ & London & City of London & $\mathrm{U}$ & 23-25 Austin Friars, EC2 & SF & & Trefoil lid & & $\mid \begin{array}{l}\text { MOLA } \\
\text { AST87236BS }\langle 991>\end{array}$ \\
\hline L0047 & Jug & & London & City of London & $\mathrm{u}$ & $\begin{array}{l}72-80 \text { Cheapside, } 83-84 \text { Queen } \\
\text { Street, 9-12 Pancras Lane, EC2 } \\
\text { and EC4 }\end{array}$ & : & & & & \begin{tabular}{|l} 
MOLA \\
CID90[764]<893>
\end{tabular} \\
\hline L0048 & Jug & & London & City of London & u & 36-37 King Street, EC2 & SF & & & & \begin{tabular}{|l|} 
MOLA \\
KNG85[2118]<32>
\end{tabular} \\
\hline L0049 & Jug (handle) & & London & City of London & u & $\begin{array}{l}\text { Billingsggate Market Lorry } \\
\text { Park, Lower Thames Street, } \\
\text { EC3 }\end{array}$ & SF & & & & \begin{tabular}{|l} 
MOLA \\
BWB83[354]<3546>
\end{tabular} \\
\hline Lo050 & Jug (handle) & & London & City of London & U & \begin{tabular}{|l|} 
General Post Office, 81 \\
Newgate Street, ECI
\end{tabular} & SF & & & & $\begin{array}{l}\text { MOLA } \\
\text { GPO75[9164]<2168> }\end{array}$ \\
\hline
\end{tabular}




\begin{tabular}{|c|c|c|c|c|c|c|c|c|c|c|}
\hline L0051 & Jug (handle) & & London & City of London & u & \begin{tabular}{|l} 
11-11A Pudding Lane \\
Nomura House), $121-127$ \\
Lower Thames Street, 33-36 \\
Fish Street Hill, 22-26 \\
Monument Stree, $711 \mathrm{~A}$ \\
King's Head Court, EC3
\end{tabular} & SF & & Zoomorphic dolphin handle & $\begin{array}{l}\text { MOLA } \\
\text { PDN81[1761]<620> }\end{array}$ \\
\hline L0052 & Vessel (mount) & & London & City of London & u & 27-30 Lime Street, EC3 & SF & Zoomorphic aquatic bird hanging vessel mount & & MOLA \\
\hline L0053 & Bucket & & London & City of London & $\mathrm{u}$ & $\begin{array}{l}\text { 5-12 Fenchurch Street, } 1 \\
\text { Philpot Lane, EC3 }\end{array}$ & SF & & & $\begin{array}{l}\text { MOLA } \\
\text { FEN83[2142]<214> }\end{array}$ \\
\hline L0054 & Bucket & & London & City of London & $\mathrm{U}$ & 12-15 Finsbury Circus, EC2 & SF & & & \begin{tabular}{|l|l|} 
MOLA \\
FIB88 103$\}<2>$
\end{tabular} \\
\hline L0055 & Bowl & Irchester & London & City of London & u & River Thames & $\mathrm{SF}$ & & & BM 1891.0320.10 \\
\hline L0056 & Jar & & London & City of London & $\mathrm{u}$ & London Wall & $\mathrm{SF}$ & inscription reads "EX OF COR" & & $\begin{array}{l}\text { Hassall \& Tomlin } 1984 . \\
\text { 3444 (35); RIB II } \\
2415.33\end{array}$ \\
\hline L0057 & \begin{tabular}{|l}
$\begin{array}{l}\text { Handled Pan } 2 \\
\text { (handle) }\end{array}$ \\
\end{tabular} & & London & City of London & u & $\begin{array}{l}\begin{array}{l}\text { Queen Victoria Street and } \\
\text { Bucklersbury }\end{array} \\
\end{array}$ & SF & & inscription "SANGVSF" & 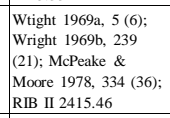 \\
\hline L0058 & Bowl & & London & City of London & $\mathrm{u}$ & Creed Lane & SF & & & BM 1855.0804.24 \\
\hline M0001 & Jug & & Greater Manchester & Manchester & M & & SF & & & Bruton 1929, 162 (24) \\
\hline м0002 & Jug (lid) & Eggers 128 & Greater Manchester & Manchester & M & Barton Street & SF & & & Gregory 2007, $134(2)$ \\
\hline MONoool & Handled Pan 2 & Eggers 140 & Monmouthshire & Gelligar & M & Fort & SF & & Concentric cirlce handle medallion & Eggers 1968, 103 (14) \\
\hline MON0002 & Vessel (mount) & & Monmouthshire & Mathern & $\mathrm{RU}$ & & PAS & $\begin{array}{l}\text { Zoomorphic dragonesque ring hooks; Floral five } \\
\text { pointed leaf medallion }\end{array}$ & & PAS PUBLIC-74C930 \\
\hline MON0003 & Vessel (fragment) & & Monmouthshire & Usk & м & & PAS & $\begin{array}{l}\text { Geomertic pelta shaped bucket foot, two nodes on } \\
\text { each side of crescent and triangle in the midddele }\end{array}$ & & PAS PUBLIC-749A73 \\
\hline MON0004 & Jug (lid) & Eggers 123-126 & Monmouthshire & Usk & M & & PAS & Floral leaf shaped jug lid & & PAS PUBLIC-CF7051 \\
\hline MON0005 & Vessel (fragment) & & Monmouthshire & Lantrisant Fawr & $\mathrm{RU}$ & & PAS & Zoomorphic bull's head spout & & PAS PUBLIC-699346 \\
\hline MON0006 & Vessel (mount) & & Monmouthshire & Caerwent & u & & PAS & Zoomorphic boar shaped mount & & PAS NMGW-2FC205 \\
\hline MON0007 & Jug & & Monmouthshire & Llantilio Pertholey & $\mathrm{RU}$ & & GD & & Zoomorphic leopard handle with silver spot inlays & PAS NMGW-9A9D16 \\
\hline MON0008 & Jug (handle) & & Monmouthshire & Usk & M & Burrium, Fortress Well & SF & & & $\begin{array}{l}\text { Manning et alia 1995, } \\
192 \text { (1) }\end{array}$ \\
\hline MON0009 & Jug (lid) & Eggers $123-126$ & Monmouthshire & Usk & M & Burrium, Fortress Ditch & SF & Zoomorphic bird lid statuette & & $\begin{array}{l}\text { Manning et alia 1995, } \\
194(2)\end{array}$ \\
\hline MON0010 & Jug (lid) & Eggers $123-126$ & Monmouthshire & Usk & M & Burrium, Fortress Latrine & SF & Floral leaf shaped jug lid & & $\begin{array}{l}\text { Manning et alia } 1995, \\
194(3)\end{array}$ \\
\hline MON0011 & Jug (lid) & Eggers $123-126$ & Monmouthshire & Usk & M & Burrium, Fortress Pit & SF & Floral leaf shaped jug lid & & $\begin{array}{l}\text { Manning et alia } 1995, \\
194(4)\end{array}$ \\
\hline MON0012 & Jug (lid) & Eggers 123-126 & Monmouthshire & Usk & M & Burrium, Third-century Pit & SF & Floral leaf shaped jug lid & & $\begin{array}{l}\text { Manning et alia 1995, } \\
194 \text { (5) }\end{array}$ \\
\hline MON0013 & Handled Pan 2 & Eggers $145-147$ & Monmouthshire & Usk & M & Burrium, Fortress Latrine & SF & Concentric circle umbo and omphalos dot & Trefoil suspension loop & $\begin{array}{l}\text { Manning et alia 1995, } \\
194(6)\end{array}$ \\
\hline MON0014 & Vessel (fragment) & & Monmouthshire & Usk & м & Burrium, Fortress Pit & SF & Concentric circle body band & & $\begin{array}{l}\text { Manning et alia } 1995, \\
196(9)\end{array}$ \\
\hline MON0015 & Vessel (fragment) & & Monmouthshire & Usk & M & Burrium, Fortress Pit & SF & Concentric circle body band & & $\begin{array}{l}\text { Manning et alia } 1995, \\
196(10)\end{array}$ \\
\hline MON0016 & Vessel (mount) & & Monmouthshire & Usk & M & Burrium & SF & Geometric triangular hanging vessel mount & & $\begin{array}{l}\text { Manning et alia } 1995 \text {, } \\
106(13)\end{array}$ \\
\hline MON0017 & Handled Pan 4 & Coptic & Monmouthshire & Usk & M & $\begin{array}{l}\text { Burrium, Drainage Gully near } \\
\text { via principalis of Fortress }\end{array}$ & SF & & & $\begin{array}{l}\text { Manning et alia } 1995 \text {, } \\
198(16)\end{array}$ \\
\hline
\end{tabular}




\begin{tabular}{|c|c|c|c|c|c|c|c|c|c|c|}
\hline NE0001 & Bucket & & Newport & Langstone & RS & & PAS & & & PAS NMGW-07F2B4 \\
\hline NE0002 & Vessel (mount) & & Newport & Langstone & RS & & PAS & Anthropomorphic reclining banquetor mount & & PAS NMGW-F4A3F6 \\
\hline NE0003 & Vessel (mount) & & Newport & Caerleon & M & & PAS & Zoomorphic ox head mount & & PAS NMGW-DBBD23 \\
\hline NE0004 & Bowl & Rose Ash & Newport & Langstone & RS & Bog/Swamp & SD & & & $\begin{array}{l}\text { PAS NMGW-9C0216; } \\
\text { Worrell 2009, 285-287 }\end{array}$ \\
\hline NE0005 & Bowl & Rose Ash & Newport & Langstone & RS & Bog/Swamp & SD & & & $\begin{array}{l}\text { PAS NMGW-9C0216; } \\
\text { Worrell 2009, 285-287 }\end{array}$ \\
\hline NE0006 & Strainer & & Newport & Langstone & RS & Bog/Swamp & SD & & & $\begin{array}{l}\text { PAS NMGW-9C0216; } \\
\text { Worrell 2009, 285-287 }\end{array}$ \\
\hline NE0007 & Handled Pan 2 & Eggers 140 & Newport & Caerleon & M & Fort & $\mathrm{SF}$ & & Concentric cirlce handle medallion & Eggers 1968, 104 (23) \\
\hline NE0008 & Bucket (fragment) & & Newport & Caerleon & M & Fort, beneath frigidarium & SF & Geometric pelta shaped bucket foot & & $\begin{array}{l}\text { Zienkiewicz 1986, } 173 \\
(8)\end{array}$ \\
\hline $\begin{array}{l}\text { NE0009 } \\
\text { NE0010 }\end{array}$ & \begin{tabular}{|l|}
$\begin{array}{l}\text { Handled Pan } 2 \\
\text { (handle) }\end{array}$ \\
Vessel (mount)
\end{tabular} & Eggers 139-144 & \begin{tabular}{|l|} 
Newport \\
Newport
\end{tabular} & \begin{tabular}{|l|} 
Carleon \\
Caerleon
\end{tabular} & \begin{tabular}{|l}
$\mathrm{M}$ \\
$\mathrm{M}$
\end{tabular} & & \begin{tabular}{|l}
$\mathrm{SF}$ \\
$\mathrm{SF}$
\end{tabular} & Geometric sub-triangular hanging vessel mount & Concentric cirlce handle medallion & \begin{tabular}{|l|l} 
Evans 2000, $350(25)$ \\
Evans 2000, $350(26)$
\end{tabular} \\
\hline NE0011 & Vessel (mount) & & Newport & Caerleon & $\mathrm{M}$ & & SF & $\begin{array}{l}\text { Geomertic sub-riangular hanging vessel mount } \\
\text { Zoomorphic bird's head rim attachment to vessel } \\
\text { mount, possibly duck or swan }\end{array}$ & & Evans 2000, 352 (29) \\
\hline NE0012 & Bucket (fragment) & & Newport & Caerleon & M & & SF & 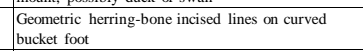 & & Evans 2000, 352 (30) \\
\hline NE0013 & \begin{tabular}{|l|l|} 
Handled Pan 1 \\
(handle)
\end{tabular} & Eggers 154155 & Newport & Caerleon & M & Alstone Cottage & SF & & Zoomorphic ram's head handle terminal & $\begin{array}{l}\text { Casey \& Hoffiman } 1995 \\
89(7)\end{array}$ \\
\hline NE0014 & Handled Pan 2 & Eggers 144 & Newport & Caerleon & M & Well & SF & $\begin{array}{l}\text { Floral leaf body band; Concentric circle umbo and } \\
\text { foot-ring; etched inscription "LVCCA" }\end{array}$ & $\begin{array}{l}\text { Concentric cricle handle loop; stamped } \\
\text { inscriptions "MATVRVS F", "ALA I ITH"; punch } \\
\text { dot inscripion "LL" }\end{array}$ & $\begin{array}{l}\text { Hassall \& Tomilin } 1985 \\
330.311(41) \text {; RIB II } \\
2415.39\end{array}$ \\
\hline NHo001 & Bucket (fragment) & & Northamptonshire & Irchester & RS & & SD & & & Kennett 1971, 1284.1 \\
\hline NHooo2 & Bowl & Irchester & Northamptonshire & Irchester & RS & & SD & & & Kennett 1971, 1284.2 \\
\hline NH0003 & Bowl & Bassin à bord godronné & Northamptonshire & Irchester & RS & & SD & & & Kennett 1971, 1284.3 \\
\hline NHo004 & Bowl & Irchester & Norhamptonshire & Irchester & RS & & SD & & & Kennett 1971, 1304.4 \\
\hline NHo005 & Bowl & Irchester & Northamptonshire & Irchester & RS & & SD & & & Kennett 1971, 1304.5 \\
\hline NHooo6 & Bowl & Irchester & Northamptonshire & Irchester & RS & & SD & & & Kennett 1971, 1304.6 \\
\hline NHo007 & Handled Pan 2 & & Northamptonshire & Irchester & RS & & $\mathrm{SD}$ & & & Kennett 1971, 1304.7 \\
\hline NHo008 & Strainer & & Northamptonshire & Irchester & RS & & SD & $\begin{array}{l}\begin{array}{l}\text { Geometric (strainer punch dots of base and wall of } \\
\text { body in circle, cross, trangular, and cable-knit } \\
\text { decoration), Symetrical, Umbo ring }\end{array} \\
\end{array}$ & & Kennet 1971, 1304.8 . \\
\hline NHo009 & Strainer & & Northamptonshire & Irchester & RS & & SD & $\begin{array}{l}\text { Geemetric cstrainer punch dots of base and wall of } \\
\text { body in circle, cross, and waved decoration,, } \\
\text { Symetrical, Umbo punch dot ring inside punch dot } \\
\text { six pointed star }\end{array}$ & $\begin{array}{l}\text { Geometric handle cross line engraving in } \\
\text { diamonds and triangles, Flared hande terminal }\end{array}$ & Kennett 1971, 1304.9 \\
\hline NHO010 & Vessel (fragment) & & Northamptonshire & Higham Ferrers & RS & & SF & Concentric circle body bands & & $\begin{array}{l}\text { Lawrence \& Smith } \\
2009,241 \text { (357) }\end{array}$ \\
\hline NHO011 & Vessel (fragment) & & Northamptonshire & Higham Ferrers & RS & & SF & & & $\begin{array}{l}\text { Lawrence \& Smith } \\
\text { 2009, } 241(358)\end{array}$ \\
\hline NH0012 & Vessel (fragment) & & Northamptonshire & Higham Ferrers & RS & & $\mathrm{sF}$ & Concentric circle body bands & & $\begin{array}{l}\text { Lawrence \& Smith } \\
2009,241(360)\end{array}$ \\
\hline NLIN0001 & \begin{tabular}{|l|l|}
$\begin{array}{l}\text { Handled Pan 2 } \\
\text { (fragment) }\end{array}$ \\
\end{tabular} & Rudge Cup Type & North Lincolnshire & Crowle & RS & & PAS & $\begin{array}{l}\begin{array}{l}\text { Geometric celtic swirls and circles, triskele within a } \\
\text { circular field }\end{array} \\
\end{array}$ & & PAS FAKL-9900E3 \\
\hline NLIN0002 & $\begin{array}{l}\text { Vesesel (fragment) } \\
\text { Vsel }\end{array}$ & & North Lincolnshire & $\begin{array}{l}\text { Winteringham } \\
\text { with }\end{array}$ & $\begin{array}{ll}\text { RS } \\
\text { Re }\end{array}$ & & PAS & Zoomorphic panther's head spout & & PAS NLM-E3E502 \\
\hline NLIN0003 & Vessel (fragment) & & North Lincolnshire & Winteringham & RS & & PAS & $\begin{array}{l}\text { Geometric pelta shaped vessel foot } \\
\text { Zoomorphic lion head at the top of handle; }\end{array}$ & & PAS NLM-A2CB67 \\
\hline NLLN0004 & Jug (handle) & Eggers 125 & North Lincolnshire & Appleby & RS & & PAS & $\begin{array}{l}\text { Zoomorphic lion head at the top of handle; } \\
\text { zoomorphic lion's foot handle medallion }\end{array}$ & & PAS SWYOR-E54DB2 \\
\hline
\end{tabular}




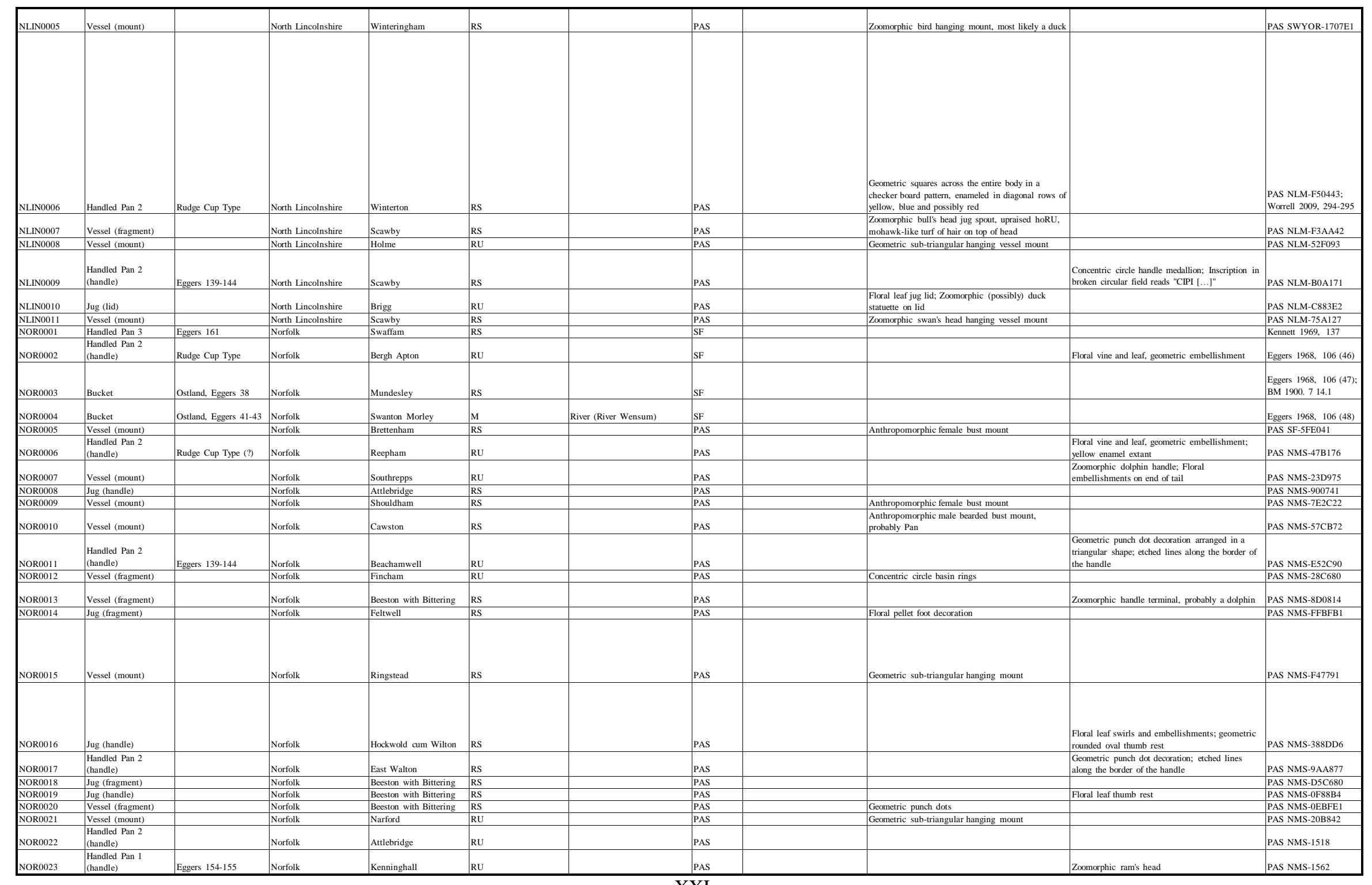




\begin{tabular}{|c|c|c|c|c|c|c|c|c|c|c|c|}
\hline NOR0024 & $\begin{array}{l}\begin{array}{l}\text { Handled Pan } 2 \\
\text { (handle) }\end{array} \\
\end{array}$ & Eggers 150 & Norfolk & Tacolneston & $\mathrm{RU}$ & & PAS & & & \begin{tabular}{|l}
$\begin{array}{l}\text { Geometric keyhole shaped handle loop, pellet at } \\
\text { handle terminal }\end{array}$ \\
\end{tabular} & PAS NMS-199 \\
\hline NOR0025 & Vessel (mount) & & Norfolk & Hockwold cum Wilton & RS & & PAS & & \begin{tabular}{|l} 
Anthropomorphic female bust mount; floral five \\
pointed leaf backing
\end{tabular} & & PAS NMS-134 \\
\hline 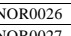 & \begin{tabular}{|l|l|} 
Vessel (mount) \\
Vosel (mont)
\end{tabular} & & \begin{tabular}{|l|l} 
Norfolk \\
$N$ Nofke
\end{tabular} & 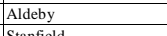 & RS & & PAS & & & & PAS NMS-D75F24 \\
\hline $\begin{array}{l}\text { NOR0027 } \\
\text { NoR028 }\end{array}$ & \begin{tabular}{|l|l|l|l} 
Vessel (mount) \\
Jug handle)
\end{tabular} & & \begin{tabular}{|l|l|l|l} 
Norfolk \\
Norfolk
\end{tabular} & $\begin{array}{l}\text { Stanfield } \\
\text { Themelthorpe }\end{array}$ & $\frac{\mathrm{RU}}{\mathrm{RU}}$ & & $\begin{array}{l}\text { PAS } \\
\text { PAS }\end{array}$ & & \begin{tabular}{|l|} 
Geometric triangular hanging vessel mount \\
Florar leaf handle medallion
\end{tabular} & & PAS NMS1310 \\
\hline NOR0029 & Jug (handle) & & Norfolk & Colkirk & $\mathrm{RU}$ & & PAS & & & & $\begin{array}{l}\text { PAS NMSDA1831 } \\
\text { PAS NMS1924 }\end{array}$ \\
\hline NOR0030 & Bowl & & Norfolk & Caistor-on-Sea & M & & $\mathrm{SF}$ & & & & $\begin{array}{l}\text { Darling \& Gurney } \\
\text { 1993, 96 (355) }\end{array}$ \\
\hline NOR0031 & \begin{tabular}{|l} 
Handdded Pan 2 \\
(handle)
\end{tabular} & Eggers 139-144 & Norfolk & Oxnead Mill & $\mathrm{RU}$ & Brampton & $\mathrm{SF}$ & & & & Frere 1985, 294 \\
\hline NOR0032 & \begin{tabular}{|l|}
$\begin{array}{l}\text { Handled Pan } 2 \\
\text { (handle) }\end{array}$ \\
\end{tabular} & Eggers $139-144$ & Norfolk & Walsingham & $\mathrm{RU}$ & near Roman temple site & $\mathrm{SF}$ & & & $\begin{array}{l}\text { Concentric circle handle medallion and } \\
\text { suspension loop }\end{array}$ & Smith 1999, 39 \\
\hline NOR0033 & Cauldron & & Norfolk & Weeting & RS & $\begin{array}{l}\text { Northern flood-plain of River } \\
\text { Ouse }\end{array}$ & SD & NOR0033-NOR0040 & & & Gregory $1977.265(1)$ \\
\hline NOR0034 & Bowl & Bassin a bord godrone & Norfolk & Weeting & RS & $\begin{array}{l}\text { Northern flood-plain of River } \\
\text { Ouse }\end{array}$ & SD & NOR0033-NOR0040 & Geometric 'godronne' decoration along rim & & Gregory $1977,267(2)$ \\
\hline NOR0035 & Bowl & Bassin uni & Norfolk & Weeting & RS & $\begin{array}{l}\text { Northern flood-plain of River } \\
\text { Ouse }\end{array}$ & SD & NOR0033-NOR0040 & & & Gregory 1977, 268 (3) \\
\hline NOR0036 & Cauldron & & Norfolk & Weeting & RS & $\begin{array}{l}\text { Northern flood-plain of River } \\
\text { Ouse }\end{array}$ & SD & NOR0033-NOR0040 & & & Gregory 1977, 268 (4) \\
\hline NOR0037 & Cauldron & & Norfolk & Weeting & RS & $\begin{array}{l}\text { Northern flood-plain of River } \\
\text { Ouse }\end{array}$ & SD & NOR0033-NOR0040 & & & Gregory 1977, 268 (5) \\
\hline NOR0038 & Bowl & Irchester & Norfolk & Weeting & RS & $\begin{array}{l}\text { Northern flood-plain of River } \\
\text { Ouse }\end{array}$ & SD & NOR0033-NOR0040 & & & Gregory 1977, 268 (6) \\
\hline NOR0039 & Bowl & Irchester & Norfolk & Weeting & RS & $\begin{array}{l}\text { Northern flood-plain of River } \\
\text { Ouse }\end{array}$ & SD & NOR0033-NOR0040 & & & Gregory $1977.268(7)$ \\
\hline NOR0040 & Bowl & & Norfolk & Weeting & RS & $\begin{array}{l}\text { Northern flood-plain of River } \\
\text { Ouse }\end{array}$ & SD & NOR0033-NOR0040 & & & Gregory $1977,269(8)$ \\
\hline NOR0041 & \begin{tabular}{|l} 
Handled Pan 2 \\
(handle)
\end{tabular} & Eggers $139-144$ & Norfolk & Saham Toney & M & $\begin{array}{l}\text { near suspected fort at Sand } \\
\text { Hills, Woodcock Hall }\end{array}$ & $\mathrm{SF}$ & & & \begin{tabular}{|l} 
Concentric circle handle loop; punch dot \\
inscription "C PRIMI"
\end{tabular} & $\begin{array}{l}\text { Hassall \& Tomlin } 1978 . \\
\text { 480 (61); RIB II } \\
2415.59\end{array}$ \\
\hline NOR0042 & Jug & & Norfolk & Mundesley & RS & & $\mathrm{SF}$ & & & & BM 1900.0717.1 \\
\hline NOR0043 & $\begin{array}{l}\begin{array}{l}\text { Handled Pan } 2 \\
\text { (handle) }\end{array} \\
\end{array}$ & Rudge Cup Type & Norfolk & Gunthorpe & $\mathrm{RU}$ & & PAS & & & Inscription "BEBE SESE", enameled & $\begin{array}{l}\text { PAS NMS-7BC635; } \\
\text { Worrell 2012, 73-74 }\end{array}$ \\
\hline NoT0001 & Handled Pan 2 & Eggers 144 & Nottinghamshire & Broxtowe & M & & $\mathrm{SF}$ & & & inscription "ALBANVS" & \begin{tabular}{|l|} 
Oswald 1939, 441; \\
Eggers 1968. 110 (87); \\
Mceeake \& Moore \\
1978, 333 (3); RIB II \\
2415.3
\end{tabular} \\
\hline кото002 & $\mid \begin{array}{l}\text { Handled Pan } 2 \\
\text { (fiagment) }\end{array}$ & Eggers 139-144 & Nottinghamshire & Winthorpe & $\mathrm{RU}$ & & PAS & & & Concentric circle handle medallion loop & PAS SWYOR-4FC184 \\
\hline NOT0003 & Vessel (fragment) & & Nottinghamshire & Hawton & $\mathrm{RU}$ & & PAS & & Geometric circle band on rim & & PAS DENO-CCC324 \\
\hline NOT0004 & Vessel (mount) & & Nottinghamshire & Collingham & $\mathrm{RU}$ & & PAS & & Floral leaf shaped hanging vessel mount & & PAS DENO-149754 \\
\hline NoT0005 & \begin{tabular}{|l|}
$\begin{array}{l}\text { Handled Pan } 2 \\
\text { (handle) }\end{array}$ \\
\end{tabular} & Rudge Cup Type & Nottinghamshire & Brough-on-Fosse & M & & SF & & & Floral vines enameled in blue, green and red & Moore 1978, 319-327 \\
\hline Nu0001 & Vessel (fragment) & & Northumberland & Great Chesters & M & & $\mathrm{SF}$ & & & & Eggers 1966, $10859 \mathrm{a}$ \\
\hline Nuo0oo2 & Vessel (fragment) & & Northumberland & Great Chesters & M & & SF & & Concentric circle umbo & & Eggers $1966,10859 \mathrm{~b}$ \\
\hline vu0003 & Strainer & Eggers 161 & Northumberland & Great Chesters & M & Fort (Aesica) & SD & NU0003-NU0005 & Geometric sunburst strainer dot pattern & & Eggers 1966, $10859 \mathrm{c}$ \\
\hline vuooou & Strainer & Eggers 161 & Northumberland & Great Chesters & M & Fort (Aesica) & SD & NU0003-NU0005 & Geometric sunburst strainer dot pattern & & Eggers 1966, $10859 \mathrm{~d}$ \\
\hline vuo005 & Handled Pan 3 & Eggers 161 & Northumberland & \begin{tabular}{|l} 
Great Chesters \\
\end{tabular} & M & Fort (Aesica) & SD & NU0003-NU0005 & & & Eggers $1966,10859 \mathrm{e}$ \\
\hline Nuooor & Handled Pan 2 & Eggers 139-144 & Northumberland & Bardon Mill & M & $\mid \begin{array}{l}\text { Housesteads Fort } \\
\text { (Borcovicium/Vercovicium) }\end{array}$ & $\mathrm{SF}$ & & Concentric circle umbo & & Eggers 1968, 10860 \\
\hline vuoooo7 & \begin{tabular}{|l|}
$\begin{array}{l}\text { Handled Pan } 2 \\
\text { (handle) }\end{array}$ \\
\end{tabular} & Eggers $145-147$ & Northumberland & Bardon Mill & M & \begin{tabular}{|l}
$\begin{array}{l}\text { Housesteads Fort } \\
\text { (Borcovicium/Vercovicium) }\end{array}$ \\
\end{tabular} & SF & & & Geometric trefoil hole in handle medallion & Eggers 1968, 10860 \\
\hline vuooos & \begin{tabular}{|l|}
$\begin{array}{l}\text { Handled Pan } 2 \\
\text { (handle) }\end{array}$ \\
\end{tabular} & Eggers 139-144 & Northumberland & Bardon Mill & M & \begin{tabular}{|l|}
$\begin{array}{l}\text { Housesteads Fort } \\
\text { (Borcovicium/Vercovicium) }\end{array}$ \\
\end{tabular} & $\mathrm{SF}$ & & & Geomerric circles and border & Eggers 1968, 10860 \\
\hline vuooog & Vessel (fragment) & & Northumberland & Bardon Mill & M & $\begin{array}{l}\text { Housesteads Fort } \\
\text { (Borcovicium/Vercovicium) }\end{array}$ & $\mathrm{SF}$ & & & & Eggers 1968, 10860 \\
\hline Nuoolo & $\begin{array}{l}\begin{array}{l}\text { Handled Pan } 2 \\
\text { (handle) }\end{array} \\
\end{array}$ & Eggers 139-144 & Northumberland & Bardon Mill & M & $\begin{array}{l}\begin{array}{l}\text { Housesteads Fort } \\
\text { (Borcovicium/Vercovicium) }\end{array} \\
\end{array}$ & $\mathrm{SF}$ & & & Geometric circles and border & Eggers 1968, 10860 \\
\hline
\end{tabular}




\begin{tabular}{|c|c|c|c|c|c|c|c|c|c|c|c|}
\hline Nuo0 12 & \begin{tabular}{|l}
$\begin{array}{l}\text { Handled Pan } 2 \\
\text { (handle) }\end{array}$ \\
\end{tabular} & Eggers 139-144 & Northumberland & Chesters & $\mathrm{M}$ & Fort (Corstopitum) & SF & & & $\begin{array}{l}\text { Geometric circles and border, inscription } \\
\text { "ANSIEPAIPIHR[ODITI]" }\end{array}$ & \begin{tabular}{|l|} 
Eggers 1968,10861 \\
Eggers 1968, 108 62; \\
Wright \& Hassal 1991, \\
301 (76); RIB II \\
2415.6 \\
\end{tabular} \\
\hline \begin{tabular}{|l} 
NuO0013 \\
Nuo014 \\
\end{tabular} & \begin{tabular}{|l} 
Vessel (fragment) \\
Vessel (fragment)
\end{tabular} & & \begin{tabular}{|l} 
Northumberland \\
Northumberland \\
\end{tabular} & \begin{tabular}{|l|} 
Corbridge \\
Rudchester \\
\end{tabular} & $\frac{\mathrm{M}}{\mathrm{M}}$ & \begin{tabular}{|l|} 
Fort (Corstopitum) \\
Fort (Vindobala) \\
\end{tabular} & $\frac{\mathrm{SF}}{\mathrm{SF}}$ & & Concentic cirlce umbo & & \begin{tabular}{|l} 
Eggers 1968, 10862 \\
Eggers 1968. $10864 \mathrm{ag}$
\end{tabular} \\
\hline Nuo015 & Handled Pan 3 & Eggers $160-161$ & Northumberland & Rudchester & M & Fort (Vindobala) & SF & & & & Eggers $1968,10864 \mathrm{~b}$ \\
\hline Nuo016 & Handled Pan 2 & Eggers 144 & Northumberland & South Shields & M & & SF & & $\begin{array}{l}\text { Concentric circle umbo, floral and geometric band } \\
\text { under rim }\end{array}$ & Concentric circle handle ring, punch dots & $\begin{array}{l}\text { Bosanquet \& Richmond } \\
\text { 1936, 139-151; Eggers } \\
\text { 1968, 108 66a } \\
\end{array}$ \\
\hline Nuo017 & $\begin{array}{l}\text { Handled Pan } 3 \\
\text { (handle) }\end{array}$ & Eggers $160-161$ & Northumberland & South Shields & M & & sF & & & & Eggers 1968, 108 (66c) \\
\hline Nuoo18 & Vessel (fragment) & & Northumberland & South Shields & M & & SF & & & & Eggers 1968, 108 (66d) \\
\hline Nuo019 & Handled Pan 2 & Eggers 139 & Northumberland & Ponteland & $\mathrm{RU}$ & Prestwick Carr, Swamp & SD & YOR0019-YOR0025 & Concentric circle umbo & $\begin{array}{l}\text { Concentric circle handle ring, stylised caducaeus } \\
\text { on handle with trefoil points and punch dot } \\
\text { embellishments; incription "DRACCIVS F" }\end{array}$ & $\begin{array}{l}\text { Eggers 1968, } 108 \\
\text { 65a); McPeake \& } \\
\text { Moore 1978, } 334 \text { (21); } \\
\text { RIB II 2415.32 }\end{array}$ \\
\hline Nuo020 & Handled Pan 2 & Eggers $140-141$ & Northumberland & Ponteland & $\mathrm{RU}$ & Prestwick Carr, Swamp & SD & YOR0019-YOR0025 & Concentric circle umbo & & Eggers 1968, 108 (65b) \\
\hline Nu0021 & Handled Pan 2 & Eggers $140-141$ & Northumberland & Ponteland & $\mathrm{RU}$ & Prestwick Carr, Swamp & SD & YOR0019-YOR0025 & Concentric circle umbo & & Eggers 1968, 108 (65c) \\
\hline Nu0022 & Bowl & Eggers 68 & Northumberland & Ponteland & RU & Prestwick Carr, Swamp & sD & YOR0019-YOR0025 & & & Eggers 1968, 108 (65d) \\
\hline Nu0023 & Bowl & Eggers 70 & Northumberland & Ponteland & $\mathrm{RU}$ & Prestwick Carr, Swamp & SD & YOR0019-YOR0025 & & $\begin{array}{l}\text { punch dot inscriptions below rim "T TTIRONIS } \\
\text { CRIISCRII SNT SENECIONIS" and } \\
\text { "TKANDIANI VANNI" }\end{array}$ & $\begin{array}{l}\text { Eggers 1968, } 108 \\
\text { (65e); RIB II 2415.63 }\end{array}$ \\
\hline Nuo024 & Cauldron & $\begin{array}{l}\text { Westland, Eggers 12- } \\
13 \text { (c.f.) }\end{array}$ & Northumberland & Ponteland & $\mathrm{RU}$ & Prestwick Carr, Swamp & SD & YOR0019-YOR0025 & & & Eggers 1968, $108(65 f)$ \\
\hline Nuo025 & Bowl & Eggers 101-105 & Northumberland & Ponteland & $\mathrm{RU}$ & Prestwick Carr, Swamp & SD & YOR0019-YOR0025 & Concentric circle footring & & Eggers 1968, $108(65 \mathrm{~g})$ \\
\hline Nuo026 & \begin{tabular}{|l}
$\begin{array}{l}\text { Handled Pan } 2 \\
\text { (fragment) }\end{array}$ \\
\end{tabular} & $\begin{array}{l}\begin{array}{l}\text { Rudge Cup Type, } \\
\text { Eggers } 157\end{array} \\
\end{array}$ & Northumberland & Harwood & $\mathrm{RU}$ & & SF & & Geometric dots, scallops, and shields, enameled & & $\begin{array}{l}\text { Eggers 1968, } 108(65 \\
\text { A); BM 1852.1001.1 }\end{array}$ \\
\hline Nu0027 & Strainer & Eggers 160 & Northumberland & Hexham & RS & (Whitfield) & SF & & Geometric circle and scallops strainer dot pattern & & Eggers 1968, $108(63)$ \\
\hline Nuo028 & Vessel (fragment) & Eggers $140-144$ & Northumberland & Bolton & $\mathrm{RU}$ & & SF & & $\begin{array}{l}\text { Concentric circle umbo, omphalos dot, concentric } \\
\text { circle foot ring }\end{array}$ & & $\begin{array}{l}\text { Eggers 1968, } 109 \text { (67); } \\
\text { Newcastle Museum } \\
\text { 1956, 130 A }\end{array}$ \\
\hline Nu0029 & Jar & & Northumberland & Whittington & $\mathrm{RU}$ & & SD & Nuo030 & $\begin{array}{l}\text { Floral oval half leaf band running under the rim; } \\
\text { Concentric circle umbo }\end{array}$ & & PAS NCL-33CC76 \\
\hline NUO030 & Handled Pan 2 & Eggers 139-144 & Northumberland & Whittington & $\mathrm{RU}$ & & SD & NU0029 & Concentric circle umbo and foot ring & Concentric circle handle medallion loop & PAS NCL-335745 \\
\hline Nuo031 & $\begin{array}{l}\text { Handled Pan } 22 \\
\text { (fragment) }\end{array}$ & Eggers $145-147$ & Northumberland & Birdoswald & M & Fort (Birdoswald) & SF & & Trefoil suspension loop in handle medallion & & $\begin{array}{l}\text { Wilmott 1997, } 290 \\
\text { (125) }\end{array}$ \\
\hline Nu0032 & Jug (fragment) & & Northumberland & Birdoswald & M & Fort (Birdoswald) & SF & & & & $\begin{array}{l}\begin{array}{l}\text { Wilmott 1997, } 290 \\
\text { (126) }\end{array} \\
\end{array}$ \\
\hline Nu0033 & Jar (fragment) & & Northumberland & Bardon Mill & M & $\begin{array}{l}\begin{array}{l}\text { Housesteads Fort } \\
\text { (Borcovicium/Vercovicium) }\end{array} \\
\end{array}$ & SF & & $\begin{array}{l}\begin{array}{l}\text { Geometric enameled triangle and zig zag body } \\
\text { bands }\end{array} \\
\text { a }\end{array}$ & & Wilson 2002, 79 \\
\hline Nuo0 34 & Jug (fragment) & & Northumberland & Corbridge & M & Fort (Corstopitum) & SF & & & Floral leaf-shaped handle medallion & 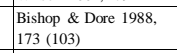 \\
\hline Nuo035 & Jug & Eggers 128a & Northumberland & Corbridge & M & $\begin{array}{l}\text { Fort (Corstopitum), north of } \\
\text { Stanegate }\end{array}$ & SD & $160 \mathrm{Cn}[\mathrm{g}], 2 \mathrm{Cn}[\mathrm{ca}]$ & & & $\begin{array}{l}\text { Forester et al. 1912, } \\
\text { 154; McDonald 1912, } 1 \\
\text { 12; Abdy 2002, 35 (19) } \\
\end{array}$ \\
\hline Nu0036 & Jug & & Northumberland & Corbridge & M & $\begin{array}{l}\text { Fort (Corstopitum), east end of } \\
\text { field, Site XLIIII }\end{array}$ & $\mathrm{SF}_{\mathrm{SF}}$ & & & $\begin{array}{l}\begin{array}{l}\text { Floral leaf embellishments; Anthropomorphic } \\
\text { (possible) handle medallion }\end{array}\end{array}$ & $\begin{array}{l}\text { Forester et al. } 1913, \\
235\end{array}$ \\
\hline Nu0037 & Vessel (fragment) & & Northumberland & Corbridge & M & $\begin{array}{l}\text { Haltonchesters Roman Fort } \\
\text { (Hunnum) }\end{array}$ & SF & & Concentric circle umbo & & Dore 2010, 147 (26) \\
\hline Nuo038 & Jar & & Northumberland & Bardon Mill & M & $\begin{array}{l}\begin{array}{l}\text { Housesteads Fort } \\
\text { (Borcovicium/Vercovicium) }\end{array} \\
\end{array}$ & SF & & $\begin{array}{l}\text { Geometric triangle, wave, and linear bands; } \\
\text { enameled in green and turquoise }\end{array}$ & & $\begin{array}{l}\text { Rushmorth 2009, } 438 \\
\text { (50) }\end{array}$ \\
\hline Nu0039 & Vessel (fragment) & & Northumberland & Bardon Mill & M & $\begin{array}{l}\text { Housesteads Fort } \\
\text { (Borcovicium/Vercovicium) }\end{array}$ & $\mathrm{SF}$ & & Concentriec circle rim & & $\begin{array}{l}\text { Rushmorth 2009, } 438 \\
(51)\end{array}$ \\
\hline Nu0040 & Vessel (mount) & & Northumberland & Bardon Mill & M & 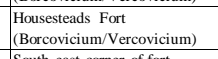 & SF & & & Geomertic pelta shaped hanging vessel mount & $\begin{array}{l}\begin{array}{l}\text { Rushmorth 2009, } 438 \\
\text { (54) }\end{array} \\
\end{array}$ \\
\hline Nu0041 & Vessel (fragment) & & Northumberland & South Shields & $\mathrm{M}$ & South east corner of fort & SF & & Concentric circle rim & & Miket 1983, $111(41)$ \\
\hline
\end{tabular}




\begin{tabular}{|c|c|c|c|c|c|c|c|c|c|c|c|}
\hline Nuo042 & Vessel (fragment) & & Northumberland & South Shields & M & Southwest gate ditches & SF & & $\begin{array}{l}\begin{array}{l}\text { Concentric circle umbo and umphalos dot; three } \\
\text { undecorated feet }\end{array} \\
\end{array}$ & & \begin{tabular}{|l|}
$\begin{array}{l}\text { Bidwell \& Speak 1994, } \\
186(45)\end{array}$ \\
\end{tabular} \\
\hline Nuo043 & \begin{tabular}{|l}
$\begin{array}{l}\text { Handled Pan } 22 \\
\text { (handle) }\end{array}$ \\
\end{tabular} & Eggers 139-144 & Northumberland & Bardon Mill & M & $\begin{array}{l}\text { Vindolanda (2003/2004 Site } \\
\text { A) }\end{array}$ & SF & & & & $\begin{array}{l}\text { Birley \& Blake 2005, } \\
256 \text { (9161) }\end{array}$ \\
\hline Nuo044 & Bowl & & Northumberland & Bardon Mill & M & Vindolanda (2003 Area B) & SF & & & & $\begin{array}{l}\text { Birley \& Blake 2005, } \\
259 \text { (8955) }\end{array}$ \\
\hline Nuo045 & Handled Pan 1 & Eggers 154-155 & Northumberland & Bardon Mill & M & Vindolanda (Area A 2005) & SF & & & Zoomorphic canine handle terminal & $\begin{array}{l}\text { Birley \& Blake 2007, } \\
221(10035)\end{array}$ \\
\hline Nuo046 & Handled Pan 2 & Eggers $139-144$ & Northumberland & Bardon Mill & M & Vindolanda (2005 Area B) & SF & & & & $\begin{array}{l}\text { Birley \& Blake } 2007, \\
228 \text { (9845) }\end{array}$ \\
\hline Nu0047 & \begin{tabular}{|l}
$\begin{array}{l}\text { Handled Pan } 2 \\
\text { (handle) }\end{array}$ \\
\end{tabular} & Eggers 139-144 & Northumberland & Bardon Mill & M & Vindolanda & SF & & & Inscription in punch dots reads "[...] ERIORIS" & $\begin{array}{l}\text { Burnham et alia } 2005 \text {, } \\
492\end{array}$ \\
\hline Nuo048 & $\begin{array}{l}\begin{array}{l}\text { Handled Pan } 2 \\
\text { (handle) }\end{array} \\
\end{array}$ & Eggers $139-144$ & Northumberland & Bardon Mill & M & Vindolanda & SF & & & Inscription in punch dots reads "BRO" & $\begin{array}{l}\text { Burnham et alia } 2005 \text {, } \\
492\end{array}$ \\
\hline Nuo049 & Jar & & Northumberland & Corbridge & M & Burial 508 & $\mathrm{GD}$ & 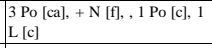 & & & $\begin{array}{l}\text { Casey \& Hoffman, } \\
\text { 1995b, 24 (6) }\end{array}$ \\
\hline Nuo050 & Handled Pan 2 & Eggers 139-144 & Northumberland & Matfen & $\mathrm{RU}$ & Ingoe township & SD & NU0050-NU0051 & & 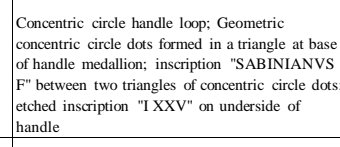 & ; $\begin{array}{l}\text { Wright 1969a, 1-5; RIB } \\
\text { II 2415.44 }\end{array}$ \\
\hline \begin{tabular}{|l} 
NU0051 \\
NYRO0001
\end{tabular} & \begin{tabular}{|l|} 
Handled Pan 2 \\
Handled Pan 2
\end{tabular} & \begin{tabular}{|l|} 
Eggers $139-144$ \\
Eggers $139-144$
\end{tabular} & \begin{tabular}{|l|} 
Northumberland \\
North Yorkshire \\
\end{tabular} & \begin{tabular}{|l} 
Maten \\
Malton
\end{tabular} & \begin{tabular}{|l}
$\mathrm{RU}$ \\
$\mathrm{RS}$
\end{tabular} & Ingoe township & $\frac{\text { SD }}{\text { PAS }}$ & NU0050-NU0051 & Concentric cirlce umbo and foot ring & 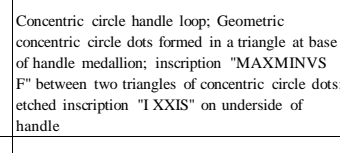 & $\begin{array}{l}\text { Wright 1969a 1-5; } \\
\text { McPeake \& Moore } \\
1978,334 \text { (30); RIB II } \\
2415.40 \\
\text { PAS NLM-6A7473 } \\
\end{array}$ \\
\hline NYR0002 & $\begin{array}{l}\text { Handled Pan } 2 \\
\text { (handle) }\end{array}$ & Eggers $139-144$ & North Yorkshire & Brough with St. Giles & RU & & PAS & & Concentric cirlce umbo and foot ring & $\begin{array}{l}\text { Geometric punch dots arranged in a triangular } \\
\text { shape at base of handle medallilion; Concentric } \\
\text { circle handle medallion loop }\end{array}$ & PAS NCL-782251 \\
\hline NYR003 & Handled Pan 2 & & North Yorkshire & Linton & RS & & SD & NYR0004 & & $\begin{array}{l}\text { Geometric triangular protrusion from end of } \\
\text { handle }\end{array}$ & PAS SWYOR-E51F57 \\
\hline NYR0004 & Bowl & & North Yorkshire & Linton & RS & & SD & NYR0003 & & & PAS SWYOR-E4D7DO \\
\hline NYR0005 & Handled Pan 2 & & \begin{tabular}{|l} 
North Yorkshire \\
\end{tabular} & Hawkswick & $\mathrm{RU}$ & & PAS & & Concentric circle umbo and rings in interior basin & & PAS LVPL-F9BE12 \\
\hline NYR0006 & \begin{tabular}{|l|l|l} 
Vessel (mount) \\
Vsent
\end{tabular} & & $\begin{array}{l}\text { North Yorkshire } \\
\end{array}$ & Claxton & $\mathrm{RU}$ & & PAS & & Geometric sub-triangular hanging vessel mount & & $\begin{array}{l}\text { PAS DUR-510214 } \\
\text { PSDP }\end{array}$ \\
\hline NYR0007 & Vessel (mount) & & North Yorkshire & Bedale & $\mathrm{RU}$ & & PAS & & Anthropomorphic face, possibly Cupid or cherub & & PAS DUR-EID6C7 \\
\hline NYR0008 & Bucket & Ostland, Eggers $37-40$ & North Yorkshire & Knaresborough & $\mathrm{RU}$ & & SD & NYR0008-NYR0028 & & & \begin{tabular}{|l} 
Kennett 1971, $1346.1 ;$ \\
Eggers $1966 \quad 107(551)$
\end{tabular} \\
\hline NYR0009 & Strainer & Eggers 161 & North Yorkshire & Knaresborough & $\mathrm{RU}$ & & SD & NYR0008-NYR0028 & Geometric swirl strainer dot pattern & & $\begin{array}{l}\text { Kennett 1971, } 1346.2 ; \\
\text { Eggers } 1966 \quad 137 \text { (55 2) }\end{array}$ \\
\hline NYR0010 & Strainer & Eggers 161 & North Yorkshire & Knaresborough & $\mathrm{RU}$ & & SD & NYR0008-NYR0028 & Geometric swirl strainer dot pattern & & $\begin{array}{l}\text { Kennett 1971, } 1346.3 ; \\
\text { Eggers } 1966 \quad 107(553)\end{array}$ \\
\hline NYR0011 & Strainer & & North Yorkshire & Knaresborough & $\mathrm{RU}$ & & SD & NYR0008-NYR0028 & & & $\begin{array}{l}\text { Kennett 1971, } 1346.4 ; \\
\text { Eggers } 1966 \quad 107 \text { (55 4) }\end{array}$ \\
\hline NYR0012 & Strainer & & North Yorkshire & Knaresborough & $\mathrm{RU}$ & & SD & NYR0008-NYR0028 & & Geometric swirl strainer dot pattern & \begin{tabular}{|l} 
Kennett 1971, $1346.5 ;$ \\
Eggers $1966 \quad 107(555)$
\end{tabular} \\
\hline NYR0013 & Strainer & & North Yorkshire & Knaresborough & $\mathrm{RU}$ & & SD & NYR0008-NYR0028 & & $\begin{array}{l}\text { Floral strainer dot pattern in the shape of multi- } \\
\text { petaled flower }\end{array}$ & $\begin{array}{l}\text { Kennett 1971, } 1346.6 ; \\
\text { Eggers } 1966 \quad 107(556)\end{array}$ \\
\hline NYR0014 & Strainer & & North Yorkshire & Knaresborough & $\mathrm{RU}$ & & SD & NYR0008-NYR0028 & & $\begin{array}{l}\text { Geometric wave, swirl, flourish, swastika, and } \\
\text { triskele strainer dot pattern }\end{array}$ & $\begin{array}{l}\text { Kennett 1971, } 1346.7 \text {; } \\
\text { Eggers } 1966 \quad 107 \text { (55 7) }\end{array}$ \\
\hline NYR0015 & Bowl & & North Yorkshire & Knaresborough & $\mathrm{RU}$ & & SD & NYR0008-NYR0028 & Concentric circle umbo & & $\begin{array}{l}\text { Kennett 1971, } 1356.8 \text {; } \\
\text { Eggers } 1966 \quad 107 \text { (55 8) }\end{array}$ \\
\hline NYR0016 & Bowl & & North Yorkshire & Knaresborough & RU & & SD & NYR0008-NYR0028 & & & \begin{tabular}{|l} 
Kennett $1971,1356.9 ;$ \\
Eggers $1966107(559)$
\end{tabular} \\
\hline
\end{tabular}




\begin{tabular}{|c|c|c|c|c|c|c|c|c|c|c|c|}
\hline NYR0017 & Bowl & Bassin à bord godroné & North Yorkshire & Knaresborough & $\mathrm{RU}$ & & SD & NYR0008-NYR0028 & $\begin{array}{l}\text { Geometric linear rim decoration, many punch dots } \\
\text { on underside of umbo }\end{array}$ & & \begin{tabular}{|l} 
Kennett 1971, 135 \\
$6.10 ;$ Eggers 1966107 \\
(55 10) \\
\end{tabular} \\
\hline NYR0018 & Bowl & Irchester & North Yorkshire & Knaresborough & $\mathrm{RU}$ & & SD & NYR0008-NYR0028 & & & $\begin{array}{l}\text { Kennett 1971, } 135 \\
6.11 ; \text { Eggers } 1966107 \\
(5511)\end{array}$ \\
\hline NYR0019 & Bowl & Irchester & North Yorkshire & Knaresborough & $\mathrm{RU}$ & & SD & NYR0008-NYR0028 & & & \begin{tabular}{|l} 
Kennett 1971, 135 \\
$6.12 ;$ Eggers 1966107 \\
$(55$ 12)
\end{tabular} \\
\hline NYR0020 & Bowl & Irchester & North Yorkshire & Knaresborough & $\mathrm{RU}$ & & SD & NYR0008-NYR0028 & & & \begin{tabular}{|l|l|}
$\begin{array}{l}\text { Kennett 1971, } 135 \\
\text { 6.13; Eggers } 1966 \\
\text { (55 13) }\end{array} 107$ \\
\end{tabular} \\
\hline NYR0021 & Bowl & Irchester & North Yorkshire & Knaresborough & $\mathrm{RU}$ & & SD & NYR0008-NYR0028 & & & \begin{tabular}{|l|} 
Kennett 1971, 135 \\
6.14; Eggers 1966107 \\
(55 14) \\
\end{tabular} \\
\hline NYR0022 & Bowl & Irchester & North Yorkshire & Knaresborough & $\mathrm{RU}$ & & SD & NYR0008-NYR0028 & & & 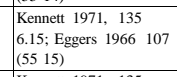 \\
\hline NYR0023 & Bowl & Irchester & North Yorkshire & Knaresborough & $\mathrm{RU}$ & & SD & NYR0008-NYR0028 & & & 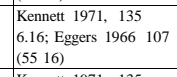 \\
\hline NYR0024 & Bowl & & North Yorkshire & Knaresborough & $\mathrm{RU}$ & & SD & NYR0008-NYR0028 & & & $\begin{array}{l}\text { Kennett 1971, } 135 \\
6.17 \text { : Eggers } 1966107 \\
(55 \text { 17) }\end{array}$ \\
\hline NYR0025 & Vessel (fragment) & & North Yorkshire & Knaresborough & $\mathrm{RU}$ & & SD & NYR0008-NYR0028 & & & $\begin{array}{l}\text { Kennett 1971, } 1356.18 \\
20 ; \text { Eggers } 1966107 \\
(5518-20)\end{array}$ \\
\hline NYR0026 & Bowl & & North Yorkshire & Knaresborough & $\mathrm{RU}$ & & SD & NYR0008-NYR0028 & & & \begin{tabular}{|l|l|}
$\begin{array}{l}\text { Kennett 1971, } 135 \\
6.21 ; \text { Eggers } 1966107 \\
(5521)\end{array}$ \\
\end{tabular} \\
\hline NYR0027 & Handled Pan 5 & & North Yorkshire & Knaresborough & $\mathrm{RU}$ & & SD & NYR0008-NYR0028 & & Geometric linear etching & 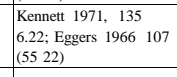 \\
\hline NYR0028 & Bowl & Bassin à bord godronné & North Yorkshire & Knaresborough & RU & & SD & NYR0008-NYR0028 & & & Kennett 1971, 1366.23 \\
\hline NYR0029 & Jar & Ostland, Eggers 40 & North Yorkshire & York & u & River, Blue Bridge Lane & SF & & $\begin{array}{l}\begin{array}{l}\text { Two incriptions on rim "C ATTISIVIIIRI" (in } \\
\text { punch dots), "C APRILI" (straight etched) post } \\
\text { manufacture }\end{array} \\
\end{array}$ & & $\begin{array}{l}\text { Eggers 1968, } 107 \\
\text { (52a); RCH 1962, } 133 \\
\text { (143); RIB II 2415.58 }\end{array}$ \\
\hline NYR0030 & Vessel (fragment) & & North Yorkshire & York & $\mathrm{U}$ & River, (River Ouse) & SF & & & & Eggers $1968, \quad 107(52 \mathrm{c})$ \\
\hline NYRo031 & Bowl & Eggers 101-105 & North Yorkshire & Matton & RS & & SF & & Concentric circle foot ring & & Eggers 1968, 107 (56a) \\
\hline NYR0032 & Handled Pan 2 & Eggers 139-144 & North Yorkshire & Matton & RS & & SF & & & $\begin{array}{l}\text { stamped inscription "ALPICVSF", punch dot } \\
\text { inscriptions "LSERVENISVSVPER" and } \\
\text { "SVPERI" under handle }\end{array}$ & $\begin{array}{l}\text { Eggers 1968, } 107 \\
\text { (56b); Mceeake \& } \\
\text { Moore 1978, 333 (4); } \\
\text { RIB II 2415.4 (2) }\end{array}$ \\
\hline 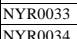 & $\begin{array}{l}\text { Vessel (fragment) } \\
\text { Juv f(fasment) }\end{array}$ & Fogers 123-126 & \begin{tabular}{|l} 
North Yorkshire \\
North Yorkhier
\end{tabular} & \begin{tabular}{|l|l|l|l|l} 
Aldborough \\
Aldbrounh
\end{tabular} & U & & SF & & \begin{tabular}{|l} 
Concentric circle umbo \\
Zomorothic duck thumbert \\
\end{tabular} & & \begin{tabular}{|l} 
Bistop 1996, $10(16)$ \\
Bishop 1996,10188
\end{tabular} \\
\hline $\begin{array}{l}\text { NYRR0034 } \\
\text { NYRO035 }\end{array}$ & $\begin{array}{l}\text { ugg (riagment) } \\
\text { Vessel (mount) }\end{array}$ & & \begin{tabular}{|l|} 
North Yorshine \\
North Yorkshire
\end{tabular} & \begin{tabular}{|l|} 
Alddbororough \\
\end{tabular} & U & & SF & & $\begin{array}{l}\text { Zoomorphic cuck thumbrest } \\
\text { Geometric sub-pelta shaped hanging vessel mount }\end{array}$ & & 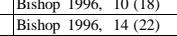 \\
\hline NYRO036 & Strainer (fragment) & & North Yorkshire & Beadlam Roman Villa & RS & Villa, Corridor 3, Building 1 & SF & & Concentric circle (possible) strainer dot pattern & & Neal 1996, $49(17)$ \\
\hline NYR0037 & $\begin{array}{l}\text { Handled Pan } 2 \\
\text { (fragment) }\end{array}$ & Rudge Cup Type & North Yorkshire & Beadlam Roman Villa & RS & $\begin{array}{l}\text { south wall of Room } 6, \\
\text { Building 1 }\end{array}$ & SD & $59 \mathrm{Un}(\mathrm{m})$ & $\begin{array}{l}\text { Floral vine (possible) body band; Inscription "[...]I I } \\
\text { CrTR" in upper register followed by a geometric zig- } \\
\text { zag line }\end{array}$ & & $\begin{array}{l}\text { Wright \& Hassall 1973, } \\
\text { 334 (38); Neal 1996, } \\
\text { 49 (23); RIB II 2415.54 }\end{array}$ \\
\hline NYR0038 & Jar & & North Yorkshire & Catterick & RS & Catterick Bypass, Site 433 & SF & & $\begin{array}{l}\text { Geometric triangle body bands above and below } \\
\text { two crescent body bands, both bands in red and blue } \\
\text { enamel }\end{array}$ & & Wilson 2002, 78 (1) \\
\hline NYR0039 & Vessel (fragment) & & North Yorkshire & York & u & Minster, Building 4 & SF & & Concentric circle umbo & & $\begin{array}{l}\begin{array}{l}\text { Philips \& Heywood } \\
\text { 1995, 385 (46) }\end{array} \\
\end{array}$ \\
\hline NYR0040 & Handled Pan 2 & Eggers 142 & North Yorkshire & Stittenham & $\mathrm{RU}$ & & SD & NYR0040-NYR0044 & & $\begin{array}{l}\begin{array}{l}\text { Concentric Circle handle loop; inscription "P } \\
\text { CIIII POLYIBI" }\end{array} \\
\end{array}$ & $\begin{array}{l}\text { Eggers 1968, 107 (54a); } \\
\text { RIB II 2415.26 }\end{array}$ \\
\hline NYR0041 & Handled Pan 2 & Eggers 142 & North Yorkshire & Stittenham & $\mathrm{RU}$ & & SD & NYR0040-NYR0044 & & $\begin{array}{l}\begin{array}{l}\text { Concentirc Circle handle loop; inscription "P } \\
\text { CIPI POLBB" }\end{array} \\
\end{array}$ & $\begin{array}{l}\text { Eggers 1968, } 107 \text { (54b); } \\
\text { RIB II 2415.19 }\end{array}$ \\
\hline
\end{tabular}




\begin{tabular}{|c|c|c|c|c|c|c|c|c|c|c|c|}
\hline NYR0042 & Handled Pan 2 & Eggers 142 & North Yorkshire & Stittenham & $\mathrm{RU}$ & & SD & NYR0040-NYR0044 & & & Eggesr 1968, 107 (54c) \\
\hline NYR0043 & Handled Pan 2 & Eggers 142 & North Yorkshire & Stittenham & $\mathrm{RU}$ & & SD & NYR0040-NYR0044 & & & Eggers 1968, 107 (54d) \\
\hline NYR0044 & Handled Pan 2 & Eggers 142 & North Yorkshire & Stitenham & $\mathrm{RU}$ & & SD & NYR0040-NYR0044 & & & Eggers 1968, 107 (54e) \\
\hline oxoool & Vessel (mount) & & Oxfordshire & Adwell & $\mathrm{RU}$ & & PAS & & $\begin{array}{l}\text { Zoomorphic duck vessel mount, likely from a jug } \\
\text { lid or other vessel lid }\end{array}$ & & PAS BH-2DA8C6 \\
\hline DX0002 & \begin{tabular}{|l} 
Vessel (mount) \\
\end{tabular} & & Oxfordshire & Letcombe Regis & $\mathrm{RU}$ & & PAS & & Anthropomorphic bust of Bacchus & & PAS BERK-C01546 \\
\hline ox0003 & Vessel (mount) & & Oxfordshire & North Leigh & RS & $\begin{array}{l}\text { Shakenoak Farm; hypocaust in } \\
\text { room XVI Building A }\end{array}$ & $\mathrm{SF}$ & & Zoomorphic bull bust hanging vessel mount & & $\begin{array}{l}\text { BrodRIB IIb et alia } \\
2005,52(1)\end{array}$ \\
\hline ox0004 & Vessel (fragment) & & Oxfordshire & North Leigh & RS & $\begin{array}{l}\text { Shakenoak Farm; north of Fish } \\
\text { Pond II }\end{array}$ & $\mathrm{SF}$ & & & & $\begin{array}{l}\text { BrodRRIB IIb et alia } \\
2005,482(250)\end{array}$ \\
\hline ox0005 & Vessel (fragment) & & Oxfordshire & North Leigh & RS & Akeman Street, Field 5651 & $\mathrm{SF}$ & & Concentric circle rim & & $\begin{array}{l}\begin{array}{l}\text { Hands et alia 2004, } 50 \\
(134)\end{array} \\
\end{array}$ \\
\hline 000001 & Handled Pan 1 & Eggers 154-155 & Powys & Caersws & RU & & $\mathrm{SF}$ & & & $\begin{array}{l}\text { Zoomorphic ram's head handle terminal, fluted } \\
\text { handle }\end{array}$ & $\begin{array}{l}\text { Eggers 1968, } 103 \\
(15 a \& b)\end{array}$ \\
\hline 00002 & Jug & & Powys & Welshpool & RS & & GD & $\begin{array}{l}\text { PO0002-PO0007, } 3 \text { Fn [f], } 1 \mathrm{~J} \\
{[\mathrm{gl}], 1 \text { B [gl], 2 V [c] }}\end{array}$ & & Anthropomorphic satyr & $\begin{array}{l}\text { Eggers 1968, } 103(16 \\
1) \text { Boon } 196113(1)\end{array}$ \\
\hline 00003 & Handled Pan 1 & Eggers $154-155$ & Powys & Welshpool & RS & & GD & $\begin{array}{l}\text { Poo0002-P0o0007, 3 Fn [f], 1 J } \\
\text { [gl], 1B [ [g] , 2V [c] }\end{array}$ & & $\begin{array}{l}\text { Anthropomorphic human bust handle terminal } \\
\text { and thumb rest }\end{array}$ & $\begin{array}{l}\text { Eggers } 1968,103(16 \\
\text { 2); Boon } 196113(2)\end{array}$ \\
\hline 00004 & Handled Pan 1 & Eggers $154-155$ & Powys & Welshpool & RS & & GD & $\begin{array}{l}\text { Poo0002-POo0007, 3 Fn [f], 1 J } \\
\text { [gl], 1 B [gl], 2 V [c] }\end{array}$ & Floral (exterior body, under handle) & $\begin{array}{l}\text { Zoomorphic ram's head handle terminal, fluted } \\
\text { handle }\end{array}$ & $\begin{array}{l}\text { Eggers 1968, } 103(16 \\
\text { 1); Boon 1961 } 13(3)\end{array}$ \\
\hline 00005 & Handled Pan 1 & Eggers $154-155$ & Powys & Welshpool & RS & & GD & 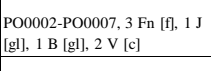 & & $\begin{array}{l}\text { Zoomorphic ram's head handle terminal, fluted } \\
\text { handle }\end{array}$ & $\begin{array}{l}\text { Eggers } 1968,103(16 \\
\text { 1); Boon } 196113(4)\end{array}$ \\
\hline 000006 & Cauldron & & Powys & Welshpool & RS & & GD & 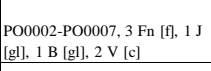 & & & $\begin{array}{l}\text { Eggers } 1968,103(16 \\
\text { 1):Boon } 1961133(5)\end{array}$ \\
\hline 00007 & Vessel (mount) & & Powys & Welshpool & RS & & GD & $\begin{array}{l}\text { Poo0002-POo0007, 3 Fn [f], 1 J } \\
\text { [gl], 1 B [gl], 2 V [c] }\end{array}$ & Zoomorphic bull's head hanging vessel mount & & $\begin{array}{l}\text { Eggers } 1968,103(16 \\
\text { 1); Boon 1961 } 13(6)\end{array}$ \\
\hline 000008 & Handled Pan 2 & Eggers 150 & Powys & Brecon & M & Fort & $\mathrm{SF}$ & & & $\begin{array}{l}\text { inscription "[..] RODITT" in rectangular field; } \\
\text { kesthole shaped handle loop }\end{array}$ & $\begin{array}{l}\text { Eggers 1968, } 103(14) \\
\text { Wheeler 1928, 107-11 } \\
\text { RII II 2415.7 }\end{array}$ \\
\hline 000009 & Bowl & & Powys & Brecon & M & $\begin{array}{l}10 \text { feet north of Building A, } \\
\text { outside the fort }\end{array}$ & $\mathrm{sF}$ & & & & Wheeler 1928, 111-112 \\
\hline 00010 & Vessel (fragment) & & \begin{tabular}{|c|c|c|c|c|} 
Powys \\
\end{tabular} & Brecon & $\mathrm{M}$ & Retentura & $\mathrm{SF}$ & & & & Wheeler 1928, 112 \\
\hline 00011 & Jug (handle) & & Powys & Brecon & M & Hutment of pratetentura & $\mathrm{SF}$ & & & \begin{tabular}{|l}
$\begin{array}{l}\text { Anthropomorphic Medusas's head handle } \\
\text { medallion }\end{array}$ \\
\end{tabular} & Wheeler 1928, 117 (1) \\
\hline RCo001 & Jug (lid) & Eggers $123-126$ & Rhondda Cynon Taf & Pont Y Clun & RU & & PAS & & & & PAS NMGW-2EECF6 \\
\hline 30001 & Vessel (fragment) & & Shropshire & Hordley & $\mathrm{RU}$ & & PAS & & Concentric circle umbo & & PAS HESH-02FED3 \\
\hline SH0002 & Jug (lid) & Eggers 123-126 & Shropshire & Sheinton & RS & & PAS & & Floral (possible) leaf jug lid & & PAS HESH-9774C3 \\
\hline $8 \mathrm{H} 0003$ & \begin{tabular}{|l|l|l} 
Vessel (mount) \\
\end{tabular} & & $\begin{array}{l}\text { Shropshire } \\
\end{array}$ & Whitchurch & RS & & PAS & & Zoomorphic ox head mount & & PAS LVPL-FIC917 \\
\hline нно004 & Jar & & Shropshire & Wroxeter & u & Well 1 & SF & & & & Atkinson 1942, 209 (1) \\
\hline зно0о5 & $\begin{array}{l}\text { Handled Pan } 2 \\
\text { (handle) }\end{array}$ & Eggers 139-144 & Shropshire & Wroxeter & $\mathrm{U}$ & East Room 1 & SF & & & Geometric 'key-hole' suspension loop & Atkinson 1942, 209 (3) \\
\hline вно0об & \begin{tabular}{|l|}
$\begin{array}{l}\text { Handled Pan } 2 \\
\text { (handle) }\end{array}$ \\
\end{tabular} & Eggers 139-144 & Shropshire & Wroxeter & u & $\begin{array}{l}\text { outside east wall of Baths, } \\
\text { Room } 2\end{array}$ & SF & & & $\begin{array}{l}\text { Concentric circle handle medallion and } \\
\text { suspension loop }\end{array}$ & Atkinson 1942, 209 (4) \\
\hline нHо0о6 & Jug (lid) & Eggers 123-126 & Shropshire & Wroxeter & $\mathrm{u}$ & East Entrance & SF & & Trefoil lid & & \begin{tabular}{|l|}
$\begin{array}{l}\text { Atkinson 1942, } 210 \\
\text { (12, A 255) }\end{array}$ \\
\end{tabular} \\
\hline нно0о7 & Jug (lid) & Eggers $123-126$ & Shropshire & Wroxeter & $\mathrm{u}$ & Courty yard west of Room A & SF & & Trefoil lid & & \begin{tabular}{|l|}
$\begin{array}{l}\text { Atkinson 1942, } 210 \\
(12, \text { A 258) }\end{array}$ \\
\end{tabular} \\
\hline нно0ов & Jug (lid) & Eggers $123-126$ & Shropshire & Wroxeter & $\mathrm{u}$ & Pit 10 & SF & & Trefoil lid & & $\begin{array}{l}\text { Atkinson 1942, } 210 \\
(12, \mathrm{~A} 257)\end{array}$ \\
\hline нно0о9 & Jug (lid) & Eggers 123-126 & Shropshire & Wroxeter & u & Dump & $\mathrm{SF}$ & & Trefoil lid & & $\begin{array}{l}\text { Altkinson 1942, } 210(12 \\
\left.A_{2} 256\right)\end{array}$ \\
\hline 30010 & Jug & Eggers $123-126$ & Shropshire & Wroxeter & U & Bath & $\mathrm{SF}$ & & Trefoil lid & & Ellis 2000, $120(235)$ \\
\hline 今н0011 & \begin{tabular}{|l}
$\begin{array}{l}\text { Handled Pan } 2 \\
\text { (handle) }\end{array}$ \\
\end{tabular} & Eggers $137-138$ & Shropshire & Wroxeter & u & House on south side of baths & $\mathrm{SF}$ & & & inscription reads "[...ESRV $[\ldots] "$ & $\begin{array}{l}\text { Wright 1958, 152 (14); } \\
\text { RiB II 2415.52 }\end{array}$ \\
\hline 800001 & Bowl & & Somerset & Somerton & RS & Bradley Hill, Building F & $\mathrm{SF}$ & & Repouse decoration and champleve enameling & & Wilson 1971, 276 \\
\hline 500002 & Handled Pan 2 & Rudge Cup Type & Somerset & Somerton & RS & Bradley Hill, Building 2 & SF & & $\begin{array}{l}\text { Floral vine and leaf swirl and 's'scroll decoration } \\
\text { with blue, green and red enamel }\end{array}$ & & Leech 1981, 210 \\
\hline SYR0001 & \begin{tabular}{|l|}
$\begin{array}{l}\text { Handled Pan } 2 \\
\text { (handle) }\end{array}$ \\
\end{tabular} & & South Yorkshire & Edlington & $\mathrm{RU}$ & & PAS & & & & PAS SWYOR-EA9393 \\
\hline
\end{tabular}




\begin{tabular}{|c|c|c|c|c|c|c|c|c|c|c|c|}
\hline SYR0002 & \begin{tabular}{|l|}
$\begin{array}{l}\text { Handled Pan } 2 \\
\text { (handle) }\end{array}$ \\
Jus (lid)
\end{tabular} & Eggers 139-144 & \begin{tabular}{|l} 
South Yorkshire \\
South Yorkshre
\end{tabular} & \begin{tabular}{|l} 
Doncaster \\
Rotherham \\
\end{tabular} & M & \begin{tabular}{|l} 
Frenchgate (Site DG) \\
Temmllobouob
\end{tabular} & $\frac{\mathrm{SF}}{\mathrm{SF}}$ & & & & \begin{tabular}{|l|}
$\begin{array}{l}\text { Buckland \& Magilton } \\
1986,85(3)\end{array}$ \\
Mav 1922,75 (1)
\end{tabular} \\
\hline SYR0004 & Jug (lid) & & $\begin{array}{l}\text { Sount rosknime } \\
\text { South Yorkhire }\end{array}$ & \begin{tabular}{|l} 
Rotherham \\
Rotherham \\
\end{tabular} & $\begin{array}{l}\mathrm{M} \\
\mathrm{M} \\
\end{array}$ & $\begin{array}{l}\text { Templeborough } \\
\text { Templeborough } \\
\end{array}$ & $\begin{array}{l}S F \\
S F \\
S F\end{array}$ & & 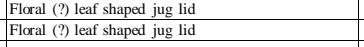 & & $\begin{array}{l}\text { May } 1922,75(1) \\
\text { May 1922, 75(9) }\end{array}$ \\
\hline STA0001 & Vessel (mount) & & Staffordshire & Shenstone & RS & & PAS & & Anthropomorphic reclining banquetor vessel mount & & PAS WMID-6553C1 \\
\hline STA0002 & Vessel (mount) & & Staffordshire & Fisherwick & $\mathrm{RU}$ & & PAS & & $\begin{array}{l}\text { Anthropomorphic male face circular mount, stylized } \\
\text { provincial features, high cheeks, almond eyes }\end{array}$ & & PAS WMII-26ACD7 \\
\hline STA0003 & $\begin{array}{l}\text { Handlded Pan } 2 \\
\text { (handle) }\end{array}$ & Eggers 139-144 & Staffordshire & Brewood & RS & & PAS & & & Concentric circle handle medallion loop & PAS WMID-6C2FE3 \\
\hline STA0004 & Handled Pan 2 & Rudge Cup Type & 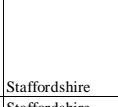 & Ilam & $\mathrm{RU}$ & Staffordshire Moorlands & PAS & & 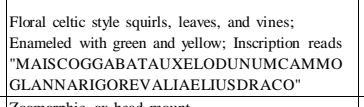 & & $\begin{array}{l}\text { PAS WMID-3FE965; } \\
\text { Jackson 2012, 41-60 }\end{array}$ \\
\hline STA0005 & \begin{tabular}{|l|} 
Vessel (mount) \\
$\begin{array}{l}\text { Handded Pan } 1 \\
\text { handde) }\end{array}$
\end{tabular} & Eggers $154-155$ & Staffordshire & Thorpe Constantine & $\mathrm{RU}$ & Otron's Farm ('shrine? & PAS & & Zoomorphic ox head mount & $\begin{array}{l}\text { Anthropomorphic 'Bacchic medusa' handle } \\
\text { terminal; zoomorphic lion bust undercarriage; } \\
\text { fluted handle }\end{array}$ & $\begin{array}{l}\text { Ferris et alia 2000, } 55 \\
\text { (10) }\end{array}$ \\
\hline TAA0007 & Vessel (mount) & & Staffordshire & Wall & RS & Castle Croft & $\mathrm{SF}$ & & Zoomorphic ox head mount & & Gould 1964, 43(8) \\
\hline STA0008 & Bowl & & Staffordshire & Wall & RS & & SD & $31 \mathrm{Cn}[\mathrm{s}]$ & Geometric Chi-rho design & & Mawer 1995, 19; RIB I \\
\hline SUF0001 & Handled Pan 2 & Eggers $137-138$ & Suffolk & Oulton & RU & Swamp & SD & SUF0002 & & \begin{tabular}{|l|}
$\begin{array}{l}\text { Geometric half sunburst on handle medallion, half } \\
\text { circle handle loop, caducaueus on handle }\end{array}$ \\
\end{tabular} & Eggers 1968, 106 (45a) \\
\hline SUF0002 & Handled Pan 2 & Eggers 144 & Suffolk & Oulton & RU & Swamp & SD & SUF0001 & 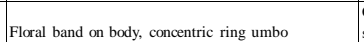 & $\begin{array}{l}\text { Concentric iricle handlde loop, goometric } \\
\text { salloping under handle menallilion }\end{array}$ & Eggers 1968, 106 (45b) \\
\hline SUF0003 & \begin{tabular}{|l|}
$\begin{array}{l}\text { Handled Pan } 1 \\
\text { (handle) }\end{array}$ \\
\end{tabular} & Eggers $154-155$ & Suffolk & Santon Downham & RS & & GD & $\begin{array}{l}\text { SUFF003-SUF0004 \& } \\
\text { SuFo051 }\end{array}$ & & Zoomorphic canine handle terminal, fluted handle & Eggers 1968, 106 (44a) \\
\hline SUF0004 & Jug & Eggers 125 & Suffolk & Santon Downham & RS & & GD & $\begin{array}{l}\text { SUFF003-SUF0004 \& } \\
\text { suF0051 }\end{array}$ & & \begin{tabular}{|l|} 
Zoomorphic canine bust thumb rest and dog foot \\
handle medallion
\end{tabular} & Eggers 1968, 106 (44b) \\
\hline SUF0005 & Handled Pan 2 & Eggers 139 & Suffolk & $\begin{array}{l}\text { Somberleyton Asshy and } \\
\text { Herringflet }\end{array}$ & $\mathrm{RU}$ & Swamp (Herringfleet) & SF & & Concentric circle foot ring & 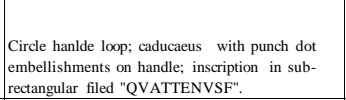 & $\begin{array}{l}\text { Eggers 1968, 106 (43); } \\
\text { Mcreake \& Moore } \\
\text { 1978, 334(32); RIB II } \\
22115.43\end{array}$ \\
\hline SUF0006 & Jug (handle) & & Suffolk & Pettistree & $\mathrm{RU}$ & & PAS & & & Geometric godronné thumb rest & PAS SF-8182F7 \\
\hline SUF0007 & Vessel (fragment) & & Suffolk & Kettlebaston & $\mathrm{RU}$ & & PAS & & $\begin{array}{l}\text { 隹ometricic pelta shaped vessel foot, "heart-shaped" } \\
\text { for in nentro of crescent }\end{array}$ & & PAS SF-7CE6CI \\
\hline SUF0008 & Vessel (mount) & & Suffolk & Hacheston & RS & & PAS & & $\begin{array}{l}\text { Floral leaf shaped hanging vessel mount } \\
\end{array}$ & & PAS SF-4E5A43 \\
\hline SUF0009 & $\begin{array}{l}\text { Handled Pan } 2 \\
\text { (handle) }\end{array}$ & Rudge Cup Type & Suffolk & Combs & $\mathrm{RU}$ & & PAS & & & $\begin{array}{l}\begin{array}{l}\text { Floral stylized leaves and spirals, enameled in } \\
\text { blue }\end{array} \\
\end{array}$ & PAS SF-0349E2 \\
\hline SUF0010 & $\begin{array}{l}\text { Handled Pan } 2 \\
\text { (handle) }\end{array}$ & Eggers 139-144 & Suffolk & Hoxne & $\mathrm{RU}$ & & PAS & & & \begin{tabular}{|} 
Concentric circle handle loop; Geometric punch \\
dots arranged in triangle
\end{tabular} & PAS SF-3C6B04 \\
\hline SUF0011 & Vessel (mount) & & Suffolk & West Stow & $\mathrm{RU}$ & & PAS & & Anthropomorphic helmeted bust, possibly Minerva & & PAS SF-040874 \\
\hline SUF0012 & Vessel (fragment) & & Suffolk & Barking & RS & & PAS & & & & PAS SF-A72D31 \\
\hline SUF0013 & \begin{tabular}{|l|}
$\begin{array}{l}\text { Handled Pan } 22 \\
\text { (handle) }\end{array}$ \\
\end{tabular} & Eggers 139-144 & Suffolk & Combs & $\mathrm{RU}$ & & PAS & & & Concentric circle handle loop & PAS SF-3DCCA7 \\
\hline SUF0014 & Vessel (mount) & & Suffolk & Arwarton & $\mathrm{RU}$ & & PAS & & Geometric sub-triangular hanging vessel mount & & PAS SF-2BC393 \\
\hline suf0015 & $\begin{array}{l}\begin{array}{l}\text { Handled Pan } 2 \\
\text { (handle) }\end{array} \\
\end{array}$ & Eggers 140-142 & Suffolk & Chediston & $\mathrm{RU}$ & & PAS & & & \begin{tabular}{|l|}
$\begin{array}{l}\text { Concentric circle handle loop; Stamped } \\
\text { inscription reads "CIPIPOL[..." }\end{array}$ \\
\end{tabular} & PAS NMS-7F1BE6 \\
\hline SUF0016 & Jug (handle) & & Suffolk & Lowestoft & RS & & PAS & & & Floral leaf handle medallion & PAS NMS-2E4838 \\
\hline SUF0017 & Vessel (mount) & & Suffolk & Mendham & $\mathrm{RU}$ & & PAS & & Zoomorphic dolphin hanging vessel mount & & PAS NMS-C40776 \\
\hline SUF0018 & Jug (lid) & Eggers 123-126 & Suffolk & Freckenham & RU & & PAS & & $\begin{array}{l}\text { Zoomorphic bird thumb rest; Floral (possible) leaf } \\
\text { shaned jug lid }\end{array}$ & & PAS SF-210633 \\
\hline SUF0019 & Vessel (mount) & & Suffolk & Mildenhall & RU & & PAS & & \begin{tabular}{|l|l|} 
Zoomorphic duck vessel lid mount \\
\end{tabular} & & PAS SF-DF4933 \\
\hline
\end{tabular}




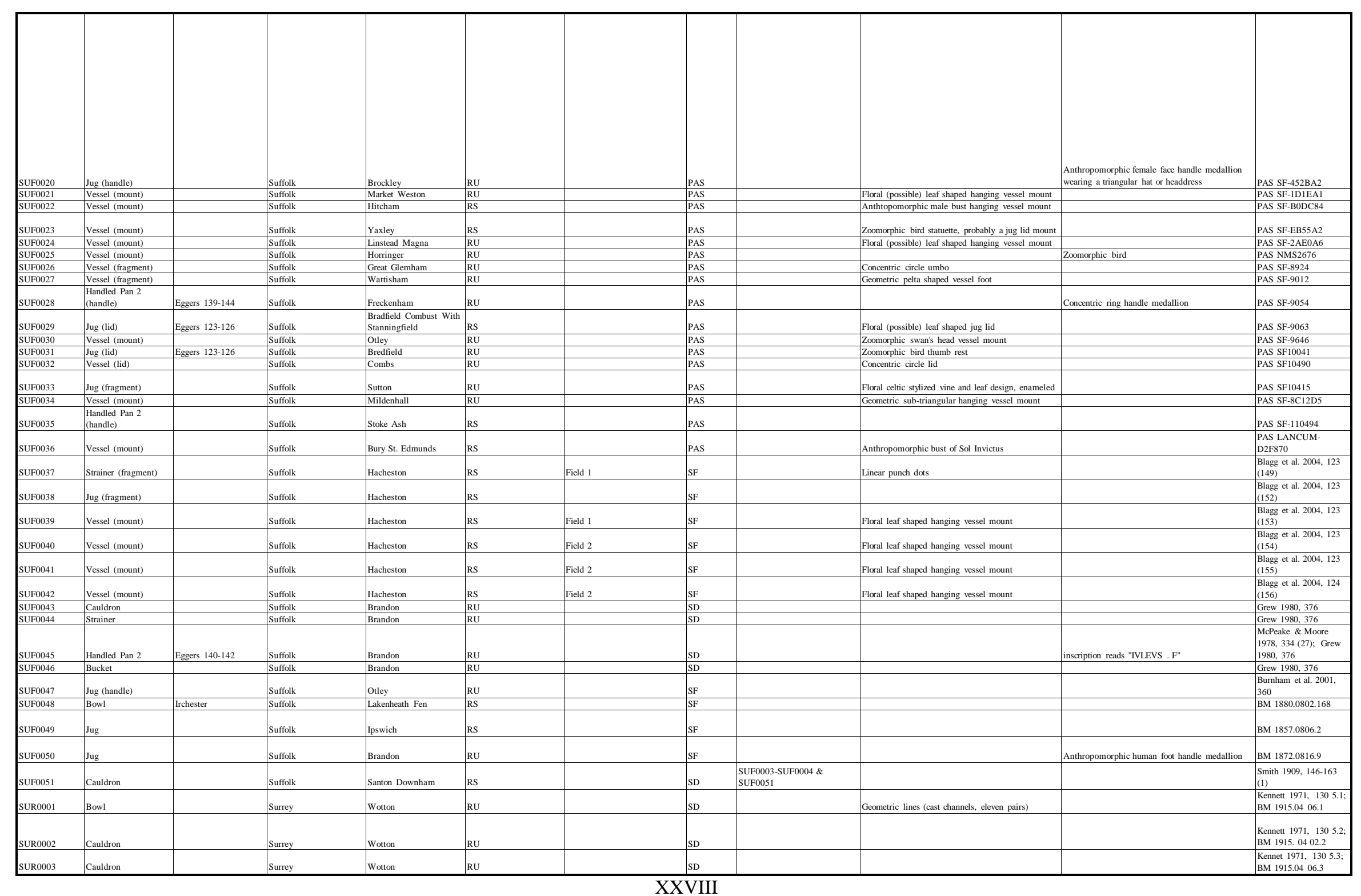




\begin{tabular}{|c|c|c|c|c|c|c|c|c|c|c|c|}
\hline SUR0004 & Bucket & & Surrey & Wotton & RU & & SD & & & & $\begin{array}{l}\text { Kennett 1971, } 130.54 \text {.4; } \\
\text { BM 1915.04 06.4 }\end{array}$ \\
\hline SURo005 & Cauldron & & Surrey & Wotton & $\mathrm{RU}$ & & SD & & & & $\begin{array}{l}\text { Kennett 1971, 130 5.5; } \\
\text { BM 1915.04 06.5 }\end{array}$ \\
\hline SURo006 & Bowl & Irchester & Surrey & Wotton & $\mathrm{RU}$ & & SD & & & & $\begin{array}{l}\text { Kennett 1971, 130 5.6; } \\
\text { BM 1915.04 06.6 }\end{array}$ \\
\hline SUR0007 & Bowl & Irchester & Surrey & Wotton & $\mathrm{RU}$ & & SD & & & & $\begin{array}{l}\text { Kennett 1971, 130.77; } \\
\text { BM 1915.04 06.7 }\end{array}$ \\
\hline SURo008 & Bowl & & Surrey & Wotton & $\mathrm{RU}$ & & SD & & \begin{tabular}{|l|}
$\begin{array}{l}\text { Geometric herringbone and square stamps on } \\
\text { interior basin }\end{array}$ \\
\end{tabular} & & $\begin{array}{l}\text { Kennett 1971, } 1325.8 ; \\
\text { BM 1915.04 } 06.8\end{array}$ \\
\hline SURo0o9 & Bowl & & Surrey & Wotton & $\mathrm{RU}$ & & SD & & & & $\begin{array}{l}\text { Kennett 1971, 132 5.9; } \\
\text { BM 1915.04 06.9 }\end{array}$ \\
\hline SURo010 & Handled Pan 4 & Coptic & Surrey & Wotton & $\mathrm{RU}$ & & SD & & & & $\begin{array}{l}\text { Kennett 1971, } 132 \\
\text { 5.10; BM 1915.04 } \\
06.10\end{array}$ \\
\hline SUR0011 & Vessel (fragment) & & Surrey & Charlwood & $\mathrm{RU}$ & & PAS & & Zoomorphic canine vessel foot & & PAS SUR-17AA03 \\
\hline SUR0012 & Bowl & & Surrey & Walton on Thames & $\mathrm{RU}$ & River Thames & $\mathrm{SF}$ & & & & BM 1909.0320.1 \\
\hline SWA0001 & Vessel (fragment) & & Swansea & Loughor & M & Leucarum Fort & SF & & & & $\begin{array}{l}\text { Marvell \& Owen-John } \\
1997,247(55)\end{array}$ \\
\hline SWA0002 & Handled Pan 2 & Eggers $140-142$ & Swansea & Loughor & M & Leucarum Fort & $\mathrm{SF}$ & & $\begin{array}{l}\text { Concentric circle umbo and omphalos dot; } \\
\text { Geometric linear etched body band; Concentric } \\
\text { circle foot ring }\end{array}$ & $\begin{array}{l}\begin{array}{l}\text { Concentric circle handle medallion and } \\
\text { suuspension loop }\end{array} \\
\text { lon }\end{array}$ & $\begin{array}{l}\text { Marvell \& Owen-John } \\
1997,247(56) \text { - }\end{array}$ \\
\hline VGL0001 & Jug (handle) & & Vale of Glamorgan & Wenvoe & RU & Whitton Cross Roads & $\mathrm{SF}$ & & & & \begin{tabular}{|l|l|l|l|l} 
Jarret \& Wrathmell \\
$1981,182(50)$
\end{tabular} \\
\hline WAR0001 & Vessel (fragment) & & \begin{tabular}{|l|} 
Warwickshire \\
\end{tabular} & Tanworth In Arden & $\mathrm{RU}$ & & PAS & & Geometric linear bands on vessel foot & & PAS WAW-FFE863 \\
\hline WARO002 & Vessel (mount) & & Warvickshire & Alcester & $\mathrm{M}$ & & PAS & & Floral (possible) leaf shaped hanging vessel mount & & PAS WAW-5036D6 \\
\hline WAR0003 & Bowl & Basin Uní & Warwickshire & Alcester & M & Southern Extramural Area & SF & & & & $\begin{array}{l}\text { Cracknell \& Mahany } \\
\text { 1994. 181. 120 }\end{array}$ \\
\hline WAR0004 & Vessel (fragment) & & Warwickshire & Alcester & M & Southern Extramural Area & SF & & & & $\begin{array}{l}\text { Cracknell \& Mahany } \\
1994,181,121\end{array}$ \\
\hline WAR0005 & Vessel (mount) & & Warwickshire & Alcester & M & Southern Extramural Area & $\mathrm{SF}$ & & Floral leaf shaped hanging vessel mount & & $\begin{array}{l}\text { Cracknell \& Mahany } \\
1994,181,122\end{array}$ \\
\hline WAR0006 & Vessel (mount) & & Warwickshire & Alcester & M & Southern Extramural Area & $\mathrm{sF}$ & & Floral (possible) leaf shaped vessel mount & & $\begin{array}{l}\text { Cracknell \& Mahany } \\
\text { 1994, 181, 123 }\end{array}$ \\
\hline WAR0007 & $\begin{array}{l}\begin{array}{l}\text { Handled Pan } 2 \\
\text { (handle) }\end{array} \\
\end{array}$ & Eggers $139-144$ & Warwickshire & Churchover & $\mathrm{RU}$ & Cave's Inn & $\mathrm{SF}$ & & & $\begin{array}{l}\text { Concentric circle handle loop; inscription } \\
\text { "MATVRVS F" }\end{array}$ & $\begin{array}{l}\text { Wright R. 1961, } 195 \\
\text { (17); Mpceake \& Moore } \\
\text { 1978, 334 (29); RIB II } \\
2415.38\end{array}$ \\
\hline wsuo001 & Vessel (mount) & & West Sussex & Eartham & $\mathrm{RU}$ & & PAS & & $\begin{array}{l}\text { Zoomorphic duck or swan's head hanging vessel } \\
\text { mount }\end{array}$ & & PAS SUSS-37ADE6 6 \\
\hline wsuo002 & Jug (fragment) & & West Sussex & Chichester & $\mathrm{u}$ & $\begin{array}{l}\text { North West Quadrent, Area } 7 . \\
\text { D 36 (685) }\end{array}$ & $\mathrm{sF}$ & & & Floral (possible) leaf handle medallion & Down 1978, 307 (153) \\
\hline WSU0003 & Vessel (fragment) & & West Sussex & Chichester & u & \begin{tabular}{|l|l|} 
Cattlemarket \\
\end{tabular} & $\mathrm{SF}$ & & Geometric linear rim bands & 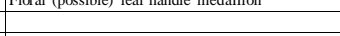 & Down 1989, $196(62)$ \\
\hline WSU0004 & Bowl (fragment) & & West Sussex & Chichester & u & County Hall & $\mathrm{SF}$ & & . & & Down 1989, $218(7)$ \\
\hline wsuooos & Bowl (fragment) & & West Sussex & Chichester & u & Greyfriars & SF & & & & $\begin{array}{l}\text { Down \& Magilton } \\
\text { 1993, } 190(23)\end{array}$ \\
\hline wsuo0o6 & Bowl (fragment) & & West Sussex & Fishbourne & RS & A27 & $\mathrm{sF}$ & & & & $\begin{array}{l}\text { Cunliffe et al. 1996, } \\
200(34)\end{array}$ \\
\hline wsu0007 & Jug (handle) & & West Sussex & Fishbourne & RS & A27 & $\mathrm{SF}$ & & & $\begin{array}{l}\text { Anthropomorophic cherub face handle medallion; } \\
\text { Floral vine and leaf, Zoomorphic cat's paw near } \\
\text { handle terminal; Floral leaf thumb rest }\end{array}$ & $\begin{array}{l}\text { Cunliffe et al. 1996, } \\
\text { 200 (45); Down \& } \\
\text { Henig 1988, 308-309 }\end{array}$ \\
\hline wsuo008 & Vessel (fragment) & & West Sussex & Fishbourne & RS & Fishbourne Roman Villa & SF & & Concentric circle rim & & $\begin{array}{l}\text { Cunlifie } 1971,120 \\
\text { (152) }\end{array}$ \\
\hline wsuo009 & Vessel (fragment) & & West Sussex & Fishbourne & RS & Fishbourne Roman Villa & $\mathrm{SF}$ & & Concentric circle rim & & $\begin{array}{l}\text { Cunliffe } 1971,120 \\
(153)\end{array}$ \\
\hline WSU0010 & Jug & & West Sussex & Chichester & u & Westergate & GD & $2 \mathrm{Po}[\mathrm{ca}],+\mathrm{V}[\mathrm{c}]$ & & & Smith, 192298 \\
\hline WYR0001 & Cauldron & & West Yorkshire & Castleford & M & Fort (Lagentium) & SF & & & & \begin{tabular}{|l} 
Cool \& Philo 1998, 81 \\
$(333)$
\end{tabular} \\
\hline
\end{tabular}




\begin{tabular}{|c|c|c|c|c|c|c|c|c|c|c|c|}
\hline WYR0002 & Vessel (fragment) & & West Yorkshire & Dalton Parlours & RS & Villa & $\mathrm{SF}$ & & Out turned rim & & $\begin{array}{l}\text { Wrathmell \& Nicholson } \\
\text { 1990, 84 (22) }\end{array}$ \\
\hline WYR0003 & Vessel (fragment) & & West Yorkshire & Dalton Parlours & RS & Villa & $\mathrm{SF}$ & & Out turned rim & & $\begin{array}{l}\text { Wrathmell \& Nicholson } \\
1990,84(23)\end{array}$ \\
\hline WYR0004 & Vessel (fragment) & & West Yorkshire & Inkley & M & Foundations of wall F, Site IV & $\mathrm{SF}$ & & \begin{tabular}{|} 
Anthropomorphic horned and bearded male bust \\
vessel mount, probably Silenus or a satyr
\end{tabular} & & \begin{tabular}{|l} 
Woodward 1925,280 \\
(I); JRS 10,185
\end{tabular} \\
\hline WYRo005 & Handled Pan 2 & & West Yorkshire & Inkley & M & Site III (lowest level) & $\mathrm{SF}$ & & & & $\begin{array}{l}\text { Woodward 1925, } 285 \\
\text { (22) }\end{array}$ \\
\hline WYR0006 & $\begin{array}{l}\text { Handled Pan } 1 \\
\text { (handle) }\end{array}$ & Eggers $154-155$ & West Yorkshire & Castleford & $\mathrm{M}$ & Fort (Lagentium) & SF & & & $\begin{array}{l}\begin{array}{l}\text { Zoomorphic ram's head handle terminal, fluted } \\
\text { handle }\end{array} \\
\end{array}$ & \begin{tabular}{|l|} 
Cool \& Philo 1998, 98 \\
$(476)$
\end{tabular} \\
\hline WYR0007 & \begin{tabular}{|l}
$\begin{array}{l}\text { Handled Pan } 2 \\
\text { (handle) }\end{array}$ \\
\end{tabular} & Eggers $140-142$ & West Yorkshire & Hebden Royd & $\mathrm{RU}$ & Slack & SF & & & $\begin{array}{l}\begin{array}{l}\text { Concentric circle handle medallion and } \\
\text { suspension loop }\end{array} \\
\end{array}$ & \begin{tabular}{|l|}
$\begin{array}{l}\text { Dodd \& Woodward } \\
\text { n.d., } 77(13)\end{array}$ \\
\end{tabular} \\
\hline WLLo001 & Vessel (mount) & & Wiltshire & Avebury & RS & & PAS & & $\begin{array}{l}\text { Zoomorphic bird hanging vessel mount, probably a } \\
\text { duck or a swan }\end{array}$ & & PAS WLLT-DSEBBS \\
\hline WLO0002 & Vessel (fragment) & & Wiltshire & Leigh & $\mathrm{RU}$ & & PAS & & & Zoomorphic depictions of a horse and a bird & PAS NMGW-C46CB4 \\
\hline WIL0003 & Jug (handle) & & Wiltshire & Codford & $\mathrm{RU}$ & & PAS & & $\begin{array}{l}\text { Anthropomorophic female face handle medallion, } \\
\text { stylized curred back blocked hair }\end{array}$ & & PAS WILT-7E0308 \\
\hline WL0004 & Handled Pan 2 & Eggers $140-142$ & Wiltshire & Kingston Deverill & RS & & SD & WILT0004-0008 & Concentric circle umbo & Concentric circle handle medallion & PAS WLLT-92B052 \\
\hline WIL0005 & Handled Pan 2 & Eggers 140-142 & Wiltshire & Kingston Deverill & RS & & SD & WILT0004-0008 & Concentric circle umbo & Concentric circle handle medallion & PAS WILT-92B052 \\
\hline WIL0006 & Handled Pan 2 & Eggers $140-142$ & Wiltshire & Kingston Deverill & RS & & SD & WILT0004-0008 & Concentric circle umbo & $\begin{array}{l}\text { Concentric cirle handle medallion: Inscription } \\
\text { "CIPIPIPOLIBI" in rectangullar field }\end{array}$ & PAS WILT-92B052 \\
\hline WL0007 & Strainer & & Wiltshire & Kingston Deverill & RS & & SD & WILT0004-0008 & Zoomorphic vessel spout & & PAS WLTT-92B052 \\
\hline WIL0008 & Strainer & & Wiltshire & Kingston Deverill & RS & & SD & WILT0004-0008 & Zoomorphic vessel spout & & PAS WLLT-92B052 \\
\hline WIL0009 & $\begin{array}{l}\text { Handled Pan } 2 \\
\text { (handle) }\end{array}$ & & Wiltshire & Wanborough & RS & & SF & & & Zoomorphic water bird's head rim attachment & \begin{tabular}{|l} 
Anderson et alia 2001, \\
$92(106)$
\end{tabular} \\
\hline WIL0010 & $\begin{array}{l}\begin{array}{l}\text { Handled Pan } 2 \\
\text { (handle) }\end{array} \\
\end{array}$ & & Wiltshire & Wanborough & RS & & $\mathrm{SF}$ & & & $\begin{array}{l}\text { Floral (possibly) leaflbeaded vine decoration near } \\
\text { rim }\end{array}$ & $\begin{array}{l}\text { Anderson et alia } 2001 \text {, } \\
93 \text { (107) }\end{array}$ \\
\hline WIL0011 & Jug (lid) & Eggers $123-126$ & Wiltshire & Wanborough & RS & & SF & & Floral (possibly) leat shaped lid & & $\begin{array}{l}\text { Anderson et alia 2001, } \\
113 \text { (242) }\end{array}$ \\
\hline WIL0012 & Vessel (fragment) & & Wiltshire & Wanborough & RS & & SF & & & & \begin{tabular}{|l|} 
Anderson et alia 2001, \\
$113(243)$
\end{tabular} \\
\hline WIL0013 & Bowl & Irchester & Wiltshire & Bishop's Cannings & $\mathrm{RU}$ & Blagan Hill & SD & $\begin{array}{l}1 \mathrm{Cn}[\mathrm{gg}], 1,646 \mathrm{Cn}[\mathrm{s]}], 5,535 \\
\text { Cn [ca], 4 Po [s], } 1 \text { Po [ca], } \\
\text { wLL013-WLL017 }\end{array}$ & & & $\begin{array}{l}\text { Burnham et al. 1994, } \\
\text { 286; Guest et al. 1997, } \\
427\end{array}$ \\
\hline WIL0014 & Bowl & Irchester & Wiltshire & Bishop's Cannings & $\mathrm{RU}$ & Blagan Hill & SD & 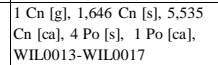 & & & $\begin{array}{l}\text { Burnham et al. 1994, } \\
\text { 288; Guest tet al. 1997, } \\
427\end{array}$ \\
\hline WIL0015 & Bow1 & Irchester & Wiltshire & Bishop's Cannings & $\mathrm{RU}$ & Blagan Hill & SD & 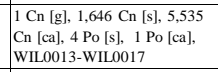 & & & $\begin{array}{l}\text { Burnham et al. 1994, } \\
\text { 286; Guest tet al. 1997, } \\
427\end{array}$ \\
\hline WIL0016 & Bow1 & Irchester & Wiltshire & Bishop's Cannings & $\mathrm{RU}$ & Blagan Hill & SD & 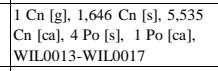 & & & $\begin{array}{l}\begin{array}{l}\text { Burnham et al. 1994, } \\
\text { 286; Guest et al. 1997, } \\
427\end{array} \\
427\end{array}$ \\
\hline WIL0017 & Bowl & Irchester & Wiltshire & Bishop's Cannings & $\mathrm{RU}$ & Blagan Hill & SD & 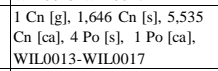 & & & \begin{tabular}{|l} 
Burnham et al. 1994, \\
286; Guest et al. 1997, \\
427
\end{tabular} \\
\hline WIL0018 & Bowl & Irchester & Wilthsire & Bishop's Cannings & $\mathrm{RU}$ & Blagan Hill & SD & 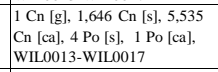 & & & Guest et al. 1997, 427 \\
\hline WLL0019 & Bowl & Irchester & Wiltshire & Bishop's Cannings & $\mathrm{RU}$ & Blagan Hill & SD & 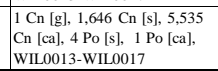 & & & Guest et al. 1997, 427 \\
\hline
\end{tabular}




\begin{tabular}{|c|c|c|c|c|c|c|c|c|c|c|}
\hline WL0020 & Jug & & Wiltshire & Bishop's Cannings & $\mathrm{RU}$ & Blagan Hill & SD & 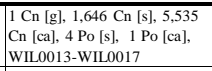 & & Guest et al. 1997, 427 \\
\hline WIL0021 & Bowl & Bassin festonné & Wiltshire & Bishop's Cannings & $\mathrm{RU}$ & Blagan Hill & SD & 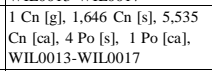 & & Guest et al. 1997, 427 \\
\hline WIL0022 & Bowl & Bassin festonné & Wiltshire & Bishop's Cannings & RU & Blagan Hill & SD & 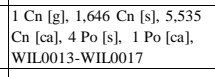 & & Guest et al. 1997, 427 \\
\hline WL0023 & Jug & Eggers 125 & Wiltshire & Roundway Hill & $\mathrm{RU}$ & & SD & & inscripition "ASPAE[...]" on inside of rim & $\begin{array}{l}\text { Taylor \& Collingwood } \\
1923,283(12) ; \text { RIB II } \\
2415.8\end{array}$ \\
\hline WL0024 & Handled Pan 2 & Rudge Cup type & Wiltshire & Rudge & $\mathrm{RU}$ & & $\mathrm{SF}$ & & 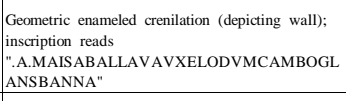 & $\begin{array}{l}\text { Allason-Jones 2012, 23- } \\
\text { 36; RIB II 2415.53 }\end{array}$ \\
\hline WOR0001 & Vessel (fragment) & & Worcestershire & Inkberrow & $\mathrm{RU}$ & & PAS & & $\begin{array}{l}\text { Geometric pelta shaped vessel foot, "heart-shaped" } \\
\text { leg in centre of crescent }\end{array}$ & PAS WAW-18C577 \\
\hline WOR0002 & Vessel (fragment) & & Worcestershire & Leigh & $\mathrm{RU}$ & & PAS & & Concentric circle rings on interior of basin & PAS WAW-07FEC3 \\
\hline WOR0003 & Bowl & & Worcestershire & Droitwich & RS & Hanbury Street & $\mathrm{SF}$ & & Flanged rim & Hurst et al. 2006, $67(9)$ \\
\hline WOR0004 & Vessel (fragment) & & Worcestershire & Worcester & RS & Deansway & SF & & & $\begin{array}{l}\text { Dalwood \& Edwards } \\
2004,410(15)\end{array}$ \\
\hline
\end{tabular}


Appendix II: Structured Deposits of Copper Alloy Vessels in Roman Britain

\begin{tabular}{|c|c|c|c|c|c|c|c|c|c|c|}
\hline Number & Form & Type & County & Site & Location & Context & Associated Finds & Decoration (body) & Decoration (handle) & Reference \\
\hline Av0002 & Handled Pan 2 & Rudge Cup Type & Avon & Bath & $\begin{array}{l}\text { Reservoir of the } \\
\text { Roman Baths, (CS } \\
\text { layer } 3 \text { sf 20,008) }\end{array}$ & RS & & $\begin{array}{l}\text { Geometric squares and triangles; } \\
\text { Geometric depiction of wall crenalations; } \\
\text { Concentric circle foot ring }\end{array}$ & $\begin{array}{l}\text { Floral (possible) leaf and/or vine motif; Geometric wave } \\
\text { shapes; punch dot inscription } \\
\text { "DIISVM[...]/CODON[...]" }\end{array}$ & $\begin{array}{l}\text { Cunliffe 1988, } 14-16(23) ; \\
\text { Hassall \& Tomlin 1981, } \\
381 \text { (20); RIB II2415.60 }\end{array}$ \\
\hline BE0001 & Bowl & Bassin festonné & Bedfordshire & Sandy & & RS & BE0001-BE0003 & Geometric scalloped edge (festonné) & & Kennet $1971 \quad 124,1.1$ \\
\hline BE0002 & Bowl & $\begin{array}{l}\begin{array}{l}\text { Bassin á bord } \\
\text { godronné }\end{array} \\
\end{array}$ & Bedfordshire & Sandy & & RS & BE0001-BE0003 & $\begin{array}{l}\text { Geometric repoussé bosses (godrons) } 59 \\
\text { in number }\end{array}$ & & Kennet $1971 \quad 124,1.2$ \\
\hline BE0003 & Bowl & Bassin uni & Bedfordshire & Sandy & & RS & BE0001-BE0003 & Geometric rim bosses & & Kennet $1971 \quad 124,1.3$ \\
\hline BUC0006 & Bowl & Irchester & Buckinghamshire & Amersham & Misbourne Valley & RS & BUC0006-BUC0011 & $\begin{array}{l}\text { Concentric circle body bands; Inscription } \\
\text { "X" and sideways "A" on exterior body of } \\
\text { vessel; Coins found in area date between } \\
\text { Trajan and Constantine I with two } \\
\text { "unidentifiable 4th century" coins }\end{array}$ & & $\begin{array}{l}\text { Farley et alia } 1988,359- \\
366 \text { (1) }\end{array}$ \\
\hline BUC0007 & Bowl & Irchester & Buckinghamshire & Amersham & Misbourne Valley & RS & BUC0006-BUC0011 & $\begin{array}{l}\text { Concentric circle body bands; omphalos } \\
\text { dot; Coins found in area date between } \\
\text { Trajan and Constantine I with two } \\
\text { "unidentifiable 4th century" coins } \\
\end{array}$ & & $\begin{array}{l}\text { Farley et alia 1988, 359- } \\
366 \text { (2) }\end{array}$ \\
\hline BUC0008 & Bowl & Irchester & Buckinghamshire & Amersham & Misbourne Valley & RS & BUC0006-BUC0011 & $\begin{array}{l}\text { Raised umbo and omphalos dot; Coins } \\
\text { found in area date between Trajan and } \\
\text { Constantine I with two "unidentifiable 4th } \\
\text { century" coins }\end{array}$ & & $\begin{array}{l}\text { Farley et alia 1988, 359- } \\
366 \text { (3) }\end{array}$ \\
\hline BUC0009 & Bowl & Irchester & Buckinghamshire & Amersham & Misbourne Valley & RS & BUC0006-BUC0011 & $\begin{array}{l}\text { Raised umbo and omphalos dot; Coins } \\
\text { found in area date between Trajan and } \\
\text { Constantine I with two "unidentifiable 4th } \\
\text { century" coins }\end{array}$ & & $\begin{array}{l}\text { Farley et alia } 1988,359- \\
366 \text { (4) }\end{array}$ \\
\hline BUC0010 & Bowl & & Buckinghamshire & Amersham & Misbourne Valley & RS & BUC0006-BUC0011 & $\begin{array}{l}\text { Coins found in area date between Trajan } \\
\text { and Constantine I with two } \\
\text { "unidentifiable 4th century" coins }\end{array}$ & & $\begin{array}{l}\text { Farley et alia 1988, 359- } \\
366 \text { (5) }\end{array}$ \\
\hline BUC0011 & Bowl & & Buckinghamshire & Amersham & Misbourne Valley & RS & BUC0006-BUC0011 & $\begin{array}{l}\text { Coins found in area date between Trajan } \\
\text { and Constantine I with two } \\
\text { "unidentifiable 4th century" coins }\end{array}$ & & $\begin{array}{l}\text { Farley et alia } 1988,359- \\
366(6)\end{array}$ \\
\hline CAR0001 & Handled Pan 2 & Eggers 139-144 & Carmarthenshire & Kyngadl & Coygan Cave & RS & CAEO002 & Geometric triskele umbo & Concentric circle handle medallion & $\begin{array}{l}\text { Eggers 1968, 103 (12a); } \\
\text { Wainwright 1967, 85-88 }\end{array}$ \\
\hline CAR0002 & Strainer & & Carmarthenshire & Kyngadl & Coygan Cave & RS & CAE0001 & Geometric circle/wave strainer dot pattern & & $\begin{array}{l}\text { Eggers 1968, } 103(12 \mathrm{~b}) ; \\
\text { Wainwright 1967, 85-88 }\end{array}$ \\
\hline CAM0001 & Handled Pan 2 & Eggers 140 & Cambridgeshire & Elm & Coldham & RS & CAM0002 & Concentric circle umbo & Circle handle loop & Eggers 1968, $106(40 \mathrm{a})$ \\
\hline CAM0002 & Handled Pan 2 & Eggers 144 & Cambridgeshire & Elm & Coldham & RS & CAM0001 & Concentric circle umbo & Circle handle loop & Eggers 1968, $106(40 \mathrm{~b})$ \\
\hline САM0003 & Jug & Eggers 125 & Cambridgeshire & Hauxton & & RS & CAM0003-СAM0005 & Trefoil mouth & $\begin{array}{l}\begin{array}{l}\text { Zoomorphic human face, perhaps cherub, on handle } \\
\text { medallion }\end{array} \\
\end{array}$ & 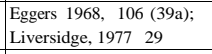 \\
\hline CAM0004 & Jug & & Cambridgeshire & Hauxton & & RS & CAM0003-САM0005 & & $\begin{array}{l}\text { Zoomorphic human face with wings above head, perhaps } \\
\text { some form of Mercury, on handle medallion; high handle } \\
\text { with floral thumb rest }\end{array}$ & $\begin{array}{l}\text { Eggers 1968, } 106(39 \mathrm{~b}) \\
\text { Liversidge 1977, 29 }\end{array}$ \\
\hline CAM0005 & Jug & & Cambridgeshire & Hauxton & & RS & CAM0003-СAM0005 & & $\begin{array}{l}\text { Zoomorphic human feet handle medallion, flat thumb } \\
\text { rest, semi-fluted handle }\end{array}$ & $\begin{array}{l}\text { Eggers } 1968,106(39 \mathrm{c}) \\
\text { Liversidge } 1977,29\end{array}$ \\
\hline CAM0010 & Cauldron & & Cambridgeshire & Burwell & natural chalk knoll & RU & CAM0011-CAM0024 & & & $\begin{array}{l}\text { Cra'ster 1970, 344; Wilson } \\
\text { 1971, 270; MAA 1969.44; } \\
\text { MAA 1994-11-11 }\end{array}$ \\
\hline CAM0011 & Bowl & $\begin{array}{l}\text { Basin á bord } \\
\text { godronne }\end{array}$ & Cambridgeshire & Burwell & natural chalk knoll & RU & CAM0011-САM0024 & Geometric repoussé bosses (godrons) & & $\begin{array}{l}\text { Cra'ster 1970, 344; Wilson } \\
\text { 1971, 270; MAA 1969.44; } \\
\text { MAA 1994-11-11 }\end{array}$ \\
\hline CAM0012 & Bowl & Irchester & Cambridgeshire & Burwell & natural chalk knoll & RU & CAM0011-CAM0024 & & & $\begin{array}{l}\text { Cra'ster 1970, 344; Wilson } \\
\text { 1971, 270; MAA 1969.44; } \\
\text { MAA 1994-11-11 }\end{array}$ \\
\hline CAM0013 & Bowl & Irchester & Cambridgeshire & Burwell & natural chalk knoll & RU & CAM0011-СAM0024 & & & $\begin{array}{l}\text { Cra'ster 1970, 344; Wilson } \\
\text { 1971, 270; MAA 1969.44; } \\
\text { MAA 1994-11-11 }\end{array}$ \\
\hline CAM0014 & Bowl & Irchester & Cambridgeshire & Burwell & natural chalk knoll & RU & CAM0011-CAM0024 & & & $\begin{array}{l}\text { Cra'ster 1970, 344; Wilson } \\
\text { 1971, 270; MAA; 1969.44; } \\
\text { MAA 1994-11-11 }\end{array}$ \\
\hline
\end{tabular}




\begin{tabular}{|c|c|c|c|c|c|c|c|c|c|c|}
\hline CAM0015 & Bowl & Irchester & Cambridgeshire & Burwell & natural chalk knoll & $\mathrm{RU}$ & CAM0011-CAM0024 & & & $\begin{array}{l}\text { Cra'ster 1970, 344; Wilson } \\
\text { 1971, 270; MAA 1969.44; } \\
\text { MAA 1994-11-11 }\end{array}$ \\
\hline САM0016 & Bowl & Irchester & Cambridgeshire & Burwell & natural chalk knoll & $\mathrm{RU}$ & CAM0011-CAM0024 & & & $\begin{array}{l}\text { Cra'ster 1970, 344; Wilson } \\
\text { 1971, 270; MAA 1969.44; } \\
\text { MAA 1994-11-11 }\end{array}$ \\
\hline CAM0017 & Bowl & Irchester & Cambridgeshire & Burwell & natural chalk knoll & $\mathrm{RU}$ & CAM0011-CAM0024 & & & $\begin{array}{l}\text { Cra'ster 1970, 344; Wilson } \\
\text { 1971, 270; MAA 1969.44; } \\
\text { MAA 1994-11-11 }\end{array}$ \\
\hline САМ0018 & Bowl & Irchester & Cambridgeshire & Burwell & natural chalk knoll & $\mathrm{RU}$ & САM0011-САM0024 & & & $\begin{array}{l}\text { Cra'ster 1970, 344; Wilson } \\
\text { 1971, 270; MAA 1969.44; } \\
\text { MAA 1994-11-11 }\end{array}$ \\
\hline CAM0019 & Bowl & & Cambridgeshire & Burwell & natural chalk knoll & $\mathrm{RU}$ & CAM0011-CAM0024 & & & $\begin{array}{l}\text { Cra'ster 1970, 344; Wilson } \\
\text { 1971, 270; MAA 1969.44; } \\
\text { MAA 1994-11-11 }\end{array}$ \\
\hline CAM0020 & Bowl & & Cambridgeshire & Burwell & natural chalk knoll & $\mathrm{RU}$ & CAM0011-CAM0024 & Floral vine circling umbo & & $\begin{array}{l}\text { Cra'ster 1970, 344; Wilson } \\
\text { 1971, 270; MAA 1969.44; } \\
\text { MAA 1994-11-11 }\end{array}$ \\
\hline САM0021 & Bowl & & Cambridgeshire & Burwell & natural chalk knoll & $\mathrm{RU}$ & САM0011-CAM0024 & Floral vine circling umbo & & $\begin{array}{l}\text { Cra'ster 1970, 344; Wilson } \\
\text { 1971, 270; MAA 1969.44; } \\
\text { MAA 1994-11-11 }\end{array}$ \\
\hline CAM0022 & Bowl & & Cambridgeshire & Burwell & natural chalk knoll & $\mathrm{RU}$ & CAM0011-CAM0024 & & & $\begin{array}{l}\text { Cra'ster 1970, 344; Wilson } \\
\text { 1971, 270; MAA 1969.44; } \\
\text { MAA 1994-11-11 }\end{array}$ \\
\hline САM0023 & Handled Pan 2 & & Cambridgeshire & Burwell & natural chalk knoll & $\mathrm{RU}$ & CAM0011-CAM0024 & & & $\begin{array}{l}\text { Cra'ster 1970, 344; Wilson } \\
\text { 1971, 270; MAA 1969.44; } \\
\text { MAA 1994-11-11 } \\
\end{array}$ \\
\hline САM0024 & Bowl & & Cambridgeshire & Burwell & natural chalk knoll & $\mathrm{RU}$ & CAM0011-CAM0024 & & & $\begin{array}{l}\text { Cra'ster 1970, 344; Wilson } \\
\text { 1971, 270; MAA 1969.44; } \\
\text { MAA 1944-11-11 }\end{array}$ \\
\hline $\mathrm{C} 0029$ & Handled Pan 2 & Eggers 140 & Cumbria & Clifton & & RS & & & inscription "TALIO F" & RIB II2415.47 \\
\hline $\mathrm{C} 0030$ & Strainer & & Cumbria & Clifton & & RS & & & & RIB II2415.47 \\
\hline DEN0001 & Handled Pan 2 & Eggers 131 & Denbighshire & Glyn Dyfrdwy & & $\mathrm{RU}$ & DEN0001-0004 & Concentric circle umbo & $\begin{array}{l}\text { Zoomorphic dual swan head handle loop, geometric } \\
\text { scalloping boarder, inscription "S MERCV" in } \\
\text { horizontal rectangualr field, inscription "CIPINICOMA" } \\
\text { in veritcal rectangular field }\end{array}$ & $\begin{array}{l}\text { Gardner 1927, 129-140; } \\
\text { Eggers 1968 104 (19a); } \\
\text { McPeake \& Moore 1978, } \\
\text { 333 (9); RIB II2415.16 }\end{array}$ \\
\hline DEN0002 & Handled Pan 2 & Eggers 137-138 & Denbighshire & Glyn Dyfrdwy & & $\mathrm{RU}$ & DEN0001-0004 & \begin{tabular}{|l|} 
Concentric circle umbo, concentric circle \\
on exterior body
\end{tabular} & $\begin{array}{l}\begin{array}{l}\text { Geometric half circle handle loop, caducaeus on handle, } \\
\text { illegible inscription }\end{array} \\
\end{array}$ & $\begin{array}{l}\text { Gardner 1927, 129-140; } \\
\text { Eggers 1968 104 (19b) }\end{array}$ \\
\hline DENO003 & Handled Pan 2 & Eggers 146 & Denbighshire & Glyn Dyfrdwy & & RU & DEN0001-0004 & Concentric circle umbo & $\begin{array}{l}\begin{array}{l}\text { Geoometric trefoil dots on handle medallion, illegible } \\
\text { inscription }\end{array} \\
\end{array}$ & \begin{tabular}{|l} 
Gardner 1927, 129-140; \\
Eggers 1968 104 (19c)
\end{tabular} \\
\hline DEN0004 & $\begin{array}{l}\begin{array}{l}\text { Handled Pan } 2 \\
\text { (fragment) }\end{array} \\
\end{array}$ & Eggers 144 & Denbighshire & Glyn Dyfrdwy & & $\mathrm{RU}$ & DENO001-0004 & $\begin{array}{l}\text { Geometric rectagular sectioned ring on } \\
\text { exterior body }\end{array}$ & & $\begin{array}{l}\text { Gardner 1927, 129-140; } \\
\text { Eggers 1968 104(19d) } \\
\end{array}$ \\
\hline DEN0008 & Bowl & & Denbighshire & Abergele & Plas Uchaf & RS & DEN0008-DEN0016 & $\begin{array}{l}\text { Concentric circle umbo; etched inscription } \\
\text { "INDVS LX" }\end{array}$ & & $\begin{array}{l}\text { Wright \& Hassall 1972, } \\
\text { 363; NMGW 71.27H; } \\
\text { RCAHM 1914, 7-8; RIB } \\
\text { II2415.61 }\end{array}$ \\
\hline DEN0009 & Bowl & & Denbighshire & Abergele & Plas Uchaf & RS & DEN0008-DEN0016 & $\begin{array}{l}\text { Concentric circle umbo; etched inscription } \\
\text { "INDVS SH(?)" }\end{array}$ & & $\begin{array}{l}\text { Wright \& Hassall 1972, } \\
\text { 363; NMGW 71.27H; } \\
\text { RCAHM 1914, 7-8; RIB } \\
\text { II2415.62 }\end{array}$ \\
\hline
\end{tabular}




\begin{tabular}{|c|c|c|c|c|c|c|c|c|c|c|}
\hline DEN0010 & Bowl & & Denbighshire & Abergele & Plas Uchaf & RS & DEN0008-DEN0016 & & & $\begin{array}{l}\text { Wright \& Hassall 1972, } \\
\text { 363; NMGW 71.27H; } \\
\text { RCAHM 1914, 7-8 } \\
\end{array}$ \\
\hline DENO011 & Bowl & & Denbighshire & Abergele & Plas Uchaf & RS & DEN0008-DEN0016 & & & $\begin{array}{l}\text { Wright \& Hassall 1972, } \\
\text { 363; NMGW 71.27H; } \\
\text { RCAHM 1914, 7-8 }\end{array}$ \\
\hline DENOO12 & Strainer & & Denbighshire & Abergele & Plas Uchaf & RS & DEN0008-DEN0016 & $\begin{array}{l}\text { Geometric meander, concentric circle and } \\
\text { floral strainer dot patterns }\end{array}$ & & $\begin{array}{l}\text { Wright \& Hassall 1972, } \\
\text { 363; NMGW 71.27H; } \\
\text { RCAHM 1914, 7-8 }\end{array}$ \\
\hline DENO013 & Handled Pan 2 & Eggers $139-140$ & Denbighshire & Abergele & Plas Uchaf & RS & DEN0008-DEN0016 & & Concentric circle handle loop & $\begin{array}{l}\text { Wright \& Hassall 1972, } \\
\text { 363; NMGW 71.27H; } \\
\text { RCAHM 1914, 7-8; }\end{array}$ \\
\hline DENO014 & Handled Pan 2 & Eggers 146-147 & Denbighshire & Abergele & Plas Uchaf & RS & DEN0008-DEN0016 & & Trefoil handle loop & $\begin{array}{l}\text { Wright \& Hassall 1972, } \\
\text { 363; NMGW 71.277; } \\
\text { RCAHM 1914, 7-8 }\end{array}$ \\
\hline DENO015 & Bowl & & Denbighshire & Abergele & Plas Uchaf & RS & DENO008-DENO016 & & & $\begin{array}{l}\text { Wright \& Hassall 1972, } \\
\text { 363; NMGW 71.277; } \\
\text { RCAHM 1914, 7-8 }\end{array}$ \\
\hline DEN0016 & Bowl & & Denbighshire & Abergele & Plas Uchaf & RS & DEN0008-DEN0016 & & & $\begin{array}{l}\text { Wright \& Hassall 1972, } \\
\text { 363; NMGW 71.27H; } \\
\text { RCAHM 1914, 7-8 }\end{array}$ \\
\hline DEV0003 & Handled Pan 2 & Eggers 139-144 & Devon & Exeter & $\begin{array}{l}\begin{array}{l}\text { Stone lined pit next } \\
\text { to timber house }\end{array} \\
\end{array}$ & U & & & & Rankov 1982, 383 \\
\hline DEV0004 & Handled Pan 2 & Eggers $139-144$ & Devon & Exeter & $\begin{array}{l}\text { Stone lined pit next } \\
\text { to timber house }\end{array}$ & $\mathrm{u}$ & & & & Rankov 1982, 383 \\
\hline DOR0001 & Bucket (fragment) & Eggers 24 & Dorset & Cranborne & Hod Hill & M & DOR0001-DOR0005 & $\begin{array}{l}\text { Anthropomorphic human bust and animal } \\
\text { (canine?) heads on rim }\end{array}$ & & Eggers $1968103(9 \mathrm{a})$ \\
\hline DOR0002 & $\begin{array}{l}\text { Handled Pan } 2 \\
\text { (handle) }\end{array}$ & Eggers 133 & Dorset & Cranborne & Hod Hill & M & DOR0001-DOR0005 & & Geometric punch dots & Eggers $1968103(9 \mathrm{~b})$ \\
\hline DOR0003 & $\begin{array}{l}\text { Handled Pan } 2 \\
\text { (handle) }\end{array}$ & Eggers 138 & Dorset & Cranborne & Hod Hill & M & DOR0001-DOR0005 & & $\begin{array}{l}\text { Geometric half sunburst on handle medallion, caducaeus } \\
\text { on handle }\end{array}$ & Eggers $1968103(9 \mathrm{c})$ \\
\hline DOR0004 & $\begin{array}{l}\begin{array}{l}\text { Handled Pan } 2 \\
\text { (handle) }\end{array} \\
\end{array}$ & Eggers $140-141$ & Dorset & Cranborne & Hod Hill & M & DOR0001-DOR0005 & & Concentric ring handle medallion & Eggers 1968103 (9d) \\
\hline DOR0005 & $\begin{array}{l}\begin{array}{l}\text { Handlde) Pan } 1 \\
\text { (handle) }\end{array} \\
\end{array}$ & Eggers $154-155$ & Dorset & Cranborne & Hod Hill & M & DOR0001-DOR0005 & & Zoomorphic ramshead handle terminal, fluted handle & Eggers $1968103(9 \mathrm{e})$ \\
\hline DUR0001 & Jug & & Durham & Sedgefield & & RS & & & $\begin{array}{l}\text { Anthropomorphic medusa handle medallion; } \\
\text { Zoomorphic birds' heads where handle frames rim; fluted } \\
\text { handle }\end{array}$ & $\begin{array}{l}\text { Burnham 2007, } 264 \text { (5, } \\
\text { Fig. 13) }\end{array}$ \\
\hline DUR0005 & Bowl & & Durham & South Shields & $\begin{array}{l}\text { South Shields } \\
\text { Roman Fort }\end{array}$ & M & & $\begin{array}{l}\text { Inscription "M A SAB APOLLINI } \\
\text { ANEXTIOMAROM" circling raised } \\
\text { umbo }\end{array}$ & & $\begin{array}{l}\text { Henig 1984, 132-133; RIB } \\
\text { II2415.55 }\end{array}$ \\
\hline DUR0006 & Handled Pan 2 & Eggers 142 & Durham & Upper Weardale & Peat bog & $\mathrm{RU}$ & DUR0006-DUR0008 & Concentric circle umbo & $\begin{array}{l}\text { Concentric circle handle loop; inscription "P. } \\
\text { CIPEPOLI" }\end{array}$ & $\begin{array}{l}\text { Egglestone 1915, 9-11; } \\
\text { Bennett \& Young 1981, } 43 \\
\text { (41); RIB II2415.20 }\end{array}$ \\
\hline DUR0007 & Handled Pan 2 & Egger 142 & Durham & Upper Weardale & Peat bog & $\mathrm{RU}$ & DUR0006-DUR0008 & $\begin{array}{l}\text { Concentric circle umbo; punch dot } \\
\text { inscription around umbo read } \\
\text { "LICINIANI" }\end{array}$ & Concentric circle handle loop; inscription "POLIBI.M" & \begin{tabular}{|l} 
Egglestone 1915, 9-11; \\
Bennet \& Young 1981, 43 \\
(42); RIB II2415.27 \\
\end{tabular} \\
\hline DUR0008 & Handled Pan 2 & Eggers 142 & Durham & Upper Weardale & Peat bog & $\mathrm{RU}$ & DUR0006-DUR0008 & & & Egglestone 1915, 9-11 \\
\hline ESUS0004 & Bucket & & East Sussex & Beachy Head & Bullock Down Farm & $\mathrm{RU}$ & $5495 \mathrm{Cn}[\mathrm{s}]$ & & & $\begin{array}{l}\text { Bland \& Johns 1979, 61- } \\
\text { 107; BM 1973.0404.1 }\end{array}$ \\
\hline EX0008 & Bowl & Irchester & Essex & Sturmer & & RU & EX0008-EX0014 & & & Kennet 1971, 1242.1 \\
\hline EX0009 & Bowl & Irchester & Essex & Sturmer & & RU & EX0008-EX0014 & & & Kennet $1971,1242.2$ \\
\hline EX0010 & Bowl & Irchester & Essex & Sturmer & & RU & EX0008-EX0014 & & & Kennet $1971,1262.3$ \\
\hline EX0011 & Handled Pan 4 & Coptic & Essex & Sturmer & & $\mathrm{RU}$ & EX0008-EX0014 & & & Kennet 1971, 1262.4 \\
\hline EX0012 & Bowl & & Essex & Sturmer & & $\mathrm{RU}$ & EX0008-EX0014 & & & Kennet $1971, \quad 1262.5$ \\
\hline EX0013 & Bowl & Bassin Uní & Essex & Sturmer & & RU & EX0008-EX0014 & Concentric circle umbo & & Kennet 1971, 1272.6 \\
\hline EX0014 & Bowl & Bassin Uní & Essex & Sturmer & & RU & EX0008-EX0014 & Concentric circle umbo & & Kennet 1971, 1282.7 \\
\hline EX0022 & Handled Pan 2 & Eggers 150 & Essex & Chesterford & Bors Field & M & EX0023 & Geometric linear rim embellishment & Geometric sub rectangular handle loop & Eggers 1968, $106(38 \mathrm{a})$ \\
\hline EX0023 & \begin{tabular}{|l} 
Strainer \\
Dan \\
\end{tabular} & Eggers 160-161 & Essex & Chesterford & Bors Field & $\mathrm{M}$ & \begin{tabular}{|l|l|} 
EX0022 \\
Ton2
\end{tabular} & Geometric strainer dots & Hilted handle & \begin{tabular}{|l|l|} 
Eggers 1968, $106(38 \mathrm{~b})$ \\
\end{tabular} \\
\hline F0001 & Bowl & & Flintshire & Halkyn & Halkyn Mountain & RS & F0001-F0008 & & & Kennet 1971, 1283.1 \\
\hline F0002 & Bucket & & Flintshire & Halkyn & Halkyn Mountain & RS & F0001-F0008 & $\begin{array}{l}\text { Geometric triangular mount fittings with } \\
\text { trefoil hoops }\end{array}$ & & Kennet 1971, 1283.2 \\
\hline F0003 & Bucket & & Flintshire & Halkyn & Halkyn Mountain & RS & F0001-F0008 & $\begin{array}{l}\text { Geometric triangular mount fittings with } \\
\text { trefoil hoops }\end{array}$ & & Kennet $1971,1283.3$ \\
\hline
\end{tabular}




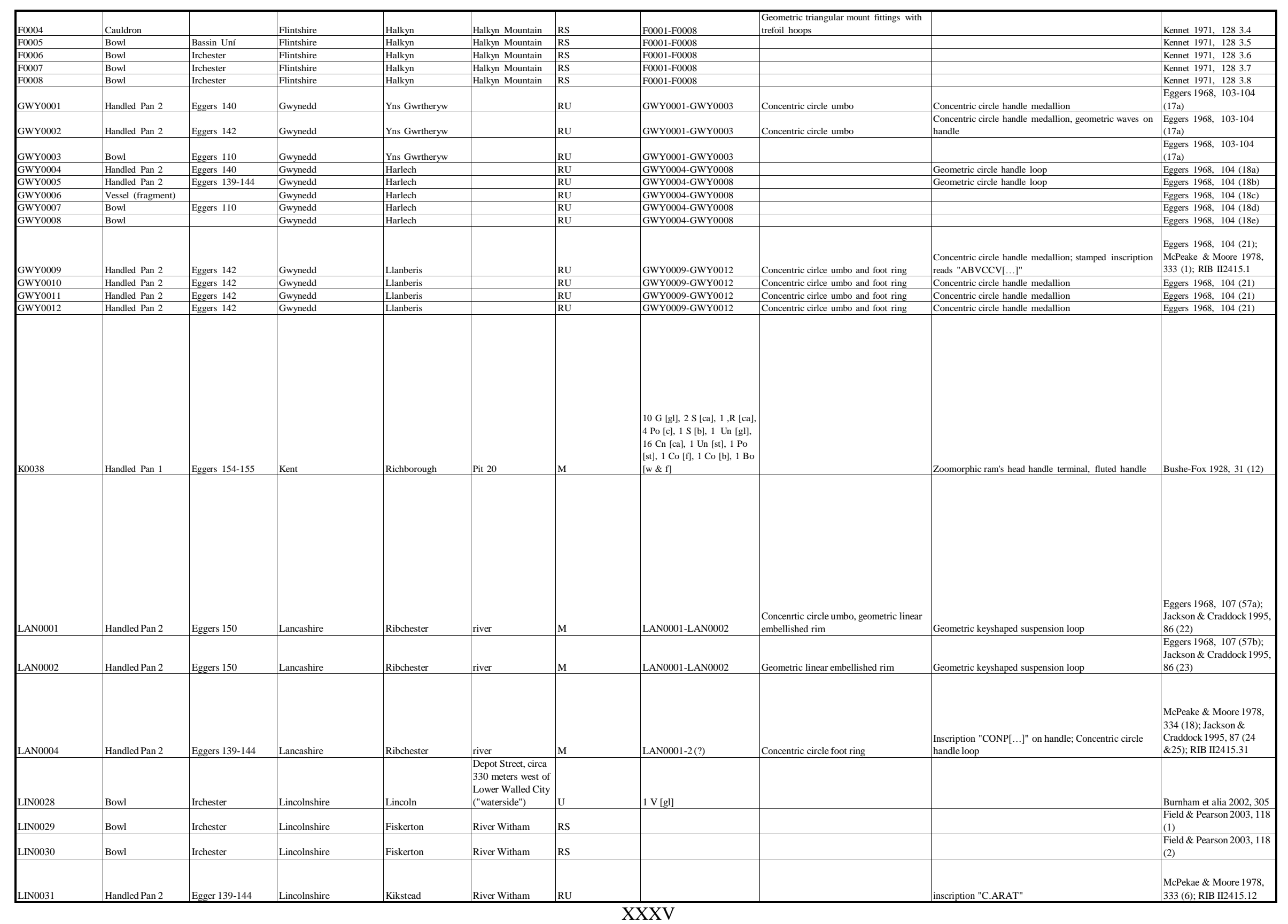




\begin{tabular}{|c|c|c|c|c|c|c|c|c|c|c|}
\hline LIN0032 & $\begin{array}{l}\text { Handled Pan } 2 \\
\text { (handle) }\end{array}$ & & Lincolnshire & Fiskerton & River Witham & RS & & & $\begin{array}{l}\text { Floral vine leaves and caducaeus; inscription "FLORVS } \\
\text { F" }\end{array}$ & $\begin{array}{l}\text { McPeake \& Moore } 1978 \\
334 \text { (26); RIB II2415.35 }\end{array}$ \\
\hline L0001 & Bowl & Irchester & London & City of London & $\begin{array}{l}\begin{array}{l}\text { Drapers' Gardens, } \\
\text { Well }\end{array} \\
\end{array}$ & $\mathrm{u}$ & L0001-L0015 & $\begin{array}{l}\begin{array}{l}\text { Zoomorphic hanging vessel mount (bear } \\
\text { or lion) }\end{array} \\
\text {. }\end{array}$ & & Gerrard 2009168854 \\
\hline L0002 & Bucket & $\begin{array}{l}\text { Hemmoor, Eggers } \\
58\end{array}$ & London & City of London & \begin{tabular}{|l}
$\begin{array}{l}\text { Drapers' Gardens, } \\
\text { well }\end{array}$ \\
\end{tabular} & $\mathrm{u}$ & L0001-L0015 & & & Gerrard 2009171821 \\
\hline L0003 & Bucket & $\begin{array}{l}\text { Hemmoor, Eggers } \\
58\end{array}$ & London & City of London & $\begin{array}{l}\text { Drapers' Gardens, } \\
\text { Well }\end{array}$ & $\mathrm{u}$ & L0001-L0015 & & & Gerrard 2009171824 \\
\hline$L 0004$ & Bucket & & London & City of London & \begin{tabular}{|l|}
$\begin{array}{l}\text { Drapers' Gardens, } \\
\text { Well }\end{array}$ \\
\end{tabular} & $\mathrm{u}$ & L0001-L0015 & & & Gerrard $2009 \quad 171820$ \\
\hline L0005 & Bucket & & London & City of London & \begin{tabular}{|l|}
$\begin{array}{l}\text { Drapers' Gardens, } \\
\text { Well }\end{array}$ \\
\end{tabular} & $\mathrm{u}$ & L0001-L0015 & & & Gerrard 2009171816 \\
\hline L0006 & Bucket & Westland & London & City of London & \begin{tabular}{|l}
$\begin{array}{l}\text { Drapers' Gardens, } \\
\text { Well }\end{array}$ \\
\end{tabular} & $\mathrm{u}$ & L0001-L0015 & & & Gerrard 2009171813 \\
\hline L0007 & Bowl & Perlandbecken & London & City of London & $\begin{array}{l}\text { Drapers' Gardens, } \\
\text { Well }\end{array}$ & $\mathrm{u}$ & L0001-L0015 & & & Gerrard 2009173818 \\
\hline L0008 & Bowl & Perlandbecken & London & City of London & $\begin{array}{l}\text { Drapers' Gardens, } \\
\text { Well }\end{array}$ & $\mathrm{U}$ & L0001-L0015 & & & Gerrard $2009 \quad 173829$ \\
\hline L0009 & Bowl & Perlandbecken & London & City of London & \begin{tabular}{|l}
$\begin{array}{l}\text { Drapers' Gardens, } \\
\text { Well }\end{array}$ \\
well
\end{tabular} & $\mathrm{U}$ & L0001-L0015 & & & Gerrard 2009172822 \\
\hline L0010 & Bowl & \begin{tabular}{|l}
$\begin{array}{l}\text { Bassinin á bord } \\
\text { godronné }\end{array}$ \\
\end{tabular} & London & City of London & \begin{tabular}{|l} 
Drapers' Gardens, \\
Well
\end{tabular} & $\mathrm{u}$ & L0001-L0015 & & & Gerrard 2009173826 \\
\hline L0011 & Bowl & $\begin{array}{l}\text { Bassin á bord } \\
\text { godronné }\end{array}$ & London & City of London & \begin{tabular}{|l} 
Drapers' Gardens, \\
Well
\end{tabular} & $\mathrm{U}$ & L0001-L0015 & & & Gerrard $2009 \quad 173853$ \\
\hline L0012 & Bowl & & London & City of London & \begin{tabular}{|l} 
Drapers' Gardens, \\
Well
\end{tabular} & $\mathrm{U}$ & L0001-L0015 & & & Gerrard $2009 \quad 173817$ \\
\hline L0013 & Bowl & & London & City of London & $\begin{array}{l}\text { Drapers' Gardens, } \\
\text { Well }\end{array}$ & $\mathrm{U}$ & L0001-L0015 & & & Gerrard 2009173819 \\
\hline L0014 & Handled Pan 2 & Eggers 161 (cf) & London & City of London & $\begin{array}{l}\text { Drapers' Gardens, } \\
\text { Well }\end{array}$ & $\mathrm{U}$ & L0001-L0015 & & & Gerrard $2009 \quad 176823$ \\
\hline L0015 & Handled Pan 2 & Eggers 160 (c.f.) & London & City of London & $\begin{array}{l}\text { Drapers' Gardens, } \\
\text { Well }\end{array}$ & $\mathrm{u}$ & L0001-L0015 & & Geometric diamond-shaped expansion & Gerrard 2009176814 \\
\hline NE0004 & Bowl & Rose Ash & Newport & Langstone & Bog/Swamp & RS & & & 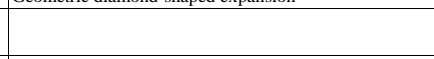 & $\begin{array}{l}\text { PAS NMGW-9C0216; } \\
\text { Worrell 2009, 285-287 }\end{array}$ \\
\hline NF0005 & Bowl & Rose Ash & Newport & Langstone & Bog/Swamp & RS & & & & $\begin{array}{l}\text { PAS NMGW-9C0216; } \\
\text { Worrell 2009 285-287 }\end{array}$ \\
\hline NE0006 & Strainer & & Newport & Langstone & Bog/Swamp & RS & & & & $\begin{array}{l}\text { PAS NMGW-9C0216; } \\
\text { Worrell 2009, 285-287 }\end{array}$ \\
\hline NH0001 & Bucket (fragment) & & Northamptonshire & Irchester & & RS & & & & Kennett 1971, 1284.1 \\
\hline NH0002 & Bowl & Irchester & Northamptonshire & Irchester & & RS & & & & Kennett $1971,1284.2$ \\
\hline NH0003 & Bowl & \begin{tabular}{|l|} 
Bassin à bord \\
godronné
\end{tabular} & Northamptonshire & Irchester & & RS & & & & Kennett 1971, 1284.3 \\
\hline NH0004 & Bowl & Irchester & Northamptonshire & Irchester & & RS & & & & Kennett 1971, 1304.4 \\
\hline NH0005 & Bowl & Irchester & Northamptonshire & Irchester & & RS & & & & Kennett 1971, 1304.5 \\
\hline NH0006 & Bowl & Irchester & Northamptonshire & Irchester & & RS & & & & Kennett 1971, 1304.6 \\
\hline NH0007 & Handled Pan 2 & & Northamptonshire & Irchester & & RS & & & & Kennett 1971, 1304.7 \\
\hline NH0008 & Strainer & & Northamptonshire & Irchester & & RS & & $\begin{array}{l}\text { Geometric (strainer punch dots of base } \\
\text { and wall of body in circle, cross, } \\
\text { triangular, and cable-knit decoration), } \\
\text { Symetrical, Umbo ring }\end{array}$ & & Kennet 1971, 130 4.8, \\
\hline NH0009 & Strainer & & Northamptonshire & Irchester & & RS & & $\begin{array}{l}\text { Geometric (strainer punch dots of base } \\
\text { and wall of body in circle, cross, and } \\
\text { waved decoration), Symetrical, Umbo } \\
\text { punch dot ring inside punch dot six } \\
\text { pointed star }\end{array}$ & $\begin{array}{l}\text { Geometric handle cross line engraving in diamonds and } \\
\text { triangles, Flared handle terminal }\end{array}$ & Kennett 1971, 1304.9 \\
\hline NOR0033 & Cauldron & & Norfolk & Weeting & \begin{tabular}{|l|}
$\begin{array}{l}\text { Northern flood- } \\
\text { plain of River Ouse }\end{array}$ \\
\end{tabular} & RS & NOR0033-NOR0040 & & & Gregory 1977, 265 (1) \\
\hline NOR0034 & Bowl & $\begin{array}{l}\text { Bassin a bord } \\
\text { godronne }\end{array}$ & Norfolk & Weeting & $\begin{array}{l}\text { Northern flood- } \\
\text { plain of River Ouse }\end{array}$ & RS & NOR0033-NOR0040 & $\begin{array}{l}\text { Geometric 'godronne' decoration along } \\
\text { rim }\end{array}$ & & Gregory 1977, 267 (2) \\
\hline NOR0035 & Bowl & Bassin uni & Norfolk & Weeting & $\begin{array}{l}\text { Northern flood- } \\
\text { plain of River Ouse }\end{array}$ & RS & NOR0033-NOR0040 & & & Gregory 1977, 268 (3) \\
\hline NOR0036 & Cauldron & & Norfolk & Weeting & $\begin{array}{l}\text { Northern flood- } \\
\text { plain of River Ouse }\end{array}$ & RS & NOR0033-NOR0040 & & & Gregory 1977, 268 (4) \\
\hline NOR0037 & Cauldron & & Norfolk & Weeting & \begin{tabular}{|l|} 
Northern flood- \\
plain of River Ouse
\end{tabular} & RS & NOR0033-NOR0040 & & & Gregory 1977, 268 (5) \\
\hline NOR0038 & Bowl & Irchester & Norfolk & Weeting & $\begin{array}{l}\text { Northern flood- } \\
\text { plain of River Ouse }\end{array}$ & RS & NOR0033-NOR0040 & & & Gregory 1977, 268 (6) \\
\hline
\end{tabular}




\begin{tabular}{|c|c|c|c|c|c|c|c|c|c|c|}
\hline NOR0039 & Bowl & Irchester & Norfolk & Weeting & \begin{tabular}{|l|}
$\begin{array}{l}\text { Northern flood- } \\
\text { plain of River Ouse }\end{array}$ \\
\end{tabular} & RS & NOR0033-NOR0040 & & & Gregory 1977, $268(7)$ \\
\hline NOR0040 & Bowl & & Norfolk & Weeting & \begin{tabular}{|l|}
$\begin{array}{l}\text { Northern flood- } \\
\text { plain of River Ouse }\end{array}$ \\
\end{tabular} & RS & NOR0033-NOR0040 & & & Gregory 1977, $269(8)$ \\
\hline NU0003 & Strainer & Eggers 161 & Northumberland & \begin{tabular}{|l} 
Great Chesters \\
\end{tabular} & Fort (Aesica) & M & NU0004, NU0005 & Geometric sunburst strainer dot pattern & & Eggers $1966,10859 \mathrm{c}$ \\
\hline NU0004 & \begin{tabular}{|l|l|} 
Strainer \\
\end{tabular} & Eggers 161 & Northumberland & \begin{tabular}{|l} 
Great Chesters \\
\end{tabular} & Fort (Aesica) & $\mathrm{M}$ & NU0003, NU0005 & Geometric sunburst strainer dot pattern & & Eggers $1966,10859 \mathrm{~d}$ \\
\hline NU0005 & Handled Pan 3 & Eggers 161 & Northumberland & Great Chesters & Fort (Aesica) & $\mathrm{M}$ & NU0003, NU0004 & & & Eggers $1966,10859 \mathrm{e}$ \\
\hline NU0019 & Handled Pan 2 & Eggers 139 & Northumberland & Ponteland & $\begin{array}{l}\text { Prestwick Carr, } \\
\text { Swamp }\end{array}$ & $\mathrm{RU}$ & YOR0019-YOR0025 & Concentric circle umbo & $\begin{array}{l}\text { Concentric circle handle ring, stylised caducaeus on } \\
\text { handle with trefoil points and punch dot embellishments; } \\
\text { incription "DRACCIVS F" }\end{array}$ & $\begin{array}{l}\text { Eggers 1968, } 108 \text { (65a); } \\
\text { McPeake \& Moore 1978, } \\
\text { 334 (21); RIB II2415.32 }\end{array}$ \\
\hline Nu0020 & Handled Pan 2 & Eggers $140-141$ & Northumberland & Ponteland & $\begin{array}{l}\text { Prestwick Carr, } \\
\text { Swamp }\end{array}$ & $\mathrm{RU}$ & YOR0019-YOR0025 & Concentric circle umbo & & Eggers 1968, 108 (65b) \\
\hline Nu0021 & Handled Pan 2 & Eggers $140-141$ & Northumberland & Ponteland & $\begin{array}{l}\text { Prestwick Carr, } \\
\text { Swamp }\end{array}$ & $\mathrm{RU}$ & YOR0019-YOR0025 & Concentric circle umbo & & Eggers 1968, $108(65 \mathrm{c})$ \\
\hline Nu0022 & Bowl & Eggers 68 & Northumberland & Ponteland & $\begin{array}{l}\text { Prestwick Carr, } \\
\text { Swamp }\end{array}$ & $\mathrm{RU}$ & YOR0019-YOR0025 & & & Eggers 1968, 108 (65d) \\
\hline Nu0023 & Bowl & Eggers 70 & Northumberland & Ponteland & $\begin{array}{l}\text { Prestwick Carr, } \\
\text { Swamp }\end{array}$ & $\mathrm{RU}$ & YOR0019-YOR0025 & & $\begin{array}{l}\text { punch dot inscriptions below rim "T TTIRONIS } \\
\text { CRIISCRII SNT SENECIONIS" and "TKANDIANI } \\
\text { VANNI" }\end{array}$ & $\begin{array}{l}\text { Eggers 1968, } 108 \text { (65e); } \\
\text { RIB II } 2415.63\end{array}$ \\
\hline NU0024 & Cauldron & $\begin{array}{l}\text { Westland, Eggers } 12 \\
13 \text { (c.f) }\end{array}$ & Northumberland & Ponteland & $\begin{array}{l}\text { Prestwick Carr, } \\
\text { Swamp }\end{array}$ & RU & YOR0019-YOR0025 & & & Eggers 1968, 108 (65f) \\
\hline Nu0025 & Bowl & Eggers 101-105 & Northumberland & Ponteland & $\begin{array}{l}\text { Prestwick Carr, } \\
\text { Swamp }\end{array}$ & RU & YOR0019-YOR0025 & Concentric circle footring & & Eggers 1968, $108(65 \mathrm{~g})$ \\
\hline NU0029 & Jar & & Northumberland & Whittington & & RU & 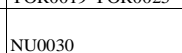 & $\begin{array}{l}\text { Floral oval half leaf band running under } \\
\text { the rim; Concentric circle umbo }\end{array}$ & & PAS NCL-33CC76 \\
\hline NU0030 & Handled Pan 2 & Eggers $139-144$ & Northumberland & Whittington & & $\mathrm{RU}$ & NuO029 & Concentric circle umbo and foot ring & Concentric circle handle medallion loop & PAS NCL-335745 \\
\hline Nu0035 & Jug & Eggers 128a & Northumberland & Corbridge & $\begin{array}{l}\begin{array}{l}\text { Fort (Corstopitum), } \\
\text { north of Stanegate }\end{array} \\
\end{array}$ & M & $160 \mathrm{Cn}[\mathrm{g}], 2 \mathrm{Cn}[\mathrm{ca}]$ & & & $\begin{array}{l}\text { Forester et al. 1912, 154; } \\
\text { McDonald 1912, 1-12; } \\
\text { Abdy 2002, 35 (19) } \\
\end{array}$ \\
\hline Nuo050 & Handled Pan 2 & Eggers 139-144 & Northumberland & Matfen & Ingoe township & $\mathrm{RU}$ & NU0050-NU0051 & & $\begin{array}{l}\text { Concentric circle handle loop; Geometric concentric } \\
\text { circle dots formed in a triangle at base of handle } \\
\text { medallion; inscription "SABINIANVS F" between two } \\
\text { triangles of concentric circle dots; etched inscription "I } \\
\text { XXV" on underside of handle }\end{array}$ & $\begin{array}{l}\text { Wright } 1969,1-5 ; \text { RIB } \\
\text { II2415.44 }\end{array}$ \\
\hline NU0051 & Handled Pan 2 & Eggers 139-144 & Northumberland & Matfen & Ingoe township & $\mathrm{RU}$ & NU0050-NU0051 & & $\begin{array}{l}\text { Concentric circle handle loop; Geometric concentric } \\
\text { circle dots formed in a triangleat base of handle } \\
\text { medallion; inscription "MAXMINVS F" between two } \\
\text { triangles of concentric circle dots; etched inscription "I } \\
\text { XXIS" on underside of handle }\end{array}$ & $\begin{array}{l}\text { Wright 1969, 1-5; } \\
\text { McPeake \& Moore 1978, } \\
\text { 334 (30); RIB II2415.40 }\end{array}$ \\
\hline \begin{tabular}{|l|l|} 
NYR003 \\
NYR0004
\end{tabular} & \begin{tabular}{|l} 
Handled Pan 2 \\
Bowl \\
\end{tabular} & & \begin{tabular}{|l|l} 
North Yorkshire \\
North Yorkshire
\end{tabular} & \begin{tabular}{|l|l|} 
Linton \\
Linton
\end{tabular} & & \begin{tabular}{|l}
$\mathrm{RS}$ \\
$\mathrm{RS}$
\end{tabular} & \begin{tabular}{|l|l|} 
NYR0004 \\
NYR0003
\end{tabular} & & Geometric triangular protrusion from end of handle & PAS SWYOR-E51F57 \\
\hline NYR0008 & Bucket & $\begin{array}{l}\text { Ostland, Eggers 37- } \\
40\end{array}$ & North Yorkshire & Knaresborough & & $\mathrm{RU}$ & NYR0008-NYR0028 & & & $\begin{array}{l}\text { Kennett 1971, } 1346.1 ; \\
\text { Eggers 1966 107 (55 1) }\end{array}$ \\
\hline NYR0009 & Strainer & Eggers 161 & North Yorkshire & Knaresborough & & $\mathrm{RU}$ & NYR0008-NYR0028 & Geometric swirl strainer dot pattern & & $\begin{array}{l}\text { Kennett } 1971,1346.2 ; \\
\text { Eggers } 1966107(552)\end{array}$ \\
\hline NYR0010 & Strainer & Eggers 161 & North Yorkshire & Knaresborough & & $\mathrm{RU}$ & NYR0008-NYR0028 & Geometric swirl strainer dot pattern & & $\begin{array}{l}\text { Kennett } 1971,1346.33 \\
\text { Eggers } 1966107 \text {; } 1053)\end{array}$ \\
\hline NYR0011 & Strainer & & North Yorkshire & Knaresborough & & RU & NYR0008-NYR0028 & & & $\begin{array}{l}\text { Kennett 1971, } 1346.4 ; \\
\text { Eggers } 1966107(554)\end{array}$ \\
\hline NYR0012 & Strainer & & North Yorkshire & Knaresborough & & RU & NYR0008-NYR0028 & & Geometric swirl strainer dot pattern & $\begin{array}{l}\text { Kennett } 1971,1346.5 ; \\
\text { Eggers } 1966107(555)\end{array}$ \\
\hline NYR0013 & Strainer & & North Yorkshire & Knaresborough & & RU & NYR0008-NYR0028 & & $\begin{array}{l}\text { Floral strainer dot pattern in the shape of multi-petaled } \\
\text { flower }\end{array}$ & $\begin{array}{l}\text { Kennett 1971, } 1346.6 ; \\
\text { Eggers } 1966107(556)\end{array}$ \\
\hline NYR0014 & Strainer & & North Yorkshire & Knaresborough & & RU & NYR0008-NYR0028 & & $\begin{array}{l}\text { Geomer } \\
\text { strainer dot wave, pwirl, flourish, swastika, and trefoil }\end{array}$ & 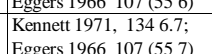 \\
\hline NYR0015 & Bowl & & North Yorkshire & Knaresborough & & RU & NYR0008-NYR0028 & Concentric circle umbo & & $\begin{array}{l}\text { Kennett 1971, } 1356.8 ; \\
\text { Eggers 1966 } 107(558)\end{array}$ \\
\hline NYR0016 & Bowl & & North Yorkshire & Knaresborough & & $\mathrm{RU}$ & NYR0008-NYR0028 & & & $\begin{array}{l}\text { Kennett 1971, } 1356.9 ; \\
\text { Eggers } 1966107(559)\end{array}$ \\
\hline NYR0017 & Bowl & $\begin{array}{l}\text { Bassin à bord } \\
\text { godronné }\end{array}$ & North Yorkshire & Knaresborough & & $\mathrm{RU}$ & NYR0008-NYR0028 & $\begin{array}{l}\text { Geometric linear rim decoration, many } \\
\text { punch dots on underside of umbo }\end{array}$ & & $\begin{array}{l}\text { Kennett 1971, 135 6.10; } \\
\text { Eggers 1966 107 (55 10) }\end{array}$ \\
\hline NYR0018 & Bowl & Irchester & North Yorkshire & Knaresborough & & $\mathrm{RU}$ & NYR0008-NYR0028 & & & \begin{tabular}{|l}
$\begin{array}{l}\text { Kennett 1971, } 1356.11 ; \\
\text { Eggers } 1966 \text { 107 (55 11) }\end{array}$ \\
\end{tabular} \\
\hline
\end{tabular}




\begin{tabular}{|c|c|c|c|c|c|c|c|c|c|c|}
\hline NYR0019 & Bowl & Irchester & North Yorkshire & Knaresborough & & $\mathrm{RU}$ & NYR0008-NYR0028 & & & \begin{tabular}{|l} 
Kennett $1971, \quad 1356.12 ;$ \\
Eggers $1966 \quad 107(5512)$
\end{tabular} \\
\hline NYR0020 & Bowl & Irchester & North Yorkshire & Knaresborough & & $\mathrm{RU}$ & NYR0008-NYR0028 & & & $\begin{array}{l}\text { Kennett t1971, } 1356.13 ; \\
\text { Eggers } 1966 \quad 107(5513)\end{array}$ \\
\hline NYR0021 & Bowl & Irchester & North Yorkshire & Knaresborough & & $\mathrm{RU}$ & NYR0008-NYR0028 & & & \begin{tabular}{|l|}
$\begin{array}{l}\text { Kennett 1971, } 1356.14 ; \\
\text { Eggers } 1966 \quad 107 \text { (55 14) }\end{array}$ \\
\end{tabular} \\
\hline NYR0022 & Bowl & Irchester & North Yorkshire & Knaresborough & & $\mathrm{RU}$ & NYR0008-NYR0028 & & & $\begin{array}{l}\text { Kennett 1971, } 1356.15 ; \\
\text { Eggers } 1966107(5515)\end{array}$ \\
\hline NYR0023 & Bowl & Irchester & North Yorkshire & Knaresborough & & RU & NYR0008-NYR0028 & & & $\begin{array}{l}\text { Kennett 1971, 1356 6.16; } \\
\text { Eggers 1966 107 (516) }\end{array}$ \\
\hline NYR0024 & Bowl & & North Yorkshire & Knaresborough & & $\mathrm{RU}$ & NYR0008-NYR0028 & & & \begin{tabular}{|l|} 
Kennett $1971,1356.17 ;$ \\
Eggers 1966 107 (55 17)
\end{tabular} \\
\hline NYR0025 & Vessel (fragment) & & North Yorkshire & Knaresborough & & $\mathrm{RU}$ & NYR0008-NYR0028 & & & $\begin{array}{l}\text { Kennett } 1971,1356.18- \\
20 \text { - Eggers } 1966 \quad 107 \text { (55 } \\
18-20)\end{array}$ \\
\hline NYR0026 & tos & & North Yorkshire & Knaresborough & & $\mathrm{RU}$ & NYR0008-NYR0028 & & & 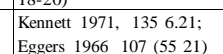 \\
\hline NYR0027 & Handled Pan 5 & & North Yorkshire & Knaresborough & & $\mathrm{RU}$ & NYR0008-NYR0028 & & Geometric linear etching & $\begin{array}{l}\text { Kennett 1971, } 1356.22 ; \\
\text { Eggers } 1966 \quad 107(5522)\end{array}$ \\
\hline NYR0028 & Bowl & \begin{tabular}{|l|} 
Bassin à bord \\
godronné
\end{tabular} & North Yorkshire & Knaresborough & & $\mathrm{RU}$ & \begin{tabular}{|l|} 
NYR0008-NYR0028 \\
\end{tabular} & & & Kennett 1971, 1366.23 \\
\hline NYR0037 & $\begin{array}{l}\text { Handled Pan } 2 \\
\text { (fragment) }\end{array}$ & Rudge Cup type & North Yorkshire & $\begin{array}{l}\text { Beadlam Roman } \\
\text { Villa }\end{array}$ & $\begin{array}{l}\text { south wall of Room } \\
\text { 6, Building } 1\end{array}$ & RS & $59 \mathrm{Un}(\mathrm{m})$ & $\begin{array}{l}\text { Floral vine (possible) body band; } \\
\text { Inscription "[...]I CITR" in upper register } \\
\text { followed by a geometric zig-zag line }\end{array}$ & & $\begin{array}{l}\text { Wright \& Hassall 1973, } \\
\text { 334 (38); Neal 1996, } 49 \\
\text { (23); RIB II2415.54 }\end{array}$ \\
\hline NYR0040 & Handled Pan 2 & Eggers 142 & North Yorkshire & Stittenham & & $\mathrm{RU}$ & NYR0040-NYR0044 & & $\begin{array}{l}\text { Concentric Circle handle loop; inscription "P CIPI } \\
\text { POLYIBI" }\end{array}$ & $\begin{array}{l}\text { Eggers 1968, } 107 \text { (54a); } \\
\text { RIB II2415.26 }\end{array}$ \\
\hline NYR0041 & Handled Pan 2 & Eggers 142 & North Yorkshire & Stittenham & & $\mathrm{RU}$ & NYR0040-NYR0044 & & $\begin{array}{l}\text { Concentirc Circle handle loop; inscription "P CIPI } \\
\text { POLIB" }\end{array}$ & $\begin{array}{l}\text { Eggers 1968, } 107 \text { (54b); } \\
\text { RIB II2415.19 }\end{array}$ \\
\hline NYR0042 & Handled Pan 2 & Eggers 142 & North Yorkshire & Stittenham & & $\mathrm{RU}$ & \begin{tabular}{|l|} 
NYR0040-NYR0044 \\
\end{tabular} & & & Eggesr 1968, $107(54 \mathrm{c})$ \\
\hline NYR0043 & Handled Pan 2 & Eggers 142 & North Yorkshire & Stittenham & & $\mathrm{RU}$ & \begin{tabular}{|l|} 
NYR0040-NYR0044 \\
\end{tabular} & & & Eggers 1968, 107 (54d) \\
\hline NYR0044 & Handled Pan 2 & Eggers 142 & North Yorkshire & Stittenham & & $\mathrm{RU}$ & NYR0040-NYR0044 & & & Eggers 1968, $107(54 \mathrm{e})$ \\
\hline STA0008 & Bowl & & Staffordshire & Wall & & RS & $31 \mathrm{Cn}[\mathrm{s}]$ & Geometric Chi-rho design & & $\begin{array}{l}\text { Mawer 1995, 19; RIB } \\
\text { II2415.64 }\end{array}$ \\
\hline SUF0001 & Handled Pan 2 & Eggers $137-138$ & Suffolk & Oulton & Swamp & $\mathrm{RU}$ & SUF0002 & & $\begin{array}{l}\text { Geometric half subburst on handle medallion, half circle } \\
\text { handle loop, caducaeus on handle }\end{array}$ & Eggers 1968, 106 (45a) \\
\hline SUF0002 & Handled Pan 2 & Eggers 144 & Suffolk & Oulton & Swamp & $\mathrm{RU}$ & SUF0001 & $\begin{array}{l}\text { Floral band on body, concentric ring } \\
\text { umbo }\end{array}$ & $\begin{array}{l}\text { Concentric circle handle loop, geometric scalloping } \\
\text { under handle medallion }\end{array}$ & Eggers 1968, $106(45 b)$ \\
\hline SUF0003 & $\begin{array}{l}\text { Handded Pan } 1 \\
\text { (handle) }\end{array}$ & Eggers $154-155$ & Suffolk & Santon Downham & & RS & SUF0004 & & Zoomorphic canine handle terminal, fluted handle & Eggers $1968,106(44 a)$ \\
\hline SUF0004 & Jug & Eggers 125 & Suffolk & Santon Dounham & & RS & SUF0003 & & $\begin{array}{l}\text { Zoomorphic canine bust thumb rest and dog foot handle } \\
\text { medallion }\end{array}$ & Eggers 1968. $106(44 \mathrm{~b})$ \\
\hline SUF0043 & Cauldron & & \begin{tabular}{|l|l|l|l|l|} 
Suffolk \\
\end{tabular} & Brandon & & $\mathrm{RU}$ & & & & Grew 1980, 376 \\
\hline SUF0044 & Strainer & & Suffolk & Brandon & & $\mathrm{RU}$ & & & & Grew 1980,376 \\
\hline SUF0045 & Handled Pan 2 & Eggers 140-142 & Suffolk & Brandon & & RU & & & inscription reads "IVLEVS. F" & $\begin{array}{l}\text { McPeake \& Moore 1978, } \\
\text { 334 (27); Grew 1980, } 376 \\
\end{array}$ \\
\hline SUF0046 & Bucket & & Suffolk & Brandon & & $\mathrm{RU}$ & & & & Grew 1980,376 \\
\hline SUF0051 & Cauldron & & Suffolk & Santon Downham & & RS & SUF0003-SUF0004 & & & Smith 1909, 146-163(1) \\
\hline SUR0001 & Bowl & & Surrey & Wotton & & $\mathrm{RU}$ & & $\begin{array}{l}\begin{array}{l}\text { Geometric lines (cast channels, eleven } \\
\text { pairs) }\end{array} \\
\end{array}$ & & $\begin{array}{l}\text { Kennett 1971, 130 5.1; } \\
\text { BM 191.50406. }\end{array}$ \\
\hline SUR0002 & Cauldron & & Surrey & Wotton & & $\mathrm{RU}$ & & & & $\begin{array}{l}\text { Kennett 1971, } 1305.2 ; \\
\text { BM 1915.04 02.2 }\end{array}$ \\
\hline SUR0003 & Cauldron & & Surrey & Wotton & & $\mathrm{RU}$ & & & & $\begin{array}{l}\text { Kennet 1971, } 1305.3 \text {; BM } \\
1915.0406 .3\end{array}$ \\
\hline SUR0004 & Bucket & & Surrey & Wotton & & RU & & & & $\begin{array}{l}\text { Kennett 196.3 1971, 130 5.4; } \\
\text { BM 1915.04 064. }\end{array}$ \\
\hline SUR0005 & Cauldron & & Surrey & Wotton & & RU & & & & $\begin{array}{l}\text { Kennett 1971, } 1305.5 ; \\
\text { BM 1915.04 06.5 }\end{array}$ \\
\hline SUR0006 & Bowl & Irchester & Surrey & Wotton & & RU & & & & $\begin{array}{l}\text { Kennett 1971, 130 5.6; } \\
\text { BM 1915.04 } 066 \text {; }\end{array}$ \\
\hline SUR0007 & Bowl & Irchester & Surrey & Wotton & & RU & & & & $\begin{array}{l}\text { Kennett 1971, 130 5.7; } \\
\text { BM 1915.04 06.7 }\end{array}$ \\
\hline SUR0008 & Bowl & & Surrey & Wotton & & $\mathrm{RU}$ & & $\begin{array}{l}\text { Geometric herringbone and square stamps } \\
\text { on interior basin }\end{array}$ & & $\begin{array}{l}\text { Kennett 1971, 132 5.8; } \\
\text { BM 1915.04 06.8 }\end{array}$ \\
\hline SUR0009 & Bowl & & Surrey & Wotton & & $\mathrm{RU}$ & & & & $\begin{array}{l}\text { Kennett 1971, 132 5.9; } \\
\text { BM 1915.04 06.9 }\end{array}$ \\
\hline SUR0010 & Handled Pan 4 & Coptic & Surrey & Wotton & & $\mathrm{RU}$ & & & & $\begin{array}{l}\text { Kennett 1971, 132 5.10; } \\
\text { BM 1915.04 06.10 }\end{array}$ \\
\hline
\end{tabular}




\begin{tabular}{|c|c|c|c|c|c|c|c|c|c|c|}
\hline WLL0004 & Handled Pan 2 & Eggers $140-142$ & Wiltshire & Kingston Deverill & & RS & WILT0004-0008 & Concentric circle umbo & Concentric circle handle medallion & PAS WLT-92B052 \\
\hline WLL0005 & Handled Pan 2 & Eggers $140-142$ & Wiltshire & Kingston Deverill & & RS & WILT0004-0008 & Concentric circle umbo & Concentric circle handle medallion & PAS WLTT-92B052 \\
\hline WIL0006 & Handled Pan 2 & Eggers $140-142$ & Wiltshire & Kingston Deverill & & RS & WILT0004-0008 & Concentric circle umbo & $\begin{array}{l}\text { Concentric cirlce handle medallion: Inscription } \\
\text { "CIPI.POLIBI" in rectangular field }\end{array}$ & PAS WLLT-92B052 \\
\hline WIL0007 & Strainer & & Wiltshire & Kingston Deverill & & RS & WILT0004-0008 & Zoomorphic vessel spouts & & PAS WLIT-92B052 \\
\hline WLL0008 & Strainer & & Wiltshire & Kingston Deverill & & RS & WILT0004-0008 & Zoomorphic vessel spouts & & PAS WILT-92B052 \\
\hline WL0013 & Bowl & Irchester & Wiltshire & Bishop's Cannings & Blagan Hill & $\mathrm{RU}$ & 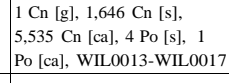 & & & $\begin{array}{l}\text { Burnham et al. 1994, 286; } \\
\text { Guest et al. 1997, 427 }\end{array}$ \\
\hline WIL0014 & Bowl & Irchester & Wiltshire & Bishop's Cannings & Blagan Hill & $\mathrm{RU}$ & $\begin{array}{l}1 \mathrm{Cn} \text { [g], 1,646 Cn [s], } \\
5,535 \mathrm{Cn} \text { [ca], } 4 \text { Po [s], } 1 \\
\text { Po [ca], WILO013-WIL0017 }\end{array}$ & & & $\begin{array}{l}\text { Burnham et al. 1994, 286; } \\
\text { Guest et al. 1997, } 427\end{array}$ \\
\hline WLL0015 & Bowl & Irchester & Wiltshire & Bishop's Cannings & Blagan Hill & $\mathrm{RU}$ & $\begin{array}{l}1 \mathrm{Cn} \text { [g], 1,646 Cn [s], } \\
5,535 \mathrm{Cn} \text { [ca], } 4 \text { Po [s], } 1 \\
\text { Po [ca], WILO0013-WIL0017 }\end{array}$ & & & $\begin{array}{l}\text { Burnham et al. 1994, 286; } \\
\text { Guest et al. 1997, } 427\end{array}$ \\
\hline WL0016 & Bowl & Irchester & Wiltshire & Bishop's Cannings & Blagan Hill & $\mathrm{RU}$ & 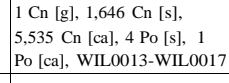 & & & $\begin{array}{l}\text { Burnham et al. 1994, 286; } \\
\text { Guest et al. 1997, 427 }\end{array}$ \\
\hline WLL0017 & Bowl & Irchester & Wiltshire & Bishop's Cannings & Blagan Hill & $\mathrm{RU}$ & 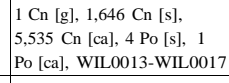 & & & $\begin{array}{l}\text { Burnham et al. 1994, 286; } \\
\text { Guest et al. 1997, } 427\end{array}$ \\
\hline WLL018 & Bowl & Irchester & Wilthsire & Bishop's Cannings & Blagan Hill & $\mathrm{RU}$ & $\begin{array}{l}1 \mathrm{Cn}[\mathrm{gg}, 1,1,646 \mathrm{Cn} \text { [s], } \\
5,535 \mathrm{Cn} \text { [ca], } 4 \text { Po [s], } \\
\text { Po [ca], WILO013-WLL0017 }\end{array}$ & & & Guest et al. 1997,427 \\
\hline WL0019 & Bowl & Irchester & Wiltshire & Bishop's Cannings & Blagan Hill & $\mathrm{RU}$ & 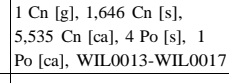 & & & Guest et al. 1997,427 \\
\hline WLL0020 & Jug & & Wiltshire & Bishop's Cannings & Blagan Hill & $\mathrm{RU}$ & $\begin{array}{l}1 \mathrm{Cn} \text { [g], 1,646 Cn [s], } \\
5,535 \mathrm{Cn} \text { [ca], } 4 \text { Po [s], } \\
\text { Po [ca], WILO013-WLL0017 }\end{array}$ & & & Guest et al. 1997,427 \\
\hline WIL0021 & Bowl & Bassin festonné & Wiltshire & Bishop's Cannings & Blagan Hill & $\mathrm{RU}$ & $\begin{array}{l}1 \mathrm{Cn}[\mathrm{gg}], 1,646 \mathrm{Cn}[\mathrm{s}] \\
5,535 \mathrm{Cn} \text { [ca], } 4 \text { Po [s], } \\
\text { Po [ca], WLL0013-WLO017 }\end{array}$ & & & Guest et al. 1997, 427 \\
\hline WL0022 & Bowl & Bassin festonné & Wiltshire & Bishop's Cannings & Blagan Hill & RU & 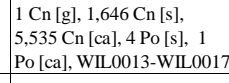 & & & Guest et al. 1997, 427 \\
\hline WIL0023 & Jug & Eggers 125 & Wiltshire & Roundway Hill & & $\mathrm{RU}$ & & $\begin{array}{l}\text { inscripition "ASPAE[...]" on inside of } \\
\text { rim }\end{array}$ & & $\begin{array}{l}\text { Taylor \& Collingwood } \\
\text { 1923, 283 (12); RIB } \\
\text { II2415.8 }\end{array}$ \\
\hline
\end{tabular}


Appendix III: Grave Deposits of Copper Alloy Vessels in Roman Britain.

\begin{tabular}{|c|c|c|c|c|c|c|c|c|c|c|}
\hline Number & Handled Pan 2 & Eggers $137-138$ & County & Shefford & Context & $\begin{array}{l}\text { Location } \\
\begin{array}{l}\text { Stanfordbury Farm, } \\
\text { Grave 1 }\end{array} \\
\end{array}$ & Associated Finds & Decoration (body) & \begin{tabular}{|l} 
Decoration (handle) \\
Geometric half sunburst on handle \\
medallion, acucaeaus on handle; \\
inscription below sunburst "[...]ESPYV" \\
\end{tabular} & \begin{tabular}{|l|} 
Reference \\
Eggers 1968 104- \\
105 (29A); RIB II \\
2415.51 \\
\end{tabular} \\
\hline BE0005 & Jug & Eggers $124-125$ & Bedfordshire & Shefford & RS & $\begin{array}{l}\text { Stanfordbury Farm, } \\
\text { Grave } 1\end{array}$ & BE0004-BE0005 & & $\begin{array}{l}\text { Zoomorphic human bust on thumbrest and } \\
\text { handle medallion }\end{array}$ & $\begin{array}{l}\text { Eggers } 1968 \text { 104- } \\
105(29 \mathrm{Ab})\end{array}$ \\
\hline BE0006 & Handled Pan 1 & Eggers 155 & Bedfordshire & Shefford & RS & $\begin{array}{l}\text { Stanfordbury Farm, } \\
\text { Grave } 2\end{array}$ & $2 \mathrm{~A}[\mathrm{c}], 1 \mathrm{~B}[\mathrm{c}]$ & Concentric ring umbo & $\begin{array}{l}\text { Zoomorphic rams head handle terminal, } \\
\text { fluted handle, floral second handle }\end{array}$ & $\begin{array}{l}\text { Eggers } 1968 \text { 104- } \\
105(29 \mathrm{Ab})\end{array}$ \\
\hline BE0007 & Jug & Eggers 125 & Bedfordshire & Shefford & RS & \begin{tabular}{|l} 
Stanfordbury Farm, \\
Grave 2
\end{tabular} & $2 \mathrm{~A}[\mathrm{c}], 1 \mathrm{~B}[\mathrm{c}]$ & & & \begin{tabular}{|l} 
Eggers 1968 104- \\
$105(29 \mathrm{Ab})$
\end{tabular} \\
\hline BUC0001 & Jug & Eggers 125 & Buckinghamshire & Thornborough & RS & \begin{tabular}{|l} 
Thornborough \\
Barrow, possible \\
family tomb
\end{tabular} & $\begin{array}{l}2 \mathrm{Am}[\mathrm{cl}, 3 \mathrm{Bo}[\mathrm{gl}], 1 \mathrm{~L}[\mathrm{ca}], 1 \mathrm{Bc}, \\
\text { BUC0001-00004 }\end{array}$ & Trefoil mouth & $\begin{array}{l}\text { Zoomorphic lion thumb rest with paw as } \\
\text { handle medallion }\end{array}$ & $\begin{array}{l}\text { Eggers 1968, } 110 \\
\text { (89a); Philpott 1991, } \\
\text { 261: Liversidge } \\
\text { 1953, 29-32 }\end{array}$ \\
\hline BUC0002 & Jug & Eggers $128 \mathrm{a}$ & Buckinghamshire & Thornborough & RS & $\begin{array}{l}\text { Thornborough } \\
\text { Barrow, possible } \\
\text { family tomb }\end{array}$ & $\begin{array}{l}2 \mathrm{Am}[\mathrm{cc}, 3 \mathrm{Bo}[\mathrm{ggl}], 1 \mathrm{~L}[\mathrm{ca}], 1 \mathrm{Bc}, \\
\text { BUC0001-0004 }\end{array}$ & & $\begin{array}{l}\text { Anthropomorphic standing figure on } \\
\text { handle medallion, floral thumb rest }\end{array}$ & $\begin{array}{l}\text { Eggers 1968, } 110 \\
\text { (89b); Philpott } \\
\text { 1991, 261; } \\
\text { Liversidge 1953, } 29 \\
32\end{array}$ \\
\hline BUC0003 & Bowl & Eggers 94 & Buckinghamshire & Thornborough & RS & \begin{tabular}{|l|} 
Thornborough \\
Barrow, possible \\
family tomb
\end{tabular} & $\begin{array}{l}2 \mathrm{Am}[\mathrm{cc}, 3 \mathrm{Bo}[\mathrm{gl}], 1 \mathrm{~L} \text { [ca], } 1 \mathrm{Bc}, \\
\text { BUC0001-0004 }\end{array}$ & & & $\begin{array}{l}\text { Philpott 1991, 261; } \\
\text { Liversidge 1953, } 29 . \\
32\end{array}$ \\
\hline BUC0004 & Handled Pan 1 & Eggers $154-155$ & Buckinghamshire & Thornborough & RS & \begin{tabular}{|l|}
$\begin{array}{l}\text { Thornborough } \\
\text { Barrow, possible } \\
\text { family tomb }\end{array}$ \\
\end{tabular} & $\begin{array}{l}2 \mathrm{Am}[\mathrm{cc}, 3 \mathrm{Bo}[\mathrm{gl}], 1 \mathrm{~L}[\mathrm{ca}], 1 \mathrm{Bc}, \\
\text { BUC0001-0004 }\end{array}$ & & Zoomorphic bear/wolf handle terminal & $\begin{array}{l}\text { Philpott 1991, 261; } \\
\text { Liversidge 1953, } 29 . \\
32\end{array}$ \\
\hline CAM0007 & Vessel & & Cambridgeshire & Cambridge & RS & $\begin{array}{l}\text { Huntington Road, } \\
\text { Gravel Hill, Grave a }\end{array}$ & $4 \mathrm{~F}[\mathrm{gl}]$, Po $3[\mathrm{st}], 1 \mathrm{~F}$ [c] & & & $\begin{array}{l}\text { Philpott 1991, 325; } \\
\text { Liversidge 1977, } 15- \\
16\end{array}$ \\
\hline CAM0008 & Jar & & Cambridgeshire & Godmanchester & RS & $\begin{array}{l}\text { Hinchingbrooke, } \\
\text { Nun's Bridge }\end{array}$ & 3 Bo [g]], $1 \mathrm{~V}[\mathrm{c}], 1 \mathrm{Cn}[\mathrm{s}]$ & $\begin{array}{l}\text { Zoomorphic busts of three beared men } \\
\text { (satyrs?) }\end{array}$ & Floral drop handle & \begin{tabular}{|l} 
Philpott 1991, 332; \\
Wilson, 1968 191 \\
(5); Liversidge 1977, \\
24-25 \\
\end{tabular} \\
\hline $\mathrm{C} 0001$ & Handled Pan 2 & Rudge Cup Type & Cumbria & Brougham & M & \begin{tabular}{|l} 
Roman Cemetary, \\
Grave 107
\end{tabular} & $\begin{array}{l}1 \mathrm{M}[\mathrm{ca}], 1 \mathrm{Un}[\mathrm{ca}], 1 \mathrm{~N}[\mathrm{f}], 2 \mathrm{Un}[\mathrm{b}], 1 \mathrm{~J} \\
{[\mathrm{cc}, 2 \mathrm{~B}[\mathrm{cc}, 1 \mathrm{C}[\mathrm{g}]}\end{array}$ & Floral and Geometric, enameled & & Cool 2004, 124-128 \\
\hline $\mathrm{C} 0002$ & Bucket (fragment) & $\begin{array}{l}\text { Hemmoor, Eggers } \\
58\end{array}$ & Cumbria & Brougham & M & $\begin{array}{l}\text { Roman Cemetary, } \\
\text { Grave } 237\end{array}$ & $\begin{array}{l}1 \mathrm{M}[\mathrm{ca}], 1 \mathrm{Un}[\mathrm{ca}], 1 \mathrm{Po}[\mathrm{g}], 4 \mathrm{Hn}[\mathrm{ff}, 1 \\
\text { Po [g]l, 25 N [f] }\end{array}$ & Floral and Geometric & & Cool 2004, 374 \\
\hline $\mathrm{C} 0003$ & Bucket & $\begin{array}{l}\text { Hemmoor, Eggers } \\
58\end{array}$ & Cumbria & Brougham & M & $\begin{array}{l}\text { Roman Cemetary, } \\
\text { Grave } 235\end{array}$ & $1 \mathrm{M}[\mathrm{ca}], 1 \mathrm{Un}[\mathrm{ca}], 11 \mathrm{~N}[\mathrm{f}], 4 \mathrm{~V}(\mathrm{~F})[\mathrm{c}]$ & & & Cool 2004, 195 \\
\hline $\mathrm{C} 0004$ & Bucket & $\begin{array}{l}\text { Hemmoor, Eggers } \\
58\end{array}$ & Cumbria & Brougham & M & $\begin{array}{l}\text { Roman Cemetary, } \\
\text { Grave } 141\end{array}$ & $20 \mathrm{Hn}[\mathrm{f}], 5 \mathrm{Po}[\mathrm{gl}], 2 \mathrm{~V}(\mathrm{~F})[\mathrm{c}]$ & & & Cool 2004, 144 \\
\hline C0005 & Bucket & $\begin{array}{l}\text { Hemmoor, Eggers } \\
58\end{array}$ & Cumbria & Brougham & M & Roman Cemetary & & Geometric Beaded rim & & Cool 2004, 374 \\
\hline C0006 & Bucket & & Cumbria & Brougham & M & \begin{tabular}{|l} 
Roman Cemetary, \\
Grave 326
\end{tabular} & $\begin{array}{l}8 \mathrm{Un}(\mathrm{F})[\mathrm{ca}], 6 \mathrm{~N}[\mathrm{f}], 1 \mathrm{~B}[\mathrm{c}], 1 \mathrm{~J}[\mathrm{c}], 26 \\
\mathrm{~V}(\mathrm{~F})[\mathrm{c}], 1 \mathrm{Po}[\mathrm{g}]\end{array}$ & & & Cool 2004, 254 \\
\hline C0007 & Bucket & & Cumbria & Brougham & M & \begin{tabular}{|l|} 
Roman Cemetary, \\
Grave 114
\end{tabular} & $\begin{array}{l}5 \mathrm{Un}(\mathrm{F})[\mathrm{ca}], 1 \mathrm{M}[\mathrm{cc}], 1 \mathrm{~N}[\mathrm{ca}], 1 \mathrm{Un}[\mathrm{f}], \\
1 \mathrm{Po}[\mathrm{gl}], 4 \mathrm{~V}(\mathrm{~F})[\mathrm{c}]\end{array}$ & & & Cool 2004, 129 \\
\hline C0008 & Bucket & & Cumbria & Brougham & M & $\begin{array}{l}\text { Roman Cemetary, } \\
\text { Grave 289 }\end{array}$ & $1 \mathrm{M}[\mathrm{b}], 1 \mathrm{~V}[\mathrm{c}]$ & & & Cool 2004, 230 \\
\hline Co009 & Cauldron (fragment) & & Cumbria & Brougham & $\mathrm{M}$ & $\begin{array}{l}\text { Roman Cemetary, } \\
\text { Grave } 198\end{array}$ & \begin{tabular}{|l|}
$\mathrm{Un}[\mathrm{b}], 4 \mathrm{Un}[\mathrm{ca}], 1 \mathrm{~N}[\mathrm{ca}], 1 \mathrm{Kn}[\mathrm{ff}], 130$ \\
$\mathrm{~N}[\mathrm{fl}], 36 \mathrm{HN}[\mathrm{f}], 1 \mathrm{Wf}[\mathrm{b}], 13 \mathrm{Ar}[\mathrm{b}], 3 \mathrm{~V}$ \\
{$[[\mathrm{~g}]$}
\end{tabular} & & & Cool 2004, 173 \\
\hline $\mathrm{C} 0010$ & Bowl & & Cumbria & Brougham & M & $\begin{array}{l}\text { Roman Cemetary, } \\
\text { Grave } 127\end{array}$ & & & & Cool 2004, 136 \\
\hline C0011 & Bowl & & Cumbria & Brougham & M & $\begin{array}{l}\text { Roman Cemetary, } \\
\text { Grave 245 }\end{array}$ & & & & Cool 2004, 203 \\
\hline $\mathrm{C} 0012$ & Bowl & & Cumbria & Brougham & M & \begin{tabular}{|l} 
Roman Cemetary, \\
Grave 239
\end{tabular} & $28 \mathrm{Un}[\mathrm{ca}], 2 \mathrm{Hn}[\mathrm{f}], 10 \mathrm{~N}[\mathrm{f}], 1 \mathrm{~V}$ [gl] & & & Cool 2004, 198 \\
\hline $\mathrm{C} 0013$ & Bowl & & Cumbria & Brougham & M & $\begin{array}{l}\text { Roman Cemetary, } \\
\text { Grave 270 }\end{array}$ & $\begin{array}{l}1 \mathrm{Un}[\mathrm{s}], 1 \mathrm{M}[\mathrm{ca}], 2 \mathrm{Un}[\mathrm{ca}], 1 \mathrm{Hn}[\mathrm{f}], 2 \mathrm{~N} \\
{[\mathrm{f}], \mathrm{A}[\mathrm{b}]}\end{array}$ & & & Cool 2004, 217 \\
\hline $\mathrm{C} 0014$ & Strainer & & Cumbria & Brougham & M & $\begin{array}{l}\text { Roman Cemetary, } \\
\text { Grave } 133\end{array}$ & $\begin{array}{l}23 \mathrm{Un}[\mathrm{ca}], 1 \mathrm{Po}[\mathrm{ff}], 17 \mathrm{~N}[\mathrm{ff}], 1 \mathrm{~V}[\mathrm{gl}], 1 \mathrm{~J} \\
{[\mathrm{cc}]}\end{array}$ & & & Cool 2004, 138 \\
\hline C0015 & Strainer & & Cumbria & Brougham & M & \begin{tabular}{|l} 
Roman Cemetary, \\
Grave 81
\end{tabular} & $2 \mathrm{~N}[\mathrm{f}], 1 \mathrm{~A}[\mathrm{~b}], 1 \mathrm{~J}[\mathrm{c}], 1 \mathrm{Mo}[\mathrm{c}]$ & & & Cool 2004, 106 \\
\hline C0016 & Strainer & & Cumbria & Brougham & M & $\begin{array}{l}\text { Roman Cemetary, } \\
\text { Grave 217 }\end{array}$ & 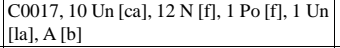 & & & Cool 2004, 185 \\
\hline C0017 & Vessel (mount) & & Cumbria & Brougham & M & $\begin{array}{l}\text { Roman Cemetary, } \\
\text { Grave } 217\end{array}$ & 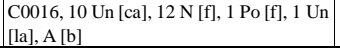 & & & Cool 2004, 185 \\
\hline
\end{tabular}




\begin{tabular}{|c|c|c|c|c|c|c|c|c|c|c|}
\hline $\mathrm{C} 0018$ & Jug & & Cumbria & Brougham & M & $\begin{array}{l}\text { Roman Cemetary, } \\
\text { Grave } 221\end{array}$ & $\mathrm{C} 0019,15 \mathrm{Un}[\mathrm{ca}], 1 \mathrm{Hn}[\mathrm{ff}, 2 \mathrm{~N}[\mathrm{f}]$ & & & Cool 2004, 188 \\
\hline C0019 & Strainer & & Cumbria & Brougham & M & $\begin{array}{l}\text { Roman Cemetary, } \\
\text { Grave } 221\end{array}$ & Coo18, 15 Un [ca], 1 Hn [f], 2 N [f] & & & Cool 2004, 188 \\
\hline $\mathrm{C} 0020$ & Jug & & Cumbria & Brougham & M & $\begin{array}{l}\text { Roman Cemetary, } \\
\text { Grave } 77\end{array}$ & $\begin{array}{l}21 \mathrm{Un}[\mathrm{ca}], 2 \mathrm{Hn}[\mathrm{f}], 15 \mathrm{~N}[\mathrm{f}], 1 \mathrm{Po}[\mathrm{gl}], 1 \\
\mathrm{~V}[\mathrm{gl}], \mathrm{A}[\mathrm{b}], 1 \mathrm{~J}[\mathrm{c}]\end{array}$ & & & Cool 204, 104 \\
\hline DOR0006 & Vessel (fragment) & & Dorset & Woodlands & RS & $\begin{array}{l}\text { Knob's Crook, Pit I, } \\
\text { Cemetary }\end{array}$ & $\begin{array}{l}28 \mathrm{~N}[\mathrm{f}],+\mathrm{V}[\mathrm{c}],+\mathrm{Un}[\mathrm{ca}], 7 \mathrm{Un}[\mathrm{f}],+\mathrm{V} \\
{[\mathrm{ggl}], 5 \mathrm{Un}[\mathrm{st}]}\end{array}$ & & & Fowler, 196536 (1) \\
\hline EX0015 & (2) & & Essex & \begin{tabular}{|l} 
Stansted \\
Mountiftchet
\end{tabular} & RS & $\begin{array}{l}\text { Standsted Airport, } \\
\text { Cremation } 24 \text { (DCS } \\
\text { 251) }\end{array}$ & $\mathrm{Bc}(\mathrm{F})[\mathrm{ca}], 13 \mathrm{~N}[\mathrm{f}], \mathrm{V}[\mathrm{c}], \mathrm{J}[\mathrm{c}]$ & Concentric circle umbo & & $\begin{array}{l}\text { Havis and Brooks } \\
2004,215\end{array}$ \\
\hline EX0016 & Jug & Eggers $123-126$ & Essex & \begin{tabular}{|l} 
Stansted \\
Mountiftchet
\end{tabular} & RS & $\begin{array}{l}\text { Stansted Airport, } \\
\text { Cremation } 25 \text { (DCS } \\
\text { 247) }\end{array}$ & 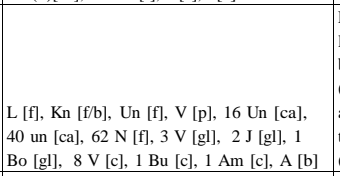 & $\begin{array}{l}\text { Floral neck with palm and trefoil } \\
\text { leaves, trefoil mouth with godronné } \\
\text { border, concentric circles on foot ring } \\
\text { (body); bust of youth/satyr, eagle atop } \\
\text { a globe, basket of fruit, thumb shaped } \\
\text { thumb rest, hoof footed rim frame } \\
\text { (handle) }\end{array}$ & & $\begin{array}{l}\text { Havis and Brooks } \\
2004,216\end{array}$ \\
\hline EX0017 & Handled Pan 1 & Eggers 154-155 & Essex & $\begin{array}{l}\text { Stansted } \\
\text { Mountiftchet }\end{array}$ & RS & \begin{tabular}{|l|} 
Standsted Airport, \\
Cremation 25 (DCS \\
247 )
\end{tabular} & 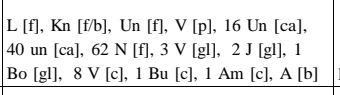 & $\begin{array}{l}\text { Floral umbo with plam and trefoil } \\
\text { leaves }\end{array}$ & $\begin{array}{l}\text { Zoomorphic handle with bear/canine/lion } \\
\text { terminal and rim ornamentation as well as } \\
\text { dolphins on the underside of the body }\end{array}$ & $\begin{array}{l}\text { Havis and Brooks } \\
2004,216\end{array}$ \\
\hline EX0018 & Handled Pan 2 & Eggers 150 & Essex & \begin{tabular}{|l} 
Stansted \\
Mountiftchet
\end{tabular} & RS & $\begin{array}{l}\text { Standsted Airport, } \\
\text { Cremation 25 (DCS } \\
\text { 247) }\end{array}$ & 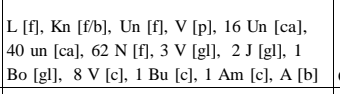 & Concentric circle foot ring & Geometric keyhole shaped handle loop & $\begin{array}{l}\text { Havis and Brooks } \\
2004,224\end{array}$ \\
\hline EX0019 & Jug & Eggers 129 & Essex & \begin{tabular}{|l} 
Stansted \\
Mountiftchet
\end{tabular} & RS & \begin{tabular}{|l} 
Standsted Airport, \\
Cremation 25 (DCS \\
247 )
\end{tabular} & 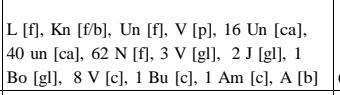 & Concentric circle foot ring & & $\begin{array}{l}\text { Havis and Brooks } \\
2004,226\end{array}$ \\
\hline EX0020 & Bowl & & Essex & $\begin{array}{l}\text { Stansted } \\
\text { Mountiftchet }\end{array}$ & RS & \begin{tabular}{|l|} 
Standsted Airport, \\
Cremation 25 (DCS \\
247 )
\end{tabular} & 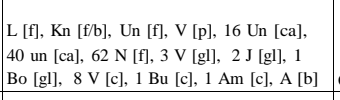 & Concentric circle umbo & & $\begin{array}{l}\text { Havis and Brooks } \\
2004,226\end{array}$ \\
\hline EX0021 & Vessel (fragment) & & Essex & \begin{tabular}{|l} 
Stansted \\
Mountiftchet
\end{tabular} & RS & $\begin{array}{l}\text { Standsted Airport, } \\
\text { Cremation } 25 \text { (DCS } \\
\text { 247) }\end{array}$ & 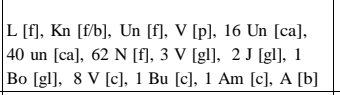 & & & $\begin{array}{l}\text { Havis and Brooks } \\
2004,226\end{array}$ \\
\hline EX0031 & $\begin{array}{l}\text { Handled Pan } 1 \\
\text { (handle) }\end{array}$ & Eggers 154-155 & Essex & Heybridge & RS & The Towers & 然 & Floral Umbo & Fluted handle & $\begin{array}{l}\text { Eggers 1968, } 105 \\
\text { (35a); Wickenden } \\
\text { 1986, 55 (4A) }\end{array}$ \\
\hline EX0034 & Handled Pan 2 & Eggers 150 & Essex & Colchester & $\mathrm{u}$ & Joslin Collection & $\begin{array}{l}10 \mathrm{~V}[\mathrm{cc}, 3 \mathrm{Bo}[\mathrm{gl}], 1 \mathrm{Bc}, 36 \mathrm{Cn}, 21 \mathrm{Ar}[\mathrm{cc}, \\
+\mathrm{Co}[\mathrm{b}]\end{array}$ & 年 & Keyhole shaped handle loop & \begin{tabular}{|l|} 
Philpott, $\quad 1991 \quad 289 ;$ \\
May 1930, 251-253 \\
(1141); Eckardt \\
1999,74-76 \\
$(40 / 1141)$ \\
\end{tabular} \\
\hline EX0035 & tion & Eggers 123-126 & Essex & Rivenhall & RS & $\begin{array}{l}\text { Barrow Field, } \\
\text { Possible Grave }\end{array}$ & fecopos & & $\begin{array}{l}\text { Zoomorphic lion's head thumb rest, } \\
\text { zoomorphic lion's paw handle medallion }\end{array}$ & $\begin{array}{l}\text { Rodwell, } 1978 \text { 15; } \\
\text { Rodwell \& Rodwwell } \\
\text { 1993, 35-36 }\end{array}$ \\
\hline EX0036 & Handled Pan 1 & Eggers 154-155 & Essex & Rivenhall & RS & $\begin{array}{l}\text { Barrow Field, } \\
\text { Possible Grave }\end{array}$ & EX0044 & Concentric circle foot ring & & \begin{tabular}{|l|} 
Rodwell, 1978 15; \\
Rodwell \& Rodwell \\
1993,35-36 \\
\end{tabular} \\
\hline EX0038 & Jug (handle) & & Essex & Heybridge & RS & The Towers & $1 \mathrm{~A}[\mathrm{c}], \mathrm{EX} 0031$ & & \begin{tabular}{|l} 
Anthropomorphic harpy thumb rest and \\
handle medallion
\end{tabular} & \begin{tabular}{|l|}
$\begin{array}{l}\text { Wickenden } 1986,55 \\
(4 \mathrm{~A})\end{array}$ \\
\end{tabular} \\
\hline EX0058 & Jug & & Essex & Ashdon & RS & $\begin{array}{l}\text { Bartlow Hills, } \\
\text { Barrow IV, } \\
\text { Cemetary }\end{array}$ & $\begin{array}{l}1 \mathrm{AM}[\mathrm{c}], 1 \mathrm{Bc}[\mathrm{w}], 1 \mathrm{Fn}[\mathrm{ca}], 2 \mathrm{St} \text { [ca] }, 5 \\
\text { Bo [gl], } 1 \mathrm{~L} \text { [ca], EX0034-EX0036 }\end{array}$ & Floral neck ring & $\begin{array}{l}\text { Zoomorphic bull skull handle medallion, } \\
\text { zoomorphic sphinx standing on storks } \\
\text { thumb rest }\end{array}$ & $\begin{array}{l}\text { Philpott, 1991 256; } \\
\text { VCH 3 (Essex) 1963 } \\
41 \text {; Eckardt 2009, } 65 \\
98\end{array}$ \\
\hline EX0059 & Handled Pan 1 & Eggers 154-155 & Essex & Ashdon & RS & $\begin{array}{l}\text { Bartlow Hills, } \\
\text { Barrow IV, } \\
\text { Cemetary }\end{array}$ & $\begin{array}{l}1 \mathrm{AM}[\mathrm{c}], 1 \mathrm{Bc}[\mathrm{w}], 1 \mathrm{Fn}[\mathrm{ca}], 2 \mathrm{St}[\mathrm{cc}], 5 \\
\text { Bo [gl], } 1 \mathrm{~L} \text { [ca], EX0034-EX0036 }\end{array}$ & & $\begin{array}{l}\text { Zoomorphic rams head handle terminal, } \\
\text { fluted handle }\end{array}$ & $\begin{array}{l}\text { Philpott, } 1991 \text { 256; } \\
\text { VCH 3 (Essex) 1963 } \\
41 \text {; Eckardt 2009, } 65 \\
98\end{array}$ \\
\hline EX0060 & Jar & & Essex & Ashdon & RS & $\begin{array}{l}\text { Bartlow Hills, } \\
\text { Barrow IV, } \\
\text { Cemetary }\end{array}$ & $\begin{array}{l}1 \mathrm{AM}[\mathrm{c}], 1 \mathrm{Bc}[\mathrm{ww}], 1 \mathrm{Fn}[\mathrm{ca}], 2 \mathrm{St} \text { [ca], }, \\
\text { Bo [gl], } 1 \mathrm{~L} \text { [ca], EX0034-EX0036 }\end{array}$ & $\begin{array}{l}\text { Floral leaves and vines, geometric } \\
\text { triangle incised bands, linear etchings } \\
\text { on rim, enameled }\end{array}$ & $\begin{array}{l}\text { Geometric linear etchings, geometric } \\
\text { tapered terminals to drop loo handle }\end{array}$ & \begin{tabular}{|l|} 
VCH 3 (Essex) 1963 \\
41 ; Eckardt 2009, \\
$65-98 ; \mathrm{BM}$ \\
1868.0801 .1
\end{tabular} \\
\hline EX0061 & Jug & Eggers 123-126 & Essex & Ashdon & RS & $\begin{array}{l}\text { Bartlow Hills, } \\
\text { Barrow III, } \\
\text { Cemetary }\end{array}$ & 3 Bo [gl], $1 \mathrm{~L}[\mathrm{f}], 1 \mathrm{~V}[\mathrm{c}]$ & & & $\begin{array}{l}\text { VCH3 3(Essex) 1963 } \\
\text { 40; Eckardt 2009, } \\
65-98\end{array}$ \\
\hline EX0062 & Handled Pan 1 & Eggers 154-155 & Essex & Ashdon & RS & \begin{tabular}{|l|}
$\begin{array}{l}\text { Bartlow Hills, } \\
\text { Barrow III, } \\
\text { Cemetary }\end{array}$ \\
\end{tabular} & $3 \mathrm{Bo}[\mathrm{g}], 1 \mathrm{~L}[\mathrm{f}], 1 \mathrm{~V}[\mathrm{c}]$ & & $\begin{array}{l}\text { Zoomorphic ram's head handle terminal, } \\
\text { fluted handle }\end{array}$ & $\begin{array}{l}\text { VCH3 (Essex) } 1963 \\
40 ; \text { Eckardt 2009, } 65 \\
98\end{array}$ \\
\hline
\end{tabular}




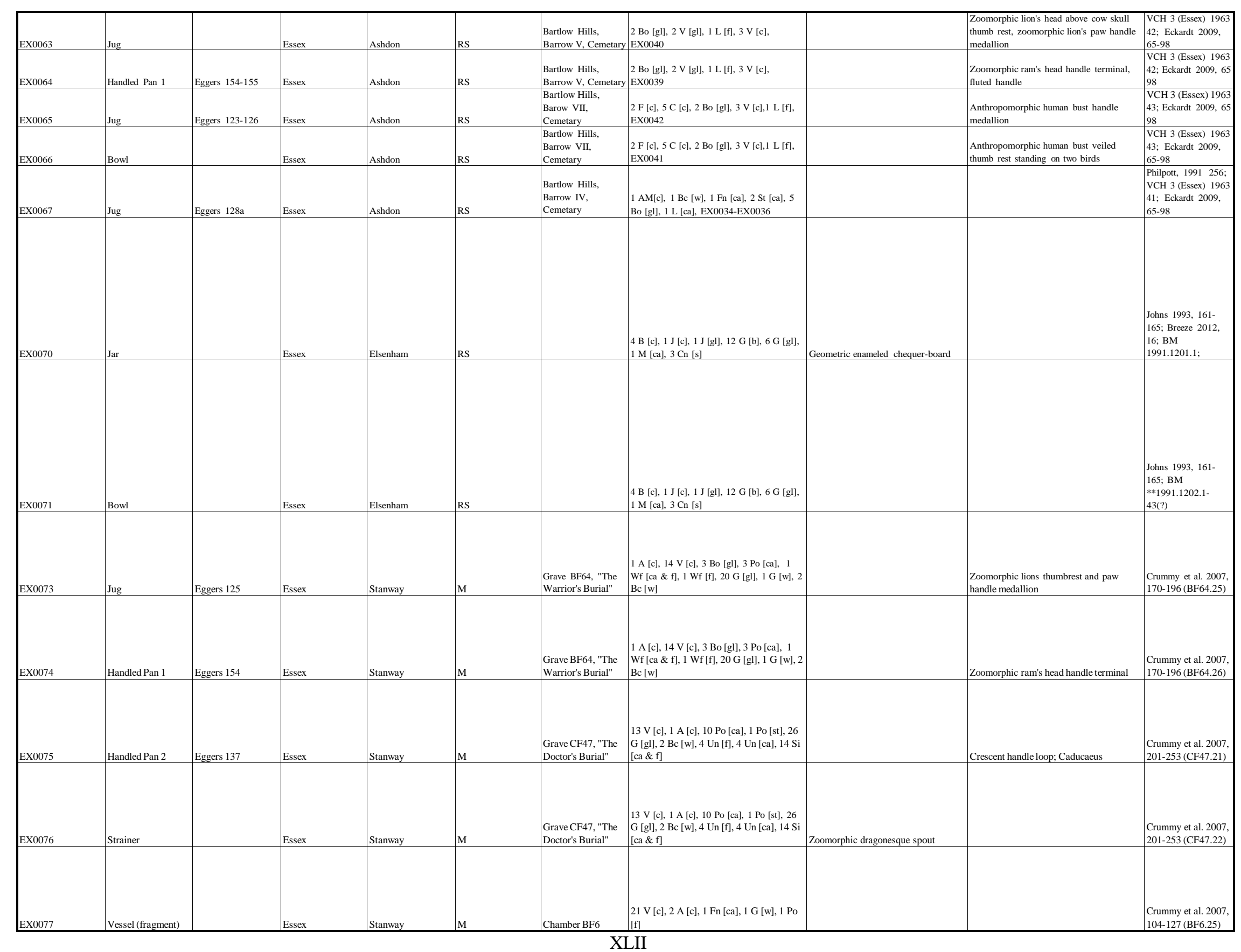




\begin{tabular}{|c|c|c|c|c|c|c|c|c|c|c|}
\hline HAM0003 & Jug & Eggers 123-126 & Hampshire & Winchester & $\mathrm{U}$ & $\begin{array}{l}\text { Grange Road, Grave } \\
2\end{array}$ & 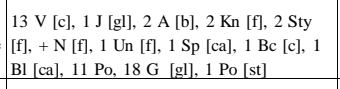 & Trefoil mouth & $\begin{array}{l}\text { Anthropomorphic female bust thumb rest } \\
\text { and handle medallion }\end{array}$ & $\begin{array}{l}\text { Philpott, 1991 270; } \\
\begin{array}{l}\text { Biddle, } 1967 \text { 230- } \\
\text { 231, 240-242 }\end{array}\end{array}$ \\
\hline HER0019 & Bowl & Eggers 98-99 & Hertfordshire & St. Albans & $\mathrm{u}$ & \begin{tabular}{|l} 
Verulamium, \\
Silchester Gate, \\
Mayne Avenue \\
(Cremation Pit) \\
\end{tabular} & $\begin{array}{l}15 \mathrm{~V} \text { [c], } 1 \text { Bo [gl], } 2 \mathrm{~J}[\mathrm{gl}], 1 \mathrm{Fn}[\mathrm{fl}], \mathrm{Co} \\
{[\mathrm{cac}, 4 \mathrm{~L}[\mathrm{c}], 20 \mathrm{G}[\mathrm{gl}]]}\end{array}$ & $\begin{array}{l}\text { Concentric circle interior and exterior } \\
\text { of body, Concentric cirlce foot ring; } \\
\text { omphalos evident }\end{array}$ & $\begin{array}{l}\text { Anthropomorphic female bust thumb rest; } \\
\text { zoomorphic birds heads; Floral leaf handle } \\
\text { and lower handle medallion }\end{array}$ & $\begin{array}{l}\text { Niblett \& Reeves } \\
\text { 1990, } 441-446 \text { (Fig. } \\
\text { 1) }\end{array}$ \\
\hline HER 0030 & Bowl & & Hertfordshire & Stanton & RS & $\begin{array}{l}\text { Ermine Street; Site } \\
\text { D }\end{array}$ & $3 \mathrm{~V}$ [c] & $\begin{array}{l}\begin{array}{l}\text { Concentric circle umbo and omphalos } \\
\text { dot }\end{array} \\
\end{array}$ & & $\begin{array}{l}\text { Potter \& Trow 1988, } \\
58-59 \text { (7) }\end{array}$ \\
\hline HER0031 & Jug & Eggers 127 & Hertfordshire & Harpenden & RS & \begin{tabular}{|l} 
Turners Hall Farm, \\
Grave 1
\end{tabular} & $\begin{array}{l}\text { HER0031-HER0040; } 30 \mathrm{~W} \text { [f], } 2 \text { B [gl], } 2 \\
\text { Bo [gl], 1 J [gl], 8 B [c] }\end{array}$ & & $\begin{array}{l}\text { Anthropomorphic Triton statuette at crest } \\
\text { of handle by rim holding a jug and pan; } \\
\text { Anthropomorphic Medusa head handle } \\
\text { medallion }\end{array}$ & $\begin{array}{l}\text { Burnham et al. 2003, } \\
327\end{array}$ \\
\hline HER0032 & Jug & . & Hertfordshire & Harpenden & RS & $\begin{array}{l}\text { Turners Hall Farm, } \\
\text { Grave } 1\end{array}$ & $\begin{array}{l}\text { HER0031-HER0040; } 30 \mathrm{~W} \text { [f], 2 B [gl], } 2 \\
\text { Bo [gl], 1 J J }[\mathrm{gl}], 8 \text { B [c] }\end{array}$ & & $\begin{array}{l}\text { Anthropomorphic Athena/Minerva handle } \\
\text { medallion (helmeted female); Shield on } \\
\text { handle; bowl of fruit atop a three footed } \\
\text { table }\end{array}$ & $\begin{array}{l}\text { Burnham et al. 2003, } \\
327\end{array}$ \\
\hline HER0033 & Jug & & Hertfordshire & Harpenden & RS & $\begin{array}{l}\text { Turners Hall Farm, } \\
\text { Grave } 1\end{array}$ & $\begin{array}{l}\text { HER0031-HER0040; } 30 \mathrm{~W} \text { [f], 2 B [gl], } 2 \\
\text { Bo [gl], 1 J [gl]], 8 B [c] }\end{array}$ & & & $\begin{array}{l}\text { Burnham et al. } 2003 \text {, } \\
327\end{array}$ \\
\hline HER0034 & Bowl & & Hertfordshire & Harpenden & RS & $\begin{array}{l}\text { Turners Hall Farm, } \\
\text { Grave } 1\end{array}$ & $\begin{array}{l}\text { HER0031-HER0040; } 30 \mathrm{~W}[\mathrm{ff} \text {, 2 B [gl], } 2 \\
\text { Bo [gl], 1 J [gl], 8 B [c] }\end{array}$ & & & $\begin{array}{l}\text { Burnham et al. } 2003 \text {, } \\
327\end{array}$ \\
\hline HER0035 & Strainer & & Hertfordshire & Harpenden & RS & $\begin{array}{l}\text { Turners Hall Farm, } \\
\text { Grave } 1 \\
\end{array}$ & $\begin{array}{l}\begin{array}{l}\text { HRR0031-HER0040; } 30 \mathrm{~W}[\mathrm{ff}, 2 \text { B [gl], } 2 \\
\text { Bo [gl], 1 J [gl], 8 B [c] }\end{array} \\
\end{array}$ & & & $\begin{array}{l}\text { Burnham et al. } 2003 \text {, } \\
327\end{array}$ \\
\hline HER0036 & Strainer & & Hertfordshire & Harpenden & RS & \begin{tabular}{|l|} 
Turners Hall Farm, \\
Grave 1
\end{tabular} & $\begin{array}{l}\text { HER0031-HER0040; } 30 \mathrm{~W}[\mathrm{ff}, 2 \mathrm{~B}[\mathrm{~g}] \mathrm{l}, 2 \\
\text { Bo [gl], 1 J [gl], 8 B [c] }\end{array}$ & & & $\begin{array}{l}\begin{array}{l}\text { Burnham et al. } 2003, \\
327\end{array} \\
327\end{array}$ \\
\hline HER0037 & Handled Pan 1 & Eggers $154-155$ & Hertfordshire & Harpenden & RS & \begin{tabular}{|l|} 
Turners Hall Farm, \\
Grave 1
\end{tabular} & $\begin{array}{l}\text { HER0031-HER0040; } 30 \mathrm{~W}[\mathrm{ff}, 2 \mathrm{~B}[\mathrm{gl}], 2 \\
\text { Bo [gl], 1 J [gl]], 8 B [c] }\end{array}$ & & $\begin{array}{l}\text { Zoomorphic ram's head terminal, fluted } \\
\text { handle }\end{array}$ & $\begin{array}{l}\text { Burnham et al. 2003, } \\
327\end{array}$ \\
\hline HER0038 & Handled Pan 2 & Eggers 150 & Hertfordshire & Harpenden & RS & $\begin{array}{l}\text { Turners Hall Farm, } \\
\text { Grave } 1\end{array}$ & $\begin{array}{l}\text { HER0031-HER0040; } 30 \mathrm{~W}[\mathrm{ff}, 2 \mathrm{~B}[\mathrm{gl}], 2 \\
\text { Bo [gl], 1 J [gl]], 8 B [c] }\end{array}$ & & & $\begin{array}{l}\text { Burnham et al. 2003, } \\
327\end{array}$ \\
\hline HER0039 & Handled Pan 2 & Eggers 150 & Hertfordshire & Harpenden & RS & $\begin{array}{l}\text { Turners Hall Farm, } \\
\text { Grave } 1\end{array}$ & $\begin{array}{l}\text { HER0031-HER0040; } 30 \mathrm{~W}[\mathrm{fl}, 2 \mathrm{~B}[\mathrm{~g}]], 2 \\
\text { Bo [gl], 1 J [gl], 8 B [c] }\end{array}$ & & & $\begin{array}{l}\text { Burnham et al. 2003, } \\
327\end{array}$ \\
\hline HER0040 & Bowl & & Hertfordshire & Harpenden & RS & $\begin{array}{l}\text { Turners Hall Farm, } \\
\text { Grave } 1\end{array}$ & $\begin{array}{l}\text { HER0031-HER0040; } 30 \mathrm{~W}[\mathrm{f}] \text {, } 2 \text { [ [gl], } 2 \\
\text { Bo [gl], 1 J [gl], 8 B [c] }\end{array}$ & & & $\begin{array}{l}\text { Burnham et al. } 2003 \text {, } \\
327\end{array}$ \\
\hline HER0041 & Jug & Eggers 127 & Hertfordshire & Harpenden & RS & $\begin{array}{l}\text { Turners Hall Farm, } \\
\text { Grave } 2\end{array}$ & $\begin{array}{l}\text { HER0041-HER0042, } 1 \text { L [ca], 5 B [c], } 2 \\
\text { Bo [gl], 1 B [gl] }\end{array}$ & & $\begin{array}{l}\text { Zoomorphic bull thumb rest; } \\
\text { Anthropomorphic gorgon head handle } \\
\text { medallion }\end{array}$ & $\begin{array}{l}\text { Burnham et al. } 2003 \text {, } \\
327\end{array}$ \\
\hline HER0042 & Jug & & Hertfordshire & Harpenden & RS & $\begin{array}{l}\text { Turners Hall Farm, } \\
\text { Grave 2 }\end{array}$ & $\begin{array}{l}\text { HER0041-HER0042, } 1 \text { L [ca], 5 B [c], } 2 \\
\text { Bo [gl], 1 B [gl] }\end{array}$ & & & $\begin{array}{l}\text { Burnham et al. } 2003 \text {, } \\
327\end{array}$ \\
\hline K0001 & Jug & & Kent & Lullingstone & RS & Temple/Mausoleum & $\begin{array}{l}\text { A 1[b], F 1[c], B 2[g]], Bo 4[g]l, G } 1[\mathrm{w}] \\
30[\mathrm{gl}] 17[\mathrm{~b}], \text { Kn 2[f], S 2[f] }\end{array}$ & & & $\begin{array}{l}\text { Meates, } 1979 \text { 122- } \\
1322\end{array}$ \\
\hline K0002 & Jug & & Kent & $\begin{array}{l}\text { Maidstone, between } \\
\text { Pested Bars and } \\
\text { Lockham, Joy } \\
\text { Wood, Cremated } \\
\text { Deposit 3, Amphora } \\
\text { X } \\
\end{array}$ & RS & Cemetary & Bo 1[gl], L 1[f], T 1[uo], Am 1[c] K0003 & & & $\begin{array}{l}\text { Philpott, } 1991 \text { 254; } \\
\text { Scott Robinson, } \\
188378 \text { (Xb); } \\
\text { Jessup, } 1958 \text { 26 (3) }\end{array}$ \\
\hline K0003 & Jug & Eggers 129 & Kent & \begin{tabular}{|l|} 
Maidstone, between \\
pested bars and \\
Lockham, Joy \\
Wood, Cremated \\
Deposit 3, Amphora \\
X \\
\end{tabular} & RS & Cemetary & Bo 1[gl], L 1[f], T 1[uo], Am 1[c] K0002 & & & $\begin{array}{l}\text { Philpott, } 1991 \text { 254; } \\
\text { Scott Robinson, } \\
\text { |883 } 78 \text { (Xc); } \\
\text { Jessup, } 1958 \text { 26 (3) } \\
\end{array}$ \\
\hline K0004 & Jug & & Kent & $\begin{array}{l}\text { Maidstone, between } \\
\text { Pested Bars and } \\
\text { Lockham, Joy } \\
\text { Wood, Cremated } \\
\text { Deposit3, Amphora } \\
\text { X** } \\
\end{array}$ & RS & Cemetary & Bo 1[gl], T 1[uo], L 1[f] K0005 & & & $\begin{array}{l}\text { Philpott, } 1991 \text { 259; } \\
\text { Jessup } 195827 \text { (5) }\end{array}$ \\
\hline K0005 & Jug & & Kent & $\begin{array}{l}\text { Maidstone, between } \\
\text { Pested Bars and } \\
\text { Lockham, Joy } \\
\text { Wood, Cremated } \\
\text { Deposit3, Amphora } \\
\mathrm{X}^{* * *} \\
\end{array}$ & RS & Cemetary & Bo 1[g]], T 1[uo], L 1[f] K0004 & & & Philpott, 1991259 \\
\hline K0006 & Vessel & & Kent & Maidstone & RS & & & & & Philpott 1991259 \\
\hline K0007 & Jug & & Kent & \begin{tabular}{|l} 
Sittingbourne, \\
Bayford
\end{tabular} & RS & & $\begin{array}{l}\text { L 1[ca], Bo 1[g]], J 1[g]], St 1[f], V 12[c], } \\
\text { A 1[b] K0007-K0010 }\end{array}$ & & $\begin{array}{l}\text { Anthropomorphic winged female (?) figure, } \\
\text { possibly arrpy or siren }\end{array}$ & Philpott 1991259 \\
\hline Ko008 & Bowl & & Kent & $\begin{array}{l}\text { Sittingbourne, } \\
\text { Bayford }\end{array}$ & RS & & $\begin{array}{l}\text { L 1 [ca], Bo 1[g]], J 1[g]], St 1[f], V 12[c], } \\
\text { A 1[b] K0007-K0010 }\end{array}$ & & & Philpott 1991259 \\
\hline
\end{tabular}




\begin{tabular}{|c|c|c|c|c|c|c|c|c|c|c|}
\hline K0009 & Jug & & Kent & \begin{tabular}{|l|}
$\begin{array}{l}\text { Sittingbourne, } \\
\text { Bayford }\end{array}$ \\
\end{tabular} & RS & & $\begin{array}{l}\text { L 1[ca], Bo 1[g], J J [g]], St 1[f], V 12[c], } \\
\text { A 1[b] K0007-K0010 }\end{array}$ & Anthropomorphic negroid faces & & Philpott 1991259 \\
\hline K0010 & Handled Pan 1 & Eggers 155 & Kent & $\begin{array}{l}\text { Sittingbourne, } \\
\text { Bayford }\end{array}$ & $\mathrm{RS}$ & & $\begin{array}{l}\text { L 1[ca], Bo 1[g]], J 1[g]], St 1[f], V 12[c], } \\
\text { A [1] K0007-K0010 }\end{array}$ & & \begin{tabular}{|l|} 
Anthropomorphiic satyr head it beard, horns \\
and pointed ears eyes have space where \\
semi-precious stones or gems may have once \\
been inserted
\end{tabular} & $\begin{array}{l}\text { Philpott 1991 259; } \\
\text { BM 1883.1213.299 }\end{array}$ \\
\hline K0011 & Jug & & Kent & $\begin{array}{l}\text { Sittingbourne, } \\
\text { Bayford }\end{array}$ & RS & & Bo 1[g]], J 1[gl], Un 3[gl], St 3[ui], L 1[f] & & $\begin{array}{l}\text { Anthropomorphic Ajax in frenzy attacking } \\
\text { zoomorphic beasts }\end{array}$ & $\begin{array}{l}\text { Philpott } 1991259 \text { 259; } \\
\text { BM 1883.1213.320 }\end{array}$ \\
\hline K0012 & Jug & & Kent & Canterbury & $\mathrm{u}$ & Martyrs Field Road & Co 1[c] 1 [ui], Po 3[c], Bo 1[g]] K0013 & & & \begin{tabular}{|l|} 
Philpott $1991 \quad 314 ;$ \\
Eggers $1996 \quad 102$; \\
Musum of \\
Canterbury $7847-$ \\
7848
\end{tabular} \\
\hline K0013 & Handled Pan 1 & Eggers 155 & Kent & Canterbury & $\mathrm{u}$ & Martyrs Field Road & Co 1[c] 1 [ui], Po 3[c], Bo 1[g]] K0012 & $\begin{array}{l}\text { Zoomorphic ram's head terminal, } \\
\text { fluted handle }\end{array}$ & & \begin{tabular}{|l|} 
Philpott 1991 314; \\
Egggers 1996102 ; \\
Museum of \\
Canterbury $7847-$ \\
7848
\end{tabular} \\
\hline Ko014 & Cauldron & & Kent & Springhead & RS & $\begin{array}{l}\text { Vagniacae, Toll } \\
\text { Gate, Cremation Pit }\end{array}$ & $18 \mathrm{~V}$ [c], G [v], K0015, K0016 & $\begin{array}{l}\text { Geometric scalloped rim, perhaps } \\
\text { depicting the evil eye }\end{array}$ & $\begin{array}{l}\text { Zoomorphic duck's head drop-loop handle; } \\
\text { Florall leaf shaped hanging vessel mount }\end{array}$ & Mynott 2008, 15 \\
\hline Ko015 & Handled Pan 1 & Eggers 155 & Kent & Springhead & RS & \begin{tabular}{|l|l|} 
Vagniacae, Toll \\
Gate, Cremation Pit
\end{tabular} & 18 V[c], G [v], K0014, K0016 & Concentric circle umbo & $\begin{array}{l}\text { Zoomorphic ram's head terminal, fluted } \\
\text { handle }\end{array}$ & Mynott 2008, 15 \\
\hline K0016 & Jug & & Kent & Springhead & RS & $\begin{array}{l}\text { Vagniacae, Toll } \\
\text { Gate, Cremation Pit }\end{array}$ & $18 \mathrm{~V}$ [c], G [v], K0014, K0015 & & $\begin{array}{l}\text { Anthropomorphic female bust thumb rest } \\
\text { and handle medallion }\end{array}$ & Mynott 2008,15 \\
\hline K0017 & Handled Pan 1 & Eggers 155 & Kent & Springhead & RS & $\begin{array}{l}\text { Vagniacae, Toll } \\
\text { Gate, Cremation Pit }\end{array}$ & $15 \mathrm{~V}[\mathrm{c}], \mathrm{K} 0018$ & $\begin{array}{l}\text { Floral flower in centre of basin circled by } \\
\text { two bands of floral wreathes }\end{array}$ & $\begin{array}{l}\text { Zoomorphic ram's head terminal, fluted } \\
\text { handle }\end{array}$ & Mynott 2008,15 \\
\hline K0018 & Jug & & Kent & Springhead & RS & $\begin{array}{l}\text { Vagniacae, Toll } \\
\text { Gate, Cremation Pit }\end{array}$ & $15 \mathrm{~V}$ [c], K0019 & & \begin{tabular}{|l|} 
Anthropomorphic female handle medallion; \\
Zoomorphic lion'd head thumb rest
\end{tabular} & Mynott 2008, 15 \\
\hline K0020 & Bowl & Eggers 99 (c.f.) & Kent & Luton & RS & & $\begin{array}{l}1 \mathrm{Bc}[\mathrm{ca}], 1 \mathrm{Bo}[\mathrm{gl}], 1 \mathrm{~B}[\mathrm{gl}], 3 \mathrm{~V} \text { [c], } 2 \mathrm{C} \\
{[\mathrm{cc}, \mathrm{K} 0027}\end{array}$ & $\begin{array}{l}\begin{array}{l}\text { Footed bowl, "AFRICANVS" } \\
\text { inscription }\end{array} \\
\end{array}$ & & 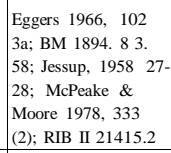 \\
\hline K0025 & Bucket & $\begin{array}{l}\text { Hemmoor, Eggers } \\
56-58\end{array}$ & Kent & Ramsgate & RS & Cremation Pit & & $\begin{array}{l}\text { Concentric cirlce rim, drop handle, } \\
\text { trefoil handle fittings }\end{array}$ & & $\begin{array}{l}\text { Eggers } 1966,102 \\
\text { 5A; Smith 1922, } 93- \\
\text { 94; BM 1901.0716.1 }\end{array}$ \\
\hline K0027 & Jug & & Kent & Luton & RS & & $\begin{array}{l}1 \mathrm{Bc}[\mathrm{ca}], 1 \mathrm{Bo}[\mathrm{gl}], 1 \mathrm{~B}[\mathrm{gl}], 3 \mathrm{~V}[\mathrm{c}], 2 \mathrm{C} \\
{[\mathrm{cc}, \mathrm{K0020}}\end{array}$ & & & $\begin{array}{l}\text { Jessup 1958, 27-28; } \\
\text { BM 1894.0803.59 }\end{array}$ \\
\hline K0028 & Bowl & & Kent & Canterbury & $\mathrm{U}$ & Palace Street & $2 \mathrm{Un}[\mathrm{f}]$ & & & Smith, 192286 \\
\hline K0043 & Jug & & Kent & Ashford & RS & Cremation Pit & K0043-K0044, 1 В [c], 1 Bo [w], $1 \mathrm{Bu}[\mathrm{w}]$ & & & \begin{tabular}{|l|} 
Burnham et alia \\
2001,382
\end{tabular} \\
\hline K0044 & Vessel (fragment) & & Kent & Ashford & RS & Cremation Pit & K0043-K0044, 1 В [c], 1 Bo [w], $1 \mathrm{Bu}[\mathrm{w}]$ & & & $\begin{array}{l}\text { Burnham et alia } \\
2001,382\end{array}$ \\
\hline MON0007 & Jug & & Monmouthshire & Llantilio Pertholey & $\mathrm{RU}$ & & & $\begin{array}{l}\text { Zoomorphic leopard handle with silver } \\
\text { spot inlays }\end{array}$ & & \begin{tabular}{|l|} 
PAS NMGW- \\
9A9D16
\end{tabular} \\
\hline NU0049 & Jar & & Northumberland & Corbridge & M & Burial 508 & $3 \mathrm{Po}[\mathrm{ca}],+\mathrm{N}[\mathrm{f}],, 1$ Po [c], $1 \mathrm{~L}[\mathrm{cc}]$ & & & $\begin{array}{l}\text { Casey \& Hoffman, } \\
\text { 1995b, } 24 \text { (6) }\end{array}$ \\
\hline PO0002 & Jug & & Powys & Welshpool & RS & & $\begin{array}{l}\text { PO0002-PO0007, } 3 \text { Fn [f], , J [gl], } 1 \text { B } \\
\text { [gl], 2 V [c] }\end{array}$ & & Anthropomorphic satyr & $\begin{array}{l}\text { Eggers 1968, } 103 \\
\text { (16 1); Boon } 1961 \\
13(1)\end{array}$ \\
\hline $\mathrm{PO} 0003$ & Handled Pan 1 & Eggers 154-155 & Powys & Welshpool & RS & & $\begin{array}{l}\text { PO0002-PO0007, } 3 \text { Fn [f], 1 J [gl], } 1 \text { B } \\
[\mathrm{g}] 1], 2 \text { V [c] }\end{array}$ & & $\begin{array}{l}\text { Anthropomorphic human bust handle } \\
\text { terminal and thumb rest }\end{array}$ & $\begin{array}{l}\text { Eggers 1968, } 103 \\
\text { (162); Boon } 1961 \\
13(2)\end{array}$ \\
\hline PO0004 & Handled Pan 1 & Eggers 154-155 & Powys & Welshpool & RS & & $\begin{array}{l}\begin{array}{l}\text { PO00002-PO0007, } 3 \mathrm{Fn}[\mathrm{ff}], 1 \mathrm{~J}[\mathrm{~g}]], 1 \mathrm{~B} \\
{[\mathrm{gl}], 2 \mathrm{~V}[\mathrm{c}]}\end{array}\end{array}$ & Floral (exterior body, under handle) & $\begin{array}{l}\text { Zoomorphic ram's head handle terminal, } \\
\text { fluted handle }\end{array}$ & $\begin{array}{l}\text { Eggers 1968, } 103 \\
(161) \text {; Boon } 1961 \\
13 \text { (3) }\end{array}$ \\
\hline PO0005 & Handled Pan 1 & Eggers 154-155 & Powys & Welshpool & RS & & $\begin{array}{l}\text { PO0002-PO0007, 3 Fn [f], 1 J [gl], } 1 \text { B } \\
[\mathrm{g}] 1], 2 \text { V [c] }\end{array}$ & & $\begin{array}{l}\text { Zoomorphic ram's head handle terminal, } \\
\text { fluted handle }\end{array}$ & $\begin{array}{l}\text { Eggers 1968, } 103 \\
\text { (16 1); Boon } 1961 \\
13(4)\end{array}$ \\
\hline PO0006 & Cauldron & & Powys & Welshpool & RS & & $\begin{array}{l}\text { PO0002-PO0007, } 3 \text { Fn [f], } 1 \mathrm{~J} \text { [gl], } 1 \text { B } \\
{[\mathrm{gl}], 2 \text { V [c] }}\end{array}$ & & & $\begin{array}{l}\text { Eggers 1968, } 103 \\
\text { (161);:Boon } 196113 \\
\text { (5) }\end{array}$ \\
\hline
\end{tabular}




\begin{tabular}{|c|c|c|c|c|c|c|c|c|}
\hline \begin{tabular}{|l|} 
Poo0007 \\
WSU0010
\end{tabular} & $\begin{array}{l}\text { Vessel (mount) } \\
\text { Juge }\end{array}$ & $\frac{\text { Powys }}{\text { Wess Sussex }}$ & \begin{tabular}{|l|l|} 
Welshpool \\
Chichester
\end{tabular} & $\frac{\mathrm{RS}}{\mathrm{U}}$ & Westergate & 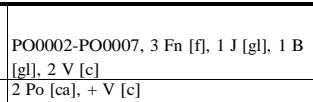 & $\begin{array}{l}\begin{array}{l}\text { Zoomorphic bull's head hanging vessel } \\
\text { mount }\end{array} \\
\end{array}$ & 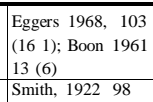 \\
\hline
\end{tabular}


Appendix IV: Site Finds of Copper Alloy Vessels in Roman Britain.

\begin{tabular}{|c|c|c|c|c|c|c|c|c|c|}
\hline Number & Form & Type & County & Site & Context & Location & Decoration (body) & Decoration (handle) & Reference \\
\hline AV0001 & Jug (fragment) & & Avon & Bath & RS & $\begin{array}{l}\text { Swallow Street, } \\
\text { Context } 390\end{array}$ & $\begin{array}{l}\text { Zoomorphic duck thumb- } \\
\text { rest }\end{array}$ & & $\begin{array}{l}\text { Davenport 1991, } 81 \\
\text { (1) }\end{array}$ \\
\hline BERK0001 & Jug & Eggers 128 & Berkshire & Thatcham & RNS & Near spring & Concentric rings on neck & & $\begin{array}{l}\text { Eggers 1966, } 103 \\
(7)\end{array}$ \\
\hline BUC0005 & Handled Pan 1 & Eggers 154-155 & Buckinghamshire & Milton Keynes & RS & Olney & $\begin{array}{l}\text { Concentric cirlce umbo and } \\
\text { foot ring }\end{array}$ & Zoomorphic bear/wolf handle terminal & Farley 1977, 485 \\
\hline CAR0003 & $\begin{array}{l}\text { Handled Pan } 2 \\
\text { (handle) }\end{array}$ & Rudge Cup Type & Carmarthenshire & Carmarthen & M & Priory Street & & Floral vine and leaf enameled in blue & $\begin{array}{l}\text { James 2003, } 317 \\
(57)\end{array}$ \\
\hline CAR0004 & Jug (lid) & Eggers 123-126 & Carmarthenshire & Carmarthen & M & Church Street & $\begin{array}{l}\text { Floral (possibly) leaf } \\
\text { shaped jug lid }\end{array}$ & & $\begin{array}{l}\text { James 2003, } 319 \\
(60)\end{array}$ \\
\hline CAR0005 & Vessel (fragment) & & Carmarthenshire & Coygan Camp & RS & & Concentric circle umbo & & $\begin{array}{l}\text { Wainwright 1967, } \\
93 \text { (24.7) }\end{array}$ \\
\hline CAR0006 & Vessel (fragment) & & Carmarthenshire & Coygan Camp & RS & & & & $\begin{array}{l}\text { Wainwright 1967, } \\
93 \text { (24.9) }\end{array}$ \\
\hline CAR0007 & Vessel (fragment) & & Carmarthenshire & Coygan Camp & RS & & & & $\begin{array}{l}\text { Wainwright 1967, } \\
93(24.10)\end{array}$ \\
\hline CAR0008 & Vessel (fragment) & & Carmarthenshire & Coygan Camp & RS & & & & $\begin{array}{l}\text { Wainwright 1967, } \\
93 \text { (24.11) }\end{array}$ \\
\hline CAR0009 & Vessel (fragment) & & Carmarthenshire & Coygan Camp & RS & & & & $\begin{array}{l}\text { Wainwright 1967, } \\
93 \text { (24.12) }\end{array}$ \\
\hline САM0006 & Vessel (mount) & Eggers 79 & Cambridgeshire & Barton & RNS & & Zoomorphic bird (dove) & & $\begin{array}{l}\text { Eggers 1968, } 106 \\
(41)\end{array}$ \\
\hline CAM0009 & Handled Pan 2 & Eggers 131-133 & Cambridgeshire & Ely & RNS & $\begin{array}{l}\text { Isle of Ely, } \\
\text { Prickwillow }\end{array}$ & & $\begin{array}{l}\text { Zoomorphic elements on top, dolphins } \\
\text { below, floral vines; inscription } \\
\text { "BODVOGENVS F" }\end{array}$ & $\begin{array}{l}\text { Smith, } 192285 ; \\
\text { RIB II } 2415.11\end{array}$ \\
\hline CER0001 & Handled Pan 2 & Eggers 139-144 & Ceredigion & Llanio & M & & $\begin{array}{l}\text { Concentric circle umbo and } \\
\text { omphalos dot }\end{array}$ & $\begin{array}{l}\text { Concentric circle handle medallion and } \\
\text { suspension loop; } 3 \text { concentric circle } \\
\text { punch dots forming a triangle where } \\
\text { handle medallion meets the handle }\end{array}$ & $\begin{array}{l}\text { Burnham et alia } \\
2005,386\end{array}$ \\
\hline CHE0001 & Cauldron & & Cheshire & Middlewich & RS & \begin{tabular}{|l|} 
King Street; Shallow \\
Hearth Pit withing \\
Building B
\end{tabular} & & & $\begin{array}{l}\text { Dunn 2008, 43-44 } \\
\text { (1) }\end{array}$ \\
\hline CHE0002 & Vessel (fragment) & & Cheshire & Middlewich & RS & $\begin{array}{l}\text { King Street; post } \\
\text { hole fill }\end{array}$ & $\begin{array}{l}\text { Floral (possibly) vines } \\
\text { circling omphalos dot; } \\
\text { geometric linear etched } \\
\text { umbo ring; concentric } \\
\text { circle foot ring }\end{array}$ & & Dunn 2008, 45 (6) \\
\hline CHE0003 & $\begin{array}{l}\text { Handled Pan } 2 \\
\text { (handle) }\end{array}$ & Eggers 139-144 & Cheshire & Chester & M & Goss Street & & $\begin{array}{l}\text { Concenrtic cirlce handle medallion and } \\
\text { suspension loop; stamped inscription } \\
\text { reads CIPI POLI[...] }\end{array}$ & $\begin{array}{l}\text { McPeake \& Moore } \\
1978,331(11) ; \text { RIB } \\
\text { II } 2415.25\end{array}$ \\
\hline $\mathrm{C} 0021$ & Jug (fragment) & Eggers 123-126 & Cumbria & Carlisle & M & South Lanes & & & $\begin{array}{l}\text { McCarthy 2000, } \\
105 \text { (C30) }\end{array}$ \\
\hline $\mathrm{C} 0022$ & Jug (fragment) & Eggers 123-126 & Cumbria & Carlisle & M & South Lanes & & & $\begin{array}{l}\text { McCarthy 2000, } \\
105 \text { (C31) }\end{array}$ \\
\hline $\mathrm{C} 0023$ & Bowl (fragment) & & Cumbria & Carlisle & M & $\begin{array}{l}\text { Blackfriars Street, } \\
\text { BLA D } 101\end{array}$ & & & $\begin{array}{l}\text { McCarthy 1990, } \\
132 \text { (95) }\end{array}$ \\
\hline $\mathrm{C} 0024$ & Handled Pan 2 & Eggers $145-147$ & Cumbria & Carlisle & M & $\begin{array}{l}\text { Blackfriars Street, } \\
\text { BLA G + }\end{array}$ & & $\begin{array}{l}\text { Trefoil suspension loop on handle } \\
\text { medallion }\end{array}$ & $\begin{array}{l}\text { McCarthy } 1990, \\
132,135(96,99)\end{array}$ \\
\hline C0025 & $\begin{array}{l}\text { Handled Pan } 2 \\
\text { (fragment) }\end{array}$ & Eggers 139-144 & Cumbria & Carlisle & M & $\begin{array}{l}\text { Blackfriars Street, } \\
\text { BLA D } 501\end{array}$ & & $\begin{array}{l}\text { Concentric circle handle medallion with } \\
\text { circular suspension loop; inscription in } \\
\text { rectangular field reads "ANSI. } \\
\text { DIODORI[...]" }\end{array}$ & $\begin{array}{l}\text { Bennet \& Young } \\
1981,44 \text { (45); } \\
\text { McCarthy 1990, } \\
135 \text { (97) }\end{array}$ \\
\hline $\mathrm{C} 0026$ & $\begin{array}{l}\text { Handled Pan } 2 \\
\text { (fragment) }\end{array}$ & Eggers 139-144 & Cumbria & Carlisle & M & $\begin{array}{l}\text { Blackfriars Street, } \\
\text { BLA B } 600\end{array}$ & & $\begin{array}{l}\text { Concentric circle handle medallion with } \\
\text { circular suspension loop; inscription in } \\
\text { rectangular field reads ".C.I.A[...]" }\end{array}$ & $\begin{array}{l}\text { Bennet \& Young } \\
1981,44 \text { (46); } \\
\text { McCarthy 1990, } \\
135 \text { (98) } \\
\end{array}$ \\
\hline
\end{tabular}




\begin{tabular}{|c|c|c|c|c|c|c|c|c|c|}
\hline $\mathrm{C} 0027$ & $\begin{array}{l}\text { Handled Pan } 2 \\
\text { (fragment) }\end{array}$ & Eggers $139-144$ & Cumbria & Watercrook & M & & & $\begin{array}{l}\text { Concentric circle handle medallion with } \\
\text { circular suspension loop; inscription "[...] } \\
\text { ANDID [...]" }\end{array}$ & $\begin{array}{l}\text { Potter 1979, } 215 \\
\text { (48); Bennet \& } \\
\text { Young 1981, } 44 \\
\text { (44); RIB II } \\
2415.13 \\
\end{array}$ \\
\hline $\mathrm{C} 0028$ & Jug & & Cumbria & Carlisle & M & & & $\begin{array}{l}\text { Anthropomorphic and zoomorphic scene } \\
\text { of magistrates sacrificing a pig in four } \\
\text { registers }\end{array}$ & $\begin{array}{l}\text { BM 1814,0705.37; } \\
\text { Henig 1984, 132- } \\
134\end{array}$ \\
\hline DEN0005 & Vessel (mount) & & Denbighshire & Rhuddlan & M & Dinorben & $\begin{array}{l}\text { Zoomorphic ox head } \\
\text { hanging vessel mount }\end{array}$ & & $\begin{array}{l}\text { Gardner \& Savoy } \\
1964,144-148\end{array}$ \\
\hline DENO006 & Vessel (mount) & & Denbighshire & Rhuddlan & M & Dinorben & $\begin{array}{l}\text { Zoomorphic ox head } \\
\text { hanging vessel mount }\end{array}$ & & \begin{tabular}{|l|} 
Gardner \& Savoy \\
$1964,144-148$
\end{tabular} \\
\hline DEN0007 & \begin{tabular}{|l|}
$\begin{array}{l}\text { Handled Pan } 2 \\
\text { (handle) }\end{array}$ \\
\end{tabular} & Rudge Cup Type & Denbighshire & Rhuddlan & M & Dinorben & & $\begin{array}{l}\text { Geometric linear etchings and swirls } \\
\text { (possibly floral vines) }\end{array}$ & \begin{tabular}{|l|} 
Gardner \& Savoy \\
$1964,148-149$ \\
\end{tabular} \\
\hline DEV0001 & Vessel (fragment) & & Devon & Exeter & $\mathrm{U}$ & Topsham & Concentric circle rim & & $\begin{array}{l}\text { Holbrook \& Bidwell } \\
1991,257 \text { (106) }\end{array}$ \\
\hline DEV0002 & Handled Pan 5 & Eggers 130 & Devon & Exeter & $\mathrm{U}$ & WS 22 & & & $\begin{array}{l}\text { Holbrook \& Bidwell } \\
1991,257 \text { (107) }\end{array}$ \\
\hline DOR0012 & Jug & & Dorset & Dorchester & $\mathrm{U}$ & Greyhound Yard & & Anthropomorphic face handle medallion & $\begin{array}{l}\text { Woodward et al. } \\
1993,127(80)\end{array}$ \\
\hline DOR0013 & Bowl & & Dorset & Dorchester & $\mathrm{U}$ & Greyhound Yard & Concentric circle umbo & & $\begin{array}{l}\text { Woodward et al. } \\
1993,127(81)\end{array}$ \\
\hline DOR0014 & Vessel (fragment) & & Dorset & Dorchester & $\mathrm{U}$ & Greyhound Yard & & & $\begin{array}{l}\text { Woodward et al. } \\
1993,127(82)\end{array}$ \\
\hline DOR0015 & Jug (fragment) & Eggers $123-126$ & Dorset & Dorchester & $\mathrm{U}$ & Greyhound Yard & $\begin{array}{l}\text { Zoomorphic dolphin thumb } \\
\text { rest on lid; Floral (possible) } \\
\text { heart shaped lid }\end{array}$ & & $\begin{array}{l}\text { Woodward et al. } \\
1993,127 \text { (83) }\end{array}$ \\
\hline DOR0016 & Jug (fragment) & & Dorset & Dorchester & $\mathrm{U}$ & Greyhound Yard & & $\begin{array}{l}\text { Zoomorphic lion's paws and tail handle } \\
\text { terminal }\end{array}$ & $\begin{array}{l}\text { Woodward et al. } \\
1993,127(84)\end{array}$ \\
\hline DOR0017 & Jug (fragment) & & Dorset & Dorchester & $\mathrm{U}$ & Greyhound Yard & & Floral (possible) leaf thumb rest & $\begin{array}{l}\text { Woodward et al. } \\
1993,127 \text { (85) }\end{array}$ \\
\hline DOR0018 & $\begin{array}{l}\text { Handled Pan } 2 \\
\text { (handle) }\end{array}$ & & Dorset & Blandford Forum & M & Hod Hill & & $\begin{array}{l}\text { Geometric scalloping on terminal of } \\
\text { handle }\end{array}$ & $\begin{array}{l}\text { Richmond 1968, } \\
114 \text { (26) }\end{array}$ \\
\hline DOR0019 & $\begin{array}{l}\text { Handled Pan } 2 \\
\text { (handle) }\end{array}$ & & Dorset & Blandford Forum & M & Hod Hill & & Geometric incised line border & \begin{tabular}{|l} 
Richmond 1968, \\
114 (27) \\
\end{tabular} \\
\hline DUR0002 & Bowl & & Durham & Hurworth-on-Tees & RS & & Concentric circle umbo & & $\begin{array}{l}\text { Burnham 2008, } \\
\text { 2286-287 (1, Fig. } \\
\text { 13) }\end{array}$ \\
\hline DUR0003 & $\begin{array}{l}\text { Handled Pan } 2 \\
\text { (handle) }\end{array}$ & Eggers 139-144 & Durham & Bishop Auckland & M & \begin{tabular}{|l|} 
Binchester Roman \\
Fort
\end{tabular} & & $\begin{array}{l}\text { Concentric circle handle medallion and } \\
\text { loop }\end{array}$ & $\begin{array}{l}\text { Ferris 2010, } 352 \\
(90)\end{array}$ \\
\hline DUR0004 & $\begin{array}{l}\text { Handled Pan } 3 \\
\text { (handle) }\end{array}$ & Eggers 160-161 & Durham & Bishop Auckland & M & $\begin{array}{l}\text { Binchester Roman } \\
\text { Fort }\end{array}$ & & & $\begin{array}{l}\text { Ferris 2010, } 352 \\
(91)\end{array}$ \\
\hline EX0001 & Strainer & & Essex & Colchester & $\mathrm{U}$ & $\begin{array}{l}\text { Lion Walk } \\
\text { (Domestic Fill) }\end{array}$ & $\begin{array}{l}\text { Geometric swastica } \\
\text { meander band }\end{array}$ & & $\begin{array}{l}\text { Crummy 1983, } 71 \\
2029\end{array}$ \\
\hline EX0002 & Bowl (fragment) & & Essex & Colchester & $\mathrm{U}$ & $\begin{array}{l}\text { Lion Walk } \\
\text { (Destruction layer) }\end{array}$ & & & $\begin{array}{l}\text { Crummy 1983, } 71 \\
2030\end{array}$ \\
\hline EX0003 & Bowl (fragment) & & Essex & Colchester & $\mathrm{U}$ & $\begin{array}{l}\text { Lion Walk } \\
\text { (Destruction Layer) }\end{array}$ & $\begin{array}{l}\text { Geometric and floral } \\
\text { beaded rim }\end{array}$ & & \begin{tabular}{|l} 
Crummy 1983, 71 \\
2031
\end{tabular} \\
\hline EX0004 & Bowl & \begin{tabular}{|l}
$\begin{array}{l}\text { Bassin Uní } \\
\text { (possibly) }\end{array}$ \\
\end{tabular} & Essex & Colchester & $\mathrm{U}$ & $\begin{array}{l}\text { Balkerne Lane } \\
\text { (Domestic fill) }\end{array}$ & & & \begin{tabular}{|l|} 
Crummy 1983, 71 \\
2034
\end{tabular} \\
\hline EX0005 & Vessel (mount) & & Essex & Colchester & $\mathrm{U}$ & Balkerne Lane & & & \begin{tabular}{|l} 
Crummy 1983, 71 \\
2040
\end{tabular} \\
\hline
\end{tabular}




\begin{tabular}{|c|c|c|c|c|c|c|c|c|c|}
\hline EX0006 & $\begin{array}{l}\text { Handled Pan } 3 \\
\text { (handle) }\end{array}$ & Eggers 160-161 & Essex & Colchester & $\mathrm{U}$ & Balkerne Lane & & & \begin{tabular}{|l|} 
Crummy 1983, 73 \\
2043
\end{tabular} \\
\hline EX0007 & Jug (handle) & & Essex & Colchester & $\mathrm{U}$ & Cups Hotel & Geometric & & $\begin{array}{l}\text { Crummy 1983, } 73 \\
2044\end{array}$ \\
\hline EX0024 & Handled Pan 2 & Eggers 196 (c.f.) & Essex & Colchester & $\mathrm{U}$ & & Concentric cirlce foot ring & $\begin{array}{l}\text { Geometric trefoil handle loop, inscription } \\
\text { in rectangular field "PCIPI.POLYBI". }\end{array}$ & $\begin{array}{l}\text { Eggers 1968, } 106 \\
\text { (37c); McPeake \& } \\
\text { Moore 1978, } 333 \\
\text { (13); RIB II 2415.23 }\end{array}$ \\
\hline EX0025 & Handled Pan 2 & Eggers 150 & Essex & Colchester & $\mathrm{U}$ & & Concentric circle foot ring & $\begin{array}{l}\text { Floral (possibly) tear drop/leaf shaped } \\
\text { handle loop }\end{array}$ & $\begin{array}{l}\text { Eggers 1968, } 106 \\
(37 f)\end{array}$ \\
\hline EX0026 & $\begin{array}{l}\begin{array}{l}\text { Handled Pan } 2 \\
\text { (handle) }\end{array} \\
\end{array}$ & Eggers 139 (c.f.) & Essex & Colchester & $\mathrm{U}$ & & & Geometric circle handle loop & $\begin{array}{l}\text { Eggers 1968, } 106 \\
(37 \mathrm{~g})\end{array}$ \\
\hline EX0027 & $\begin{array}{l}\text { Handled Pan } 2 \\
\text { (handle) }\end{array}$ & Eggers 140 & Essex & Colchester & $\mathrm{U}$ & Trinity Street & & $\begin{array}{l}\text { Geometric circle handle loop; inscription } \\
\text { "TVGIM" }\end{array}$ & $\begin{array}{l}\text { Eggers 1968, } 106 \\
\text { (37h); Wright 1944, } \\
89 \text { (12); RIB II } \\
2415.50\end{array}$ \\
\hline EX0028 & Jug (fragment) & Eggers 128 & Essex & Colchester & $\mathrm{U}$ & & $\begin{array}{l}\text { Concentric circle neck, } \\
\text { single spout, stopper }\end{array}$ & Floral thumb rest & $\begin{array}{l}\text { Eggers 1968, } 106 \\
(37 i)\end{array}$ \\
\hline EX0029 & Jug & Eggers 128 & Essex & Colchester & $\mathrm{U}$ & & $\begin{array}{l}\text { Concentric circle neck, } \\
\text { single spout, stopper }\end{array}$ & & $\begin{array}{l}\text { Eggers 1968, } 106 \\
(37 \mathrm{k})\end{array}$ \\
\hline EX0030 & Strainer & Eggers 161 & Essex & Great Wackering & RNS & & $\begin{array}{l}\text { Geometric sunburst and } \\
\text { ring strainer dot pattern }\end{array}$ & & $\begin{array}{l}\text { Eggers 1968, } 105 \\
(34)\end{array}$ \\
\hline EX0032 & Vessel (fragment) & Eggers 25 & Essex & Heybridge & RS & The Towers & & & $\begin{array}{l}\text { Eggers 1968, } 105 \\
(35 \mathrm{~b})\end{array}$ \\
\hline EX0033 & Bucket & $\begin{array}{l}\text { Ostland, Eggers 40- } \\
41\end{array}$ & Essex & Fingringhoe & RNS & $\begin{array}{l}\text { Wick Fame Gravel } \\
\text { Pits }\end{array}$ & & & $\begin{array}{l}\text { Eggers 1968, } 105 \\
(36)\end{array}$ \\
\hline EX0037 & $\begin{array}{l}\text { Handled Pan } 2 \\
\text { (handle) }\end{array}$ & Eggers 136 & Essex & Colchester & $\mathrm{U}$ & & & $\begin{array}{l}\text { Zoomorphic birds framed by floral vines, } \\
\text { two handle loops; inscription in } \\
\text { rectangular field "T.POMP.NIC" }\end{array}$ & $\begin{array}{l}\text { Smith, } 192285 \\
\text { RIB II } 2415.48\end{array}$ \\
\hline EX0053 & Vessel (fragment) & & Essex & Boreham & RS & & $\begin{array}{l}\text { Geometric 'pear-shaped' } \\
\text { suspension loop }\end{array}$ & & \begin{tabular}{|l|} 
Germany et al. \\
$2003,75(18)$ \\
\end{tabular} \\
\hline EX0054 & $\begin{array}{l}\text { Handled Pan } 2 \\
\text { (handle) }\end{array}$ & Rudge Cup type & Essex & Kelvedon & RS & & & $\begin{array}{l}\text { Geometric checker board enamel pattern } \\
\text { in black, yellow, blue and red enamel }\end{array}$ & $\begin{array}{l}\text { Rodwell, } 198857 \\
\text { (Fig. 48.43) }\end{array}$ \\
\hline EX0055 & Jug (handle) & & Essex & Little Oakley & RS & Site II & & Floral leaf shaped handle medallion & $\begin{array}{l}\text { Barford 2002, } 88 \\
\text { (CU11) }\end{array}$ \\
\hline EX0056 & Jug (handle) & & Essex & Colchester & $\mathrm{U}$ & Sheepen Hill & & & $\begin{array}{l}\text { Niblett } 1985,119 \\
(21)\end{array}$ \\
\hline EX0057 & Vessel (mount) & & Essex & Witham & RS & Ivy Chimneys & $\begin{array}{l}\text { Geometric triangualr vessel } \\
\text { mount }\end{array}$ & & $\begin{array}{l}\text { Turner 1999, } 83 \\
(47)\end{array}$ \\
\hline EX0068 & $\begin{array}{l}\begin{array}{l}\text { Handled Pan } 2 \\
\text { (handle) }\end{array} \\
\end{array}$ & Eggers 139-144 & Essex & Colchester & $\mathrm{U}$ & & & Concentric Circle handle loop & $\begin{array}{l}\text { Crummy 1992, } 156 \\
\text { (543) }\end{array}$ \\
\hline EX0069 & Jug (handle) & Eggers $123-126$ & Essex & Colchester & $\mathrm{U}$ & & & Floral (?) thumbrest; jug lid attached & $\begin{array}{l}\text { Crummy 1992, } 156 \\
(544)\end{array}$ \\
\hline EX0072 & Strainer & Eggers 160 & Essex & Great Wakering & RNS & & $\begin{array}{l}\text { Geometric waves and } \\
\text { trefoil strainer dot patterns }\end{array}$ & Hilted Handle & BM 1892.1104.14 \\
\hline F0010 & Jug (lid) & & Flintshire & Flint & RS & Pentre Farm & & & \begin{tabular}{|l|} 
O'Leary et alia \\
$1989,64(4)$
\end{tabular} \\
\hline G0001 & Strainer (fragment) & Eggers 160-162 & Gloucestershire & Lydney & RS & Lydney Park & $\begin{array}{l}\text { Geometric linear strainer } \\
\text { dot pattern }\end{array}$ & & $\begin{array}{l}\text { Eggers 1968, } 104 \\
(27)\end{array}$ \\
\hline
\end{tabular}




\begin{tabular}{|c|c|c|c|c|c|c|c|c|c|}
\hline G0002 & $\begin{array}{l}\text { Handled Pan } 2 \\
\text { (handle) }\end{array}$ & Eggers 140 & Gloucestershire & Cirencester & $\mathrm{U}$ & & & Concentric circle handle loop & $\begin{array}{l}\text { Eggers 1968, } 104 \\
(28)\end{array}$ \\
\hline G0003 & Handled Pan 2 & Eggers 146 & Gloucestershire & Kingholm & M & & $\begin{array}{l}\text { Concentric circle foot ring, } \\
\text { body band }\end{array}$ & $\begin{array}{l}\text { Concentric circle handle loop, linear } \\
\text { etched boarder }\end{array}$ & $\begin{array}{l}\text { Eggers 1968, } 104 \\
(26)\end{array}$ \\
\hline G0007 & Handled Pan 2 & Eggers 139-144 & Gloucestershire & Woodchester & RS & & \begin{tabular}{|l|} 
Geometric rectangle and \\
triangle body band; \\
Concentric circle umbo and \\
foot ring \\
\end{tabular} & $\begin{array}{l}\text { Floral wheat stalks on perimeter; } \\
\text { Concentric circle handle medallion }\end{array}$ & BM 1811.0607.9 \\
\hline G0008 & Jug (fragment) & & Gloucestershire & Cirencester & $\mathrm{U}$ & DM I 137 & & & $\begin{array}{l}\text { Wacher \& McWhirr } \\
\text { 1982, 93 (30) }\end{array}$ \\
\hline G0009 & Bucket (fragment) & & Gloucestershire & Cirencester & $\mathrm{U}$ & CY V 8 (house) & $\begin{array}{l}\text { Floral leaf-shaped hanging } \\
\text { vessel mount }\end{array}$ & & $\begin{array}{l}\text { McWhirr 1986, } 111 \\
(71)\end{array}$ \\
\hline G0010 & Jug (lid) & Eggers 123-126 & Gloucestershire & Cirencester & $\mathrm{U}$ & ARI 46 & $\begin{array}{l}\text { Floral (possible) leaf- } \\
\text { shaped jug lid }\end{array}$ & & $\begin{array}{l}\text { Holbrook 1998, } \\
318(40)\end{array}$ \\
\hline G0011 & Vessel (mount) & & Gloucestershire & Cirencester & $\mathrm{U}$ & AHVIII 26 & $\begin{array}{l}\text { Anthropomorphic reclicing } \\
\text { banqueter vessel mount }\end{array}$ & & \begin{tabular}{|l} 
Holbrook 1998, \\
$318(43)$
\end{tabular} \\
\hline G0012 & Vessel (fragment) & & Gloucestershire & Lydney & RS & Bath Building & $\begin{array}{l}\text { Floral vine pattern on body; } \\
\text { pearl scalloping on base }\end{array}$ & & $\begin{array}{l}\text { Wheeler 1932, } 87 \\
(112)\end{array}$ \\
\hline G0013 & Bowl (fragment) & & Gloucestershire & Uley & RS & Structure IX & & & $\begin{array}{l}\text { Woodward \& Leach } \\
1993,209(5)\end{array}$ \\
\hline G0014 & Jug (lid) & Eggers 128 & Gloucestershire & Uley & RS & Structure IV & Geometric circular lid & & $\begin{array}{l}\text { Woodward \& Leach } \\
1993,209(9)\end{array}$ \\
\hline G0015 & Jug (handle) & & Gloucestershire & Tewkesbury & RS & & & $\begin{array}{l}\text { Zoomorphic clawed foot handle } \\
\text { medallion }\end{array}$ & BM 1903.014.1 \\
\hline GWY0013 & $\begin{array}{l}\text { Handled Pan } 2 \\
\text { (handle) }\end{array}$ & Eggers 142 & Gwynedd & Caernarvon & $\mathrm{M}$ & & & $\begin{array}{l}\text { Concentric circle handle medallion, } \\
\text { inscription "SAGAVGVF" in rectangular } \\
\text { field }\end{array}$ & $\begin{array}{l}\text { Eggers 1968, } 104 \\
\text { (22a); Wright 1969, } \\
238 \text { (20); RIB II } \\
2415.45 \\
\end{array}$ \\
\hline HАM0001 & Handled Pan 2 & Eggers 140 (c.f.) & Hampshire & Silchester & $\mathrm{U}$ & Pit 10, Insula XXIII & Concentric circle umbo & $\begin{array}{l}\text { Concentric circle handle terminal, } \\
\text { "PIAV" inscription on handle }\end{array}$ & $\begin{array}{l}\text { Eggers 1966, } 102 \\
\text { (6a); RIB II 2415.37 }\end{array}$ \\
\hline HAM0002 & Handled Pan 1 & Eggers 154-155 & Hampshire & Silchester & $\mathrm{U}$ & Pit 10, Insula XXIII & Concentric circle umbo & Zoomorphic ram's head handle terminal & $\begin{array}{l}\text { Eggers 1966, } 102 \\
\text { (6b); Boon 1974 } \\
228-230(1)\end{array}$ \\
\hline HAM0016 & Bucket (fragment) & & Hampshire & Fareham & $\mathrm{M}$ & Portchester Castle & $\begin{array}{l}\text { Zoomorhpic ox head } \\
\text { hanging vessel mount }\end{array}$ & & $\begin{array}{l}\text { Cunliffe 1975, } 212 \\
\text { (61) }\end{array}$ \\
\hline HAM0017 & Vessel (fragment) & & Hampshire & Silchester & $\mathrm{U}$ & Forum-Basilica & $\begin{array}{l}\text { Geometric fan/'pelta' } \\
\text { shaped foot }\end{array}$ & & $\begin{array}{l}\text { Fulford \& Timby } \\
2000,345 \text { (46) }\end{array}$ \\
\hline HAM0018 & Vessel (fragment) & & Hampshire & Silchester & $\mathrm{U}$ & Forum-Basilica & Concentric circle umbo & & $\begin{array}{l}\text { Fulford \& Timby } \\
2000,350(84)\end{array}$ \\
\hline HАM0019 & Vessel (fragment) & & Hampshire & Silchester & $\mathrm{U}$ & Insula IX & $\begin{array}{l}\text { Geometric fan/'pelta' } \\
\text { shaped foot }\end{array}$ & & \begin{tabular}{|l} 
Fulford \& Clarke \\
$2011,108(46)$
\end{tabular} \\
\hline HAM0020 & Jug (handle) & & Hampshire & Silchester & $\mathrm{U}$ & Insula IX & $\begin{array}{l}\text { Anthropomorphic human } \\
\text { foot handle terminal; } \\
\text { zoomorphic leaf thumb rest }\end{array}$ & & $\begin{array}{l}\text { Fulford \& Clarke } \\
2011,114(65)\end{array}$ \\
\hline HAM0021 & Jug & Egger 129 & Hampshire & Silchester & $\mathrm{U}$ & Insula XXIII, Well & & $\begin{array}{l}\text { Anthropomorphic handle medallion of } \\
\text { bearded man, perhaps Silenus }\end{array}$ & $\begin{array}{l}\text { Boon 1974, } 85-86 \\
\& 228-229(3) \\
\end{array}$ \\
\hline HAM0022 & Cauldron & & Hampshire & Silchester & $\mathrm{U}$ & & & & \begin{tabular}{|l|} 
Boon 1974, 228- \\
$229(4)$
\end{tabular} \\
\hline HER0001 & Handled Pan 2 & Rudge Cup & Hertfordshire & Braughing & RS & & $\begin{array}{l}\text { Floral vine and leaf, } \\
\text { enameled; foot ring }\end{array}$ & & $\begin{array}{l}\text { Eggers 1968, } 105 \\
(31 \mathrm{~A})\end{array}$ \\
\hline
\end{tabular}




\begin{tabular}{|c|c|c|c|c|c|c|c|c|c|}
\hline HER0002 & Vessel (mount) & & Hertfordshire & St. Albans & $\mathrm{U}$ & Verulamium & & & Frere 1972,130 \\
\hline HER0003 & Vessel (mount) & & Hertfordshire & St. Albans & $\mathrm{U}$ & Verulamium & & & Frere 1972,130 \\
\hline HER0004 & Vessel (mount) & & Hertfordshire & St. Albans & $\mathrm{U}$ & Verulamium & & & Frere 1972,130 \\
\hline HER0005 & Jug (handle) & & Hertfordshire & St. Albans & $\mathrm{U}$ & Verulamium & Zoomorphic Dolphin & & Frere 1972, 130 \\
\hline HER0006 & Jug (handle) & & Hertfordshire & St. Albans & $\mathrm{U}$ & Verulamium & Zoomorphic Dolphin & & Frere 1972,130 \\
\hline HER0007 & Jug (handle) & & Hertfordshire & St. Albans & $\mathrm{U}$ & Verulamium & Zoomorphic Dolphin & & $\begin{array}{l}\text { Frere 1972, 130- } \\
132\end{array}$ \\
\hline HER0008 & Jug (handle) & & Hertfordshire & St. Albans & $\mathrm{U}$ & Verulamium & & & Frere 1972,132 \\
\hline HER0009 & Jug (handle) & & Hertfordshire & St. Albans & $\mathrm{U}$ & Verulamium & $\begin{array}{l}\text { Floral leaf moulding, Face } \\
\text { (female) }\end{array}$ & & Frere 1972, 132 \\
\hline HER0010 & Vessel (fragment) & & Hertfordshire & St. Albans & $\mathrm{U}$ & Verulamium & Concentric circle umbo & & Frere 1972,132 \\
\hline HER0011 & Jug & & Hertfordshire & St. Albans & $\mathrm{U}$ & Verulamium & & & Frere 1972,132 \\
\hline HER0012 & Jug & & Hertfordshire & St. Albans & $\mathrm{U}$ & Verulamium & & $\begin{array}{l}\text { Floral handle with trefoils and heart } \\
\text { shapes terminating in bulls head at vessel } \\
\text { rim }\end{array}$ & Frere 1972, 132 \\
\hline HER0013 & $\begin{array}{l}\text { Handled Pan } 1 \\
\text { (handle) }\end{array}$ & Eggers 154-155 & Hertfordshire & St. Albans & $\mathrm{U}$ & $\begin{array}{l}\text { Verulamium, } \\
\text { unstratified }\end{array}$ & & $\begin{array}{l}\text { Zoomorphic ram's head terminal, fluted } \\
\text { handle }\end{array}$ & $\begin{array}{l}\text { Frere 1972, } 138 \\
(148)\end{array}$ \\
\hline HER0014 & $\begin{array}{l}\text { Handled Pan } 1 \\
\text { (handle) }\end{array}$ & Eggers $154-155$ & Hertfordshire & St. Albans & $\mathrm{U}$ & Verulamium, B I 55 & & $\begin{array}{l}\text { Zoomorphic ram's head terminal, fluted } \\
\text { handle }\end{array}$ & $\begin{array}{l}\begin{array}{l}\text { Frere } 1972,138 \\
(149)\end{array} \\
\end{array}$ \\
\hline HER0015 & Strainer & & Hertfordshire & St. Albans & $\mathrm{U}$ & Verulamium & $\begin{array}{l}\text { Geometric punch-dot } \\
\text { circle, 'S', and 'Z' }\end{array}$ & & Frere 1972, 144 \\
\hline HER0016 & $\begin{array}{l}\text { Handled Pan } 3 \\
\text { (handle) }\end{array}$ & Eggers 160-161 & Hertfordshire & St. Albans & $\mathrm{U}$ & Verulamium & & & Frere 1984, 51173 \\
\hline HER0017 & $\begin{array}{l}\text { Handled Pan } 3 \\
\text { (handle) }\end{array}$ & Eggers 160-161 & Hertfordshire & St. Albans & $\mathrm{U}$ & Verulamium & & & Frere 1984, 51174 \\
\hline HER0018 & Jug (lid) & Eggers $123-126$ & Hertfordshire & St. Albans & $\mathrm{U}$ & Verulamium & $\begin{array}{l}\text { Zoomorphic duck sitting or } \\
\text { swimming }\end{array}$ & & Frere 1984, 51175 \\
\hline HER0029 & Handled Pan 2 & Eggers 153 & Hertfordshire & Baldock & RS & \begin{tabular}{|l|} 
Upper Wall's \\
Common, Site A \\
268
\end{tabular} & & Flared handle terminal & \begin{tabular}{|l} 
Stead \& Rigby \\
1986,139 (394)
\end{tabular} \\
\hline K0021 & Jug & & Kent & Faversham & RS & & $\begin{array}{l}\text { Concentric circle body } \\
\text { bands }\end{array}$ & $\begin{array}{l}\text { Anthropomorphic Cupid with sword and } \\
\text { sling handle medallion }\end{array}$ & $\begin{array}{l}\text { BM 1882.0405.2; } \\
\text { BM 1882.0405.3 }\end{array}$ \\
\hline K0022 & $\begin{array}{l}\begin{array}{l}\text { Handled Pan } 1 \\
\text { (handle) }\end{array} \\
\end{array}$ & Eggers 154-155 & Kent & Richborough & M & & $\begin{array}{l}\text { Zoomorphic ram's head } \\
\text { terminal, fluted handle }\end{array}$ & & $\begin{array}{l}\text { Eggers 1966, } 102 \\
5 \mathrm{a}\end{array}$ \\
\hline K0023 & Jug (fragment) & Eggers 123-126 & Kent & Richborough & M & & Floral & & $\begin{array}{l}\text { Eggers 1966, } 102 \\
5 c\end{array}$ \\
\hline K0024 & Vessel (mount) & & Kent & Richborough & M & & & & $\begin{array}{l}\text { Eggers 1966, } 102 \\
\text { 5d }\end{array}$ \\
\hline K0026 & $\begin{array}{l}\begin{array}{l}\text { Handled Pan } 2 \\
\text { (fragment) }\end{array} \\
\end{array}$ & Rudge Cup type & Kent & Canterbury & $\mathrm{U}$ & & $\begin{array}{l}\text { Geometric circles and } \\
\text { swirls inside pentagons }\end{array}$ & & $\begin{array}{l}\text { Eggers 1968, } 148 \\
(4)\end{array}$ \\
\hline K0034 & Jug (fragment) & Eggers 123-126 & Kent & Faversham & RS & & \begin{tabular}{|l} 
Floral palm pattern on neck \\
(resembles fleur-de-lys)
\end{tabular} & & BM 1882.0405 .2 \\
\hline K0035 & Vessel (mount) & & Kent & Ashford & RS & $\begin{array}{l}\text { Westhawk Farm, } \\
\text { Context } 7004\end{array}$ & \begin{tabular}{|l}
$\begin{array}{l}\text { Floral leaf shaped (five } \\
\text { pointed) hanging vessel } \\
\text { mount }\end{array}$ \\
\end{tabular} & & \begin{tabular}{|l} 
Booth et al. 2008, \\
$165(75)$
\end{tabular} \\
\hline K0036 & Jug (fragment) & Eggers 123-126 & Kent & Canterbury & $\mathrm{U}$ & Marlowe Car Park & & & \begin{tabular}{|l|} 
Blockley et alia \\
$1995,1018(336)$
\end{tabular} \\
\hline K0037 & Bowl (fragment) & & Kent & Springhead & RS & $\begin{array}{l}\text { Roman Building } \\
300522\end{array}$ & $\begin{array}{l}\text { Zoomorphic spout, highly } \\
\text { stylized and difficult to } \\
\text { ascribe; possibly a bear }\end{array}$ & & $\begin{array}{l}\text { Biddulph et al. } \\
2011,247 \text { (194) }\end{array}$ \\
\hline K0039 & Jug (fragment) & & Kent & Richborough & M & west of Site I & & Floral (possible) leaf thumb rest & \begin{tabular}{|l} 
Bushe-Fox 1932, \\
$83(49)$
\end{tabular} \\
\hline K0040 & Vessel (mount) & & Kent & Richborough & M & & & & $\begin{array}{l}\text { Bushe-Fox 1932, } \\
83 \text { (52) }\end{array}$ \\
\hline K0041 & Vessel (mount) & & Kent & Richborough & M & & & & $\begin{array}{l}\text { Bushe-Fox 1932, } \\
83 \text { (54) }\end{array}$ \\
\hline
\end{tabular}




\begin{tabular}{|c|c|c|c|c|c|c|c|c|c|}
\hline K0042 & Jug (fragment) & & Kent & Richborough & M & $\begin{array}{l}\begin{array}{l}\text { South west area of } \\
\text { Stone Fort }\end{array} \\
\end{array}$ & & $\begin{array}{l}\text { Anthropomorphic femalebust handle } \\
\text { medallion; Floral leaf thumb rest }\end{array}$ & $\begin{array}{l}\begin{array}{l}\text { Cunliffe 1968, 102- } \\
103(189)\end{array} \\
\end{array}$ \\
\hline K0045 & Jug (handle) & & Kent & Chalkwell & RNS & & & $\begin{array}{l}\text { Anthropomorphic (perhaps Gorgon?) } \\
\text { handle medallion }\end{array}$ & BM 1883.1213.369 \\
\hline LEI0001 & $\begin{array}{l}\text { Handled Pan } 2 \\
\text { (handle) }\end{array}$ & Eggers 140 & Leicestershire & Leicester & $\mathrm{U}$ & Jewry Wall & & $\begin{array}{l}\text { Concentric circle hanlde loop with dot in } \\
\text { centre }\end{array}$ & $\begin{array}{l}\text { Eggers 1968, } 107 \\
\text { (49); Kenyon 1948, } \\
260(6)\end{array}$ \\
\hline LEI0004 & $\begin{array}{l}\begin{array}{l}\text { Handled Pan } 2 \\
\text { (handle) }\end{array} \\
\end{array}$ & Rudge Cup Type & Leicestershire & Leicester & $\mathrm{U}$ & West Bridge, Site I & & $\begin{array}{l}\text { Floral (possible) vine patterns enameled } \\
\text { in blue }\end{array}$ & $\begin{array}{l}\text { Clay \& Pollard } \\
1994,147(41)\end{array}$ \\
\hline LEI0005 & Vessel (fragment) & & Leicestershire & Leicester & $\mathrm{U}$ & West Bridge, Site I & $\begin{array}{l}\text { Concentric ring umbo and } \\
\text { omphalos dot }\end{array}$ & & $\begin{array}{l}\text { Clay \& Pollard } \\
1994,147(42)\end{array}$ \\
\hline LEI0006 & Vessel (fragment) & Perlrandbecken & Leicestershire & Leicester & $\mathrm{U}$ & West Bridge, Site I & $\begin{array}{l}\text { Geometric 'pearl' beaded } \\
\text { out-turned rim }\end{array}$ & & $\begin{array}{l}\text { Clay \& Pollard } \\
1994,147 \text { (43) }\end{array}$ \\
\hline LIN0001 & Vessel (fragment) & & Lincolnshire & Osgodby & RNS & & & & $\begin{array}{l}\text { Eggers 1968, } 107 \\
\text { (50a) }\end{array}$ \\
\hline LIN0002 & Strainer (fragment) & & Lincolnshire & Osgodby & RNS & & $\begin{array}{l}\text { Geometric circle and } \\
\text { square dot strainer dots }\end{array}$ & & $\begin{array}{l}\text { Eggers 1968, } 107 \\
(50 \mathrm{~b})\end{array}$ \\
\hline LIN0003 & Vessel (fragment) & Eggers 79 & Lincolnshire & Ashby & RNS & & $\begin{array}{l}\text { Zoomorphic bird mount, } \\
\text { perhaps a dove or duck }\end{array}$ & & $\begin{array}{l}\text { Eggers 1968, } 107 \\
(51)\end{array}$ \\
\hline LIN0025 & $\begin{array}{l}\text { Handled Pan } 2 \\
\text { (handle) }\end{array}$ & Rudge Cup Type & Lincolnshire & Sleaford & RS & Near R.D.C Offices & & $\begin{array}{l}\text { Floral vine and leaf; 'heart-shaped' leaves; } \\
\text { enameled in Dark blue, red and white }\end{array}$ & $\begin{array}{l}\text { Elsdon 1997, 190- } \\
191\end{array}$ \\
\hline LIN0026 & $\begin{array}{l}\text { Handled Pan } 2 \\
\text { (handle) }\end{array}$ & Rudge Cup Type & Lincolnshire & Sleaford & RS & $\begin{array}{l}\text { Site B (near St. } \\
\text { Giles Church) }\end{array}$ & & $\begin{array}{l}\text { Floral leaf and vine; thin, oval shaped } \\
\text { leaves on either side of central vine; } \\
\text { traces of blue enamel }\end{array}$ & \begin{tabular}{|l} 
Elsdon 1997, 190- \\
191
\end{tabular} \\
\hline LIN0027 & $\begin{array}{l}\text { Handled Pan } 2 \\
\text { (handle) }\end{array}$ & & Lincolnshire & Navenby & RS & & & & $\begin{array}{l}\text { Goodburn 1979, } \\
295\end{array}$ \\
\hline LIN0033 & $\begin{array}{l}\text { Handled Pan } 2 \\
\text { (handle) }\end{array}$ & Eggers 139-144 & Lincolnshire & Humberside** & RS & & & inscription "[...]IAPPIA" & $\begin{array}{l}\text { Hassall \& Tomlin } \\
\text { 1984, 345 (49); } \\
\text { RIB II 2415.36 } \\
\end{array}$ \\
\hline LIN0034 & $\begin{array}{l}\text { Handled Pan } 2 \\
\text { (handle) }\end{array}$ & Eggers 139-144 & Lincolnshire & Louth & RNS & & & $\begin{array}{l}\text { stamped inscription "MLNFEC"; punch } \\
\text { dot inscription "C CLSENIORIS ANNI" }\end{array}$ & $\begin{array}{l}\text { Wright 1964, } 180 \\
\text { (16); RIB II 2415.41 }\end{array}$ \\
\hline LIN0035 & $\begin{array}{l}\text { Handled Pan } 2 \\
\text { (handle) }\end{array}$ & Eggers $146-147$ & Lincolnshire & Lincoln & $\mathrm{U}$ & & & $\begin{array}{l}\text { inscription "MLN [...]" on top of handle; } \\
\text { inscription "[...] C. A. S. DC" on } \\
\text { underside of handle }\end{array}$ & \begin{tabular}{|l} 
Bennett \& Young \\
1981, 44 (43); RIB \\
II 2415.42
\end{tabular} \\
\hline LIN0036 & $\begin{array}{l}\text { Handled Pan } 2 \\
\text { (handle) }\end{array}$ & Eggers 139-144 & Lincolnshire & Normanby & RNS & near Scunthorpe & & $\begin{array}{l}\text { Concentric cicle handle loop; punch dot } \\
\text { inscription reads "ALPRI" }\end{array}$ & \begin{tabular}{|l} 
Wright \& Hassall \\
1971,299 (60); RIB \\
II 2415.57 \\
\end{tabular} \\
\hline L0016 & Bowl & & London & City of London & $\mathrm{U}$ & Shadwell, Well (A) & & & $\begin{array}{l}\text { Burnham et alia } \\
\text { 2003, 347; Douglas } \\
\text { et. alia 2011 }\end{array}$ \\
\hline L0017 & Jug (lid) & & London & City of London & $\mathrm{U}$ & Paternoster Square & & & $\begin{array}{l}\text { Watson 2006, } 97 \\
\text { S37 }\end{array}$ \\
\hline L0018 & Vessel (fragment) & & London & City of London & $\mathrm{U}$ & Paternoster Square & & & \begin{tabular}{|l} 
Watson 2006, 97 \\
S38
\end{tabular} \\
\hline L0019 & Jug (handle) & & London & City of London & $\mathrm{U}$ & Paternoster Square & Zoomorphic, possibly lion & & $\begin{array}{l}\text { Watson 2006, } 97 \\
\text { S39 }\end{array}$ \\
\hline L0020 & $\begin{array}{l}\text { Handled Pan } 3 \\
\text { (handle) }\end{array}$ & Eggers 160-161 & London & Southwark & $\mathrm{U}$ & & & & \begin{tabular}{|l|} 
Drummond-Murray \\
2002,218 R11
\end{tabular} \\
\hline L0021 & Bucket (fragment) & & London & City of London & $\mathrm{U}$ & Fenchurch & & & \begin{tabular}{|l|} 
Birbeck 2009, 82 \\
3611
\end{tabular} \\
\hline
\end{tabular}




\begin{tabular}{|c|c|c|c|c|c|c|c|c|c|}
\hline L0022 & Jug (lid) & Eggers $123-126$ & London & City of London & $\mathrm{U}$ & Fenchurch & Zoomorphic Dolphin & & \begin{tabular}{|l} 
Birbeck 2009, 82 \\
468
\end{tabular} \\
\hline L0023 & $\begin{array}{l}\begin{array}{l}\text { Handled Pan } 1 \\
\text { (handle) }\end{array} \\
\end{array}$ & Eggers 154-155 & London & & $\mathrm{U}$ & & $\begin{array}{l}\text { Zoomorphic ram's head } \\
\text { terminal, fluted handle }\end{array}$ & & \begin{tabular}{|l|} 
Eggers 1966, 100 \\
1a
\end{tabular} \\
\hline L0024 & $\begin{array}{l}\text { Handled Pan } 2 \\
\text { (handle) }\end{array}$ & Eggers 142-144 & London & City of London & $\mathrm{U}$ & Christ's Hospital & & $\begin{array}{l}\text { Geometric circle terminal, linear border; } \\
\text { inscription "L[...]SI" }\end{array}$ & $\begin{array}{l}\text { Eggers 1966, } 100 \\
\text { 1b; McPeake \& } \\
\text { Moore 1978, 334 } \\
\text { (25); RIB II 2415.8 } \\
\end{array}$ \\
\hline L0025 & Handled Pan 2 & Eggers 141 & London & Bermondsey & $\mathrm{U}$ & $\begin{array}{l}\begin{array}{l}\text { Potter's Fields } \\
\text { (south London) }\end{array} \\
\end{array}$ & $\begin{array}{l}\text { Concentric circle umbo and } \\
\text { foot ring, "TRVFC" } \\
\text { inscription in rectangular } \\
\text { field }\end{array}$ & & $\begin{array}{l}\text { Eggers 1966, } 101 \\
\text { 1c; McPeake \& } \\
\text { Moore 1978, 334 } \\
\text { (33); RIB II 2415.49 }\end{array}$ \\
\hline L0026 & Jar & Eggers 39 & London & & $\mathrm{U}$ & & & & $\begin{array}{l}\text { Eggers 1966, } 101 \\
1 \mathrm{~d}\end{array}$ \\
\hline L0027 & Jug (fragment) & Eggers 125 & London & & $\mathrm{U}$ & & Concentric circles on neck & & $\begin{array}{l}\text { Eggers 1966, } 101 \\
1 \mathrm{~g}\end{array}$ \\
\hline L0028 & Handled Pan 3 & Eggers 161 & London & City of London & $\mathrm{U}$ & & & & $\begin{array}{l}\text { Eggers 1966, } 101 \\
1 \mathrm{~h}\end{array}$ \\
\hline L0029 & Bowl & Eggers 71 & London & City of London & $\mathrm{U}$ & Bucklersbury House & & & $\begin{array}{l}\text { Eggers 1966, } 101 \\
\text { (1i) }\end{array}$ \\
\hline L0030 & Vessel (fragment) & & London & & $\mathrm{U}$ & & $\begin{array}{l}\text { Anthropomorphic medusa } \\
\text { head }\end{array}$ & & $\begin{array}{l}\text { Eggers 1966, } 101 \\
(1 \mathrm{r})\end{array}$ \\
\hline L0031 & Vessel (fragment) & Eggers 160-161 & London & City of London & $\mathrm{U}$ & Bucklersbury House & & $\begin{array}{l}\text { Inscription "NDINVSF" in rectangular } \\
\text { field }\end{array}$ & $\begin{array}{l}\text { Eggers 1966, } 101 \\
\text { 1s; Wright 1969b, } \\
239 \text { (22); McPeake } \\
\text { \& Moore 1978, } 334 \\
\text { (38); RIB II } \\
2415.15\end{array}$ \\
\hline L0032 & Handled Pan 5 & Eggers 130 & London & City of London & $\mathrm{U}$ & Creed Lane & & & \begin{tabular}{|l|} 
Eggers 1966, 101 \\
lu; BM \\
1855.0804 .23
\end{tabular} \\
\hline L0033 & Strainer & Eggers $160-161$ & London & City of London & $\mathrm{U}$ & & & & $\begin{array}{l}\text { Eggers 1966, } 102 \\
1 \mathrm{w}\end{array}$ \\
\hline L0035 & Vessel (fragment) & & London & City of London & $\mathrm{U}$ & Angel Court & Possible engraved lines & & $\begin{array}{l}\text { MoL } \\
\text { ACW74[B12]<19> }\end{array}$ \\
\hline L0036 & Bowl & & London & City of London & $\mathrm{U}$ & Peninsular House & & & $\begin{array}{l}\text { MoL } \\
\text { PEN79[1142] }<53\rangle\end{array}$ \\
\hline L0037 & Handled Pan 2 & & London & City of London & $\mathrm{U}$ & $\begin{array}{l}\text { 145-146 Leadenhall } \\
\text { Street, EC3 }\end{array}$ & & & \begin{tabular}{|l} 
MoL \\
LEN89604]<55>
\end{tabular} \\
\hline L0038 & Vessel (fragment) & & London & City of London & $\mathrm{U}$ & \begin{tabular}{|l|} 
160-162 Fenchurch \\
Street, 22-3 Lime \\
Street, EC3
\end{tabular} & Concentric circle umbo & & MoL FSE76[0]<23> \\
\hline L0039 & Cauldron & & London & City of London & $\mathrm{U}$ & Blossom's Inn (well) & & & $\begin{array}{l}\text { Burnham et alia } \\
2002,329\end{array}$ \\
\hline L0040 & Jug (lid) & Eggers 123-126 & London & City of London & $\mathrm{U}$ & $\begin{array}{l}\text { 19-25 Birchin Lane, } \\
\text { Bengal Court, 1-3 } \\
\text { Castle Court, EC3 }\end{array}$ & Trefoil lid & & \begin{tabular}{|l} 
MOLA \\
BRL87[595]<67>
\end{tabular} \\
\hline
\end{tabular}




\begin{tabular}{|c|c|c|c|c|c|c|c|c|c|}
\hline L0041 & Vessel (fragment) & & London & City of London & $\mathrm{U}$ & $\begin{array}{l}\text { Southwark, Tabbard } \\
\text { Square (drain) }\end{array}$ & & & $\begin{array}{l}\text { Burnham et alia } \\
2004,301\end{array}$ \\
\hline L0042 & Jug (lid) & & London & City of London & $\mathrm{U}$ & Amphitheater & & & $\begin{array}{l}\text { Bateman 2008, } 197 \\
\text { (S82) }\end{array}$ \\
\hline L0043 & Jug (lid) & & London & City of London & $\mathrm{U}$ & $\begin{array}{l}\text { Hibernia Wharf, } \\
\text { Southwark }\end{array}$ & $\begin{array}{l}\text { Anthropomorphic (?) jug } \\
\text { lid in the shape of a conical } \\
\text { Gladiator's helmet }\end{array}$ & & $\begin{array}{l}\text { MOLA } \\
\text { HIB } 79[53]<28>\end{array}$ \\
\hline L0044 & Jug (lid) & Eggers 123-126 & London & City of London & $\mathrm{U}$ & $\begin{array}{l}\text { Hibernia Wharf, } \\
\text { Southwark }\end{array}$ & Trefoil lid & & \begin{tabular}{|l|} 
MOLA \\
HIB79[260]<80>
\end{tabular} \\
\hline L0045 & Jug (lid) & Eggers 123-126 & London & City of London & $\mathrm{U}$ & $\begin{array}{l}\text { 23-25 Austin Friars, } \\
\text { EC2 }\end{array}$ & Trefoil lid & & \begin{tabular}{|l} 
MOLA \\
AST87[233]<69>
\end{tabular} \\
\hline L0046 & Jug (lid) & Eggers 123-126 & London & City of London & $\mathrm{U}$ & $\begin{array}{l}\text { 23-25 Austin Friars, } \\
\text { EC2 }\end{array}$ & Trefoil lid & & $\begin{array}{l}\text { MOLA } \\
\text { AST87 }[236 \mathrm{BS}]<91 \\
> \\
\end{array}$ \\
\hline L0047 & Jug & & London & City of London & $\mathrm{U}$ & $\begin{array}{l}\text { 72-80 Cheapside, } 83 \\
84 \text { Queen Street, 9- } \\
12 \text { Pancras Lane, } \\
\text { EC2 and EC4 }\end{array}$ & & & $\begin{array}{l}\text { MOLA } \\
\text { CID90[764]<893> }\end{array}$ \\
\hline L0048 & Jug & & London & City of London & $\mathrm{U}$ & $\begin{array}{l}\text { 36-37 King Street, } \\
\text { EC2 }\end{array}$ & & & \begin{tabular}{|l} 
MOLA \\
KNG85[2118]<32>
\end{tabular} \\
\hline L0049 & Jug (handle) & & London & City of London & $\mathrm{U}$ & $\begin{array}{l}\text { Billingsgate } \text { Market } \\
\text { Lorry Park, Lower } \\
\text { Thames Street, EC3 }\end{array}$ & & & $\begin{array}{l}\text { MOLA } \\
\text { BWB83[354] }>3546 \\
>\end{array}$ \\
\hline L0050 & Jug (handle) & & London & City of London & $\mathrm{U}$ & $\begin{array}{l}\text { General Post Office, } \\
81 \text { Newgate Street, } \\
\text { EC1 }\end{array}$ & & & $\begin{array}{l}\text { MOLA } \\
\text { GPO75[9164]<216 } \\
8>\end{array}$ \\
\hline L0051 & Jug (handle) & & London & City of London & $\mathrm{U}$ & \begin{tabular}{|l} 
Lane (Nomura \\
House), 121-127 \\
Lower Thames \\
Street, 33-36 Fish \\
Street Hill, 22-26 \\
Monument Street, 7- \\
11A King's Head \\
Court, EC3
\end{tabular} & & Zoomorphic dolphin handle & $\begin{array}{l}\text { MOLA } \\
\text { PDN81[1761]<620 } \\
>\end{array}$ \\
\hline L0052 & Vessel (mount) & & London & City of London & $\mathrm{U}$ & $\begin{array}{l}\text { 27-30 Lime Street, } \\
\text { EC3 }\end{array}$ & $\begin{array}{l}\text { Zoomorphic aquatic bird } \\
\text { hanging vessel mount }\end{array}$ & & \begin{tabular}{|l} 
MOLA \\
IME83[376]<77>
\end{tabular} \\
\hline L0053 & Bucket & & London & City of London & $\mathrm{U}$ & $\begin{array}{l}\text { 5-12 Fenchurch } \\
\text { Street, } 1 \text { Philpot } \\
\text { Lane, EC3 }\end{array}$ & & & $\begin{array}{l}\text { MOLA } \\
\text { FEN83[2142]<214> }\end{array}$ \\
\hline L0054 & Bucket & & London & City of London & $\mathrm{U}$ & $\begin{array}{l}\text { 12-15 Finsbury } \\
\text { Circus, EC2 }\end{array}$ & & & \begin{tabular}{|l} 
MOLA \\
FIB $88[103]<2>$ \\
\end{tabular} \\
\hline L0055 & Bowl & Irchester & London & City of London & $\mathrm{U}$ & River Thames & & & BM 1891.0320.10 \\
\hline L0056 & Jar & & London & City of London & $\mathrm{U}$ & London Wall & $\begin{array}{l}\text { inscription reads "EX OF } \\
\text { COR" }\end{array}$ & & $\begin{array}{l}\text { Hassall \& Tomlin } \\
\text { 1984, } 344 \text { (35); } \\
\text { RIB II 2415.33 }\end{array}$ \\
\hline
\end{tabular}




\begin{tabular}{|c|c|c|c|c|c|c|c|c|c|}
\hline L0057 & $\begin{array}{l}\text { Handled Pan } 2 \\
\text { (handle) }\end{array}$ & & London & City of London & $\mathrm{u}$ & $\begin{array}{l}\text { Queen Victoria } \\
\text { Street and } \\
\text { Bucklersbury }\end{array}$ & & inscription "SANGVSF" & $\begin{array}{l}\text { Wtight 1969a, 5 (6); } \\
\text { Wright 1969b, 239 } \\
\text { (21); McPeake \& } \\
\text { Moore 1978, 334 } \\
\text { (36); RIB II 2415.46 } \\
\end{array}$ \\
\hline L0058 & Bowl & & London & City of London & $\mathrm{U}$ & Creed Lane & & & BM 1855.0804.24 \\
\hline M0001 & Jug & & Greater Manchester & Manchester & M & & & & $\begin{array}{l}\text { Bruton 1929, } 162 \\
\text { (24) }\end{array}$ \\
\hline M0002 & Jug (lid) & Eggers 128 & Greater Manchester & Manchester & M & Barton Street & & & $\begin{array}{l}\text { Gregory 2007, } 134 \\
\text { (2) }\end{array}$ \\
\hline MON0001 & Handled Pan 2 & Eggers 140 & Monmouthshire & Gelligaer & M & Fort & & Concentric cirlce handle medallion & $\begin{array}{l}\text { Eggers 1968, } 103 \\
\text { (14) }\end{array}$ \\
\hline MON0008 & Jug (handle) & & Monmouthshire & Usk & M & \begin{tabular}{|l|} 
Burrium, Fortress \\
Well
\end{tabular} & & & $\begin{array}{l}\text { Manning et alia } \\
1995,192(1)\end{array}$ \\
\hline MON0009 & Jug (lid) & Eggers 123-126 & Monmouthshire & Usk & M & $\begin{array}{l}\text { Burrium, Fortress } \\
\text { Ditch }\end{array}$ & $\begin{array}{l}\text { Zoomorphic bird lid } \\
\text { statuette }\end{array}$ & & $\begin{array}{l}\text { Manning et alia } \\
1995,194 \text { (2) }\end{array}$ \\
\hline MON0010 & Jug (lid) & Eggers $123-126$ & Monmouthshire & Usk & M & $\begin{array}{l}\text { Burrium, Fortress } \\
\text { Latrine }\end{array}$ & Floral leaf shaped jug lid & & $\begin{array}{l}\text { Manning et alia } \\
1995,194 \text { (3) }\end{array}$ \\
\hline MON0011 & Jug (lid) & Eggers $123-126$ & Monmouthshire & Usk & M & $\begin{array}{l}\text { Burrium, Fortress } \\
\text { Pit }\end{array}$ & Floral leaf shaped jug lid & & $\begin{array}{l}\text { Manning et alia } \\
1995,194 \text { (4) }\end{array}$ \\
\hline MON0012 & Jug (lid) & Eggers 123-126 & Monmouthshire & Usk & M & $\begin{array}{l}\text { Burrium, Third- } \\
\text { century Pit }\end{array}$ & Floral leaf shaped jug lid & & $\begin{array}{l}\text { Manning et alia } \\
1995,194 \text { (5) }\end{array}$ \\
\hline MON0013 & Handled Pan 2 & Eggers 145-147 & Monmouthshire & Usk & M & $\begin{array}{l}\text { Burrium, Fortress } \\
\text { Latrine }\end{array}$ & $\begin{array}{l}\text { Concentric circle umbo and } \\
\text { omphalos dot }\end{array}$ & Trefoil suspension loop & $\begin{array}{l}\text { Manning et alia } \\
1995,194 \text { (6) }\end{array}$ \\
\hline MON0014 & Vessel (fragment) & & Monmouthshire & Usk & M & $\begin{array}{l}\text { Burrium, Fortress } \\
\text { Pit }\end{array}$ & $\begin{array}{l}\text { Concentric circle body } \\
\text { band }\end{array}$ & & $\begin{array}{l}\text { Manning et alia } \\
1995,196(9)\end{array}$ \\
\hline MON0015 & Vessel (fragment) & & Monmouthshire & Usk & M & \begin{tabular}{|l|}
$\begin{array}{l}\text { Burrium, Fortress } \\
\text { Pit }\end{array}$ \\
\end{tabular} & $\begin{array}{l}\text { Concentric circle body } \\
\text { band }\end{array}$ & & $\begin{array}{l}\text { Manning et alia } \\
1995,196(10)\end{array}$ \\
\hline MON0016 & Vessel (mount) & & Monmouthshire & Usk & M & Burrium & $\begin{array}{l}\text { Geometric triangular } \\
\text { hanging vessel mount }\end{array}$ & & $\begin{array}{l}\text { Manning et alia } \\
1995,196 \text { (13) }\end{array}$ \\
\hline MON0017 & Handled Pan 4 & Coptic & Monmouthshire & Usk & M & $\begin{array}{l}\text { Burrium, Drainage } \\
\text { Gully near via } \\
\text { principalis of } \\
\text { Fortress }\end{array}$ & & & $\begin{array}{l}\text { Manning et alia } \\
1995,198(16)\end{array}$ \\
\hline NE0007 & Handled Pan 2 & Eggers 140 & Newport & Caerleon & M & Fort & & Concentric cirlce handle medallion & $\begin{array}{l}\text { Eggers 1968, } 104 \\
(23)\end{array}$ \\
\hline NE0008 & Bucket (fragment) & & Newport & Caerleon & M & $\begin{array}{l}\text { Fort, beneath } \\
\text { frigidarium }\end{array}$ & $\begin{array}{l}\text { Geometric pelta shaped } \\
\text { bucket foot }\end{array}$ & & $\begin{array}{l}\text { Zienkiewicz 1986, } \\
173(8)\end{array}$ \\
\hline NE0009 & $\begin{array}{l}\begin{array}{l}\text { Handled Pan } 2 \\
\text { (handle) }\end{array} \\
\end{array}$ & Eggers 139-144 & Newport & Caerleon & M & & & Concentric cirlce handle medallion & $\begin{array}{l}\text { Evans 2000, } 350 \\
(25)\end{array}$ \\
\hline NE0010 & Vessel (mount) & & Newport & Caerleon & M & & $\begin{array}{l}\text { Geometric sub-triangular } \\
\text { hanging vessel mount }\end{array}$ & & $\begin{array}{l}\text { Evans } 2000,350 \\
(26)\end{array}$ \\
\hline NE0011 & Vessel (mount) & & Newport & Caerleon & M & & $\begin{array}{l}\text { rim attachment to vessel } \\
\text { mount, possibly duck or } \\
\text { swan }\end{array}$ & & $\begin{array}{l}\text { Evans 2000, } 352 \\
(29)\end{array}$ \\
\hline NE0012 & Bucket (fragment) & & Newport & Caerleon & M & & $\begin{array}{l}\text { Geometric herring-bone } \\
\text { incised lines on curved } \\
\text { bucket foot }\end{array}$ & & $\begin{array}{l}\text { Evans 2000, } 352 \\
(30)\end{array}$ \\
\hline NE0013 & $\begin{array}{l}\text { Handled Pan } 1 \\
\text { (handle) }\end{array}$ & Eggers 154-155 & Newport & Caerleon & M & Alstone Cottage & & Zoomorphic ram's head handle terminal & $\begin{array}{l}\text { Casey \& Hoffman } \\
1995,89 \text { (7) }\end{array}$ \\
\hline NE0014 & Handled Pan 2 & Eggers 144 & Newport & Caerleon & M & Well & $\begin{array}{l}\text { Floral leaf body band; } \\
\text { Concentric circle umbo and } \\
\text { foot-ring; etched } \\
\text { inscription "LVCCA" }\end{array}$ & $\begin{array}{l}\text { Concentric cricle handle loop; stamped } \\
\text { inscriptions "MATVRVS F", "ALA I } \\
\text { TH"; punch dot inscription "LI" }\end{array}$ & $\begin{array}{l}\text { Hassall \& Tomlin } \\
\text { 1985, 330-331 (41); } \\
\text { RIB II 2415.39 }\end{array}$ \\
\hline NH0010 & Vessel (fragment) & & Northamptonshire & Higham Ferrers & RS & & $\begin{array}{l}\text { Concentric circle body } \\
\text { bands }\end{array}$ & & $\begin{array}{l}\text { Lawrence \& Smith } \\
\text { 2009, } 241 \text { (357) }\end{array}$ \\
\hline
\end{tabular}




\begin{tabular}{|c|c|c|c|c|c|c|c|c|c|}
\hline NH0011 & Vessel (fragment) & & Northamptonshire & Higham Ferrers & RS & & & & $\begin{array}{l}\text { Lawrence \& Smith } \\
2009,241 \text { (358) }\end{array}$ \\
\hline NH0012 & Vessel (fragment) & & Northamptonshire & Higham Ferrers & RS & & $\begin{array}{l}\text { Concentric circle body } \\
\text { bands }\end{array}$ & & $\begin{array}{l}\text { Lawrence \& Smith } \\
2009,241(360)\end{array}$ \\
\hline NOR0001 & Handled Pan 3 & Eggers 161 & \begin{tabular}{|l|} 
Norfolk \\
\end{tabular} & Swaffam & RS & & & & Kennett 1969, 137 \\
\hline NOR0002 & $\begin{array}{l}\text { Handled Pan } 2 \\
\text { (handle) }\end{array}$ & Rudge Cup & Norfolk & Bergh Apton & RNS & & & $\begin{array}{l}\text { Floral vine and leaf, geometric } \\
\text { embellishment }\end{array}$ & $\begin{array}{l}\text { Eggers 1968, } 106 \\
(46)\end{array}$ \\
\hline NOR0003 & Bucket & Ostland, Eggers 38 & Norfolk & Mundesley & RS & & & & $\begin{array}{l}\text { Eggers 1968, } 106 \\
\text { (47); BM 1900. } 7 \\
14.1\end{array}$ \\
\hline NOR0004 & Bucket & $\begin{array}{l}\text { Ostland, Eggers 41- } \\
43\end{array}$ & Norfolk & Swanton Morley & M & $\begin{array}{l}\text { River (River } \\
\text { Wensum) }\end{array}$ & & & $\begin{array}{l}\text { Eggers 1968, } 106 \\
(48)\end{array}$ \\
\hline NOR0030 & Bowl & & Norfolk & Caistor-on-Sea & M & & & & $\begin{array}{l}\text { Darling \& Gurney } \\
1993,96(355)\end{array}$ \\
\hline NOR0031 & $\begin{array}{l}\text { Handled Pan } 2 \\
\text { (handle) }\end{array}$ & Eggers 139-144 & Norfolk & Oxnead Mill & RNS & Brampton & & & Frere 1985, 294 \\
\hline NOR0032 & $\begin{array}{l}\begin{array}{l}\text { Handled Pan } 2 \\
\text { (handle) }\end{array} \\
\end{array}$ & Eggers $139-144$ & Norfolk & Walsingham & RNS & $\begin{array}{l}\text { near Roman temple } \\
\text { site }\end{array}$ & & $\begin{array}{l}\text { Concentric circle handled medallion and } \\
\text { suspension loop }\end{array}$ & Smith 1999, 39 \\
\hline NOR0041 & $\begin{array}{l}\text { Handled Pan } 2 \\
\text { (handle) }\end{array}$ & Eggers 139-144 & Norfolk & Saham Toney & M & $\begin{array}{l}\text { near suspected fort } \\
\text { at Sand Hills, } \\
\text { Woodcock Hall } \\
\end{array}$ & & $\begin{array}{l}\text { Concentric circle handle loop; punch dot } \\
\text { inscription "C PRIMI" }\end{array}$ & $\begin{array}{l}\text { Hassall \& Tomlin } \\
\text { 1978, } 480 \text { (61); RIB } \\
\text { II } 2415.59\end{array}$ \\
\hline NOR0042 & Jug & & Norfolk & Mundesley & RS & & & & BM 1900.0717.1 \\
\hline NOT0001 & Handled Pan 2 & Eggers 144 & Nottinghamshire & Broxtowe & M & & & inscription "ALBANVS" & $\begin{array}{l}\text { Oswald 1939, 441; } \\
\text { Eggers 1968, 110 } \\
\text { (87); McPeake \& } \\
\text { Moore 1978, } 333 \\
\text { (3); RIB II 2415.3 }\end{array}$ \\
\hline NOT0005 & $\begin{array}{l}\text { Handled Pan } 2 \\
\text { (handle) }\end{array}$ & Rudge Cup Type & Nottinghamshire & Brough-on-Fosse & M & & & $\begin{array}{l}\text { Floral vines enameled in blue, green and } \\
\text { red }\end{array}$ & $\begin{array}{l}\text { Moore 1978, 319- } \\
327\end{array}$ \\
\hline NU0001 & Vessel (fragment) & & Northumberland & Great Chesters & M & & & & $\begin{array}{l}\text { Eggers 1966, } 108 \\
\text { 59a }\end{array}$ \\
\hline NU0002 & Vessel (fragment) & & Northumberland & Great Chesters & M & & Concentric circle umbo & & $\begin{array}{l}\text { Eggers 1966, } 108 \\
59 \mathrm{~b}\end{array}$ \\
\hline NU0006 & Handled Pan 2 & Eggers 139-144 & Northumberland & Bardon Mill & M & \begin{tabular}{|l|} 
Housesteads Fort \\
(Borcovicium/Verco \\
vicium)
\end{tabular} & Concentric circle umbo & & $\begin{array}{l}\text { Eggers 1968, } 108 \\
60\end{array}$ \\
\hline NU0007 & $\begin{array}{l}\text { Handled Pan } 2 \\
\text { (handle) }\end{array}$ & Eggers $145-147$ & Northumberland & Bardon Mill & M & \begin{tabular}{|l|} 
Housesteads Fort \\
(Borcovicium/Verco \\
vicium)
\end{tabular} & & $\begin{array}{l}\text { Geometric trefoil hole in handle } \\
\text { medallion }\end{array}$ & $\begin{array}{l}\text { Eggers 1968, } 108 \\
60\end{array}$ \\
\hline NU0008 & $\begin{array}{l}\text { Handled Pan } 2 \\
\text { (handle) }\end{array}$ & Eggers 139-144 & Northumberland & Bardon Mill & M & \begin{tabular}{|l|} 
Housesteads Fort \\
(Borcovicium/Verco \\
vicium)
\end{tabular} & & Geometric circles and border & $\begin{array}{l}\text { Eggers 1968, } 108 \\
60\end{array}$ \\
\hline NU0009 & Vessel (fragment) & & Northumberland & Bardon Mill & M & \begin{tabular}{|l|} 
Housesteads Fort \\
(Borcovicium/Verco \\
vicium)
\end{tabular} & & & $\begin{array}{l}\text { Eggers 1968, } 108 \\
60\end{array}$ \\
\hline NU0010 & $\begin{array}{l}\text { Handled Pan } 2 \\
\text { (handle) }\end{array}$ & Eggers 139-144 & Northumberland & Bardon Mill & M & \begin{tabular}{|l|} 
Housesteads Fort \\
(Borcovicium/Verco \\
vicium)
\end{tabular} & & Geometric circles and border & $\begin{array}{l}\text { Eggers 1968, } 108 \\
60\end{array}$ \\
\hline NU0011 & Vessel (fragment) & & Northumberland & Chesters & M & Fort (Cilurnum) & & & $\begin{array}{l}\text { Eggers 1968, } 108 \\
61\end{array}$ \\
\hline NU0012 & $\begin{array}{l}\text { Handled Pan } 2 \\
\text { (handle) }\end{array}$ & Eggers 139-144 & Northumberland & Corbridge & M & Fort (Corstopitum) & & $\begin{array}{l}\text { Geometric circles and border; inscription } \\
\text { "ANSIEPA[P]HR[ODITI]" }\end{array}$ & $\begin{array}{l}\text { Eggers 1968, } 108 \\
62 ; \text { Wright \& } \\
\text { Hassal 1991, } 301 \\
\text { (76); RIB II 2415.6 }\end{array}$ \\
\hline NU0013 & Vessel (fragment) & & Northumberland & Corbridge & M & Fort (Corstopitum) & & & $\begin{array}{l}\text { Eggers 1968, } 108 \\
62\end{array}$ \\
\hline
\end{tabular}




\begin{tabular}{|c|c|c|c|c|c|c|c|c|c|}
\hline NU0014 & Vessel (fragment) & & Northumberland & Rudchester & M & Fort (Vindobala) & Concentic cirlce umbo & & \begin{tabular}{|l} 
Eggers 1968, 108 \\
$64 a$
\end{tabular} \\
\hline NU0015 & Handled Pan 3 & Eggers 160-161 & Northumberland & Rudchester & M & Fort (Vindobala) & & & $\begin{array}{l}\text { Eggers 1968, } 108 \\
64 \mathrm{~b}\end{array}$ \\
\hline NU0016 & Handled Pan 2 & Eggers 144 & Northumberland & South Shields & M & & $\begin{array}{l}\text { Concentric circle umbo, } \\
\text { floral and geometric band } \\
\text { under rim }\end{array}$ & Concentric circle handle ring, punch dots & \begin{tabular}{|l|} 
Bosanquet \& \\
Richmond 1936, \\
139-151; Eggers \\
1968, 108 66a \\
\end{tabular} \\
\hline NU0017 & $\begin{array}{l}\begin{array}{l}\text { Handled Pan } 3 \\
\text { (handle) }\end{array} \\
\end{array}$ & Eggers 160-161 & Northumberland & South Shields & M & & & & $\begin{array}{l}\text { Eggers 1968, } 108 \\
(66 \mathrm{c})\end{array}$ \\
\hline NU0018 & Vessel (fragment) & & Northumberland & South Shields & M & & & & \begin{tabular}{|l} 
Eggers 1968, 108 \\
(66d)
\end{tabular} \\
\hline NU0026 & $\begin{array}{l}\text { Handled Pan } 2 \\
\text { (fragment) }\end{array}$ & $\begin{array}{l}\text { Rudge Cup, Eggers } \\
157\end{array}$ & Northumberland & Harwood & RNS & & $\begin{array}{l}\text { Geometric dots, scallops, } \\
\text { and shields, enameled }\end{array}$ & & $\begin{array}{l}\text { Eggers 1968, } 108 \\
(65 \mathrm{~A}) ; \mathrm{BM} \\
1852.1001 .1 \\
\end{array}$ \\
\hline NU0027 & Strainer & Eggers 160 & Northumberland & Hexham & RS & (Whitfield) & $\begin{array}{l}\text { Geometric circle and } \\
\text { scallops strainer dot pattern }\end{array}$ & & \begin{tabular}{|l} 
Eggers 1968, 108 \\
$(63)$
\end{tabular} \\
\hline NU0028 & Vessel (fragment) & Eggers 140-144 & Northumberland & Bolton & RNS & & $\begin{array}{l}\text { Concentric circle umbo, } \\
\text { omphalos dot, concentric } \\
\text { circle foot ring }\end{array}$ & & \begin{tabular}{|l|} 
Eggers 1968, 109 \\
(67); Newcastle \\
Museum 1956, 130 \\
A \\
\end{tabular} \\
\hline NU0031 & \begin{tabular}{|l|}
$\begin{array}{l}\text { Handled Pan } 2 \\
\text { (fragment) }\end{array}$ \\
\end{tabular} & Eggers $145-147$ & Northumberland & Birdoswald & M & Fort (Birdoswald) & $\begin{array}{l}\text { Trefoil suspension loop in } \\
\text { handle medallion }\end{array}$ & & $\begin{array}{l}\text { Wilmott 1997, } 290 \\
(125)\end{array}$ \\
\hline NU0032 & Jug (fragment) & & Northumberland & Birdoswald & M & Fort (Birdoswald) & & & $\begin{array}{l}\text { Wilmott 1997, } 290 \\
\text { (126) }\end{array}$ \\
\hline NU0033 & Jar (fragment) & & Northumberland & Bardon Mill & M & $\begin{array}{l}\text { Housesteads Fort } \\
\text { (Borcovicium/Verco } \\
\text { vicium) }\end{array}$ & $\begin{array}{l}\text { Geometric enameled } \\
\text { triangle and zig zag body } \\
\text { bands }\end{array}$ & & Wilson 2002, 79 \\
\hline NU0034 & Jug (fragment) & & Northumberland & Corbridge & M & Fort (Corstopitum) & & Floral leaf-shaped handle medallion & \begin{tabular}{|l|} 
Bishop \& Dore \\
1988, 173 (103)
\end{tabular} \\
\hline NU0036 & Jug & & Northumberland & Corbridge & M & $\begin{array}{l}\text { Fort (Corstopitum), } \\
\text { east end of field, } \\
\text { Site XLIII }\end{array}$ & & $\begin{array}{l}\text { Floral leaf embellishments; } \\
\text { Anthropomorphic (possible) handle } \\
\text { medallion }\end{array}$ & $\begin{array}{l}\text { Forester et al. 1913, } \\
235\end{array}$ \\
\hline NU0037 & Vessel (fragment) & & Northumberland & Corbridge & M & \begin{tabular}{|l|}
$\begin{array}{l}\text { Haltonchesters } \\
\text { Roman Fort } \\
\text { (Hunnum) }\end{array}$ \\
\end{tabular} & Concentric circle umbo & & Dore 2010,147 (26) \\
\hline NU0038 & Jar & & Northumberland & Bardon Mill & M & $\begin{array}{l}\text { Housesteads Fort } \\
\text { (Borcovicium/Verco } \\
\text { vicium) }\end{array}$ & $\begin{array}{l}\text { Geometric triangle, wave, } \\
\text { and linear bands; enameled } \\
\text { in green and turquoise }\end{array}$ & & $\begin{array}{l}\text { Rushmorth 2009, } \\
438 \text { (50) }\end{array}$ \\
\hline NU0039 & Vessel (fragment) & & Northumberland & Bardon Mill & M & $\begin{array}{l}\text { Housesteads Fort } \\
\text { (Borcovicium/Verco } \\
\text { vicium) }\end{array}$ & Concentric circle rim & & $\begin{array}{l}\text { Rushmorth 2009, } \\
438 \text { (51) }\end{array}$ \\
\hline NU0040 & Vessel (mount) & & Northumberland & Bardon Mill & M & $\begin{array}{l}\text { Housesteads Fort } \\
\text { (Borcovicium/Verco } \\
\text { vicium) }\end{array}$ & & $\begin{array}{l}\text { Geometric pelta shaped hanging vessel } \\
\text { mount }\end{array}$ & $\begin{array}{l}\text { Rushmorth 2009, } \\
438 \text { (54) }\end{array}$ \\
\hline NU0041 & Vessel (fragment) & & Northumberland & South Shields & M & $\begin{array}{l}\text { South east corner of } \\
\text { fort }\end{array}$ & Concentric circle rim & & $\begin{array}{l}\text { Miket 1983, } 111 \\
(41)\end{array}$ \\
\hline NU0042 & Vessel (fragment) & & Northumberland & South Shields & M & $\begin{array}{l}\text { Southwest gate } \\
\text { ditches }\end{array}$ & $\begin{array}{l}\text { Concentric circle umbo and } \\
\text { omphalos dot; three } \\
\text { undecorated feet }\end{array}$ & & \begin{tabular}{|l} 
Bidwell \& Speak \\
1994, 186 (45)
\end{tabular} \\
\hline NU0043 & $\begin{array}{l}\begin{array}{l}\text { Handled Pan } 2 \\
\text { (handle) }\end{array} \\
\end{array}$ & Eggers 139-144 & Northumberland & Bardon Mill & M & $\begin{array}{l}\text { Vindolanda } \\
\text { (2003/2004 Site A) }\end{array}$ & & & \begin{tabular}{|l|} 
Birley \& Blake \\
$2005,256(9161)$
\end{tabular} \\
\hline NU0044 & Bowl & & Northumberland & Bardon Mill & M & $\begin{array}{l}\text { Vindolanda (2003 } \\
\text { Area B) }\end{array}$ & & & \begin{tabular}{|l|} 
Birley \& Blake \\
$2005,259(8955)$
\end{tabular} \\
\hline NU0045 & Handled Pan 1 & Eggers 154-155 & Northumberland & Bardon Mill & M & $\begin{array}{l}\text { Vindolanda (Area A } \\
\text { 2005) }\end{array}$ & & Zoomorphic canine handle terminal & \begin{tabular}{|l|} 
Birley \& Blake \\
2007, 221 (10035) \\
\end{tabular} \\
\hline
\end{tabular}




\begin{tabular}{|c|c|c|c|c|c|c|c|c|c|}
\hline NU0046 & Handled Pan 2 & Eggers 139-144 & Northumberland & Bardon Mill & M & $\begin{array}{l}\text { Vindolanda (2005 } \\
\text { Area B) }\end{array}$ & & & \begin{tabular}{|l} 
Birley \& Blake \\
2007, 228 (9845)
\end{tabular} \\
\hline NU0047 & $\begin{array}{l}\text { Handled Pan } 2 \\
\text { (handle) }\end{array}$ & Eggers 139-144 & Northumberland & Bardon Mill & M & Vindolanda & & $\begin{array}{l}\text { Inscription in punch dots reads "[...] } \\
\text { ERIORIS" }\end{array}$ & \begin{tabular}{|l} 
Burnham et alia \\
2005,492
\end{tabular} \\
\hline NU0048 & $\begin{array}{l}\text { Handled Pan } 2 \\
\text { (handle) }\end{array}$ & Eggers 139-144 & Northumberland & Bardon Mill & M & Vindolanda & & Inscription in punch dots reads "BRO" & $\begin{array}{l}\text { Burnham et alia } \\
2005,492\end{array}$ \\
\hline NYR0029 & Jar & Ostland, Eggers 40 & North Yorkshire & York & $\mathrm{U}$ & $\begin{array}{l}\text { River, Blue Bridge } \\
\text { Lane }\end{array}$ & $\begin{array}{l}\text { Two incriptions on rim "C } \\
\text { ATTISIIVIIRI" (in punch } \\
\text { dots), "C APRILI" (straight } \\
\text { etched) post manufacture } \\
\end{array}$ & & $\begin{array}{l}\text { Eggers } 1968,107 \\
\text { (52a); RCH 1962, } \\
133 \text { (143); RIB II } \\
2415.58\end{array}$ \\
\hline NYR0030 & Vessel (fragment) & & North Yorkshire & York & $\mathrm{U}$ & River, (River Ouse) & & & $\begin{array}{l}\text { Eggers 1968, } 107 \\
(52 c)\end{array}$ \\
\hline NYR0031 & Bowl & Eggers 101-105 & North Yorkshire & Malton & RS & & Concentric circle foot ring & & $\begin{array}{l}\text { Eggers 1968, } 107 \\
\text { (56a) }\end{array}$ \\
\hline NYR0032 & Handled Pan 2 & Eggers 139-144 & North Yorkshire & Malton & RS & & & $\begin{array}{l}\text { stamped inscription "ALPICVSF", punch } \\
\text { dot inscriptions "LSERVENISVSVPER" } \\
\text { and "SVPERI" under handle }\end{array}$ & $\begin{array}{l}\text { Eggers 1968, } 107 \\
\text { (56b); McPeake \& } \\
\text { Moore 1978, 333 } \\
\text { (4); RIB II 2415.4 }\end{array}$ \\
\hline NYR0033 & Vessel (fragment) & & North Yorkshire & Aldborough & $\mathrm{U}$ & & Concentric circle umbo & & $\begin{array}{l}\text { Bishop 1996, } 10 \\
(16)\end{array}$ \\
\hline NYR0034 & Jug (fragment) & Eggers $123-126$ & North Yorkshire & Aldborough & $\mathrm{U}$ & & \begin{tabular}{|l|}
$\begin{array}{l}\text { Zoomorphic duck } \\
\text { thumbrest }\end{array}$ \\
\end{tabular} & & $\begin{array}{l}\text { Bishop 1996, } 10 \\
\text { (18) }\end{array}$ \\
\hline NYR0035 & Vessel (mount) & & North Yorkshire & Aldborough & $\mathrm{u}$ & & $\begin{array}{l}\text { Geometric sub-pelta shaped } \\
\text { hanging vessel mount }\end{array}$ & & $\begin{array}{l}\text { Bishop 1996, } 14 \\
(22)\end{array}$ \\
\hline NYR0036 & Strainer (fragment) & & North Yorkshire & $\begin{array}{l}\text { Beadlam Roman } \\
\text { Villa }\end{array}$ & RS & $\begin{array}{l}\text { Villa, Corridor 3, } \\
\text { Building 1 }\end{array}$ & $\begin{array}{l}\begin{array}{l}\text { Concentric circle (possible) } \\
\text { strainer dot pattern }\end{array} \\
\end{array}$ & & Neal 1996, $49(17)$ \\
\hline NYR0038 & Jar & & North Yorkshire & Catterick & RS & $\begin{array}{l}\text { Catterick Bypass, } \\
\text { Site } 433\end{array}$ & \begin{tabular}{|l|} 
Geometric triangle body \\
bands above and below two \\
crescent body bands, both \\
bands in red and blue \\
enamel
\end{tabular} & & $\begin{array}{l}\text { Wilson 2002, } 78 \\
\text { (1) }\end{array}$ \\
\hline NYR0039 & Vessel (fragment) & & North Yorkshire & York & $\mathrm{U}$ & Minster, Building 4 & Concentric circle umbo & & \begin{tabular}{|l} 
Philips \& Heywood \\
1995, 385 (46)
\end{tabular} \\
\hline OX0003 & Vessel (mount) & & Oxfordshire & North Leigh & RS & $\begin{array}{l}\text { Shakenoak Farm; } \\
\text { hypocaust in room } \\
\text { XVI Building A }\end{array}$ & $\begin{array}{l}\text { Zoomorphic bull bust } \\
\text { hanging vessel mount }\end{array}$ & & $\begin{array}{l}\text { BrodRIB IIb et alia } \\
2005,52(1)\end{array}$ \\
\hline OX0004 & Vessel (fragment) & & Oxfordshire & North Leigh & RS & $\begin{array}{l}\text { Shakenoak Farm; } \\
\text { north of Fish Pond } \\
\text { II }\end{array}$ & & & $\begin{array}{l}\text { BrodRIB IIb et alia } \\
2005,482(250)\end{array}$ \\
\hline OX0005 & Vessel (fragment) & & Oxfordshire & North Leigh & RS & \begin{tabular}{|l} 
Akeman Street, \\
Field 5651
\end{tabular} & Concentric circle rim & & $\begin{array}{l}\text { Hands et alia 2004, } \\
50(134)\end{array}$ \\
\hline PO0001 & Handled Pan 1 & Eggers 154-155 & Powys & Caersws & RNS & & & $\begin{array}{l}\text { Zoomorphic ram's head handle terminal, } \\
\text { fluted handle }\end{array}$ & $\begin{array}{l}\text { Eggers 1968, } 103 \\
(15 \mathrm{a} \& b)\end{array}$ \\
\hline PO0008 & Handled Pan 2 & Eggers 150 & Powys & Brecon & $\mathrm{M}$ & Fort & & $\begin{array}{l}\text { inscription "[...] RODITI" in rectangular } \\
\text { field; keyhole shaped handle loop }\end{array}$ & $\begin{array}{l}\text { Eggers 1968, } 103 \\
\text { (14); Wheeler 1928, } \\
\text { 107-111; RIB II } \\
2415.7\end{array}$ \\
\hline PO0009 & Bowl & & Powys & Brecon & $\mathrm{M}$ & $\begin{array}{l}10 \text { feet north of } \\
\text { Building A, outside } \\
\text { the fort }\end{array}$ & & & $\begin{array}{l}\text { Wheeler 1928, 111- } \\
112\end{array}$ \\
\hline PO0010 & Vessel (fragment) & & Powys & Brecon & M & Retentura & & & Wheeler 1928,112 \\
\hline PO0011 & Jug (handle) & & Powys & Brecon & $\mathrm{M}$ & $\begin{array}{l}\text { Hutment of } \\
\text { praetentura }\end{array}$ & & $\begin{array}{l}\text { Anthropomorphic Medusa's head handle } \\
\text { medallion }\end{array}$ & $\begin{array}{l}\text { Wheeler 1928, } 117 \\
(1)\end{array}$ \\
\hline
\end{tabular}




\begin{tabular}{|c|c|c|c|c|c|c|c|c|c|}
\hline SH0004 & Jar & & Shropshire & Wroxeter & $\mathrm{U}$ & Well 1 & & & $\begin{array}{l}\text { Atkinson 1942, } 209 \\
\text { (1) }\end{array}$ \\
\hline SH0005 & $\begin{array}{l}\text { Handled Pan } 2 \\
\text { (handle) }\end{array}$ & Eggers 139-144 & Shropshire & Wroxeter & $\mathrm{U}$ & East Room 1 & & Geometric 'key-hole' suspension loop & $\begin{array}{l}\text { Atkinson 1942, } 209 \\
\text { (3) }\end{array}$ \\
\hline SH0006 & $\begin{array}{l}\text { Handled Pan } 2 \\
\text { (handle) }\end{array}$ & Eggers 139-144 & Shropshire & Wroxeter & $\mathrm{U}$ & $\begin{array}{l}\text { outside east wall of } \\
\text { Baths, Room } 2\end{array}$ & & $\begin{array}{l}\text { Concentric circle handle medallion and } \\
\text { suspension loop }\end{array}$ & $\begin{array}{l}\text { Atkinson 1942, } 209 \\
\text { (4) }\end{array}$ \\
\hline SH0006 & Jug (lid) & Eggers $123-126$ & Shropshire & Wroxeter & $\mathrm{U}$ & East Entrance & Trefoil lid & & $\begin{array}{l}\text { Atkinson 1942, } 210 \\
(12, \text { A 255) }\end{array}$ \\
\hline SH0007 & Jug (lid) & Eggers 123-126 & Shropshire & Wroxeter & $\mathrm{U}$ & $\begin{array}{l}\text { Courty yard west of } \\
\text { Room A }\end{array}$ & Trefoil lid & & $\begin{array}{l}\text { Atkinson 1942, } 210 \\
\text { (12, A 258) }\end{array}$ \\
\hline SH0008 & Jug (lid) & Eggers 123-126 & Shropshire & Wroxeter & $\mathrm{U}$ & Pit 10 & Trefoil lid & & $\begin{array}{l}\text { Atkinson 1942, } 210 \\
\text { (12, A 257) }\end{array}$ \\
\hline SH0009 & Jug (lid) & Eggers $123-126$ & Shropshire & Wroxeter & $\mathrm{U}$ & Dump & Trefoil lid & & $\begin{array}{l}\text { Atkinson 1942, } 210 \\
\text { (12 A 256) }\end{array}$ \\
\hline SH0010 & Jug & Eggers $123-126$ & Shropshire & Wroxeter & $\mathrm{U}$ & Bath & Trefoil lid & & $\begin{array}{l}\text { Ellis 2000, } 120 \\
(235)\end{array}$ \\
\hline SH0011 & $\begin{array}{l}\text { Handled Pan } 2 \\
\text { (handle) }\end{array}$ & Eggers 137-138 & Shropshire & Wroxeter & $\mathrm{U}$ & $\begin{array}{l}\text { House on south side } \\
\text { of baths }\end{array}$ & & inscription reads "[...]ESRV[...]" & $\begin{array}{l}\text { Wright 1958, } 152 \\
\text { (14); RIB II } 2415.52\end{array}$ \\
\hline SO0001 & Bowl & | & Somerset & Somerton & RS & \begin{tabular}{|l} 
Bradley Hill, \\
Building F
\end{tabular} & $\begin{array}{l}\text { Repouse decoration and } \\
\text { champleve enameling }\end{array}$ & & Wilson 1971, 276 \\
\hline SO0002 & Handled Pan 2 & Rudge Cup Type & Somerset & Somerton & RS & $\begin{array}{l}\text { Bradley Hill, } \\
\text { Building } 2\end{array}$ & $\begin{array}{l}\text { Floral vine and leaf swirl } \\
\text { and 's' scroll decoration } \\
\text { with blue, green and red } \\
\text { enamel }\end{array}$ & & Leech 1981,210 \\
\hline SYR0002 & $\begin{array}{l}\text { Handled Pan } 2 \\
\text { (handle) }\end{array}$ & Eggers 139-144 & South Yorkshire & Doncaster & $\mathrm{M}$ & $\begin{array}{l}\text { Frenchgate (Site } \\
\text { DG) }\end{array}$ & & & $\begin{array}{l}\text { Buckland \& } \\
\text { Magilton 1986, } 85 \\
\text { (3) }\end{array}$ \\
\hline SYR0003 & Jug (lid) & & South Yorkshire & Rotherham & M & Templeborough & $\begin{array}{l}\text { Floral (?) leaf shaped jug } \\
\text { lid }\end{array}$ & & May 1922, 75 (1) \\
\hline SYR0004 & Jug (lid) & & South Yorkshire & Rotherham & M & Templeborough & $\begin{array}{l}\begin{array}{l}\text { Floral (?) leaf shaped jug } \\
\text { lid }\end{array} \\
\end{array}$ & & May 1922, 75 (9) \\
\hline STA0006 & $\begin{array}{l}\text { Handled Pan } 1 \\
\text { (handle) }\end{array}$ & Eggers 154-155 & Staffordshire & Rocester & RS & $\begin{array}{l}\text { Otron's Farm } \\
\text { ('shrine?') }\end{array}$ & & $\begin{array}{l}\text { Anthropomorphic 'Bacchic medusa' } \\
\text { handle terminal; zoomorphic lion bust } \\
\text { undercarriage; fluted handle }\end{array}$ & $\begin{array}{l}\text { Ferris et alia 2000, } \\
55(10)\end{array}$ \\
\hline STA0007 & Vessel (mount) & & Staffordshire & Wall & RS & Castle Croft & $\begin{array}{l}\text { Zoomorphic ox head } \\
\text { mount }\end{array}$ & & Gould 1964, 43 (8) \\
\hline SUF0005 & Handled Pan 2 & Eggers 139 & Suffolk & $\begin{array}{l}\text { Somberleyton } \\
\text { Ashby and } \\
\text { Herringfleet }\end{array}$ & RNS & $\begin{array}{l}\text { Swamp } \\
\text { (Herringfleet) }\end{array}$ & Concentric circle foot ring & $\begin{array}{l}\text { Circle hanlde loop; caducaeus with } \\
\text { punch dot embellishments on handle; } \\
\text { inscription in sub-rectangular filed } \\
\text { "QVATTENVSF". }\end{array}$ & $\begin{array}{l}\text { Eggers 1968, } 106 \\
\text { (43); McPeake \& } \\
\text { Moore 1978, } 334 \\
\text { (32); RIB II 2415.43 }\end{array}$ \\
\hline SUF0037 & Strainer (fragment) & & Suffolk & Hacheston & RS & Field 1 & Linear punch dots & & $\begin{array}{l}\text { Blagg et al. 2004, } \\
123(149)\end{array}$ \\
\hline SUF0038 & Jug (fragment) & & Suffolk & Hacheston & RS & & & & $\begin{array}{l}\text { Blagg et al. 2004, } \\
123(152)\end{array}$ \\
\hline SUF0039 & Vessel (mount) & & Suffolk & Hacheston & RS & Field 1 & $\begin{array}{l}\text { Floral leaf shaped hanging } \\
\text { vessel mount }\end{array}$ & & $\begin{array}{l}\text { Blagg et al. 2004, } \\
123 \text { (153) }\end{array}$ \\
\hline SUF0040 & Vessel (mount) & & Suffolk & Hacheston & RS & Field 2 & $\begin{array}{l}\text { Floral leaf shaped hanging } \\
\text { vessel mount }\end{array}$ & & $\begin{array}{l}\text { Blagg et al. 2004, } \\
123 \text { (154) }\end{array}$ \\
\hline SUF0041 & Vessel (mount) & & Suffolk & Hacheston & RS & Field 2 & $\begin{array}{l}\text { Floral leaf shaped hanging } \\
\text { vessel mount }\end{array}$ & & $\begin{array}{l}\text { Blagg et al. 2004, } \\
123 \text { (155) }\end{array}$ \\
\hline SUF0042 & Vessel (mount) & & Suffolk & Hacheston & RS & Field 2 & $\begin{array}{l}\text { Floral leaf shaped hanging } \\
\text { vessel mount }\end{array}$ & & $\begin{array}{l}\text { Blaggg et al. 2004, } \\
124(156)\end{array}$ \\
\hline SUF0047 & Jug (handle) & & Suffolk & Otley & RNS & & & & \begin{tabular}{|l|} 
Burnham et al. \\
2001,360
\end{tabular} \\
\hline SUF0048 & Bowl & Irchester & Suffolk & Lakenheath Fen & RS & & & & BM 1880.0802.168 \\
\hline
\end{tabular}




\begin{tabular}{|c|c|c|c|c|c|c|c|c|c|}
\hline SUF0049 & Jug & & Suffolk & Ipswich & RS & & & & BM 1857.0806.2 \\
\hline SUF0050 & Jug & & Suffolk & Brandon & RNS & & & $\begin{array}{l}\text { Anthropomorphic human foot handle } \\
\text { medallion }\end{array}$ & BM 1872.0816.9 \\
\hline SUR0012 & Bowl & & Surrey & Walton on Thames & RNS & River Thames & & & BM 1909.0320.1 \\
\hline SWA0001 & Vessel (fragment) & & Swansea & Loughor & M & Leucarum Fort & & & \begin{tabular}{|l} 
Marvell \& Owen- \\
John 1997, 247 (55)
\end{tabular} \\
\hline SWA0002 & Handled Pan 2 & Eggers 140-142 & Swansea & Loughor & $\mathrm{M}$ & Leucarum Fort & $\begin{array}{l}\text { Concentric circle umbo and } \\
\text { omphalos dot; Geometric } \\
\text { linear etched body band; } \\
\text { Concentric circle foot ring }\end{array}$ & $\begin{array}{l}\text { Concentric circle handle medallion and } \\
\text { suspension loop }\end{array}$ & $\begin{array}{l}\text { Marvell \& Owen- } \\
\text { John 1997, } 247 \text { (56) }\end{array}$ \\
\hline VGL0001 & Jug (handle) & & Vale of Glamorgan & Wenvoe & RNS & $\begin{array}{l}\text { Whitton Cross } \\
\text { Roads }\end{array}$ & & & $\begin{array}{l}\text { Jarrett \& Wrathmell } \\
\text { 1981, 182 (50) }\end{array}$ \\
\hline WAR0003 & Bowl & Basin Uní & Warwickshire & Alcester & $\mathrm{M}$ & $\begin{array}{l}\text { Southern Extramural } \\
\text { Area }\end{array}$ & & & \begin{tabular}{|l|} 
Cracknell \& \\
Mahany 1994, 181, \\
120
\end{tabular} \\
\hline WAR0004 & Vessel (fragment) & & Warwickshire & Alcester & $\mathrm{M}$ & $\begin{array}{l}\text { Southern Extramural } \\
\text { Area }\end{array}$ & & & \begin{tabular}{|l|} 
Cracknell \& \\
Mahany 1994, 181, \\
121
\end{tabular} \\
\hline WAR0005 & Vessel (mount) & & Warwickshire & Alcester & M & $\begin{array}{l}\text { Southern Extramural } \\
\text { Area }\end{array}$ & $\begin{array}{l}\text { Floral leaf shaped hanging } \\
\text { vessel mount }\end{array}$ & & \begin{tabular}{|l|} 
Cracknell \& \\
Mahany 1994, 181, \\
122
\end{tabular} \\
\hline WAR0006 & Vessel (mount) & & Warwickshire & Alcester & M & $\begin{array}{l}\text { Southern Extramural } \\
\text { Area }\end{array}$ & $\begin{array}{l}\text { Floral (possible) leaf } \\
\text { shaped vessel mount }\end{array}$ & & \begin{tabular}{|l|} 
Cracknell \& \\
Mahany 1994, 181, \\
123
\end{tabular} \\
\hline WAR0007 & $\begin{array}{l}\text { Handled Pan } 2 \\
\text { (handle) }\end{array}$ & Eggers 139-144 & Warwickshire & Churchover & RNS & Cave's Inn & & $\begin{array}{l}\text { Concentric circle handle loop; inscription } \\
\text { "MATVRVS F" }\end{array}$ & $\begin{array}{l}\text { Wright R. 1961, } \\
195 \text { (17); Mcpeake } \\
\text { \& Moore 1978, } 334 \\
\text { (29); RIB II 2415.38 } \\
\end{array}$ \\
\hline WSU0002 & Jug (fragment) & & West Sussex & Chichester & $\mathrm{U}$ & $\begin{array}{l}\text { North West } \\
\text { Quadrent, Area 7. D } \\
36 \text { (685) }\end{array}$ & & Floral (possible) leaf handle medallion & $\begin{array}{l}\text { Down 1978, } 307 \\
\text { (153) }\end{array}$ \\
\hline WSU0003 & Vessel (fragment) & & West Sussex & Chichester & $\mathrm{U}$ & Cattlemarket & Geometric linear rim bands & & $\begin{array}{l}\text { Down 1989, } 196 \\
(62)\end{array}$ \\
\hline WSU0004 & Bowl (fragment) & & West Sussex & Chichester & $\mathrm{U}$ & County Hall & & & $\begin{array}{l}\text { Down 1989, } 218 \\
\text { (7) }\end{array}$ \\
\hline WSU0005 & Bowl (fragment) & & West Sussex & Chichester & $\mathrm{U}$ & Greyfriars & & & $\begin{array}{l}\text { Down \& Magilton } \\
1993,190(23)\end{array}$ \\
\hline WSU0006 & Bowl (fragment) & & West Sussex & Fishbourne & RS & A27 & & & $\begin{array}{l}\text { Cunliffe et al. 1996, } \\
200(34)\end{array}$ \\
\hline WSU0007 & Jug (handle) & & West Sussex & Fishbourne & RS & A27 & & $\begin{array}{l}\text { Anthropomorphic cherub face handle } \\
\text { medallion; Floral vine and leaf; } \\
\text { Zoomorphic cat's paw near handle } \\
\text { terminal; Floral leaf thumb rest }\end{array}$ & $\begin{array}{l}\text { Cunliffe et al. 1996, } \\
200 \text { (45); Down \& } \\
\text { Henig 1988, 308- } \\
309\end{array}$ \\
\hline WSU0008 & Vessel (fragment) & & West Sussex & Fishbourne & RS & \begin{tabular}{|l|} 
Fishbourne Roman \\
Villa
\end{tabular} & Concentric circle rim & & $\begin{array}{l}\text { Cunliffe } 1971,120 \\
(152)\end{array}$ \\
\hline WSU0009 & Vessel (fragment) & & West Sussex & Fishbourne & RS & $\begin{array}{l}\text { Fishbourne Roman } \\
\text { Villa }\end{array}$ & Concentric circle rim & & $\begin{array}{l}\text { Cunliffe 1971, } 120 \\
\text { (153) }\end{array}$ \\
\hline WYR0001 & Cauldron & & West Yorkshire & Castleford & $\mathrm{M}$ & Fort (Lagentium) & & & $\begin{array}{l}\text { Cool \& Philo 1998, } \\
81 \text { (333) }\end{array}$ \\
\hline WYR0002 & Vessel (fragment) & & West Yorkshire & Dalton Parlours & RS & Villa & Out turned rim & & $\begin{array}{l}\text { Wrathmell \& } \\
\text { Nicholson 1990, } 84 \\
\text { (22) }\end{array}$ \\
\hline
\end{tabular}




\begin{tabular}{|c|c|c|c|c|c|c|c|c|c|}
\hline WYR0003 & Vessel (fragment) & & West Yorkshire & Dalton Parlours & RS & Villa & Out turned rim & & $\begin{array}{l}\text { Wrathmell \& } \\
\text { Nicholson 1990, } 84 \\
\text { (23) } \\
\end{array}$ \\
\hline WYR0004 & Vessel (fragment) & & West Yorkshire & Ilkley & M & $\begin{array}{l}\text { Foundations of wall } \\
\text { F, Site IV }\end{array}$ & $\begin{array}{l}\text { Anthropomorphic horned } \\
\text { and bearded male bust } \\
\text { vessel mount, probably } \\
\text { Silenus or a satyr }\end{array}$ & & $\begin{array}{l}\text { Woodward 1925, } \\
280 \text { (I); JRS 10, } 185\end{array}$ \\
\hline WYR0005 & Handled Pan 2 & & West Yorkshire & Ilkley & M & \begin{tabular}{|l|} 
Site III (lowest \\
level)
\end{tabular} & & & $\begin{array}{l}\text { Woodward 1925, } \\
285 \text { (22) }\end{array}$ \\
\hline WYR0006 & $\begin{array}{l}\text { Handled Pan } 1 \\
\text { (handle) }\end{array}$ & Eggers 154-155 & West Yorkshire & Castleford & $\mathrm{M}$ & Fort (Lagentium) & & $\begin{array}{l}\text { Zoomorphic ram's head handle terminal, } \\
\text { fluted handle }\end{array}$ & \begin{tabular}{|l|} 
Cool \& Philo 1998, \\
$98(476)$
\end{tabular} \\
\hline WYR0007 & $\begin{array}{l}\text { Handled Pan } 2 \\
\text { (handle) }\end{array}$ & Eggers $140-142$ & West Yorkshire & Hebden Royd & RNS & Slack & & $\begin{array}{l}\text { Concentric circle handle medallion and } \\
\text { suspension loop }\end{array}$ & $\begin{array}{l}\text { Dodd \& Woodward } \\
\text { n.d., } 77 \text { (13) }\end{array}$ \\
\hline WIL0009 & $\begin{array}{l}\text { Handled Pan } 2 \\
\text { (handle) }\end{array}$ & & Wiltshire & Wanborough & RS & & & $\begin{array}{l}\text { Zoomorphic water bird's head rim } \\
\text { attachment }\end{array}$ & $\begin{array}{l}\text { Anderson et alia } \\
2001,92(106)\end{array}$ \\
\hline WIL0010 & $\begin{array}{l}\text { Handled Pan } 2 \\
\text { (handle) }\end{array}$ & & Wiltshire & Wanborough & RS & & & $\begin{array}{l}\text { Floral (possibly) leaf/beaded vine } \\
\text { decoration near rim }\end{array}$ & \begin{tabular}{|l} 
Anderson et alia \\
2001,93 (107)
\end{tabular} \\
\hline WIL0011 & Jug (lid) & Eggers $123-126$ & Wiltshire & Wanborough & RS & & $\begin{array}{l}\text { Floral (possibly) leaf } \\
\text { shaped lid }\end{array}$ & & \begin{tabular}{|l} 
Anderson et alia \\
$2001,113(242)$
\end{tabular} \\
\hline WIL0012 & Vessel (fragment) & & Wiltshire & Wanborough & RS & & & & $\begin{array}{l}\text { Anderson et alia } \\
2001,113(243)\end{array}$ \\
\hline WIL0024 & Handled Pan 2 & Rudge Cup type & Wiltshire & Rudge & RNS & & $\begin{array}{l}\text { Geometric enameled } \\
\text { crenilation (depicting wall); } \\
\text { inscription reads } \\
\text { ".A.MAISABALLAVAVX } \\
\text { ELODVMCAMBOGLAN } \\
\text { SBANNA" }\end{array}$ & & $\begin{array}{l}\text { Allason-Jones 2012, } \\
\text { 23-36; RIB II } \\
2415.53\end{array}$ \\
\hline WOR0003 & Bowl & & Worcestershire & Droitwich & RS & Hanbury Street & Flanged rim & & \begin{tabular}{|l} 
Hurst et al. 2006, 67 \\
$(9)$
\end{tabular} \\
\hline WOR0004 & Vessel (fragment) & & Worcestershire & Worcester & RS & Deansway & & & $\begin{array}{l}\text { Dalwood \& } \\
\text { Edwards 2004, } 410 \\
\text { (15) }\end{array}$ \\
\hline
\end{tabular}


Appendix V: Single Finds of Copper Alloy Vessels from Roman Britain reported through the Portable Antiquities Scheme.

\begin{tabular}{|c|c|c|c|c|c|c|c|c|}
\hline Number & Form & Type & County & Site & Context & Decoration (body) & Decoration (handle) & Reference \\
\hline BERK0002 & Vessel (mount) & & Berkshire & Chieveley & RU & $\begin{array}{l}\text { Zoomorphic non-aquatic bird } \\
\text { mount }\end{array}$ & & PAS BERK-291567 \\
\hline DER0001 & Vessel (fragment) & & Derbyshire & Ripley & RU & Slightly bulged rim & & $\begin{array}{l}\text { PAS DENO- } \\
\text { D72802 }\end{array}$ \\
\hline DOR0007 & Jug (fragment) & & Dorset & Castleton & RS & & $\begin{array}{l}\text { Anthropomorphic feet handle } \\
\text { terminal medallion }\end{array}$ & PAS DOR-2DCB21 \\
\hline DOR0008 & $\begin{array}{l}\text { Handled Pan } 2 \\
\text { (handle) }\end{array}$ & Eggers 139-144 & Dorset & Nether Compton & RS & & $\begin{array}{l}\text { Concentric ring handle } \\
\text { medallion }\end{array}$ & $\begin{array}{l}\text { PAS SOMDOR- } \\
\text { 0FE673 }\end{array}$ \\
\hline DOR0009 & Jug (fragment) & Eggers 128 & Dorset & Corfe Castle & RS & Geometric circular lid & & $\begin{array}{l}\text { PAS WAW- } \\
\text { 4B7FA1 }\end{array}$ \\
\hline DOR0010 & Bucket (fragment) & & Dorset & Stoke Abbott & M & $\begin{array}{l}\text { Geometric guilloche running } \\
\text { along top of foot }\end{array}$ & & $\begin{array}{l}\text { PAS SOMDOR- } \\
\text { 53DF91 }\end{array}$ \\
\hline DOR0011 & Vessel (fragment) & & Dorset & Compton Abbas & RS & $\begin{array}{l}\text { Zoomorphic bull's head } \\
\text { mount }\end{array}$ & & $\begin{array}{l}\text { PAS SOMDOR- } \\
\text { B23561 }\end{array}$ \\
\hline ESUS0001 & Jug (handle) & & East Sussex & Etchingham & RU & & $\begin{array}{l}\text { Anthropomorphic cherub } \\
\text { with prominent curls and } \\
\text { possible phrygian cap }\end{array}$ & PAS SUSS-C411A6 \\
\hline ESUS0002 & Bucket (fragment) & & East Sussex & St. Ann Without & RU & $\begin{array}{l}\text { Geometric fan design, } \\
\text { probably foot }\end{array}$ & & PAS SUSS-85A5E2 \\
\hline ESUS0003 & $\begin{array}{l}\text { Handled Pan } 2 \\
\text { (handle) }\end{array}$ & Rudge Cup Type & East Sussex & Southease & RU & & $\begin{array}{l}\text { Geometric etched lines form } \\
\text { diamond and triagular } \\
\text { shapes; possible enameling }\end{array}$ & PAS SUR-4DE0E1 \\
\hline EX0039 & Bucket (fragment) & & Essex & $\begin{array}{l}\text { Greenstead Green } \\
\text { And Halstead Rural }\end{array}$ & RU & Geometric 'pelta' bucket foot & & PAS ESS-8C8A14 \\
\hline EX0040 & Vessel (mount) & & Essex & Ugley & RU & $\begin{array}{l}\text { Geometric sub-triangular, } \\
\text { top shaped mount }\end{array}$ & & PAS BH-118825 \\
\hline EX0041 & Vessel (mount) & & Essex & Birch & RU & $\begin{array}{l}\text { Geometric sub-triangular, } \\
\text { top shaped mount }\end{array}$ & & PAS ESS-7F6EB2 \\
\hline EX0042 & Vessel (mount) & & Essex & Wix & RU & & & PAS ESS-332F71 \\
\hline EX0043 & $\begin{array}{l}\text { Handled Pan } 3 \\
\text { (handle) }\end{array}$ & Eggers 160 & Essex & Great Bentley & RU & & Hilted Handle & PAS ESS-6BE383 \\
\hline EX0044 & $\begin{array}{l}\text { Handled Pan } 1 \\
\text { (handle) }\end{array}$ & Eggers $154-155$ & Essex & Steeple Bumpstead & RU & & $\begin{array}{l}\text { Zoomorphic handle terminal, } \\
\text { either lion or a bear }\end{array}$ & PAS SF-9C7EA4 \\
\hline EX0045 & Handled Pan 4 & Coptic & Essex & Broxted & RU & & & PAS ESS-1D3342 \\
\hline EX0046 & Vessel (fragment) & & Essex & Roxwell & RU & $\begin{array}{l}\text { Zoomorphic birds head } \\
\text { suspension hook, probably a } \\
\text { swan or duck }\end{array}$ & & PAS ESS-E58103 \\
\hline EX0047 & Bowl & & Essex & Manningtree & $\mathrm{RU}$ & & & PAS ESS-45C445 \\
\hline EX0048 & Vessel (mount) & & Essex & Elsenham & RU & $\begin{array}{l}\text { Floral (possibly) leaf shaped } \\
\text { mount }\end{array}$ & & PAS ESS-333B24 \\
\hline
\end{tabular}




\begin{tabular}{|c|c|c|c|c|c|c|c|c|}
\hline EX0049 & Vessel (mount) & & Essex & Fordham & RU & & & PAS ESS-874B53 \\
\hline EX0050 & Vessel (fragment) & & Essex & Fingringhoe & RU & $\begin{array}{l}\text { Zoomorphic birds head } \\
\text { suspension hook, probably a } \\
\text { swan or duck }\end{array}$ & & PAS ESS-2B77F7 \\
\hline EX0051 & Vessel (mount) & & Essex & Good Easter & RU & $\begin{array}{l}\text { Floral leaf shaped hanging } \\
\text { vessel mount, five points }\end{array}$ & & PAS ESS-DD8738 \\
\hline EX0052 & Vessel (mount) & & Essex & Ardleigh & RU & Geometric pelta vessel foot & & PAS ESS-A61324 \\
\hline EY0001 & Vessel (handle) & & East Riding of Yorkshire & Fridaythorpe & RU & & Zoomorphic swan's head & PAS NLM-596735 \\
\hline EY0002 & $\begin{array}{l}\text { Handled Pan } 2 \\
\text { (handle) }\end{array}$ & & East Riding of Yorkshire & Humbleton & RU & & & PAS FAKL-58F455 \\
\hline EY0003 & Vessel (mount) & & East Riding of Yorkshire & North Cave & RU & Geometric fan-shaped mount & & $\begin{array}{l}\text { PAS SWYOR- } \\
\text { 53D721 }\end{array}$ \\
\hline EY0004 & Handled Pan 4 & Coptic & East Riding of Yorkshire & Hayton & RU & & & $\begin{array}{l}\text { PAS RESEARCH- } \\
\text { 230A51 }\end{array}$ \\
\hline EY0005 & Handled Pan 2 & Eggers 139-144 & East Riding of Yorkshire & Shipton Thorpe & RS & & Geometric framing lines & $\begin{array}{l}\text { PAS SWYOR- } \\
\text { 8F20A5 }\end{array}$ \\
\hline EY0006 & Jar & & East Riding of Yorkshire & Shipton Thorpe & RS & $\begin{array}{l}\text { Geometric punch dots along } \\
\text { base of the neck }\end{array}$ & & $\begin{array}{l}\text { PAS YORYM- } \\
103 E 35 \\
\end{array}$ \\
\hline EY0007 & Bowl & & East Riding of Yorkshire & Thwing & RS & & & PAS NCL-55ECD6 \\
\hline EY0008 & Vessel (mount) & & East Riding of Yorkshire & North Dalton & RS & $\begin{array}{l}\text { Geometric pelta shaped } \\
\text { vessel foot }\end{array}$ & & PAS NCL-B86E85 \\
\hline EY0009 & Vessel (mount) & & East Riding of Yorkshire & North Dalton & RS & $\begin{array}{l}\text { Geometric pelta shaped } \\
\text { vessel foot }\end{array}$ & & $\begin{array}{l}\text { PAS YORYM- } \\
6 \text { A0083 }\end{array}$ \\
\hline F0009 & Vessel (mount) & & Flintshire & Flint & RS & & & $\begin{array}{l}\text { PAS HESH- } \\
\text { A0AE36 }\end{array}$ \\
\hline G0004 & Vessel (mount) & & Gloucestershire & Twyning & RU & $\begin{array}{l}\text { Zoomorphic dragonesque } \\
\text { mount }\end{array}$ & & $\begin{array}{l}\text { PAS WAW- } \\
\text { CE0AC5 }\end{array}$ \\
\hline G0005 & Vessel (mount) & & Gloucestershire & Leigh & RS & Zoomorphic duck mount & & PAS WAW-C7F0F1 \\
\hline G0006 & Vessel (mount) & & Gloucestershire & Sudeley & RS & Zoomorphic ox head mount & & PAS GLO-63AEF2 \\
\hline HAM0004 & Bucket (fragment) & & Hampshire & Beaulieu & RU & $\begin{array}{l}\text { Geometric fan/'pelta' shaped } \\
\text { foot }\end{array}$ & & $\begin{array}{l}\text { PAS HAMP- } \\
\text { EFA6E4 }\end{array}$ \\
\hline HAM0005 & Jug (lid) & Eggers $123-126$ & Hampshire & Medstead CP & RS & $\begin{array}{l}\text { Zoomorphic duck statuette, } \\
\text { feather design encompasses } \\
\text { surface of lid }\end{array}$ & & $\begin{array}{l}\text { PAS HAMP- } \\
258 \mathrm{E} 52\end{array}$ \\
\hline HAM0006 & $\begin{array}{l}\text { Handled Pan } 1 \\
\text { (handle) }\end{array}$ & Eggers $154-155$ & Hampshire & Ropley & RS & $\begin{array}{l}\text { Zoomorphic ram's head } \\
\text { handle terminal }\end{array}$ & & $\begin{array}{l}\text { PAS HAMP- } \\
\text { D46597 }\end{array}$ \\
\hline HAM0007 & Vessel (fragment) & & Hampshire & King's Worthy & RU & & $\begin{array}{l}\text { Floral leaf thumb rest, } \\
\text { possibly palm }\end{array}$ & $\begin{array}{l}\text { PAS HAMP- } \\
\text { D685D2 }\end{array}$ \\
\hline HAM0008 & Vessel (fragment) & & Hampshire & Broughton & RU & $\begin{array}{l}\text { Zoomorphic bird statuette on } \\
\text { lid, perhaps a duck or } \\
\text { chicken }\end{array}$ & & PAS SUR-411370 \\
\hline HAM0009 & Vessel (mount) & & Hampshire & Ropley & RS & Floral leaf shaped mount & & PAS SUR-934DA8 \\
\hline HAM0010 & Vessel (mount) & & Hampshire & Ropley & RS & $\begin{array}{l}\text { Floral (possible) leaf shaped } \\
\text { mount }\end{array}$ & & PAS SUR-2D6151 \\
\hline
\end{tabular}




\begin{tabular}{|c|c|c|c|c|c|c|c|c|}
\hline HAM0011 & Vessel (fragment) & & Hampshire & Damerham & RS & & $\begin{array}{l}\text { Geometric etched lines on } \\
\text { handle sides }\end{array}$ & PAS WILT-149137 \\
\hline HAM0012 & Vessel (fragment) & & Hampshire & Owslebury & RS & & & $\begin{array}{l}\text { PAS FASW- } \\
4 \text { CB045 }\end{array}$ \\
\hline HAM0013 & Vessel (fragment) & & Hampshire & Owslebury & RS & Zoomorphic duck statuette & & PAS HAMP3382 \\
\hline HАM0014 & Vessel (fragment) & & Hampshire & $\begin{array}{l}\text { Corhampton And } \\
\text { Meonstoke }\end{array}$ & RU & $\begin{array}{l}\text { Geometric fan/'pelta' shaped } \\
\text { foot }\end{array}$ & & PAS HAMP2278 \\
\hline HAM0015 & Vessel (fragment) & & Hampshire & Wherwell & RU & & $\begin{array}{l}\text { Zoomorphic birds head } \\
\text { terminal, perhaps a swan }\end{array}$ & PAS HAMP336 \\
\hline HER0020 & Vessel (mount) & & Hertfordshire & St. Michael & RU & $\begin{array}{l}\text { Concentric circle punch dots, } \\
\text { circle handle loop }\end{array}$ & & PAS BH-5EC1F6 \\
\hline HER0021 & Vessel (mount) & & Hertfordshire & Clothall & RS & $\begin{array}{l}\text { Geometric triangular shaped } \\
\text { mount }\end{array}$ & & PAS BH-FB17E1 \\
\hline HER0022 & Vessel (mount) & & Hertfordshire & Clothall & RS & $\begin{array}{l}\text { Anthropomorphic bust, } \\
\text { round headed male with } \\
\text { wide nose, large eyes, and } \\
\text { wavy hair, possibly satyr or } \\
\text { Pan }\end{array}$ & & PAS BH-C 89753 \\
\hline HER0023 & Jug (lid) & Eggers $123-126$ & Hertfordshire & Watton-at-Stone & RS & $\begin{array}{l}\text { Floral leaf (possibly) shaped } \\
\text { lid }\end{array}$ & & PAS BH-57C9D5 \\
\hline HER0024 & Vessel (mount) & & Hertfordshire & Clothall & RS & Zoomorphic bird mount & & PAS BH-231086 \\
\hline HER0025 & Vessel (mount) & & Hertfordshire & Ashwell & RS & $\begin{array}{l}\text { Zoomorphic bird mount, } \\
\text { appears to be a duck }\end{array}$ & & PAS BH-5D2737 \\
\hline HER0026 & Bucket (fragment) & & Hertfordshire & Albury & RU & $\begin{array}{l}\text { Geometric crescent bucket } \\
\text { foot }\end{array}$ & & PAS BH-4FF557 \\
\hline HER0027 & Vessel (mount) & & Hertfordshire & Much Hadham & RU & $\begin{array}{l}\text { Anthropomorphic bust, } \\
\text { female with two buns in hair, } \\
\text { possibly Diana }\end{array}$ & & PAS ESS-C55282 \\
\hline HER0028 & Vessel (mount) & & Hertfordshire & Wymondley & RS & $\begin{array}{l}\text { Zoomorphic bull's head } \\
\text { hanging vessel mount }\end{array}$ & & PAS BH-1729A7 \\
\hline IOW0001 & Vessel (fragment) & & Isle of Wight & Newport & RS & $\begin{array}{l}\text { Geometric crescent shaped } \\
\text { bucket foot }\end{array}$ & & PAS IOW-9CE336 \\
\hline IOW0002 & Vessel (mount) & & Isle of Wight & Newport & RS & $\begin{array}{l}\text { Zoomorphic bird mount, } \\
\text { perhaps a swan or duck }\end{array}$ & & PAS IOW-BDD755 \\
\hline
\end{tabular}




\begin{tabular}{|c|c|c|c|c|c|c|c|c|}
\hline IOW0003 & Vessel (mount) & & Isle of Wight & Bembridge & RU & $\begin{array}{l}\text { Anthropomorphic mount of a } \\
\text { reclining male banquetor }\end{array}$ & & PAS IOW-2F7DD1 \\
\hline K0029 & Vessel (mount) & & Kent & Lyminge & RU & $\begin{array}{l}\text { Geometric sub-triangular } \\
\text { mount }\end{array}$ & & PAS KENT-9604E7 \\
\hline K0030 & $\begin{array}{l}\text { Handled Pan } 1 \\
\text { (handle) }\end{array}$ & Eggers $154-155$ & Kent & Eynsford & RS & & $\begin{array}{l}\text { Zoomorphic ram's head } \\
\text { handle terminal, fluted } \\
\text { handle }\end{array}$ & PAS LON-B47821 \\
\hline K0031 & $\begin{array}{l}\text { Handled Pan } 3 \\
\text { (handle) }\end{array}$ & Eggers $160-161$ & Kent & Lenham & RU & & Hilted Handle & $\begin{array}{l}\text { PAS KENT- } \\
\text { 8DAE18 }\end{array}$ \\
\hline K0032 & Bowl & & Kent & Chislet, Upstreet & RU & & & $\begin{array}{l}\text { PAS KENT- } \\
\text { 6E89B4 }\end{array}$ \\
\hline K0033 & Jug & & Kent & Chislet, Upstreet & RU & Concentric circle foot ring & $\begin{array}{l}\text { Anthropomorphic bust } \\
\text { handled terminal, appears to } \\
\text { be youthful male with hat } \\
\text { (perhaps Phrygian); Floral } \\
\text { leaf thumb rest }\end{array}$ & $\begin{array}{l}\text { PAS KENT- } \\
\text { 6E5FE6 }\end{array}$ \\
\hline LAN0003 & $\begin{array}{l}\text { Handled Pan } 3 \\
\text { (handle) }\end{array}$ & Eggers $160-161$ & Lancashire & Slyne with Hest & RU & & Hilted handle & $\begin{array}{l}\text { PAS LANCUM- } \\
101193\end{array}$ \\
\hline LEI0002 & Vessel (fragment) & & Leicestershire & Gaddesby & RU & $\begin{array}{l}\text { Geometric pelta shaped } \\
\text { bucket foot, duel-globular } \\
\text { "heart-shaped" foot in centre } \\
\text { of cresent }\end{array}$ & & PAS LEIC-92A461 \\
\hline LEI0003 & Vessel (fragment) & & Leicestershire & Leire & RU & $\begin{array}{l}\text { Gemoemtric pelta shaped } \\
\text { bucket foot }\end{array}$ & & PAS LEIC-055A12 \\
\hline LIN0004 & Vessel (fragment) & & Lincolnshire & Ancaster & RS & & Zoomorphic bird's head & $\begin{array}{l}\text { PAS WMID- } \\
\text { E86F58 }\end{array}$ \\
\hline LIN0005 & Handled Pan 1 & Eggers 155 & Lincolnshire & Scotton & RU & Concentric circle foot ring & & \begin{tabular}{|l|} 
PAS SWYOR- \\
FB6262 \\
\end{tabular} \\
\hline LIN0006 & Vessel (fragment) & & Lincolnshire & North Thoresby & RU & $\begin{array}{l}\text { Geometric pelta bucket foot, } \\
\text { 'Heart-shaped" foot in centre } \\
\text { of crescent }\end{array}$ & & PAS NLM-D01851 \\
\hline LIN0007 & Bowl & & Lincolnshire & Torksey & RU & & & $\begin{array}{l}\text { PAS SWYOR- } \\
54 \mathrm{~B} 841\end{array}$ \\
\hline LIN0008 & Vessel (fragment) & & Lincolnshire & Nettleton & RU & & $\begin{array}{l}\text { Zoomorphic bird's head, } \\
\text { swan }\end{array}$ & PAS LIN-567032 \\
\hline LIN0009 & Vessel (fragment) & & Lincolnshire & Nettleton & RU & & Zoomorphic bird's head, duck & PAS LIN-565C52 \\
\hline
\end{tabular}




\begin{tabular}{|c|c|c|c|c|c|c|c|c|}
\hline LIN0010 & $\begin{array}{l}\text { Handled Pan } 2 \\
\text { (handle) }\end{array}$ & & Lincolnshire & Thonock & RU & & & PAS LIN-D5C4E1 \\
\hline LIN0011 & Bowl & & Lincolnshire & Scotter & RU & & & $\begin{array}{l}\text { PAS SWYOR- } \\
8 \mathrm{E} 4 \mathrm{C} 25\end{array}$ \\
\hline LIN0012 & Vessel (fragment) & & Lincolnshire & Weston & RU & $\begin{array}{l}\text { Geometric pelta shaped } \\
\text { bucket foot, "Heart shaped" } \\
\text { foot in centre of crescent }\end{array}$ & & $\begin{array}{l}\text { PAS SWYOR- } \\
\text { A88651 }\end{array}$ \\
\hline LIN0013 & Jug (lid) & Eggers $123-126$ & Lincolnshire & Folkingham & RU & $\begin{array}{l}\text { Floral leaf (possible) shaped } \\
\text { jug lid }\end{array}$ & & PAS LIN-6C2E02 \\
\hline LIN0014 & Vessel (fragment) & & Lincolnshire & Bilsby & $\mathrm{RU}$ & $\begin{array}{l}\text { Zoomorphic bull's head } \\
\text { vessel spout }\end{array}$ & & PAS LIN-F8BC42 \\
\hline LIN0015 & Jug (handle) & & Lincolnshire & Lincoln & $\mathrm{U}$ & & $\begin{array}{l}\text { Zoomorphic shell or floral } \\
\text { palm handle medallion }\end{array}$ & PAS LIN-51C7A7 \\
\hline LIN0016 & $\begin{array}{l}\text { Handled Pan } 2 \\
\text { (handle) }\end{array}$ & Eggers 139-144 & Lincolnshire & Keelby & RU & & $\begin{array}{l}\text { Inscription in rectangular } \\
\text { field, broken "(..)VG" }\end{array}$ & PAS LIN-3EED71 \\
\hline LIN0017 & Vessel (fragment) & & Lincolnshire & Wickenby & RS & $\begin{array}{l}\text { Zoomorphic bull's head } \\
\text { vessel spout }\end{array}$ & & PAS NLM-5DF5D6 \\
\hline LIN0018 & Vessel (mount) & & Lincolnshire & Crowland & RU & $\begin{array}{l}\text { Zoomorphic dolphin hook, } \\
\text { "S" shaped }\end{array}$ & & PAS NLM-4255 \\
\hline LIN0019 & Vessel (fragment) & & Lincolnshire & $\begin{array}{l}\text { Stainton By } \\
\text { Langworth }\end{array}$ & $\mathrm{RU}$ & $\begin{array}{l}\text { Geometric pelta shaped } \\
\text { bucket foot }\end{array}$ & & PAS NLM-224 \\
\hline LIN0020 & Vessel (fragment) & & Lincolnshire & Gate Burton & RU & $\begin{array}{l}\text { Floral palm shaped handle } \\
\text { loop }\end{array}$ & & PAS LVPL-1244 \\
\hline LIN0021 & Vessel (fragment) & & Lincolnshire & Marston & RU & $\begin{array}{l}\text { Geometric pelta shaped } \\
\text { vessel foot }\end{array}$ & & PAS LIN-85A3A3 \\
\hline LIN0022 & Vessel (mount) & & Lincolnshire & Spilsby & RS & $\begin{array}{l}\text { Zoomorphic bull's head } \\
\text { vessel spout }\end{array}$ & & PAS LIN-40CE20 \\
\hline
\end{tabular}




\begin{tabular}{|c|c|c|c|c|c|c|c|}
\hline LIN0023 & Vessel (mount) & & Lincolnshire & Caistor & M & $\begin{array}{l}\text { Anthropomorphic female } \\
\text { bust, two buns in hair, } \\
\text { probably Diana or Luna }\end{array}$ & PAS NLM-AEA444 \\
\hline LIN0024 & Vessel (mount) & & Lincolnshire & Revesby & RU & $\begin{array}{l}\text { Geometric trefoil triangular } \\
\text { hanging vessel mount }\end{array}$ & PAS NCL-249C60 \\
\hline L0034 & Vessel (mount) & & London & Rainham & & $\begin{array}{l}\text { Geometric sub-triangular } \\
\text { mount }\end{array}$ & PAS ESS-1DAE38 \\
\hline MON0002 & Vessel (mount) & & Monmouthshire & Mathern & RU & $\begin{array}{l}\text { Zoomorphic dragonesque } \\
\text { ring hooks; Floral five } \\
\text { pointed leaf medallion }\end{array}$ & $\begin{array}{l}\text { PAS PUBLIC- } \\
\text { 74C } 930\end{array}$ \\
\hline MON0003 & Vessel (fragment) & & Monmouthshire & Usk & M & $\begin{array}{l}\text { Geometric pelta shaped } \\
\text { bucket foot, two nodes on } \\
\text { each side of crescent and } \\
\text { triangle in the middle }\end{array}$ & $\begin{array}{l}\text { PAS PUBLIC- } \\
\text { 749A73 }\end{array}$ \\
\hline MON0004 & Jug (lid) & Eggers $123-126$ & Monmouthshire & Usk & M & Floral leaf shaped jug lid & $\begin{array}{l}\text { PAS PUBLIC- } \\
\text { CF7051 }\end{array}$ \\
\hline MON0005 & Vessel (fragment) & & Monmouthshire & Llantrisant Fawr & RU & $\begin{array}{l}\begin{array}{l}\text { Zoomorphic bull's head } \\
\text { spout }\end{array} \\
\end{array}$ & $\begin{array}{l}\text { PAS PUBLIC- } \\
699346\end{array}$ \\
\hline MON0006 & Vessel (mount) & & Monmouthshire & Caerwent & $\mathrm{U}$ & $\begin{array}{l}\text { Zoomorphic boar shaped } \\
\text { mount }\end{array}$ & $\begin{array}{l}\text { PAS NMGW- } \\
\text { 2FC205 }\end{array}$ \\
\hline NE0001 & Bucket & & Newport & Langstone & RS & & $\begin{array}{l}\text { PAS NMGW- } \\
\text { 07F2B4 }\end{array}$ \\
\hline NE0002 & Vessel (mount) & & Newport & Langstone & RS & $\begin{array}{l}\text { Anthropomorphic reclining } \\
\text { banquetor mount }\end{array}$ & $\begin{array}{l}\text { PAS NMGW- } \\
\text { F4A3F6 }\end{array}$ \\
\hline NE0003 & Vessel (mount) & & Newport & Caerleon & M & Zoomorphic ox head mount & $\begin{array}{l}\text { PAS NMGW- } \\
\text { DBBD23 }\end{array}$ \\
\hline NLIN0001 & $\begin{array}{l}\text { Handled Pan } 2 \\
\text { (fragment) }\end{array}$ & Rudge Cup Type & North Lincolnshire & Crowle & RS & $\begin{array}{l}\text { Geometric swirls and circles, } \\
\text { triskele within a circular } \\
\text { field }\end{array}$ & PAS FAKL-9900E3 \\
\hline NLINO002 & Vessel (fragment) & & North Lincolnshire & Winteringham & RS & & PAS NLM-E3E502 \\
\hline NLINO003 & Vessel (fragment) & & North Lincolnshire & Winteringham & RS & $\begin{array}{l}\text { Geometric pelta shaped } \\
\text { vessel foot }\end{array}$ & PAS NLM-A2CB67 \\
\hline NLINO004 & Jug (handle) & Eggers 125 & North Lincolnshire & Appleby & RS & $\begin{array}{l}\text { Zoomorphic lion head at the } \\
\text { top of handle; zoomorphic } \\
\text { lion's foot handle medallion }\end{array}$ & $\begin{array}{l}\text { PAS SWYOR- } \\
\text { E54DB2 }\end{array}$ \\
\hline NLIN0005 & Vessel (mount) & & North Lincolnshire & Winteringham & RS & $\begin{array}{l}\text { Zoomorphic bird hanging } \\
\text { mount, most likely a duck }\end{array}$ & $\begin{array}{l}\text { PAS SWYOR- } \\
\text { 1707E1 }\end{array}$ \\
\hline
\end{tabular}




\begin{tabular}{|c|c|c|c|c|c|c|c|c|}
\hline NLIN0006 & Handled Pan 2 & Rudge Cup Type & North Lincolnshire & Winterton & RS & $\begin{array}{l}\text { Geometric squares across the } \\
\text { entire body in a chequer } \\
\text { board pattern, enameled in } \\
\text { diagonal rows of yellow, } \\
\text { blue and possibly red }\end{array}$ & & $\begin{array}{l}\text { PAS NLM-F50443; } \\
\text { Worrell 2009, 294- } \\
295\end{array}$ \\
\hline NLIN0007 & Vessel (fragment) & & North Lincolnshire & Scawby & RS & $\begin{array}{l}\text { Zoomorphic bull's head jug } \\
\text { spout, upraised horns, } \\
\text { mohawk-like turf of hair on } \\
\text { top of head }\end{array}$ & & PAS NLM-F3AA42 \\
\hline NLIN0008 & Vessel (mount) & & North Lincolnshire & Holme & RU & $\begin{array}{l}\text { Geometric sub-triangular } \\
\text { hanging vessel mount }\end{array}$ & & PAS NLM-52F093 \\
\hline NLIN0009 & $\begin{array}{l}\text { Handled Pan } 2 \\
\text { (handle) }\end{array}$ & Eggers $139-144$ & North Lincolnshire & Scawby & RS & & $\begin{array}{l}\text { Concentric circle handle } \\
\text { medallion; Inscription in } \\
\text { broken circular field reads } \\
\text { "CIPI" }\end{array}$ & PAS NLM-B0A171 \\
\hline NLIN0010 & Jug (lid) & & North Lincolnshire & Brigg & RU & $\begin{array}{l}\text { Floral leaf jug lid; } \\
\text { Zoomorphic (possibly) duck } \\
\text { statuette on lid }\end{array}$ & & PAS NLM-C883E2 \\
\hline NLIN0011 & Vessel (mount) & & North Lincolnshire & Scawby & RS & $\begin{array}{l}\text { Zoomorphic swan's head } \\
\text { hanging vessel mount }\end{array}$ & & PAS NLM-75A127 \\
\hline NOR0005 & Vessel (mount) & & Norfolk & Brettenham & RS & $\begin{array}{l}\text { Anthropomorphic female } \\
\text { bust mount }\end{array}$ & & PAS SF-5FE041 \\
\hline NOR0006 & $\begin{array}{l}\text { Handled Pan } 2 \\
\text { (handle) }\end{array}$ & Rudge Cup Type & Norfolk & Reepham & RU & & $\begin{array}{l}\text { Floral vine and leaf, } \\
\text { geometric embellishment; } \\
\text { yellow enamel extant }\end{array}$ & PAS NMS-47B176 \\
\hline NOR0007 & Vessel (mount) & & Norfolk & Southrepps & RU & & $\begin{array}{l}\text { Zoomorphic dolphin handle; } \\
\text { Floral embellishments on end } \\
\text { of tail }\end{array}$ & PAS NMS-23D975 \\
\hline NOR0008 & Jug (handle) & & Norfolk & Attlebridge & RS & & & PAS NMS-900741 \\
\hline NOR0009 & Vessel (mount) & & Norfolk & Shouldham & RS & $\begin{array}{l}\text { Anthropomorphic female } \\
\text { bust mount }\end{array}$ & & PAS NMS-7E2C22 \\
\hline NOR0010 & Vessel (mount) & & Norfolk & Cawston & RS & $\begin{array}{l}\text { Anthropomorphic male } \\
\text { bearded bust mount, } \\
\text { probably Pan }\end{array}$ & & PAS NMS-57CB72 \\
\hline
\end{tabular}




\begin{tabular}{|c|c|c|c|c|c|c|c|c|}
\hline NOR0011 & $\begin{array}{l}\text { Handled Pan } 2 \\
\text { (handle) }\end{array}$ & Eggers 139-144 & Norfolk & Beachamwell & RU & & $\begin{array}{l}\text { Geometric punch dot } \\
\text { decoration arranged in a } \\
\text { triangular shape; etched lines } \\
\text { along the border of the } \\
\text { handle }\end{array}$ & PAS NMS-E52C 90 \\
\hline NOR0012 & Vessel (fragment) & & Norfolk & Fincham & RU & Concentric circle basin rings & & PAS NMS-28C680 \\
\hline NOR0013 & Vessel (fragment) & & Norfolk & $\begin{array}{l}\text { Beeston with } \\
\text { Bittering }\end{array}$ & RS & & $\begin{array}{l}\text { Zoomorphic handle terminal, } \\
\text { probably a dolphin }\end{array}$ & PAS NMS-8D0814 \\
\hline NOR0014 & Jug (fragment) & & Norfolk & Feltwell & RS & Floral pellet foot decoration & & PAS NMS-FFBFB 1 \\
\hline NOR0015 & Vessel (mount) & & Norfolk & Ringstead & RS & $\begin{array}{l}\text { Geometric sub-triangular } \\
\text { hanging mount }\end{array}$ & & PAS NMS-F47791 \\
\hline NOR0016 & Jug (handle) & & Norfolk & $\begin{array}{l}\text { Hockwold cum } \\
\text { Wilton }\end{array}$ & RS & & $\begin{array}{l}\text { Floral leaf swirls and } \\
\text { embellishments; geometric } \\
\text { rounded oval thumb rest }\end{array}$ & PAS NMS-388DD6 \\
\hline NOR0017 & $\begin{array}{l}\text { Handled Pan } 2 \\
\text { (handle) }\end{array}$ & & Norfolk & East Walton & RS & & $\begin{array}{l}\text { Geometric punch dot } \\
\text { decoration; etched lines } \\
\text { along the border of the } \\
\text { handle }\end{array}$ & PAS NMS-9AA877 \\
\hline NOR0018 & Jug (fragment) & & Norfolk & $\begin{array}{l}\text { Beeston with } \\
\text { Bittering }\end{array}$ & RS & & & PAS NMS-D5C680 \\
\hline NOR0019 & Jug (handle) & & Norfolk & $\begin{array}{l}\text { Beeston with } \\
\text { Bittering }\end{array}$ & RS & & Floral leaf thumb rest & PAS NMS-0F88B4 \\
\hline NOR0020 & Vessel (fragment) & & Norfolk & $\begin{array}{l}\text { Beeston with } \\
\text { Bittering }\end{array}$ & RS & Geometric punch dots & & PAS NMS-0EBFE1 \\
\hline NOR0021 & Vessel (mount) & & Norfolk & Narford & RU & $\begin{array}{l}\text { Geometric sub-triangular } \\
\text { hanging mount }\end{array}$ & & PAS NMS-20B 842 \\
\hline NOR0022 & $\begin{array}{l}\text { Handled Pan } 2 \\
\text { (handle) }\end{array}$ & & Norfolk & Attlebridge & RU & & & PAS NMS-1518 \\
\hline NOR0023 & $\begin{array}{l}\text { Handled Pan } 1 \\
\text { (handle) }\end{array}$ & Eggers $154-155$ & Norfolk & Kenninghall & RU & & Zoomorphic ram's head & PAS NMS-1562 \\
\hline NOR0024 & $\begin{array}{l}\text { Handled Pan } 2 \\
\text { (handle) }\end{array}$ & Eggers 150 & Norfolk & Tacolneston & RU & & $\begin{array}{l}\text { Geometric keyhole shaped } \\
\text { handle loop, pellet at handle } \\
\text { terminal }\end{array}$ & PAS NMS-199 \\
\hline NOR0025 & Vessel (mount) & & Norfolk & $\begin{array}{l}\text { Hockwold cum } \\
\text { Wilton }\end{array}$ & RS & $\begin{array}{l}\text { Anthropomorphic female } \\
\text { bust mount; floral five } \\
\text { pointed leaf backing }\end{array}$ & & PAS NMS-134 \\
\hline NOR0026 & Vessel (mount) & & Norfolk & Aldeby & RS & $\begin{array}{l}\text { Geometric triangular } \\
\text { hanging vessel mount }\end{array}$ & & PAS NMS-D75F24 \\
\hline NOR0027 & Vessel (mount) & & Norfolk & Stanfield & RU & $\begin{array}{l}\text { Geometric triangular } \\
\text { hanging vessel mount }\end{array}$ & & PAS NMS1310 \\
\hline
\end{tabular}




\begin{tabular}{|c|c|c|c|c|c|c|c|c|}
\hline NOR0028 & Jug (handle) & & Norfolk & Themelthorpe & RU & Floral leaf handle medallion & & PAS NMS-DA1851 \\
\hline NOR0029 & Jug (handle) & & Norfolk & Colkirk & RU & & & PAS NMS1924 \\
\hline NOR0043 & $\begin{array}{l}\text { Handled Pan } 2 \\
\text { (handle) }\end{array}$ & Rudge Cup Type & Norfolk & Gunthorpe & PAS & & Inscription "BEBE SESE" & $\begin{array}{l}\text { NMS-7BC635; } \\
\text { Worrell } 2012\end{array}$ \\
\hline NOT0003 & Vessel (fragment) & & Nottinghamshire & Hawton & RU & Geometric circle band on rim & & $\begin{array}{l}\text { PAS DENO- } \\
\text { CCC324 }\end{array}$ \\
\hline NOT0004 & Vessel (mount) & & Nottinghamshire & Collingham & RU & $\begin{array}{l}\text { Floral leaf shaped hanging } \\
\text { vessel mount }\end{array}$ & & PAS DENO-149754 \\
\hline NYR0001 & Handled Pan 2 & Eggers 139-144 & North Yorkshire & Malton & RS & $\begin{array}{l}\text { Concentric cirlce umbo and } \\
\text { foot ring }\end{array}$ & & PAS NLM-6A7473 \\
\hline NYR0002 & $\begin{array}{l}\text { Handled Pan } 2 \\
\text { (handle) }\end{array}$ & Eggers 139-144 & North Yorkshire & $\begin{array}{l}\text { Brough with St. } \\
\text { Giles }\end{array}$ & RU & & $\begin{array}{l}\text { Geometric punch dots } \\
\text { arranged in a triangular shape } \\
\text { at base of handle medallion; } \\
\text { Concentric circle handle } \\
\text { medallion loop }\end{array}$ & PAS NCL-782251 \\
\hline NYR0005 & Handled Pan 2 & & North Yorkshire & Hawkswick & RU & $\begin{array}{l}\text { Concentric circle umbo and } \\
\text { rings in interior basin }\end{array}$ & & PAS LVPL-F9BE12 \\
\hline NYR0006 & Vessel (mount) & & North Yorkshire & Claxton & RU & $\begin{array}{l}\text { Geometric sub-triangular } \\
\text { hanging vessel mount }\end{array}$ & & PAS DUR-510214 \\
\hline NYR0007 & Vessel (mount) & & North Yorkshire & Bedale & RU & $\begin{array}{l}\text { Anthropomorphic face, } \\
\text { possibly Cupid or cherub }\end{array}$ & & PAS DUR-E1D6C7 \\
\hline OX0001 & Vessel (mount) & & Oxfordshire & Adwell & RU & $\begin{array}{l}\text { Zoomorphic duck vessel } \\
\text { mount, likely from a jug lid } \\
\text { or other vessel lid }\end{array}$ & & PAS BH-2DA8C6 \\
\hline OX0002 & Vessel (mount) & & Oxfordshire & Letcombe Regis & RU & $\begin{array}{l}\text { Anthropomorphic bust of } \\
\text { Bacchus }\end{array}$ & & PAS BERK-C01546 \\
\hline RC0001 & Jug (lid) & Eggers $123-126$ & Rhondda Cynon Taf & Pont Y Clun & RU & & & $\begin{array}{l}\text { PAS NMGW- } \\
\text { 2EECF6 }\end{array}$ \\
\hline SH0001 & Vessel (fragment) & & Shropshire & Hordley & RU & Concentric circle umbo & & $\begin{array}{l}\text { PAS HESH- } \\
02 \text { FED3 }\end{array}$ \\
\hline SH0002 & Jug (lid) & Eggers $123-126$ & Shropshire & Sheinton & RS & Floral (possible) leaf jug lid & & PAS HESH-9774C3 \\
\hline SH0O03 & Vessel (mount) & & Shropshire & Whitchurch & RS & Zoomorphic ox head mount & & PAS LVPL-F1C917 \\
\hline SYR0001 & $\begin{array}{l}\text { Handled Pan } 2 \\
\text { (handle) }\end{array}$ & & South Yorkshire & Edlington & RU & & & $\begin{array}{l}\text { PAS SWYOR- } \\
\text { EA9393 }\end{array}$ \\
\hline STA0001 & Vessel (mount) & & Staffordshire & Shenstone & RS & $\begin{array}{l}\text { Anthropomorphic reclining } \\
\text { banquetor vessel mount }\end{array}$ & & $\begin{array}{l}\text { PAS WMID- } \\
6553 \mathrm{C} 1\end{array}$ \\
\hline
\end{tabular}




\begin{tabular}{|c|c|c|c|c|c|c|c|c|}
\hline STA0002 & Vessel (mount) & & Staffordshire & Fisherwick & RU & $\begin{array}{l}\text { Anthropomorphic male face } \\
\text { circular mount, stylized } \\
\text { provincial features, high } \\
\text { cheeks, almond eyes }\end{array}$ & & $\begin{array}{l}\text { PAS WMID- } \\
\text { 26ACD7 }\end{array}$ \\
\hline STA0003 & $\begin{array}{l}\text { Handled Pan } 2 \\
\text { (handle) }\end{array}$ & Eggers 139-144 & Staffordshire & Brewood & RS & & $\begin{array}{l}\text { Concentric circle handle } \\
\text { medallion loop }\end{array}$ & $\begin{array}{l}\text { PAS WMID- } \\
\text { 6C2FE3 }\end{array}$ \\
\hline STA0004 & Handled Pan 2 & Rudge Cup Type & Staffordshire & Ilam & RU & \begin{tabular}{|l|} 
Floral celtic style squirls, \\
leaves, and vines; Enameled \\
with green and yellow; \\
Inscription reads \\
"MAISCOGGABATAUXEL \\
ODUNUMCAMMOGLAN \\
NARIGOREVALIAELIUSD \\
RACO"
\end{tabular} & & $\begin{array}{l}\text { PAS WMID- } \\
\text { 3FE965; Jackson } \\
\text { 2012, 41-60 }\end{array}$ \\
\hline STA0005 & Vessel (mount) & & Staffordshire & Thorpe Constantine & RU & Zoomorphic ox head mount & & PAS WAW-342131 \\
\hline SUF0006 & Jug (handle) & & Suffolk & Pettistree & RU & & $\begin{array}{l}\text { Geometric godronné thumb } \\
\text { rest }\end{array}$ & PAS SF-8182F7 \\
\hline SUF0007 & Vessel (fragment) & & Suffolk & Kettlebaston & RU & $\begin{array}{l}\text { Geometric pelta shaped } \\
\text { vessel foot, "heart-shaped" } \\
\text { foot in centre of crescent }\end{array}$ & & PAS SF-7CE6C1 \\
\hline SUF0008 & Vessel (mount) & & Suffolk & Hacheston & RS & $\begin{array}{l}\text { Floral leaf shaped hanging } \\
\text { vessel mount }\end{array}$ & & PAS SF-4E5A43 \\
\hline SUF0009 & $\begin{array}{l}\text { Handled Pan } 2 \\
\text { (handle) }\end{array}$ & Rudge Cup Type & Suffolk & Combs & RU & & $\begin{array}{l}\text { Floral celtic stylized leaves } \\
\text { and spirals, enameled in blue }\end{array}$ & PAS SF-0349E2 \\
\hline SUF0010 & $\begin{array}{l}\text { Handled Pan } 2 \\
\text { (handle) }\end{array}$ & Eggers 139-144 & Suffolk & Hoxne & RU & & $\begin{array}{l}\text { Concentric circle handle } \\
\text { loop; Geometric punch dots } \\
\text { arranged in triangle }\end{array}$ & PAS SF-3C6B04 \\
\hline SUF0011 & Vessel (mount) & & Suffolk & West Stow & RU & $\begin{array}{l}\text { Anthropomorphic helmeted } \\
\text { bust, possibly Minerva }\end{array}$ & & PAS SF-040874 \\
\hline SUF0012 & Vessel (fragment) & & Suffolk & Barking & RS & & & PAS SF-A72D31 \\
\hline SUF0013 & $\begin{array}{l}\text { Handled Pan } 2 \\
\text { (handle) }\end{array}$ & Eggers 139-144 & Suffolk & Combs & RU & & Concentric circle handle loop & PAS SF-3DCCA7 \\
\hline SUF0014 & Vessel (mount) & & Suffolk & Arwarton & RU & $\begin{array}{l}\text { Geometric sub-triangular } \\
\text { hanging vessel mount }\end{array}$ & & PAS SF-2BC393 \\
\hline SUF0015 & $\begin{array}{l}\text { Handled Pan } 2 \\
\text { (handle) }\end{array}$ & Eggers $140-142$ & Suffolk & Chediston & RU & & $\begin{array}{l}\text { Concentric circle handle } \\
\text { loop; Stamped inscription } \\
\text { reads "CIPIPOL[...]" }\end{array}$ & PAS NMS-7F1BE6 \\
\hline SUF0016 & Jug (handle) & & Suffolk & Lowestoft & RS & & Floral leaf handle medallion & PAS NMS-2E4838 \\
\hline
\end{tabular}




\begin{tabular}{|c|c|c|c|c|c|c|c|c|}
\hline SUF0017 & Vessel (mount) & & Suffolk & Mendham & RU & $\begin{array}{l}\text { Zoomorphic dolphin hanging } \\
\text { vessel mount }\end{array}$ & & PAS NMS-C40776 \\
\hline SUF0018 & Jug (lid) & Eggers $123-126$ & Suffolk & Freckenham & RU & $\begin{array}{l}\text { Zoomorphic bird thumb rest; } \\
\text { Floral (possible) leaf shaped } \\
\text { jug lid }\end{array}$ & & PAS SF-210633 \\
\hline SUF0019 & Vessel (mount) & & Suffolk & Mildenhall & RU & $\begin{array}{l}\text { Zoomorphic duck vessel lid } \\
\text { mount }\end{array}$ & & PAS SF-DF4933 \\
\hline SUF0020 & Jug (handle) & & Suffolk & Brockley & RU & & $\begin{array}{l}\text { Anthropomorphic female } \\
\text { face handle medallion } \\
\text { wearing a triangular hat or } \\
\text { headdress }\end{array}$ & PAS SF-452BA2 \\
\hline SUF0021 & Vessel (mount) & & Suffolk & Market Weston & RU & $\begin{array}{l}\text { Floral (possible) leaf shaped } \\
\text { hanging vessel mount }\end{array}$ & & PAS SF-1D1EA1 \\
\hline SUF0022 & Vessel (mount) & & Suffolk & Hitcham & RS & $\begin{array}{l}\text { Anthtopomorphic male bust } \\
\text { hanging vessel mount }\end{array}$ & & PAS SF-B0DC84 \\
\hline SUF0023 & Vessel (mount) & & Suffolk & Yaxley & RS & $\begin{array}{l}\text { Zoomorphic bird statuette, } \\
\text { probably a jug lid mount }\end{array}$ & & PAS SF-EB55A2 \\
\hline SUF0024 & Vessel (mount) & & Suffolk & Linstead Magna & RU & $\begin{array}{l}\text { Floral (possible) leaf shaped } \\
\text { hanging vessel mount }\end{array}$ & & PAS SF-2AE0A6 \\
\hline SUF0025 & Vessel (mount) & & Suffolk & Horringer & RU & & Zoomorphic bird & PAS NMS2676 \\
\hline SUF0026 & Vessel (fragment) & & Suffolk & Great Glemham & RU & Concentric circle umbo & & PAS SF-8924 \\
\hline SUF0027 & Vessel (fragment) & & Suffolk & Wattisham & RU & $\begin{array}{l}\text { Geometric pelta shaped } \\
\text { vessel foot }\end{array}$ & & PAS SF-9012 \\
\hline SUF0028 & $\begin{array}{l}\text { Handled Pan } 2 \\
\text { (handle) }\end{array}$ & Eggers 139-144 & Suffolk & Freckenham & RU & & $\begin{array}{l}\text { Concentric ring handle } \\
\text { medallion }\end{array}$ & PAS SF-9054 \\
\hline SUF0029 & Jug (lid) & Eggers $123-126$ & Suffolk & $\begin{array}{l}\text { Bradfield Combust } \\
\text { With Stanningfield }\end{array}$ & RS & $\begin{array}{l}\text { Floral (possible) leaf shaped } \\
\text { jug lid }\end{array}$ & & PAS SF-9063 \\
\hline SUF0030 & Vessel (mount) & & Suffolk & Otley & RU & $\begin{array}{l}\text { Zoomorphic swan's head } \\
\text { vessel mount }\end{array}$ & & PAS SF-9646 \\
\hline SUF0031 & Jug (lid) & Eggers $123-126$ & Suffolk & Bredfield & RU & Zoomorphic bird thumb rest & & PAS SF10041 \\
\hline SUF0032 & Vessel (ffragment) & & Suffolk & Combs & RU & Concentric circle lid & & PAS SF10490 \\
\hline
\end{tabular}




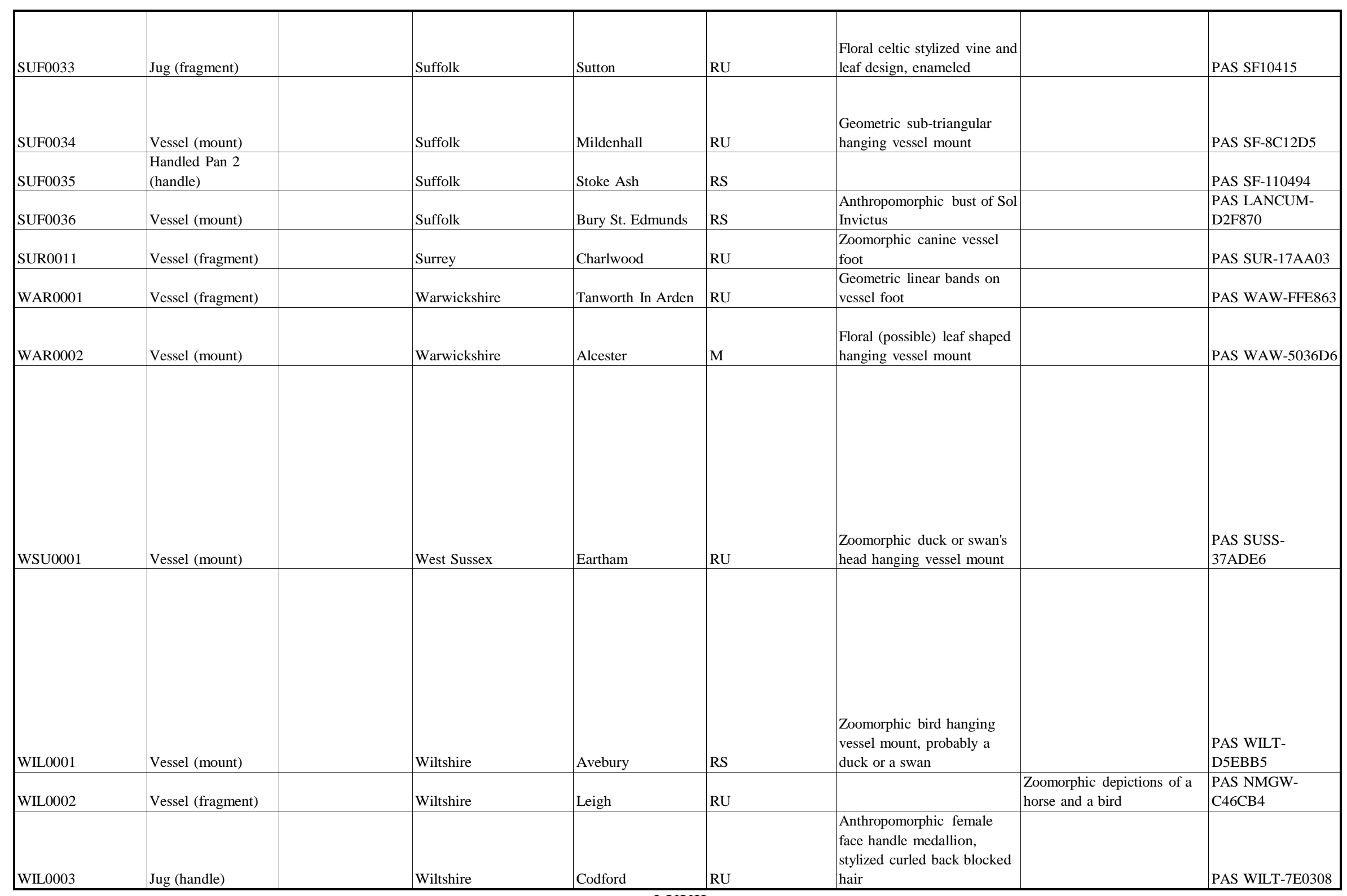




\begin{tabular}{|l|l|l|l|l|l|l|l|}
\hline & & & & & & \\
\hline & & & & & & \\
& & & & & \\
WOR0001 & Vessel (fragment) & & Worcestershire & Inkberrow & RU & $\begin{array}{l}\text { Geometric pelta shaped } \\
\text { vessel foot, "heart-shaped" } \\
\text { leg in centre of crescent }\end{array}$ & $\begin{array}{l}\text { Concentric circle rings on } \\
\text { interior of basin }\end{array}$ \\
\hline WOR0002 & Vessel (fragment) & & Worcestershire & Leigh & RU & & PAS WAW-18C577 \\
\hline
\end{tabular}


Appendix VI: Graves from Brougham, Cumbria with Copper Alloy Vessels

\begin{tabular}{|c|c|c|}
\hline Findspot & Copper Alloy Vessels & Associated Finds \\
\hline Grave 107 & C0001 (Handled Pan 2) & $\begin{array}{l}1 \text { copper alloy mount, } 1 \\
\text { undiagnostic copper alloy } \\
\text { fragment, } 1 \text { iron nail, } 2 \\
\text { undiagnostic bone objects, } 1 \\
\text { ceramic jar, } 2 \text { ceramic bowls, } \\
1 \text { glass cup }\end{array}$ \\
\hline Grave 237 & C0002 (Bucket) & $\begin{array}{l}1 \text { copper alloy mount, multiple } \\
\text { undiagnostic copper alloy } \\
\text { fragments, } 1 \text { gold earring, } 4 \\
\text { iron hobnails, } 1 \text { glass bead, } 25 \\
\text { iron nails }\end{array}$ \\
\hline Grave 235 & C0003 (Bucket) & $\begin{array}{l}1 \text { copper alloy mount, } 1 \\
\text { undiagnostic copper alloy } \\
\text { fragment, } 11 \text { iron nails, } 4 \\
\text { fragments of ceramic vessels }\end{array}$ \\
\hline Grave 141 & C0004 (Bucket) & $\begin{array}{l}20 \text { iron hobnails, } 5 \text { glass } \\
\text { beads, } 2 \text { fragments of ceramic } \\
\text { vessels }\end{array}$ \\
\hline Unstratified in cemetery & C0005 (Bucket) & \\
\hline Grave 326 & C0006 (Bucket) & $\begin{array}{l}8 \text { undiagnostic copper alloy } \\
\text { fragments, } 6 \text { iron nails, } 1 \\
\text { ceramic bowl, } 1 \text { ceramic jar, } \\
26 \text { fragments of ceramic } \\
\text { vessels, } 1 \text { glass bead }\end{array}$ \\
\hline Grave 114 & C0007 (Bucket) & $\begin{array}{l}\text { multiple undiagnostic copper } \\
\text { alloy fragments, } 1 \text { copper } \\
\text { alloy mount, } 1 \text { copper alloy } \\
\text { nail, } 1 \text { iron nail, } 1 \\
\text { undiagnostic iron fragment, } \\
\text { melted glass bead fragments, } 4 \\
\text { fragments of ceramic vessels }\end{array}$ \\
\hline Grave 289 & C0008 (Bucket) & $\begin{array}{l}1 \text { bone mount, } 1 \text { fragment of a } \\
\text { ceramic vessel }\end{array}$ \\
\hline Grave 198 & C0009 (Cauldron) & $\begin{array}{l}1 \text { undiagnostic bone fragment, } \\
4 \text { undiagnostic copper alloy } \\
\text { fragments, } 1 \text { copper alloy nail, } \\
1 \text { iron knife, } 130 \text { iron nails, } 36 \\
\text { iron hobnails, } 1 \text { bone scabbard } \\
\text { fitting, } 13 \text { bone/antler fittings, } \\
3 \text { glass vessels }\end{array}$ \\
\hline Grave 127 & C0010 (Bowl) & \\
\hline Grave 245 & C0011 (Bowl) & $\begin{array}{l}10 \text { undiagnostic copper alloy } \\
\text { fragments, } 12 \text { iron hobnails, } 1\end{array}$ \\
\hline
\end{tabular}




\begin{tabular}{|c|c|c|}
\hline & & iron nail \\
\hline Grave 239 & C0012 (Bowl) & $\begin{array}{l}28 \text { undiagnostic copper alloy } \\
\text { fragments, } 2 \text { iron hobnails, } 10 \\
\text { iron nails, } 1 \text { glass vessel }\end{array}$ \\
\hline Grave 270 & C0013 (Bowl) & $\begin{array}{l}1 \text { undiagnostic silver } \\
\text { fragment, } 1 \text { copper alloy } \\
\text { mount, } 2 \text { undiagnostic copper } \\
\text { alloy fragments, } 1 \text { iron } \\
\text { hobnail, } 2 \text { iron nails, } 1 \text { bone } \\
\text { fetish }\end{array}$ \\
\hline Grave 133 & C0014 (Strainer) & $\begin{array}{l}23 \text { undiagnostic copper alloy } \\
\text { fragments, } 1 \text { iron brooch, } 17 \\
\text { iron nails, } 1 \text { fragment of vessel } \\
\text { glass, } 1 \text { ceramic jar }\end{array}$ \\
\hline Grave 81 & C0015 (Strainer) & $\begin{array}{l}2 \text { iron nails, } 1 \text { bone fitting, } 1 \\
\text { ceramic jar, } 1 \text { ceramic } \\
\text { mortaria }\end{array}$ \\
\hline Grave 217 & $\begin{array}{l}\text { C0016 (Strainer) } \\
\text { C0017 (Vessel mount) }\end{array}$ & $\begin{array}{l}10 \text { undiagnostic copper alloy } \\
\text { fragments, } 12 \text { iron nails, } 1 \text { iron } \\
\text { brooch, } 1 \text { undiagnostic lead } \\
\text { alloy fragment, } 1 \text { bone fitting }\end{array}$ \\
\hline Grave 221 & $\begin{array}{l}\text { C0018 (Jug) } \\
\text { C0019 (Strainer) }\end{array}$ & $\begin{array}{l}15 \text { undiagnostic copper alloy } \\
\text { fragments, } 1 \text { iron hobnail, } 2 \\
\text { iron nails }\end{array}$ \\
\hline Grave 77 & C0020 (Jug) & $\begin{array}{l}21 \text { undiagnostic copper alloy } \\
\text { fragments, } 2 \text { iron hobnails, } 15 \\
\text { iron nails, } 1 \text { glass bead, } 1 \\
\text { fragment of vessel glass, } \\
\text { 1bone fitting, } 1 \text { ceramic jar }\end{array}$ \\
\hline
\end{tabular}


Appendix VII: Romano-British Graves in Essex with Copper Alloy Vessels

\begin{tabular}{|c|c|c|}
\hline Findspot/Date & Copper Alloy Vessels & Associated Finds \\
\hline $\begin{array}{l}\text { Stansted Airport (Cremation } \\
\text { 24)/ 99-150 CE }\end{array}$ & EX0015 (Bowl) & $\begin{array}{l}1 \text { copper alloy box fitting, } 13 \\
\text { iron nails, } 1 \text { ceramic bowl, } 1 \\
\text { ceramic jar }\end{array}$ \\
\hline $\begin{array}{l}\text { Stansted Airport (Cremation } \\
\text { 25)/ 99-150 CE }\end{array}$ & $\begin{array}{l}\text { EX0016 (Jug) } \\
\text { EX0017 (Handled Pan 1) } \\
\text { EX0018 (Handled Pan 2) } \\
\text { EX0019 (Jug) } \\
\text { EX0020 (Bowl) } \\
\text { EX0021 (Vessel) }\end{array}$ & $\begin{array}{l}1 \text { iron lamps, } 1 \text { iron knife with } \\
\text { bone handle, } 1 \text { undiagnostic } \\
\text { iron fragment, } 1 \text { pewter plate } \\
\text { (with cremated bone), } 16 \\
\text { undiagnostic copper alloy } \\
\text { fragments, } 62 \text { iron nails, } 3 \\
\text { glass bowls, } 2 \text { glass jugs, } 1 \\
\text { glass bottle, } 8 \text { ceramic bowls, } \\
1 \text { ceramic bucket, } 1 \text { jug, } 1 \text { bone } \\
\text { fitting }\end{array}$ \\
\hline $\begin{array}{l}\text { Heybridge (The Towers)/ } 1^{\text {st }} \text { - } \\
\text { 2nd }\end{array}$ & $\begin{array}{l}\text { EX0031 (Handled Pan 1) } \\
\text { EX0038 (Jug) }\end{array}$ & 1 ceramic statuette \\
\hline Colchester/ 1st & EX0034 (Handled Pan 2) & $\begin{array}{l}10 \text { ceramic bowls, } 3 \text { glass } \\
\text { bottles, } 1 \text { wooden box, } 36 \\
\text { Coins, } 21 \text { ceramic statuettes, } \\
\text { multiple bone fragments of } \\
\text { cosmetic tools }\end{array}$ \\
\hline $\begin{array}{l}\text { Rivenhall (Barrow Field)/ } 1^{\text {st }}- \\
2^{\text {nd }}\end{array}$ & $\begin{array}{l}\text { EX0035 (Jug) } \\
\text { EX0036 (Handled Pan1) }\end{array}$ & \\
\hline $\begin{array}{l}\text { Bartlow Hills (Barrow IV) } 1^{\text {st }} \\
\text { 2nd }\end{array}$ & $\begin{array}{l}\text { EX0058 (Jug) } \\
\text { EX0059 (Handled Pan 1) } \\
\text { EX0060 (Jar) } \\
\text { EX0067 (Jug) }\end{array}$ & $\begin{array}{l}1 \text { amphora, } 1 \text { wooden box, } 1 \\
\text { copper alloy furniture mount, } \\
2 \text { copper alloy strigils, } 5 \text { glass } \\
\text { bottles, } 1 \text { copper alloy lamp }\end{array}$ \\
\hline $\begin{array}{l}\text { Bartlow Hills (Barrow III) } 1^{\text {st }}- \\
2^{\text {nd }}\end{array}$ & $\begin{array}{l}\text { EX0061 (Jug) } \\
\text { EX0062 (Handled Pan 1) }\end{array}$ & $\begin{array}{l}3 \text { glass bottles, } 1 \text { iron lamp, } 1 \\
\text { ceramic bowl }\end{array}$ \\
\hline $\begin{array}{l}\text { Bartlow Hills (Barrow V) } 1^{\text {st }} \\
2^{\text {nd }}\end{array}$ & $\begin{array}{l}\text { EX0063 (Jug) } \\
\text { EX0064 (Handled Pan 1) }\end{array}$ & $\begin{array}{l}2 \text { glass bottles, } 2 \text { glass bowls, } \\
1 \text { iron lamp, } 3 \text { ceramic bowls }\end{array}$ \\
\hline $\begin{array}{l}\text { Bartlow Hills (Barrow VII) } 1^{\text {st }}- \\
2^{\text {nd }}\end{array}$ & $\begin{array}{l}\text { EX0065 (Jug) } \\
\text { EX0066 (Bowl) }\end{array}$ & $\begin{array}{l}2 \text { ceramic jugs, } 5 \text { ceramic } \\
\text { cups, } 2 \text { glass bottles, } 3 \\
\text { ceramic bowls, } 1 \text { iron lamp }\end{array}$ \\
\hline Elsenham $/ 1^{\text {st }}-2^{\text {nd }}$ & $\begin{array}{l}\text { EX0070 (Jar) } \\
\text { EX0071 (Bowl) }\end{array}$ & $\begin{array}{l}4 \text { ceramic bowls, } 1 \text { ceramic } \\
\text { jar, } 1 \text { glass jug, } 12 \text { bone game } \\
\text { counters, } 6 \text { glass game } \\
\text { counters ( } 5 \text { black, } 1 \text { white), } 1 \\
\text { copper alloy box fitting, } \\
\text { multiple copper alloy \& iron } \\
\text { studs and nails, } 3 \text { coins }\end{array}$ \\
\hline
\end{tabular}




\begin{tabular}{|c|c|c|}
\hline $\begin{array}{l}\text { Stanway (Warroir's Burial)/ } \\
1^{\text {st }}\end{array}$ & $\begin{array}{l}\text { EX0073 (Jug) } \\
\text { EX0074 (Handled Pan 1) }\end{array}$ & $\begin{array}{l}1 \text { amphora, } 14 \text { ceramic } \\
\text { vessels, } 3 \text { glass bottles, } 2 \\
\text { copper alloy brooches, } 1 \\
\text { copper alloy arm ring, } 1 \text { iron } \\
\text { and copper alloy shield boss, } 1 \\
\text { iron lancehead, } 20 \text { glass game } \\
\text { counters, } 1 \text { wooden game } \\
\text { board, } 2 \text { wooden boxes }\end{array}$ \\
\hline Stanway (Doctor's Burial)/1 ${ }^{\text {st }}$ & $\begin{array}{l}\text { EX0075 (Handled Pan 2) } \\
\text { EX0076 (Strainer) }\end{array}$ & $\begin{array}{l}13 \text { ceramic vessels, } 1 \\
\text { amphora, } 2 \text { copper alloy } \\
\text { brooches, } 8 \text { copper alloy rings, } \\
1 \text { jet bead, } 26 \text { glass game } \\
\text { counters, } 2 \text { wooden boxes, } 4 \\
\text { iron rods, } 4 \text { copper alloy rods, } \\
14 \text { surgical instruments }\end{array}$ \\
\hline Stanway (Chamber BF6) $/ 1^{\text {st }}$ & EX0077 (Vessel) & $\begin{array}{l}21 \text { ceramic vessels, } 2 \\
\text { amphorae, } 1 \text { copper alloy } \\
\text { pedestal, } 1 \text { wooden game } \\
\text { board, } 1 \text { iron garment fitting, } \\
\text { multiple copper alloy strips }\end{array}$ \\
\hline
\end{tabular}


Appendix VIII: Romano-British Grave Deposits from the South Eastern Counties of England containing Copper Alloy Vessels.

\begin{tabular}{|c|c|c|}
\hline Findspot/Date & Copper Alloy Vessels & Associated Finds \\
\hline $\begin{array}{l}\text { Winchester, Hampshire } \\
\text { (Grange Road, Grave 2)/ 85- } \\
95 \mathrm{CE}\end{array}$ & HAM0003 (Jug) & $\begin{array}{l}13 \text { ceramic bowls, } 1 \text { glass jug, } \\
2 \text { bone fittings, } 2 \text { iron knives, } \\
2 \text { iron styluses, multiple iron } \\
\text { nails, } 1 \text { undiagnsotic iron } \\
\text { fragment, } 1 \text { copper alloy } \\
\text { spoon, } 1 \text { copper alloy box } \\
\text { fitting, } 1 \text { copper alloy bell, } 11 \\
\text { beads, } 18 \text { glass gaming pieces, } \\
1 \text { stone amulet }\end{array}$ \\
\hline $\begin{array}{l}\text { Lullingstone, Kent/ 275-325 } \\
\text { CE }\end{array}$ & K0001 (Jug) & $\begin{array}{l}\text { 1bone fitting, } 1 \text { ceramic jug, } 2 \\
\text { glass bowls, } 4 \text { glass bottles, } 1 \\
\text { wooden game board. } 30 \text { glass } \\
\text { game counters, } 17 \text { bone game } \\
\text { counters, } 2 \text { iron knives, } 2 \text { iron } \\
\text { spoons }\end{array}$ \\
\hline $\begin{array}{l}\text { Maidstone, Kent (Cremated } \\
\text { Deposit 3, Amphora X) }\end{array}$ & $\begin{array}{l}\text { K0002 (Jug) } \\
\text { K0003 (Jug) } \\
\text { K0004 (Jug) } \\
\text { K0005 (Jug) }\end{array}$ & $\begin{array}{l}1 \text { glass bottle, } 1 \text { iron lamp, } 1 \\
\text { amphora, textile fragments }\end{array}$ \\
\hline Maidstone, Kent & K0006 (Vessel) & \\
\hline $\begin{array}{l}\text { Sittingbourne (Bayford), Kent/ } \\
\text { 175-300 CE }\end{array}$ & $\begin{array}{l}\text { K0007 (Jug) } \\
\text { K0008 (Bowl) } \\
\text { K0009 (Jug) } \\
\text { K0010 (Handled Pan 1) }\end{array}$ & $\begin{array}{l}1 \text { copper alloy lamp, } 1 \text { glass } \\
\text { bottle, } 1 \text { glass jug, } 1 \text { iron } \\
\text { strigil, } 12 \text { ceramic bowls, } \\
\text { 1bone fitting }\end{array}$ \\
\hline $\begin{array}{l}\text { Sittingbourne (Bayford), Kent/ } \\
\text { 175-300 CE }\end{array}$ & K0011 (Jug) & $\begin{array}{l}1 \text { glass bottle, } 1 \text { glass jug, } 3 \\
\text { undiagnostic glass fragments, } \\
3 \text { iron strigils, } 1 \text { iron lamp }\end{array}$ \\
\hline $\begin{array}{l}\text { Canterbury, Kent (Martyr's } \\
\text { Field Road)/ } 2^{\text {nd }}\end{array}$ & $\begin{array}{l}\text { K0012 (Jug) } \\
\text { K0013 (Handled Pan 1) }\end{array}$ & $\begin{array}{l}2 \text { coins, } 3 \text { ceramic beads, } 1 \\
\text { glass bottle }\end{array}$ \\
\hline $\begin{array}{l}\text { Springhead, Kent } \\
\text { (Tollgate) } / 1^{\text {st }}-2^{\text {nd }}\end{array}$ & $\begin{array}{l}\text { K0014 (Cauldron) } \\
\text { K0015 (Handled Pan 1) } \\
\text { K0016 (Jug) }\end{array}$ & $\begin{array}{l}18 \text { ceramic bowls, } 1 \text { copper } \\
\text { alloy brooch, } 1 \text { wooden table } \\
\text { with multiple copper alloy } \\
\text { fittings, } 1 \text { wooden gaming } \\
\text { board with copper alloy drop- } \\
\text { loop handles, } 23 \text { glass gaming } \\
\text { pieces, } 1 \text { bone dice }\end{array}$ \\
\hline $\begin{array}{l}\text { Springhead, Kent (Tollgate)/ } \\
1^{\text {st }}-2^{\text {nd }}\end{array}$ & $\begin{array}{l}\text { K0017 (Handled Pan 1) } \\
\text { K0018 (Jug) }\end{array}$ & $\begin{array}{l}14 \text { ceramic bowls, } 1 \text { copper } \\
\text { alloy brooch, } 1 \text { wooden box, } 1 \\
\text { copper alloy palette, } 1 \text { copper } \\
\text { alloy spatula probe, } 1 \text { wooden } \\
\text { gaming board, } 4 \text { undiagnostic }\end{array}$ \\
\hline
\end{tabular}




\begin{tabular}{|c|c|c|}
\hline & & iron fragments \\
\hline Luton, Kent $/ 1^{\text {st }}-2^{\text {nd }}$ & $\begin{array}{l}\text { K0020 (Bowl) } \\
\text { K0027 (Jug) }\end{array}$ & $\begin{array}{l}1 \text { copper alloy box fitting, } 1 \\
\text { glass bottle, } 1 \text { glass bowl, } 3 \\
\text { ceramic bowls, } 2 \text { ceramic cups }\end{array}$ \\
\hline Ramsgate, Kent/ $3^{\text {rd }} \mathrm{CE}$ & K0025 (Bucket) & \\
\hline $\begin{array}{l}\text { Canterbury, Kent (Palace } \\
\text { Street) } / 1^{\text {st }}-2^{\text {nd }}\end{array}$ & K0028 (Bowl) & 2 undiagnostic iron fragments \\
\hline Ashford, Kent/ $1^{\text {st }}-2^{\text {nd }}$ & $\begin{array}{l}\text { K0043 (Jug) } \\
\text { K0044 (Vessel) }\end{array}$ & $\begin{array}{l}1 \text { ceramic bowl, } 1 \text { wooden } \\
\text { box, } 1 \text { wooden bucket }\end{array}$ \\
\hline $\begin{array}{l}\text { Chichester, West Sussex } \\
\text { (Westergate) / circa } 150 \mathrm{CE}\end{array}$ & WSU0010 (Jug) & $\begin{array}{l}2 \text { copper alloy brooches, } \\
\text { multiple ceramic vessel } \\
\text { fragments }\end{array}$ \\
\hline
\end{tabular}


Appendix IX: Romano-British Site Finds of Copper Alloy Vessels from the South Eastern Counties of England.

\begin{tabular}{|c|c|}
\hline Findspot (Site Type) & Vessel/Catalogue Number \\
\hline Great Wackering, Essex (RU) & Strainer/EX0030 \\
\hline Heybridge, Essex (RS) & Vessel(fragment)/EX0032 \\
\hline Fingringhoe, Essex (RU) & Bucket/EX0033 \\
\hline Boreham, Essex (RS) & Vessel(fragment)/EX0053 \\
\hline Kelvedon, Essex (RS) & Handled Pan 2/EX0054 \\
\hline Little Oakley, Essex (RS) & Jug(handle)/EX0055 \\
\hline Witham, Essex (RS) & Vessel(mount)/EX0057 \\
\hline Great Wakering, Essex (RU) & Strainer/EX0072 \\
\hline Silchester, Hampshire (U) & $\begin{array}{c}\text { Handled Pan 2/HAM0001 } \\
\text { Handled Pan 1/HAM0002 } \\
\text { Vessel(fragment)/HAM0017 } \\
\text { Vessel(fragment)/HAM0018 } \\
\text { Vessel(fragment)/HAM0019 } \\
\text { Jug(handle)/HAM0020 } \\
\text { Jug/HAM0021 } \\
\text { Cauldron/HAM0022 }\end{array}$ \\
\hline Faversham, Kent (RS) & $\begin{array}{l}\text { Jug/K0021 } \\
\text { Jug/K0034 }\end{array}$ \\
\hline Richborough, Kent (M) & $\begin{array}{c}\text { Handled Pan 1/K0022 } \\
\text { Jug/K0023 } \\
\text { Vessel(mount)/K0024 } \\
\text { Jug/K0039 } \\
\text { Vessel(mount)/K0040 } \\
\text { Vessel(mount)/K0041 } \\
\text { Jug/K0042 } \\
\end{array}$ \\
\hline Canterbury, Kent (U) & $\begin{array}{c}\text { Handled Pan 2/K0026 } \\
\text { Jug/K0036 }\end{array}$ \\
\hline Springhead, Kent (RS) & Bowl/K0037 \\
\hline Chalkwell, Kent (RU) & Jug(handle)/K0045 \\
\hline Swaffam, Norfolk (RS) & Handled Pan 3/NOR0001 \\
\hline Bergh Apton, Norfolk (RU) & Handled Pan 2/NOR0002 \\
\hline Mundesley, Norfolk (RS) & $\begin{array}{c}\text { Bucket/NOR0003 } \\
\text { Jug/NOR0042 } \\
\end{array}$ \\
\hline Swanton Morley, Norfolk (M) & Bucket/NOR0004 \\
\hline Caistor-on-Sea, Norfolk (M) & Bucket/NOR0030 \\
\hline Oxnead Mill, Norfolk (RU) & Handled Pan 2/NOR0031 \\
\hline Walsingham, Norfolk (RU) & Handled Pan 2/NOR0032 \\
\hline Saham Toney, Norfolk (M) & Handled Pan 2/NOR0041 \\
\hline $\begin{array}{l}\text { Somberleyton Ashby and Herringfleet, Suffolk } \\
(\mathrm{RU})\end{array}$ & Handled Pan 2/SUF0005 \\
\hline
\end{tabular}




\begin{tabular}{|c|c|}
\hline Hacheston, Suffolk (RS) & Strainer/SUF0037 \\
& Jug/SUF0038 \\
& Vessel(mount)/SUF0039 \\
& Vessel(mount)/SUF0040 \\
& Vessel(mount)/SUF0041 \\
& Vessel(mount)/SUF0042 \\
\hline Otley, Suffolk (RU) & Jug(handle)/SUF0047 \\
\hline Lakenheath Fen, Suffolk (RS) & Bowl/SUF0048 \\
\hline Ipswich, Suffolk (RS) & Jug/SUF0049 \\
\hline Brandon, Suffolk (RU) & Jug/SUF0050 \\
\hline Walton on Thames, Surrey (RU) & Bowl/SUR0012 \\
\hline Chichester, West Sussex (U) & Jug/WSU0002 \\
& Vessel(fragment)/WSU0003 \\
& Bowl/WSU0004 \\
& Bowl/WSU0005 \\
\hline Fishbourne Roman Villa, West Sussex (RS) & Bowl/WSU0006 \\
& Jug/WSU0007 \\
& Vessel(fragment)/WSU0008 \\
& Vessel(fragment)/WSU0009 \\
\hline
\end{tabular}


Appendix X: Single Finds of Copper Alloy Vessels of Roman date reported through the Portable Antiquities Scheme in East Anglia and the South East of England.

\begin{tabular}{|c|c|}
\hline Findspot (Site type) & Vessel/Catalogue Number \\
\hline Etchingham, East Sussex (RU) & Jug(handle)/ESUS0001 \\
\hline St. Ann Without, East Sussex (RU) & Bucket/ESUS0002 \\
\hline Southease, East Sussex (RU) & Handled Pan 2/ESUS0003 \\
\hline $\begin{array}{l}\text { Greenstead Green And Halstead Rural, Essex } \\
\text { (RU) }\end{array}$ & Bucket/E0039 \\
\hline Ugley, Essex (RU) & Vessel(mount)/E0040 \\
\hline Birch, Essex (RU) & Vessel(mount)/E0041 \\
\hline Wix, Essex (RU) & Vessel(mount)/E0042 \\
\hline Great Bentley, Essex (RU) & Handled Pan 3/E0043 \\
\hline Steeple Bumpstead, Essex (RU) & Handled Pan 1/E0044 \\
\hline Broxted, Essex (RU) & Handled Pan 4/E0045 \\
\hline Roxwell, Essex (RU) & Vessel(fragment)/E0046 \\
\hline Manningtree, Essex (RU) & Bowl/EX0047 \\
\hline Elsenham, Essex (RU) & Vessel(mount)/E0048 \\
\hline Fordham, Essex (RU) & Vessel(mount)/E0049 \\
\hline Fingringhoe, Essex (RU) & Vessel(fragment)/E0050 \\
\hline Good Easter, Essex (RU) & Vessel(mount)/E0051 \\
\hline Ardleigh, Essex (RU) & Vessel(mount)/E0052 \\
\hline Beaulieu, Hampshire (RU) & Bucket/HAM0004 \\
\hline Medstead CP, Hampshire (RS) & Jug(lid)/HAM0005 \\
\hline Ropley, Hampshire (RS) & Handled Pan 1/HAM0006 \\
\hline King's Worthy, Hampshire (RU) & Vessel(fragment)/HAM0007 \\
\hline Broughton, Hampshire (RU) & Vessel(fragment)/HAM0008 \\
\hline Ropley, Hampshire (RS) & $\begin{array}{l}\text { Vessel(mount)/HAM0009 } \\
\text { Vessel(mount)/HAM0010 }\end{array}$ \\
\hline Damerham, Hampshire (RS) & Vessel(fragment)/HAM0011 \\
\hline Owslebury, Hampshire (RS) & $\begin{array}{l}\text { Vessel(fragment)/HAM0012 } \\
\text { Vessel(fragment)/HAM0013 }\end{array}$ \\
\hline Corhampton And Meonstoke, Hampshire (RU) & Vessel(fragment)/HAM0014 \\
\hline Wherwell, Hampshire (RU) & Vessel(fragment)/HAM0015 \\
\hline Newport, Isle of Wight (RS) & Vessel(fragment)/IOW0001 \\
\hline Newport, Isle of Wight (RS) & Vessel(mount)/IOW0002 \\
\hline Bembridge, Isle of Wight (RU) & Vessel(mount)/IOW0003 \\
\hline Lyminge, Kent (RU) & Vessel(mount)/K0029 \\
\hline Eynsford, Kent (RS) & Handled Pan 1/K0030 \\
\hline Lenham, Kent (RU) & Handled Pan 3/K0031 \\
\hline Chislet Upstreet, Kent (RU) & $\begin{array}{c}\text { Bowl/K0032 } \\
\text { Jug/K0033 }\end{array}$ \\
\hline Rainham, Greater London & Vessel(mount)/L0034 \\
\hline Brettenham, Norfolk (RS) & Vessel(mount)/NOR0005 \\
\hline
\end{tabular}




\begin{tabular}{|c|c|}
\hline Reepham, Norfolk (RU) & Handled Pan 2/NOR0006 \\
\hline Southrepps, Norfolk (RU) & Vessel(mount)/NOR0007 \\
\hline Attlebridge, Norfolk (RS) & $\begin{array}{c}\text { Jug(handle)/NOR0008 } \\
\text { Handled Pan 2/NOR0022 }\end{array}$ \\
\hline Shouldham, Norfolk (RS) & Vessel(mount)/NOR0009 \\
\hline Cawston, Norfolk (RS) & Vessel(mount)/NOR0010 \\
\hline Beachamwell, Norfolk (RU) & Handled Pan 2/NOR0011 \\
\hline Fincham, Norfolk (RU) & Vessel(fragment)/NOR0012 \\
\hline Beeston with Bittering, Norfolk (RS) & $\begin{array}{c}\text { Vessel(fragment)/NOR0013 } \\
\text { Jug/NOR0018 } \\
\text { Jug(handle)/NOR0019 } \\
\text { Vessel(fragment)/NOR0020 }\end{array}$ \\
\hline Feltwell, Norfolk (RS) & Jug/NOR0014 \\
\hline Ringstead, Norfolk (RS) & Vessel(mount)/NOR0015 \\
\hline Hockwold cum Wilton, Norfolk (RS) & $\begin{array}{c}\text { Jug/NOR0016 } \\
\text { Vessel(mount)/NOR0025 }\end{array}$ \\
\hline East Walton, Norfolk (RS) & Handled Pan 2/NOR0017 \\
\hline Narford, Norfolk (RU) & Vessel(mount)/NOR0021 \\
\hline Kenninghall, Norfolk (RU) & Handled Pan 1/NOR0023 \\
\hline Tacolneston, Norfolk (RU) & Handled Pan 2/NOR0024 \\
\hline Aldeby, Norfolk (RS) & Vessel(mount)/NOR0026 \\
\hline Stanfield, Norfolk (RU) & Vessel(mount)/NOR0027 \\
\hline Themelthorpe, Norfolk (RU) & Jug(handle)/NOR0028 \\
\hline Colkirk, Norfolk (RU) & Jug(handle)/NOR0029 \\
\hline Gunthorpe, Norfolk (RU) & Handled Pan 2/NOR0043 \\
\hline Pettistree, Suffolk (RU) & Jug(handle)/SUF0006 \\
\hline Kettlebaston, Suffolk (RU) & Vessel(fragment)/SUF0007 \\
\hline Hacheston, Suffolk (RS) & Vessel(mount)/SUF0008 \\
\hline Combs, Suffolk (RU) & $\begin{array}{c}\text { Handled Pan 2/SUF0009 } \\
\text { Handled Pan 2/SUF0013 } \\
\text { Vessel(fragment)/SUF0032 }\end{array}$ \\
\hline Hoxne, Suffolk (RU) & Handled Pan 2/SUF0010 \\
\hline West Stow, Suffolk (RU) & Vessel(mount)/SUF0011 \\
\hline Barking, Suffolk (RS) & Vessel(fragment)/SUF0012 \\
\hline Arwarton, Suffolk (RU) & Vessel(mount)/SUF0014 \\
\hline Chediston, Suffolk (RU) & Handled Pan 2/SUF0015 \\
\hline Lowestoft, Suffolk (RS) & Jug(handle)/SUF0016 \\
\hline Mendham, Suffolk (RU) & Vessel(mount)/SUF0017 \\
\hline Freckenham, Suffolk (RU) & Jug(lid)/SUF0018 \\
\hline Mildenhall, Suffolk (RU) & $\begin{array}{l}\text { Vessel(mount)/SUF0019 } \\
\text { Vessel(mount)/SUF0034 }\end{array}$ \\
\hline Brockley, Suffolk (RU) & Jug(handle)/SUF0020 \\
\hline Market Weston, Suffolk (RU) & Vessel(mount)/SUF0021 \\
\hline Hitcham, Suffolk (RS) & Vessel(mount)/SUF0022 \\
\hline Yaxley, Suffolk (RS) & Vessel(mount)/SUF0023 \\
\hline
\end{tabular}




\begin{tabular}{|c|c|}
\hline Linstead Magna, Suffolk (RU) & Vessel(mount)/SUF0024 \\
\hline Horringer, Suffolk (RU) & Vessel(mount)/SUF0025 \\
\hline Great Glemham, Suffolk (RU) & Vessel(fragment)/SUF0026 \\
\hline Wattisham, Suffolk (RU) & Vessel(fragment)/SUF0027 \\
\hline Freckenham, Suffolk (RU) & Handled Pan 2/SUF0028 \\
\hline Bradfield Combust With Stanningfield, Suffolk & Jug(lid)/SUF0029 \\
\hline (RS) & Vessel(mount)/SUF0030 \\
\hline Otley, Suffolk (RU) & Jug(lid)/SUF0031 \\
\hline Bredfield, Suffolk (RU) & Jug/SUF0033 \\
\hline Sutton, Suffolk (RU) & Handled Pan 2/SUF0035 \\
\hline Stoke Ash, Suffolk (RS) & Vessel(mount)/SUF0036 \\
\hline Bury St. Edmunds, Suffolk (RS) & Vessel(fragment)/SUR0011 \\
\hline Charlwood, Surrey (RU) & Vessel(mount)/WSU0001 \\
\hline Eartham, West Sussex (RU) & \\
\hline
\end{tabular}


Appendix XI: Finds associated with Copper Alloy Vessels of Roman date reported through the Portable Antiquities Scheme.

\begin{tabular}{|c|c|c|c|c|c|c|c|c|c|c|}
\hline 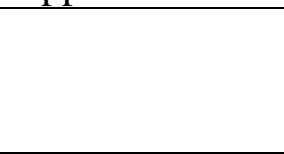 & Coins & Brooch/PO & Mounts & Weights & $\begin{array}{l}\text { Spindle } \\
\text { Whorls }\end{array}$ & $\begin{array}{l}\text { Ceramic } \\
\text { Vessels }\end{array}$ & $\begin{array}{l}\text { Glass } \\
\text { Vessels }\end{array}$ & $\begin{array}{l}\text { Lead- } \\
\text { Alloy } \\
\text { Vessels }\end{array}$ & $\begin{array}{l}\text { Architectural } \\
\text { Remains }\end{array}$ & Misc. \\
\hline $\begin{array}{l}\text { Steeple } \\
\text { Bumpstead } \\
\text { (Essex) }\end{array}$ & 205 & 29 & 13 & 1 & 0 & 1 & 0 & 1 & 1 & 4 \\
\hline $\begin{array}{l}\text { Good Easter } \\
\text { (Essex) }\end{array}$ & 46 & 61 & 15 & 28 & 2 & 11 & 0 & 0 & 0 & 40 \\
\hline $\begin{array}{l}\text { Ardleigh } \\
\text { (Essex) }\end{array}$ & 93 & 30 & 0 & 0 & 0 & 0 & 0 & 0 & 0 & 4 \\
\hline $\begin{array}{l}\text { Fridaythorpe } \\
\text { (East Riding of } \\
\text { Yorkshire) }\end{array}$ & 136 & 51 & 3 & 3 & 0 & 7 & 0 & 0 & 0 & 10 \\
\hline $\begin{array}{l}\text { Shipton Thorpe } \\
\text { (East Riding of } \\
\text { Yorkshire) }\end{array}$ & 343 & 6 & 2 & 0 & 0 & 0 & 0 & 0 & 0 & 0 \\
\hline $\begin{array}{l}\text { Ropley } \\
\text { (Hampshire) }\end{array}$ & 115 & 7 & 1 & 1 & 0 & 0 & 0 & 0 & 0 & 2 \\
\hline $\begin{array}{l}\text { Wherwell } \\
\text { (Hampshire) }\end{array}$ & 122 & 6 & 4 & 1 & 0 & 1 & 0 & 0 & 1 & 3 \\
\hline $\begin{array}{l}\text { Clothall } \\
\text { (Hertfordshire) }\end{array}$ & 82 & 21 & 0 & 9 & 0 & 1 & 2 & 0 & 0 & 14 \\
\hline $\begin{array}{l}\text { Watton-at- } \\
\text { Stone } \\
\text { (Hertfordshire) }\end{array}$ & 125 & 7 & 2 & 6 & 0 & 0 & 0 & 0 & 0 & 8 \\
\hline $\begin{array}{l}\text { Gaddesby } \\
\text { (Leicestershire) }\end{array}$ & 110 & 28 & 2 & 0 & 0 & 0 & 0 & 0 & 0 & 3 \\
\hline $\begin{array}{l}\text { Ancaster } \\
\text { (Lincolnshire) }\end{array}$ & 186 & 12 & 1 & 1 & 0 & 0 & 0 & 0 & 0 & 4 \\
\hline $\begin{array}{l}\text { Thonock } \\
\text { (Lincolnshire) }\end{array}$ & 560 & 112 & 28 & 3 & 0 & 1 & 0 & 0 & 0 & 38 \\
\hline $\begin{array}{l}\text { Keelby } \\
\text { (Lincolnshire) }\end{array}$ & 133 & 29 & 0 & 1 & 0 & 2 & 0 & 0 & 0 & 9 \\
\hline $\begin{array}{l}\text { Wickenby } \\
\text { (Lincolnshire) }\end{array}$ & 54 & 49 & 6 & 2 & 1 & 53 & 0 & 0 & 3 & 10 \\
\hline
\end{tabular}




\begin{tabular}{|l|l|l|l|l|l|l|l|l|l|l|}
\hline $\begin{array}{l}\text { Stainton by } \\
\text { Langworth } \\
\text { (Lincolnshire) }\end{array}$ & 104 & 8 & 1 & 0 & 4 & 0 & 0 & 0 & 0 & 0 \\
\hline $\begin{array}{l}\text { Shouldham } \\
\text { (Norfolk) }\end{array}$ & 793 & 21 & 2 & 0 & 0 & 0 & 0 & 0 & 0 & \\
\hline $\begin{array}{l}\text { Beeston with } \\
\text { Bittering } \\
\text { (Norfolk) }\end{array}$ & 155 & 46 & 11 & 4 & 0 & 2 & 0 & 0 & 0 & 6 \\
\hline $\begin{array}{l}\text { Letcombe } \\
\text { Regis (Oxford) }\end{array}$ & 94 & 21 & 6 & 5 & 0 & 7 & 0 & 0 & 0 & 23 \\
\hline $\begin{array}{l}\text { West Stow } \\
\text { (Suffolk) }\end{array}$ & 883 & 23 & 1 & 1 & 0 & 12 & 0 & 0 & 0 & 5 \\
\hline $\begin{array}{l}\text { Barking } \\
\text { (Suffolk) }\end{array}$ & 347 & 25 & 3 & 2 & 0 & 15 & 0 & 0 & 1 & 4 \\
\hline Otley (Suffolk) & 61 & 54 & 0 & 3 & 0 & 13 & 3 & 0 & 0 & 10 \\
\hline $\begin{array}{l}\text { Sutton } \\
\text { (Suffolk) }\end{array}$ & 323 & 36 & 0 & 1 & 0 & 0 & 0 & 0 & 0 & 21 \\
\hline $\begin{array}{l}\text { Brockley } \\
\text { (Suffolk) }\end{array}$ & 58 & 22 & 0 & 4 & 2 & 31 & 0 & 0 & 0 & 13 \\
\hline $\begin{array}{l}\text { Hitcham } \\
\text { (Suffolk) }\end{array}$ & 45 & 22 & 5 & 4 & 0 & 6 & 0 & 0 & 2 & 4 \\
\hline $\begin{array}{l}\text { Alcester } \\
\text { (Warwickshire) }\end{array}$ & 153 & 47 & 0 & 2 & 0 & 3 & 0 & 0 & 0 & 23 \\
\hline $\begin{array}{l}\text { Eartham (West } \\
\text { Sussex) }\end{array}$ & 217 & 42 & 2 & 4 & 3 & 1 & 0 & 0 & 0 & 13 \\
\hline
\end{tabular}


Appendix XII: Inscriptions on Copper Alloy Vessels from Roman Britain.

\begin{tabular}{|c|c|c|c|c|c|c|}
\hline Number & Form (type) & Site (County) & Context (Site Type) & Body Inscription & Handle Inscription & Reference \\
\hline AV0002 & $\begin{array}{l}\text { Handled Pan } 2 \\
\text { (Rudge Cup Type) }\end{array}$ & Bath (Avon) & SD (RS) & & $\begin{array}{l}\text { "DIISVM[...]/CODON[...]" De(ae) } \\
\text { Su(li) M[in(ervae)] Codon[...] }\end{array}$ & $\begin{array}{l}\text { Cunliffe 1988, 14- } \\
16 \text { (23); Hassall \& } \\
\text { Tomlin 1981, 381 } \\
\text { (20); RIB II } \\
2415.60\end{array}$ \\
\hline BE0004 & $\begin{array}{l}\text { Handled Pan } 2 \\
\text { (Eggers 137-138) }\end{array}$ & Shefford (Bedfordshire) & GD (RS) & & $\mathrm{t}$ "[...]ESPYV" & $\begin{array}{l}\text { Eggers 1968 104- } \\
105 \text { (29Aa); RIB II } \\
2415.51\end{array}$ \\
\hline BUC0006 & Bowl (Irchester) & $\begin{array}{l}\begin{array}{l}\text { Amersham } \\
\text { (Buckinghamshire) }\end{array} \\
\end{array}$ & SD (RS) & "X"; sideways "A" & & $\begin{array}{l}\text { Farley et al. 1988, } \\
359-366 \text { (1) }\end{array}$ \\
\hline CAM0009 & $\begin{array}{l}\text { Handled Pan } 2 \\
\text { (Eggers 131-133) }\end{array}$ & Ely (Cambridgeshire) & $\mathrm{SF}(\mathrm{RU})$ & & "BODVOGENVS F" Bodvogenus f(ecit) & $\begin{array}{l}\text { Smith, } 192285 ; \\
\text { RIB II 2415.11 } \\
\end{array}$ \\
\hline CHE0003 & $\begin{array}{l}\text { Handled Pan } 2 \\
\text { (Eggers 139-144) }\end{array}$ & Chester (Chesire) & $\mathrm{SF}(\mathrm{M})$ & & "CIPI POLI[...]" Cipi Poly (bi) & $\begin{array}{l}\text { McPeake \& Moore } \\
\text { 1978, } 331 \text { (11); RIB } \\
\text { II } 2415.25\end{array}$ \\
\hline $\mathrm{C} 0025$ & $\begin{array}{l}\text { Handled Pan } 2 \\
\text { (Eggers 139-144) }\end{array}$ & Carlisle (Cumbria) & $\mathrm{SF}(\mathrm{M})$ & & "ANSI. DIODORI[...]" & $\begin{array}{l}\text { Bennet \& Young } \\
\text { 1981, } 44 \text { (45); } \\
\text { McCarthy 1990, } \\
135 \text { (97) }\end{array}$ \\
\hline $\mathrm{C} 0026$ & $\begin{array}{l}\text { Handled Pan } 2 \\
\text { (Eggers 139-144) }\end{array}$ & Carlisle (Cumbria) & SF (M) & & ".C.I.A[ ...]" & $\begin{array}{l}\text { Bennet \& Young } \\
\text { 1981, } 44 \text { (46); } \\
\text { McCarthy 1990, } \\
135 \text { (98) }\end{array}$ \\
\hline $\mathrm{C} 0027$ & $\begin{array}{l}\text { Handled Pan } 2 \\
\text { (Eggers 139-144) }\end{array}$ & Watercrook (Cumbria) & SF (M) & & 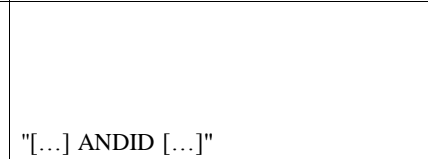 & $\begin{array}{l}\text { Potter 1979, } 215 \\
\text { (48); Bennet \& } \\
\text { Young 1981, } 44 \\
\text { (44); RIB II } \\
2415.13\end{array}$ \\
\hline $\mathrm{C} 0029$ & $\begin{array}{l}\text { Handled Pan } 2 \\
\text { (Eggers 140) }\end{array}$ & Clifton (Cumbria) & SD (RS) & & "TALIO F" Talio f(ecit) & RIB II 2415.47 \\
\hline DEN0001 & $\begin{array}{l}\text { Handled Pan } 2 \\
\text { (Eggers 131) }\end{array}$ & Glyn Dyfrdwy (Denbighshire) & $\mathrm{SD}(\mathrm{RU})$ & & $\begin{array}{l}\text { "S MERCV" [Sor]s Mercur[i] ; } \\
\text { "CIPINICOMA" P(ubli) Cipi } \\
\text { Nicoma[chi] }\end{array}$ & $\begin{array}{l}\text { Gardner 1927, 129- } \\
\text { 140; Eggers 1968 } \\
\text { 104 (19a); McPeake } \\
\text { \& Moore 1978, } 333 \\
\text { (9); RIB II 2415.16 }\end{array}$ \\
\hline DEN0002 & $\begin{array}{l}\text { Handled Pan } 2 \\
\text { (Eggers 137-138) }\end{array}$ & Glyn Dyfrdwy (Denbighshire) & SD (RU) & & "A[...]VIT" & $\begin{array}{l}\text { Gardner 1927, 129- } \\
\text { 140; Eggers 1968 } \\
\text { 104 (19b); RIB II } \\
2415.10\end{array}$ \\
\hline DEN0003 & $\begin{array}{l}\text { Handled Pan } 2 \\
\text { (Eggers 146) }\end{array}$ & Glyn Dyfrdwy (Denbighshire) & $\mathrm{SD}(\mathrm{RU})$ & & 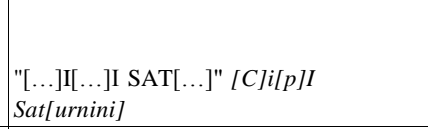 & $\begin{array}{l}\text { Gardner 1927, 129- } \\
\text { 140; Eggers 1968 } \\
\text { 104 (19c); RIB II } \\
2415.28\end{array}$ \\
\hline DUR0005 & Bowl & South Shields (Durham) & $\mathrm{SD}(\mathrm{M})$ & $\begin{array}{l}\text { "M A SAB APOLLINI ANEXTIOMAROM" } \\
\text { Apollini Anextiomaro M(arcus) A(...) } \\
\text { Sab(inus?) }\end{array}$ & & $\begin{array}{l}\text { Henig 1984, 132- } \\
\text { 133; RIB II 2415.55 }\end{array}$ \\
\hline DUR0006 & $\begin{array}{l}\text { Handled Pan } 2 \\
\text { (Eggers 142) }\end{array}$ & Upper Weardale (Durham) & $\mathrm{SD}(\mathrm{RU})$ & & "P. CIPEPOLI" P(ubli) Cipi Poli(bi) & $\begin{array}{l}\text { Egglestone 1915, 9- } \\
11 ; \text { Bennett \& } \\
\text { Young 1981, } 43 \\
\text { (41); RIB II } \\
2415.20\end{array}$ \\
\hline
\end{tabular}

LXXXVII 


\begin{tabular}{|c|c|c|c|c|c|c|}
\hline DUR0007 & $\begin{array}{l}\text { Handled Pan } 2 \\
\text { (Eggers 142) }\end{array}$ & Upper Weardale (Durham) & $\mathrm{SD}(\mathrm{RU})$ & "LICINIANI" & "POLYBI.M" Polybi m(anu) & $\begin{array}{l}\text { Egglestone 1915, 9- } \\
11 ; \text { Bennet \& } \\
\text { Young 1981, 43 } \\
\text { (42); RIB II } \\
2415.27\end{array}$ \\
\hline EX0024 & $\begin{array}{l}\text { Handled Pan } 2 \\
\text { (Eggers 196) }\end{array}$ & Colchester (Essex) & $\mathrm{SF}(\mathrm{U})$ & & "PCIPI.POLYBI" P(ubli) Cipi Polybi & $\begin{array}{l}\text { Eggers 1968, 106 } \\
\text { (37c); McPeake \& } \\
\text { Moore 1978, 333 } \\
\text { (13); RIB II } \\
2415.23\end{array}$ \\
\hline EX0027 & $\begin{array}{l}\text { Handled Pan } 2 \\
\text { (Eggers 140) }\end{array}$ & Colchester (Essex) & $\mathrm{SF}(\mathrm{U})$ & & "TVGIM" Tugi m(anu) & $\begin{array}{l}\text { Eggers 1968, } 106 \\
\text { (37h); Wright 1944, } \\
89 \text { (12); RIB II } \\
2415.50\end{array}$ \\
\hline EX0037 & $\begin{array}{l}\text { Handled Pan } 2 \\
\text { (Eggers 136) }\end{array}$ & Colchester (Essex) & $\mathrm{SF}(\mathrm{U})$ & & "T.POMP.NIC" & $\begin{array}{l}\text { Smith 1922, 85; } \\
\text { RIB II } 2415.48\end{array}$ \\
\hline GWY0009 & $\begin{array}{l}\text { Handled Pan } 2 \\
\text { (Eggers 142) }\end{array}$ & Llanberis (Gwynedd) & $\mathrm{SD}(\mathrm{U})$ & & "ABVCCV[...]" Аbuccu[s f(ecit)] & $\begin{array}{l}\text { Eggers 1968, 104 } \\
(21) ; \text { McPeake \& } \\
\text { Moore 1978, } 333 \\
\text { (1); RIB II 2415.1 }\end{array}$ \\
\hline GWY0013 & $\begin{array}{l}\text { Handled Pan } 2 \\
\text { (Eggers 142) }\end{array}$ & Caernarvon (Gwynedd) & $\mathrm{SF}(\mathrm{M})$ & & $\begin{array}{l}\text { "SAGAVGVF" Sag(untinus?) } \\
\text { Augu(stinus?) f(ecit) }\end{array}$ & $\begin{array}{l}\text { Eggers 1968, } 104 \\
\text { (22a); Wright 1969, } \\
238 \text { (20); RIB II } \\
2415.45\end{array}$ \\
\hline HАM0001 & $\begin{array}{l}\text { Handled Pan } 2 \\
\text { (Eggers 140) }\end{array}$ & Silchester (Hampshire) & SF (U) & & "PIAV" & $\begin{array}{l}\text { Eggers 1966, } 102 \\
\text { (6a); RIB II } 2415.37\end{array}$ \\
\hline K0020 & Bowl (Eggers 99) & Luton (Kent) & GD (RS) & "AFRICANVS" & & $\begin{array}{l}\text { Eggers 1966, } 102 \\
\text { 3a; BM 1894. } 83 . \\
\text { 58; Jessup, } 195827 \\
\text { 28; McPeake \& } \\
\text { Moore 1978, 333 } \\
\text { (2); RIB II 2415.2 }\end{array}$ \\
\hline LAN0004 & $\begin{array}{l}\text { Handled Pan } 2 \\
\text { (Eggers 139-144) }\end{array}$ & Ribchester (Lancashire) & $\mathrm{SD}(\mathrm{M})$ & & "CONP[...]" & $\begin{array}{l}\text { McPeake \& Moore } \\
\text { 1978, } 334 \text { (18); } \\
\text { Jackson \& } \\
\text { Craddock 1995, } 87 \\
\text { (24 \&25); RIB II } \\
2415.31\end{array}$ \\
\hline LIN0016 & $\begin{array}{l}\text { Handled Pan } 2 \\
\text { (Eggers 139-144) }\end{array}$ & Keelby (Lincolnshire) & PAS (RU) & & $"(\ldots) \mathrm{VG}^{\prime}$ & PAS LIN-3EED71 \\
\hline LIN0031 & $\begin{array}{l}\text { Handled Pan } 2 \\
\text { (Eggers 139-144) }\end{array}$ & Kirkstead (Lincolnshire) & $\mathrm{SD}(\mathrm{RU})$ & & "C.ARAT" & $\begin{array}{l}\text { McPekae \& Moore } \\
\text { 1978, } 333 \text { (6); RIB } \\
\text { II 2415.12 }\end{array}$ \\
\hline LIN0032 & $\begin{array}{l}\text { Handled Pan } 2 \\
\text { (Eggers 139-144) }\end{array}$ & Fiskerton (Lincolnshire) & SD (RS) & & "FLORVS F" Florus f(ecit) & $\begin{array}{l}\text { McPeake \& Moore } \\
\text { 1978, } 334 \text { (26); RIB } \\
\text { II 2415.35 }\end{array}$ \\
\hline LIN0033 & $\begin{array}{l}\text { Handled Pan } 2 \\
\text { (Eggers 139-144) }\end{array}$ & Humberside (Lincolnshire) & $\mathrm{SF}(\mathrm{RS})$ & & "[ ...]IAPPIA" & $\begin{array}{l}\text { Hassall \& Tomlin } \\
\text { 1984, } 345 \text { (49); } \\
\text { RIB II 2415.36 }\end{array}$ \\
\hline
\end{tabular}




\begin{tabular}{|c|c|c|c|c|c|c|}
\hline LIN0034 & $\begin{array}{l}\text { Handled Pan } 2 \\
\text { (Eggers 139-144) }\end{array}$ & Louth (Lincolnshire) & $\mathrm{SF}(\mathrm{RU})$ & & $\begin{array}{l}\text { "MLNFEC" M(arcus) } L(\ldots) N(\ldots) \text { fec(it); } \\
\text { "C CLSENIORIS ANNI" (centuria) } \\
\text { C(audii) Senioris Ann(i)i }\end{array}$ & $\begin{array}{l}\text { Wright 1964, } 180 \\
\text { (16); RIB II } \\
2415.41 \\
\end{array}$ \\
\hline LIN0035 & $\begin{array}{l}\text { Handled Pan } 2 \\
\text { (Eggers 146-147) }\end{array}$ & Lincoln (Lincolnshire) & $\mathrm{SF}(\mathrm{U})$ & & "MLN [...]"; "[...] C. A. S. DC" & $\begin{array}{l}\text { Bennett \& Young } \\
\text { 1981, } 44 \text { (43); RIB } \\
\text { II 2415.42 }\end{array}$ \\
\hline LIN0036 & $\begin{array}{l}\text { Handled Pan } 2 \\
\text { (Eggers 139-144) }\end{array}$ & Normanby (Lincolnshire) & $\mathrm{SF}(\mathrm{RU})$ & & "ALPRI" A(e)li Pri( ...) & $\begin{array}{l}\text { Wright \& Hassall } \\
\text { 1971, } 299 \text { (60); RIB } \\
\text { II 2415.57 } \\
\end{array}$ \\
\hline L0024 & $\begin{array}{l}\text { Handled Pan } 2 \\
\text { (Eggers 142-144) }\end{array}$ & Cit of London (London) & $\mathrm{SF}(\mathrm{U})$ & & 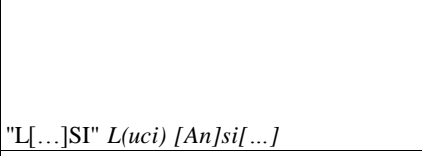 & $\begin{array}{l}\text { Eggers 1966, } 100 \\
\text { 1b; McPeake \& } \\
\text { Moore 1978, 334 } \\
\text { (25); RIB II 2415.8 }\end{array}$ \\
\hline L0025 & $\begin{array}{l}\text { Handled Pan } 2 \\
\text { (Eggers 141) }\end{array}$ & Bermondsey (London) & $\mathrm{SF}(\mathrm{U})$ & "TRVFC" & & $\begin{array}{l}\text { Eggers 1966, } 101 \\
\text { 1c; McPeake \& } \\
\text { Moore 1978, 334 } \\
\text { (33); RIB II } \\
2415.49\end{array}$ \\
\hline L0031 & $\begin{array}{l}\text { Vessel (Eggers 160- } \\
161 \text { ) }\end{array}$ & City of London (London) & $\mathrm{SF}(\mathrm{U})$ & & "[...] NDINVSF" [...]ndinus f(ecit) & $\begin{array}{l}\text { Eggers 1966, } 101 \\
\text { 1s; Wrght 1969b, } \\
239 \text { (22); McPeake } \\
\& \text { Moore 1978, } 334 \\
\text { (38); RIB II } \\
2415.15\end{array}$ \\
\hline L0056 & Jar & City of London (London) & $\mathrm{SF}(\mathrm{U})$ & "EX OF COR" ex off(ficina) Cor(...) & & \begin{tabular}{|l|} 
Hassall \& Tomlin \\
1984, 344 (35); \\
RIB II 2415.33 \\
\end{tabular} \\
\hline L0057 & $\begin{array}{l}\text { Handled Pan } 2 \\
\text { (Eggers 139-144) }\end{array}$ & City of London (London) & $\mathrm{SF}(\mathrm{U})$ & & "SANGVSF" Sangus f(ecit) & $\begin{array}{l}\text { Wtight 1969a, } 5 \text { (6); } \\
\text { Wright 1969b, 239 } \\
\text { (21); McPeake \& } \\
\text { Moore 1978, } 334 \\
(36) ; \text { RIB II } \\
2415.46\end{array}$ \\
\hline NE0014 & $\begin{array}{l}\text { Handled Pan } 2 \\
\text { (Eggers 144) }\end{array}$ & Caerleon (Newport) & $\mathrm{SF}(\mathrm{M})$ & "LVCCA" & $\begin{array}{l}\text { "MATVRVS F" Maturus f(ecit) ; "ALA I } \\
\text { TH" Ala I Th(racum); "LI" (51) }\end{array}$ & $\begin{array}{l}\text { Hassall \& Tomlin } \\
\text { 1985, 330-331 (41); } \\
\text { RIB II 2415.39 }\end{array}$ \\
\hline NOR0041 & $\begin{array}{l}\text { Handled Pan } 2 \\
\text { (Eggers 139-144) }\end{array}$ & Saham Toney (Norfolk) & $\mathrm{SF}(\mathrm{M})$ & & "C PRIMI" (centuria) Primi & $\begin{array}{l}\text { Hassall \& Tomlin } \\
\text { 1978, } 480 \text { (61); RIB } \\
\text { II } 2415.59\end{array}$ \\
\hline NOR0043 & $\begin{array}{l}\text { Handled Pan } 2 \\
\text { (Rudge Cup Type) }\end{array}$ & Gunthorpe (Norfolk) & PAS (RU) & & "BEBE SESE" & $\begin{array}{l}\text { PAS NMS-7BC635; } \\
\text { Worrell 2012, 73- } \\
74\end{array}$ \\
\hline NOT0001 & $\begin{array}{l}\text { Handled Pan } 2 \\
\text { (Eggers 144) }\end{array}$ & Broxtowe (Nottinghamshire) & $\mathrm{SF}(\mathrm{M})$ & & "ALBANVS" & $\begin{array}{l}\text { Oswald 1939, 441; } \\
\text { Eggers 1968, 110 } \\
\text { (87); McPeake \& } \\
\text { Moore 1978, } 333 \\
\text { (3); RIB II 2415.3 }\end{array}$ \\
\hline
\end{tabular}




\begin{tabular}{|c|c|c|c|c|c|c|}
\hline NU0012 & $\begin{array}{l}\text { Handled Pan } 2 \\
\text { (Eggers 139-144) }\end{array}$ & Corbridge (Northumberland) & $\mathrm{SF}(\mathrm{M})$ & & $\begin{array}{l}\text { "ANSIEPA[P]HR[ODITI]" Ansi } \\
\text { Epa[p]hr[oditi] }\end{array}$ & $\begin{array}{l}\text { Eggers 1968, } 108 \\
62 \text {; Wright \& } \\
\text { Hassal 1991, } 301 \\
\text { (76); RIB II 2415.6 }\end{array}$ \\
\hline NU0019 & $\begin{array}{l}\text { Handled Pan } 2 \\
\text { (Eggers 139) }\end{array}$ & Ponteland (Northumberland) & $\mathrm{SD}(\mathrm{RU})$ & & "DRACCIVS F" Draccius f(ecit) & $\begin{array}{l}\text { Eggers } 1968,108 \\
\text { (65a); McPeake \& } \\
\text { Moore 1978, } 334 \\
\text { (21); RIB II } \\
2415.32\end{array}$ \\
\hline NU0023 & Bowl (Eggers 70) & Ponteland (Northumberland) & $\mathrm{SD}(\mathrm{RU})$ & & $\begin{array}{l}\text { "T TTIRONIS CRIISCRII SNT } \\
\text { SENECIONIS" t(urma)Tironis } \\
\text { Crescresnt(is) ; "TKANDIANI VANNI" } \\
\text { t(urma) Kandiani Vanni }\end{array}$ & $\begin{array}{l}\text { Eggers 1968, } 108 \\
\text { (65e); RIB II } \\
2415.63\end{array}$ \\
\hline NU0047 & $\begin{array}{l}\text { Handled Pan } 2 \\
\text { (Eggers 139-144) }\end{array}$ & $\begin{array}{l}\text { Bardon Mill } \\
\text { (Northumberland) }\end{array}$ & $\mathrm{SF}(\mathrm{M})$ & & "[...] ERIORIS" & $\begin{array}{l}\text { Burnham et al. } \\
2005,492\end{array}$ \\
\hline NU0048 & $\begin{array}{l}\text { Handled Pan } 2 \\
\text { (Eggers 139-144) }\end{array}$ & $\begin{array}{l}\text { Bardon Mill } \\
\text { (Northumberland) }\end{array}$ & $\mathrm{SF}(\mathrm{M})$ & & "BRO" & $\begin{array}{l}\text { Burnham et al. } \\
2005,492\end{array}$ \\
\hline NU0050 & $\begin{array}{l}\text { Handled Pan } 2 \\
\text { (Eggers 139-144) }\end{array}$ & Matfen (Northumberland) & $\mathrm{SD}(\mathrm{RU})$ & & $\begin{array}{l}\text { "SABINIANVS F" Sabinianus f(ecit); ; I } \\
\text { XXV" }\end{array}$ & $\begin{array}{l}\text { Wright 1969a, 1-5; } \\
\text { RIB II 2415.44 }\end{array}$ \\
\hline NU0051 & $\begin{array}{l}\text { Handled Pan } 2 \\
\text { (Eggers 139-144) }\end{array}$ & Matfen (Northumberland) & $\mathrm{SD}(\mathrm{RU})$ & & $\begin{array}{l}\text { "MAXMINVS F" Maxminus f(ecit); "I } \\
\text { XXIS" }\end{array}$ & $\begin{array}{l}\text { Wright 1969a, 1-5; } \\
\text { McPeake \& Moore } \\
\text { 1978, } 334 \text { (30); } \\
\text { RIB II 2415.40 }\end{array}$ \\
\hline NYR0029 & Jar (Eggers 40) & York (North Yorkshire) & $\mathrm{SF}(\mathrm{U})$ & $\begin{array}{l}\text { "C ATTISIIVIIRI" (centuria) Att(i)i Severi ; "C } \\
\text { APRILI" (centuria) Capril(i)i }\end{array}$ & & $\begin{array}{l}\text { Eggers 1968, } 107 \\
\text { (52a); RCH 1962, } \\
\text { 133 (143); RIB II } \\
2415.58\end{array}$ \\
\hline NYR0032 & $\begin{array}{l}\text { Handled Pan } 2 \\
\text { (Eggers 139-144) }\end{array}$ & Malton (North Yorkshire) & SF (RS) & & $\begin{array}{l}\text { "ALPICVSF" Alpicus f(ecit); } \\
\text { "LSERVENISVSVPER" L(ucius) } \\
\text { Servenius Super; "SVPERI" Superi }\end{array}$ & $\begin{array}{l}\text { Eggers 1968, } 107 \\
\text { (56b); McPeake \& } \\
\text { Moore 1978, } 333 \\
\text { (4); RIB II 2415.4 } \\
\end{array}$ \\
\hline NYR0037 & $\begin{array}{l}\text { Handled Pan } 2 \\
\text { (Rudge Cup Type) }\end{array}$ & $\begin{array}{l}\text { Beadlam Roman Villa (North } \\
\text { Yorkshire) }\end{array}$ & SD (RS) & "[...]I CITR" [Fel]icit $(e) r$ & & $\begin{array}{l}\text { Wright \& Hassall } \\
\text { 1973, 334 (38); } \\
\text { Neal 1996, 49 (23); } \\
\text { RIB II 2415.54 }\end{array}$ \\
\hline NYR0040 & $\begin{array}{l}\text { Handled Pan } 2 \\
\text { (Eggers 142) }\end{array}$ & Stittenham (North Yorkshire) & $\mathrm{SD}(\mathrm{RU})$ & & "P CIPI POLYIBI" P(ubli) Cipi Polyibi & $\begin{array}{l}\text { Eggers 1968, } 107 \\
(54 a) ; \quad \text { RIB II } \\
2415.26\end{array}$ \\
\hline NYR0041 & $\begin{array}{l}\text { Handled Pan } 2 \\
\text { (Eggers 142) }\end{array}$ & Stittenham (North Yorkshire) & $\mathrm{SD}(\mathrm{RU})$ & & "P CIPI POLIB" P(ubli) Cipi Polib $(i)$ & $\begin{array}{l}\text { Eggers 1968, } 107 \\
(54 b) ; \text { RIB II } \\
2415.19\end{array}$ \\
\hline PO0008 & $\begin{array}{l}\text { Handled Pan } 2 \\
\text { (Eggers 150) }\end{array}$ & Brecon (Powys) & $\mathrm{SF}(\mathrm{M})$ & & $\begin{array}{l}\text { "[...] RODITI" [L(uci) Ansi } \\
\text { Epaph]roditi }\end{array}$ & $\begin{array}{l}\text { Eggers 1968, 103 } \\
\text { (14); Wheeler 1928, } \\
\text { 107-111; RIB II } \\
2415.7\end{array}$ \\
\hline SH0011 & $\begin{array}{l}\text { Handled Pan } 2 \\
\text { (Eggers 137-138) }\end{array}$ & Wroxeter (Shropshire) & $\mathrm{SF}(\mathrm{U})$ & & "[..]ESRV[...]" & $\begin{array}{l}\text { Wright } 1958,152 \\
\text { (14); RIB II } \\
2415.52\end{array}$ \\
\hline STA0004 & $\begin{array}{l}\text { Handled Pan } 2 \\
\text { (Rudge Cup Type) }\end{array}$ & Ilam (Staffordshire) & PAS (RU) & $\begin{array}{l}\text { "MAISCOGGABATAUXELODUNUMCAM } \\
\text { MOGLANNARIGOREVALIAELIUSDRACO" }\end{array}$ & & $\begin{array}{l}\text { PAS WMID- } \\
\text { 3FE965; Jackson } \\
\text { 2012, 41-60 }\end{array}$ \\
\hline
\end{tabular}




\begin{tabular}{|c|c|c|c|c|c|c|}
\hline SUF0005 & $\begin{array}{l}\text { Handled Pan } 2 \\
\text { (Eggers 139) }\end{array}$ & $\begin{array}{l}\text { Somberleyton Ashby and } \\
\text { Herringfleet (Suffolk) }\end{array}$ & $\mathrm{SF}(\mathrm{RU})$ & & "QVATTENVSF" Quattenus f(ecit) & \begin{tabular}{|l|} 
Eggers 1968, 106 \\
(43); McPeake \& \\
Moore 1978, 334 \\
(32); RIB II \\
2415.43 \\
\end{tabular} \\
\hline SUF0015 & $\begin{array}{l}\text { Handled Pan } 2 \\
\text { (Eggers 140-142) }\end{array}$ & Chediston (Suffolk) & PAS (RU) & & "CIPIPOL[...]" & PAS NMS-7F1BE6 \\
\hline SUF0045 & $\begin{array}{l}\text { Handled Pan } 2 \\
\text { (Eggers 140-142) }\end{array}$ & Brandon (Suffolk) & $\mathrm{SD}(\mathrm{RU})$ & & "IVLEVS . F" Juleus f(ecit) & $\begin{array}{l}\text { McPeake \& Moore } \\
1978,334(27) ; \\
\text { Grew } 1980,376 \\
\end{array}$ \\
\hline WAR0007 & $\begin{array}{l}\text { Handled Pan } 2 \\
\text { (Eggers 139-144) }\end{array}$ & Churchover (Warwickshire) & $\mathrm{SF}(\mathrm{RU})$ & & "MATVRVS F" Maturus f(ecit) & $\begin{array}{l}\text { Wright R. 1961, } \\
195 \text { (17); Mcpeake } \\
\& \text { Moore 1978, } 334 \\
\text { (29); RIB II } \\
2415.38\end{array}$ \\
\hline WIL0006 & $\begin{array}{l}\text { Handled Pan } 2 \\
\text { (Eggers 140-142) }\end{array}$ & $\begin{array}{l}\begin{array}{l}\text { Kingston Deverill } \\
\text { (Wilthshire) }\end{array} \\
\end{array}$ & SD (RS) & & "CIPI.POLIBI" & PAS WILT-92B052 \\
\hline WIL0023 & Jug (Eggers 125) & Roundway Hill (Wilthsire) & $\mathrm{SD}(\mathrm{RU})$ & "ASPAE[...]" & & \begin{tabular}{|l|} 
Taylor \& \\
Collingwood 1923, \\
283 (12); RIB II \\
2415.8 \\
\end{tabular} \\
\hline WIL0024 & $\begin{array}{l}\text { Handled Pan } 2 \\
\text { (Rudge Cup Type) }\end{array}$ & Rudge (Wiltshire) & $\mathrm{SF}(\mathrm{RU})$ & $\begin{array}{l}\text { ".A.MAISABALLAVAVXELODVMCAMBO } \\
\text { GLANSBANNA" A Mais Aballava } \\
\text { Uxelod(un)um Camboglan(ni)s Banna }\end{array}$ & & $\begin{array}{l}\text { Allason-Jones 2012, } \\
\text { 23-36; RIB II } \\
2415.53\end{array}$ \\
\hline
\end{tabular}


Associated finds table:

Object:

(F)- indicates fragment

Amphora- Am

Animal remains- A

Art- Ar

Bell- B1

Bottle- Bo

Bowl- B

Box/Casket/Chest- Bc

Bucket- Bu

Cauldron- $\mathrm{Ca}$

Coin- $\mathrm{Cn}$

Cosmetic Object- Co

Cup- C

Flagon/Flask- F

Food remains- Fr

Furniture- Fn

Game/Recreation objects: $G$

Hob Nails- HN

Jug/ Jar- J

Knife- Kn

Lamp- L

Mount/ Ring/ Fitting- M

Mortaria- Mo

Nail/ Fittings- N

Personal Adornment, Jewelry- Po

Rod- R

Spoon- S

Strigil- St

Stylus- Sty

Surgical Instruments- Si

Textiles- T

Unidentified- Un

Vessel/Tableware- V

Weapon/Weapon Fitting- Wf
Material:

Bone: [b]

Ceramic: [c]

Copper-alloy: [ca]

Glass: [gl]

Gold: [g]

Iron/Ferrous: [f]

Leather: [1]

Lead-alloy: [la]

Pewter: [p]

Silver: [s]

Stone: [st]

Wood: [w]

Unidentified in-organic: [ui]

Unidentified organic: [uo]

Various: [v] 